\title{
National Symposium
}

on

\section{Developments in \\ Irradiation Testing \\ Technology}

held at Sandusky, Ohio

September 9-11, 1969

\section{EXPORT CONTROLLED INFORMATION}

Contains technical data whose export is restricted by statute. Violations may result in administrative, covil, or criminal penalties. Limit dissemination to U.S.

Department of Energy, DOE contractors, and DOE-cleared personnel, and to other

U.S. Government agencies The cognizant program manager must approve other

dissemination. This notice shall not be separated from the attached document.
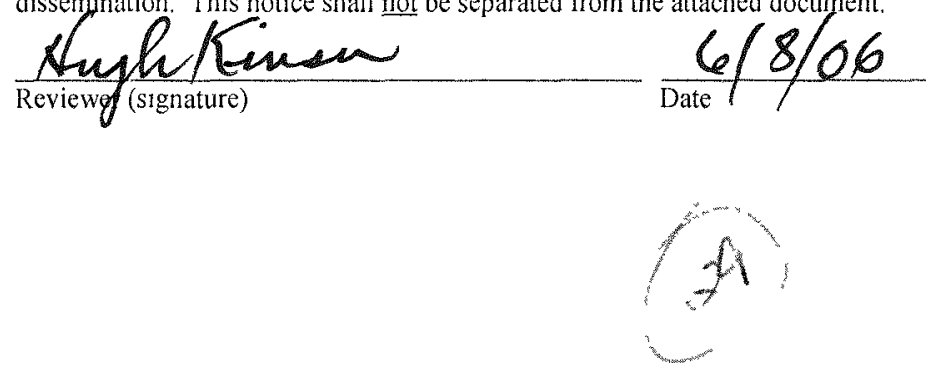

\section{United States}

Atomic Energy Commission

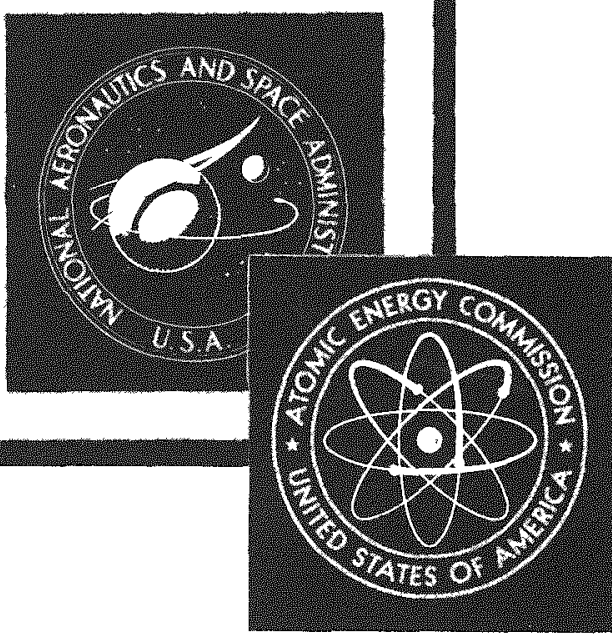




\section{LEGAL NOTICE}

This report was prepsred as an account of Government sponsored work. Neither the Unifed States, nor the Commission, nor any person acting on behalf of the Commission:

A. Makes any warranty or representation, expressed or implied, with respect to the accuracy, completeness, or usefulness of the information contained in this report, or that the use of any information, apparatus, method, or process disclosed in this report may not infringe privately owned rights; or

B. Assumes any liablitties with respect to the use of, or for damages resulting from the use of any information, apparatus, method, or process disclosed in this report.

As used in the above, "person acting on behalf of the Commission" includes any employee or contractor of the Commission, or employee of such contractor, to the extent that such employee or contractor of the Commission, or employee of such contractor prepares, disseminates, or provides access to, any information pursuant to his employment or contract with the Commission, or his employment with such contractor.

This report has been reproduced directly from the best available copy.

Printed in USA. Price $\$ 3.00$. Available from the Clearinghouse for Federal Scientific and Technical Information, National Bureau of Standards, U. S. Department of Commerce, Springfield, Virginia 22151. 


\section{DISCLAIMER}

This report was prepared as an account of work sponsored by an agency of the United States Government. Neither the United States Government nor any agency Thereof, nor any of their employees, makes any warranty, express or implied, or assumes any legal liability or responsibility for the accuracy, completeness, or usefulness of any information, apparatus, product, or process disclosed, or represents that its use would not infringe privately owned rights. Reference herein to any specific commercial product, process, or service by trade name, trademark, manufacturer, or otherwise does not necessarily constitute or imply its endorsement, recommendation, or favoring by the United States Government or any agency thereof. The views and opinions of authors expressed herein do not necessarily state or reflect those of the United States Government or any agency thereof. 


\section{DISCLAIMER}

Portions of this document may be illegible in electronic image products. Images are produced from the best available original document. 
CONF-690910

METALS, CERAMICS, AND

MATERIAIS

(TID-4500)

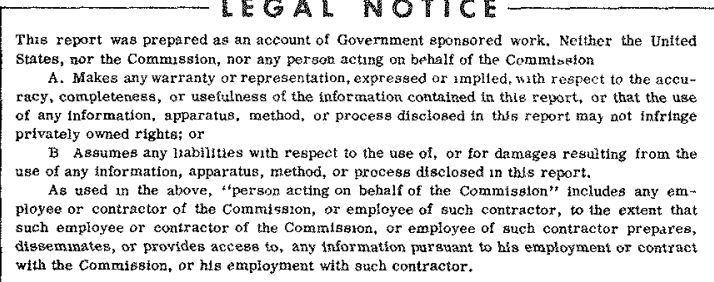

\title{
National Symposium
}

on

\section{Developments in}

Irradiation Testing

\section{Technology}

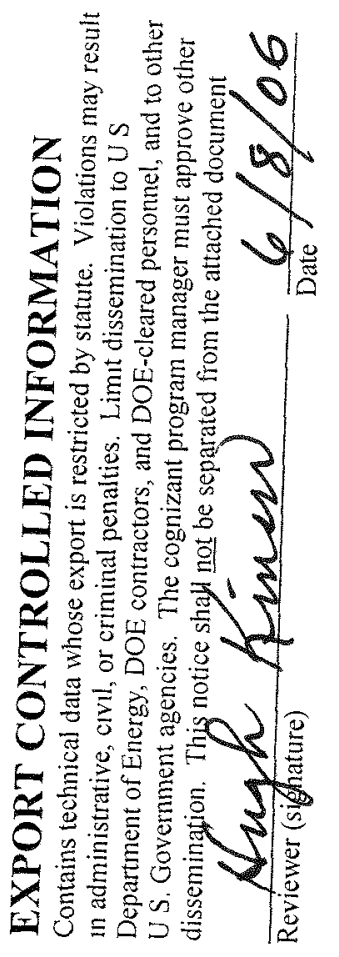

\author{
Sponsored by
}

U.S. ATOMIC ENERGY COMMISSION

and

NATIONAL AERONAUTICS

AND

SPACE ADMINISTRATION

on

September 9 through 11, 1969

NASA Lewis Research Center

Plum Brook Station

Sandusky, Ohio 44879 


\title{
SYMPOSIUM COMMITTEE
}

\author{
General Chairman ............ R. H. BrickZey \\ (NASA-LeRC) \\ Technical Program.......... S. Goldsmith \\ (BNW) \\ Finance.............. J. Crooks \\ (NASA-LeRC) \\ Facilities, Registration.......... J. Bonn \\ (NASA-LeRC) \\ Arrangements ............... . . Steinke \\ (NASA-LeRC)
}

Symposium Originated by

Fuels and Materials Branch Division of Reactor Development and Technology

Atomic Energy Commission

and

Reactor Division

Plum Brook Station

-

National Aeronautics and Space Administration 


\section{PREFACE}

This document contains the papers presented at the third symposium devoted to Irradiation Testing Technology. It records many of the developments which have occurred in this field since the previous two meetings which were held in October 1963 and May 1966.

Evaluation of the papers presented and the discussion held at this symposium, and their comparison with the papers of the previous two meetings suggests that the appreciable gains realized since 1963 may best be characterized as a shift in emphasis from the design and manufacture of irradiation capsules to developments which contribute to controlling and determining the irradiation environment. This period has also seen increased attention to continuous data acquisition methods and equipment. Another shift in emphasis worth noting has been from post-irradiation examination technique development to those methods which can be employed during interim examination. It is contemplated that fuller use of this latter approach will yield a more timely and accurate estimation of damage accumulation and rate effects.

The program of the symposium and a list of attendees is included as an appendix to this report. The meeting was a joint effort of the Atomic Energy Commission and the National Aeronautics and Space Agency and was held at the latter's Lewis Research Center's Plum Brook Station. Special credit for the success of the meeting is extended to the Symposium Committee of the hosting facility, S. Goldsmith of PNL, the technical chairman and to R. H. Brickley of NASA, the general chairman. 


\section{CONTENTS}

SESSION I

CAPSULE DESIGN AND EXPERIMENTAL TECHNIQUES FOR FAST REACTOR IRRADIATIONS

Influence of Neutron Spectrum on Helium Atom Generation in

Stainless Steels, J. Moteff . . . . . . . . . . . . . .

Fatigue Specimen Capsules, EBR-11 Nark B-7 Subassembly, M. W.

Ellingford, J. M. Beeston, and C. R. Brinkman ...........

Capsules and Sodium Loops Used in Argonne National Laboratory Reactor

Safety Experiments in the TREAT Reactor, I. E. Robinson, R. Ivins,

and C. E. Dickeman .....................

Use of Thermal TREAT Reactor for Fast Reactor Safety Studies, C. E. Dickeman, R. O. Ivins, A. B. Rothman, and J. F. Bolánd .......

Xenon Tagging of Instrumented Fuel Capsules for Use in EBR-11, D.W. Walker, P. J. Halfman, and J. H. Sanecki...............

Instrumented Subassembly System and In-Core Instrument Test Facility for EBR-11, E. Hutter, O. S. Seim, and A. Smaardyk. . . . . . . .

Characterization of Advanced Irradiation Testing Vehicles for EBR-11, O. S. Seim, W. R. Ware, and E. Hutter..............

Unrestrained Swelling and Fission Gas Release Experiments, M. H. Montgomery, C. J. Grando, and A. A, Strasser. . . . . . . . . .

Analysis of the Validity of Fast Reactor Fuel Tests in Existing Test Reactors, A. R. Olsen, R. B. Fitts, and C.M. Cox..........

SESSION II

ENVIRONMENTAL CONTROI

A Reactor Facility for Neutron Irradiations at Temperatures Ranging from -193 to $725^{\circ} \mathrm{C}, \mathrm{C}$. W. Chen and R. G. Struss ...........

Achieving Uniform Specimen Temperatures in an Irradiation Capsule Using Heat Pipes, W. J. Zielenbach and N. E. Miller . . . . . . .

Apparatus for Measuring In-Pile Creep of IMFBR Candidate Fiels, R. A. Robinson and S. J. Basham, Jr....................

Development and Feasibility Tests of Isothermal Irradiators, C. V. Weaver, A. J. Patrick, and W. A. Ranken 
Compact $30 \mathrm{KW}$ Circulating Helium Capsule for Fuel Element Tests in

NASA Plum Brook Reactor, P. M. Finnegan, M. C. Swanson, G. E.

McDonald, I. Homyak, and H. A. Putre............ 197

Experiment Design Studies for Fuel Pin Development, R. Stein, R.

DeFayette, and I. Honyak. . . . . . . . . . . 210

Concept for a Gas Buffered Annular Heatpipe Fuel Irradiation Cap-

sule, J. D. Lee and R. W. Werner. .............. 221

\section{SESSTON III}

\section{ENVIRONIVENTAL DETERVIINATION}

In-Pile Gamma Dosimetry Measurements with Oxalic Acid, J. I. Power. . . 251

Development of Fission Gas Pressure Transducers at Argonne National

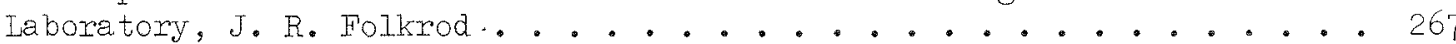

Continuous Monitoring of Fission Gas Release During Irradiation of

Nuclear Fuel Specimens, I. L. Yarger, N. E. Miller, and J. W. Ray . . . 281

The Measurement of Internal Pressure Buildup in Frul Sized Fuel Rods

During Irradiation. . . . . . . . . . . . . 296

High Temperature Thermal Cycling and Drift Test of W95 Re5 versus W74

Re26 Thermocouples for Use in Fuel Material Irradiation Capsules, D. E.

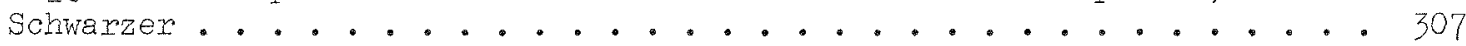

Grooved Melt Wires for Temperature Measurement of Reactox Fuel, P. G.

Salgado, B. J. Thamer, and R. I. Rudman ............. 330

Measured Energy Deposition Rates for Various Nonfissile Materials in a

Reactor Environment, P. J. Zimmerman. . . . . . . . . . . . 350

Recent Advances in Fast Response Miniature Neutron Flux Monitors, D. B.

Stillman and R. L. Chaney ................. 366

Observation of Fuel Failure Behavior During In-Pile Meltdown Tests Using High-Speed Photography and a Transparent Autoclave, J. J. Barghusen, L. J. Hamison, R. O. Ivins, and L. E. Robinson ......... 378

\section{SESSION IV}

\section{POST-IRRADIATION EXANINATION}

Irradiated Fuel Examination and Measurement, K. B. Sowa . . . . . . 403

Special Hot Cell Equipment for Space Reactor Fuel Examination, S. E.

Dismuke . . . . . . . . . . . . . . . . . 4 413

Techniques and Apparatus for Inspection, Handling, and Annealing High

Radioactive Euel Microspheres, M. I. Morgan and R. I. Towns . . . . . 420

Post-Irradiation Analys is of High Temperature, Low Burnup Tungsten-UO2

Test Plates, I. I. Betll and D. W. Sheibley............. 435 
Decay Heat Generation and Removal from Fuel Test Bundles, H. F.

Daugherty, M. Doumas, and R. M. Lieberman . . . . . . . . . . 446

Post-Irradiation Microanalysis, E. D. Albrecht, J. M. Johnson, and

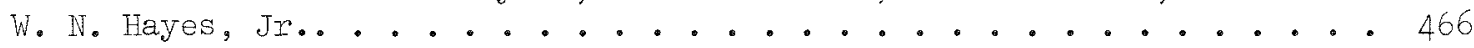

\author{
SESSION $V$ \\ TECHNIQUES AND APPLICATIONS - GENERAL
}

A System for Calculation of Fission Power in Irradiation Tests, R. M.

Lieberman . . . . . . . . . . . . . . . 473

Damage-Function Analysis of Neutron-Energy and Spectrum Effects Upon

the Radiation Embrittlement of Steels, C. Z. Serpan, Jr., and W. N.

MoElroy . . . . . . . . . . . . . . . . . . 4 491

Use of Nuclear Explosions for the Study of Radiation Damage Processes,

R. I. Carter. . . . . . . . . . . . . . . 506

A Microprobe Study of the Retention of the Fission Gas Xenon in Irradi-

ated Uranium Fuels Dispersed in Aluminum Clad Juclear Reactor Fuel

Plates, W. F. Zelezny, G. W. Gibson, and M. J. Graber . . . . . . 516

Some Applications of Neutron Radiography in Irradiation Testing Tech-

nology, J. W. Ray ................... 533

Neutron Radiographic Inspection of Irradiated SNAP fuel, O. R. Hillig . . 540

Reliability of High-Temperature Themocouples in Irradiation Capsules,

N. I. Sandefur, J.S. Steibel, R. J. Grenda, and F. D. Carpenter. . . . 551

CRICET, A System for Remote Measurement of Fuel Element Temperatures,

J. S. Buck. . . . . . . . . . . . . . . . 583

Irradiation Studies of Uranium Nitride, E. D. Albrecht, A. J. Rothman,

J. D. Lee, J. M. Johnson, and W. N. Hayes, Jr........... 595

SESSION VI

CAPSULE DESIGN, FABRICATION, AND OPERATING EXPERIENCE

Experience with Instrumented Capsules for High Temperature Irradiations

of Fhel Forms, H. J. Reilly . . . . . . . . . . . . 631

Assemblies for Themal and Fast Flux Irradiation of (UPu) N Fhels, E. O.

From and $\mathbb{N}$. E. Miller. . . . . . . . . . . . . . . . 637

A Retractable-Replaceable Thermocouple for In-Pile Experiments, J.D.

Heckelman . . . . . . . . . . . . . . . 652

Flowing Sodium Capsules in the General Electric Test Reactor, G. W.

TunnelI and D. I. Brown . . . . . . . . . . 669

Fast Reactor Fuel Testing in Forced Convection Capsules, P. E. Novak,

H. C. Pfefferlen, R. E. Murata, and G. W. Tunnell . . . . . . . . 679

Design of a Lead-Type Capsule System and Test Program to Investigate

the Radiolysis of Water, D. W. Sheibley ............. 690 
A Double Telescoping Design of a Nuclear Reacton Experiment Containing Eight Variables, D. W. Sheibley, A. G. Holms, and S. M. Sidik..... 698 Fast Reactor Sodiun-Bonded Fuel Rod Evaluation in a Themal Reactor, M. H. Montgomery, C. J. Grando, and A. A. Strasser. . . . . . . . 733

A Capsule Design for Dynamic In-Pile Friction Tests - Plum Brook Experiment 62-04. . . . . . . . . . . . . . . . 742

Remote Encapsulation and TRFAT Capsule Handling Techniques, G. E. Culley and M. E. McMahan ............... 759

The Design and In-Pile Operation of Non-Iead Instrumented Capsules, R. G. Rohal . . . . . . . . . . . . . . 774 List of Attendees . . . . . . . . . . . . . . . 784 


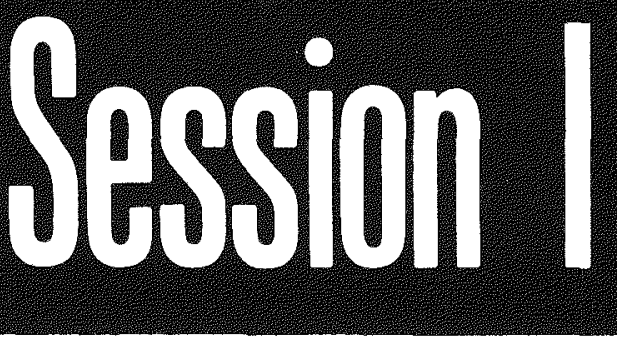

Capsule Design and

Experimental Techniques

for Fast Reactor

Irradiations 
-

- 
THE INFLUENCE OF NEUTRON SPECTRUM ON HELIUM ATON GENERATION IN STAINLESS STEELS

J. Moteff ,

General Electric Company

\&uclear Systems Programs

L. Lincinnati, Ohio

\section{INTRODUCTION}

The problems of reactor cladding materials as they are currently defined, are (1) reduced ductility, or "irradiation embrittlement", in structural alloys tested at elevated temperatures and (2) reduced density, or "clad swelling", in alloys irradiated to high fast neutron fluences at specific temperatures. These effects are considered as two of the more important problem areas for high performance reactor designs and, in particular, for liquid metal fast breeder reactor (LMFBR) or liquid metal space power systems. This is true not only from the adverse changes in mechanical properties, per se, but also from the fact that these effects are not sufficiently understood to the extent that an optimum solution has been demonstrated.

As pointed out by Weir, et. al. (1) there appears to be three important irradiation temperature regions where different irradiation induced changes occurs in the mechanical properties of the austenitic stainless steels when tested at temperatures which are approximately the same as the irradiation temperature. In the low-temperature range $(\mathrm{T}<0.40 \mathrm{~T})$, there is an increase in yield strength and reduction of work-hardening coefficient and uniform strain. These effects result primarily from the interaction of dislocations with irradiation-produced defects. At intermediate temperatures $\left(0.40 \mathrm{~T}_{\mathrm{m}}<\mathrm{T}<0.55 \mathrm{~T}_{\mathrm{m}}\right)$ 
irradiation-produced changes in the precipitation process become

important. In this temperature range the formation of voids and dislocation loops after irradiation to high fast neutron fluences cause large increases in yield strength and large reductions in ductility parameters. At high-irradiation $\left(T>0.55 T_{m}\right)$ temperatures strength properties are not affected; however, ductility is severely reduced. These effects are attributed to the presence of helium atoms produced by various $(n, \alpha)$ reactions.

Although many investigators ${ }^{(1-4)}$ have proposed specific mechanisms which could qualitatively account for the observed changes in the strengths and ductilities of some irradiated cladding materials, there is no real evidence of any consistent quantitative agreement between theory and experiment. As a matter of fact, fundamental understanding of the intermediate temperature plastic deformation behavior of structural alloys, without the added variable of the presence of irradiation induced defects, is very inadequate. Even if the present creep theories were widely applied by those investigators in the radiation effects programs, the true nature and migration behavior of the irradiation induced defects are not sufficiently known to be able to predict the interaction of dislocations with these defects. Solutions to the problems of elevated temperature embrittlement therefore are a matter of conjecture at present inasmuch as the causes are not clearly defined. The embrittlement can be attributed to both the displacement type of damage, including the radiation enhanced nucleation of precipitates, and to the helium bubble mechanism, helium being a product of transmutations of the alloy elements. There is convincing evidence pro and con for these hypotheses. 
In order to assess the real influence of neutron radiation on the elevated temperature mechanical properties of structural metals and alloys, it is essential that some consistent technology be established in the areas of (1) atom displacements and (2) transmutations, and on (3) the dependence of these two on neutron spectrum. The (4) radiation enhanced nucleation of precipitates and (5) the migration kinetics of the displaced atoms and transmutation products needs further investigations. In addition, it is important to establish a better understanding of the (6) plastic deformation mechanisms (dislocation motion and/or multiplication) in the temperature regions of about 0.3 to $0.7 \mathrm{~T}$ and finally the (7) influence of radiation induced defects on dislocation mobility in the metals within this temperature region. The discussion to follow will consider the second and third item with particular emphasis on the formations of helium atoms in various reactor spectra.

The influence of helium atoms or bubbles in the elevated temperature embrittlement of stainless steels and nickel base alloys has been firmly established. (1-4) In addition, it has also been proposed that helium atoms may be responsible for the stabilization of cavities ${ }^{(5,6)}$ during the early nucleation periods. The chief contribution to the helium formation in thermal reactors and in the blanket and reflector region of fast reactors is made by the thermal neutron capture of $B^{10}$ present as trace quantities. In the core regions of fast reactors, however, the $(n, \alpha)$ threshold reactions with the constituents of the alloy, particularly iron, nickel, and chromium, play the dominant role. These types of reactions are possible only with high energy neutrons, essentially those lying above one MeV.

Alter and Weber ${ }^{(7)}$ have calculated expected amounts of helium generation in 304 SS and 316 SS using fission spectrum averages $(n, \alpha)$ cross 
sections. De Pino ${ }^{(8)}$ has utilized Alter and Webex's cross sections to determine helium production in the Advanced Test Reactor (ATR) and Fast Flux Test Facility (FFTF) neutron environments, while at the same time pointing out that although Alter and Weber's work has made a very significant contribution in this field, there is still a dire need for accurate $(n, \alpha)$ cross section data. The former further points out that the error involved in these types of calculations is derived chiefly from inaccuracies in the cross sections of the various elements which make up the alloys.

A further investigation ${ }^{(9)}$ of the cross section problem has been made and the expected helium generation in 304 and 316 sS has been calculated for different positions in several reactors using new "spectrum corrected" fission spectrum cross section values. For core positions in each reactor facility, the fast neutron flux density of $10^{16} \mathrm{n} \mathrm{cm}^{-2} \mathrm{sec}^{-1}$ chosen by Alter and Weber as representative of a reasonable upper limit for future fast reactors was adopted. A time period of two years was also used to coincide with the choice of these authors.

\section{CROSS SECTION DETERMINATIONS}

The reported fast neutron spectra ( $E>0.01 \mathrm{MeV})$ typical of the Engineering Test Reactor (ETR) ${ }^{(11)}$, the Dounreay Reactor (DFR) ${ }^{(12)}$, and the Experimental Breeder Reactor $(E B R-I I)^{(13)}$ are presented in Figures 1 , 2, and 3. All are plotted on the same scale for sake of comparison and were normalized to unity above one MeV.

Utilizing these spectra and the documented ${ }^{(14)}(n, \alpha)$ cross section versus neutron energy data, spectrum averaged cross sections for each of these reactor environments were determined for the following five reactions: 


$$
\begin{aligned}
& \mathrm{Fe}^{54}(\mathrm{n}, \alpha) \mathrm{Cr}^{51} \\
& \mathrm{~N}^{14}(\mathrm{n}, \alpha) \mathrm{B}^{11} \\
& \mathrm{Al}^{27}(\mathrm{n}, \alpha) \mathrm{Na}^{24} \\
& \mathrm{Co}^{59}(\mathrm{n}, \alpha) \mathrm{Mn}^{56} \\
& \mathrm{Nb}^{93}\left(\mathrm{n}, \alpha\left(\mathrm{Y}^{90}\right.\right.
\end{aligned}
$$

The above isotopes were chosen because they are the only ones which have the differential $(n, \alpha)$ cross sections spanning the neutron energies of importance. It is important to point out that the spectrum averaged cross sections will depend on the type of spectra normalization employed in the calculations. Since much of the irradiation effects data on changes in mechanical properties are reported as functions of the fluence of neutrons greater than one $\mathrm{MeV}\left[\int_{1}^{\infty} \phi(E) \mathrm{dE}\right]$, or of the total number of neutrons $\left[\int_{0}^{\infty} \phi(E) \mathrm{dE}\right]$, the spectrum averaged cross sections were determined for these two cases from the following relation:

$$
\begin{array}{rlrl}
\left(\frac{\text { reactions }}{\text { atom } \mathrm{sec}}\right) & =\left(\frac{\text { reactions } \mathrm{cm}^{2}}{\text { atom neutron }}\right)\left(\frac{\text { neutrons }}{\mathrm{cm}^{2} \sec \mathrm{MeV}}\right)(\mathrm{MeV}) \\
\mathrm{R} / \mathrm{A} & =\int_{0}^{\infty} \sigma(E) \phi(E) \mathrm{d} E & \\
& =\bar{\sigma}_{1} \int_{1}^{\infty} \phi(E) \mathrm{d} E & \text { for } E 1 \mathrm{MeV} \\
& =\bar{\sigma}_{0} \int_{0}^{\infty} \phi(E) \mathrm{dE} \quad \text { for total neutrons }
\end{array}
$$

where $\bar{\sigma}_{1}$ is the spectrum averaged cross section for the case when the fluence $(\phi t)$ is reported as the number of neutrons with energies above one $\mathrm{MeV}$ and $\bar{\sigma}_{\mathrm{o}}$ that for total neutrons.

With these two adopted methods of normalization in mind, the integral neutron fluxes $\int_{E}^{\infty} \phi(E) d E$ normalized to unity above one MeV were calculated for these reactor positions and the results are presented in Figure 4. These plots enable one to change from one type of spectrum normalization 
to another merely by multiplying or dividing the spectrum by the value obtained from the $\gamma$ intercept of the integral curves. For example, the spectrum averaged cross section $\left(\bar{\sigma}_{1}\right)$ for $\mathrm{Al}^{27}$ on the core midplane in row 2 of the EBR-II is 0.68 millibarns $\left(E_{n}>1 \mathrm{MeV}\right)$. To convert to a spectrum averaged cross section $\left(\bar{\sigma}_{0}\right)$ normalized to total neutrons, one obtains a factor of 4.0 from the intercept of the integral flux curve for this particular reactor position; dividing $0.68 \mathrm{millibarns}$ by this factor of 4 yields 0.17 millibarns, normalized to total neutrons. As a further example, if a user of the EBR-II wished to convert a total fluence of $5 \times 10^{22}$ in the core region of Row 2 to a fast fluence $\left(E_{n}>1 \mathrm{MeV}\right)$, he would use the factor of 4.0 from the integral flux curve and obtain $1.25 \times 10^{22}$ for the fast fluence ( $E>1 \mathrm{MeV}$ ) in this particular position.

Figures 5 and 6 illustrate the manner in which the cross sections change with distance from the core midplane in EBR-II. Since the five reactions considered range in atomic number from fourteen up to ninetythree, and since all the spectrum averaged cross sections fell within the band of values as shown, one may be able to say that, to a first approximation, all the spectrum averaged $(n, \alpha)$ cross sections for isotopes of interest will behave in the manner depicted in the figures.

As shown in Figure 5 , the spectrum averaged cross sections are decreased by about a factor of three in going from positions within the core to a position about twenty-seven inches from the core midplane in the EBR-II. On the other hand, in comparing the spectrum averaged cross sections at different positions within this reactor with the corresponding fission spectrum averaged cross sections reported by Alter and Weber, it is apparent, as shown in Figure 6, that overestimates by average factors 
ranging from 3.5 up to about 450 can be made in the calculation of helium atoms generated if the fission spectrum averaged cross sections of Alter and Weber are used. Table 1 shows how these factors vary from one reactor to another applying both types of normalization to the spectrum averaged cross sections. Thus if one uses Alter and Weber's cross sections weighted (reduced) by the average factor taken from Table 1 for that particular reactor position, the calculated amount of helium atoms formed will be much more accurate. Table 2 lists the spectrum average cross sections for the $\mathrm{B}^{10}(\mathrm{n}, \alpha) \mathrm{Li}^{7}$ reactions. The entire spectrum of each reactor system was used in these calculations since the boron cross section extends down to the thermal neutron energies.

In the evaluation of the $(n, \alpha)$ cross sections in this report the experimentally determined fission spectrum averaged cross sections of Weitman and Daverhög ${ }^{(15)}$ were used. These values are more reliable than those of Alter and Weber whose data were taken from the theoretical work of Ray and Hawton. (16) Roy and Hawton admit that at best their results are accurate only within a factor of three. Consequently, for the helium atom calculations in various reactors, it has been decided to use Alter and Weber's cross sections weighted (reduced) by the appropriate factor except in the case of the iron, nickel, and chromium isotopes, in which cases the Studsvik values will be used weighted by these same appropriate factors. It is felt that this procedure is the best possible considering the status of current $(n, \alpha)$ cross section data.

\section{HELIUM ATOM CALCULATIONS}

The following convention will be adopted in the calculation of the helium atom generation rate 


$$
A=N_{0}\left[1-\exp \left(-t \int_{0}^{\infty} \sigma(E) \phi(E) d E\right)\right]
$$

and for small values of the argument of the exponential, equation (4)

becomes

$$
A=N_{0} t \int_{0}^{\infty} \sigma(E) \phi(E) d E=N_{0} \bar{\sigma}_{0} t \int_{0}^{\infty} \phi(E) d E=N_{0} \bar{\sigma}_{1} t \int_{1}^{\infty} \phi(E) d E
$$

where $A=$ helium atom $\mathrm{cm}^{-3}$

$$
\begin{aligned}
\mathrm{N}_{0}= & \text { target (isotope) atoms } \mathrm{cm}^{-3} \\
\sigma(E)= & \text { energy differential }(\mathrm{n}, \alpha) \text { cross section, millibarns } \\
\phi(E)= & \text { neutron flux density, neutrons } \mathrm{cm}^{-2} \mathrm{sec}^{-1} \mathrm{MeV}^{-1} \\
\bar{\sigma}_{0}= & \text { spectrum averaged cross section for total flux density normalized } \\
& \text { to unity, millibarns. } \\
\bar{\sigma}_{1}= & \text { spectrum averaged cross section for flux density normalized to } \\
& \text { unity above one MeV, millibarns. } \\
t= & \text { time of irradiation at constant power, seconds }
\end{aligned}
$$

Using these relationships and the "weighted" cross sections discussed in the last section, the calculated quantity of helijm atoms generated by irradiation in various positions of the three given reactors was obtained for 304 SS and 316 SS. The chemical composition of the two steels is given in Table 3. The theoretical results are presented in Table 4 . The calculations were performed initially using just Alter and Weber values. Then the work was repeated using weighted Alter and Weber cross sections for the respective reactor positions plus the spectrum averaged cross section for $B^{10}$ for that reactor. The value of this latter cross section for the reactor positions under consideration is given in Table 2 . Finally the calculations were performed using weighted Alter and Weber values except for iron, nickel, and chromium, in which instances the weighted Studsvik values were used. Again the spectrum averaged value for $\mathrm{B}^{10}$ was applied. The results of the three calculations are indicated in columns a), b), and c) of Table 4 respectively. The fluences for the 
various positions in the EBR-II were based on the assumption of $6.3 \times 10^{23}$ fluence $(E>1 \mathrm{MeV})$ in the core midplane of row 2 .

One notes from Table 4 that the ordering of the reactor spectra with respect to the helium formation is dependent upon which cross sections are applied. When Alter and Weber's values are used, the core regions of the Dounreay, ETR, and EBR-II all produce the same ppm quantities of helium. However, when weighted by the factors discussed in this report the order with respect to helium generation [as is evident from both columns (b) and (c) of Table 4] is ETR > Dounreay > EBR-II.

The percent contributions from the various constituents change considerably in going from the Alter and Weber to the Weitman and Daverhög cross section values. About $99 \%$ of the helium generated in the blanket regions of the EBR-II comes about as a result of the burnup of the boron. Since the steels used in this study are assumed to contain 0.001 weight percent natural boron, there are only $10 \mathrm{ppm} \mathrm{B}^{10}$ available for transmutation to helium. In the EBR-II blanket regions only seven or eight ppm helium has been formed, indicating that complete burnup has not been achieved, e.g., the equivalent thermal neutron fluence has not reached the point of saturation. On the other hand, in the core of the thermal neutron facility ETR, complete burnup of the boron has been achieved. Yet here the boron contributes only 4-7 percent of the total helium formed.

\section{CONCLUSIONS}

The study of the effect of helium generation due to neutron irradiation increases in importance as new theories of void formation and subsequent embrittlement develop. Up until now, the opinion has been that only a rough calculation of the amount of helium atoms formed in $(n, \sigma)$ reactions could be made. The present work is a step in the direction of obtaining more reliable 
calculations in this field. The cross sections discussed here are by no means final. Much more work is required, particularly in the area of experimental measurement of the more important $(n, \alpha)$ cross sections, e.g., iron, nickel, chromium, nitrogen, etc. The work of Alter and Neber enabled initial studies to be made. Weitman and Daverhög have made the primary experimental contribution. The work at this laboratory combines these results obtained thus far. Furthermore, experimental techniques have been develoned for measuring directly the amount of helium generation in irradiated materials by Farrar ${ }^{(17)}$. Comparisons between calculated and directly measured cuantities of helium are now heing made.

Py (1) irradiating high purity elements in several different reactor facilities where detailed information on the neutron spectrum is available (i.e. Monte Carlo calculations) and (2) measuring directly the helium atom generation, then (3) effective spectrum averaged cross sections or (4) empirical differential cross sections (using cross section unfolding programs ${ }^{18}$ ) may be obtained.

It is belicved that the proposed correction factors to the fission spectrum averaged cross sections, as discussed in this paper, renresents an interim method for calculations of the helium atoms generated in various fast spectrum facilities.

\section{ACKNOWLEDGEMFNTS}

This paper originated from work sponsored by the Fuels and Materials Branch, U.S. Atomic Energy Commission, under Contract AT(40-1)-2847. 


\section{REFERENCES}

1. J。R。 Weir, J. O. Stieglex, and E。E. Bloom, US-AEC DTICONF-680419, Dec. 1968.

2. D。R。 Harries, Journal of the British Nuclear Society, January 1966. pp. $74-87$.

3. R. S。 Barnes, Nature, No. 4991, June 26, 1965. p. 1307.

4. J。 Moteff, F。 D。 Robertshaw and F。D。Kingsbury, J. Nucl. Matls. 17. 245 (1965).

5. R. Bullough and R. Co Perrin, IAEA Symposium on Radiation Damage, Vienna, June (1969).

6. R. S. Nelson and D。 J。 Mazey, IAEA Symposium on Radiation Damage, Vienna, June (1969).

7. H. Alter and C. E。 Weber, Journal of Nuclear Materials, 16 (1965), p. 68.

8. A. DePino, Nuclear Applications, 3 (1967) p. 620.

9. J. F. Scherrer and J. Moteff, to be published.

10. J. Moteff, to be published.

11. L. S。 Burns, Private communication, GE-NSP.

12. I. R。 Birss and T。 P. Bishop, TRG Report, TRG 1039 (D), 1966.

13. W. E。 Edwards and W. B。 Henderson, to be published.

14. J. R. Stehn, M。D。Goldberg, B。A。 Magurno, R. Werner-Chasman, Brookhaven Report, BNL 325, Second Edition, No. 2, Vol. 1, 1964.

15. Jo Weitman and N. Daverhog, Second Conference on Neutron Cross Sections and Technology, March 4-7, 1968, Washington, D.C.

16. J. C. Roy and J. J. Hawton, CRC Report, CRC 1003, 1960.

17. H. Farrar and C. H. Knox, Transactions of the American Nuclear Society, 11, No. 2, 1968, p. 503

18. W. M. McElroy, PNWL, Private Communications. 
TABLE 1

RATIO OF FISSION SPECTRUM AVERAGED TO SPECTRUM AVERAGED

CROSS SECTIONS FOR SEVERAL REACTOR POSITIONS

A. Normalized to Neutrons Above One $\mathrm{MeV}$

Reactor

Isotopes

\begin{tabular}{llllll} 
& & \multicolumn{4}{c}{ Isotopes } \\
\hline $\mathrm{N}^{14}$ & $\mathrm{Fe}^{54}$ & $\mathrm{Al}^{27}$ & $\mathrm{Co}^{59}$ & $\mathrm{Nb}^{93}$ & $\mathrm{Avg}$. \\
3.6 & 4.2 & 3.7 & 3.9 & 4.3 & 4.0 \\
270 & 550 & 370 & 420 & 770 & 480 \\
8.2 & 9.9 & 9.0 & 9.4 & 9.6 & 9.2 \\
450 & 380 & 320 & 330 & 390 & 370 \\
2.3 & 2.3 & 2.4 & 2.4 & 2.3 & 2.3 \\
4.3 & 4.4 & 2.3 & 2.8 & 3.4 & 3.2
\end{tabular}

EBR-II Row 2 Core

EBR-II Row 2 Blanket

EBR-II Row 7 Core

EBR-II Row 7 Blanket

ETR Core

Dounreay Core

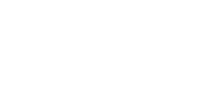

B. Normalized to Total Neutrons

\begin{tabular}{lllllll} 
Reactor & \multicolumn{1}{c}{$\mathrm{N}^{14}$} & $\mathrm{Fe}^{54}$ & $\mathrm{Al}^{27}$ & $\mathrm{Co}^{59}$ & $\mathrm{Nb}$ & $\mathrm{Avg}$. \\
EBR-II Row 2 Core & 0.88 & 1.1 & 0.93 & 0.99 & 1.1 & 1.0 \\
EBR-II Row 2 Blanket & 2.4 & 5.0 & 3.3 & 3.8 & 5.2 & 3.9 \\
EBR-II Row 7 Core & 1.2 & 1.6 & 1.4 & 1.5 & 1.5 & 1.4 \\
EBR-II Row 7 Blanket & 2.9 & 2.6 & 2.0 & 2.2 & 2.6 & 2.5 \\
ETR Core & 0.81 & 0.82 & 0.87 & 0.84 & 0.83 & 0.83 \\
Dounreay Core & 0.97 & 0.75 & 0.52 & 0.61 & 0.74 & 0.71
\end{tabular}


TABLE 2

SPECTRUM AVERAGED CROSS SECTIONS FOR $B^{10}$

\begin{tabular}{|c|c|c|c|c|c|}
\hline Reactor & Cross Section ${ }^{a)}$ & (barns) & Cross & Section $\left.{ }^{b}\right)$ & (barns) \\
\hline EBR-II Row 2 Core & 4.6 & & & 1.2 & \\
\hline EBR-II Row 2 Blanket & 2300 & & & 21 & \\
\hline EBR-II Row 7 Core & 15 & & & 2.4 & \\
\hline EBR-II Row 7 Blanket & 3000 & & & 20 & \\
\hline ETR Core & 2100 & & & 750 & \\
\hline Dounreay Core & 2.0 & & & 0.44 & \\
\hline
\end{tabular}

a) Neutron flux density above one MeV normalized to unity:

$$
\bar{\sigma}_{1}=\frac{\int_{0}^{\infty} \sigma(E) \phi(E) \mathrm{d} E}{1 \phi(E) \mathrm{dE}}
$$

b) Total neutron flux density normalized to unity:

$$
\bar{\sigma}_{0}=\frac{\int_{0}^{\infty} \sigma(E) \phi(E) d E}{0_{0}^{\infty} \phi(E) d E}
$$


TABLE 3

COMPOSYTION OF 304 SS AND 316 SS (WT.\%)

$\begin{array}{lll}\text { ELENENT } & 304 \mathrm{SS} & 316 \mathrm{SS} \\ \mathrm{Ni} & 9.15 & 13.31 \\ \mathrm{Cr} & 18.42 & 17.39 \\ \mathrm{Fe} & 68.514 & 64.474 \\ \mathrm{C} & 0.08 & 0.04 \\ \mathrm{Mn} & 2.0 & 1.8 \\ \mathrm{Si} & 1.0 & 0.66 \\ \mathrm{Cu} & 0.27 & 0.07 \\ \mathrm{Mo} & 0.5 & 2.2 \\ \mathrm{P} & 0.025 & 0.017 \\ \mathrm{~S} & 0.01 & 0.008 \\ \mathrm{~B} & 0.001 & 0.001 \\ \mathrm{~N} & 0.03 & 0.03\end{array}$

TABLE 4

HELIUM ATOM PRODUCTION, ATOMIC PPM

\begin{tabular}{|c|c|c|c|c|c|c|c|c|}
\hline \multirow[b]{2}{*}{ Reactor } & \multirow[b]{2}{*}{ Tntal } & \multirow{2}{*}{$\frac{\text { Fluence }\left(\mathrm{n} \mathrm{cm} \mathrm{cm}^{-2}\right)}{(\mathrm{E}>1 \mathrm{MeV})}$} & \multicolumn{3}{|c|}{$304 \mathrm{~S}_{.} \mathrm{S}$} & \multicolumn{3}{|c|}{316 S.S. } \\
\hline & & & a) & b) & c) & a) & b) & c) \\
\hline EBR-II Row 2 Core & $2.5 \times 10^{24}$ & $6.3 \times 10^{23}$ & 320 & 83 & 140 & 320 & 84 & 170 \\
\hline EBR-II Row 7 Core & $1.9 \times 10^{24}$ & $3.7 \times 10^{23}$ & 190 & 25 & 39 & 190 & 25 & 47 \\
\hline ETR Core & $1.8 \times 10^{24}$ & $6.3 \times 10^{23}$ & 320 & 140 & 230 & 320 & 150 & 280 \\
\hline Dounreay Core & $2.7 \times 10^{24}$ & $6.3 \times 10^{23}$ & 320 & 100 & 170 & 320 & 100 & 200 \\
\hline Fission Spectrum & $8.8 \times 10^{23}$ & $6.3 \times 10^{23}$ & 320 & 320 & 530 & 320 & 320 & 650 \\
\hline
\end{tabular}

a) Unweighted Alter and Weber values used for all isotopes.

b) Weighted AIter and Weber values used for all isotopes except ${ }^{10}$, for which spectrum averaged values were used.

c) Weighted Alter and Weber values used for all isotopes except $B^{10}$, for which spectrum averaged values were used, and for the iron, nickel, and chromium isotopes, for which the weighted Weigman and Daverhög values were used. 


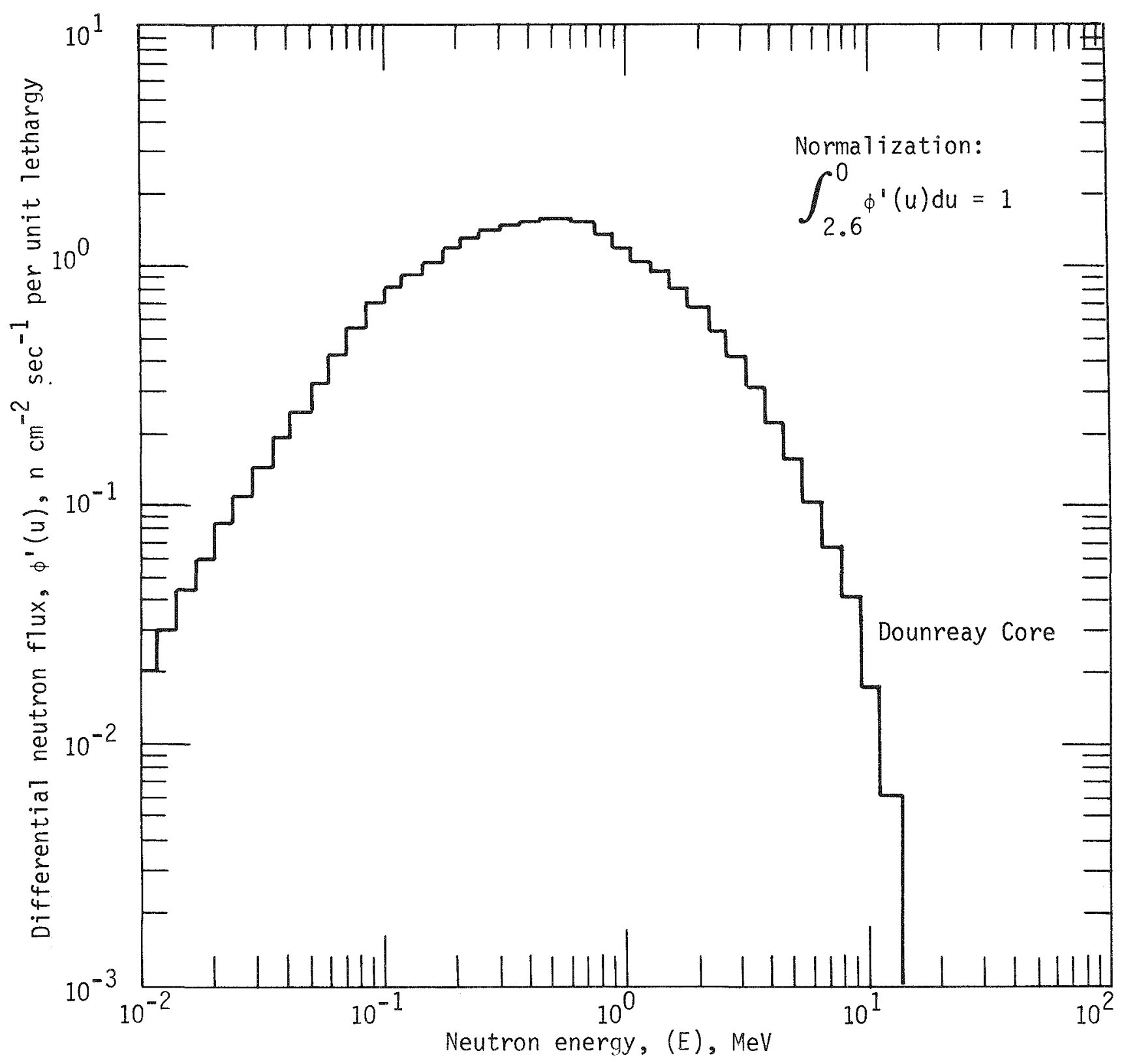

Figure 1A - Differential neutron fiux in the Dounreay core. 


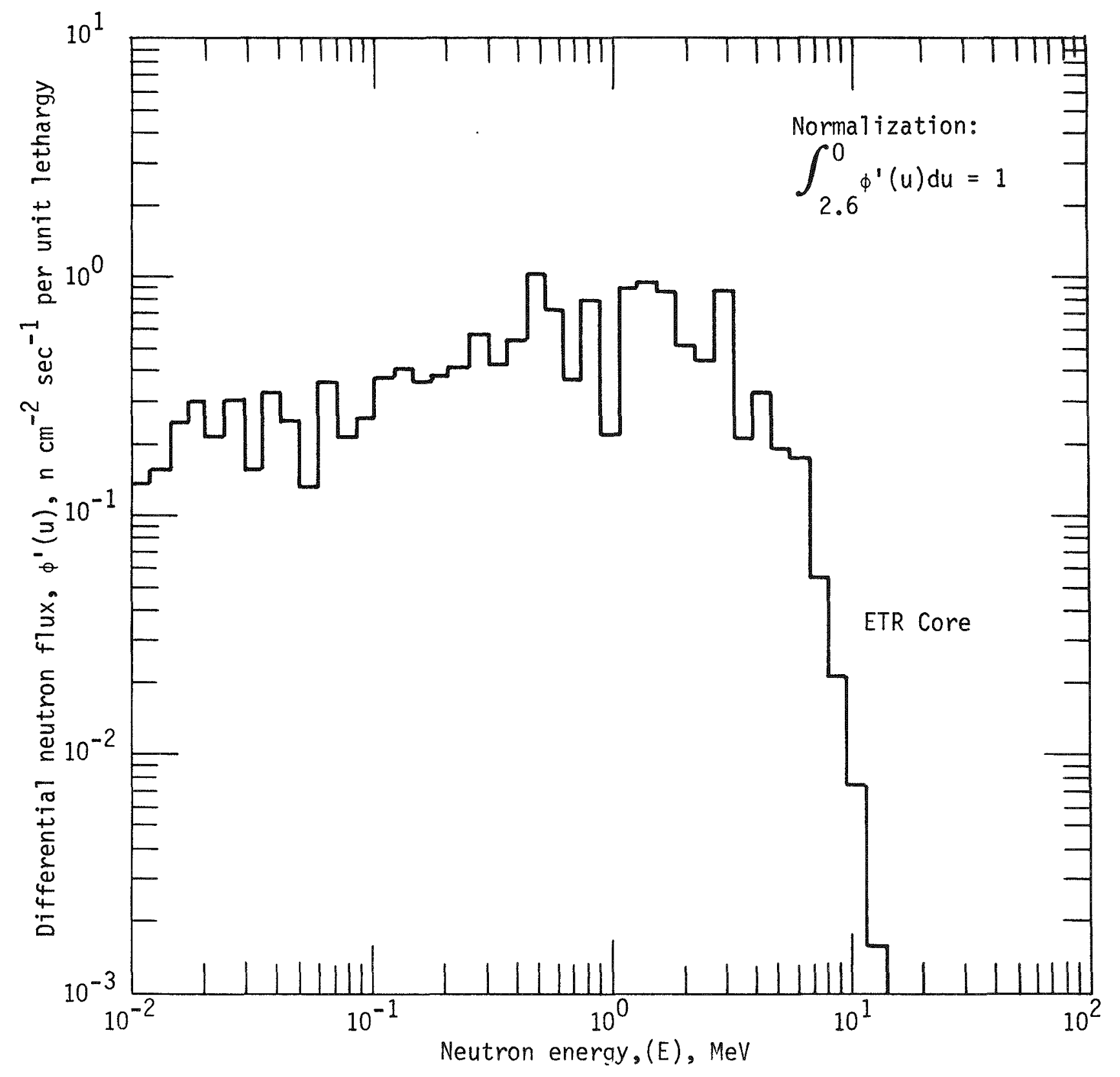

Figure 1B - Differential neutron flux in the ETR core. 


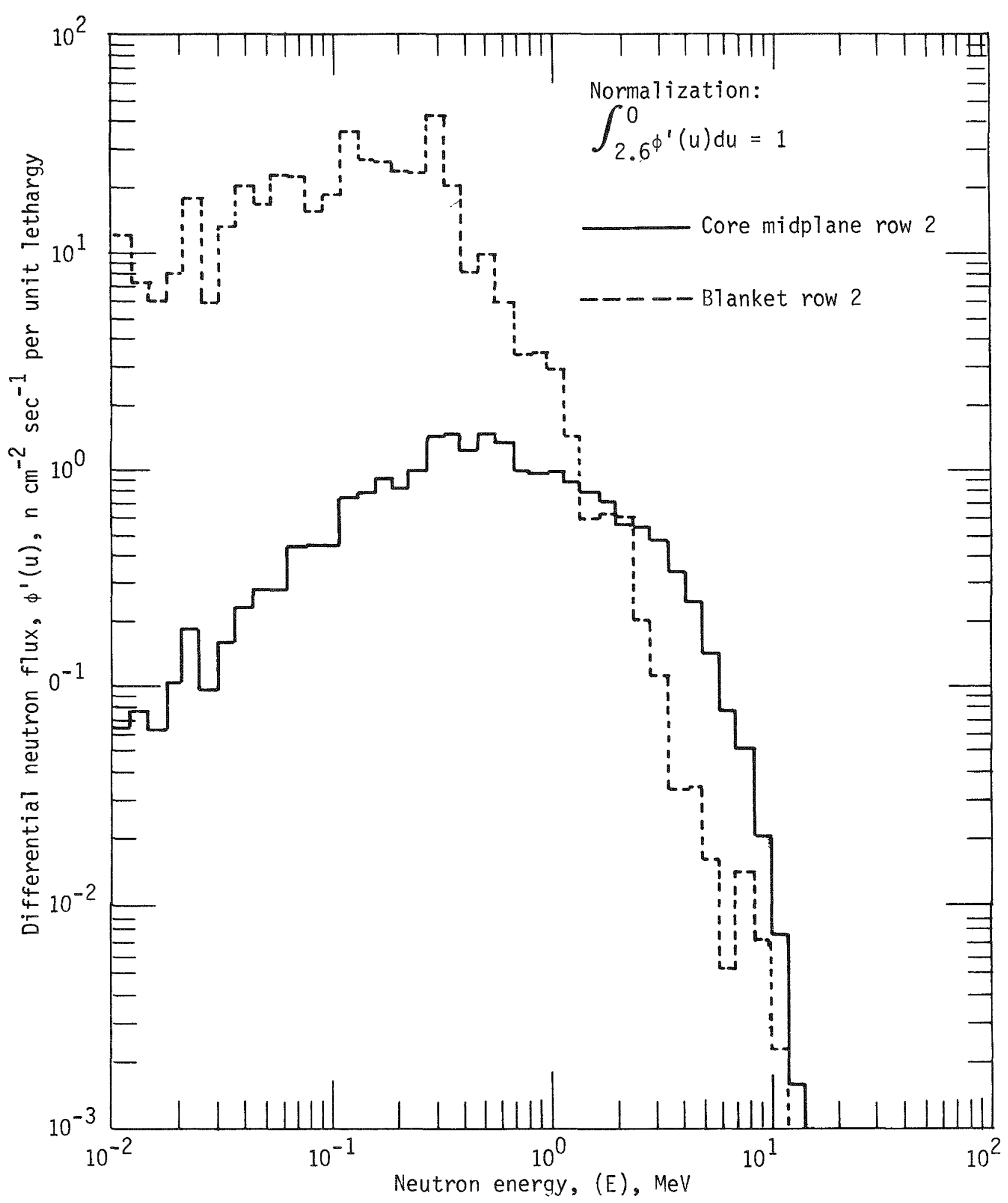

Figure 2 - Monte Carlo calculated differential neutron flux in EBR-II at the midplane and blanket positions of row 2 . 


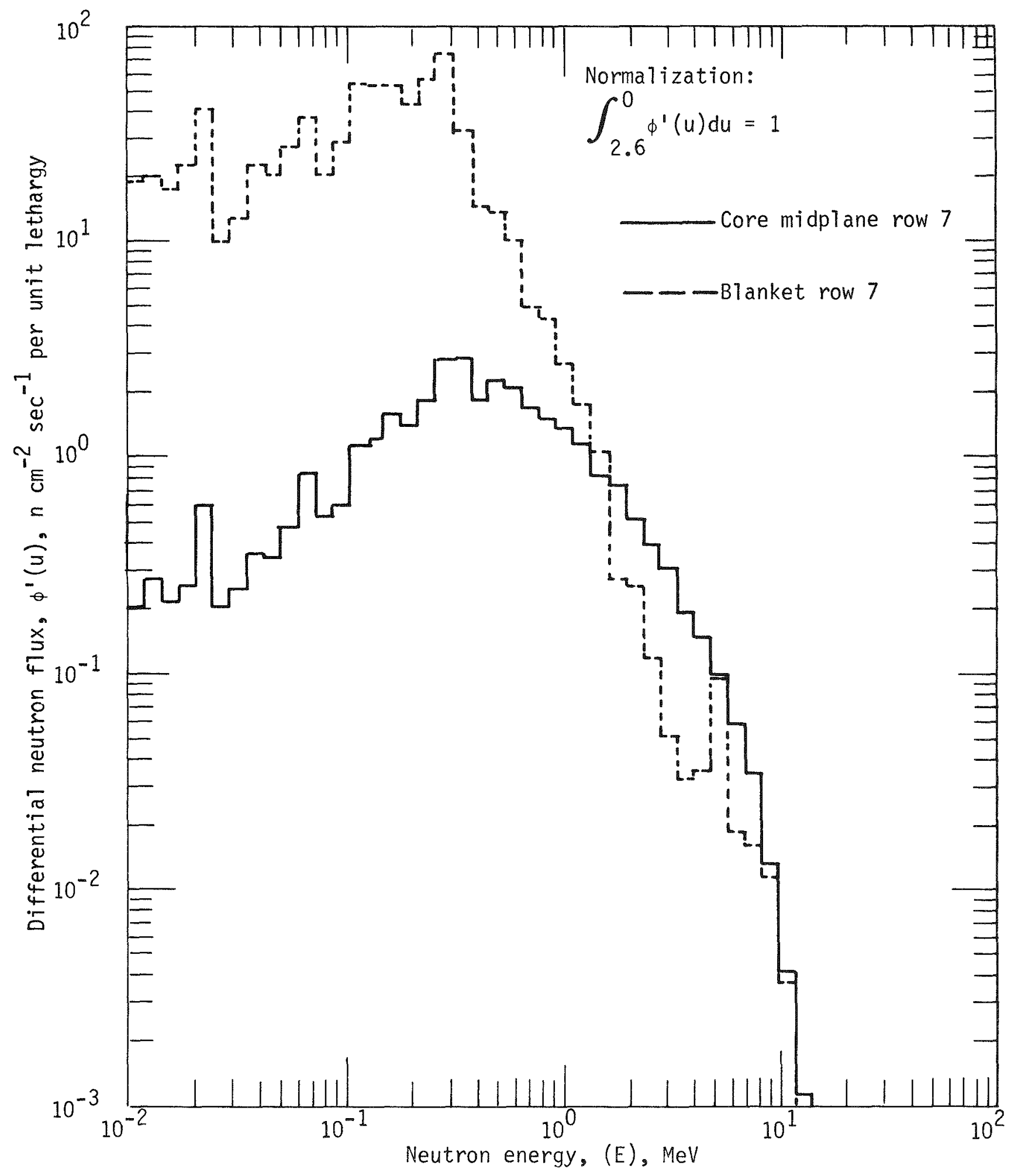

Figure 3 - Monte Carlo calculated differential neutron flux in EBR-II at the midplane and blanket positions of row 7 . 

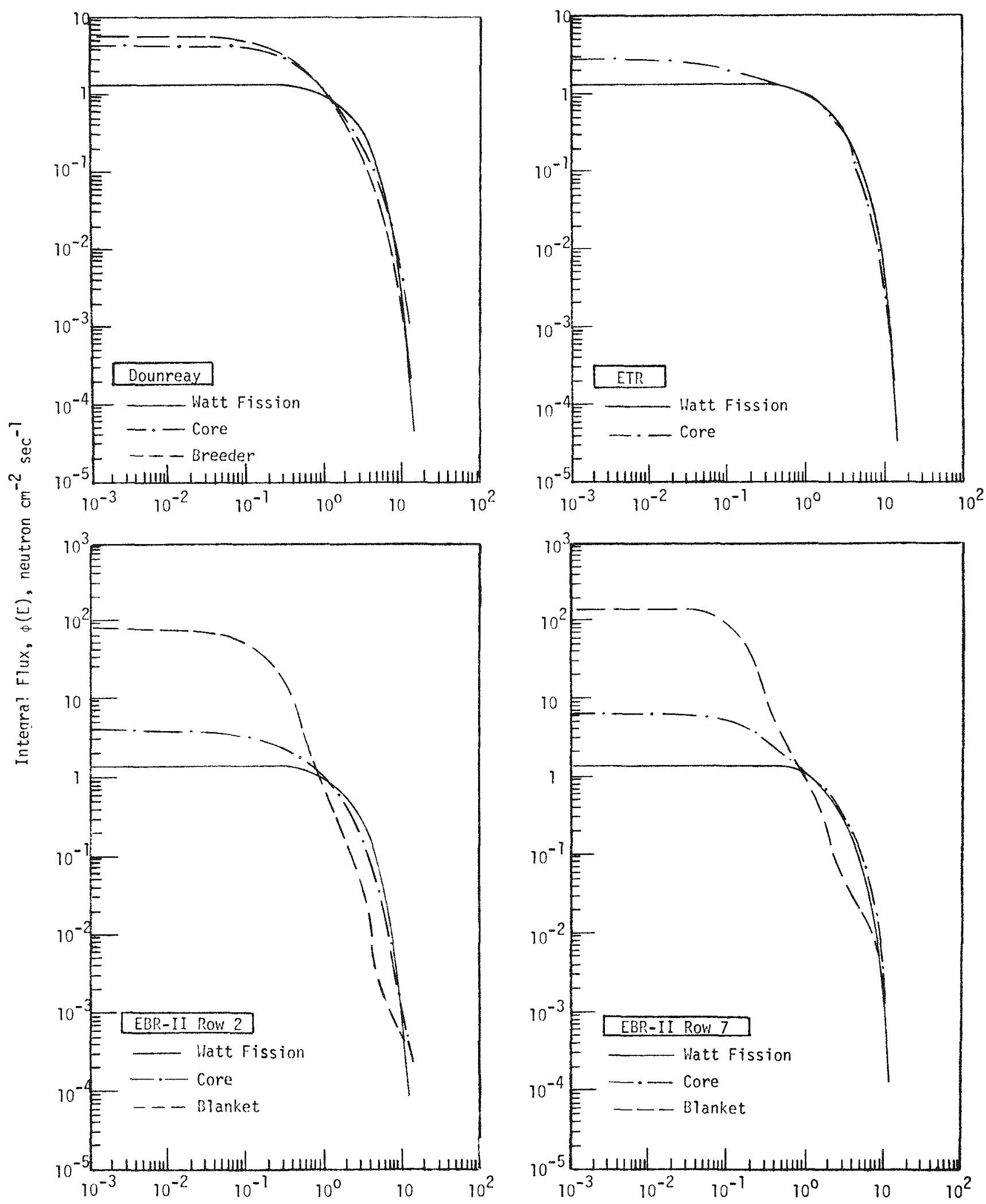

lleutron [nergy, (E), MeV

Figure 4. Integral lleutron Fluxes Ilomalized to Unity Above 1 "leV. 


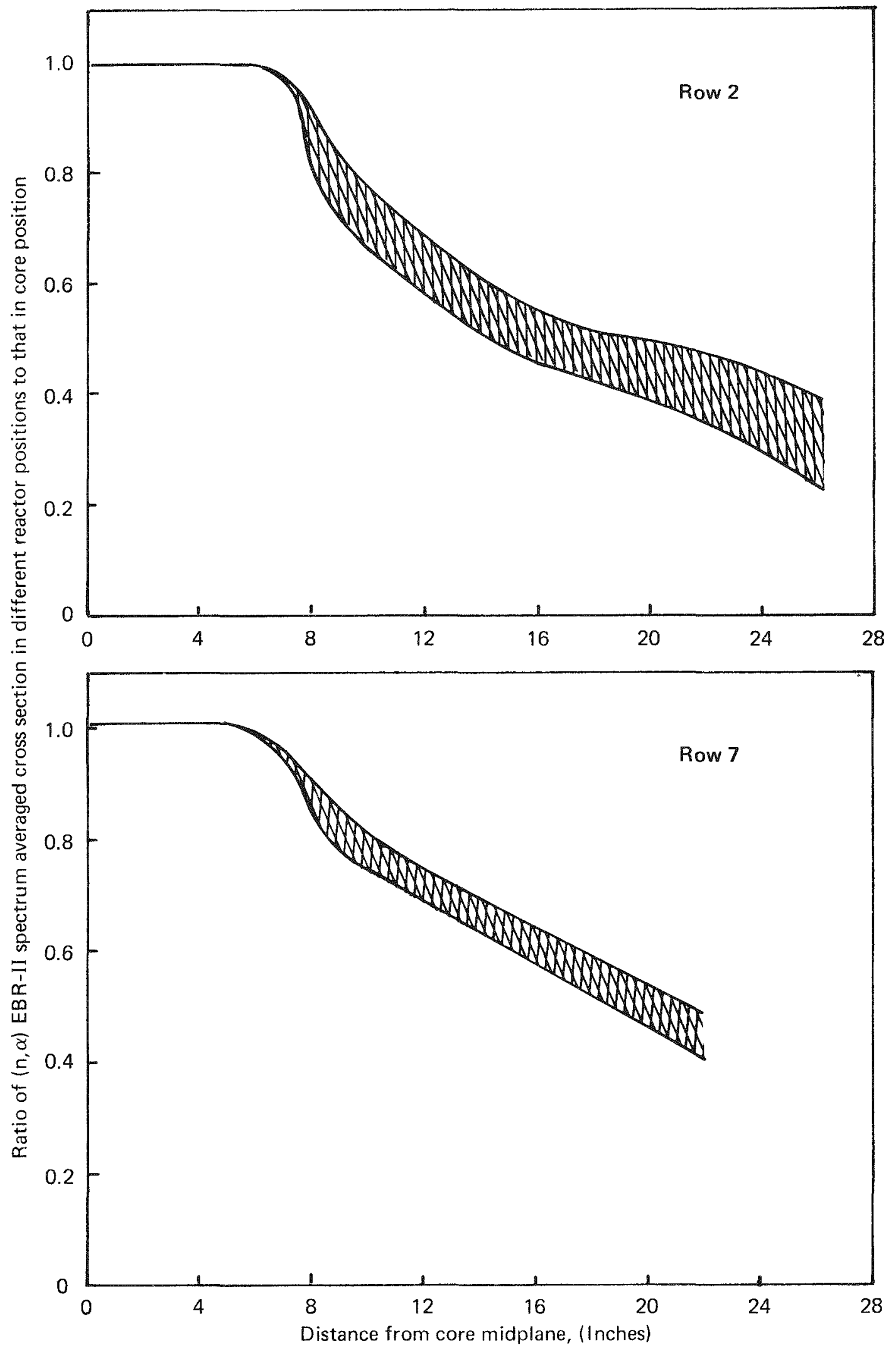

Figure 5. Ratio of $(n, \alpha)$ EBR-II spectrum averaged cross section in different reactor positions to that in core position as a function of distance from the core midplane. 


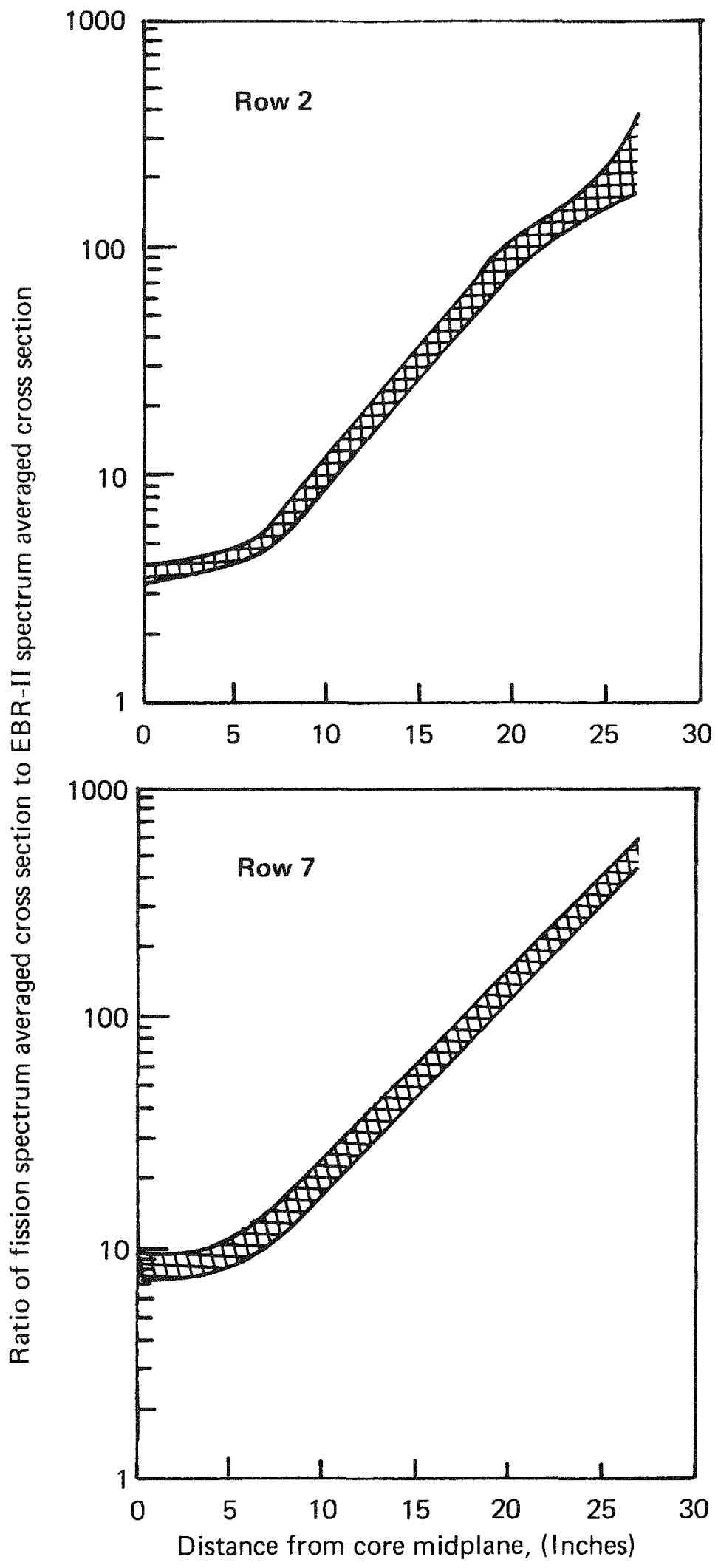

Figure 6. Ratio of $(n, \alpha)$ fission spectrum averaged cross section to EBR-II spectrum averaged cross section as a function of distance from the core midplane. 
J.M。 Beeston

M. W. Ellingford

C.R. Brinkman

Idaho Nuclear Corperation

Idaho Falls, Itaho

\section{Abstract ${ }^{l}$}

Des:-gn, fabrication, and operating experience from irradiation of fatigue specimen capsules in an EBR-II MARK B-7 subassembly are discussed. The effect of specimen size and shape, and of program criteria on the design is evaluated. Heat transfer considerations that influence the design are reriewed. The assembly to meet quality assurance of parts and performance and the sperating experience from 2864 MWd in EBR-II on one subassembly are given. The cperating temperature of the specimens in sodium at $50 \mathrm{MW}$ was about $400^{\circ} \mathrm{C}$ and in argon was $750^{\circ} \mathrm{C}$.

* Work performed under the auspices of the U. S. Atomic Energy Commission under Contract AT(10-1)-1230

Paper prepared for presentation in the National Symposium on Developments in Irradiation Testing Technology, September 9-11, 1969 Lewis Research Center's Plum Brook Station, Sandusky, Ohio. 
The fatigue specimen capsules design, fabrication, and operating experzence for irradiation in an EBR -II MARK B $=7$ Subassembly are discussed in this paper. Variability in capsule design for experimental irradiations is often required by nonuniformity in test samples. Standard test specimens for irradiated mechanical property measurement help to alleviate capsule design variations, as well as help to achieve better correlation and interpretation of data. However, even recommended standard specimens for fatigue testing would require several kinds and shapes depending upon whether testing in bending, rotation, or push-pull. Other testing criteria such as tolerances, alignments, number of repeats, and sensitivity of measurements tend to influence specimen size and shape. Obviously, the specimen size and shape affects the capsule design.

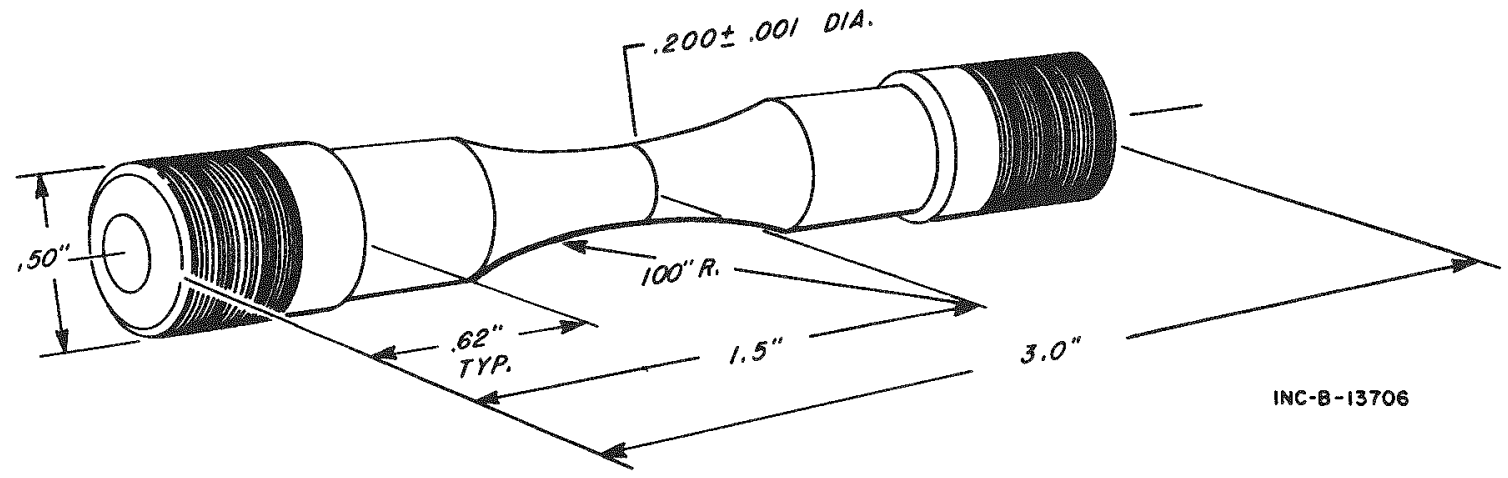

FIG. I

Fatigue Hourglass Shaped Specimen Details

\section{DESIGN}

High temperature axial fatigue testing requires a system in which buckling stresses are minimized The specimen which is the component at high temperature exhibits the effects of any buckling or lateral stresses, and in fact may tend to magnify them. In order to reduce these effects, the specimens seiected for testing are hourglass shaped, three inches long, with a 0.200 inch minimum gage section, Figure 1. The measurement of specimen deflection is made on the gage section diameter with a diametral extensometer.

The reacto: position also has a major effect on the capsule design.

* Work performed under the ausplces of the U.S.Atomic Energy Commission under Contract AT(10-1)-1230. 
Types of positions are to a certain extent dictated by the reactor design. In the EBR-II the HARK B-F subassembly accommodates the maximum possible test volume and is used for structural irradiations, Figure 2. This subassembly used exterior parts such as the upper and lower adaptor and hexagonal tube that are identical to the standard reactor fuel subassembiles. The capsules which contain the experimental assemblies have an overall length of 57.875 inches and an outside diameter of 0.8055 inch. The capsule has an internal length of 55.750 inches to accommodate the experimental assembly. This length from a neutron flux standpoint is conveniently divided into three regions; a core elevation region and two blanket elevation regions, with the core region about $131 / 2$ inches long.

\section{A Criteria}

The inctial design criteria required the specimens to be irradiated over a range of temperatures and in both sodium and gas environment. The irradiation temperature was to vary between reactor ambient $\left(370-400^{\circ} \mathrm{C}\right.$ ) for the sodium bonded specimens and a high temperature of $700^{\circ} 100^{\circ} \mathrm{C}$ produced by a thermal gas bond. The three reactor regions; core, upper, and lower blanket provided in the desigr natural regions for evaluating the effects of flowing reactor sodium as related to purified sodium, and an inert atmosphere. The equivalence of the flux in the upper and lower blanket regions would allow the effects of other parameters to be evaluated. The effects of irradiation environment, temperature, and fluence (integrated flux) could thus be investigated on specimens of different materials to study the influence of fabrication methods and metallurgical factors (cold work, microstructure or grain size) on the fatigue properties. The fatigue properties being measured are the cyclic strain, stress, fatigue life, and hysteresis energy, so that data curves for reactor design may be prepared. Test parameters also being investigated will be the effect of environment, strain rate. state of stress, cycle hold times, and temperature.

\section{B. Heat Transfer Calculations}

For purposes of the design, it was assumed that a reactor position such as $7 \mathrm{~N} 3$ or $7 \mathrm{~N} 5$ would be available and this placed the radial distance of the axial centerline position at 12.28 inches from the reactor axial centerline. The gamma heating at the reactor midplane was taken as 2 watts/g, Figure 3. Figure 3B gives a relative heat absorption for each specimen location depending on distance from the midplane.

The specimens in the upper and lower reflector regions were to be irradiated at close to reactor ambient temperature in a sodium environment. The heat transfer calculations were thus limited to the center insert in the core region in which the specimens were to be irradiated at temperatures of $600-800^{\circ} \mathrm{C}$ in inert gas.

Due to the specimen shape calculations were initially made on the basis of one dimensional radial conduction from the specimen to the sodium coolant. Two gases, helium and argon, were considered in the gas barrier. The calculation indicated that a ballast, Figure 4, which surrounds the specimen would even out the specimen temperature and provide the mass required for the gamma heating to bring the temperature to $700^{\circ} \mathrm{C}$. The final 


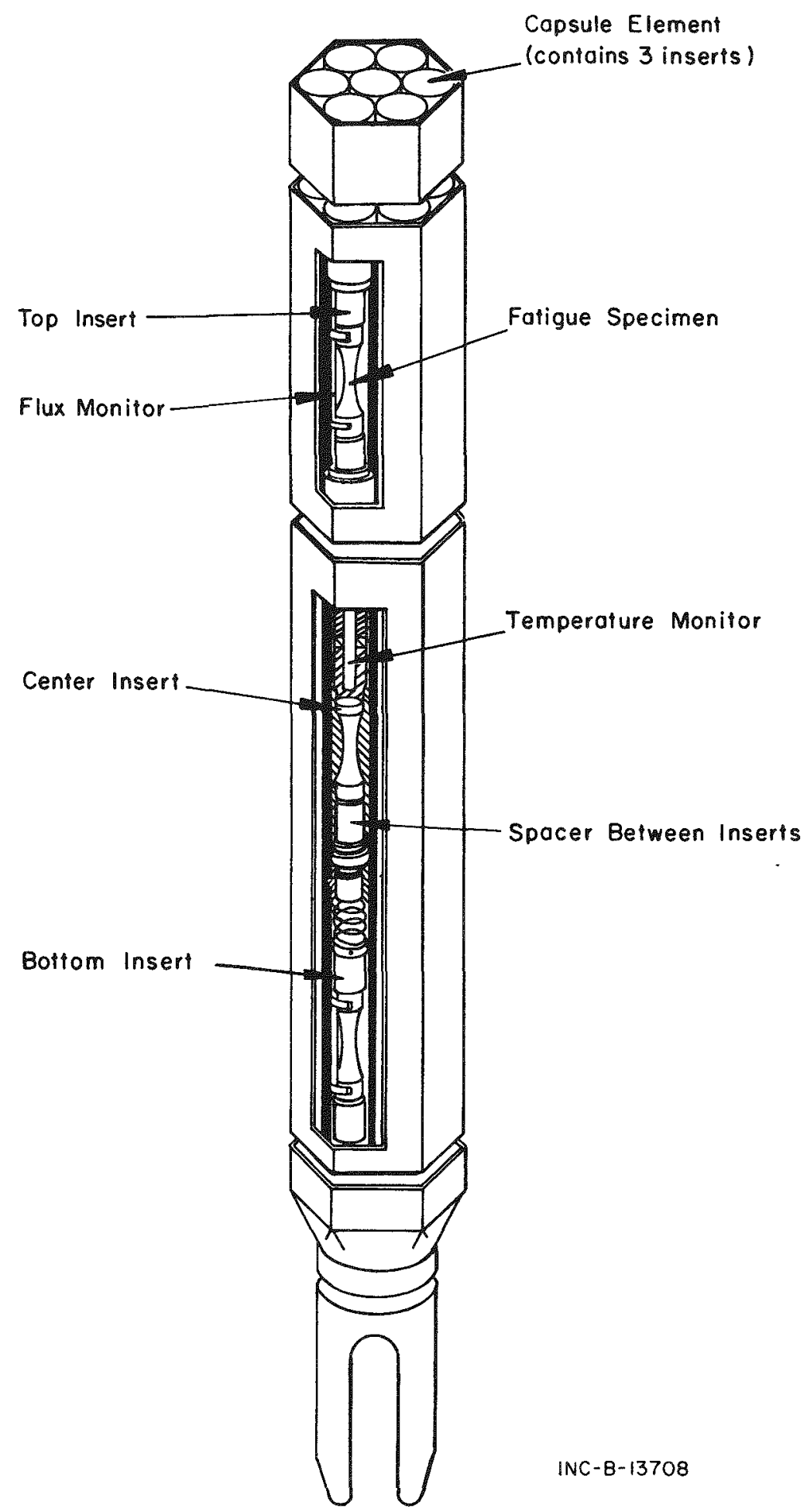

Figure 2, Fatigue Specimen EBRII MARK B-7 Subassembly 

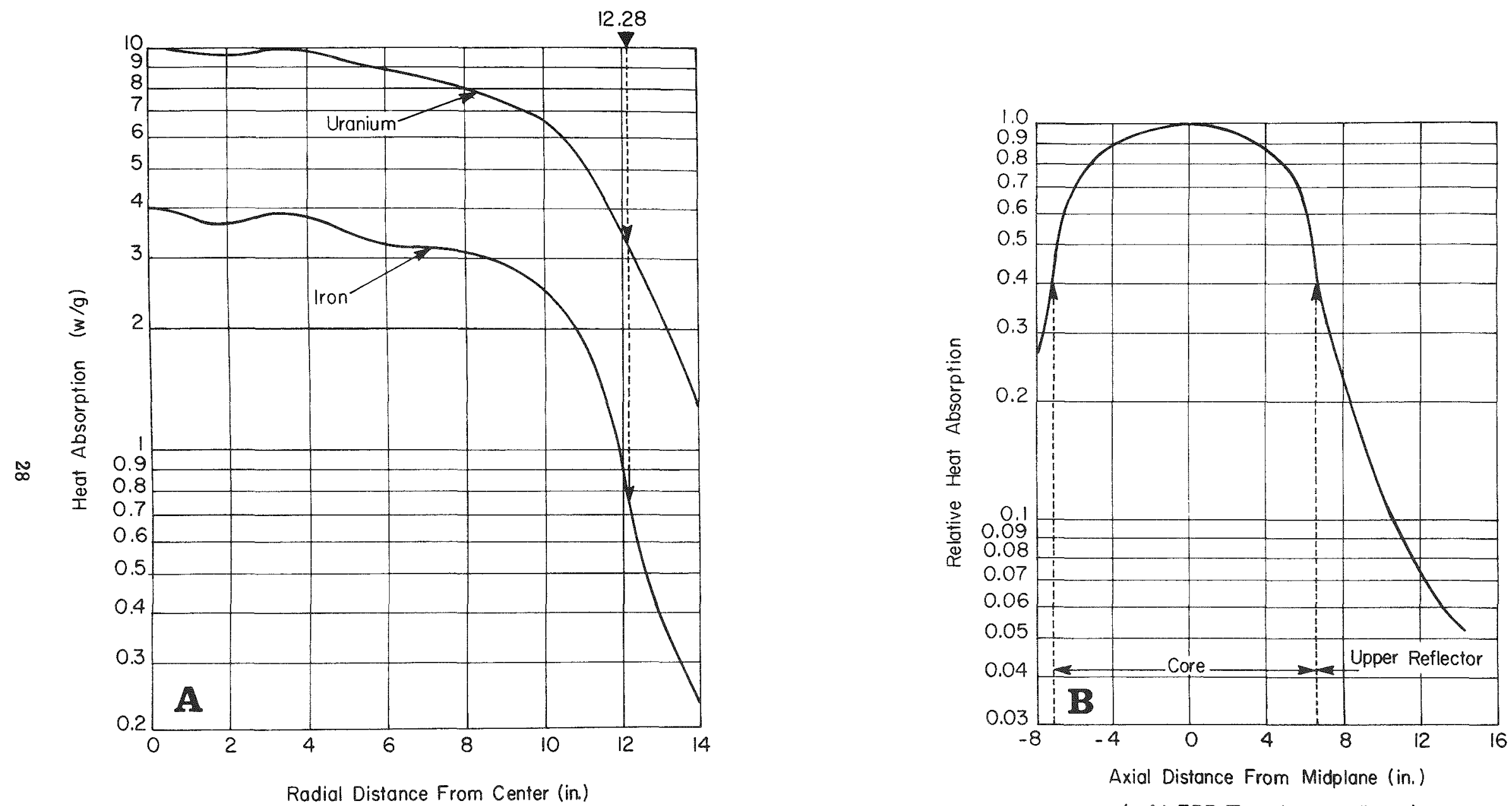

(ref.: EBR II project handbook)

ING-C - 13705

Figure 3, Gamma Heating at Reactor Midplane And

Relative Gamma Heating in Axial Direction. 


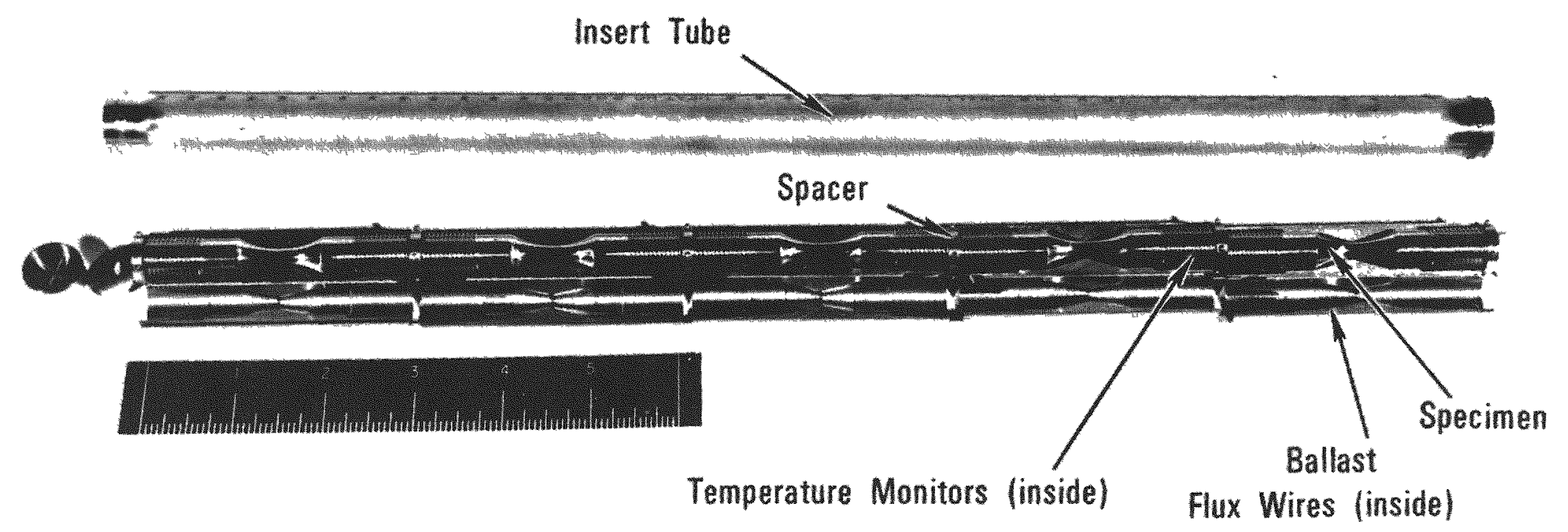

(A) High Temperature Inserts

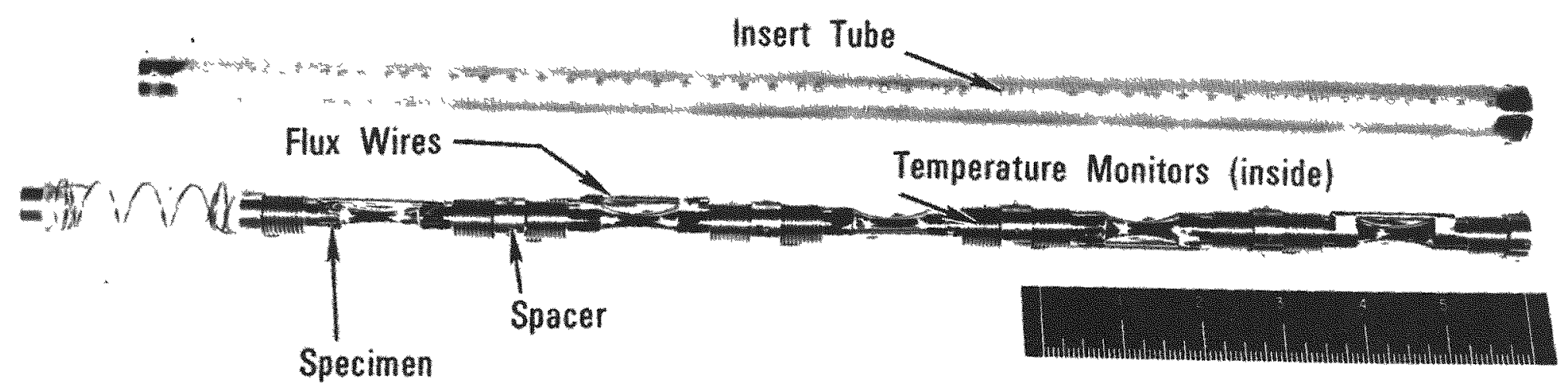

(B) Sodium Bonded Inserts

Figure 4, Photograph of Parts For Gas And Sodium Bonded Inserts. 
calculations considered the radiation heat transfer coupled with conduction to arrive at a final temperature of $715^{\circ} \mathrm{C}$.

The heat generated in the subassembly was removed by $12 \mathrm{gpm}$ of flowing sodium as provided by the standard orifices in the bottom of the subassembly. The coolant outlet temperature from this subassembly was calculated as $377^{\circ} \mathrm{C}$. Adjacent coolant outlet temperatures in the blanket are calculated to be higher' (about $480^{\circ} \mathrm{C}$ ), hence there will be a heat flow into this subassembly which will give higher temperatures.

\section{General Description}

To accommodate the cxiteria three capsule inserts were designed and are -ntained in an element tube, Figure 2 and 4. The center insert is gas bonded and occupies the center region at reactor core elevation. The top and bottom inserts contain sodium as the heat transfer medium. The top insert is filled with purified sodium within one-half inch of the upper end cap at maximum operating temperatures. In the cold $\left(30^{\circ} \mathrm{C}\right)$ condition the level stands at 2.2 inches from the bottom of the top end cap. The bottcm inserts in subass mblies 3 and 4 contain weep holes through which approximately $1 \mathrm{gpm}$ of reactor sodium flows. The velocity past the speclmen gage section is two feet per second. The cover gas in the sodium filled top inserts is a. gon. The center inserts contain argon or helium as the specimen environment Each insert contains five specimens. There are seven element tubes per subassembly. To produce a uniform temperature over the specimen gage section in the gas bonded inserts, ballasts were designed to distrıbute the mass. The ballasts were made in two halves, contoured to the shape of the specımens with contact points at the specimen shoulders (not threaded section) and the gage section (smallest diameter). The measurement of temperature was accomplished by insertion of fusible sentinels (melting wires) and silicon carbide monitors in drilled holes at the ends of the specimens. Flux monitor wires were attached to the shoulders of the spcrimens for the determination of the fluence each specimer received.

\section{FABRICATION}

\section{A. Parts Procurement}

The hardware obtained from ANL for the Type B-7 Irradiation Subassembly consisted of seven each of the element tube, plug end, and capsule tip. The fabrication of the insert parts and the specimens was accomplished by the commercial vendor. The specimen material was furnished the vendor. Quality Assurance inspection of all parts and certification of all materials was required.

\section{B. Assembly}

The assembly was principally performed in inert atmosphere in special glove boxes in which the oxygen content was measured by Model 80 Beckman Analyzer to be less than $1 \mathrm{ppm}$, Figure 5 All fabricated parts were cleaned in ace one and rinsed with alcohol immediately prior to assembly, then handled only w:th clean gloves. 


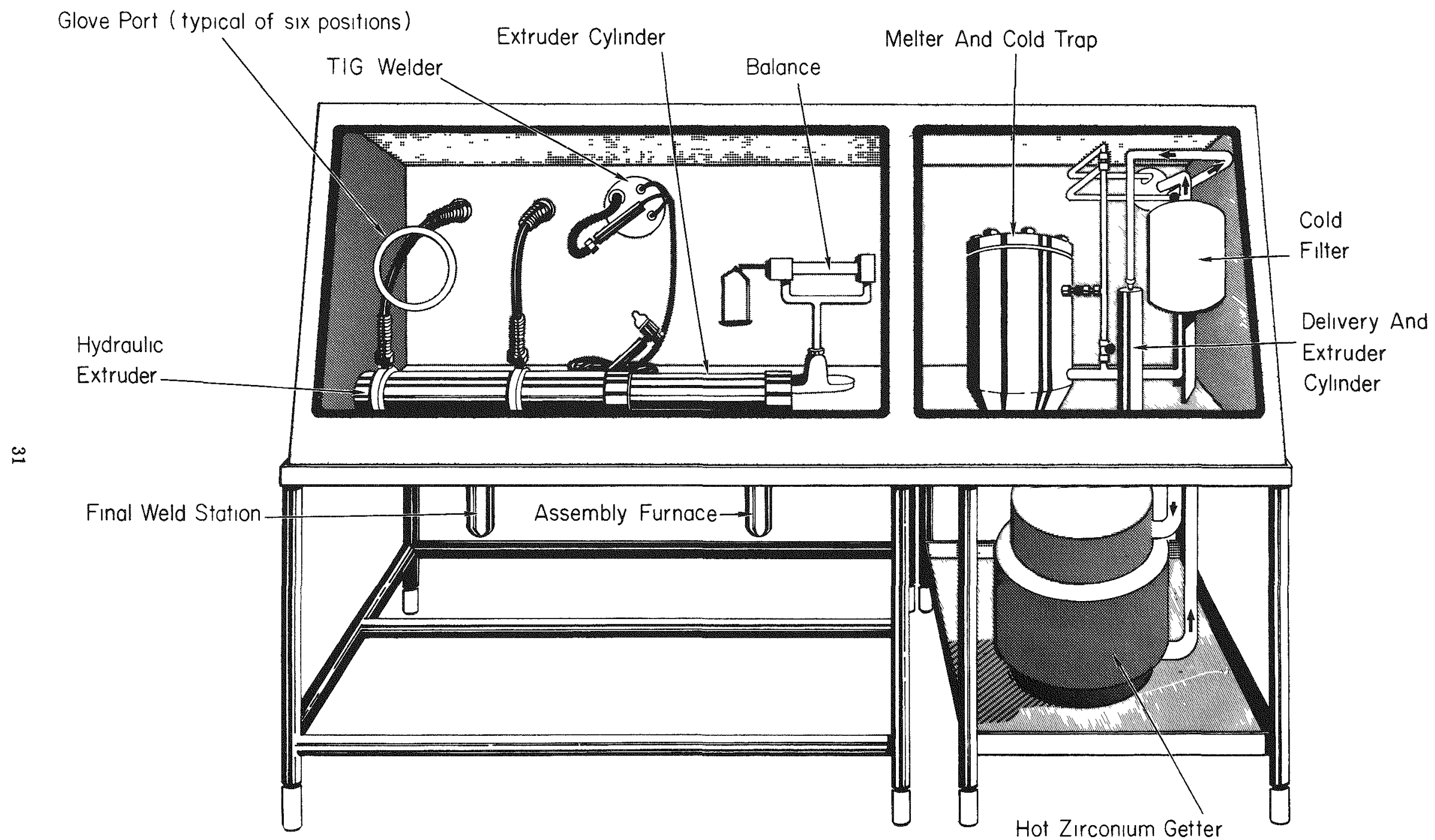

Figure 5, Sodium Purification System And Capsule Assembly Box. wc c 370 
The sodium of ACS grade was melted and purified before dispensing into the sodium bonded inserts. The sodium was purified by passing through a stainless steel system with a $125^{\circ} \mathrm{C}$ cold trap, 10 micron filter, and a zirconium getter at $650^{\circ} \mathrm{C}$. The purified sodium was delivered into the extruder cylinder which was transferred inside the inert gas atmosphere to the extruder. The sodium was extruded into slugs and weighed to 47.6 grams. The slugs were then dropped into the insert tube through a polyethylene sleeve and melted in the assembly furnace. The specimen train previously assembled was lowered into the sodium. The procedure of heating the sodium to $125^{\circ} \mathrm{C}$ and slowly lowering the specimen train, especially with the learances, provided of $.050^{\prime \prime}$ on the major surfaces, insured wetting of all steel surfaces (other surfaces involved pins and rounded ends).

All assembly steps including welding were accomplished inside the inert $\exists t m o s p h e r e . A I l$ welds were made in accordance with Idaho Nuclear Corporation welding specifieation IN-W-1-COM, Manual of Welding of Austenitic Stainfess Steel in Nuclear Reactor Experiment Systems (presently Procedure No. S-2.0 which satisfies RDT Standard F6-1)。 The welding process was tungsten inert gas (TIG) with filler material ER308I. The inspection of the welds was made in accordance with specification IN-W-10-COM, Liquid Penetrant Inspection in Nuclear Experiment Systems and final radicgraphy. All defects found were repaired by grinding out the defect and rewelding.

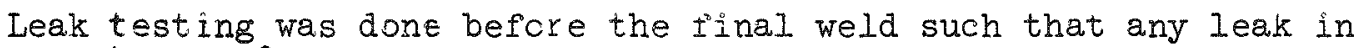
excess of $4 \times 10^{-6}$ oc per second was unacceptable. After the final we:d, the insert capsule was placed in a hellum chamber at 300 psig for eight hours then removed and checked by helium leak detector at the same unaceptable level.

The final assembly consisted of welding the insert capsules inside the element tubes in which had been machined weep holes.

\section{OPERATING EXPERIENCE}

A. Insertion and D2sassembly

The insertion, irradiation period, and subsequent removal from the reactor of the MARK B-7 Fatigue Subassemblies was completed according to $E B R=I I$ normal procedure. The operating experience with the capsules has been good. One subassembly has been removed from the reactor after 2864 MWd, and three other subassemblies have been fabricated and inserted in the reactor. After ixradiation, subassembly one was transferred to the interbuilding cofin for cleaning. Sodlum was removed by flowing moist argon gas over the subassembly to initiate a slow, controlled cxidation process. Next, moist air was flowed over the subassembly to complete the conversion of sodium to sodium hydroxide. Demineralized water flushes removed the sodium oxide and sodium hydroxide. A flow of dry alr was used to remove the moisture and the subassembly was then transferred to the air cell for disassembly

In the air cell, the element tubes were removed from the hex tube by making a circumferential cut just above the point where the tube is 
welded to the lower adaptor. The hex tube and top end fixture was then withdrawn to expose the seven element tubes, and allow removing the individual element tubes from the T-bar grid.

The element tubes were transferred to the TAN Hot Cell for completion of disassembly. The capsules were removed from the element tubes by cutting circumferentially through the tubes just below the top welds using a thin abrasive wheel. The capsules slipped freely from the tubes. The capsules were opened in the same manner as the element tubes.

The sodium-filled capsules were mmersed in butyl-alcohol and later in water to remove the sodlum. The hourglass specimens were washed in acetone to quickly remcve the water and finally stored in containers filled with water soluble oil. Flux monitor pods were removed and were stored in marked vials tc maintain identity. Marked vials were also used to store the sentinel wire temperature monitors, silicon carbide temperature monitors, and the boron flux monitors. All of the specimens in the sodium bonded capsules appeared to have as bright a finish as before Irradiation.

For the most part, the sperimens in the argon bonded inserts also retained their metallic luster and straightness With those specimens that were damaged, the ballast had warped a greater amount than the clearance between the ballast and thread and had seized the threaded section. The selzing had occurred on several of the first ballasts welded. The ballasts, made in two halves, were welded together with 1/8" long welds in three places using no filler material. Penetration of the weld was too deep in the first welds and distorted the ends of the ballast caising interference with the specimen threads, Figure 6. The welder's technlque improved with subsequent ballasts and no damage of this type occurred, Figure $6 B$. During temperature cycling in the reactor eight out of 105 specimens were fatigue cracked to varying depths because of the previously described distorted ballasts.

The tack welds on the ballast ends have been eliminated on later assemblies. A circumferential groove is machined at each end of the ballasts which are held together with a spring at each end and a center weld。

\section{B. Temperature Determination}

In order to provide a measurement of specimen irradiation temperature, several types of temperature monitors were included in the capsules. Fusible sentinel wires were included in the sodium bonded capsules while both sentinel wires and "Crusilite" (Beta SiC slugs) specimens were included in the argon bonded capsules. The crusilite slugs measured $.125=.001$ inch in diameter and were .500 \pm .01 inch long.

Dimensional changes induced by fast-neutron damage in silicon carbide have been shown to be a reliable means of establishing the irradiation temperature in experiments subjected to high fluences. Thorne 2 et al. irradiated $\beta-$ SiC up to a fast neutron fluence of $1.8 \times 10^{21}$ at $250^{\circ} \mathrm{C}, 475^{\circ} \mathrm{C}$ and $700^{\circ} \mathrm{C}$ and found growth saturation values of $.73 \%$, $.44 \%$, and $.31 \%$ respectively. Further, they found that the dimensional changes 

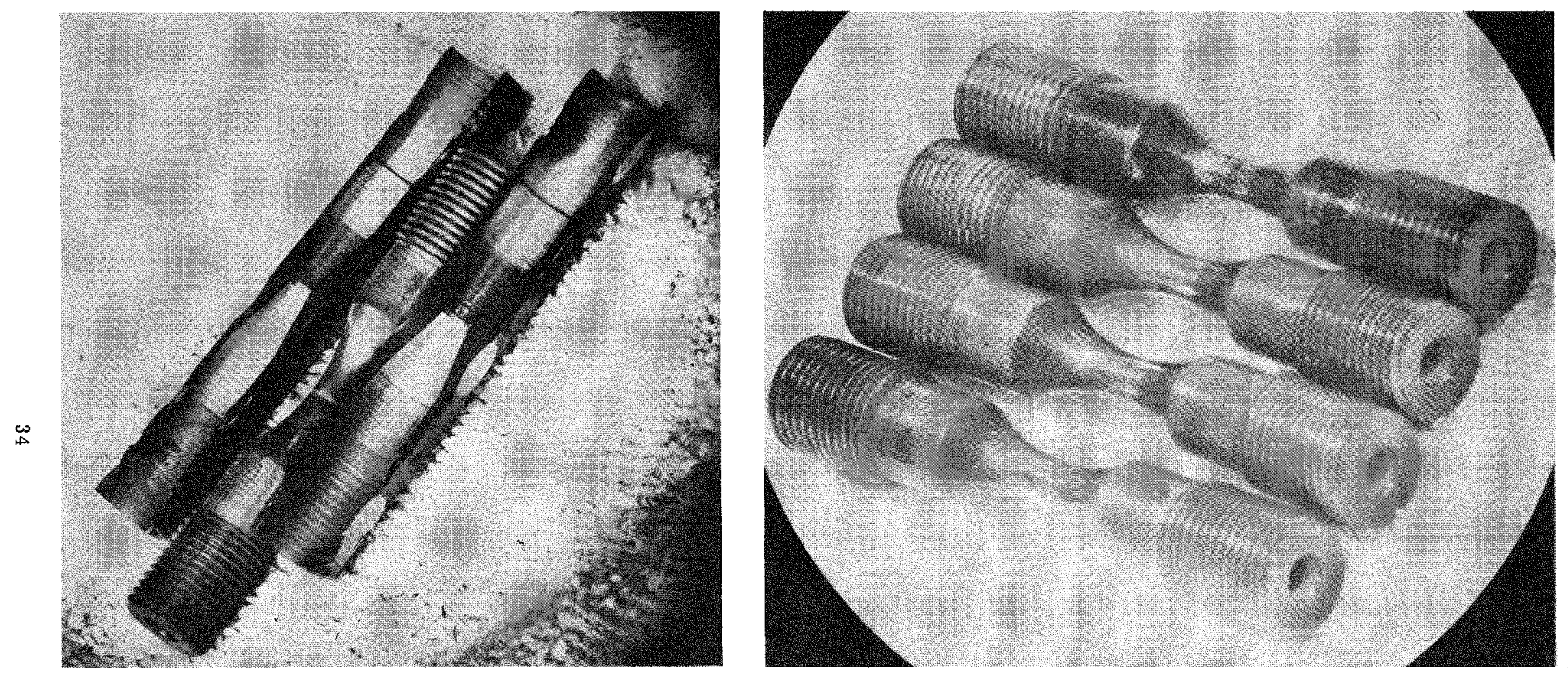

(A) Unsatisfactory

(B) Satisfactory

Figure 6, Appearance of Fatigue Specimens After Irradiation at $750^{\circ} \mathrm{C}$. 
tended to saturate after about $3 \times 10^{21} \mathrm{n} / \mathrm{cm}^{2}$ with no further change up to a fluence of $2 \times 10^{21} \mathrm{n} / \mathrm{cm}^{2}$. Upon annealing, they showed that substantial maounts of shrinkage did not occur until the annealing temperature exceeded the irradiation temperature. A linear change in dimensions then occurred with increasing temperature. "Break points" thus reflected irradiation temperatures. However, as equilibrium saturation concentrations are rapidly established, the "break points" reflect maximum temperatures attained and say nothing regarding the length of time that the specimens were at that temperature.

Sllicon carbide slugs included in the argon bonded inserts were subjected to a series of one hour isochronal anneals at a pressure of $10^{-5}$ torr. All measurements were made with a Praft \& Whitney Opt-0-Iinit Indicator that had been previously calibrated with standard gauge blocks. Sereral measurements were made on the slugs at room temperature with a precision of $\$ 50$ micro-inches. The isochronal annealing curves generated as length change in percent from the room temperature value are shown in Figume 7. Growth saturation values (approximately the same as length change percent) of the slugs ranged from .24\% to $.34 \%$ with an average of $.28 \%$, while the average fluence was $5.1 \times 10^{20} \mathrm{n} / \mathrm{cm}^{2} \mathrm{E}>\mathrm{MMeV}$. No relationship between growth saturation and fluence variations was found, (the variation appears random see figure) indicating that the carbide slugs were all irradiated into a growth saturation range. Heat transfer calculations based on reactor full power of $45 \mathrm{MW}$ predicted specimen centerline temperatures of $700=100^{\circ} \mathrm{C}$. However, the reactor operated at a full power of $50 \mathrm{MW}$ for approximately $29 \%$ ( $392 \mathrm{hrs}$ ) of the irradiation time. Three of the five "break points" in Figure 7 indicate a maximum temperature ranging from $710-750^{\circ} \mathrm{C}$. Fuslble sentinel wires placed in the positions shown in Figure $7 \mathrm{~B}$ indicated a temperature of around $750^{\circ} \mathrm{C}$ as all of the wires with a melting point of $710^{\circ} \mathrm{C}$ or lower were melted and several of the $750^{\circ} \mathrm{C}$ wires showed indications of having melted. Figure 8 shows pictures of two wires from the S-1I position, one of which has clearly melted and the other has not. Based on the behavior of these two different temperature monitors it was, therefore, concluded that the maximum specimen temperature in the argon bonded capsules during irradiation was around $750^{\circ} \mathrm{C}$.

Sentinel wires placed in the upper and lower structural steel inserts indicated that the highest temperature attained was higher that the preirradiation estimate of $380^{\circ} \mathrm{C}$. One of the wires $\left(360^{\circ} \mathrm{C}\right.$ ) in each pair clearly melted, suffered a marked change in shape or otherwise deteriorated indicative of melting. The temperature of the sodium thus appears to be about $400^{\circ} \mathrm{C}$. The fluence on the specimens varied between $3 \times 10^{18}$ and $8 \times 10^{20} \mathrm{n} / \mathrm{cm}^{2} \mathrm{E}>1 \mathrm{MeV}$.

\section{REFERENCES}

1 Guide For Irradiation Experiments in EBR-II, EBR-II project, ANL, Argonne Illinols, page 66.

$2 \mathrm{R}$. P. Thorne, $\mathrm{V} . \mathrm{C}$. Howard and B.Hope. "Radiation-Induced cnanges in Porous Cubic Silzcon Carbide"。 TRG Report 1024 (c), 1965. 


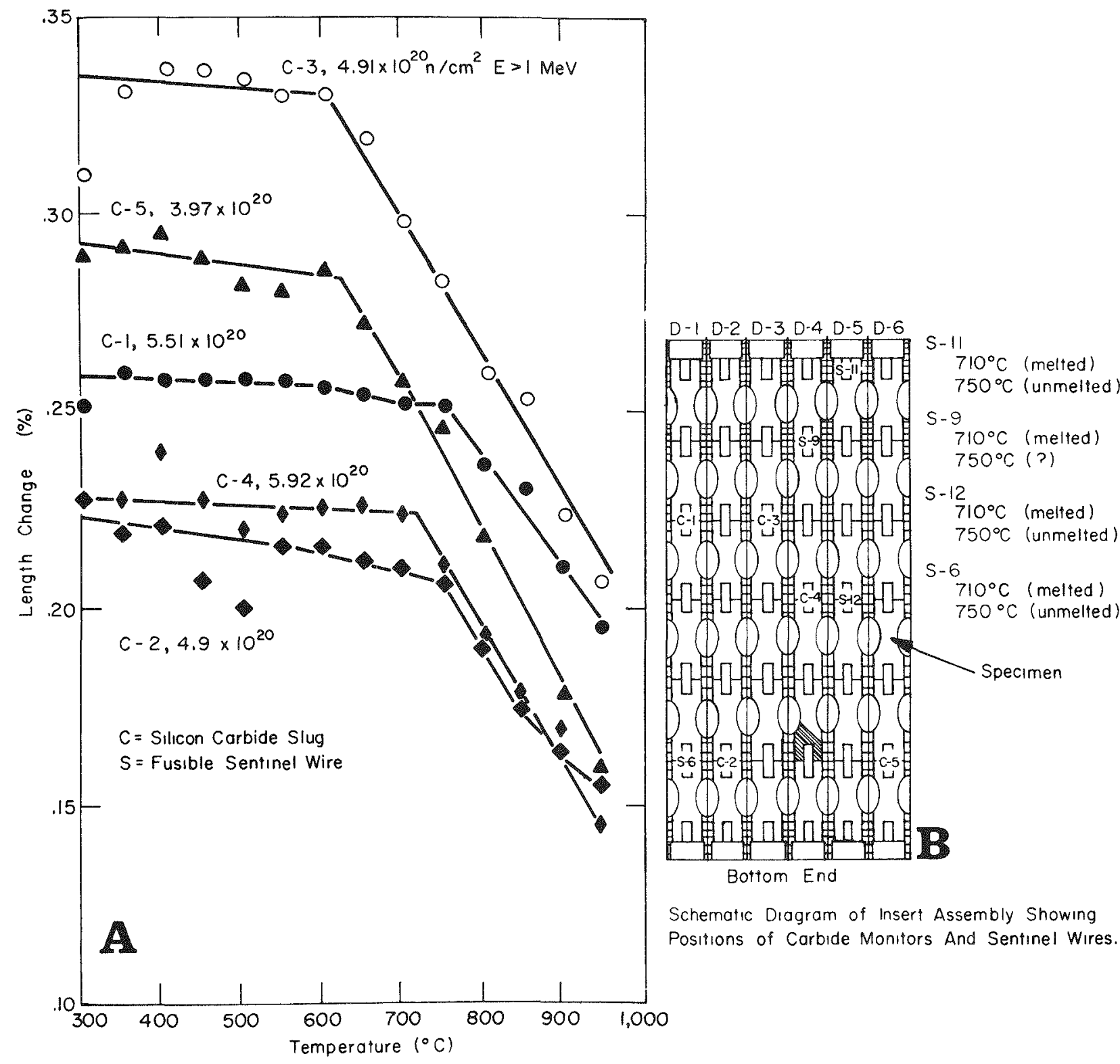

Figure 7, Isochronal Annealing Curves of The Silicon Carbide Slugs Included in The Argon Bonded Inserts of Subassembly $\times 037$. 


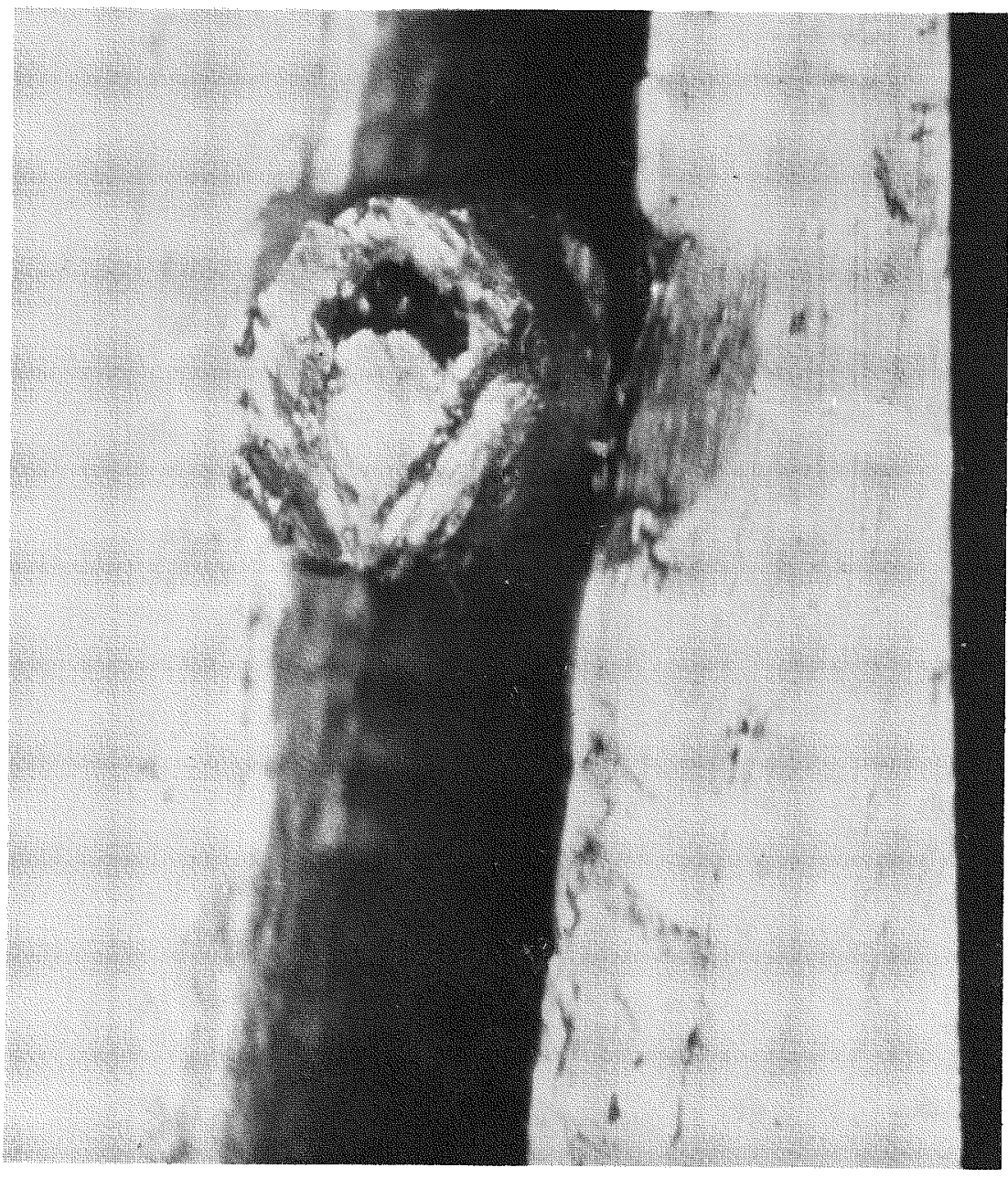

(A) Sentinel Wire Melted (M.P. $710^{\circ} \mathrm{C}$ )

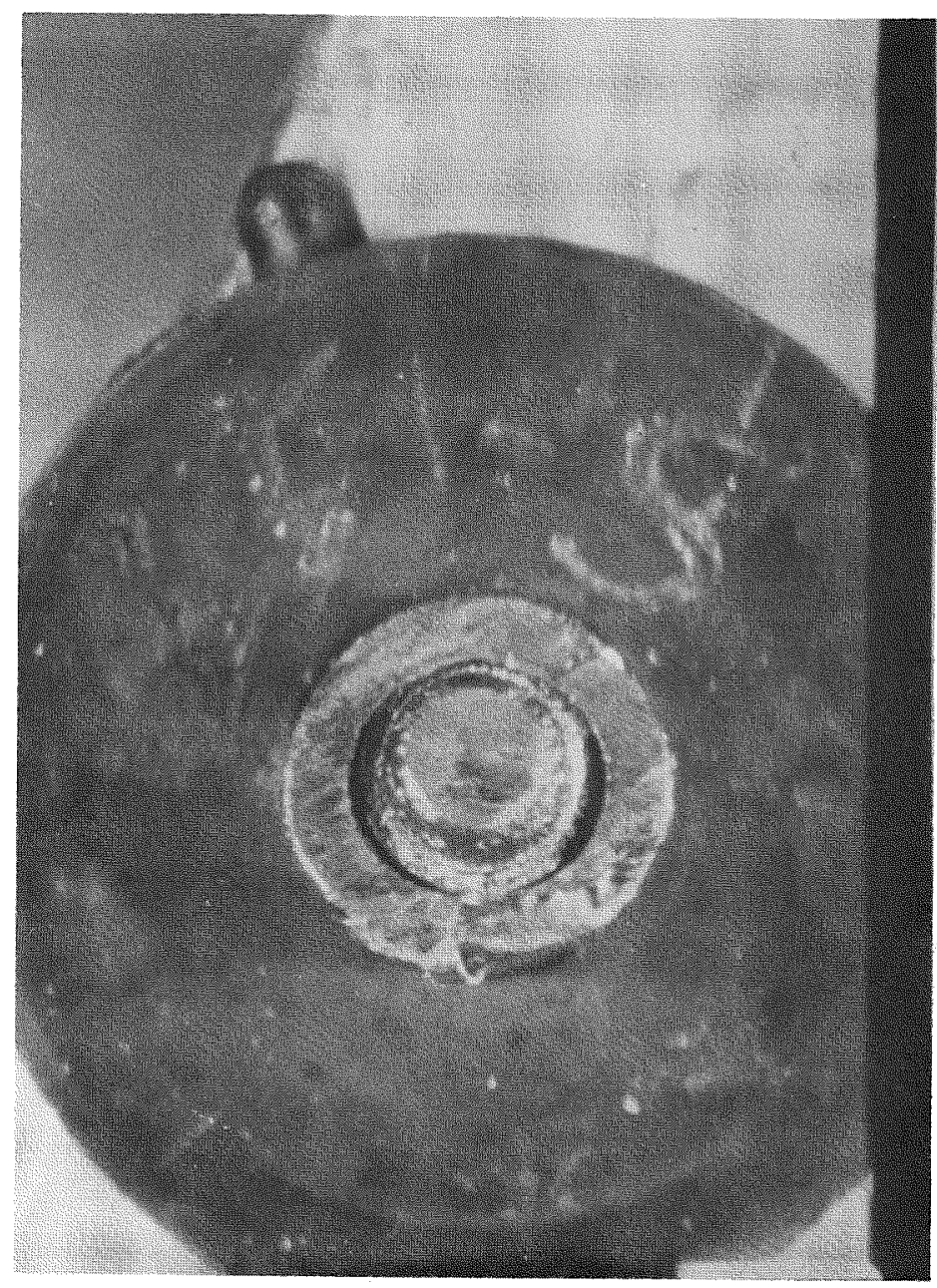

(B) Sentinel Wire Unmelted (M.P. $750^{\circ} \mathrm{C}$ )

Figure 8, Fusible Sentinel Wires From Post Irradiated Insert-Position S-11. 


\section{$X$ Capsules and Sodium Loops I'sed in Argonne National Laboratory Reactor Safety Experiments in the TREAT Reactor \\ L. E. Robinson, R. Ivins and C. E. Dickerman \\ 0639000 \\ Argonne National Laboratory, Argonne, I1linois}

Argonne National Laboratory is engaged in reactor safety experiments using the Transient Reactor Test (TREAT) facility. In these tests, reactor fuel specimens are subjected to power bursts to study the modes and mechanisms of failure, failure thresholds and the conseauences of fuel failure (including behavior of coolant). Originally, the work was divided between fast, liauid metal-cooled reactor safety studies and thermal, water-cooled reactor safety studies: now, the total effort is in sumnort of the Liauid Metal Fast Breeder Reactor (IMFBR) program. Throughout the TREAT experimentation, there has been a consistent requirement that exnerimental apparatus he canable of routinely accommodating conditions that normally are considered to be accident conditions. Hence, an important feature of the normal design criteria is that the apparatus contain safely large accumulations of molten fuel, and nossihle pressure pulses arising from sodium vaporization and "sodium hammer" impact.

The first experiments were simnle, and were performed on fuel pins contained in inert gas environment, using sealed stainless steel capsules whose walls were lined by protective gfaphite crucibles that served to freeze fuel meltdown products safely in situ. These cansules were originally used for uranium metal specimens, (meiting noint $\sim 14 n n^{\circ} \mathrm{K}$ ) but were later extended to oxide fuel (melting point $\sim 3100^{\circ} \mathrm{K}$ ). Instrumentation normally was limited to fuel thermocouples. Figure 1 shows the basic design of this capsule. The cylindrical capsule walls are of $0.15 \mathrm{~cm}$ thick stainless steel. The flat end (original) version had steel end plates $0.3 \mathrm{~cm}$ thick. Later versions with higher pressure ratings used hemispherical ends of the same thickness as the walls. This capsule was designed to freeze molten metal or ceramic fuel inside the graphite without overheating the steel walls. A special TREAT experimental dummy fuel element holds the cansule inside a $4.45 \mathrm{~cm}$ diameter zircaloy centering tube. The dummy element serves as a backup contaiment, as a holder inside the TREAT core, and as a convenient adaptor permitting use of the standard TREAT fuel handling apparatus and procedures. 
Water reactor experimentation hegan with stainless steel autoclaves designed to hold fuel and water with inert gas cover and to contain hoth fuel meltdown and the pressure generated hy metal water reactions. 2 Pressure and temperature measurements were made. Poth metal and oxide fuel pins were studied. Figure 2 shows the autoclave design. The hasic autoclave design 2 provided an experimental space $15 \mathrm{~cm}$ long hy $46 \mathrm{~cm}$ diameter. The stainless steel autoclave body was sealed to the stainless steel top plug by an asbestos-stainless steel flexitalic-type gasket. A typical sample arrangement consisted of a short test pin, inside a multiwall crucible composed of alumina, stainless steel, and zirconia layers, and a $50 \mathrm{cc}$ fill of distilled water. Total volume inside the autoclave was $200 \mathrm{cc}$. The autoclave was contained inside a stainless steel-clad TREAT dummy element. Autoclave instrumentation included a pressure transducer attached to the bottom, thermocouples, and a gas sampling valve to permit analysis of hydrogen produced by metal-water reactions inside the autoclave.

The next step in expanding experimental capabilities consisted of developing transparent capsules which could he viewed by high speed cameras with system capability of color photography at speeds un to 4000 frames per sec. 3 Figure 3 is a cutaway drawing of the hasic transparent design. 3 The sample, proper, is held by a sample frame inside a zircaloy cansule. This cansule, approximately $5.1 \mathrm{~cm}$ wite hy $50 \mathrm{~cm}$ high by 50 on deep is made from two $0.06 \mathrm{~cm}$ thick cansule subassemhlies. For exneriments in which thermal neutron shielding is required, the shielding material is inserted between the double zircaloy walls. The back end of the capsule has a mirror set at an angle with the viewing camera line of sight in order to provide a rear view of the sample. The front end of the cansule contains a window of high-purity fused silica. Graphite and zircaloy surround the zircaloy capsule as a backup in case of failure of the capsule (no cansule has yet failed). An outer, stainless steel can $0.15 \mathrm{~cm}$ thick provides a sealed containment for an inert gas purge and continuous cover. The entire assembly is slid into the TREAT reactor core, using one of the large core viewing slots.

Two basic modifications were made of the original in-pile apparatus: one for experiments with pre-irradiated fast reactor fuel in inert gas enviromment, ${ }^{4}$ and one for themal reactor fuels in water with inert gas cover. 5 These modifications are discussed in a companion paper. 6

Stagnant sodium autoclaves were also developed to permit attainment of a simple liauid metal environment for fast reactor fuel pin experiments. 7 Autoclaves and fuel were instrumented with thermocouples. 
This design used the basic opaaue capsule, with a sodium-containing inner cansule that held the fuel samnle. Flectrical heaters liauified the sodium and brought the sample to a pre-transient temperature of $260^{\circ} \mathrm{C}$. Figure 4 is a line drawing of this cansule, showing a pressure transducer attached to the inner capsule. Internal thermocouples could be substituted for the pressure sensor. To first order, the inner capsule was isolated thermally from the rest of the assembly. Thus, the temperature rises recorded for the outer surface of the inner capsule could be used to measure the sample energy released in the experiment.

Study of effects of prior Irradiation on fuel limits and post-failure phenomena is an integral part of the safety experimentation. Fngineering for remote operations was performed on the basic graphite-lined capsules, 7,8 and metal-water autoclaves, 9 as well as the transparent capsules. 4

With attainment of a basic canahility to study LMFBR fuel failure, it became necessary to develop apparatus providing more realistic core environment simulation, and permitting the study of coolant expulsion, generation of coolant pressure pulses, and secondary movements of coolant and fuel. Three different types of such advanced apparatus have been developed.

The first was an integral sodium loop, designed to hold a cluster of seven fuel pins of 0.174 in diameter. The loop package - including electromagnetic pump: and simple instrumentation including thermocouples, pressure transducer and electromagnetic flowmeter - fits within the $10 \mathrm{~cm}$ by $10 \mathrm{~cm}$ cross section of a TREAT fuel element.10,11

Figure 5 is a drawing of the Mark I 10op. Sodium flows unward from the Faraday-type electromagnetic pump, through the fuel holder, then down the return annulus around the test section through the twin return tubes until it reenters the pump section. In Fig. 6 is shown the cross section of the loop test section, showing the placement of a 7-pin cluster with spiral spacer wires wound around the pins. Figure 7 shows the final result of the most severe meltdown experiment conducted with this loop - a high energy meltdown of seven pins with no sodium flow. 12 In this case, a small loop failure occurred in the thin-walled pump duct, but all the fuel was held within the loop, and all of the sodium escaping from the loop was contained safely inside the sealed $0.15 \mathrm{~cm}$ thick steel secondary container. Although deposits of high density fuel occur throughout the loop, little fuel actually remains within the test section. This 1oop has a capability of 4 meters/sec sodium flow through the test section. Originally, the operating temperature was $260^{\circ} \mathrm{C}$, but this was raised, as the loops were modified during use, to $400^{\circ} \mathrm{C}$. About 10 experiments were carried out using the kark I loop. 
Next was a high pressure sodium autoclave designed for studies on coolant pressure pulses and voiding resulting from high energy input to refractory fuel samples. The autoclave is instrumented with a pressure transducer, thermocouples, and a linear motion detector to measure the transient expulsion of a piston above the sodium level.13 As shown in Fig. 8, the facility consists of an inner, or primary autoclave containing the fuel sample, sodium, and the transducers for temperature, pressure and linear motion, and an outer or secondary autoclave. The primary autoclave has a design pressure rating of $6000 \mathrm{psi}$ at $600^{\circ} \mathrm{F}$, with a safety burst valve designed to runture at the rated pressure. The secondary autoclave has a design rating of 600 psi at $600^{\circ} \mathrm{F}$, and provides a douhle-containment for the sodium at rupture of the safety burst valve. Provision is made for clusters of up to nine fuel pins of 0.29 in diameter, usually in a sauare array. As in the case of previously described capsules, the autoclave is designed to fit into the $10 \mathrm{~cm}$ sauare cross section of a single TRFAT fuel element. A series of experiments on stainless-steel clad $\mathrm{UO}_{2}$ fuel have been carried out in the sodium-filled piston autoclave, with the 9-pin cluster consisting of a sauare array of 5 fueled and 4 dumm rods. Each of the rods was 0.29 in in outer diameter, 12 in long, and had 15-mil walls: the fueled rods contained 41 grams of $10 \%$ enriched $\mathrm{UO}_{2}$ in a 5 in long column of 0.25 in diameter pellets helium-bonded to the cladding, below an upper blanket section designed to collanse under an external pressure of 6000 psi. The dummy pins were filled with 15 psi helium.

In the most recent experiment, a $650 \mathrm{cal} / \mathrm{g} \mathrm{UO}_{2}$ transient caused the rupture and destruction of the fueled nins, and resulted in a maximum pressiure pulse of about 2000 psi, with a maximum event temperature of $3500^{\circ} \mathrm{C}$.

The latest apparatus is a second-generation integral sodium loon, the Mark II, designed to contain the destructive meltdown of a cluster of seven refractory fuel pins of 0.29 in diameter in flowing sodium. Figure 9 is a line drawing of the Mark II loop. This loop fits within a cross section of $10 \mathrm{~cm}$ hy $20 \mathrm{~cm}$, the equivalent of two TRFAT fue 1 elements. Built in two versions, the Mark II has an order of magnitude higher pressure capability than the Mark I integral loop. The Mark IIA version has a design pressure rating of 5000 psi internal pressure at $1000^{\circ} \mathrm{F}$, while the Mark IIB version is rated at 4000 psi pressure at the same temperature. The prototype Mark IIB has heen proof-tested out-ofpile to $6200 \mathrm{psi}$ at $800^{\circ} \mathrm{F}$, with a sodium flow of 4 meters $/ \mathrm{sec}$. 
A maximum sodium flow of ahout 6 meters/sec is provided in the Mark II loop by an Annular Linear Induction Pump (ALIP), a polyphase traveling wave electromagnetic pump. 15 Experimental instrumentation consists of thermocouples, pressure transducers and electromagnetic flowmeters at the inlet and outlet of the test section. Safety against internal over-pressure is provided by a hurst-disc valve which is designed to rupture at the rated pressure of the loop and vent the molten sodium into the expansion space of a high-pressure safety tank. The integral loop is contained within a secondary vessel of $50 \mathrm{mil}$ stainless steel, with the control and instrumentation center, power supply and coolant sources well removed from the facility during its operation.

The prototype Mark II loop has been operated in the TREAT facility and checkout experiments on $\mathrm{UO}_{2}$ fuel are in progress at present. 


\section{REFERENCES}

1. C. E. Dickerman, E. S. Sowa, D. Okrent, J. H. Monaweck and L. B. Miller, "Studies of Fast Reactor Fuel Element Behavior Inder Transient Heating Leading to Failure. I. Initial Fxperiments on Metallic Samples in the Absence of Coolant," ANL-6334 (1.961).

2. R. C. Liimatainen, R. O. Ivins, M. F. Deerwester and F. J. Testa, "Studies of Metal-Water Reactions at High Temperatures. II. TRFAT Experiments. Status Report on Results with Aluminum, Stainless-Steel304, Uranium and Zircaloy-2," ANL-625n (1962).

3. G. H. Golden, C. E. Dickerman and L. E. Rohinson, "Facility for Photographing In-Pile Meltdown Experiments in TRFAT," ANL-6457 (1962).

4. L. E. Robinson and C. August, "Apparatus for High Speed Photographic Safety Experiments on Irradiated Fast Reactor Fue1," ANL (to be published).

5. L. J. Harrison and R. O. Ivins, "A Photographic Study of the Transient Meltdown Behavior of Aluminum/Iranium Fuel Plates in Water," Trans. Amer. Nuc1. Soc. 9, 561 (1966).

6. J. J. Barghusen, L. J. Harrison, R. O. Ivins and L. E. Robinson, "Observation of Fuel Failure Behavior During In-Pile Meltdown Tests Using High Speed Photographing and a Transparent Autoclave," Proc. this conference.

7. C. E. Dickerman, E. S. Sowa, J. H. Monaweck and A. Barse11, "In-Pile Experiments on Meltdown of EBR-II Mark I Fuel Elements in Stagnant Sodium," Nucl. Sci. Eng. 18, 319 (1964).

8. F. L. Willis, R. J. Schiltz and J. H. Monaweck, "Remote Encapsulation of Pre-Irradiated Fuel Elements for Exposure in TREAT," Reactor Physics Division Annual Report, July 1, 1964 to June 30, 1965, ANL-7110, 280 (1965).

9. R. C. Liimatainen, R. O. Ivins and F. J. Testa, "Reaction of Uranium with Water in TREAT Meltdown Excursion," Trans. Amer. Nucl. Soc. $\underline{8}$, No. 1,306 (1965).

10. E. S. Sowa and J. C. Heap, "The Development of a Small Integral Loop for In-Pile Fuel Failure Studies in the Presence of Flowing Sodium," Trans. Amer. Nuc1. Soc. $\underline{8}$, No. 2, 559 (1965). 


\section{REFERFNCES (Contd.)}

11. L. E. Robinson, R. T. Purviance and F. L. Willis, 'Morification of the TREAT Integral Sodium Loops," Reactor Physics Division Annual Report, July 1, 1964 to June 3n, 1965, ANL-7110, 268 (1965).

12. L. E. Robinson, C. E. Dickerman and F. L. Willis, "Initial (7-Pin) EBR-II Mark I Scale Up Cluster Fast Reactor Safety Integral Loop Experiments in TREAT," Trans. Amer. Nuc1. Soc. 10, (2) 697 (1967).

13. J. F. Boland, R. C. Liimatainen and F. J. Testa, "TREAT Studies of Fuel Meltdown in Sodium," Chemical Engineering Division Semiannual Report July-December 1966, ANL-7325, 170 (1967).

14. L. E. Robinson, "Design of the Mark II Integral Transient Reactor Test (TREAT) Facility Sodium Loop," Reactor Physics Division Annual Report, July 1, 1964 to June 30, 1965, ANL-7110, 279 (1965).

15. L. E. Robinson and R. D. Carison, "Development of Pumps for Use in Fast-Reactor-Safety Integral-Loop Experiments," ANL-7369, 1968. 


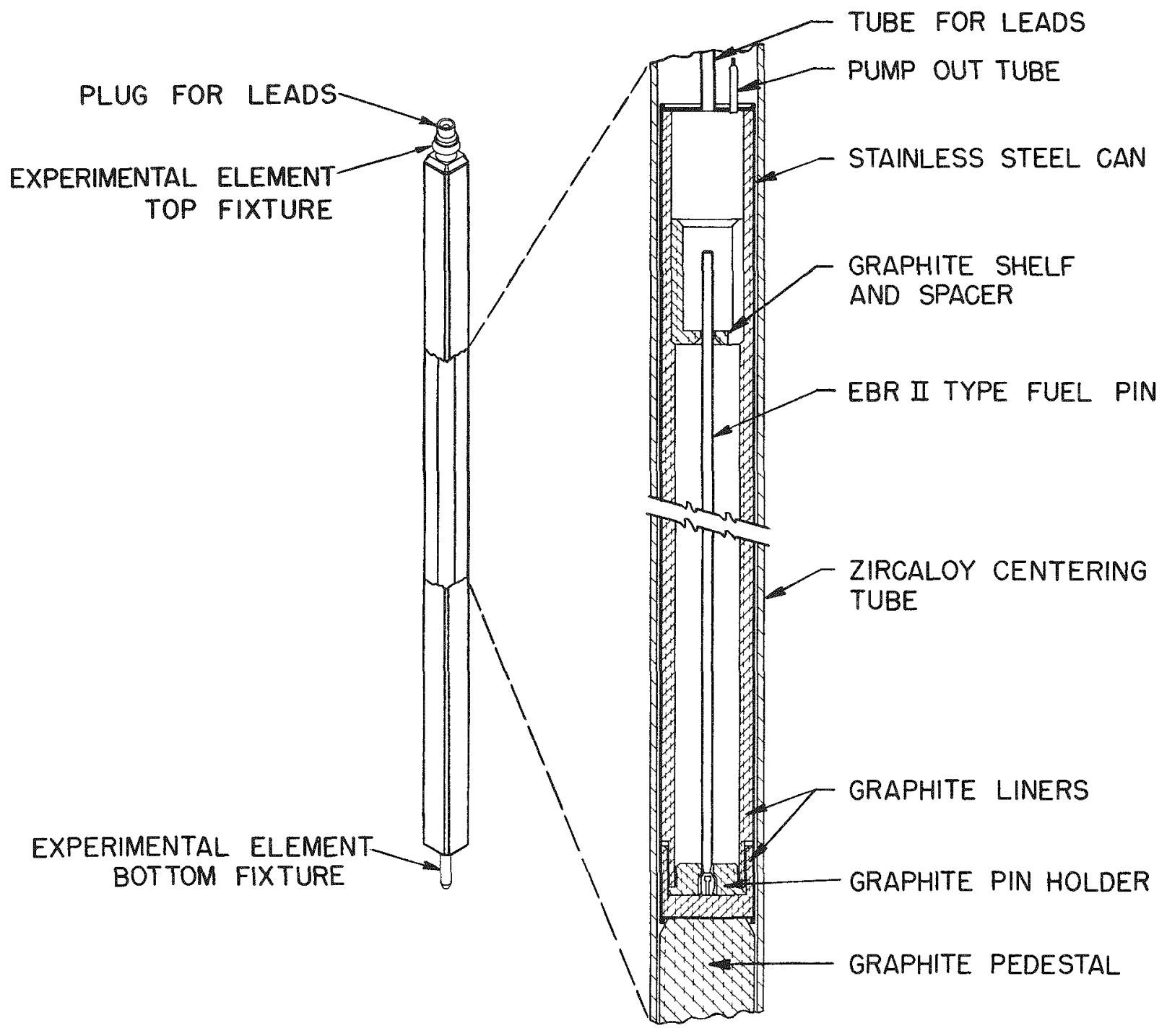

Figure I Dry, Opaque, Single Element Cassule 


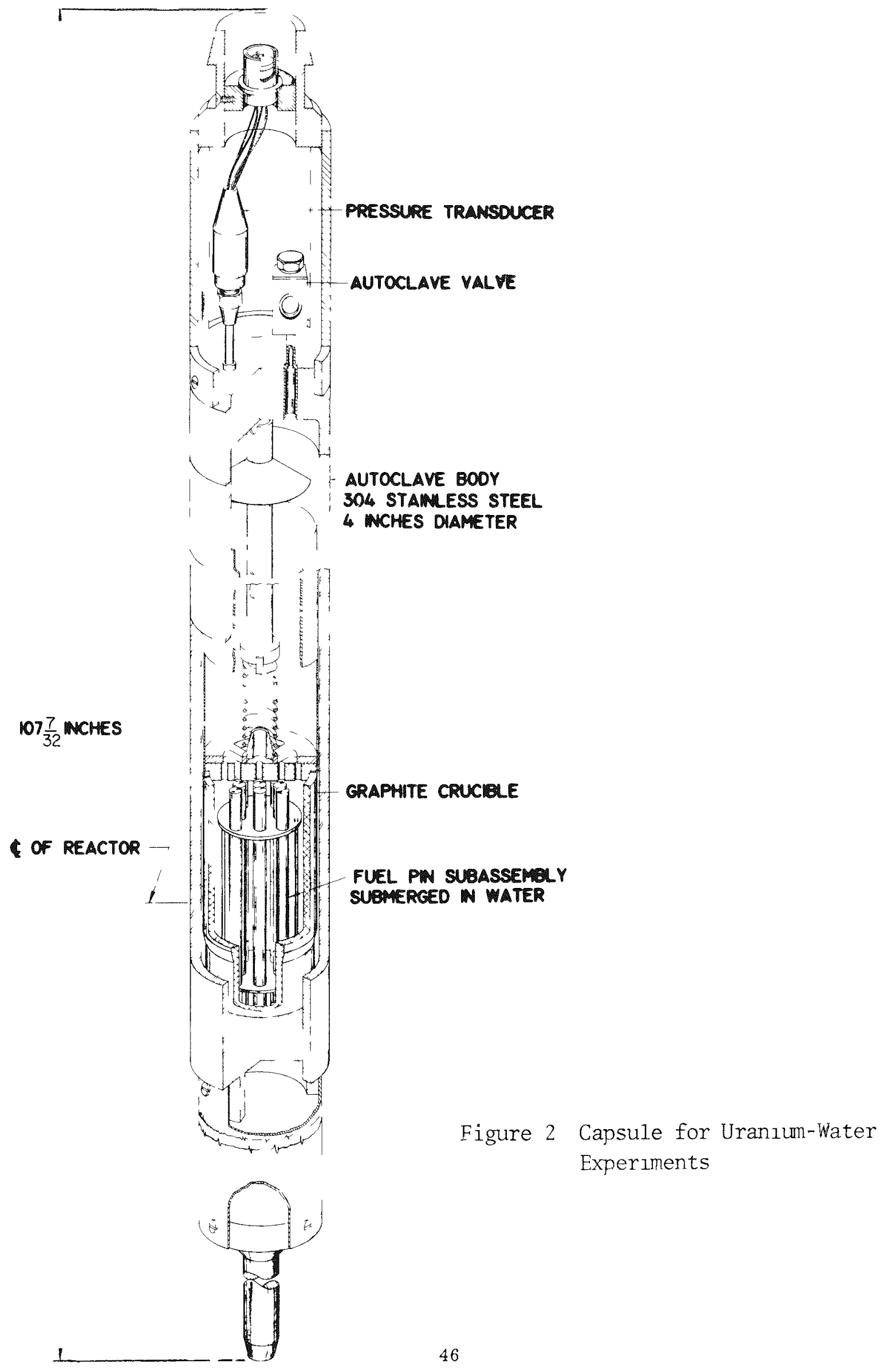




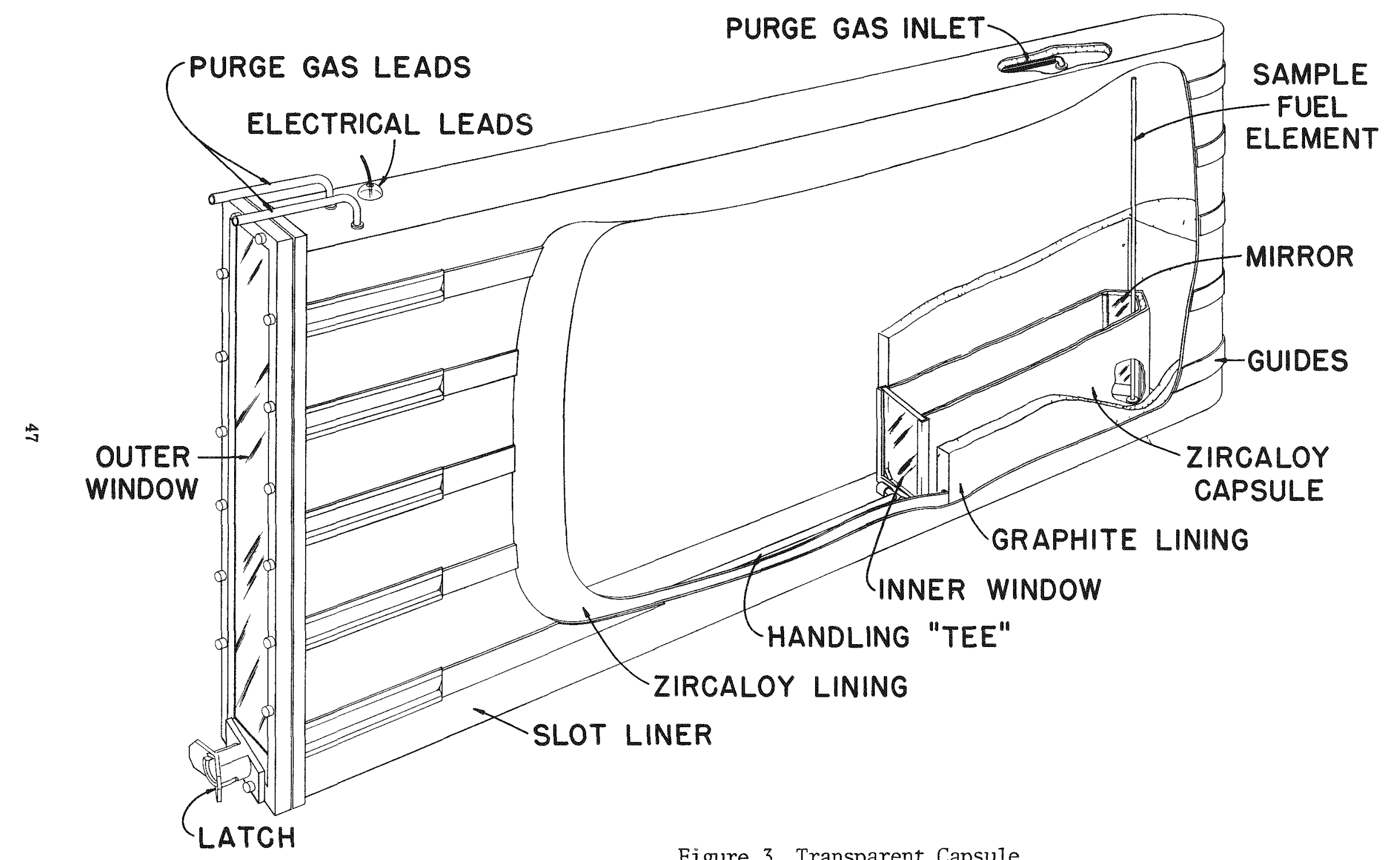

Figure 3 Transparent Capsule 


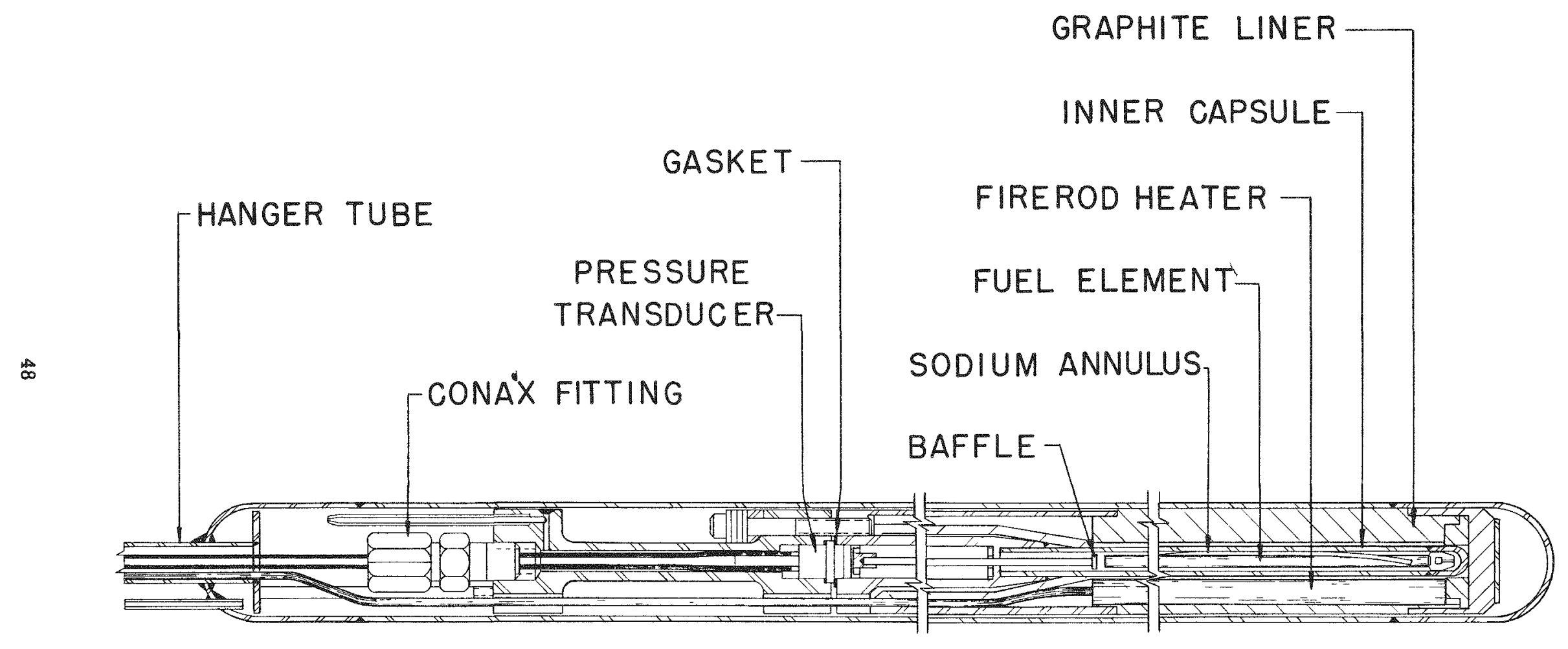

Figure 4 Stagnant Sodium Capsule 
1. OUTER CONNECTOR

2. GRIPPER CAP

3. INNER CONNECTOR

4. UPPER FLANGE

5. TREAT FUEL CAN

6. LOOP SEAL FLANGE

7. FUEL HOLDER EXTENSION

8. RUPTURE DISK

9. SAFETY TANK

10. UPPER PRESSURE TRANSDUCER

11. TEST SECTION HEATER

12. FUEL HOLDER

13. FUEL HOLDER SEAL

14. HARP HEATER

15. LOWER PRESSURE TRANSDUCER

16. PUMP COILS

17. LEAK DETECTOR

18. PUMP TRANSFORMER

19. FLOWMETER

20. EM PUMP MAGNET

21. EM PUMP SECTION

DIMENSIONS IN CENTIMETERS

Figure 5 Mark I Loop

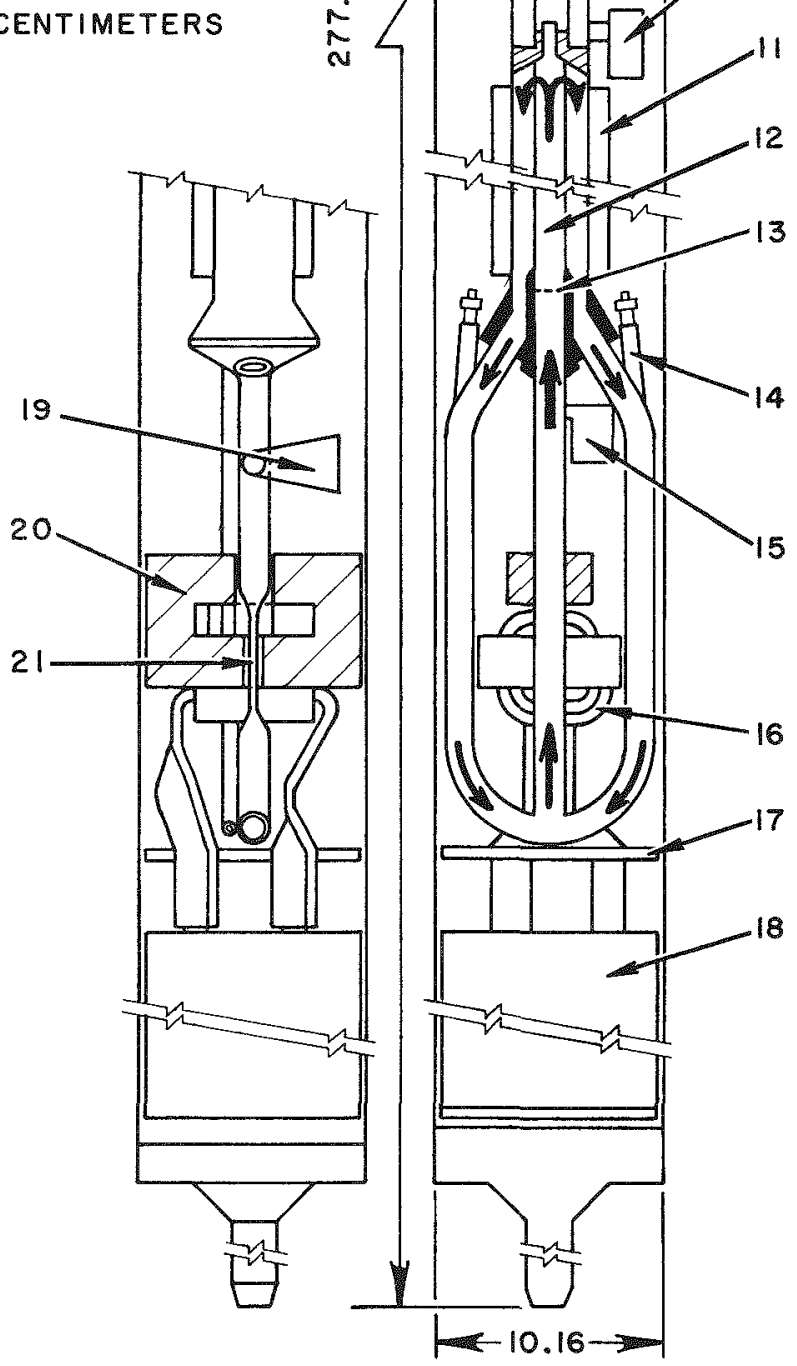



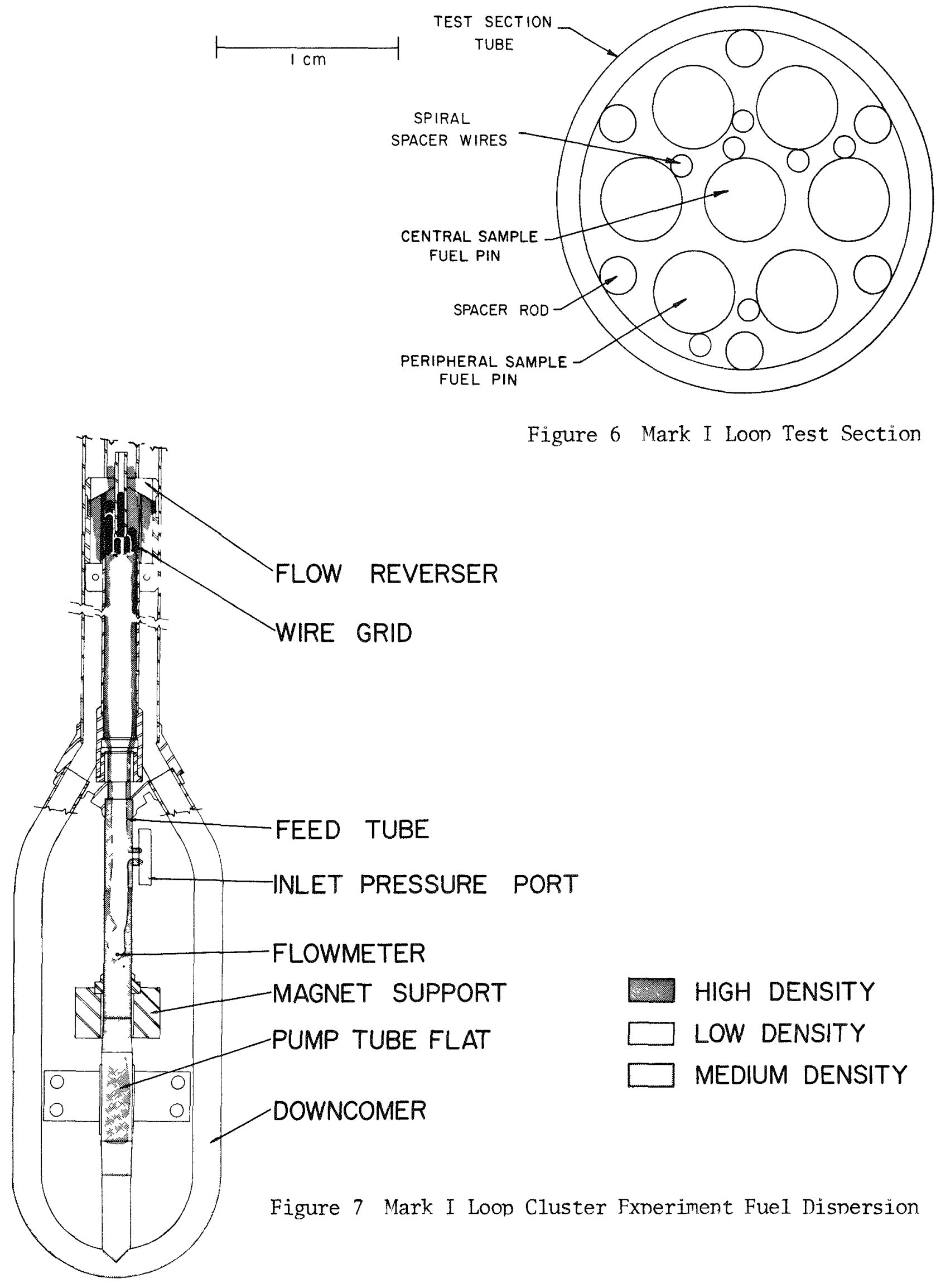

Figure 6 Mark I Loon Test Section 


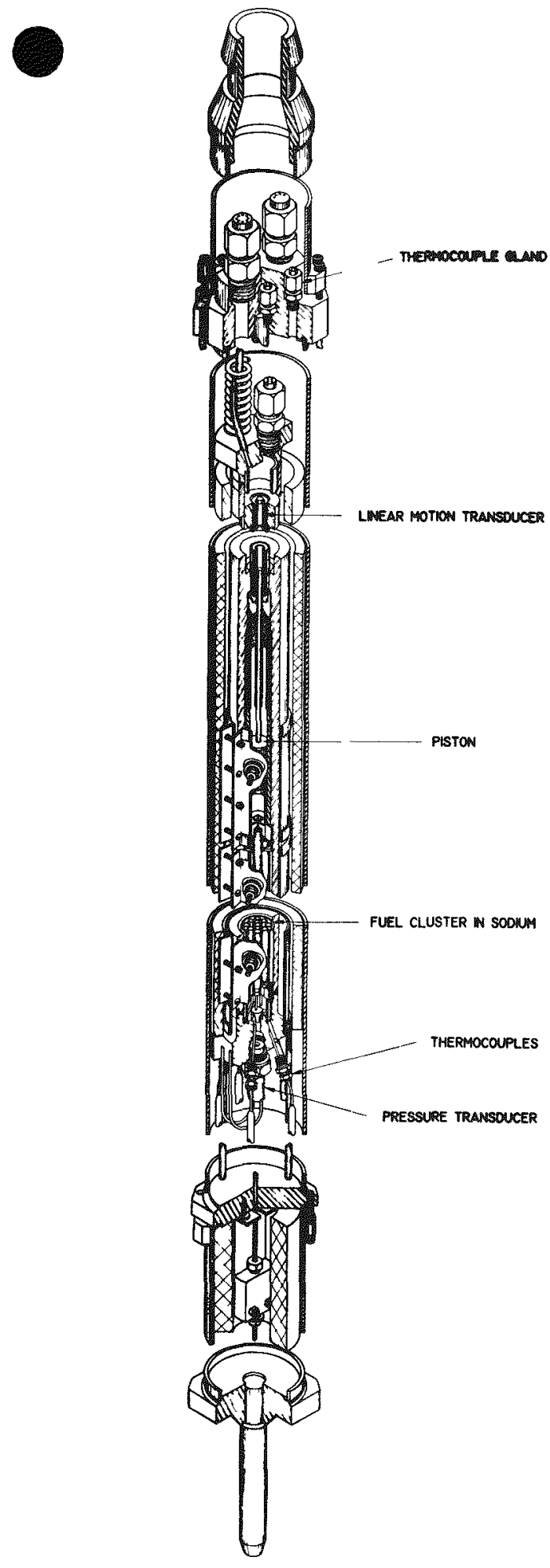

Figure 8 Sodium-Filled Piston Autoclave

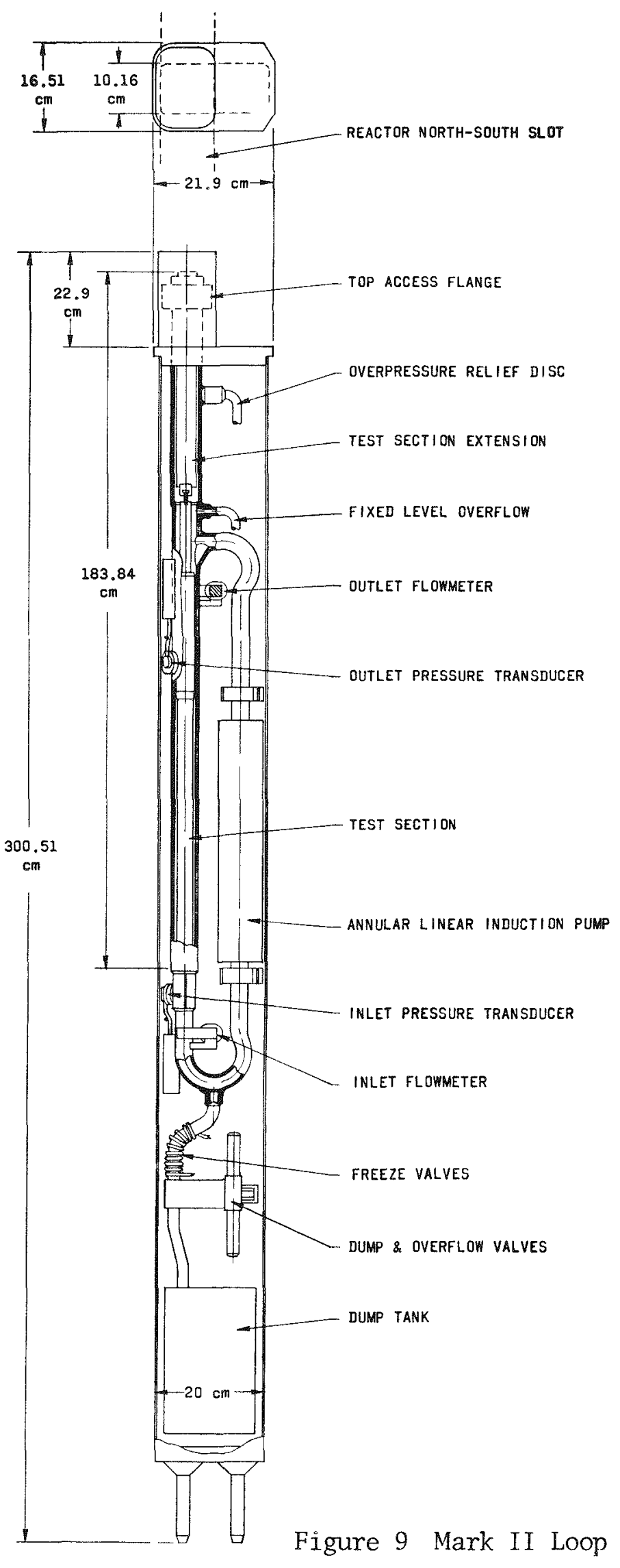


X Use of the Thermal TREAT Reactor for Fast Reactor Safety Studies

C. E. Dickerman, R. O. Ivins, A. R. Rothman and J. F. Roland Argonne National Laboratory, Argonne, Illinois

The Transient Reactor Test (TRFAT) facility was designed and built by Argonne National Laboratory specifically for use in ISAEC fast reactor safety research. Principal design goal was attainment of the maximu feasible integrated thermal neutron flux over a sizeable core volume, in a short reactor power burst. 1 The reactor fuel consists of fully-enriched urania particles dispersed in a graphite matrix, with a ratio of carbon to 2351 atoms of 10,000 . Thus this design provides a large (carbon) heat sink in intimate contact with the fuel. In addition, as the graphite heats un during a power burst, it raises the effective themal neutron temperature, which raises the ratio of leakage to fission and generates a sizeable prompt negative temperature coefficient of reactivity. Renorts on the reactor proper include the engineering report, ${ }^{2}$ reactor physics measurements ${ }^{3}$ and a kinetics analysis of the reactor behavior in use as a transient testing reactor. ${ }^{4}$ Under the current limit of $600^{\circ} \mathrm{C}$ maximum core temperature, the maximum (unperturbed) integrated themal neutron flux is about $9 \times 10^{15} \mathrm{nvt}$.

Core fuel elements are of $10 \mathrm{~cm}$ souare cross section. One or more elements can be removed to provide a test space. Special slotted elements can be loaded to provide a viewing slot extending into the core center. Figure 1 shows a cutaway view of the reactor. The zircaloy-clad core is $120 \mathrm{~cm}$ high. Each TPEAT fuel element has two $60 \mathrm{~cm}$ long, aluminum-clad graphite axial reflector sections, one attached at the bottom of the core section and one attached above. The core cavity inside the concrete shielding is lined with a $120 \mathrm{~cm}$ thick stacked, permanent graphite reflector. Aluminum- or zircaloyclad graphite radial reflector elements are interchangeable with the TREAT fuel elements, and provide flexibility in core loaring. Also shown in the Fig. is a viewing slot extending into the core center. A mirror system, pulsed Xe light, and high speed camera are used to take motion pictures of meltdown experiments conducted in the core. 5 On the reactor face opnosite the high speed (optical) camera is a fast neutron hodoscope device. When the viewing slot is extended completely through the core, the hodoscone can he user to detect the horizontal and vertical distribution of fissioning test samnle fuel as a function of time. Figure 2 is a cutaway view of the horoscone system.

Reactor power burst durations ranging from $0.05 \mathrm{sec}$ to tens of seconds are possible. These make it possible to study fuel 
failure limits and consequences over a wide range of conditions between the limiting cases of millisec reactor periods and steady state operation. Figure 3 shows power vs time curves for typical TREAT transients, grouped for convenience in one graph. Each power curve is labeled by the initiating reactivity input, in $\Delta \mathrm{K}$. Within the current operating limits, i.e., a temperature limiter transient at $600^{\circ} \mathrm{C}, \mathrm{k}_{\mathrm{ex}}(0)$ can be raised to approximately $5 \%$. The maximum transient conducted in TREAT produced a maximum power of about 17,000 $\mathrm{M}^{\mathrm{TT}}$, and an integrater nower at $60 \mathrm{sec}$ of 2,150 MW-sec, for an input of $4.9 \% \mathrm{k} .7$ It is possible to obtain "flattened" power bursts, in which the reactor power is sustained near the temperature feedback-limited peak value by pre-programmed withdrawal of a control rod to compensate approxinately for the negative feedback of the reactor. In typical experiments, power variations during the "flattened" portion are within about $\pm 15 \%$ 。

A neutron radiographic facility ${ }^{8}$ was installed at TREAT during 1967 to permit nondestructive examination of pre-test and post-test condition of samples run in TREAT (and also the nearhy EBR-II). Typical data from this radiographic facility include fuel rearrangement inside the sample cladding, and fuel configuration inside a steel capsule resulting from post failure motions.

Effects of extended irradiation (such as structural damage, fuel restructuring and fission gas inventories) on transient behavior can be studied by use of samples irradiated previously in a suitahle environment. Undesirable, unrepresentative transient temperature distributions produced by thermal neutron flux depressions inside a test sample can be avoided by use of low enrichments, thermal neutron flux filters, or by suitably matching power burst durations to the test sample heat transfer time constant. 9 For experiments on clusters of pins, the approaches thus far have been to use relatively low enrichments, with either: (1) a variation in the enrichment of individual pins, to compensate for neutron flux depressions at specific radial positions in the cluster, 10 or (2) uniform enrichment ${ }^{11}$ depending upon experimental requirements.

The relatively simple modular TRFAT core design and simple air cooling system provide a flexible system suitable for transient studies involving a variety of experimental apparatus ranging from simple capsules through flowing sodium loops. Fven though true steady state operation is not possible, snecial "limiting case models" have heen used in setting up 10op experiments with flattened power hursts to provide data for analyses of the short term conseauences of fuel nin failure under steady state conditions. 12

The first large scale use of information from TREAT exneriments was the incorporation of failure threshold, failure mode, and kinetics of failure data for FRR-II driver fuel nins into analyses of the spreads in fuel failure times across an FRR-II like reactor under conditions of flow decay, coolant hlockage, and power excursions. These calculations showed that consireration of realistic fallure data, along with real variations in specific nower and flow rates across a reactor, can produce a significant decrease in the rate of failure. Conseauently the calculated rates of meltdown-produced reactivity 
changes or rates of attack of adjacent narts of the core are significantly lower than the rates predicted previously, even when the time scales associated with a single subassembly are considered. Phenomena of coolant expulsion and reentry, along with associated impact "sodium hammer" pulses have been demonstrater in loop experiments. 14 Loop 10 and stagnant sodium autoclave 11 experiments have confirmer existence of spreads in fuel pin failure times for small clusters, and demonstrated secondary movements of fue 1 and coolant. Loon experiments have also been performed to confirm the canability of EBR-II driver fuel to survive a loss of thermal bond early in irradiation.12 Atomics International Investigators observed extensive post-failure dispersal of fuel and showed how this could reduce the severity of a fast reactor accident.15 General Electric nersonnel have sturied fuel behavior 16,17 up to and including failure, for both unirradiated and pre-irradiated mixed oxide fuel pins. A program of transient experiments on pins from the Fast Fuel Test Reactor develonment nrogram is heginning, as direct support for the FFTF project.18

An advanced TREAT control system, consisting, of two servo-hydraulic control rod drives under closed-1oon computer control, is being procure to provide extended capabilities for programmed transients. Within certain practical limits, this system will make possible arbitrary TREAT power burst shapes, as long as the maximum core temperature remains below $600^{\circ} \mathrm{C}$. Precision and repeatibility of flattened power bursts will be significantly improved. Design and calculation work is underway on a converter region that would produce a fast neutron flux environment in the core center, in order to make nossible experiments on clusters of plutonium-bearing fuel pins without unacceptable power depressions. 19,20

\section{REFERENCES}

1. G. A. Freund, H. P. Iskenderian and D. Okrent, "TREAT, A Pulsed Graphite-Moderated Reactor for Kinetic Experiments," Proc. 2nd Int. Conf. Peaceful Ises of Atomic Fnergy, Geneva, 1958, Vol. X, 461, United Nations, New York (1959).

2. G. A. Freund, P. Elias, D. R. MacFarlane, J. D. Geier and J. F. Boland, "Design Summary Report on the Transient Reactor Test Facility (TREAT)," ANL-6034 (1960).

3. F. Kirn, J. F. Boland, R. D. Cook and H. Lawroski, "Reactor Physics Measurements in TREAT," ANL-6173 (1960).

4. C. E. Dickerman, "Kinetics of the Transient Reactor Test Facility (TREAT)," Reactor Kinetics and Control AEC Symposium Series, p. 419, USAEC, Nak Ridge (1964).

5. C. E. Dickerman, G. H. Golden and L. F. Robinson, "In-Pile Photographic Studies of FBR-II Mark I and Fermi Core A Sample Meltdown," Nuc1. Sci. Eng. 14, 30 (1962). 
6. A. DeVolpi, R. Pecina, C. H. Freese, E. Rolnicki, D. Travis and G. Larsen, "Extended Capability for Fast Neutron Hodoscone Isen at TREAT," IEEE Trans. Nuc1. Sci. NS-15(1), 77 (1968).

7. J. F. Boland, "Operating Experience with TRFAT," Proc. Conf. Reactor Cperating Experience, Cctober 1-3, 1969, San Juan, Puerto Rico, Supplement to Vo1. 12, Trans. Amer. Nucl. Soc., 15 (1969).

8. J. F. Boland, "Neutron Radiography - A Tool for Nuclear Materials Safeguards," Nuc1. News 11, 8, 48 (1968).

9. C. E. Dickerman, "Use of Present TRFAT Core as a Fast-Flux Loop Meltdown Facility," Trans. Amer. Nucl. Soc. 9, No. 2, 551 (1966).

10. L. E. Robinson, C. E. Dickerman and F. L. Willis, "Initial (7-Pin) EBR-II Mark I Scale Up Cluster Fast-Reactor Safety Integral Loon Experiments in TREAT," Trans. Amer. Nucl. Soc. 10, No. 2, 697 (1967).

11. J. J. Barghusen, D. R. Anderson, J. F. Bolant, R. W. Mouring and J. C. Hesson, "Fuel Failure Behavior During Transient Meltdown in a Sodium-Filled Piston Autoclave," Trans. Amer. Nucl. Soc. 12 (2) (1969) to be puhlished.

12. C. E. Dickerman, F. L. Willis, R. R. Smith, P. B. Henault, R. Purviance, J. F. Boland, A. DeVolpi, R. A. Noland, J. Regis, A. B. Cohen and C. M. Walter, "TREAT Sodium Loop Experiments on Performance of Unbonded, Inirradiated FBR-II Mark I Fuel Elements," (to be published in Nucl. Eng. and Des., Decemher 1969).

13. D. V. Gopinath and C. E. Dickerman, "Calculations of Coherence of Failure for Hypothetical Meltdown Accidents in an FBR-II Like Reactor," ANL-6844 (1964).

14. C. E. Dickerman, R. Purviance, L. E. Rohinson, W. Stephany and F. L. Willis, "Summary and Analyses of TRFAT Sodium Loop Experiments on Behavior of Single EBR-II Mark I Pins Inder Accident Conditions," Nucl. Eng. Design, 7, 442 (1968).

15. S. J. Stachiva, R. N. Cordy and M. Silberherg, "Iranium Carbide Transient Heating Studies - Phase II," Trans. Amer. Nucl. Soc. $\underline{8}(1), 305(1965)$.

16. G. R. Thomas and J. H. Field, "Destructive Examination of an Axially-Restrained Pelleted Mixed-nxide Specimen That Failed During Transient Nverpower Conditions," Trans. Amer. Nuc1. Soc. 11 (2) 697 (1968).

17. G. R. Thomas and J. H. Field, "Transient Performance of MixedOxide Power Fuel with un to $60 \%$ Melting," Trans. Amer. Nucl. Soc. 12 (1), 343 (1969). 
18. C. A. Burgess, J. E. Hanson and B. R. Hayward, "FFTF Fuel Development Program," Proc. National Tonical Meeting on Fast Reactor Systems, Materials and Components, Southwestern (hio Section, Amer. Nucl. Soc., Cincinnati, 1968 (1969).

19. A. P. Gavin, "Preliminary Engineering Design of the TREAT Fast Neutron Converter," Reactor Physics Divison Annual Progress Report for July 1, 1968 to June 30,-1969, to be nublished.

20. H. P. Iskenderian, "Reactor Physics Calculations on Preliminary Engineering Design of TREAT Fast Neutron Converter," Reactor Physics Division Annual Report for July 1, 1968 to June 30, 1969, to be published.

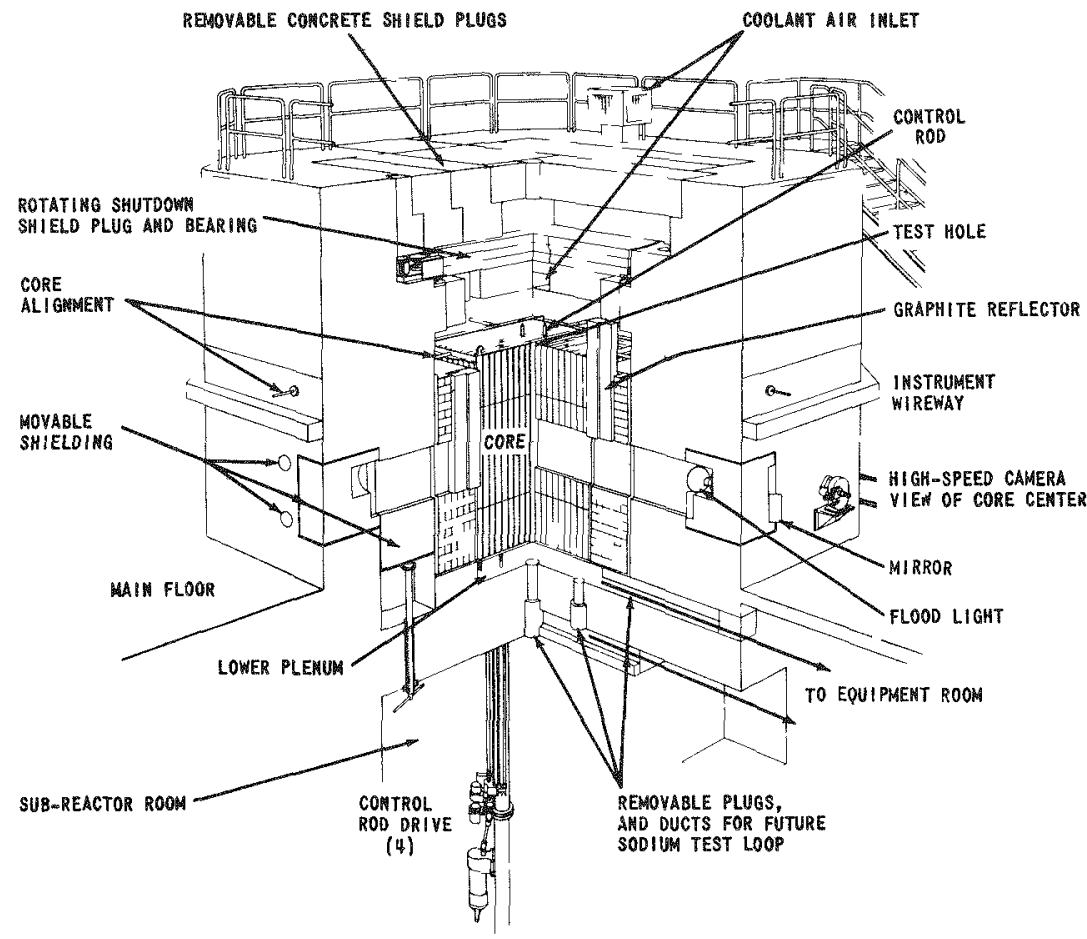

Figure 1 Cutaway View of the TREAT Reactor 


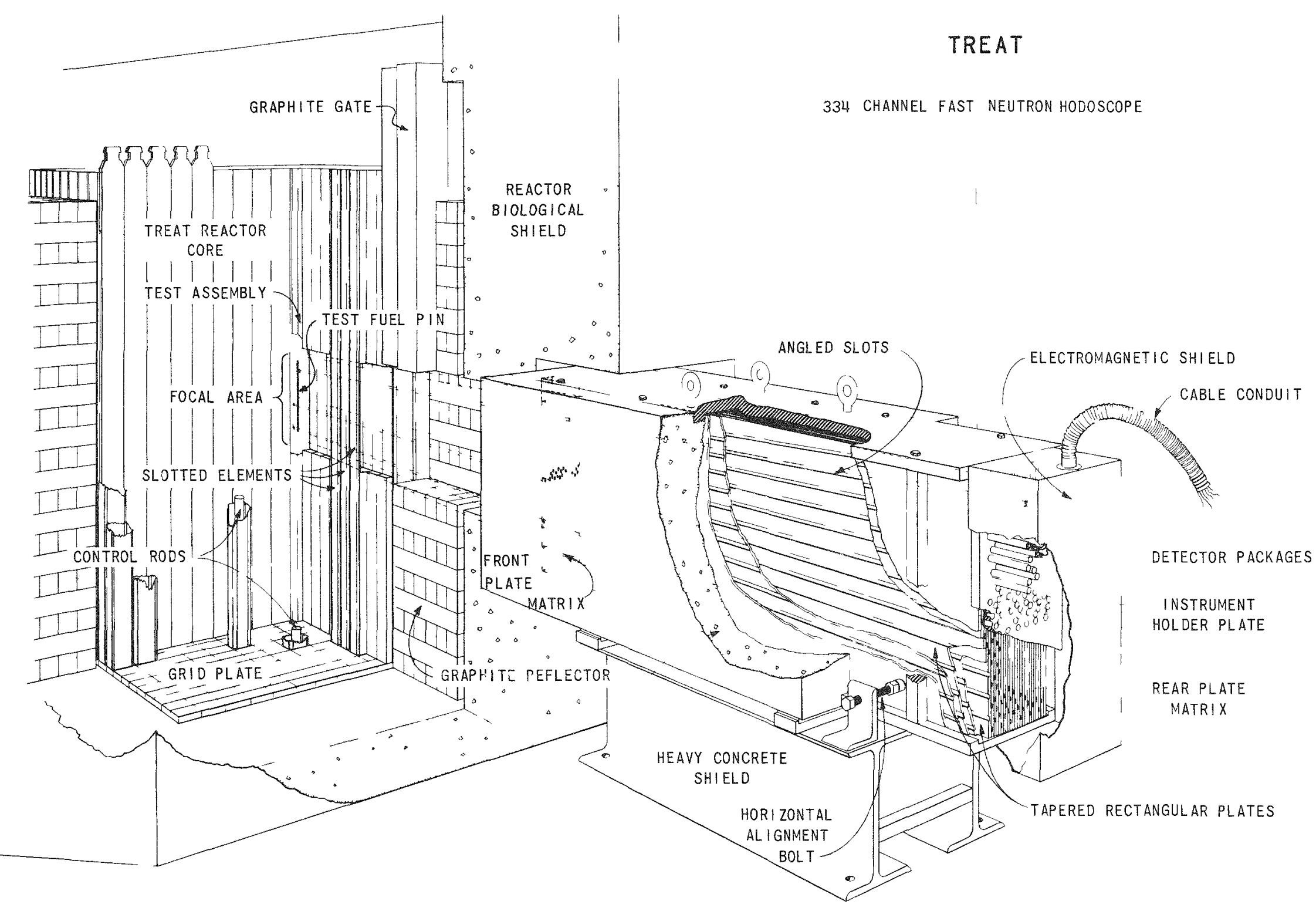

Figure 2 View of the Fast Neutron Hodoscone at TREAT 


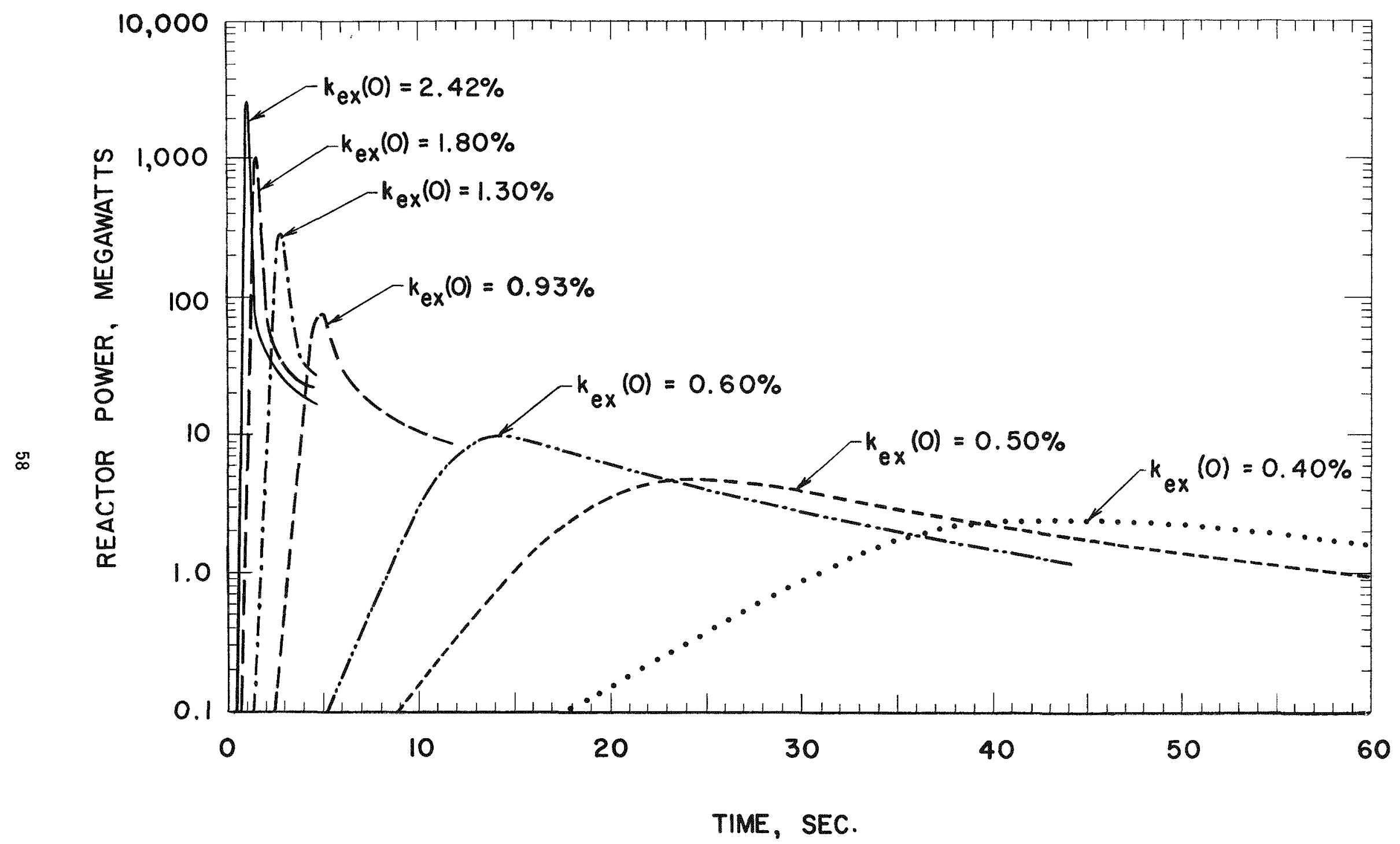

Figure 3 Typical Power vs Time Curves for TRFAT 


\section{$X$ XENON TAgging OF INSTRUMENTED FUEL CAPSULES FOR USE IN EBR-II* by D. E. Walker, P.J. Halfmon, and J. H. Sanecki \\ Argonne National Laboratory \\ $06 \% 9800$ 9700 South Cass Avenue Argonne, Illinois 60439}

\section{INTRODUCTION}

Personnel of the Reactor Engineering Division at Argonne National Laboratory have designed an instrumented subassembly, containing some fueled elements, for use in EBR-II. The sensors in this subassembly will be capable of providing signals for (1) fuel-center temperatures up to $2000^{\circ} \mathrm{C}$, (2) cladding temperature, (3) fuel-element internal pressure, (4) sodium inlet and outlet temperatures, (5) sodium flow rate, and (6) neutron flux. Its present design incorporates 19 elements and will allow the use of 22 instrument leads.

Searches for the sources of indicated fission-product releases in EBR-II have customarily been conducted by removing one or more of the most suspect subassemblies and then operating the reactor. This procedure is repeated until the source of the release is found. When removing the instrumented subassembly from the reactor, however, a11 its instrument leads will have to be cut thereby destroying the utility of the instrumentation. Therefore, in the event of a fission-gas release in the reactor, it will be very desirable to determine if any of the elements in the instrumented subassembly have failed without removing the subassembly from the reactor. To permit rapid and positive indication that the subassembly contains a failed or defected fuel element, each element will be tagged by injecting approximately $1 \mathrm{ml}$ of a gas containing a unique mixture of xenon isotopes to the helium bond gas. The resulting gas mixture consists of approximately 14 vol\% of xenon in helium.

Other experimental subassemblies being inserted in EBR-II also are xenon tagged with other unique mixtures of xenon isotopes. If an element in any of these subassemblies should fail in the reactor, the tag gas would be liberated to the reactor's argon cover gas, where it would be detected. An evaluation of the xenon isotopic ratio would identify the subassembly containing the failed element.

*This work was performed under the auspices of the United States Atomic Energy Commission. 
Table I compares the isotopic compositions of natural xenon and the special xenon tag gas used in the elements in the instrumented subassembly. since natural xenon is present in the argon cover gas, a unique isotopic mixture for each experimental subassembly must be established. In the tag shown, the ratio of $129 \mathrm{Xe}$ to $124 \mathrm{Xe}$ has been characterized. The $124 \mathrm{Xe}$ present in the natural xenon is negligible and therefore will not significantly change the established ratio in the cover gas. Considerable work has been done by Coleman and Bernstein at Argonne to develop these unique mixtures.

\section{DESCRIPTION OF THE XENON-TAGGING PROBLEM}

Fuel elements for test 2 of the EBR-II instrumented subassembly contain uranium dioxide pellets that are $44.5 \%$ enriched in $235 \mathrm{U}$. These pellets are clad with Type 304 stainless steel and are bonded with helium at $1 \mathrm{~atm}$. The elements are encapsulated in a second Type 304 stainless steel tube that is sodium bonded to the element. One milliliter of xenon tag gas at 1 atm must be charged into the helium in the element just before the element is welded closed. After the element has been tagged and welded, there is no way of determining if it contains xenon. Therefore, a reliable method of injecting the tag had to be used. The method had to ensure that at least $75 \%$ of the required tag would be retained in each element. (The amount retained is called the amount of "xenon recovery.")

\section{DESCRIPTION OF XENON-TAGGING EQUIPMENT AND ITS OPERATION}

The xenon-tagging method developed by Coleman and Bernstein was used. with this method, the tag is inserted into the element by a hypodermic syringe fitted with a long needle that is placed into the element. After the insertion, the element is quickly capped and welded closed.

This method requires a minimum of equipment other than that normally needed to assemble and weld experimental fuel elements. Figure 1 is a schematic illustration of the equipment required. The equipment consists of an inertatmosphere welding chamber equipped with glove ports, an external xenon supply system that is integral with the welding chamber, and a rubber septum that separates the xenon supply system from the welding chamber. Not shown is the hypodermic syringe, a high-quality, gas-tight model of 5.0-ml capacity and graduated in tenths of a $\mathrm{ml}$. The rubber septum is a special three-layer selfhealing rubber that will permit many penetrations by the needle and still maintain an effective seal.

Figure 2 shows the external xenon supply system (left) attached to the welding chamber. The xenon tag gas was supplied in a $30-\mathrm{ml}$ stainless steel cylinder (A) capped with a leaktight bellows-seal valve. Two other important parts of the system are the pressure gage and the small-diameter cylinder (B) to the right of the supply cylinder. This small cylinder has a volume of approximately $0.8 \mathrm{ml}$.

In use, the system beyond the supply cylinder is evacuated up to the rubber septum, which is inside the welding chamber. The xenon gas in the supply cylinder is allowed to fill the complete system, after which a reading of the total system pressure is obtained. Liquid nitrogen is then applied to the outside of the small cylinder, causing all the xenon gas in the system to be cryogenically pumped into the cylinder. When all the xenon has solidified in the sma11 cylinder, the pressure gage indicates the vacuum in the system. Next, 
the valve isolating the small cylinder from the system is closed, the liquid nitrogen is removed, and the small cylinder is allowed to warm up to room temperature. This causes the xenon in the cylinder to gasify. If the pressure in the large supply cylinder was $1 \mathrm{~atm}$, the resulting pressure in the small cylinder will be of the order of $450 \mathrm{psi}$. The large supply cylinder is then isolated from the system, and the high-pressure xenon is bled through a needle valve from the small cylinder into that part of the system containing the rubber septum. Once the pressure is adjusted to $1 \mathrm{~atm}$, or to be equal to that in the welding chamber, the system is ready for use and xenon may be withdrawn with the hypodermic syringe. Figure 3 shows the inside of the welding chamber with the hypodermic needle entering the metal guide and adapter that holds the rubber septum. Other parts visible in this photograph are the rotating chuck for the welding operation and the welding torch.

\section{XENON TAGGING THE INSTRUMENTED FUEL ELEMENTS}

The most significant departure from a routine fuel element was the presence of sensor leads up to 62-in. long that are part of the top closure caps of the elements. Figures 4 and 5 illustrate the fuel-center thermocouple and the pressure-sensor elements, respectively.

The assembly of the fuel-center thermocouple and cap was preassembled into the annular fuel pellets in the element, and the top cap was left sufficiently open to permit insertion of the needle. This preassembly step was performed before installing the elements in the welding chamber. Final placement of the top cap was accomplished immediately after the xenon injection. The assembly of the pressure-sensor element and cap did not require any unusual handling.

A detailed process description of the steps required to tag these elements is given as an appendix. However, the following is a general description of the procedure used:

1. Load each element with fuel, and weld to bottom closure.

2. Place the elements (up to five of them) in the welding chamber (include the top caps and the hypodermic syringe and needle).

3. Evacuate the welding chamber and xenon system, and purge with helium gas four times.

4. Leave the xenon system under vacuum.

5. Fill the welding chamber with helium to a pressure of $5 \mathrm{in} . \mathrm{wg}$.

6. Transfer the xenon from the supply cylinder to the small cylinder.

7. Adjust the pressure in the rubber-septum portion of the xenon system to the same pressure as in the welding chamber.

8. Work through the glove ports and withdraw sufficient xenon through the septum to provide $1 \mathrm{~m} 7$ for each element. Readjust the xenon pressure to equalize the chamber pressure before withdrawing the needle from the septum.

9. Place the needle down into the element as far as it will go (about $8 \mathrm{in.}$ ) and carefully eject $1 \mathrm{ml}$ of xenon in 7 to $70 \mathrm{sec}$.

10. Cap the element quickly. This should be done in $10 \mathrm{sec}$ or less.

11. Weld the cap in place.

12. Repeat the injection, capping, and welding steps for each of the remaining elements. 
Before the actual fuel elements are tagged, a number of sample elements were tagged to evaluate the xenon recovery that could be achieved by the procedure. These sample elements simulated actual fuel elements in size and design. The plenum size determines the depth to which the hypodermic needle can be inserted. The design of the top closure affects the time and ease of capping and closing the element.

Figure 6 shows a sampling tube developed to obtain representative samples of the tag from the elements. To facilitate transfer of the sample to the mass spectrometer for analysis, a brass ground joint and a special valve were installed on the bottom end of the tube. This valve permitted the sample tube to fit down through the chuck when putting the element in position for welding.

Table II lists some of the results obtained with the method of tagging used. Once the top cap has been inserted, diffusion is no Ionger a problem. The xenon recovery averages $80.2 \%$. No trouble was encountered in injecting the tag in 7-10 sec and in capping the element in $10 \mathrm{sec}$.

A running account of the amount of xenon consumed is readily maintained by summing the amounts withdrawn with the hypodermic syringe. In addition, the amount of xenon remaining in the 30-ml supply cylinder, and thus what has been consumed, can be calculated from the total system pressure after each withdrawal. Figure 7 compares the results of the calculations using the two methods. When the xenon-supply cylinder was installed in the xenon system, the pressure of the xenon in the cylinder was not known because some of the gas had been removed for an analysis to determine its purity. The volume of xenon at room temperature and $1 \mathrm{~atm}$ in the cylinder at the start of the tagging operation was calculated to be $30 \mathrm{ml}$ and is indicated by extrapolating the 1 ine for the calculated volume to zero pressure. The xenon volume indicated by the line representing the actual volume removed with the hypodermic syringe extrapolates to an initial volume of approximate $7 y 27 \mathrm{ml}$. This discrepancy is probably due to inaccuracy in reading the total system pressure. The last set of points indicates the system pressure limit for withdrawing xenon at $1 \mathrm{~atm}$. For this size system, it is $5.6 \mathrm{in.} \mathrm{Hg}$ absolute. At this point, the cumulative volume of xenon removed at $1 \mathrm{~atm}$ was 20 and $27.6 \mathrm{ml}$, respectively. This amount represents the maximum yolume of xenon that can be removed from the original 30-ml supply cylinder using the existing total xenon system. The smaller the volume in the system, the greater will be the xenon use factor.

The use of liquid nitrogen to transfer xenon by means of cryogenic pumping was very successful. In addition, it provided a process for purification of the xenon in case contamination with air or other gases should occur. Table III lists some important physical properties of some common environmental gases associated with fuel-element manufacture. Liquid nitrogen, with a boiling point of $-195.8^{\circ} \mathrm{C}$, will freeze xenon solidly. Should oxygen, helium, argon, or nitrogen be contaminants, they can be pumped off the frozen xenon under vacuum. 0xygen and argon, however, will liquefy at liquid-nitrogen temperatures, so they will be harder to remove. Table III shows also that xenon is more than 30 times heavier than helium--a fact that is significant in the success of the hypodermicsyringe method of introducing a controlled amount of xenon into a helium-filled fuel element. The heavier xenon, introduced through the top of the element, sinks through the helium. 
TABLE I. Comparison of Isotopic Compositions, in Percent, of Natural Xenon and the Xenon-tag-gas Mixture for the Elements of the EBR-II Instrumented Subassembiy

\begin{tabular}{lccccccccc}
\hline & \multicolumn{10}{c}{ Xenon Isotope } \\
\cline { 2 - 10 } & 124 & 126 & 128 & 129 & 130 & 131 & 132 & 134 & 136 \\
\hline Natural Xenon & 0.096 & 0.090 & 1.92 & 26.44 & 4.08 & 21.18 & 26.89 & 10.44 & 8.87 \\
Tag Xenon & 2.05 & 0.85 & 4.32 & 32.20 & 3.88 & 18.08 & 22.65 & 8.66 & 7.30 \\
\hline
\end{tabular}

TABLE II. Xenon Recovery and Injection Times

\begin{tabular}{|c|c|c|c|c|c|}
\hline $\begin{array}{l}\text { Sample } \\
\text { No. }\end{array}$ & $\begin{array}{l}\text { Injection } \\
\text { Time, sec }\end{array}$ & $\begin{array}{l}\text { Time to Cap } \\
\text { Element, sec }\end{array}$ & $\begin{array}{l}\text { Total Time to } \\
\text { Tag and Close } \\
\text { Element, sec }\end{array}$ & $\begin{array}{c}\text { Xenon } \\
\text { Recovery, \% }\end{array}$ & Remarks \\
\hline $513-1$ & 9 & 6 & 50 & 80.4 & Experimental Sample \\
\hline-2 & 9 & 6 & 38 & 78.7 & Experimental Sample \\
\hline-3 & 9 & 4 & $105 *$ & 77.9 & Experimental Sample \\
\hline-4 & 7.5 & 3.5 & $555^{*}$ & Bad Weld & Experimental Sample \\
\hline-5 & 8 & 6 & 43 & 76.4 & Experimental Sample \\
\hline $514-2$ & - & -- & -- & 79.1 & Initial Proof Sample \\
\hline 522 & 9 & 5 & 39 & 88.7 & Final Proof Sample \\
\hline 3 & 8 & 4 & 50 & & \multirow{4}{*}{$\begin{array}{l}\text { Elements for test-2 } \\
\text { Instrumented Sub- } \\
\text { assembly }\end{array}$} \\
\hline 7 & 7.5 & 4.5 & 48 & & \\
\hline 10 & 7 & 4 & 38 & & \\
\hline 15 & 7 & 5 & 35 & & \\
\hline
\end{tabular}

*Experienced trouble with arc starting in pure helium atmosphere.

TABLE III. Important Physical Properties of Environmental Gases

\begin{tabular}{lcrrrr}
\hline Property & Xe & He & Ar & 0 & $\mathrm{~N}$ \\
\hline Atomic Weight & 131.3 & 4.00 & 39.95 & 15.99 & 14.01 \\
Boiling Point, ${ }^{\circ} \mathrm{C}$ & -108 & -268.6 & -185.7 & -183.0 & -195.8 \\
Freezing Point, ${ }^{\circ} \mathrm{C}$ & -111.8 & -272.2 & -189.2 & -218.4 & -209.9 \\
Density, g/1iter & 5.897 & 0.177 & 1.784 & 1.429 & 1.251 \\
\hline
\end{tabular}


Fig. 1. Schematic of inert-atmosphere welding chamoer with xenon tagging system.
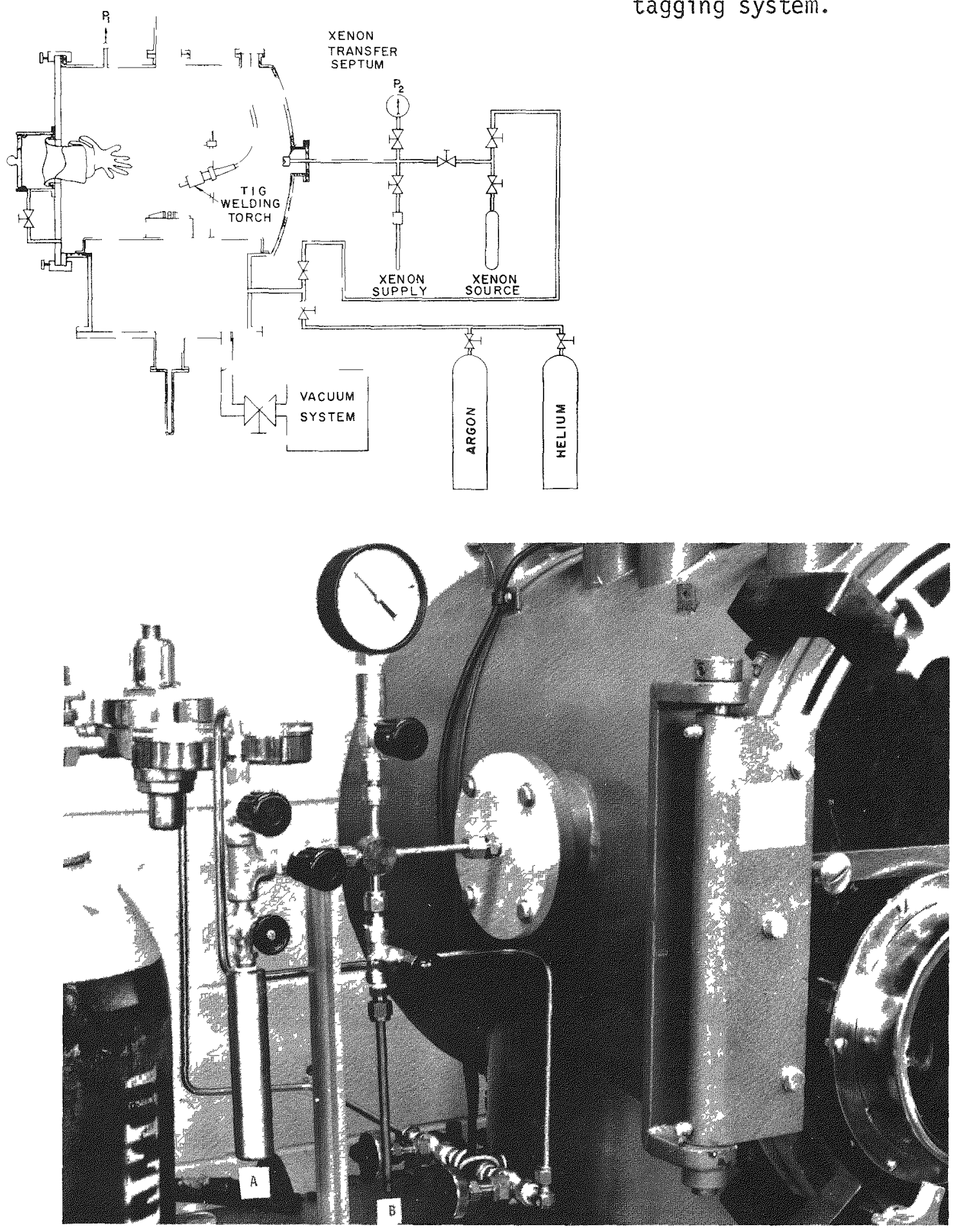

Fig. 2. Photograph of xenon-supply system attached to welding chamber (right). Xenon-supply cylinder and high-pressure cylinder into which xenon is pumped cryogenically are identified by $A$ and $B$, respectively. 


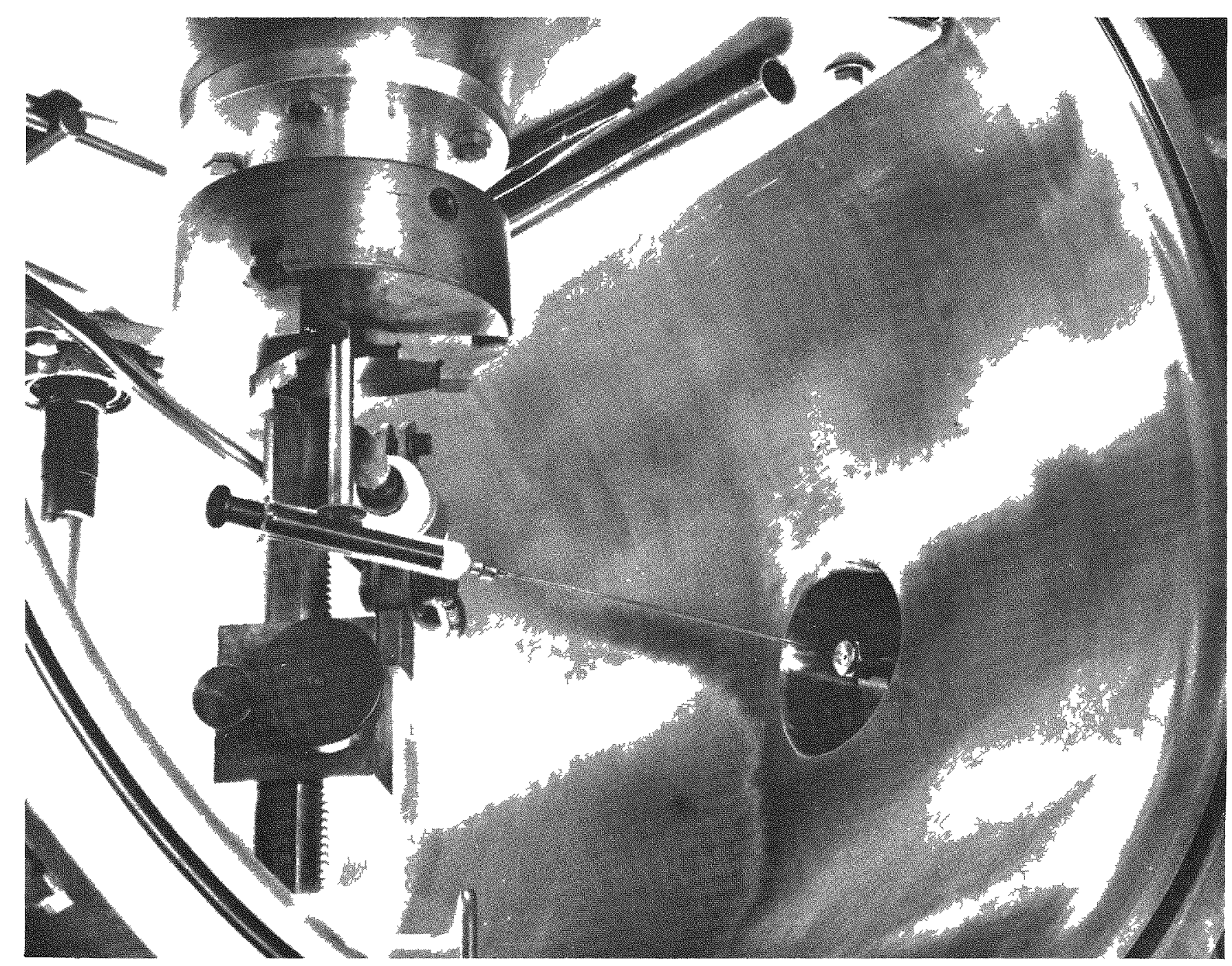

Fig. 3. Inside of welding chamber, showing hypodermic in position to withdraw xenon tag from external xenon-supply system. 

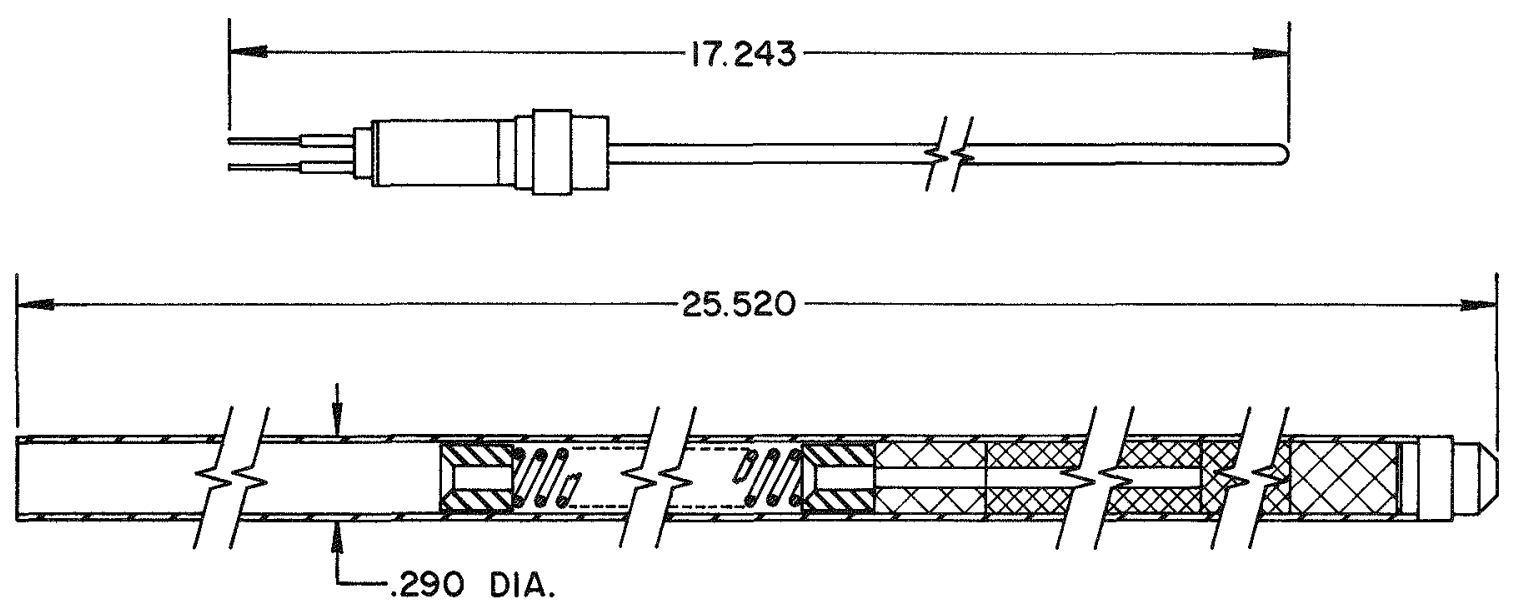

Fig. 4. Fuel-center thermocouple element (bottom) and top cap assembly.
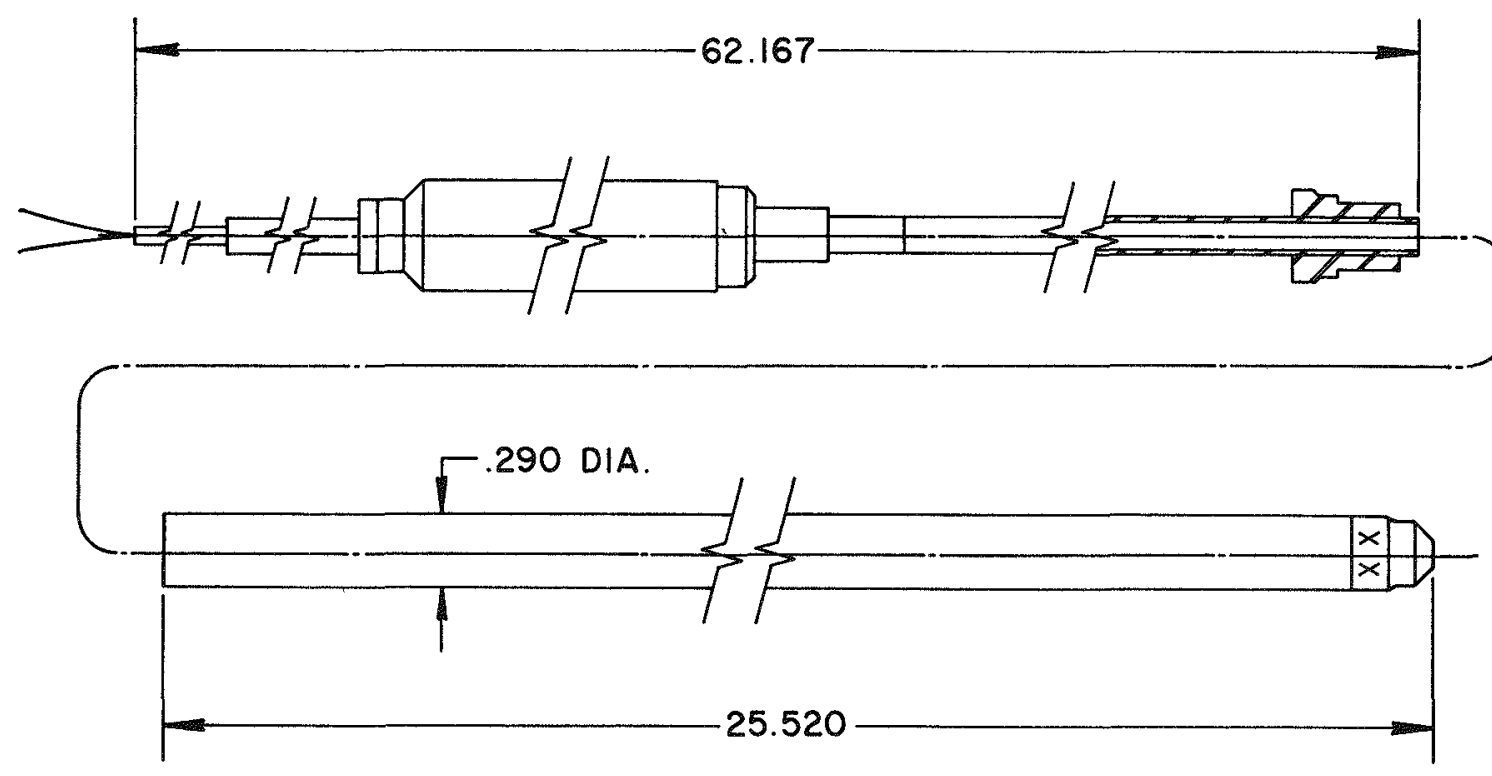

Fig. 5. Pressure-sensor element (bottom) and top cap assembiy.

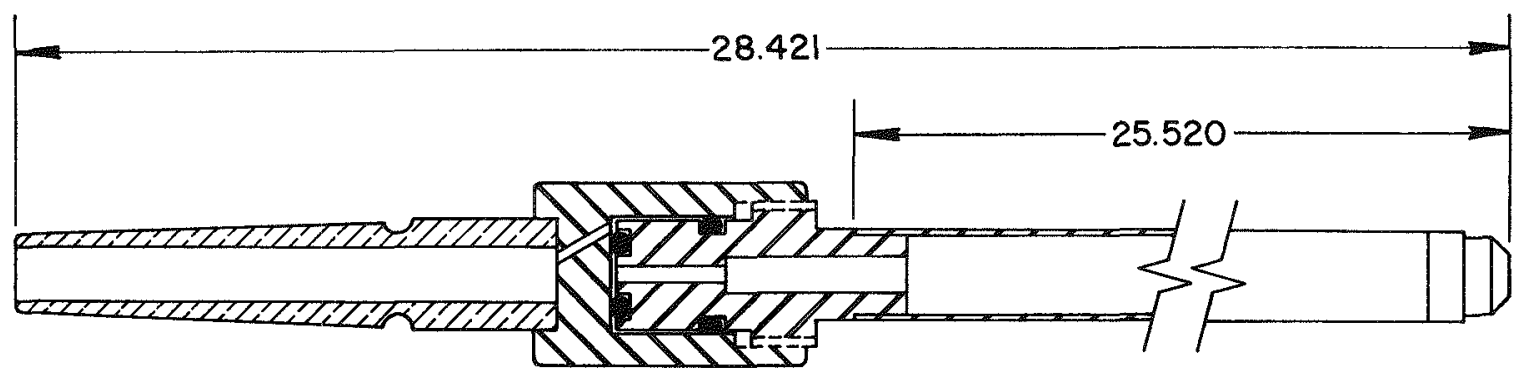

Fig. 6. Cross section of gas-sampling tube. 


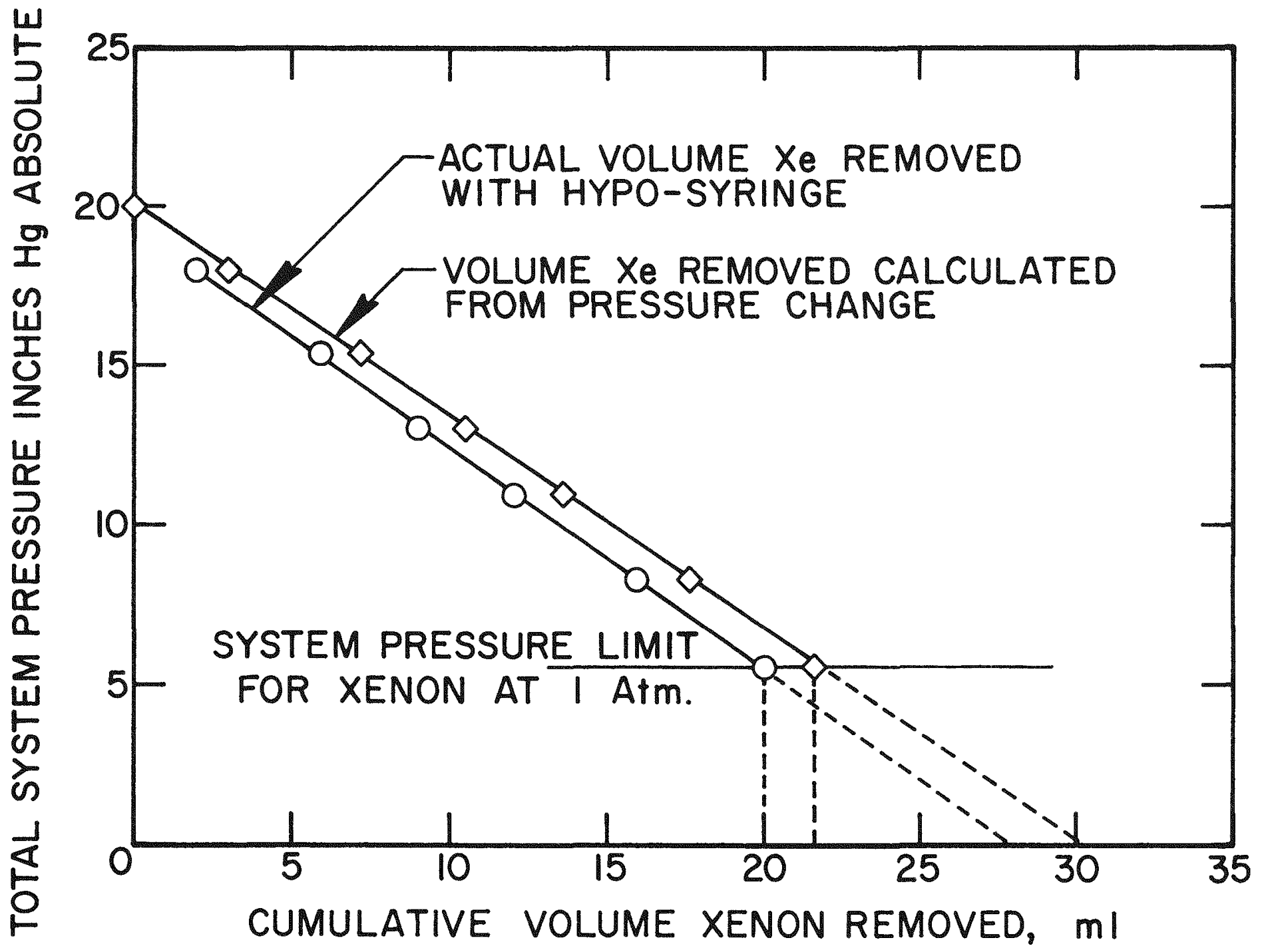

Fig. 7. Comparison of actual and calculated amounts of xenon removed from system. 
APPENDIX

Procedure 320-6710.2-1. 15 Rev. 1

Xenon Tagging Fuel Elements in the

Vacuum-Atmosphere Welding Chamber

Purpose: Leaking test elements in the reactor must be removed. The reactor downtime required to locate the unknown leaker has been excessive. Xenon tags of a specific isotopic ratio can be used to identify test elements and in the event of a failure, this xenon will quickly identify the location of the leaking element.

Procedure: 1. Place the loaded elements into the welding chamber. The bottom closure will have been welded in place and inspected.

2. Place the element top caps, the hypodermic syringe, extra tungsten welding electrodes, and the hypodermic needle cap into the chamber.

3. Close the welding chamber and open the glove port valve.

4. Evacuate the welding chamber and purge with helium to produce a pure atmosphere.

\section{Purge Sequence}

a. Evacuate the chamber to $4 \times 10^{-4} \mathrm{~mm}$.

b. Back fill to $1 \mathrm{~atm}$. with helium and hold for 5 minutes.

c. Evacuate the chamber to $4 \times 10^{-4} \mathrm{~mm}$.

d. Back fill to 1 atm. with helium and hold for 5 minutes.

e. Evacuate the chamber to $4 \times 10^{-4} \mathrm{~mm}$.

f. Back fill to 1 atm. with helium and hold for 5 minutes.

g. Evacuate the chamber to $4 \times 10^{-4} \mathrm{~mm}$.

$h$. Open the valves between the chamber and the xenon system and evacuate the chamber and the xenon system to $3 \times 10^{-4} \mathrm{~mm}$. When the xenon system has been opened to air, start the purge sequence with this valve open.

$i$. Freeze the xenon in the $30-m 7$ source cylinder with liquid nitrogen.

$j$. When the xenon is frozen, open the xenon source cylinder to the vacuum in the system and pump out any gaseous contamination. Record the pressure in the system as observed on gage, P-2.

$k$. Close the valves between the xenon system and the welding chamber.

1. Remove the liquid nitrogen from the 30-ml source cylinder and allow the cylinder to warm up to room temperature.

m. Record the xenon pressure in the system as observed on gage, $P-2$.

$n$. Use liquid nitrogen and transfer the xenon into the small supply finger. The transfer is complete when the system returns to its original vacuum $(P-2)$. If the transfer is not complete, freeze both the finger and the supply cylinder and evacuate the system. The second transfer will usually be complete. 
Procedure 320-6710.2-1. 15 Rev. 1

o. Close the source cylinder and the supply finger valves and after warming the finger to room temperature, open the finger valve and increase the system pressure to 5 inches water gage.

p. Back fill the welding chamber to 5 inches water gage pressure with helium, and maintain an equal pressure between the xenon system and the welding chamber.

5. Close the glove port evacuation valve and open the glove ports.

6. Working through the glove ports, adjust the element top elevation and the tungsten welding electrode to the desired position.

7. Use the syringe to withdraw sufficient xenon through the septum to provide $1 \mathrm{ml}$ of xenon per element.

a. Adjust the xenon system pressure to equal the chamber pressure before withdrawing the needle from the septum.

b. Record the pressures indicated in the welding chamber and xenon system.

8. Place the hypodermic needle into the open end of the element as far as possible and carefully inject $1 \mathrm{ml}$ of xenon at a rate of the order of $1 \mathrm{~m} 7$ per 7 to 10 seconds.

a. After each injection, quickly insert the top cap into the open end of the element and weld closed. The time after injection until the top cap is in place should not exceed 10 seconds.

b. Before removing the tagged element and replacing with the next one, store the syringe needle in the cap to prevent xenon loss from the syringe by pressure-change-pumping caused by hand movements of the gloves.

c. Record the injection times as follows:

Time to inject xenon

Time to effect cap closure

Time to effect weld closure

9. Repeat steps 6,7 , and 8 for the remaining elements.

Xenon Storage:

1. After the tagging and welding sequence is complete, open the valve from the xenon supply finger and allow the xenon to fill the system external 
Procedure 320-6710.2-1. 15 Rev. 1

to the $30-\mathrm{m} 1$ source cylinder.

2. Open the valve to the $30-\mathrm{m} 1$ xenon source cylinder and allow the xenon to fill into the cylinder.

a. When the xenon pressure reaches $1 \mathrm{~atm}$ in the total system, close the valve to the xenon supply finger.

b. When the pressure has equalized in the total system, read and record the system pressure observed on gage, P-2.

3. With liquid nitrogen, transfer the remaining xenon gas to the $30-\mathrm{ml}$ supply cylinder.

a. When transfer is complete, close the valve for storage.

4. Open the valves separating the welding chamber from the external xenon system and establish 1 atm helium in the xenon system.

5. Close the valves to isolate the xenon system from the welding chamber.

6. Open the chamber and remove the tagged and welded elements. 
Instrumented SubassembIy System and In-Core Instrument Test Facility for EBR-II

by E. Hutter, O.S. Seim and A. Smaardyk

Argonne National Laboratory

$0.54+83$

Argonne, Illinois 60439

SUMMARY

EBR-II (Experimental Breeder Reactor No. 2) was originally designed and operated by ANL as a demonstration of a fast breeder central station reactor. After successful demonstration of this goal, including a "quick turn-around" fuel cycle, the reactor was converted into an irradiation test facility.

Numerous types of irradiation subassemblies were designed and constructed. In addition, two types of experimental facilities will serve to provide instrument read-out data during the life of the experiments, while the reactor is operating. The two types are "The Instrumented Subassembly System", and "The In-Core Instrument Test Facility".

The "Instrumented Subassembly System" supplies the means of measuring operating parameters of specimen fuels, instruments, and related components in the EBR-II core. The system consists of an instrumented subassembly, an extension tube with seals and connections for the instrument lead wires, a drive to make it compatible with fuel handling operations, and recording and data logging equipment. The instrumented subassembly is located in the fifth row of the core (replacing a control rod), is cooled by flowing sodium of $700^{\circ}-900^{\circ} \mathrm{F}$, and is exposed to a total flux of approximately $1.9 \times 10^{15} \mathrm{n} / \mathrm{cm}^{2} \mathrm{sec}$ at $50 \mathrm{MW}$ reactor power.

The subassembly consists of sixteen capsules containing clad uraniumoxide fuel specimens and two capsules containing structural materials. The instruments include one inlet coolant thermocouple, three outlet coolant thermocouples, one electromagnetic flowmeter, one 3/16 in. diameter tube for future flux monitors, seven fuel-centerline thermocouples having $W-3 \% \operatorname{Re} / W-25 \%$ Re thermo-elements, and four spacer-wire thermocouples, four fission-gas pressure transducers and two structural materials centerline thermocouples. 
The "In-Core Instrument Test Facility" provides direct access into the reactor core to accomplish operational testing of in-core instrumentation under simulated LMFBR conditions. This project is in the conceptual design study stage and construction has not yet been approved. It is planned to provide a "dry thimble" which will extend from the top of the biological shielding, through the bulk coolant sodium into the reactor core. It will be located in, and exposed to a similar environment as the instrumented subassembly. Instrument sensors will be inserted into this thimble and exposed to a gas environment; or the sensors could be individually encapsulated in a sodium environmenta1, or any other medium that could be contained in such capsules. The study shows that temperatures of $900^{\circ}-1350^{\circ} \mathrm{F}$ could be attained during reactor operation. Temperatures above and below this range would cause heat transfer or stress problems. It is intended to make it possible to remove and insert individual tests without effecting the remaining instruments being tested.

\section{Plant Description}

EBR-II is an integrated nuclear power plant, 1,2 located at the National Reactor Testing Station in Idaho. Its main facilities are the reactor plant, the Fuel Cycle Facility, the sodium-boiler plant, the power plant, and the laboratory and service building. The reactor, the primaxy sodium-coolant system, and several ancillary systems are housed in the reactor plant (Figure 1), which is a steel containment vessel, $80 \mathrm{ft}$ in diameter, $140 \mathrm{ft}$ high. The fuel reprocessing is done in the Fuel Cycle Facility, which contains a circular shielded cell. The major part of the secondary sodium system, including the ac electromagnetic pump and the sodium-to-water steam-generating equipment, is located in the sodium-boiler plant. The power plant houses the steam turbine and generator and the associated electrical system, as well as the control room for the reactor.

\section{A. Reactor Description}

The reactor and the entire primary system (including the heat exchanger) are contained in a large vessel (primary tank) and operate completely submerged in the bulk-sodium coolant. The primary-sodium coolant is pumped directly from the bulk sodium into the reactor vessel and up through the core. The effluent coolant flows from the vessel, through the shell side of the heat exchanger, and back to the bulk sodium. The secondary-sodium system transfers the heat to a steam generator, which supplies the steam for a turbine-generator.

The reactor assembly (Figure 2) consists of the reactor vesse1, the grid-plenum assembly, and the vessel cover. The assembly is surrounded by the neutron shield and is submerged beneath approximately $10 \mathrm{ft}$ of sodium.

The grid-plenum assembly is a core grid structure, which supports and locates the subassemblies and incorporates the coolant inlet plena. The grid structure accomnodates 637 hexagonal subassemblies spaced on a triangular pitch of $2.320 \mathrm{in}$. The nominal core loading consisted of 47 
enriched-uranium core subassemblies, six enriched-uranium inner-blankettype subassemblies, two safety subassemblies, 12 control subassemblies, 60 inner-blanket subassemblies, and 510 outer-blanket subassemblies during power demonstration runs. Each subassembly contains a number of fuel elements or blanket elements of size and shape appropriate to its particular type (Figures 3 and 4).

A single fuel-subassembly size is employed, resulting in a closely-packed reactor geometry (Figure 5). Each hexagonal fuel-subassembly tube is 2.290 in. across external flats, with a 0.040 -in. wall thickness. A nominal clearance of $0.030 \mathrm{in}$. between fuel subassemblies facilitates their removal from the reactor. The upper adaptor on each type of fuel subassembly is identical, so that all fuel subassemblies are acconmodated by the same handling and transfer mechanisms. The lower adaptors are of different sizes to distinguish between the three basic types of fuel subassemblies, and are of different configurations to accommodate the two coolant plena.

The reactor-vessel cover, serving as a neutron shield as well as a closure, is clamped to the vessel flange by three holddown clamps. When the cover is lowered, it forms the reactor upper plenum from which the coolant flows to the heat exchanger.

The control-rod drive shafts operate through seals and guide bearings in the vessel cover. Each control rod is operated independently by an electromechanical drive mounted on top of the rotating-shield plug (Figure 6). In the event of a reactor scram, the 12 control rods operate simultaneously.

Normal operational control is not effected by the two safety rods, which are a separate part of the system. The main purpose of these rods is to provide available negative reactivity during reactor shutdown.

The primary tank contains the reactor vesse1, the two primary sodium pumps, the heat exchanger, the storage rack, various instruments, mechanisms, and ancillary systems, as well as 80,000 gal of sodium. The primary tank is of double-wall construction and is $26 \mathrm{ft}$ in diameter. The cover of the primary tank is 39 in. deep and contains shielding material and thermal insulation. The region above the bulk sodium is filled with inert argon gas.

The primary tank has no side or bottom openings but allows access to its interior through 67 nozzles in the cover and one large circular opening in the center of the cover. Each nozzle accommodates one, in most cases removable, primary-system component, and the central opening accommodates the rotating shield plugs.

The primary tank and its contents and the components that are connected to the primary-tank cover are supported by six hangers, which in turn transfer these loads to the top structure beams. Each hanger is supported by a roller so that differential radial expansion between the top structure and the primary-tank cover will not produce additional stresses 
in the system. The primary-tank design and the method of support are arranged to provide radial expansion about the vertical centerline of the system. The most critical units, the reactor, the rotating plugs, which index the control-rod drives, and part of the fuel-unloading mechanisms, are centered on the physical centerline of the system. Differential vertical expansion is reduced by using identical material for all equipment in the system, and maintaining isothermal conditions wherever possible.

\section{B. Fuel-handling Description}

The fuel-handling system (Figure 6) is designed to perform fuelsubassembly transfers in two phases: The first phase begins immediately after reactor shutdown and includes all transfer steps between the reactor and the storage rack, both of which are submerged in the sodiumfilled primary tank. The fuel subassemblies remain below the bulk sodium surface at all times, making the transfers relatively simple since no special cooling requirements are needed. Thus, the first phase of fuel handling is fast and interrupts reactor operation for a short time only. The subsequent steps in fuel handling can be performed during reactor operation, including approximately a 15-day cooling-off period for spentfuel subassemblies in the storage rack. This permits the decay of fissionheat generation to a level at which a fuel subassembly can be conveniently gas-cooled. The second phase of fuel handling includes the transfers between the storage rack, the fuel-unloading machine, the interbuilding coffin, and the Fuel Cycle Facility. The timing of these transfers is affected by the cooling-off period in the storage rack.

\section{Control Subassembly and Drive}

Each of the twelve control subassemblies consists of a guide thimble and a control rod (Figure 4). The guide thimble is hexagonal in cross section and occupies a unit core lattice identical with that occupied by the various subassemblies. The control rod, a modified fuel subassembly, is enclosed in a hexagonal tube which is smaller than the guide thimble by the equivalent of one row of fuel elements in the core section. Thus the core section of each control rod contains 61 pin-type fuel elements as compared with 91 elements in the core section of a fuel subassembly. Reactor control is effected by vertical movement of the control rod, to adjust the proportion of fuel in the active core region. The lower portion of the control rod is comprised of a bottom adaptor with coolant entrance holes, and guide bearings which fit into matching sections in the guide thimble and which are also part of the hydraulic holddown of the control rod. The upper end of the control rod consists of a mushroom shaped upper adaptor which is identical to the upper adaptors of all other subassemblies so that they can be accommodated by the same handling and transfer mechanisms.

Each control rod is operated independently by a control rod drive. This is an electromechanical drive mechanism mounted on top of the rotating shield plug (Figure 7). Each of the twelve drives extends a vertical shaft assembly through a nozzle in the rotating shield plug into the sodium 
inside the primary tank and through seals and guide bearings installed in the reactor vessel cover. The drive shaft assembly terminates in the reactor vessel upper plenum where it actuates agripper mechanism. During reactor operation this gripper mechanism grasps the control rod upper adaptor and thus imparts vertical motion to the control rod. During fuel handling operations, when rotation of the shield plugs is required, the gripper releases the control rod and thus separates the moving drive shaft assembly from the control rod which remains in the reactor ${ }^{3}$.

The Instrumented-Subassembly System and the In-Core Instmument Test Facility, which are the subject of this report, each occupy the location of a control rod and drive mechanism. This necessitates that the reactor will have to be controlled by less than 12 control rods, depending how many of these or similar irradiation facilities will be operated simultaneously. Activities are underway to provide the reactor with "Higher Worth Control Rods" which will make it possible to reduce the number of the 12 original control rods by more than is presently possible.

\section{Instrumented-Subassemb1y System}

\section{A. Genera1}

The Instrumented-Subassembly System ${ }^{4}$ provides the means of inserting an instrumented irradiation subassembly into EBR-II and to monitor the various instrument readings while the reactor is operating (Figure 8). This requires the extension of instrument leads from the reactor core region to the outside of the primary tank where they can be connected to conventional data logging equipment. In addition the system has to comply with all safety considerations and must be compatible with the already existing reactor components and fuel handling mechanisms. Because of the compact nature of the reactor, space considerations are of a major concern. The Instrumented-Subassembly System consists basically of two major portions:

1. The "semi-permanent portion", comprised of the drive system and other components, which can be reused for subsequent irradiation subassemblies.

2. The "replaceable portion", comprised of the instrumented subassembly and its extension, which are replaced with each new experiment.

One current intent of the EBR-II project is to provide a vehicle for capsules, supplied by experimenters, to be incorporated in a common subassembly which is monitored during reactor operation. In order to demonstrate the capability and versatility of the overall system, a prototype subassembly has been fabricated using ANL supplied irradiation samples and instruments. The instrumentation used in the prototype is intended to give a general indication of the measurements that can be 
performed in the reactor core. However, the system is in no way limited to such instruments and it is fully intended to incorporate more advanced measuring devices in future experiments.

\section{B. Description}

The major components of the system are:

1) The subassembly, containing capsules with experimental irradiations (primarily fuel) and a variety of instrument sensors to measure pertinent operating and experimental parameters.

2) The extension of the subassembly, consisting of a $29 \mathrm{ft}$ long tube assemb1y incorporating a gripper at its bottom end and an electrical terminal box on the top end (Figure 9). The extension serves as the connecting link between the subassembly and the drive system. The space between the concentric tubes, sealed at the bottom end, provides a sodium free dry-well for routing the instrument leads out of the reactor and primary tank to the terminal box, where they are connected to lines leading to appropriate receiving instruments (Figure 10).

3) The bellows seal assembly which seals the opening in the rotating plug through which the extension tube assembly protrudes the sodium filled primary tank.

4) The drive system imparts an eight foot vertical motion to the subassembly in order to pull it out of, and reinsert it into the reactor core. This allows plug rotation and makes fuel handling possible during the life of the instrumented subassembly, while the instrument leads remain intact. The drive system consists of a motor and gear train which rotate two lead screws to raise or lower an elevator arm. The elevator arm is engaged to a vertical guide track and supports the extension tube assembly. Several safety devices prevent damage to other systems due to malfunctions, e.g. a force transducer and an electrically checked spring support prevent excessive pull or push forces on the subassembly and extension tube, and electrically interlocked sliding yokes hold the subassembly in its full-up or full-down position.

5) The data logging system records continuously the output from the in-core instruments (22 signals) plus one neutron flux signal from the reactor instrumentation on analog strip charts using 2-pen recorders. Also, the information is digitally recorded on a data acquisition system located outside of the reactor building. The recorders are capable of mming at a fast speed to record data during initial escalation to power or at other desired times, otherwise the recorders are switched to low chart speed. In addition, event markers are provided and these are synchronized for appropriate marking of the strip charts. The data acquisition system is capable of recording on magnetic tape at high speed. 


\section{Operation}

The Instrumented Subassembly System has been designed so as to minimize changes to the reactor system and interference with standard reactor operations. The drive system is electrically interlocked with the fuel handling control system thus preventing any improper or outof-sequence activities. Only a small part of the drive system has to be removed to allow insertion of a fresh instrumented subassembly and extension tube. Since this is a new assembly no irradiation hazard exists, however, the subassembly is preheated and the opening into the primary tank is properly sealed off. After installation of this subassembly and its connection to the drive end to the instrument readout equipment, reactor operation proceeds in the normal manner. When fuel handling is required during the lifetime of the instrumented subassembly, the drive raises the subassembly and its extension tube $8 \mathrm{ft}$, which allows it to clear all reactor internals so that standard fuel handling operations can take place. Because these activities include shield plug rotations, the instrument connections between the terminal box and the data logging equipment are temporarily disconnected.

After the instrumented subassembly irradiation experiment has been completed, the subassembly must be removed from the reactor. By that time the subassembly has had a significant residence time in the reactor so that its radiation level requires a substantial shielding coffin and sealing provisions. Because of this, the standard removal activities do not employ reversed installation procedures. Instead, a cutting device is inserted into the center of the extension tube. The cutting blades of the device come to rest on top of the subassembly top adaptor shoulder. A rotary motion of the drive shears the instrument leads where they protrude from the top adaptor shoulder. Now the extension tube can be removed from the primary tank by lifting it into a removal coffin that is temporarily mounted above the bellows seal on the rotating plug. A gate valve which is attached to the bellows flange, seals the opening into the primary tank, while the coffin is transferred to an appropriate storage location. In the meantime the instrumented subassembly remains in the guide thimble in the reactor. Since the subassembly has been severed from the extension tube, its contour is almost exactly like that of a control rod. Thus the instrumented subassembly can be transferred from the reactor to the storage basket by the standard fuel handling mechanisms according to the standard procedures. After a cooling period of approximately 15 days in the storage basket, the subassembly is further transferred via the fuel-unloading machine ${ }^{5}$ and interbuilding coffin ${ }^{6}$ to the Fuel Cycle Facility. There a dismant1ing machine separates the irradiated experimental capsules from the subassembly enclosure. The capsules are to be subjected to post-irradiation analysis or to be shipped to the experimenter's laboratory,

\section{Instrumented Subassembly Prototype}

In order to demonstrate the versatility of the system, a variety of different instruments has been incorporated in the subassembly prototype (see Table I). The instrument application is divided into. two major categories. 
1. Instrumentation measuring conditions within the subassembly.

2. Instrumentation measuring conditions within capsules containing fuels or other materials.

The instrument selection was made on the basis of availability and anticipated types which are likely to be used in future instrumented subassembly experiments. The prototype incorporates:

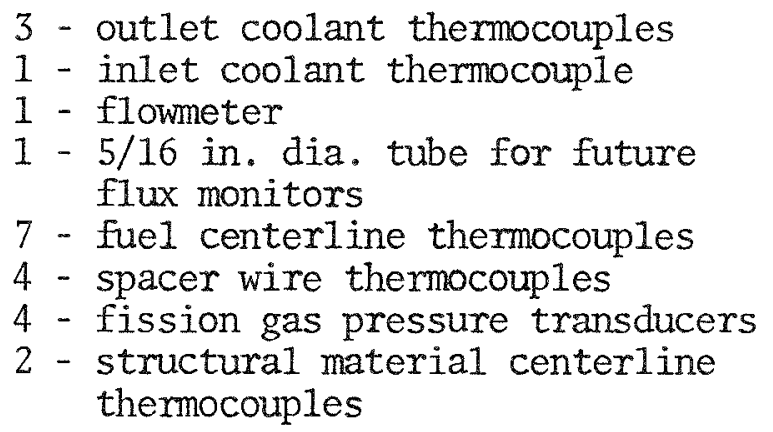

The prototype subassembly (Figure 11) contains 19 capsule positions. Sixteen capsules contain uranium oxide fuel, two contain structural material, and one capsule is replaced by a tube that serves as a conduit for instrument leads. All capsules are 40-13/16 in. long, 0.357 in. dia. and have a 0.041 in. dia. spacer wire helically wound on the outside.

The fuel centerline thermocouples are encased in tantalum sheath, have thoria insulation and $W-3 \% \operatorname{Re} / W-25 \% R e$ thermo-elements . The fuel centerline temperatures at the point of measurement are expected to be in the range of $1700-2000^{\circ} \mathrm{C}$ (Figure 12a).

The coolant thermocouples are sheathed in 304 stainless steel, have alumina insulation and chromel/alumel thermo-elements.

The fission gas pressure transducers are pressure balancing instruments (Figure 12b). Two small stainless steel bellows are welded to a common coupling disk and their opposite ends are welded to a housing wall. The inside of the lower bellows is open to the fuel element gas space via a connecting tube. The inside of the upper bellows is filled with a reference gas (argon) and is connected via a long stainless steel tube to controlling and recording equipment. A metal probe in the upper bellows makes electrical contact with the coupling disk. Buildup of fission gas pressure elongates the lower bellows and tends to push the coupling disk upward. This reduces the resistance of the electrical circuit (between probe and coupling disk) which via a solenoid valve increases the reference gas pressure forcing the coupling disk away from the probe until the equilibrium condition is reached. In this way the recorded reference gas pressure balances the fission gas pressure inside the fuel element. 
TABLE I: $\quad$ REFERENCE DESIGN CRITERIA FOR EBR-II INSTRUMENTED SUBASSEMBLY PROTOTYPE

Subassembly

Number

Location (Control Rod Position)

Configuration

Material

Dimensions, in.

Across flats

Inside flats

Overa11 length

Less lead length

Inc. $28-1 / 2$ in. lead

Capsule

Number/subassembly

Material

Dimensions, in.

Diameter

Wa11 Thickness

Length

Spacer Wire

Element

Diameter, in.

Clad Material

Clad Dimensions, in.

Diameter

Wa11 thickness

Fue1

Length

Material

Est. U-235 enxichment, :

Method of fabrication

Dimensions, in.

Diameter

Length

Theoretical density, :

Power Output, kW

Coolant Temperature at Inlet, ${ }^{\circ} \mathrm{F}$

Flow Rate Through Subassembly, gpm

Coolant Temperature Rise, ${ }^{\circ} \mathrm{F}$

Vertical Travel for Fuel Handling, in.

Speed of Travel for Fuel Handling, in/min

Extension Tube

Material

Diameter (nomina1), in.

Wa1l thickness (nominal), in.

Overall length (Subassembly, Extension Tube,

Terminal Box), ft. nominal

Estimated Total Weight, $1 \mathrm{~b}$.

No. of Sheathed Leads

1

No. 6

Hex. tube

304 SS

$1.902 / 1.912$

$1.824 / 1.830$

$93-37 / 64$

$122-5 / 64$

18

304 SS

$0.357 \pm 0.001$

$0.020 \pm 0.0005$

$40-13 / 16$

$0.041 \pm 0.0005$

304 SS

$0.290 \pm 0.001$

$0.020 \pm 0.0005$

27.68

$\mathrm{UO}_{2}$

$44.5 \pm 1.0$

Pressed \& Sintered

$0.246 \pm 0.001$

$14.22 \pm 1 / 16$

$95 \pm 2.0$

162

700

32

130

96

16.1

304 SS

2.5

0.120

36

ح350

23 
The flowmeter is of the permanent magnet (PM) type (Figure 13). The magnetic field is produced by two Alnico 5 magnets operating in parallel. The pole pieces are fully annealed soft iron. The flowmeter is contained in a stainless steel structure that is filled with helium. The expected sensitivity is app. $0.325 \mathrm{Mv} / \mathrm{gpm}$ at $800^{\circ} \mathrm{F}$ or about 10.5 millivolts output at a flowrate of 32 gallons per minute.

The $3 / 16$ in. OD $\times 0.016$ in. wall monitoring tube anticipates future interest in in-core neutron-monitoring devices. It extends from the terminal box to the capsule support grid and is sealed at the bottom. Thus it provides a dry-well for a monitor sensor that could be moved to various elevations to provide flux profiles, or be removed from the high flux zone between measurements in order to increase its lifetime.

\section{E. Irradiation of Experiment}

The instrumented subassembly system prototype is to be installed in a control rod and control drive location in calendar year 1969. It will be placed into the fifth row of the core and cooled by flowing sodium of $700^{\circ} \mathrm{F}-900^{\circ} \mathrm{F}$. At this location the total flux is approximately $1.9 \times 10^{15}$ $\mathrm{n} / \mathrm{cm}^{2} \mathrm{sec}$ at $50 \mathrm{MW}$ reactor power. In order to demonstrate its reliability an irradiation length of 2 reactor runs (app. 50 days of reactor operation) is planned. A burnup of about $0.4 \mathrm{a} / \mathrm{O}$ will be achieved at this time.

After removal from the reactor, the prototype subassembly will be dismantled and post-irradiation examinations of the fuel and structural materials will be conducted.

\section{F. Future Instrumented Subassemblies}

Plans and activities are underway to build successors to the prototype subassembly. The first experimenter subassembly will contain 36 plutonium-uranium oxide elements. Besides coolant flow and inlet and outlet temperatures it will measure the temperatures within the coolant spaces in the subassembly at two elevations. Another instrumented subassembly will measure creep rates of various materials under irradiation.

It is expected that future improvements in instrument techniques will further enhance the usefulness of the instrumented subassembly system as an advanced irradiation facility. However, to a large extent, the system itself will contribute significantly to the improvement of fuels and instruments.

III. In-Core Instrument Test Facility (INCOT)

\section{A. General}

The In-Core Instrument Test Facility will make it feasible to insert instrument sensors into the reactor core, expose them to a high fast 
flux and high temperature environment and record their performance during the duration of such tests or experiments.

In order to extend into the core, many components of the facility have to be located in a very congested region of the reactor system. This poses severe space restrictions on the design, in addition to the requirement of being compatible with the fuel handiing system and to comply with all safety considerations.

Testing of sensors in liquid metal causes complications. The liquid metal, or the chemical compounds thereof, may attack the sensors and alter the true experimental results. In addition, adherence of radioactive liquid metals to sensors might make interim-recalibrations or post-irradiation examinations more difficult. Thus, the design basis for INCOT is that of a "dry-well". The environment of the sensors is gas. Should an experimenter be interested in testing a sensor in liquid metal, the sensors can be encapsulated in liquid metal. This approach has the advantage that the chemistry of the liquid metal environment can be closely controlled.

\section{B. Description}

The major components of the system are (Figure 14):

1) The thimble assembly (approx. $35 \mathrm{ft}$ long) which extends from the top of the rotating plug into the primary tank and into the reactor core, forming a "dry well". The thimble assembly must have adequate space for test sensors, yet it must fit through various existing guidebushings, such as the $2.559 \mathrm{in}$. dia. opening in the reactor vessel cover. In addition, it must have internal shielding and means for heating and cooling. Since the methods of temperature control have a profound effect on the design, this matter will be discussed in a later chapter.

2) The bellows seal which seals the opening in the rotating plug, through which the thimble assembly extends into the primary tank. This seal prevents the sodium blanket gas (argon) from escaping out of the primary tank.

3) The drive system, which lifts the thimble assembly about eight feet above its operating level during fuel handling operations. Its purpose is the same as in the instrumented subassembly system, and its design is essentially similar.

4) The data acquisition system, that will transmit the output of the test sensors. Other supporting instrumentation will depend on the test sensor types and on the requirements of the experimenters.

5) The handling container, which is able to accept the radioactive sensors during or after their experimental lifetime. Two types 
are being studied. One is a long and straight coffin-type container, and the other is a reel-type container which wraps the sensor lead around a storage reel of approx. $4 \mathrm{ft}$. dia. Both container types are affected by the very limited space that is available above the thimble assembly.

\section{Thimble Assembly Temperature Contro1}

Heating and cooling of the thimble assembly interior is essential in order to provide the desired thermal environment for the sensor tests. Numerous alternates were considered before choosing the system that appears to be most advantageous.

Thimble Assembly Heating: Circulating loops of sodium, sodiumpotassium, argon, helium, air, water or oil are potential heating systems. Electrical heaters provide another possibility. However, radiation, shielding, safety or space problems make these solutions either impossible or undesirable. Gamma heating has favorable aspects. The heat loss through an insulating gas space is so small that addition of very little heat is required to achieve very high temperatures. Calculations indicate that any desired temperature (to $1400^{\circ} \mathrm{F}$ ) can be reached by gamma heating. Thus, it is the method best suited for the facility.

gated:

Thimble Assembly Cooling: The following concepts were investi-

Gas Cooled

$\mathrm{NaK}$ Convection Cooled

Primaxy Sodium Cooled

The gas cooled concept requires gas to be circulated inside the thimble assembly. The pumping and cooling system is located on the operating floor, with flexible hose connections to the thimble assembly. Helium or air can be used as coolant gas; argon would require very heavy shielding. The large space needed for the coolant passages restricts the sensor test thimble to about 0.5 in. inside diameter. The compressor motor size is about $7.5 \mathrm{HP}$. The gas cooled concept has an advantage since it provides the lowest environmental temperature of about $600^{\circ} \mathrm{F}$ for the sensor tests. It has the significant disadvantages of providing space for only one or two test sensors at one time, requiring major equipment on the crowded operating floor, and needs flexible quick-disconnect lines for high temperature radioactive coolant gase's which will not pose a safety hazard.

The NaK convection cooled concept provides a method to achieve sensor environment temperatures near $700^{\circ} \mathrm{F}$. A dual system (primary and secondary loops) is required to avoid shielding problems. NaK is used in both loops and no pumps are needed since free convection is relied upon for circulation. However, a heat exchanger on top of the rotating plug is required, the sensor test thimble size is small. While this NaK convection cooled concept may be useful in special applications where the importance of low environment temperatures is sufficient to downgrade other considerations, it does not appear promising as a convenient, flexible facility for general use. 
The primary sodium cooled concept utilizes the reactor coolant to control the temperature rise of the test sensors due to gamma heating. It lends itself for use in two variations:

In Model 1, an open ended sensor tube (1.402 ID) contains several small individual sensor thimbles which are closed on the bottom and each of which houses a sensor kept in a helium or argon environment. Primary sodium cools the individual sensor thimbles by entering the open ended sensor tube, flowing upward and exiting into the primary tank sodium approximately $13 \mathrm{ft}$. above the reactor core centerline.

In Model 2, a large sensor thimble (1.402 ID), which is closed at the bottom, contains several test sensors which are kept in a helium or argon environment. Primary sodium coolant flows upward in an annulus on the outside of the large sensor thimble and leaves the assembly at the same elevation as in Model 1.

These options offer flexibility for handling a variety of sensor sizes and for meeting a range of temperature requirements. The choice of sensor thimble model depends on the size of the sensors to be tested and the test temperature range desired. It is anticipated that most temperature objectives can be reached by combining coolant flow control with the necessary degree of built-in thermal coupling to the sodium coolant.

Both models permit vertical movement of the sensors in order to expose them to a wide range of neutron flux levels. Vertical motion can be accomplished either by moving the whole assembly upward as much as $40 \mathrm{in}$. or by moving an individual sensor to different levels. The sensor test tube has a relatively large inside diameter of $1.402 \mathrm{in}$. Flow control can provide means for varying sensor temperatures perhaps by as much as $350-400^{\circ} \mathrm{F}$. No external coolant components are required, however, it may be difficult to reach temperature levels below $900^{\circ} \mathrm{F}$.

An evaluation of the various concepts shows the Primary Sodium Cooled Concept to be the most favorable. It is preferable concerning space limitations on top of the shield plug, thimble assembly complexity, maximum sensor test temperatures, facility operation, safety considerations and costs. However, it is not able to reach as low temperatures as the other two systems $\left(900^{\circ} \mathrm{F}\right.$ vs, about $\left.700^{\circ} \mathrm{F}\right)$.

\section{Test Thimble Assembly}

Based on the selection of the most favorable heating and cooling concepts two models have been developed of the gamma heating and primary sodium cooling approach.

Mode1 1 (Figures 15 and 16) pertains to a thimble assembly which essentially consists of three concentric tubes. The two outer tubes serve mainly for insulating purposes (insulating gas tube assembly) and the innermost, called sensor tube ( 1.402 ID and 1.500 OD), acts as a coolant flow 
guide. The sensor tube extends approx. 4.5 inches below the bottom of the reactor core where it is attached to a coolant flow control valve and labyrinth seal that fits into the INCOT guide tube assembly. (This is a guide tube which replaces the control rod guide tube.) The sensor tube contains several sensor thimbles which are held in place by sensor thimble spacers. Each sensor thimble is filled with gas and contains one test sensor. Each test sensor in this assembly can be moved vertically within its sensor thimble without disturbing the other sensor or thimbles. The thimble assembly is approx. $35 \mathrm{ft}$. Iong and extends from the sensor lead teminal box (which is located above the rotating shield plugs) into the primary tank, reactor vessel, and reactor core. Shielding slugs are incorporated at the level of the rotating plug shielding. The coolant flow control valve near the lower end of the assembly, is regulated by rotating the sensor tube over a small angle. The degree of rotation (which covers or uncovers small flow holes within the valve) is accomplished by the flow control valve operator that is connected at the sensor lead terminal box.

The coolant flow control valve regulates and directs coolant from the reactor high pressure plenum into the sensor tube and hence around each individual sensor thimble. The coolant flows from the sensor tube and thimble assembly into the primary tank bulk sodium via coolant exit holes. These holes are located well above the reactor vessel cover, thus providing the sensor thimbles with a nearly isothermal environment for about seven feet above the reactor core.

Mode1 2 (Figure 17) is similar to Model 1 with one important exception. Instead of several individual sensor thimbles (one for each sensor), Model 2 employs one common thimble (1.402 ID, 1.500 OD) which is closed at the bottom. It contains several individual small sensor tubes each of which contains one sensor and its leads. The small sensor tubes are open ended at the bottom, so that the sensors may extend into the argon filled bottom cavity of the common thimble, called sensor thimble. Cooling is accomplished by sodium that flows through an annulus between the sensor thimble outside and the inside of the insulating gas tube assembly.

\section{E. Conclusion}

The conducted studies and analyses lead to the conclusion that it is feasible and desirable to design and fabricate an In-Core Instrument Test Facility for use in the EBR-II reactor for the purpose of testing various sensors. This facility will enhance the usefulness of EBR-II as a high flux irradiation facility and will contribute significantly to the test programs of LMFBR instruments. 


\section{REFERFNCES}

1. L.J. Koch et al, Hazard Summary Report, Experimental Breeder Reactor II (EBR-II), ANL-5719 (May 1957) and Addendum to Hazard Summary Report, Experimental Breeder Reactor II (EBR-II), ANL-5719 (Addendum)

2. J.C. Hesson, M.J. Feldman, and I. Burris, Description and Proposed Operation of the Fuel Cycle Facility for the second Experimental Breeder Reactor (EBR-II), ANL-6605 (Apri1 1963)

3. E. Hutter and G. Giorgis, Design and Performance Characteristics of EBR-II Control Rod Drive Mechanisms, ANL-6921 (August 1964)

4. A. Smaardyk, C.J. Divona, R.H. Olp and E. Hutter, Design and Analysis of Instrumented Subassembly System for EBR-II, ANL-7423 (June 1968)

5. E. Hutter, P. Elias, D.J. Veith, and W.R. Ware, EBR-II Fuel-UnZoading Machine: Design and Performance Characteristics, ANL-7201 (June 1966)

6. G.J. Bernstein, A.A. Chilenskas and R.F. Malecha, Interbuilding Euel Transfer Coffin for the EBR-II Reactor, ANL-6934 (September 1964) 


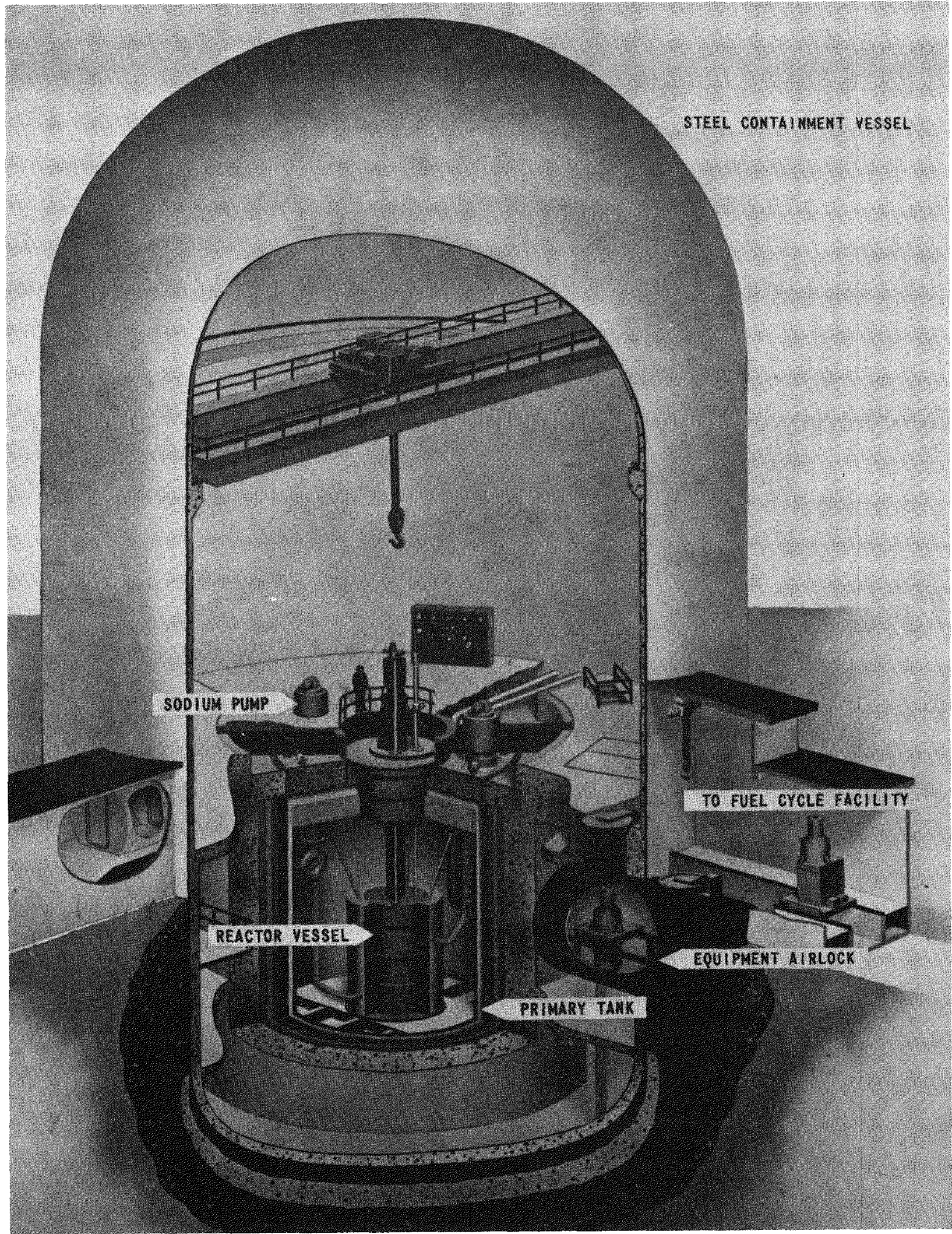

Fig. 1. EBR-II Reactor Plant 


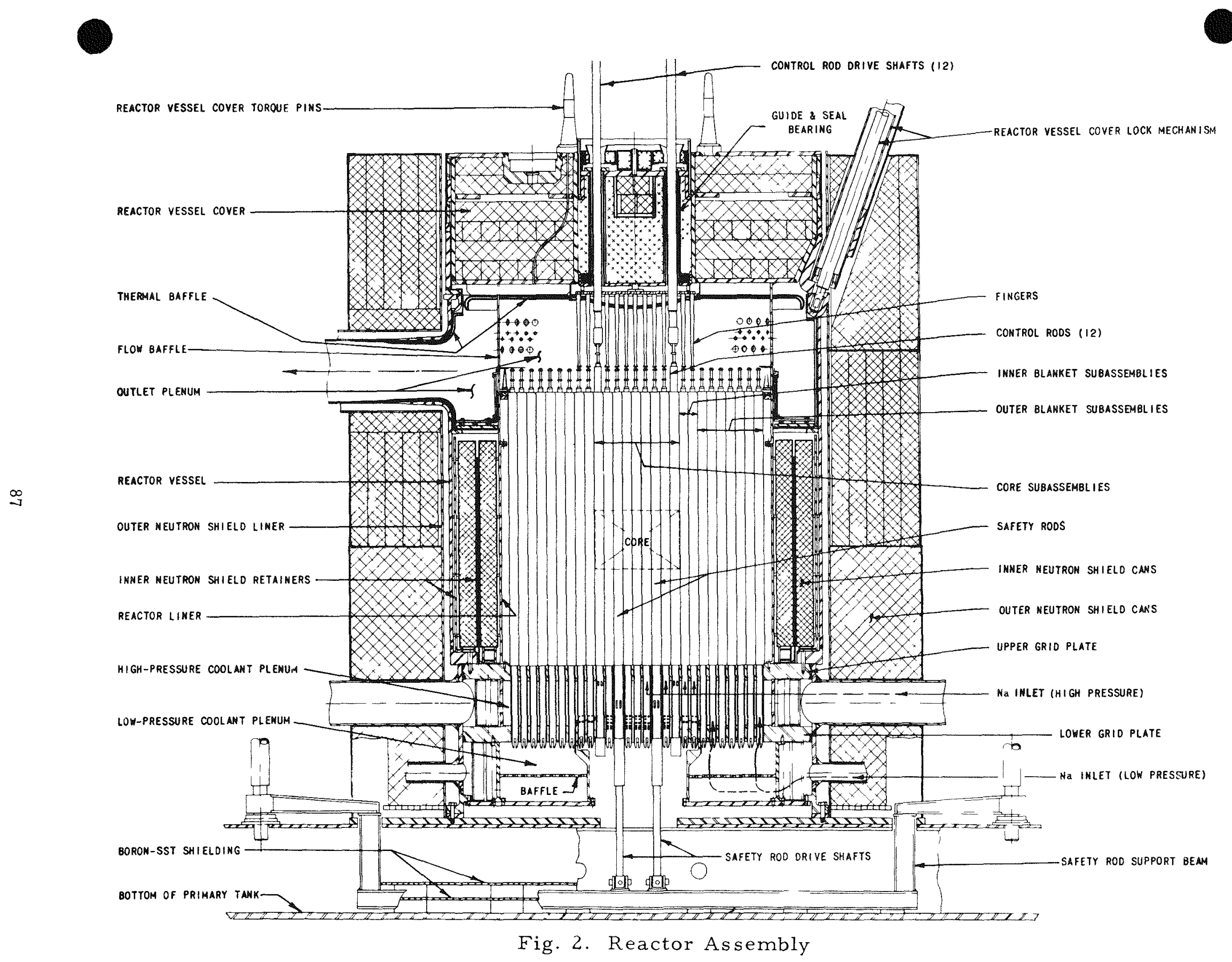




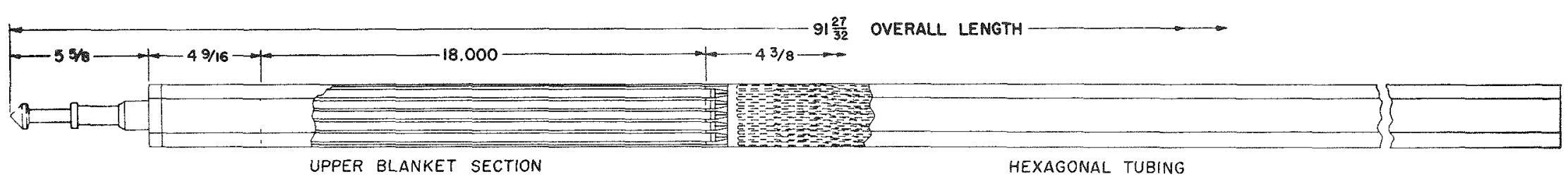

HEXAGONAL TUBING

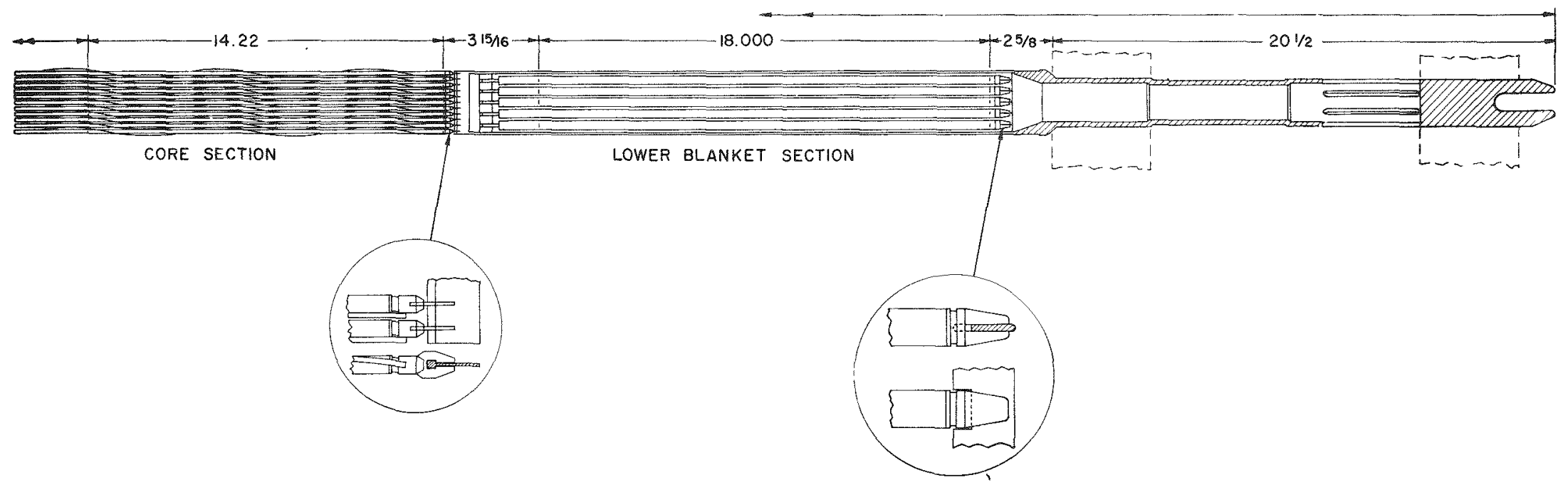

Fig. 3. Core Subassembly 


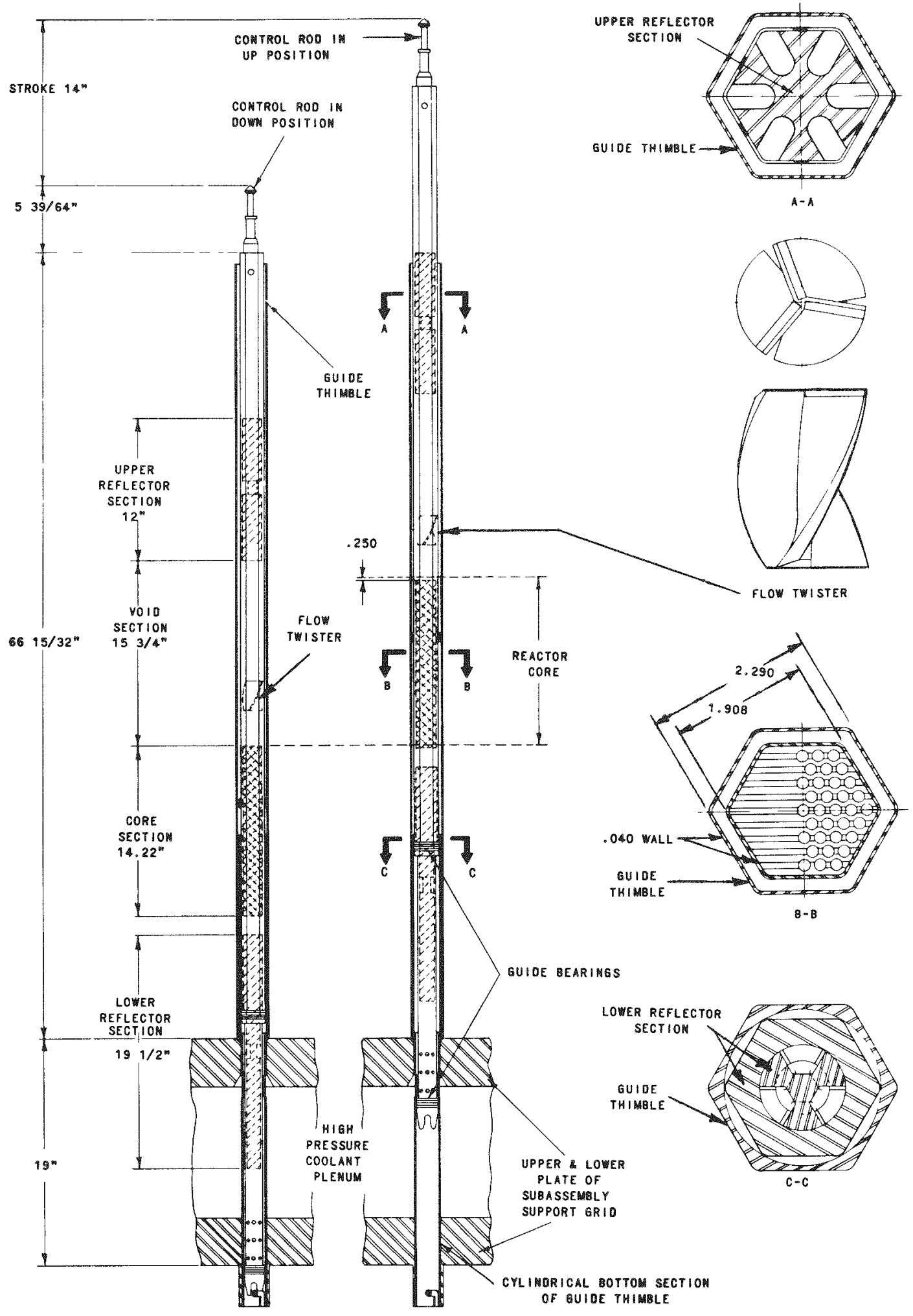

Fig. 4. Control Subassembly 


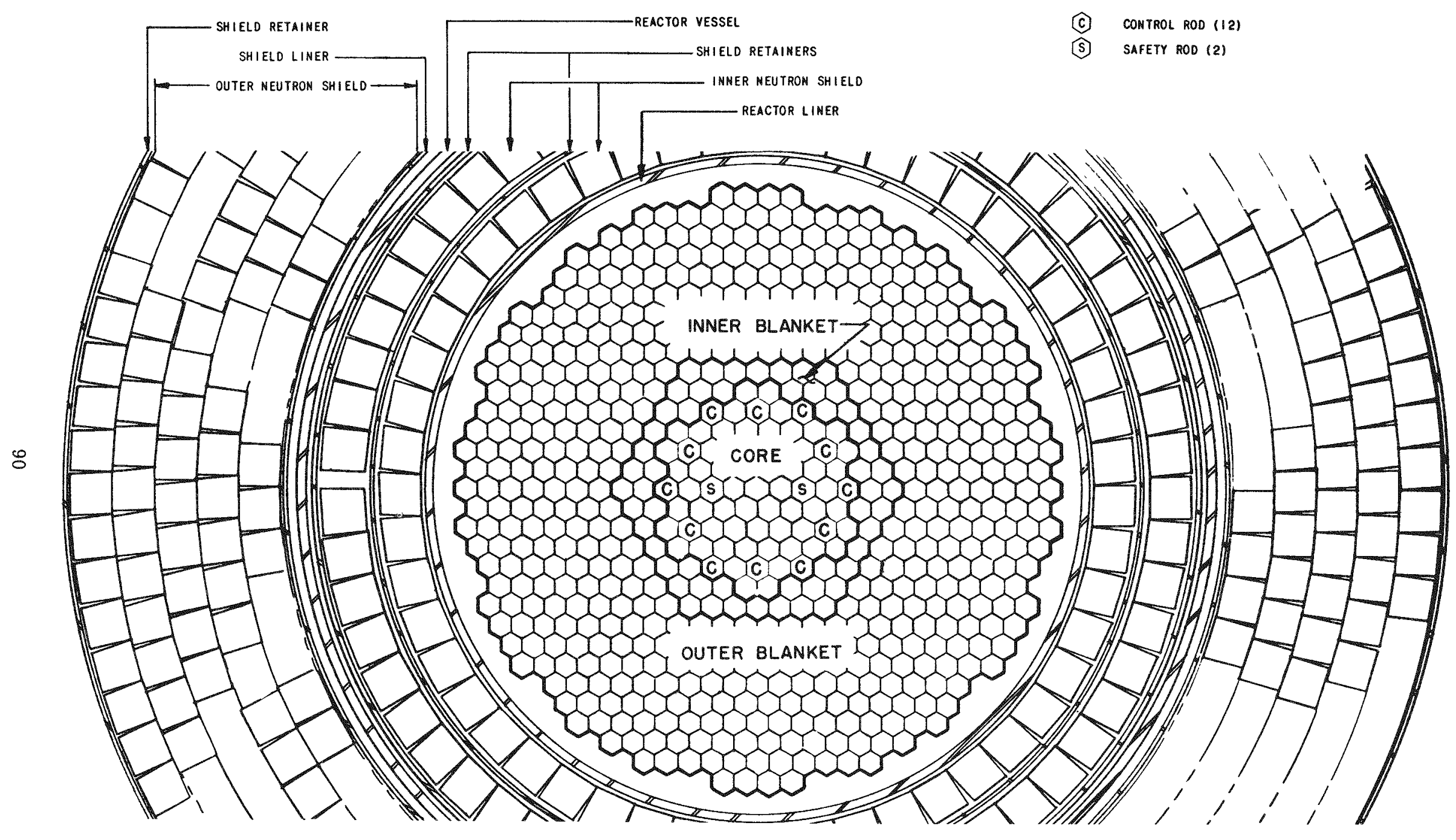

Fig. 5. Reactor Arrangement 


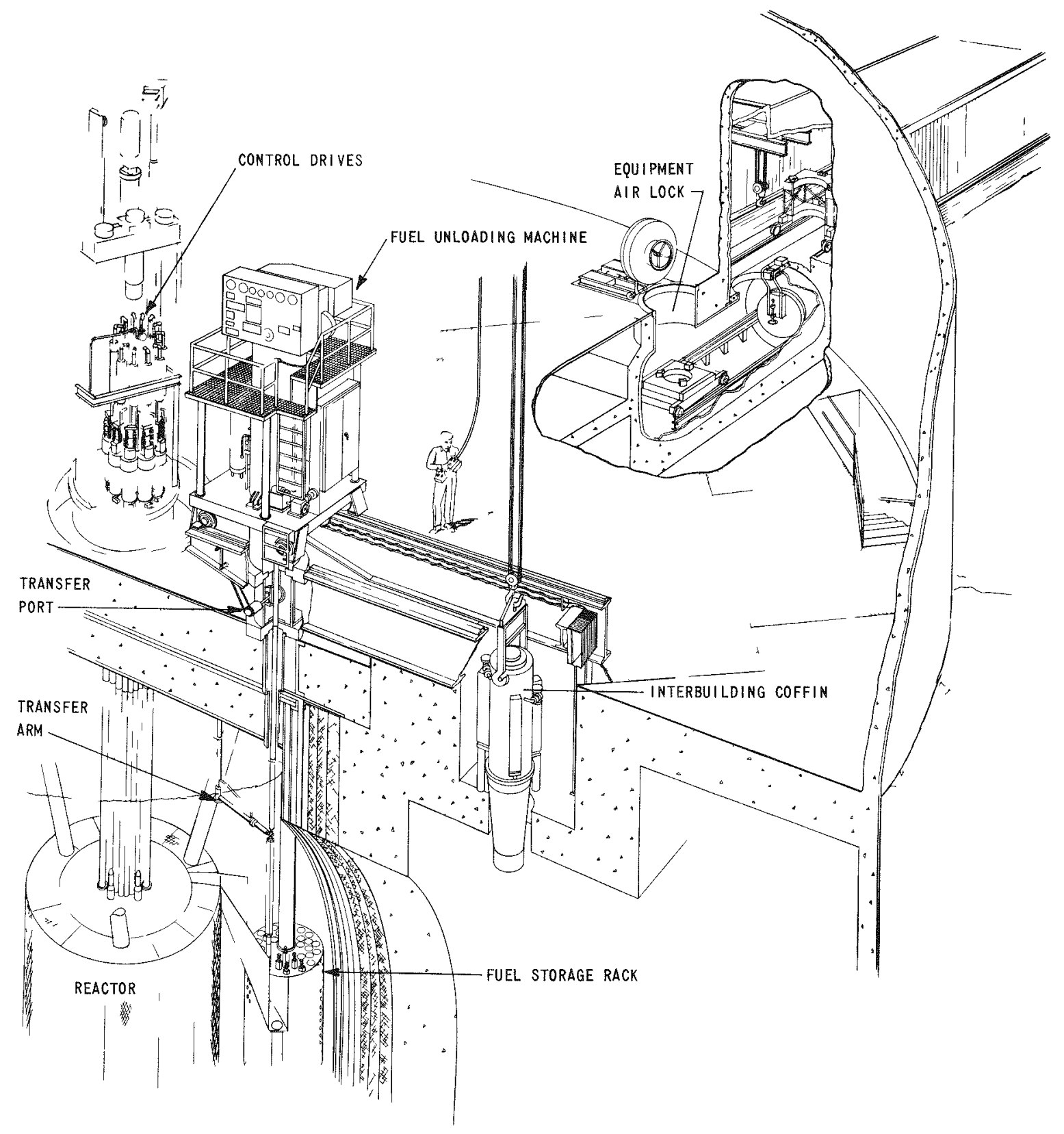

Fig. 6. Fuel-handling System 


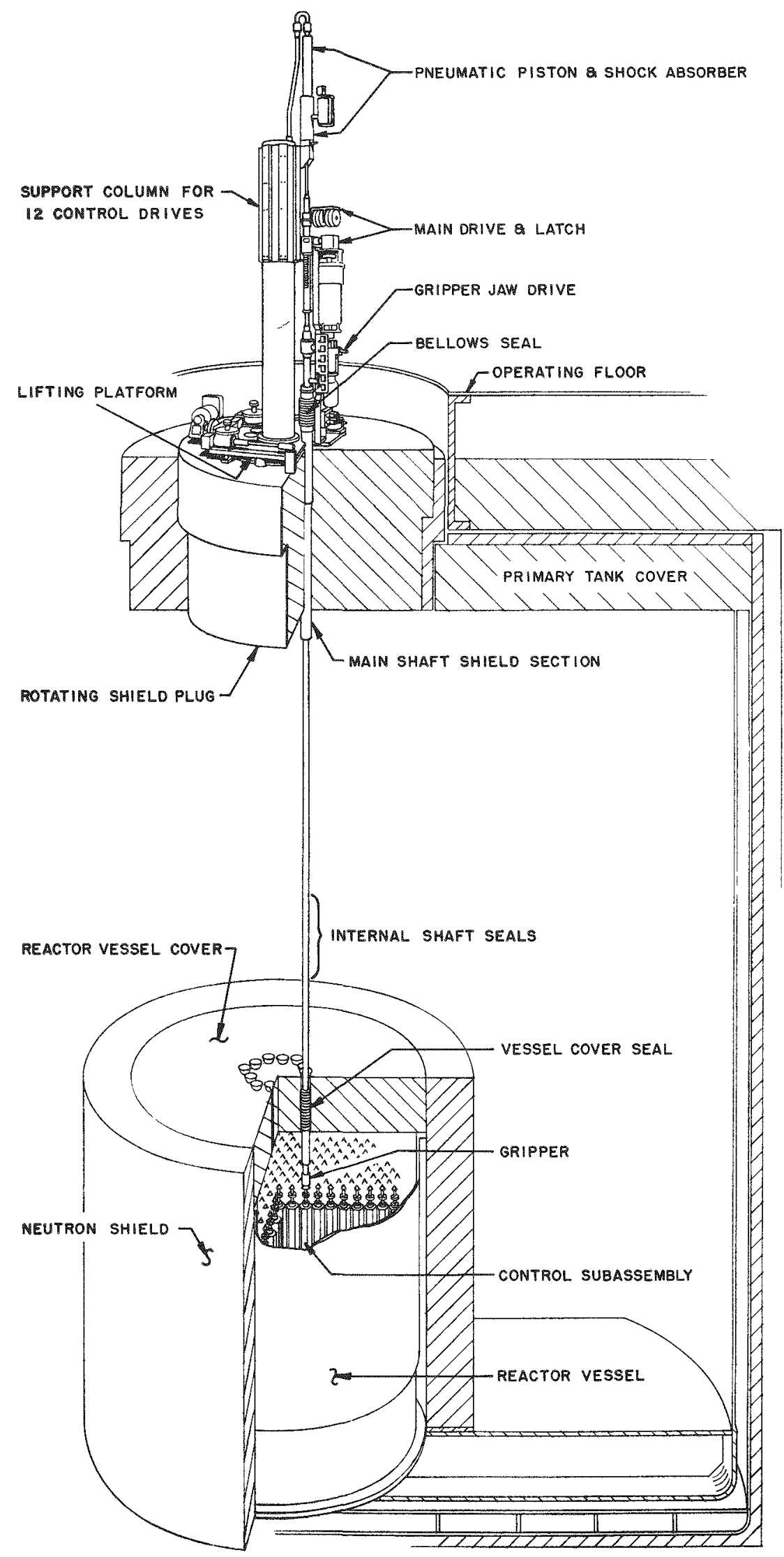

Fig. 7. Control-rod Drive 


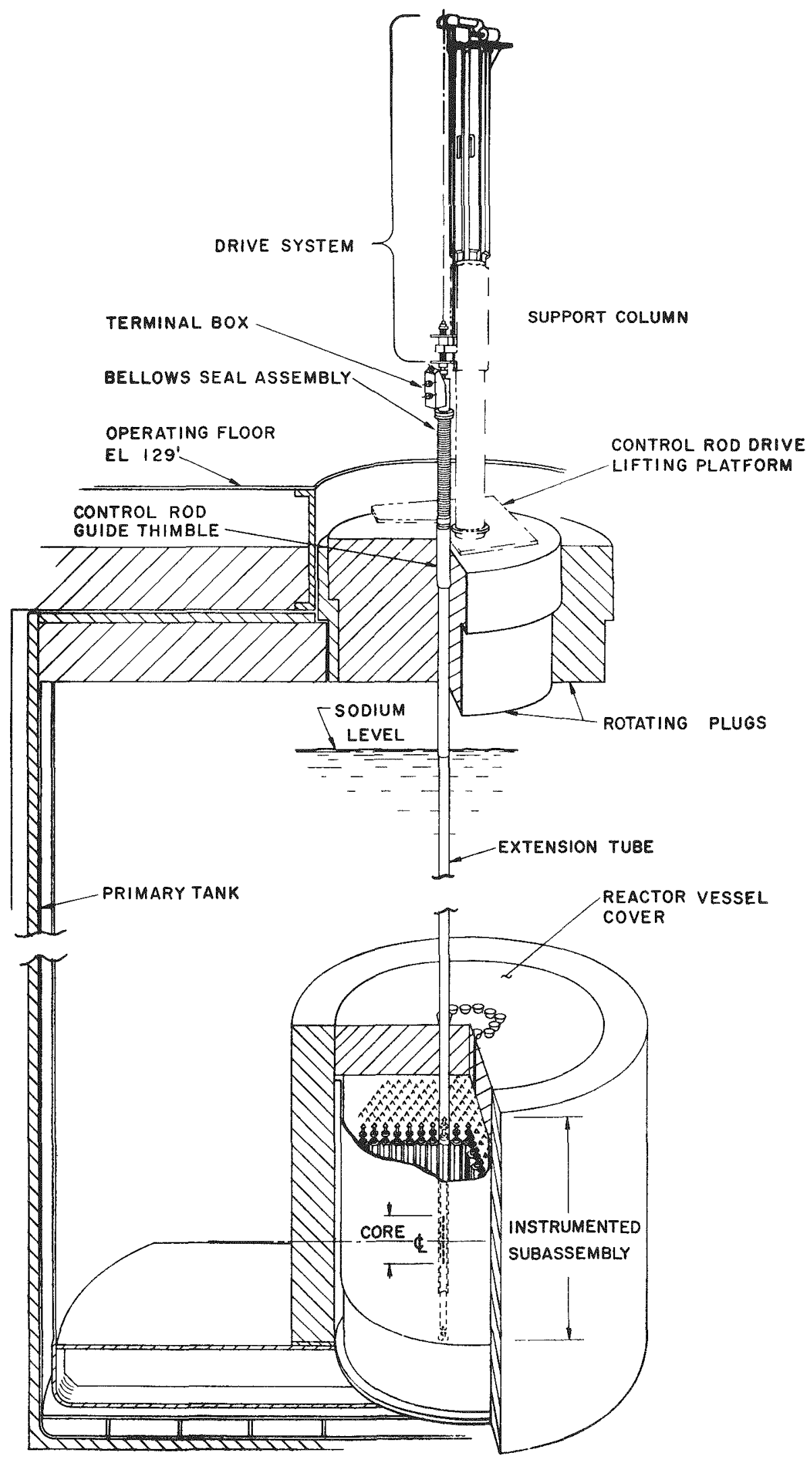

Fig. 8. Instrumented Subassembly Installation 


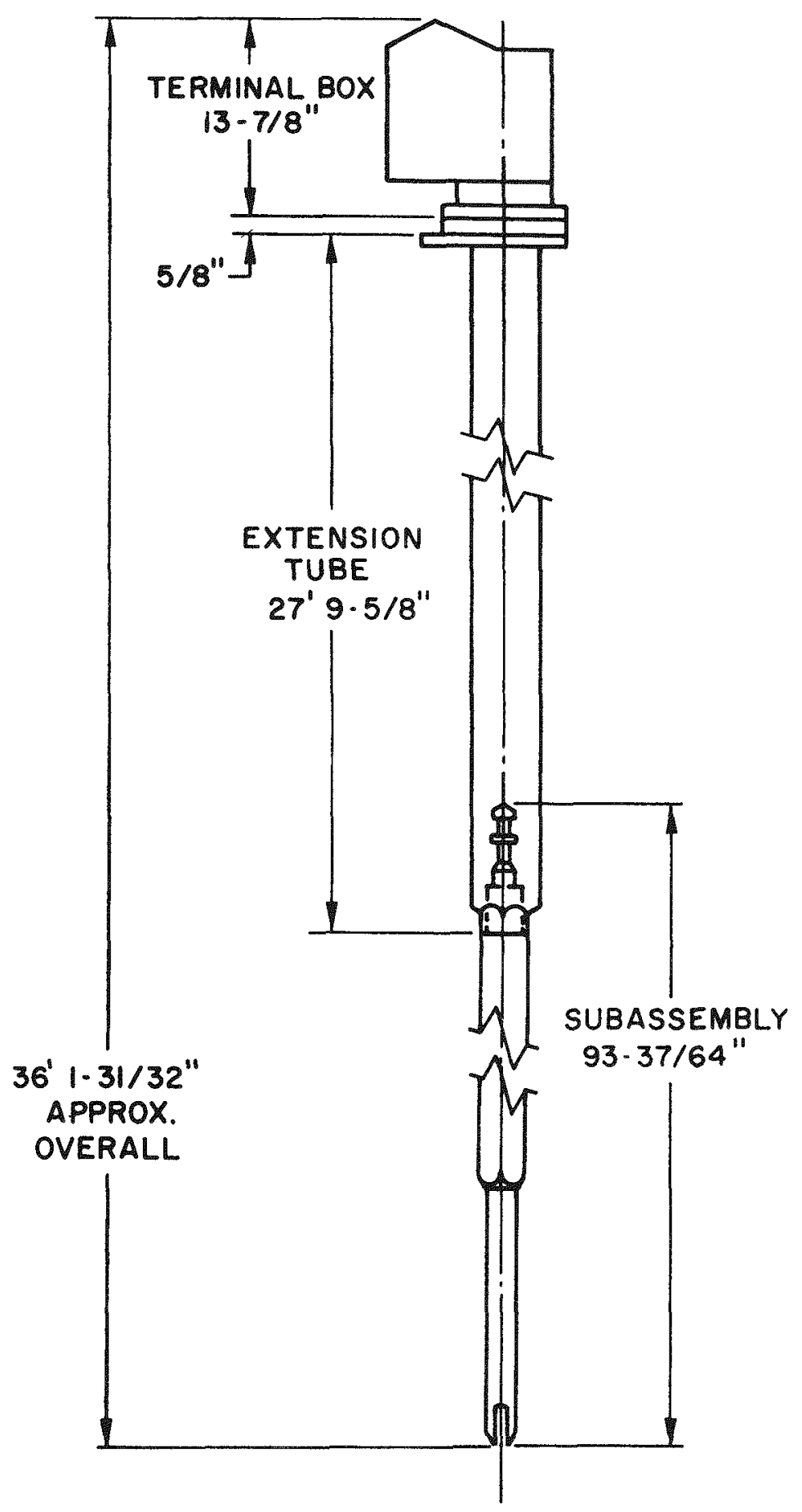

FIG. 9 SUBASSEMBLY AND EXTENSION 


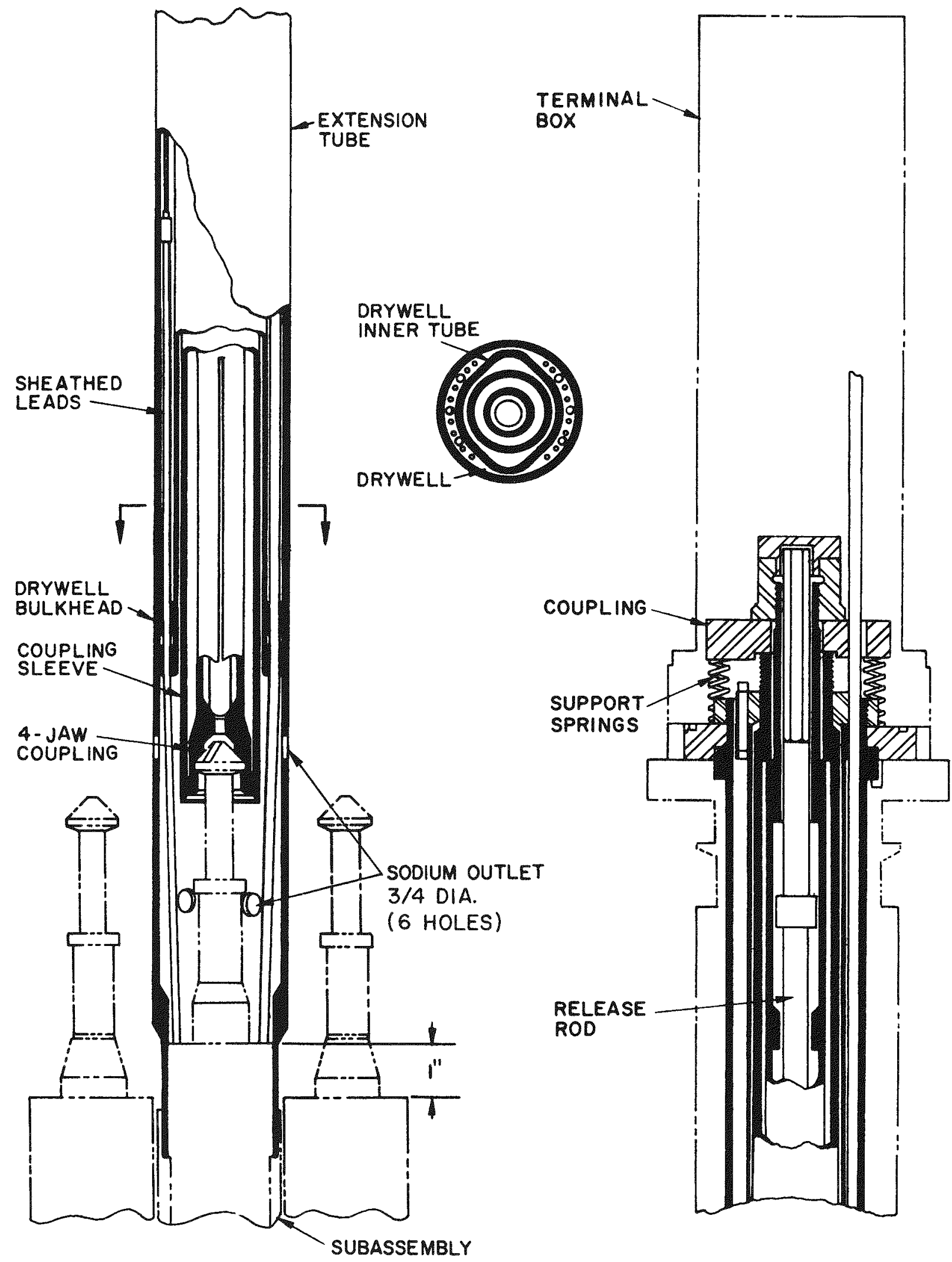

FI6. 10 EXTENSION TUBE 


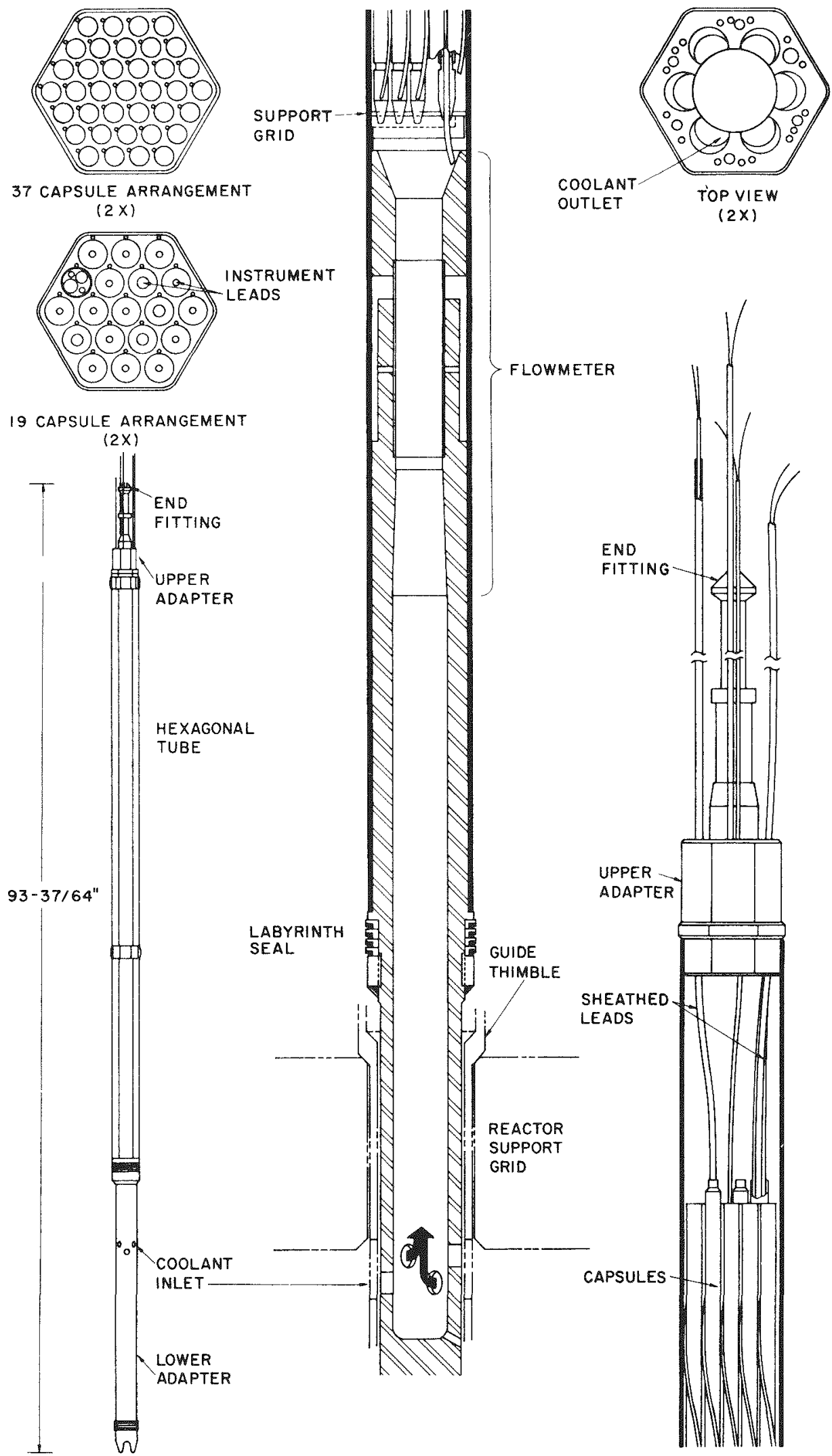

FIG. II INSTRUMENTED SUBASSEMBLY 


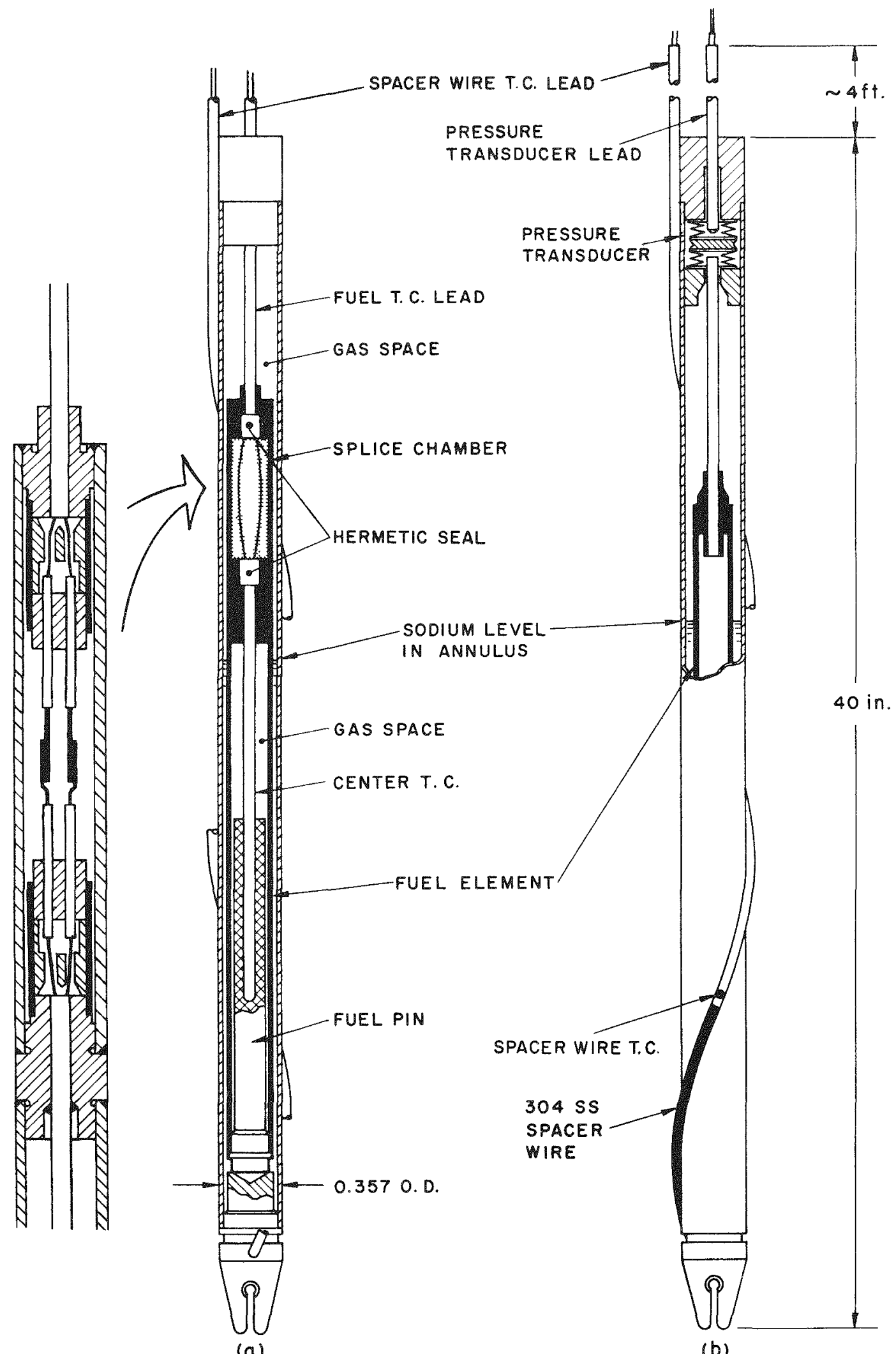

FI6. 12 INSTRUMENTED CAPSULES 


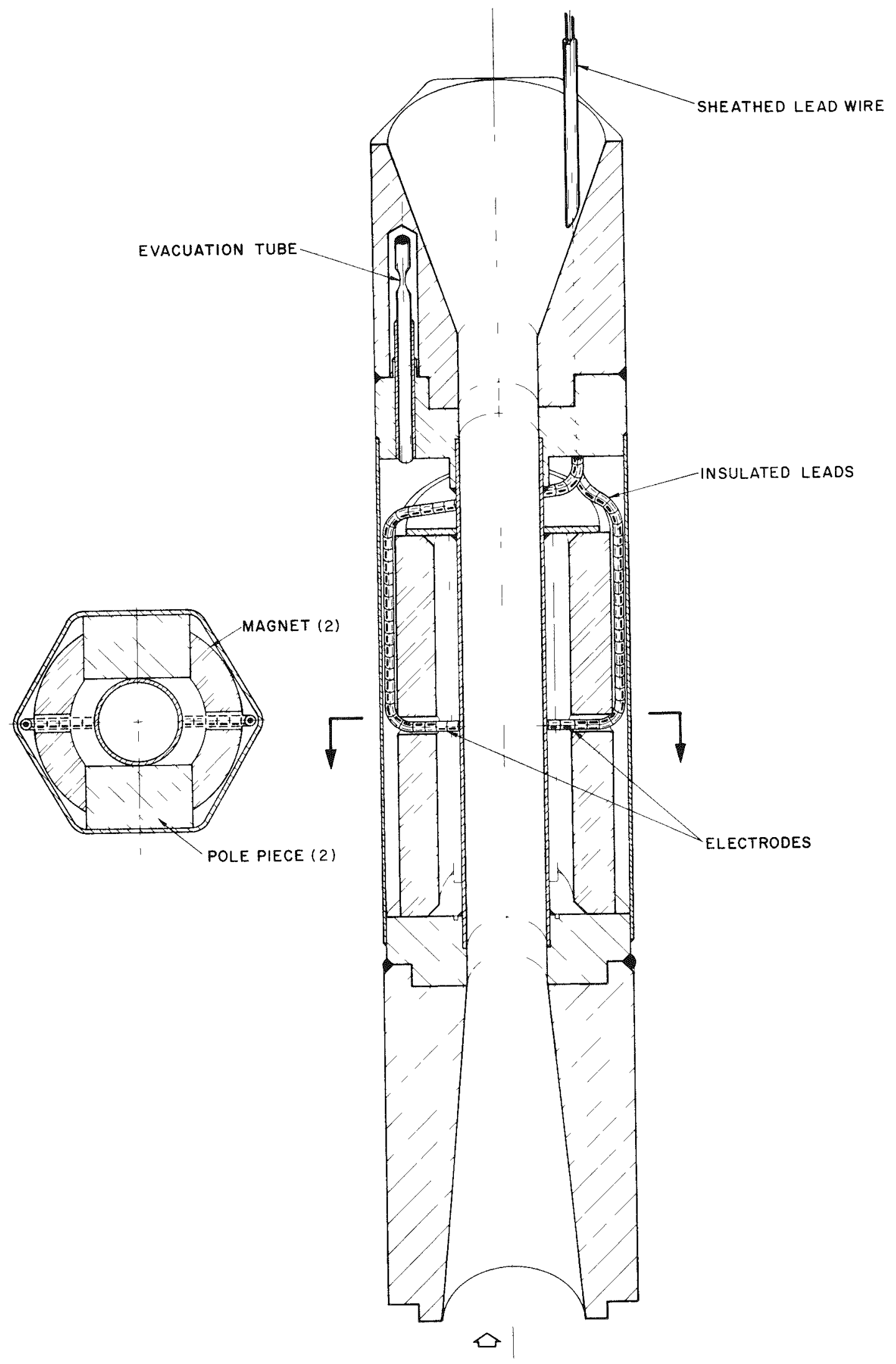

FIG. I3 FLOWMETER 


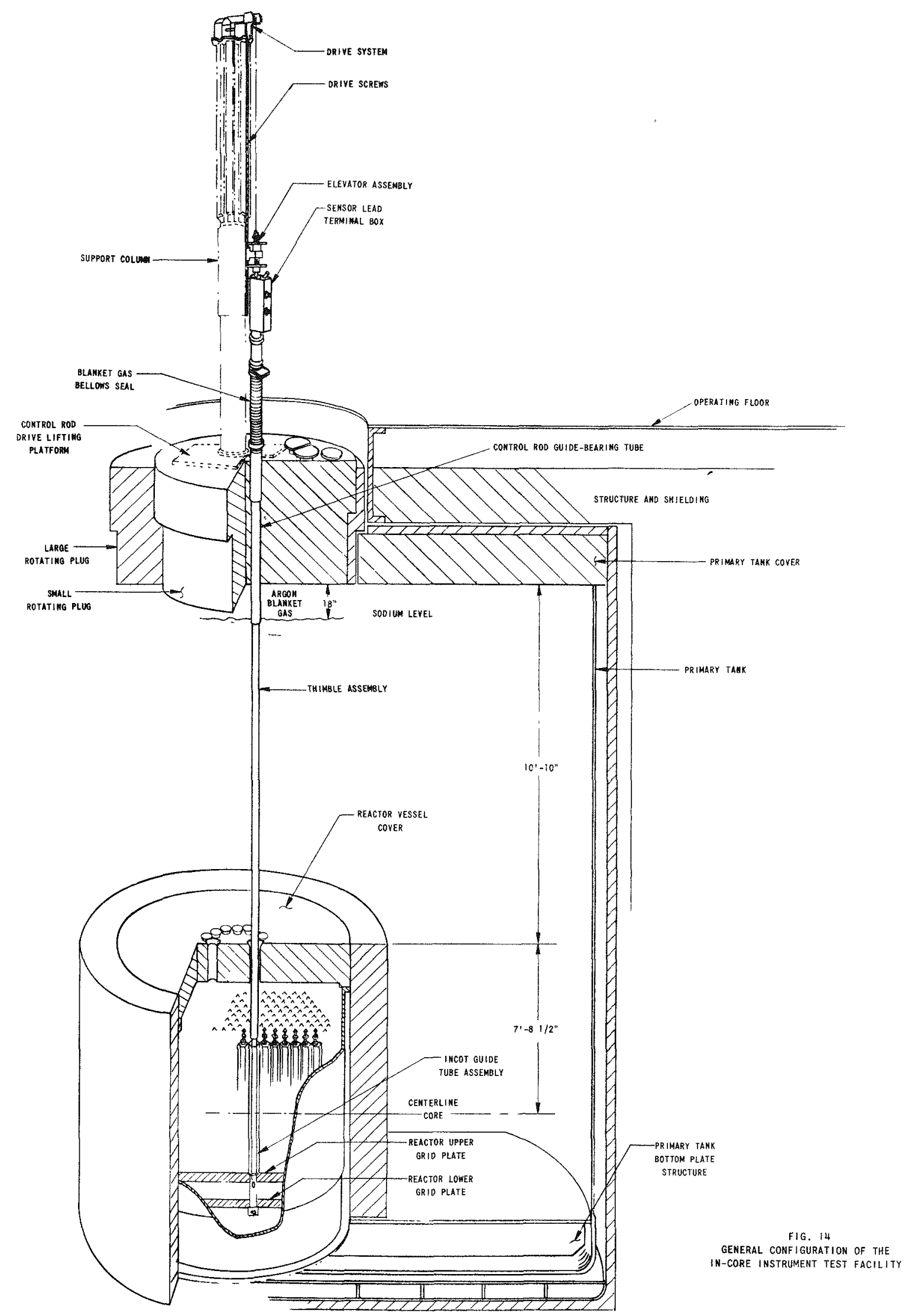




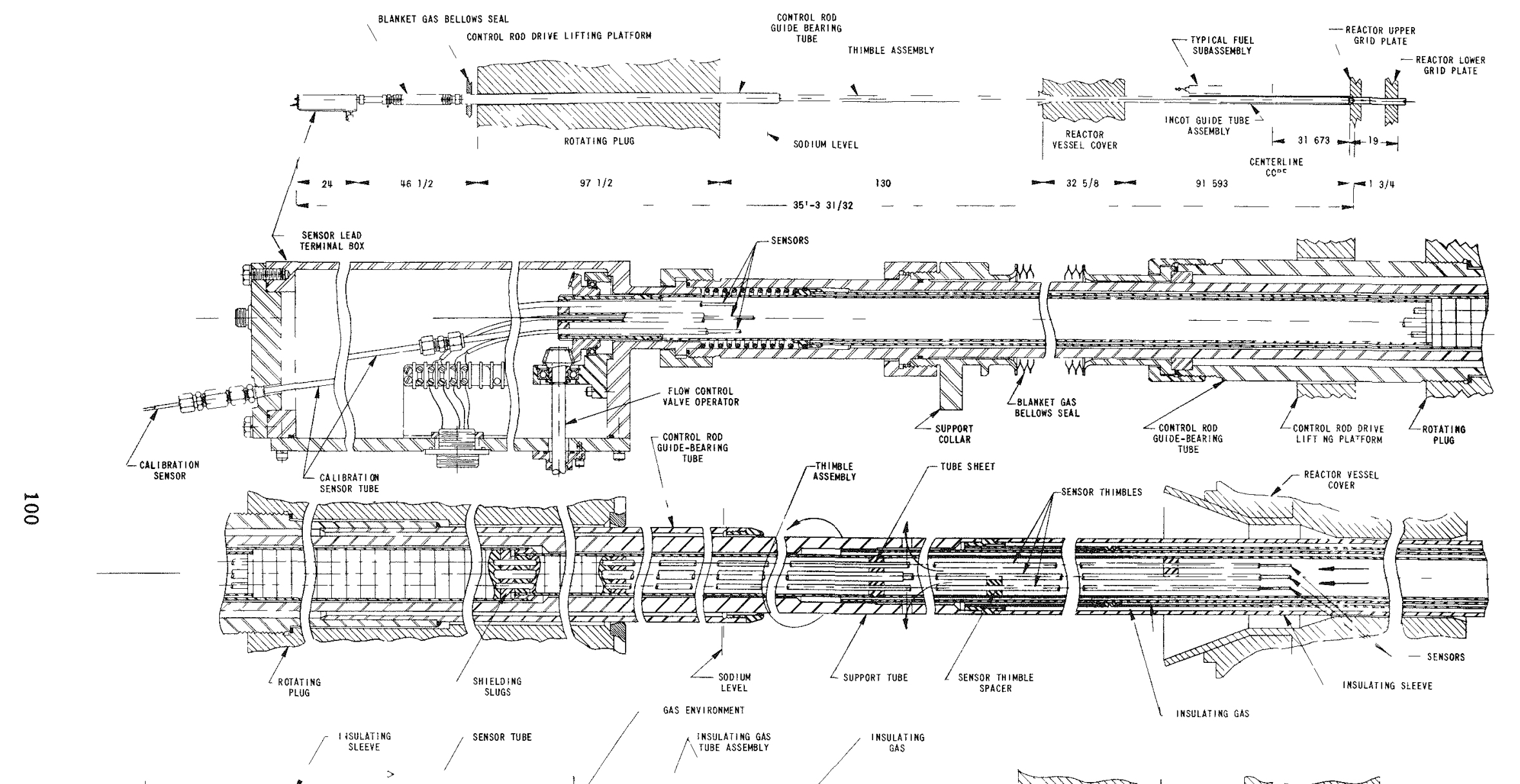

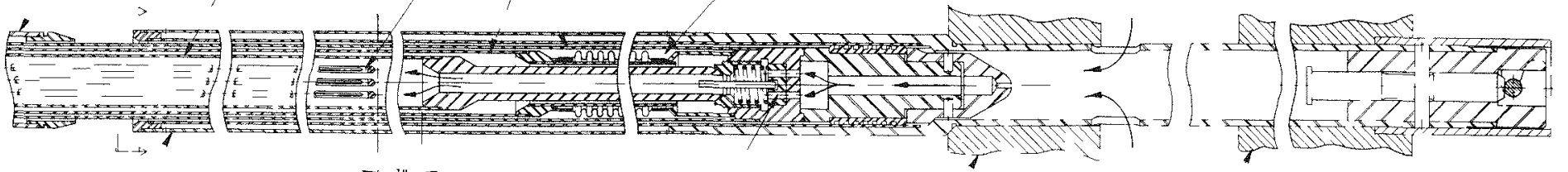

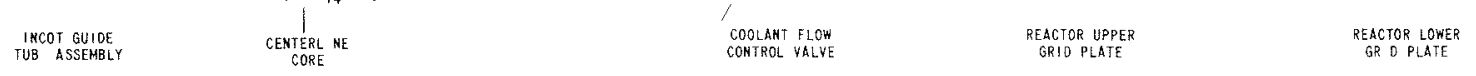

F16 15

INCOT THIMBLE ASSE ABLY

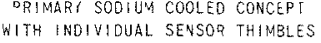
WITH INOIVIDUAL SENSOQ THIMBLES
(MOOEL 1$)$ 
INCOT GUIDE TUBE ASSEMBLY 2.027 I.D.

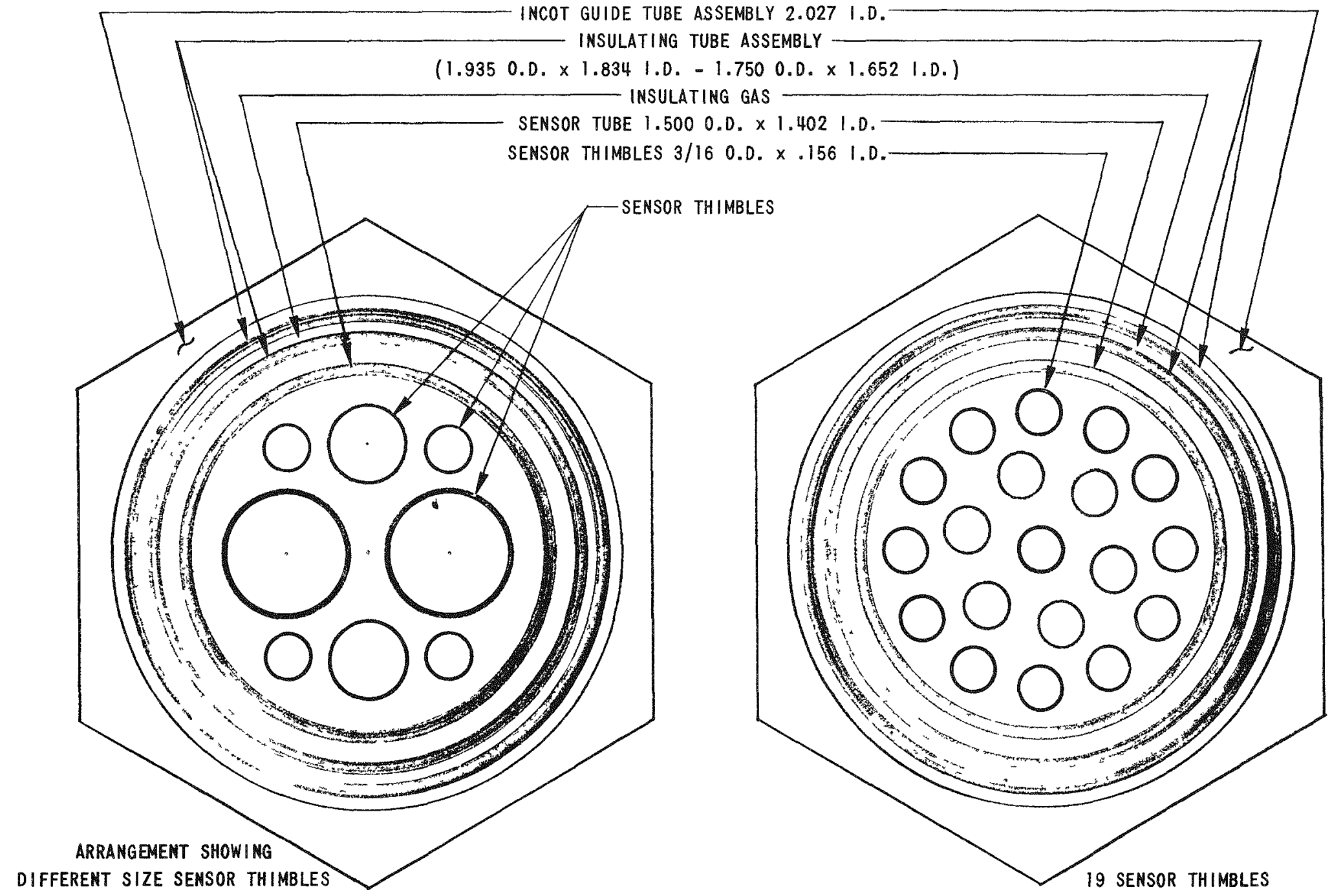

FIG. 16

INCOT THIMBLE ASSEMBLY

WITH INDIVIDUAL SENSOR THIMBLES (MODEL I)

CROSS SECTIONS 


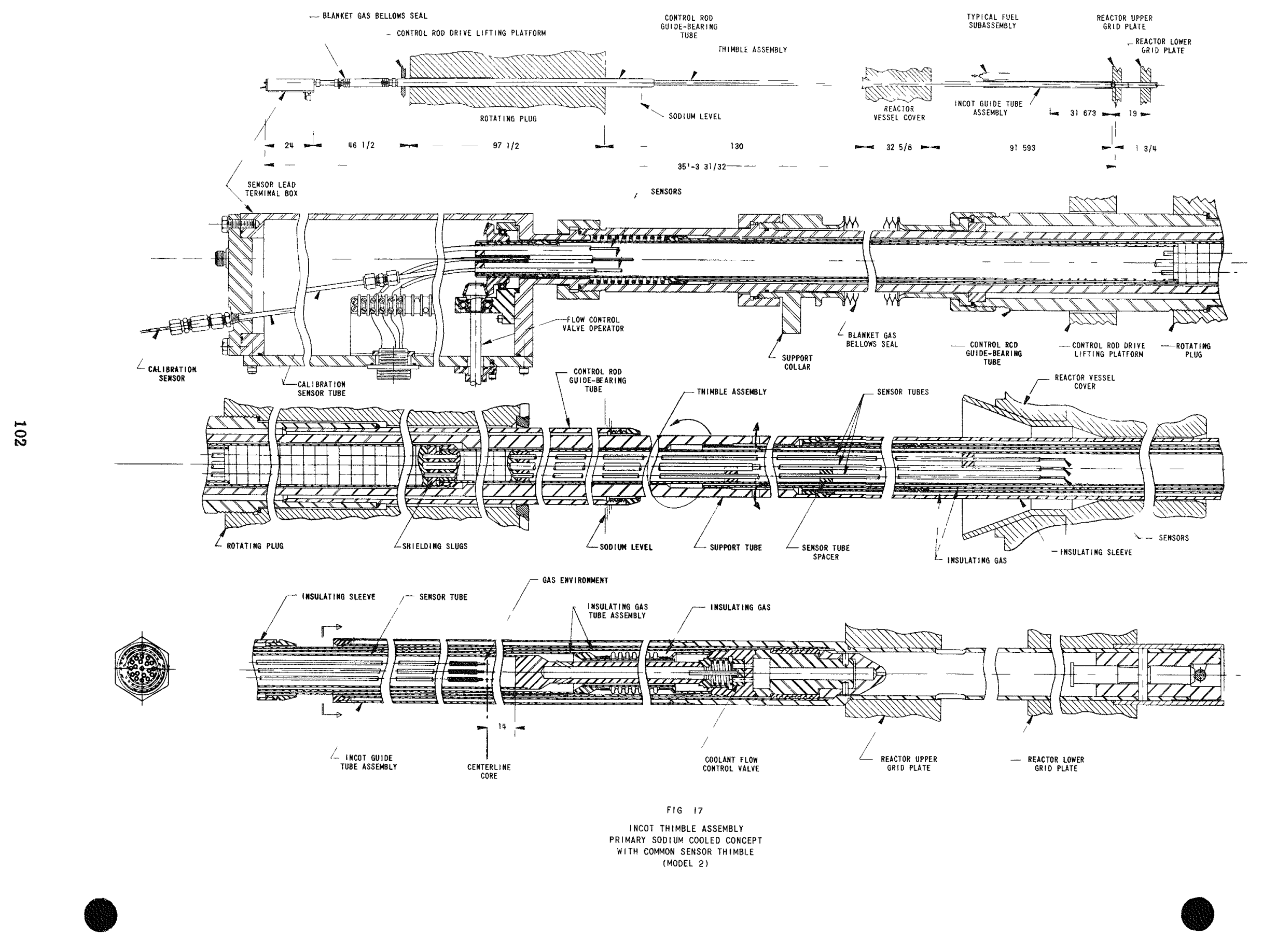




\section{* CHaRaCTERIzation OF ADVANCED IRRADIATION TESTING VEHICLES FOR EBR-II}

O.S. Seim, W.R. Ware, \& E. Hutter

Argonne National Laboratory Argonne, I1Iinois 60439

The EBR-II (Experimental Breeder Reactor No. II) was originally designed and operated by the Argonne National Laboratory to demonstrate a sodium-cooled fast-breeder reactor power plant. An aerial view of the EBR-II Facility located at the National Reactor Test Station near Idaho Falls, Idaho, is presented in Figure 1. The reactor building with its hemispherical top is visible in the center of the figure. It contains the reactor and its primary coolant system. A section through the reactor building is shown in Figure 2. The reactor vessel is situated at the bottom of the 26 foot diameter primary tank located slightly off center in the reactor building. The entire primary sodium coolant system (with the exception of the small purification system) is contained within the double walled primary tank. The normal reactor inlet coolant temperature is $700^{\circ} \mathrm{F}$ and the outlet temperature is about $900^{\circ} \mathrm{F}$. The flux level is about $2(10)^{15} \mathrm{n} / \mathrm{cm}^{2} \mathrm{sec}$.

A vertical section through the reactor vessel is presented in Figure 3. Currently the reactor core consists of the first six rows of hexagonal fuel subassemblies. The first seven rows of subassemblies receive coolant sodium from the high pressure inlet plenum. The seventh reactor row is an inner reflector. The surrounding reactor rows ( 8 through 16) are outer reflector and blanket. The core portion of the reactor is approximately 14 inches high and 24 inches in diameter. The reactor vessel cover lifts for fuel subassembly handling, and remains suspended from the rotating plugs during these activities. The rotating plugs form part of the biological shielding of the primary tank cover and enable the fuel handling mechanisms to reach any fuel location in the reactor vessel.

Following the successful demonstration of the plant as a fast breeder power p1ant, EBR-II has been operating as an irradiation test facility. The present emphasis in the nation's reactor development program on fast 
breeder technology makes this the key experimental facility for virtually all concemed with fast breeder development. This facility is shared by many different experimenters.

A total of sixty-eight experimental irradiation subassemblies have been placed in the reactor. Each irradiation subassembly type has been designed and developed to serve as an irradiation vehicle to contain experimental fuel elements and materials of interest, and provide the required irradiation conditions. The sixteen types of irradiation subassemblies which have been designed to permit these experimental irradiations are shown in Table I. All these vehicles are identical in exterior shape and dimensions, to the standard fuel subassemblies.

Each irradiation subassembly contains numerous irradiation experiments. Each subassembly may contain from seven to 61 capsules or elements. A capsule is a sealed container for a test element or a number of structural test specimens. The capsule usually contains sodium to achieve good heat transfer between the irradiating specimens and the reactor primary sodium coolant. An element is the fuel material inserted and sealed inside its jacket. Usually this duplicates the manner the fuel is expected to be employed in a future actual fast reactor application.

Most new fuel element designs having untried features, or fuel irradiations to relatively high burnup, are placed in sealed capsules to obtain a secondary protection against jacket failure and subsequent release of fission products to the reactor coolant. This satisfies reactor safety requirements.

Encapsulated fuel experiments and structural test specimens obviously contain less fuel than would be in the driver fuel they replace. This deficiency in reactivity is overcome by enlarging the core size. Originally, when the reactor was used for power demonstration, the core consisted of only slightly more than five radial rows of driver fuel. The present core size consists of six radial rows of driver and irradiation subassemblies. Thus the core now contains about 30 more core subassemblies than in its original configuration.

The design objectives for irradiation subassemblies are shown schematically in Figure 4. The first type provides 19 capsules each surrounded by a shroud tube (A). The shroud tube provides equal coolant flow around each of the capsules. The second type accommodates more irradiation capsules within each subassembly by using helical spacing wires $(B, F, H)$. Several new capsule or element diameters wire selected for the 7, 19, 37 , and 61 arrays which precipitate from a triangular pitch within a hexagonal enclosure. To permit higher irradiation temperatures, the third type employs a coolant bypass inside the subassembly $\left(C_{3} J\right)$. The bypassing sodium is mixed with the outlet from the irradiation capsules within the subassembly to obtain an acceptable subassembly outlet temperature. The fourth type is an irradiation subassembly having debris collection devices above and below the test elements (E). The intent is to contain any particulates leaving vented irradiation test fuel elements or high burnup singly encapsulated fuel elements. 
The first irradiation subassembly was Mark A-19 (Figure 5). Its 0.375 in. OD capsule size is particularly useful for encapsulated fuel element irradiations. Each capsule fits into an enclosing shroud tube to assure a uniform flow distribution to each capsule. The shroud tube has dimpled indents to center the capsule. The space between the shroud tubes is blocked off. Orificing, internal to this irradiation subassembly, controls the coolant exit temperatures of the adjacent subassemblies.

The Mark B was the second type irradiation subassembly designed. Four models of the Mark B irradiation subassembly, each comprised of a different configuration of elements, are provided to accommodate different irradiation experiments. The four types are identified by the number of elements: $\mathrm{B}-7, \mathrm{~B}-19, \mathrm{~B}-37$, and $\mathrm{B}-61$.

Figure 6 illustrates a Mark B irradiation subassembly with B-7 type elements. A hexagonally shaped grid support plate is mounted at the top of the lower adaptor. The bottom of the grid support plate can be supplied with an open bottom to provide maximum coolant flow; or it can be supplied with an orifice plate to conform to the requirements of the experimenter for the desired amount of coolant flow in a similiar manner as described for the Mark A irradiation subassembly. The test specimens can be placed in positions inside the capsules so as to obtain the most desirable irradiation conditions.

The Mark C irradiation subassembly (Figure 7) was developed to provide a higher temperature environment than that available with the Mark A or Mark B subassemblies. It permits sodium coolant temperatures of up to $1200^{\circ} \mathrm{F}$ in the specimen container without exceeding the thermal stress limits of the subassembly structure. Simultaneously it prevents the coolant from exiting into the reactor vessel outlet plenum at too high temperatures by mixing it with the cooler sodium flowing through the subassembly. The bypass flow design also provides higher total sodium flow rates than are possible with a standard subassembly. The greatest advantage of this design is that separate total flow and bypass flow orificing allow selection of the desired specimen temperature. The internal structures incorporate an internal hexagonal tube and liner within the outer hexagonal tube, a bypass flow channel, a flow divider, and a mixer section. The Mark $\mathrm{C}$ irradiation subassembly figure illustrates the Mark C-19 and Mark C-37 subassemblies.

The Mark B-61A, Mark E, Mark F, Mark $H$ and the Mark $J$ irradiation subassemblies are advanced irradiation vehicles and they are intended to accommodate unencapsulated fuel elements. The designs accept the specific element diameter and pitch-to-diameter ratios of interest for a specific future reactor application.

The Mark B-61A irradiation subassembly is a special modification of the Mark B-61 to accept unencapsulated fuel elements proposed for a specific reactor design. The type B-61A elements have an overall length of 60-7/8 in. and an outside diameter of 0.230 in. A 0.040 in. diameter spacer wire is wound helically around the outside of the element on a. 12 in. pitch. The clearances are such that a one percent diametral growth 
of the elements and spacer wires can be accommodated before the clearances fall below the standard values used in most subassemblies. This is desirable when at high burnup the elements grow one percent more than the enclosing hexagonal tube, or when the test elements are to be reassembled in new hardware for return to the reactor, following examination after high burnup. The hexagonal tube is indented to cluster the elements tightly at their top, and provide more than the standard guidance with limited indentations just above and below the core.

The Mark E-61 is one of the newest irradiation subassembly vehicles. The purpose is to serve as a vehicle for the irradiation of fuel elements with known defects and/or to irradiate to much higher than standard allowable burnup. The Mark E-61 design, as shown in Figure 8, uses multiple hexagonal tubing in the core region to avoid failure propagation to adjacent subassemblies. Particle catcher devices are placed above and below the core. Another Mark E type subassembly is being designed in an effort to promote interchangeability of capsules from other irradiation subassemblies while retaining the extra containment feature.

The Mark $\mathrm{F}$ irradiation subassembly was designed to accept elements which are singly encapsulated (one cladding thickness between the fuel and the EBR-II primary sodium coolant). The Mark F-37 assembles 37 elements of 0.250 in. OD with an 0.0625 spacing wire of 6 or 12 in. pitch. This provides a specific fuel element pitch to fuel element diameter ratio requested by an experimenter. Figure 9 shows the optional orificing, the grid support, and the grid assembly just above the lower adaptor. An internal hexagonal tube of special size is used for dimensional control and lateral support of the elements.

The Mark $\mathrm{H}$ irradiation subassembly was designed to accept singly encapsulated elements. It is identical in feature to the Mark $F$. The Mark H-37 assembles 37 elements of 0.250 in. OD with a 0.040 in. spacing wire of 6 or 12 in. pitch. This provides an additional (in addition to the Mark F-37) specific fuel element pitch to diameter ratio requested by an experimenter.

The Mark $J$ irradiation subassembly (Figure 10 ) is an irradiation vehicle to permit irradiation temperatures above normal EBR-II temperatures. This is possible because of the coolant bypass provided inside the subassembly. A predetermined quantity of coolant flows upward between the tubes which enclose the nineteen irradiation capsules and the standard external hexagonal tube. This bypass flow annulus is necessarily quite small when nineteen capsules of $0.375 \mathrm{in}$. dia. are accommodated. The intent is to reduce the maximum coolant temperature of 1200 oF to the acceptable fuel subassembly outlet temperature. This is accomplished by mixing the coolant stream with the bypass stream. Near the top of the lower axial reflector, the flow is divided into a coolant bypass stream by sizing orifices. The nineteen capsules are fastened at their base with tips that slide over cylindrical top grid bars. Dual hexagonal tubes designed to provide a trapped insulating gas space between them within the core region enclose the cluster of capsules. The insulating gas space below the core is wider so as to provide a reservoir of gas accommodating primary system pressure 
variations. The insulating gas along the core serves to diminish the temperature distribution existing horizontally across the cluster of capsules. The dual hexagonal tube construction extends above the core in order to retain acceptable thermal stresses.

Various modifications of and/or new irradiation subassemblies will continue to be designed to meet the ever changing need for new irradiation vehicles, so as to enhance the capability of EBR-II as an irradiation facility. 
TABLE I

EBR-II IRRADIATION SUBASSEMBIIES

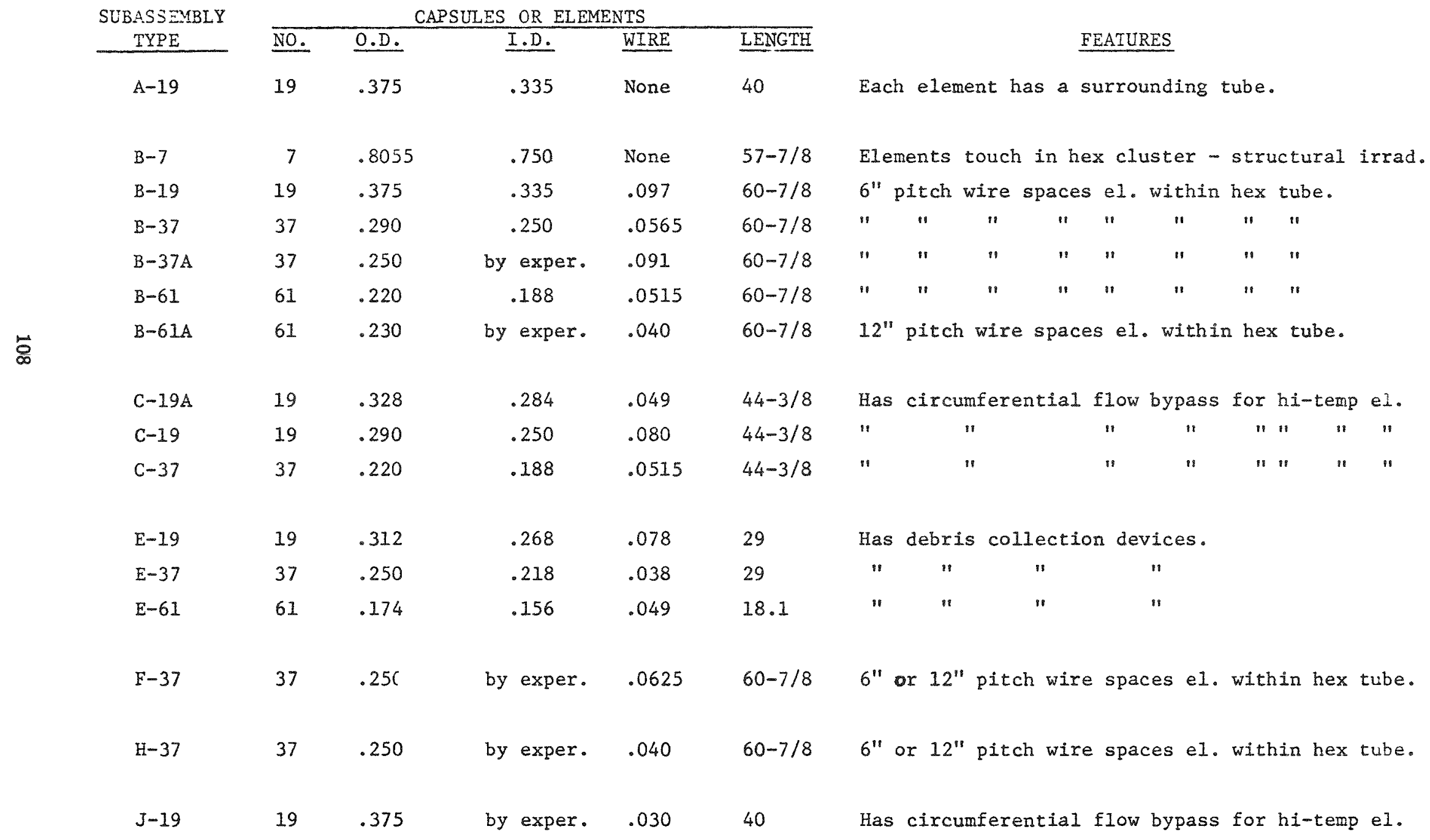




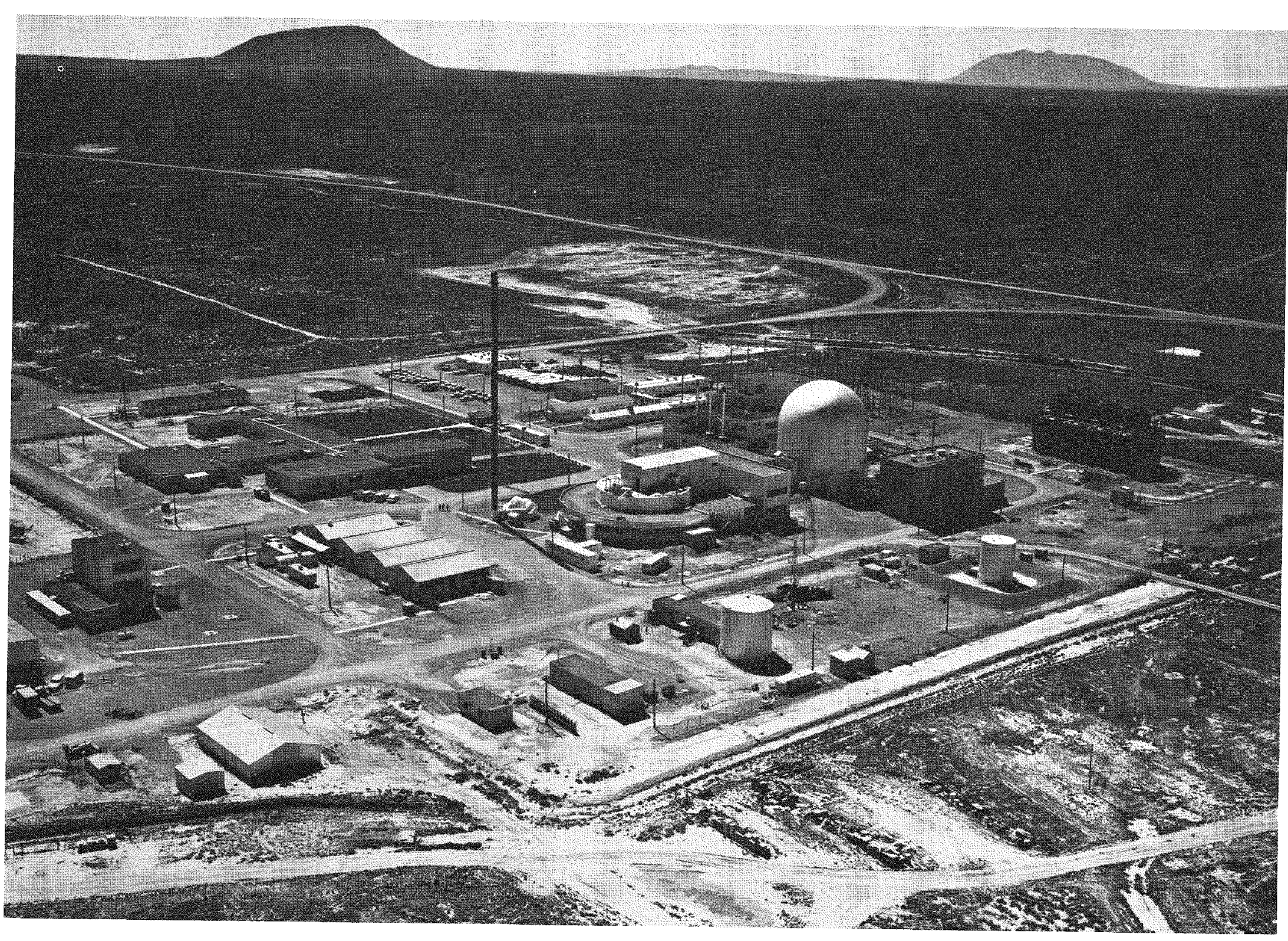

FIG. 1 AERIAL VIEW OF EBR-II FACILITY 


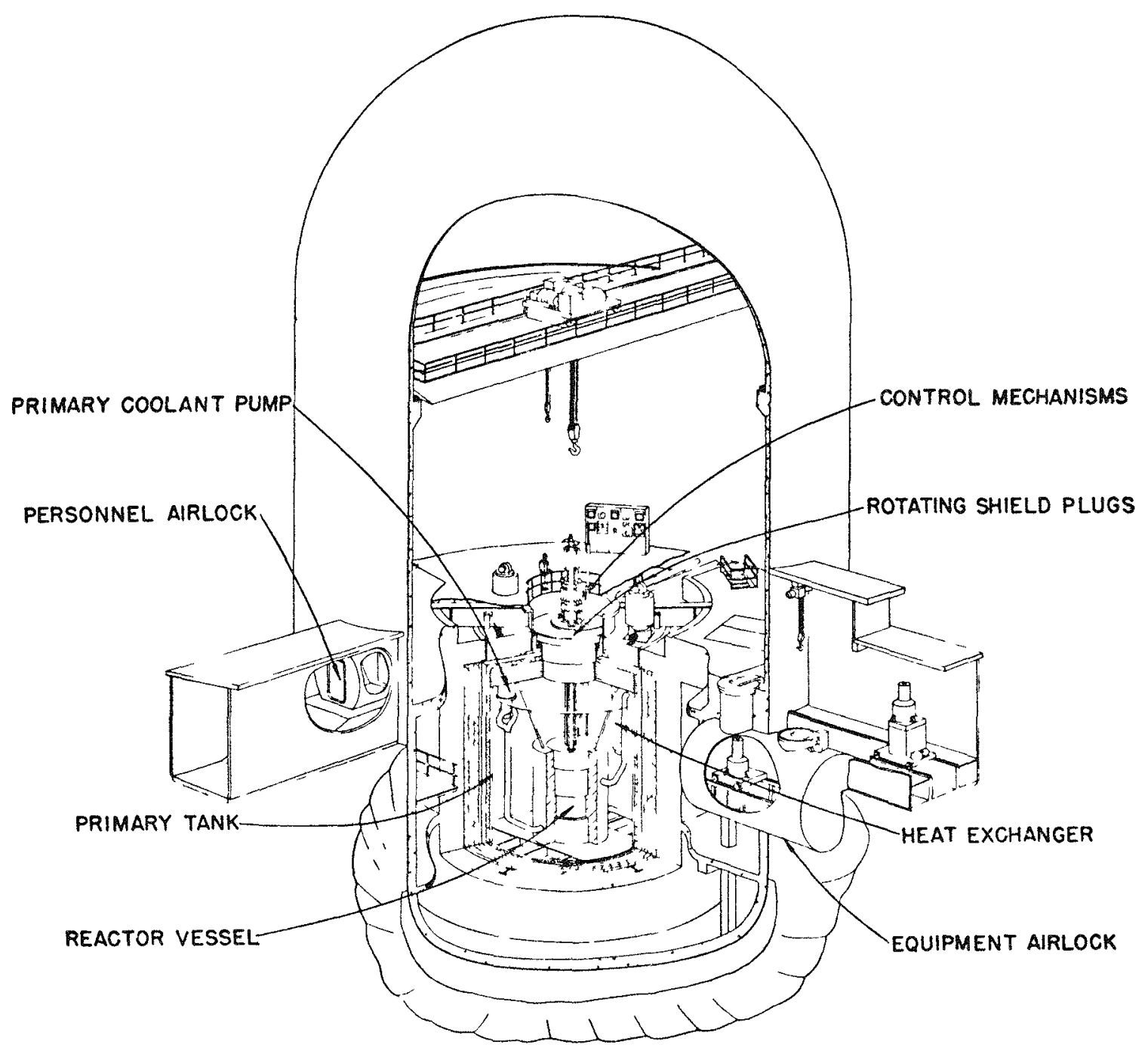

FIG. 2 SECTION THROUGH EBR-II REACTOR BUILDING 


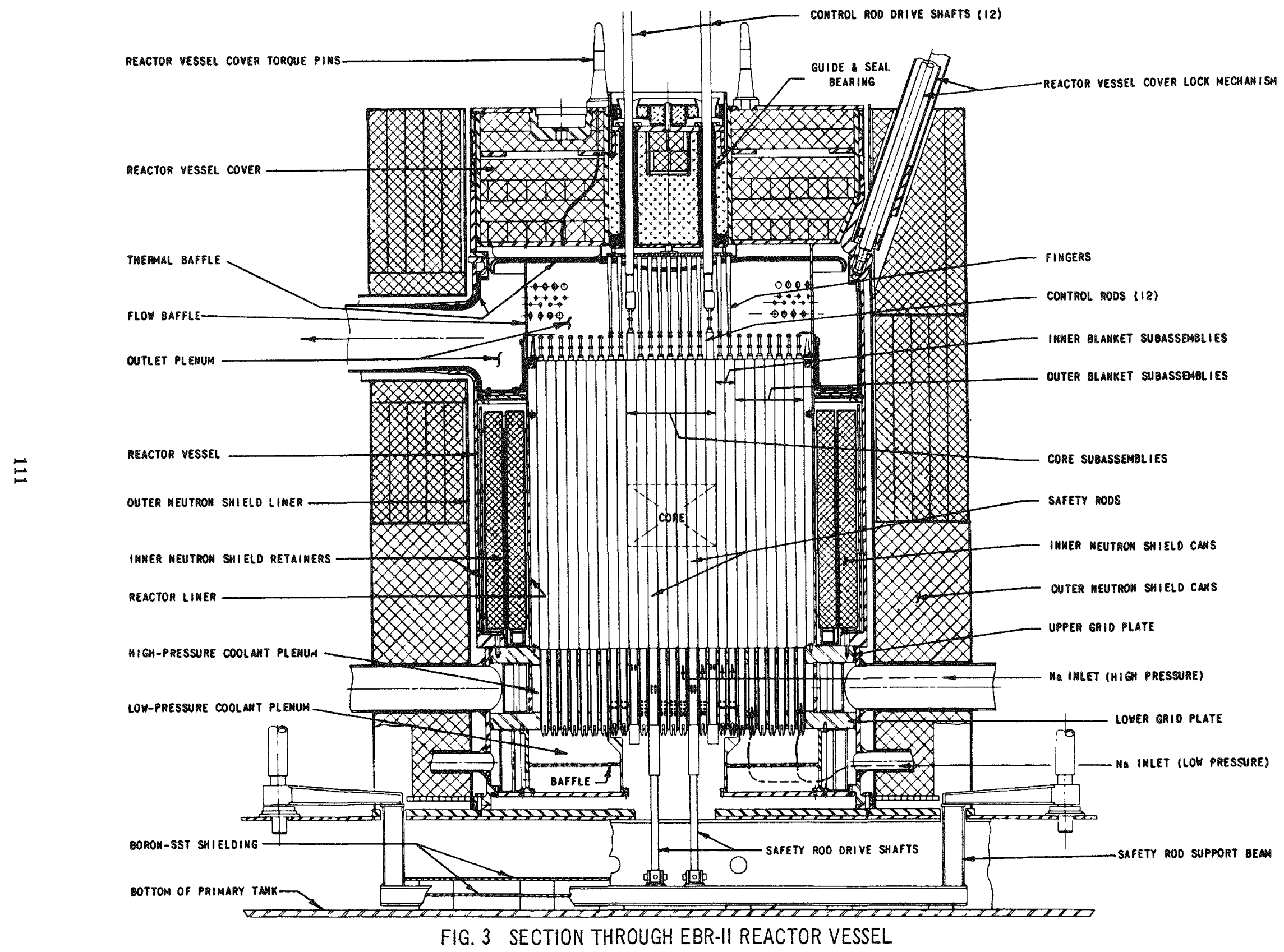




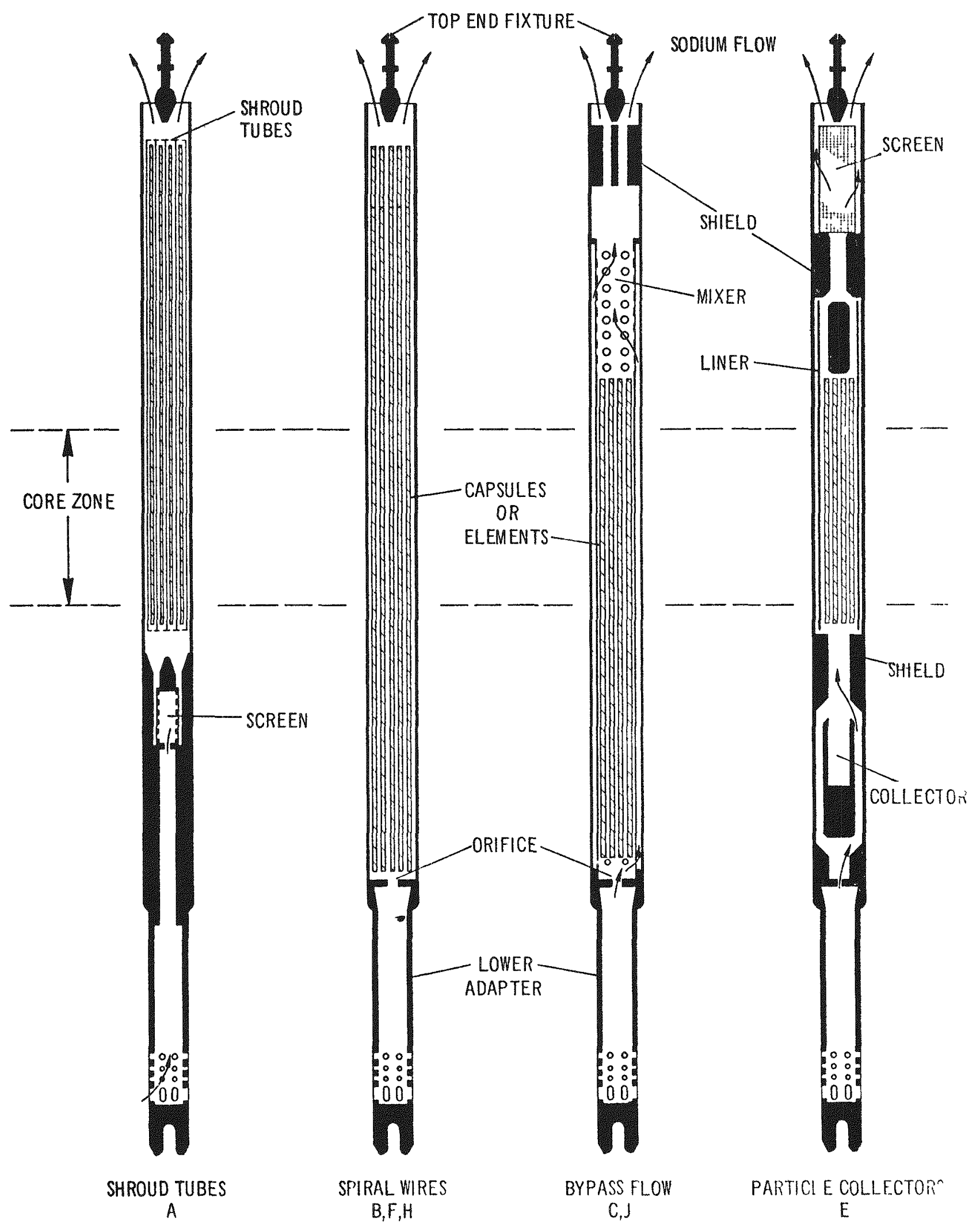

FIG. 4 EBR-II IRRADIATION SUBASSEMBLY TYPES 


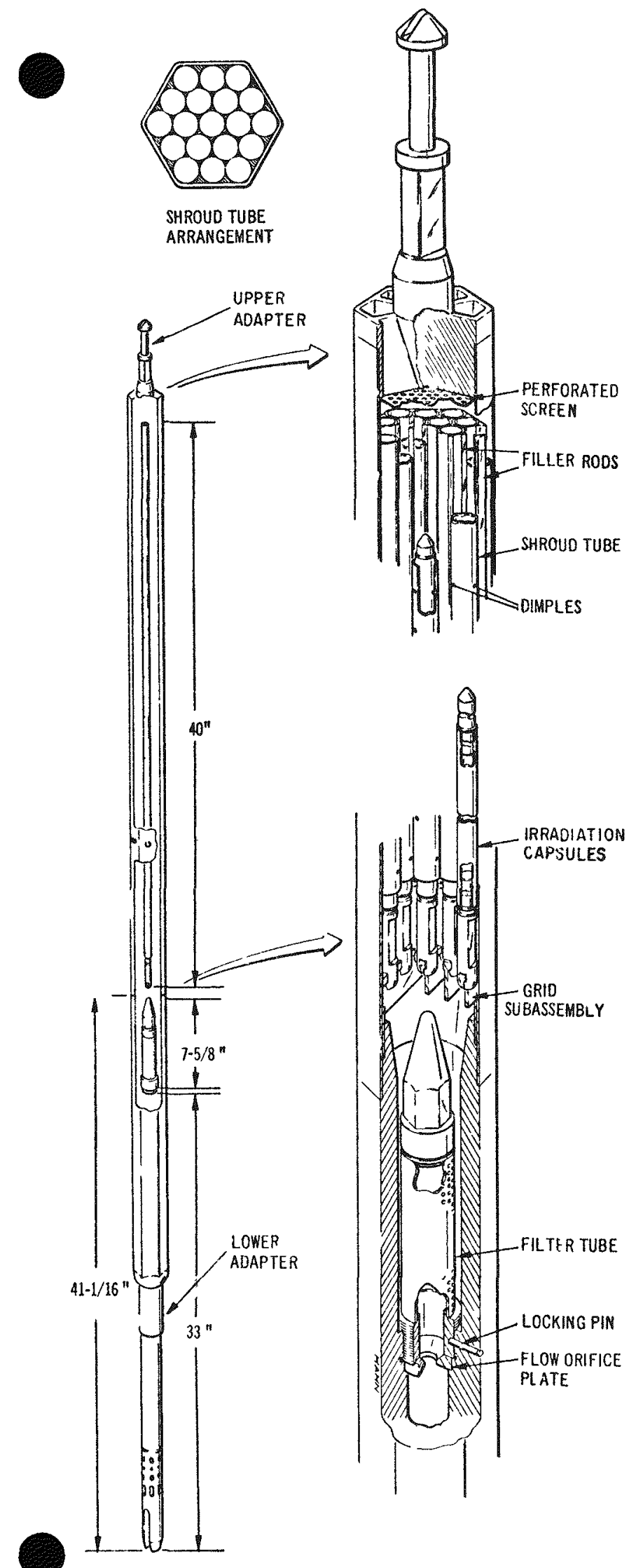

FIG. 5 MARK A IRRADIATION SUBASSEMBLY
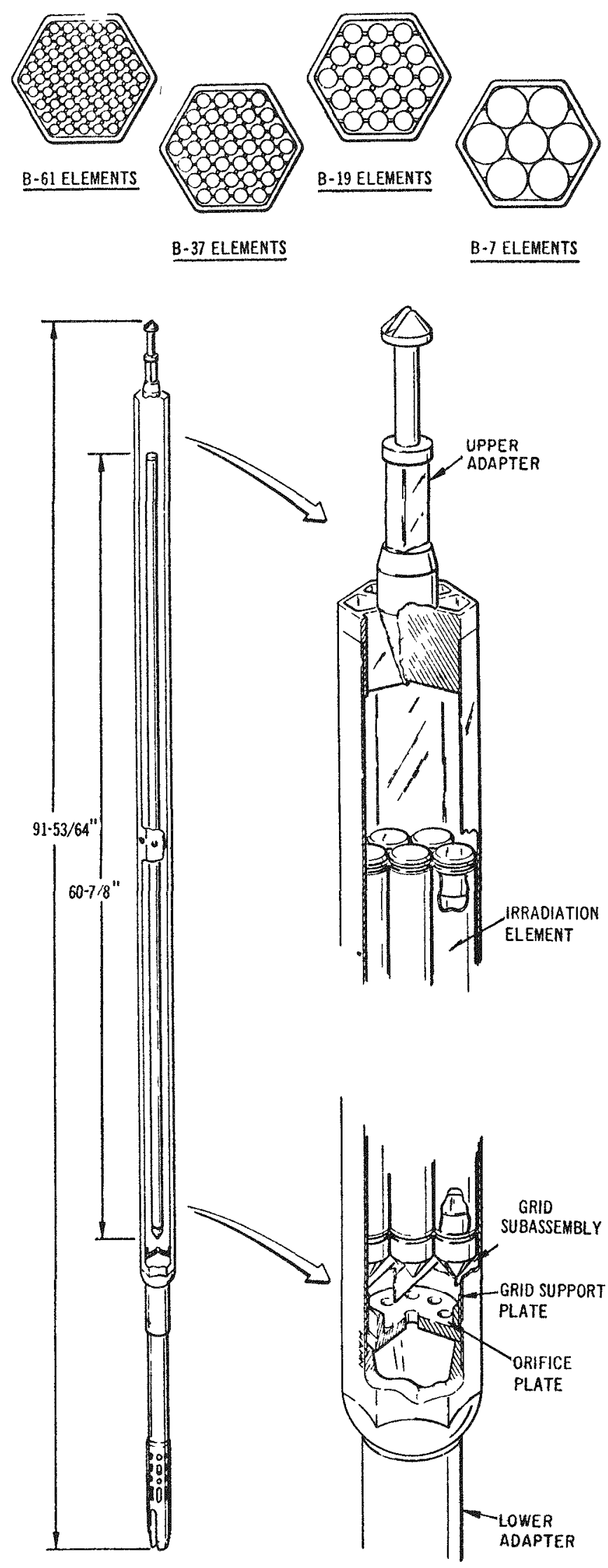

FIC. 6 MARK BIRRADIATION SUBASSEMBLY 


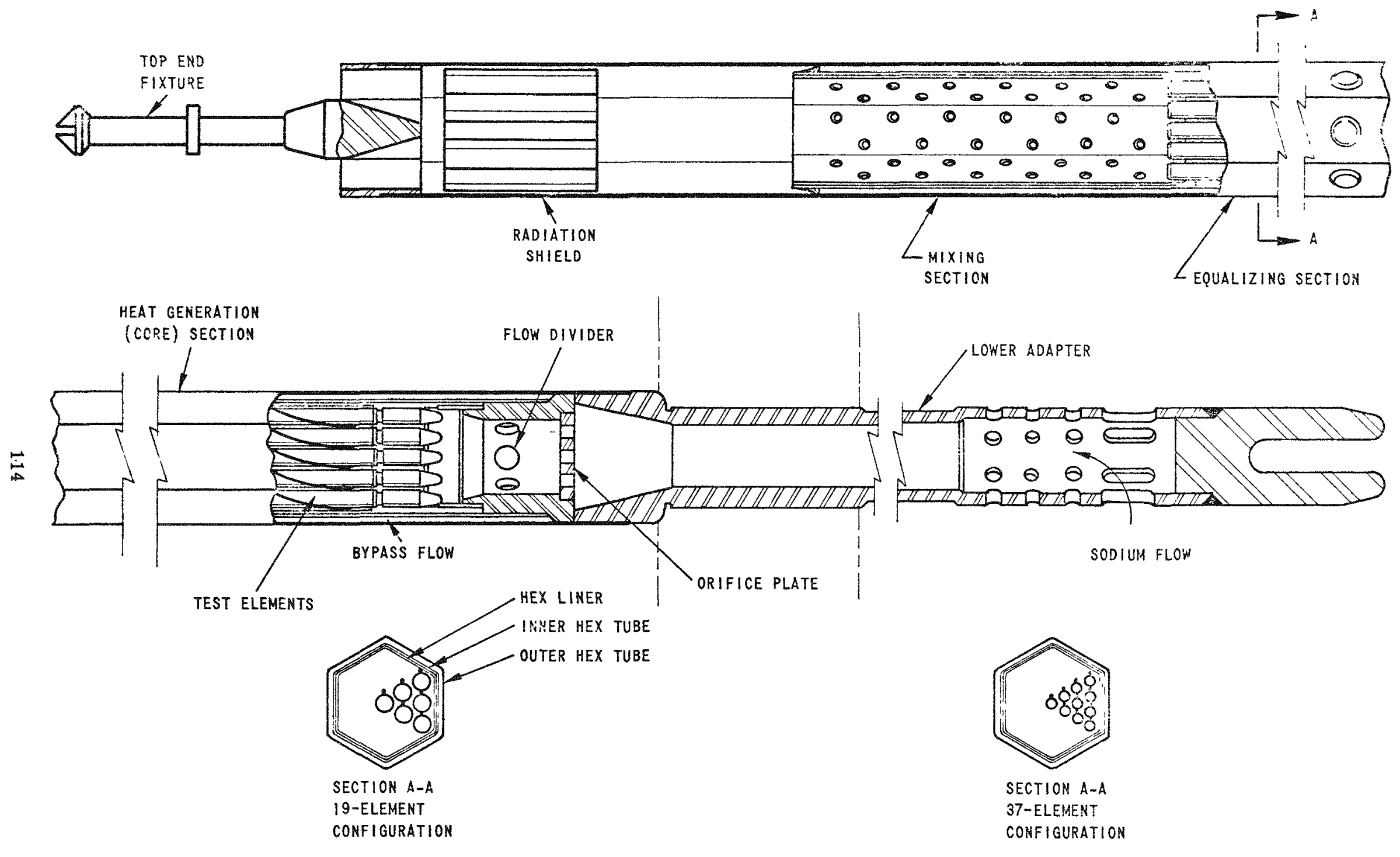

FIG. 7 MARK C IRRADIATION SUBASSEMBLY 

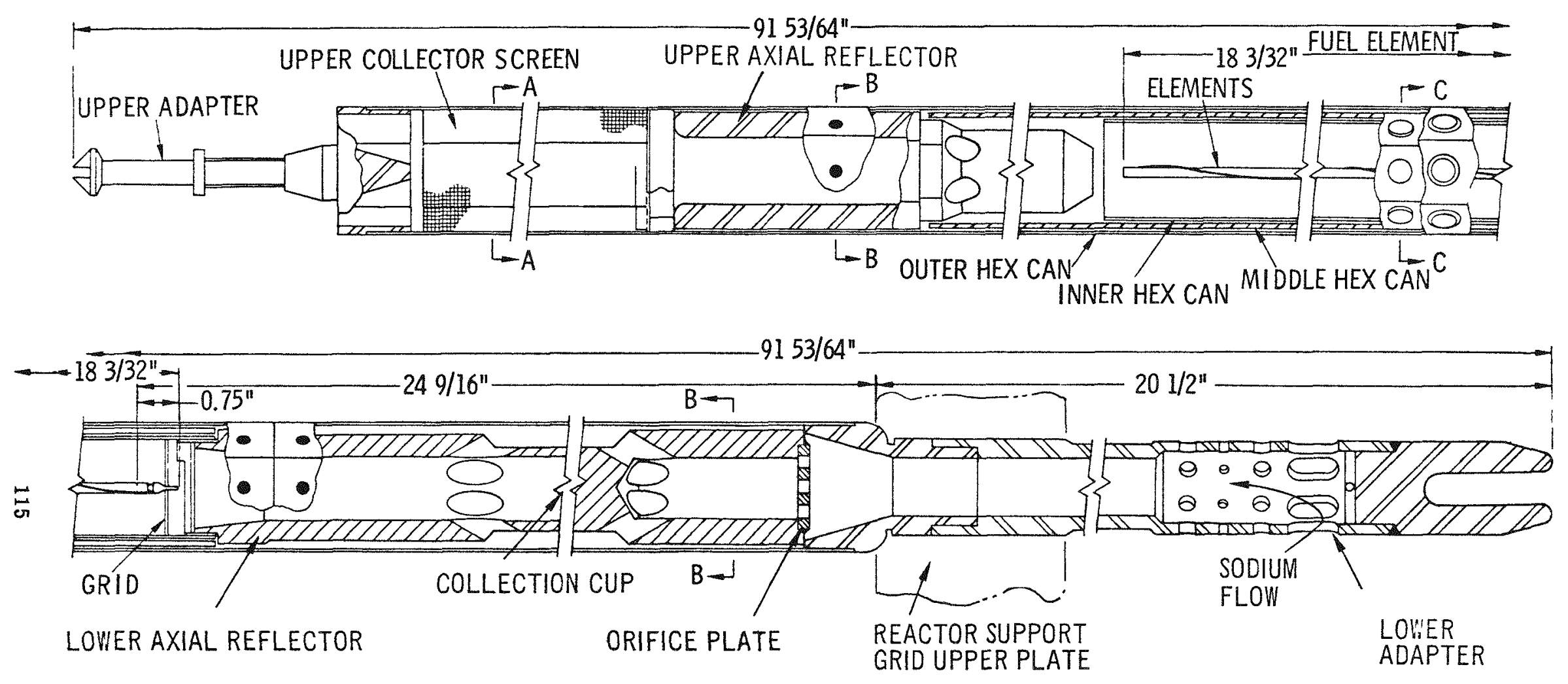

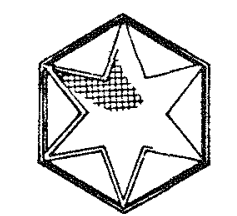

SECTION A-A

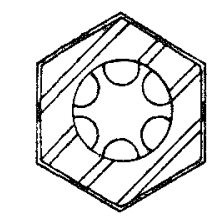

SECTION B-B

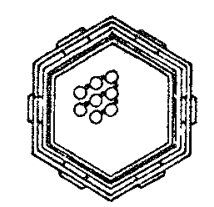

SECTION C-C

FIG. 8 MARK E-61 IRRADIATION SUBASSEMBLY 

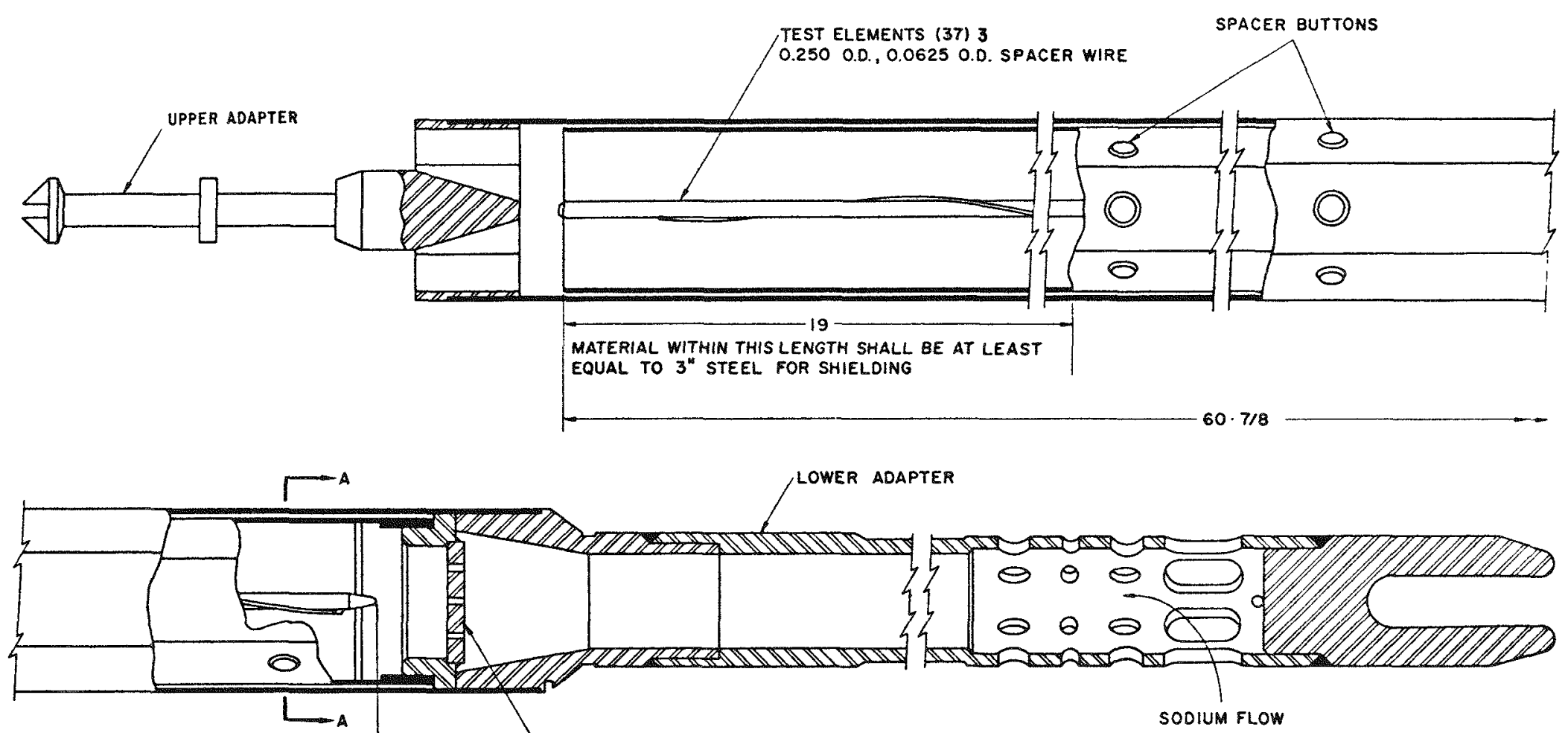

ORIFICE PLATE
(OPTIONAL)

SODIUM FLOW

FIG. 9 MARK FIRRADIATION SUBASSEMBLY 

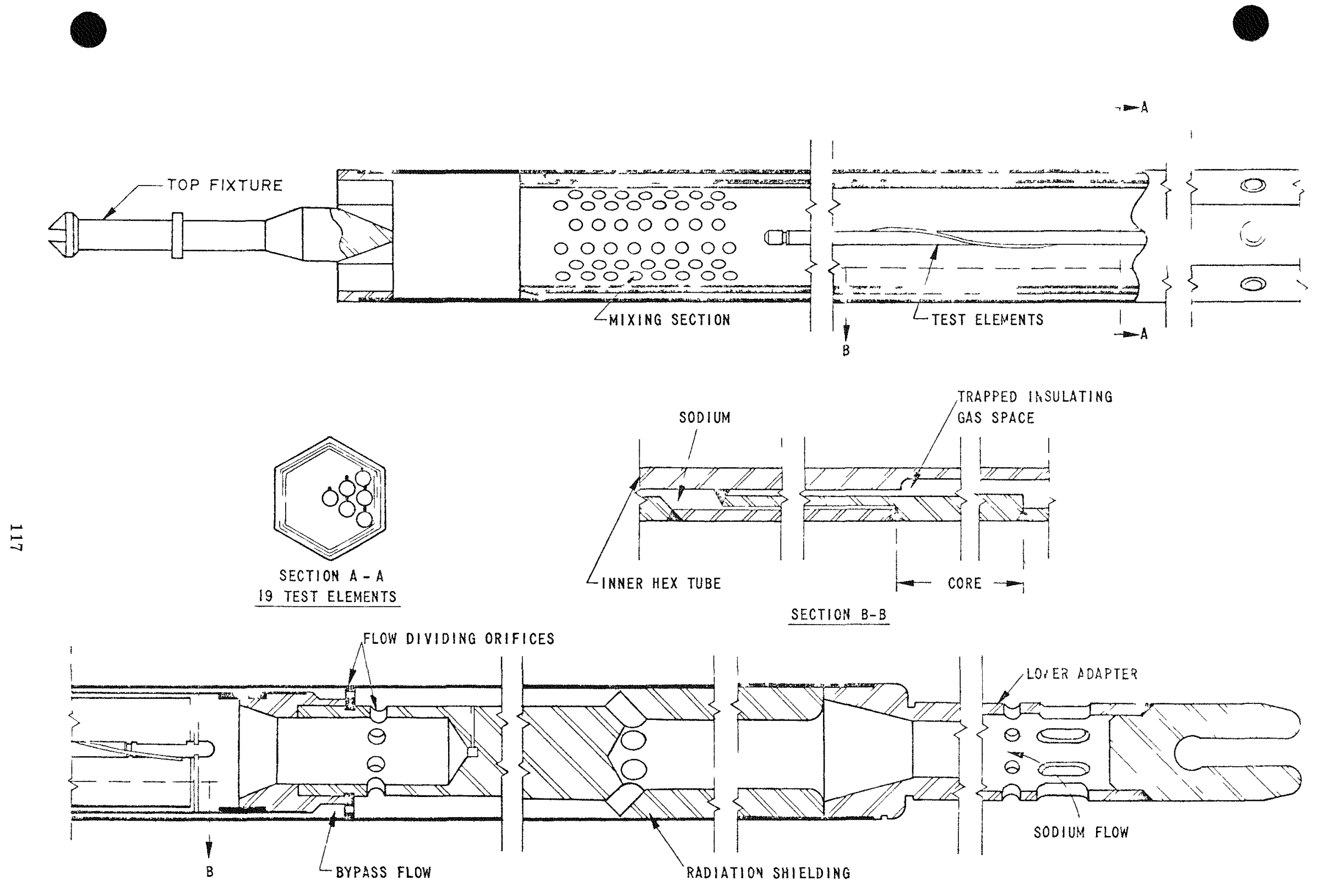

FIG 10 MARK J IRRADIATION SUBASSEMELY 


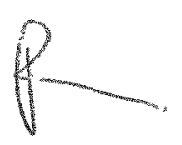

Michael H. Montgomery, Carl J. Grando, and Alfred A. Strasser

United Nuclear Corporation

The most critical problem of high-burnup, high-power fuels is the accommodation of fuel swelling without rupturing the clad. Swelling data for uraniumplutonium carbide fuel to date are based on the testing of clad samples and represent the restrained swelling rate of the fuel. A comparison of the unrestrained swelling rate in combination with the high-temperature creep rate of various fuel structures and compositions will help to design a fuel rod for maximum life. A second important factor in fuel rod design is fission gas release to estimate the fission gas pressure component of the clad strain, and provide for the proper size fission gas plenum.

Both fuel swelling and fission gas release rates are strongly dependent on temperature. The lack of instrumentation is a serious handicap in the study of these properties in present fast reactors. Such studies are best done with extensive instrumentation and flexibility of reactor position, available today in thermal reactors only. The axial and radial flux changes in highly enriched fast reactor fuels irradiated in thermal reactors can be minimized by the use of small cross-section samples operated at essentially isothermal conditions. Isothermal tests have the advantage that they may pinpoint critical temperature boundaries beyond which large fuel swelling and fission gas release rates occur.

The United Nuclear Corporation has designed a basic instrumented capsule able to screen a large number of fuel compositions and structures at varying 
temperatures in a minimum amount of time and reactor space under well-controlled, comparable conditions. This capsule concept makes use of a capsule train of which there are two types, unrestrained swelling and fission gas release. Each capsule train consists of five capsule assemblies, each containing two specimen assemblies. Cutaway views of the capsule train assembly, capsule assembly, and specimen assembly are shown in Figs. 1, 2, and 3.

The unrestrained swelling fuel specimens consist of 0.060 -in. diameter by 0.50 -in. long pins, held at the ends in tantalum-carbide supports with space provided for axial expansion. There are eight pins located in each tantalum-clad specimen assembly. These unclad pin specimens are free to expand radially in a helium annulus with clearances of $0.010 \mathrm{in}$, and $0.020 \mathrm{in}$., respectively, between the pins and the inner and outer annulus wall. These pins were irradiated to determine the extent of unrestrained swelling as a function of temperature and burnup.

The fission gas release fuel specimens are 0.060-in. wall thickness annular rings, 0.375 -in. OD by 0.50 -in. long, supported at the ends by tantalum-carbide disks with space provided for axial expansion. One annular specimen is contained in each tantalum specimen assembly. The fission gas released by the fuel during irradiation is contained within the tantalum can. These fuel specimens were irradiated to determine the amount of fission gas released from the fuel as a function of temperature and burnup.

The fuel materials and operating conditions are summarized in Table 1. These fuel materials were selected for their various technical and economic potentials. Variables include stoichiometry, fuel density, and oxygen content. The fuel specimens were prepared by cold pressing and sintering to a tolerance of \pm 0.001 in. on all dimensions.

Design of the capsule required that fuel temperatures be controlled to with- 
TABLE 1 - SUMMARY OF CAPSULE FUEL MATERIALS AND OPERATING DATA

Unrestrained Swelling Capsule Train

\begin{tabular}{|c|c|c|c|c|c|c|}
\hline \multirow[b]{2}{*}{ Capsule } & \multirow[b]{2}{*}{ Fue 1* } & \multirow[b]{2}{*}{ ICT.D. } & \multicolumn{2}{|c|}{ Fuel Temperature, ${ }^{2} \mathrm{C}$} & \multicolumn{2}{|c|}{ Burnup, $\times 10^{-3} \mathrm{Mwd} / \mathrm{T}$} \\
\hline & & & Design & Operating & Goal & Calculated \\
\hline UNC 116A & $\mathrm{MC}+\mathrm{M}_{2} \mathrm{C}_{3}+$ & 98 & 1300 & 1289 & 20 & 14 \\
\hline $116 \mathrm{~B}$ & $\mathrm{MC}$ & 84 & 1300 & 1340 & & \\
\hline $117 \mathrm{~A}$ & $\mathrm{MC}$ & 93 & 1300 & 1325 & & \\
\hline $117 \mathrm{~B}$ & $\mathrm{M}(\mathrm{OC})$ & 93 & 1300 & 1360 & & \\
\hline $118 \mathrm{~A}$ & $\mathrm{MO}_{2}$ & $>90$ & 1600 & 1366 & & \\
\hline $118 \mathrm{~B}$ & $\mathrm{MO}_{2}$ & $>90$ & 1600 & 1578 & & \\
\hline $119 \mathrm{~A}$ & $\mathrm{MC}+\mathrm{M}_{2} \mathrm{C}_{3}$ & 98 & 1600 & $\S$ & & \\
\hline $119 \mathrm{~B}$ & $\mathrm{MC}$ & 84 & 1600 & $1304 \pi$ & 1 & 1 \\
\hline
\end{tabular}

Fission Gas Release Capsule Train

\begin{tabular}{rlrrr|r} 
UNC 120A & $M C+M_{2} C_{3}$ & 98 & 1000 & 1003 & 20 \\
$120 B$ & $M C$ & 84 & 1000 & 978 & \\
$121 A$ & $M C+M_{2} C_{3}$ & 98 & 1300 & 1275 \\
$121 B$ & $M C$ & 84 & 1300 & 1373 & \\
$122 A$ & $M C$ & 93 & 1300 & 1308 \\
$122 B$ & $M C$ & 75 & 1300 & 1375 \\
$123 A$ & $M_{2} C_{3}$ & 93 & 1300 & 1288 \\
$123 B$ & $M(O C)$ & 93 & 1300 & 1398 \\
$124 A$ & $M C+M_{2} C_{3}$ & 98 & 1600 & $1298 \pi$ & \\
$124 B$ & $M C$ & 84 & 1600 & $1320 \pi$ &
\end{tabular}

${ }^{*} \mathrm{M}=\left(\mathrm{U}_{0.85} \mathrm{Pu}_{0.15}\right), \mathrm{U}=93 \% \mathrm{U}^{235}$.

f"A" specimen is the control specimen and is operated at $\pm 25^{\circ} \mathrm{C}$ of the design temperature. $\$ 90 \mathrm{v} / 0 \mathrm{MC}+10 \mathrm{v} / 0 \mathrm{M}_{2} \mathrm{C}_{3}$.

8Thermocouple failed. Control is on " $\mathrm{B}$ " specimen.

TOperating temperature is below design due to control rod shadowing. 


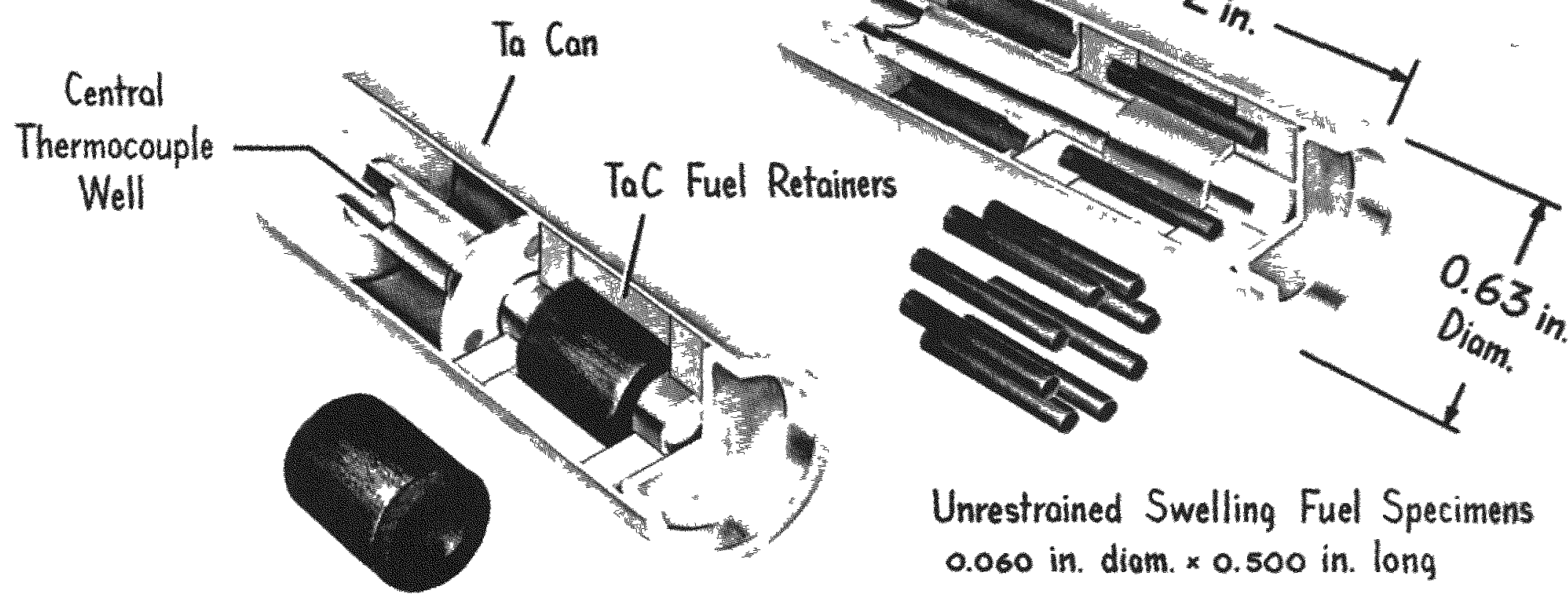

Fission Gas Release Fuel Specimen

0.375 in. $0.0 . \times 0.255$ in. $1.0 . \times 0.500 \mathrm{in}$. lonq

Fig. 1 - Carbide Fuel Irradiation Experiment - Specimen Assembly 


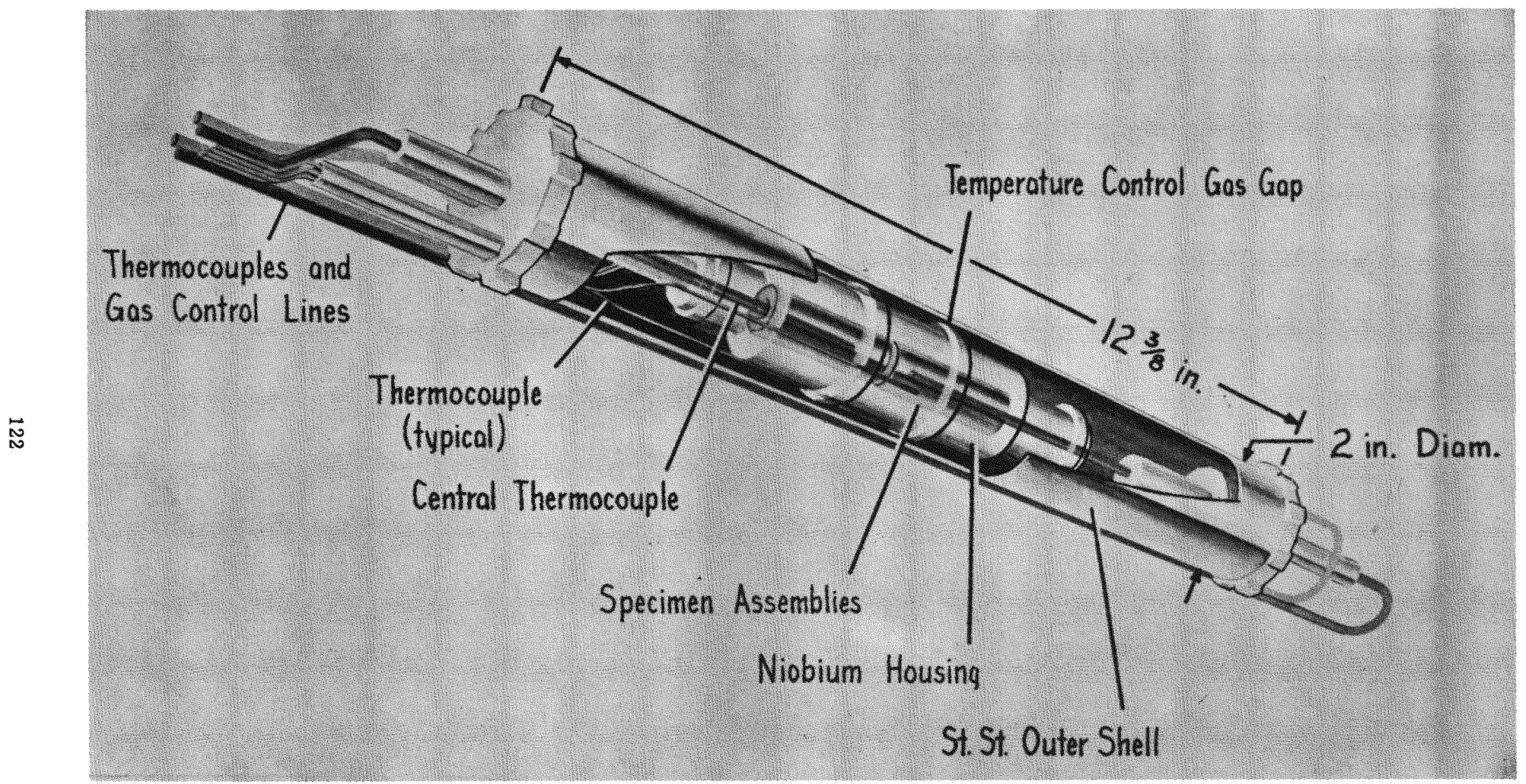

Fig. 2 - Carbide Fuel Irradiation Experiment - Capsule Assembly 


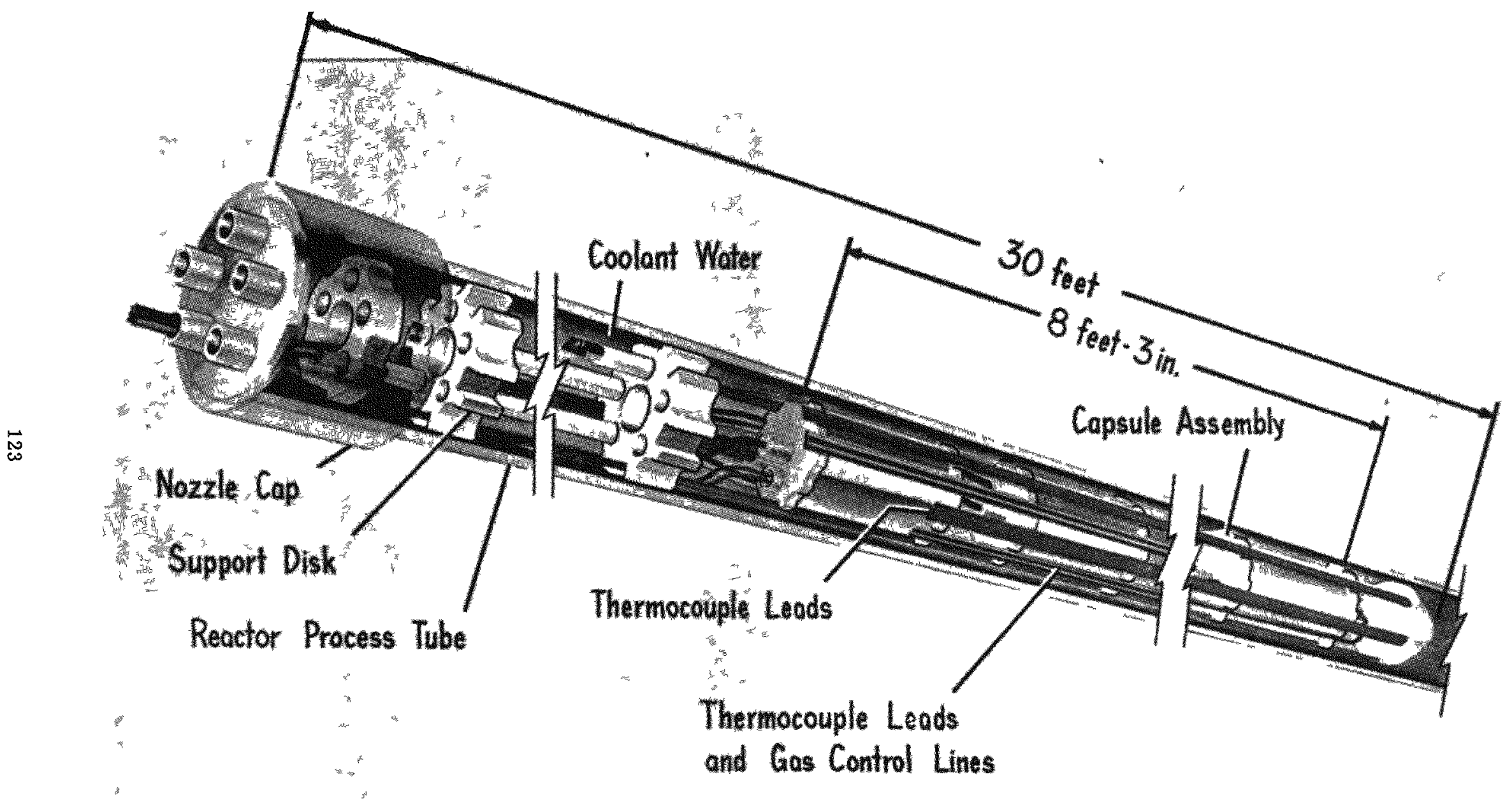

Fig. 3 - Carbide Fuel Irradiation Experiment - Capsule Train 
in $\pm 25^{\circ} \mathrm{C}$ of design with a fuel $\Delta \mathrm{T}$ of $<53^{\circ} \mathrm{C}$. To achieve this, several features were incorporated into the capsule design. First, the physical dimensions of the fuel were kept small so that the temperature gradient across the fuel would be small. Second, the annular gap located just inside the capsule outer shell was filled with an argon-helium gas mixture whose composition could be varied during irrradiation so as to keep fuel temperatures constant. This control device enables one to off set fuel temperature changes that otherwise would occur as fuel swelling and fission gas buildup occurred. Gas purity was maintained at less than 1 ppm oxygen plus moisture by employing a purification train of molecular sieves and hot titanium chips.

Third, fuel temperature is monitored constantly by means of a $1 / 8$-in. diameter tungsten-rhenium thermocouple located at the center of the fuel specimen assembly and by two 1/16-in. diameter Chromel-Alumel and two 1/16-in. diameter tungsten-rhenium thermocouples embedded in the niobium housing. Control gas adjustments are based on thermocouple readings. Finally, the DUN Irradiation Testing Facility test positions exhibit a nearly constant neutron flux profile along the length of the experiment and over an irradiation cycle.

Each capsule assembly is a complete experiment within itself containing its own binary gas system for temperature control, central thermocouple in each specimen assembly for fuel temperature measurement, and peripheral thermocouples in the niobium housing for backup in the event of a central thermocouple failure.

The 1/16-in. Chromel-Alumel and tungsten-rhenium thermocouples are located next to each other. Correlation between the two types was good, indicating little, if any, drift of the tungsten-rhenium thermocouples.

The 1/8- and 1/16-in. high temperature thermocouples were made of braided wire, $W / 25 \operatorname{Re}-W / 3$ Re alloys. The hot end was assembled with BeO insulator 
beads in a tantalum sheath. The long cold lead was a swaged stainless steel sheath with $\mathrm{Al}_{2} \mathrm{O}_{3}$ insulation. The Chromel-Alumel thermocouples were in swaged stainless steel sheaths with $\mathrm{Al}_{2} \mathrm{O}_{3}$ insulation.

The experiments were fabricated without any undue difficulty, despite the very close tolerances necessary to maintain the proper temperature control gas gaps. Information based on irradiation results indicates that the capsule design was quite accurate.

All unrestrained swelling capsules operated at design temperature except for one which achieved $\sim 80 \%$ of design. Power depression in this capsule was attributed to flux shadowing by a reactor control rod which covers that region of the reactor. This capsule later developed complications which led to discharging the entire capsule train after accumulating $\sim 14,000 \mathrm{Mwd} / \mathrm{T}\left(4.9 \times 10^{20} \mathrm{fiss} / \mathrm{cm}^{3}\right)$. Twenty-one of 24 thermocouples remained operational throughout the test. Fig. 4 illustrates the fuel thermal history of a representative unrestrained swelling capsule.

The fission gas release capsule train achieved its goal burnup of $20,000 \mathrm{Mwd} /$ $\mathrm{T}\left(7.0 \times 10^{20} \mathrm{fiss} / \mathrm{cm}^{3}\right)$. All capsules except one operated at design temperature. Here again, a $20 \%$ power depression in this capsule was attributed to flux shadowing by a reactor control rod. Twenty-five of the original 30 thermocouples remained operational over the duration of the test. Fig. 5 illustrates the fuel thermal history of a typical fission gas release capsule. 


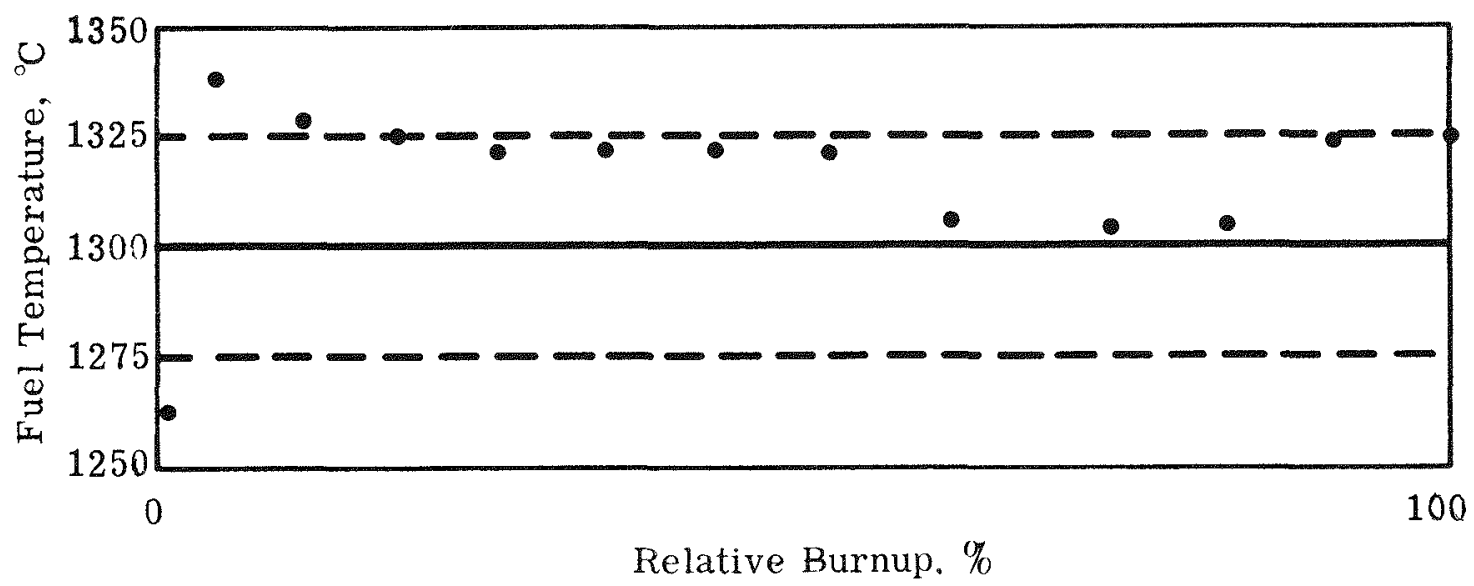

Fig. 4 - Typical Unrestrained Swelling Capsule Temperature History (Accumulated Exposure: $14,000 \mathrm{Mwd} / \mathrm{T}$ )

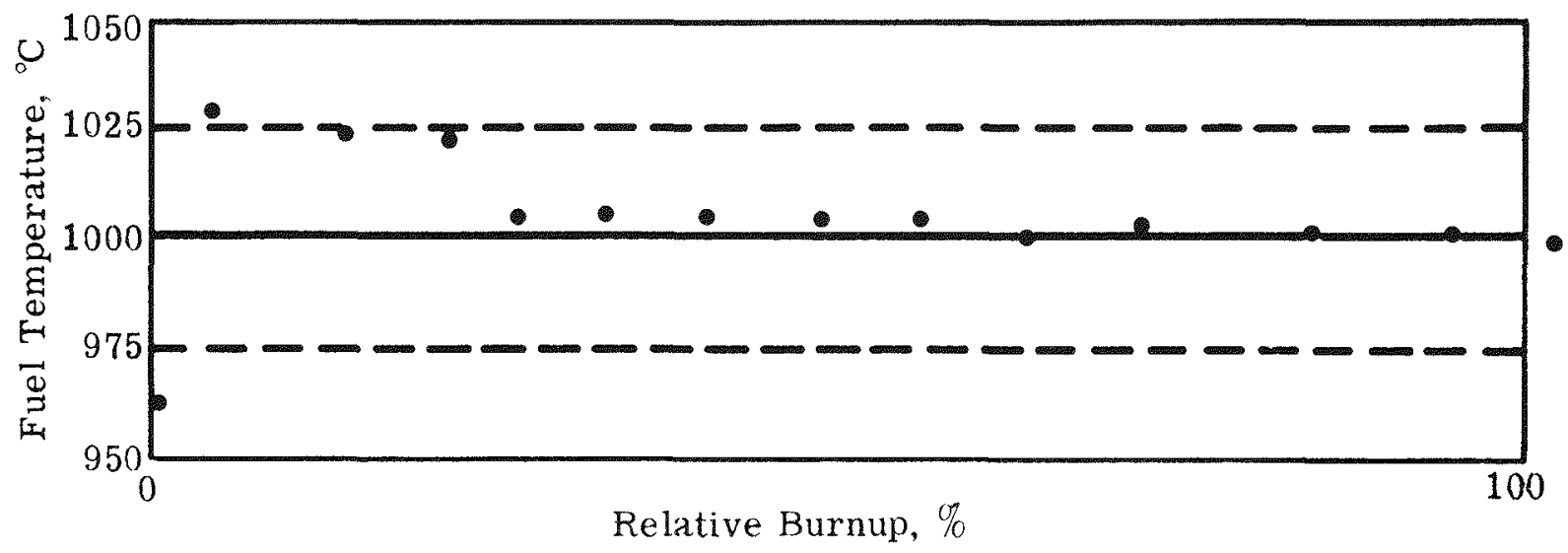

Fig. 5 - Typical Fission Gas Release Temperature History (Accumulated Exposure: 20,000 Mwd/T) 


\title{
QRNLTM-271 -6
}

ANALYSIS OF THE VALIDITY OF FAST REACTOR FUEL TESTS

IN EXISTING TEST REACTORS ${ }^{1}$

A. R. Olsen, R. B. Fitts, and C. M. Cox

Metals and Ceramics Division

Oak Ridge National Laboratory

Oak Ridge, Tennessee 37830

\begin{abstract}
This paper compares the neutronic and thermal environments for a stainless steel-clad ( $U, \mathrm{Pu} \mathrm{O}_{2}$ fuel rod operating in a typical proposed commercial fast reactor with test conditions currently available in both thermal and fast flux test reactors. Calculations were performed to describe the cladding fluence, fuel radial power distribution, fuel and cladding temperature distributions, fuel restructuring, and fission product yields as functions of fuel burnup.

The calculations indicate that properly designed and interpreted thermal flux experiments can simulate the reference fuel operating conditions in terms of temperature distributions and average fission product yields. The cladding fast neutron fluences are very low, and the fuel radial power distribution is atypical except at high burnup.

The EBR-II fast flux test simulation is, in general, quite good except for difficulties arising from the relatively low flux in the reactor which leads to the use of fully enriched uranium to obtain the $16 \mathrm{kw} / \mathrm{ft}$ peak heat rate desired. The consequences of this high enrichment are an appreciable change in certain of the fission product yields which may affect the oxygen activity in the fuel and a decrease by about a factor of 3 for the cladding fluence to fuel burnup ratio. However, the higher knock-on displacement cross section arising from the harder EBR-II spectrum tends to increase the damage per unit of fluence.
\end{abstract}

\section{INTRODUCTION}

This paper will describe some of the considerations that should be included in planning, conducting, and analyzing irradiation tests of fast reactor fuel pin concepts using currently available test reactors. In particular, we shall compare the results of calculations for typical fuel pin tests in the Engineering Test Reactor (ETR) and the Experimental Breeder Reactor (EBR-II), with a reference Liquid Metal Cooled Fast Breeder Reactor (LMFBR). Fach irradiation test group uses some scheme 
to investigate the effects of flux perturbation, burnup, fuel restructuring, and neutron spectra on the test conditions. The degree of . sophistication in these analyses is increasing rapidly, due primarily to the need for improved understanding of the tests so that the results can be extrapolated to fuel rod operating conditions not currently available.

In our case, this extrapolation is done by the use of computerized mathematical models now being developed to predict the performance of fuel rods, particularly LMFBR fuel rods. These models require an understanding of the interrelationships between fuel cladding, and reactor environment. In this paper, we have concentrated on the effects of the neutron spectra of different test reactors on the fuel thermal and chemical environments together with the corresponding gross effects on fuel cladding. We will not at this time consider the fuel-cladding-coolant interactions, the effects of cladding corrosion by coolant, or the effects of nonuniform heat removal.

\section{BASIC ASSUMPTIONS AND CALCULATION PROCEDURES}

We have chosen an early $1000 \mathrm{MW}(\mathrm{e})$ reactor design proposed by the General Electric Company ${ }^{2}$ as our reference reactor. This choice was arbitrary; but the selection of any of the more recent designs being studied would have little effect on the general comparisons. This choice then set our (U,Pu) $\mathrm{O}_{2}$ fuel pin design with a 0.250 -in.-OD $X$ 0.015-in.-wall type 316 stainless steel cladding. We have also arbitrarily chosen an oxide fuel composition containing $80 \% \mathrm{U}$ and $20 \% \mathrm{Pu}$ with a fuel smear density of $85 \%$ of theoretical. Again, a change in fuel composition will affect the details but not the general comparison. The exact starting fuel compositions used are given in Table $I$. The enriched fuel composition to achieve the desired heat rate was derived from tests we now have in the EBR-II. Normally an EBR-II test would be operated at essentially a constant flux; therefore, we have also chosen to operate the reference reactor fuel at a constant flux. The thermal reactor tests are periodically moved to higher flux positions to maintain more comparable fuel operating temperature profiles. We have selected a load factor of $80 \%$ for all reactors, but have again arbitrarily set the operating sequence at approximately 37 days at full power and approximately 9 days of shutdown for each step. This sequence fits none of the test reactor schedules or the normal operation of a power reactor, but was chosen to provide information at burnup intervals of approximately $1 \%$ fissions per initial actinide metal atom (FIMA). With these basic assumptions, we have utilized available computer codes to determine the operating conditions for the fuel pins.

The neutron spectra in the various reactors were calculated by using the ANISN ${ }^{3}$ discrete ordinates neutron transport code. The material cross sections were derived as spectrum averaged cross sections from the 99-group GAM-II ${ }^{4}$ library for the fast reactors and by coupling 5 the GAM-II library with the 30-group THERM $\phi S^{6}$ (thermal) library for the ETR. Justification for confidence in these calculations results from comparisons of test results with predictions for some of our thermal flux irradiation tests." The power distributions within the fuel pins were determined with the ANISN code. 
Table I. Staxting Fuel Used in Each Test Calculation

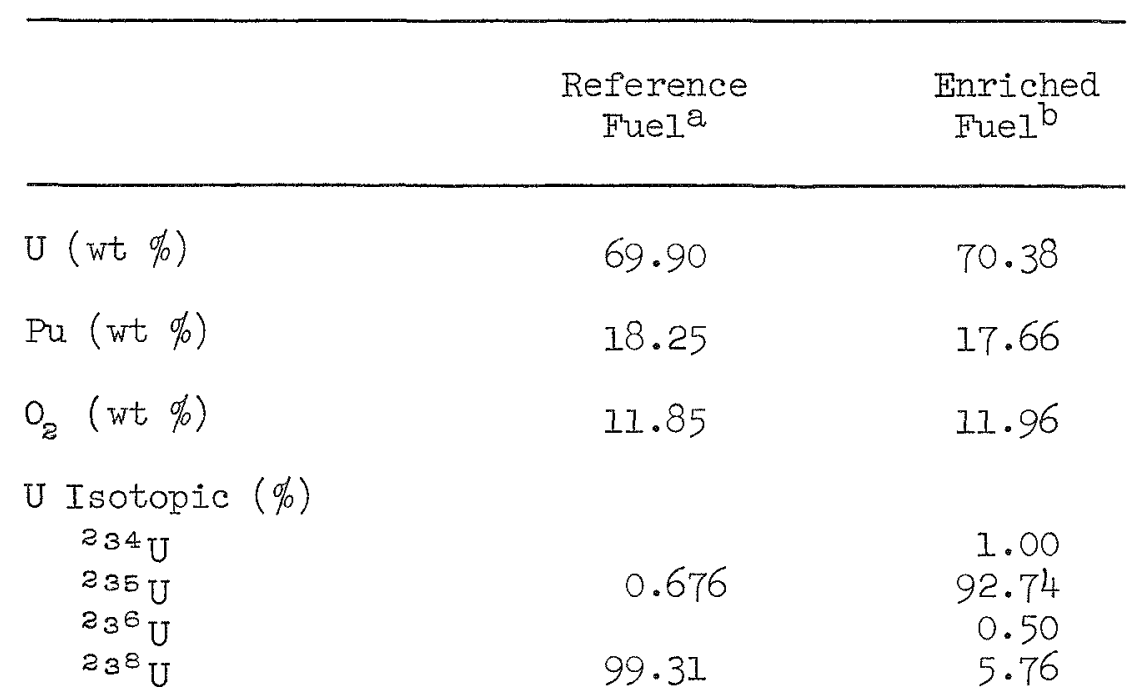

Pu Isotopic (\%)

$$
\begin{aligned}
& 23^{9} \mathrm{Pu} \\
& 240 \mathrm{Pu} \\
& 241 \mathrm{Pu} \\
& 242 \mathrm{Pu}
\end{aligned}
$$

9.93

1.39

0.101

90.51

8.58

0.84

0.044

Theoretical

Density $\left(\mathrm{g} / \mathrm{cm}^{3}\right)$

11.06

10.95

Initial Smear

Density $\left(\mathrm{g} / \mathrm{cm}^{3}\right)$

$$
\text { (\% T.D.) }
$$

8.28

85

8.19

85

$a_{\text {This fuel is used in calculations of performance }}$ in the reference reactor (fast flux) and the ETR (thermal flux).

$b_{\text {This }}$ fuel is used in calculations of performance in the EBR-II (existing fast flux test reactor). 
Fuel temperature distributions and restructuring were calculated

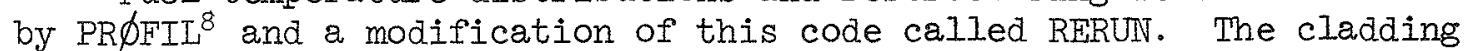
outer surface temperature was set at $550^{\circ} \mathrm{C}$ for the reference reactor fuel pin operating at $16 \mathrm{kw} / \mathrm{ft}$. These parameters were used with a published $(\mathrm{U}, \mathrm{Pu}) \mathrm{O}_{2}$ thermal conductivity function and a fuel-to-cladding gap conductance of $1.14 \mathrm{w} \mathrm{cm}^{-2}{ }^{\circ} \mathrm{C}^{-1}$, appropriate for pellets with a $0.0005-$ in. gap. ${ }^{9}$ A cladding inside surface temperature of $605^{\circ} \mathrm{C}$ and a fuel surface temperature of $870^{\circ} \mathrm{C}$ were calculated for the reference and EBR-II pins. In the thermal flux tests which operate at higher linear heat rates to offset the effects of radial flux depression, the same fuel surface temperature of $870^{\circ} \mathrm{C}$ is achieved with a cladding inside surface temperature of $550^{\circ} \mathrm{C}$ and an outside surface temperature of $495^{\circ} \mathrm{C}$. We have assumed that at these linear heat rates fuel thermal restructuring will be essentially complete before a burnup of $1 \%$ FIMA is achieved. Therefore, the temperature profiles and heat generation rate profiles are all based on thermally restructured fuel. We have made one further simplifying assumption in that we have not taken into account any fission product induced fuel swelling. We have assumed that the available porosity in the fuel will accommodate this swelling. The heat transfer and fuel restructuring calculations assumed a $97 \%$ density for the columnar grain region at temperatures above $1700^{\circ} \mathrm{C}$, where essentially all the fission gas escapes and a $92 \%$ density for the equiaxed grain region, which retains more fission gas, at temperatures above $1400^{\circ} \mathrm{C}$.

Burnup calculations were made by using a modification of IS $\phi \mathrm{CRUNCH}{ }^{10}$ called DEPLETE. In the thermal flux tests, because of the radial flux gradient, we subdivided the fuel region into five equal volume radial zones with six increments of burnup rate in each zone. ANISN provided the burnup rate for each increment, and the volume averaged rate for each zone was used with spectrum averaged cross sections for each zone in calculating the burnup. The burnup variations in each increment change the flux perturbation and thus the burnup rate in all increments continuously. Since a neutron transport depletion code was not available, we arbitrarily adjusted the rates at approximately $3 \%$ FIMA volume averaged intervals by repeating the ANISN and RERUN calculations. The revised incremental fission rates were then used for the next 3\% FIMA. Smaller burnup intervals would provide a better approximation of the actual condition; but since the corrections were less than $10 \%$, the frequency of adjustment is adequate for generalized discussion. Similar adjustments were not necessary for the fast flux conditions where the flux is essentially constant across the fuel and the power varies directly with the fuel density and burnup.

\section{CALCULATIONAL RESULTS}

We calculated the effects of linear heat rate on center fuel temperatures for fuels in both a thermal and fast flux environment, as shown in Fig. 1. A fuel center temperature of approximately $2360^{\circ} \mathrm{C}$ is achieved in a restructured fuel operated at a linear heat rate of $16 \mathrm{kw} / \mathrm{ft}$ in a fast flux environment. A comparable center temperature can be obtained in the same fuel in a thermal flux environment at $18.5 \mathrm{kw} / \mathrm{ft}$ linear heat rate. 
ORNL-DWG 69-9493

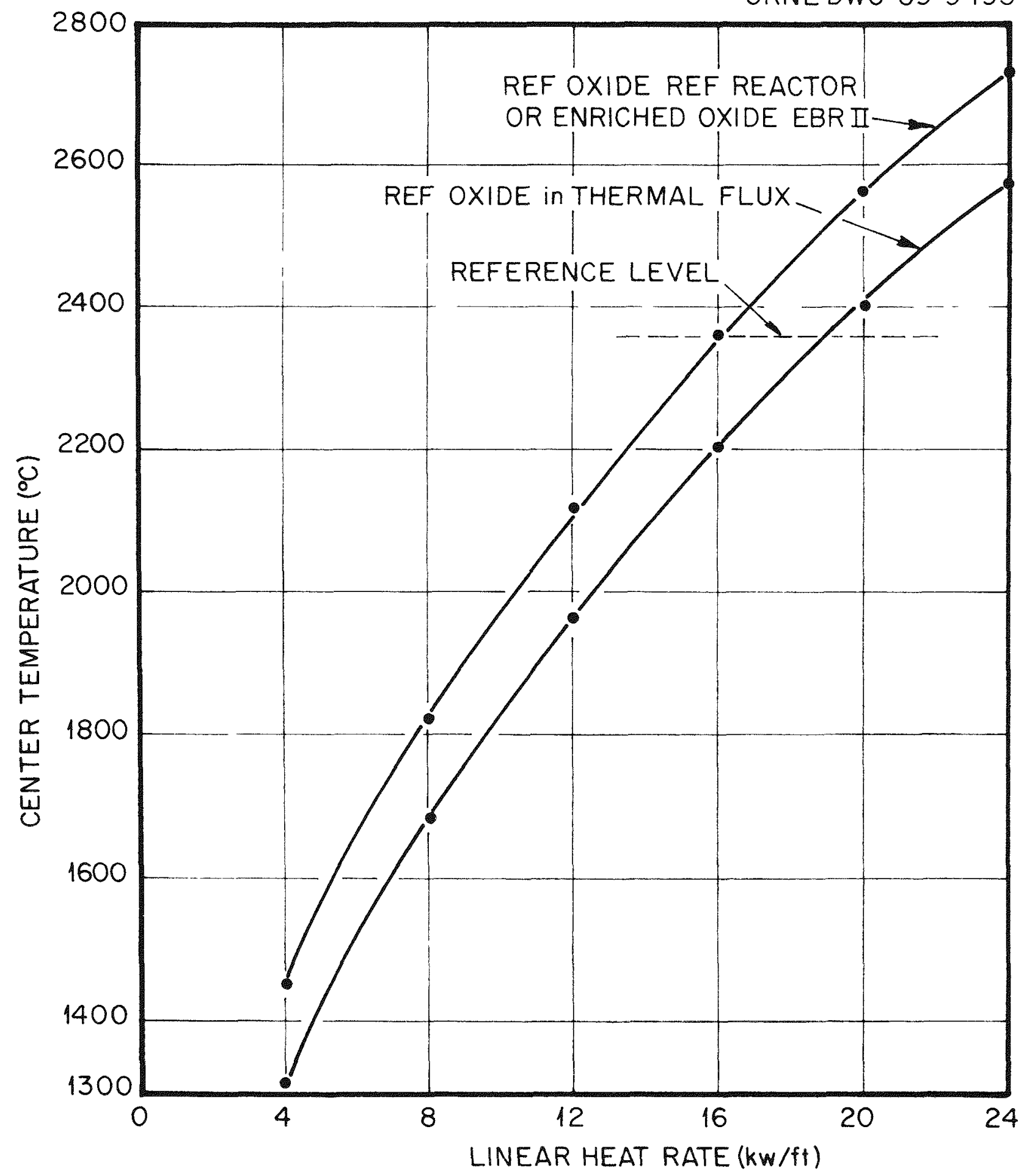

Fig. 1. Fuel Central Temperatures During Irradiation in a Fast or Thermal Flux as a Function of Linear teat Rate for Initially $85 \%$ Dense (U-20\% $\mathrm{Pu}_{\mathrm{O}} \mathrm{O}_{2}$, 0.230-in.-OD Pellets after Thermal Restructuring to $97 \%$ Density at Temperatures above $1700 \mathrm{C}$ and $92 \%$ Density at Temperatures above $1400 \mathrm{C}$. 
Using these linear heat rates as our starting conditions, we calculated the initial power generation and fuel temperature profiles for typical fuel pin cross sections. These data are plotted in Fig. 2 along with the restructured fuel densities for the three cases being considered.

Using these thermally modified starting structures, we calculated the burnup of the fuel for the two fast flux environments assuming a constant flux. The resulting fission product production, burnup, and periodic linear heat rates are given in Tables II and III.

The thermal flux environment calculations were more complex in that they required periodic reassessment of the flux distribution throughout the fuel; and we stepped the flux periodically to maintain the fuel temperature profiles similar to the fast flux cases. The data for the integrated fission product accumulation, burnup, and linear heat rates during each time interval are given in Table IV. Two linear heat rates are reported for the final period at each flux: one which describes the predicted heat rate based on the starting flux perturbation, and a second corrected for the effects of burnup on flux perturbation at the end of the time interval.

Power and temperature profiles were calculated at each of the steps indicated on Tables II, III, and IV at roughly equivalent burnup levels of 4,7 , and 10\% FIMA. The profiles for all three test conditions at each burnup level are compared in Figs. 3, 4, and 5. The profile plotted for the thermal flux cases is that at the end of the burnup interval with corrected flux perturbation and before stepping the unperturbed flux level to proceed with the next interval.

A fourth case was also calculated in which the reference fuel was exposed in the EBR-II environment to the same flux as the enriched oxide (Table III). This resulted in a significantly lower heat generation rate and burnup rate. The burnup data.for this case are listed in Table $V$.

In Fig. 6 we have plotted the burnup as a function of effective full power days of operation for all four cases. Figure 7 is a plot of the burnup as a function of fast fluence ( $>0.1 \mathrm{Mev}$ ) seen by the cladding for all four cases. In this plot the fast flux for the thermal flux irradiation test is negligible ( $1 \times 10^{21}$ neutrons $/ \mathrm{cm}^{2}$ at $10.4 \%$ FIMA).

One additional calculation was made for each of the two fast flux environments. A modification of the $\mathrm{RIBD}^{11}$ code was used together with the irradiation history to calculate the production and decay of various fission products. These data are presented in Tables VI and VII. Although the distribution of fission product yields will vary with the neutron energy spectrum, the same spectrum was assumed for both cases. For this paper, no attempt was made to perform the similar but more complex calculations for the various fuel zones in the thermal flux case. However, based on currently available information, ${ }^{12}$ the variations in yield for thermal and fast flux fissioning are small for each of the fissile elements. 

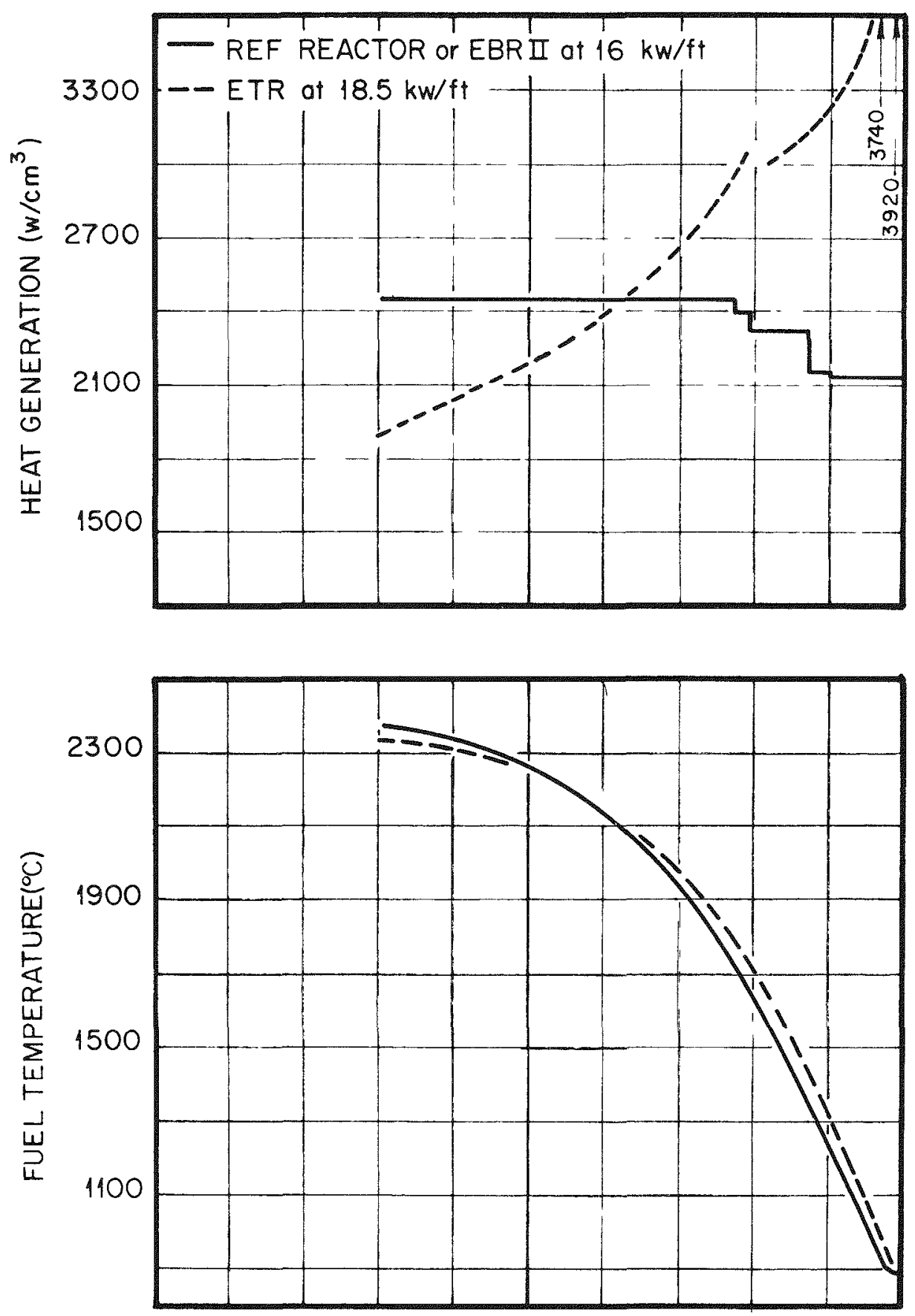

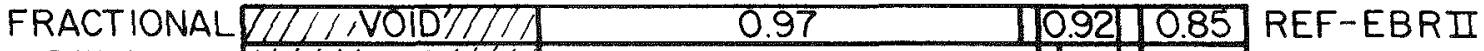

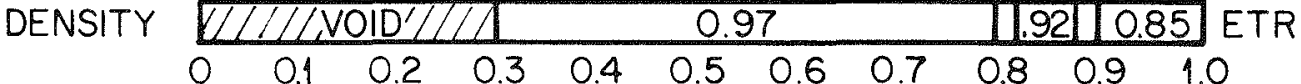
$\notin$ FRACTION OF FUEL RADIUS SURFACE

Fig. 2. Power, Temperature, and Density as a Function of the Frel Radius Fraction at O\% FIMA for Thermally Restructured $85 \%$ Dense (U,Pu) $\mathrm{O}_{2}$ Fuel in Thermal and Fast Flux Environments. 
Table II. Reference Fuel Irradiated in the Reference Reactor at

$$
\int \phi(E) d E=7.7 \times 10^{15} \text { neutrons } / \mathrm{cm}^{2} / \mathrm{sec}
$$

\begin{tabular}{|c|c|c|c|c|c|c|}
\hline $\begin{array}{l}\text { Cumulat } \\
\text { Operating } \\
\text { EFS } \\
\left(x 10^{-6}\right)\end{array}$ & $\begin{array}{l}\text { Time } \\
\text { EFPD }\end{array}$ & $\begin{array}{c}\text { Fission Product } \\
\text { Pairs per cm of } \\
\text { Fuel Rod } \\
\left(\times 10^{\approx 0}\right)\end{array}$ & $\frac{\text { Burnup, }}{\text { IncrementaI }}$ & $\frac{\% \text { FTMA }}{\text { Accumulative }}$ & $\begin{array}{c}\text { Linear } \\
\text { Heat } \\
\text { Rate } \\
\text { (kw/ft) }\end{array}$ & $\begin{array}{l}\text { Profiles } \\
\text { Plotted }\end{array}$ \\
\hline 0 & 0 & 0 & 0 & 0 & 16.0 & Fig. 2 \\
\hline 3.2 & 37 & 0.5 & 1.01 & 1.0 & 15.6 & \\
\hline 6.4 & 74 & 1.0 & 0.99 & 2.0 & $15 \cdot 3$ & \\
\hline 9.6 & 111 & 1.5 & 0.97 & 3.0 & 15.0 & \\
\hline 12.8 & 148 & 2.0 & 0.95 & $3 \cdot 9$ & 14.8 & Fig. 3 \\
\hline 16.0 & 185 & 2.5 & 0.93 & 4.8 & 14.5 & \\
\hline 19.2 & 222 & 3.0 & 0.92 & 5.8 & 14.2 & \\
\hline 22.4 & 259 & 3.4 & 0.90 & 6.7 & 14.0 & Fig. 4 \\
\hline 25.6 & 296 & $3 \cdot 9$ & 0.88 & 7.5 & 13.7 & \\
\hline 28.8 & 333 & $4 \cdot 3$ & 0.87 & 8.4 & 13.5 & \\
\hline 32.0 & 370 & 4.8 & 0.86 & $9 \cdot 3$ & $13 \cdot 3$ & \\
\hline 35.2 & 407 & 5.2 & 0.84 & 10.1 & 13.1 & Fig. 5 \\
\hline
\end{tabular}

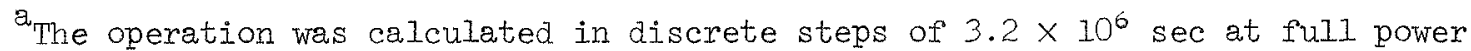
and $8 \times 10^{5} \mathrm{sec}$ at zero power for each step. EFPS = effective full power sec; EFPD = effective full power days. 
Table III. Enriched Fuel Irradiated in the FBR-II at $\int \phi(E) d E=2.5 \times 10^{15}$ neutrons $/ \mathrm{cm}^{2}-\mathrm{sec}$

\begin{tabular}{|c|c|c|c|c|c|c|}
\hline \multicolumn{2}{|c|}{$\begin{array}{c}\text { Cumulative } \\
\text { Operating Time }\end{array}$} & $\begin{array}{c}\text { Fission Product } \\
\text { Pairs per cm of } \\
\text { Fuel Rod } \\
\left(\times 10^{-0}\right)\end{array}$ & $\frac{\text { Burnup }}{\text { Incremental }}$ & $\frac{\% \text { FTMA }}{\text { Accumulative }}$ & $\begin{array}{l}\text { Linear } \\
\text { Heat } \\
\text { Rate } \\
(\mathrm{kw} / \mathrm{ft})\end{array}$ & $\begin{array}{l}\text { Profiles } \\
\text { Plotted. }\end{array}$ \\
\hline 0 & 0 & 0 & 0 & 0 & 16.0 & Fig. 2 \\
\hline 3.2 & 37 & 0.5 & 1.01 & 1.0 & 15.8 & \\
\hline 6.4 & 74 & 1.0 & 1.00 & 2.0 & 15.6 & \\
\hline 9.6 & 111 & 1.5 & 0.99 & 3.0 & 15.5 & \\
\hline 12.8 & 148 & 2.1 & 0.98 & 4.0 & $15 \cdot 3$ & Fig. 3 \\
\hline 16.0 & 185 & 2.5 & 0.97 & 4.9 & 15.1 & \\
\hline 19.2 & 222 & 3.0 & 0.95 & 5.9 & 14.9 & \\
\hline 22.4 & 259 & 3.5 & 0.94 & 6.8 & 14.7 & Fig. 4 \\
\hline 25.6 & 296 & 4.0 & 0.93 & 7.8 & 14.6 & \\
\hline 23.8 & 333 & 4.5 & 0.92 & 8.7 & 14.4 & \\
\hline 32.0 & 370 & 4.9 & 0.91 & 9.6 & 14.2 & \\
\hline 35.2 & 407 & 5.4 & 0.90 & 10.5 & 14.1 & Fig. 5 \\
\hline
\end{tabular}

${ }^{2}$ The operation was calculated in discrete steps of $3.2 \times 10^{6} \mathrm{sec}$ at full power and $8 \times 10^{5} \mathrm{sec}$ at zero power for each step. EFPS = effective full power sec; EPPD = effective full power days.

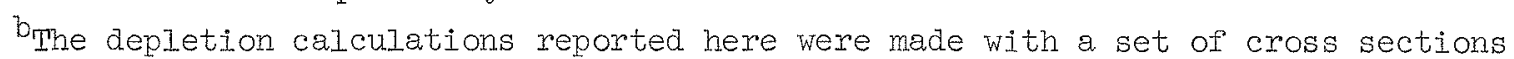
derived for an EBR-II core without experimental subassemblies. Similar cross sections calculated for a core containing a currently typical distribution of experimental subassemblies are generally higher by approximately $10 \%$. 
Table IV. Reference Fuel Irradiated in the ETR at the Unperturbed Flux Indicated.

\begin{tabular}{|c|c|c|c|c|c|c|c|c|}
\hline \multicolumn{2}{|c|}{$\begin{array}{c}\text { Cumulative } \\
\text { Operating Time }\end{array}$} & \multirow{2}{*}{$\begin{array}{c}\oint \text { Unperturbed } \\
2000 \\
\left(\text { neutrons } / \mathrm{cm}^{2} / \mathrm{sec}\right) \\
\left(\times 10^{-14}\right)\end{array}$} & \multirow{2}{*}{$\begin{array}{l}\text { Fission Product } \\
\text { Pairs per cm of } \\
\text { Fuel Rod } \\
\left(\times 10^{-20}\right)\end{array}$} & \multicolumn{2}{|c|}{ Burnup, of FIMA } & \multicolumn{2}{|c|}{$\begin{array}{c}\text { Linear Heat Rate, } \\
\mathrm{kw} / \mathrm{ft}\end{array}$} & \multirow{2}{*}{$\begin{array}{l}\text { Profiles } \\
\text { Plotted }\end{array}$} \\
\hline$\left(\times 10^{-6}\right)$ & & & & & & Direct & Corrected & \\
\hline 0 & 0 & 0.93 & 0 & 0 & 0 & 18.5 & 18.5 & Fig. 2 \\
\hline $3 \cdot 2$ & 37 & 0.93 & 0.6 & 1.25 & 1.2 & 18.3 & & \\
\hline 6.4 & 74 & 0.93 & 1.2 & 1.13 & 2.4 & 16.7 & & \\
\hline 9.6 & 111 & 0.93 & 1.7 & 1.04 & 3.4 & $15 \cdot 3$ & 16.6 & Fig. 3 \\
\hline 12.8 & 148 & 1.11 & 2.4 & 1.27 & 4.7 & 18.5 & & \\
\hline 16.0 & 185 & 1.11 & 2.9 & 1.13 & 5.8 & $17 \cdot 3$ & & \\
\hline 19.2 & 222 & 1.11 & 3.5 & 1.01 & 6.8 & 14.8 & 17.1 & Fig. 4 \\
\hline 22.4 & 259 & 1.23 & 4.0 & 1.07 & $7 \cdot 9$ & 15.4 & & \\
\hline 25.6 & 296 & 1.23 & 4.5 & 0.94 & 8.8 & 13.6 & & \\
\hline 28.8 & 333 & 1.23 & 4.9 & 0.83 & 9.7 & 13.1 & & \\
\hline 32.0 & 370 & 1.23 & 5.3 & 0.74 & 10.4 & 10.8 & & Fig. 5 \\
\hline
\end{tabular}

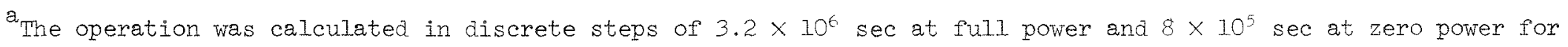
each step. EFPS = effective full power sec; EFPD = effective full power days.

bCorrected linear heat rates represent reanalysis of the flux perturvation, assuming the burnup rates were as predicted in the depletion calculation. More frequent intervals would produce slightly higher accumulative burnups and linear heat rates between the direct and corrected values. 

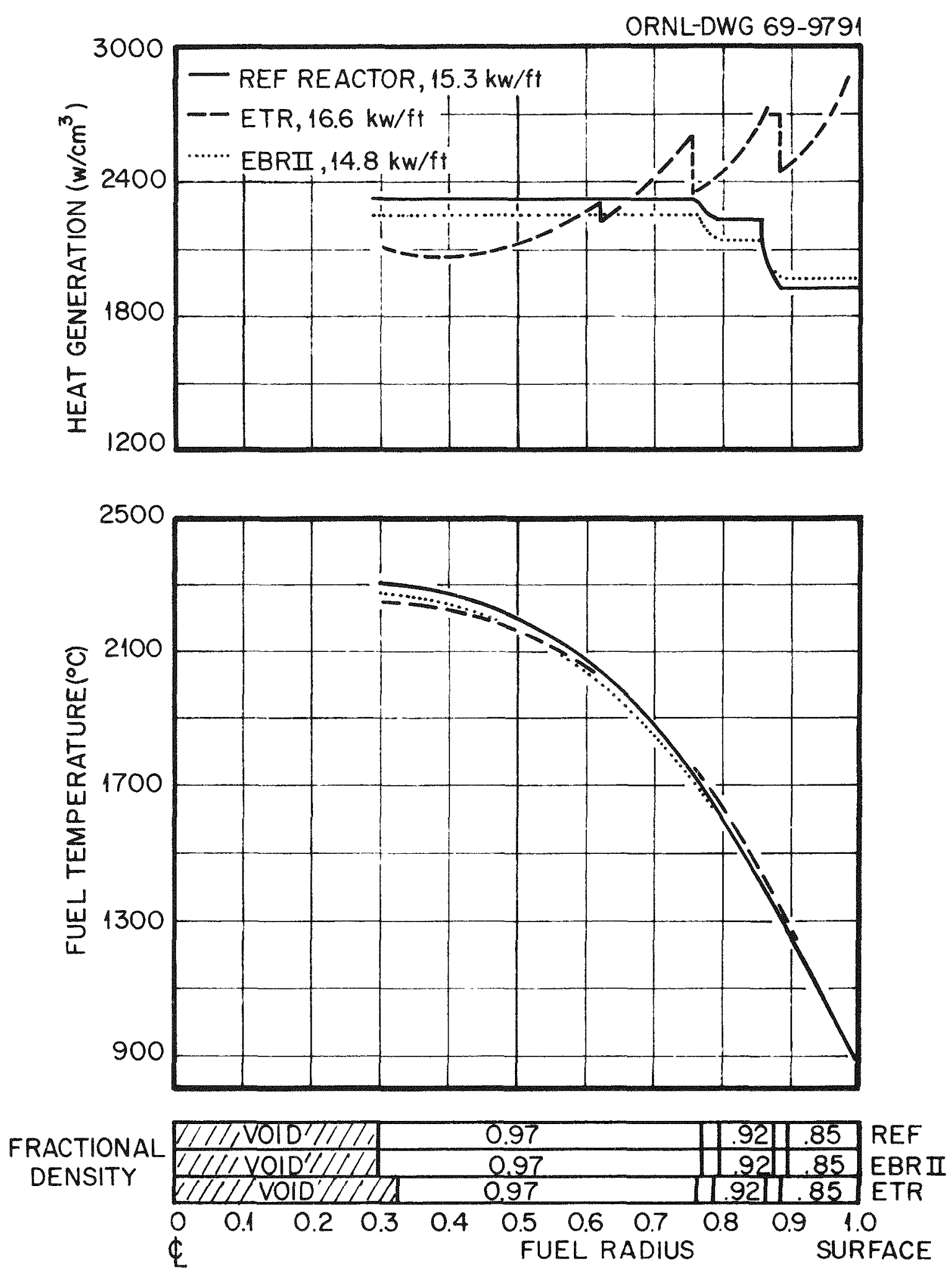

Fig. 3. Power, Temperature, and Density as a Function of the Fuel Radius Fraction at $4 \%$ FIMA for Thermally Restructured $85 \%$ Dense (U, Pu) $\mathrm{O}_{2}$ Fuel in Thermal and Fast Flux Environments. 

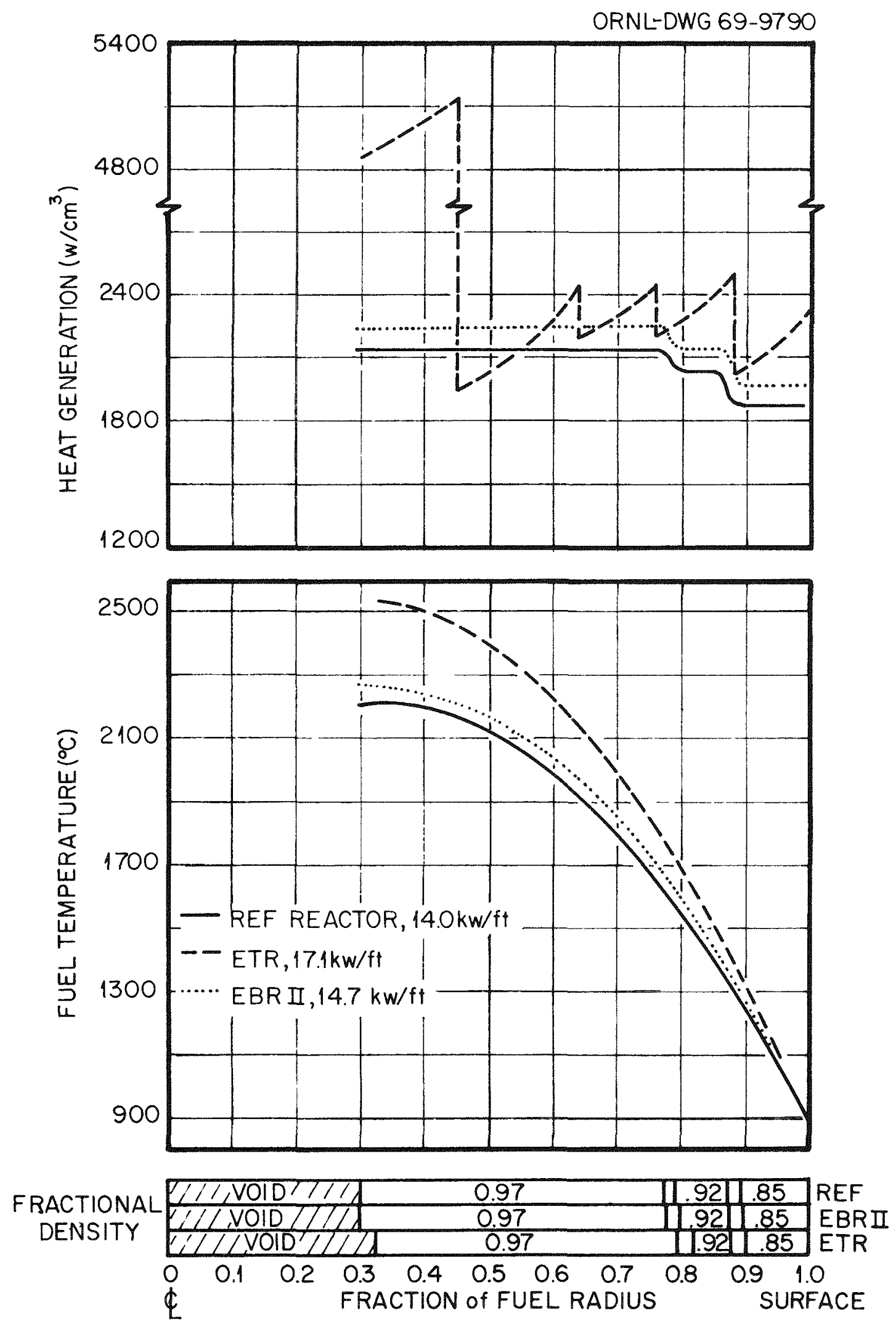

Fig. 4. Power, Temperature, and Density as a Function of the Fuel Radius Fraction at $7 \%$ FIMA for Thermally Restructured $85 \%$ Dense (U, Pu) $\mathrm{O}_{2}$ Fuel in Thermal and Fast Flux Environments. 

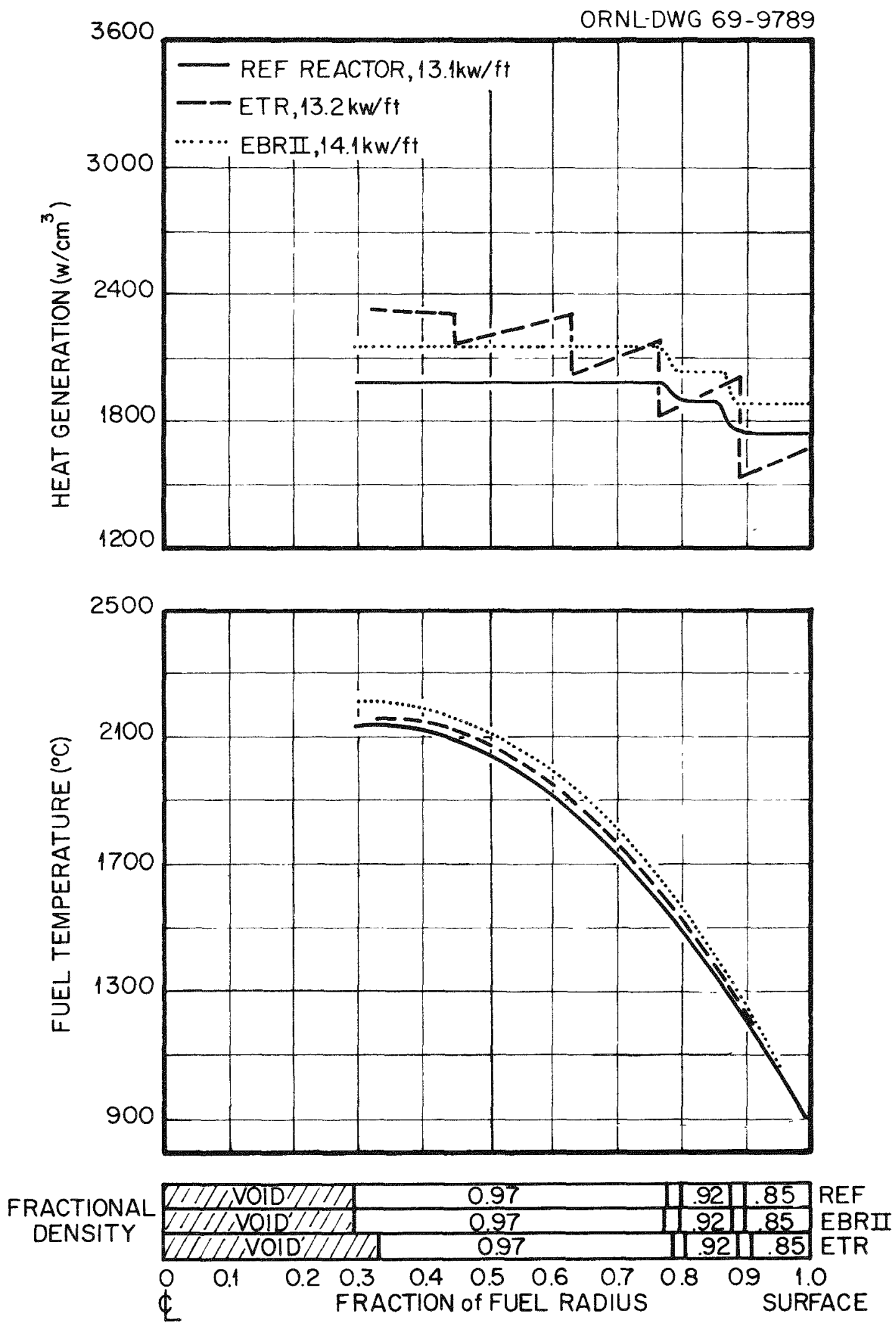

Fig. 5. Power, Temperature, and Density as a Function of the Frel Radius Fraction at 10\% FIMA for Thermally Restructured $85 \%$ Dense (U, Pu) $\mathrm{O}_{2}$ Fuel in Thermal and Fast Flux Environments. 
Table V. Reference Fuel Irradiated in the EBR-II at

$$
\int \phi(E) d E=2.5 \times 10^{15} \text { neutrons } / \mathrm{cm}^{2}-\mathrm{sec}
$$

\begin{tabular}{|c|c|c|c|c|c|}
\hline \multicolumn{2}{|c|}{$\begin{array}{c}\text { Cumulative } \\
\text { Operating Time }\end{array}$} & \multirow{2}{*}{$\begin{array}{c}\text { Fission Product } \\
\text { Pairs per cm of } \\
\text { Fuel Rod } \\
\left(\times 10^{-20}\right)\end{array}$} & \multicolumn{2}{|c|}{ Burnup, \% FTMA } & \multirow{2}{*}{$\begin{array}{l}\text { Linear } \\
\text { Heat } \\
\text { Rate } \\
\text { (kw/ft) }\end{array}$} \\
\hline $\begin{array}{l}\text { EFPS } \\
\left(\times 10^{-\infty}\right)\end{array}$ & EPPD & & Incremental & Accumulative & \\
\hline 0 & 0 & 0 & 0 & 0 & 5.3 \\
\hline 3.2 & 37 & 0.17 & 0.34 & 0.3 & 5.2 \\
\hline 6.4 & 74 & 0.34 & 0.33 & 0.7 & 5.2 \\
\hline 9.6 & 111 & 0.51 & 0.33 & 1.00 & 5.2 \\
\hline 12.8 & 148 & 0.68 & 0.33 & 1.3 & 5.1 \\
\hline 16.0 & 185 & 0.85 & 0.33 & 1.7 & 5.1 \\
\hline 19.2 & 222 & 1.01 & 0.32 & 2.0 & 5.0 \\
\hline 22.4 & 259 & 1.18 & 0.32 & 2.3 & 5.0 \\
\hline 25.6 & 296 & 1.34 & 0.32 & 2.6 & 5.0 \\
\hline 28.8 & 333 & 1.51 & 0.32 & 2.9 & 4.9 \\
\hline 32.0 & 370 & 1.67 & 0.31 & $3 \cdot 3$ & 4.9 \\
\hline 35.2 & 407 & 1.83 & 0.31 & 3.6 & 4.9 \\
\hline 38.4 & 444 & 1.99 & 0.31 & $3 \cdot 9$ & 4.8 \\
\hline 41.6 & 481 & 2.14 & 0.31 & 4.2 & 4.8 \\
\hline 44.8 & 519 & 2.30 & 0.31 & 4.5 & 4.8 \\
\hline 48.0 & 555 & 2.46 & 0.30 & 4.8 & 4.7 \\
\hline
\end{tabular}

The operation was calculated in discrete steps of $3.2 \times 10^{6} \mathrm{sec}$ at full power and $8 \times 10^{5} \mathrm{sec}$ at zero power for each step. EFPS = effective full power sec; EFPD = effective full power days.

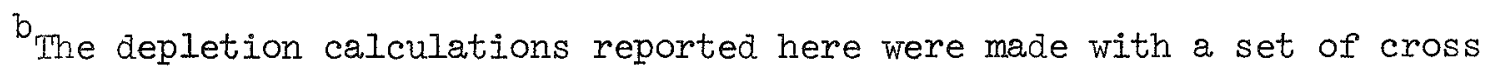
sections derived for an EBR-II core without experimental subassemblies. Similar cross sections calculated for a core containing a currently typical distribution of experimental subassemblies are generally higher by approximately $10 \%$. 


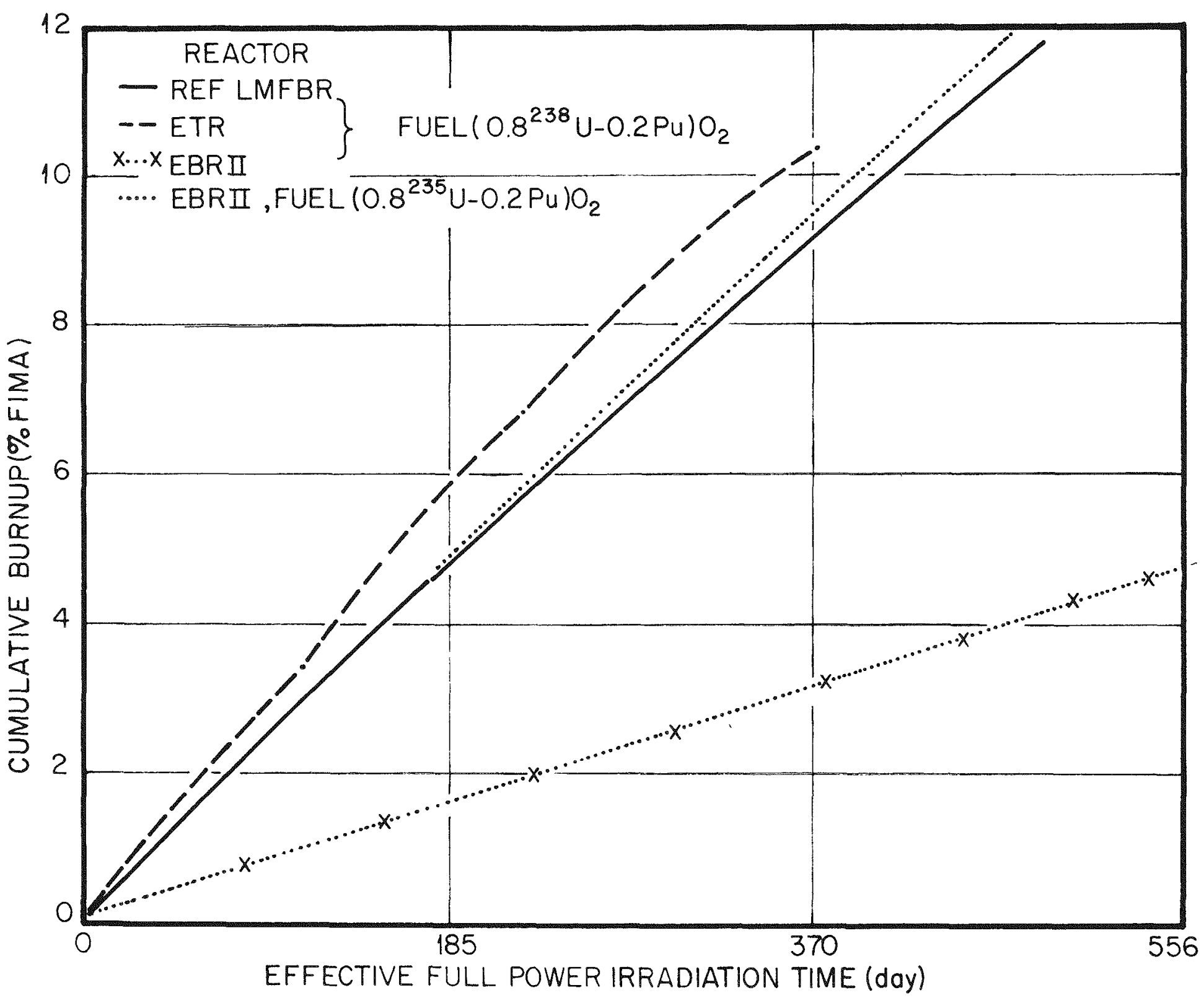

Fig. 6. Burnup for (U,Pu) $\mathrm{O}_{2}$ Fuel Irradiated in Various Reactors with Comparable Fuel Temperature Profiles as a Function of Full Power Days of Trradiation. 


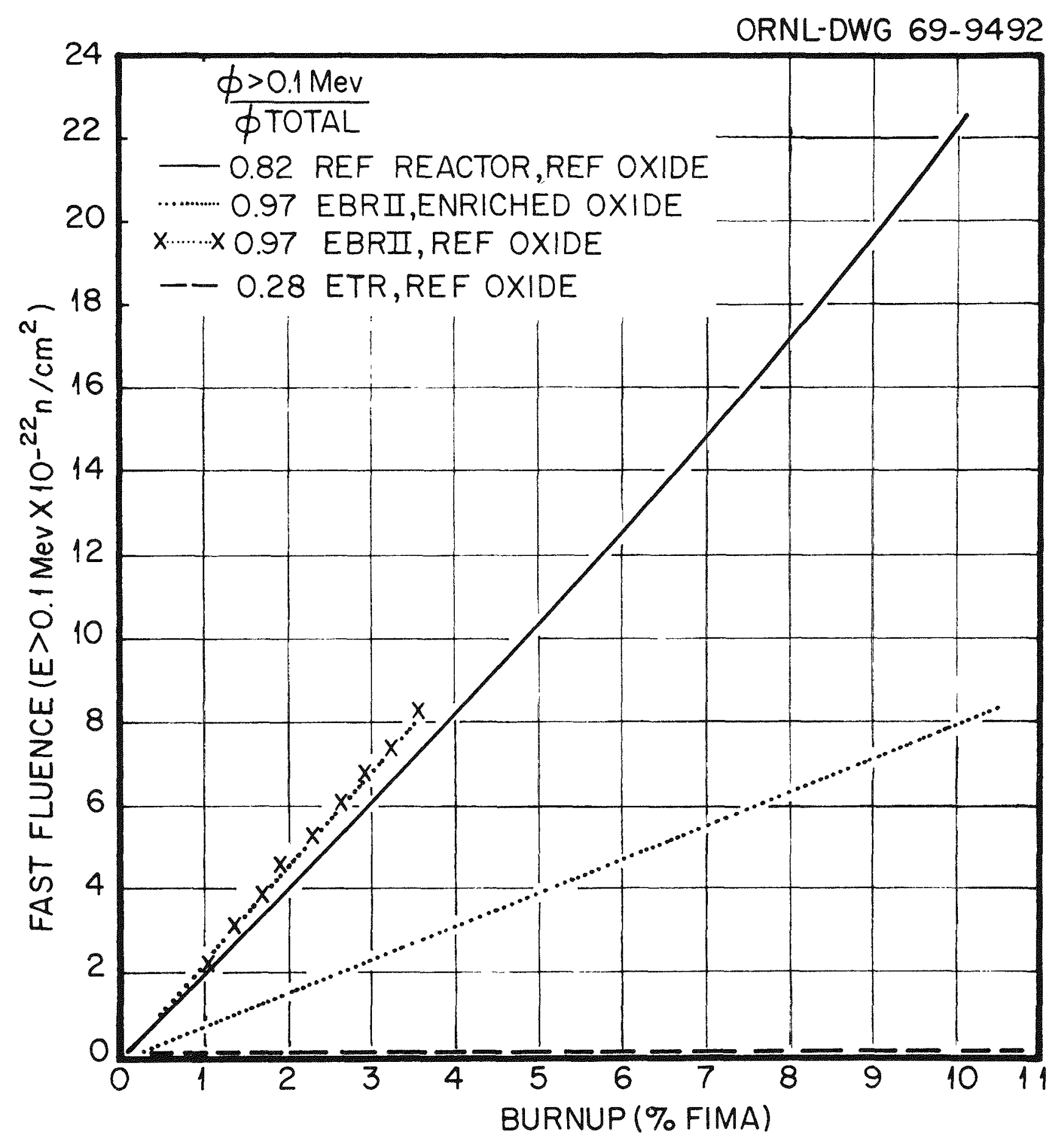

Fig. 7. Fast Fluence $\left(>0.1 \mathrm{Mev}\right.$ ) as a Function of Burnup for $(\mathrm{U}, \mathrm{Pu}) \mathrm{O}_{2}$ Fuels Irradiated in Various Reactors. 
Table VI. Fission Product Composition at 10.1\% FIMA from Reference Fuel Irradiated in the Reference Reactor

\begin{tabular}{|c|c|c|c|c|}
\hline \multirow{2}{*}{ Element } & \multicolumn{4}{|c|}{ Concentration ${ }^{2}$} \\
\hline & Shutdown & $60 \mathrm{sec}$ & 120 days & $1 \mathrm{yr}$ \\
\hline $30 \mathrm{ZN}$ & $4.189 \mathrm{E}-09$ & $4.188 \mathrm{E}-09$ & $9.529 \mathrm{E}-28$ & 0.0 \\
\hline $31 \mathrm{GA}$ & $1.282 \mathrm{E}-09$ & $1.282 \mathrm{E}-09$ & $4.145 \mathrm{E}-28$ & 0.0 \\
\hline $32 \mathrm{GE}$ & $8.912 E-07$ & 8.912E-07 & $8.964 \mathrm{E}-07$ & $8.964 E-07$ \\
\hline $33 \mathrm{AS}$ & $2.692 \mathrm{E}-07$ & $2.692 \mathrm{E}-07$ & $2.683 \mathrm{E}-07$ & $2.683 E-07$ \\
\hline $34 \mathrm{SE}$ & $3.029 \mathrm{E}-06$ & $3.028 \mathrm{E}-06$ & $3.023 E-06$ & $3.023 \mathrm{E}-06$ \\
\hline $35 \mathrm{BR}$ & $1.136 \mathrm{E}-06$ & $1.134 \mathrm{E}-06$ & $9.680 \mathrm{E}-07$ & $9.680 E-07$ \\
\hline $36 \mathrm{KR}$ & $1.649 \mathrm{E}-03$ & $1.649 \mathrm{E}-03$ & $1.646 \mathrm{E}-03$ & $1.641 \mathrm{E}-03$ \\
\hline $37 \mathrm{RB}$ & $8.653 \mathrm{E}-04$ & $8.653 E-04$ & $8.681 \mathrm{E}-04$ & $8.736 \mathrm{E}-04$ \\
\hline $38 \mathrm{SR}$ & $2.731 \mathrm{E}-03$ & $2.731 E-03$ & $2.582 \mathrm{E}-03$ & $2.525 \mathrm{E}-03$ \\
\hline $39 \mathrm{Y}$ & $1.325 \mathrm{E}-03$ & $1.325 \mathrm{E}-03$ & $1.242 \mathrm{E}-03$ & $1.208 \mathrm{E}-03$ \\
\hline $40 \mathrm{ZR}$ & $1.464 \mathrm{E}-02$ & $1.464 E-02$ & $1.431 \Xi-02$ & $1.420 \mathrm{E}-02$ \\
\hline $41 N B$ & $4.026 \mathrm{E}-04$ & $4.026 \mathrm{E}-04$ & $2.033 E-04$ & I. $788 \mathrm{E}-05$ \\
\hline $42 \mathrm{MO}$ & $1.754 \mathrm{E}-02$ & $1.754 \mathrm{E}-02$ & $1.827 \mathrm{E}-02$ & $1.865 \mathrm{E}-02$ \\
\hline $43 \mathrm{TC}$ & $4.669 \mathrm{E}-03$ & $4.669 \mathrm{E}-03$ & $4.710 \mathrm{E}-03$ & $4.710 \mathrm{E}-03$ \\
\hline $44 \mathrm{RU}$ & $1.718 \mathrm{E}-02$ & $1.718 \mathrm{E}-02$ & $1.624 \mathrm{E}-02$ & $1.547 \mathrm{E}-02$ \\
\hline $45 \mathrm{RH}$ & $4.031 \mathrm{E}-03$ & $4.031 \mathrm{E}-03$ & $4.460 \mathrm{E}-03$ & $4.523 \mathrm{E}-03$ \\
\hline $46 \mathrm{PD}$ & $9.390 \mathrm{E}-03$ & $9.390 \mathrm{E}-03$ & $9.890 \mathrm{E}-03$ & $1.060 \mathrm{E}-02$ \\
\hline $47 \mathrm{AG}$ & $2.36 I E-03$ & $2.36 \geq E-03$ & $2.355 E-03$ & $2.355 \mathrm{E}-03$ \\
\hline $48 \mathrm{CD}$ & $6.622 \mathrm{E}-04$ & $6.622 \mathrm{E}-04$ & $6.723 E-04$ & $6.725 \mathrm{E}-04$ \\
\hline $49 \mathrm{IN}$ & $7.061 \mathrm{E}-06$ & $7.061 \mathrm{~F}-06$ & $7.171 \mathrm{E}-06$ & $7.205 \mathrm{E}-06$ \\
\hline $50 \mathrm{SN}$ & $4.282 \mathrm{E}-04$ & $4.282 \mathrm{E}-04$ & $4.236 \mathrm{E}-04$ & $4.233 E-04$ \\
\hline $51 \mathrm{SB}$ & $1.960 \mathrm{E}-04$ & $1.960 \mathrm{E}-04$ & $1.801 \mathrm{E}-04$ & $1.638 \mathrm{E}-04$ \\
\hline $52 \mathrm{TE}$ & $3.117 \mathrm{E}-03$ & $3.117 \mathrm{E}-03$ & $2.986 E-03$ & $2.981 \mathrm{E}-03$ \\
\hline $53 I$ & $2.693 \mathrm{E}-03$ & $2.693 \mathrm{E}-03$ & $2.670 E_{-}-03$ & $2.691 \mathrm{E}-03$ \\
\hline $54 \mathrm{XE}$ & $2.332 \mathrm{E}-02$ & $2.332 \mathrm{E}-02$ & $2.334 E-02$ & $2.334 E-02$ \\
\hline $55 \mathrm{CS}$ & $2.093 \mathrm{E}-02$ & $2.093 \mathrm{E}-02$ & $2.102 \mathrm{E}-02$ & $2.091 E-02$ \\
\hline $56 \mathrm{BA}$ & $7.044 \mathrm{E}=03$ & $7.044 E-03$ & $6.884 \mathrm{E}-03$ & $6.993 E-03$ \\
\hline 57 IA & $6.188 \mathrm{E}-03$ & $6.188 \mathrm{E}-03$ & $6.155 \mathrm{E}-03$ & $6.155 \mathrm{E}-03$ \\
\hline $58 \mathrm{CE}$ & $1.393 \mathrm{E}-02$ & $1.393 \mathrm{E}-02$ & $1.306 \mathrm{E}-02$ & $1.224 E-02$ \\
\hline $59 \mathrm{PR}$ & $5.748 \mathrm{E}-03$ & $5.748 \mathrm{E}-03$ & $6.019 \mathrm{~F}-03$ & $6.061 \mathrm{E}-03$ \\
\hline $60 \mathrm{ND}$ & $1.688 \mathrm{E}-02$ & $1.688 \mathrm{E}-02$ & $1.766 \mathrm{E}-02$ & $1.844 \mathrm{E}-02$ \\
\hline $61 \mathrm{PM}$ & $1.968 \mathrm{E}-03$ & $1.968 \mathrm{E}-03$ & $1.869 \mathrm{E}-03$ & $1.572 \mathrm{E}-03$ \\
\hline $62 \mathrm{SM}$ & $3.843 \mathrm{E}-03$ & $3.843 E-03$ & $4.020 \mathrm{E}-03$ & $4.312 \mathrm{E}-03$ \\
\hline $63 \mathrm{EU}$ & $8.473 \mathrm{E}-04$ & $8.473 E-04$ & $7.914 \mathrm{E}-04$ & $7.143 \mathrm{E}-04$ \\
\hline $64 \mathrm{GD}$ & 1.057E-03 & $1.057 \mathrm{E}-03$ & $1.115 \mathrm{E}-03$ & $1.193 \mathrm{E}-03$ \\
\hline $65 \mathrm{~TB}$ & $2.499 \mathrm{E}-04$ & $2.499 \mathrm{E}-04$ & $2.482 E-04$ & $2.477 \mathrm{E}-04$ \\
\hline $66 \mathrm{DY}$ & $7.246 E-05$ & $7.246 \mathrm{E}-05$ & $7.760 \mathrm{E}-05$ & $8.224 \mathrm{E}-05$ \\
\hline $67 \mathrm{HO}$ & $1.469 \mathrm{E}-06$ & $1.469 \mathrm{E}-06$ & $1.466 \mathrm{E}-06$ & $1.466 \mathrm{E}-06$ \\
\hline \multirow{2}{*}{$\begin{aligned} 68 & \text { ER } \\
& \text { TOTAI }\end{aligned}$} & $8.669 \mathrm{E}-07$ & $8.669 E-07$ & $8.792 \mathrm{E}-07$ & $8.792 \mathrm{E}-07$ \\
\hline & $1.860 \mathrm{E}-01$ & $1.860 \mathrm{E}-01$ & $1.860 \mathrm{E}-01$ & $1.860 \mathrm{E}-01$ \\
\hline
\end{tabular}

${ }^{a}$ Concentration in gram per centimeter of fuel rod at specified times of decay. 
Table VII. Fission Product Composition at 10.5\% FIMA from Enriched Fuel Irradiated in the EBR-II

\begin{tabular}{|c|c|c|c|c|}
\hline \multirow{2}{*}{ Element } & \multicolumn{4}{|c|}{ Concentrationa } \\
\hline & Shutdown & $60 \mathrm{sec}$ & 120 days & $1 \mathrm{yr}$ \\
\hline $30 \mathrm{ZN}$ & $6.360 \mathrm{E}-09$ & $6.351 \mathrm{E}-09$ & $1.44 I \mathrm{E}-27$ & 0.0 \\
\hline $31 \mathrm{GA}$ & $5.997 \mathrm{E}-09$ & $5.931 \mathrm{E}-09$ & $6.270 \mathrm{E}-28$ & 0.0 \\
\hline $32 \mathrm{GE}$ & $3.507 \mathrm{E}-05$ & $3.507 \mathrm{E}-05$ & $3.499 \mathrm{E}-05$ & $3.499 \mathrm{E}-05$ \\
\hline 33 AS & $4.211 \mathrm{E}-05$ & $4.211 \mathrm{E}-05$ & $4.182 \mathrm{E}-05$ & $4.182 E-05$ \\
\hline $34 \mathrm{SE}$ & $4.712 E-04$ & $4.712 \mathrm{E}-04$ & $4.716 \mathrm{E}-04$ & $4.716 \mathrm{E}-04$ \\
\hline $35 \mathrm{BR}$ & $1.513 \mathrm{E}-04$ & $1.513 \mathrm{E}-04$ & $1.509 \mathrm{E}-04$ & $1.509 \mathrm{E}-04$ \\
\hline $36 \mathrm{KR}$ & $3.514 \mathrm{E}-03$ & $3.514 \mathrm{E}-03$ & $3.509 \mathrm{E}-03$ & $3.501 \mathrm{E}-03$ \\
\hline $37 \mathrm{RB}$ & $2.584 \mathrm{E}-03$ & $2.584 \mathrm{E}-03$ & $2.588 \mathrm{E}-03$ & $2.597 \mathrm{E}-03$ \\
\hline $38 \mathrm{SR}$ & $7.058 E-03$ & $7.058 \mathrm{E}-03$ & $6.626 \mathrm{E}-03$ & $6.470 \mathrm{E}-03$ \\
\hline $39 Y$ & $3.526 \mathrm{E}-03$ & $3.526 \mathrm{E}-03$ & $3.369 \mathrm{E}-03$ & $3.293 E-03$ \\
\hline $40 \mathrm{ZR}$ & $2.423 \mathrm{E}-02$ & $2.423 \mathrm{E}-02$ & $2.411 E-02$ & $2.409 \mathrm{E}-02$ \\
\hline $41 \mathrm{NB}$ & $5.085 \mathrm{E}-04$ & $5.085 \mathrm{E}-04$ & $2.586 \mathrm{E}-04$ & $2.277 \mathrm{E}-05$ \\
\hline $42 \mathrm{MO}$ & $1.987 \mathrm{E}-02$ & $1.987 \mathrm{E}-02$ & $2.078 \mathrm{E}-02$ & $2.127 \mathrm{E}-02$ \\
\hline $43 \mathrm{TC}$ & $5.274 \mathrm{E}-03$ & $5.274 \mathrm{E}-03$ & $5.323 \mathrm{E}-03$ & $5.323 \mathrm{~F}-03$ \\
\hline $44 \mathrm{RU}$ & $1.267 \mathrm{E}-02$ & $1.267 \mathrm{E}-02$ & $1.215 \mathrm{E}-02$ & $1.180 \mathrm{E}-02$ \\
\hline $45 \mathrm{RH}$ & $2.860 \mathrm{E}-03$ & $2.860 \mathrm{E}-03$ & $3.172 \mathrm{E}-03$ & $3.217 \mathrm{E}-03$ \\
\hline $46 \mathrm{PD}$ & $4.766 \pm-03$ & $4.766 \mathrm{E}-03$ & $4.982 E-03$ & $5.280 \mathrm{E}-03$ \\
\hline $47 \mathrm{AG}$ & $6.427 \mathrm{E}-04$ & $6.427 \mathrm{E}-04$ & $6.403 \mathrm{E}-04$ & $6.402 E-04$ \\
\hline $48 \mathrm{CD}$ & $3.572 \mathrm{E}-04$ & $3.572 \mathrm{E}-04$ & $3.604 \mathrm{E}-04$ & $3.604 \mathrm{E}-04$ \\
\hline $49 \mathrm{IN}$ & $3.295 \mathrm{E}-05$ & $3.295 \mathrm{E}-05$ & $3.346 \mathrm{E}-05$ & $3.353 \mathrm{E}-05$ \\
\hline $50 \mathrm{SN}$ & $5.922 \mathrm{E}-04$ & $5.922 \mathrm{E}-04$ & $5.891 \mathrm{E}-04$ & $5.887 \mathrm{E}-04$ \\
\hline $51 \mathrm{SB}$ & $2.249 \mathrm{E}-04$ & $2.249 \mathrm{E}-04$ & $2.083 \mathrm{E}-04$ & $1.948 \mathrm{E}-04$ \\
\hline $52 \mathrm{TE}$ & $3.865 \mathrm{E}-03$ & $3.865 \mathrm{E}-03$ & $3.716 \mathrm{E}-03$ & $3.707 \mathrm{E}-03$ \\
\hline $53 I$ & $2.991 \mathrm{E}-03$ & $2.991 \mathrm{E}-03$ & $2.969 \mathrm{E}-03$ & $2.991 \mathrm{E}-03$ \\
\hline $54 \mathrm{XE}$ & $2.586 \bar{E}-02$ & $2.586 \mathrm{E}-02$ & $2.587 \mathrm{E}-02$ & $2.587 \mathrm{E}-02$ \\
\hline $55 \mathrm{CS}$ & $2.307 \mathrm{E}-02$ & $2.307 \mathrm{E}-02$ & $2.318 \mathrm{E}-02$ & $2.305 E-02$ \\
\hline $56 \mathrm{BA}$ & $7.740 \mathrm{E}-03$ & $7.740 \mathrm{E}-03$ & $7.500 \mathrm{E}-03$ & $7.626 \mathrm{~F}-03$ \\
\hline 57 IA & $7.393 \mathrm{E}-03$ & $7.393 E-03$ & $7.349 \mathrm{E}-03$ & $7.349 \mathrm{E}-03$ \\
\hline $58 \mathrm{CE}$ & $1.930 \mathrm{E}-02$ & $1.930 \mathrm{E}-02$ & $1.792 \mathrm{E}-02$ & $1.649 \mathrm{E}-02$ \\
\hline $59 \mathrm{PR}$ & $7.038 \mathrm{E}-03$ & $7.038 \mathrm{E}-03$ & $7.351 \mathrm{E}-03$ & $7.405 \mathrm{E}-03$ \\
\hline $60 \mathrm{ND}$ & $2.300 \mathrm{E}-02$ & $2.300 \mathrm{E}-02$ & $2.430 \mathrm{E}-02$ & $2.568 \mathrm{E}-02$ \\
\hline $61 \mathrm{PM}$ & $2.622 \mathrm{E}-03$ & $2.622 \mathrm{E}-03$ & $2.496 \mathrm{E}-03$ & $2.099 \mathrm{E}-03$ \\
\hline $62 \mathrm{SM}$ & $3.712 \mathrm{E}-03$ & $3.712 \mathrm{E}-03$ & $3.954 \mathrm{E}-03$ & $4.347 \mathrm{E}-03$ \\
\hline $63 \mathrm{EU}$ & $4.178 \mathrm{E}-04$ & $4.178 \mathrm{E}-04$ & $3.996 \mathrm{E}-04$ & $3.737 \mathrm{E}-04$ \\
\hline 64 GD & $3.672 \mathrm{E}-04$ & $3.672 \mathrm{E}-04$ & $3.882 \mathrm{~F}-04$ & $4.169 \mathrm{E}-04$ \\
\hline $65 \mathrm{~TB}$ & $7.362 \mathrm{E}-05$ & $7.362 \mathrm{E}-05$ & $7.070 \mathrm{E}-05$ & $7.041 \mathrm{E}-05$ \\
\hline 66 DY & $1.076 \mathrm{E}-04$ & $1.076 \mathrm{E}-04$ & $1.113 \mathrm{E}-04$ & $1.125 \mathrm{E}-04$ \\
\hline $67 \mathrm{HO}$ & $3.450 \mathrm{E}-07$ & $3.450 \mathrm{E}-07$ & $3.444 E-07$ & $3.444 E-07$ \\
\hline \multirow{2}{*}{$68 \mathrm{ER}$} & $2.127 \mathrm{E}-07$ & $2.127 \mathrm{E}-07$ & 2.15 IE-O7 & $2.151 \mathrm{E}-07$ \\
\hline & $2.170 \mathrm{E}-01$ & $2.170 \mathrm{E}-01$ & $2.170 \mathrm{E}-01$ & $2.170 \mathrm{E}-01$ \\
\hline
\end{tabular}
decay .

${ }^{a}$ Concentration in gram per centimeter of fuel rod at specified times of 


\section{DISCUSSION OF RESULTS}

In analyzing the validity of any fuel test, one must consider a variety of factors. These factors include, but are not limited to, such things as fuel operating temperatures, the relative fuel burnup to cladding damage ratios, and the effects of fissioning on the fuel composition.

Let us first examine the usefulness of thermal flux test reactors. Because of the high thermal neutron fission cross sections for the plutonium, prototypic linear heat rates can be obtained with the exact fuel composition proposed for the reference reactor.

From Fig. 2 it is apparent that a fast reactor fuel can be tested in a thermal flux environment under conditions which will closely approximate not only the fuel center and surface temperatures but also the temperature gradient in the fuel. Figures 3 through 5 show that this comparative temperature profile can be maintained during extended exposure periods. Thus, if one wishes to study the thermal restructuring of fuel, the thermal reactor provides a good test site. Since such tests can be readily instrumented, the effective in-reactor thermal conductivity can be measured and the effects of restructuring on thermal conductivity determined. Care must be used in interpreting such data because of fuel compositional changes and the nonuniform heat generation as a function of radial position, particularly in the early stages of irradiation. As indicated by the heat generation plots in Figs. 2 through 5 , the nonuniform burnup rates gradually modify this heat generation distribution until at about 10\% FIMA the fast reactor and thermal reactor profiles are essentially identical.

A more detailed calculation using more fuel zones and shorter burnup increments will undoubtedly smooth out the heat generation profiles and may show that the duplication of the fast flux profile by the thermal flux test occurs at a lower burnup level. We are currently making these rather tedious calculations. A knowledge of this fission rate distribution and the associated burnup is critical in evaluating such things as fission gas release or fission product and fissile element redistribution as a function of fuel temperature, the temperature gradient in an increment, and the fuel structure of the increments.

Since a test is usually conducted in a reactor where there is an axial flux profile which changes with time during each reactor cycle, it is apparent that the data from thermal reactor tests of fast reactor fuels require considerable analysis before it can be properly interpreted. However, the large amount of test reactor space available, relative ease in instrumenting such tests, and the generally high operating efficiency of such reactors make them very useful in conducting tests where the mechanical interaction between the fuel and the cladding are not a first priority objective. This limitation derives from examination of the plot of cladding fast fluence and fuel burnup for the ETR tests shown in Fig. 7, which clearly shows that there is minimal fast fluence exposure for the cladding. Thus, the cladding is not in a prototypical condition.

The advantages of tests in a fast flux reactor such as the EBR-II are apparent. The fission rates as a function of radial position in the fuel closely approximate the reference reactor. The fast flux damage 
to the cladding may be comparable, as we shall see later, and other environmental considerations, such as flowing sodium coolant, cladding temperatures, and heat flux levels, can be obtained. The disadvantages are the Iimited amount of such test space, the current lack of instrumentation capabilities, and the relatively low flux levels currently available in such test reactors.

In analyzing the validity of tests in such reactors, it is the low flux level and its effect on the required fuel composition which are most important. The fact that the EBR-II and Dounreay Fast Reactor (DFR) have a different neutron energy spectrum than proposed commercial fast reactors, as shown by the partial flux-versus-lethargy plots in Fig. 8, may be an advantage.

For the reference fuel, the fluence to FIMA ratio (see Fig. 7) is approximately three times that for an enriched fuel in the EBR-II. This would indicate a difference in the cladding damage to fuel burnup ratio for the two cases. However, recent analyses of the neutron spectrum effects 13,14 indicate that the harder spectrum reactors yield approximately three times as much damage per unit of fluence. This factor then means the ratios of fuel burnup to cladding displacement damage for the reference fuel rod and tests in the EBR-II with enriched fuel may be similar. The gas production in the cladding in the EBR-II from $(n, p)$ and $(n, \alpha)$ reactions is also up by a factor of about 2 . Validating the data on mechanical interaction of fuel and irradiated cladding for EBR-II tests will require an improved understanding of the neutron energy effects on cladding damage and transmutation and a detailed knowledge of the flux energy spectrum in each test location, as well as an improved understanding of the effects of irradiation on such cladding characteristics as creep.

A more subtle and as yet undefined effect of neutron energy is the effect on fission product yields. This effect is probably too small to significantly influence our current understanding of changes in fuel composition and their effects on fuel chemical and thermophysical properties. This subtle difference is insignificant when one considers the fact that the current EBR-II tests of oxide fuels generally involve enriched uranium in the fuel. Certain fission product yields of uranium are significantly different from those of plutonium. These account for most of the differences shown in Tables VI and VII. The possible effects of the two- to threefold higher yields of $\mathrm{Rb}, \mathrm{Sr}, \mathrm{Y}$, and particularly $\mathrm{Zr}$ on the oxygen activity ${ }^{15}-2 \mathrm{I}$ in the fuel is beyond the scope of this paper, but may be significant in evaluating fuelcladding chemical interactions. Certainly, the lower yields of Tc, Ru, $\mathrm{Rh}$, and $\mathrm{Pd}$ in the enriched fuels will result in fewer metallic inclusions in metallographic cross sections of enriched fuel irradiation tests. Detailed analysis by microprobe techniques to evaluate fission product and fissile element migration and redistribution require improved fission product yield and cross-section information for quantitative evaluation. A quantitative understanding is needed to establish the effects of burnup and fuel temperature on fuel swelling and fuel-cladding compatibility. 
ORNL-DWG 69-9490

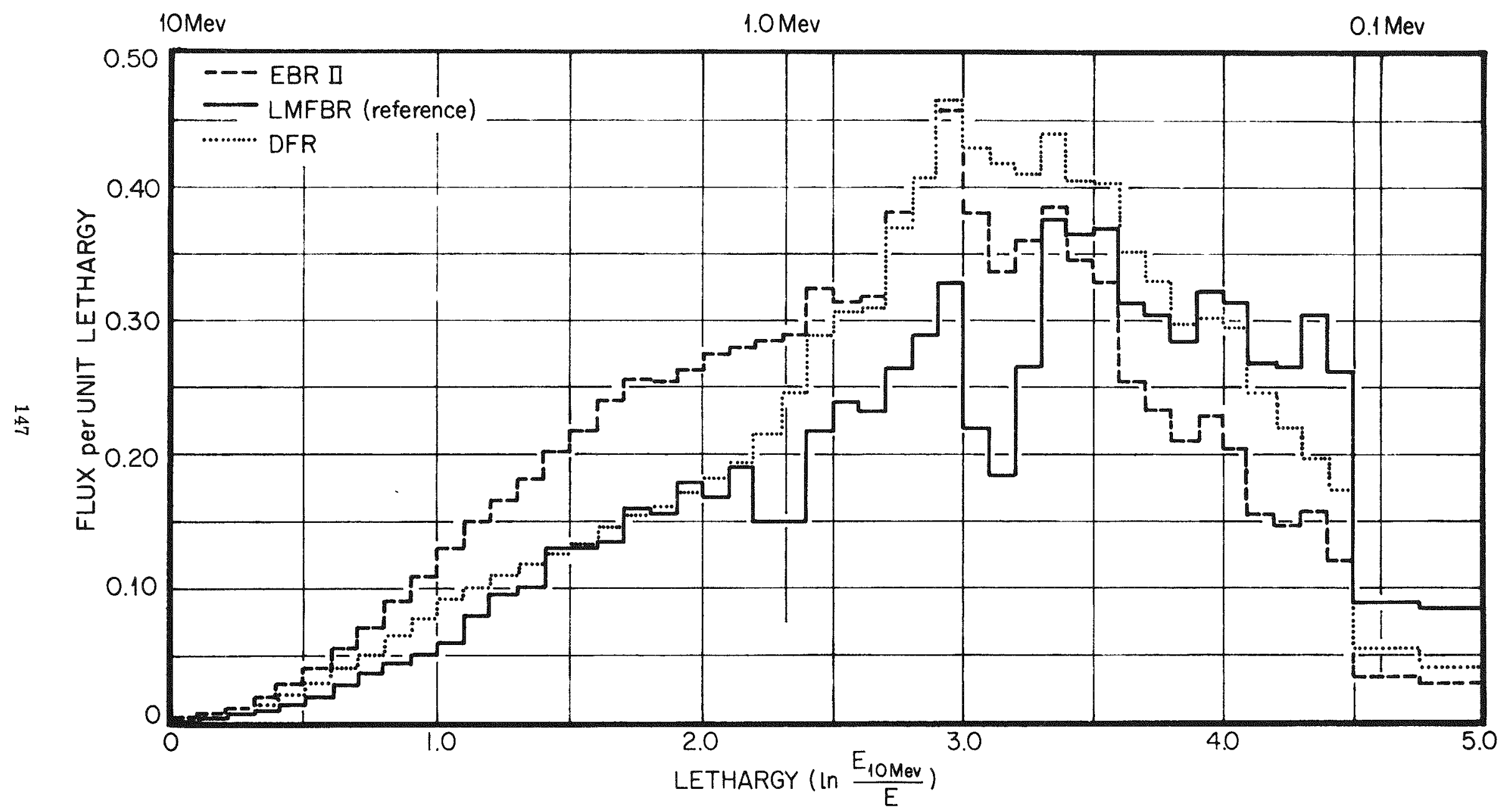

Fig. 8. Comparative Neutron Energy Spectra for a Reference Design LNFBR, the EBR-II, and the DFR Reactors Calculated by GAM-II. 
No test conducted in currently available test reactors can provide valid information on all of the fuel rod performance characteristics that are needed by reactor designers. In this paper we have presented some calculational results to show the types of analyses that must be applied to the data obtained from any irradiation test of potential IMFBR fuel rods. Using such analytical techniques, valid tests of specific limited characteristics can be designed and conducted in both the thermal and fast flux test reactors.

Specifically, a large amount of valid testing of fuel properties can be done in a thermal flux environment: these include fuel thermal restructuring kinetic studies, in-reactor thermal conductivity measurements, and fission gas release measurements.

The available fast flux test environments appear to provide a reasonable location for testing fuel-cladding mechanical interactions involving the irradiation damage parameters. Currently, the need for uranium enrichment in the fast test reactors such as the EBR-II means that changes in fuel composition with burnup are not typical of proposed LMPBR fuel. Interpretation of postirradiation microprobe analysis of such tests requires better fission product yield information than that currently available.

The introduction of new higher flux fast reactors will alleviate many of the restrictions on testing of LMFBR fuel rods. However, a continuing analysis of the neutronic environment differences between the test reactor and the proposed power reactor will have to be maintained to make the test results truly valid.

\section{ACKNOWLEDGMENTS}

Studies of this type are essentialiy impossible without the cooperation and assistance of many others. In particular, the authors would like to thank: J. D. Jenkins, who did a large part of the physics analyses and provided the cross-section data and flux characterizations, E. D. Arnold, who revised the RIBD code and assisted in conducting the calculations, J. E. Bigelow, who provided us with his "CALLSPT" modification of "IS $\phi$ CRUNCH" from which our "DEPLETE" code evolved, F. J. Homan, who revised the "PR $\phi F I L "$ code to provide us with the needed "RERUN" version, and G. R. Bates, W. L. Clements, and D. E. Gates for their invaluable assistance in actually doing the calculations and reducing the output to the tabular and graphical forms used in this report.

\section{REFERENCES}

1. Research sponsored by the U.S. Atomic Energy Commission under contract with the Union Carbide Corporation.

2. Liquid Metal Fast Breeder Reactor Design Study, GEAP-4418 (January 1964 ).

3. W. W. Engle, Jr., A User's Manual for ANISN: A One-Dimensional Discrete Ordinate Transport Code with Anisotropic Scattering, K-1693 (March 1967). 
4. G. D. Joanou and J. S. Dudek, GAM-II - A B Code for the Calculation of Fast-Neutron Spectra and Associated Multigroup Constants, GA-4265 (September 1963).

5. J. D. Jenkins, Oak Ridge National Laboratory, personal communication, November 1968.

6. H. C. Honeck, THERMOS - A Thermalization Transport Theory Code for Reactor Iattice Calculations, BNL-5826

7. A. R. Olsen, Fuels and Materials Development Program Quarterly Progress Report for Period Ending March 31, 1969, ORIL-4420, pp. $24-39$.

8. C. M. Cox and F. J. Homan, PR $\phi F I L-A$ One-DimensionaI F $\phi R T R A N$ IV Program for Computing Steady-State Temperature Distributions in Cylindrical Ceramic Fuels, ORNI-TM-2443 (March 1969) and Addendum (August 1969).

9. W. E. Baily, E. A. Aitken, R. R. Asamoto, and C. I. Craig, "Thermal Conductivity of Uranium-Plutonium Oxide Fuels," pp. 293-308 in International Symposium on Plutonium Fuels Technology, Scottsdale, Arizona, 1967, Nucl. Met. 13, American Institute of Mining, MetalIurgical, and Petroleum Engineers, New York, 1968.

10. C. W. Friend and J. R. Knight, ISOCRUNCH - Modifications to the CRUNCH Program for the IBM-7090, ORNL-3689 (January 1965).

11. J. L. Rash, Use of Computer Code RIBD for Fission Product Analysis, RI-INRD-610 (November 1965).

12. M. E. Meek and B. F. Rider, Sunmary of Fission Product Yields for $\mathrm{U}^{235}, \mathrm{U}^{238}, \mathrm{Pu}^{239}$, and $\mathrm{Pu}^{24} \mathrm{I}$ at Thermal, Fission Spectrum and 14 Mev Neutron Energies, APED-5398 (March 1, 1968).

13. R. E. Dah1, "Correlation of Burnup and Clad Damage for Fast Reactor Fuel Burnup," Trans. Am. Nucl. Soc. 12(1), 139-140 (June 1969).

14. J. D. Jenkins, "Primary Recoil Spectra and Displacement Damage from ENDF/B Data," Trans. Am. Nucl. Soc. 12(1), 139 (June 1969).

15. M. H. Rand and I.E.J. Roberts, "Thermochemistry and Nuclear Engineering, "pp. 3-3I in Thermodynamics, Vol. I, International Atomic Energy Agency, Vienna, 1966.

16. T. I. Markin and E. J. McIver, "Thermodynamic and Phase Studies for Plutonium and Uranium-Plutonium Oxides with Application to Compatibility Calculations," pp. 845-857 in Plutonium 1965, ed. by A. E. Kay and M. B. Waldron, Chapman and Hall, London, 1967. 
17. T. L. Markin, "Thermodynamic Data for Uranium and Uranium/PIutonium Oxides Applied to Fuel Preparation Problems," pp. 43-49 in Preparation of Nuclear Fuels, Nuclear Engineering - Pt. XVIII, Chem. Eng. Progr. Symp. Ser. 80, American Institute of Chemical Engineers, New York, 1967.

18. M. H. Rand and T. I. Markin, Some Thermodynamic Aspects of $(\mathrm{U}, \mathrm{Pu}) \mathrm{O}_{2}$ Solid Solutions and Their Use as Nuclear Fuels, AERE-R-5560

(August 1967); also pp. 637-650 in Thermodynamics of Nuclear Materials, International Atomic Energy Agency, Vienna, 1968.

19. K. E. Spear, A. R. Olsen, and J. M. Leitnaker, Thermodynamic Applications to (U,Pu) $\mathrm{O}_{2} \pm x$ Fuel Systems, ORNL-TM-2494 (Apri1 1969).

20. K. E. Spear, J. M. Leitnaker, and N. Engel, Fuels and Materials Development Program Quarterly Progress Report for Period Ending June 30, 1969, ORNL-4440 (in preparation).

21. K. E. Spear and J. M. Leitnaker, "Chemical Effects of Nuclear Burnup on Fast Reactor Fuels," Invited paper to be presented at the American Nuclear Society Winter Meeting, San Francisco, November 30December 4, 1969. 


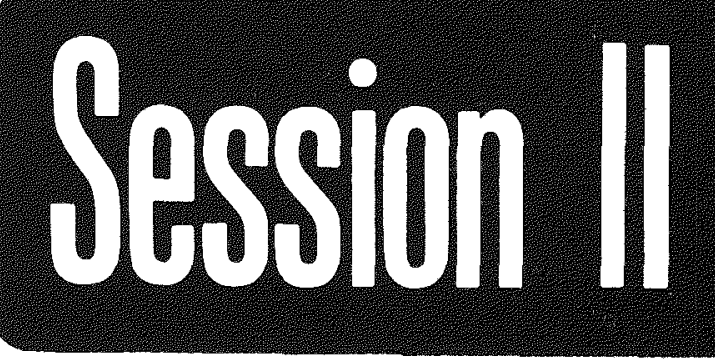

Environmental Control 
-

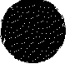




\section{X a reactor facility for neUtron irRadiations} AT TEMPERATURES RANGING FROM -193 TO $725^{\circ} \mathrm{C}$

R. G. Struss and C. W. Chen

426625 Institute for Atomic Research and Ames Laboratory 054560 lowa State University Ames, lowa 50010

A facility has been installed and operated in the Ames Laboratory Research Reactor which provides a variable temperature environment in a fast neutron flux. This facility has been in semi-continuous operation for more than a year and has provided a very useful irradiation facility at this reactor. The combination of using a flux converter in the reactor in conjunction with the temperature control in a cryostat is described in this paper.

The Ames Laboratory Research Reactor is a heterogeneous, heavy water moderated and cooled reactor, using enriched uranium fuel elements. The operating power level of the reactor is $5 \mathrm{MW}$ which produces a maximum measured thermal flux of $1.0 \times 10^{14} \mathrm{n} / \mathrm{cm}^{2} \mathrm{sec}$ in the central thimble of the reactor core. The facility described in this paper is installed in a side vertical thimble which had a measured thermal flux of $1.6 \mathrm{x}$ $1013 \mathrm{n} / \mathrm{cm}^{2} \mathrm{sec}$ before the installation of the facility.

The flux converter was constructed of CP-5 type cylindrical fuel tubes. An assembly of three concentric fuel tubes containing an aluminumuranium fuel section enriched in $235 \mathrm{U}$, with suitable end fittings, makes up the converter. This assembly has a total mass of $170 \mathrm{~g}$ of $235 \mathrm{U}$ distributed in the three tubes over a $66.8 \mathrm{~cm}$ height. The design of the flux converter provides a hollow fuel array which is cooled by forced flow of the heavy water. The flux converter assembly as installed in the reactor then allows the cryostat to be installed inside of this fuel array. The total flux measured inside this flux converter shortly after installation in the reactor was $1.5 \times 10^{13} \mathrm{n} / \mathrm{cm}^{2} \mathrm{sec}$ with a cadmium ratio of about 5 for gold. A maximum fast flux of $6 \times 10^{12} \mathrm{n} / \mathrm{cm}^{2} \mathrm{sec}$ with E>IMeV was recorded at a position which was $14 \mathrm{~cm}$ from the bottom of the converter.

The cryostat system was designed to provide a facility for use in radiation damage studies. Since the fast neutron fluxes required for producing detectable radiation damage in metals are generally much higher than those for semiconductors and non-metallic substances, a total exposure of $10^{19}$ nvt with E $>1$ MeV was taken as the upper limit for the design of the present system. An assumed time interval of 10 weeks was used as the 
practical irradiation time. Using the average reactor operating time at $5 \mathrm{MW}$, the 10 week period would provide an actual irradiation time of $4.5 \times 10^{6} \mathrm{sec}$. This then requires an assumed maximum dose rate of $2.2 \times 10^{12} \mathrm{n} / \mathrm{cm}^{2} \mathrm{sec}$. The preliminary converter facility calculations indicated the capability of providing these dose rates, and after the entire system was installed the flux was measured at 3.5 to $4 \times 10^{12}$ $\mathrm{n} / \mathrm{cm}^{2} \mathrm{sec}$ with ElMeV.

The cryostat was designed for use in the flux converter fuel array which then provided the parameters to consider in the design. The maximum outside diameter of the cryostat was limited to just less than $5 \mathrm{~cm}$ by the fuel tubes. The fuel tubes surrounding the cryostat would also cause an extra burden in gamma heating from the prompt fission gamma rays. The presence of intense ionizing radiation was also considered a problem in this design from the standpoint of explosions in irradiated liquid nitrogen.

The cryostat design used in this installation was based on the thermosiphon principle of operation. 1 This principle was first applied to the operation of a cryostat in a reactor at the Grenoble Nuclear Research Center, France. 2 Under this principle the liquid nitrogen cools the samples by boiling off an appropriate mass and the vapor produced in this cooling effort is condensed by a heat exchanger with the condensate returning to the sample region by gravity. This principle of operation allows the use of high purity oxygen free nitrogen in a closed primary system. This closed system also eliminates the problem of constant ventilation of irradiated nitrogen gas in an open system.

The cryostat system is then designed with a vacuum insulated dewar vessel of $3.8 \mathrm{~cm}$ inside diameter and $4.8 \mathrm{~cm}$ outside diameter. This inner tube carries the sample container and the high purity nitrogen during an irradiation. At the upper end of this sample tube is the heat exchanger which is designed to allow sample insertion, withdrawal or instrumentation through the center. The heat exchanger condenses the high purity nitrogen as it consumes commercial liquid nitrogen circulated through it. The commercial liquid nitrogen is gravity fed to the heat exchanger from a small dewar on the reactor top. A 500 liter dewar is used on the reactor floor as the supply to automatically fill the smaller dewar. A vacuum pumping system is used to maintain vacuum insulation and a small purification system is also incorporated to remove moisture and oxygen from the gaseous nitrogen fed to the cryostat.

The cryostat is fabricated from Type 304 stainless steel and utilizes metal 0-rings for vacuum seals. The heat exchanger design is based on many small tubes to present a large surface area to volume ratio, with a total condensing area of $3,865 \mathrm{~cm}^{2}$. The inner tube has four copper-constantan thermocouples attached at intervals in the sample region to monitor temperatures during operation. The sample region is shielded from thermal neutrons with a cadmium jacket of $0.076 \mathrm{~cm}$ thickness incorporated into the vacuum jacket surrounding the sample tube. This describes the flux converter and cryostat that is installed in the reactor.

After the installation of the flux converter and cryostat had been 
completed in the reactor the fast neutron flux was found to vary only between 3.5 and $4 \times 10^{12} \mathrm{n} / \mathrm{cm}^{2} \mathrm{sec}$ in the bottom $30 \mathrm{~cm}$ section of the dewar vessel. Because of a slight overpressure required to condense the high purity nitrogen gas during operation, the sample temperature can be maintained at a minimum value of $-193^{\circ} \mathrm{C}$ instead of $-196^{\circ} \mathrm{C}$ for atmospheric pressures. The consumption of commercial liquid nitrogen is about 11.8 liters/hr, from which the gamma flux in the converter is estimated at about $10^{8} \mathrm{R} / \mathrm{hr}$. This magnitude of gamma flux is slightly higher than expected but the operation of the cryostat at low temperatures is not altered.

A preliminary measurement has been made subsequent to the initial operation of the cryostat to support the estimation of gamma flux in the cryostat. A nuclear heat calorimeter, utilizing the principle of constant temperature difference along a heat path, was irradiated in the reactor. The measurements show a nuclear heating load at 5 MW reactor power level of 0.1 and 0.12 watts/g of stainless steel and lead, respectively, in a side thimble of the reactor without a converter. A measurement which simulates the thimble with a converter showed a nuclear heating load of 1.1 watts $/ \mathrm{g}$ in lead at $5 \mathrm{MW}$ power level. This latter heating load can be used to calculate an apparent gamma flux of $3.7 \times 10^{8} \mathrm{R} / \mathrm{hr}$ using reported absorption coefficients. The gamma flux was then measured at $5 \mathrm{MW}$, using cobalt glass techniques, at $2.5 \mathrm{x}$ $10^{8} \mathrm{R} / \mathrm{hr}$. At these values of gamma flux, it was felt that sufficient gamma heating could be utilized to use the facility as installed in the reactor for higher temperature irradiations.

To test this possibility the cryostat was loaded with samples and then filled with dry nitrogen or helium which could be varied in pressure during reactor operation. The gamma heating produced temperatures up to $4000 \mathrm{C}$ in the initial operations of the system. Following this indication that the sample temperature could be elevated significantly, some further work was done on the sample container preparation. The final results that have been achieved successfully were samples run in the reactor at $725^{\circ} \mathrm{C}$. The temperature control is done by calculating the heat transfer out of the sample in initially designing the package, and then the gas pressure is varied in the cryostat facility to provide a variable heat transfer during irradiation. The combination of these two factors allows the variation of temperature over a very wide range. of course, the variation of the temperature of the samples at low temperature can be done by varying the pressure of the liquid nitrogen which affects the boiling point.

The intermediate range of temperatures is available when a liner tube is inserted in the cryostat. The liquid nitrogen can then be cooling this tube and the sample temperature is controlled by heat transfer through a gas as described for the higher temperatures. When operating in this temperature range the heat transfer gas needs to be helium to avoid the condensation of gas inside the liner tube.

The installation of this facility in the reactor has provided a versatile tool in the irradiation of inorganic specimens, but there was one instance of difficulty that is worthy of mention. A small or mild explosion occurred once in the cryostat about an hour after 
reactor startup following a sample change. The nature and cause of this explosion is discussed fully elsewhere, 3 but can briefly be described here. The sample change preceding this excursion followed the possible condensation of liquid oxygen in the cryostat. This is the only factor differing from the other runs, which never caused difficulty. This oxygen apparently was converted into ozone by the intense ionizing radiation and when a critical level was exceeded the explosion occurred and the cryostat inner tube was ruptured. No change in temperature was recorded prior to this point. The cryostat has not been operated with the condensed liquid nitrogen open to the atmosphere since that time, and no further difficulty has been encountered.

\section{References :}

1. L. Bewilogua and R. Knöner, Cryogenics 2, 46(1961).

2. L. Bochirol, J. Donlat, and L. Weil, Cryogenics 1, 44 (1960).

3. C. W. Chen and R. G. Struss, Cryogenics 9, 131 (1 $\overline{9} 69)$. 


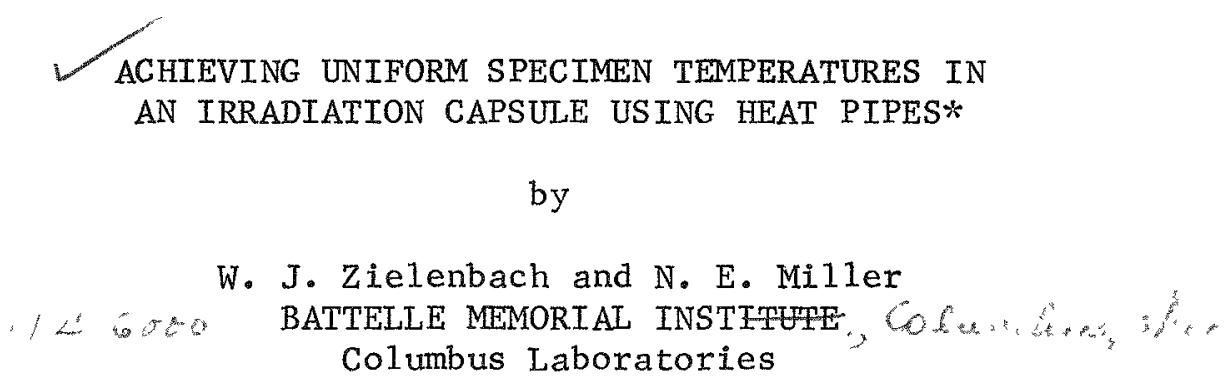

\section{ABSTRACT}

Laboratory studies indicate that a lithium-filled annular heat pipe is feasible as a means of obtaining near isothermal conditions axially along the surface on the cladding of a short fuel sample in an in-pile fuel-irradiation capsule. At a $1500 \mathrm{C}$ operating temperature the axial spread of temperatures was less than $20 \mathrm{C}$ along a three inch length in an experiment simulating the irradiation capsule. The annular heat pipe would be incorporated into the current capsule configuration employed in the high-temperature-fuels program to achieve uniform specimen cladding temperatures of 1600 to $1900 \mathrm{C}$.

* Work performed under AEC Contract W-7405-eng-92. 


\section{INTRODUCTION}

In irradiation capsule experiments, samples of experimental fuels are subjected to anticipate reactor service conditions to obtain operational performance data. These experiments are becoming more complex and represent a large investment of time and money; therefore, emphasis is placed on maximizing the information to be gained in individual experiments. Unfortunately, not al1 parameters can be evaluated in a single experiment. The use of heat pipes is considered a possible way of circumventing some of the problems created by increasing the number of samples in a given irradiation experiment at the expense of sample length. The axial temperature gradients caused by end effects in short fuel specimens are generally much larger than temperature gradients that would be expected in actual fuel-element service. The gradients are further affected by the vertical flux profile within the test facility. High gradients may influence fuel-swelling mechanisms and cause unrealistic fuel performance.

A study is in progress to evaluate the feasibility of incorporating an annular heat pipe in an irradiation capsule to achieve a uniform temperature over the length of fuel sample. The heat-pipe principle presents two aspects which favor its consideration:

(1) A near isothermal temperature exists along the surface of the evaporator.

(2) The heat is transferred under relatively isothermal conditions to a condenser away from the fuel surface, where the system temperature can be monitored.

* Work performed under AEC Contract W-7405-eng-92. 


\section{PROPOSED CAPSULE SYSTEM}

In the proposed application a 0.250-inch-diameter $\times 1-7 / 16-$ long tungsten-26 rhenium cladding will be sealed within a 0.5 -inchdiameter $\times 0.035$-inch-wall $\times 2$ in.-long molybdenum tube to form an annular heat pipe. The outer surface of the cladding (the evaporator) and the inner surface of the tube (the condenser) will be covered with severa1 layers of refractory-metal wire cloth to form a wick to return the lithium working fluid from the condenser to the boiler. Noncondensible gases will be removed from the system prior to capsule assembly. Thermocouples will be attached to the outer surface of the molybdenum tube to monitor specimen operating temperatures. Heat will be rejected from the condenser of the heat pipe sequentially through an argon-filled gap, a 0.7 inch ID molybdenum primary containment, a binary gas gap, and a stainless steel secondary containment to the reactor process water.

\section{OUT-OF-PILE MOCKUP STUDY}

\section{Mockup Design}

A mockup experiment was conducted to evaluate the proposed application of the in-pile heat pipe; a sketch of this assembly is shown in Figure 1 and a photograph in Figure 2. The assembly was fabricated of molybdenum. The annular heat pipe (left side of Figure 1 ) is surrounded by a stainless steel she11 to simulate the capsule containment wall. The inner tube simulates the specimen cladding and acts as the evaporator; the condenser encloses the evaporator and is about one inch longer. These lengths are scaled up from the in-pile version, but the operation of this model is indicative of in-pile performance. The inner surface of the condenser was covered with 3 layers of 100-mesh molybdenum wire cloth. The cloth was held in place by a 0.02-inch-diameter molybdenum wire spiral. The surface of the evaporator was covered with 3 layers of 200-mesh molybdenum wire cloth which extended about 1/2-inch above the evaporator; the wire cloth was flared out at both ends of the evaporator to contact with the 100 mesh screen on the condenser to provide a flow path for the return of condensed lithium to each end of the evaporator.

The heat source for the annular heat pipe was a tubular heat pipe (on the right in Figure 1). A heat-pipe heat source offered a practical method to supply the desired heat rate to the annular heat pipe, in that it was easily capable of converting the low-flux radiant heat input to a large-area evaporator into a high-flux heat output in a smal1 condenser. The latter simulated the actual heat flux from the fuel sample to the cladding. A tantalum-tube heater, surrounded by molybdenum baffles, was employed to heat the molybdenum tubular heat pipe. 


\section{Lithium Loading}

Original lithium loadings were calculated on the basis of the void volume in the wicking within each heat pipe. A weighed amount of solid lithium was loaded into the tubular heat pipe in a heliumfilled dry box; the heat pipe was sealed by TIG welding the end cap in place and welding shut the end of the tube. The non-condensible gases were removed by placing the tubular heat pipe in an electron-beam welding chamber, evacuating the chamber, melting the seal at the end of the tube with the electron beam, and pumping the helium out with the chamber's vacuum system. The end of the tube was then resealed with the electron beam before removing the assembly from the chamber. The tubular heat pipe was operated at $1100 \mathrm{C}$ in a vacuum to verify its performance before loading the annular heat pipe.

It was evident that loading the lithium into the annular heat pipe in a similar manner and keeping it away from the end-cap weld zone would be most difficult owing to the small internal volume. Therefore, the end cap was welded in place and the lithium was loaded in liquid form through the tube in the end cap. This was accomplished by melting the lithium in a stainless steel chamber to which was joined a hypodermic syringe. The annular heat pipe was heated to about $500 \mathrm{C}$ to promote wetting of the molybdenum wicking by the 1ithium. The helium gas in the hypodermic syringe was compressed under the plunger, and the melted lithium was forced through the hypodermic needle, which was fully inserted in the end cap tube, into the annular heat pipe as shown in Figure 3. The loading tube was checked to insure that it was free of lithium, and it was sealed by TIG welding. The non-condensible gases were removed in the $\mathrm{EB}$ welding chamber as described previously.

\section{Experimental Facility}

The heat pipe was centered within the tantalum tube heater and the assembly was mounted in a vacuum facility developed for laboratory testing. Three vertical rods supported the centering assembly; nylon insulators were employed in the centering assembly to isolate the heat pipe electrically from the heater, one side of which was grounded to the test facility. The heater was connected to water-cooled copper electrodes, which penetrated the base plate of the facility; one of these electrodes was electrically insulated by a double 0-ring vacuum seal in the base plate. A welding transformer was employed to convert the output of an auto-transformer to a low-voltage input to the heater. Five molybdenum radiation baffles were installed around the heater. The connecting neck between the two heat pipes was surrounded by a fivelayer molybdenum-foil baffle to restrict heat loss in this region. Five horizontal molybdenum-foil baffles were installed around the neck baffle to shield the annular heat pipe from radiant heating by the heater. The stainless steel tube, which simulated the capsule inner she11, was hung from the centering supports. 
The assembly was enclosed within a heavy-wall Vycor tube which was sealed to the end plates with flat elastomer rings. The interior of the tube was evacuated through the top seal plate. A series of foil baffles rested on top of the centering supports to shield the upper elastomer seal from thermal radiation. Temperatures at selected locations on the exterior of the heat pipes (denoted by $X^{\prime} \mathrm{S}$ in Figure 1) were monitored with an optical pyrometer through holes in the surrounding shields.

\section{Experiment Operation and Results}

Experiments were conducted by applying the desired heater power quickly and holding it constant until the surface of the annular heat pipe reached a steady-state temperature. It general1y required about 2 minutes for the bottom of the annular heat pipe to reach an optically measurable temperature and an additional 20 seconds for this heat pipe to attain near isothermal conditions along its 3-inch length. The variation in temperature along this length was usually less than $20 \mathrm{C}$ which is within the accuracy attainable with an optical pyrometer. Measurements indicated that the temperature of the tubular heat pipe was 150 to $200 \mathrm{C}$ higher than that of the annular heat pipe, but this was thought to be in error owing to reflection of the heater illumination from the tubular heat-pipe surface.

The temperatures monitored at various heat inputs are summarized in Table 1.

\section{CONCLUSIONS}

Because of the inaccessibility of the simulated fuel cladding it was not practical to measure its temperature uniformity directly. However, the experiment was conducted with the belief that if reliable operation of the annular heat pipe could be demonstrated one could expect isothermal conditions to exist in the evaporator region unless burnout occurred. At the heat fluxes of interest, nominally 75 watts $/ \mathrm{cm}^{2}$, one would not anticipate burnout in a lithium heat pipe.

A clad fuel specimen norma11y has an unfueled plenum region at one end, where there is no heat input to the cladding. This nonuniform heating in the axial direction is largely responsible for the axial temperature nonuniformity. The heat input to the simulated cladding at the condenser end of the tubular heat pipe was believed to be similar qualitatively to that which occurs in a fissioning fuel specimen. Some noncondensible gases (adsorbed gases released from the walls, dissolved gases released from the lithium, and residual gases from the atmosphere) would inevitably be present in the tubular heat pipe used to supply heat to the simulated cladding. These gases would be pumped to the end of 
the condenser region by the action of the heat pipe and produce a region in which there would be reduced heat input near the end of the simulated cladding.

In spite of this nonuniform heat input to the interior of the simulated cladding (condenser of the tubular heat pipe), a uniform temperature condition occurs on the exterior of the simulated cladding (evaporator of the annular heat pipe) by preferential evaporation of the lithium at a rate governed by the uniform vapor pressure above its surface. The near isothermal conditions measured along the full length of the condenser during operation of the experimental mockup were good evidence that the annular heat pipe was functioning properly and that isothermal conditions could be expected on the evaporator.

These initial results are very encouraging in the application of an annular heat pipe to irradiation capsule for the purpose of producing axially uniform fuel cladding temperatures. The techniques developed for assembling and loading the small annular heat pipe with very small annular spaces for wicking and working fluid can be applied to the actual geometry of a fuel specimen in an irradiation capsule. No difficulties were encountered upon initial or subsequent startups of the heat pipe operation. Experimentation is planned to be continued to investigate the performance of the heat pipe as a function of heat load and temperature before the technique is applied to an actual fuel irradiation experiment. 
TABLE 1. OPTICALLY MONITORED TEMPERATURES IN THE MOCKUP HEAT PIPE EXPERIMENT

\begin{tabular}{|c|c|c|c|c|c|c|c|}
\hline \multirow[b]{2}{*}{ Run No. } & \multicolumn{7}{|c|}{ Optical1y Measure Temperature, $C$ (1) } \\
\hline & 1 & 2 & 3 & 4 & 5 & 6 & 7 \\
\hline 1 & $\mathrm{~N} \cdot \mathrm{M} \cdot(2)$ & $\mathrm{N} \cdot \mathrm{M}$ & 771 & 838 & 868 & 1043 & 1143 \\
\hline 2 & 907 & 960 & 971 & 1013 & 1085 & 1143 & 1215 \\
\hline 3 & 1121 & 1140 & 1157 & 1232 & 1252 & 1268 & 1351 \\
\hline 4 & 1240 & 1240 & 1257 & 1293 & 1337 & 1377 & 1460 \\
\hline 5 & 1360 & 1351 & 1363 & 1368 & 1421 & 1343 & 1607 \\
\hline 6 & 1410 & 1399 & 1404 & 1368 & 1377 & 1354 & 1571 \\
\hline 7 & 1527 & 1527 & 1521 & 1471 & 1488 & $N \cdot M$ & 1727 \\
\hline $8(3)$ & $\mathrm{N}, \mathrm{M}$ & 1566 & 1543 & $N \cdot M$ & $\mathrm{~N} . \mathrm{M}$ & N.M. & N.M. \\
\hline
\end{tabular}

(1) Refer to Figure 1 for position of temperature readings.

(2) Not measured.

(3) Experiment interrupted by lithium leak. 


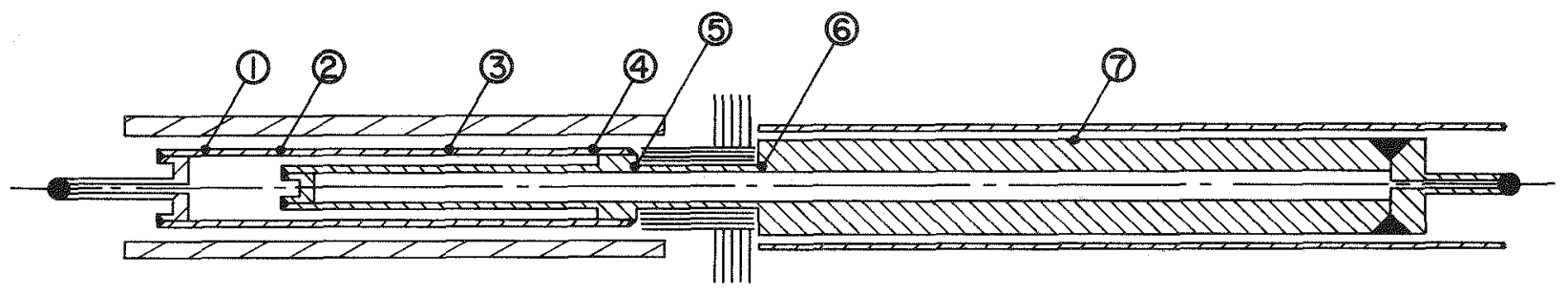

Annular Heat Pipe

Tubular Heat Pipe

FIGURE 1. OUT-OF-PILE MOCKUP TO EVALUATE PROPOSED ANNULAR HEAT PIPE

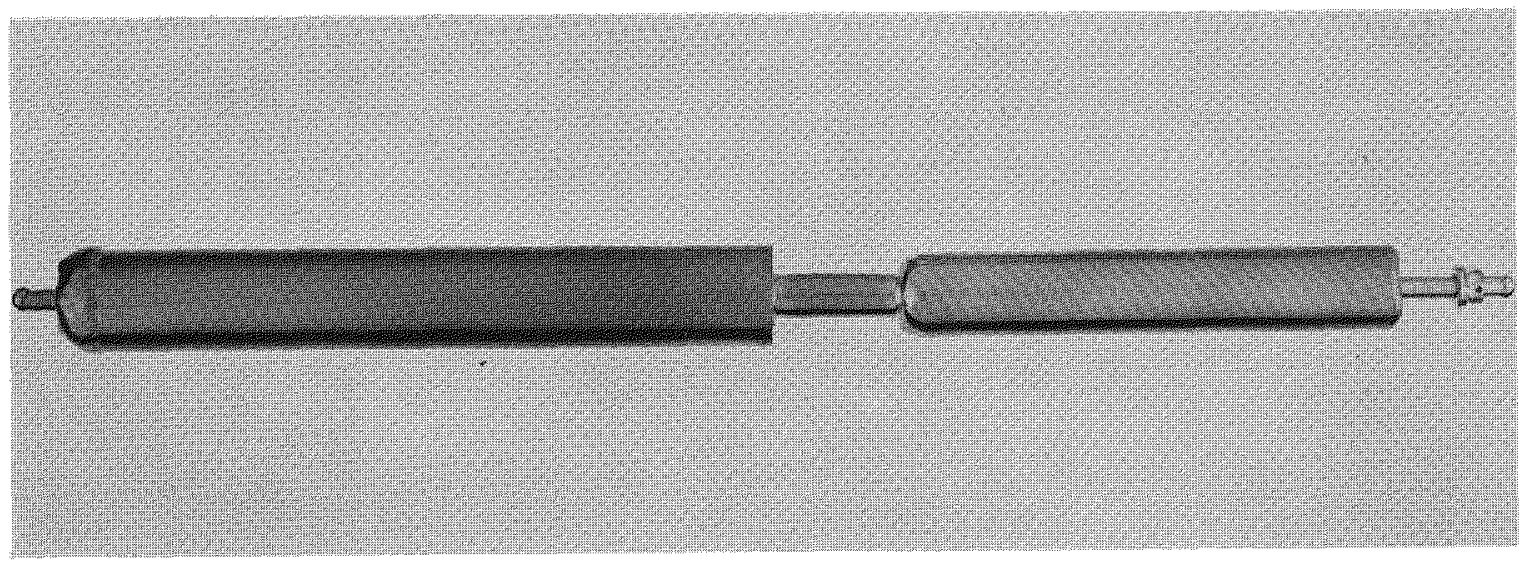

FIGURE 2. ASSEMBLED ANNULAR HEAT PIPE MOCKUP EXPERIMENT

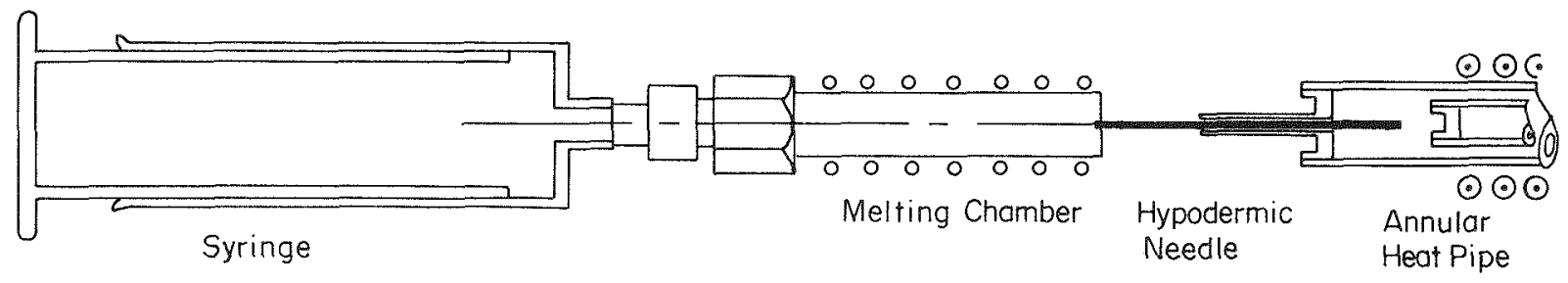

FTGURE 3. SCHEMATIC REPRESENTATION OF METHOD FOR LOADING LITHIUM IN ANNUT,AR HEAT PIPE 


\title{
X APPARATUS FOR MEASURING IN-PILE CREEP OF LMFBR CANDIDATE FUELS*
}

by

R. A. Robinson and S. J. Basham, Jr. $1 / 46 \%$ BATTELLE MEMORIAL INSTITUTE

Columbus Laboratories

\begin{abstract}
$\underline{\text { ABSTRACT }}$
This paper describes the design and operation of an apparatus to obtain data on the compressive creep of ceramic fuel materials during irradiation. The specimen $\left(\mathrm{UO}_{2}\right.$ or $\left.\mathrm{UO}_{2}-\mathrm{PuO}_{2}\right)$ is fabricated in the shape of an annular cylinder to reduce temperature gradients caused by fission heating. A compressive load is applied to the specimen through a bellows-sealed, gas-actuated piston. Specimen temperatures are maintained by a resistance-wire heater. Axial creep of the fuel is measured by transmission of the motion through bellows-sealed probes that are linked to a variable-area-orifice, pneumatic displacement transducer.

The equipment is designed to operate with fuel temperatures in the range 900 to $1300 \mathrm{C}$, stress levels of 3,000 to $12,000 \mathrm{psi}$, and fission rates ranging up to $2 \times 10^{13}$ fissions/cc-sec. Data are presented on the in-pile and out-of-pile creep characteristics of $\mathrm{UO}_{2}$ specimens as measured in initial tests to check the operation of the equipment.
\end{abstract}

* Work performed under AEC Contract W-7405-eng-92. 


\section{INTRODUCTION}

Dimensional changes that occur in fuel elements during reactor operation are strongly dependent on the mechanical properties of both the fuel and the cladding. Properties of most common cladding materials, both in and out of radiation environments, are either known or are already being studied intensively. However, comparatively little is known about the mechanical behavior of candidate mixed-oxide fuel materials in the anticipated radiation, temperature, and stress environments of a liquid-metal-cooled fast-breeder power reactor (LMFBR).

Battelle-Columbus is conducting a programk* with the objective of measuring the in-pile compressive creep behavior of ceramic fuels as affected by fission rate, stress, temperature, and burnup. (1) The development of an apparatus to accomplish this task is described in this paper.

\section{DESIGN CRITERIA}

Requirements which had a major influence on the design of the apparatus include the following:

$$
\begin{aligned}
\text { Temperature - } & \text { Specimen surface temperatures up to } 1300 \mathrm{C} \text { are } \\
& \text { desired. In any creep experiment, it is essential } \\
& \text { that the specimen temperature be maintained }
\end{aligned}
$$

\footnotetext{
* Work performed under AEC Contract W-7405-eng-92.

** Monitored by Argonne National Laboratory and carried out under Contract W-7405-eng-92 for the AEC Division of Reactor Development and Technology.
} 
uniform throughout the volume and constant with time. This necessitated the use of (1) heaters capable of keeping the specimen at the operating temperature, even during reactor shutdowns and scrams, (2) thin-section specimens to minimize transverse temperature gradients, and (3) irradiation facilities where the neutron $f l u x$ is constant and uniform over the specimen length to minimize axial temperature gradients.

Stress - Compressive loading was to be used, with controlled constant stress at any level from zero to 13,000 psi.

Fission Rate - Initial creep experiments at low fission rates (up to $2 \times 10^{13}$ fissions/cc-sec) were to be run in the BRR. Subsequently, creep tests in high-flux environments ( $10^{14}$ or more fissions/cc-sec) are to be run in high-flux facilities like the ETR, ATR, or EBR-II. (Design fission rates for full-scale LMFBR's are about $5 \times 10^{13}$ fissions/cc-sec.)

Burnup - For LMFBR designs, burnups of about 100,000 MWD/tonne are expected. This value will be the target for creep specimens in the high-flux facilities. However, the initial experiments in the BRR were to be run to burnups of only about 10 percent of this, which could be obtained in a reasonable period (three 3-week cycles).

Specimen

Configuration - Fabrication, heat transfer, and loading considerations dictate the use of specimens in the form of right cylinders 0.25 -in. in diameter $\times 0.75$-in. in length, with $0.15-i n$. diameter holes through centers (0.05-in.-thick wa11).

Specimen Material - $\mathrm{UO}_{2}$ ( 24.48 percent enrichment) was to be used for initial experiments during the apparatus-development stage. Subsequently, a fuel of greater practical interest, $\mathrm{UO}_{2}-22 \mathrm{w} / \mathrm{o} \mathrm{PuO}_{2}$ is to be tested.

\section{CAPSULE DESIGN}

\section{General Description}

Based on the above criteria and the results of tests on laboratory mockups, the design shown schematically in Figure 1 was developed. Because of space limitations and the necessity for fissionproduct isolation in a fueled in-pile capsule, the techniques employed 


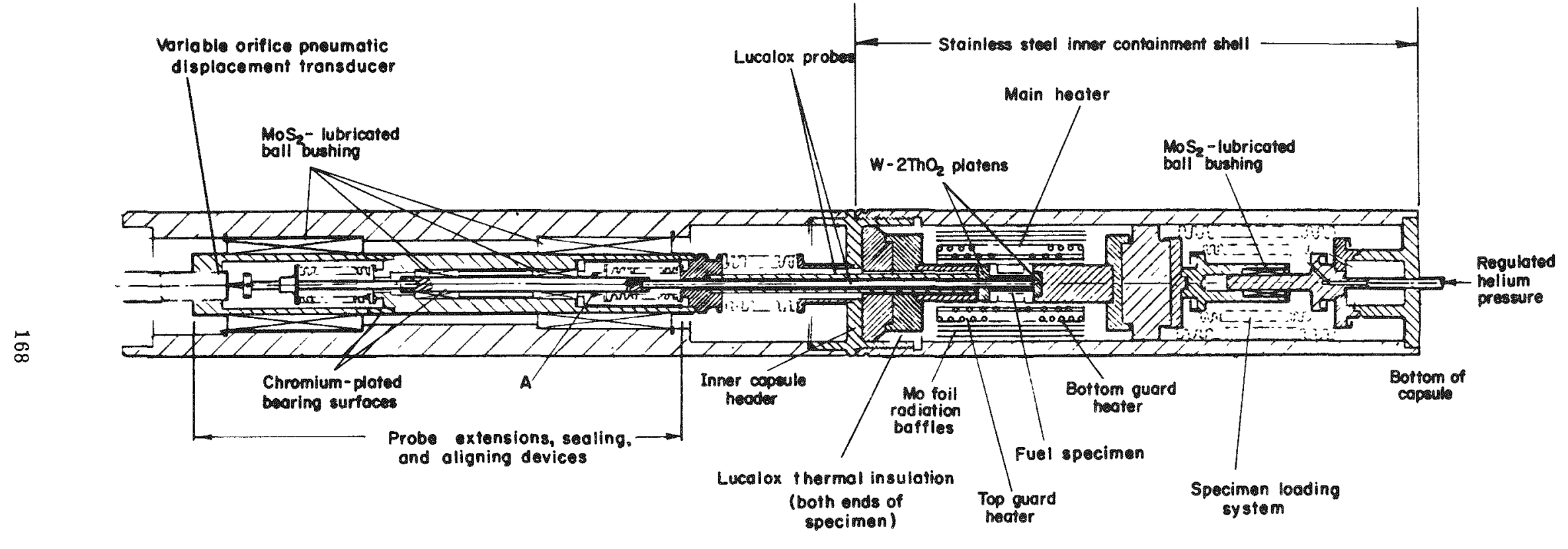

FIGURE 1. SCHEMATIC OF IN-PILE CREEP APPARATUS 
in this device deviate somewhat from standard creep-measurement methods. A double-bellows-sealed, helium-gas-loaded piston applies controlled static compressive loads to the fuel specimen. The desired specimen temperatures are maintained with the heater unit and can be adjusted by varying the power input to the heaters. Axial dimensional changes of the fuel are measured by the relative movement of rods foupling the specimen ends to a commercial pneumatic transducer. $(2,3)$

\section{Heater Unit}

The specimen heaters consist of two concentric alumina tubes that surround the specimen, with stranded tungsten- $25 \mathrm{w} / \mathrm{o}$ rhenium wire wrapped in fully recessed grooves on the OD of each tube. The tubes are about three times as long as the specimen. The inner tube supports the primary specimen heater and the outer tube holds the top and bottom guard heaters. The three heaters are grounded to the capsule wa11, and power to each is controlled independently.

\section{Thermocouples}

Three tungsten-rhenium thermocouples (tungsten- $26 \mathrm{w} / \mathrm{o}$ rhenium versus tungsten-5 w/o rhenium) are attached to the OD of the fuel specimen to monitor the midplane surface temperature and the surface-temperature at each end. A thermocouple is made by stranding together eight 3-mil wires to form each leg of the junction. The final diameter of each stranded $1 \mathrm{eg}$ is about $10 \mathrm{mils}$. It has been our experience that such stranded wires have considerably more flexibility and higher integrity than solid wires. A thermocouple junction is formed by simply tying the two wires in a square knot. This knot is held firmly against the specimen surface by wrapping the ends beyond the knot around the fuel specimen and tying them tightly on the opposite side.

Twenty-four thermocouples are placed at strategic locations within the assembly to gain data needed to interpret the results of the irradiation. The thermocouples and heater sheaths (not shown in Figure 1) are braze sealed at their point of emergence from the inner capsule header (center of drawing) and are located in grooves on the exterior of the upper portion of the inner shell. 


\section{Strain Measurement}

Changes in length of the fuel specimen are detected by a transducer that converts linear motion into changes in pressure drop of a gas flowing through a variable-area orifice. The axial motion of the top surface of the fuel specimen relative to that of the bottom surface is transmitted by two probes which mechanically link these reference planes to a needle and an orifice, respectively. As the bottom surface of the specimen moves toward the top surface (i.e., as the compressed specimen gets shorter), the needle moves farther into the orifice hole and causes the pressure drop across the orifice to increase.

A plot of the pressure drop across the orifice versus the relative position of the needle in the orifice hole (Figure 2) provides a calibration curve for translating measured pressure drops into strains. Although the manufacturer 1 ists $120 \mathrm{mils}$ as the operating range, the useful creep-sensing range of this particular transducer is limited to about $90 \mathrm{mils}$ because of the insensitivity of the output to linear motion at either end of the travel. The manufacturer's quoted resolution of $\pm 0.1 \mathrm{mil}$ has been verified for the transducer at various temperatures, specified supply pressure, and atmospheric exhaust pressure.

Because of the effects of gamma heating $(0.3 \mathrm{watt} / \mathrm{gm})$ in-pile, the transducer housing will operate at a temperature somewhat above the ambient pool-water temperature. To compensate for the uncertainty in operating temperature, the motion transducer was calibrated over a temperature range that includes the value expected in-pile.

Bellows joints are installed in the transducer gas lines immediately above the top of the inner capsule to permit unrestrained axial movement of the transducer housing.

\section{Containment of Gaseous Fission Products}

During an in-pile creep test of an unclad fuel specimen, gaseous fission products will be released from the specimen surfaces to the surrounding space inside the capsule. For safety, the system design provides multiple barriers between this gas space and the exterior of the assembly. Some of these containment features are as follows (refer to Figure 1): 


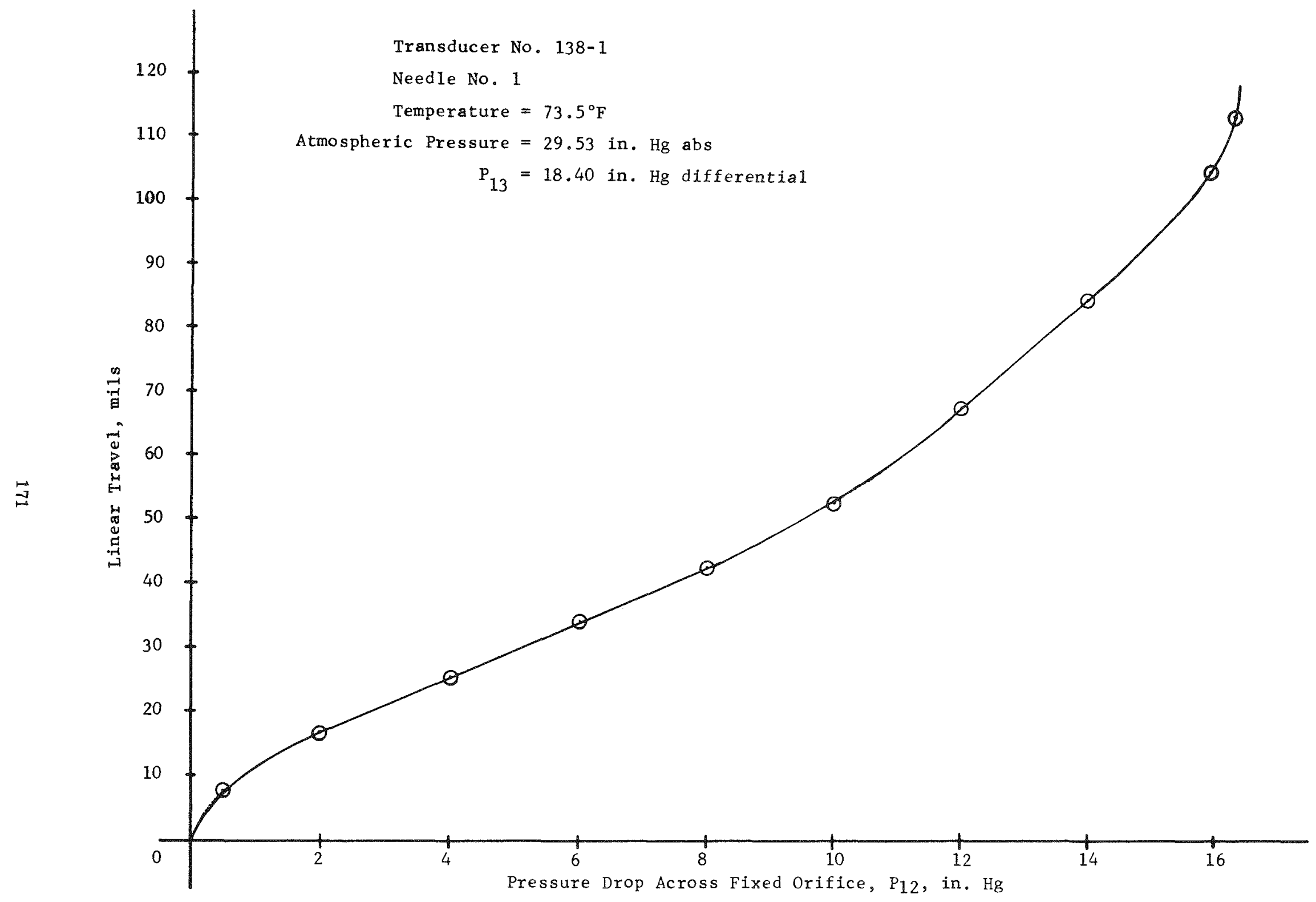

FIGURE 2. CALIBRATION CURVE FOR LINEAR MOTION TRANSDUCER 
(1) At gas-pressure loading end (bottom)

Primary containment - outer bellows surrounding gas-loading piston.

Secondary containment - inner bellows surrounding gas-loading piston.

Tertiary containment - helium supply system.

(Note that this triple containment allows the loading-gas system to be located in an unshielded environment to simplify control, maintenance, and data recording.)

(2) At transducer end (top)

Primary containment - bellows surrounding transducer probes and welded joint between probe and bellows flange (see $A$ in Figure 1).

Secondary containment - inner transducer-bearing sleeve and topmost bellows.

Tertiary containment - transducer nitrogen supply system.

(Previous note also applies to transducer-gas system.)

(3) Outer she11

The 2-1/2-in.-OD inner shell (Figure 1 ) is enclosed in a 2-3/4-in.-OD, 1/8-in.-wall outer shell (not shown) that contacts the reactor cooling water. This shell provides secondary containment in case of leaks in the inner shell. It extends about 2 ft above the inner shell.

(4) Thermocouple and heater leads

The use of unsealed thermocouples and heater elements necessarily allows diffusion of fission products up through the insulation in the leads. The fission-product gases in these regions are contained by providing one or more potted junctions in the lead-out tube at a distance of about $6 \mathrm{ft}$ above the core (to eliminate radiation damage to the potting compounds) for the BRR irradiation experiments.

\section{Calculation of Temperature Pattern}

Concurrent with the mechanical design of the creep capsule, analyses were made of the fuel heat generation and heater power requirements to establish the temperature distribution in the fuel and the various capsule components. 
$\operatorname{ANISN}^{(4)}$ (a one-dimensional, discrete-ordinate transport code with anisotropic scattering) was used to calculate neutron fission distributions and fuel specimen heat generations for several types of fuels, enrichments, and flux levels in the BRR. The results were used primarily as inputs in the heat-transfer analysis. Also, they indicated that the power and radial fission distribution could be matched between the 24.48 percent enriched $\mathrm{UO}_{2}$ and the $\mathrm{UO}_{2}-22$ percent $\mathrm{PuO}_{2}$ to be used.

From the computed heat-generation rates, the temperature in the specimen region of the capsule was calculated. TRAHT-3, an explicit, two-dimensional transient heat-transfer code, adapted from NURLOC(5) for the solution of steady-state problems, was used. With the therma1insulation materials and capsule geometry described, the maximum temperature variation along the length of the specimen was calculated to be about $100 \mathrm{C}$ for operating levels in the 900-1300 C range with fission rates of the order of $10^{12}$ fissions/cc-sec.

\section{Heater-Thermocoup1e Mockup Experiment}

A 1aboratory mockup was constructed to test heater and thermocouple compatibility and performance. This test was designed to generate sufficient information to permit a choice between platinum-platinum-rhodium and tungsten-rhenium thermocouples, check the performance of a tantalum-wire heater (the initial choice for the resistance element of the heater) on an alumina shell at the temperature of interest, and check the heat removal characteristics of the selected geometry. The test was terminated prematurely when the heaters failed. Severe carbon, oxygen, and nitrogen contamination found in the heater wire, alumina insulation pieces, radiation baffles, and other parts of the heated zone suggested that the failure was caused by a gas leak. The main heater had operated continuously for 24 days at temperatures between 900 and $1300 \mathrm{C}$ when the test was terminated.

The results showed, however, that bare tungsten-rhenium thermocouples are more compatible with unclad $\mathrm{UO}_{2}$ than are platinum-platinum-rhodium thermocouples. Thus, all thermocouples in the "hot" zone of the capsule were specified to be tungsten-rhenium (tungsten-26 w/o rhenium versus tungsten $-5 \mathrm{w} / 0$ rhenium). Since there was some concern about the compatibility of tantalum with $\mathrm{UO}_{2}-\mathrm{PuO}_{2}$, a new heater was constructed of stranded tungsten-25 w/o rhenium wire. Also, all carbon-bearing materials were eliminated. 
The mockup was reassembled and operated at a specimen temperature of $1300 \mathrm{C}$. The axial temperature gradient was measured to be about $30 \mathrm{C}$ from the centerline of the specimen to one end, which was considered acceptable. The alumina insulation restricting the axial heat flow from the test section was effective in reducing hot-zone temperatures to an ambient level in very short distances. The results of the mock-up test indicated that the selected design would be satisfactory.

\section{Laboratory Prototype Experiment}

A laboratory prototype creep mechanism was then assembled to check the thermal and mechanical design of the proposed in-pile apparatus. The assembly was made, insofar as possible, identical to that intended for the first in-pile creep capsule. Figure 3 shows the principal components of the creep mechanism. Information gained from this experiment provided considerable insight into the design, assembly, operation, and related problem areas of later in-pile creep capsules.

In addition to providing increased confidence in the design of the in-pile system, specific items were defined by the operation of the laboratory prototype experiment:

(1) An optimum assembly procedure was developed.

(2) The initial differential thermal expansion of the creep sensing probes was found to be smal1 enough to preclude the need for a repositioning device for the transducer housing.

(3) It is very difficult to monitor pressures in the static helium environment surrounding the specimen in the in-pile mechanism due to the presence of fissionproduct gases. Therefore, pressure changes in this region as a function of specimen temperature were made in the prototype. This provided additional design information needed to more accurately determine loads on the specimen in the in-pile apparatus.

(4) Examination of the load-transmitting surfaces near the specimen showed no evidence of yielding or cracking from local stress concentrations. 


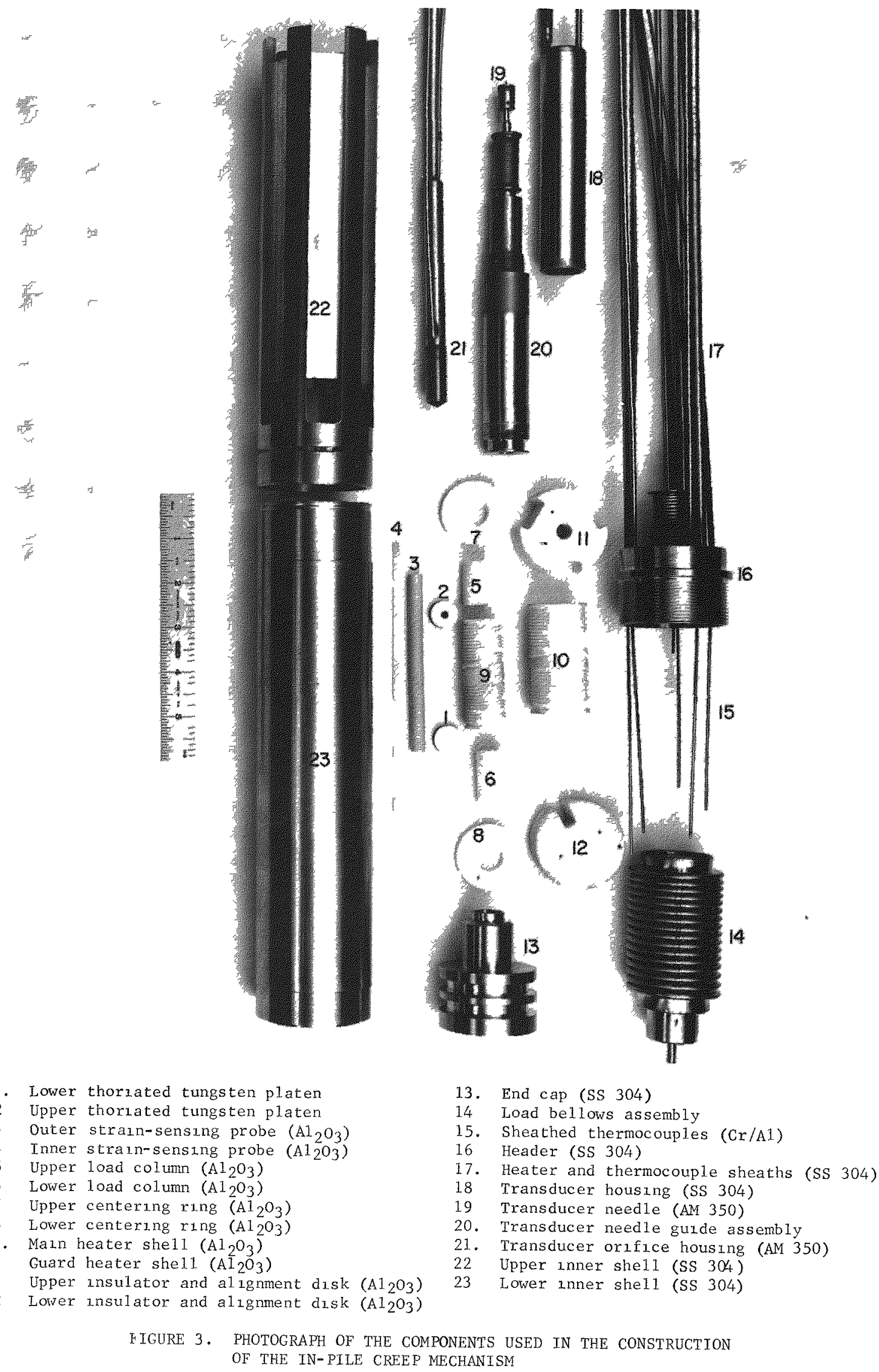


(5) Since the heat rejection mode for the laboratory mechanism was made identical to that of the capsule in-pile at the $B R R$, the temperatures measured in and around the heated zone gave a good indication of the operating temperatures of such key items as the load bellows, isolation bellows, alignment bearings, etc. The temperatures of the stainless steel components that are located above and below the heated zone were in the range of 150 to $350 \mathrm{~F}$. Thus, the alumina insulators and molybdenum radiation baffles were able to adequately insulate the loading and creep-sensing systems from the hot zone that surrounds the specimen.

(6) The power requirements for a given specimen temperature and the temperature distribution in the heated zone were similar to those obtained in the heater mockup test.

A creep test was successfully performed in the prototype on a $\mathrm{UO}_{2}$ specimen at a temperature of $1300 \mathrm{C}$ and a stress level of 3500 psi. During the startup of the test, temperature distributions in the device were also recorded for specimen temperature levels of 900 and $1100 \mathrm{C}$.

The variable-orifice transducer system indicated a movement of $0.3 \mathrm{mil}$ during the $1-\mathrm{hr}$ period that the load was being applied to the specimen. A total deformation of $4.8 \pm 0.2 \mathrm{mils}$ was indicated over a 147-hr period while the specimen was fully loaded. A posttest measurement of the specimen length showed that the actual permanent deformation was $4.6 \pm 0.1 \mathrm{mi} 1 \mathrm{~s}$. Thus, it was concluded that the transducer measurement system had performed with the required accuracy.

A posttest examination of the heater windings and lead connections was conducted to determine the integrity of these key design areas. The stranded tungsten-rhenium heater elements were a dull gray color on the surfaces exposed to the static helium atmosphere. Although this gray color indicates that the windings were slightly affected by the atmosphere, the observed changes in power requirements were nominal and were not detrimental to the performance of the heater. The lead connections to the heaters were found to be as strong as in the pretest condition.

The loading system and the temperature-control system performed satisfactorily. A posttest examination of the loaded members in the heated zone indicated that there was no problem of misalignment or local stress concentration. 
After the successful prototype experiments had verified the ability of the apparatus to perform as required, Creep Capsule I was constructed. The following procedures vere evolved for a complete creep test in the BRR.

1. The creep capsule is completely assembled, leak checked, suspended in the pool at some distance from the core, and a11 auxiliary support systems connected (power, instrumentation, controls, pressurizing gas, transducer gas, safety devices).

2. Power is supplied to the heaters to bring the fuel specimen up to the desired test temperature.

3. A neutron radiograph is taken of the hot zone to check component alignment and specimen integrity, and to provide a pretest record of specimen length for comparison with measurements from radiographs taken during the progress of subsequent creep testing.

4. An actual creep test is run on the specimen (still in the pool but out of the neutron flux) by applying the desired load, reading the transducer differential pressures as a function of time, and using the transducer calibration curve to convert these readings to specimen strains. The results of this test provide a basis for comparing in-capsule with out-of-pile creep rates.

5. Final1y, the assembly is moved into the neutron flux field, and the in-pile creep tests are performed until either the specimen fails or the useful transducer range is covered.

After Creep Capsule I was completely assembled and ready for service, it was found that five of the six tungsten-rhenium thermocouples on or near the specimen surface were not functioning properly. Study indicated that the difficulty was most likely caused by shorting of the wires on the metal sheath surrounding the insulation. This problem was eliminated in Creep Capsule II by providing more positive insulation at the point where the bare wires emerge from the sheath.

Both Creep Capsules I and II were operated in accordance with the procedures outlined above. Specimen temperatures in Creep Capsule I were inferred from a combination of readings of the remaining tungstenrhenium thermocouple (in a region about $200 \mathrm{C}$ cooler than the specimen) and heater power-specimen temperature correlations obtained from Creep Capsule II.

Figure 4 is an enlargement of a neutron radiograph of the specimen region of Creep Capsule II. The tubular $\mathrm{UO}_{2}$ fuel specimen is clearly visible, as are the tungsten-rhenium heater windings and the thoriated tungsten platens at the ends of the specimen. 
Figures 5, 6, 7, and 8 are deformation--time curves for $\mathrm{UO}_{2}$ specimens $G 2$ and $G 5$ that were tested in Creep Capsules I and II, respectively. Figure 5 is a plot of the data obtained in the first creep test performed in Capsule $I$. Part of the scatter in the data in the initial region of primary creep can be explained by the fact that the specimen temperature could not be controlled closely owing to a heater controller malfunction. Figure 6 shows the data obtained in a later in-pile creep test performed at a fission rate of $1.7 \times 10^{12}$ fissions/cc-sec. The capability to follow specimen deformation very closely (well within 0.1 mil) with the pneumatic-transducer system is evident. Figures 7 and 8 show data typical of that obtained in creep tests performed in Creep Capsule II. The indicated stepwise change in specimen length of about 2 mils deformation shown in Figure 8 after the reactor scram is not readily explainable. Other creep tests that were interrupted by reactor scrams did not show any apparent discontinuity in the rate of deformation.

\section{ACKNOWLEDGMENTS}

Significant contributions to the capsule design described in this paper were made by W. A. Carbiener, N. E. Miller, and W. J. Zielenbach of the Nuclear Engineering and Analysis Division at Battelle-Columbus.

\section{References}

(1) Keller, D. I., et.al., "Progress Relating to Civilian Applications During July through September, 1968", Section C, Report No. BMI- 1851.

(2) Fidleris, V., et.al., "In-Reactor Creep Machines", AECL, Presented at the International Symposium on In-Pile Irradiation Equipment and Techniques at A.E.R.E. Harwe11, England, May 10-12, 1966.

(3) McQuilkin, F. R., et.al., "A Dilatometer for Measuring Axial Displacement of Fuel During Irradiation", Presented at the International Symposium on Developments in Irradiation Capsule Technology, Conf-660511, Pleasanton, California, May 3-5, 1966.

(4) Engle, W. W., Jr., "A Users Manual for ANISN", Oak Ridge National Laboratory Report K-1693 (March 30, 1967).

(5) Walters, C. T., and Genco, J. M., "NURLOC-1.0 A Digital Computer Program for Thermal Analysis of a Nuclear-Reactor Loss-of-Coolant Accident", BMI-1807 (July 6, 1967). 


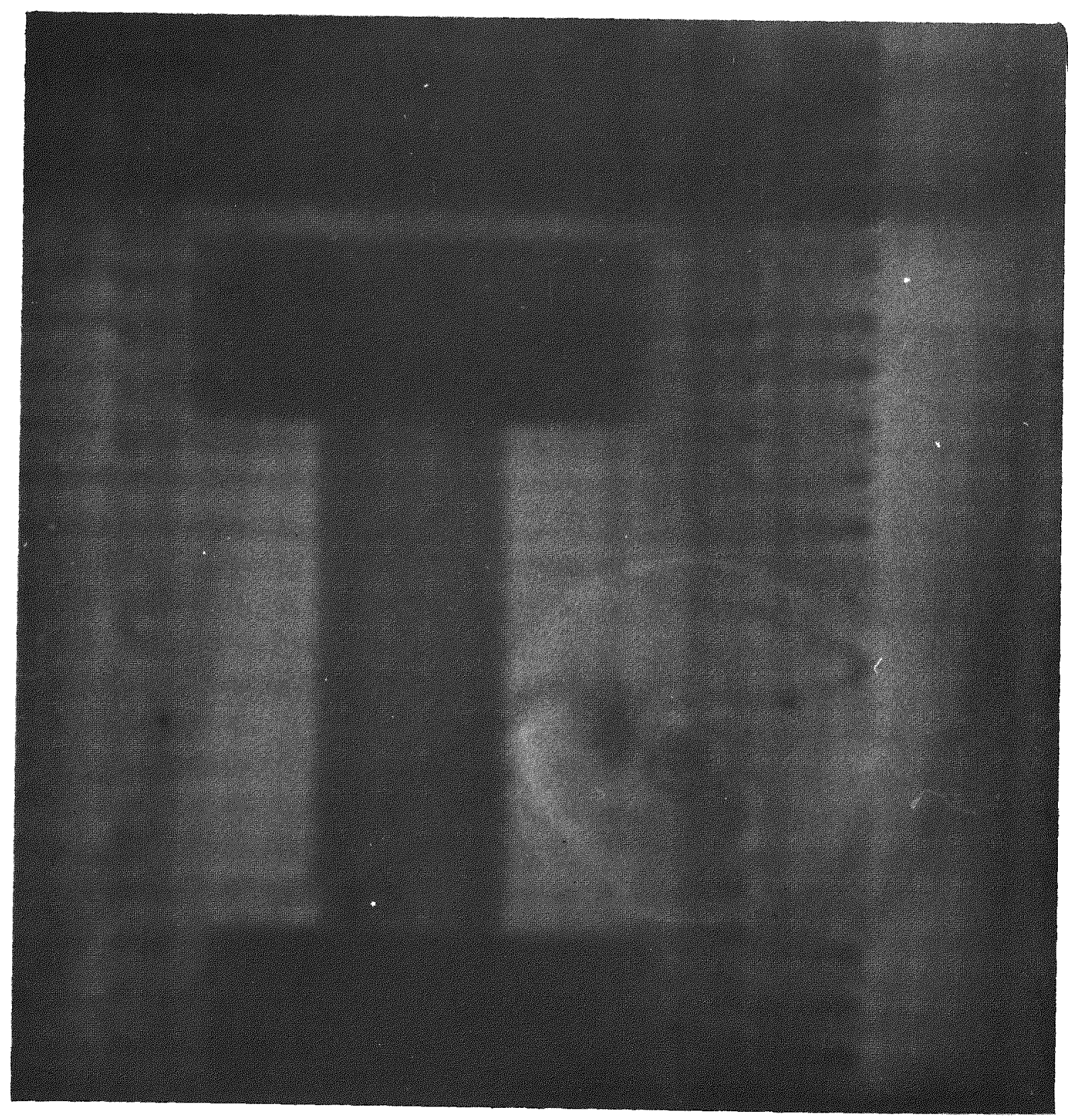

FIGURE 4. A NEUTRON RADIOGRAPH OF CREEP CAPSULE II TAREN AT A SPECIMEN STRESS OF 3500 PSI AND A TEMPERATURE OF $1300 \mathrm{C}$ 


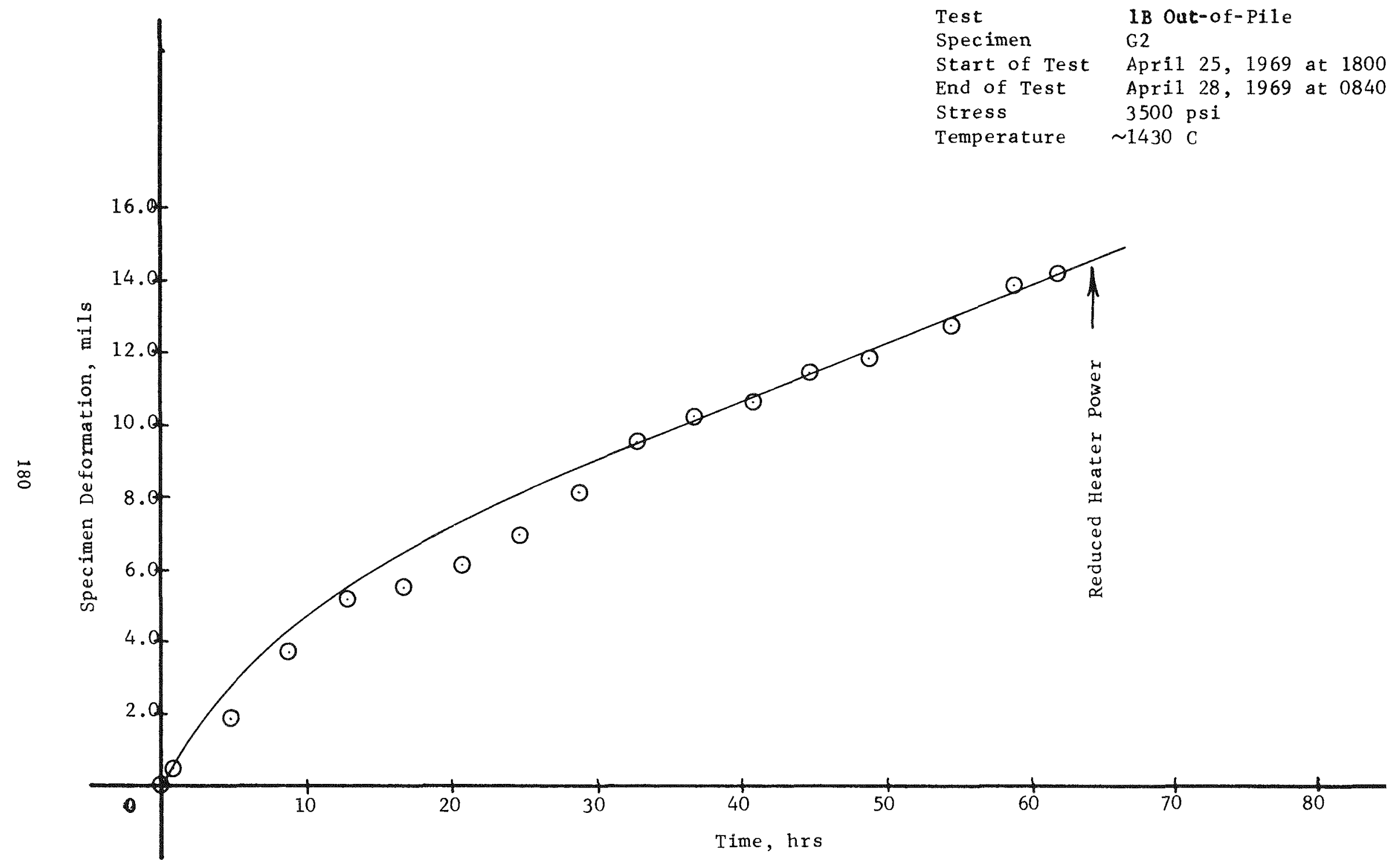

FIGURE 5. A PLOT OF SPECIMEN DEFORMATION VERSUS TIME OUT-OF-PILE AT A COMPRESSIVE STRESS OF 3500 PSI AND AN AVERAGE TEMPERATURE OF $1430 \mathrm{C}$. STEADY STATE STRAIN RATE IS $2.2 \times 10^{-4} \mathrm{HR}^{-1}$ 


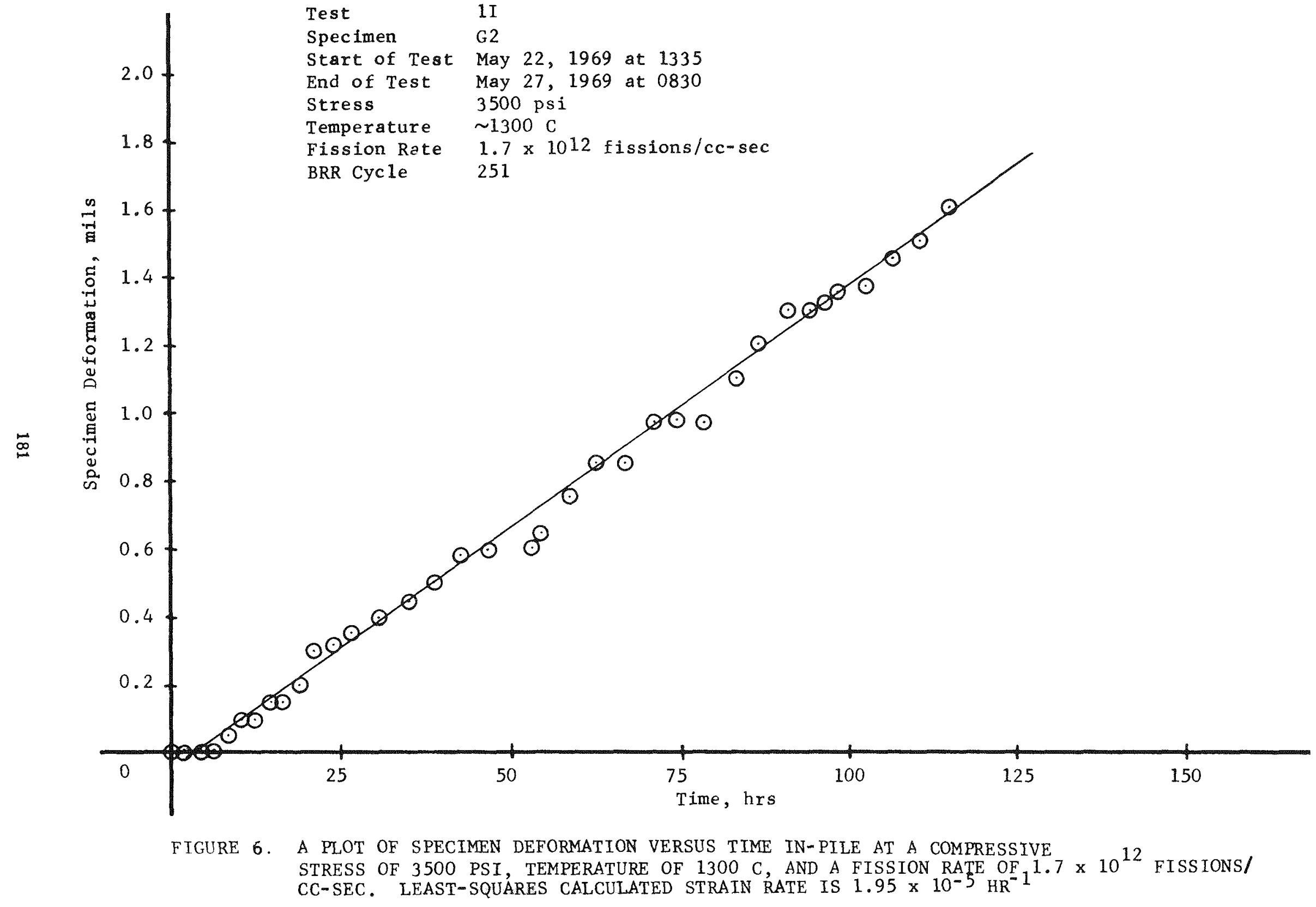




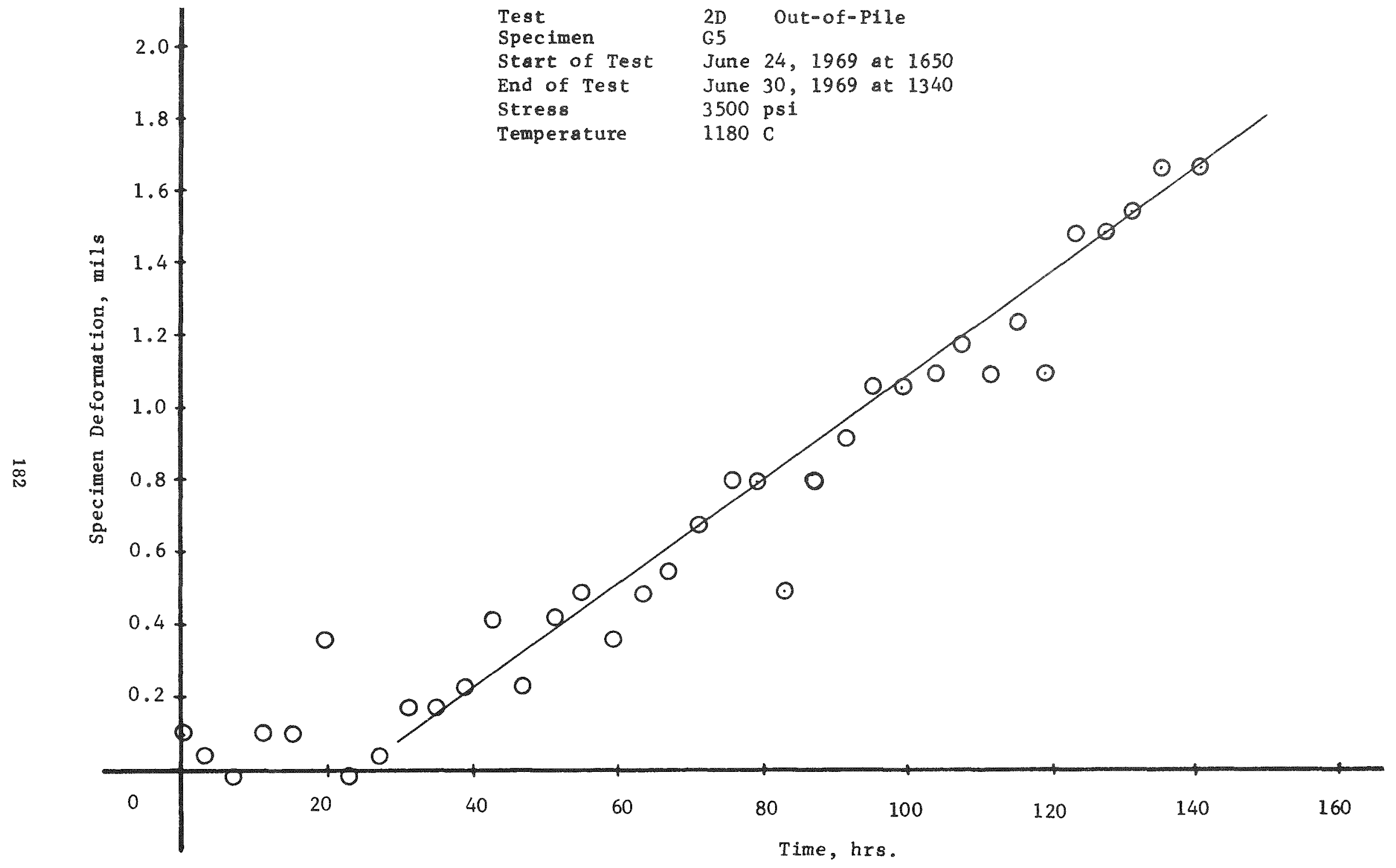

FIGURE 7. A PLOT OF SPECIMEN DEFORMATION VERSUS TIME OUT-OF-PILE AT A COMPRESSIVE STRESS OF 3500 PSI AND AN AVERAGE TEMPERATURE OF 1180 C. LEAST-SQUARES CALCULATED 


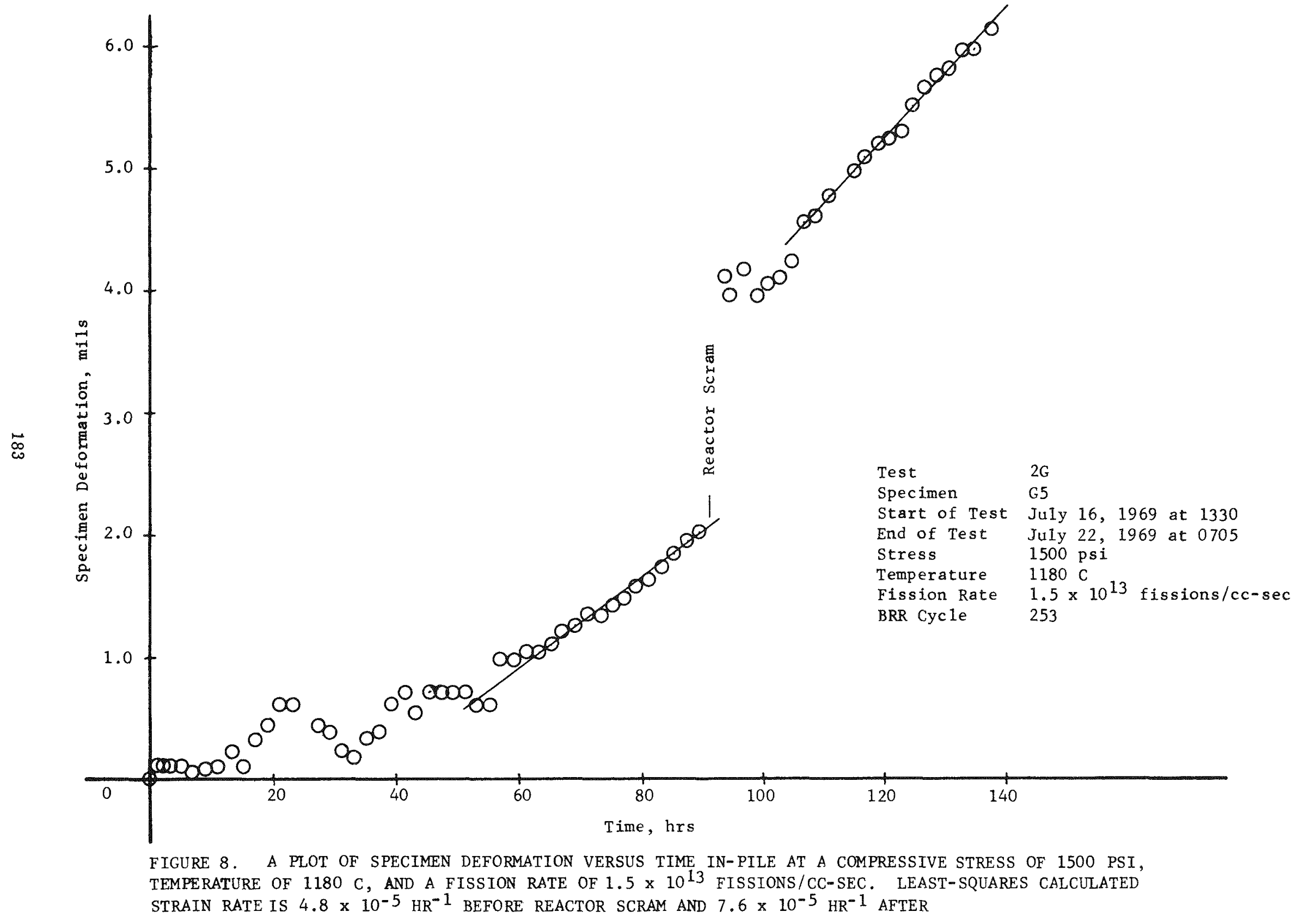


DEVELOPNENT AND FEASIBIIITY TESTS OF ISOTHERMAI IRRADIATORS ${ }^{*}$

\author{
C. V. Weaver, A. J. Patrick, and W. A. Ranken \\ Los Alamos Scientific Laboratory \\ $180 \%$ Lniversity of California. \\ Los Alamos, New Mexico 87544
}

\title{
INTRODUCTION
}

In some nuclear irradiation facilities, the temperatures in experimental capsules are difficult, if not impossible, to measure directly or to control. Self-contained test assemblies have therefore been developed. in which temperatures may be preset and maintained within fairly close limits for widely varying reactor power levels, often without prior accurate knowledge of the $\gamma$-ray heat-generation rates in the testing region.

An experimental device was used to conduct parametric tests to prove the design concept. The principles were fully verified by an instrumented experiment in the Omega West Reactor. Based on these successful results, a self regulated-constant temperature irradiation experiment with ceramic specimens is presently underway in the EBR-II reactor.

\section{PRINCIPLE OF OPERATION AND DESIGN OF ISOTHERMAL IRRADIATOR}

The principle of operation of an "isothermal regulator" (I) may be explained by referring to an experimental assembly shown schematically in Fig. 1. The irradiator contains a heat pipe (2) which, in addition to the working fluid, is filled with an inert (noncondensable) gas. Heat generated by $\gamma-f l u x$ in the evaporator section of the heat pipe is transported by an evaporation-vapor condensation cycle in an axial direction to the heatdump region. The vapor-transport mechanism pumps the inert buffer gas into the heat-dump section where an interface forms between the working fluid and the gas. This interface forms just far enough inside the heat-dump section to provide the thermal-conductance path to the reactor coolant that is required to remove the heat delivered by vapor condensation to the heat pipe wall adjacent to the interface. The radial thermal conductance of the heat-dump section is made large (compared to that of the evaporator section) so that the irradiator will loose most of its heat through a narrow zone just at the entrance to the heat dump.

\footnotetext{
* Work performed under the auspices of the U. S. Atomic Energy Commission.
} 
If the $y$-heating rate is doubled, the temperature and therefore the pressure of the working fluid vapor increases. Consequently, the gasvapor interface moves farther into the heat-dump section until the thermal conductance is nearly doubled. If the thermal conductance in the heat-dump region can vary rapidly enough with interface displacement, the buffer gas may be compressed by no more than a few percent until a new equilibrium is established. A small increase in the temperature of the working fluid will cause a large increase in its vapor pressure. For example, the vapor pressure of sodium at $800^{\circ} \mathrm{C}$ varies as the tenth power of the temperature. Therefore, doubling the input heat rate may finally require an increase in the temperature of the vapor (and of the experimental section of the assembly) of only a few degrees. This temperatureregulating feature can be improved either by increasing the relative volume of the inert gas or by making the thermal conductance of the heat dump section vary more rapidly with the displacement of the interface.

The temperature about which regulation occurs is determined by the amount of buffer gas initially introduced into the heat pipe. The fill pressure, $P_{1}$, of the gas is determined by the familiar relationship:

where

$$
\frac{P_{1} V_{1}}{T_{1}}=\frac{P_{2} V_{2}}{T_{2}}
$$

$\mathrm{P}_{1}=$ the pressure of the inert gas at ambient temperature.

$V_{I}=$ the total. volume of the space inside the heat pipe after the working fluid has been introduced.

$\mathrm{T}_{1}=$ ambient temperature.

$\mathrm{P}_{2}=$ the pressure of the buffer gas at equilibrium which must be equal to the vapor pressure of the working fluid at the design temperature of the irradiator.

$\mathrm{V}_{2}=$ the volume occupied by the buffer gas at equilibrium. Because the gas-vapor interface forms near the entrance to the heatdump section, for practical purposes $V_{2}$ is just the inside volume of the heat pipe in this section.

$T_{2}=$ the temperature of the buffer gas at equilibrium. (Prior determination of $\mathrm{T}_{2}$ for design purposes depends upon the temperature of the reactor coolant and the melting point of the working fluid. Necessary practical considerations for establishing $T_{2}$ will be discussed below.)

\section{OPERATIONAL TESTS OF ISOTHERMAL IRRADIATORS}

\section{Bench Tests}

Parametric bench tests of the experimental isothermal ixradiator shown in Fig. I were made to verify the principle of the device and to define design and operational criteria. The irradiator itself was a stainless-steel heat pipe with sodium as the working fluid. It was filled initially with 40 Torr of neon. When the gas-vapor interface is located as shown, and the temperature of the buffer gas is $250^{\circ} \mathrm{C}$, its pressure 
should be 198 Torr, which corresponds to the vapor pressure of sodium at $750^{\circ} \mathrm{C}$ - - the design temperature of the experiment. Gamma heating was simulated by induction-heating the entire heat pipe. Heat was removed. from the heat-dump section through a helium-filled gap to a water calorimeter. Because the calorimeter water temperature was $25^{\circ} \mathrm{C}$ as compared to $96{ }^{\circ} \mathrm{C}$, the melting point of sodium, the thermal conductance of the gas gap had to be adjusted so that the temperature arop ( $\Delta T$ ) across the heat-dump section was large enough to keep the sodium molten. The thermal conductance of the gap was varied by controlling the pressure of the helium.

Figure 2 shows the temperature of the test section of the experimental irradiator at various heat input rates and with the helium in the gas gap at different pressures. With pressures in the gas gap of 3.0 Torr or above, i.e., when the overall thermal conductance of the heat-dump section was relatively high, the irradiator began regulating at an input power level of $\sim 175 \mathrm{~W}$ and at a temperature of $\sim 770^{\circ} \mathrm{C}$. This was within $3 \%$ of the design temperature of $750^{\circ} \mathrm{C}$. Below $175 \mathrm{~W}$, the input heat rate was not high enough, neither to establish temperatures in the desired range nor to provide sufficient axial heat flow for temperature regulation. Above $175 \mathrm{~W}$ up to $\sim 300 \mathrm{NW}$, however, and with the gas-vapor interface well established in the heat-dump region, the temperature of the test section increased only $3 \%$ for an increase of $100 \%$ in the heat input rate. (An exception for input power levels $>300 \mathrm{~W}$ is discussed below.)

Reducing the helium pressure in the gas gap to 0.2 Torr established a large $\Delta T$ across the heat-dump section. Temperatures in the test section of the irradiator reached $900^{\circ} \mathrm{C}$ (vs a design temperature of $750^{\circ} \mathrm{C}$ ) with an input power level of only $100 \mathrm{~W}$. There was no evidence of temperature regulation. Figure 3 shows the axial temperature distribution in the experimental irradiator under these conditions. The temperature of the buffer gas (estimated from the temperature of the surrounding heat-pipe walls) was $\sim 500^{\circ} \mathrm{C}$ compared to the design value of $250^{\circ} \mathrm{C}$. Consequently, at equilibrium, the buffer gas occupied a larger volume and was at a higher pressure than intended. The gas-vapor interface thus did not form at the intended location. Instead of forming in the heat-dump region where a small displacement would have corresponded to a large change in the thermal-conductance path to the coolant, it formed in the smallerdiameter evaporator section of the heat pipe where the opposite effect prevails. The higher pressure of the buffer gas caused the heat-pipe temperatures to exceed design values. (The lower temperature at the extreme end of the evaporator section of the heat pipe may have been caused by decreased electromagnetic coupling at this point, in addition to radia.tion and thermal-conductance end losses.)

A significant design consideration was illustrated by the above results. The experimental isothermal irradiator duplicated the condition where the temperature of the reactor coolant is lower than the melting point of the heat-pipe working fluid. Obviously, the $\Delta T$ across the heatdump section must be large enough to keep the fluid in a liquid state. This can be accomplished with a gas gap, as in this experiment. On the other hand, the equilibrium temperature of the buffer gas must never greatly exceed the temperature upon which the irradiator design is based. With these imposed conditions the rate at which heat will be transferred 
to the coolant must be determined with the best possible accuracy so that the heat dump section can be designed to keep the operating temperature of the buffer gas within safe limits.

When the temperature of the reactor coolant is higher than the melting point of the working fluid, the problem of freezing the working fluid does not exist. A high thermal conductance in the dump section, e.g., direct metallic coupling to the coolant, will keep the temperature of the buffer gas relatively constant about the temperature of the coolant over a wide range of input heat rates.

Further tests with the experimental isothermal irradiator at higher power levels revealed another problem which may be avoided by proper design of the irradiator. Figure 4 shows the axial temperature-distribution a.Iong the irradiator with a helium pressure in the gas gap of 30 Torr and for various heat-input rates up to $500 \mathrm{~W}$. Below $300 \mathrm{~W}$, the entire isothermal irradiator behaved quite normally. At higher power levels, the temperature of the heat-pipe section farthest from the heat-dump region increased at a much faster rate with increased input power than the temperature of the section nearest to the heat-dump zone which continued to increase at a rate of $\sim 3 \%$ for a $100 \%$ increase in power. The following, as yet unverified explanation of this effect, may be offered. The heatpipe wick used in the experimental isothermal irradiator consisted of a cylindrical stainless-steel structure with interconnected fine pores $(25 \mu)$ to provide the capillary forces required for circulation of the working fluid. (2) Between this mesh and the heat-pipe wall was an unrestricted annulus which provide a return path for the condensed sodium to the evaporator section. Referring to Fig. 4, it can be seen that by far the largest part of the heat in the sodium vapor is lost by condensation in a relatively small area at the entrance to the heat-dump section (between Thermocouples 7 and 10). The fine pore-small area section of the wick through which most of the condensed sodium must pass to reach the return annuIus represents a restriction in the condensation-vapor transport-evaporation cycle which limits the amount of sodium being circulated. At the higher input power levels, the returning sodium may be depleted by evaporation before an amount sufficient to effectively remove the generated heat can reach the more distant section of the heat pipe. Consequently, the pipe tends to overheat in these sodium-depleted areas. For high-capacity heat pipes, pore sizes of the order of $25 \mu$ may be necessary for fluid circulation. (3) For the isothermal irradiators, however, which are usually devices of relatively low capacity, current heat-pipe technology indicates that wicks with pores as large as $250 \mathrm{w}$ would be practicable and would offer considerably less resistance to the sodium transport cycle. Irradiators with wicks of larger pore size are being investigated to determine whether isothermal radiators can be made to operate at the higher power levels without overheating.

\section{Omega West Reactor Tests}

The bench tests demonstrated the feasibility of the isothermal irradiator assembly, designated OWR-II, was placed in the core of the Omega West Reactor to evaluate its performance in a nuclear environment. (The assembly also contained insulator test specimens.) The OWR-II irradiator, shown in Fig. 5, was very similar in size and detail to the experimental assembly 
used in the parametric studies. It also consisted of a stainless-steel heat pipe containing sodium as the working fluid. It was filled with 40 Torr of neon which should have resulted in a temperature of $\sim 750^{\circ} \mathrm{C}$.

OWR-II dumped its heat to the reactor coolant water at $45^{\circ} \mathrm{C}$. To keep the temperature inside the heat pipe safely above the melting point of the sodium working fluid, a neon-filled gas gap was located in the heat-dump section.

Gamma heating rates in the Omega West Reactor are relatively low. Therefore, all radial heat losses in the evaporator section of the assembly has to be minimized to provide sufficient axial heat flow for starting the heat pipe and for temperature regulation. This was accomplished by placing a dewar around the evaporator section of the pipe.

The OWR-II irradiator was provided with a multilayer wick of rolled stainless-steel screen in which the openings had a diameter of $\sim 100 \mu$. However, input heat rates were expected to be well below the level at which the fine-pore $(25-14)$ wick of the experimental assembly had caused overheating. No such malfunction was therefore anticipated.

The OWR-II irradiator, which remained in the reactor for a total of $4850 \mathrm{~h}$, initially did not operate as intended. Significant temperatures in the irradiator at various reactor power levels at different times during irradiation are shown in Fig. 6. With the heat-dump gas gap filled with neon, the temperature at the midpoint of the test section at full reactor power $(8 \mathrm{MN})$ was $860^{\circ} \mathrm{C}$ instead of the design temperature of $750^{\circ} \mathrm{C}$. There was no evidence of temperature regulation. It was found that the operating temperature of the buffer gas, which should have been $250^{\circ} \mathrm{C}$, was at least $100^{\circ} \mathrm{C}$ higher. OWR-II performance at full reactor power corresponded to that of the experimental irradiator at a helium pressure in the gap of 0.2 Torr (see above). The failure of OWR-II to operate as planned was probably due to the dubious accuracy of the heat-transfer-rate calculations which were based on unreliable $\gamma$-heating rates in that part of the Omega West Reactor core.

The neon in the gas gap was subsequently replaced by helium to increase the radial thermal conductance of the heat dump. The results are shown in Fig. 6. When the Omega West Reactor was at 4 MW the isothermal irradiator began regulating properly at a temperature of $680^{\circ} \mathrm{C}$. When the reactor power increased from 4 to $8 \mathrm{MW}$, the temperature of the isothermal section increased to $730^{\circ} \mathrm{C}--$ a change of $7 \%$ in heat-pipe temperature for a $100 \%$ change in input heat rate. The irradiator was now operating within $3 \%$ of the design temperature.

Subsequent operational data indicated that the OWR-II irradiator continued to regulate effectively, but that the reactor power, at which regulation began, slowly increased with time, as shown in Fig. 6. This increase was found to be due to a gradual deterioration of the dewar which allowed an increasing amount of heat to be lost radially from the evaforator section. (A post-irradiation examination of the dewar showed that the gold-plated reflecting surfaces were badly corroded.) To compensate, i.e., to start the heat pipe and to move the gas-vapor interface into a 
portion of the heat dump-zone with good heat-regulating properties, more reactor power was required.

\section{EBR-II Reactor Tests}

The successful operation of the instrumented OWR-II isothermal irradiator appeared to justify the design and assembly of an experiment to investigate the effects of long term irradiation on ceramic insulating materials. The EBR-II reactor was preferred as the irradiation facility because its neutron spectrum is similar to that of many thermionic reactor designs. This reactor is not accessable during operation and consequently temperature measurement or control is not possible. Therefore an isothermal irradiator was used to provide the necessary temperature regulation for the experiment.

Figure 7 shows the general design of the first EBR-II isothermal irradiator assembIy, IADI-I, currently being irradiated. In size and detail, this irradiator is very similar to the OWR-II assembly. A significant difference is in the design of the heat dump section. In OWR-II, the reactor coolant was water at a temperature of $45^{\circ} \mathrm{C}$. In the EBR-II reactor, the coolant is sodium at $360^{\circ} \mathrm{C}$ which is well above the melting point $\left(96^{\circ} \mathrm{C}\right)$ of the sodium working fluid in the heat pipe. Consequently, no gas gap in the heat dump section is required to keep the sodium molten. Thus a reoccurrence of the problems initially encountered in the OWR-II irradiator is unlikely. This experiment has accumulated $\sim 5000$ h of irradiation time.

\section{CONCLUSIONS}

The laboratory and reactor tests described above confirmed that specimen temperatures in irradiation experiments can be established and regulated through the use of inert-gas-filled heat pipes. When properly constructed, such pipes will last almost indefinitely and will require no servicing. With the wide choice of available working fluids, there is no real restriction on the operating temperature of such devices other than the temperature of the reactor coolant.

\section{RFFERENCES}

1. C. V. Weaver and W. A. Ranken, "Reactor Tests of Isothermal Irradiators," IEEE Proceedings of the 1968 Thermionic Conversion Specialist Conference, p. 96.

2. G. M. Grover, T. P. Cotter, and G. F. Exickson, "Structures of Very High Thermal Conductance," J. Appl. Phys. 35, 1990 (1964).

3. J. E. Kemme, "Ultimate Heat Pipe Performance," IEEE Transactions on Electron Devices, August 1969. 


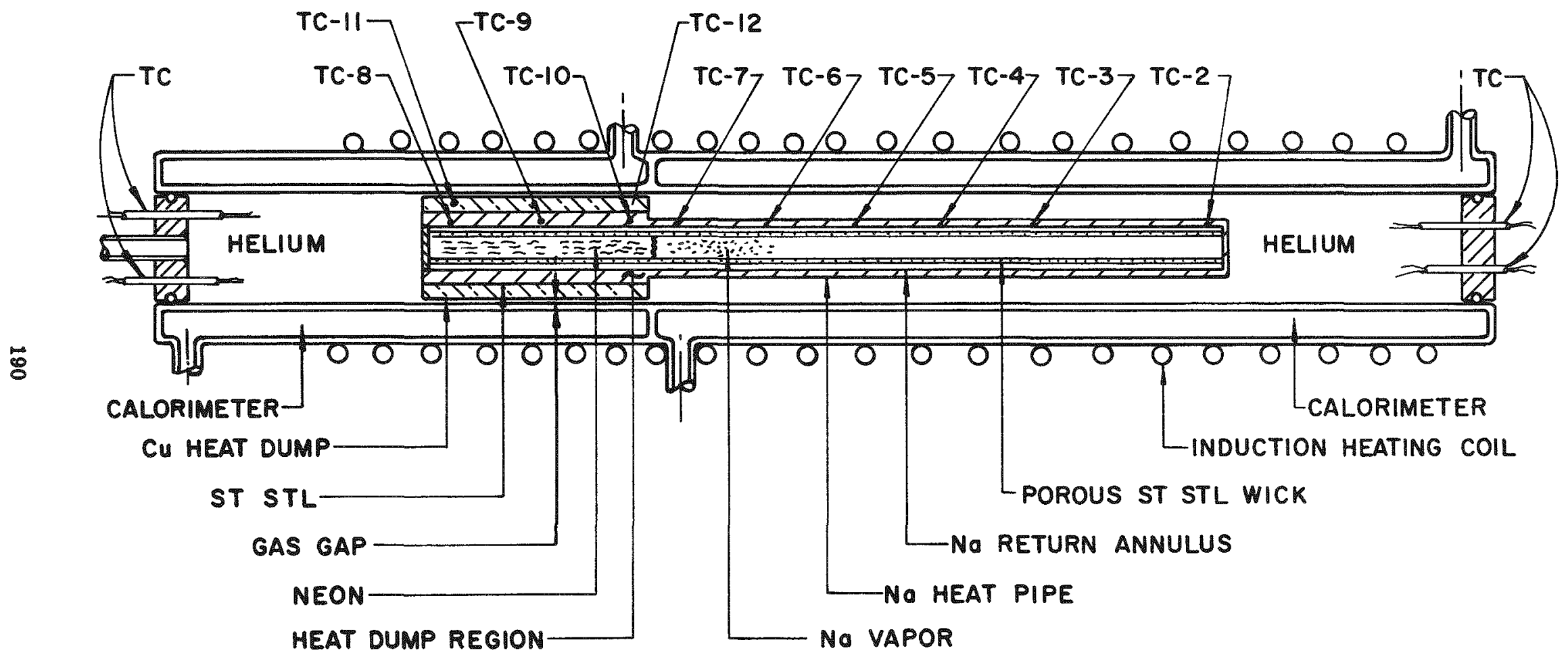

Fig. 1. Experimental isothermal irradiator for parametric studies. 


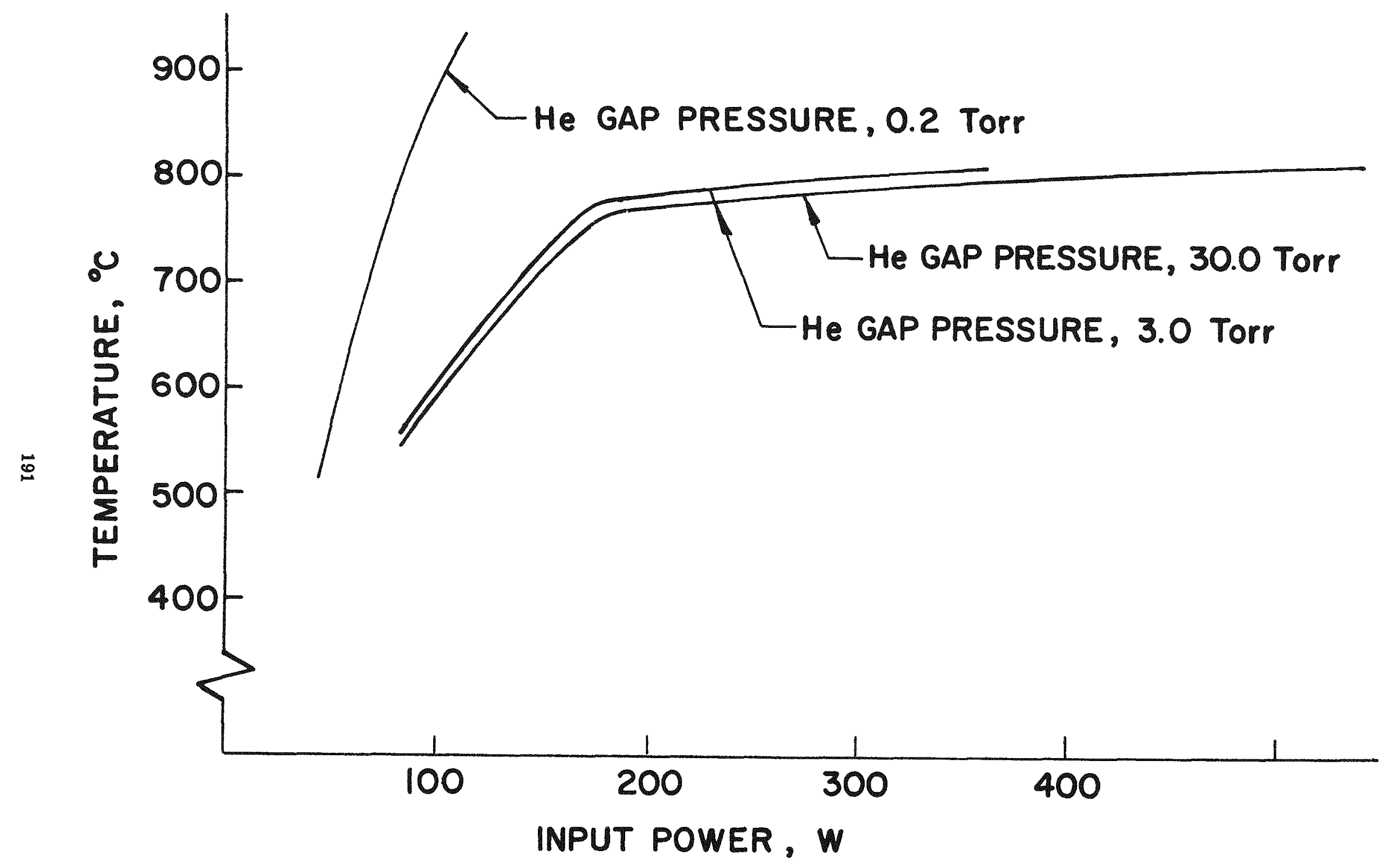

Fig. 2. Temperatures in the experimental isothermal irradiator for various heat input rates and for different helium pressures in the gas gap of the heat dump section. 


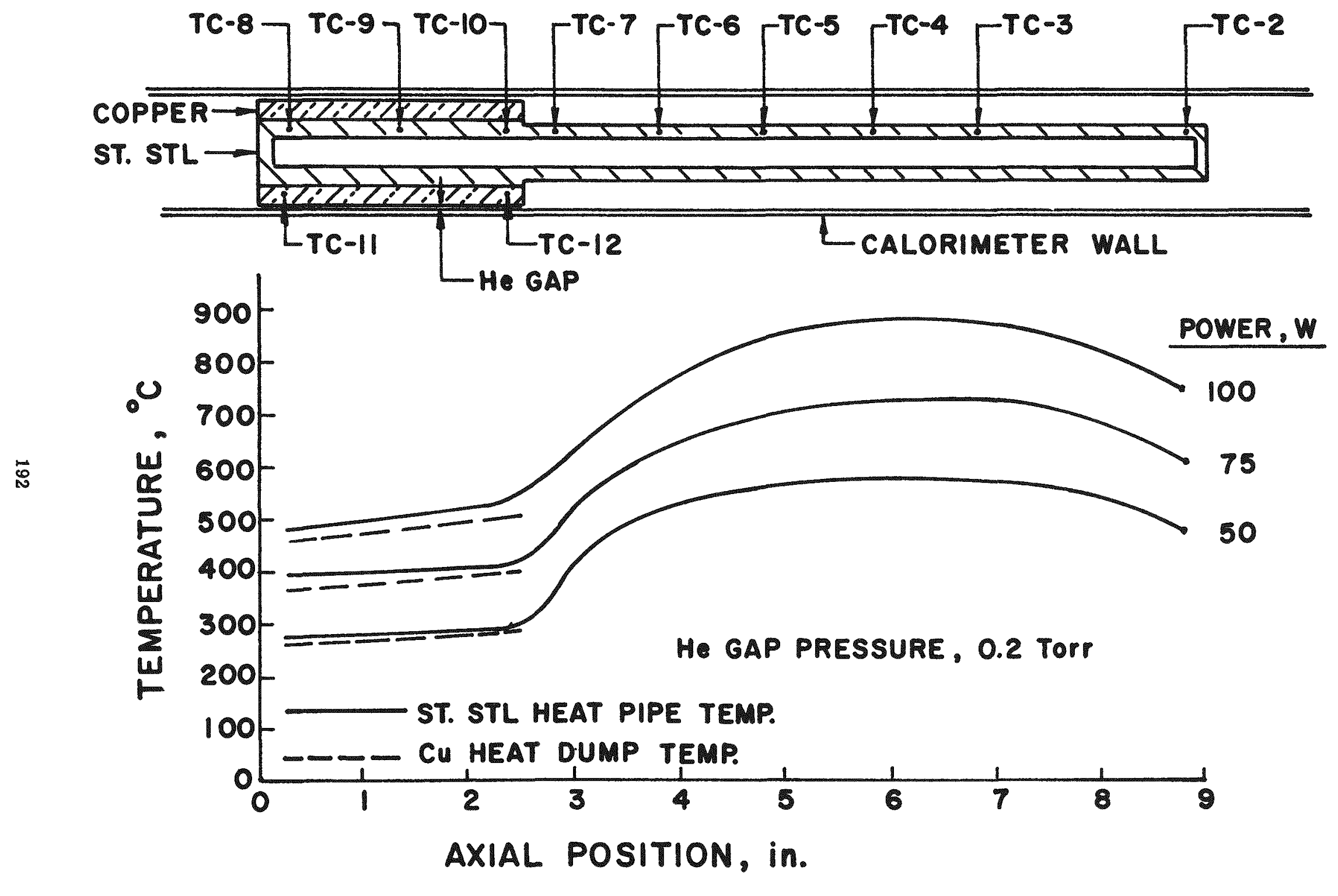

Fig. 3. Temperature distribution in an experimental isothermal irradiator for various heat input rates. Helium pressure in the gas gap is 0.2 Torr. 

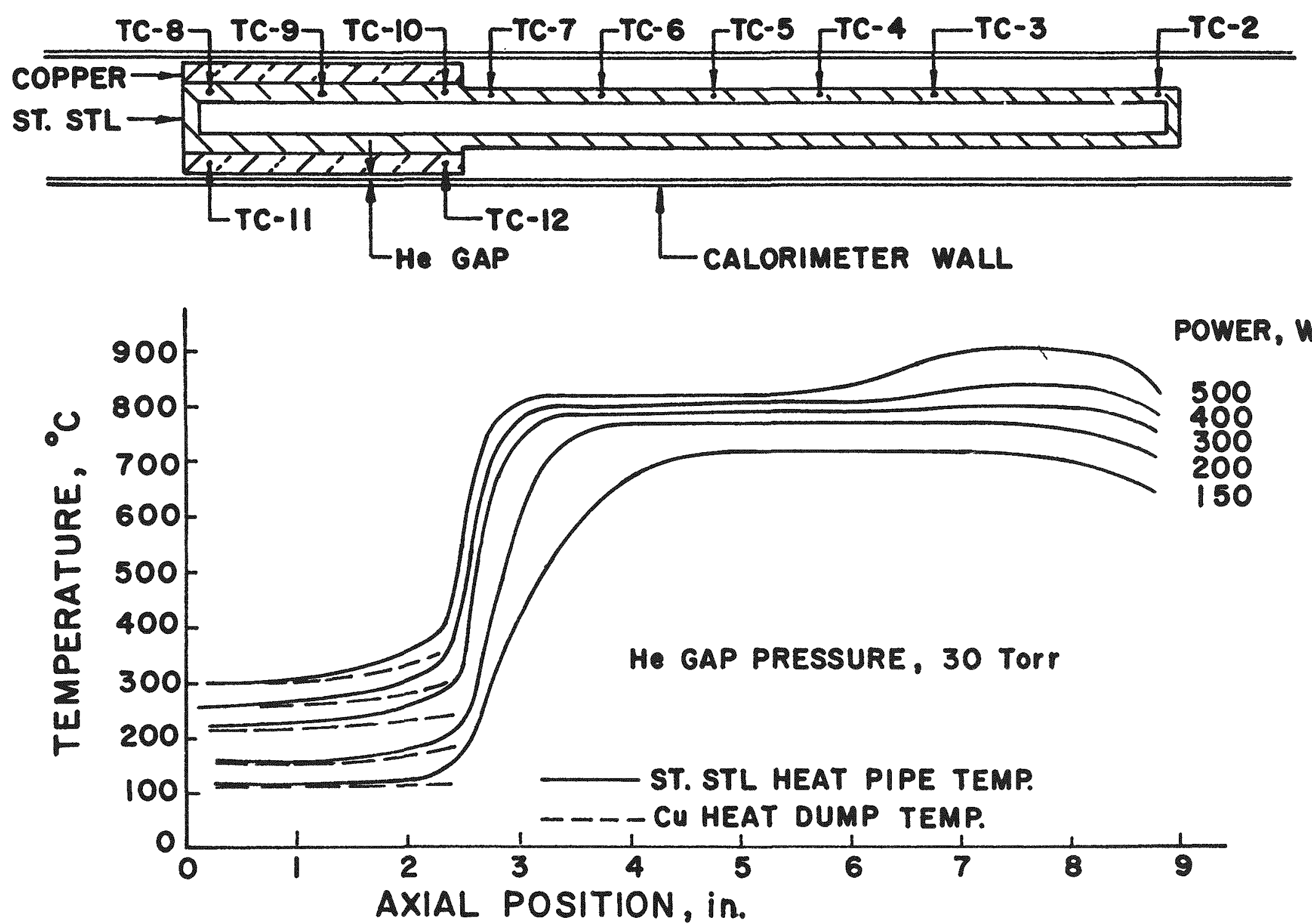

Fig. 4. Temperature distribution in an experimental isothermal irradiator for various heat input rates. Helium pressure in the gas gap is 30.0 Torr. 


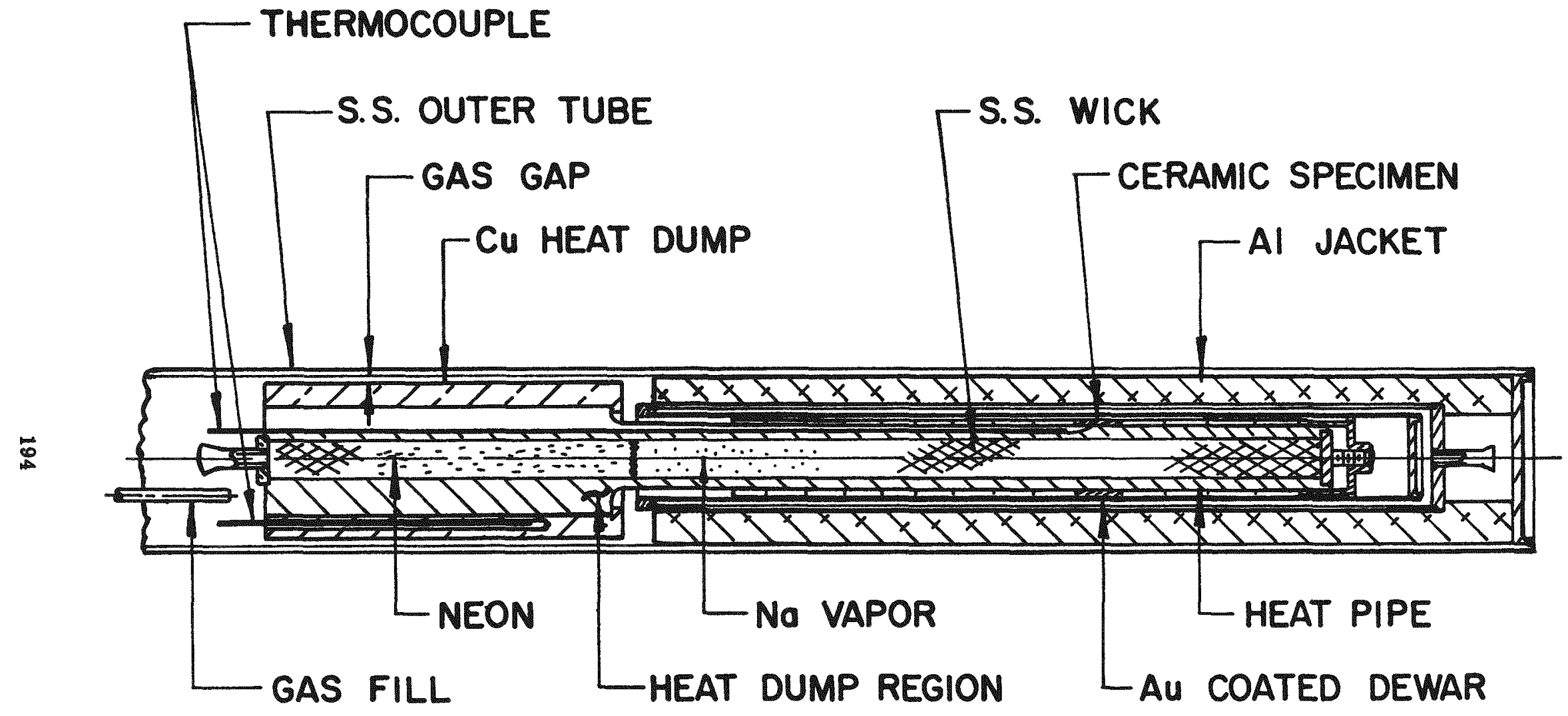

Fig. 5. An instrumented isothermal irradiator for testing in the Omega West Reactor. 


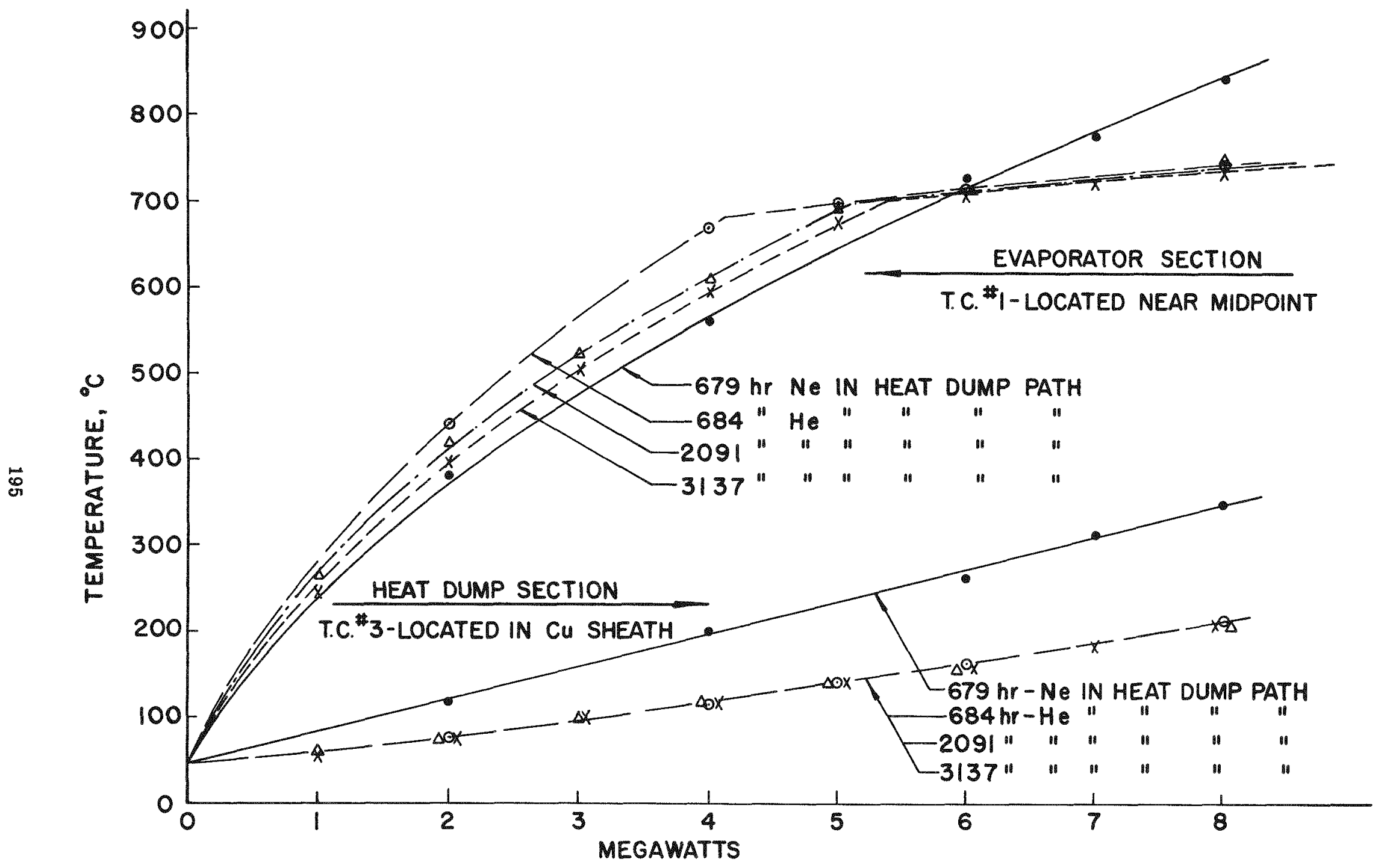

Fig. 6. Temperature distribution in the OWR-II isothermal irradiation unit for various reactor power levels. 


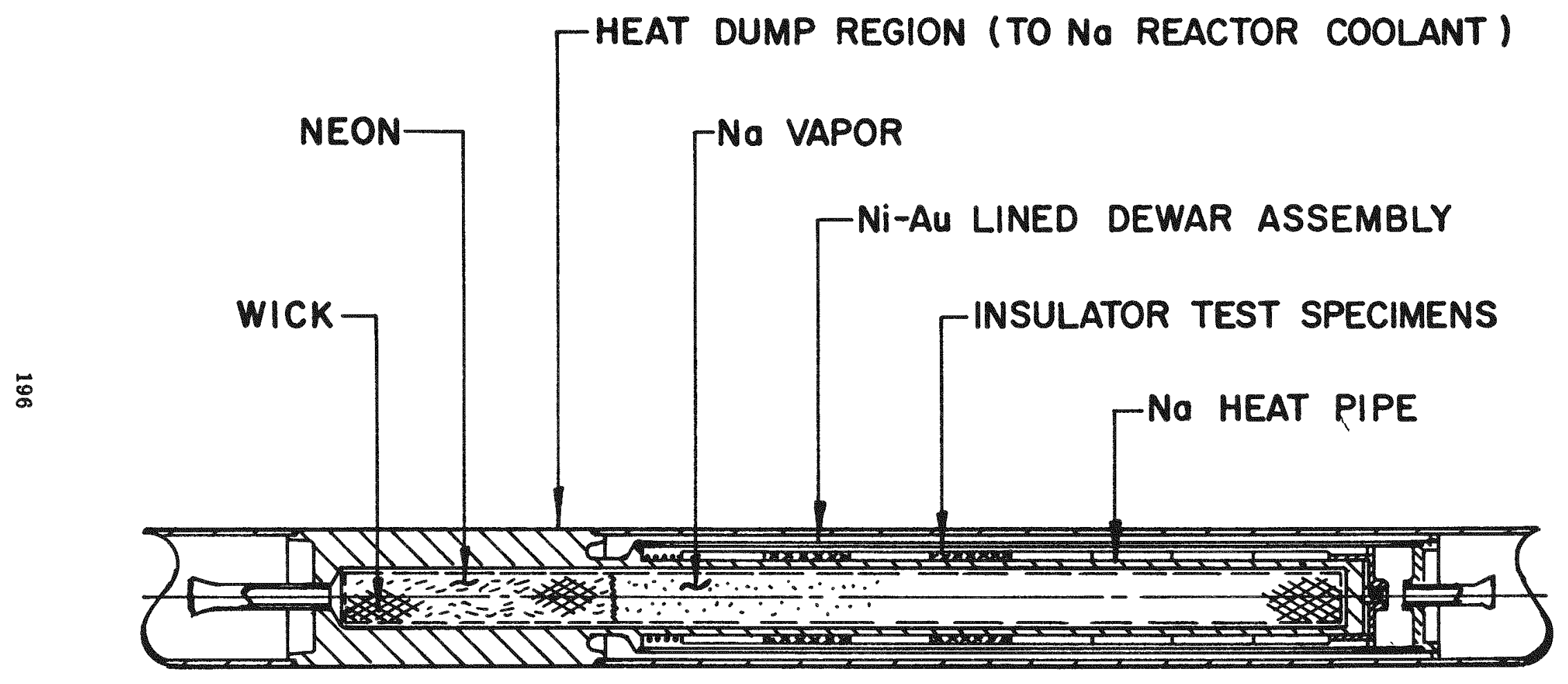

Fig. 7. Isothermal irradiator design for temperature control in the irradiation of ceramic specimens. 
COMPACT $30 \mathrm{~kW}$ CIRCULATING HELIUM CAPSUle FOR FUEL

ELEMENT TESTS IN NASA PLUM BROOK REACTOR

by G. E. McDonald, H. A. Putre, M. C. Swanson, and P. M. Finnegan 54920 Lewis Research Center

Cleveland, Ohio

\author{
and L. Homyak
}

Plum Brook Station

Sandusky, Ohio

\begin{abstract}
A capsule has been endurance tested out of pile and two capsules have been run in the Plum Brook Reactor. The capsule operates at pressures to $2500 \mathrm{psi}$, motor speed to $3000 \mathrm{rpm}$, flow rates to $0.09 \mathrm{lb} \mathrm{He} / \mathrm{sec}$, capsule wall temperatures to $1000^{\circ} \mathrm{F}$, specimen powers to $30 \mathrm{kw}$ with $180^{\circ} \mathrm{F}$ cooling water. Demonstrated out-of-pile life is at least 3000 hours. Fuel specimen surface heat fluxes of $2 \mathrm{kw} / \mathrm{in}^{2}$ have been obtained in pile. The capsule is 18 feet long with 4.3 inch maximum $0 . D$. and $2.875 \mathrm{O} . \mathrm{D}$. in the test section. It is composed of a variable speed electric motor driven vane pump, concentric tube heat exchanger, and a fuel element test section. The capsule is made of modified commercially available parts. Provisions are made for 15 thermocouples. Pressure leads used for filling are also used to measure gas flow rate and monitor coolant gas for fissiorı product leaks. A loss of coolant accident can be contained without reactor scram for specimen powers of $30 \mathrm{kw}$ or less. Capsules are relatively inexpensive and can be discarded when the test is completed.
\end{abstract}




\section{SUMMARY}

The Lewis Research Center is conducting gas cooled fuel specimen tests in the Plum Brook Reactor. This report describes the capsule used to test the specimens. A capsule has been endurance tested out of pile and two capsules have been run in the Plum Brook Reactor.

The capsule operates at pressures to 2500 psi, motor speeds to $3000 \mathrm{rpm}$, flow rates to $0.09 \mathrm{Ib} \mathrm{He} / \mathrm{sec}$, capsule wall temperatures to $1000^{\circ} \mathrm{F}$, and specimen powers to $30 \mathrm{kw}$ with $180^{\circ} \mathrm{F}$ cooling water. Demonstrated out-ofpile life is at least 3000 hours. The capsule is 18 feet long and 4.3 inch maximum O.D. and is composed of a variable speed electric motor driven vane pump, concentric tube heat exchanger, and a fuel element test section. The capsule outside diameter is 4.3 inches in the motorcompressor section and 2.875 inches in the heat exchanger and fuel element test section. The capsule is made of schedule 80 ss pipe, and uses a modified commercially available motor and pump. Provisions are made for 15 thermocouples. Pressure leads used for filling are also used to measure gas flow rate and monitor coolant gas for fission product leaks. A loss of gas coolant accident can be contained without resort to reactor scram for specimen powers of $30 \mathrm{kw}$ or less. Capsules are relatively inexpensive and can be discarded when the test is completed.

The first in-pile capsule ran for 294 hours in the Plum Brook Reactor and was stopped due to a bearing failure for reasons which have been corrected. The total operating time at the end of the test was 632 hours. The second in-pile test was terminated due to a fuel specimen fission product leak after onlyeight hours of operation. The capsule had, however, operated satisfactorily for more than 600 hours prior to irradiation and has continued to operate for 200 hours since fuel specimen failure. Fuel specimen surface heat fluxes of $2 \mathrm{kw} / \mathrm{in}^{2}$ were obtained in pile. 
The out-of-pile endurance tests are continuing and demonstrated lifetime is above 3000 hours. The endurance test is at $2400 \mathrm{rpm}, 1550 \mathrm{psi}$, 6 psi pressure drop, and $0.049 \mathrm{lb} / \mathrm{sec}$ helium flow rate. Bench test capsules are being fabricated for endurance tests at higher flow rates and pressure drops.

\section{INTRODUCTION}

The Lewis Research Center is studying gas cooled reactors for mobile nuclear power application. One important problem is establishing the probable fuel element lifetime and burnup at the contemplated reactor operating conditions. Specimens of candidate fuel elements are being tested in the PBR (Plum Brook Reactor). This paper describes the design characteristics and the performance capability of the circulating gas capsule in which the tests are conducted. A circulating capsule was required because the fuel specimen surface heat flux was greater than $2 \mathrm{kw} / \mathrm{in}^{2}$ which was higher than could be obtained in a natural convection capsule. The capsule has been endurance tested out of pile and two capsules have been operated in the Plum Brook Reactor.

The capsule is composed of an electric motor driven vane pump, concentric tube heat exchanger, and a fuel element test section. The capsule is made of standard pipe and uses a modified commercially available motor and pump. As a result the capsules are relatively inexpensive and can be discarded when the test is complete.

\section{GENERAL DESCRIPTION OF CAPSULE}

The capsule system, shown schematically in figure 1, consists of the capsule and its control console. The capsule, with its most important components identified, is shown in a water-cooled reactor test hole. A capsule assembly drawing is presented in figure 2. The capsule assembly consists of the sealed pressure vessel with the fuel element test section at the reactor end, the gas circulator at the shield end, and the heliumto-water heat exchanger connecting these ends. The long heat exchanger keeps the motor-compressor in the low radiation region when the test section is in the high flux region. The capsule length is 18 feet and is the maximum that can be handled in the water quadrant surrounding the PBR test hole. The capsule diameters were determined by the requirement that two capsules could be inserted into a nine inch I.D. water-cooled test hole. Helium is used as the gas coolant; however, any inert gas could be used.

The capsule operates in the following manner. High pressure helium is pumped through the center tube of the concentric tube heat exchanger to the fuel element, where it removes the nuclear heat and then flows back through the annular passage of the heat exchanger where this heat is transferred to the reactor facility water system. Helium pumping power is provided by a circulator consisting of a vane type pump driven by a variable speed electric motor. A wide range of helium flow rates for fuel element temperature control can be obtained by varying helium pressure and motor speed. The capsule operation is monitored and 
controlled from a console which contains the variable frequency motor power supply, coolant flow rate controls, radiation monitor controls, pressure and temperature recorders, and alarms.

The circulator end of the capsule is located outside the reactor shield in the quadrant water sufficiently far from the reactor core to assure low radiation levels and minimize radiation damage to the circulat or components (see fig. 3). The heat exchanger diameter is kept to a minimum to reduce streaming. To detect specimen fission product leak, a small amount of specimen coolant is circulated past a radiation monitor.

Personnel safety is provided by the reactor quadrant water. The quadrants are kept full during fuel element testing, and permit safe charging or withdrawal of the capsule from the reactor core with the reactor at full power. The sealed capsule design together with the quadrant water shield permits testing to fuel specimen fission product leakage, in which case the capsule gas lines are closed and the capsule is withdrawn from the reactor core.

Damage to the capsule pressure shell in event of fuel element meltdown, resulting from accidental loss of helium flow at full capsule power was regarded as the major safety problem. This problem was further complicated by an experiment ground rule that reactor scram not be used as a primary safety operation. This problem was solved by designing a graphite tray wrapped with tungsten sheet to catch materials that may melt in the event of helium flow loss, and to safely redistribute the nuclear heat without damage to the capsule pressure shell (see fig. 4). At present the catch tray can disipate $30 \mathrm{kw}$. This established the maximum allowable capsule power rating. However, the pump and the heat exchanger have the capability to go to higher powers.

Instrumentation has been provided to protect the experiment data. When high fuel element and/or gas temperatures are detected, alarms sound. Higher temperatures cause the capsule to automatically withdraw from the reactor. Still higher temperatures cause the reactor to scram. This instrumentation was not used for safety purposes because in-pile tests of several thousand hours were contemplated and thermocouple reliability for this time in the test environment could not be established prior to the test.

\section{DESIGN CHARACTERISTICS}

The following figures give an overall view of the capsule system. Capsule system layout and dimensions are presented in figures 1,2 and 3 . Figure 4 shows the graphite catch tray and tungsten wrapper. Figure 5 is a photograph of the motor-compressor parts. The thermal-hydraulic characteristics are described in figures 6 and 7 . The design and oper. ating characteristics are summarized below. The capsule is still being out-of-pile tested and its performance may be uprated as more experience is obtained. 


\section{General}

1. Out-of-pile demonstrated Iifetime is at least 3000 hours at 0.049 lbs/sec flow rate, with 6 psi pressure differential across the pump.

2. Alarms, table abort, and reactor scram protect experiment data in case of motor-circulator malfunction.

3. The capsule can be safely operated without thermocouple monitoring after operating conditions for test have been established.

\section{Nuclear}

1. Maximum fission and gamma power in fuel specimen and holder is $30 \mathrm{kw}$.

2. Maximum allowable radiation dose in motor compressor section is $10^{6}$ rads to grease and motor insulation.

\section{Safety}

1. Specimen coolant is continuously monitored for fission product leaks.

2. Fuel specimens can be tested until a fission product leak occurs without exceeding safe radiation levels in the working area above the quadrant.

3. Loss of coolant accident including fuel specimen meltdown can be contained without resort to reactor scram for power levels of $30 \mathrm{kw}$ or less. The catch tray used to contain drippings from melted fuel element is shown in figure 4.

\section{Mechanical Design}

1. System layout and dimensions are shown in figures 1, 2, and 3 . Figure 4 is a schematic of the catch tray and tungsten wrapper. Figure 5 is a photograph of motor compressor parts prior to assembly.

2. Motor speed range, 1500 - $5000 \mathrm{rpm}$.

3. Present maximum motor operating speed is $3000 \mathrm{rpm}$.

4. Maximum coolant pressure is $2500 \mathrm{psi}$ at $1000^{\circ} \mathrm{F}$ wall temperature based on ASME, pressure vessel code.

5. Compressor displacement is $3.5 \mathrm{in}^{3} /$ revolution.

6. Present maximum pumping capacity is .09 1bs/sec helium at 2500 psi, $3000 \mathrm{rpm}$, and $12.5 \mathrm{psi}$ pressure drop. (Determined with a typical fuel specimen installed in the capsule.) 
7. Maximum allowable grease temperature is $475^{\circ} \mathrm{F}$. Grease is GE Versilube G-300.

8. Maximum allowable motor temperature is $350^{\circ} \mathrm{F}$ (Class $\mathrm{H}$ insulation).

9. Maximum allowable coolant temperature into pump is $250^{\circ} \mathrm{F}$. Temperature restricted to minimize temperature gradients. Individual parts can operate to $350^{\circ} \mathrm{F}$.

10. Coolant flow can be monitored during test.

11. Up to 15 thermocouples can be used in the capsule.

12. Motor capacity is $2 \mathrm{hp}$ at $10,000 \mathrm{rpm}$ (maximum rated $\mathrm{hp}$ ) and $3 / 4$ hp at $3000 \mathrm{rpm}$.

13. Motor speed is measured by a rotating magnet tachometer in the motorcompress or shaft.

14. Catch tray has sufficient volume to contain melted test specimen and holder.

15. Catch tray surface area is sufficient to redistribute $30 \mathrm{kw}$ of nuclear heat without damage to the tray or to the capsule wall. Maximum tray temperature equals $4500^{\circ} \mathrm{F}$ (assuming radiation cooling only) which is within the strength capability of both graphite and tungsten.

16. Compressor vanes are made of Graphitar No. 2751 graphite. Wear rate was very sensitive to type of graphite and each batch of material must be tested for wear rate prior to use in a capsule. Wear rate is about one percent in 1500 hours at $2400 \mathrm{rpm}$.

Thermal - Hydraulics

1. Present maximum coolant temperature is $1000^{\circ} \mathrm{F}$ with potential for higher temperatures.

2. Coolant temperature into pump at $30 \mathrm{kw}$ is about $50^{\circ} \mathrm{F}$ above water temperature. Maximum allowable temperature into pump is $250^{\circ} \mathrm{F}$.

3. Maximum gas to water heat flux is about $140,000 \mathrm{Btw} / \mathrm{ft}^{2} \mathrm{hr}$ at $30 \mathrm{~kW}$ and $1000^{\circ} \mathrm{F}$ outlet gas temperature. Assumes gas side heat transfer coefficient increased by a factor of 2 at the heat exchanger inlet.

4. Maximum wall temperature at $30 \mathrm{kw}$ and $1000^{\circ} \mathrm{F}$ gas temperature is about $500^{\circ} \mathrm{F}$. Maximum wall temperature gradient is about $1200^{\circ} \mathrm{F} /$ in. Assumes water temperature is $150^{\circ} \mathrm{F}$ and gas side heat transfer coefficient is increased by a factor of two at the heat exchanger inlet.

5. The calculated heat exchanger pressure drop and the gas temperature at pump inlet and at test section inlet and outlet are shown in figure 6 for $30 \mathrm{kw}$. 
6. The measured flow rate and loop pressure drop as a function of pressure and motor speed is shown in figure 7.

\section{INSTRUMENTATION AND CONTROL}

Figure 8 shows the instrumentation and flow control diagram. All instrumentation and control apparatus, including the solid-state variable frequency motor power supply, is contained in a three-bay console as shown in figure 9. Instrumentation can be divided into three groups with sometimes overlapping functións. These are control, data gathering, and experiment safety.

\section{Control Apparatus and Instrumentation}

Coolant flow rate is manually controlled by adjusting coolant pressure and motor speed. Motor speed is set via a multi-turn potentiometer which is connected to the variable frequency power supply. The flow rate is measured by a venturi. The pressure, motor speed, motor current, and venturi pressure differential are recorded. Meters show motor voltage, current, and frequency. Alarms are provided to signal motor overcurrent and gas pressure too high or too low. Alarm conditions are displayed on a resettable alarm panel.

Experiment Data Instrumentation

Experiment data instrumentation consists of temperature, pressure, venturi $\triangle P$, and coolant radiation recorders. Provision is made for a maximum of 15 thermocouples. As a minimum, gas temperatures are measured at compressor inlet and fuel specimen inlet and outlet. Fuel specimen temperatures are measured at the hot and cold ends of the specimens and these temperatures are used in the maximum clad temperature calculations. The remaining thermocouples can be used to measure either gas or metal. temperatures. The radiation monitor is used to detect pin failure. All of the above data is recorded and in addition, pressure, venturi $\triangle P$, and coolant radiation level are also shown on meters.

The experiment data is protected by alarm, table abort, and reactor scram instrumentation. These systems receive their input from the thermocouple circuits. Should the thermocouples deterioriate with time in the irradiation environment, motor rpm and or current can be used as input. Alarms are displayed on a resettable alarm panel.

\section{Safety Instrumentation}

Safety instrumentation consists of a pressure relief valve to assure capsule cannot be overpressured, a coolant radiation monitor to detect release of fission products into the coolant gas, and a alarm on the radiation monitoring gas flow to assure there is a satisfactory amount of gas being circulated past the radiation monitor. 


\section{PRODUCTION MODEL TESTS}

Prior to capsule fabrication, the motor-compressor is assembled and rurin for at least 50 hours in a bench test capsule at the Lewis Research Center. When the run-in test is complete, the capsule flow rate, current and voltage are measured over the full pressure and rpm operating range and ground (HYPOT) and resistance tests are performed on motor and tachometer leads. The motor-compressor is then shipped to the capsule fabricator.

Each test capsule undergoes the following test sequence following completion of fabrication.

1. Radiography of all pressure vessel welds.

2. Pneumatic pressure test under collision mats to 3750 psig (2.16 $\mathrm{x}$ $10^{7} \mathrm{~N} / \mathrm{m}^{2}$ )。

3. Helium mass spectrometer leak test at 1500 psig $\left(1.04 \times 10^{7} \mathrm{~N} / \mathrm{m}^{2}\right)$.

4. Ground (HYPOT) and resistance tests on all motor and tachometer leads.

5. Ground and resistance tests of all thermocouples.

6. Bake-out and simultaneous evacuation of the assembly for ten hours to $180^{\circ} \mathrm{F}\left(360^{\circ} \mathrm{K}\right)$ and 50 microns $\left(6.6 \mathrm{~N} / \mathrm{m}^{2}\right)$ absolute pressure. This is the minimum pressure to prevent the bearing lubricant from boiling.

7. Operation of the motor at fixed speed from three-phase $60 \mathrm{H}_{z}$ supply. Stethoscopy for unusual noise.

Following these tests the assembly is backfilled with pure helium to approximately 25 psig, crated and shipped to Lewis.

The capsule is returned to Lewis and is run-in for at least 50 hours at contemplated in-pile operating conditions. The capsule is then calibrated and shipped to Plum Brook. Calibration consists of measuring motor current and flow rate as a function of capsule pressure and rpm over the full operating range of the capsule.

\section{CONCLUDING REMARKS}

Two capsules have been operated in the Plum Brook Reactor. The first in-pile capsule ran for 294 hours in pile. The test was stopped due to a bearing failure for reasons which have been corrected. Total operating time on the first capsule at the end of the test was 632 hours. The second in-pile capsule test was terminated due to a fuel specimen fission product leak after only eight hours of operation. The second capsule had, however, operated satisfactorily for more than 600 hours during pre- 
irradiation checkout and has continued to operate satisfactorily for more than 200 hours since fuel pin failure. Specimen surface heat fluxes of $2 \mathrm{kw} /$ in $^{2}$ were obtained in the in-pile test. The out-of-pile endurance tests are continuing and demonstrated lifetime is above 3000 hours. The endurance test is at $2400 \mathrm{rpm}, 1550 \mathrm{psi}, 6 \mathrm{psi}$ pressure drop, and $0.049 \mathrm{lbs} / \mathrm{sec}$ helium flow rate. Bench test capsules are being fabricated for endurance tests at higher flow rates and pressure drops. 


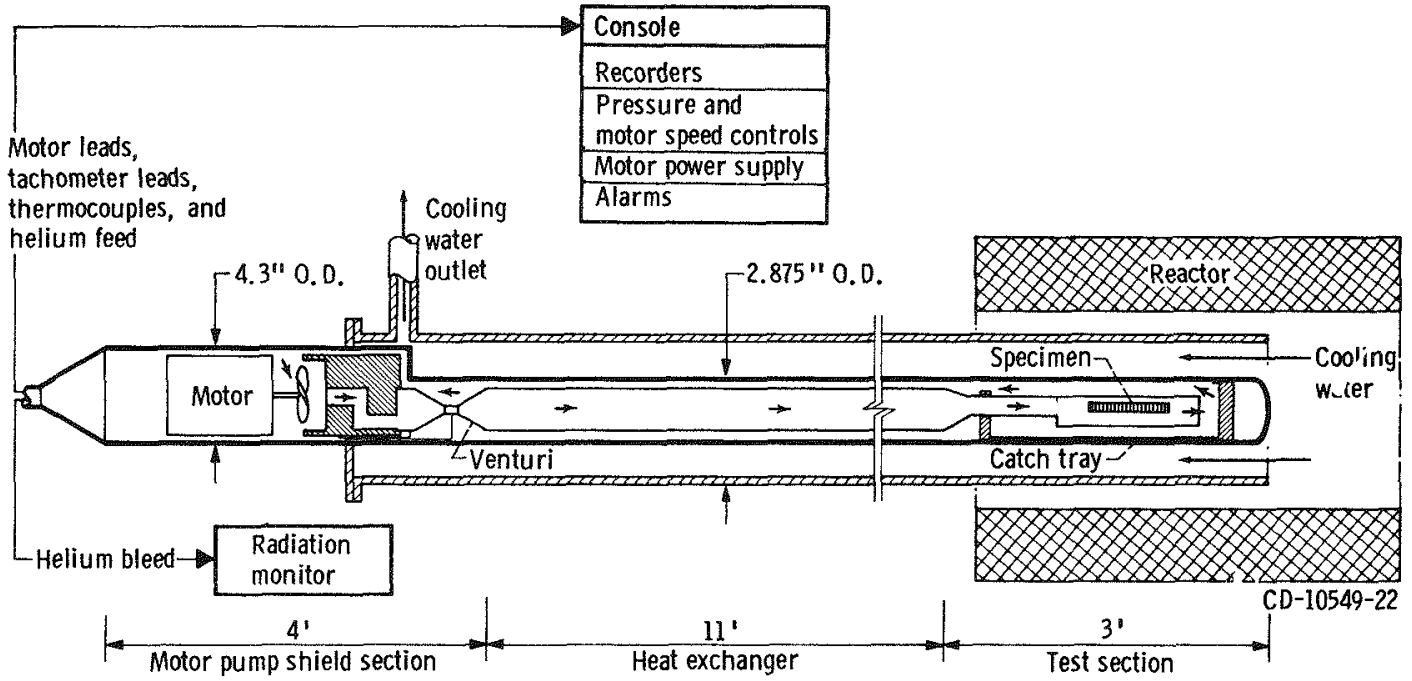

Figure 1. - Circulating capsule schematic.
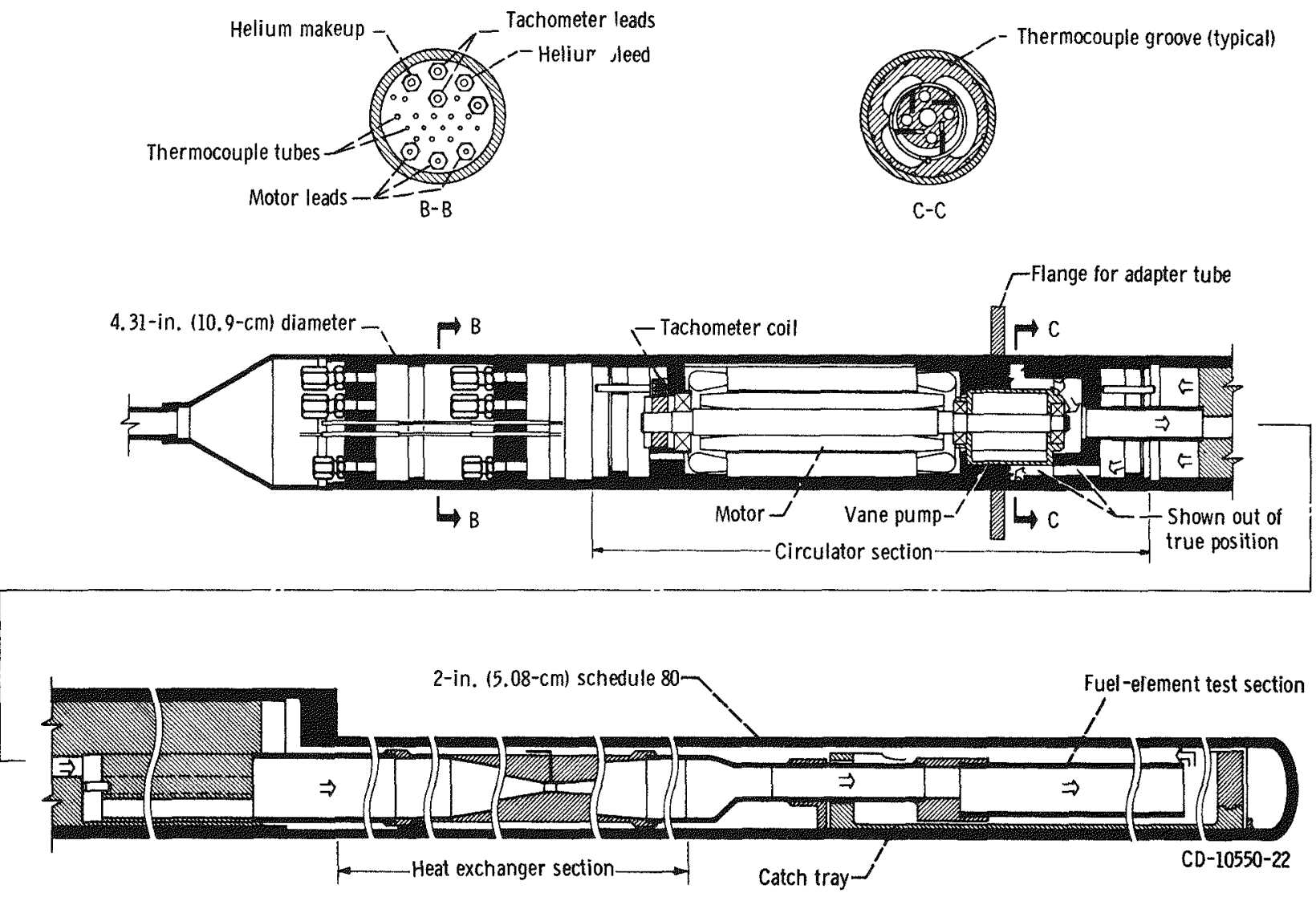

Figure 2. - Capsule assembly. 


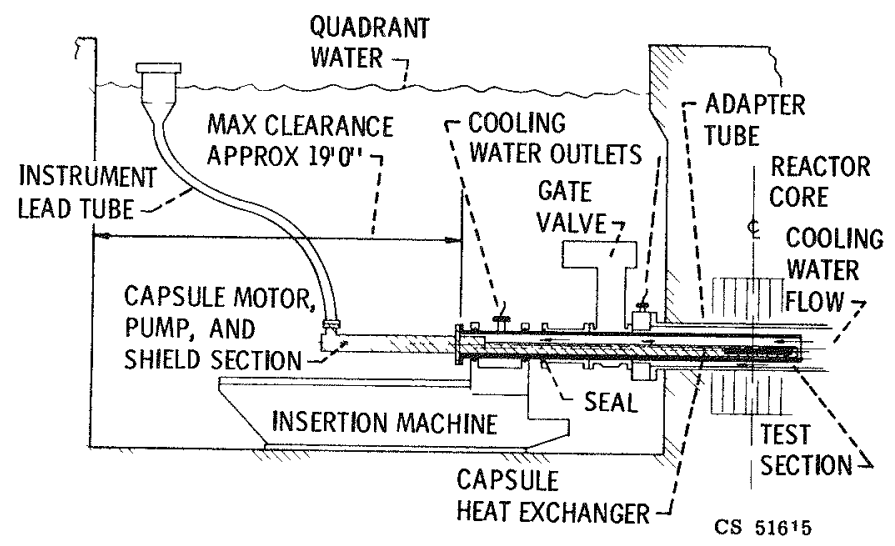

Figure 3 - Capsule in adapter tube on reactor insertion machıne
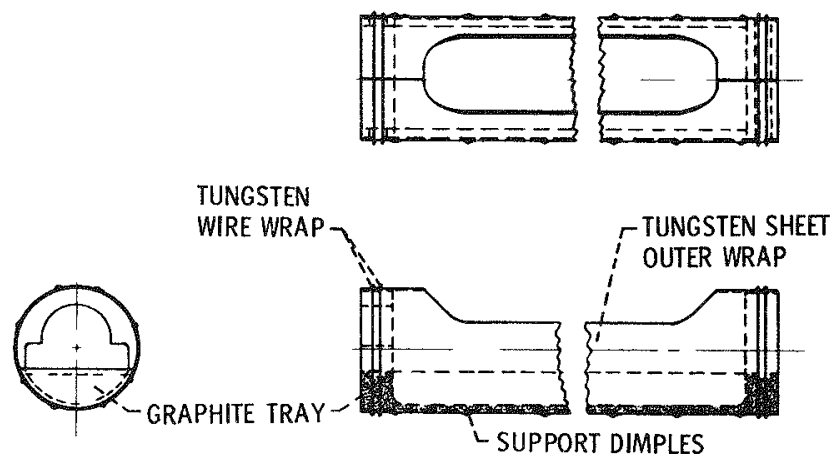

CS 51616

Figure 4 - Graphite catch tray with tungsten wrap

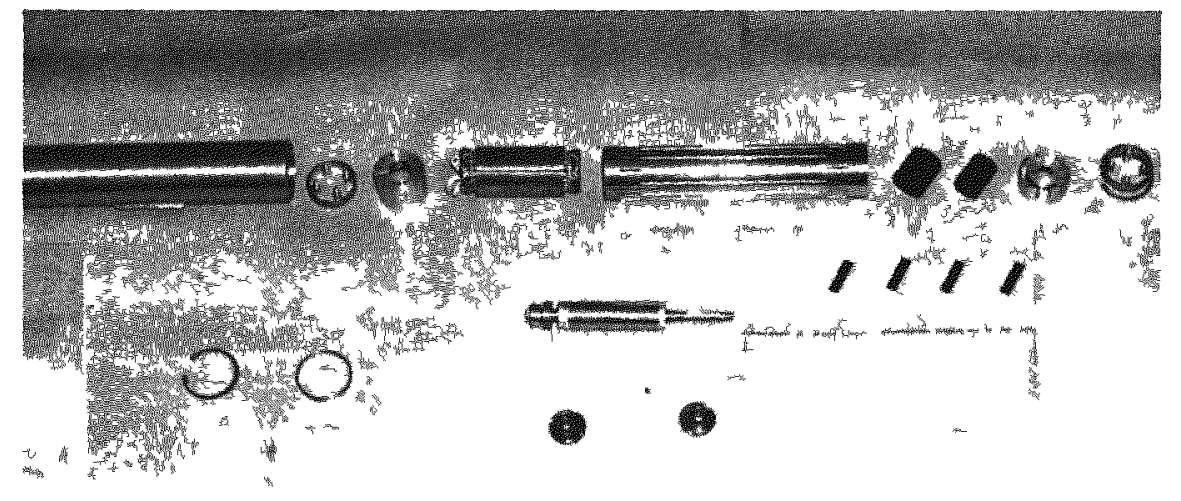

Figure 5 - Layout showing circulator parts 


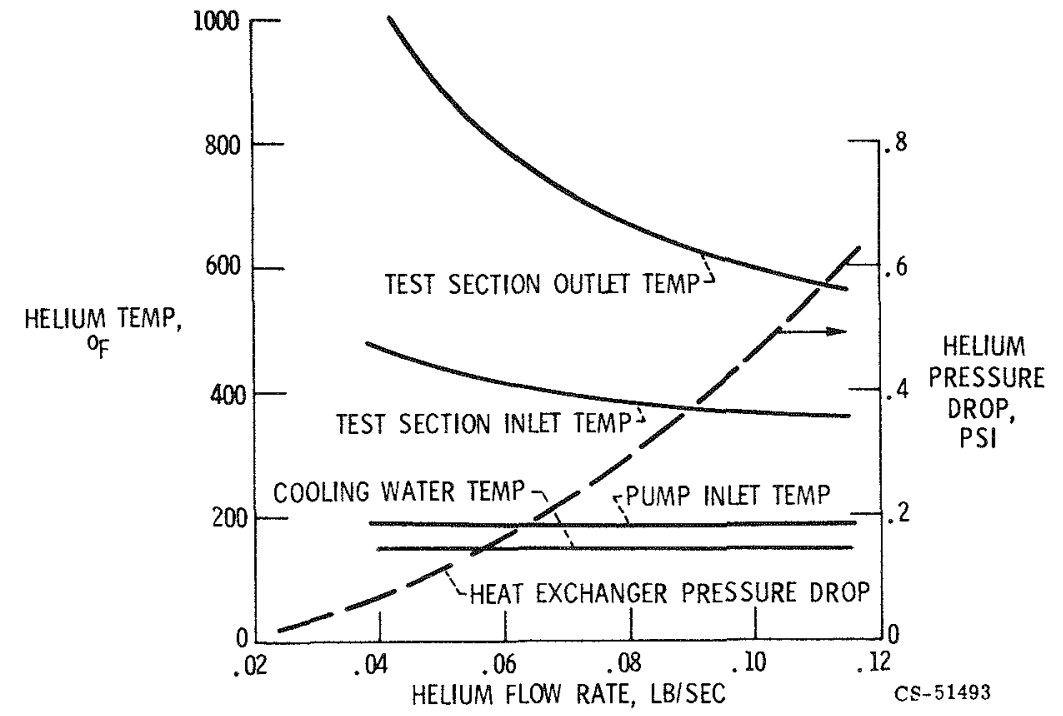

Figure $b_{0}$ - Helium temperature and pressure drop in heat exchanger for various flow rates, Power, $30 \mathrm{~kW}$, pressure, 1500 psi.

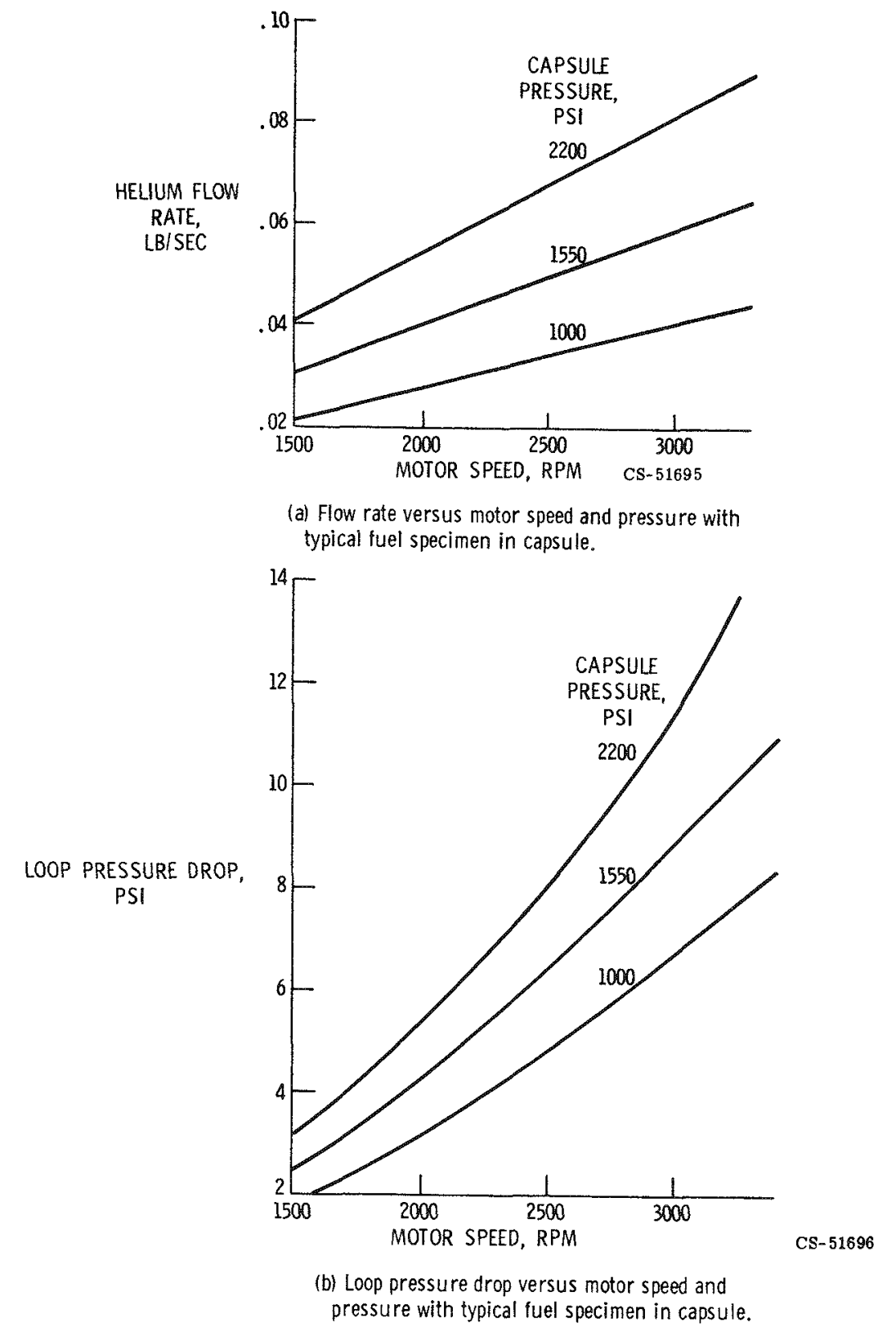

Figure 7 

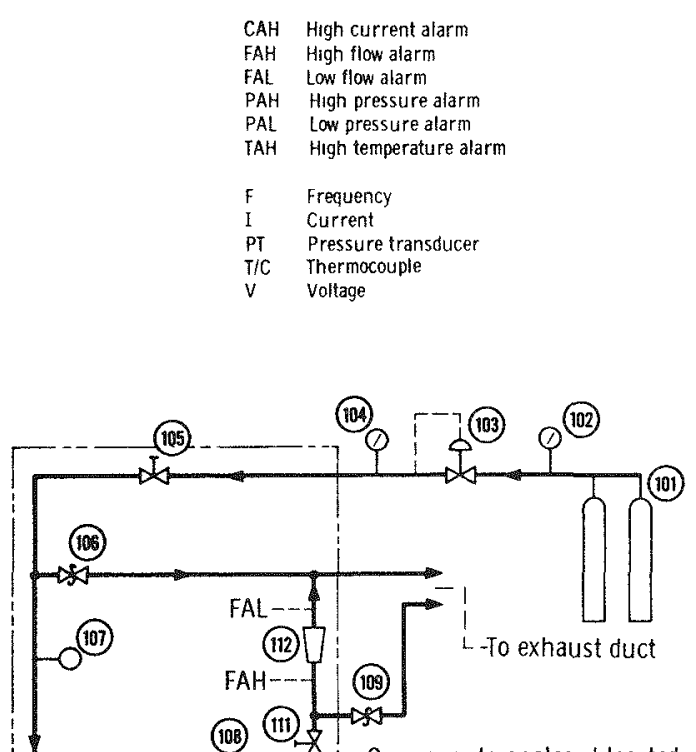

To recorder PAH PAL

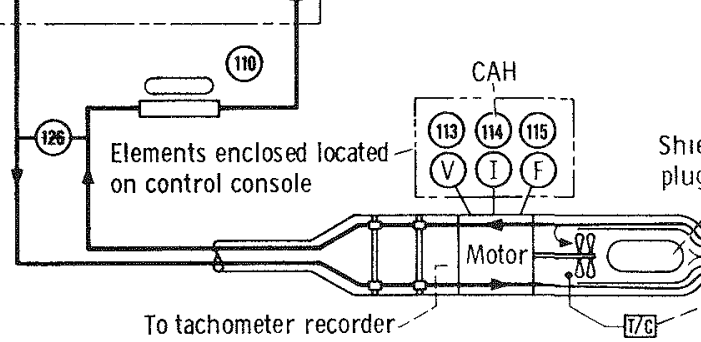

To tachometer recorder

\begin{tabular}{|c|c|c|c|c|}
\hline llem & Item & $\begin{array}{l}\text { Type and } \\
\text { size }\end{array}$ & $\begin{array}{c}\text { Working } \\
\text { pressure, } \\
\text { psia }\end{array}$ & Remarks \\
\hline 101 & Heluum manufold & $\cdots$ & 3020 & At reactor \\
\hline 102 & Hellum mantufold pressure gage & $\ldots$ & 4000 & On regulator 103 \\
\hline 103 & Helium supply regulator & $\frac{1}{4}$ NPT & 6000 & In line \\
\hline 104 & Helium supply pressure gage & - ……... & 4000 & On regulator 103 \\
\hline 105 & Helium supply shutoff valve & $\ldots$ & 30000 & | \\
\hline 100 & Helium pressure relief valve & $-\cdots$ & 3000 & Set 110 percent of capsule pressure \\
\hline 107 & Helium pressure gage & $\frac{1}{4}$ NPT & 5000 & - \\
\hline 108 & Helsum pressure transducer & mancose & 6000 & High and low pressure alarms \\
\hline 109 & Retref valve & $\ldots$ & 3000 & Set at $25 \mathrm{psig}\left(275 \times 10^{5} \mathrm{~N}^{2} \mathrm{~m}^{2}\right)$ \\
\hline 110 & Radratron monitor & $\cdots$ & - - & At reactor \\
\hline 111 & Flow control valve & ...- & 3000 & Micrometer adjustment \\
\hline 112 & Flow indicator & $115-6$ & 200 & High and low flow alarms \\
\hline 113 & Voltmeter & $4 \frac{1}{2}$ & $\cdots$ & 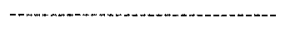 \\
\hline 114 & Ammeter & $4 \frac{1}{2}$ & -- & High-current alarm \\
\hline 115 & Frequency meter & $4 \frac{1}{2}$ & $\cdots$ & 0 to $180 \mathrm{cps}$ \\
\hline 116 & Gas temperature thermocouple & $\mathrm{CA}$ & -- & Pange $0^{\circ}$ to $100^{\circ}=10$ to $800 \mathrm{~K}$ \\
\hline 117 & Gas temper ature thermocouple & $C A$ & $-\cdots$ & $\{$ with high temperature alarm \\
\hline 118 & Gas temperature thermocouple & C A & $\cdots-$ & \\
\hline 119 & Element temperature thermocouple & W-W-Re & -- & \\
\hline 120 & & & $\ldots$ & \\
\hline 121 & & & $\cdots$ & Range $0^{\circ}$ to $3000^{\circ} \mathrm{F}(1460 \mathrm{~K})$ \\
\hline 122 & & & $\cdots$ & with high temperature alarm \\
\hline 123 & & & $-\cdots$ & \\
\hline 124 & & & - & \\
\hline 125 & 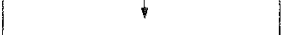 & 1 & -- & \\
\hline 126 & Differential pressure transducer & & & \\
\hline
\end{tabular}

To thermocouple recorder $1-x$
Flow
plug $\rightarrow$ measuring
(196) 而 TAH

\section{venturt}

(118)

To thermocouple recorder 1
To thermocouple recorder 2

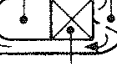

Fuel element

Figure 8. - Instrumentation and flow schematic.

(119) to (125)

$T A H$

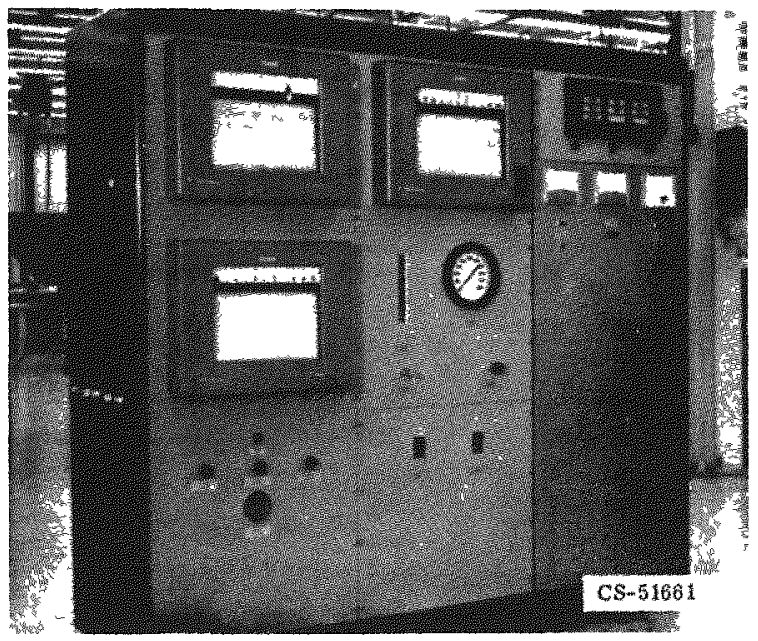

Figure 9. - Control console. 


\section{$\checkmark$ EXPERIMENT DESIGN STUDIES FOR FUEL PIN DEVELOPMENT \\ By Ra1ph Stein, Len Homyak, and Robert DeFayette \\ $54+5^{*}$ Lewis Research Center}

\section{SUMMARY}

A method is described for simulating a fuel pin proposed for irradiation in the Plum Brook Reactor. This simulation utilizes fully enriched uranium metal sheets which are sandwiched between various thicknesses of aluminum foil and rolled around a spindle.

The measurement of power density ratios by using gold flux wires is described. The importance of these ratios in the interpretation of the fuel pin performance is discussed.

A series of measurements was made to obtain the necessary fuel enrichments for the correct burnup. Fuel enrichment versus power density curves were drawn. These curves show that above certain enrichments, which are dependent upon fuel pin size, there is little increase in the power density of the fuel pins. These enrichments are $10 \%$ for our ful1 size pin and $20 \%$ for a half-size pin。

Because the power density ratios were not within specified limits, it was necessary to shape the flux in an attempt to alter the gradients. This was done with a hafnium shield. Results are presented comparing the fuel pin power densities with a hafnium shield and without it. In effect, the hafnium reduced the axial gradients from about 2.6 to 1.5 , and reduced the circumferential or radial gradients from 1.5 to 1.3 .

\section{ABSTRACT}

A mockup of a uranium nitride fuel pin proposed for a Space Power Reactor was designed and tested in the Plum Brook Reactor Facility MockUp Reactor. The method used to simulate the UN and to measure the power gradients and power densities is described. Results are presented showing the effect of a hafnium shield in reducing the power gradients. 
One of NASA's objectives in the development of space power conversion systems is to have available the technology for a compact, fast spectrum, liquid metal cooled reactor capable of operating at high temperatures for long periods of time. Obviously, a very important part of this technology is the development of a suitable fuel-clad combination.

At Lewis Research Center, we are engaged in research pointed at development of the technology for compatible fuel-clad materials. Through other programs, a candidate fuel-clad combination has been selected, a tantalum alloy for the clad and uranium nitride for the fuel. The selection of these materials was based on their desirable out-of-pile characteristics; however, information regarding their in-pile performance at the condition of interest was quite sparse. Accordingly, an irradiation program for the tantalum, UN combination was conceived to be performed in the PBR. As a prelude to this irradiation, a series of measurements was made in the Mock-Up Reactor of the Plum Brook Reactor Facility, to experimentally design the fuel capsules to be irradiated in the PBR.

The MUR measurements included a determination of fuel enrichments required for operation of the UN fuel pins at specific power levels, measurement of the axial and circumferential power density ratios, and design of thermal flux shadow shields to shape the power profiles to meet the operating conditions. The measurement of the power density ratios, and design of shields to shape the power profiles, are particularly important. The application of the UN fuel pin irradiation data is in the design of a fast reactor where the power density ratios in the fuel pins will be much lower than in a thermal reactor. To be of value, the irradiation fuel pins under test must acceptably simulate the power profiles predicted for the Space Power Reactor fuel pins.

For the Mock-Up Reactor irradiations, two fuel pin sizes were selected for irradiation. One size test pin, called a D pin, would operate at the reactor design burnup rate; the other fuel pin, one-half 
size, called a D/2 pin, would operate at twice the reactor design burnup rate. The object of the faster burnup rate was to obtain performance data earlier. The criteria for axial and circumferential power density ratio (maximum to minimum) was set at 1.5 , with a desired ratio of 1.3 . In addition, the maximum enrichment for the larger pin was set at $12 \%$, and $16 \%$ for the half-size pin. Enrichments above these limits were calculated to result in unacceptable high power density ratios across the thickness of the fuel.

The selection of these ratios was somewhat arbitrary. The application for the fuel pins is in a fast reactor where the power density across the fuel pin will be low, approaching 1. The axial gradients for short lengths will also be low, again approaching 1 . The gradients selected, then, had a lot of judgement factor in them. They represent the best compromise of what we believed could be obtained in the thermal reactor and still give applicable data in relation to the fast reactor.

\section{MUR PROGRAM}

To accomplish the objectives of the MUR irradiation, a program was established to:

(1) Determine the core location which provides sufficient neutron flux, within the range of enrichment criteria to achieve average fuel pin powers of 154 watts/Cc in the D-pin, and 308 watts/cc in the D/2-pin;

(2) Determine the exact fuel enrichment required; and

(3) Minimize the maximum-to-minimum axial and circumferential power density ratio to at least 1.5 , and try to achieve a desired power density ratio of 1.3 . 


\section{DESCRIPTION OF A MOCKUP FUEL CAPSULE}

Fuel used for the mockup capsule was in the form of $93.16 \%$ enriched uranium meta1 foils. Each foil measured about 5 by $10 \mathrm{~cm}$ on a side, and 2.5 thousands of a $\mathrm{cm}$ thick; and contained approximately 2.5 grams of $\mathrm{U}-235$.

The mockup reactor fuel pin and capsule are shown in figure 1.

Fue1 foils, sandwiched with various thicknesses of aluminum foil to distribute the fuel throughout the fuel volume, were rolled around an aluminum rod. The aluminum rod simulated the fuel center annulus and provided a means to position center flux wires.

The number of fuel sheets used were varied to provide fuel pins of various enrichment. Gold flux wires were taped to the outside of the rolled fuel sandwich to measure the circumferential power density. The flux wires were taped on the north, south, east, and west positions of the fuel pins - - the north position faces the core. Axial power densities were measured by cutting the flux wires into about $0.3 \mathrm{~cm}$ long segments and measuring each segment individual1y. To make certain that the gold wires were giving a good representation of the power density, several uranium fuel sheets were cut into one-half inch segments. These segments were then counted for the La-140 peak and the ratios of these results compared with the gold results. Agreement was obtained to within a few percent, thereby showing that the gold could be used to give accurate results.

The rolled fuel-aluminum foils were slipped into a tantalum cylinder and the tantalum cylinder slipped into a stainless steel can. This assembly is called a capsule. Three capsules fit into a capsule holder for irradiation in a test facility. The capsules of a holder consist of one D-size pin ( $2 \mathrm{~cm}$ in diameter and $5.08 \mathrm{~cm}$ long) and two D/2 size pins (1 cm in diameter and $5.08 \mathrm{~cm}$ long)。 
JUSTIFICATION OF MODEL

For the PBR irradiations, the fuel will consist of homogeneous depleted uranium blended to the proper enrichment with U-235. To insure that the mockup reactor fuel capsule was a true nuclear simulation of the PBR fuel pin, an evaluation was made of the U-235 distribution within the fuel volume, using a 20-group transport code (TDSN Code).

The results showed that the mockup reactor capsule design agreed within $5 \%$ of the nuclear analysis made for the PBR fuel capsule. This technique of wrapping the fuel-aluminum foils into fuel forms was particularly interesting because of the ease in changing the fuel enrichments, and the precision of the model.

\section{RESULTS AND DISCUSSION}

Fuel capsules with various fuel enrichments and full and half-size pins were irradiated at a known flux location in the MUR. A power vs. enrichment plot was derived from the results and is shown in figure 2 . These curves are a compilation of several MUR irradiations.

We found that the desired power of about 150 watts/cc and 300 watts/cc could be obtained with a D-pin enrichment of $5.6 \%$, and $\mathrm{D} / 2$ pin enrichment of $8.2 \%$ in a $B$-row reflector test hole.

Note also the shape of the two curves. They both level off; the D-pin at about $10 \%$ enrichment, and the D/2 pin at about $20 \%$ enrichment. This means that increasing the enrichments above these values in the $B$-row reflector will not result in more power. This characteristic is due to self-absorption of the capsules.

Based on the enrichment measurements, fuel pins were assembled and an irradiation was performed to obtain a power density profile across the fuel for a D-pin. The results are shown in figure 3.

Flux wires were located on the north and south surfaces of the fuel pins as well as in the center. The north tangent faces the core. 
All the flux values were normalized to the lowest flux value measured by the center flux wires. Basically, the purpose of these curves is to illustrate the magnitude of the power density ratios.

The maximum-to-minimum ratios of the axial power density were measured to be $\sim 2.5$, and of the circumferential power density to be $\sim 1.6$. Since these ratios were outside the desired limits for the experiment, tests were performed to flatten the fluxes, using a thermal neutron shield.

Hafnium was selected as the shield material because of its desirable nuclear properties and its compatibility with the primary cooling water. Based on the shape of the power density profiles shown in figure 3, a shield was needed to reduce the flux at the bottom of the capsule as well as at the capsule side facing the core. Irradiation tests and analyses with various configurations resulted in selection of the shadow shield shown in figure 4.

This shield consists of a half-cylinder covering the lower half of the capsule holder and a finger projecting up in front of the D-pin. The half-cylinder attenuates the thermal flux at the bottom of the capsules while the finger reduces the front-to-back ratio of the thermal flux. Hafnium disks are placed on the bottom of the fuel pins to reduce thermal neutron flux entering the fuel from the bottom.

A power density profile across a D-pin of a capsule holder using the shadow shield is shown in figure 5.

Extensive flux measurements were made on this capsule. In this case a11 the power density ratios were normalized to the center fuel pin flux. The figure illustrates clearly the flattening of the power density ratio caused by the hafnium shields. This flattening of the power density ratio profiles can be more readily seen in figure 6 . This figure shows the axial power density ratio of a D-pin at the center with and without the shadow shield. Use of the shield resulted in achieving the criteria of 1.5 established for the axial power density ratio.

The shape of the curves in the figure is characteristic of the flux depressions that occur when an absorbing material is placed in a thermal 
flux enviroment. The exceptionally high power peaking at the bottom of the fuel pin is mainly the result of the large unperturbed flux gradient that exists in the test location. In addition, some contribution to the power peaking at the ends is probably due to thermal neutrons entering the ends of the fuel pins. The shielding, which is placed primarily on the bottom half of the capsule, reduces the unperturbed flux considerably, and therefore helps to reduce the large power peak.

The circumferential power density ratios of the fuel pins were also measured. The ratio was a maximum value of 1.6 when no hafnium shields were used. The hafnium shields reduced the maximum value to 1.3.

The effectiveness of the hafnium shields in reducing the circumferential power density ratios can be attributed to the location of the fuel pins in the reflector region of the reactor where much of the thermal neutron $f 1 u x$ is due to fast flux thermalization. The hafnium absorbs thermal neutrons on the core side of the fuel pin but has little effect on the fast flux thermalized on the side of the fuel pin away from the core. The net effect is that the front-to-back thermal flux ratio is reduced. It is of interest to note that even though the enrichments were wel1 below the maximum established by the criteria, the radial power density, from surface to center, was about 1.3. This is a larger ratio than reported elsewhere. For a similar fuel configuration, another facility reports that the surface-to-center ratio is $1.05-1.1$ based on calculations.

\section{CONCLUSIONS}

A number of low power irradiations in the NASA Mock-Up Reactor were performed on simulated fuel pins. These irradiations enabled us to define the nuclear environment in which the actual pins will operate, and to modify that environment to achieve established criteria. Axial power density ratios were reduced from 2.5 to 1.5 and radial power density ratios were reduced from 1.6 to 1.3 . In addition to this, and 
we believe of significant importance, is the value of these measurements as a diagnostic tool for interpreting the results of the fuel pin performance. More fuel burnup will occur in the areas of higher power densities which in turn will result in greater fission product pressure on the clad.

This could result in premature failure of the fuel pin. By knowing the power densities at a 11 locations in the fuel pin, dimensional changes can be more accurately diagnosed. A dimensional change in a thermal reactor does not necessarily mean that the fuel pin would undergo the same change in the fast reactor because, as mentioned, the power gradients are not expected to be so severe in that application. 


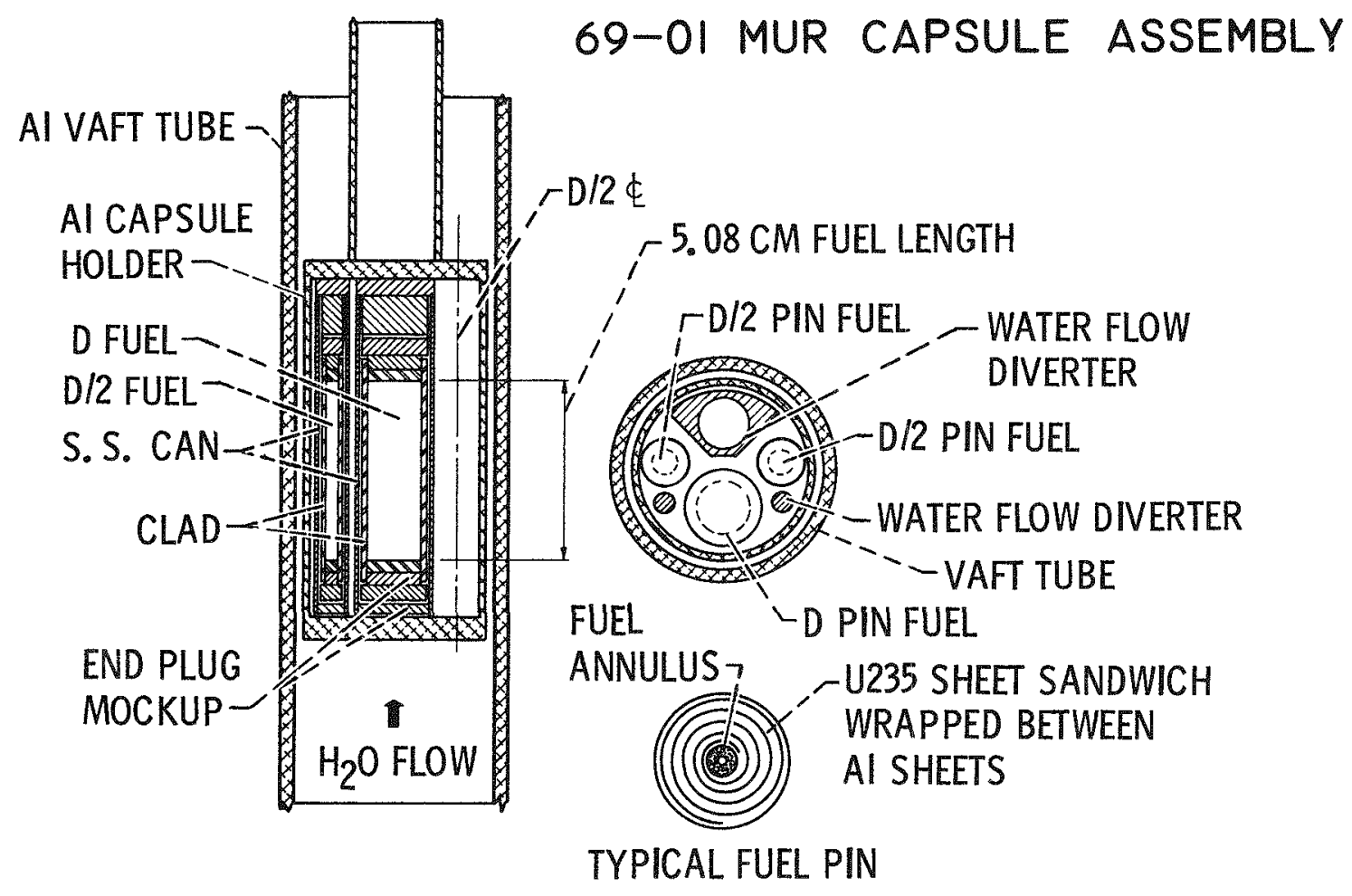

Figure 1

\section{9-OI MUR CAPSULE \\ POWER VS ENRICHMENT}

ENRICHMENT RATIO, D/2/D $\simeq 2.0$

AVERAGE POWER, W/CC

Figure 2

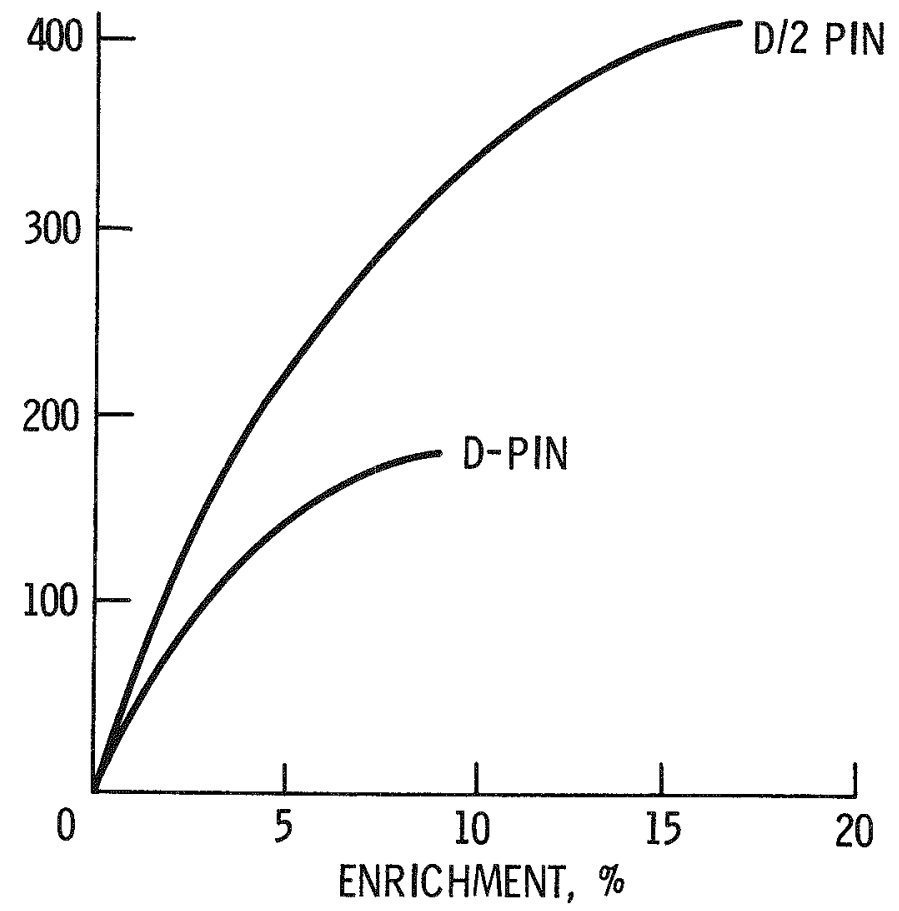


NONSHIELDED CAPSULE HOLDER

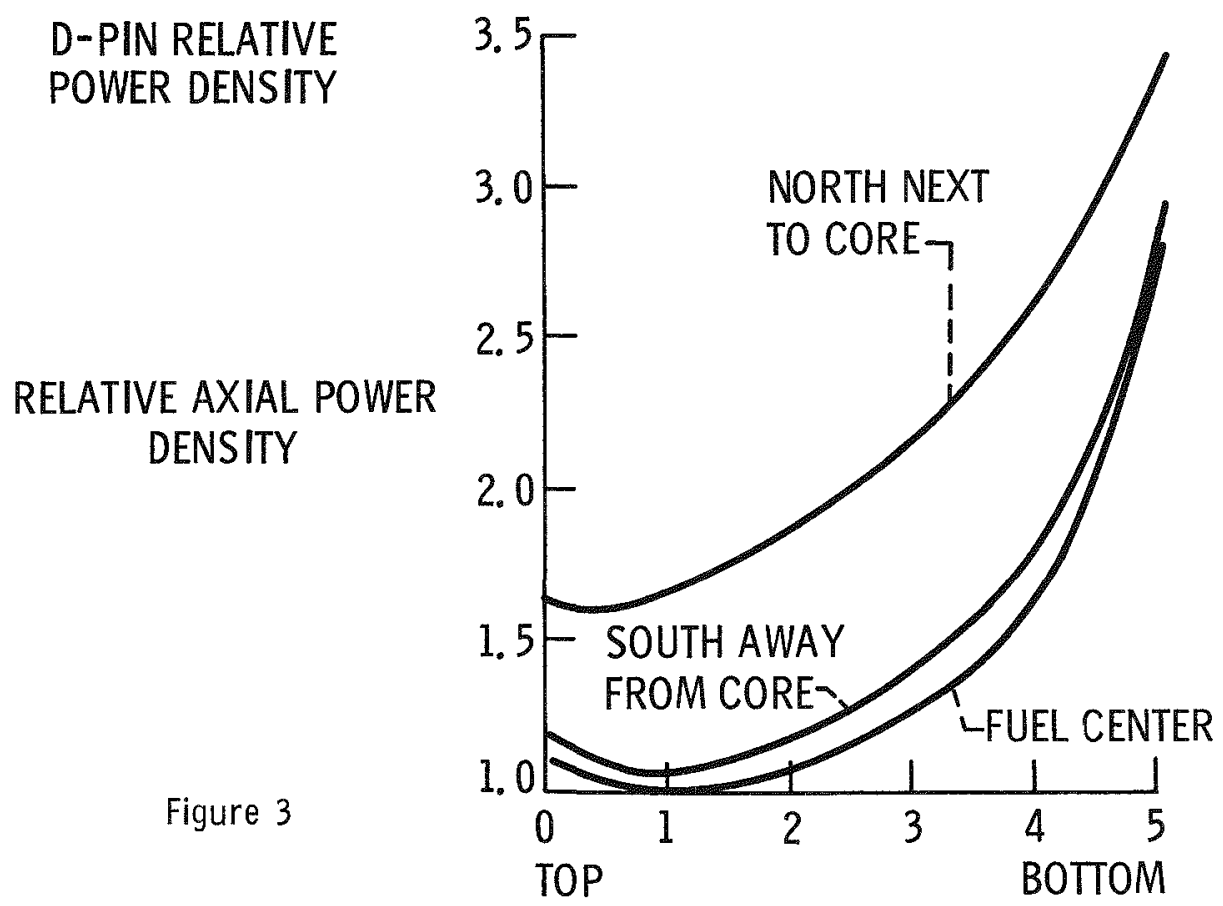

FUEL PIN LENGTH, CM

69-OI CAPSULE HOLDER SHADOW SHIELD

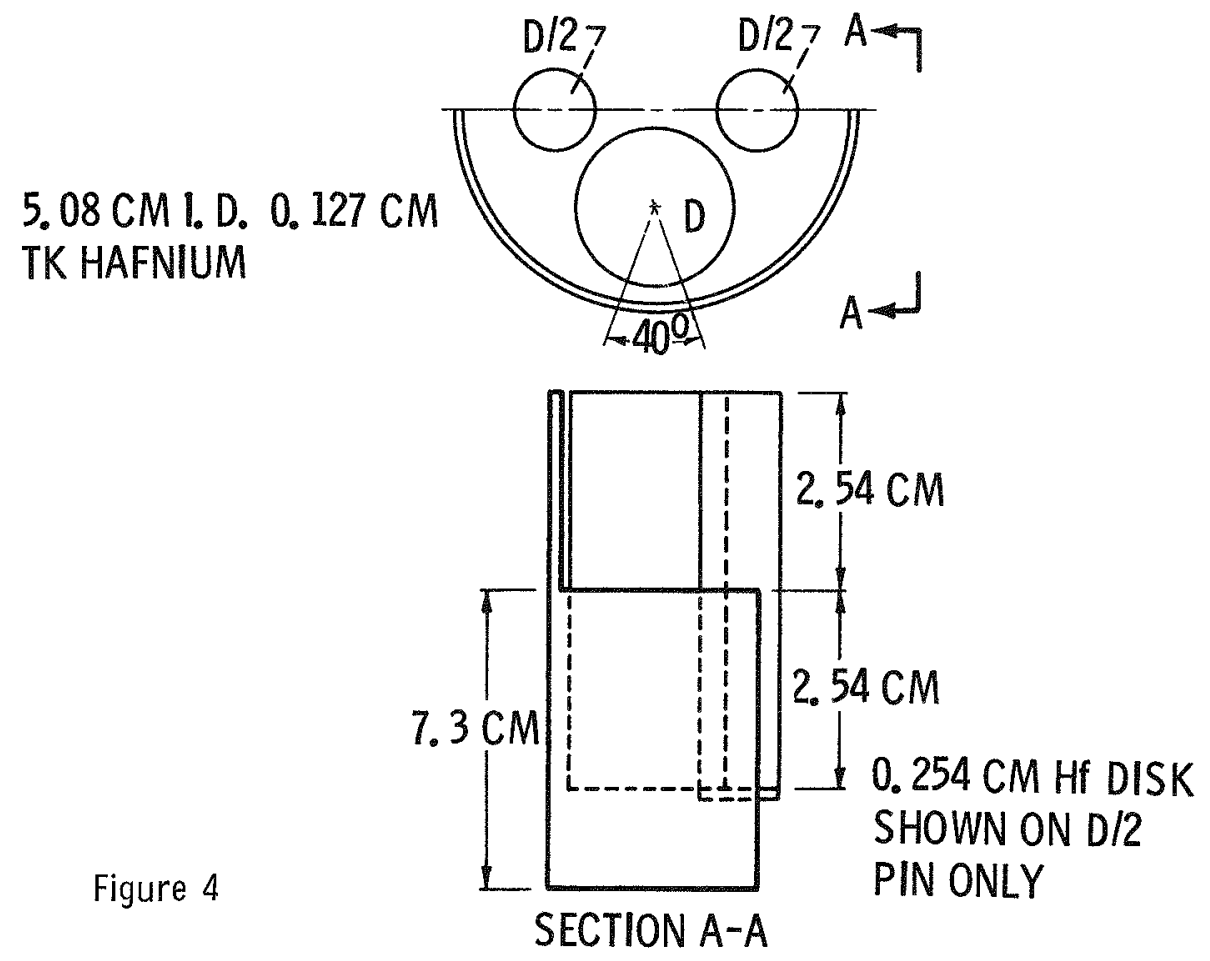




\section{SHIELDED CAPSULE HOLDER}

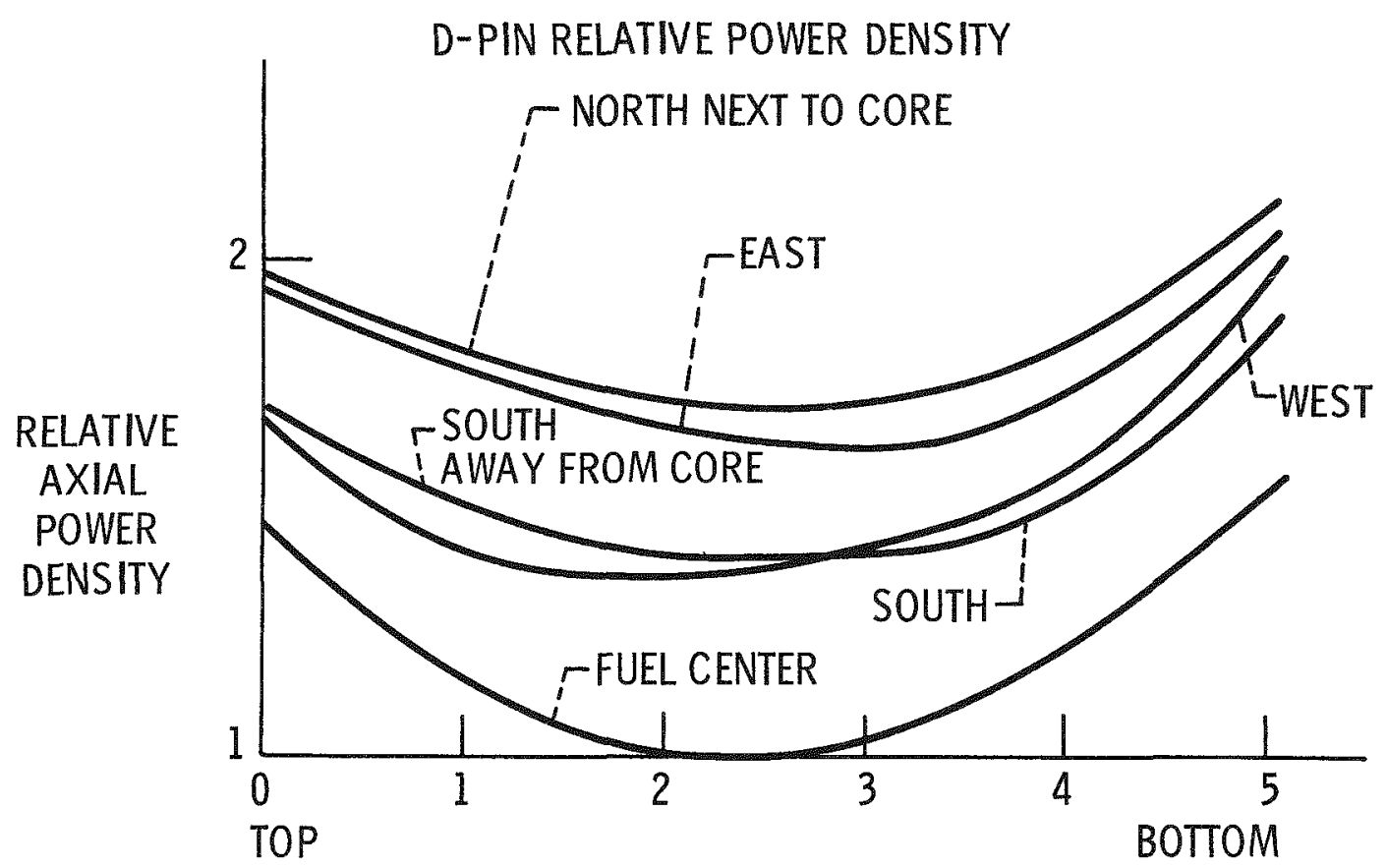

FUEL PIN LENGTH, CM

Figure 5

COMPARISON OF RELATIVE AXIAL POWER DENSITY WITH AND WITHOUT HAFNIUM SHIELD

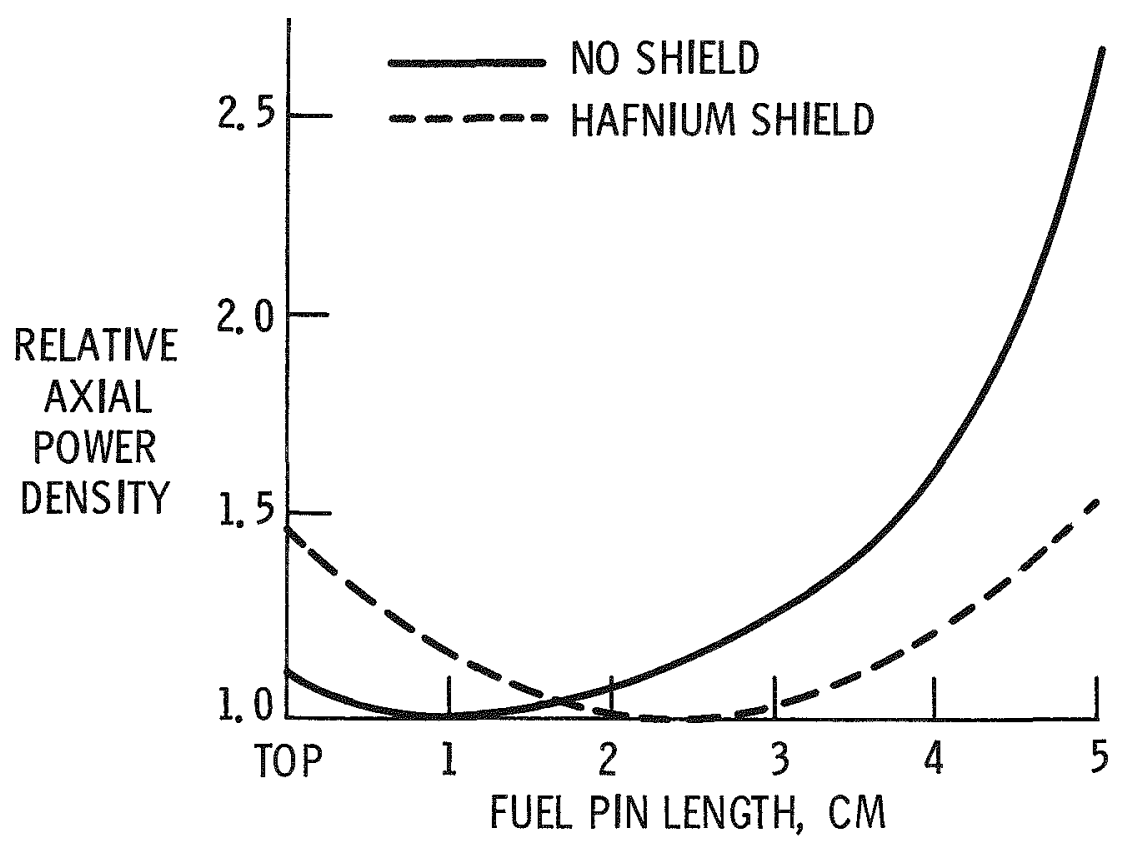

Figure 6 


\begin{abstract}
7) $x_{\text {CONCEPT FOR A GAS BUFFERED ANNULAR }}$ HEATPIPE FUEL IRRADIA TION CAPSULE*

$J$. D. Lee and R. W. Werner

Lawrence Radiation Laboratory, University of California

Livermore, California 94550
\end{abstract}

\begin{abstract}
A nuclear fuel irradiation capsule capable of handling large $\left(\approx 300 \mathrm{~W} / \mathrm{cm}^{2}\right)$ and varying fuel specimen heat fluxes while inherently maintaining nearly constant specimen temperature was conceived and two bench models successfully tested using potassium and sodium as the working fluids. Test temperatures were in the $700^{\circ} \mathrm{C}$ range and heat source temperatures were preserved within $\pm 15^{\circ} \mathrm{C}$ while undergoing factors of three changes in power.
\end{abstract}

\title{
Introduction
}

Part of the LRL Advanced Space Reactor Technology Program was a fundamental high temperature fue] swelling study. In support of this study a High Temperature Fuel Irradiation Capsule Development Program was initiated to provide the required fuel specimen environment.

Some of the more demanding specimen conditions were:

1. Temperatures in the 1000 to $2000^{\circ} \mathrm{C}$ range.

2. Temperature control and measurement accurate and dependable ( $t 1$ percent desired).

3. Specimen surface radial heat fluxes up to $\approx 300 \mathrm{~W} / \mathrm{cm}^{2}$.

4. Specimens and specimen train axially as isothermal as possible ( \pm 1 percent desired).

5. Specimen train $25 \mathrm{~cm}$ or longer.

6. Specimen time at temperature $>1000 \mathrm{hr}$.

It quickly became apparent that the standard binary gas controlled conduction type capsule could not satisfy items 2 thru 5 of the above conditions because of typical shifts in reactor flux profile during each cycle due to control rod movement. Figure 1 shows this profile shift

\footnotetext{
W Work performed under the auspices of the U. S. Atomic Energy Commission and published as UCRL-50510, July 25, 1968.
} 


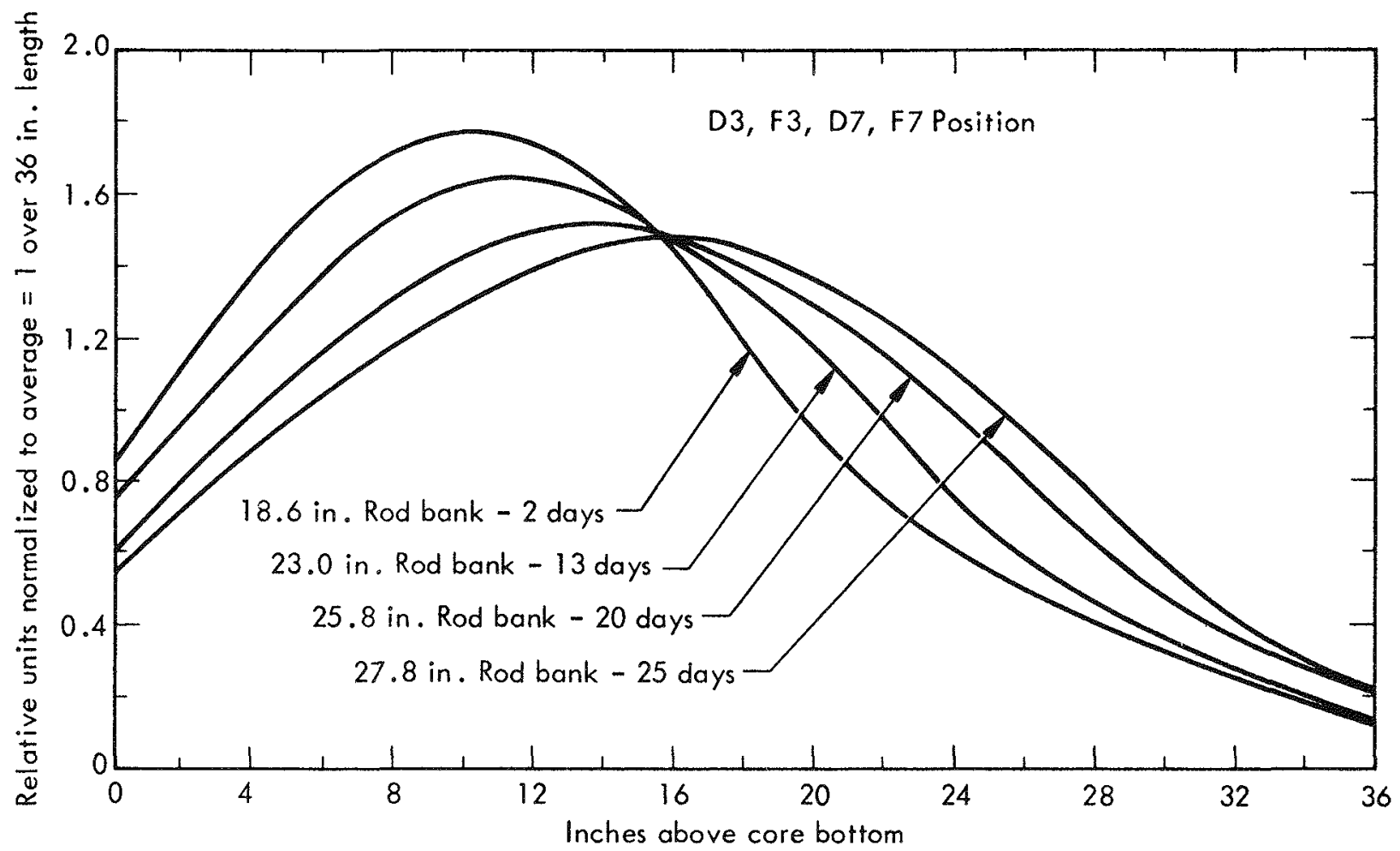

Fig. 1. Flux profile shift in General Electric test reactor. 
in GETR ${ }^{1}$ core capsule positions. Such shifts cause similar shifts in the specimen-train power profile and therefore create undesirable changes in axial temperature profiles of conduction type capsules. Uncertainties in unperturbed fluxes and capsule flux perturbation calculations and specimen swelling also create uncertainties in specimen temperatures.

The gas buffered annular heatpipe fuel irradiation capsule was conceived to approach the desired conditions under such adverse conditions.

\section{Description and Operation of Gas Buffered} Annular Heatpipe Capsule

Referring to Fig. 2, the gas buffered annular heatpipe capsule (AHP) basically consists of three concentric tubes, 2., 4. and 12. The center tube, 12., contains the fuel specimens, 13., (clad or unclad) being irradiated. These specimens are thermally coupled to the center tube i.d. with liquid metal. The center tube's o.d. is wrapped with a capillary wick structure, 11., (such as layers of screen) which supplies liquid metal from a liquid metal sump, 14.

Specimen fission heat is conducted to the wick's surface where it vaporizes liquid metal. The liquid metal vapor then flows radially and axially to the i.d. of the middle tube, 4., (which is also wrapped with screen) where it condenses. The latent heat of condensation is then conducted from the middle tube, across a helium gas gap, 3 ., to the outer containment tube, 2 , then into the reactor cooling water, 1. The condensed liquid metal returns to the evaporator wick via the sump or the condensate return insert, 9.

Specimen temperatures are maintained nearly constant over a wide range of total capsule power and power profiles by maintaining a constant vapor pressure in the annular heatpipe. As total capsule power varies, the vapor annulus length changes automatically, exposing required condenser area, thus rejecting the new heatload at the same temperature.

Vapor pressure in the annular heatpipe is maintained with a noncondensing gas buffer (such as argon). The buffer gas is kept out of the evaporator region of the heatpipe by the pumping action of the axial flowing and condensing vapor. Therefore, the vapor pressure in the evaporator region is equal to the total pressure. The pressure of the buffer gas can be controlled by connection to an external control system, or the pressure can be maintained as constant as required by providing enough free volume in the capsule itself, thus making the capsule self-contained as well as self-controlling.

Temperature sensors such as thermocouples are not required because the AHP temperature is exactly defined by the system pressure and 


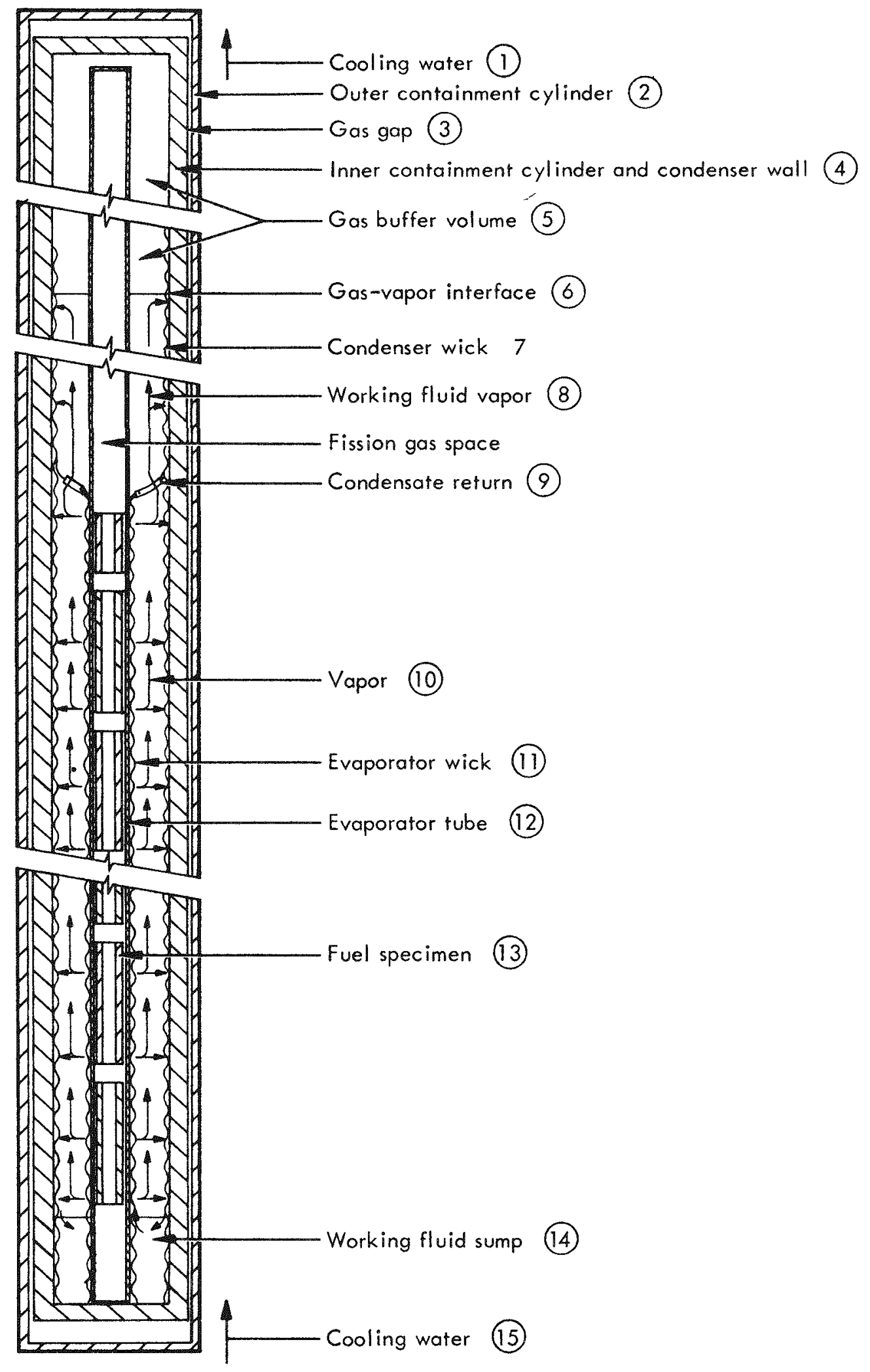

Fig. 2. A variable volume gas buffered annular heat pipe fuel irradiation capsule. 
the vapor pressure-temperature relationship of the working flund. The system pressure can be measured externally or maintained within desired limits inherently by design of an integral gas buffer.

This gas buffering principle, which stabilizes specimen temperatures against time variations in power profile can be illustrated schematically as shown in Fig. 3 .

Imagine a cylinder of relatively small volume, coupled to a container of inert gas. Let this cylinder be a heatpipe with fixed evaporator length $Z_{\mathrm{E}}$ This length relates to the specimen stack from which energy is being removed. Let the condenser length of the heatpipe, $\mathrm{Z}_{\mathrm{C}}$, be a variable and a function of the heat load from the specimens and the pressure of the vapor and the gas. At equilibrium for a given value of $Q$ input, there will be a particular value of $Z_{C}$ Consider a change in input power from $Q$ to $Q+\Delta Q$ below the burnout capacity. The change will cause the condenser length to adjust an appropriate amount $\Delta Z_{\mathrm{C}}$ to match the new load condition and preserve the temperature. Since the large gas volume effectively controls the system pressure, the temperature is stable. The gas, being non-condensible, is always pumped to the extreme condensing end of the heatpipe.

\section{Advantages of the Gas Buffered Annular Heatpipe Capsule}

1. Can handle high specimen heat fluxes allowing accelerated burnup rates.

2. Can maintain nearly constant desired specimen temperature under adverse conditions such as varying and uncertain flux profiles, uncertain flux perturbation calculations, and specimen swelling.

3. Can be made self-contained allowing capsule to be run in reactor locations where instrumentation leads are not allowed.

4. Can allow free-standing unrestrained specimen swelling if desired.

5. Has an inherent bullt-in temperature sensor (working fluid vapor pressure) eliminating dependence on undependable thermocouples.

6. Can make more efficient use of the reactor test holes by allowing longer specimen train lengths (compared to conduction type capsules) while still maintaining all specimens at the desired temperature

\section{Heat Transfer Limitations}

Standard (axial) "heatpipes" are concelvably limited by the following conditions:

1. Maximum capillary pumping.

2. Incipient boiling in the wick structure. 


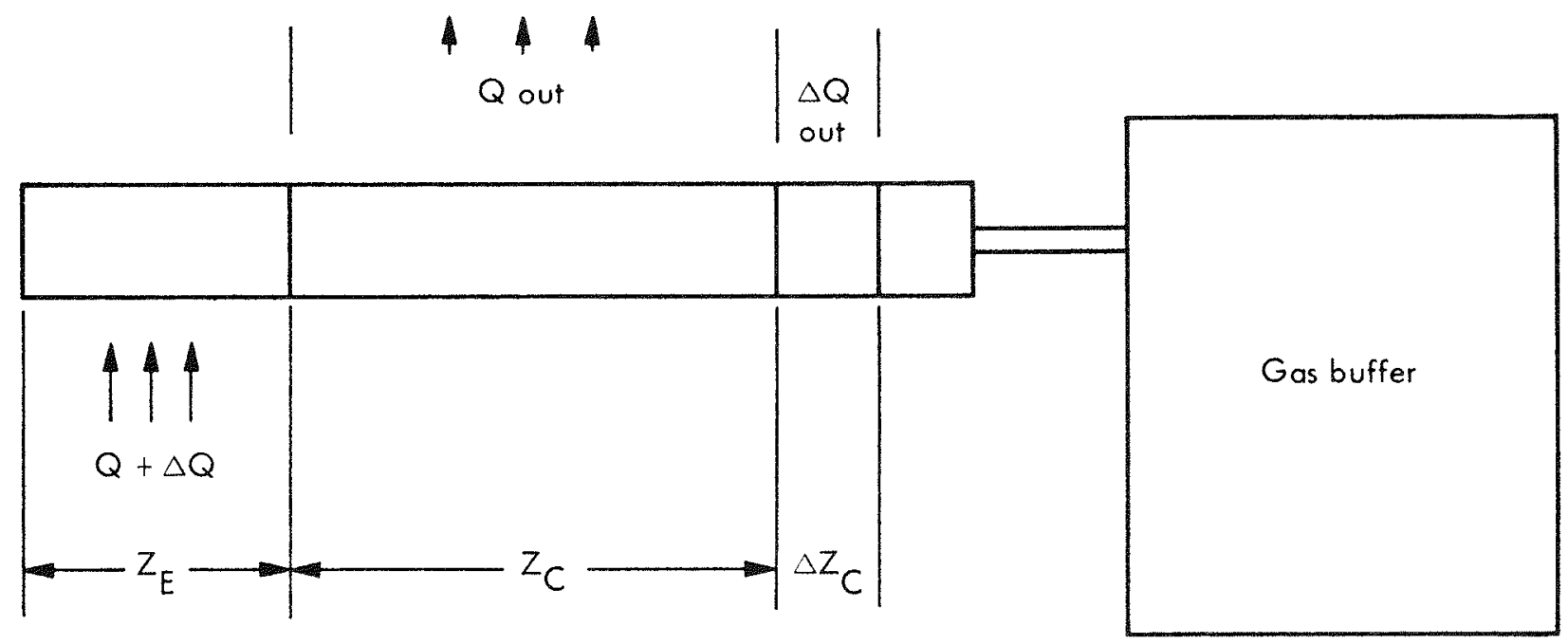

Fig. 3. Gas buffering principle. 
3. Vapor reaching sonic velocity.

4. The existence of an interfacial shear stress between the liquid in the wick and vapor such that as the counier flowing vapor velocity increases entrainment of the liquid in the vapor occurs.

The annular heatpipe, because of its geometry, is assumed to be

limited by only the first three conditions:
1 . The basic heatpipe equation as developed by Cotter ${ }^{2}$ states that the pressure rise of the capillary must be equal to or greater than the sum of the pressure drops because of vapor flow $\left(\Delta P_{v}\right)$, liquid flow $\left(\Delta \mathrm{P}_{\ell}\right)$, and gravity head $\left(\Delta \mathrm{P}_{\mathrm{g}}\right)$, that is:

$$
\Delta \mathrm{P}_{\mathrm{c}} \gtrsim \Delta \mathrm{P}_{\mathrm{v}}+\Delta \mathrm{P}_{\ell}+\Delta \mathrm{P}_{\mathrm{g}}
$$

In an annular heatpipe it is apparent that the pressure drop, because of viscous drag in the liquid, will be the same as that in a conventional pipe. Unlike the axial heatpipe case the flow of vapor in the annular pipe is substantially all radial and flows from a lesser to a larger surface area. This then is like free evaporation and the pressure drop in the vapor to a first approximation can be considered zero. Therefore, for the annular heatpipe:

$\Delta \mathrm{P}_{\mathrm{c}}=\frac{2 \cdot \gamma \cdot \cos \theta}{\mathrm{r}_{\mathrm{c}}}$

$\Delta \mathrm{P}_{\mathrm{g}}=\rho_{\mathrm{f}} \cdot \mathrm{g} \cdot \sin \phi$

$\Delta P_{\ell}=(b \cdot \eta \cdot Q \cdot l) /\left[2 \cdot \pi\left(r_{v}^{2}-r_{\mathrm{w}}^{2}\right) \rho_{\mathrm{f}} \cdot \mathrm{e} \cdot \mathrm{r}_{\mathrm{c}}^{2} \cdot \mathrm{L}\right]$

$\Delta \mathrm{P}_{\mathrm{V}} \cong 0$ (because of annular geometry)

where

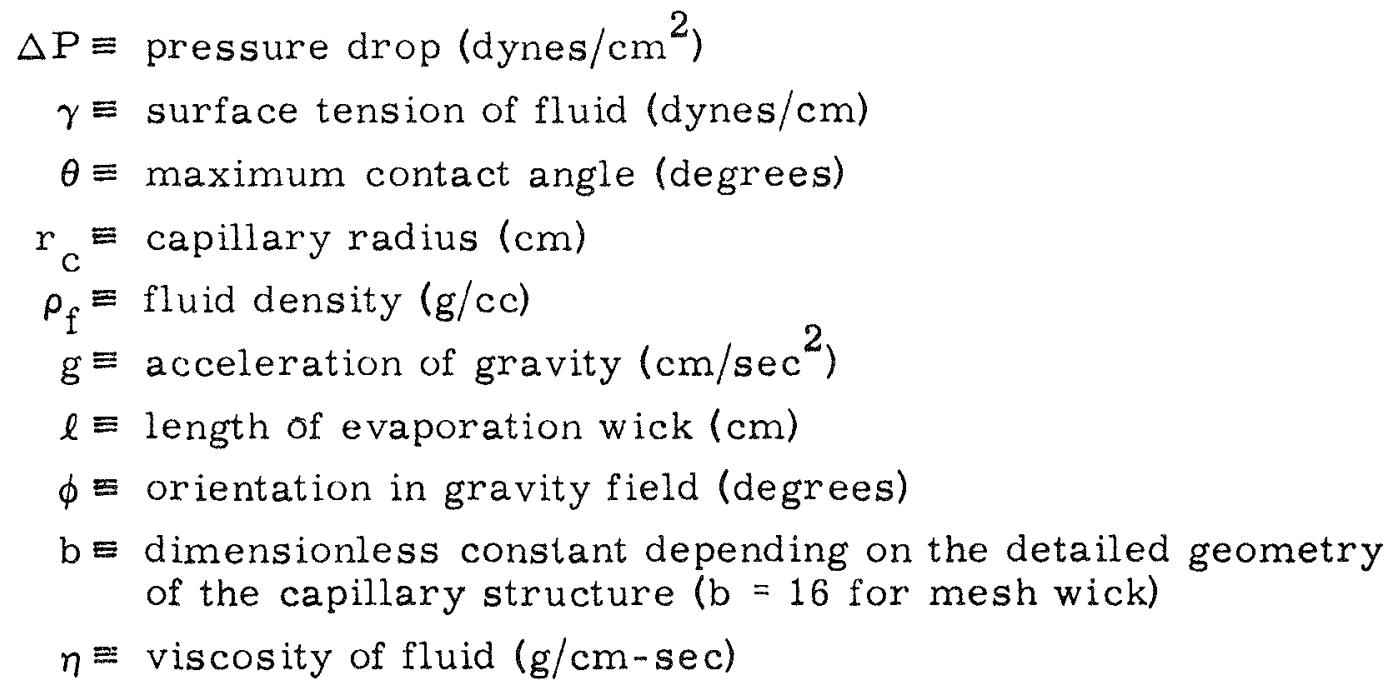




$$
\begin{aligned}
\mathrm{Q} & \equiv \text { heat transferred }(\mathrm{W}) \\
\mathrm{A} & \equiv \pi\left(\mathrm{r}_{\mathrm{V}}^{2}-\mathrm{r}_{\mathrm{W}}^{2}\right) \equiv \text { wick area }\left(\mathrm{cm}^{2}\right) \\
\mathrm{r}_{\mathrm{W}} & \equiv \text { inner radius of wick } \\
\mathrm{r}_{\mathrm{V}} & \equiv \text { outer radius of wick } \\
\mathrm{e} & \equiv \text { fraction of wick volume occupied by fluid } \\
\mathrm{L} & \equiv \text { latent heat of vaporization }(\mathrm{W}-\mathrm{sec} / \mathrm{g})
\end{aligned}
$$

Rearranging Eq. (1) and setting $\cos \theta=1$ gives

$$
Q_{\text {MAX }}=\left(\frac{2 \cdot \gamma \cdot 1}{r_{c}}-\rho \cdot g \cdot \ell \cdot \sin \phi\right)\left(\frac{\rho \cdot 2 \cdot A \cdot e \cdot r_{c}^{2} \cdot L}{b \cdot \eta \cdot \ell}\right)
$$

Equation (2) gives maximum heat transfer as limited by capillary pumping, pressure drop considerations. Addition of a condensate return insert at the top of the wick as shown in Fig. 2 should increase this limit somewhat if necessary.

2. Radial transfer of heat from the inner wall across the wick to the vapor liquid interface requires a temperature gradient across the the wick which in turn requires the liquid in the wick to be superheated. The upper limit of radial heat transfer is therefore defined by the maximum liquid super heat the wick can allow. Too much super heat would cause incipient boiling in the wick (burn out).

Maximum heat transfer as limited by super heat considerations is given by:

$$
\mathrm{Q}_{(\mathrm{SH} \text { MAX })}=\frac{\Delta \mathrm{T}_{(\mathrm{SH})} \cdot 2 \pi \cdot \mathrm{k} \cdot \ell}{\ln r_{\mathrm{V}} / r_{\mathrm{W}}}
$$

where

$$
\begin{aligned}
\Delta \mathrm{T}_{(\mathrm{SH})} & \equiv \text { maximum allowed super heat } \\
\mathrm{k} & \equiv \text { wick thermal conductivity. }
\end{aligned}
$$

Values of $\Delta T(\mathrm{SH})$ must be determined and experimentally verified for wick structures of interest. Values such as $20^{\circ} \mathrm{C}$ for $\mathrm{K}, 40^{\circ} \mathrm{C}$ for $\mathrm{Na}$ and $78^{\circ} \mathrm{C}$ for Li for LAP Surface Conditions were suggested in Ref. 3. An optimum QMAX would then be found by solving Eqs. (2) and (3) for the optimum wick thickness.

Tests of standard axial heat pipes have run successfully with high radial heat fluxes (in the 400 to $500 \mathrm{~W} / \mathrm{cm}^{2}$ range) indicating that similar radial heat fluxes should be obtainable in the annular geometry. 
3. The third limitation, vapor reaching sonic velocity, is a conditional limitation in that given a desired operating temperature (which in turn defines the vapor pressure for a given fluid) maximum heat transfer would be limited by a flow rate somewhat less than sonic.

Unlike the capillary pumping and wick boiling limits, which if exceeded cause the heat pipe to fail, exceeding the heat transfer limit imposed by the sonic velocity only causes the vapor pressure and temperature to rise above that desired.

\section{Bench Model Tests}

To verify the concept and operating characteristics of an annular heatpipe two series of tests were run.

A test model annular heatpipe was designed and built to use potassium or sodium as the working fluid. A surface heat flux of $\sim 100 \mathrm{~W} / \mathrm{cm}^{2}$ was obtainable with electrical heaters. Maximum design temperature was $1000^{\circ} \mathrm{K}$. Means of measuring wick temperatures, heater temperature, heat input and output and system pressures were provided.

The annular heatpipe test model (Fig. 4) is made up of two concentric, cylindrical, stainless steel (type 316$), 45 \mathrm{~cm}$ long tubes. The center tube $(2.67 \mathrm{~cm}$ o.d. $\times 0.165 \mathrm{~cm}$ wall) contains the electrical heater. The heater is thermally bonded to the tube with liquid potassium or sodium under sufficient pressure to preclude its boiling. Stainless steel screen (five layers of $100 \times 100 \times 0.003 \mathrm{in}$. screen) is wrapped around the o.d. of the center tube and spot welded. This screen forms the capillary structure which supplies liquid metal to the evaporator (heat source) region of the center tube. The liquid metal evaporates and flows radially and axially to the inside wall of the outer tube where it condenses and returns by capillary flow to the evaporator. The condenser is also covered with stainless steel screen. The heat is removed from the condenser by conduction across a $0.075 \mathrm{~cm}$ helium gap to a water cooled jacket. A schematic of the system is shown in Fig. 5.

Initial tests on the annular heatpipe were conducted at temperature levels of $830^{\circ} \mathrm{K}, 920^{\circ} \mathrm{K}$, and $970^{\circ} \mathrm{K}$ with potassium and a $10 \mathrm{~cm}$ heater. Six independent test runs were made. Run No. 1 was a nondata gathering run at low power $(400 \mathrm{~W})$ for system "bake out" and check out. Data was obtained during runs $2,4,5$, and 6 . Run 3 was for demonstration only. Total time with power on was approximately six hours.

Figure 6 is a reproduction of a portion of the two strip charts which recorded temperatures observed by evaporator thermocouples 2 and 3 (see Fig. 4). Note that as the power was increased from $1500 \mathrm{~W}$ to $3000 \mathrm{~W}$ the temperature of both thermocouples 2 and 3 remained substantially constant at $870^{\circ} \mathrm{K}$, changing only about 5 or $6^{\circ}$, or less than one percent. Figure 6 suggests that operation in the heatpipe regime is not accomplished until the power input is at least $1500 \mathrm{~W}$. This 


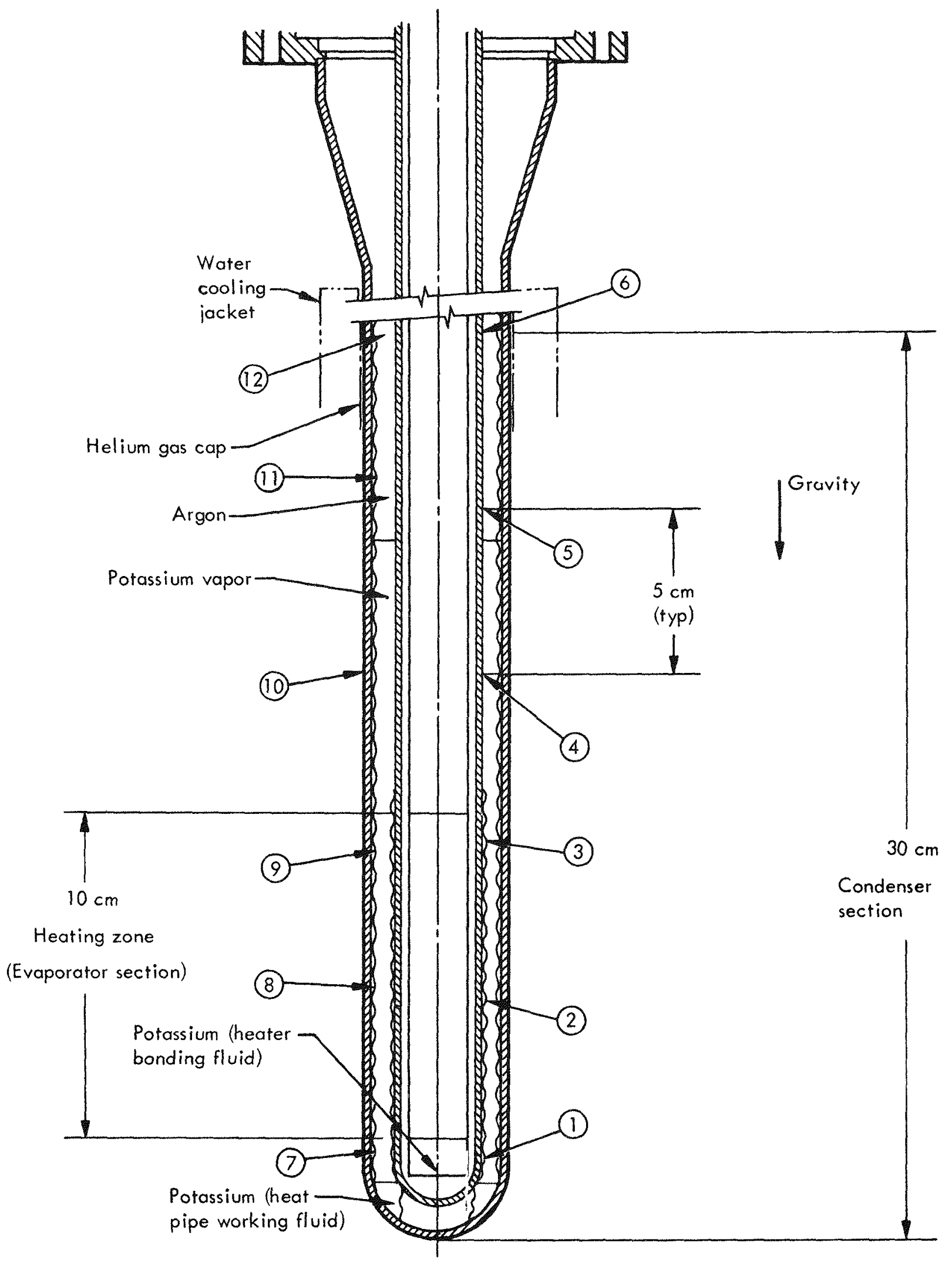

Fig. 4. Sketch of annular heatpipe. Circled numbers indicate location of thermocouples. 


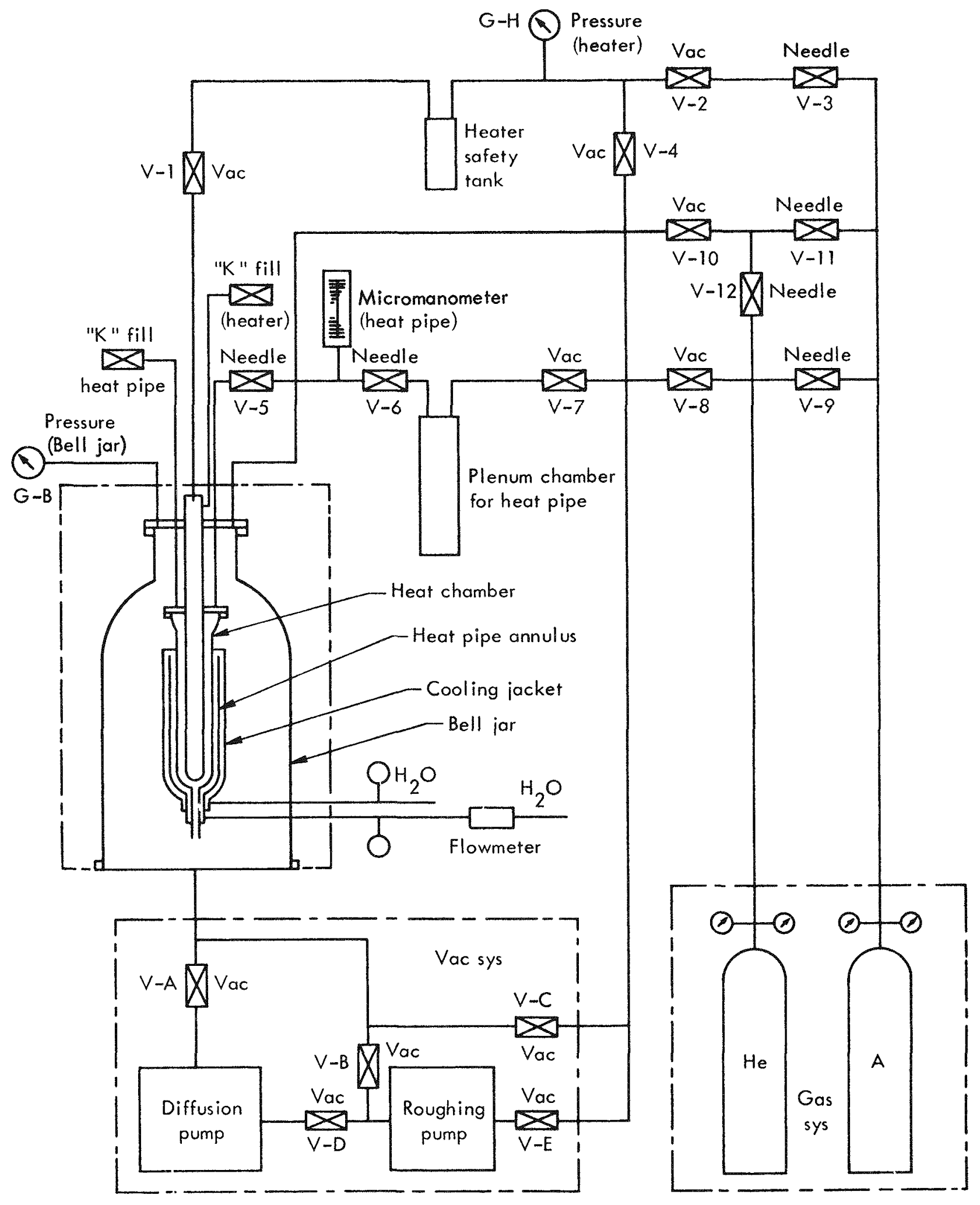

Fig. 5. Schematic diagram of annular heatpipe experimental arrangement. 

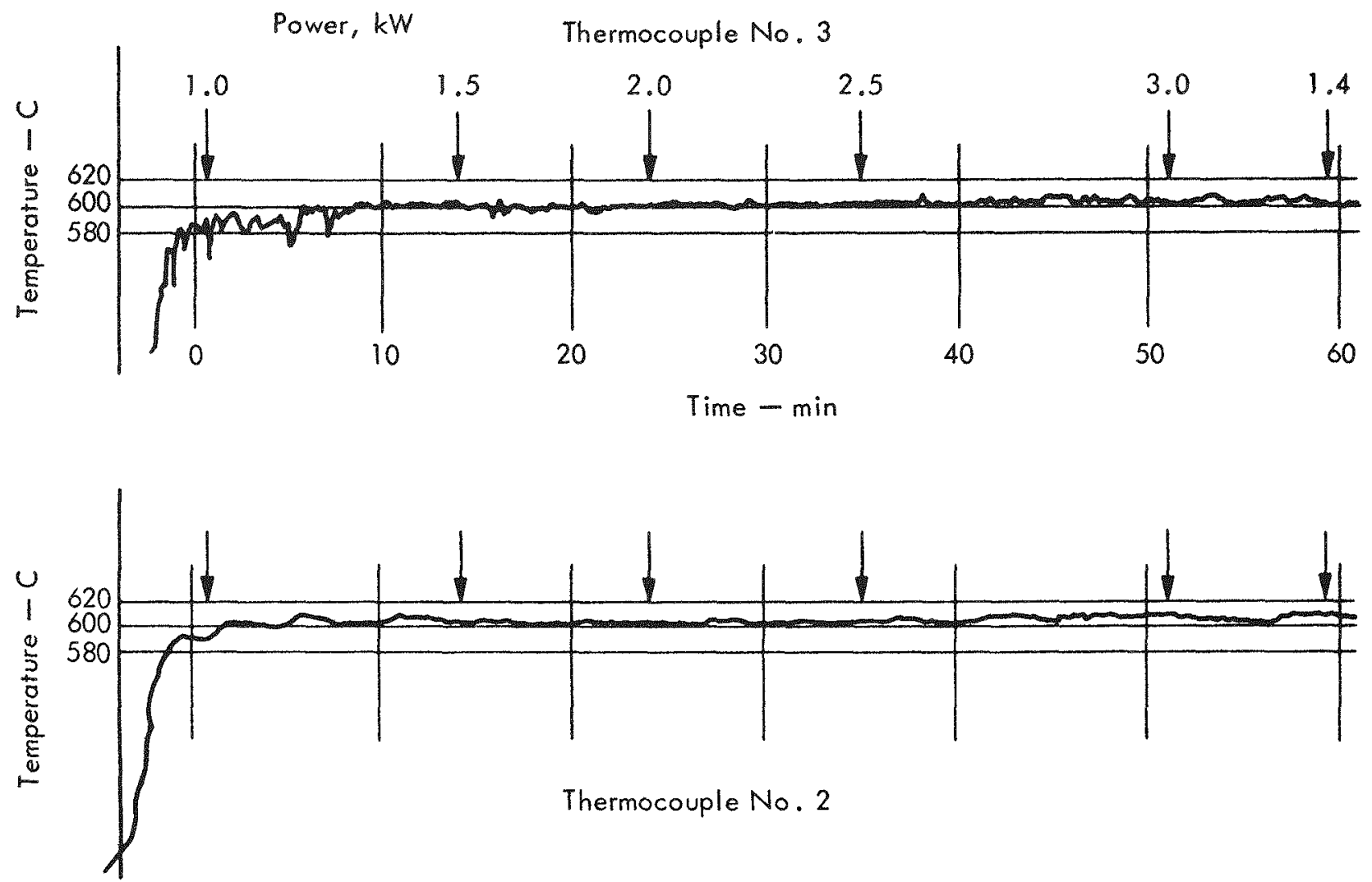

Fig.6. Reproduced temperature traces of thermocouples 2 and 3 during run No. 4 of the annular heatpipe with gas plenum. Working fluid was potassium and the plenum gas was argon. 
observation is substantiated in Fig. 7 where thermocouples 8 and 9, and the first two in the condenser section did not come into temperature agreement with the evaporator couples 2 and 3 until $1800 \mathrm{~W}$ of power input. Figure 7 shows how the vapor gas interface moves up the heatpipe as a function of power. Figure 8 demonstrates the effect of power input on isothermal length and the ability to preserve constant operating temperature. Figure 9 is a comparison of the pressures observed by a micromanometer connected to the heatpipe with the vapor pressure curve for potassium. (See Ref. 4)

Burn out at the top of the evaporator wick occurred at $\sim 3000 \mathrm{~W} . \mathrm{Q}_{\text {MAX }}$ calculated with Eq. (2) was $2650 \mathrm{~W}$.

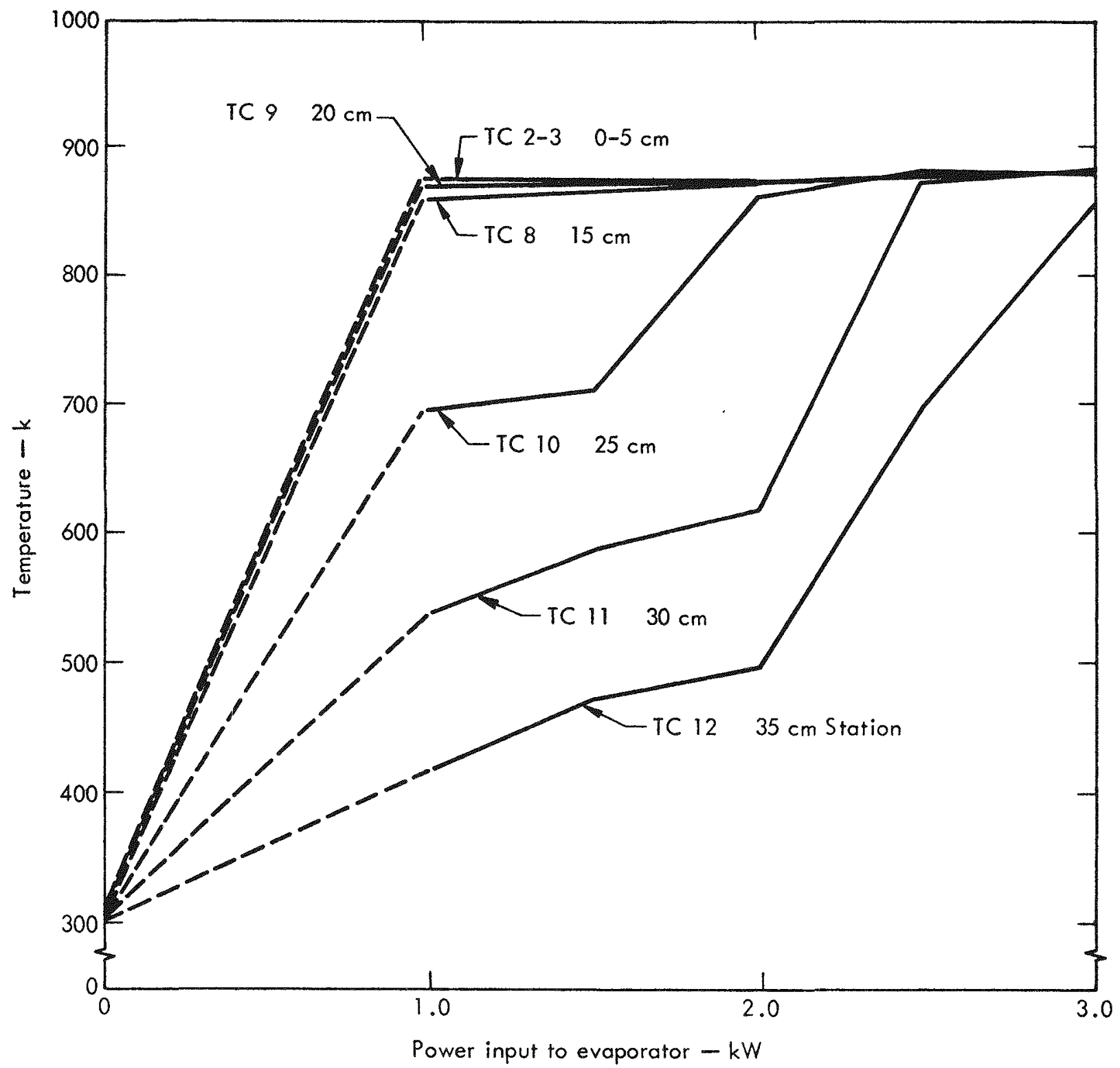

Fig. 7. Heatpipe with gas plenum. Run No. 4. Temperature at stations along heatpipe as a function of power. 


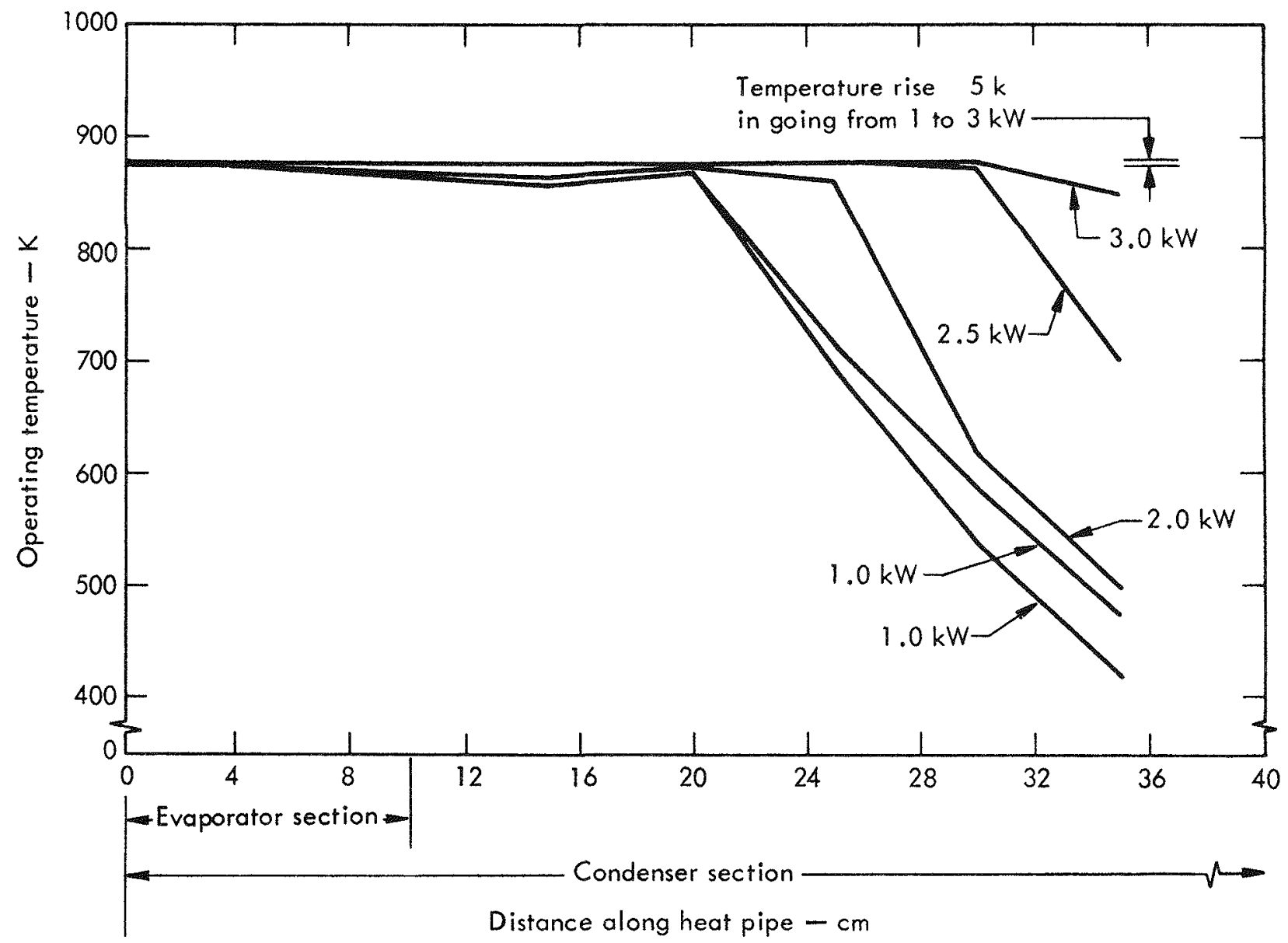

Fig. 8. Annular heatpipe with gas plenum. Run No.4. Effect of power input on isothermal length and ability to preserve constant operating temperature 


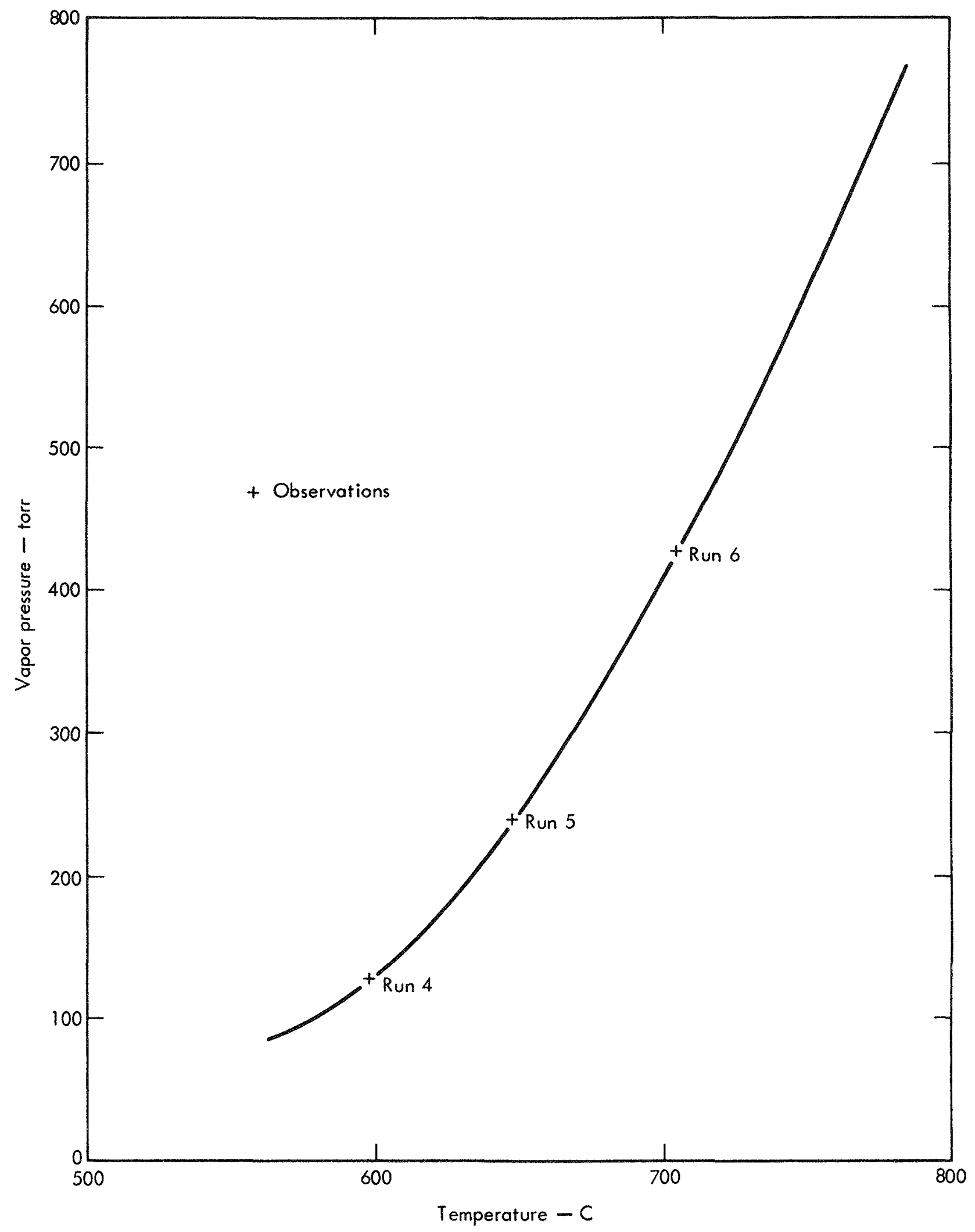

Fig. 9. Annular heatpipe with gas plenum. Comparison of experimental vapor pressures from potassium heatpipe micromanomete $r$ readings with consolidated data from Ref. 4. 


\section{Test Series No. 2}

The second series of tests was conducted with sodium as the working fluid and a $20 \mathrm{~cm}$ long heater. Thermocouples ( $\mathrm{T} \mathrm{C}^{\prime} \mathrm{s}$ ) were also placed in the heater bonding fluid to monitor heater temperature.

As in the first series this series of tests displaced the existence of the vapor-gas interface and as before the measured vapor temperatures and the measured buffer gas pressure were in agreement with the working fluids (sodium) vapor pressure-temperature relationship.

Figures 10 and 11 are copies of the strip chart records taken during a typical run of two T C's (A and $E$ ) placed in the heater bonding fluid. $\mathrm{T} C \mathrm{No}$. A was located near the middle of the heater while T C No. E was $\approx 3 \mathrm{~cm}$ above the heater.

The T C No. A record is an excellent display of the annular heatpipe's ability to maintain a heat source at nearly a constant temperature while its power varies over a wide range $(2000 \mathrm{~W}$ to $6000 \mathrm{~W}$ in this case). (Fig. 10.)

Predicted burn out of the evaporator wick was calculated with Eq. (2) to occur at $5830 \mathrm{~W}$. Observed burn out occurred between $5500 \mathrm{~W}$ and $6000 \mathrm{~W}$ as indicated by T C No. E's temperature rise (Fig. 11).

The temperature response to pressure changes was also tested during this run. Note the (Fig. 10) pressure changes from 72 torr to 152 torr (after an over shot) then later to 52 torr. The $\Delta \mathrm{T}$ response to these pressure changes followed the changes in the vapor temperature exactly.

In another test run a power ramp was applied (from 0 to $5000 \mathrm{~W}$ in about four sec) to the annular heatpipe at room temperature (work fluid in solid state) to observe its temperature response. Figures 12 and 13 show $\mathrm{T} \mathrm{C}^{\prime} \mathrm{s} \mathrm{A}$ and $\mathrm{E}$ measurement of this response. The $5000 \mathrm{~W}$ power level was maintained for three hours with no ill effects showing up.

The final test run was a $150-\mathrm{hr}$ endurance $\mathrm{run}$ at $5000 \mathrm{~W}$ and $700^{\circ} \mathrm{C}$ during which time the AHP performed flawlessly. At the end of $150 \mathrm{hr}$ power was increased to $5200 \mathrm{~W}$, then $5500 \mathrm{~W}$ and finally to $5800 \mathrm{~W}$ where burn out occurred as before. 


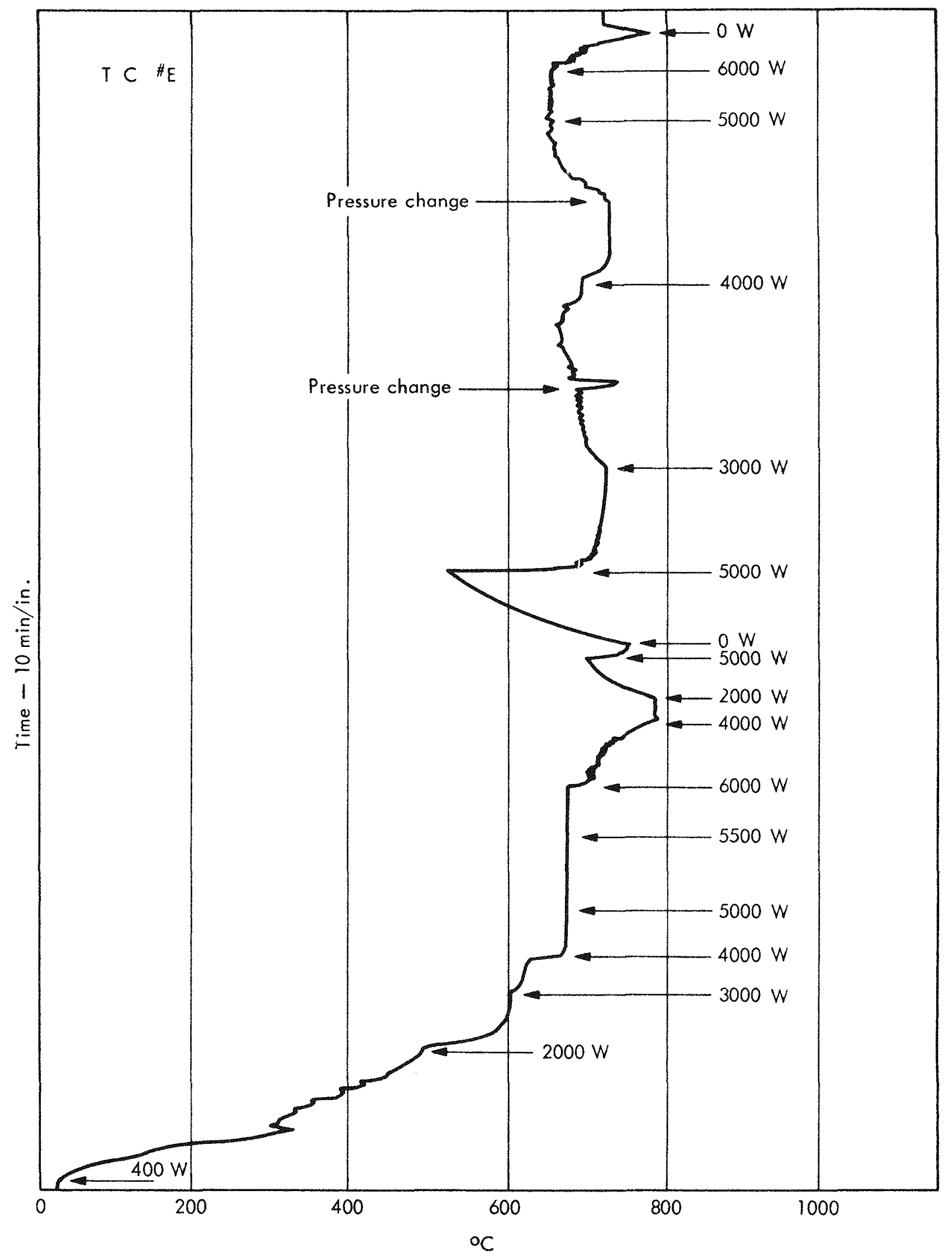

Fig. 10. Heater T C (No, A) temperature record. 


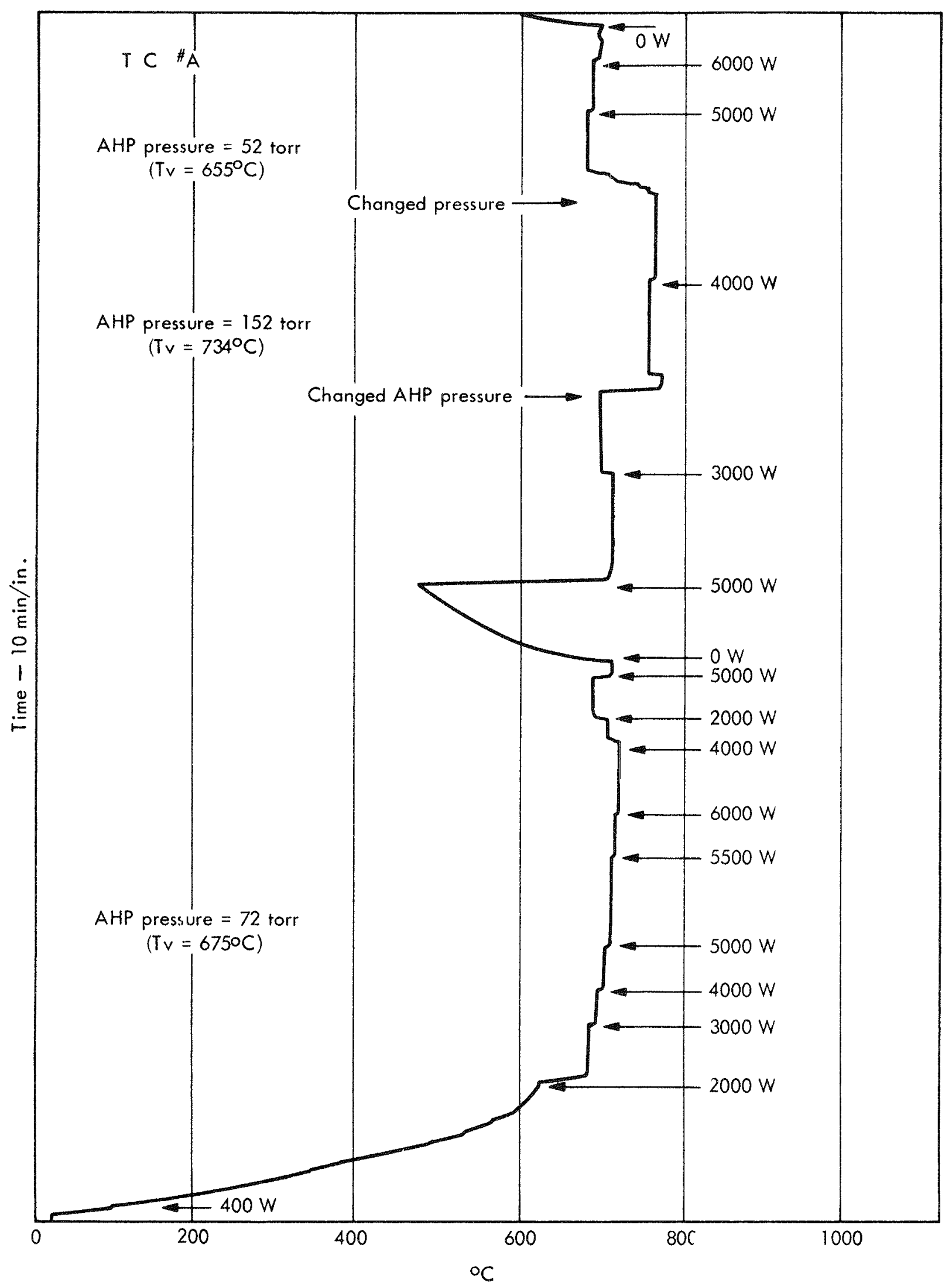

Fig. 11. Heater T C (No. E). 


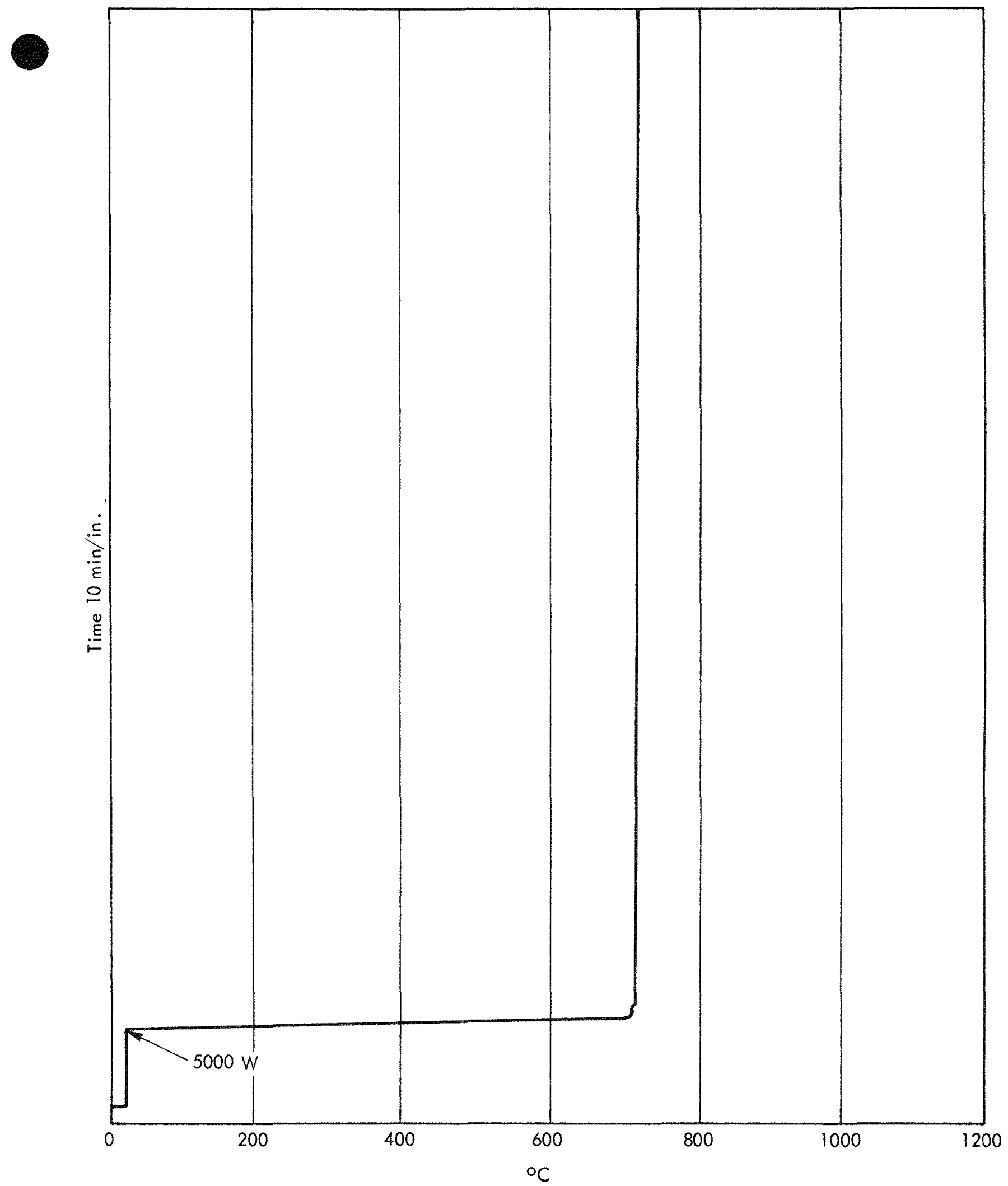

Fig. 12. Heater $\mathrm{T} \mathrm{C}$ (No. A). Response to a fast power ramp $(0-5000 \mathrm{~W}$ in $\sim 4 \mathrm{sec})$. 


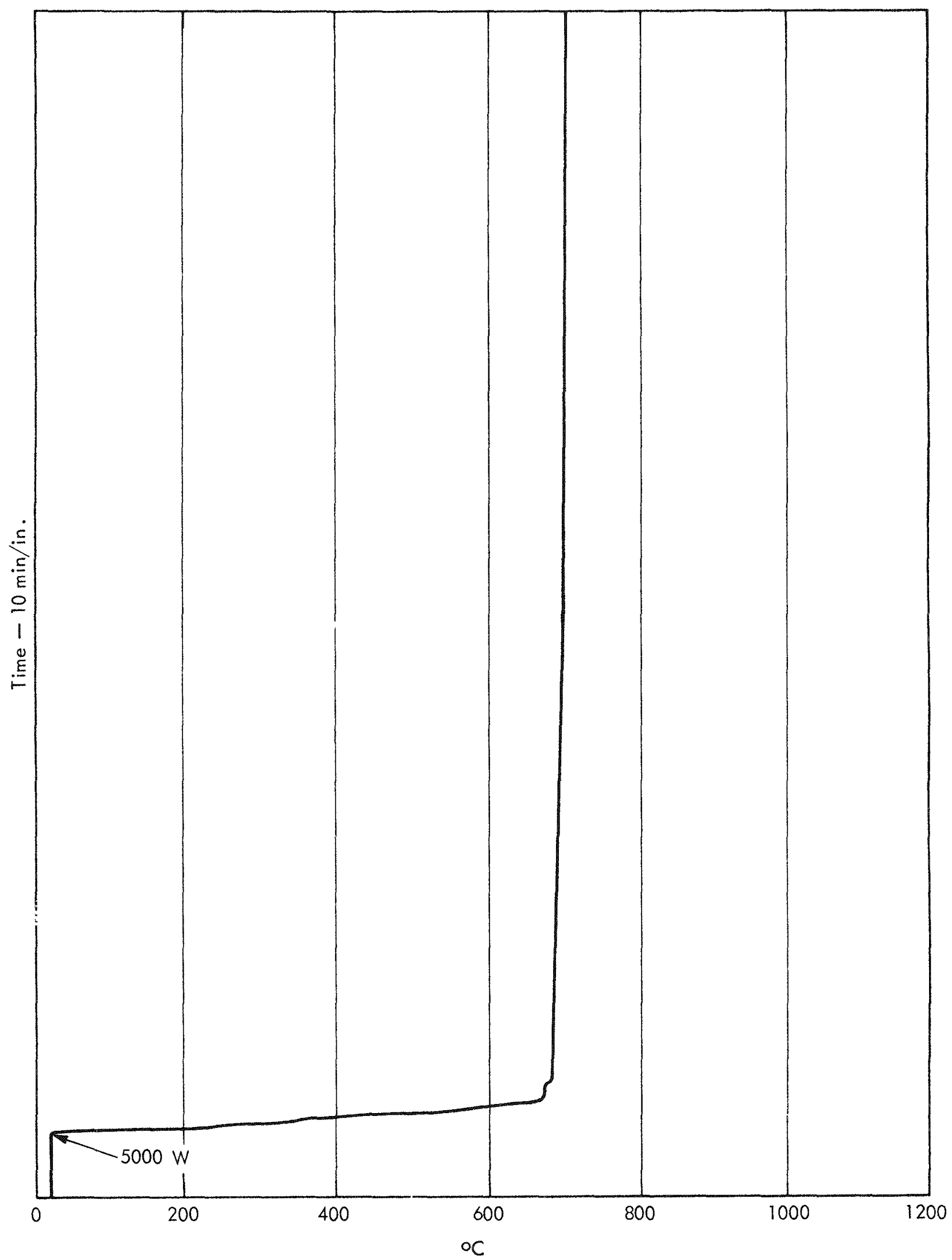

Fig. 13. T C (No, E). Response to a fast power ramp $(0-5000 \mathrm{~W}$ in $\sim 4$ sec $)$. 


\section{Conclusion}

Testing of the bench model successfully demonstrated the concept of the gas buffered annular heatpipe and displayed the concept's potential for in-pile capsule testing of nuclear fuels and materials.

By proper choice of working fluids and materials, tests over a wide temperature range $\left(\approx 900-2500^{\circ} \mathrm{K}\right)$ with this capsule concept are possible.

Before a specific capsule can be committed to long term in-pile tests the question of mass transport of the wick material by the working fluid and of the working fluid by diffusion in the buffer gas must be resolved as well as capillary pumping and wick boiling limitations.

Because of termination of the LRL reactor program, work has now stopped on implementing the gas buffered annular heatpipe fuel irradiation capsule concept. It is hoped that others in the fuel development field might find this capsule concept useful and complete its development.

\section{References}

1. General Electric Test Reactor Applications Data and Customer Service Guide AP10-1021 Class I (General Electric, April 1966).

2. T. P. Cotter, Theory of Heat Pipes, Los Alamos Scientific Laboratory, Los Alamos, New Mexico, Rept. LA-3246-MS (1965).

3. R. W. Werner, Service Operating Limits on Heat Pipes, Lawrence Radiation Laboratory, Livermore, Rept. SPN 293 (1968).

4. J. L. Watts, High Temperature Properties of Liquid Metals, Lawrence Radiation Laboratory, Livermore, Rept. SPN 227 (1967).

Appendix A

A Pictorial Record of the Program 


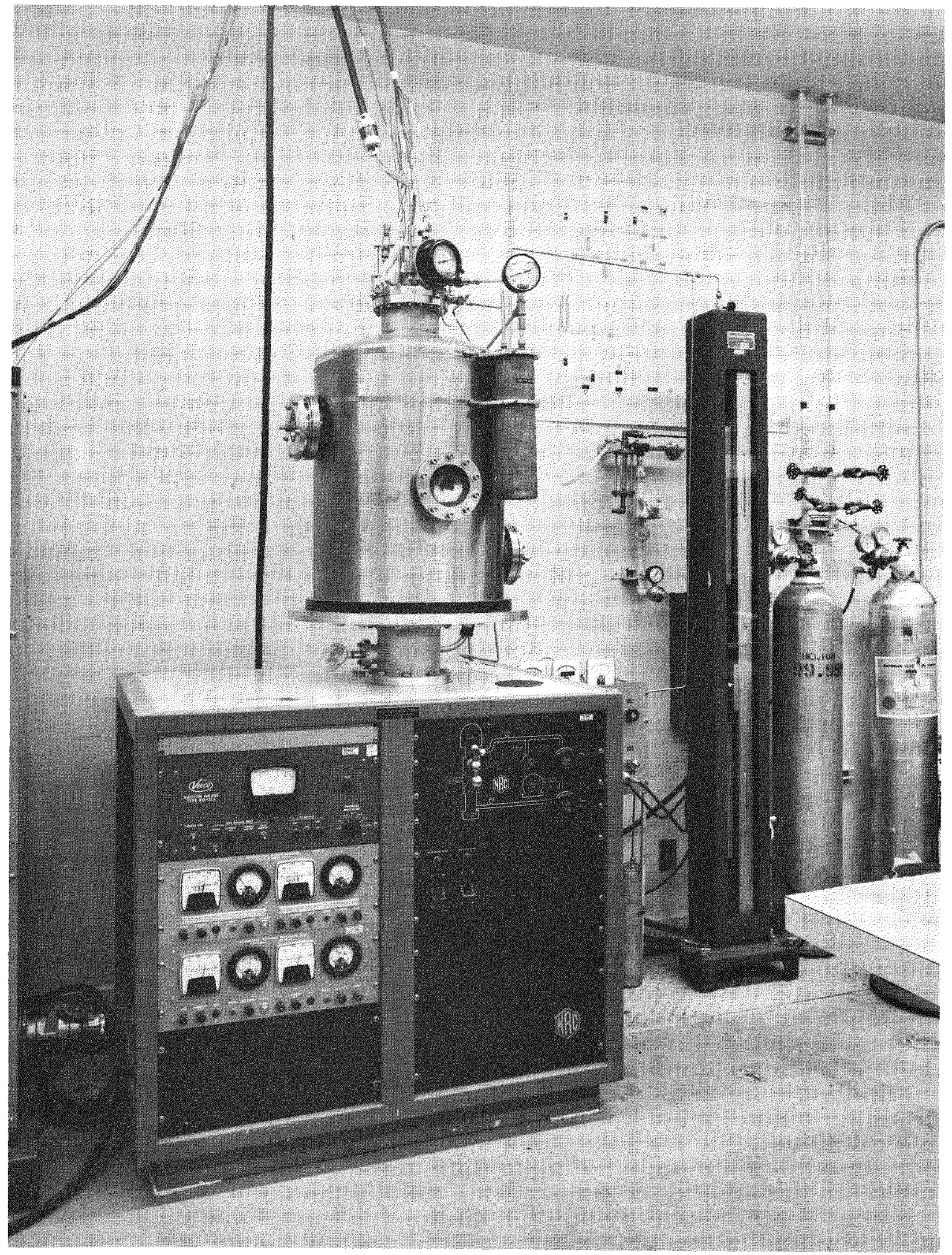

Fig. A-1. Facility ready for testing. 


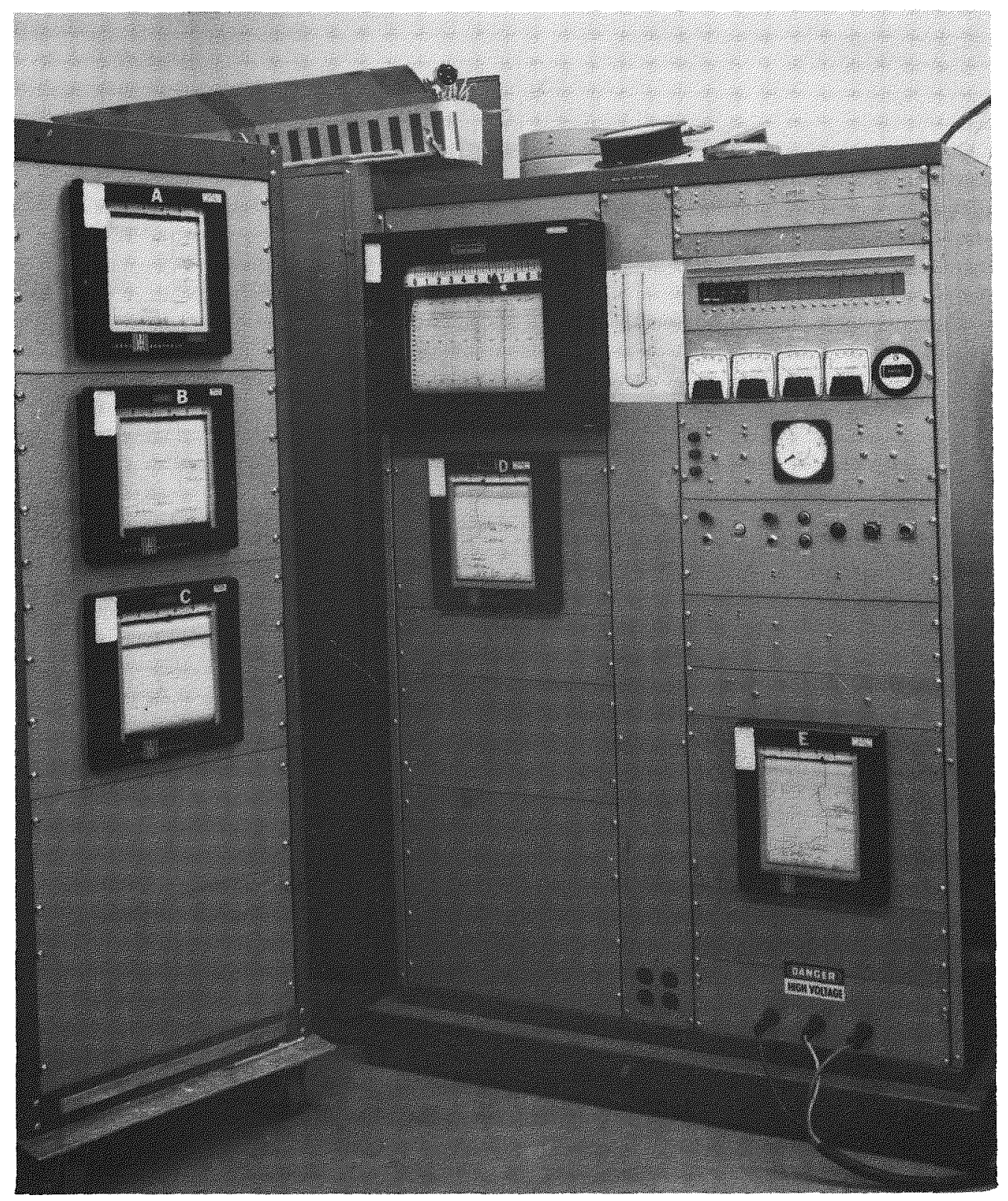

Fig. A-2. Facility ready for testing. 


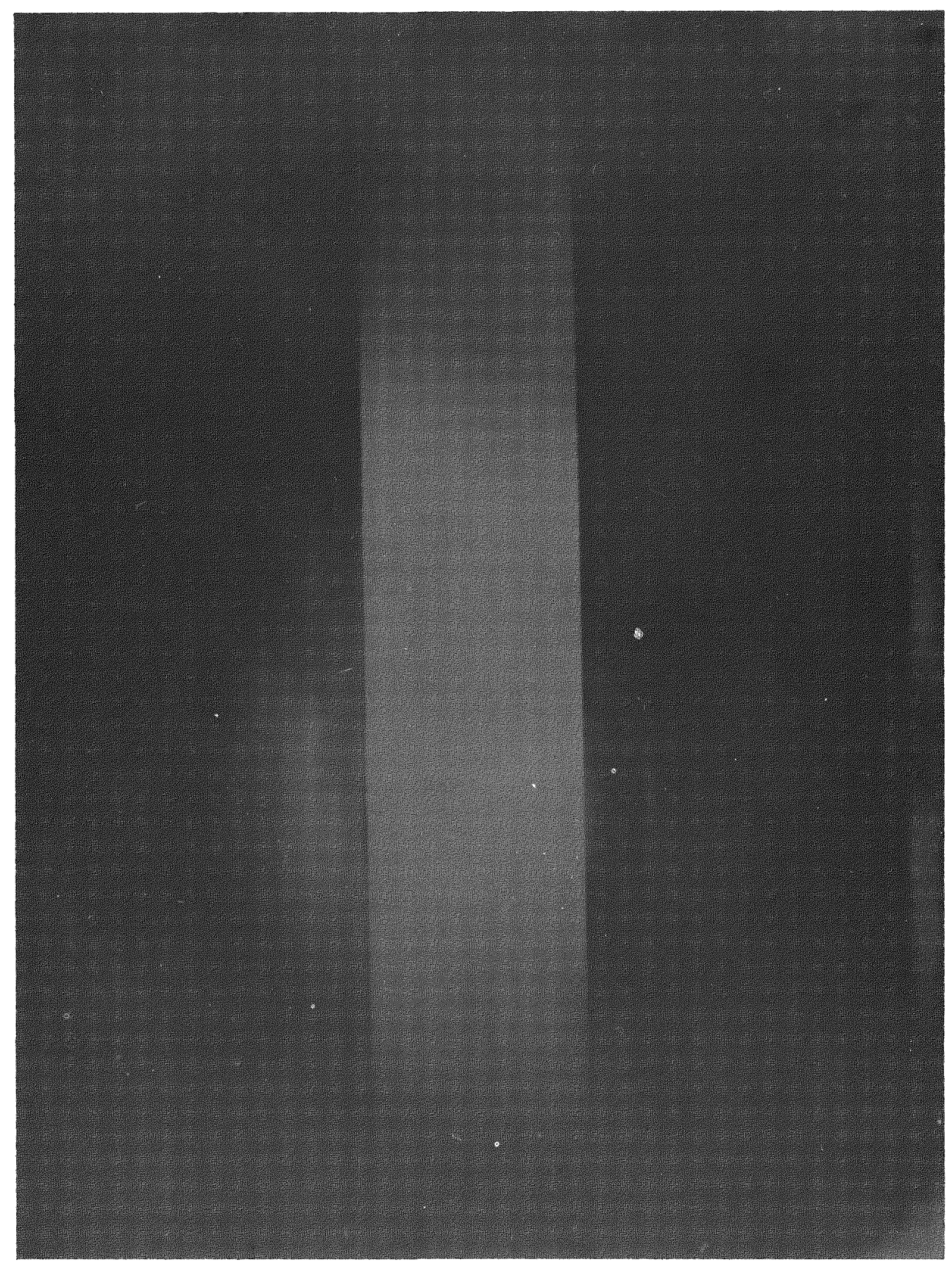

Fig. A-3. Self-illumination pictures of the test model annular heatpipe operating without its water jacket and at low power levels. 


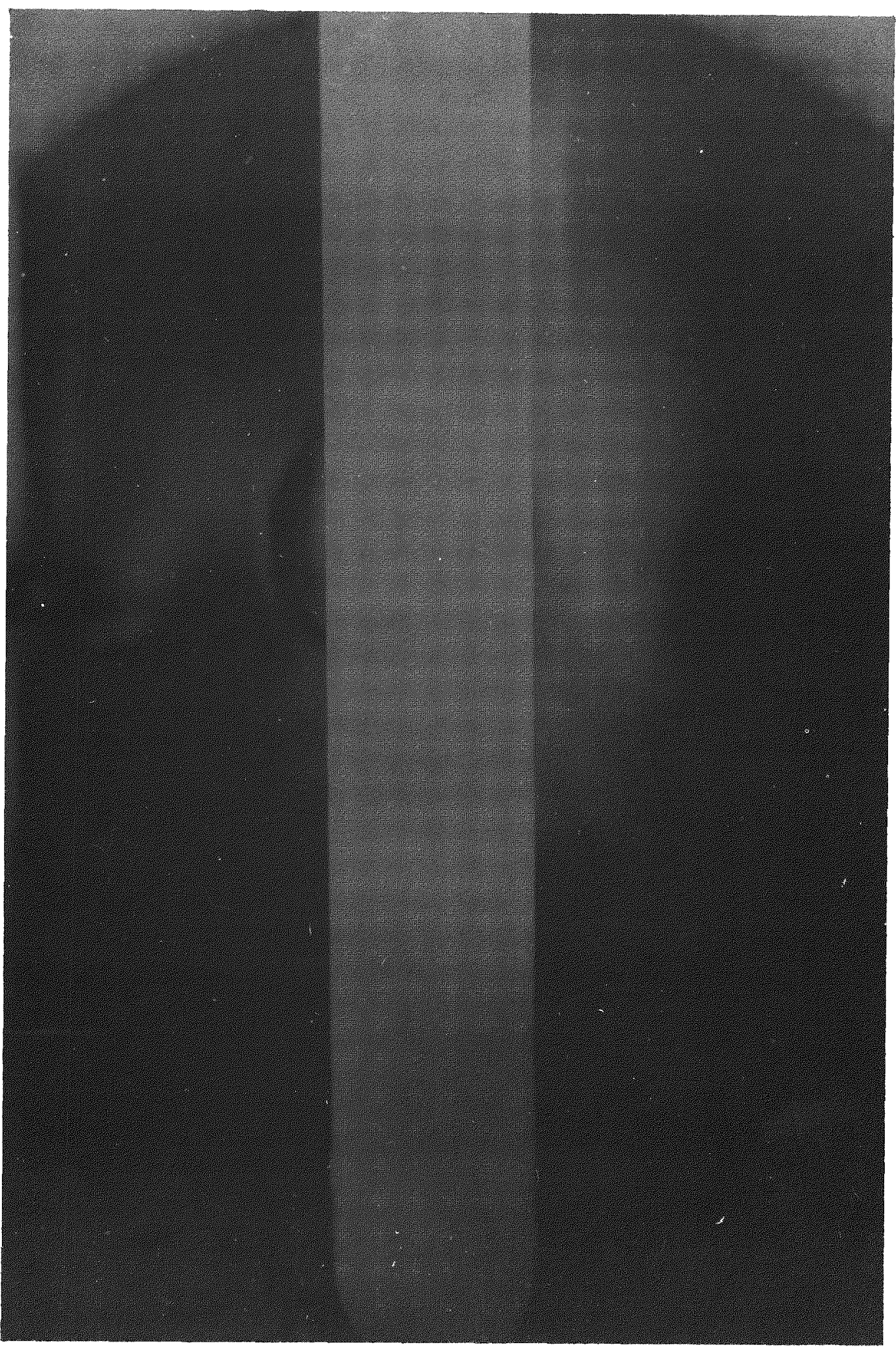

Fig. A-4. Self-illumination pictures of the test model annular heatpipe operating without its water jacket and at low power levels. 


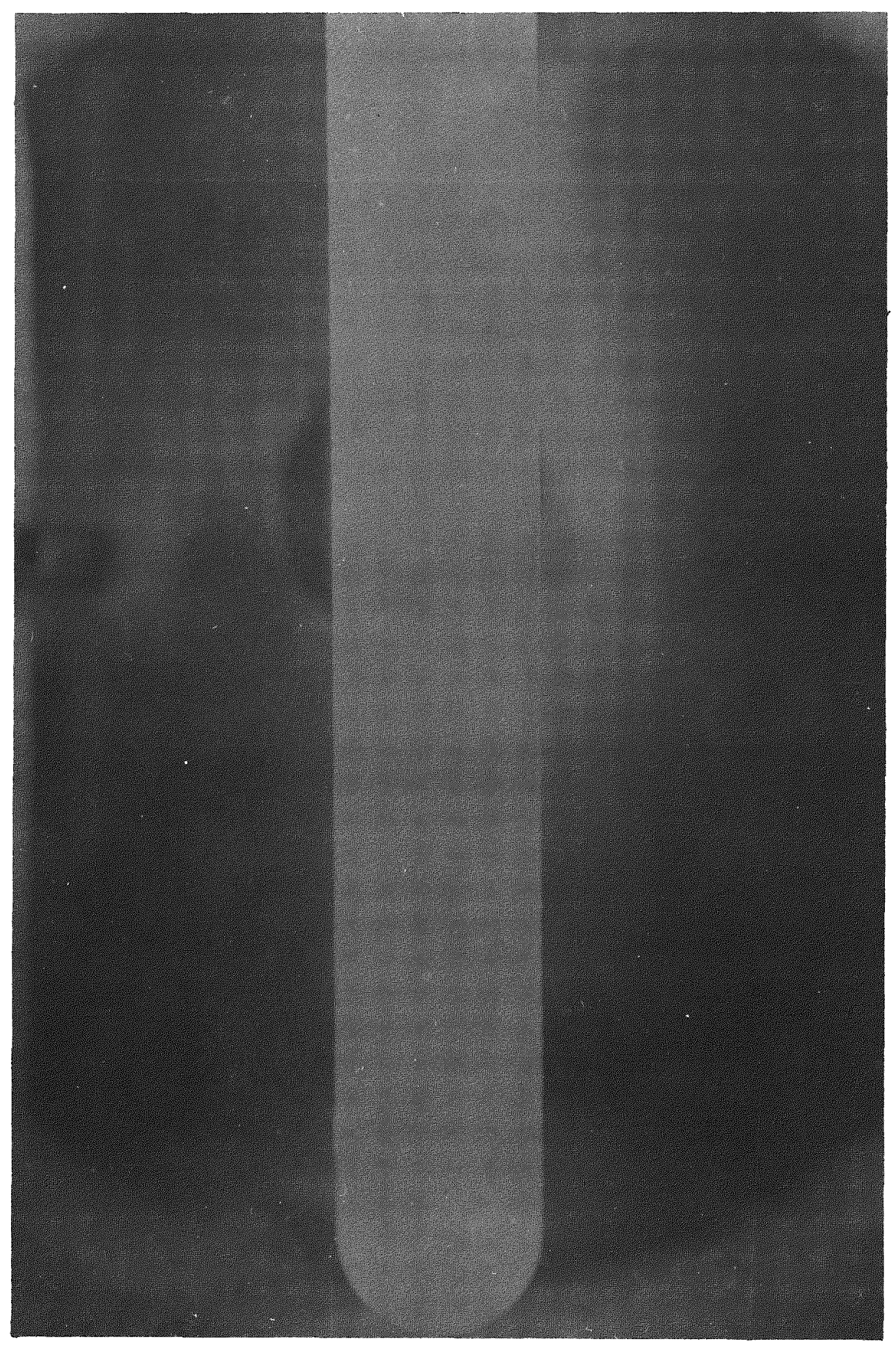

Fig. A-5. Self-illumination pictures of the test model annular heatpipe operating without its water jacket and at low power levels. 


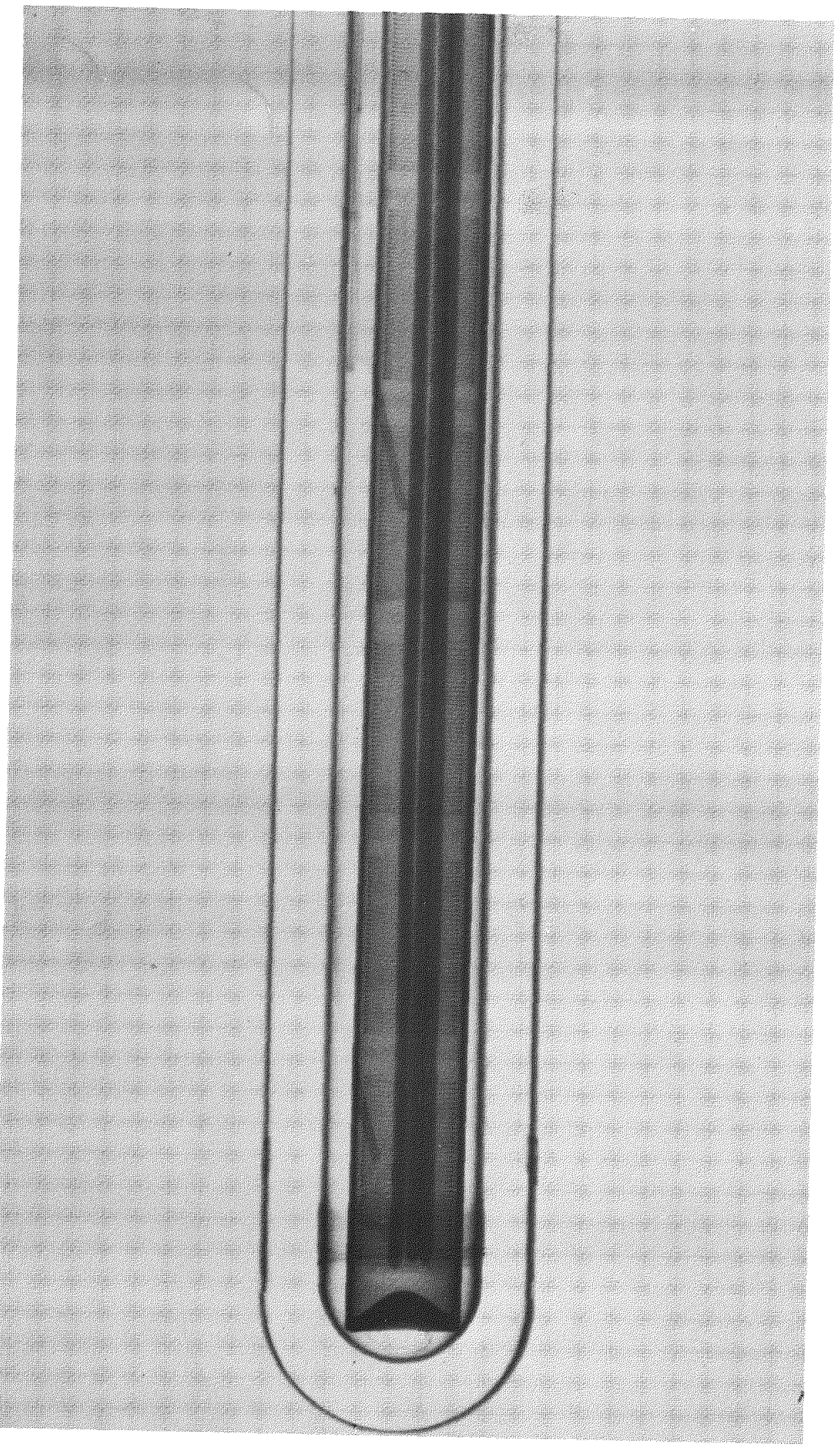

Fig. A-6. A radiograph of the test model annular heatpipe after completion of the second test series.

247 
-

○ 
Environmental Determination

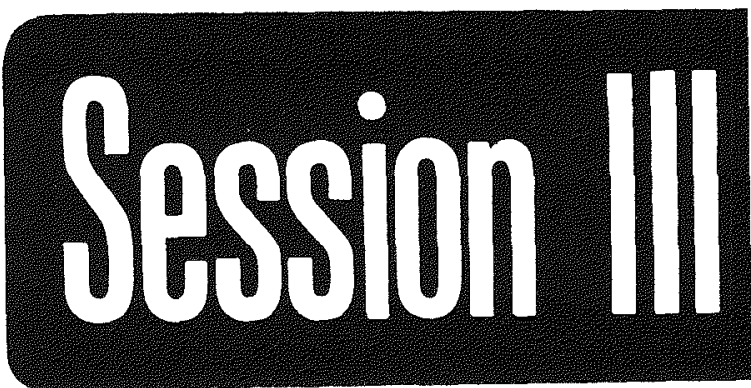


O

O 
IN - PILE GAMMA DOSIMETRY MEASUREMENTS WITH OXALIC ACID

\author{
by J. L. Power \\ $\$ 4+C$ Lewis Research Center \\ Cleveland, Ohio
}

$\underline{\text { Introduction }}$

The purpose of the work here reported was to measure $\gamma$ dose rates in the NASA-Plum Brook Reactor. Measurements in an aqueous environment were required in an in-core location during reactor operation at full power of $60 \mathrm{Mw}$. and at $40 \mathrm{Mw}$. This meant that $\gamma$ dose rates up to $4 \mathrm{w} . \mathrm{g} .^{-1}$ would be encountered. The present study is the first in which chemical dosimeters have been used to directly measure $\gamma$ dose rates in the PBR during operation at power.

Concentrated oxalic acid $\left(\mathrm{H}_{2} \mathrm{Ox}\right)$ was chosen as the only chemical $\gamma$ dosimeter both compatible with the lead-type capsule system to be used and capable of measuring the high $\gamma$ dose rates present. Draganic et al ${ }^{1-5}$ have pioneered and recommended the use of oxalic acid for $\gamma$ dosimetry measurements in reactors and have reported ${ }^{1}$ that measurements of total doses up to $1.6 \times 10^{8}$ rads with it are feasible. Thus at $4 \mathrm{w} . \mathrm{g}^{-1}$ an $\mathrm{H}_{2} \mathrm{Ox}$ dosimeter sample can be irradiated for up to 6.7 minutes.

The stoichiometric decomposition of oxalic acid according to the simple equation,

$$
\mathrm{H}_{2} \mathrm{C}_{2} \mathrm{O}_{4} \stackrel{\gamma}{\rightarrow} \mathrm{H}_{2}+2 \mathrm{CO}_{2}
$$

does not actually take place under $\gamma$ irradiation, although Markovic and 
Dragani $\mathrm{c}^{4}$ have recently reported that their data support the conclusion that the decomposition is a first order rate process. Instead the decomposition proceeds by a series of free radical reactions yielding less than two molecules of $\mathrm{CO}_{2}$ per molecule of $\mathrm{H}_{2} \mathrm{Ox}$ decomposed and variable amounts of $\mathrm{H}_{2}$ depending on the irradiation conditions. ${ }^{1}$ The presence of other carbonaceous (presumably polymeric) products in the irradiated dosimeter solutions is immediately evident from the deep yellow to brownish color of the solutions. $\mathrm{CO}$ is not produced in the decomposition and was not cbserved in the present work.

The decomposition yield from the $\gamma$ irradiation of $\mathrm{H}_{2} \mathrm{Ox}$ is reported ${ }^{2}$ to be independent of the $\gamma$ dose rate for dose rates up to $10^{10}$ rads sec $^{-1}$. In addition the yield is independent of the sample temperature at least up to $80^{\circ} \mathrm{C}$. and unaffected by impurities in the oxalic acid. ${ }^{3}$ No significant change in the $\mathrm{pH}$ of concentrated $\mathrm{H}_{2} \mathrm{Ox}$ occurs under irradiation, even to $90 \%$ decomposition, so this variable does not affect the radiation yield.

The irradiation facility used in this study allowed the sampling and gas chromatographic analysis of the off-gas from the dosimeter samples during irradiation. Hence the $\mathrm{CO}_{2}$ production rate, as well as that of $\mathrm{H}_{2}$ and $\mathrm{O}_{2}$, could be measured as a function of time during irradiations of more than a few minutes duration. An important objective of the present work was to determine whether such $\mathrm{CO}_{2}$ measurements could be used to determine constant (and varying) $\gamma$ dose rates.

\section{Experimental}

The irradiations in this study were performed in the lead-type capsule irradiation facility constructed for PBR Experiment 64-06. The capsule is made of Zircaloy-2 and is situated in core piece LB-11 of the PBR. Fig. 1, a top view of the reactor core, shows this location. The capsule is mounted vertically in a "vertical adjustable facility tube" (VAFT), which allows its elevation to be adjusted as desired over a $9 "$ range. Fig. 2 shows a side elevation view of the PBR core and indicates the vertical position of the capsule relative to the fueled region of the reactor with the VAFT full in and full out.

A simplified plumbing schematic of the 64-06 facility is shown in Fig. 3. All the plumbing apart from the capsule and the three $50 \mathrm{ft}$. long lines leading to it is located in a control panel on the lily pad above the reactor pressure vessel and is suitably shielded. Apart from aqueous samples inserted in it, the facility is always maintained helium-filled. 
An air-free sample is loaded into the charging tank and from there is charged down into the capsule by a suitable helium pressure head. At the conclusion of the irradiation an increased pressure head is applied to force the sample back up into the receiving tank. From there the sample may be discharged to the hot drain or collected for later analysis. Except during sample transfer operations and special irradiations, a constant pressure of 120 psia. is maintained in the system by the back pressure regulator.

More detailed views of the capsule are shown in Fig.4. As indicated, downward flowing primary coolant water is utilized to cool the outside of the capsule. The two chromel-alumel thermocouples measure the wall and sample temperatures. On charging an aqueous sample into the helium-filled capsule (situated at a fixed VAFT position) the temperature recorded by the sample thermocouple undergoes a sharp drop as the liquid cools the thermocouple junction. This decrease was used in the dosimeter irradiations to mark the beginning of each irradiation. Similarly, on discharging a sample from the capsule the temperature read by the sample thermocouple undergoes a rapid increase. This was used to mark the end of each irradiation. These times were easily determined to an accuracy of a few seconds.

A $40 \mathrm{ml}$. sample fills the capsule to about the extent shown in Fig. 4. During irradiation the sample can be sparged (as is indicated) with helium gas which is introduced through the bottom line at any desired rate. This rate is read on one of the inflow rotometers. When the gas chromatograph is used to analyze the off-gas from the sample being irradiated, the effluent gas stream is routed up the top capsule line, and the side line is closed off. Inside the $0.085^{\prime \prime} i d$. top line about $2^{\prime \prime}$ above the top of the capsule the effluent gas stream encounters the end of a $0.008 " i$.d. capillary tube (indicated in the bottom drawing of Fig. 4). A constant flow of gas from the effluent stream passes into and up the capillary tube and finally through the gas chromatograph sampling valve, as shown in Fig. 3. The constant flow rate through the capillary tube is determined by the 105 psi. pressure drop from the bottom end to the sampling valve. The remainder of the effluent gas flow passes on up the top capsule line, out of the system through the back pressure regulator, and through a flow transducer which accurately measures its mass flowrate.

When a gas chromatographic analysis is desired of the off-gas stream passing through the capillary tube, the sampling valve is turned $180^{\circ}$. This introduces a $0.5 \mathrm{cc}$. sample of the off-gas into the carrier gas stream leading to the absorption column of the chromatograph. A column filled with Porapak $\mathrm{T}$ and operated at $40^{\circ} \mathrm{C}$. was used in this work to separate the $\mathrm{H}_{2}$, 
$\mathrm{O}_{2}$, and $\mathrm{CO}_{2}$ in the samples analyzed. The gas chromatograph employs a standard thermal conductivity detector to measure the separated gases as each comes off the end of the column, and the detector response is continuously recorded on a strip chart. Since the $\mathrm{H}_{2}$ and $\mathrm{O}_{2}$ in each sample come off the column rapidly while the $\mathrm{CO}_{2}$ appears only after a considerable delay, several successive off-gas analyses can be initiated at constant time intervals and thus overlapped without obscuring any of the peaks recorded. A typical series of analyses overlapped in this manner is shown in Fig. 5. Here the initiation of each analysis is indicated by a low, broad pressure peak with a sharp leading edge. The sharp $\mathrm{H}_{2}$ and $\mathrm{O}_{2}$ peaks appear, in that order, within 1.5 minutes. Not until about 11 minutes after the start of the analysis does the $\mathrm{CO}_{2}$ peak occur. This delay allowed a total of seven analyses to be started at 1.5 minute intervals before the first $\mathrm{CO}_{2}$ peak appeared. Of course, then no more analyses could be initiated until all the $\mathrm{CO}_{2}$ peaks had been recorded.

The intensity of each recorded gas chromatograph peak was measured by the peak amplitude. This was then compared with analyses of standard gas mixtures to obtain the absolute concentration of the gas in the sample analyzed. Several precautions were observed and minor corrections made in analyzing the data to assure the validity and accuracy of this approach. Since the helium flow rate through the sample under irradiation was always known, the percent composition of $\mathrm{H}_{2}, \mathrm{O}_{2}$, and $\mathrm{CO}_{2}$ measured in the gas chromatograph analyses could be directly converted to absolute production rates of each of these gases in the dosimeter sample.

The dosimeter samples irradiated were $0.60 \mathrm{M} \mathrm{H}_{2} \mathrm{Ox}$ solutions prepared from reagent grade $\mathrm{H}_{2} \mathrm{Ox} \cdot 2 \mathrm{H}_{2} \mathrm{O}$ using deionized water. The concentration of $\mathrm{H}_{2} \mathrm{Ox}$ remaining in each irradiated sample, as well as that in the starting solution, was determined by titration of a $10 \mathrm{ml}$. aliquot with $\mathrm{CO}_{2}$ free, standardized $\mathrm{NaOH}(0.25 \mathrm{~N})$. Prior to titration all possible dissolved $\mathrm{CO}_{2}$ was removed from the sample by boiling for 30 minutes. Titration was done to a phenolphthalein endpoint and al so followed with a $\mathrm{pH}$ meter in order to observe any anomalous behavior in the titration-pH curve. Apart from the reduced amount of $\mathrm{H}_{2} \mathrm{Ox}$ present, the titration-pH curves obtained from the irradiated dosimeter samples were found to be indistinguishable from that of the starting solution. However, a slow downward drifting of the equilibrium $\mathrm{pH}$ was observed at $\mathrm{pH}$ 's above $\sim 6$ during the titration of irradiated samples. This was attributed to the slow neutralization of weakly acidic groups in the high molecular weight products present in the irradiated samples, the same products responsible for the characteristic deep yellow to 
brownish color of the irradiated solutions. By performing the titrations in less than 5 minutes this effect was minimized and could be neglected.

Many of the irradiated samples also showed a distinct haziness and a few had a small amount of a fine yellowish precipitate. The samples also showed a small but definite amount of zirconium $\gamma$ activity. These precipitation effects are most likely due to the formation of zirconium oxalates from the action of the $\mathrm{H}_{2} \mathrm{Ox}$ on the zirconium in the capsule. Thus they may cause a slight loss of $\mathrm{H}_{2} \mathrm{Ox}$ in the irradiated samples. However, the magnitude of and error due to these effects was estimated to be negligible.

Experimental details concerning the irradiation facility and the dosimeter irradiations performed in this study are summarized in Table $I$.

\section{Results and Discussion}

Draganic's ${ }^{2}$ original empirical equation relating total $\gamma$ dose to amount of $\mathrm{H}_{2} \mathrm{Ox}$ decomposed in a dosimeter sample may be rewritten,

$$
\log \mathrm{D}=\log \overline{\mathrm{R}} \bar{\rho}^{i} \mathrm{t}=\mathrm{a} \log \mathrm{C}+\mathrm{b}
$$

where

$\mathrm{D}=$ total absorbed $\gamma$ dose in w. min. $\mathrm{ml}^{-1}$,

$\mathrm{R}=\gamma$ dose rate in $\mathrm{w} \cdot \mathrm{g} .^{-1}$, assumed constant during irradiation,

$\bar{\rho}^{\prime}=$ average density at $25^{\circ} \mathrm{C}$, in $\mathrm{g} \cdot \mathrm{ml}{ }^{-1}$, of dosimeter sample during irradiation,

$\mathrm{t}=$ irradiation time in min, ,

$\mathrm{C}=$ molarity of $\mathrm{H}_{2} \mathrm{Ox}$ decomposed in sample during irradiation.

Values of the constants $a$ and $b$ for $0.60 \mathrm{M} \mathrm{H}_{2} \mathrm{Ox}$ dosimeter samples have been recalculated in the above units from the values given by Draganic ${ }^{2}$ and are,

$$
\begin{aligned}
& \text { for } 0.004<C<0.158, a=0.999, b=1.530 \\
& \text { for } 0.158<C<0.531, a=1.400, b=1.850
\end{aligned}
$$

The constants given in equation $(2 \mathrm{~b})$ cover a decomposition range from $\sim 26$ to $89 \%$ of the initial $0.60 \mathrm{M} \mathrm{H}_{2} \mathrm{Ox}$ concentration. The irradiations in this study produced decompositions of from 55 to $93 \%$. Therefore equation (2) 
with the constants given in equation (2b) was used to calculate the constant $\gamma$ dose rate, $R$, for each test from the measured values of $C$ and $t$ and the correct value of $\bar{\rho}$ ' obtained from tables. ${ }^{6} \mathrm{C}$ was of course determined from the titration of the irradiated sample. The results are presented in Fig. 6 , in which the calculated $\gamma$ heating rate $R$ is plotted vs. the average height of each sample above the core horizontal midplane. A straight line has been drawn to fit the results obtained. Available PBR reference values for the $\gamma$ heating in LB-11 are also shown in the figure, with their $20 \%$ error bars. These reference values were all calculated from data obtained in the PBR mock-up reactor.

All the values plotted in Fig. 6 are corrected to $60 \mathrm{Mw}$ 。 reactor power (nominal full power) and to 24" rod bank height (the approximate height at mid-cycle). The reactor power correction was made by assuming the $\gamma$ dose rate to be proportional to the power level. The corrections for rod bank height were made using correction factors calculated from $\gamma$ heating values for a reactor location similar to LB-11. These values were available for rod bank heights of $16^{\prime \prime}$ (early cycle) and $28^{\circ \prime}$ (late cycle) and covered the whole vertical range of interest.

Fig. 6 shows that the $\gamma$ dose rate values measured in this study, calculated as indicated above, are lower than but in fair agreement with the sparse PBR reference values available. The values obtained in this work show a nearly linear decrease with increasing height over the region studied. The slope and zero intercept of this linear relationship, however, are significantly different from those suggested by the PBR reference data.

Fig. 7 compares the line drawn to fit the $\gamma$ dose rate values in Fig. 6 with temperature rise data obtained during earlier runs with water samples at $60 \mathrm{Mw}$. reactor power and 24 " rod bank height. The temperature rise referred to is the difference between the readings of the wall and the sample thermocouples in the capsule. The temperature data which is plotted was taken at a high helium sparge rate to help ensure that turbulent heat-transfer conditions existed for all the measurements. Under these conditions the temperature rise should be approximately proportional to the heating rate, i.e., the $\gamma$ dose rate. Fig. 7 shows this prediction fairly well borne out. The extrapolated zero intercept of the temperature rise curve is at $\sim 18^{\prime \prime}$ above the core midplane, while the zero intercept of the $\gamma$ heating line is at $\sim 19^{\prime \prime}$ 。

In 1968, Markovic and Draganic ${ }^{4}$ proposed a different equation than equation (2) to relate the total $\gamma$ dose to the extent of $\mathrm{H}_{2} \mathrm{Ox}$ decomposition in $\mathrm{H}_{2} \mathrm{Ox}$ dosimeter samples. This new relationship may be witten as follows, using the same symbols as in equation (2), 


$$
\mathrm{D}=\mathrm{R} \bar{\rho}^{\imath} \mathrm{t}=\mathrm{cC_{0 }} \log \frac{\mathrm{C}_{0}}{\left(\mathrm{C}_{0}-\mathrm{C}\right)}
$$

where

$$
\begin{aligned}
& \mathrm{C}_{0}=\text { initial } \mathrm{H}_{2} \mathrm{Ox} \text { molarity in dosimeter sample, } \\
& \mathrm{c}=\mathrm{a} \text { constant }=66.7 \mathrm{w} . \mathrm{min} . \mathrm{mmol}^{-1} \text { (best value). }
\end{aligned}
$$

The new equation fits their data reasonably well and has the advantages (a) of describing the decomposition as a first order rate process and (b) of applying to any initial $\mathrm{H}_{2} \mathrm{Ox}$ concentration, $\mathrm{C}_{0}$, in the dosimeter sample. However, the validity of the new relationship is only claimed up to $60 \%$ decomposition of the $\mathrm{H}_{2} \mathrm{Ox}$, compared to $89 \%$ for the original equation, (2). Fig. 8 shows a comparison between relations (2) and (3) for values of $R \bar{\rho}^{\prime}=0.921 \mathrm{w} . \mathrm{ml}{ }^{-1}$ and $C_{0}=0.60 \mathrm{M}$. The percent decomposition of $\mathrm{H}_{2} \mathrm{Ox}$ is plotted vs. the time of irradiation. For reference purposes the plots of both relations (2) and (3) are extended beyond the respective 89 and $60 \%$ decomposition limits given for them.

Since nearly all of the dosimeter samples irradiated in this study were decomposed to an extent greater than $60 \%$, and many to $\sim 90 \%$, only relation (2), with the constants given in (2b), was used to calculate $\gamma$ dose rates from the decomposition data. Satisfactory agreement was obtained between the dose rates calculated for samples irradiated to substantially different decomposition levels under similar test conditions. However, not enough such comparisons are available in these tests to definitely substantiate the validity of equation (2) and the constants given in (2b). Nor is such a verification provided by the general consistency shown in Figs. 6 and 7 between the $\gamma$ dose rates from this work and both the available PBR reference data and the temperature rise measurements given.

The time derivative of relation (2) gives a prediction of the $\mathrm{CO}_{2}$ generation rate as a function of time during the irradiation of $0.60 \mathrm{M} \mathrm{H}_{2} \mathrm{Ox}$. For a constant $\gamma$ dose rate $R$, one can obtain the derivative expression

$$
\log \dot{C}^{\prime}=-\frac{a-1}{a} \log t+\frac{1}{a} \log R \bar{\rho}^{\prime}-\log \frac{a}{k}-\frac{b}{a}
$$

where

$\dot{C}^{\prime}=$ mmols. $\mathrm{CO}_{2}$ generated per min. per ml。in the sample (with the sample volume measured at $25^{\circ} \mathrm{C}$.), 
$\mathrm{k}=$ number of $\mathrm{CO}_{2}$ molecules generated per molecule of $\mathrm{H}_{2} \mathrm{Ox}$ decomposed.

The quantity $\mathrm{k}$, which is just the ratio $\dot{\mathrm{C}}^{\prime} / \dot{\mathrm{C}}$, may be a constant or may vary with $C$. Dragani $\mathrm{c}^{-1}$ has reported it to have the constant value of 1.6 in air-free samples for $\mathrm{H}_{2} \mathrm{Ox}$ concentrations $>0.025 \mathrm{M}$, but it is unclear whether this applies throughout irradiation or only initially.

The time derivative of relation (3) gives an alternate prediction of the $\mathrm{CO}_{2}$ generation rate as a function of irradiation time, namely

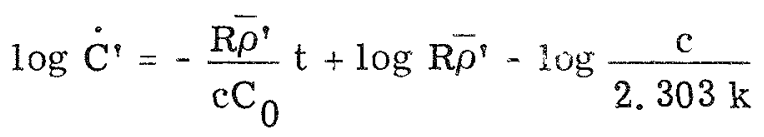

in which all the symbols have been previously identified and again the $\gamma$ dose rate $R$ is taken as constant.

Four identical, lengthy irradiations of $0.60 \mathrm{M} \mathrm{H}_{2} \mathrm{Ox}$ dosimeter samples were performed in which the $\mathrm{CO}_{2}$ generation rate was determined at frequent intervals covering the whole irradiation time by the gas chromatographic technique described earlier. The $\gamma$ dose rate during these irradiations, as calculated from the extent of $\mathrm{H}_{2} \mathrm{Ox}$ decomposition using relation (2), was $0.91 \pm 0.01 \mathrm{w} . \mathrm{g}^{-1}$. The recorded gas chromatographic response curve from one of these runs is reproduced in Fig. 5 and shows 12 separate $\mathrm{CO}_{2}$ determinations. The $\mathrm{CO}_{2}$ analyses from all the runs are plotted in Fig. 9, in which $\dot{C}^{\prime}$, the $\mathrm{CO}_{2}$ generation rate, is plotted as a function of the irradiation time.

Also indicated on Fig. 9 are the curves for $\dot{C}^{\prime}$ predicted by the derivative relations (4) and (5), assuming a constant value of $\mathrm{k}=1.6$. These have both been displaced to the right on the abscissa by 2 minutes to account for the time required for a gas sample to travel from the dosimeter solution under irradiation up the capillary tube to the gas chromatograph sampling valve. The estimate of 2 minutes for this delay time was made from a knowledge of the gas generation rates, the helium sparge rate $(\sim 100 \mathrm{sccm}$.$) ,$ the gas volume above the solution and below the end of the capillary tube $(\sim 12.5 \mathrm{cc}$ 。), the volume of the capillary tube and the expansion line at the top end of it $(\sim 2.8 \mathrm{cc}$ 。), the flow rate through the capillary tube $(\sim 36 \mathrm{sccm}$.$) ,$ and the temperatures and pressures in the system.

The percent decomposition of the $0.60 \mathrm{M} \mathrm{H}_{2} \mathrm{Ox}$ is indicated in Fig. 9 on the abscissa. This has been calculated from relation (2) and also includes the 2-minute gas sampling delay。 
As seen in Fig. 9, the derivative expression (4), together with the constants given in (2a) and $(2 \mathrm{~b})$, predicts the $\mathrm{CO}_{2}$ generation rate $\dot{C}^{\prime}$ will drop rapidly at about $26 \% \mathrm{H}_{2} \mathrm{Ox}$ decomposition from a previously constant value to a value $\sim 70 \%$ of this, and then will continue to decrease at higher decomposition levels. Also as shown in the figure the derivative relation (5) predicts a continuous exponential decline of $\dot{C}^{\prime}$ with increasing irradiation time. Again for comparison purposes this curve is carried beyond the $60 \% \mathrm{H}_{2} \mathrm{Ox}$ decomposition limit given for the validity of the parent relation (3)。

From the rise of the experimental data at the beginning of the irradiations it is evident that a substantial reservoir mixing effect is included in the data, so that the gas samples analyzed were only approximately representative of gas mixtures actually in equilibrium with the solutions under irradiation. This effect is believed to occur principally in the gas volume $1 \mathrm{~mm}$ ediately above the solutions in the capsule. Its magnitude in the runs plotted in Fig. 9 was reduced somewhat from that in previous runs by increasing the sample volume (to reduce the gas volume) and by increasing the helium sparge rate.

From Fig. 9 it will be noted that the maximum experimental values of $\dot{C}$ ' approach within $10 \%$ the constant value predicted from (4) with the constants in (2a). Also, the curves drawn from (4) and (5) with $k=1.6$ generally agree within $\sim 20 \%$ with the experimental data after the initial rise of the measured $\dot{C}^{\prime}$ values to their maximum. Taking account of the reservoir mixing effect mentioned above, the actual agreement in this region is even better than this for the portions of the two predicted curves lying below the experimental values. If the value of $\mathrm{k}$ is allowed to assume the theoretical maximum of two (see eq. (1)) near the maximum experimental values of $\dot{C}^{\prime}$ and decrease slowly to about one for the values measured at the end of the irradiations, then the decrease in the experimental values of $\dot{C}^{\prime}$ with $t$, including the estimated mixing effect, can be exactly fitted by relation (4) with the constants in $(2 b)$. Such a decrease in $k$ with irradiation time is plausible in view of the build-up of high molecular weight products during irradiation. On the other hand, the data with the same mixing effect are also well fitted by equation (5) with a constant value of $\mathrm{k} \approx 1.8$.

It will also be observed that a linear relationship between $\dot{C}^{\prime}$ and $t$ will empiricaily fit the decreasing $\dot{C}^{\prime}$ values in Fig. 9 very well. Rather sparse gas chromatograph data obtained at other dose rates indicate that the slope of such linear fits is directly related but not proportional to the $\gamma$ dose rate. 
Plotted in Figs. 10 and 11 are the measurements of $\dot{\mathrm{C}}_{\mathrm{H}_{2}}$ and $\dot{\mathrm{C}}_{\mathrm{O}_{2}}$ the generation rates of $\mathrm{H}_{2}$ and $\mathrm{O}_{2}$-- made during the same four runs in which the $\mathrm{CO}_{2}$ data in Fig. 9 were obtained. As in the case of the $\mathrm{CO}_{2}$ measurements, $\dot{\mathrm{C}}_{\mathrm{H}_{2}}$ and $\dot{\mathrm{C}}_{\mathrm{O}_{2}}$ refer to the millimoles of $\mathrm{H}_{2}$ or $\mathrm{O}_{2}$ generated per minute per $\mathrm{ml}$. in the sample (with the sample volume measured at $25^{\circ} \mathrm{C}$.). The very low $\mathrm{O}_{2}$ generation rate follows the pattern of the $\mathrm{CO}_{2}$ generation rate quite closely, with the ratio of the $\mathrm{O}_{2}: \mathrm{CO}_{2}$ production rates $\approx 1.4 \times 10^{-3}$ at their maxima $(t \approx 7 \mathrm{~min}$. $)$ and $\approx 1.8 \times 10^{-3}$ at the end of the irradiations ( $\mathrm{t} \approx 32 \mathrm{~min}$.). The $\mathrm{H}_{2}$ generation rate, however, is inversely related to the $\mathrm{CO}_{2}$ rate, the ratio of the $\mathrm{H}_{2}: \mathrm{CO}_{2}$ rates rising from $\sim 0.11$ after 7 minutes irradiation time to $\sim 0.42$ after 32 minutes.

A number of factors and effects of possible importance have been neglected in this study and should be mentioned. These include the local effects on the $\gamma$ dose rate in LB-11 due to changes or anomalies in the fuel loading of the immediately adjacent fuel element LB-10 and due to the operation of the diagonally adjacent regulating and shim rods (the former being generally locked in a nearly full-out position). Also neglected was the contribution of other species than $\gamma$ rays, in particular of recoil protons, to the radiation damage in the dosimeter samples. Similarly, the possible effect of sparging the dosimeter solutions with helium during irradiation has been neglected due to the relatively low sparge rates used. And, finally, the effect of the Zircaloy- 2 capsule on the radiation environment in the dosimeter samples was disregarded. None of these effects is believed to have possibly been so large as to invalidate the results obtained in this study or the analysis made of them.

\section{Conclusions}

The major conclusions reached in this study are as follows:

--Concentrated oxalic acid can be reliably and readily used as a $\gamma$ dosimeter in reactor environments with $\gamma$ dose rates at least as high as $4 \mathrm{w} . \mathrm{g} .{ }^{-1}$.

- For $0.60 \mathrm{M} \mathrm{H}_{2} \mathrm{Ox}$ dosimeter samples decomposed between 50 and $90 \%$, the $\gamma$ dose rates calculated from the original Draganic relationship (2) are consistent with each other, with the available PBR reference data for LB-11, and with temperature rise data obtained on similar samples.

- Determinations of $\gamma$ dose rates by the decomposition of concentrated $\mathrm{H}_{2} \mathrm{Ox}$ have excellent reproducibility.

-- Titration with $\mathrm{NaOH}$ in a $\mathrm{CO}_{2}$-free aqueous environment is a convenient, accurate, and simple analytical method for determining the $\mathrm{H}_{2} \mathrm{Ox}$ 
remaining in irradiated dosimeter samples.

--Gas chromatograph measurements of the $\mathrm{CO}_{2}$ generation rate during irradiations of $0.60 \mathrm{M} \mathrm{H}_{2} \mathrm{Ox}$ dosimeter samples may be usable for determining $\gamma$ dose rates providing (a) the $\mathrm{CO}_{2}$ generation rate is known over the whole course of several similar irradiations at accurately known $\gamma$ dose rates and (b) enough measurements of the $\mathrm{CO}_{2}$ generation rate can be made to at least determine the (constant) rate of decrease of the generation rate with time.

- The technique just described may be capable of extension at low $\gamma$ dose rates $\left(<1 \mathrm{w}, \mathrm{g}_{\circ}{ }^{-1}\right)$ to the measurement of several different, constant $\gamma$ dose rates with the same concentrated $\mathrm{H}_{2} \mathrm{Ox}$ dosimeter sample.

\section{References}

1. I. Draganic: J. Chim. Phys, 56, 9-20 (1959).

2. I. Draganic: Nucleonics 21, 33-35 (1963).

3. I. Draganić, B. Radak, and V. Marković: Intern. J. Appl. Radiation Isotopes 16, 145-152 (1965).

4. V. Marković and I. Draganić: Radiation Res. 35, 587-595 (1968).

5. V. Marković and I. Draganic: Radiation Res. 36, 588-600 (1968).

6. Handbook of Chemistry and Physics, 44th edit.; (Chemical Rubber Publishing Co., 1962): p. 2052. 
Table I: Experimental Details Concerning Irradiation Facility and

\section{$\mathrm{H}_{2} \mathrm{Ox}$ Dosimeter Ir radiations}

capsule material: Zircaloy-2.

capsule location: core piece LB-11.

capsule vertical position: adjustable over $9^{\prime \prime}$ range by a vertical

adjustable facility tube.

average sample height range above core horizontal midplane for $40 \mathrm{ml}$.

sample: $6.25-15.25^{\prime \prime}$.

dosimeter samples: $28-45 \mathrm{ml}$. $0.60 \mathrm{M} \mathrm{H}_{2} \mathrm{Ox}$ (air-free).

extent of $\mathrm{H}_{2} \mathrm{Ox}$ decomposition in irradiated samples: $55-93 \%$.

irradiation time: 4-35 min.

capsule filling time: $\sim 1$ min。

capsule emptying time: $<1$ min.

reactor power level: 40 and $60 \mathrm{Mw}$.

$\gamma$ dose rates measured: $0.8-3.3 \mathrm{w} . \mathrm{g} .{ }^{-1}$.

sample temperature during irradiation: $55-88^{\circ} \mathrm{C}$.

pressure during irradiation: held constant at 120 psia.; supplied by helium . sample sparging rate during irradiation: $55-110 \mathrm{sccm}$ (helium). 

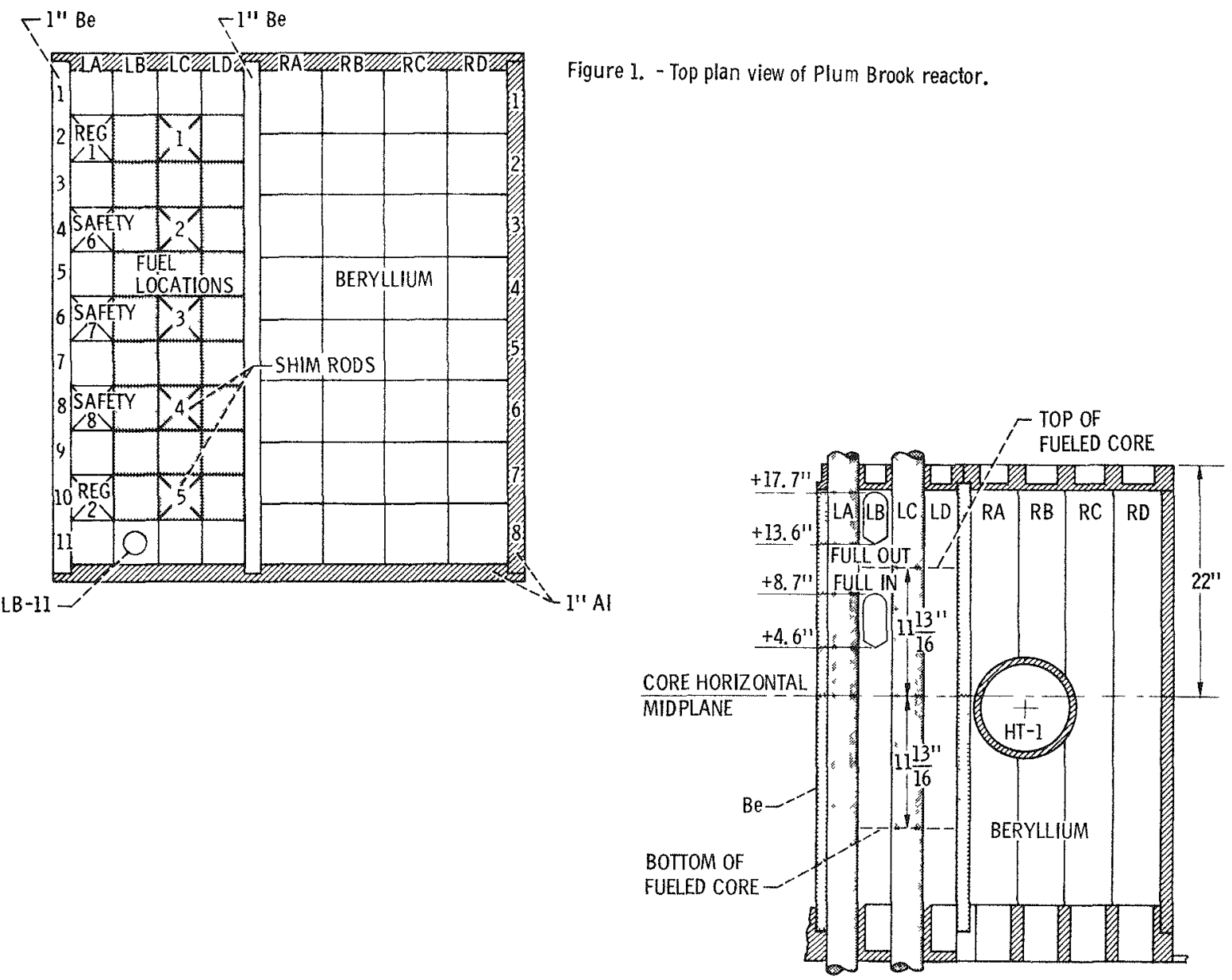

Figure 2. - Side view of Plum Brook reactor showing experiment 64-06 capsule elevations in LB-II.

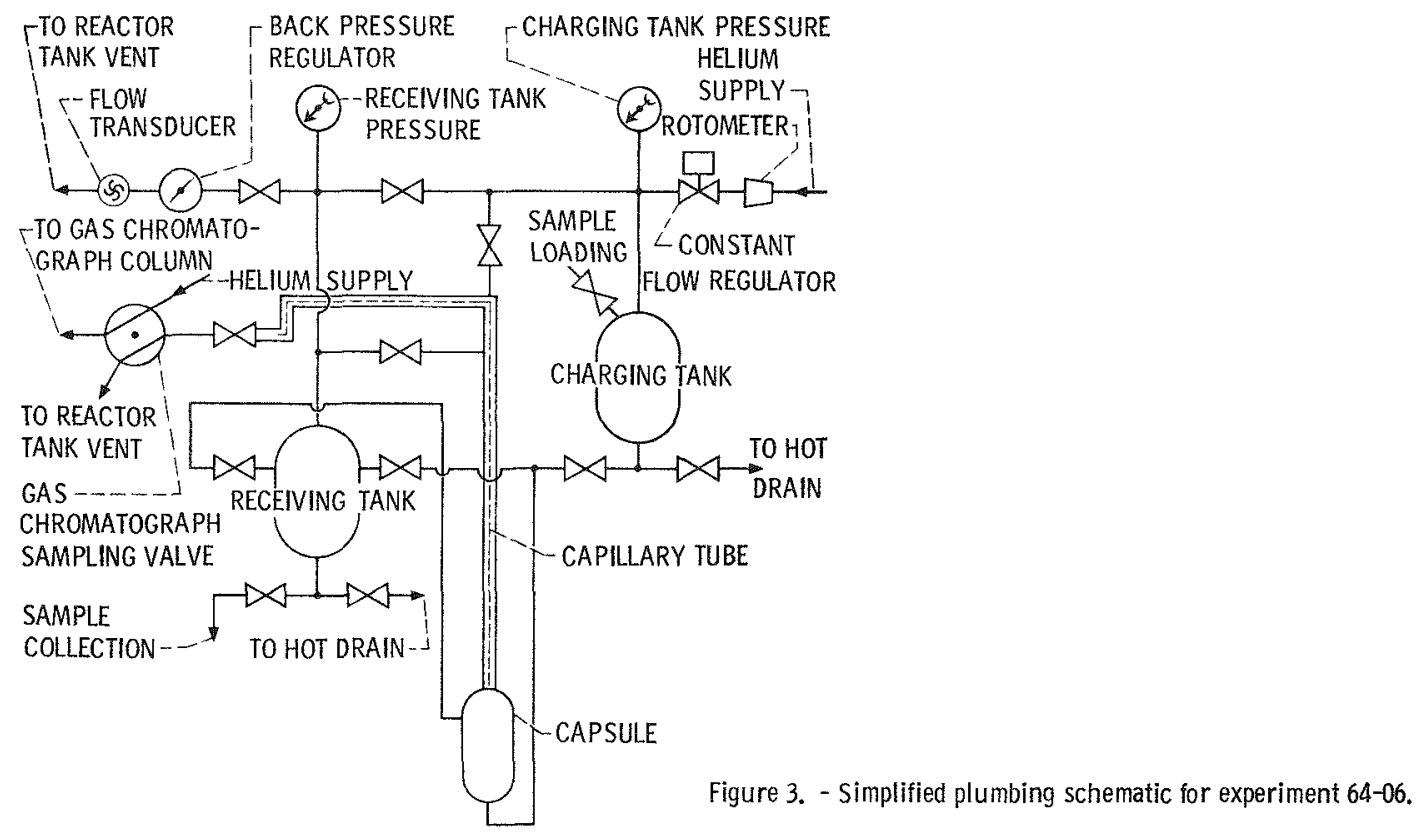



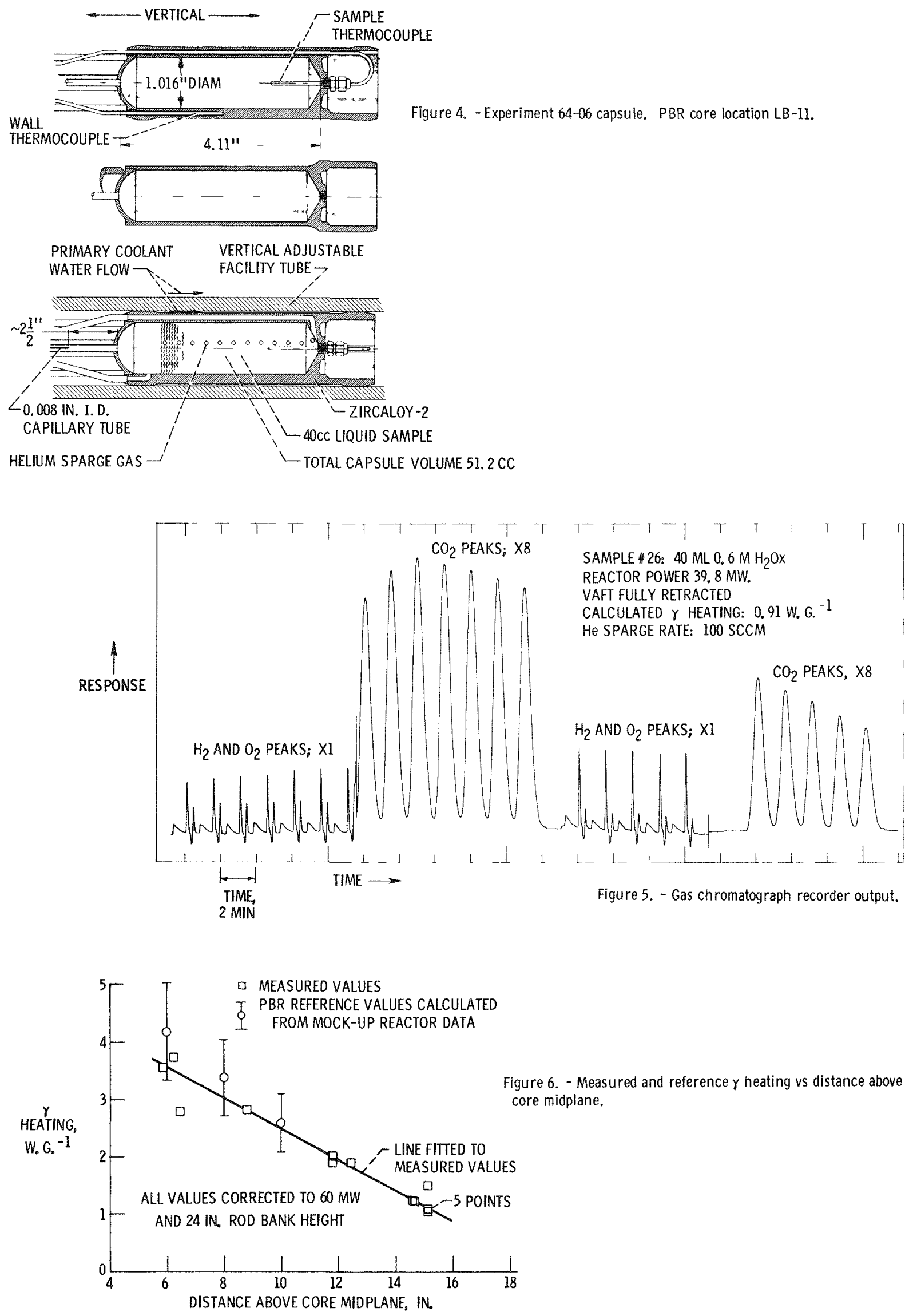
Figure 7. - y heating and sample temperature rise measurements vs distance above core midplane.
ALL VALUES FOR $60 \mathrm{MW}$ AND $24 \mathrm{IN}$. ROD 8ANK HEIGHT; $\triangle T$ MEASUREMENTS AT HIGH HE SPARGE RATE

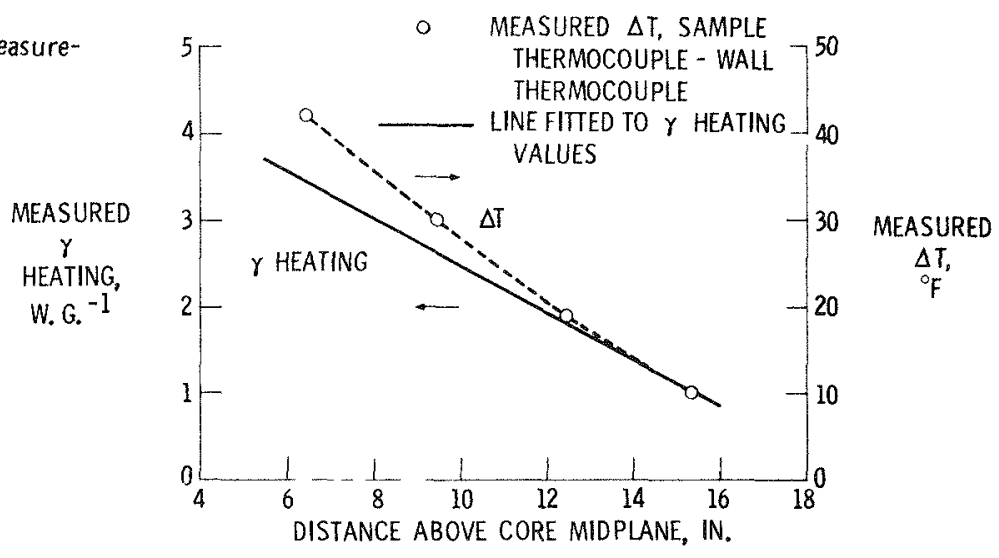

Figure 8. - Comparison of $\mathrm{H}_{2} \mathrm{Ox}$ dose equations.

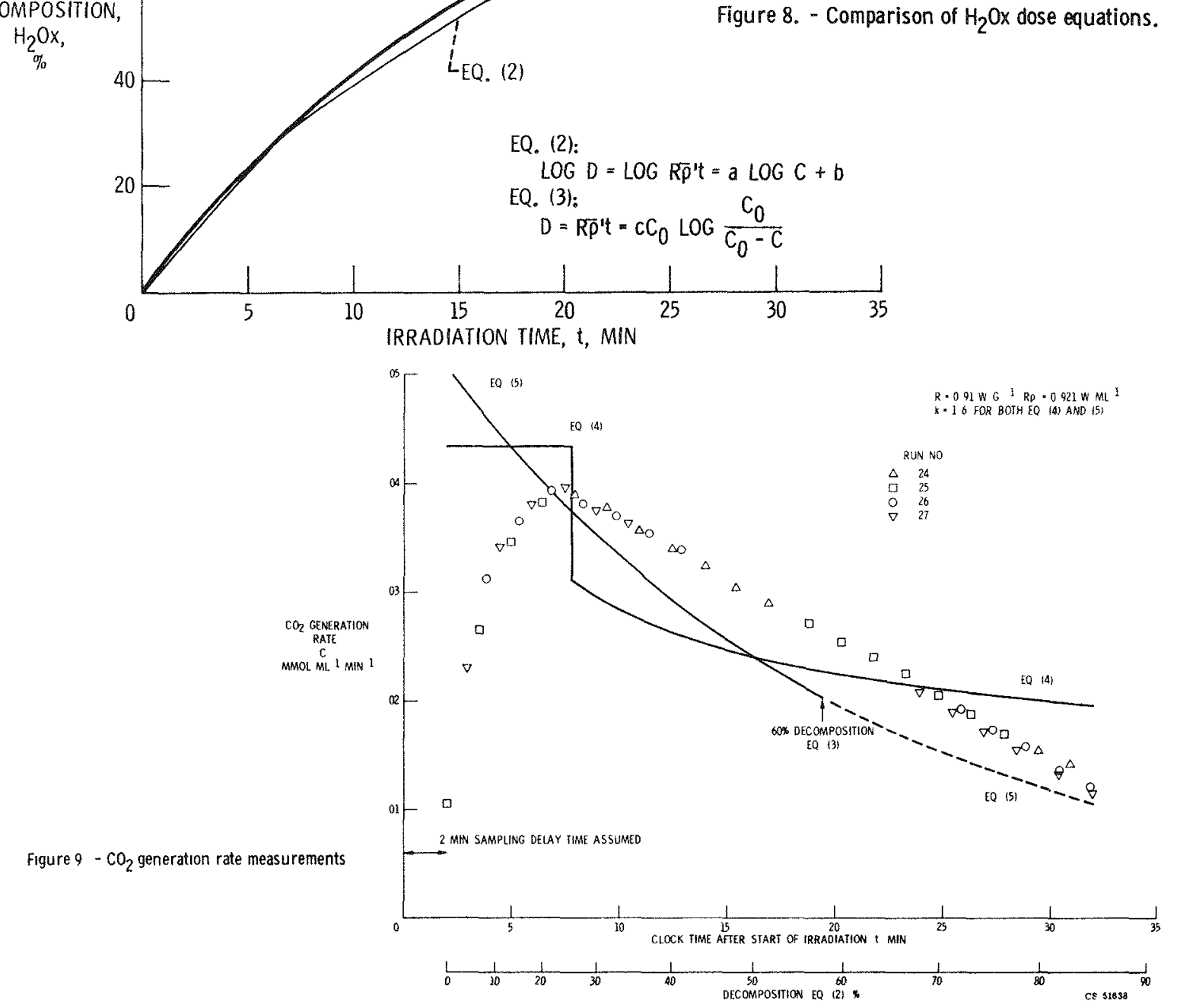




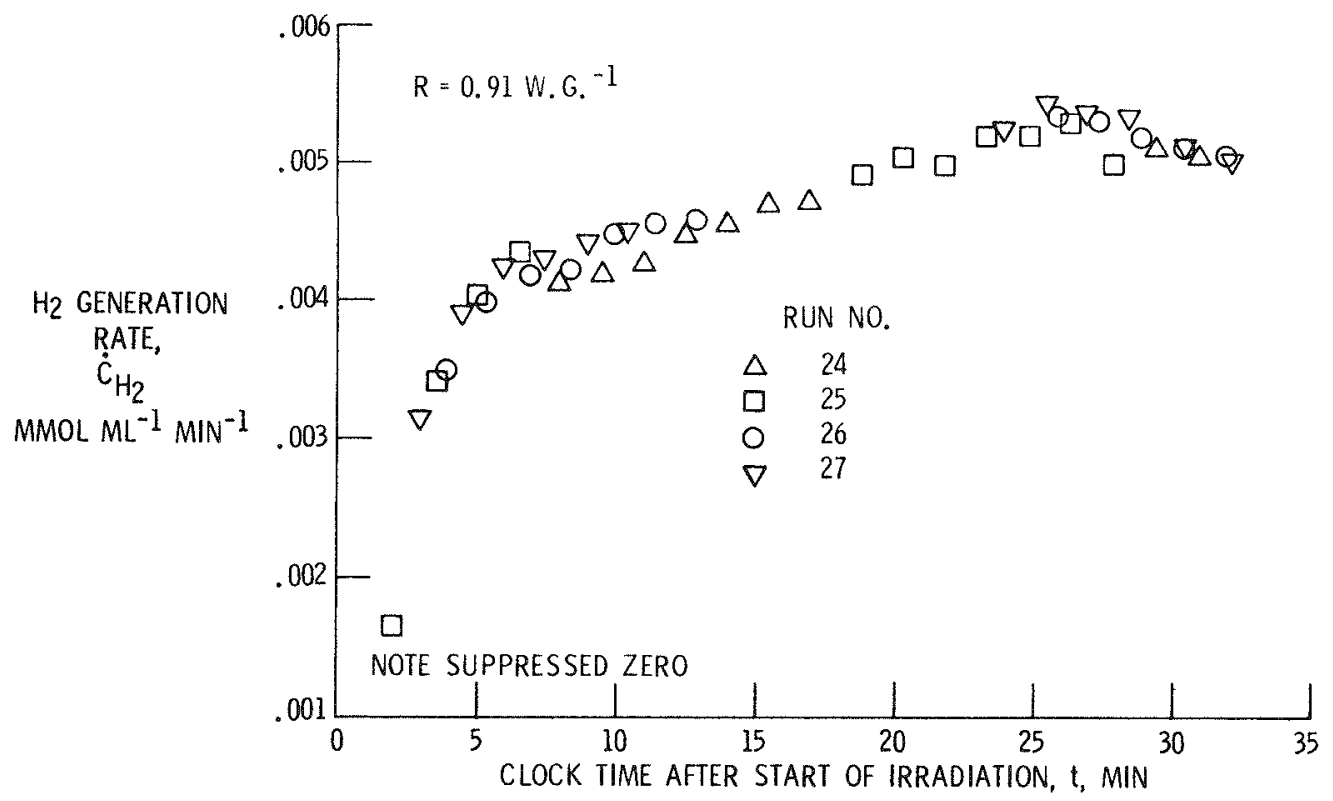

Figure 10. $-\mathrm{H}_{2}$ generation rate measurements.

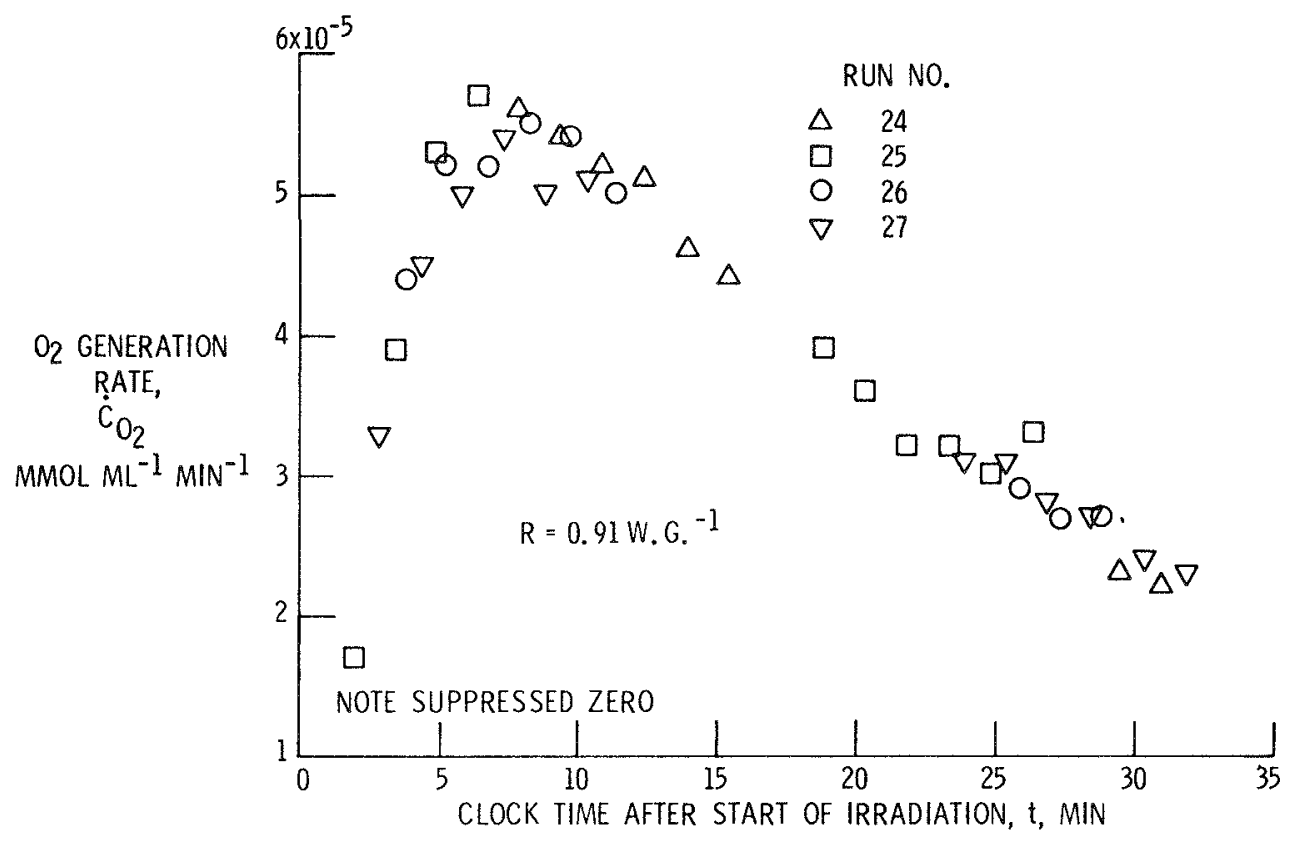

Figure 11. $-\mathrm{O}_{2}$ generation rate measurements. 


\section{AT ARGONNE NATIONAL LABORATORY \\ by \\ J. R. Folkrod \\ $[3=7, y=4$ Reactor Engineering Division}

X DEVELOPMENT OF FISSION GAS PRESSURE TRANSDUCERS

Concentration for development of fission gas pressure transducers centered around two areas at ANL. They are the nul1-balance and fluid-filled bellows-capillary types. The null-balance type, which most people are familiar with, works on the principle of a moving diaphragm which makes contact with a probe. Contact with the probe closes an electrical circuit and allows a gas solenoid valve to open. The gas, known as the reference gas, pressurizes the system and causes the diaphragm to break probe-diaphragm contact to close the valve. The pressure of the reference gas is then equivalent to the fission gas pressure. Further operation of the valve will not take place until the fission gas increases in pressure. The fluid-filled bellows-capillary transducer system may not be too familiar. It is, however, analogous to the large diaphragm-capillary fluid-filled systems built by Taylor and other well known like companies. The only difference being that the large diaphragm is replaced by a bellows.

Referring back to the null-balance transducer, the type built and tested at ANL is shown by Fig. 1. This is a replica of transducers designed and used by Atomics International. (1) Therefore, there is nothing new about this transducer. Those not too familiar with the designs should notice the feature of two bellows for double containment. The spring rate of the double bellows is low so that one can use a small. overall diameter for the transducer body which is close to a fuel pin size in some cases. A heavy coupling disk joins the ends of the two bellows and the opposite ends are welded to the housing. The probe is a thermocouple. The bare junction makes contact with the disk and acting as a thermocouple can give the temperature at contact. Very little movement of the disk is required for contact because the location of the disk is set very close to the probe end during assembly.

The unique feature of ANL's system is the method of transducer control and the reason for reporting about a null-balance system. The conventional method for control is by making and breaking probe-disk contact. This method works conveniently for pressurization in one direction. If the controller is set up to control for increasing pressures and the gas pressure to be measured is always increasing and does

(1) Jackson Kinzer, E. J. Babbe, Trans. Am. Nuc1. Soc., 11, 337 (1964). 
not decrease--this is fine. However, in practice one does not find this ideal condition so the controller has to switch from the increasing operation mode to one for detecting decreasing pressures. This is generally accomplished by an automatic mechanical switching device which periodically switches to the second mode. One can readily see that decreased pressurization would be missed when it occurs during the wrong time of the controller's cycle.

There is an electrical resistance at the probe-disk contact and this resistance varies with the amount of contact force generated by the pressure difference of the two gases. To make full use of this effect, it was made part of an electrical circuit for control (Fig. 2). As the fission gas pressure increases, the contact resistance decreases, causing the circuit voltage to decrease. A millivolt meter switch closes and activates a solenoid valve to allow reference gas to enter. This action reduces the disk force on the probe causing the voltage to increase and open the switch to close the valve. The same operation takes place for controlling the reference gas pressure. Also, a point of interest is that the probe is always in contact with the disk. RC circuits are used to delay the signals so that the controller will not overreact. Controls are available for the RC circuits so that one can adjust for a "quiet" operation.

The nul1-balance transducer system with the above described controller was extensively tested at ANL at the design temperature and pressures of the transducer for eight months. Report ANL 7573, "Design and Performance Characteristics of a Nu11 Balance Pressure Transducer," describes in detail all aspects of the test. An all 304L SST 3/8-inch $0 . D$. by 3-inch-long transducer was tested at $900^{\circ} \mathrm{F}$ and $350 \mathrm{psig}$. The transducer was built by Metal Bellows Corporation in California. They built the welded bellows and assembled them into the body. ANL finished the assembly and supplied the controller. Pressure was allowed to vary between about 100 psig to 350 psig (Fig. 3). A complete cycle took about $11 / 2$ hours. The line curve represents the simulated fission gas pressure which was automatically varied. Transducer output was represented by the wide curve. A band width was formed by action of the controller pressurizing and venting the system.

Calibration curves were generated giving a resulution of $\pm 1 / 4$ psig. However, a \pm 8 psig calibration curve spread was experienced over the life of the test (Fig. 4). Part of this spread is attributed to end flattening of the probe which was rather weak. Future designs will have a heavy probe and will not be a thermocouple because it was found to be superfluous. There was no temperature-pressure calibration relationship to be found which was the intention of the thermocouple probe design.

Temperature shock tests were made and the results are shown by Fig. 5. Temperature was decreased from $900^{\circ} \mathrm{F}$ to $400^{\circ} \mathrm{F}$ in about $21 / 2$ minutes at a pressure of 350 psig and cycled about 250 times. The hump in the curve represents dimensional changes taking place between the probe and disk. Initial dimensions reappear after temperature equilibrium and the true equilibrium was reestablished. 
The transducer was then tested to failure by heating to $1300^{\circ} \mathrm{F}$ at 350 psig for six weeks. Both bellows failed at a weld. A transducer of the same type was also tested at the CP-5 reactor at ANL as part of a fuel pin experiment. The transducer did a good job and indicated pin failure during a reactor scram. Information of the experiment can be obtained from ANL Transactions Vo1. 12, p. 78 (1969) by W. N. Beck. Also, four transducers have been installed in the EBR II instrumented subassemb1y.

The second phase of development work was extended to a fluidfilled bellows-capillary system. The fluid used was NaK and the pressure readout device was a CEC strain-gauge pressure transducer which read the fluid pressure caused by the deflection of the bellows reacting from the outside gas pressure (Fig. 6).

Several capillary systems were constructed consisting of $1 / 8-$ inch, 1/4-inch, and 5/8-inch-diameter bellows. Capillaries of $0.012-$ inch I.D. were used with the 1/8-inch and 1/4-inch bellows. A 1/16inch I.D. capillary was used with the 5/8-inch bellows. Small bellows have large spring rates and the backup pressures due to the expansion of the hot NaK caused by a hot environment can be extremely high even with the small capillary tube. However, with further development, this problem can be resolved. One of the problems first encountered and solved was filling the system adequately. No voids could be tolerated because the small bellows volume was not adequate to compensate for voids. It was found that if the bellows and the 24-foot, 0.012inch capillary were etched and heated to $700^{\circ} \mathrm{F}$ with $\mathrm{NaK}$ flowing through the capillary a complete fill could be accomplished and the transducer system would work. To date I have not been able to test the smaller sizes because of time allotted. I can say, however, that the response time is very poor. The 1/4-inch bellows used were welded bellows of AM 350 SST while the 1/8-inch size was made by the nickel-deposited method. Nickel was not the best material to use. It was possible to obtain a 1/8-inch welded bellows but was very costly. Since nickel bellows was inexpensive it was used for the general development work.

A 5/8-inch welded bellows having 100 convolutions with $1 / 16-$ inch I.D., 24-foot-long capillary was easy to produce since I was relatively successful on the first try. This was also the first transducer built and there were things to learn. The bellows had a closed end, therefore, I could only evacuate 24 feet from the bellows and I could not flow NaK through the system. I was, however, able to evacuate the bellows to 1 micron vacuum. This left the system with some minute voids but did not hinder the transducer's operation because the large bellows volume was capable of overriding the effect. It must hinder the response time compared to a system with no voids, however, at present I have not had the opportunity to test a perfectly filled system for comparison. Future systems should have the capability of flowing NaK through the capillary and bellows to achieve a complete fill. After sealing the fill tube, the bellows and 8 feet of the capillary was heated to above $1400^{\circ} \mathrm{F}$ to generate a temperature effect on the zero. A curve (Fig. 7), was generated showing a near plateau after $800^{\circ} \mathrm{F}$. This is attributed to the change in the metallic properties of 
the metal bellows decreasing the spring rate. The transducer was then tested at $1200^{\circ} \mathrm{F}$ for three months and pressure cycled many times to 100 psig. A typical calibration curve is shown by Fig. 8 .

Void volume in the system was also measured by measuring the deflection of the bellows and recording the resultant pressure. The curve generated is shown by Fig. 9 and is compared to calculation results and a curve expected with a system having no voids. The method of calculation and all aspects covering the design of a bellows-capillary system can be obtained through ANL 7574 report, "Design Analysis and Performance Characteristics of a Fluid-Filled Bellows-Capillary Transducer System." The bellows was then placed in a chamber along with a CEC transducer such as the one on the end of the capillary tube. A gas valve was suddenly opened and a curve generated (Fig. 10) giving the $\triangle \mathrm{P}$ between the two CEC transducers. The peak to the left is attributed to the inertia effect of the chamber transducer which is assumed to be quickly dampened out by the time the peak is reached to the right. This peak represents a lag of the capillary system of only 1.15 psig behind the chamber pressure and this occurs 0.375 seconds after chamber pressurization. It takes about 4 seconds for zero $\Delta \mathrm{P}$ and 10 seconds for complete equilization.

Two transducers of the 5/8-inch type have been used with a differential pressure transducer for an orifice-type flowmeter. The differential pressures measured were in parts of inches of water and made a very effective flowmeter in a sodium loop. 


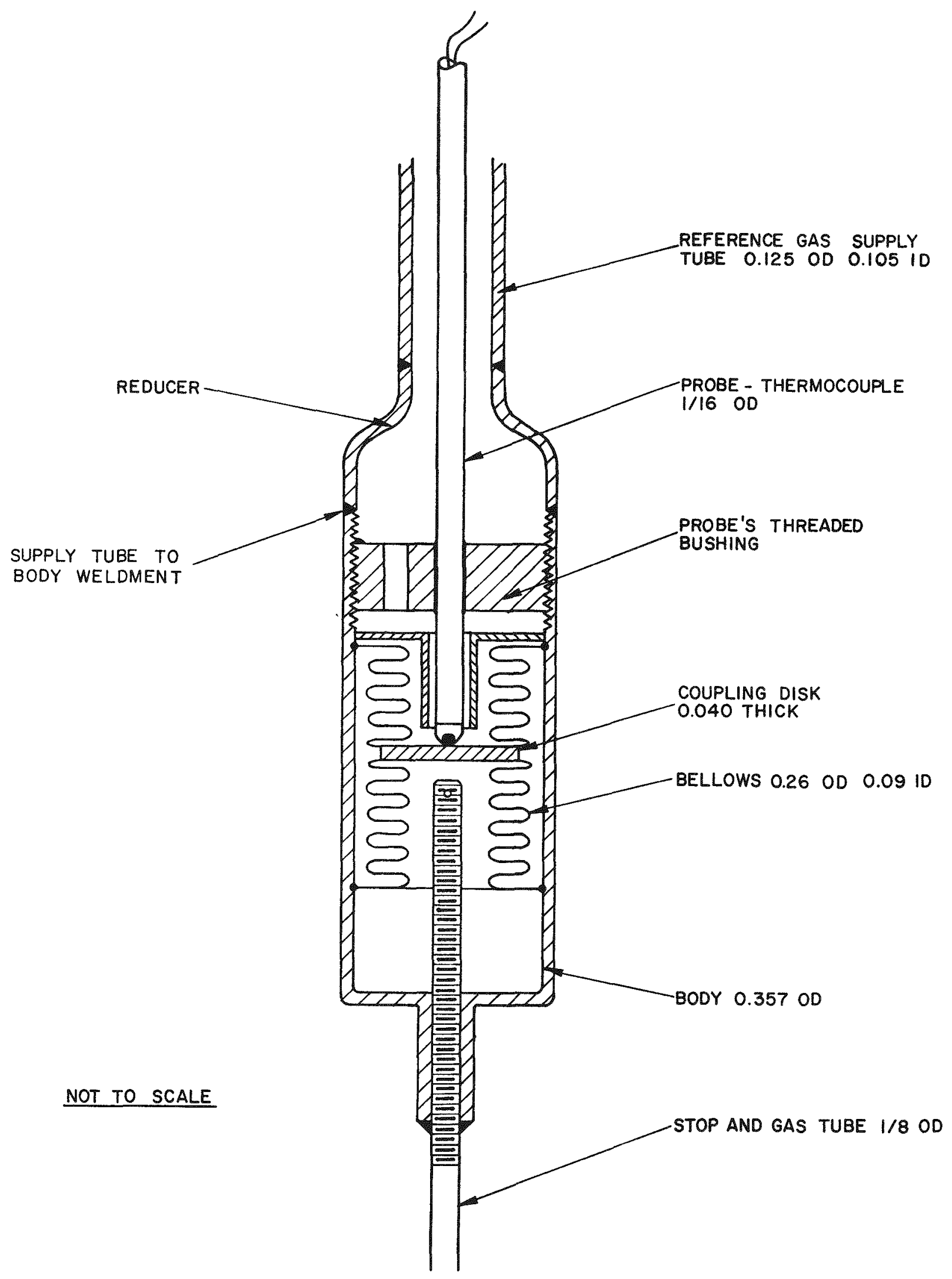

FIG. 1 NULL BALANCE PRESSURE TRANSDUCER 


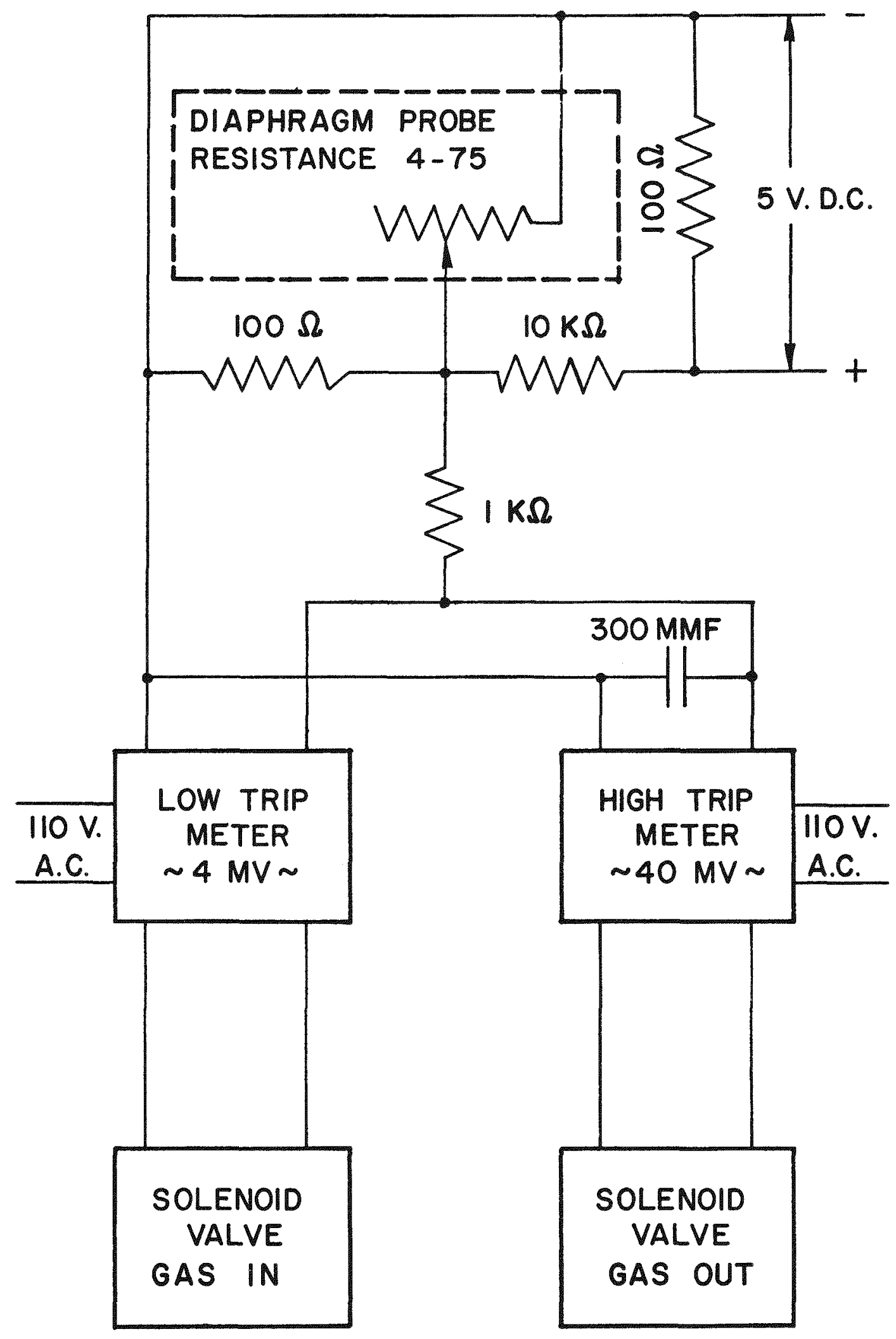

FIG. 2 NULL-BALANCE PRESSURE TRANSDUCER CONTROLLER SCHEMATIC 


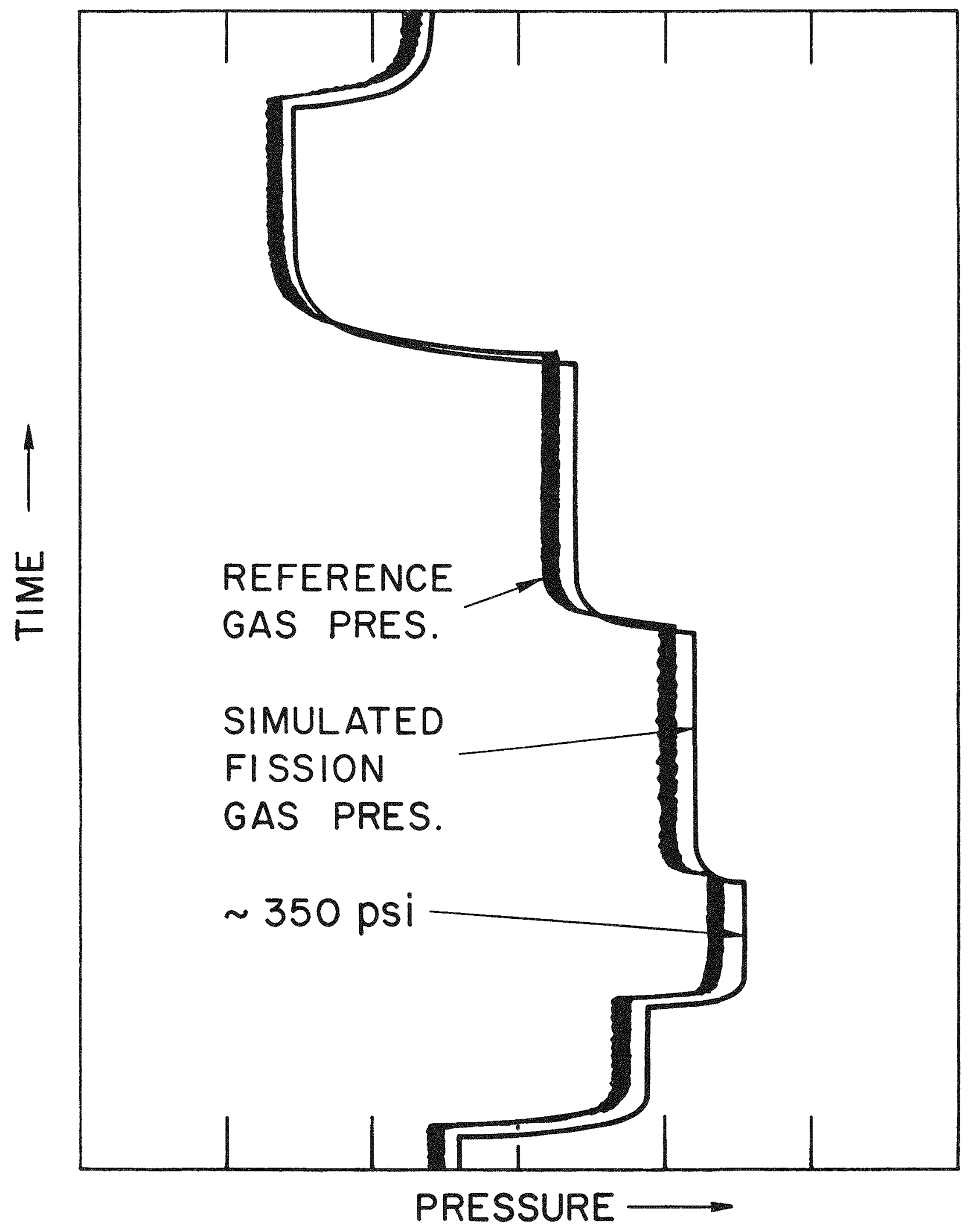

FIG. 3 ANL'S NULL - BALANCE TRANSDUCER SYSTEM 


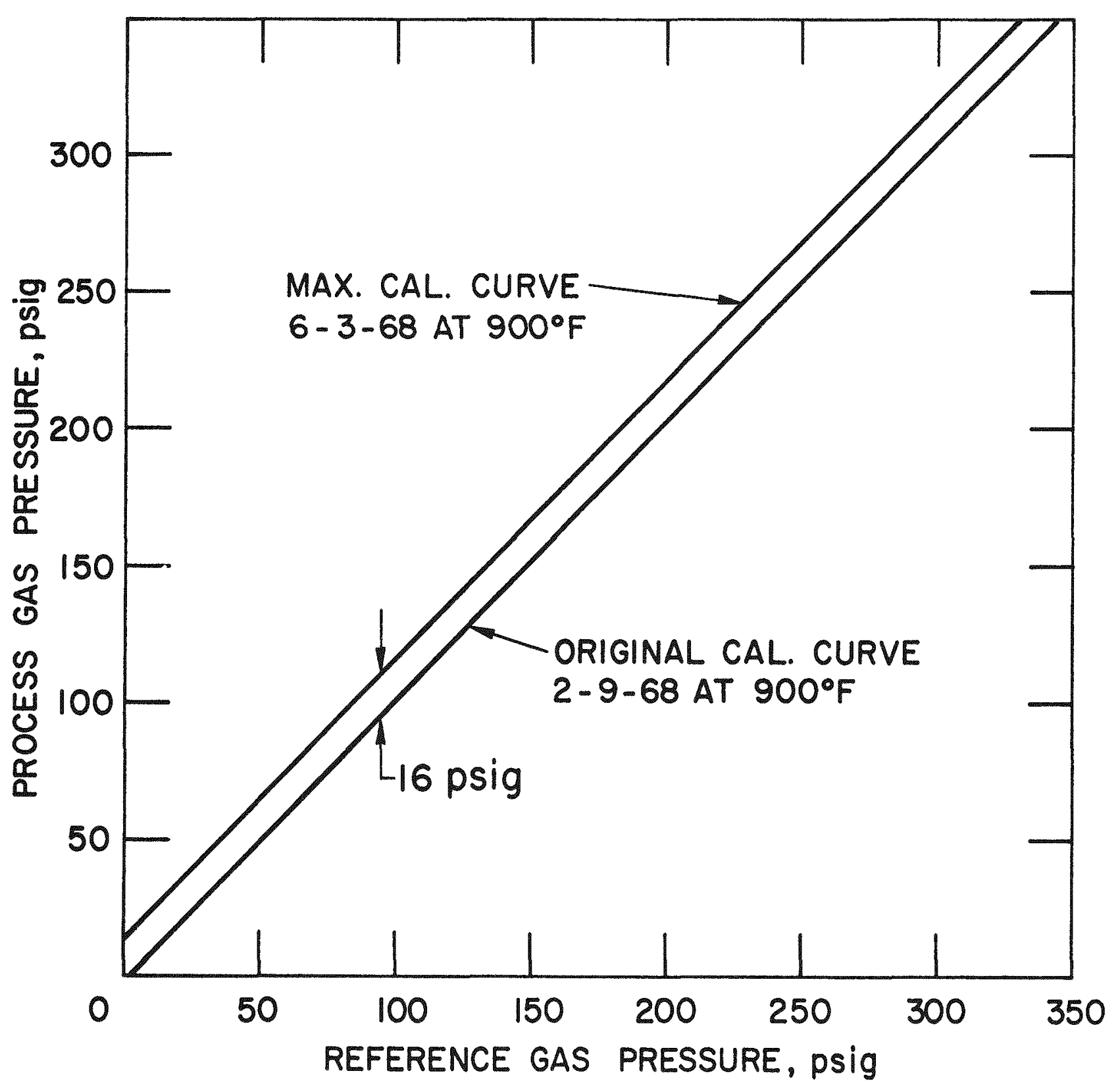

FIG. 4 MAX. AND MIN. CURVES FOR NULL - BALANCE PRESSURE TRANSDUCER 


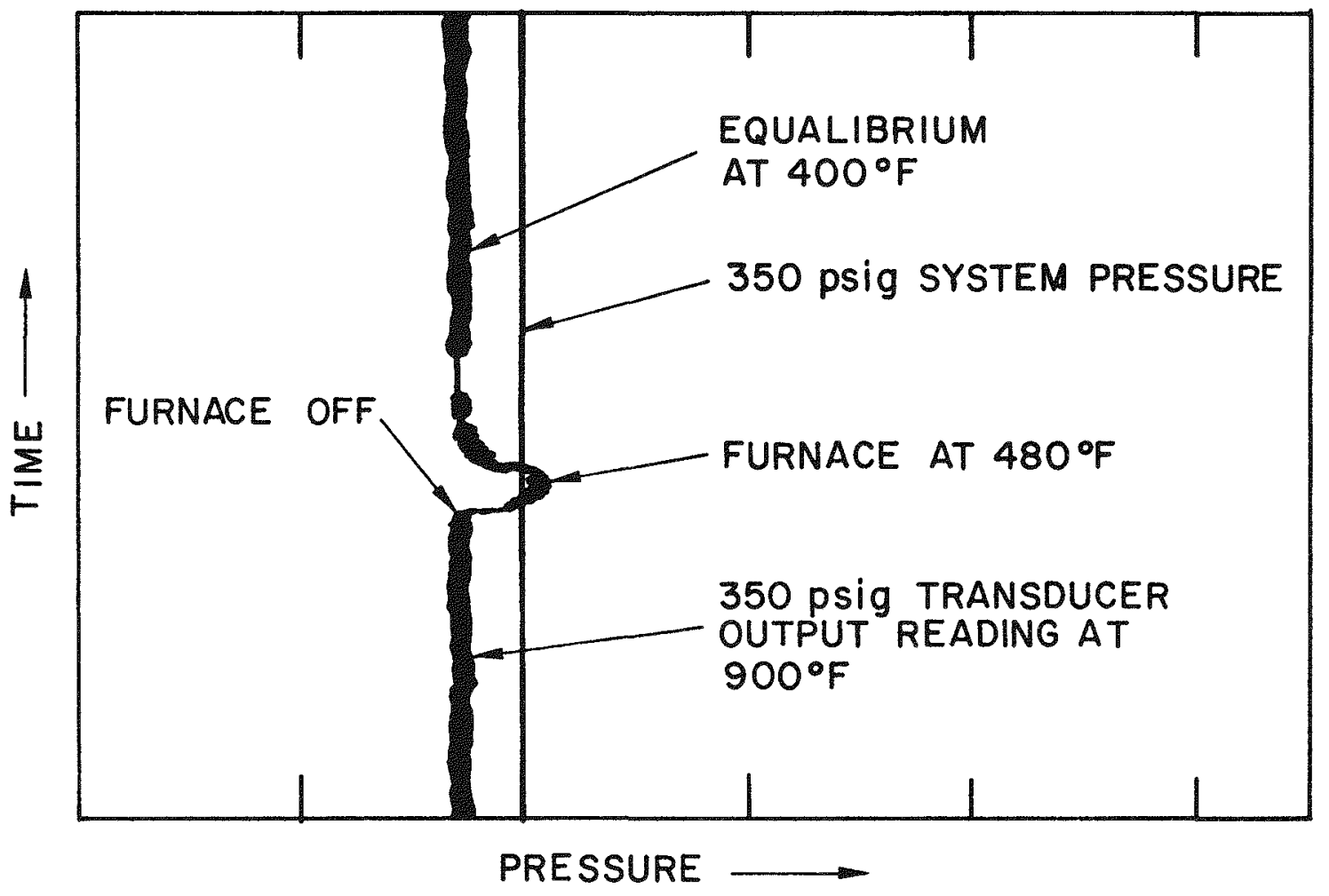

FIG. 5 THERMAL SHOCKS EFFECTS $900^{\circ} \mathrm{F}$ TO $400^{\circ} \mathrm{F}$ 


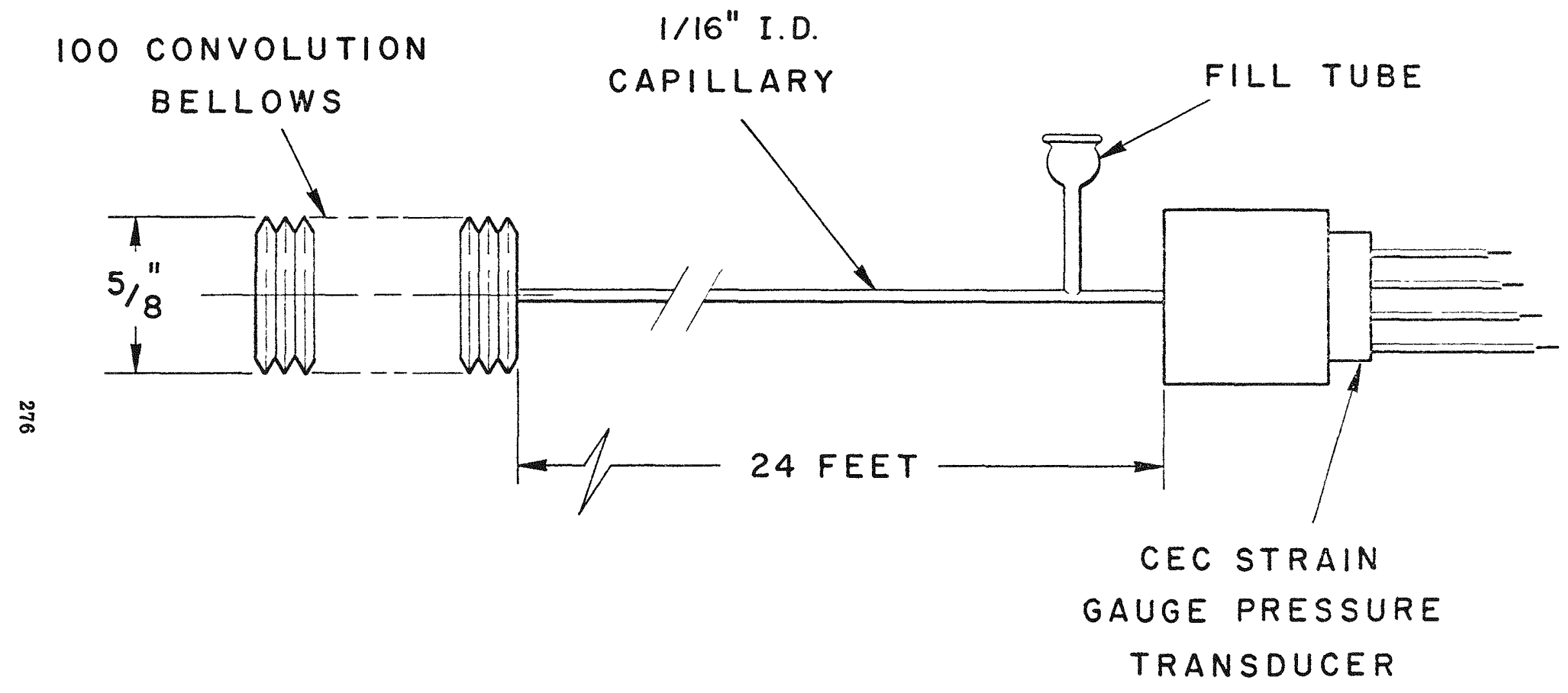

FIG. 6 A BELLOWS - CAPILLARY SYSTEM 


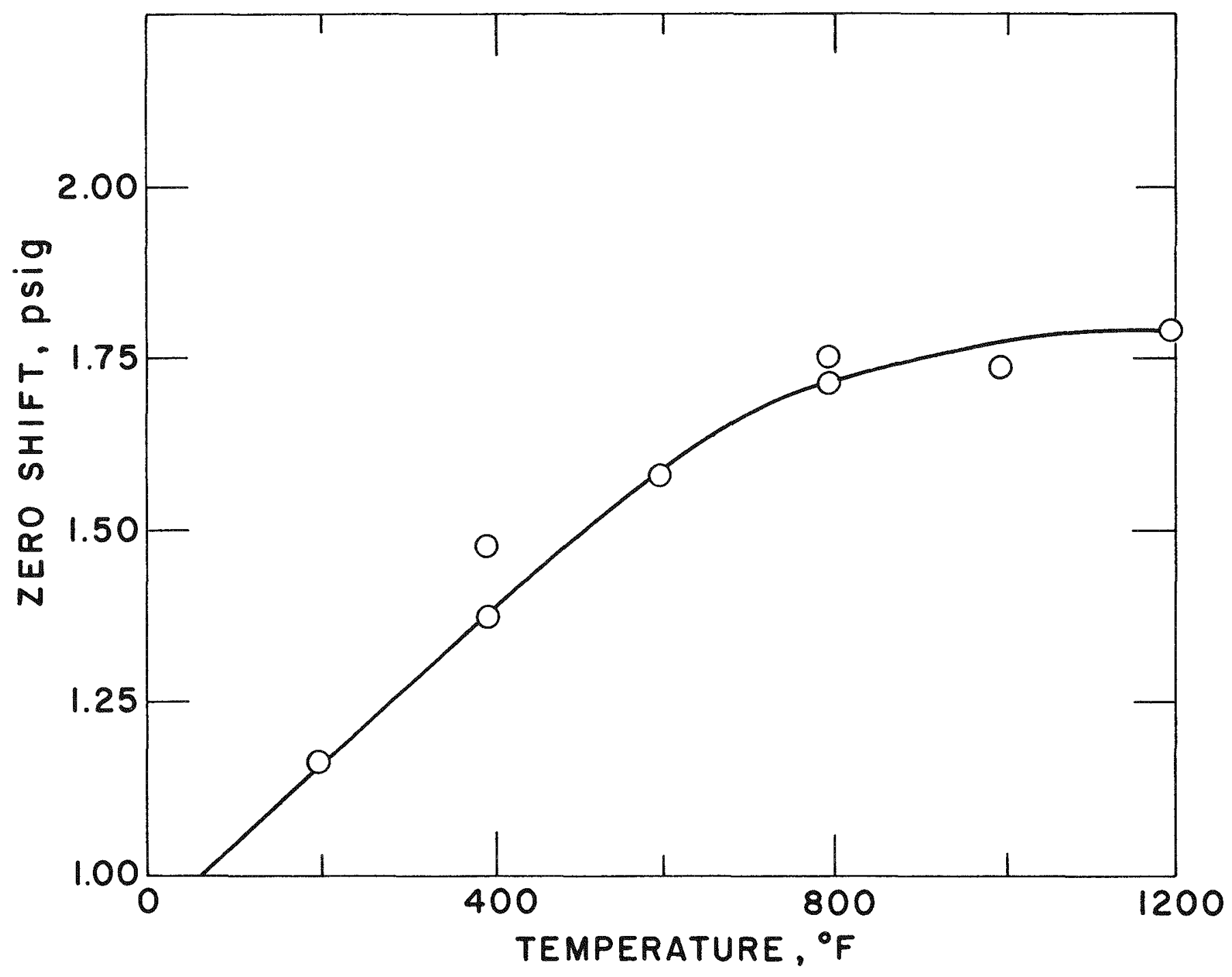

FIG. 7 TEMPERATURE EFFECTS ON ZERO REFERENCE (5/8 DIA. BELLOWS) 


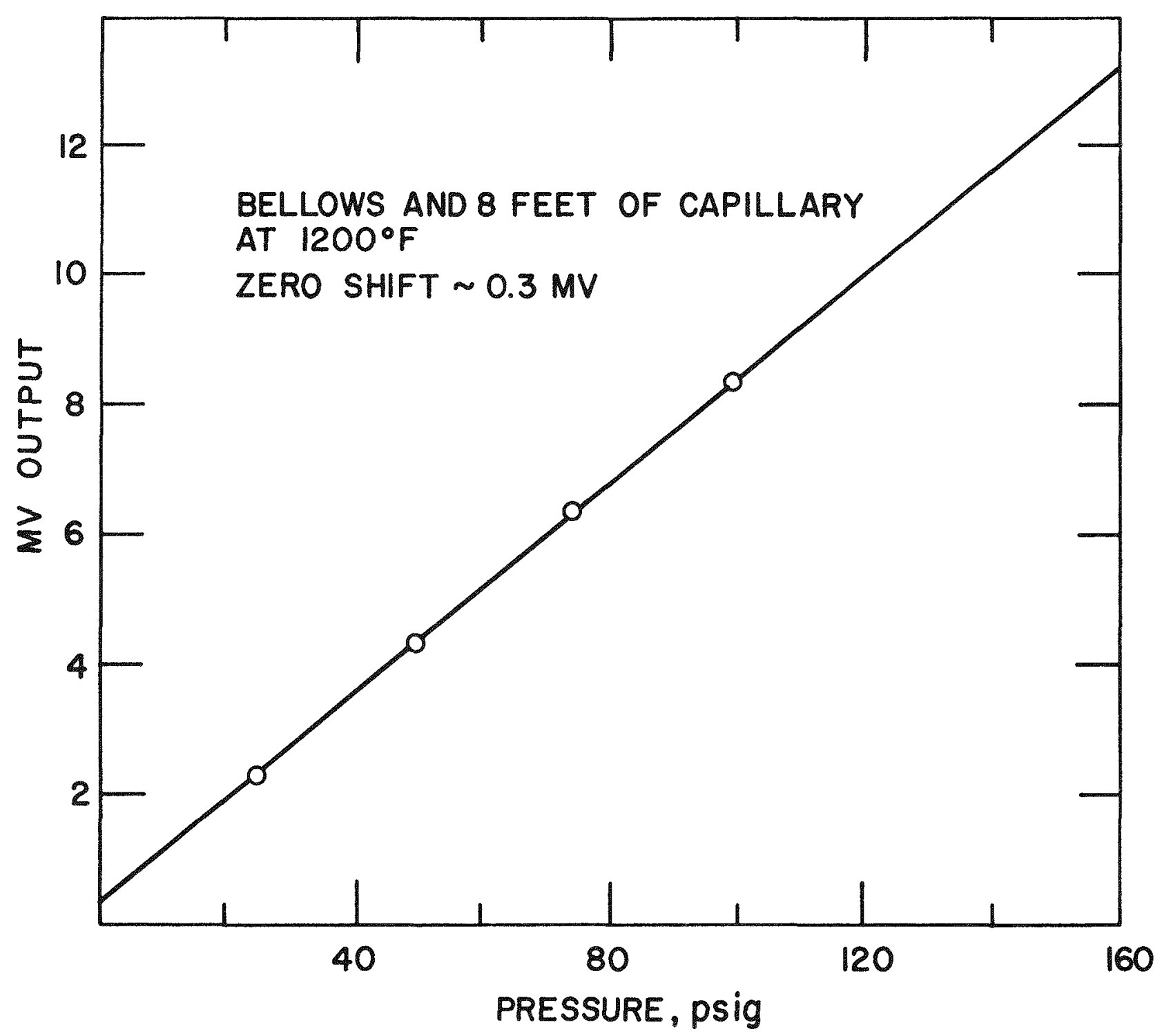

FIG. 8 5/8"BELLOWS-ID CAPILLARY SYSTEM CALIBRATION AT $1200^{\circ} \mathrm{F}$ 


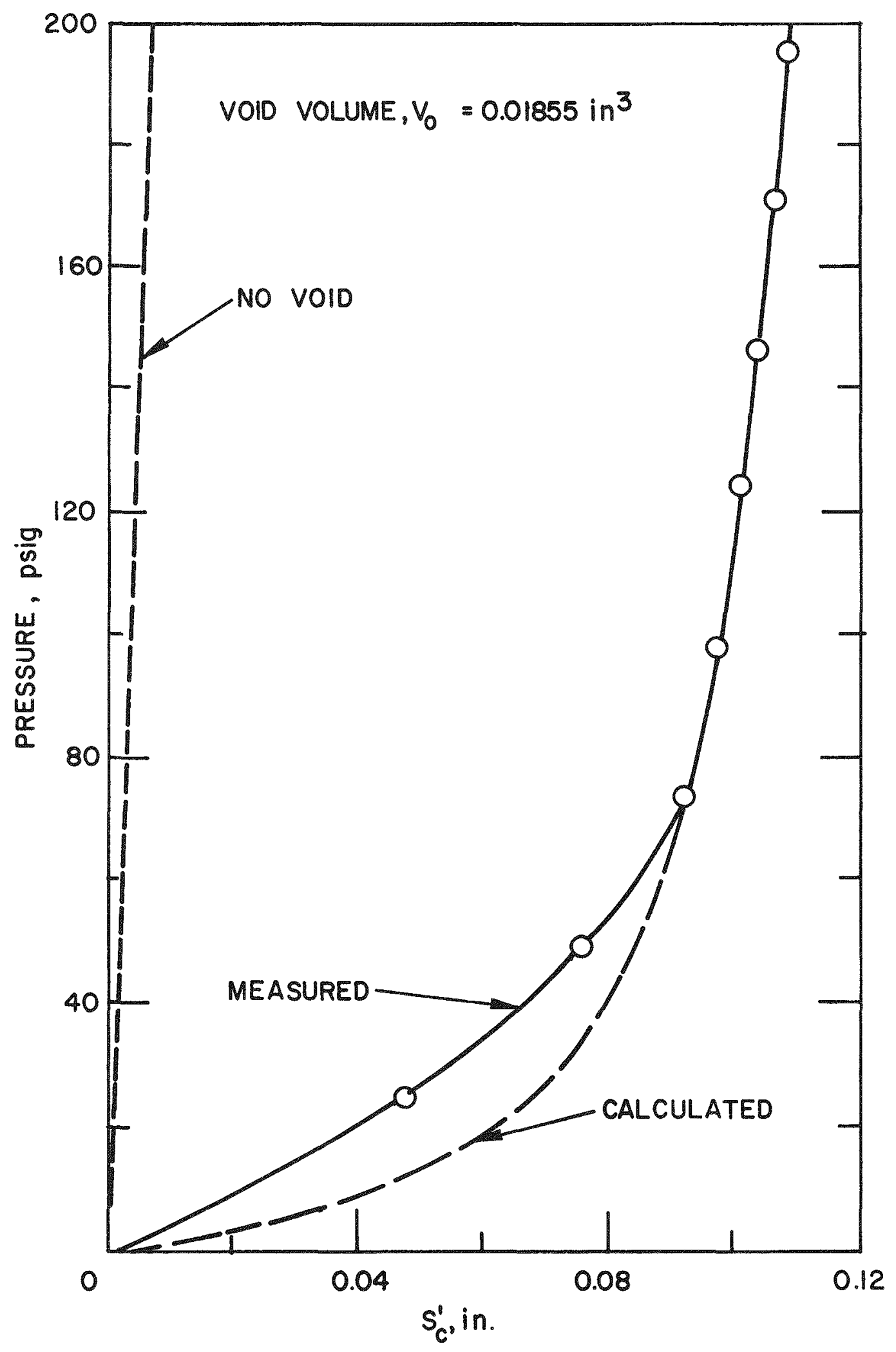

FIG. 9 MEASURED BELLOWS DEFLECTION V.S. CALCULATED DEFLECTION WITH AND WITHOUT VOIDS 


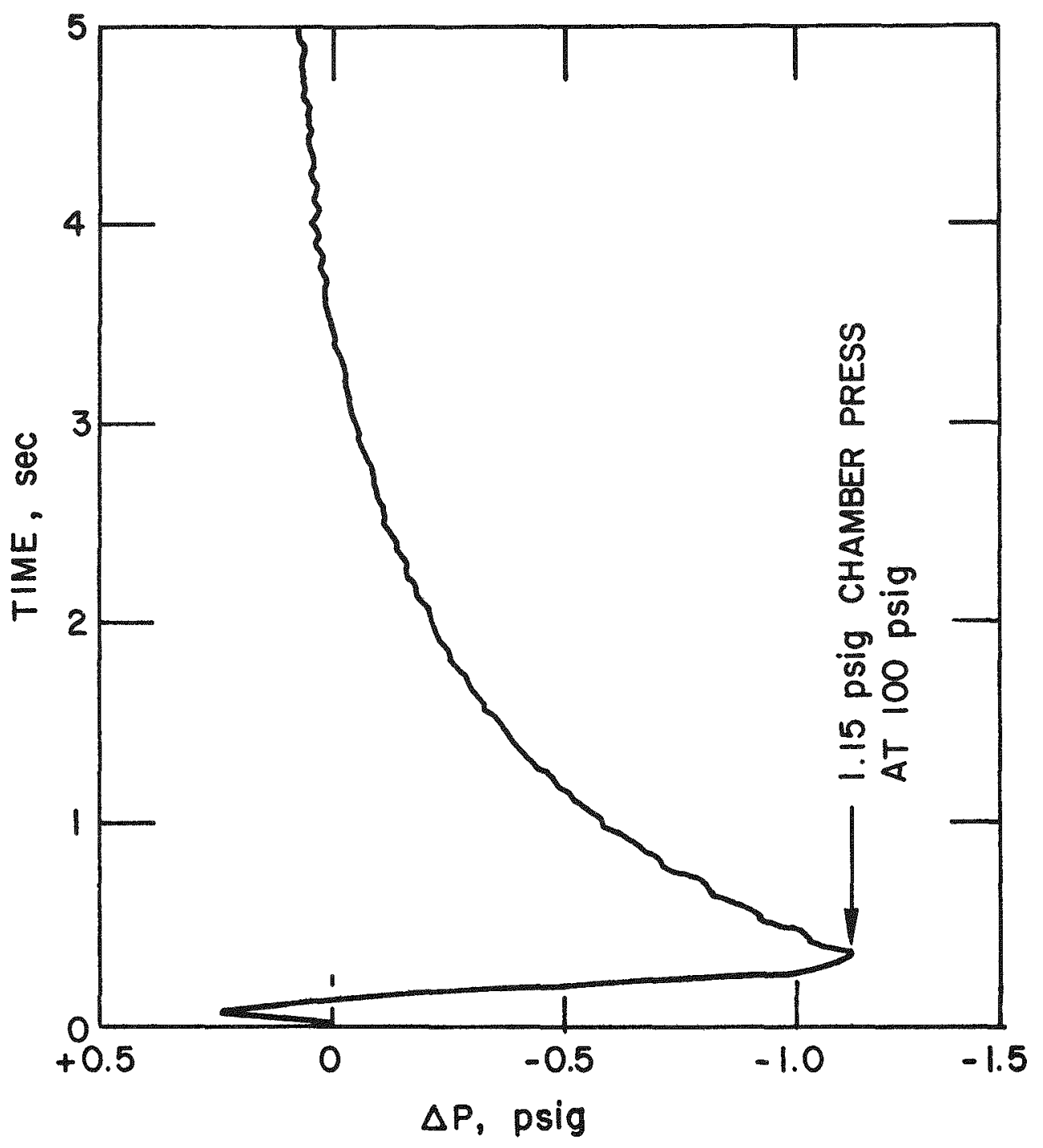

FIG 10 CHAMBER PRESSURIZATION INCREASE TO 100 PSIG FROM O PSIG 
CONTINUOUS MONITORING OF FISSION GAS RELEASE DURING IRRADIATION OF NUCLEAR FUEL SPECIMENS*

\author{
by \\ L. I. Yarger, N. E. Miller, and J.W. Ray \\ BATTELLE MEMORIAL INSTITUTE \\ Columbus Laboratories
}

\title{
ABSTRACT
}

A program is in progress at Battelle-Columbus which is aimed at developing a basic understanding of the mechanisms and processes which contribute to the phenomenology of swelling and fission gas release in nuclear fuel materials during irradiation. As part of this program, neutron radiography has been used to obtain data on the dimensional changes of fuel specimens periodically during irradiation. This data is used to develop plots of fuel swelling as a function of burnup for the specimens under irradiation. These data show a decided nonlinearity of swelling with accumulated burnup for many specimens, and it has been postulated that the shape of the curves is due, at least in part, to the gas release characteristics of the specimens. Accordingly, it was considered advantageous to develop a system to continuously monitor fission gas release from the fuel during irradiation.

The system developed consists of a nul1-balance pressure transducer attached directly to a clad fuel specimen in the capsule. Fission gases escaping from the fuel collect within the cladding and exert pressure on a diaphragm in the transducer. A balancing pressure is introduced on the other side of the diaphragm and the nu11-balance position of the diaphragm is detected by an electrical contact which is part of a detection circuit. Instrumentation is provided to monitor the balance pressure, and the fission gas temperature and pressure is correlated with the known fission gas generation rate to calculate the fractional release from the fuel. In addition to monitoring fission gas release, the system also provides information on cladding integrity. Cladding failure is detected as a large reduction in pressure while operating at essentially constant temperature.

This system has been used successfully in two irradiation experiments at fue 1 and cladding temperatures in excess of $1700 \mathrm{C}$. The data it provides on fission gas release has been used in conjunction with swelling data from neutron radiography to provide considerable insight into the phenomenology of the behavior of nuclear fuels during irradiation.

* Work performed under AEC Contract W-7405-eng-92. 


\section{INTRODUCTION}

A program is in progress at Battelle-Columbus which is aimed at developing a basic understanding of the mechanisms and processes which contribute to the phenomenology of swelling and fission gas release in nuclear fuel materials during irradiation. As part of this program, neutron radiography has been used to obtain data on the dimensional changes of fuel specimens periodically during irradiation. This data is used to develop plots of fuel swelling as a function of burnup for the specimens under irradiation. These data show a decided nonlinearity of swelling with accumulated burnup for many specimens, and it has been postulated that the shape of the curves is due, at least in part, to the gas release characteristics of the specimens. Accordingly, it was considered advantageous to include a means of continuously monitoring gas release from the fuel during irradiation.

Studies were made of several systems with potential capabilities for monitoring fission gas release continuously during the experiment. The system which was finally selected is felt to be most suitable for monitoring gas release from the standpoint of:

(1) Monitoring release directly from the fuel itself;

(2) Highest reliability; and

(3) Least difficulty in preventing activity release.

The system selected consists of a null-balance pressure transducer attached directly to a clad fuel specimen in the capsule. Fission gases escaping from the fuel collect within the cladding and exert

* Work performed under AEC Contract W-7405-eng-92. 
pressure on a diaphragm in the transducer. A balancing pressure is introduced on the other side of the diaphragm and the nu11-balance position of the diaphragm is detected by an electrical contact which is part of a detection circuit. Instrumentation is provided to monitor the balance pressure, and the fission gas temperature and pressure is correlated with the known fission gas generation rate to calculate the fractional release from the fuel. Since thermal cycles can play a considerable role in the release of fission gas from fuel materials, data is usually taken during reactor startup and shutdown as well as during irradiation.

In addition to monitoring fission gas release, the system also provides information on cladding integrity. Cladding failure is detected as a large reduction in pressure while operating at essentially constant temperature.

\section{CAPSULE DESCRIPTION}

Before discussing the method of monitoring the fission gas release during irradiation of nuclear fuel specimens a brief description of the typical irradiation capsule used to contain the specimens will be given. Figure 1 shows the irradiation capsule utilized in the present program. The capsule is designed to operate with temperatures of nominally $1700 \mathrm{C}$ at the surface of the specimen claddings. A temperature control capability of near $100 \mathrm{C}$ is affected by changing the composition of a mixture of gases, usually nitrogen and helium, in the outer annulus of the capsule. The specimens are separated from the chamber wall and each other by spacers designed to provide a minimum path for heat conduction from the specimens to the inner she11. Thermocouples are connected directly to the specimen claddings to monitor the surface temperatures.

\section{TRANSDUCER DESIGN}

The design of the pressure transducer was made within the constraints imposed by its application to the irradiation capsule. In general, the pressure transducer had to be small to fit inside the capsule lead tube (less than 0.75 inch in diameter), have good reliability so the experiment would not be jeopardized by its use, and be capable of detecting a nominal 10-psi pressure difference on the diaphragm. Figure 2 shows a drawing of the transducer. The transducer body is $2.5 \mathrm{in.}$ long by $0.566 \mathrm{in}$. diameter and is made of Type 304 stainless stee1. The diaphragm is $0.001 \mathrm{in}$. thick by $0.566 \mathrm{in}$. in diameter and is made of Type 302 stainless stee1. The diaphragm edge is welded to the transducer wall. The chamber housing the diaphragm is very shallow so that a 0.005 in. deflection in one direction will cause the diaphragm to contact the chamber wall; no deflection is permitted in the opposite direction. Thus, excessive pressures in either direction will cause the diaphragm to contact the chamber wall and prevent excessive strain in the diaphragm. 
The part of the transducer body above the diaphragm has a $0.375 \mathrm{in.} \mathrm{hole} \mathrm{drilled} \mathrm{in} \mathrm{it} \mathrm{and} \mathrm{is} \mathrm{threaded} \mathrm{to} \mathrm{accommodate} \mathrm{a} \mathrm{stainless}$ steel bolt. This bolt is bored to a 0.200 in. inside diameter and a small glass insulated terminal is attached to the bottom of the bolt. The terminal is machined so that its bottom portion is 0.030 in. in diameter. This serves as the electrical contact mentioned previously. A copper lead wire is soldered to the top side of the terminal and fiberglass insulation is placed over the lead wire. The threaded bolt is slotted to allow passage of the balance gas to the top side of the diaphragm. A $0.625 \mathrm{in.} \mathrm{diameter} \mathrm{stainless} \mathrm{steel} \mathrm{cap} \mathrm{to} \mathrm{protect}$ the bolt head is placed over and welded to the transducer body. A $0.375 \mathrm{in}$. diameter by $6 \mathrm{ft}$. Iong stainless stee1 tube is then welded to the cap to allow passage of the lead wire out of the radiation field present in the reactor. The wire is bared and sealed in epoxy as it passes out of the top of the 0.375 in. tube. Plastic insulation covers the remainder of its length. A 0.125 in. diameter stainless steel tube used to transport the balance gas from its supply point is silver soldered to the 0.375 in. tube just below the epoxy seal.

The deflection of the diaphragm is detected by the diaphragm touching an insulated contact and completing an electric circuit. The current flow through the contact is limited to 1 milliamp to reduce the risk of burning the diaphragm. The diaphragm dimensions were selected as a result of parametric studies which showed that this combination of thickness and diameter will allow the required deflection with a minimal amount of load (pressure differential) and with a resulting stress which is less than that which would be expected to cause fatigue failure of the diaphragm. Bench tests of prototype transducers showed that the devices are capable of withstanding thousands of cycles with severe pressure differentials across the diaphragm. This is important from the standpoint of safety since the diaphragm is part of the primary containment of the capsule.

In order not to complicate the capsule design, only the top specimen in an irradiation capsule has been selected to be continuously monitored for fission gas release. Little modification is required to make the adaptation for monitoring the top specimen. This is shown in Figure 3. A smal1 bore tungsten/26 rhenium tube, 0.070 in. outside diameter by $0.035 \mathrm{in.}$ inside diameter by $2.5 \mathrm{in.}$ long, was fabricated integral with the specimen cladding of the same material. The hole in the tube was formed by Electrical Discharge Machining. The top of this tube was inserted into a counter-bored 0.125 in. OD by 0.027 in. ID, Type 304 stainless steel tube and brazed with a copper brazing materia1. The temperature of this junction during full power operation is $\sim 500 \mathrm{~F}$ and the difference in thermal expansion between the two materials at this temperature is relatively insignificant. The stainless steel tube passes through the capsule header along with the thermocouples and other required tubes and is inserted into a counter-bored hole in the transducer body and welded. The transducer is located about four inches above the header so that the system volume may be minimized. Note that the specimen, instead of resting on the molybdenum support as shown in 
Figure 1, is now suspended by the tube passing from the specimen to the transducer. This part of the system is backfilled with one atmosphere of helium and sealed.

\section{SYSTEM OPERATION}

Figure 4 shows a sketch of the specimen, transducer, and associated instrumentation and components. Operation of the system is initiated when the reactor first starts up with the capsule in its test position. Since an insignificant quantity of fission gases is available during the initial stages of reactor startup, the initial pressure increase on the specimen side of the diaphragm is caused by an increase in the temperature of the residual helium. This ratio of the pressure monitored at full reactor power with essentially no fission gases present to the pressure in the system prior to operation (i.e., atmospheric) provides a correction factor to be used in determining the ratio of actual release of fission gases to the quantity which would be present with 100 percent release.

With the increase in pressure on the specimen side of the system, the diaphragm will be deflected until contact of the diaphragm is made with the electrical contact. This contact completes the low current electric circuit and is a signal for balance gas to be introduced through an inlet solenoid valve at a controllable rate. When the balance gas deflects the diaphragm away from the electric contact, the circuit is broken which closes the inlet solenoid valve and opens the outlet solenoid valve. The balance gas is then slowly bled off for a time preset on an electric timer. The range of this time is 0 to 30 seconds and the time is usually established to release only enough balance gas to result in a $\Delta \mathrm{P}$ across the diaphragm no greater than 30 psi. When the first timer completes its cycle, it triggers a second timer which has a range from 0 to 60 minutes. This timer establishes the time between balance cycles. During reactor startup, this timer is bypassed so that a balance cycle may begin immediately when the first timer completes its cycle. A large amount of data may then be obtained during the time when rapid pressure changes are most likely. Once equilibrium power is reached, the second timer is generally set to provide about a 5 to 10 minute delay between cycles. During this time, the inlet and outlet solenoid valves are both closed and a static balance system exists.

A pressure gauge equipped with a potentiometer is used to monitor the balance gas pressure since the balance pressure at the null point is also the pressure produced by fission gases and the residual helium on the specimen side of the diaphragm. A strip chart recorder is attached to the output of the pressure gauge to provide a continuous readout of the balance pressure. 
The balance gas is bled to the reactor stack after passing through an activity monitor. Activation of the alarm on the beta monitor would provide closure of both the inlet and outlet solenoids to contain any activity in the system.

\section{RESULTS}

Thus far, the system has been used successfully in two irradiation capsules at fuel and cladding temperatures in excess of $1700 \mathrm{C}$. The system was first used in conjunction with an annular $\mathrm{UO}_{2}$ specimen irxadiated at a cladding surface temperature near $1900 \mathrm{C}$. The fuel specimen was $0.210 \mathrm{in.}$ OD $\times 0.110 \mathrm{in.}$. ID $\times 1.0 \mathrm{in.}$ long. The fuel was 97 percent dense and was 20 percent enriched in U-235. Figure 5 shows the pressure of the system as a function of burnup. Note that cladding failure occurred at an estimated burnup of $0.56 \times 10^{20}$ fissions/cc. Correlation of the system pressure at the time of cladding failure with the calculated pressure for 100 percent release of the fission gases over the period shown on the plot indicated that all the fission gases produced within the fuel were released. Figure 6 shows neutron radiographs obtained at estimated burnups of $0,0.29$, and $0.59 \times 10^{10} \mathrm{fissions} / \mathrm{cc}$. Note the large redistribution of fuel inside the cladding. This large degree of relocation is typical in $\mathrm{UO}_{2}$ specimens which have high fission gas releases. Note also that the cladding failure is not visible in the radiograph implying a smal1 leak. Failure of the cladding on the top specimen did not prohibit the experiment from being irradiated to its normal target burnup of about $2 \times 10^{20}$ fissions/cc.

The second capsule which used the nul1-balance pressure system to monitor the fission gas release contained a series of UN specimens. The transducer was attached to a UN specimen, 20 percent enriched in U-235 and 85 percent dense. Its dimensions were identical to those of the $\mathrm{UO}_{2}$ specimen mentioned above except that the UN specimen had no centerline hole. This specimen was irradiated at a cladding surface temperature near $1700 \mathrm{C}$. Figure 7 shows the plot of system pressure as a function of burnup for this specimen. Note the significant increase in gas release rate at a burnup of about $0.90 \times 10^{20}$ fissions/cc and the step increase in pressure at an estimated burnup of $1.0 \times 10^{20}$ fissions/cc. At the burnup of $0.90 \times 10^{20}$ fissions/cc, the reactor was started up following a scheduled shutdown; the step increase in pressure at $1.0 \times 10^{20}$ fissions/cc also occurred after a startup following a reactor scram. The temperature of the specimen following this startup was $120 \mathrm{C}$ higher than that prior to the scram. This increase in temperature produced approximately 30 percent of the pressure increase in the step, the remaining 70 percent resulting from a release of fission gas, presumably caused by the thermal cycle from the scram. Note also that after startup from this scram, gas was released at a higher rate than before the scram. At an estimated burnup of $1.14 \times 10^{20}$ fissions/cc, the cladding of this specimen failed. A very small crack is probable since the system pressure decreased slowly 
for some 2.5 hours after the leak began to a value too low to be detected by the balance system. Figure 8 shows neutron radiographs of this specimen at estimated burnups of $0,0.27,0.59,0.90$, and $1.23 \times 10^{20}$ fissions/cc. Correlation of the system pressure with calculated pressure for 100 percent release of fission gases from the initiation of the irradiation to the beginning of higher gas release indicated about 5 percent of the fission gases produced in the fuel were released. Correlation over the entire irradiation period until cladding failure indicated about 9 percent release of the fission gas.

\section{CONCLUSIONS}

Based on the two experiments discussed above, it is seen that the data on fission gas release can be obtained with the use of a nu11balance pressure transducer system. These data can be used in conjunction with swe11ing data from neutron radiography to provide considerable insight into the phenomenology of the behavior of nuclear fuels during irradiation. The method also provides a means for determining the loss of cladding integrity. In comparison to other systems which could be used to monitor fission gas release (such as directly monitoring the activity of radioactive fission gases released from fuel by a radiation detector), this system is relatively simple to fabricate and install and is inexpensive. It is also reliable and safe. Its successful use in the two experiments above dictate its continued use in future irradiation capsules. 


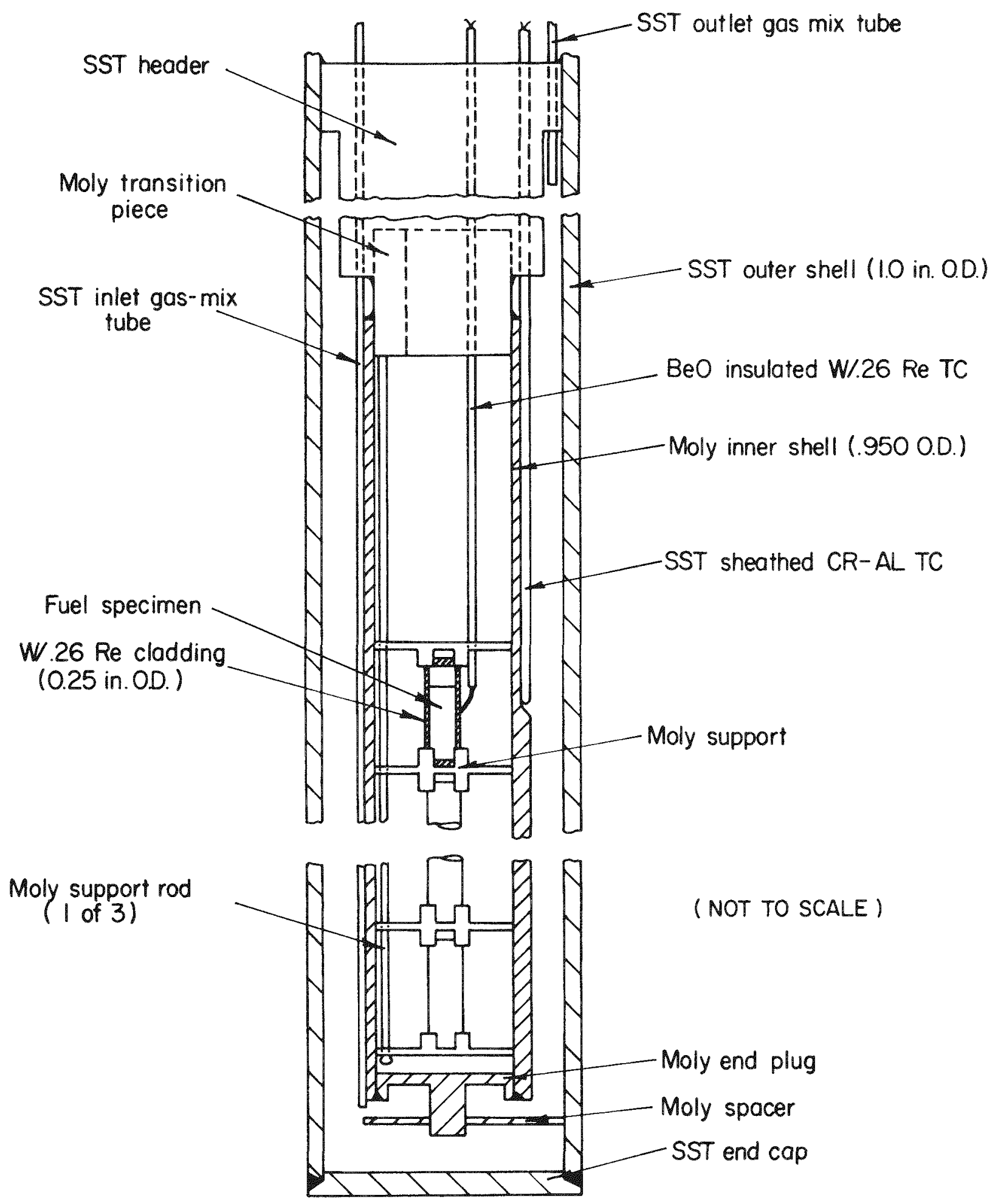

FIGURE 1. TYPICAI IRRADIATION CAPSULE USED IN PRESENT PROGRAM 


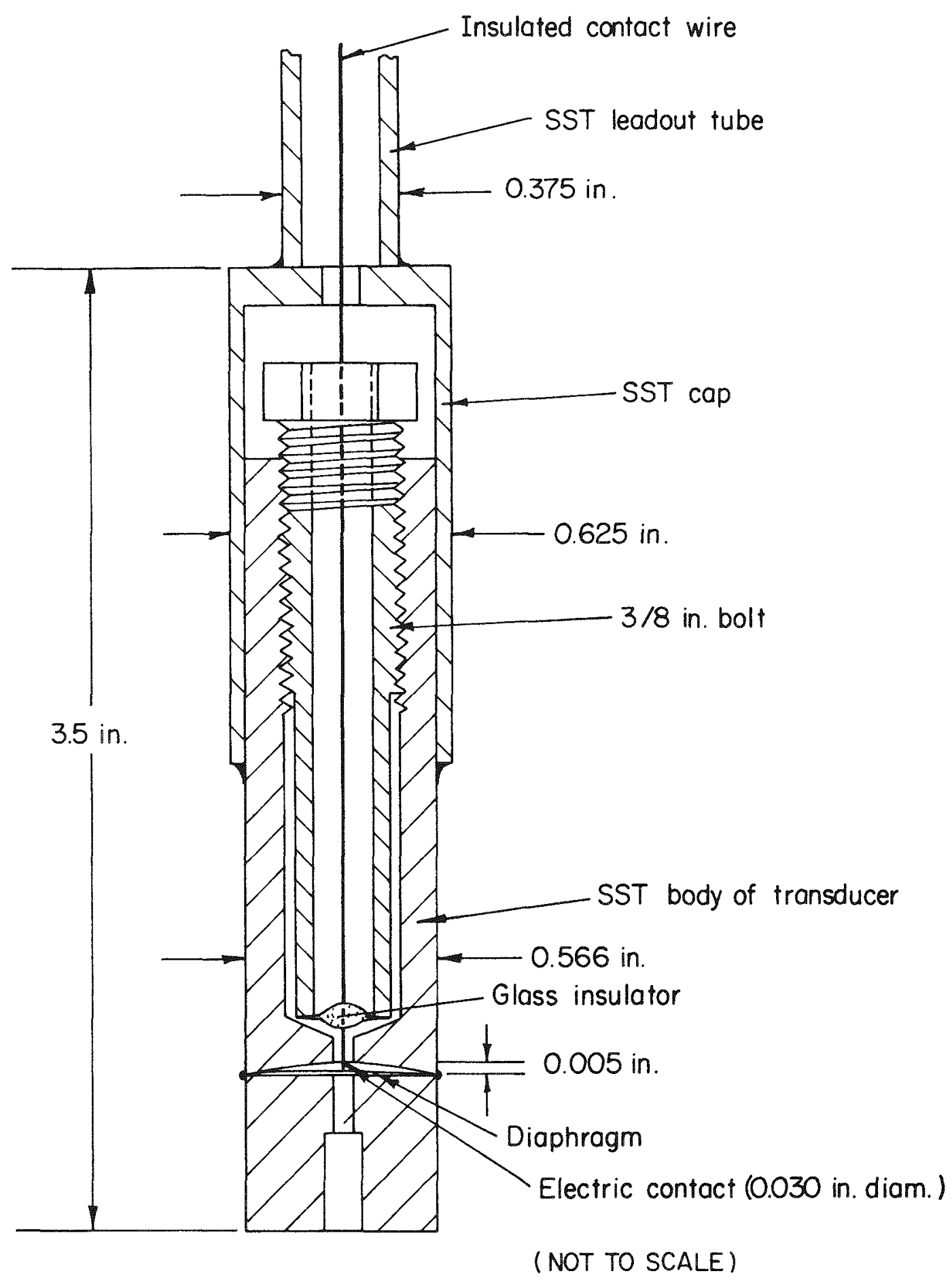

FIGURE 2. PRESSURE TRANSDUCER 

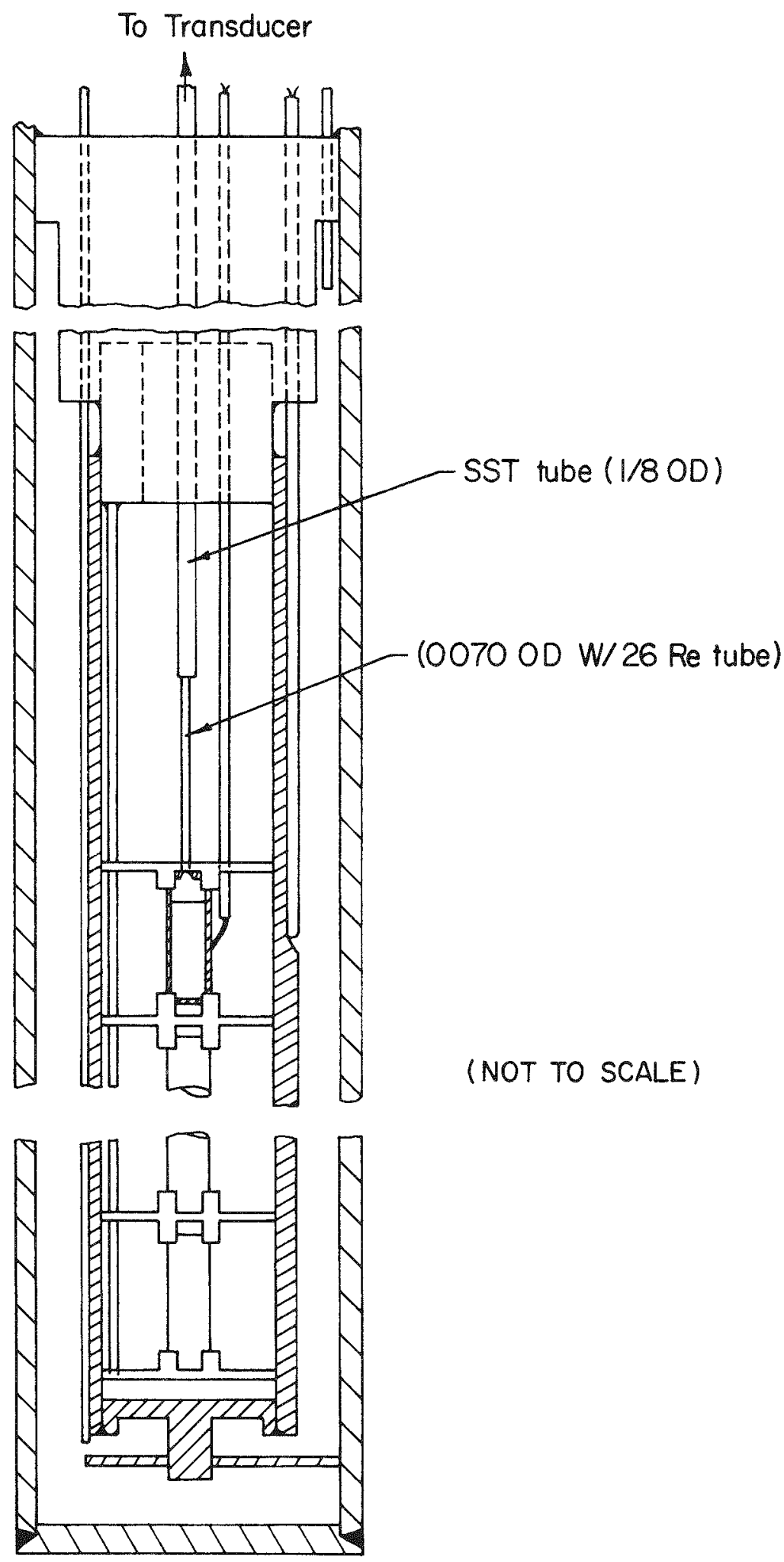

FIGURE 3. TYPICAL CAPSULE MODIFIED TO MONITOR PRESSURE OF TOP SPECIMEN 


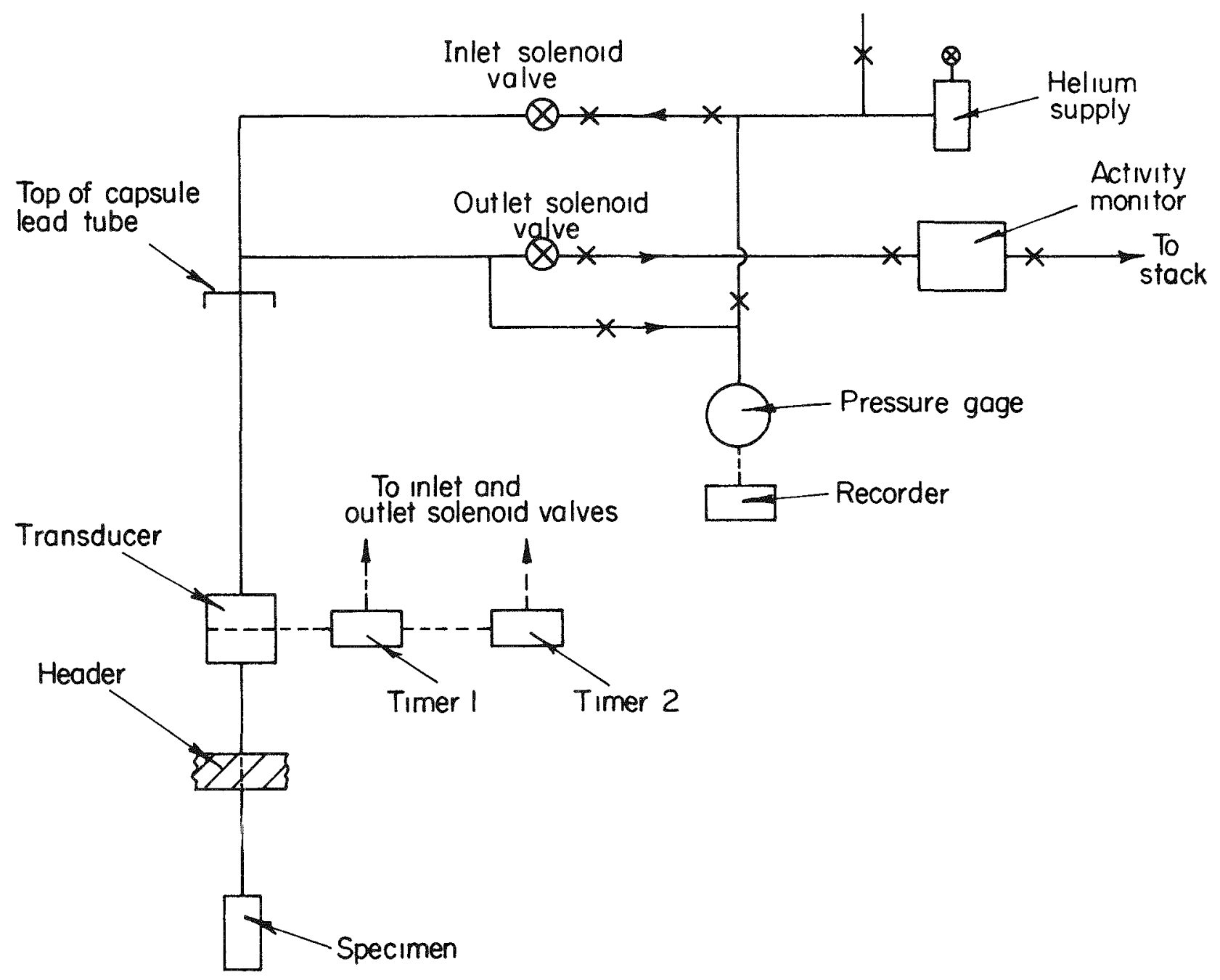

FIGURE 4. SKETCH OF SYSTEM COMPONENTS 


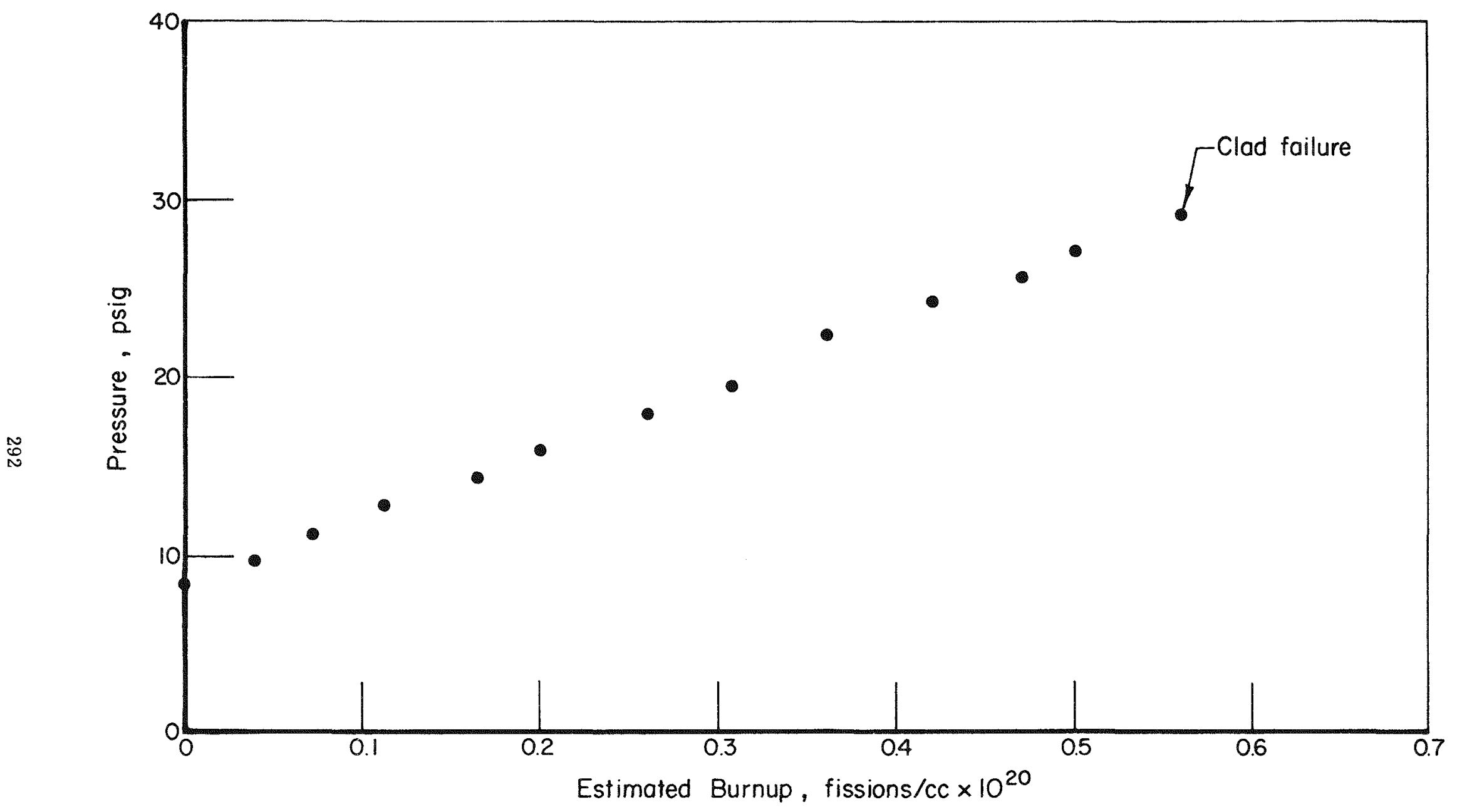

FIGURE 5. PRESSURE VS. BURNUP FOR UO 2 SPECIMEN-TRANSDUCER SYSTEM 
Estimated Burnup, fissions/cc $\times 10^{20}$
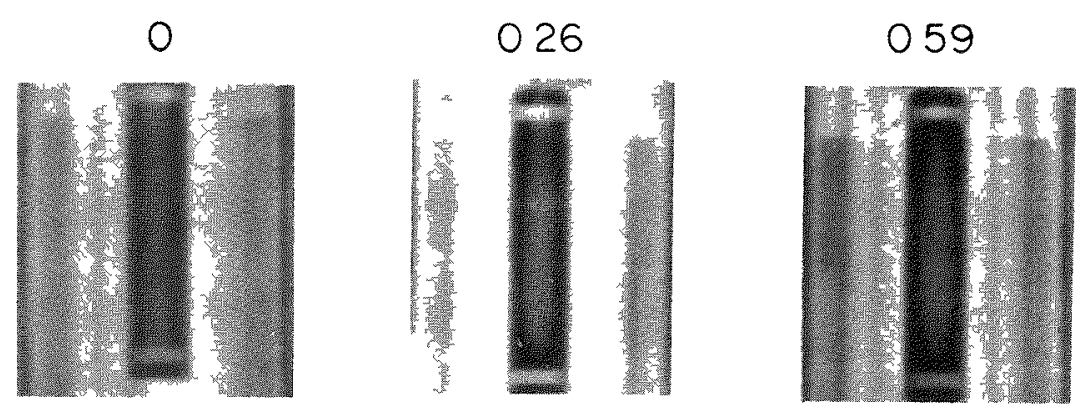

\section{FIGURE 6. NEUTRON RADIOGRAPHS TAKEN AT VARIOUS STAGES OF BURNUP FOR UO ${ }_{2}$ SPECIMEN}




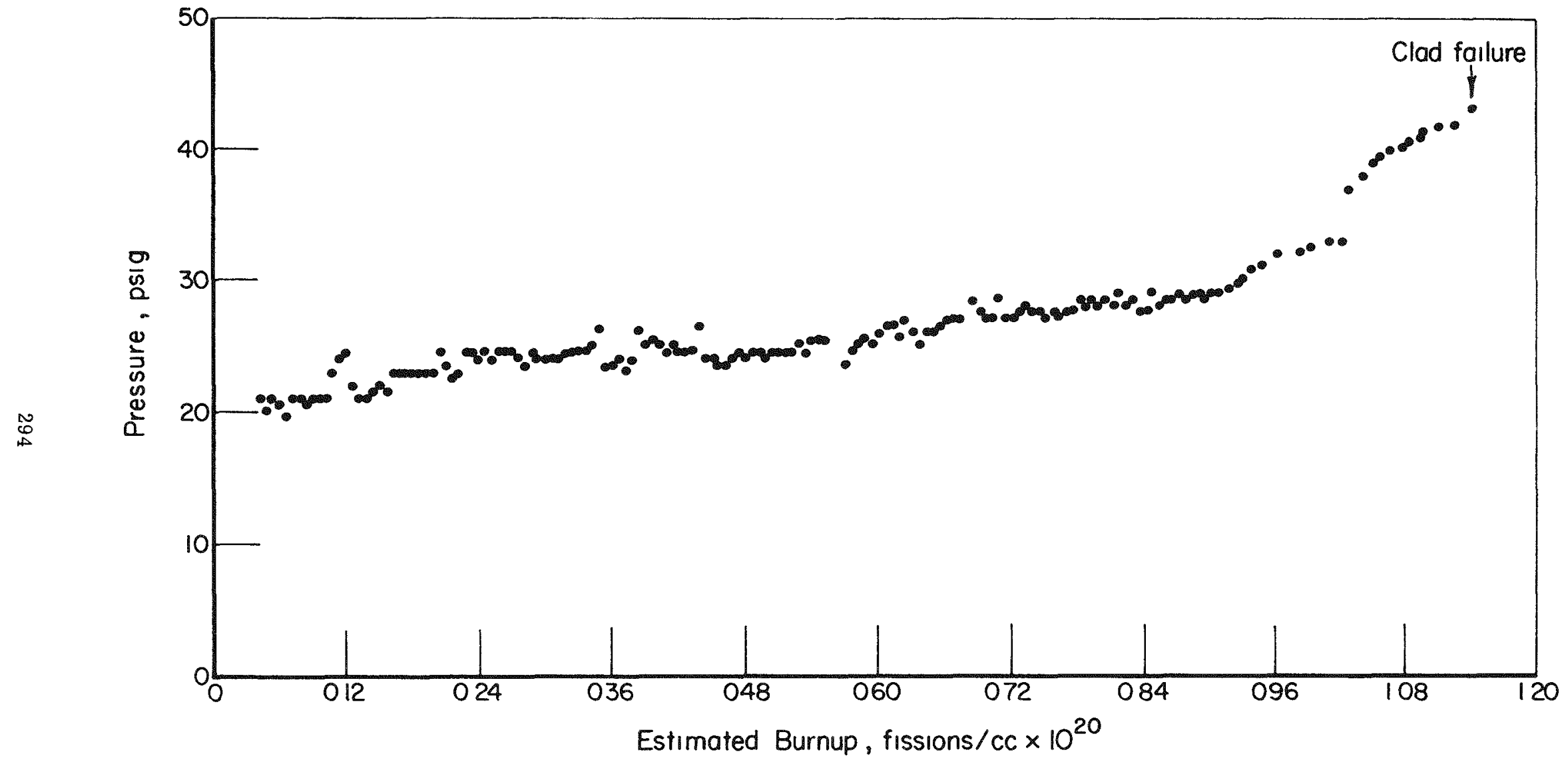

FIGURE 7. PRESSURE VS. BURNUP FOR UN SPECIMEN-TRANSDUCER SYSTEM 


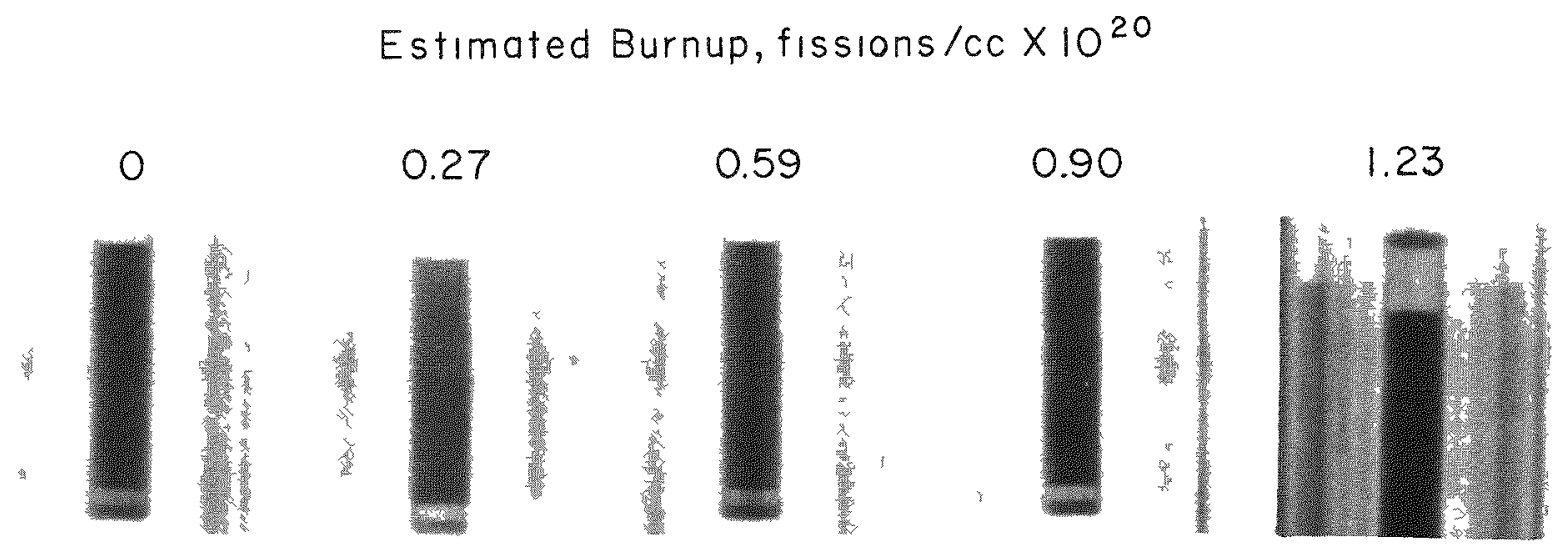

FIGURE 8. NEUTRON RADIOGRAPHS TAKEN AT VARIOUS STAGES OF BURNUP FOR UN SPECIMEN 
The Measurement of Intemal Pressure Buildup In Full Sized Fuel Rods During Irradiation

T. B. Burley

Battelle Memorial Institute, Pacific Northwest Laboratory 11586,2 Richland, Washington

\section{ABSTRACT}

Full-sized mixed-oxide fuel rods were instrumented to monitor internal gas pressures and plenum gas temperature during irradiation in the Plutonium Recycle Test Reactor (PRTR) at Battelle-Northwest. The successful operation of the experiments under different operating conditions for over fifteen months in the reactor provided information related to the sorbed gas behavior and fission gas release fraction as affected by fuel temperature and burnup.

Introduction

One criterion in determining fuel element residence time in commercial reactors is the allowable internal fuel rod pressure during irradiation. This pressure arises from the initial fill gas, and an accumulation of tine desorbed gases and released fission gases generated during irradiation.

The behavior of insoluable gases in ceramic materials has been extensively studied and the kinetics of gas release are reasonably well understood for certain idealized conditions. An accurate analytical determination of the pressure arising from fission gas requires experimental verification because of the non-uniform axial and radial gas generation, complex temperature distributions, and the steep thermal gradients characteristic of full-sized operating fuel rods. 
Experiments were conducted at Battelle-Northivest Laboratory in which internal gas pressures and plenum gas temperature were measured in full-sized fuel rods during normal reactor operation in the Plutonium Recycle Test Reactor (PRTR). Four instrumented Zircaloy-clad vibrationally compacted, mixed-oxide ( $\left.\mathrm{UO}_{2}-2 \mathrm{wt} \% \mathrm{PuO}_{2}\right)$ fuel rods were included in these experiments. Internal gas pressures were monitored with Booth-cromer type, null-balance pressure transducers. Plenum gas temperatures were measured with chromel-alumel thermocouples. The simplicity of the overall instrumentation system permitted internal pressure buildup to be determined during fifteen months of reactor operation in PRTR. Maximum internal pressures monitored were approximate $7 y 150$ psi after peak rod burnups of 10,000 Megawattdays per tonne of metal (10,000 MUd/MTM).

These experiments provided information related to the sorbed gas release behavior and fission gas release fraction as affected by fuel temperature and burnup.

Experimental Description

Four instrumented fuel rods were included in these experiments. Two each of the four Zircaloy-clad, vibrationa $17 y$ compacted, $\mathrm{UO}_{2}-2 \mathrm{wt} \% \mathrm{PuO}_{2}$ fuel rods were charged into two standard high power density nineteen-rod cluster elements (Figure 1). The elements containing the instrumented rods could therefore be manipulated by nearly standard techniques during charge-discharge operations and during fuel element inspections. Each instrumented fuel rod contained a chrome 1-alumel thermocouple to measure plenum gas temperature and a null-balance pressure transducer to measure internal gas pressure.

The stainless-steel clad thermocouple was positioned at the geometric center of the gas plenum. It was sealed into the Zircaloy-clad fuel rod by torch brazing, using a Ni-13\% Cr-10\% p brazing alloy, to a 304 stainless-steel adapter, then diffusion brazing the thermocouple-adapter assembly to the Zircaloy-2 fuel rod end plug (Fiqure 2). The diffusion brazing was accomplished by electron-beam heating the mated region of the stainless-steel-Zircaloy assembly in vacuum to slightly above the eutectic temperature. Unon cooling, the liquid stainless-steel-Zircaloy eutectic at the interface solidified to form a leak-tight joint. Thermocouple-end plug assemblies joined by these techniques withstood pressures and temperatures of 10,000 psi and $300^{\circ} \mathrm{C}$. 
The 304 stainless steel null-balance pressure transducers attached to each rod to measure internal gas pressure were the same basic type as that developed by Reynolds (Fiqure 3). This transducer utilizes a pressure differential to deflect a thin diaphragm toward the low pressure side which breaks contact and opens an electrical circuit. Full deflection of the 0.750 inch diameter, 0.002 inch thick 304 stainless steel diaphragm inside a 0.005 inch deep semilenticular transducer cavity results from a pressure differential across the diaphragm of less than 1 psi. pressure transducers were attached to the Zircaloy-2 clad fuel rods by tungsten-inert gas welding the primary pressure tube and protector shroud tube to the stainless steel end plug adapter (Figure 2).

\section{Experiment Instrumentation}

During irradiation, pressure resultina from the initial fill gas, desorbed gases and moisture, and released fission gases ( $\mathrm{Xe}+\mathrm{Kr}$ ) was exerted on the fuel rod side of the transducer diaphragms.

Internal fuel rod pressures were determined by applying helium gas to the secondary side of the transducer diaphragm through a manually operated pressure regulating valve until a null-balance condition across the diaphragm was obtained (Fiqure 4). Null-balance was indicated by an infinite resistance signal on a continuity meter as the diaphragm deflected from the electrical contact. The balancing pressure required to open the circuit was then read directly from a large-face pressure gage attached to the balancing gas tubing.

\section{Safety system}

During normal operation, all of the fuel rod gases were contained on the fuel side of the transducer diaphram. The transducers were sealed inside of the reactor process channels so that exposure of personnel to fission gases during operation was not a problem. In the event of a diaphragm failure, fuel rod fission gases could flow into the secondary pressure tubes, thus exposing operating personnel to the gamma emmiting fission product gases. A safety system was developed, however, which prevented this possibility from occurring (Figure 4). Upon failure of a transducer diaphragm, the fuel rod fission gases would flow through the failed diaphragm into a filter. The filter consisted of porous stainless steel plates at each end of a compartment of activated charcoal, all contained in a stainless steel body. Some of the gases, primarily iodine, would be adsorbed by the activated charcoal, with the remainder passing through the filter into the secondary tubing. Receipt of a fission gas signal by a gamma radiation activity monitor, which was attached to the secondary pressure tubing, deactivated a normally closed 
solenoid valve. The monitor and the fail-safe valve was positioned at the edge of the shielded portion of the secondary pressure tube so that closure of the valve isolated the tubing containing the released fission gases from the unshielded portion of the system. Upon receipt of the gamma radiation signal an audible alarm in the activity monitor would alert operating personnel, permitting them to evacuate the area in which the instrumentation was located before being exposed to the potentially radioactive secondary pressure tubes.

The radioactive gases could be removed from the system by vacuum pumping through a normally closed safetv discharge pressure tube after a time period suitable to allow highly radioactive iodine to decay off. Thus, the isolated fission gases could be removed from the fuel rod assemblies sufficiently to allow the safe removal of the defected fuel element from the reactor. The safety system was not employed during the course of the irradiations because none of the transducer diaphragms failed.

Experiment Technique

Ine of the instrumented fuel elements was placed in the highest flux region of the reactor. The other instrumented element was placed in a low flux region of the reactor. In this manner internal pressure data could be obtained for fuel rods operating at both high and low power conditions. The two instrumented fuel rods in each of the two elements were positioned so that they would operate at nearly identical conditions. Internal pressure and plenum gas temperature measurements were obtained at regular time intervals during irradiation. Pressure and temperature data were a 150 collected during periods when reactor power was either raised or lowered in a stepwise manner. The normal sequence of data collection during reactor operation was as follows:

1. Just prior to startun

2. At intervals during stepwise increase

3. At full power

4. Every 24 hours during steady power operation

5. At full power just before shutdown

6. At intervals during stepwise power reduction

7. At zero oower

8. Every 24 hours during shutdown periods. 
The raw data, i.e., pressure and temperature periods were related to fuel rod power and burnup. Samples of the pressure-reactor power relationship and plenum gas temperature data for the four intstrumented fuel rods are shown in Figure 5 and 6 .

\section{References}

1. M. B. Reynolds, "The Measurement of Fission Gas Pressure in Operating Fuel Elements," GEAP-4135, GEAPED, San Jose, California. 


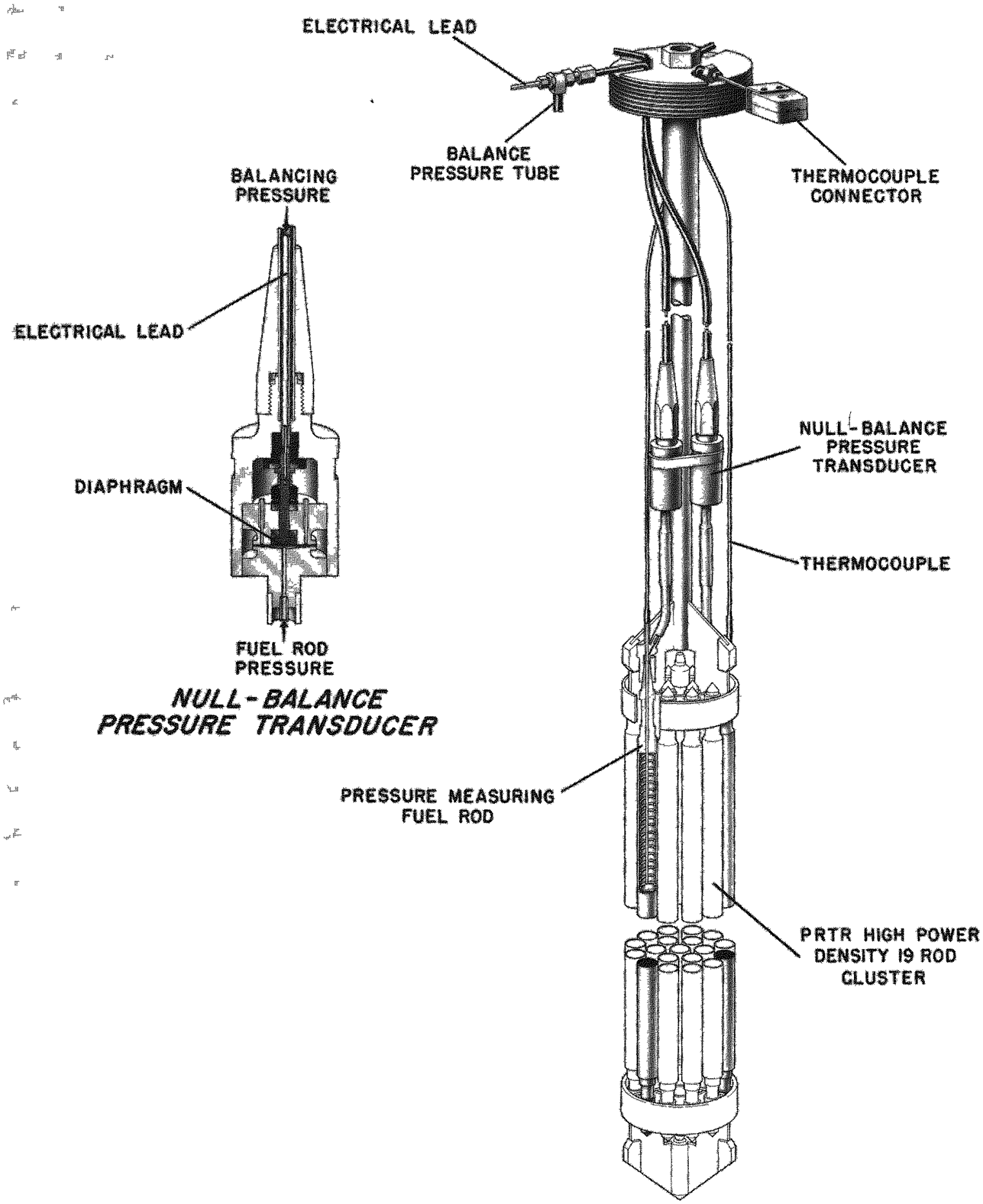

FIGURE 1

Plenum Temperature and Pressure Instrumented PRTR Fuel Element 


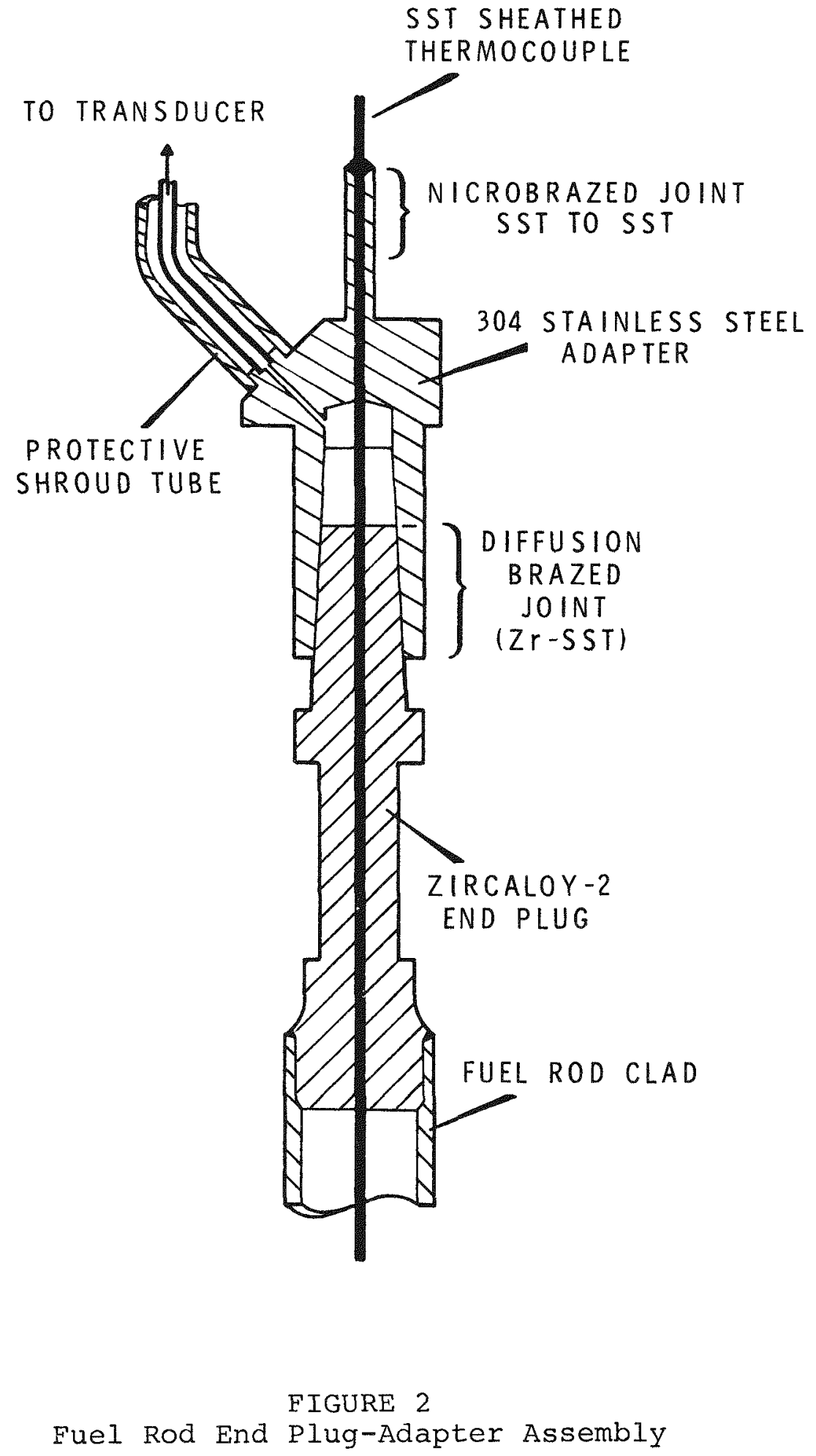




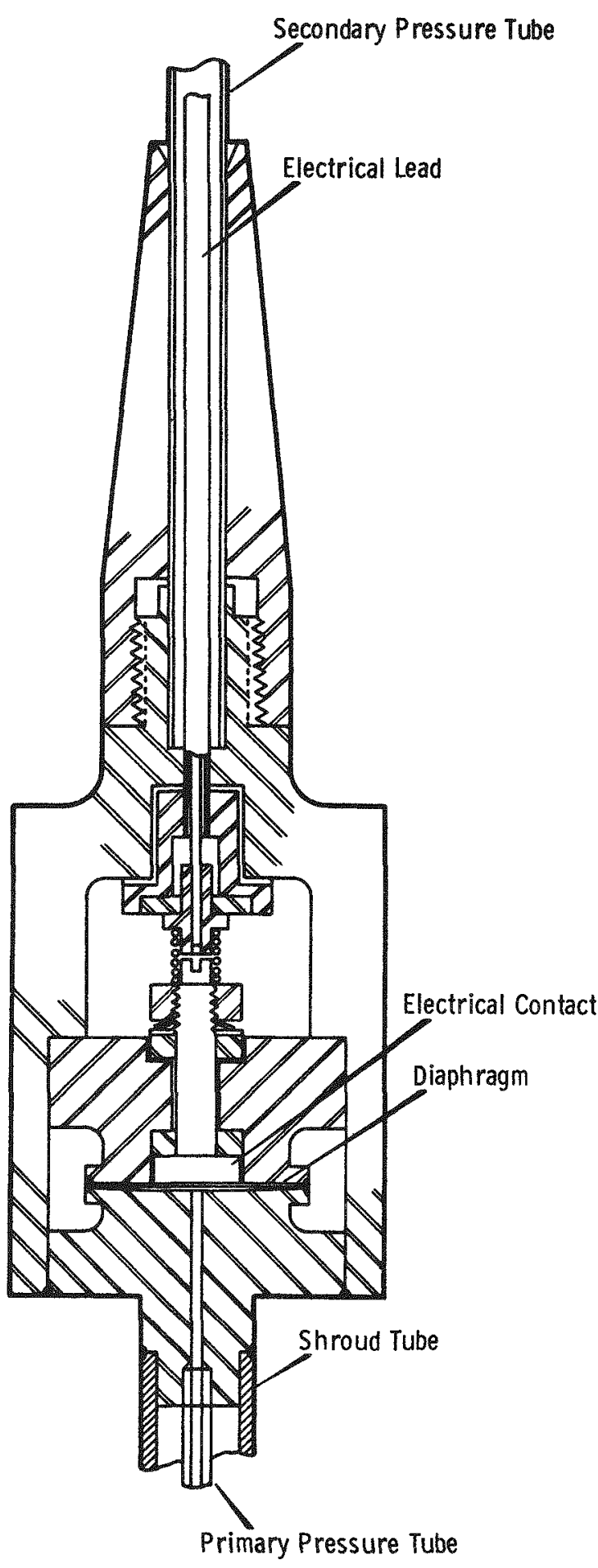

EIGURE 3

Longitudinal Section of Null Balance Pressure Transducer 


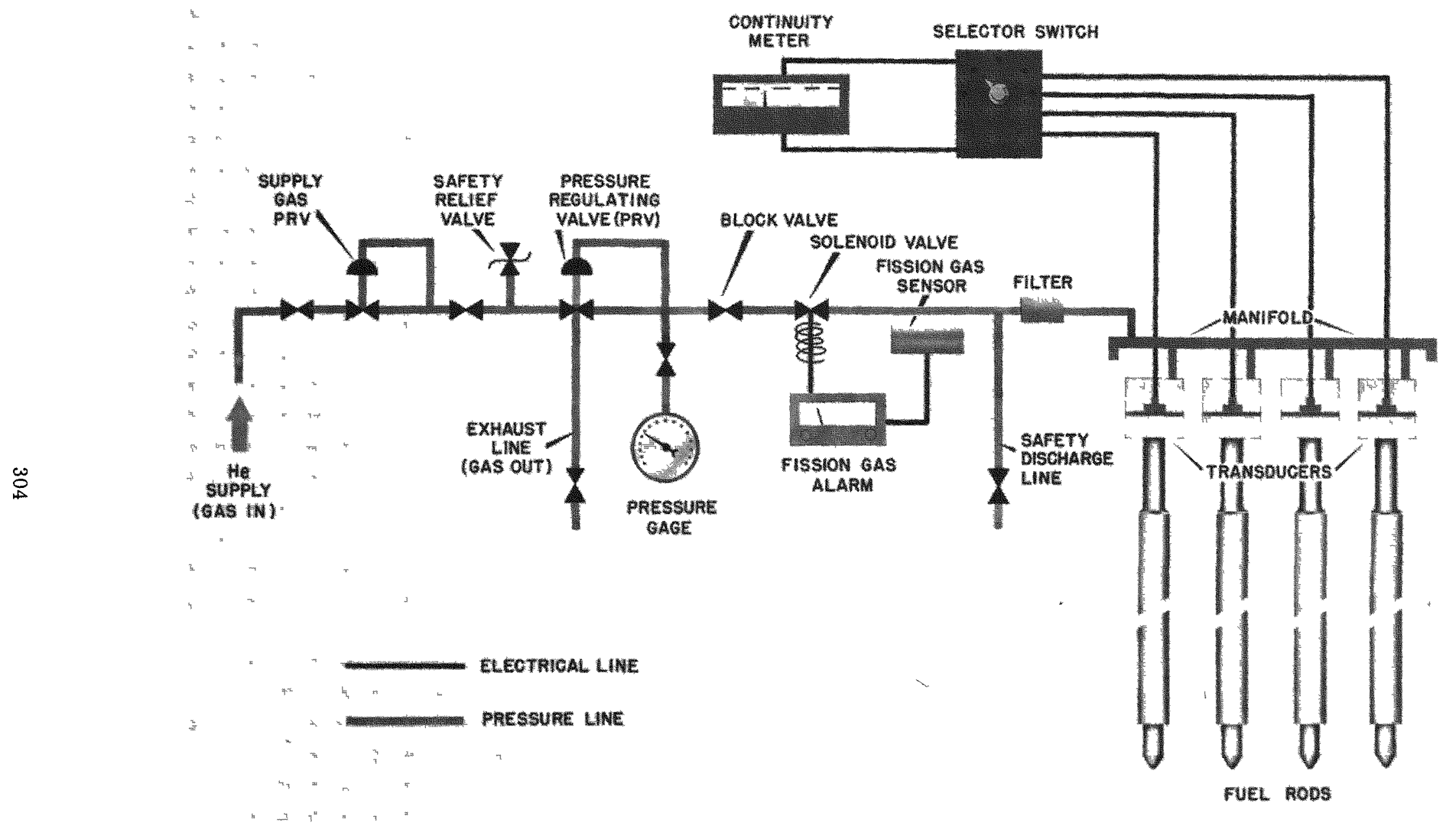

FIGURE 4

Schematic of Pressure Measuring Experiment Instrumentation 

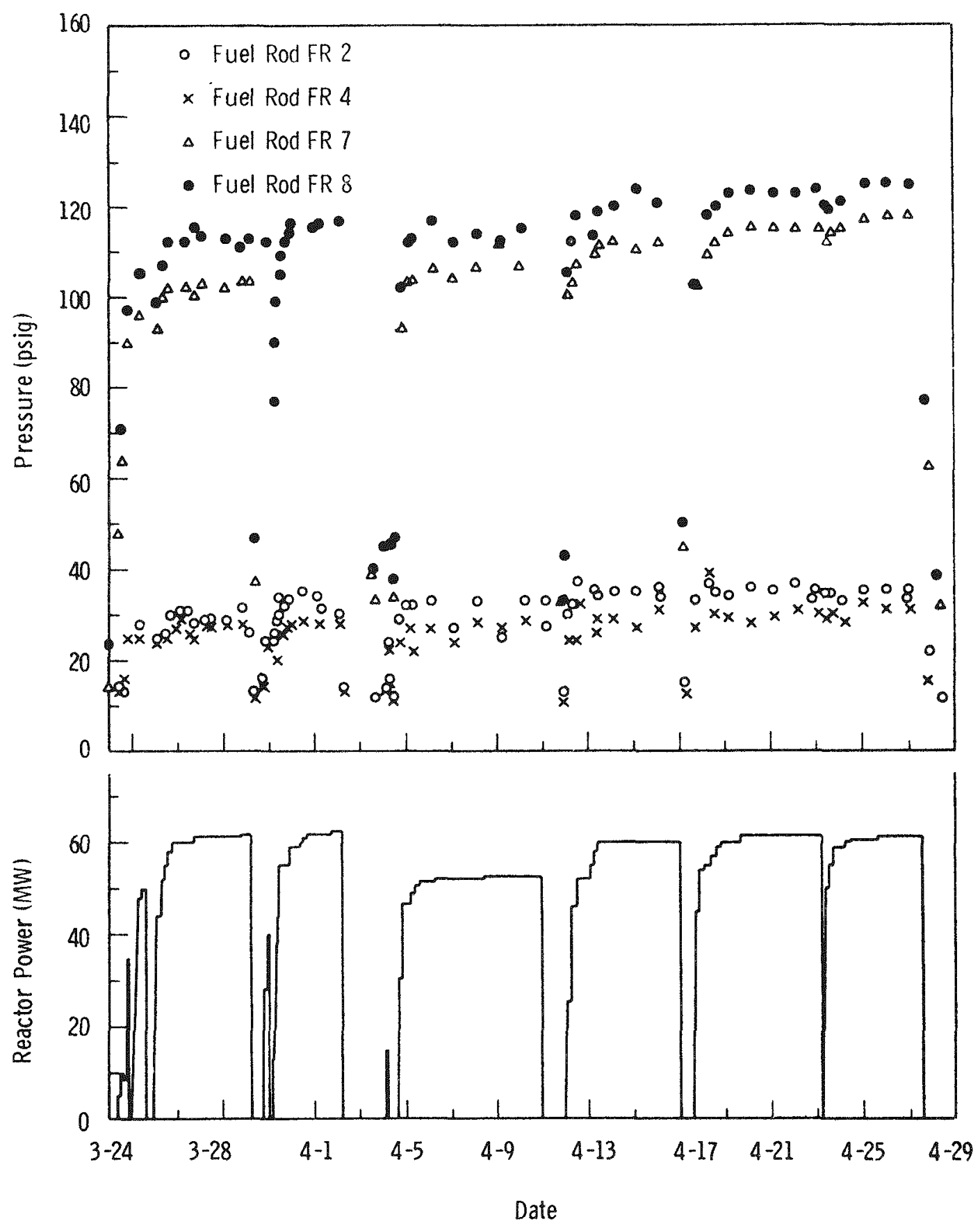

FIGURE 5

Pressure Data Plotted Against Reactor Power 


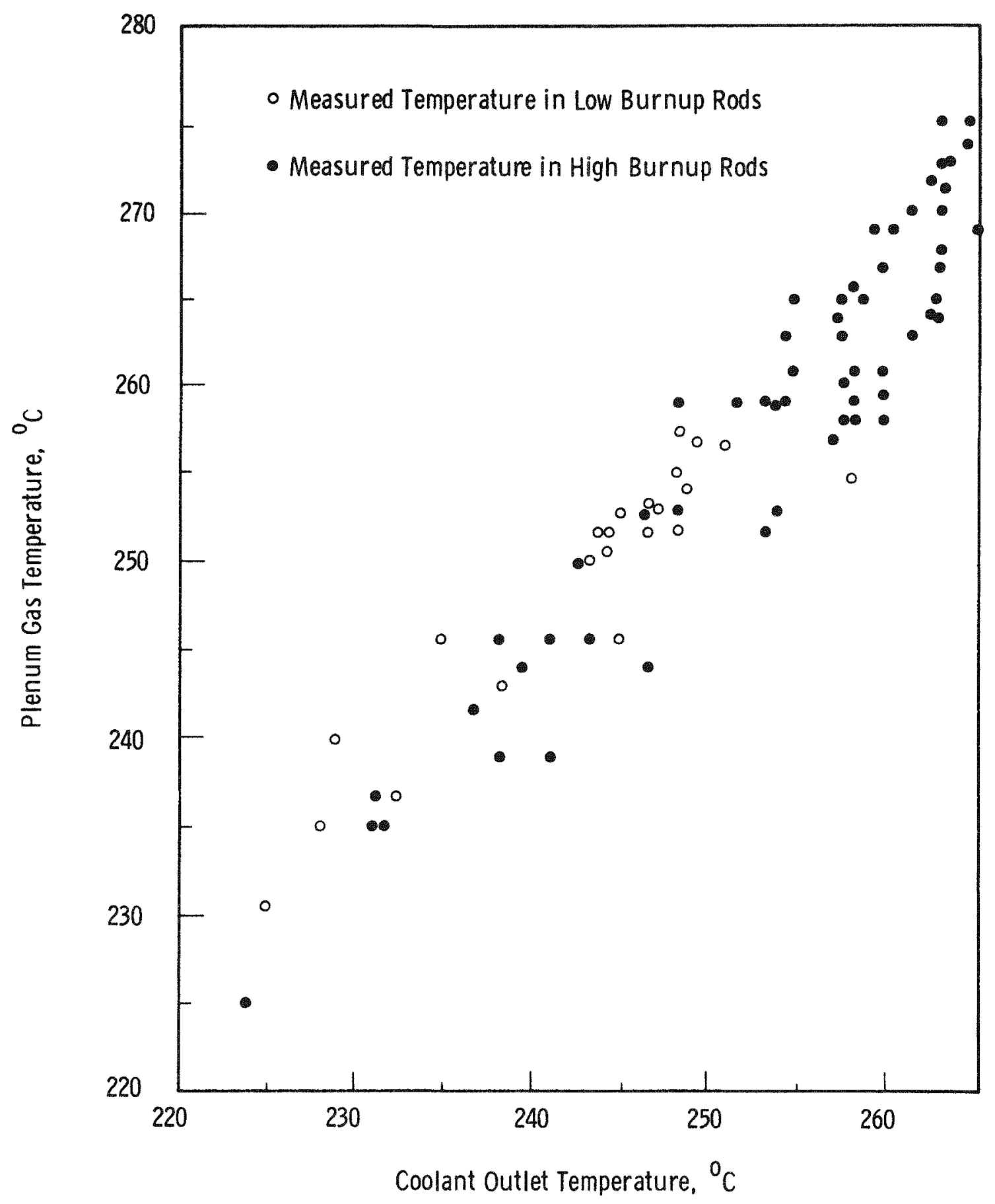

FIGURE 6

Plenum Gas Temperature Versus Maximum Coolant Temperature 
K HIGH TEMPERATURE THERMAI CYCLING AIVD DRIFT TEST OF W95 Re5 VERSUS W74 Re26 THERIMOCOUPIES*.

by

D. E. Schwarzer

$\begin{aligned} 31 \% & \text { Gulf Ceneral Atomic, Incorporated } \\ & \text { San Diego, California }\end{aligned}$

ABSTRACT

The requirement to monitor the fuel temperatures of in-pile fuel material irradiations necessitated the use of high temperature thermocouples. Due to the high loss rate of commercially fabricated thermocouple units, Gulf General Atomic proceeded to develop and test their "in-house" fabricated thermocouples using W95 Re5 versus W74 Re26 sensor wires. A tantalum swaged hot junction with various multi-sensor wire configurations is described as are the test results. Thermocouple calibrations were accomplished prior to thermal cycling from $1800^{\circ} \mathrm{C}$ to $<500^{\circ} \mathrm{C}$, after thermal cycling and after a 500 hour life test at $1650^{\circ} \mathrm{C}$. Test results indicated that the thermal emf of the tested thermocouples were in agreement with the published millivolt output data and that stable output was maintained after many thermal cycles and for long periods of time at temperature. The test apparatus is described as are the post-test examination results of the thermocouples, showing the impurities in two different samples of beryllia used in the testing. Metallographic examination of the swaged tantalum plug and sensor wires showed no contamination and also that a good contact was maintained between adjacent thermocouple wires. Two short term tests of 155 and 100 hours at $1650^{\circ} \mathrm{C}$ using $W_{97} R_{3}$ versus W75 Re25 as the sensor wires are briefly discussed. These sensor wires were in the same four wire, four slot configuration as those used for the 500 hour life test. The test results also agreed with published data and were stable over the lifetime of the test. No failure occurred during any of the testing.

INTRODUCTION

A test to determine the effects of thermal cycling and time at temperature $\left(1650^{\circ} \mathrm{C}\right)$ on the stability of the thermal emf outputs of four wire assemblies of W95 Re5 versus W74 Re26 thermocouples using a tantalum swaged hot junction of various hole configurations was required for a specific in-pile fuel

*This work was sponsored by the National Aeronautics and Space Administration under contract NAS 3-6471. 
material irradiation. Four wire thermocouples are useful since in the event that one of the wires detaches from the hot junction, there are still two dissimilar wire combinations remaining, using the three remaining wires.

\section{DESCRIPTION OF APPARATUS}

The facility shown in Fig. I was designed and built at Gulf General Atomic primarily for thermocouple calibrations at temperatures ranging from $1000^{\circ}$ to $2000^{\circ} \mathrm{C}$ at a pressure of $1 \times 10^{-6}$ torr.

A closed ended tungsten cylinder horizontally mounted and heated by electron bombardment from an internal filament is externally shielded with five layers of tantalum. There are thirteen $3 / 4$ inch deep holes in the cylinder annulus, in which the sheathed thermocouples are inserted. Hohlraums of $1 / 32$ inch diameter and 0.34 inch depth are drilled through the closed end of the cylinder to permit the temperature at the hot junction end of the thermocouple sheath to be read with an optical pyrometer (Fig, 1).

The vacuum containment and pumping system consists of an 18 inch glass bell jar, a stainless steel base plate with feedthroughs, an ion gauge, a water-cooled chevron baffle and an oil diffusion pump. The thermocouple wire feedthrough is made up of continuous 0.005 inch diameter $W_{95} \operatorname{Re}_{5}$ and $W_{74}$ Re$_{26}$ wires from inside the vacuum chamber to a terminal strip at room temperature outside of the vacuum system at which point Hoskins alloy 205/260 compensated lead wires are joined for the run to the instrumentation. A digital voltmeter having a resolution of 0.01 millivolts is used to measure the thermocouple millivolt output.

A movable shield is located between the hot tungsten cylinder and the viewing window to forestall deposition from the hot cylinder onto the window. This deposition would give incorrect pyrometer temperature readings.

\section{THERMOCOUPLE AND SHEATH FABRICATION}

The sheaths into which the thermocouples are inserted are tubes fabricated from vapor deposited $\mathrm{W}_{74} \mathrm{Re}_{2} 6$ of $1 / 16$ inch $0 . \mathrm{D}$. X 0.010 inch wall thickness X 6 inches long. Prior to closing the end of the sheath by welding in a controlled argon gas atmosphere, the tubes were degreased and etched. in a solution of 20 parts of lactic acid, 3 parts of nitric acid, 1 part of hydrofluoric acid and 25 parts of water, and then thoroughly washed in distilled water and dried. The weld and the length of tube were then leak checked using a mass spectrometer leak detector having a sensitivity of $2 \times 10^{-10} \mathrm{std} \cdot \mathrm{cc} / \mathrm{sec}$.

The hot junctions consist of 0.005 inch diameter $W_{95} R_{5}$ and $W_{74} R_{26} 6$ wires from the same lots inserted into holes in a tantalum plug which was then swaged. Two hole or four hole hard fired beryllia insulator beads are strung over the thermocouple wires from the hot junction to a point $1 / 16$ inch past the end of the sheath. This assembly is then inserted into the sheath and installed in the test apparatus. 
Three types of tantalum plugs were incorporated in the test, as shown in Fig. 2. A single 0.013 inch diameter hole on center, Fig. 2a; two 0.013 inch diameter holes equally spaced on an 0.017 inch bolt circle, Fig. 2b; and four equally spaced 0.007 inch wide slots X 0.015 inch deep, Fig. 2c. Slots were used in the last case to simplify the fabrication of the plug. All the above mentioned plugs were 0.040 inch in diameter X $3 / 32$ inch long prior to the swaged operation.

The fabricated thermocouples were given identification numbers as follows:

$$
\begin{aligned}
& C-116 \\
& \text { two dissimilar wires in a one hole plug (Fig. 2a) } \\
& \mathrm{C}-117,118 \\
& (2+2)-(\text { Fig. } 2 b) \\
& \text { C-119, } 120 \\
& (2+2)=(\text { Fig. 2C) }
\end{aligned}
$$$$
\text { two dissimilar wires per hole in a two-hole plug }
$$

Within these five units there were a total of nine dissimilar wire pairs being tested.

\section{TEEST RESULTS}

Prior to starting the test and again at the end of the test window calibration curves were taken to check any changes that might have occurred due to the possible deposition of materials from the hot test cylinder onto the window. No differences were noticed between the two window calibrations, Fig. 3 .

The five thermocouple assemblies were inserted in appropriate holes in the test cylinder and connected to the thermocouple output measuring instrumentation. The test chamber was then evacuated to a pressure of $1 \times 10^{-6}$ torr and the thermocouples heated to a temperature of $1800^{\circ} \mathrm{C}$ and aged at this temperature for three hours. At the end of the three hour aging period a calibration (Calibration No. I, Figs. 5 through 9) curve was run on each thermocouple from approximately $1800^{\circ}$ to $1300^{\circ} \mathrm{C}$ at intervals of $100^{\circ} \mathrm{C}$. The millivolt outputs were equal, within the measurement limitations, among thermocouples common to one tantalum plug. The room temperature at the transition between the thermocouple wire and the compensated lead wire was $23^{\circ} \pm 2^{\circ} \mathrm{C}$ during the test and the appropriate room temperature compensation was added to the thermocouple millivolt output. The cycling part of the test was then started and the thermocouples were thermaliy cycled 40 times from $<500^{\circ} \mathrm{C}$ to $1800^{\circ} \mathrm{C}$ and then back to $<500^{\circ} \mathrm{C}$ with a total cycle time of approximately 45 minutes. All heater power was removed to start the 15 minute decreasing part of the cycle, Fig. 4. The temperature was then brought back up to $1800^{\circ} \mathrm{C}$ over a period of 15 minutes and allowed 15 minutes to stabilize at $1800^{\circ} \mathrm{C}$ before starting another cycle. The thermocouples were brought to room temperature at the conclusion of each day and remained in vacuo during the cycling part of the test.

At the completion of the thermal cycling test all nine of the thermocouples were again calibrated (Calibration No.2, Figs. 5 through 9) from $1800^{\circ}$ to 
$1300^{\circ} \mathrm{C}$. No appreciable changes were noted when the points were plotted and compared to the standard curve.

The 500 hour life test at $1650^{\circ} \mathrm{C}$ was started and data taken every 24 hours. No special attempt was made to maintain the temperature other than normal control. Periodic checks throughout the test showed that the temperature remained at $1650^{\circ} \mathrm{C} \pm 25^{\circ} \mathrm{C}$. The steady state test data for each thermocouple as plotted in Figs. 5 through 9 shows no apparent drift from the calibration curves. No differences were noted in the millivolt outputs of the thermocouples common to one tantalum plug until 320 hours. At this time it was noted that between the dissimilar wire pairs common to a tantalum swaged hot junction there was an 0.02 millivolt output difference which remained until the test ended at 500 hours. The thermocouples were again calibrated at the conclusion of the 500 hour test (Calibration No. 3, Figs. 5 through 9) and agreed with the standard curve and the previous two calibrations. The temperature difference was never greater than $5^{\circ} \mathrm{C}$ for any dissimilar wire pair common to the same hot junction.

Figure 10 is a plot of all the data presented as Calibrations 1 , 2, and 3 in.Figs. 5 through 9. It can be readily seen that from $1300^{\circ}$ to $1800^{\circ} \mathrm{C}$ the deviations are in the order of $\pm 25^{\circ} \mathrm{C}$.

\section{POST-TEST EXAMTNATION}

\section{Visual Inspection}

The thermocouples were carefully removed from the test block and visually inspected, Fig. 11. All W74 Re26 sheaths were bright where they had been inserted into the test cylinder and had been subjected to high temperatures. There was a light brownish discoloration from the point where the sheath left the cylinder going into a darker discoloration at a point $3-3 / 8$ inches from the welded tip. This is the point at which the rear radiation shield. was installed and the darkening was probably due to the material transport from the hot filament and cylinder to the cooler areas. The sheaths from all the thermocouples, except $\mathrm{C}-119$, were helium leak checked and found to be leak tight. The sheath of $\mathrm{C}-119$ had been broken $3 / 4$ inch from the welded end during a required filament change near the end of the test. The sheaths are quite brittle after being heated to $1800^{\circ} \mathrm{C}$ and a slight shock could easily have broken it. The $\mathrm{C}-116$, 119, and 120 thermocouple assemblies were easily removed from the sheath. The tantalum plugs of assemblies $\mathrm{C}-117$ and 118 had bonded to the sheath. $\mathrm{C}-117$ was broken away from the sheath by pulling on the wires. C-118 was left for further examination if required at a later date.

Upon disassembly of the thermocouples it was found that the beryllia beads used for C-116 (two hole) were slightly discolored for the first 1-5/8 inches from the hot end then went into a very shiny black discoloration for the next $1-5 / 8$ inches. The remaining $2-3 / 4$ inches was just off-white. The beryllia used for thermocouples $\mathrm{C}-117$ through $\mathrm{C}-120$ (four hole) was white with some light gray areas for the first $1-3 / 8$ inches from the hot end then changed into a flat black color for about $1 / 2$ inch with a definite white deposit buildup. The remainder of the insulator length was dark gray, Fig. 12. 
A test, duplicating the sheath and tantalum plug materials and the calibration environment, was conducted and it was determined that the deposition of the foreign matter found on the beryllia did not originate from either the tantalum plug or from the $W_{74} R_{26}$ sheath.

Chemical Analysis

The following is the chemical analysis of the two hole and four hole beryllia beads (PPM):

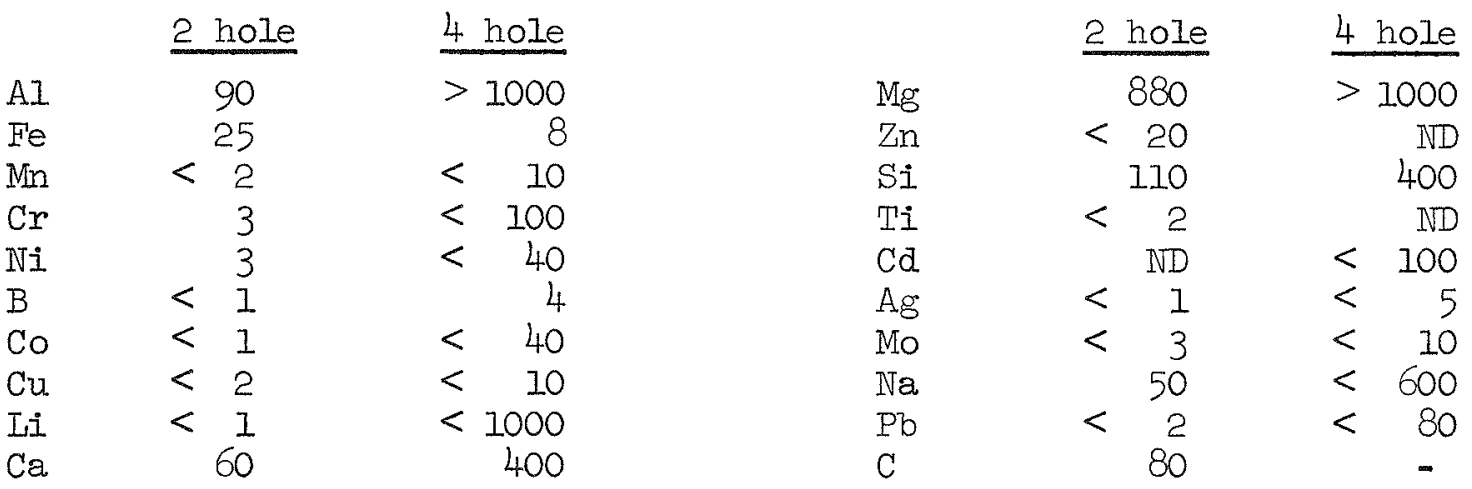

The two hole beryllia was purchased as $99.8 \%$ purity with a certified chemical analysis from one supplier and the four hole beryllia was purchased from another supplier as $99.9 \%$ purity but without a chemical analysis.

The chemical analysis of the four hole beryllia was done at Gulf General Atomic. The analysis, though not as complete as that for the two hole beryllia, is acceptable for comparison purposes and shows that the four hole beryllia at the claimed $99.9 \%$ purity is not as pure as the two hole beryllia at $99.8 \%$ purity. A later order for the four hole beryllia was made from the same vendor, with the supplied chemical analysis data in agreement with that obtained at Gulf Ceneral Atomic.

Microprobe Analysis

Samples of the deposits from C-116 and C-119 were sent for microprobe analysis. Excerpts from the analysis are as follows:

Specimen C-116 (Two hole beryllia)

Carbon was just barely detected and had no particular pattern of distribution. It was borderline in conductivity depending on the beam current used for the microprobe.

Magnesium was found in the dark grains but was not detected in the white colored grains at the hot junction end. Going away from the hot junction the magnesium gradually increased in amount and was found more frequently until the magnesium was present in all of the grains. 
Aluminum was detected only occasionally and only in that half of the specimen which was away from the hot junction.

Silicon was detected only occasionally and was usually present at a low concentration level except for some very small high silicon specks.

Chromium was very rare but did appear in the dark grains in the discolored region and was more common toward the hot junction end of this region where it reached a value of $3 \%$ in some of the grains.

Iron was detected occasionally in very small amounts in very small areas.

Not detectable were nickel, sulfur, chlorine, potassium, calcium, titanium, tungsten, vanadium, manganese, cobalt, copper, and zinc.

A complete spectral scan for all elements heavier than sodium was made on one of the dark grains with only chromium being detected.

Specimen C-119 (Four hole beryliia)

Carbon was found to be generally very high in the black circumferential ring and increased from this very high value to an even higher value as the hot junction end was approached. Going in the other direction from the black ring, carbon decreased but was always present. This specimen was found to be highly conductive with carbon probably present in an amount in excess of $10 \%$.

Magnesium was only occasionally detected in the portion from the black ring away from the hot junction end.

Aluminum was sought specifically. There was no aluminum in the beryllia at the hot junction end and was very low in the gray area approaching the black ring increasing steadily until large amounts were found in the gray area past the black ring, beyond. which it decreased again to very small amounts.

Silicon was not detected at the hot junction end or in the gray material approaching the black ring. Silicon began to appear in the black ring: and throughout the gray material following the black ring and then decreased to zero.

Chromium was not detected over the entire length of the sample.

Iron in very small amounts was detected and then only in the black ring.

Nickel occurred only rarely as small specks but was always present.

Elements sought specifically over the whole surface and not detected were sulfur, chlorine, potassium, calcium, titanium, tungsten, vanadium, manganese, cobalt, copper, and zinc.

A complete spectral scan was made on the black ring and only silicon and iron were detected. 
A complete spectral scan for all elements heavier than sodium was made on the white deposit, Fig. 12, and only aluminum and nickel were detected. Visual examination with a bench microscope indicated that the white deposit has substantial thickness. Microprobe analysis indicated that this material must be an element or elements below magnesium in the periodic table including a large amount of carbon as already noted.

Metallographic Examinations

Figure 13 shows the post-test appearance of the tantalum plugs of the one hole, two hole and four-slot configurations. Figures 14, 15, and 16 show respectively the microstructures of the cross sections of these plugs. From these photographs, the following conclusions can be drawn:

1. The tantalum has a very clean grain structure, indicating no significant contamination has occurred during the test.

2. There are pores and voids around each thermocouple wire on all three configurations. Some of them could have originated from the gap existing between the thermocouple wire and the wall of the cavity; the others may be due to the Kirkendall effect associated with the interdiffusion of metallic components. The thickmesses of the diffusion layers between the tantalum and the thermocouple wires, as estimated from these figures, are of the order of $1 / 2$ to $1 \mathrm{mil}$. In spite of these voids and pores, the test data indicated that good contact existed among the thermocouple wires. This good contact is evident from the fact that interdiffusion between the thermocouples wires has occurred for the configurations where two wires are in direct contact.

3. Electron microprobe studies indicate that the diffusion layer between the tantalum and the thermocouple wires has a high concentration of tantalum plus some tungsten and rhenium. It is interesting that the diffusion layer is present where no direct contact between tantalum and thermocouple wires exist. In all probability the tantalum reached there either by vapor transport or by surface diffusion. 


\section{APPENDIX A}

At a later date it was decided to substitute $W_{97} \operatorname{Re}_{3}$ versus $W_{75} \operatorname{Re}_{25}$ for the $W_{95} R_{5}$ versus $W_{74} R_{26}$ previously tested. The reason for this change was because it is generally thought that the W97 Re3 versus W75 Re25 thermocouple is more reliable at elevated temperatures.

The author has used $W_{95}$ Re$_{5}$ versus $W_{74}$ Re$_{26}$ as fuel temperature thermocouples for many thousands of hours in the thermionic converters being tested in the GuIf General Atomic Incorporated IRIGA reactor core. These in-pile thermocouples are the same as thermocouple type C-116 tested here, consisting of two dissimilar wires in a single holed tantalum plug. The thermocouples do operate for the se long durations although they are in a helium atmosphere and a high radiation field. However, a downward drift of $100^{\circ} \mathrm{C}$ over a period of 500 hours at $1750^{\circ} \mathrm{C}$ has been noticed for the se thermocouples on the basis of temperatures calculated from thermionic performance. Thermocouples of this same type are also used in the out-of-pile thermionic converter test program. With the input power to the converter kept at a constant value, thermocouple outputs have linearly drifted downward $70^{\circ} \mathrm{C}$ over a period of 8000 hours at $1750^{\circ} \mathrm{C}$ in a vacuum of $4 \mathrm{X} 10^{-8}$ torr.

During the fabrication of the actual four wire thermocouple $(2+2)$ to be used in the Plum Brook irradiation capsule, extensions of the thermocouple wires were removed and the cormon ends junctioned using the four slot (Fig. 2c) tantalum swage as in test pieces $\mathrm{C}-119$ and $\mathrm{C}-120$. The se test thermocouples were identified as $\mathrm{C}-121, \mathrm{C}-122$, and $\mathrm{C}-123$. They were installed in the calibration apparatus previously described. It was decided to forego the thermal cycling and to shorten the total number of hours at temperature as it was felt that nothing new could be realized over the first test.

A second set of similar thermocouples was fabricated and again sections were removed, identified as $\mathrm{C}-124, \mathrm{C}-125$, and $\mathrm{C}-126$. The se, in turn, were tested. The test hours for the two sets were 155 and 100 hours, respectively at $1650^{\circ} \mathrm{C} \pm 25^{\circ} \mathrm{C}$. The test calibration data are shown in Fig. 17 and Fig. 18. The results from these short term tests are in general agreement with that observed for W95 Re 5 versus W75 Re25 thermocouples. 


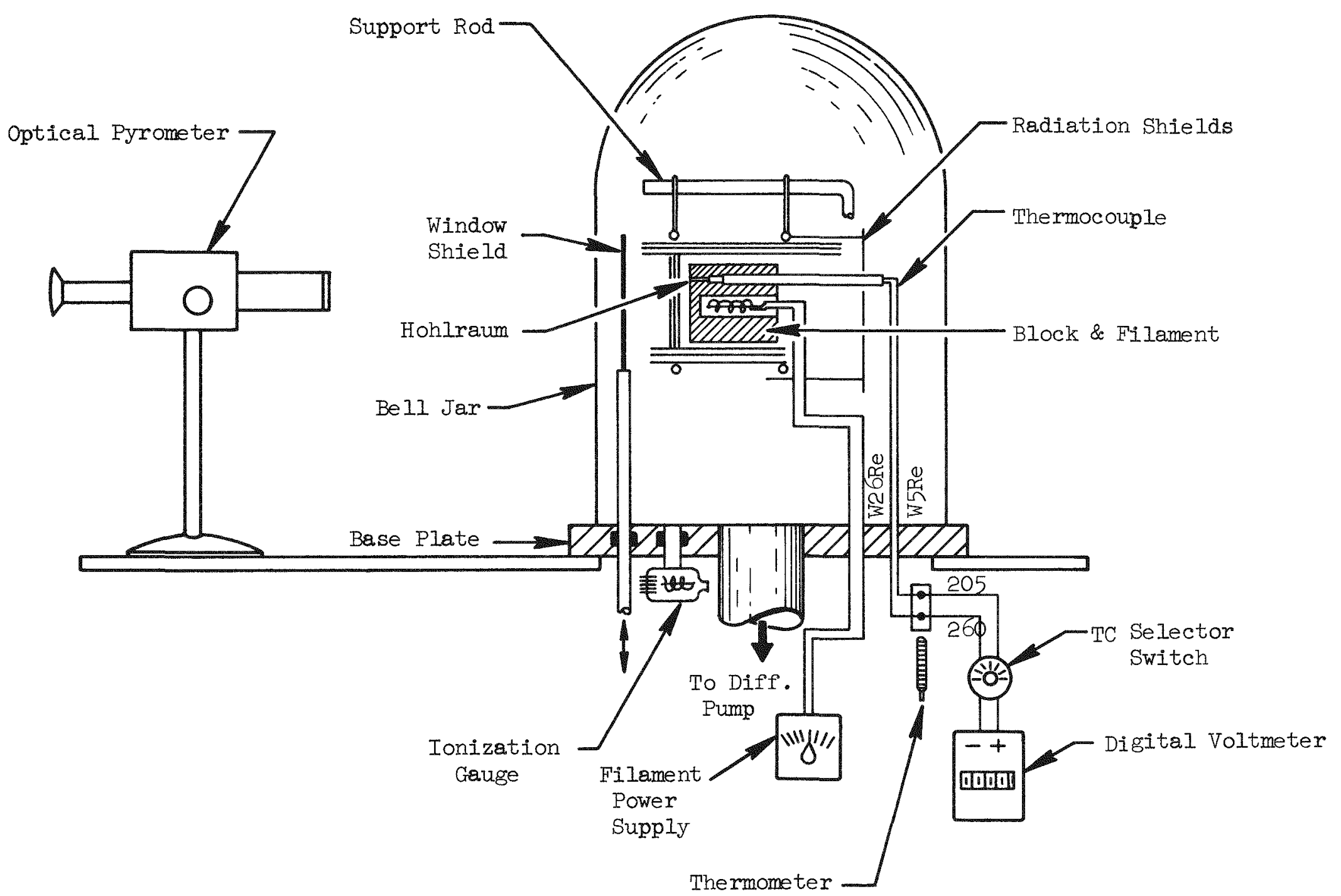

Fig. 1--Calibration and test assembly schematic 


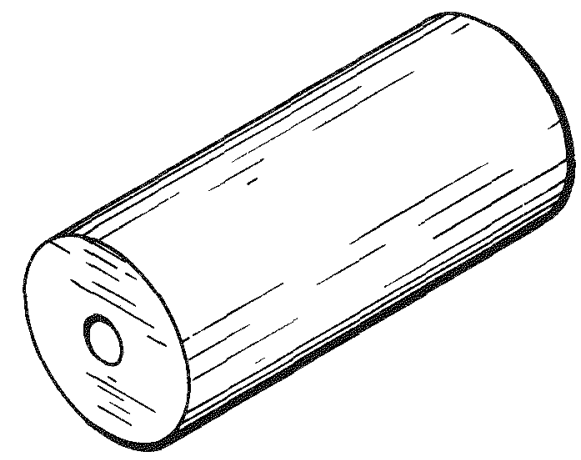

(a)

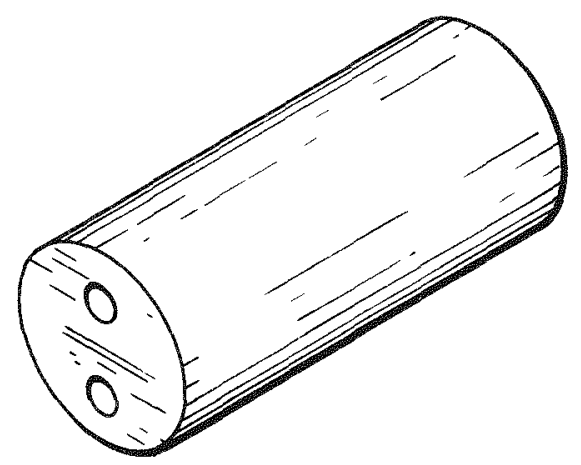

(b)

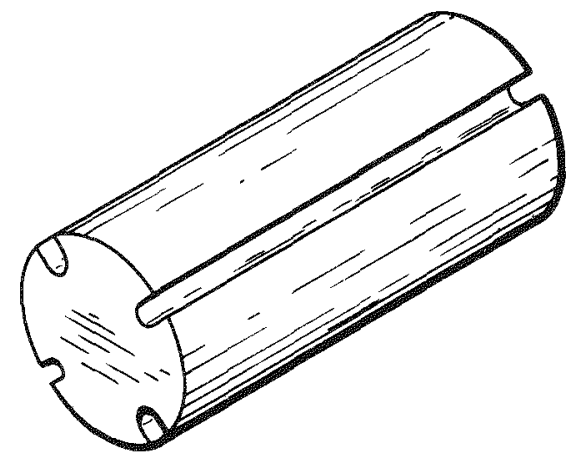

(c)

Approximately $20 \mathrm{x}$

Fig. 2--Thermocouple junction crimp plugs 


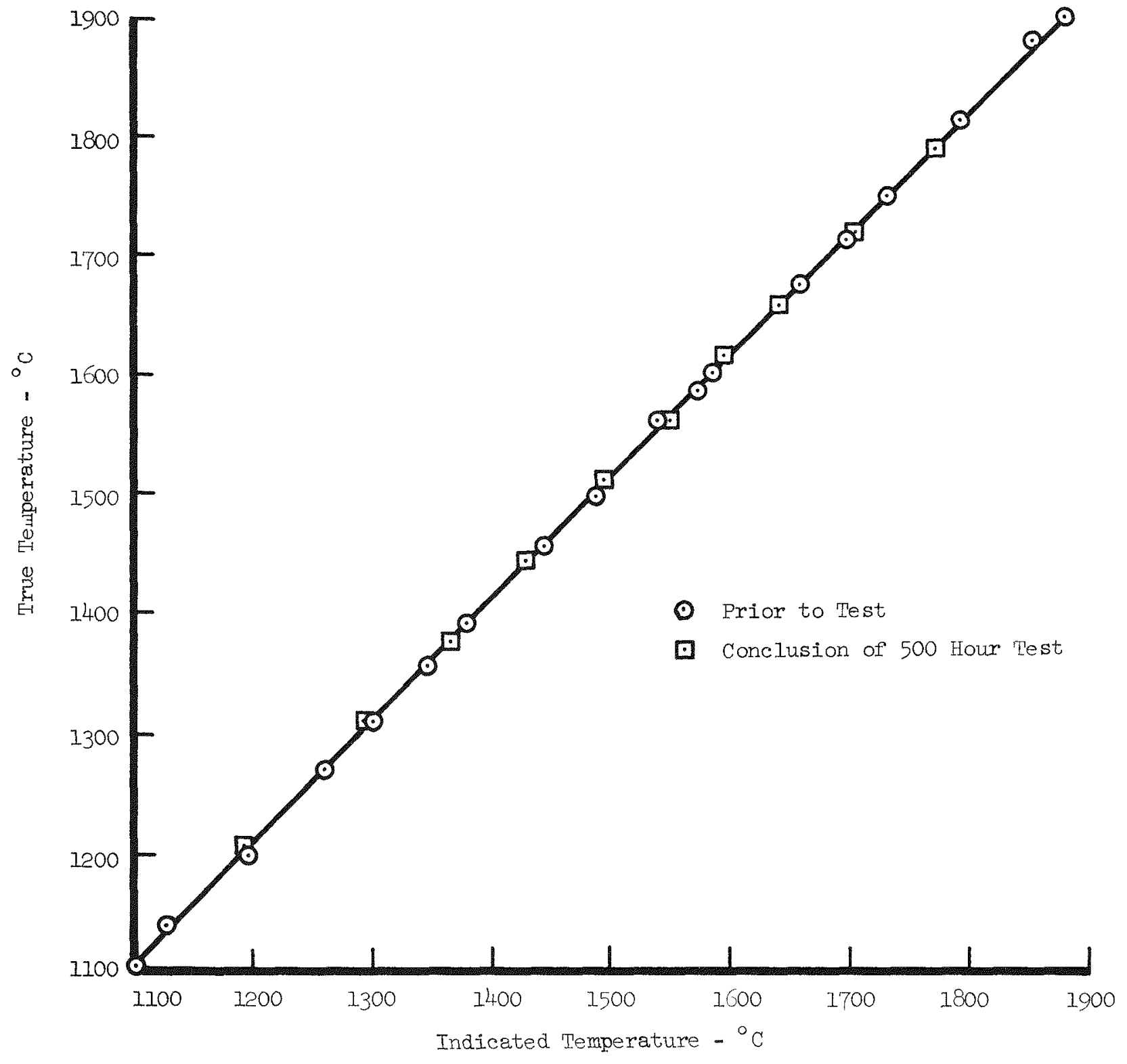

Fig. 3--Optical Window Correction 


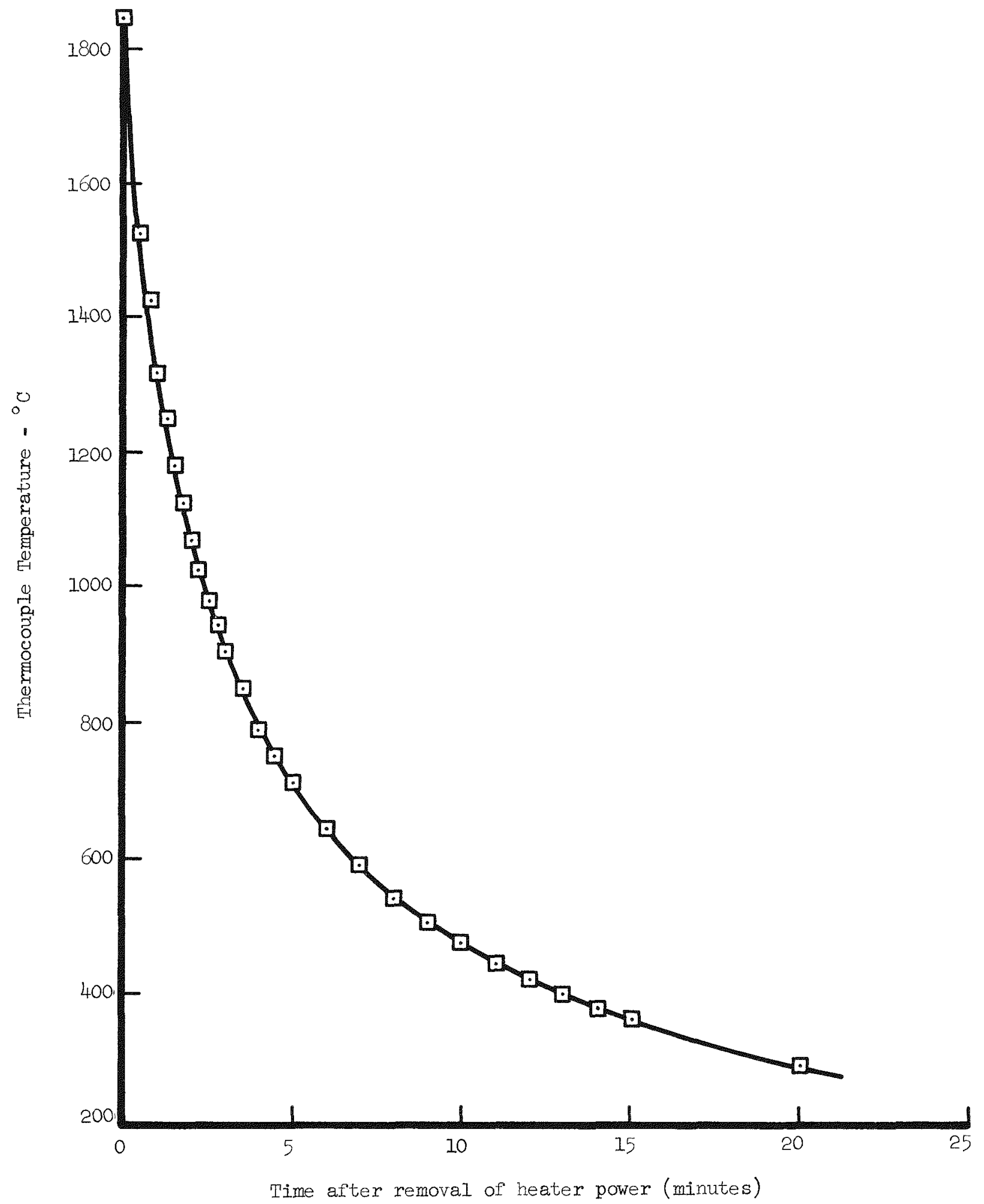

Fig. 4--Typical curve for decreasing temperature during thermal cycling 


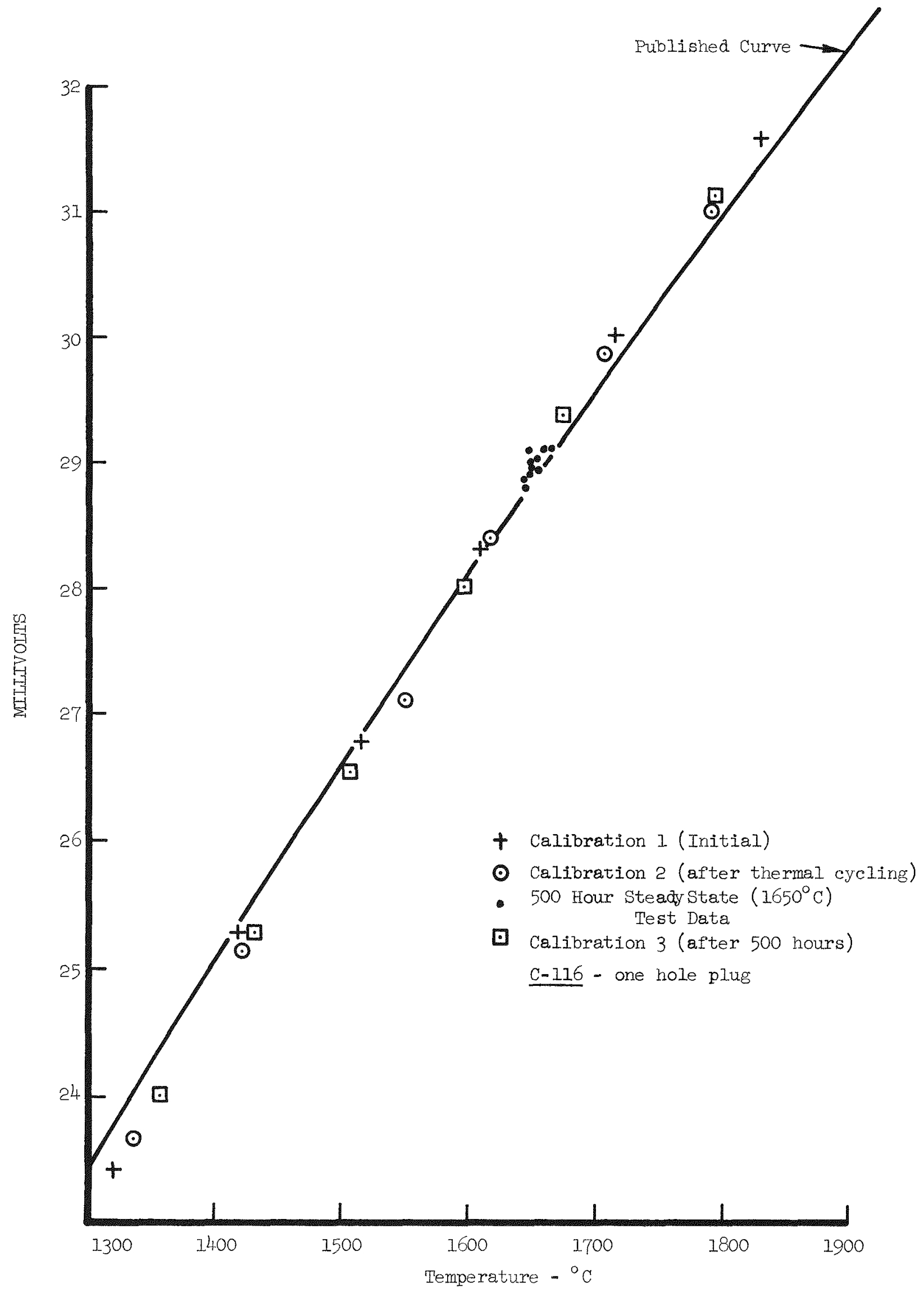

Fig. 5--Test results on $\mathrm{C}-116$ 


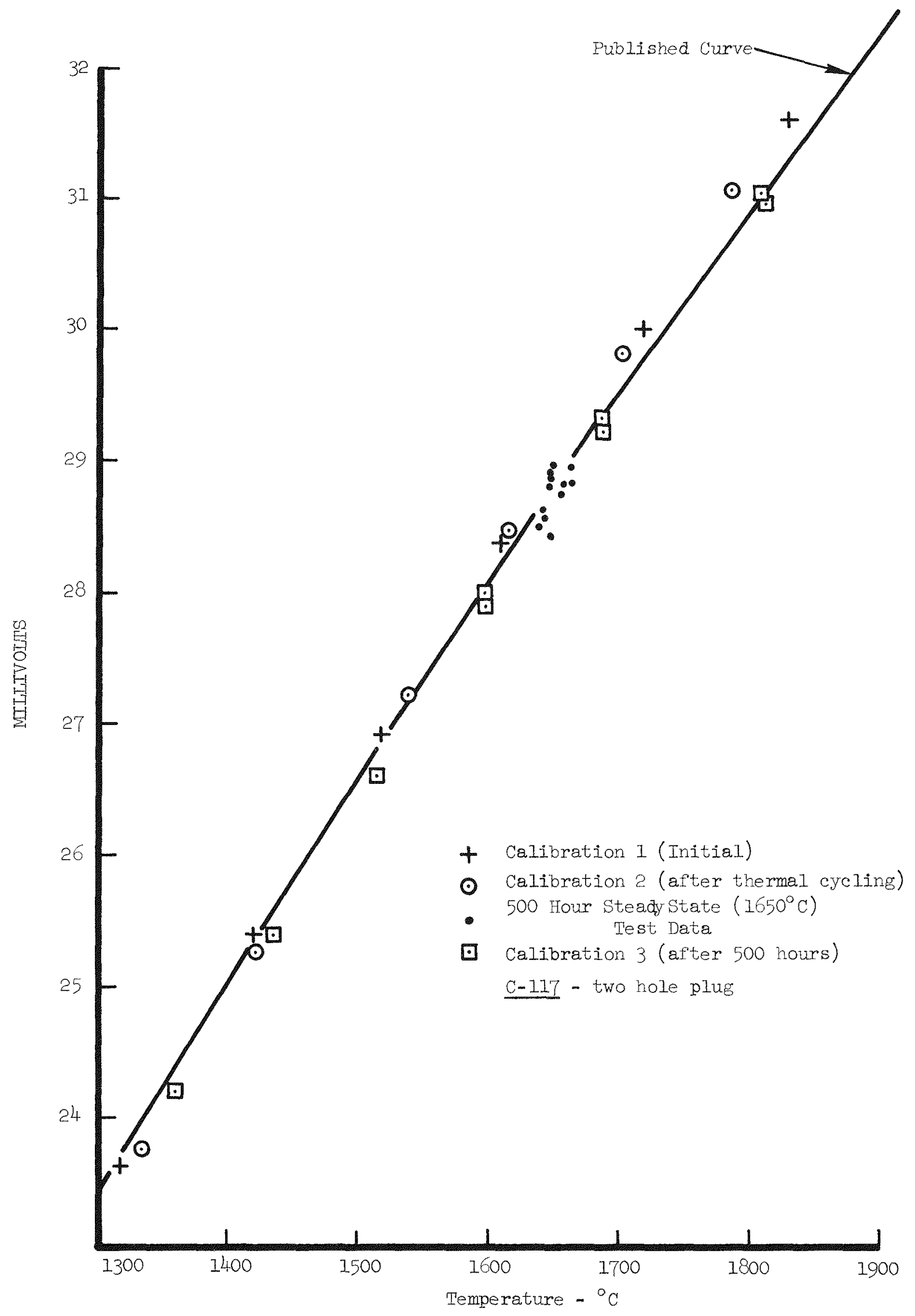

Fig. 6--Test results on $\mathrm{C}-117$ 


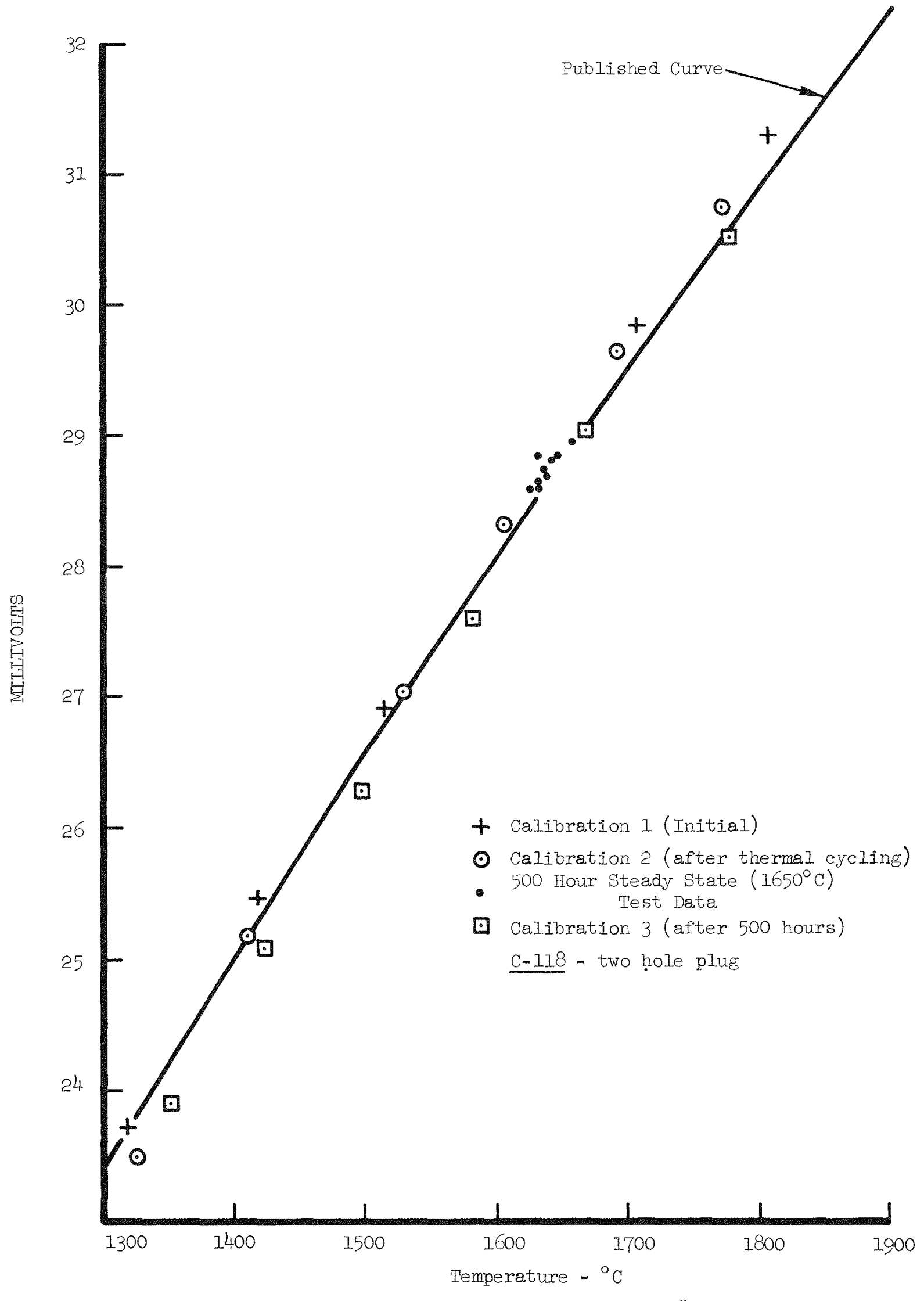

Fig. 7--Test results on $\mathrm{C}-118$ 


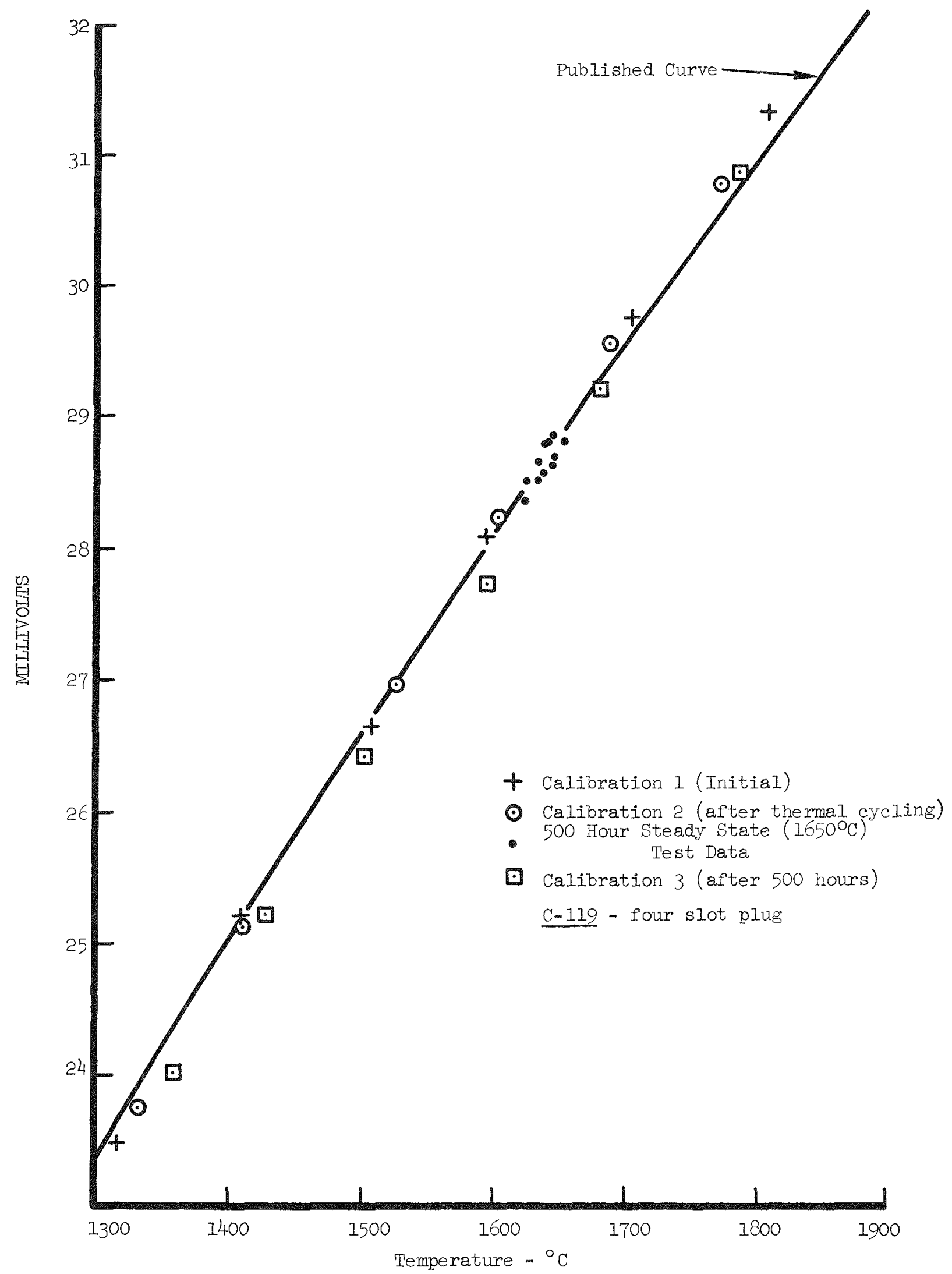

Fig. 8--Test results on $\mathrm{C}-119$ 


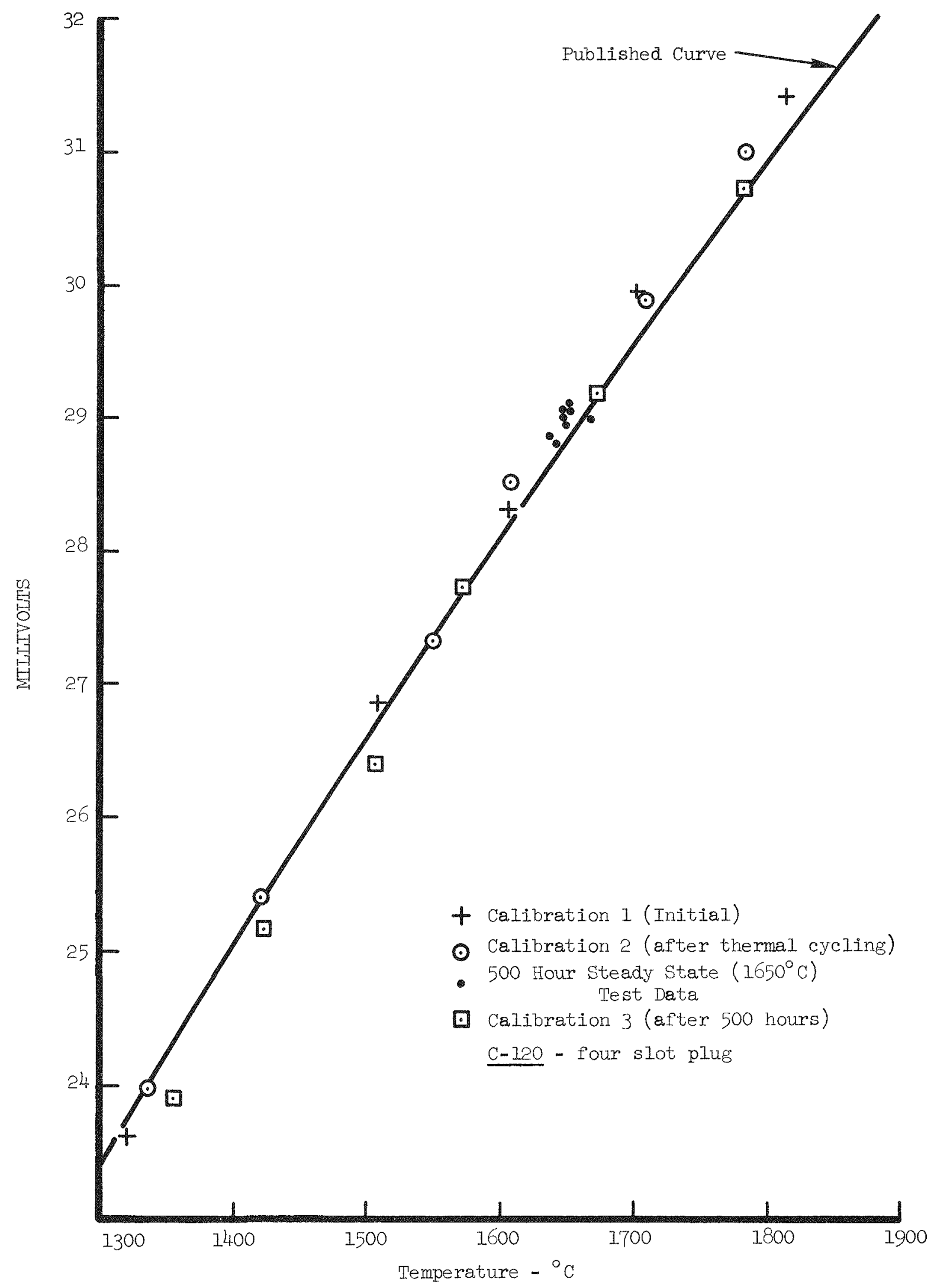

Fig. 9--Test results on $\mathrm{C}-120$ 


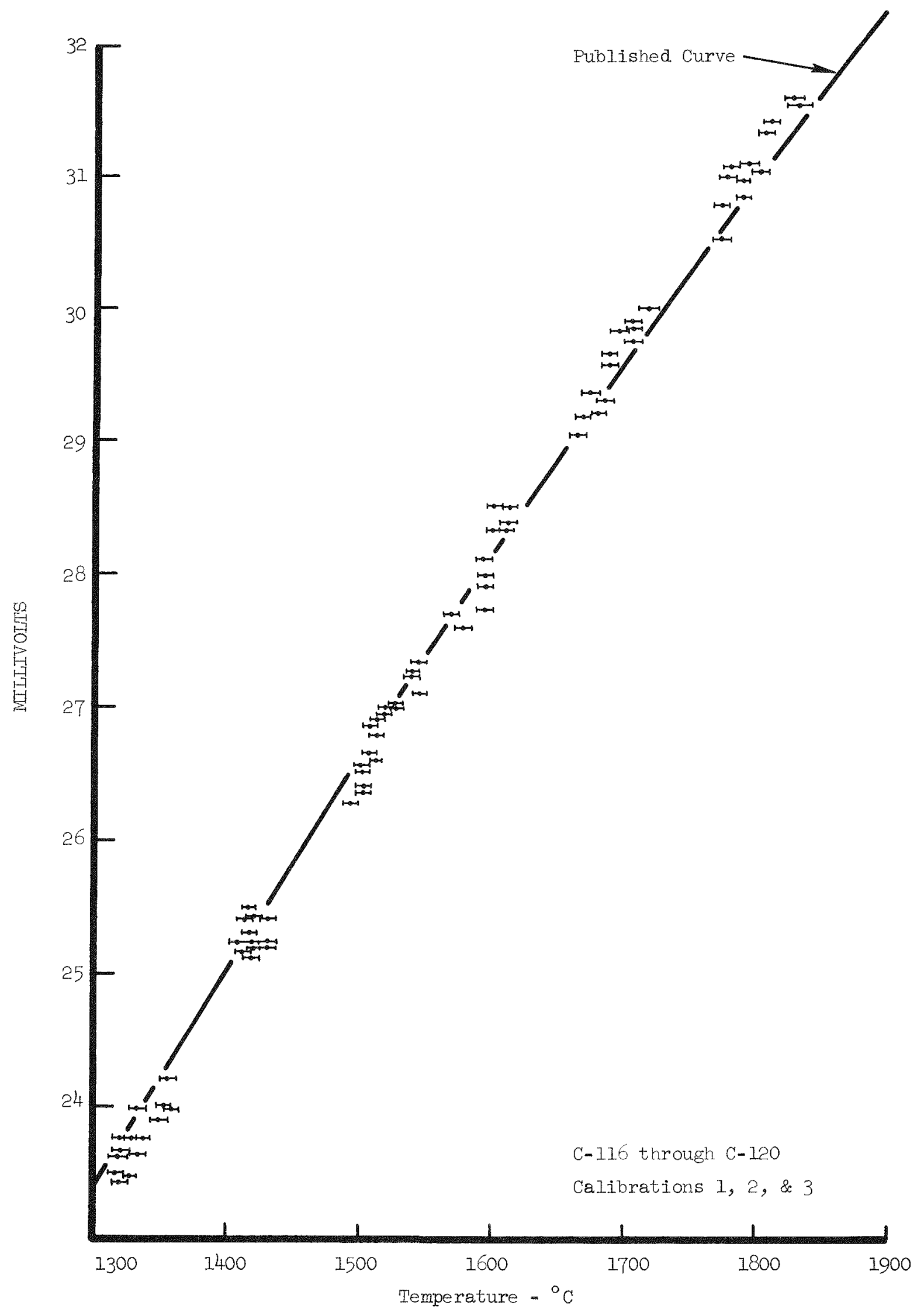

Fig. 10--Sumary plot of test results 


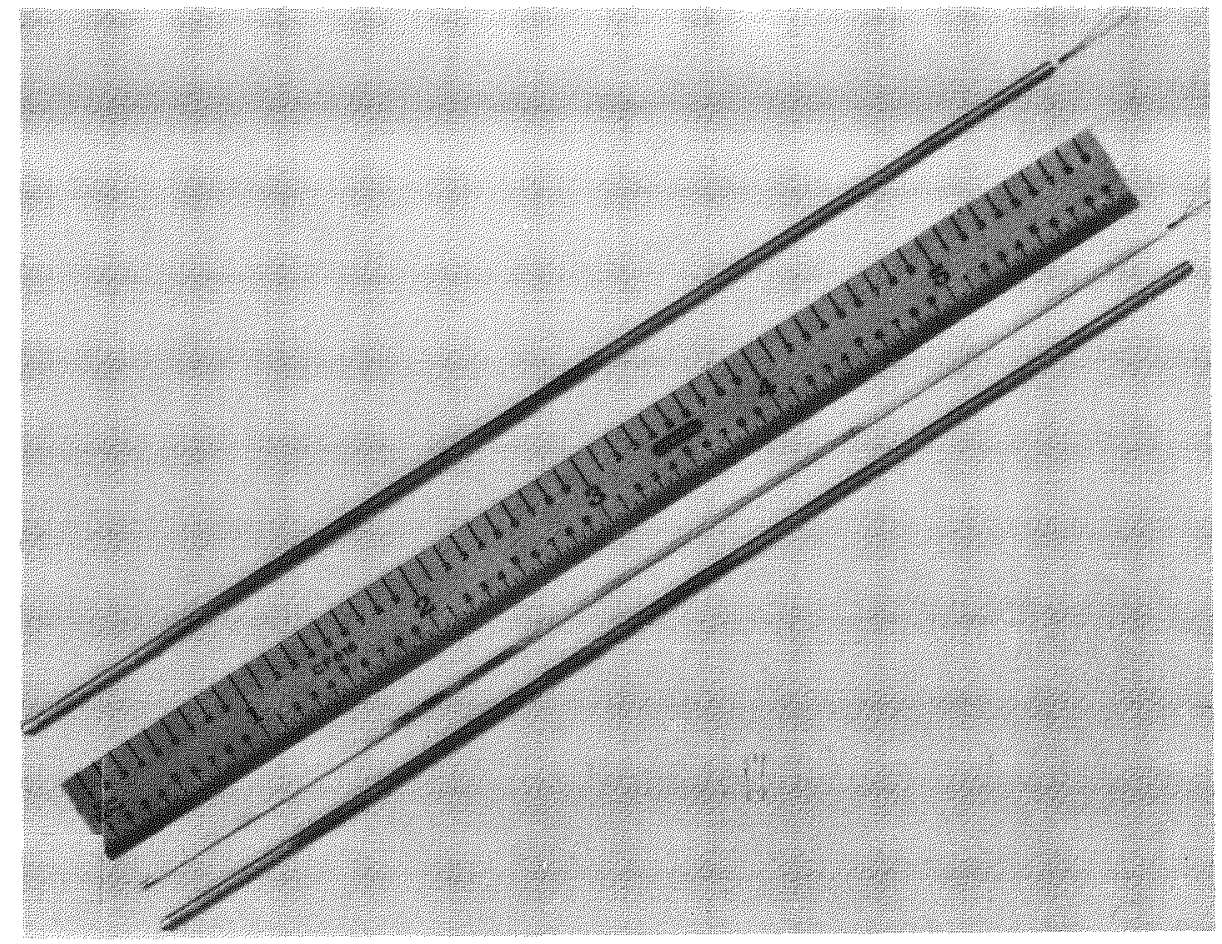

Fig. 11--Thermocouple assembly of $\mathrm{C}-120$

Section above the scale shows the complete test assembly while below the scale is the thermocouple assembly consisting of the sensor wires, tantaIum swaged hot junction and the beryllia insulating beads showing the deposit as described for C-119. Also shown is the $W_{74} R_{26}$ sheath.

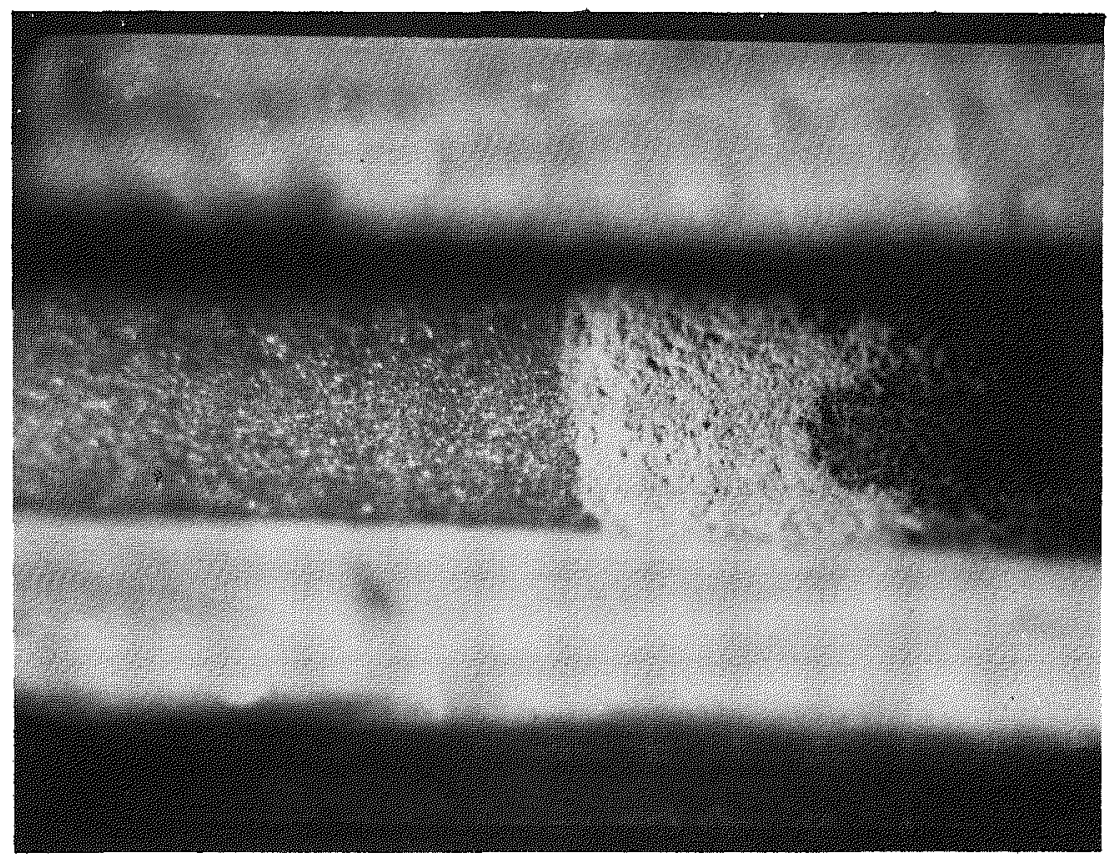

Fig. 12--White deposit bulldup on beryllia beads of thermocouple $\mathrm{C}-119$. Hot junction end was to the left of picture. 


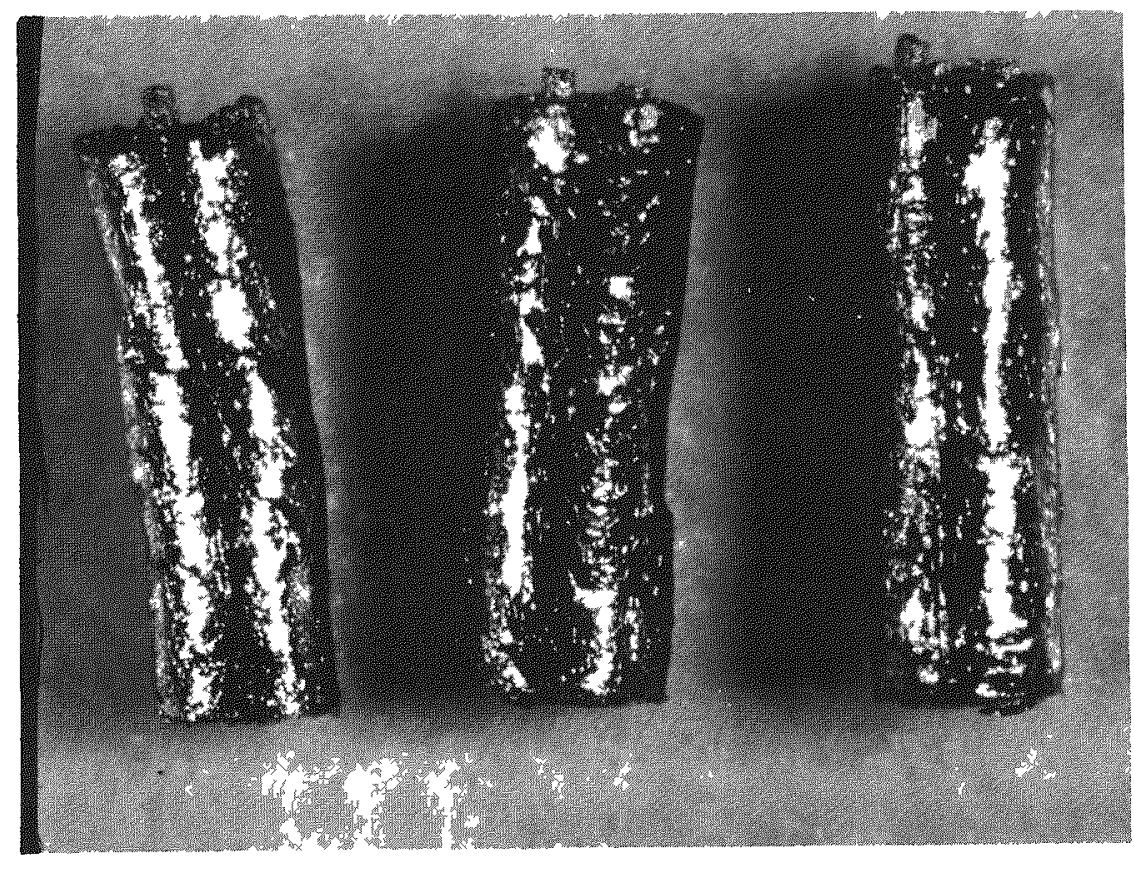
$\mathrm{C}-1.16$
C- -117
$\mathrm{C}-119$
$24 X$

Fig. 13--Appearance of Tantalum Plugs after the Test
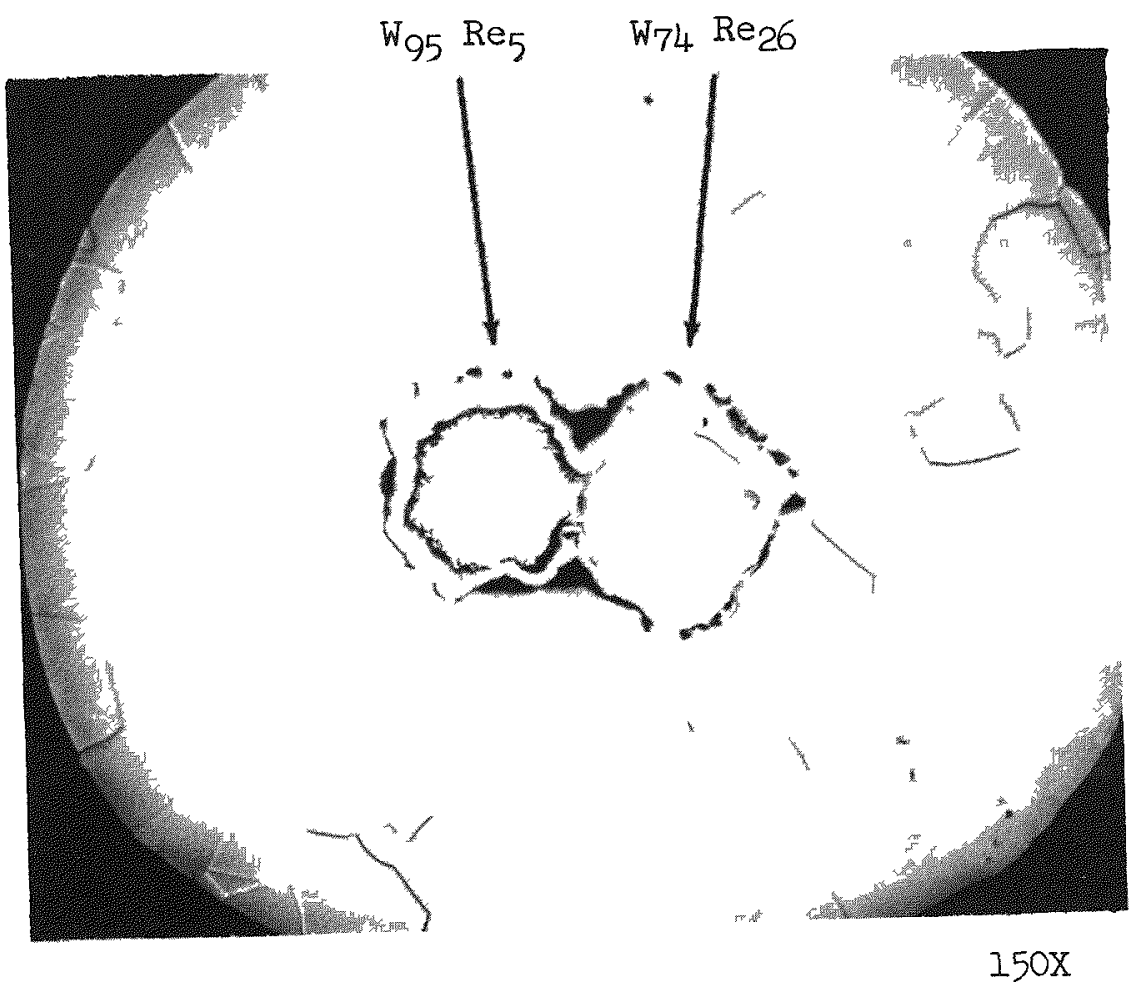

Fig. 14--Microstructures of the Cross Section of $\mathrm{C}-116$ 


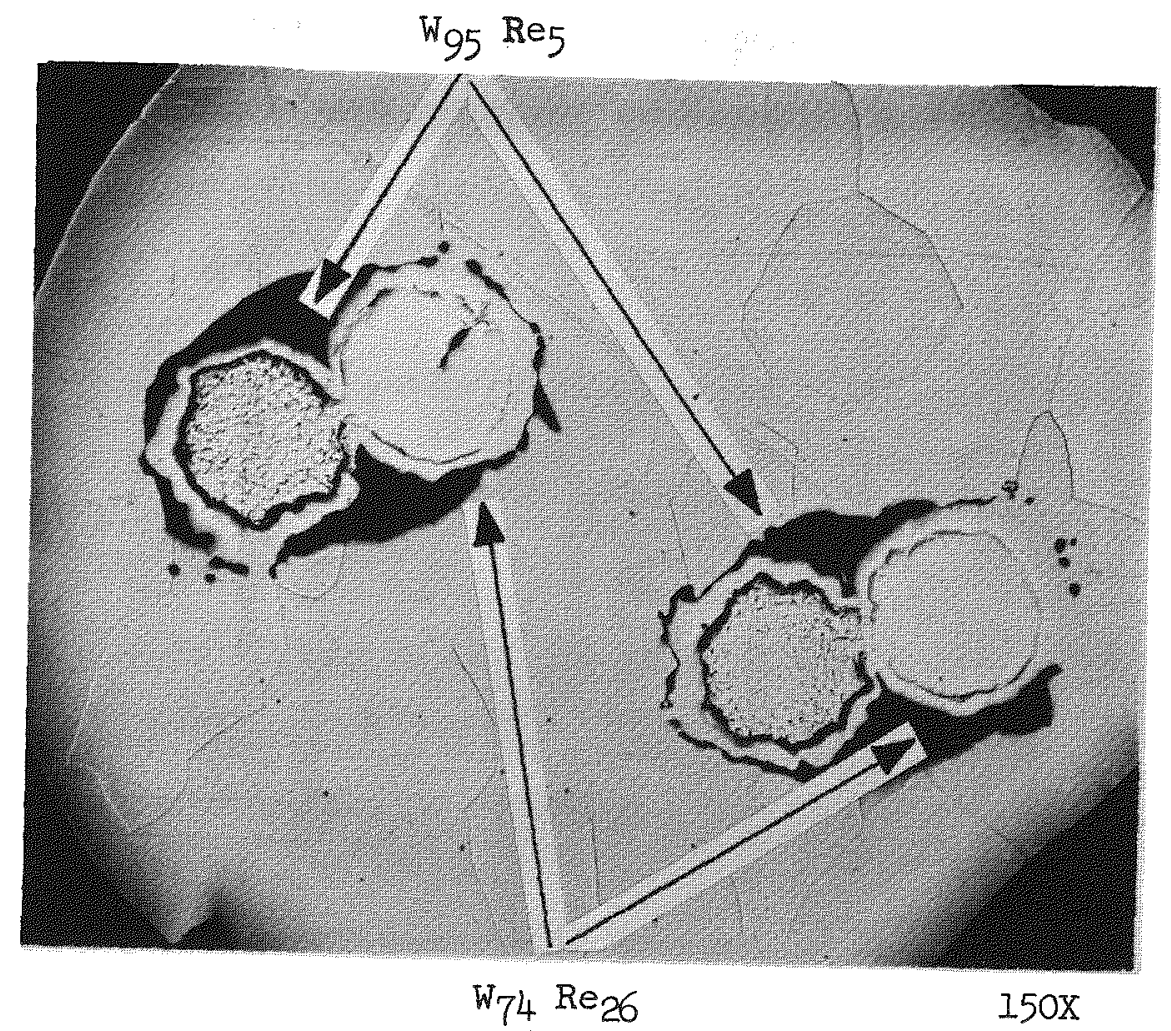

Fig. 15--Microstructures of the Cross Section of C-117

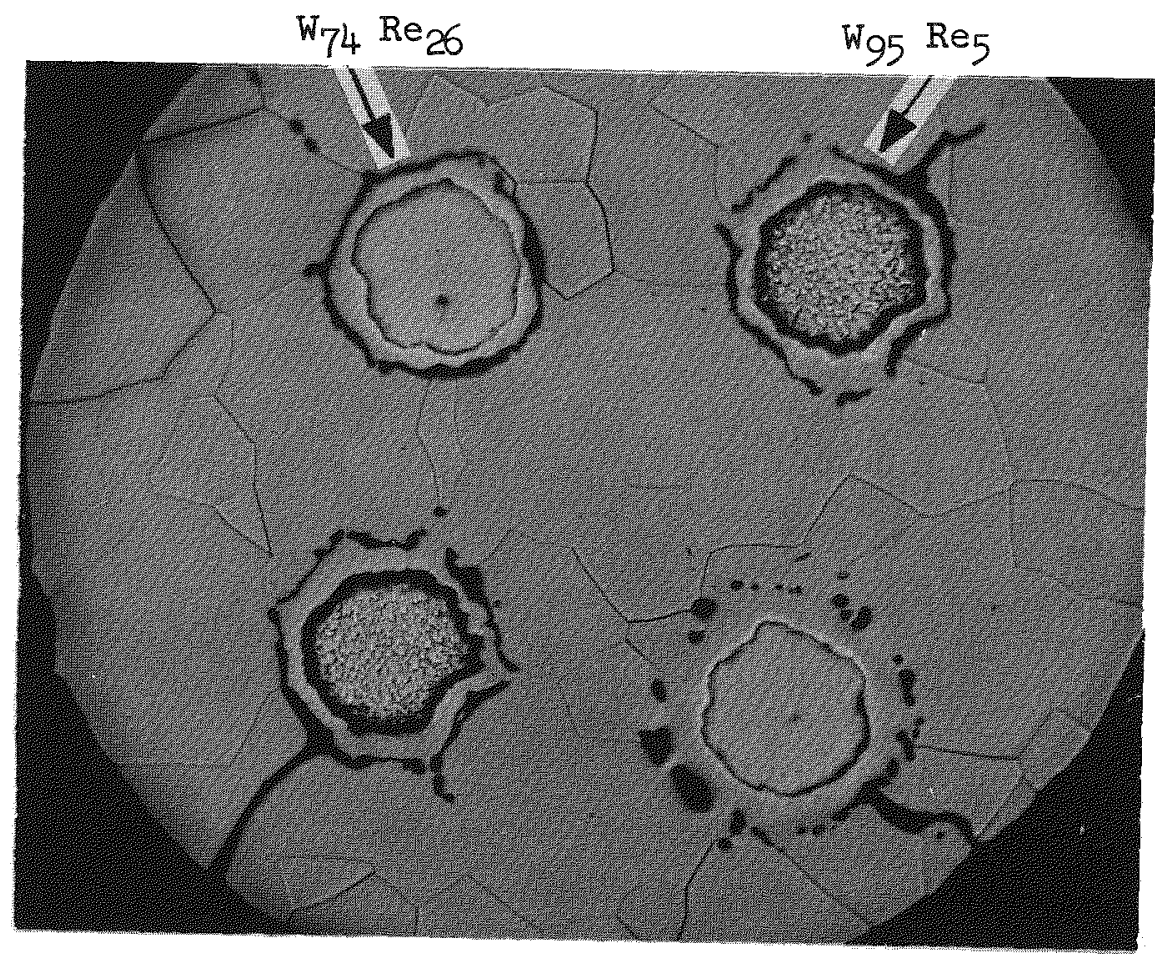

$150 \mathrm{X}$

Fig. 16--Microstructures of the Cross Section of C-119. Note Remnants of the Slots in the Tantalum 


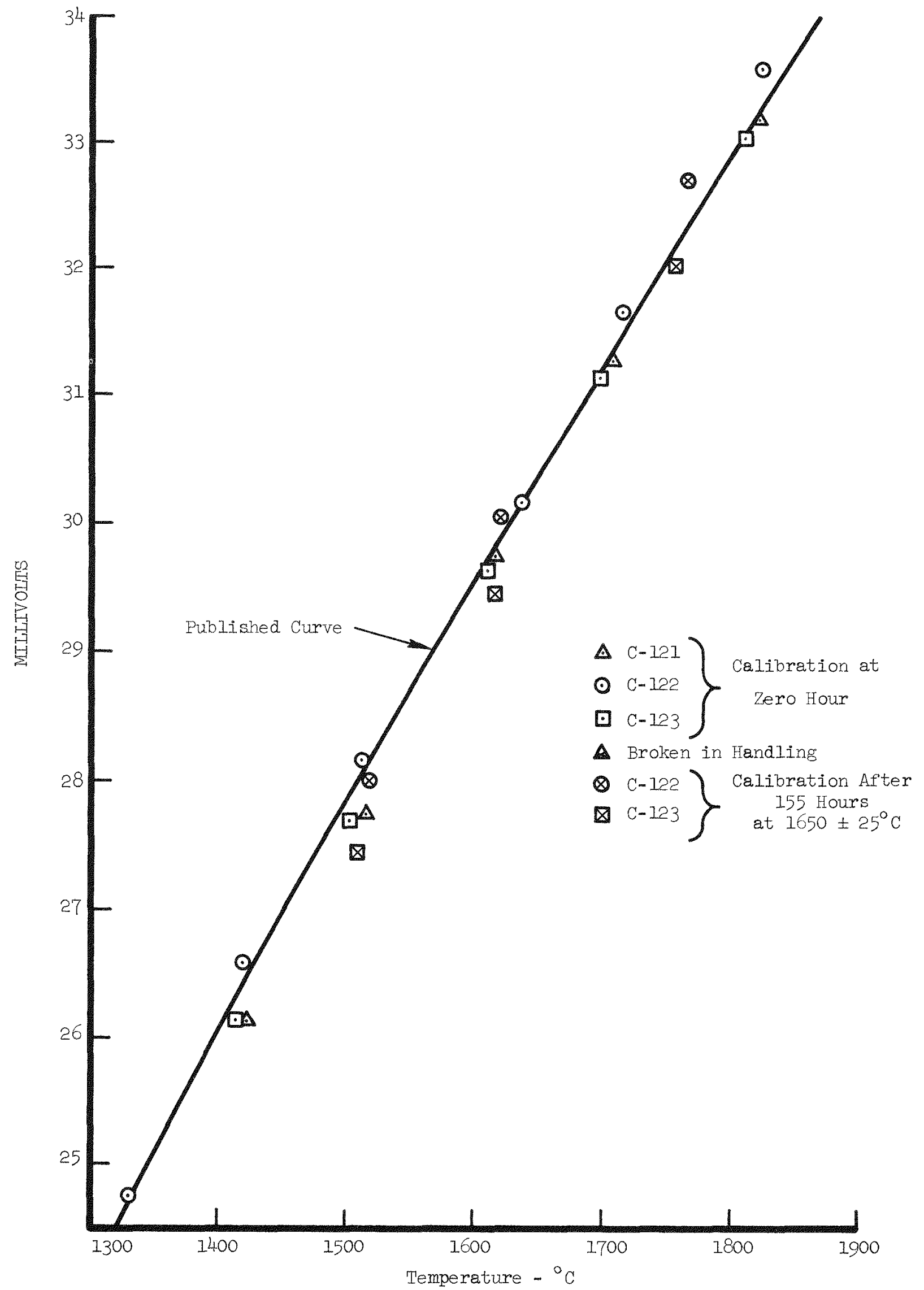

Fig. 17-mest results on $\mathrm{C}-121-123$ 


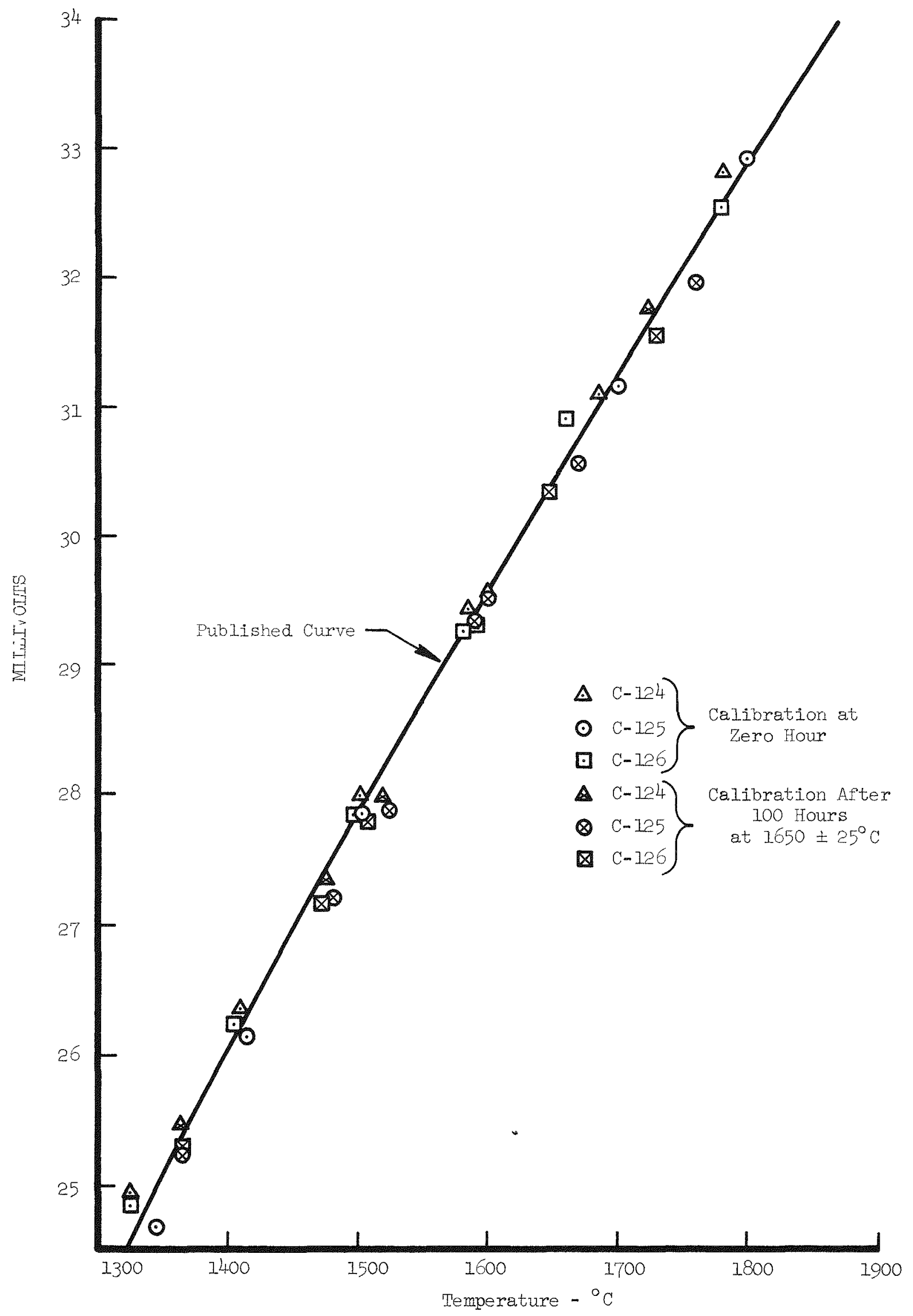

Fig. 18--Test results on $\mathrm{C}-124-126$ 


\section{GROOVED MELT WIRES FOR TEMPERATURE MEASUREMENT OF REACTOR FUEL*}

$\begin{array}{ll} & \text { P. G. Salgado } \\ & \text { B. J. Thamer } \\ & \text { R. L. Rudman } \neq\end{array}$

\section{ABSTRACT}

The U1tra High Temperature Reactor Experiment (UHTREX) is being conducted at thelLos Alamos Scientific Laboratorylas a part of the AEC gascooled reactor program. One of the features of the reactor is the ability to refuel while operating at full power, accomplished in part by rotating the central core of the reactor. The already difficult job of measuring fue 1 temperature is thus made even more difficult because connecting tubes or wires cannot be used. As a means for measuring temperatures in UHTREX, certain fuel elements have been examined after test to see which of an implanted array of melt wires have melted, thus determining the temperatures within limits at various locations. Recent work at the Los Alamos Scientific Laboratory has developed grooved melt wires of a variety of compositions covering the temperature range of $275-3000^{\circ} \mathrm{F}$. Melting can be determined easily and with good precision by radiographing the exposed fuel element or by removing the me1t wires and examining them visually.

* Work performed under the auspices of the U. S. Atomic Energy Commission.

* Mr. R. L. Rudman is presently associated with the Nuclear Engineering Laboratory, University of California at Los Angeles. 


\section{$\underline{1.0 \quad \text { Introduction }}$}

In June of 1969 , the UHTREX reactor ${ }^{(1)}$ reached its design conditions; the helium coolant attained a temperature of $2400^{\circ} \mathrm{F}$ at a power level of 3 megawatts, and fuel and moderator temperatures were correspondingly high.

One of the unique features of the reactor (Fig. 1) is the rotating core, a hollow graphite cylinder about four feet high, six feet in diameter with an inside diameter of about two feet. The core rests upon a pillar of reflector and insulating carbon, which is supported on a steel support plate and is rotated on ball bearings. Extending radially from the center of the core cylinder are 13 layers of fue 1 channels, 24 in each layer. See Fig. 2. Each channe1, which is 1.1 in. in diameter, can contain four UHTREX fuel elements. The fuel, pyrocarbon coated, ${ }^{2} 3{ }^{3} \mathrm{UC}_{2}$ particles in a graphite matrix, is also in cylindrical form: 1-in. in diameter with a 1/2-in. hole extending through the 5.5-in. length of the element. See Fig. 3.

The rotating core and other functional components of this reactor permit fue 1 to be added and removed during operation. The fuel loader is a ram system which pushes one element into a fuel channel from the outer diameter and expels an old fuel element into the center hole. Elements, in this way, proceed through the channel until they are removed at the center.

The usefulness of this reactor is associated with its ability to attain high temperatures (for fuel testing and fission-product studies) and the

* Work performed under the auspices of the U. S. Atomic Energy Commission.

* Mr. R. L. Rudman is presently associated with the Nuclear Engineering Laboratory, University of California at Los Angeles.

(1) Ultra High Temperature Reactor Experiment (UHTREX) Facility Description and Safety Analysis Report, LA-3556 Revised, April 1967. 
temperature reached in the fuel elements is an important parameter. However, with the core rotating from time to time and with such mobile fuel elements it is not feasible to use instrumentation which requires connecting tubes or leads such as thermocouples to observe the temperature of the fuel. The carbon of the insulation and reflector around the core is laced with thermocouple wire, namely, niobium-sheathed, berylia-insulated thermocouples of $95 \% \mathrm{~W}-5 \% \mathrm{Re}$ vs. $74 \% \mathrm{~W}-26 \% \mathrm{Re}$ and in cooler areas stainless-steel-sheathed, magnesia-insulated, chrome1-alume1 thermocouples. In addition there are thermocouples in the core, within the rotating cylinder. The signals are transmitted through sliding contact blocks between the base of the pressure vessel and the core support plate. Unfortunately, none of these thermocouples will indicate the temperature of the fuel elements.

Among several techniques suggested to make this measurement the use of fusible metals (melt wires) to indicate the attainment of particular temperatures, the melting points, was selected. It seemed simple, easy to develop and reliable. Tests have indicated that available temperaturesensitive paints are not suitable after unsatisfactory results such as flaking, powdering, indistinct indications, and indicated temperatures considerably at variance with the label. See Figs. 4 and 5 . These poor results were probably related to the inert coolant gas, helium, or to reaction with the graphite surface to which they were applied. The paints may respond well in air on an inert surface. Further, the paints contain appreciable neutron poison in the form of boron.

\subsection{The Me1t-Wire Method}

The melt-wire method is an inference technique. One can infer that the temperature at the location of a melt wire exceeded the melting point if the wire is melted. Conversely, one can infer that the maximum temperature remained below the melting point if the wire did not melt. Using a succession of wires with different melting points one can obtain sufficient data to plot a curve of maximum temperature versus location.

The wires should melt as sharply and reproducibly as possible. Generally, elements and eutectic compositions are employed. Although the melting temperatures are not expected to be as sharp, the use of solid solutions greatly increases the number of available materials of different melting temperatures.

The melt wires were formed into 0.040-in.-diameter cylinders, 1/4in.-long, to be secreted within the fuel element walls. They were made smal1 so that a minimum of fue 1 would be displaced and the system would experience a minimum perturbation in flux and temperature. Small containers of spectroscopic graphite, compatible with the fuel elements, were prepared to house the wire and to contain the melted metal. These also simplified removal from the fuel elements at the completion of an experiment. As discussed below quartz capsules were also used for many wires.

The graphite capsules were 0.300 -in.-1ong and 0.068 -in. in outside diameter. The 0.070-in.-diameter holes in the fuel elements corresponded to a fue 1 loss of about $2 \%$. 
When a wire melts it tends to assume the shape of the vessel in which it is contained. Since the wire and the capsule holes were cylindrical it might have been difficult to observe a melt. The wires were therefore machined with a pointed end in early experiments and later with a squarecornered groove. It was soon observed that pointed wire often retained the pointed end even after melting. In the case of certain metals which were difficult to machine and tended to gall, such as lead, the original points were somewhat rounded and often showed little difference in shape after melting at temperatures only slightly above the melting point. See Figs. 6,7 , and 8 . Grooved wires were found to be easier to machine and more sensitive to temperatures just over the melting point. The grooves, located in the axial center of the wire, were between 0.005 and 0.010-in.deep and were 0.010-in.-wide. The bottom corners were required to have a radius not exceeding $0.001-i n$. See Fig. 9 .

\subsection{Melt-Wire Characterization}

To assure that the published melting point ${ }^{2}$ ) of each metal was retained under the condition of its intended use, characterization tests were conducted on every material used. In these tests, 12 wires of the same composition were placed into a graphite disc with two to four thermocouples. See Fig. 10. The disc was maintained at temperatures below, at, or above the published melting point for ten minutes. The percentage of the wires melted was plotted as a function of the temperature. See Fig. 11. The spread of temperatures, between $100 \%$ melted and $0 \%$ melted, was considered the uncertainty of the measurement. This technique compensated for the errors which might arise from impure materials, incorrect alloy compositions or the effects of capsule/wire interactions.

During the characterization tests of materials which melted below $2000^{\circ} \mathrm{F}$ (Fig. 12), it was observed that many wires exhibited temperature errors or deviations from their published melting points in an irregular fashion. The helium surrounding the wires contained the outgassing products of graphite $\left(\mathrm{CO}, \mathrm{CO}_{2}, \mathrm{H}_{2} \mathrm{O}, \mathrm{H}_{2}, \mathrm{CH}_{4}\right.$, etc. $)$ which might have reacted to cause gross changes in the apparent melting temperature of some metals. To preclude this possibility, the wires were therefore encapsulated in quartz under vacuum. The wide and erratic deviations from published melting points ceased to occur. See Fig. 13.

Examination to determine whether a melt had occurred was accomplished by either or both of two methods, namely, visual or radiographic. See Fig. 14. Since the wires were intended to infer temperatures of irradiated fuel elements, a radiation hazard was involved during visual inspection at the completion of a reactor experiment. The radiographic technique offered the better protection from the hazard even though fuel in the element tended to interfere with the melt wire's silhouette and the slight changes in a wire groove were sometimes difficult to observe. Usually, however, it was not necessary to examine a groove very closely; the wire was eithex unmelted or it was a glob. When close examination was necessary, visual inspection was required and hot-cell techniques were used. When a wire was separated from the element it could be examined safely under a microscope, if done quickly.

${ }^{(2)}$ M. Hansen, "Constitution of Binary Alloys," McGraw-Hi11, 1958. 
During the characterization of the $\mathrm{Cu}-\mathrm{Mn}$ alloy and silver, smoky deposits formed on the inside surface of the quartz capsules. The melting temperature of the $\mathrm{Cu}-\mathrm{Mn}$ alloy was quite far from the published melting point although that of silver remained close. After some experimentation it was learned that the smoky deposit did not appear when silver was first placed in hydrogen for one hour at $800^{\circ} \mathrm{C}$, presumably removing the oxygen. The deviation in melting temperature of the $\mathrm{Cu}-\mathrm{Mn}$ alloy and the smoky deposit were eliminated by a ten-second dip in $10 \mathrm{v} / 0$ aqueous HCl which was believed to remove surface oxide.

Copper indicated a very wide temperature spread during temperature characterization. The spread was narrowed by the hydrogen treatment but it was more easily eliminated by using a graphite capsule which probably deoxidized the metal.

Since quartz devitrification seemed to occur at about $2200^{\circ} \mathrm{F}$, and copper, with a melting point of $1981^{\circ} \mathrm{F}$, required a graphite capsule, all wires which melted at temperatures above the melting point of copper were encapsulated in graphite. The melting temperatures of some metals above $2000^{\circ} \mathrm{F}$ were, therefore, actually melting points of carbide eutectics.

The characterization of wires melting above $2000^{\circ} \mathrm{F}$ (Fig. 15) took on some new aspects. Visual inspection gave very poor results. Surface smoothing of the machining marks and extrusion grooves occurred well below the melting point. The square grooves remained square until the melting point was reached but the groove was usually filled with graphite dust and visual observation would have required ultrasonic cleaning procedures. Radiography was therefore the most effective method for observing melting when the wires were encapsulated in graphite.

The characterization results shown in Figs. 12 and 15, are listed in Table I. The width of the temperature range or the uncertainty of measurement averaged $19^{\circ} \mathrm{F}$ and the deviation from the published melting point averaged $20^{\circ} \mathrm{F}$. These values generally increased at higher temperatures. This may have been due, in part, to contamination of the platinum thermocouples although they were replaced after every third test. Perhaps the dependence of the results on the formation of carbides also contributed.

There is often recrystallization within a melt wire below its melting point in time, but seldom is it mistaken for melting. A potentially more misleading phenomenon is that of "scratch smoothing". ( ${ }^{3}$ ) In the 1atter the corners of the groove may become rounded in time if there is sufficient material transport via volume diffusion, surface diffusion or vapor deposition of the melt-wire material. Estimates just below the melting point have been made for melt wires of materials for which there are sufficient data. (4) The time has been estimated for the bottom corner of the groove to become filled to a depth of one mil measured diagonally from the corner. This is roughly the change in the shape of the groove to be confused with melting. The smoothing of zinc and silver wires, which are vacuum-sealed in quartz, is almost entirely due to vaporization-condensation with a mean

(3)

(4) B. J. Thamer, to be published.

See, for example, W. W. Mullins, J.App1. Phys., 30, 77 (1959) 
TABLE I

Performance of Grooved Melt Wires, in the Temperature Range of 250 to $3000^{\circ} \mathrm{F}$

\begin{tabular}{|c|c|c|c|c|c|}
\hline $\begin{array}{l}\text { Materia1, } \\
\text { Weight \% } \\
\end{array}$ & $\begin{array}{l}\text { Maximum } \\
\text { No-Melt } \\
\text { Temp., }{ }^{\circ} \mathrm{F} \\
\end{array}$ & $\begin{array}{c}\begin{array}{c}\text { Minimum } \\
\text { Melt }\end{array} \\
\text { Temp., }{ }^{\circ} \mathrm{F} \\
\end{array}$ & $\begin{array}{l}\text { Published } \\
\text { Melting } \\
\text { Point, }{ }^{\circ} \mathrm{F} \\
\end{array}$ & $\begin{array}{l}\text { Range } \\
\text { Width, }{ }^{\circ} \mathrm{F} \\
\end{array}$ & $\begin{array}{c}\text { Deviation } \\
\text { from Published } \\
\text { Melting Point, }{ }^{\circ} \mathrm{F} \\
\end{array}$ \\
\hline $\begin{array}{ll}52 & \mathrm{Bi} \\
48 & \mathrm{~Pb}\end{array}$ & 262 & 265 & 257 & 3 & +8 \\
\hline $\begin{array}{ll}57 & \mathrm{Bi} \\
43 \mathrm{Sn}\end{array}$ & To be & characterized & 281 & - & -- \\
\hline $\begin{array}{ll}44 & \mathrm{~T} 1 \\
56 & \mathrm{Sn}\end{array}$ & To be & characterized & 339 & - & --- \\
\hline $\begin{array}{ll}38 & \mathrm{~Pb} \\
62 & \mathrm{Sn}\end{array}$ & To be & characterized & 361 & - & --- \\
\hline $100 \mathrm{Sn}$ & 442 & 452 & 451 & 10 & +1 \\
\hline $100 \mathrm{Bi}$ & 522 & 534 & 520 & 12 & +14 \\
\hline $100 \mathrm{~Pb}$ & 630 & 635 & 621 & 5 & +14 \\
\hline $100 \mathrm{Zn}$ & 785 & 797 & 787 & 12 & +10 \\
\hline $\begin{array}{l}56 \mathrm{Ag} \\
44 \mathrm{Sb}\end{array}$ & 904 & 912 & 905 & 8 & +7 \\
\hline $\begin{array}{ll}88 \mathrm{Al} \\
12 \mathrm{Si}\end{array}$ & 1079 & 1090 & 1071 & 11 & +19 \\
\hline $100 \mathrm{~A} 1$ & 1215 & 1230 & 1220 & 15 & +10 \\
\hline $\begin{array}{l}72 \mathrm{Ag} \\
28 \mathrm{Cu}\end{array}$ & 1441 & 1452 & 1434 & 11 & +18 \\
\hline $\begin{array}{l}65 \mathrm{Cu} \\
35 \mathrm{Mn}\end{array}$ & 1598 & 1612 & 1598 & 14 & +14 \\
\hline $100 \mathrm{Ag}$ & 1755 & 1776 & 1762 & 21 & +14 \\
\hline $100 \mathrm{Cu}$ & 1972 & 1999 & 1981 & 27 & +18 \\
\hline $100 \mathrm{Fe}$ & 2095 & 2111 & 2107 & 16 & +3 \\
\hline $\begin{array}{l}60 \mathrm{Pd} \\
40 \mathrm{Cu}\end{array}$ & 2181 & 2210 & --- & 29 & +18 \\
\hline $\begin{array}{l}60 \mathrm{Pd} \\
40 \mathrm{Ni}\end{array}$ & 2253 & 2277 & 2259 & 24 & +18 \\
\hline $\begin{array}{ll}77 & \mathrm{Pd} \\
23 & \mathrm{Cu}\end{array}$ & 2343 & 2358 & ---- & 15 & -14 \\
\hline $100 \mathrm{Ni}$ & 2403 & 2422 & 2404 & 19 & +18 \\
\hline $\begin{array}{l}88 \mathrm{Pd} \\
12 \mathrm{Cu}\end{array}$ & 2449 & 2474 & --- & 25 & -78 \\
\hline $100 \mathrm{Pd}$ & 2599 & 2642 & 2732 & 43 & -90 \\
\hline $\begin{array}{ll}50 & \mathrm{Pd} \\
50 \mathrm{Pt}\end{array}$ & 2872 & 2885 & 2912 & 13 & -27 \\
\hline $100 \mathrm{Rh}$ & 2935 & 3002 & 3002 & 67 & 0 \\
\hline
\end{tabular}


free path in the vapor that is long compared to the ten mil height of the groove. The times for zinc and silver are estimated to be somewhat less than a day and more than a month, respectively. In addition to being volatile, zinc has a tendency to form a surface film that can interfere with the collapse of the groove wall during actual melting.

With the exception of wires of zinc and silver, volume diffusion in the solid predominates in the material transport of the present wires at temperatures just below the melting point. Calculated smoothing of copper, nicke1, and platinum melt wires by volume diffusion in the metal, gave smoothing times of a few months to a few years at the metal-carbon eutectic temperature. For the remaining melt wire materials, the time to smoothing is expected to be in the range of one to ten years. The effect of scratch smoothing should therefore be negligible in reactor usage.

The radiation enhancement of diffusion $(5)$ is negligible in UHTREX.

\subsection{Reactor Experience}

The melt-wire technique has been used in UHTREX four times. During the startup of UHTREX, transient experiments provided two opportunities although the melt-wire inference method was designed for steady state operation. During the rise to power two more opportunities were provided, one at the intermediate power level and the other at the high temperature level. Melt wires were selected to cover the anticipated temperature profile, which was predicted from heat transfer calculations, and previous experience. Each wire was identified with up to three very shallow cuts which permitted eight material identifications.

The wires were placed onto a cardboard backing (Fig. 16) in the same order as they were to be placed into a fuel element and were photographed and radiographed.

After the walls of UHTREX fuel elements were drilled with three, equispaced 0.070-in. holes, the elements were hot-gas leached to remove any exposed uranium. The wires were next inserted into graphite capsules (Fig. 17) and then into the fuel-element holes with a 1/4-in. graphite spacer between adjacent wires. The ends of the holes were sealed with graphite cement and cured, the curing temperature being below the melting point of all wires. The fuel elements were radiographed three times, once with each of the three melt-wire holes directly over the film.

The elements were loaded into the reactor for the duration of the experiment. It was always necessary to be sure that the temperature profile of the channel was such that the elements did not pass through a temperature zone higher than that which the element would be measuring. A similar requirement was necessary during unloading. Generally, the

${ }^{(5)}$ R. Sizman and V. Daunert, "Radiation-Induced Diffusion in Metals,"

p. 351 in "Radiation Damage in Solids," Part I, Proc. of the Symposium at Venice, May 7-11, 1962, International Atomic Energy Agency, Vienna, 1962 . 
loaded elements were installed as late as possible and were withdrawn as soon after an experiment as possible. The effects of long exposure to high temperatures below the melting point of a wire have been mentioned; there appear to be no such difficulties in the usage in UHTREX.

As pointed out previously, the uranium fuel often interferred with the clear indication of sharp groove corners in the melt wires. This was usually harmless since wire melting, when it occurred, tended to be complete and the melting was very obvious. When this was the case the radiographic detection of melting was sufficient and satisfactory. However, in all four UHTREX inference tests it was desirable to remove wires to examine one or more of them. This operation in a hot cell was very time consuming. When the wires were removed from all of the elements, they were transferred to a ventilated hood and observed directly. In a very few cases the direct, visual examination upset a decision previously made by studying the radiographs. The wires were replaced onto a cardboard backing or on a glass slide and rephotographed. See Fig. 18.

The gamma activities encountered during these operations are shown in Table II.

\section{TABLE II}

Activities Encountered in UHTREX

Melt Wire Experiments

Operation

Fuel element directly from UHTREX

Fuel element in hot cell (decrease due to decay)

Melt wires (together in plastic tubes)

Individual melt wires
Activity at Contact

$$
\begin{aligned}
& 100 \text { to } 200 \mathrm{R} / \text { hour } \\
& 10 \text { to } 20 \mathrm{R} / \text { hour } \\
& 5 \text { to } 10 \mathrm{R} / \text { hour } \\
& 0.0 \text { to } 1 \mathrm{R} / \text { hour }
\end{aligned}
$$

In the first experiment, (the slow transient with a 40 \& reactivity insertion) the radiographs (Fig, 19) indicated that fuel elements remained below the melting temperatures of all but the lowest melting wire, $\mathrm{Bi}-\mathrm{Pb}$ alloy at $265^{\circ} \mathrm{F}$. The temperature at a single location was determined.

In the second transient experiment the entire profile (Fig. 20) was inferred from melt-wire data. A few data were ignored as being an effect of the transient nature of the experiment. The data not used were all low and in each element came from the same hole. It was postulated that that hole was resting on the channel and lost heat more rapidly than did the other, more insulated holes.

During the rise to the intermediate power leve1, melt wires were used to determine the temperature of a typical channel. Temperature limits were set but the temperature was established at only one position. See Fig. 21. During the rise to the high-temperature plateau the reactor exceeded the temperatures expected and very few wires remained unmelted. The unmelted ones established the temperature at one location. 
The success of determining temperatures with me1t wires seems to depend on two factors; 1) that the temperature profile be guessed or calculated sufficiently closely to enable the inclusion of many wires of similar composition, and 2) the availability of melt wires with small temperature gaps between them. The best measure of temperature occurs when one of two adjacent wires of the same composition melts and the other does not. If the temperature profile cannot be guessed closely, a wide range of melt wires must be used and the distance between two similar wires must increase. This increases the uncertainty of the location of a particular temperature.

There is a limited number of suitable compositions for melt wires although the use of solid solutions greatly increases the number of materials of different melting temperatures. The latter have been quite sensitive and characterizations of three such wires have given acceptable uncertainties of from 15 to $30^{\circ} \mathrm{F}$. Twenty-four wires have been developed for use in the melt-wire method of temperature inference and the search for more materials is continuing. The wires developed were needed for the startup of UHTREX. It will next be necessary to narrow the temperature gaps between wires with adjacent melting points. This will be the subject of some future work. 


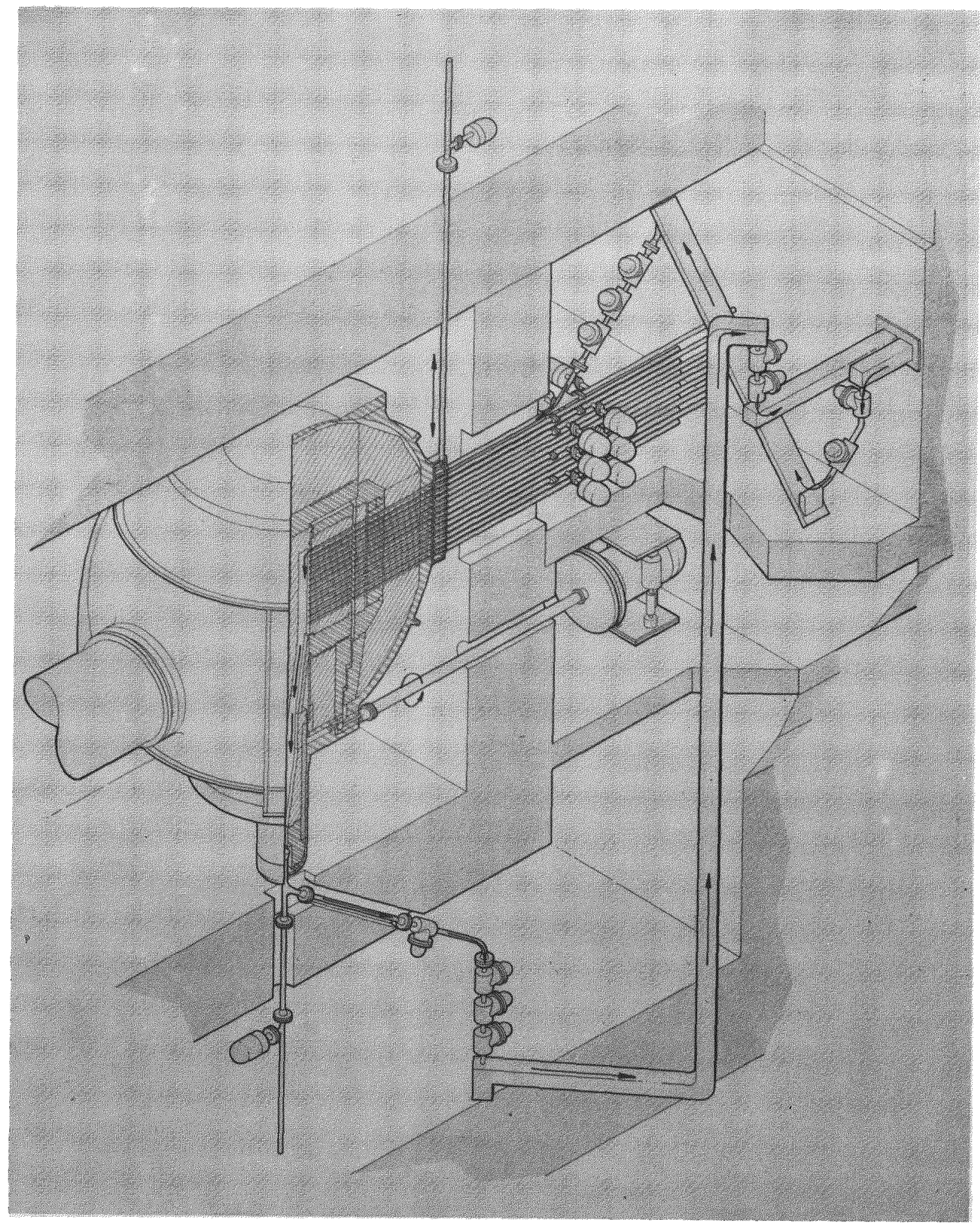

Fig. 1. Fuel Handling System in the UHTREX Reactor. Fuel elements are loaded and unloaded during operation as indicated by the arrows in the photograph. The rotating core permits alignment of any circumferential position with the elevator assembly. Graphite rams push elements, properly aligned, into the core. 


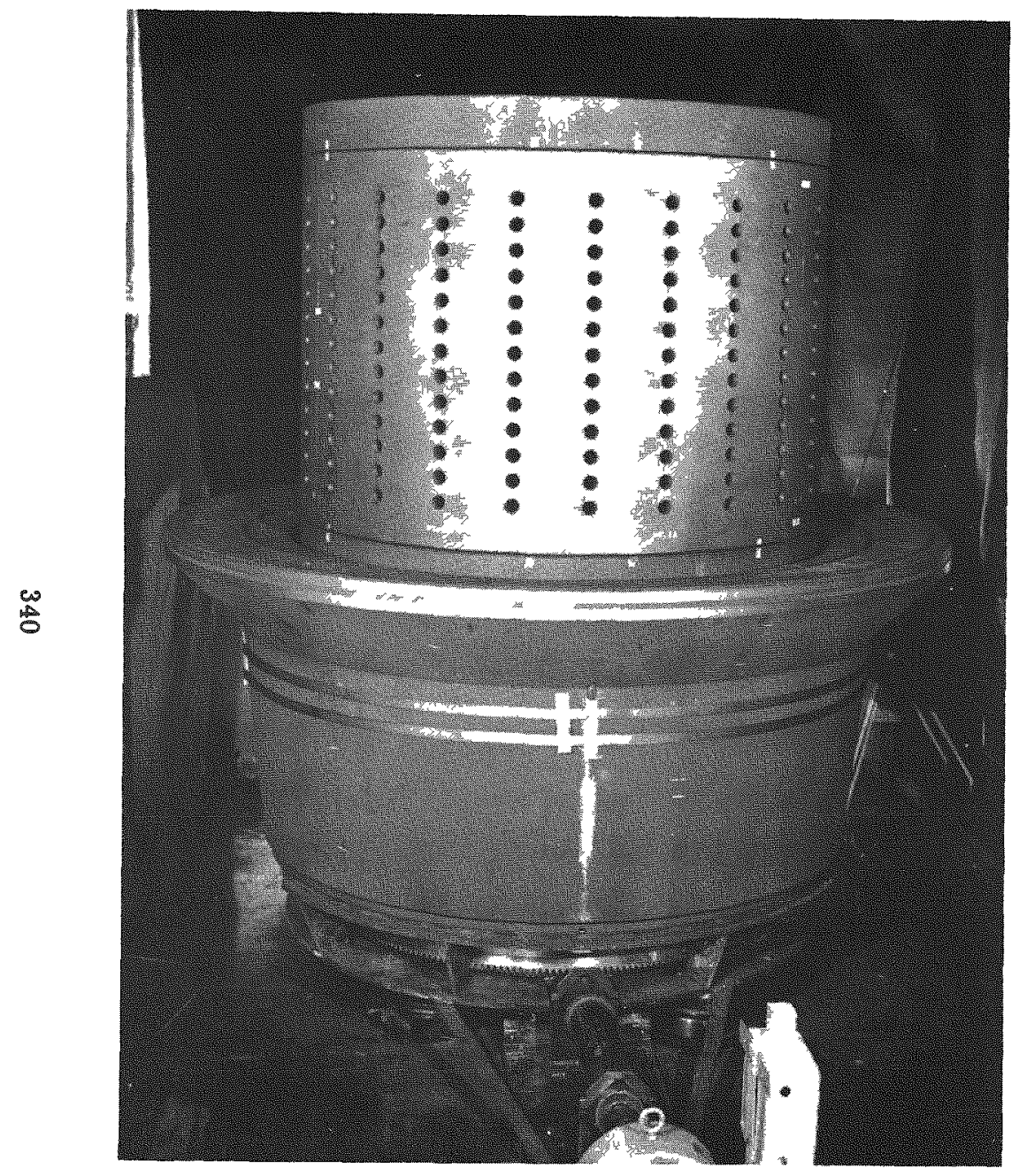

Fig. 2. UHTREX Core and Drive Mechanism. The UHTREX core is supported on a stee1 plate which can be rotated during operation.

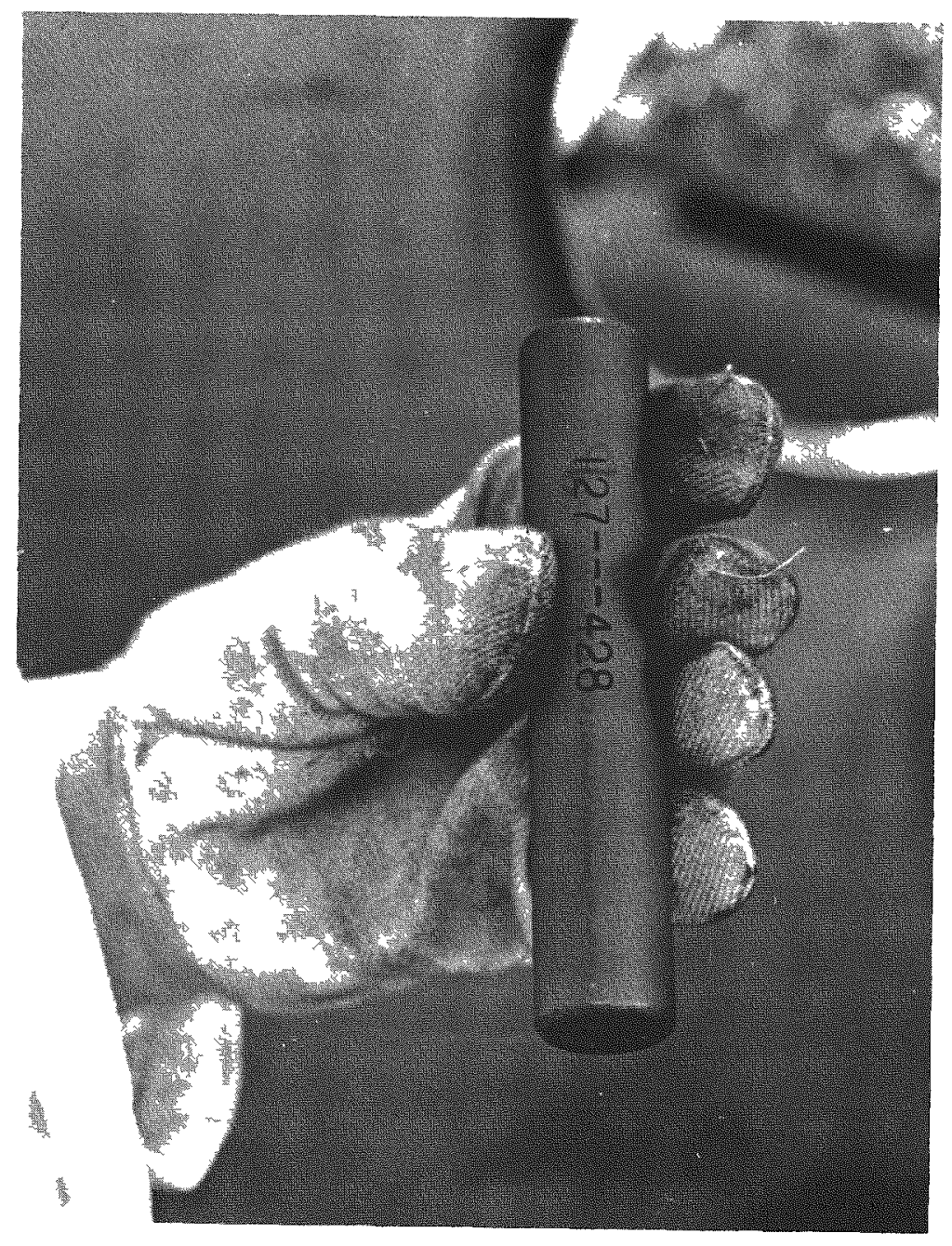

Fig. 3. UHTREX Fuel Element.

Shown here as a typical UHTREX fuel element is the element which provided the final reactivity for criticality. 

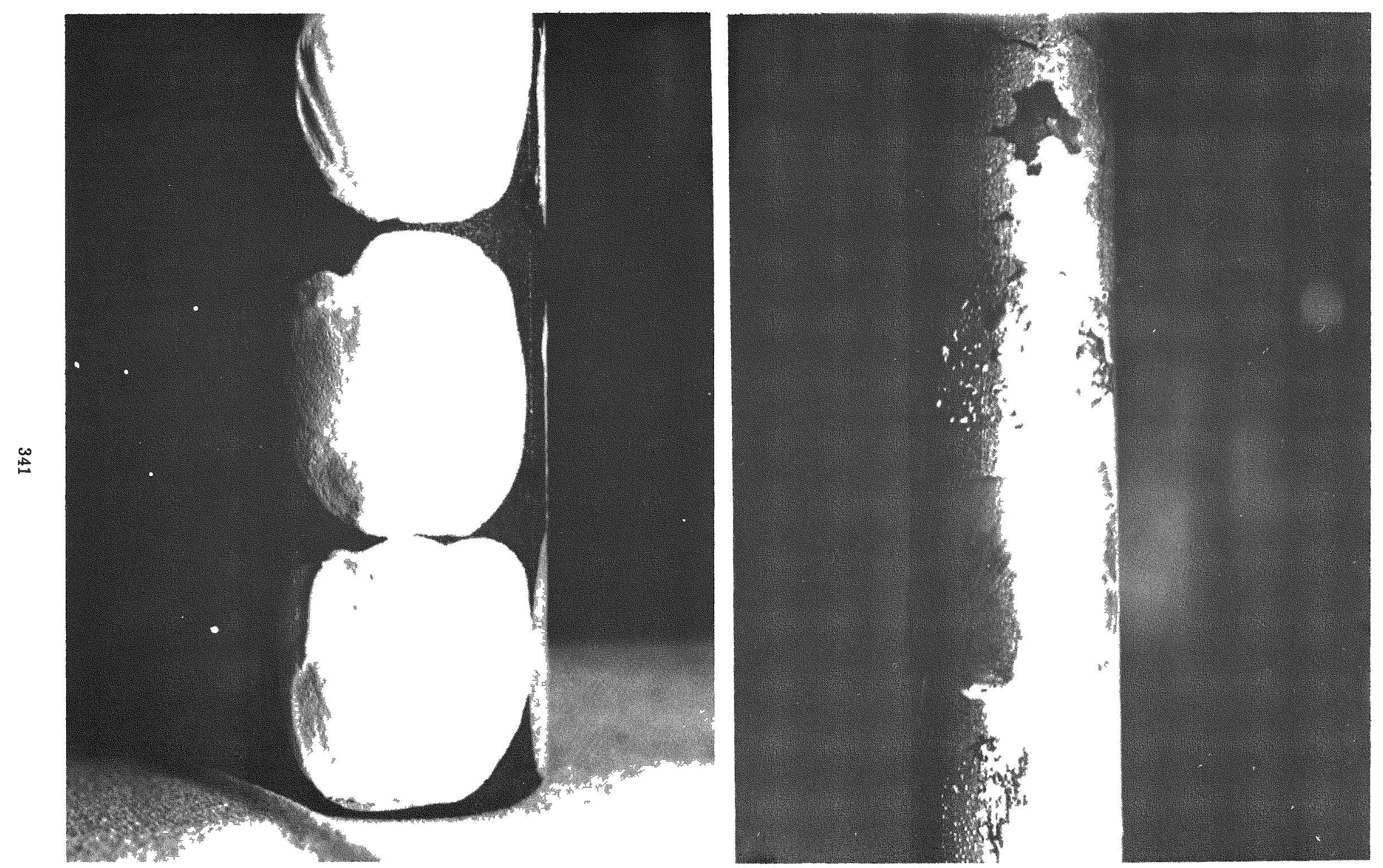

Figs. 4 and 5. Temperature-Sensitive Paints on Unheated and Heated UHTREX Dummy Fuel Elements. The attempt to use temperature-sensitive paints on UHTREX fuel elements was not successful. 

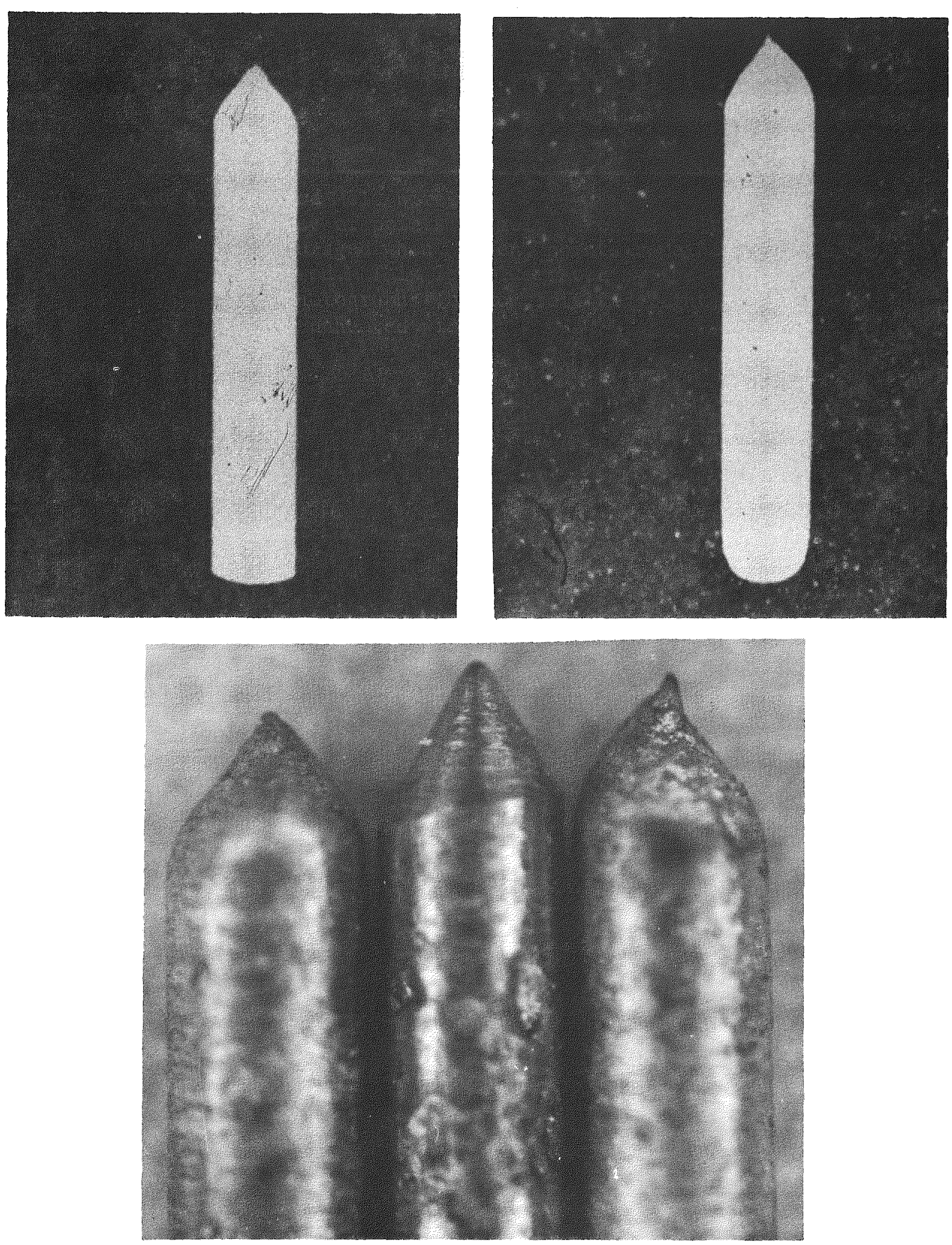

Figs. 6, 7, and 8. Pointed Melt Wires.

The use of points on melt wires as melt indicators was discouraged when, often, points remained after melting had occurred. This is illustrated by the radiographs (top) and the photographs (bottom left and right). The bottom center is an unheated wire. 


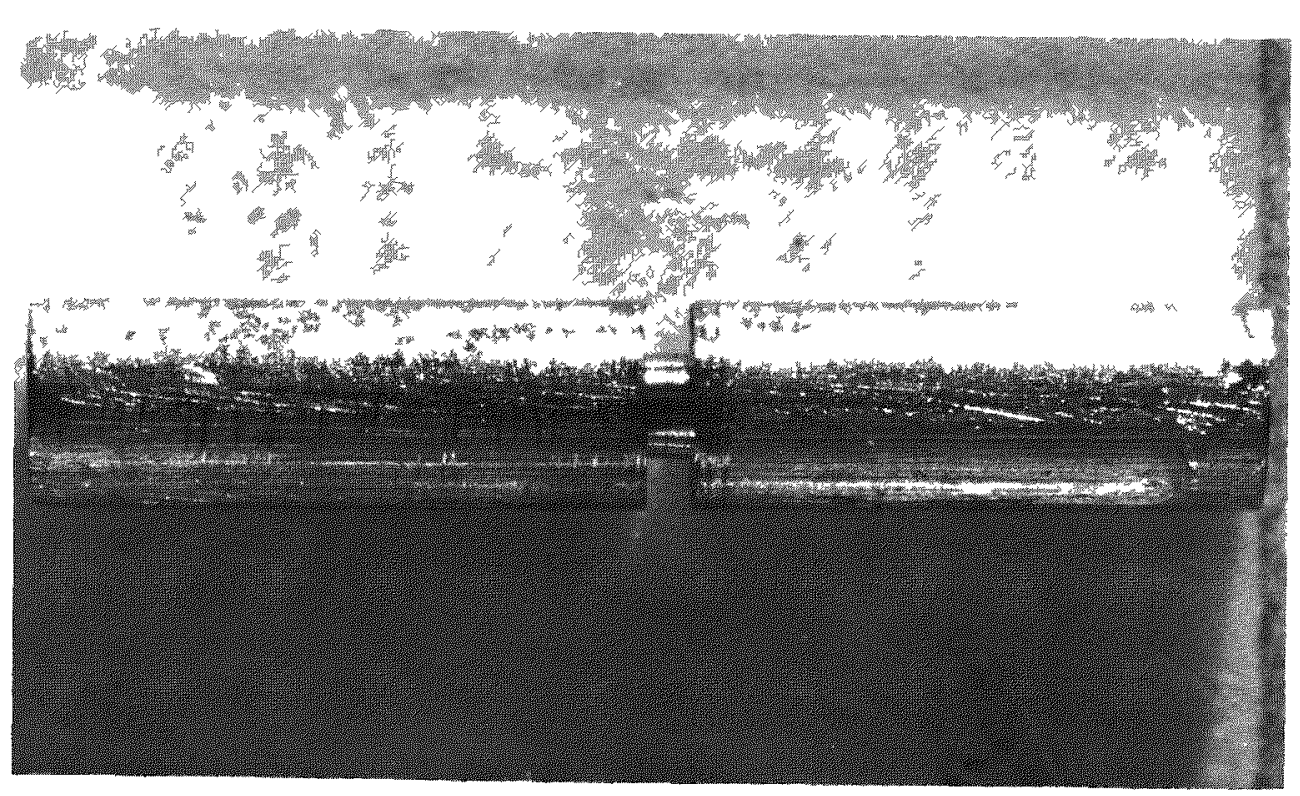

Fig. 9. Grooved Melt Wire.

Grooved melt wires were found to be easy to machine, sensitive to temperatures only slightly above melting and relatively reproducible.

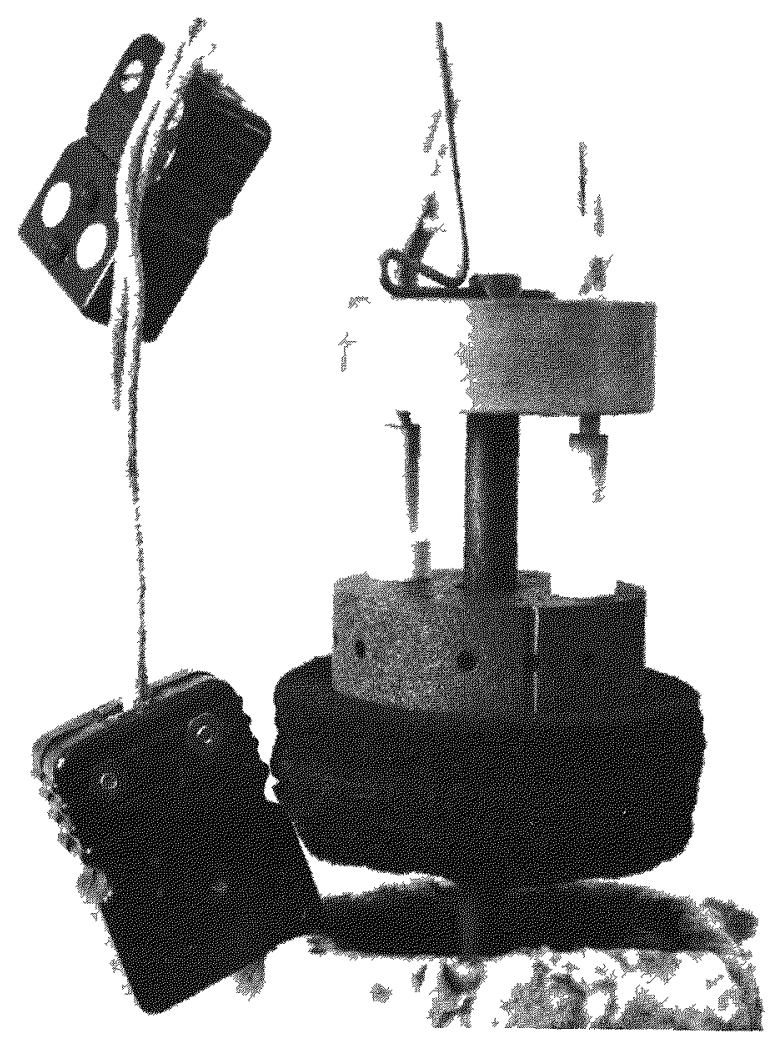

Fig. 10. Graphite Disc Assembly for Melt Wire Characterization Tests. Approximately one-hundred wires of each material, used for melt wire, were tested to establish the melting temperature. In each test, twelve wires are placed in radial holes shown and heated while the two thermocouples sense the temperature. 


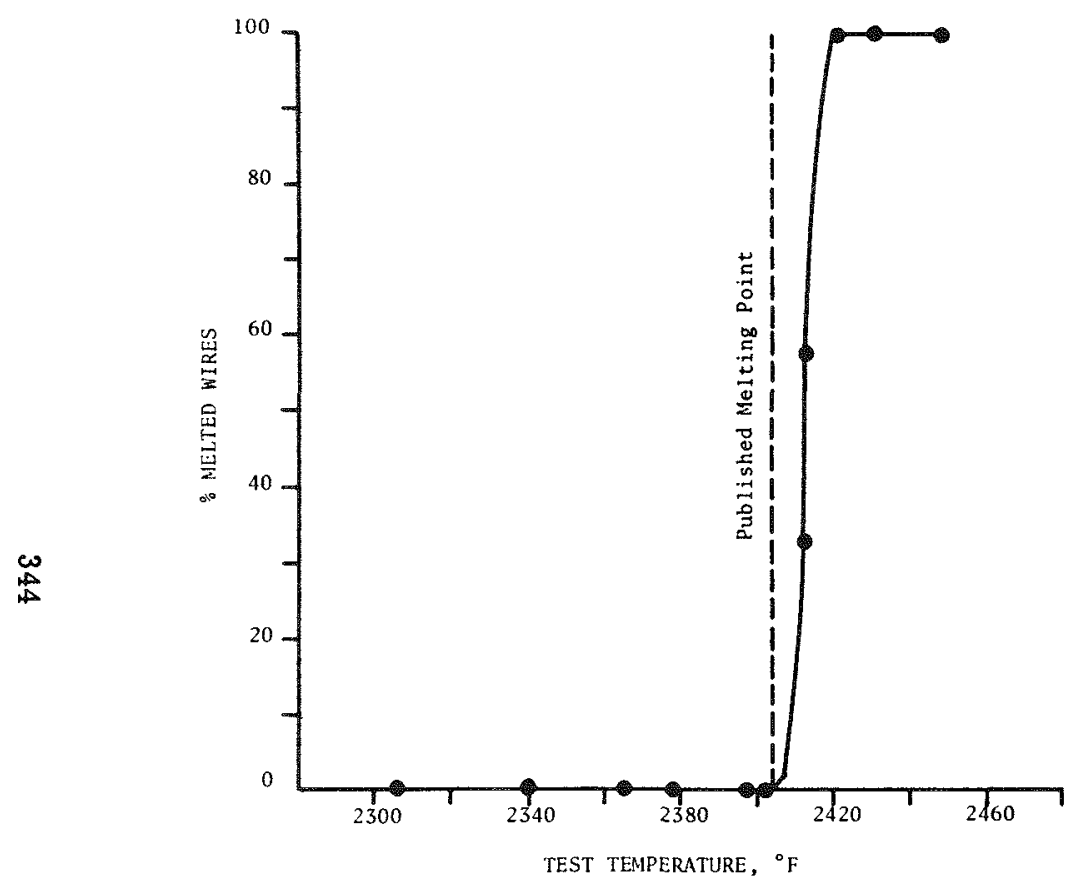

Characterization CuRve for Nickel Wire

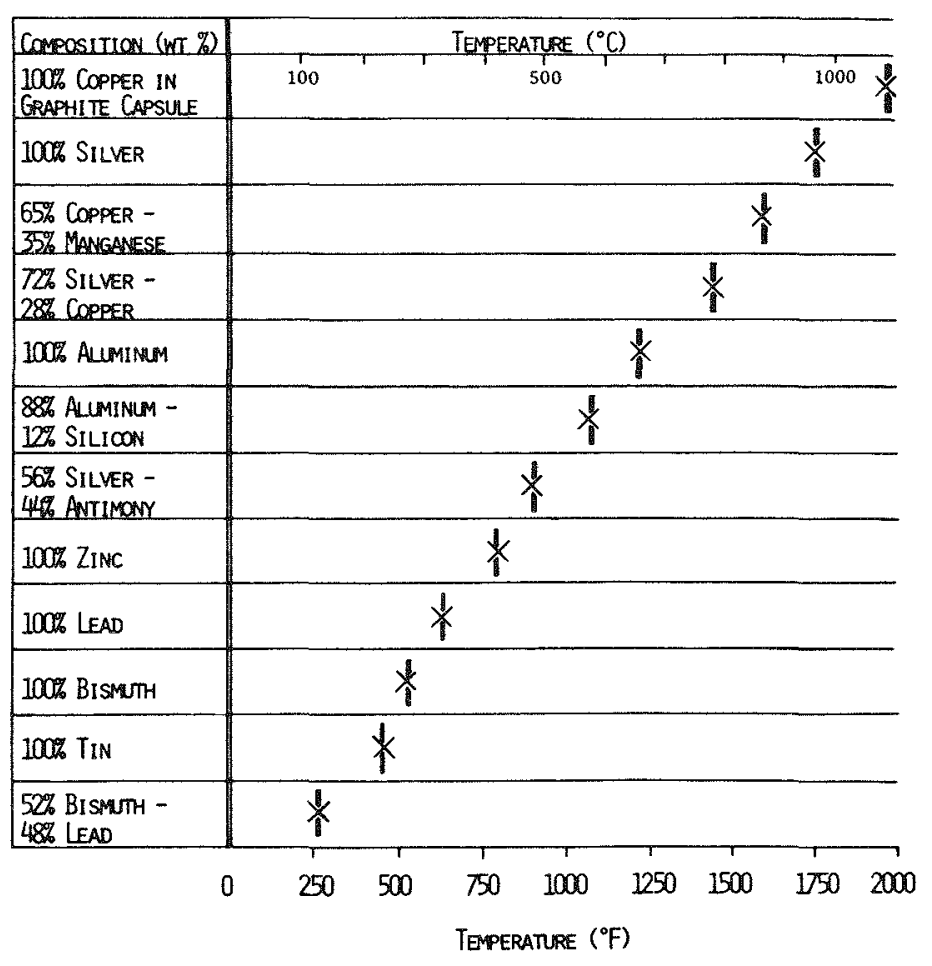

Fig. 12. Results of Characterization of Wires Melting Below $2000^{\circ} \mathrm{F}$.
The published melting point is shown at the intersection of $X$ and the bar width illustrates the temperature spread between $0 \%$ melts and $100 \%$ melts in heating tests of 12 wires each. tes
Fig. 11. Typical Charactexization Curve. The results of a typical characterization test is shown. Each point represents a single twelve-wire test. The observation of a melt is made both radiographically and visually. 


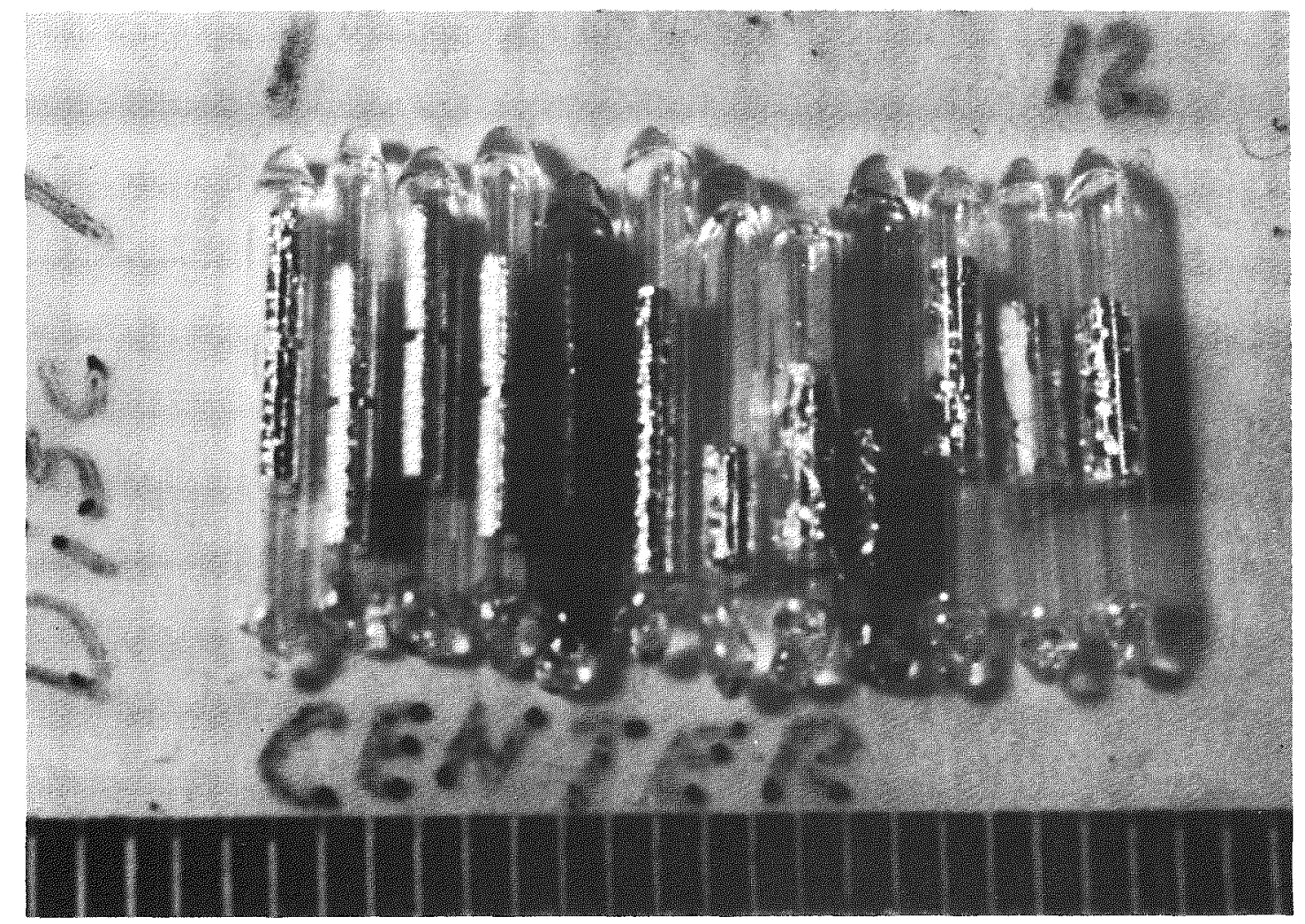

Fig. 13. Typical Quartz Encapsulated Wires After Heating. Quartz encapsulation eliminated wide deviations from published melting points observed without quartz.
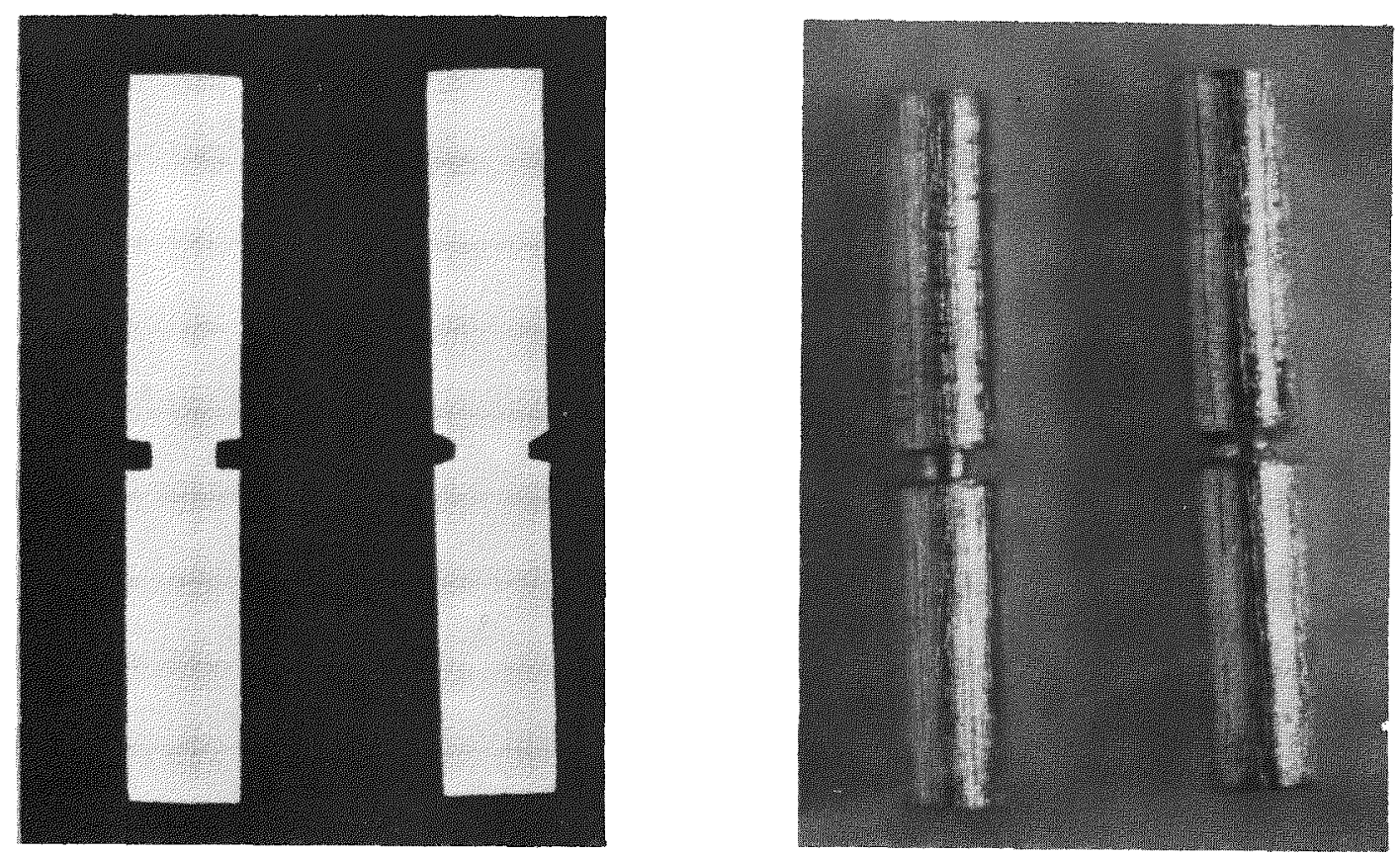

Fig. 14. Grooved Melt Wire, Betore and After Heating. The melting sensitivity of the groove is shown in the photograph and radiograph. 
Melt Wires in

Graphite Capsules

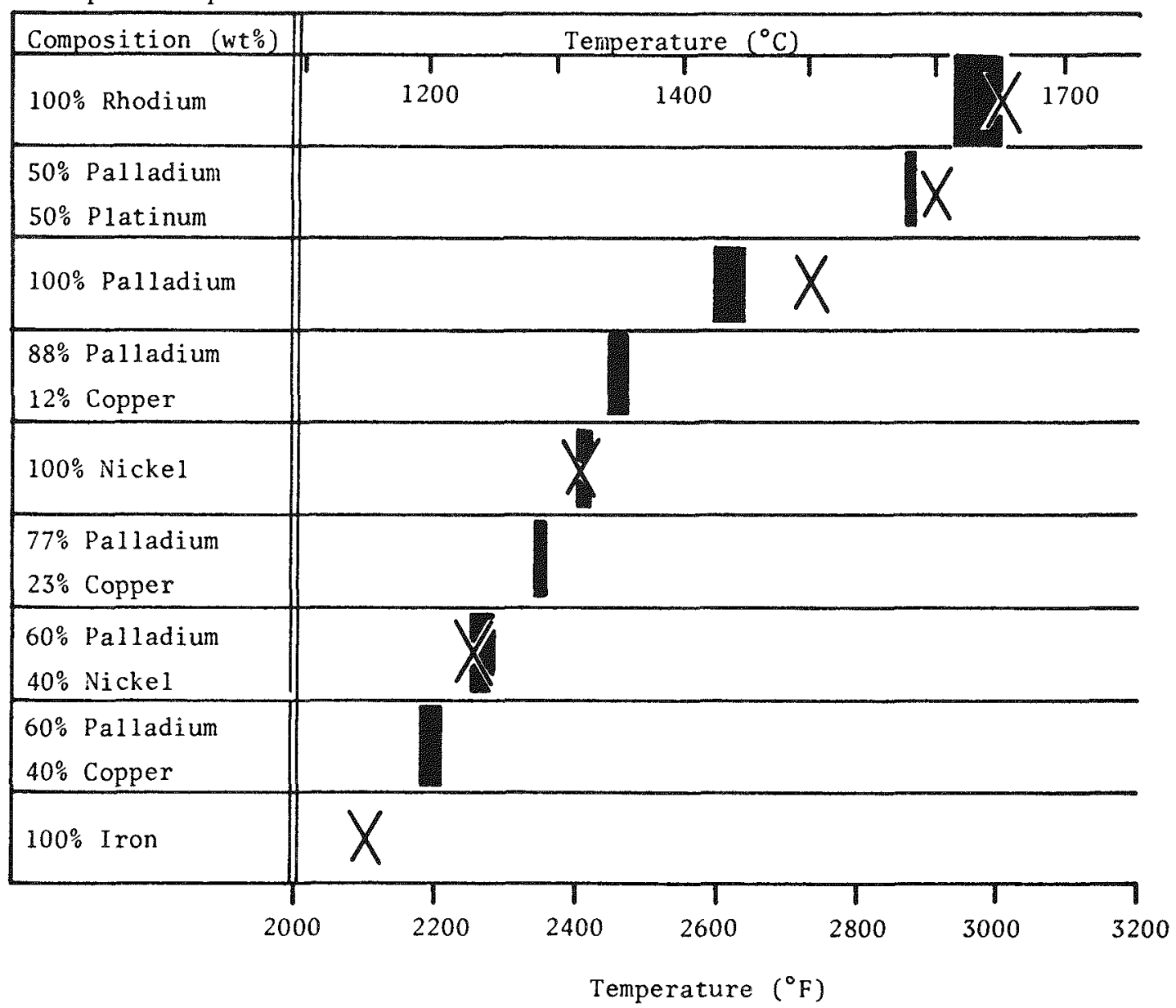

Fig. 15. Results of Characterization of Wires Melting Above $2000^{\circ} \mathrm{F}$.

The published melting point is shown at the intersection of $X$ and the bar width illustrates the temperature spread between $0 \%$ melts and $100 \%$ melts in heating tests of 12 wires each. (Fe characterization was not completed when the figure was made. Bar width - 2095-2111.) 

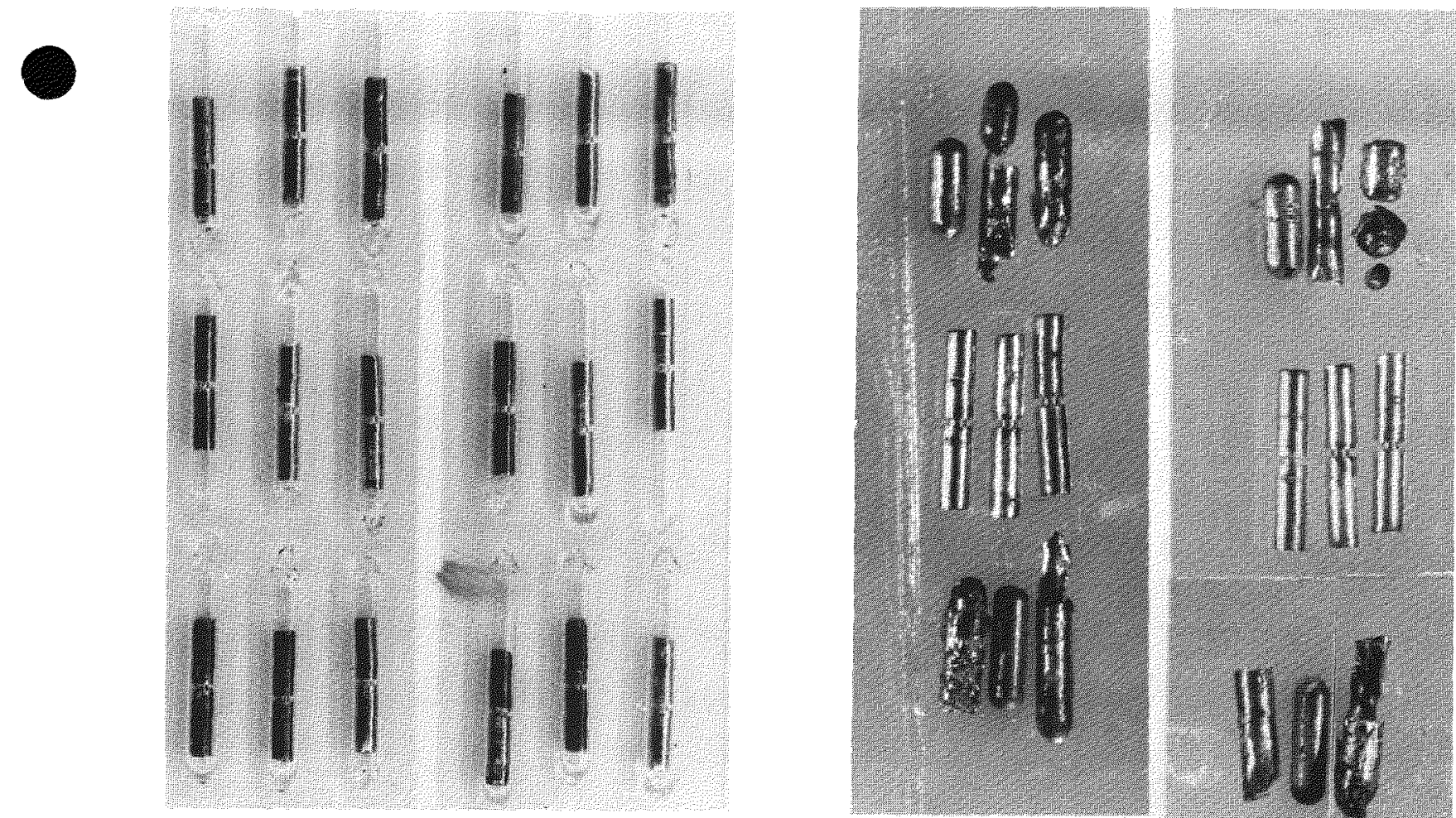

Figs. 16 and 18. Melt Wires Before Insertion and After Removal from a UHTREX Fuel Element.

These wires show the normal effects of an experiment. All melts are clear and unequivocal. Some identification marks are apparent.

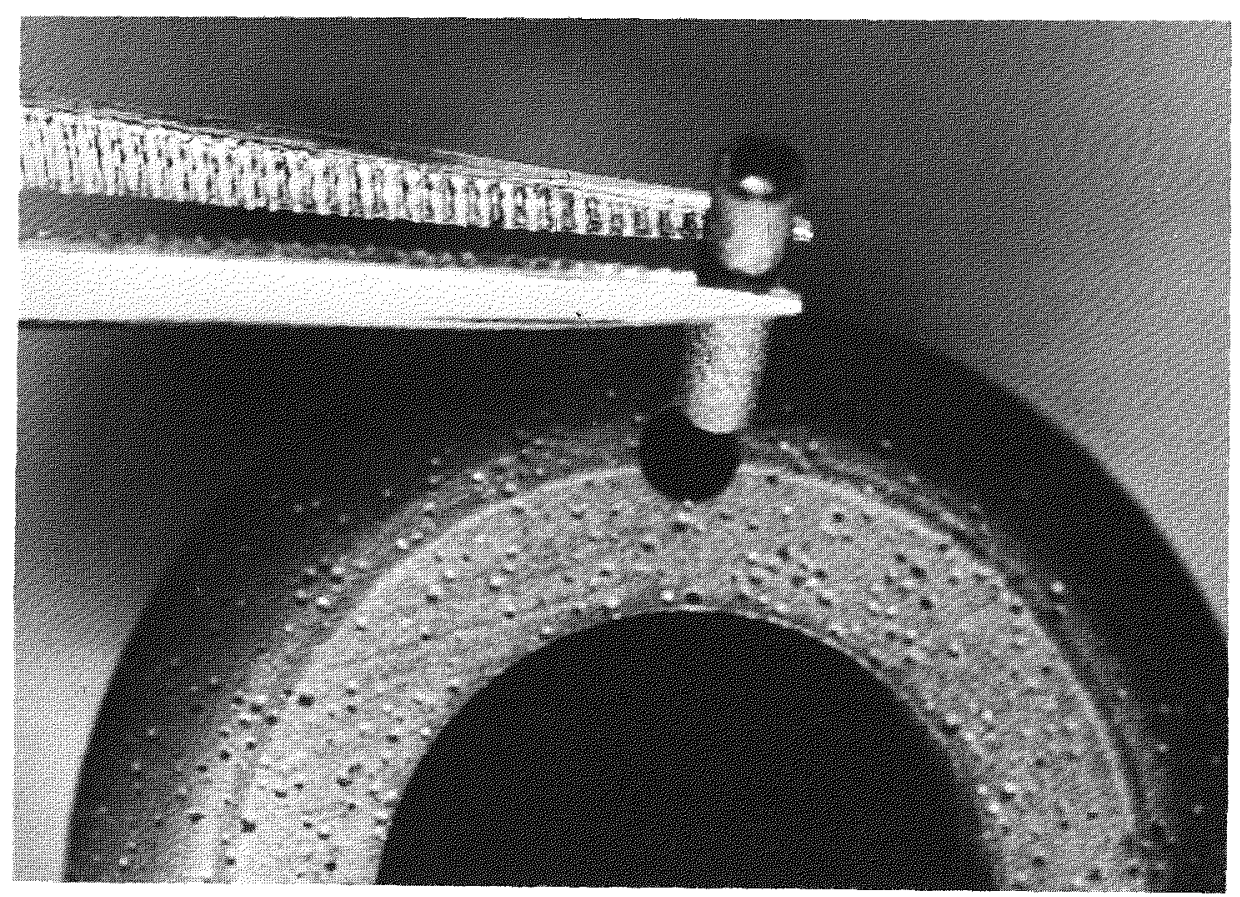

Fig. 17. Insertion of Melt Wire into UHTREX Fuel Element.

The wires are first placed into graphite cups and then inserted into the element. Graphite spacers, inserted between capsules, function as lids to the open cups. 
Distance from

Core ID (Fuel

Outlet), inches $1-5 / 8$
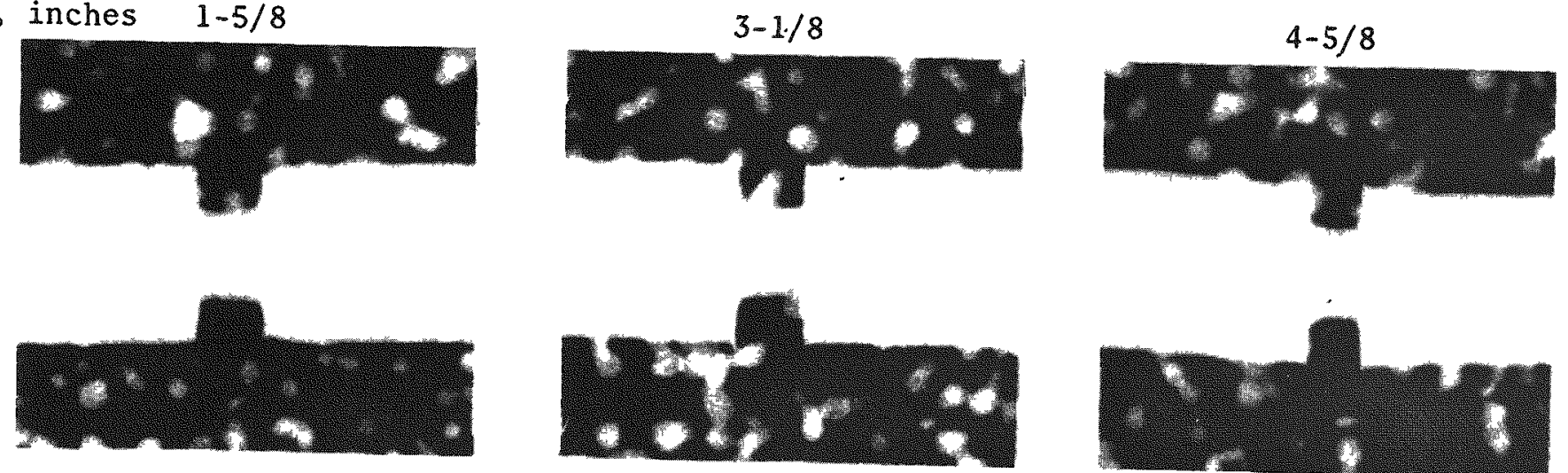

BEFORE

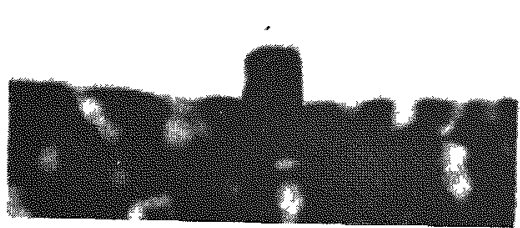

BEFORE

$\underset{\infty}{\infty}$
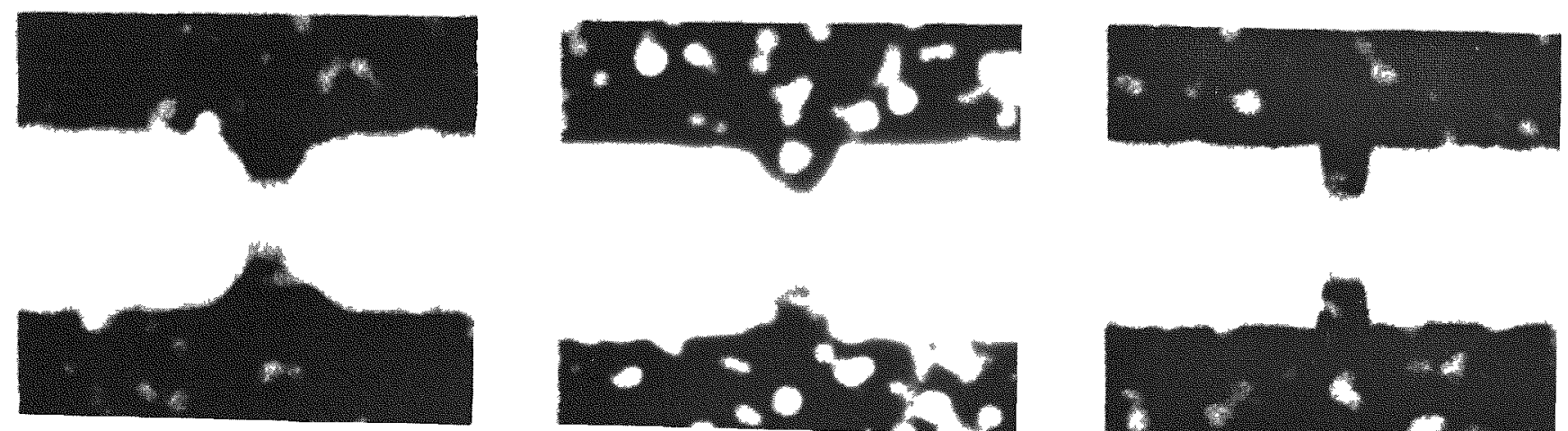

AFTER

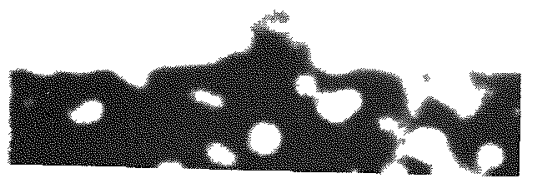

AFTER

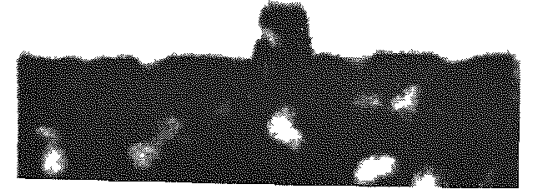

AFTER Fig. 19. Radiographic Results of the Melt Wire Test After the Forty-Cent Reactivity Insertion of the Slow Transient
Experiments in UHTREX.

The two wires on the left are seen to have melted. The wire on the right did not. A temperature at a known location was thus established. Note the interference of observation by fuel particles. 


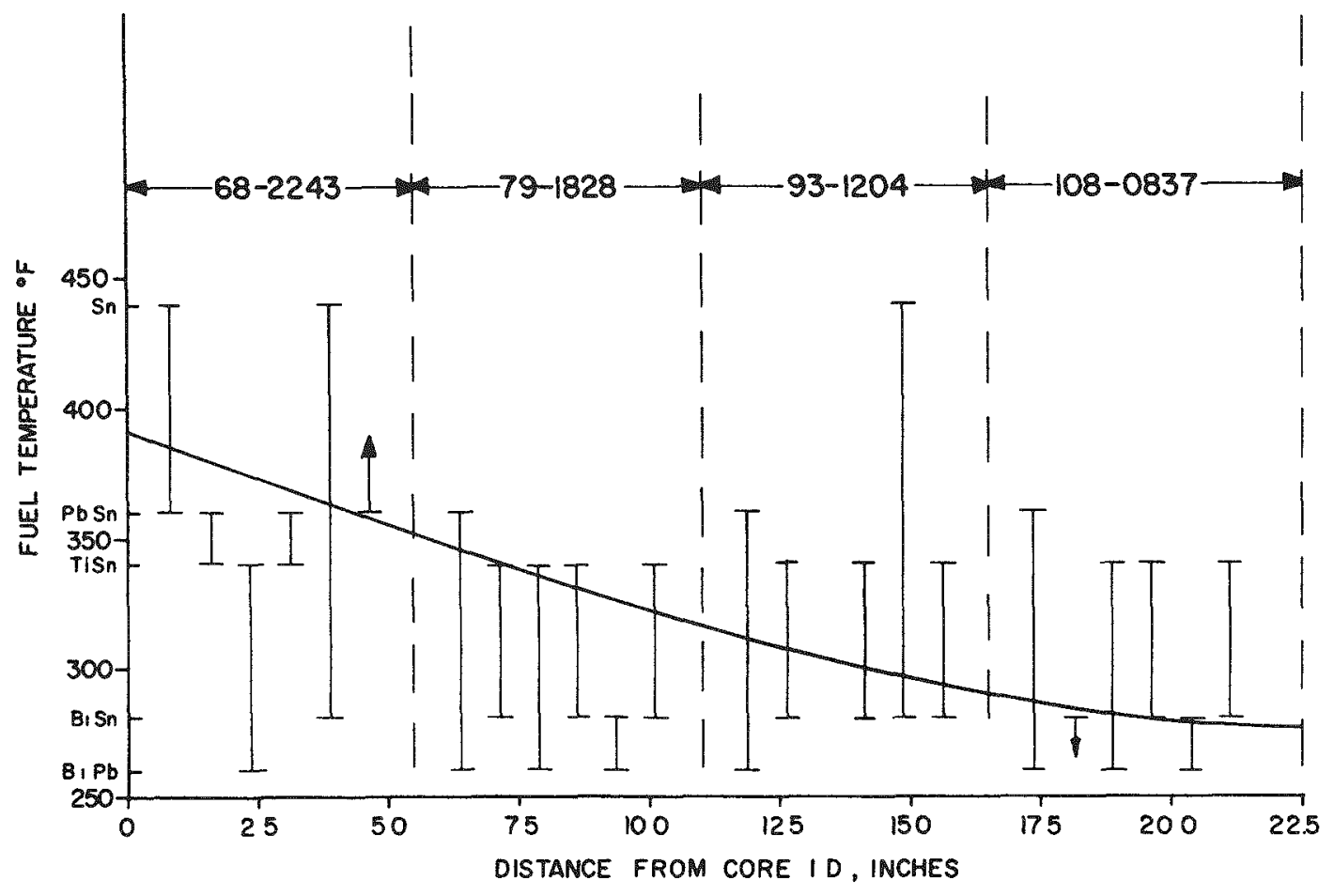

FIg. 20. Temperature Profile During a Transient Experiment As Inferred by the Melt Wire Method.

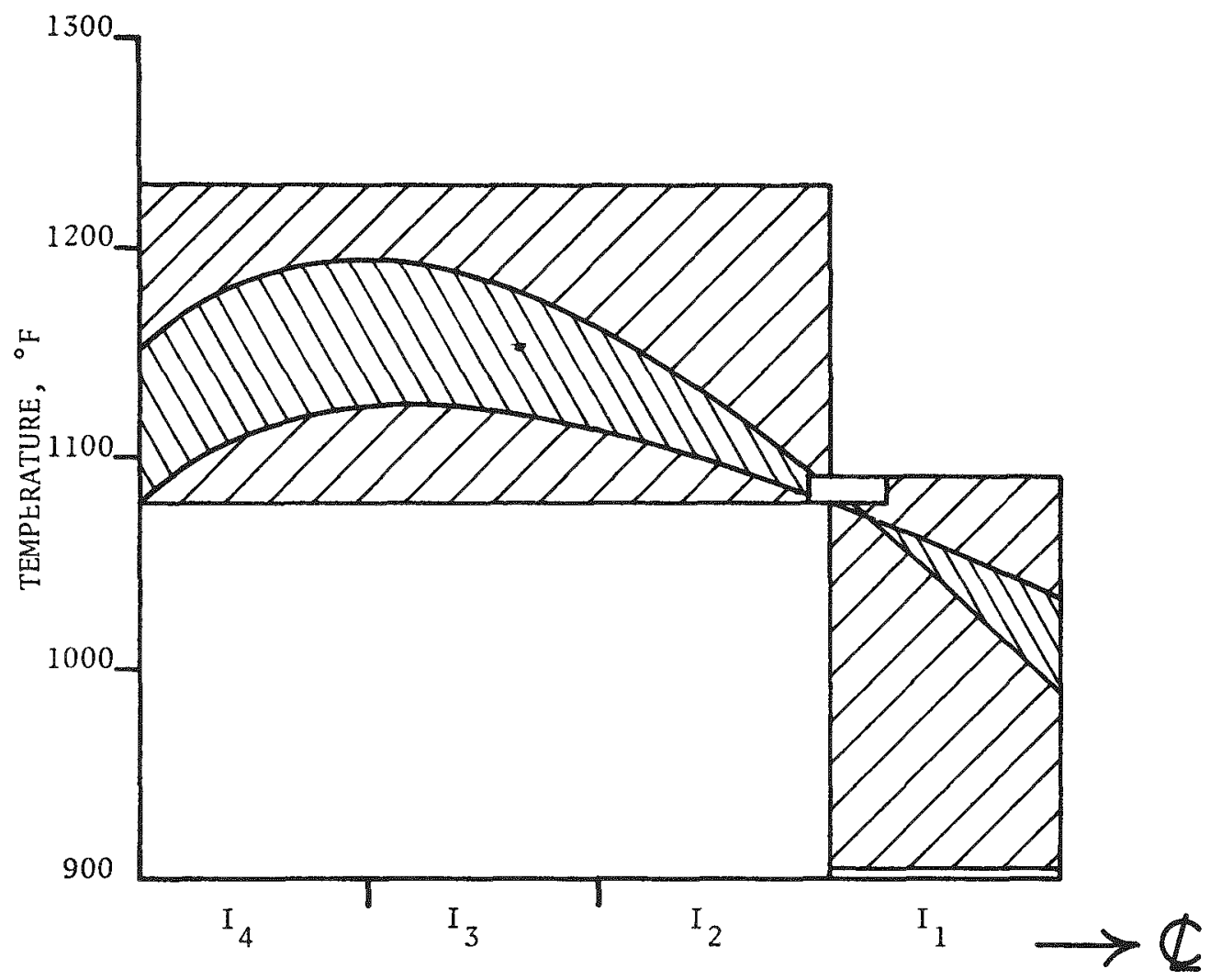

Fig. 21. Temperature Profile During a Steady State Experiment as Inferred by the Melt Wire Method. 


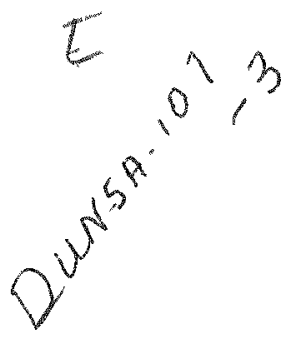

\title{
$X$ MeAsured enERGY DePOSImion RATES FOR VARIOUS NONFISSIONABIE MATIERIALS IN A NUCLEAR REACTOR ENVIRONMENT
}

\author{
Paul J. Zimmerman \\ Lit $1, \ldots$, Douglas United Nuclear, Inc.
}

\section{INTRODUCTION}

All materials experience some amount of internal heat generation when placed in a nuclear reactor environment. The energy that is deposited or generated is dependent upon the physical and geometric characteristics of the material and upon the nature of the nuclear reactor environment. Sources of heat generation include the absorption of gamma photons, neutrons, betas, positrons, and fission fragments.

Fission heating is normally of prime interest; however, special cases arise where the heat generation in nonfissionable materials is of extreme importance. Heat generation rates in reactor shielding, structural materials, and, generally, any material placed in a nuclear environment must be known to some degree of accuracy depending on the specific case. In-reactor testing of various materials and devices requires some knowledge of energy deposition rates in order to arrive at a successful and safe design.

Calculational techniques exist for predicting energy deposition rates due to gamma and neutron interactions with various materials in a known environment. These techniques range from complex and expensive computer solutions to order of magnitude hand calculations. The method for obtaining the desired information will depend upon the degree of accuracy required and resources available.

A calorimeter was designed which could be used to measure heat generation rates for many materials at relatively low cost utilizing existing facilities. The device was designed to operate in a horizontal test facility and measure heat generation rates from a few watts to several hundred watts.

Since a large number of samples could be tested, materials with a wide range of specific physical characteristics were chosen. Materials were chosen to either satisfy a specific customer interest or to give data to aid in separation of heat generation into gamma and neutron contributions, respectively. 
A calorimeter was developed which successfully measured the heat generation rate of many materials in a reactor test facility environment. Measurements were made for materials of sufficiently different properties so that it was possible to separate the heat generation by gamma interactions from thermal neutron interactions. Gamma heating values ranged from $0.16 \mathrm{~W} \mathrm{~g}^{-1}$ for titanium to $0.25 \mathrm{~W} \mathrm{~g}^{-1}$ for bismuth. Heat generation due to nuclear reactions of thermal neutrons was determined by subtracting the gamma heating from the total measured values. The thermal neutron contribution varied from 0 to $9.78 \mathrm{~W} \mathrm{~g}^{-1}$. Thermal neutron contributions exhibited a large dependence on geometry as was expected. Thermal neutron absorptions occurring in the vicinity of the material in question increase the gamma flux significantly above that contributed by fissioning material in the surrounding lattices. Thus the measurement of heat generation rates in materials in a nuclear reactor environment can yield information which can be far from representative of heat generation rates in other portions of the same reactor core. This is especially true in a thermal reactor since the potential for nuclear reactions is much greater than in a fast reactor system. Experimentally determined heat generation rates are valuable for checking calculational techniques for a given facility of fixed geometry and fixed surroundings. Deviations from these values by an order of magnitude may occur because of changes of materials in close proximity to the volume of interest. Thus, care should be exercised in the use of experimental measurements.

\section{REACTOR ENVIRONIENT AND ENERGY DEPOSITION MECHANISM}

\section{General}

The Hanford reactors are large graphite-moderated, water-cooled facilities whose primary purpose is the production of plutonium. Extensive irradiation testing facilities such as shown in Figure 1 are also available. These penetrate through the reactor orthogonal to the process channels. Figure 2 shows a typical cross-section of a test facility and its location within the reactor lattice. The heat generation data reported herein were obtained in a region of the reactor in which the neutron and gamma flux had been tailored specially for this set of measurements.

Gamma Radiation

Accurate calculation of energy deposition due to gamma dose rates in any material in a given environment requires that the magnitude and spectral distribution of the gamma photons be known. This is necessary since there is a significant variation in the energy absorption mass attenuation coefficient $\mu$ with material and photon energy. No data 


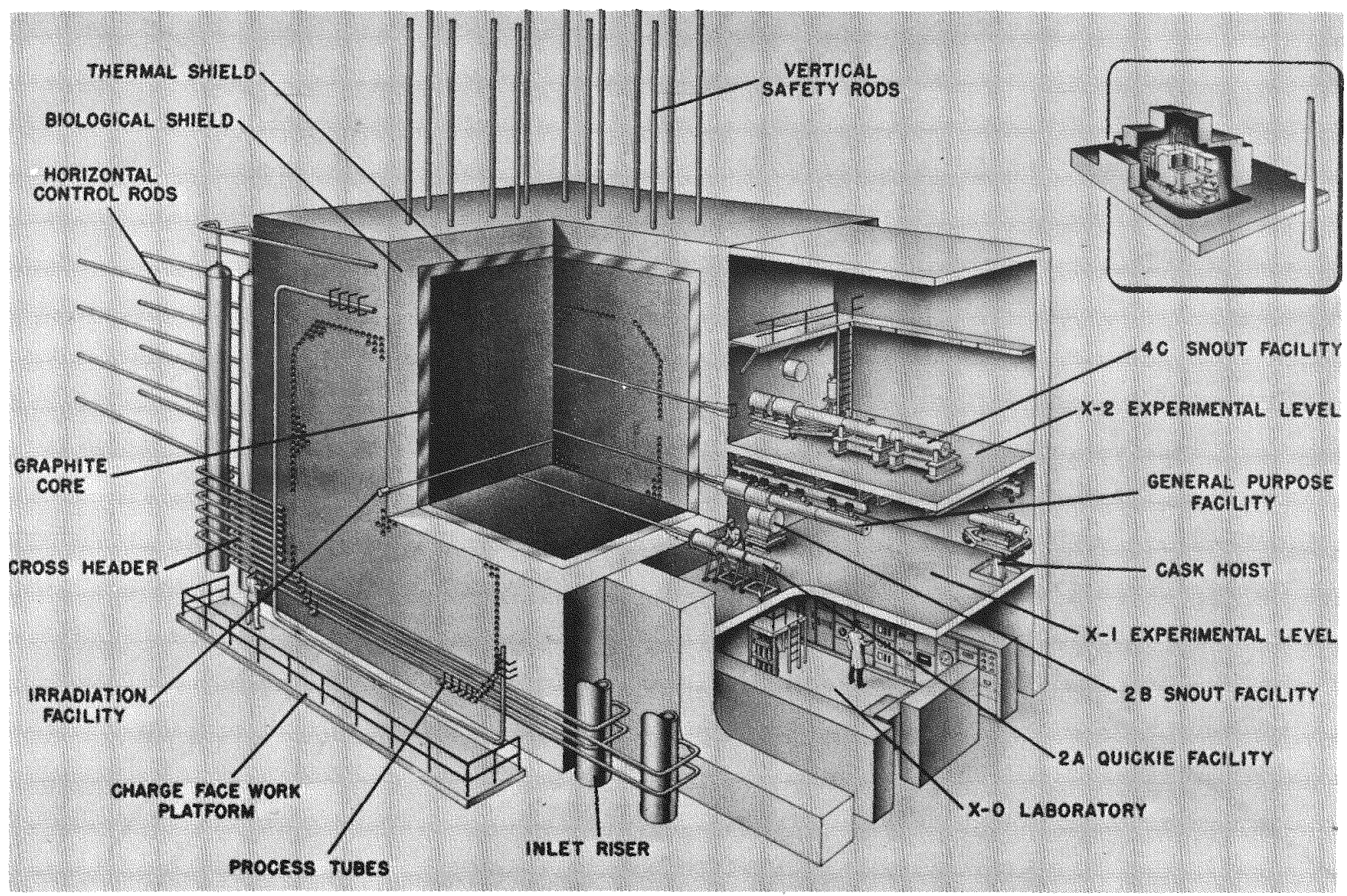

FIGURE I

SPECIAL IRRADIATION FACILITIES IN THE HANFORD PRODUCTION REACTORS 


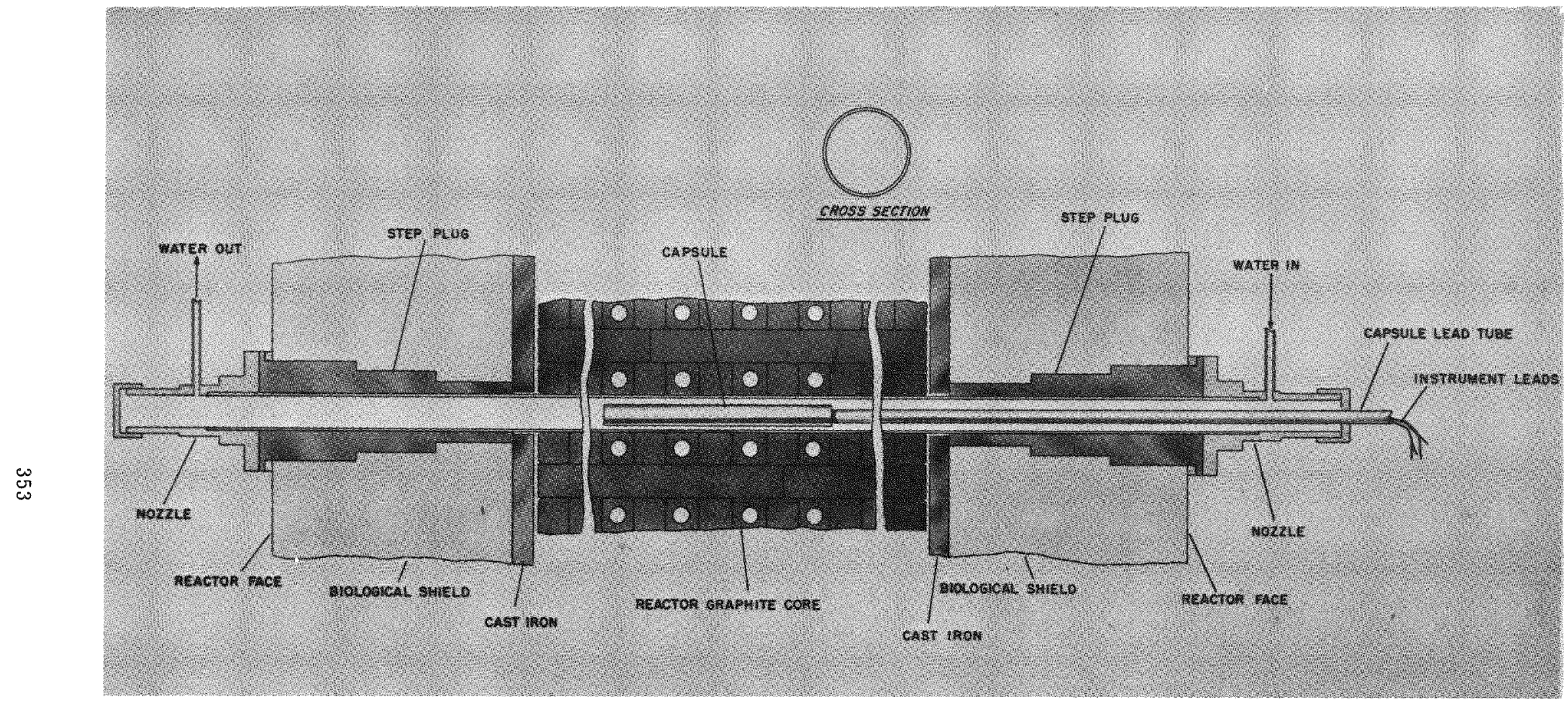

FIGURE 2 
were available on the magnitude or spectrum for any Hanford facilities tailored or otherwise. The spectrum in the calorimeter was calculated by considering the sources of gammas within the reactor core, the surrounding facilities, and the calorimeter.

The gamma dose rate and spectrum at the point of interest were calculated by superimposing the contribution from the surrounding fuel lattices on the local effect caused by thermal neutron absorptions in the facility sourroundings and calorimeter.

Surprisingly, the locally produced contribution was large compared to the surrounding fuel contribution, even though the materials were relatively transparent to neutrons. The resulting gamma flux and. spectrum were primarily from absorptions in aluminum. A heavy absorber located in the close proximity to the material in question would be expected to change the gamma heating or dose rates by an order of magnitude or more. Calorimeter No. 1 utilized a magnesium heat sleeve to minimize the local thermal neutron absorptions; however, Calorimeter No. 2 used an aluminum sleeve to increase the reliability of the device. The ganma flux at the sample location for the aluminum case was calculated to be $19 \%$ greater than for magnesium.

The three principal processes for the absorption of energy from gamma radiation are the photo-electric process, Compton scattering, and the pair production process. These three absorption mechanisms vary with material and photon energy but are reflected in the energy absorption mass attenuation coefficients $(\mu / \rho)$ for the materials. To accurately calculate the gamma contribution to the total energy deposition, the $(\mu / \rho)$ was weighted according to the calculated incident gamma photon spectrum.

An additional correction was necessary due to the significant attenuation of the gamma flux within the different samples. This effect was corrected for by assuming the average chord length in the sample to be equal to the diameter, the decrease in flux to be exp $[-(\mathrm{u} / \mathrm{\rho}) \mathrm{p} \mathrm{avg}$
and by integration, finding the average flux in the sample.

\section{Thermal Neutrons}

Energy deposition caused by thermal neutron interactions is wholly nuclear since by definition thermal neutrons cannot transfer any net energy by scattering. Usually thermal neutron absorption results in 4 to $10 \mathrm{MeV}$ energy release due to the binding energy of the neutron for the particular atom. This energy is given up by emission of one or more prompt capture gammas. There are other reactions which must be considered in some cases. The $(n, \alpha)$ reaction for boron is a case of interest in this series of measurements since all of the energy of the $\alpha$ is given up in the sample. Thermal neutron absorptions also 
can result in production of radioactive isotopes which give up decay energy, usually as gamma or betas or a combination of these. Interaction probabilities or cross-sections vary from essentially zero to several million barns. Sample materials were chosen such that heat generation rates due to thermal neutron reactions could vary over a considerable range of values. The unperturbed thermal neutron flux within the calorimeter was approximately $5.7 \times 10^{13} \mathrm{nv}$.

\section{CALORIMETER DESIGN}

General Description

Calorimeter measurements were to be taken in a horizontal, water-cooled test facility. The device was to be capable of testing a number of materials with a large variation in heat generation rates.

An isothermal, steady-state design was chosen over an adiabatic calorimeter because of relatively high heat generation rates and physical limitations of the test space. Figure 3 shows a cross-section of Calorimeter No. 2 with a sample in place.

The operation of the calorimeter is based on thermally insulating the heat sleeve in all directions except toward the heat sink end. The difference in temperature between Points $A$ and $B$ is a direct measure of the heat transferred along the sleeve. Since the temperatures were measured close to the heat sink, the $\Delta \mathrm{T}$ produced was independent of the degree and location of contact between the sample and the heat sleeve. This was considered of great importance because the remoteness of the operation made close tolerances difficult. An electrical calibrating heater was wrapped around the sleeve and the sample to be measured was placed inside the sleeve. A one-to-one correspondence was assumed between the temperature difference produced by the calibration heater and by the samples.

The calorimeter was attached eccentrically to a lead tube which contained another tube of sufficiently smaller diameter to provide a space between for thermocouple and gas leads. Samples were charged and discharged through the inner tube into the calorimeter. The heat sleeve was made from magnesium for Calorimeter No. 1 and aluminum for Calorimeter No. 2 and was press fit into the heat sink block. Thermal insulation was provided by a combination of a gas gap and an insulating material. Marinite-23 was used for insulation because it has a low thermal conductivity $(0.05 \mathrm{Btu} / \mathrm{hr}-\mathrm{ft} F)$, holds up under radiation, and is readily machinable. The calibration heater was No. 24 Nichrome wire shielded in alumina spaghetti with leads of No. 16 copper transformer wire shielded in fiberglass spaghetti. Three Chromel-Alumel thermocouples were located at Point $A$ and one at Point B. Thermocouples for Calorimeter No. I were made from glass-coated thermocouple wire whereas Calorimeter No. 2 used $1 / 16^{\prime \prime}$ custom made, stainless steel sheathed 

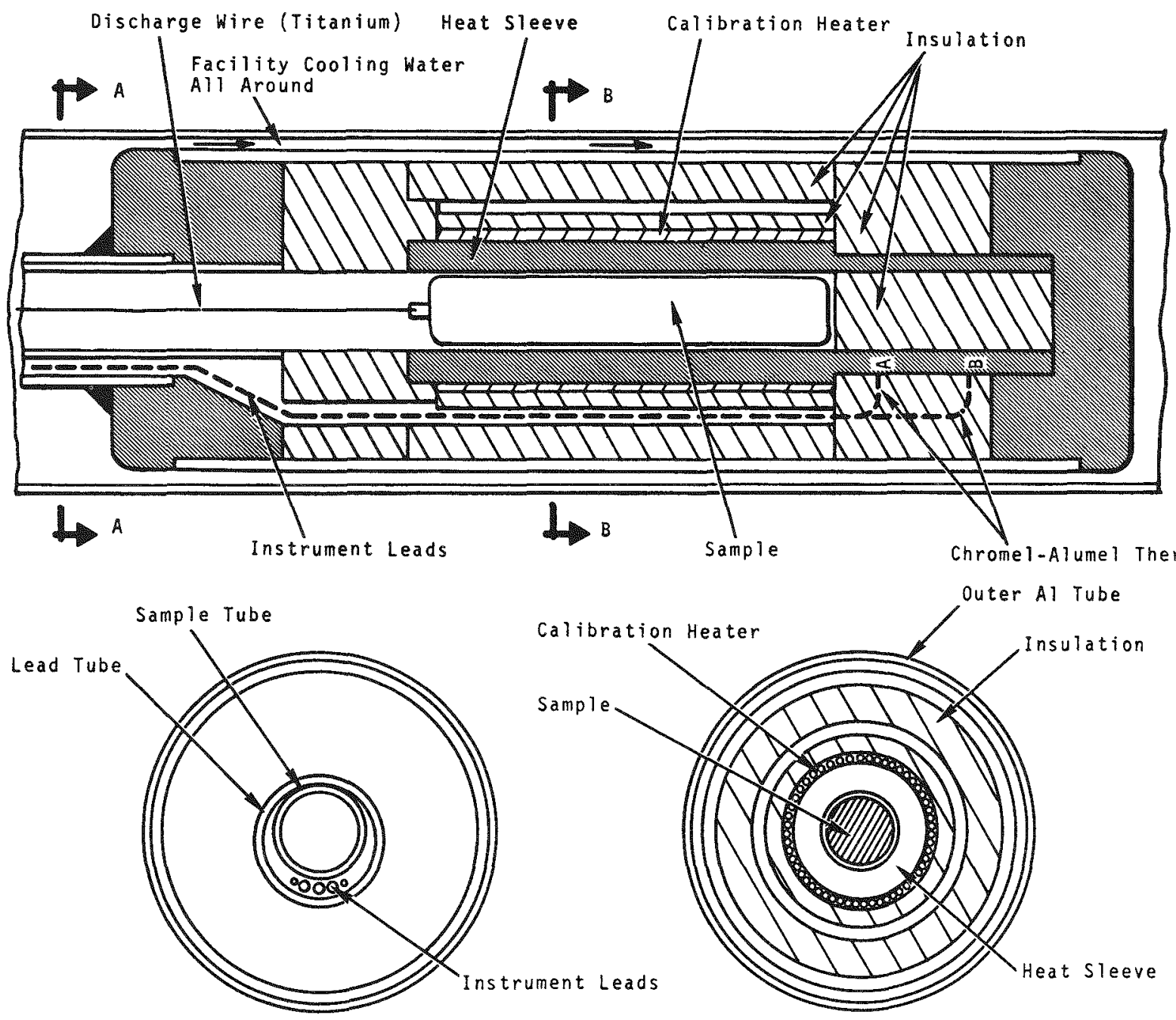

$\operatorname{Sec} A-A$

$\operatorname{Sec} B-B$

Figure 3. Calorimeter No. 2 details. 
couples. The latter were to provide added reliability. A 1/16" helium gas line was provided on Calorimeter No. 2 to reduce oxidation of the inner surface of the heat sleeve and the samples. The samples were attached to the end of a $0.040^{\prime \prime}$ diameter titanium wire which served to push the sample into the calorimeter and to pull it back out following the irradiation. Titanium was used because it has very little residual induced activity, it can withstand high temperatures, and it is relatively stiff.

\section{Instrumentation}

The necessary measurements for this series of tests were (a) the electrical power input to the calibration heaters and (b) the readout from the four thermocouples. The electrical power to the heater was supplied by a Kepco output 0-160 V, 0-4 ampere Model SM-160-4. The power input to the heater circuit was determined by measuring the circuit current, the voltage drop across the heater circuit, and correcting for the resistance of the leads. The current was measured using a precision resistor Honeywell Model 1162, Series 4062, 0.1 ohm @ 3.0 amperes with $\pm 0.04 \%$ accuracy in conjunction with a Fluke Differential DC Voltmeter Model 801B, Series $1697 \pm 0.01 \%$ accuracy. This voltmeter was also used to measure the voltage drop across the system.

Thermocouple e.m.f. values were read using a Leeds and Northrup Model 789563 Milivolt Box with $\pm 0.01 \mathrm{mV}$ accuracy.

\section{Samples}

Sample exterior dimensions were approximately $1.5 \mathrm{~cm}$ OD by $8.9 \mathrm{~cm}$ long. Detailed descriptions of the samples are contained in Table $I$. The materials to be measured were chosen either to satisfy a particular customer request or for a specific physical characteristic. Materials were chosen which would experience little or no thermal neutron absorptions or fast neutron interactions and thus determine the gamma heating contribution. Other materials with a wide range of thermal neutron absorption cross-sections were selected to evaluate this effect. Some of the materials being tested required encapsulation to contain radioactive products or to assure a given geometry. Encapsulation was provided by sealed aluminum containers. A correction was necessary to determine the heat generation of the sample. Samples $1,2,3,4,7$, 8 , and 9 in Table I utilized aluminum containers; samples 5, 6, 10, 11 , and 12 were machined directly from the material.

\section{Heat Transfer Characteristics}

The overall heat transfer from the calorimeter to the facility coolant water yielded a maximum surface heat flux from the capsule of less than $6600 \mathrm{Btu} / \mathrm{hr}-\mathrm{ft}^{2}$. At the normal coolant flow rate and temperature, this would result in a $5^{\circ} \mathrm{F}$ temperature rise across the capsule-water interface. Temperature distributions inside the calorimeter were difficult 
to calculate since heat was transferred radially and longitudinally with several different materials and interfaces in each direction. An existing steady-state heat transfer program (8, p. 1-36) was utilized to calculate the temperatures within the calorimeter for expected heat generation rates. The purposes of the calculations were to predict maximum temperatures for various heat generations and to check the validity of the assumption that a given heat generation rate would produce the same $\Delta T$ regardless of whether the heat were generated in the calibration heater or in the sample.

The deviation between the two assumed positions of heat generation was about 1\%. The inaccuracy of the assumptions which were used as input to the program plus the nonconvergence error in the solution would be expected to be significantly more than the deviation calculated. A limited amount of out-of-reactor data also tended to verify that the deviation was small. The data using the internal heater covered a limited range; however, agreement with the standard calibration heater was within about $3 \%$.

\section{Operational Procedure}

The calorimeters were calibrated outside the reactor before insertion. These calibrations were to check for effects such as gas composition in the device and noncorrespondence between sample and calibration heater induced $\Delta T$ as discussed in the previous section.

Calibration runs were made with the calorimeter in the reactor at the test location with no sample inside. The sample was then charged and the resulting $\Delta T$ was noted. Periodically, additional calibrations were made by superimposing electrical heat on the sample. The samples were attached to a $0.040^{\prime \prime}$ titanium wire which was used to charge and discharge each sample. Each sample was pulled out of the reactor and into a shielded section to allow induced activity to decay. Following the decay period, each sample was disposed of so that the next one could be charged.

\section{RESUITS AND MEASUREMENTS}

\section{Procedure and Measurement Experience}

Table II is a summary of the data obtained from measurements taken in the specially tailored portion of the reactor. The data reported were taken either out of the reactor core or at position $B$ where the environmental conditions have been significantly altered from representative values in the Hanford reactors. 
TABLE I

DESCRIPTION OF SAMPLES

\begin{tabular}{|c|c|c|c|c|}
\hline $\begin{array}{l}\text { Sample } \\
\text { Number }\end{array}$ & Material & $\begin{array}{c}\text { Material } \\
\text { Dimensions } \\
\end{array}$ & $\begin{array}{l}\text { Meterial } \\
\text { Weight } \\
\text { (grams) } \\
\end{array}$ & $\begin{array}{l}\text { Apparent } \\
\text { Density } \\
(g / c c) \\
\end{array}$ \\
\hline 1 & Lead & $\begin{array}{l}\text { Solid Cylinder } \\
1.40 \mathrm{~cm} \text { diam by } \\
\quad 7.3 \mathrm{~cm}\end{array}$ & 131.23 & 11.9 \\
\hline 2 & $\begin{array}{l}\text { Bismuth } \\
\mathrm{Bi}_{3} \mathrm{O}_{2}\end{array}$ & $\begin{array}{c}1.40 \mathrm{~cm} \text { diam by } \\
7.6 \mathrm{~cm} \text { long }\end{array}$ & 46.63 & 4.2 \\
\hline 3 & Niobium & $\begin{array}{l}3-0.635 \mathrm{~cm} \text { OD } \\
\text { by } 5.34 \mathrm{~cm} \\
\text { long rods }\end{array}$ & 59.66 & 8.6 \\
\hline 4 & Molybdenum & $\begin{array}{l}1-0.95 \mathrm{~cm} \text { diam } \\
\text { rod by } 5.1 \mathrm{~cm} \\
\text { long }\end{array}$ & 38.89 & 10.2 \\
\hline 5 & $\begin{array}{l}\text { Stainless } \\
\text { Steel }\end{array}$ & $\begin{array}{c}1.50 \mathrm{~cm} \text { diam by } \\
9.5 \mathrm{~cm} \text { long }\end{array}$ & 132.38 & 8.0 \\
\hline 6 & Titanium & $\begin{array}{c}1.56 \mathrm{~cm} \text { diam by } \\
9.5 \mathrm{~cm} \text { long }\end{array}$ & 81.97 & 4.5 \\
\hline 7 & Wolfram & $\begin{array}{l}7-1 / 8^{\prime \prime} \text { diam } \\
\text { by } 5.3 \mathrm{~cm} \text { long }\end{array}$ & 75.15 & 12.5 \\
\hline 8 & Tentalum & $\begin{array}{l}0.005^{\prime \prime} \text { thick } \\
\text { foil by } 7.1 \mathrm{~cm} \\
\text { Iong }\end{array}$ & 20.38 & 16.6 \\
\hline 9 & $\begin{array}{l}\text { Dysprosium- } \\
\text { Nickel Cermet }\end{array}$ & $\begin{array}{l}1.40 \mathrm{~cm} \text { diam by } \\
7.4 \mathrm{~cm} \text { long rod }\end{array}$ & 98.84 & 7.8 \\
\hline 10 & $\begin{array}{l}\text { Graphite }+ \\
6.7 \% \text { Boron }\end{array}$ & $\begin{array}{c}1.50 \mathrm{~cm} \text { diam by } \\
9.5 \mathrm{~cm} \text { long }\end{array}$ & 27.34 & 1.63 \\
\hline 11 & Aluminum & $\begin{array}{l}1.52 \mathrm{~cm} \text { diam by } \\
9.5 \mathrm{~cm} \text { long - } \\
\text { hollow }\end{array}$ & 14.89 & 2.7 \\
\hline 12 & Graphite & $\begin{array}{c}1.50 \mathrm{~cm} \text { diam by } \\
9.5 \mathrm{~cm} \text { long }\end{array}$ & 29.44 & 1.75 \\
\hline
\end{tabular}


TABLE II. EXPERIMENTAL DATA

\begin{tabular}{|c|c|c|c|c|c|c|}
\hline Run No. & Position & Material & $\begin{array}{c}\text { Electrical } \\
\text { Input } \\
\text { (Watts) }\end{array}$ & $\vec{T}_{1}$ & $T_{2}$ & $\Delta T$ \\
\hline \multicolumn{7}{|c|}{ Calorimeter No. I } \\
\hline 1 & Ex-Reactor & None & 283.0 & 394.0 & 157.3 & 236.7 \\
\hline 2 & Ex-Reactor & None & 194.2 & 304.5 & 130.0 & 174.5 \\
\hline 3 & Ex-Reactor & None & 123.0 & 222.0 & 106.0 & 116.0 \\
\hline 4 & Ex-Reactor & None & 41.9 & 121.0 & 76.0 & 45.0 \\
\hline 5 & $\mathrm{~B}$ & Empty & 0 & 123.8 & 84.0 & 39.8 \\
\hline 6 & $\mathrm{~B}$ & Titanium & 0 & 163.9 & 93.7 & 70.2 \\
\hline 7 & $B$ & Magnesium & 0 & 128.9 & 86.5 & 42.4 \\
\hline 8 & B & Dysprosium & 0 & $259 \cdot 3$ & 123.3 & 136.0 \\
\hline
\end{tabular}

Calorimeter No. II

\begin{tabular}{rllrrrr}
9 & Ex-Reactor & None & 0 & 57.7 & 57.7 & \multicolumn{1}{c}{0} \\
10 & Ex-Reactor & None & 101.33 & 183.4 & 95.5 & 87.9 \\
11 & Ex-Reactor & None & 213.39 & 312.2 & 148.1 & 164.1 \\
12 & B & None & 0 & 131.1 & 86.2 & 44.9 \\
13 & B & None & 99.40 & 247.5 & 128.7 & 118.8 \\
14 & B & None & 208.18 & 368.0 & 173.0 & 195.0 \\
15 & B & Bismuth & 0 & 149.3 & 92.5 & 56.8 \\
16 & B & Bismuth & 97.33 & 264.2 & 136.0 & 128.2 \\
17 & B & Niobium & 0 & 153.3 & 93.7 & 59.6 \\
18 & B & Niobium & 97.17 & 267.5 & Bad & Data \\
19 & B & Niobium & 44.30 & 205.5 & 113.0 & 92.5 \\
20 & B & Lead & 0 & 169.8 & 99.6 & 70.2 \\
21 & B & Lead & 44.35 & 222.0 & 119.4 & 102.6 \\
22 & B & Lead & 0 & 170.5 & 100.1 & 70.4 \\
23 & B & Tantalum & 0 & 156.3 & 95.4 & 60.9 \\
24 & B & Tantalum & 96.72 & 129.7 & 136.3 & 133.4 \\
25 & B & Tantalum & 31.39 & 192.5 & 107.5 & 85.0 \\
26 & B & Aluminum & 0 & 136.9 & 39.1 & 47.8 \\
27 & B & Aluminum & 32.32 & 176.2 & 103.6 & 12.6 \\
28 & B & Aluminum & 97.70 & 253.3 & 130.5 & 122.8 \\
29 & B & Stainless Steel & 0 & 196.5 & 110.8 & 85.7 \\
30 & B & Stainless Steel & 32.51 & 234.5 & 124.7 & 109.8 \\
31 & B & Tungsten & 0 & 203.1 & 112.6 & 90.5 \\
32 & B & Tungsten & 33.05 & 242.7 & 127.5 & 115.2 \\
33 & B & Molybdenum & 0 & 154.9 & 95.4 & 59.5 \\
34 & Ex-Reactor & Molybdenum & 134.02 & 226.0 & 119.1 & 106.9 \\
35 & Ex-Reactor & Molybdenum & 46.01 & 119.3 & 80.0 & 39.3 \\
36 & B & No Sample & 0 & 132.5 & 87.7 & 44.8 \\
37 & B & Graphite + & & & & \\
38 & B & Boron & 0 & 433.1 & 198.0 & 235.1 \\
39 & B & Graphite & 0 & 140.6 & 90.3 & 50.3 \\
& & Graphite & 0 & 139.6 & 90.2 & 49.4
\end{tabular}


Limited data were obtained with Calorimeter No. I because of mechanical failures. The calibration heater failed shortly after the first inreactor data were obtained so that electrical energy could not be superimposed upon the radiation induced heating. The fourth sample tested contained dysprosium-nickel cermet and became stuck in the calorimeter, thus terminating the series of measurements. Calorimeter No. 2 was built to measure additional samples. Design modifications included the use of an aluminum heat sleeve instead of magnesium, better restraints on the heater, and more reliable thermocouples. Calorimeter No. 2 functioned perfectly for the planned series of measurements.

Ex-reactor calibration data were taken to get the general overall temperature differential as a function of energy input. The $\Delta T$ was measured with the calorimeter at the reference in-reactor position with no sample charged. All energy deposition measurements for samples and electrical calibration were superimposed on and referenced to this value. It was assumed that reactor conditions and calorimeter physical characteristics remained constant for the test series.

Run No. I was the reference run for Calorimeter No. 1, with Runs No. 12 and 36 reference runs for Calorimeter No. 2. In Table II, $\vec{T}_{1}$ is the average reading of the three thermocouples located at that position; $\mathrm{T}_{2}$ is the temperature measured by the single thermocouple located at the "downstream" position. The column labeled " $\Delta \mathrm{T}$ " is the difference between $\bar{T}_{1}$ and $T_{2}$. The energy deposition by a particular sample was arrived at by subtracting the $\Delta T$ produced in the reference case from that measured for the sample. The resulting $\Delta T$ was divided by a calibration value to yield total heat added. A correction was then made if the sample was enclosed in an aluminum container. Results are listed in Table III.

\section{Accuracy of Measurements}

Electrical energy input rates were measured with instrumentation described in Section 2.4 which have an estimated overall accuracy of $\pm 1 \%$. Temperature measurements were accurate to $\pm 2 \%$ on absolute basis; however, relative values or changes in temperature were measurable to better than $\pm 1 \%$.

Good data reproducibility was demonstrated on Runs 20 and 22 where the agreement was better than $0.5 \%$. These measurements were taken about one hour apart with some calorineter movement involved between readings. This agreement is better than would normally be expected since reactor environmental changes could well be several percent. This overall accuracy of heat generation rates measuned was estimated to be $\pm 10 \%$ for Calorimeter No. 2 and $\pm 20 \%$ for Calrolmeter No. 1 . Because of the very low heat generation rate of magnesium, measurement error could be much greater. 
TABLE III

EXPERIMENTAL HEAT GENERATION RATES

Material

Lead
Bismuth
Niobium
Aluminum
Molybdenum
Stainless Steel
Titanium
Wolfram

Tantalum

Dysprosium-Nickel Cermet

Graphite $+6.7 \%$ Boron

\begin{tabular}{|c|c|c|c|}
\hline $\begin{array}{c}\bar{T}_{1} \\
\left(\bar{o}_{F}\right) \\
\end{array}$ & $\begin{array}{c}\Delta \mathrm{T} \\
\left({ }^{\circ} \mathrm{F}\right) \\
\end{array}$ & $\begin{array}{c}\text { Calibration } \\
\text { Factor } \\
\left({ }^{\circ} \mathrm{F} \mathrm{W} \mathrm{W}^{-1}\right) \\
\end{array}$ & $\begin{array}{l}\text { Total Heat } \\
\text { Generation } \\
\text { (W) }\end{array}$ \\
\hline 169.8 & 25.3 & 0.760 & 33.3 \\
\hline 149.3 & 11.9 & 0.765 & 15.6 \\
\hline 153.3 & 14.7 & 0.765 & 19.2 \\
\hline 136.9 & 2.9 & 0.770 & 3.8 \\
\hline 154.9 & 14.6 & 0.764 & 19.1 \\
\hline 196.5 & 40.8 & 0.755 & 54.0 \\
\hline 163.9 & 30.4 & 0.790 & 39.0 \\
\hline 203.1 & 45.6 & 0.752 & 60.7 \\
\hline 156.3 & 16.0 & 0.764 & 20.9 \\
\hline 259.3 & 136.0 & 0.760 & 179.2 \\
\hline 433.1 & 190.2 & 0.70 & 272.0 \\
\hline
\end{tabular}

Sample Heat

Generation (W)

29.5

11.8

15.4

3.8

15.3

54.0

39.0

56.9

17.1

175.4

272.0
Heat Generation

Sample

Weight

(g)

$\left(W-g^{-1}\right)$

$131.23 \quad 0.225$

$46.63 \quad 0.253$

$59.66 \quad 0.258$

$14.89 \quad 0.255$

$38.89 \quad 0.393$

$132.38 \quad 0.408$

$81.87 \quad 0.476$

$75.15 \quad 0.757$

$20.38 \quad 0.840$

$98.84 \quad 1.77$

$29.34 \quad 9.96$ 
Reactor neutron and gamma flux were not measured in this environment but were estimated from other measurements. The estimated thermal neutron flux is probably within $\pm 15 \%$ of the true value. The gamma flux or spectrum were calculated from basic data. However, energy deposition measurements have terded to verify these calculations for other reactor locations.

\section{INTERPRETATION OF MEASUREMENTS}

\section{Energy Deposition Rates}

By using the calibration data and the measured $\Delta T$ for each sample, a total energy deposition rate for each sample was calculated. Samples which utilized aluminum encapsulation required a correction to account for the contribution from the container. Run No. 26 evaluated the contribution of the container. The energy deposition rate of the sample material was then divided by its weight to arrive at the units normally quoted in practice. Table IV lists the experimental values of total energy deposition rate.

\section{Garma Contribution}

Essentially all of the energy deposition rates measured for bismuth and lead were from gamma interactions as discussed previously. The gamma contributions in all other samples was inferred by reference to these representative values. Two factors were used to calculate gamma heat generation rates relative to bismuth for all samples. The first factor was arrived at by assuming energy deposition rate in a material due to a given incident gamma radiation to be proportional to its energy absorption mass attenuation coefficient $(\mu / \rho)$. This factor was weighted according to the calculated incident gamma spectrum. The second factor was used to account for the fact that significant gamma flux depressions occurred in several of the samples. The gamma heating contribution for each material was found by normalizing the product of these factors for each material to that for bismuth. Table IV lists the total measured heat generation rates, the inferred gamma contribution, and the resulting neutron contribution.

Thermal Neutron Contribution

The thermal neutron contribution to the total heating effect was found experimentally by subtracting the gamma contribution from the total. The theoretical heating due to thermal neutrons was calculated by determining the thermal neutron interaction rate, considering the energy given up per absorption, and then finding the portion of the energy release which was absorbed in the sample and heat sleeve. 
TABLE IV

HEAT GENERATION RATES AND CONTRIBUTORS

Experimental Heat Generation

\begin{tabular}{|c|c|c|}
\hline $\begin{array}{l}\text { Total } \\
\text { Measured } \\
\left(\mathrm{W} \mathrm{g}^{-1}\right) \\
\end{array}$ & $\begin{array}{c}\text { Gamms } \\
\left(\mathrm{W} \mathrm{g}^{-1}\right) \\
\end{array}$ & $\begin{array}{l}\text { Thermal } \\
\text { Neutron } \\
\left(\mathrm{W} \mathrm{g}^{-1}\right) \\
\end{array}$ \\
\hline 0.225 & 0.225 & 0 \\
\hline 0.253 & 0.253 & 0 \\
\hline 0.258 & 0.182 & 0.076 \\
\hline 0.393 & 0.187 & 0.206 \\
\hline 0.408 & 0.170 & 0.238 \\
\hline 0.476 & 0.162 & 0.314 \\
\hline 0.757 & 0.239 & 0.518 \\
\hline 0.840 & 0.245 & 0.595 \\
\hline 1.77 & 0.175 & 1.60 \\
\hline 9.96 & 0.174 & 9.78 \\
\hline 0.255 & 0.173 & 0.082 \\
\hline 0.174 & 0.174 & 0 \\
\hline 0.10 & 0.10 & 0 \\
\hline
\end{tabular}

Magnesium

Graphite +

$6.7 \%$ Boron

Aluminum

0.10

0.10 


\section{BIBLIOGRAPHY}

1. Anderson, A. R. and J. R. Waite. The calorimetric measurement of energy absorbed from reactor radiation in BEPO. $1960.76 \mathrm{p}$. (Atomic Energy Research Establishment C/R 2253).

2. Argonne National Laboratory. Reactor physics constants. United States Atomic Energy Commission, 1963. 527 p. (ANL 5800)

3. Blizzard, E. P. and Lorraine S. Abbott (eds). Reactor handbook, vol. 3, part B, shielding. 2nd ed. New York, Interscience, 1962. $283 \mathrm{p}$.

4. Boyd, A. W. Atomic Energy of Canada Limited. Personal communication. Chalk River, Ontario. June 14, 1968.

5. Engle, R. I., J. Greenborg and M. M. Hendrickson. ISOSHLD - A computer code for general purpose isotope shielding analysis. June 1966. 143 p. (BNWL-236).

6. Etherington, Harold (ed). Nuclear engineering handbook. New York, McGraw-Hill Book Company, Inc. 1958.

7. Mollerus, F. J., Jr. Users manual for STHTP - A steady-state heat transfer program for the 7090 computer. 1962.36 p. (Atomic Energy Commission HW-73668).

8. Primak, W. Gamma-ray dosage in inhomogeneous nuclear reactors. Journal of Applied Physics 27:54-62. 1956. 
W "RECENT ADVANCES IN FAST-RESPONSE MTNIATURE NEUTRON FLUX MONITORS

by

D. B. STILLMAN and R. L. CHANEY

ABSTRACT

$480 \quad 800$

A fast response miniature neutron detector has been developed at the Los Alamos jcientific Laboratory (IA.jL) to measure temperature-time history of fissile materials under intense neutron irradiation. The fissile-sensing element of the fission couple neutron detector is approximately $0.030 \mathrm{in}$. in diameter. The detector sensitivity for thermal neutrons is approximately $10^{9} \mathrm{n} / \mathrm{cm}^{2}-{ }^{\circ} \mathrm{C}$. To determine any limitations in response time, fission couples were placed $3 \mathrm{~m}$ from a nuclear device in an underground nuclear weapons test at the Nevada Test Site. The detector accurately followed the change in neutron population (alpha measurement) as the device exploded. This rapid change in neutron population had an e-folding time of 20 nsec.

A number of experiments were performed in various reactors so that such a detector could be developed. These experiments were conducted in a particular sequence and were aimed at investigating the characteristics, possible uses, and limitations of the fission couple. Jome experimental results are discussed.

The significance of the results obtained with fission couples suggests numerous applications in neutron diagnostics and reactor controls. The most obvious application for this instrument would be mapping of the neutron flux in and around a reactor. Other possible applications are also discussed. The miniature size and fast response of a fission couple combine to offer unique advantages as a new tool. in reactor diagnostics and control.

\section{INTRODUCTION}

Numerous neutron detectors are available with wide ranges of sensitivities and sizes. The fission couple $e^{l}$ neutron detector effort has been primarily directed toward development of miniature instruments capable of detecting neutrons in the presence of strong gamma radiation. In addition, the detector should be accurate and respond rapidy to changes in neutron flux. The fission couple development has accomplished this goal.

The fissile-sensing element of the fission couple is approximately $0.030 \mathrm{in.}$ in diameter. The sensitivity of the detector in a thermal neutron flux is about $10^{9} \mathrm{n} / \mathrm{cm}^{2}-{ }^{\circ} \mathrm{C}$. To determine any limitations in response time, fission couples were placed $3 \mathrm{~m}$ from a nuclear device in an underground nuclear weapons test at the Nevada Test Site. The detector accurately followed the change in neutron population (alpha measurement) as the device exploded. This rapid change in neutron population had an e-folding time of 20 nsec.

II. THEORY

The fission couple technique arose with the requirement to measure the temperature-time history of fissile materials under intense pulsed neutron irradiation. The first approach to this problem involved welding a thermocouple junction directly to the fissile materiel as shown in Fig. 1. This technique had inherent time-response problems. The desired measurement was the temperature-time history of the fissile material, but the actual measurement was the temperature-time history of the junction of the thermocouple. This configuration produced delays in time for heat to flow from the fissile material 


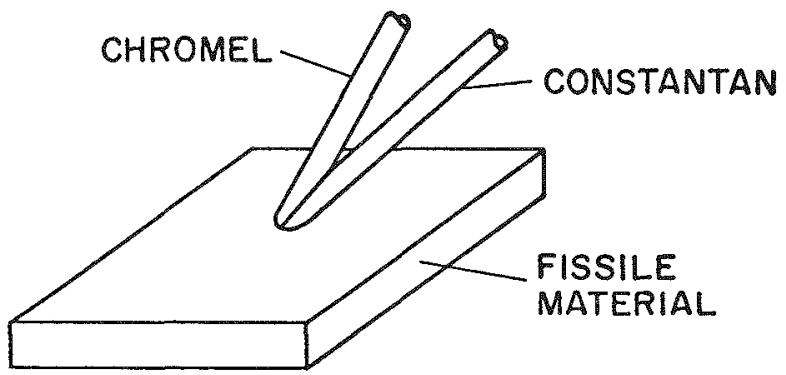

Fig. 1. Conventional thermocouple junction. into the thermocouple junction. Another shortcoming was that the observed measurement also included noise signals inherent in neutron shock-heated fissile material. Thus, a slow-response temperature measurement could be made, although it included other unwanted signels.

The second approach to the problem has come to be known as the intrinsic thermocouple (Fig. 2). Thermocouple wires were individually welded to the fissile material so that a $0.060-i n$. separation existed between each thermocouple wire. This effectively made the fissile material the junction of the thermocouple. The time response was improved over the technique shown in Fig. 1 , but the noise signals from thermal shocks were still present.

These noise signals could be eliminated if the fissile junction of the thermocouple were mechanically decoupled from the rest of the fissile material. The temperature of the fissile material would still be the same due to its location in the neutron flux. This concept evolved into the third approach to the problem, and the resultant detector was labeled a fissicn couple.

Conventional thermocouples indicate only the temperature of the hot junction. Unfortunately, this is not necessarily the same temperature as that of the adjacent fissile material, particularly

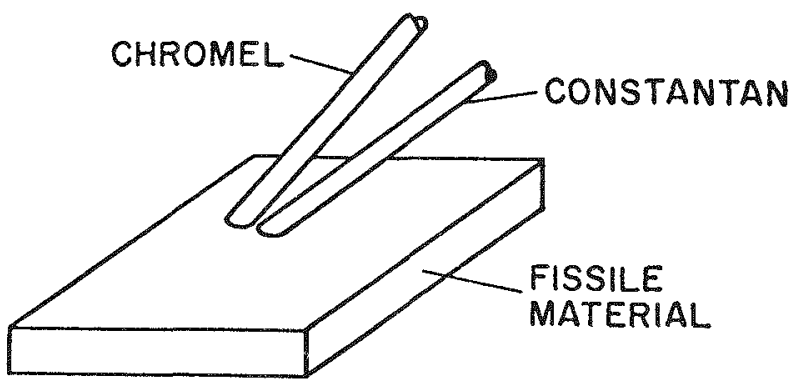

Fig. 2. Intrinsic thermocouple junction. if the temperature of the fissile material is changing rapidly. To attain an accurate temperature reading, time must be allowed for the body to reach thermal equilibrium and that equilibrium must be maintained. Therefore, a lag time in response to the actual temperature-time history of the fissile material must be expected when using the conventional configuration of Fig. $3(a)$.

The outstanding advantage of the fission couple configuration of Fig. $3(\mathrm{~b})$ is that the material of interest is the thermocouple junction. Heat is now actually generated within and flows away from, rather than into, the junction. This situation implies that the more rapid the temperature change in the fissile junction, the more accurate is the measurement.

With this measurement technique, the most obvious error is due to heat losses from the sensing junction. The two mechanisms for heat loss are thermal radiation from the bead, and heat conduction from the bead to the lead wires. If the efficiency of uranium to radiate as a blackbody is $30 \%$, then the temperature loss in $5 \mathrm{msec}$ for an initial temperature rise of $727^{\circ} \mathrm{C}$ in a 0.016 -in. diam ${ }^{235} \mathrm{U}$ bead is approximately $0.4^{\circ} \mathrm{C}$. Heat loss from a 0.016-in.-diam uranium bead along two lead wires (Chromel-Alumel) of 1/2-mil diam was calculated. For an initial bead temperature rise of $700^{\circ} \mathrm{C}$, the temperature drop in $5 \mathrm{msec}$ was approximately $0.1^{\circ} \mathrm{C}$. Therefore, both mechanisms of heat loss appear to be negligible in these time scales. In fact, the higher the rate of energy deposition in the bead, the lower the error in the measurement. Thus, the fission couple output is more accurate as the energy input approaches a step function.

The fission couple gives a direct measurement of the integrated fission density existing at the

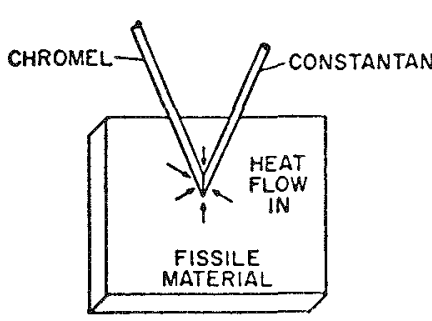

(a)

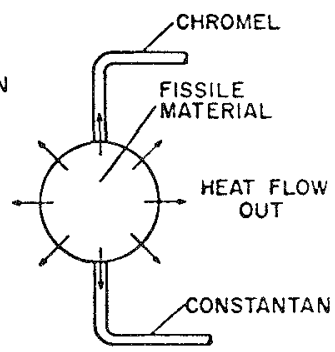

(b)
Fig. 3. Comparison of conventional with fission couple junction. 
location of the junction. Neutrons entering the fissile bead cause fissions within the bead. The resultant fission framents deposit their energy within the bead, causing an increase in bead temperature. Since the bead is the junction of the thermocouple, thermolectric voltage output, due to temperature increase, is proportional to the integrated neutron flux. Thus, the fission couple can be placed at strategic locations within or around a reactor to give a direct reading of reactor power.

\section{EXPERIMENIAL RESULTS}

To carry out a meaningful and useful program in the developnent of eission couples, a number of experiments were performed in different reactors. The aim of these experiments was to investigate the characteristies and the possible uses of the fission couple. These experiments included studying bead size restrictions, conductive heat losses, improvement of packaging techniques, therropile configuretions, gamma heating in various materials, and fission couple performance in steady-state radiation environments.

Most of the experiments were conducted at the Super KUKIA Prompt Burst Reactor ${ }^{2}$ located at the Nevada Test Site. Other reactors used were the jandia Pulsed Reactor $\mathrm{II}^{3}$ in Albuquerque, N.M., the FRAN Prompt Burst Reactor ${ }^{4}$ at the Nevada Test Site, the White Sands Missile Range Fast Burst Reactor (Molly-G) the SNAPTRAN-2 Reactor at the National Reactor Test jite in Idaho, and the Omega West Reactor at IASI. Fission couples were also used in nuclear weapon environments to successfully monitor the weapon alpha vs time.

A. Bead size study

The uniform heating of the fissile sensing element is of critical importance. This requirement places a restriction on the dimensions of the fissile junction. The correct size for the fissile bead is a function of the bead material and the neutron energy spectrum to be measured. The minimum dimensions appear to be limited by the fission fragment range in the material, while the maximum dimension is limited by self-shielding in the material which will produce nonumiform heating of the junction.

A number of uranium beads fully enriched in
${ }^{235} \mathrm{U}$, ranging in diameter from 0.010 to $0.07 \%$ in. were instrumented as fission couples using Chromel and Alumel thermocouple wires. Chromel-Alumel thermocouple lead wires, $1 / 2 \mathrm{mil}$ in dianeter, were welded to beads of diameters less than $0.030 \mathrm{in}$. while 1-mil-diam Chromel-Alumel thermocouple lead wires were welded to beads of diameters greater than $0.030 \mathrm{in.}$ The various sized fission couples were tested in three neutron energy spectra.

(1) Normal neutron leakage spectrum from the Super KUKIA Prompt Burst Reactor (average neutron energy approximately $0.7 \mathrm{MeV}$ ).

(2) Normal neutron leakage spectrum from the Super KUKIA Prompt Burst Reactor excluding neutron energies below $0.4 \mathrm{eV}$.

(3) Thermal neutrons only.

Since the beads were not perfectly spherical, the resultant bead temperature rises were plotted as a function of an equivalent spherical diameter. This data is displayed in Figs. 4, 5, and 6 .

The conclusion from this experiment indicated the range of ${ }^{205} U$ bead diameters that should be used within ranges of incident neutron energy spectra. For the neutron energy spectrum in case (I) above, the optimum bead diameter was found to be $0.015 \mathrm{in.;}$ for case (2), 0.020 to 0.040-in.-diam beals shoula be used. In case (3), the fissile bead diameter is critical when subjected to a flux of thermal neutrons only (Fig. 6). This strong dependence of the temperature rise as a function of the bead diameter in a thermal neutron flux is interpreted as follows. The temperature measurement seen by the fission couple technique is the average of the temperature across the bead from point I to point 2 as shown in Fig. 7. In a thermal neutron filux, self-shielding becomes important in choosing the size of the bead diameter. If self-shielding occurs, as it would in Fig. $7(a)$, then the bead temperature would not be in equilibrium. The outer spherical shell would be at a higher temperature, $\mathrm{T}_{1}$, than the inside ball tem perature, $\mathrm{T}_{2}$. The fission couple voltage output would then be due to the average temperature across the bead and then would correspond to temperature, $\mathrm{T}_{3}$, so that $\mathrm{T}_{2}>\mathrm{T}_{3}>\mathrm{T}_{2}$.

In Fig. 7(b), self-shielding would not occur across the smaller diameter bead and the fission couple voltage output would correspond to the uniform temperature, $T_{1}$. Thus, the fission couple 


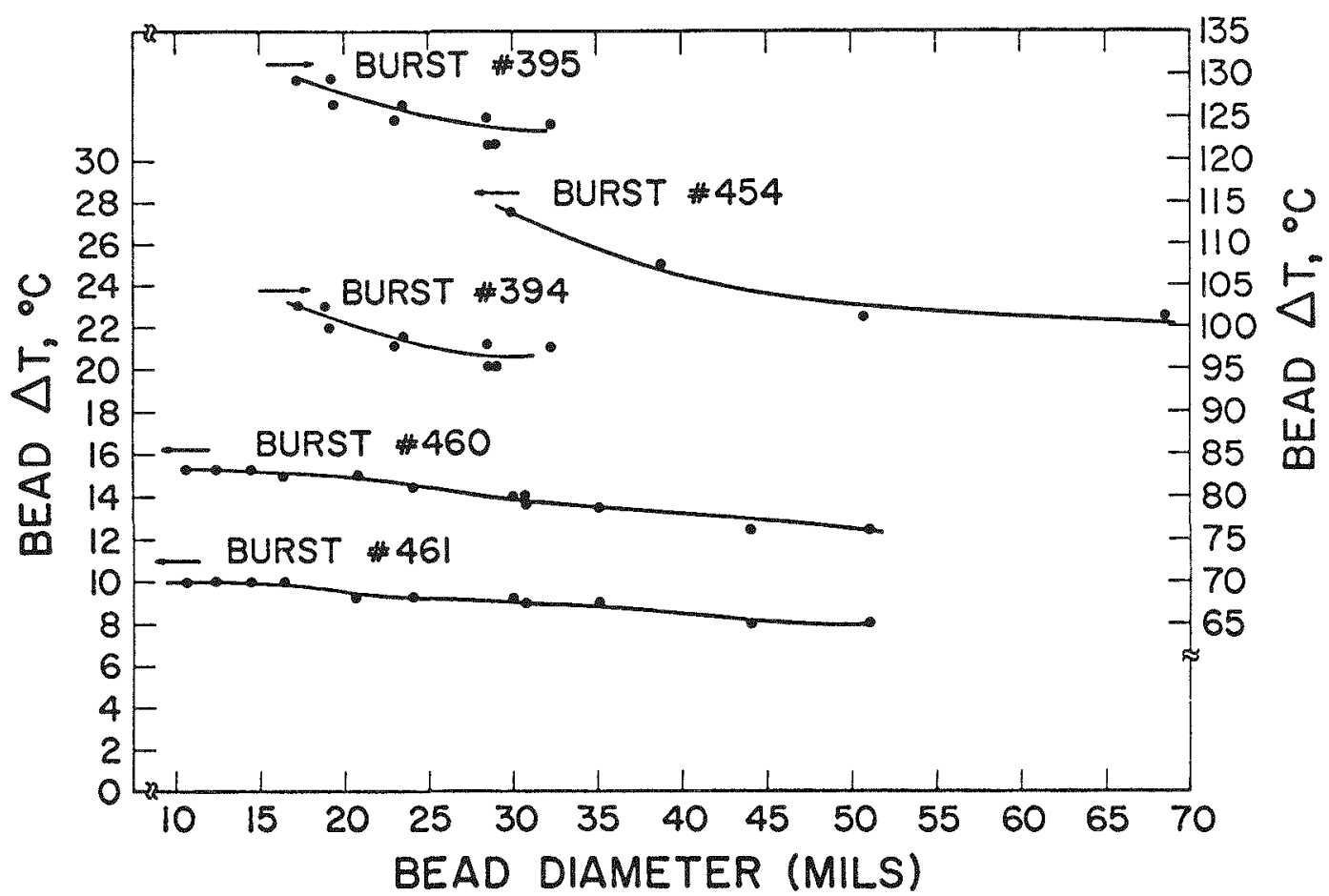

Fig. 4. ${ }^{235} \mathrm{U}$ bead $\mathrm{TP}$ vs bead diameter in the normal neutron leakage spectrum from super KUKIA Reactor (Case 1).

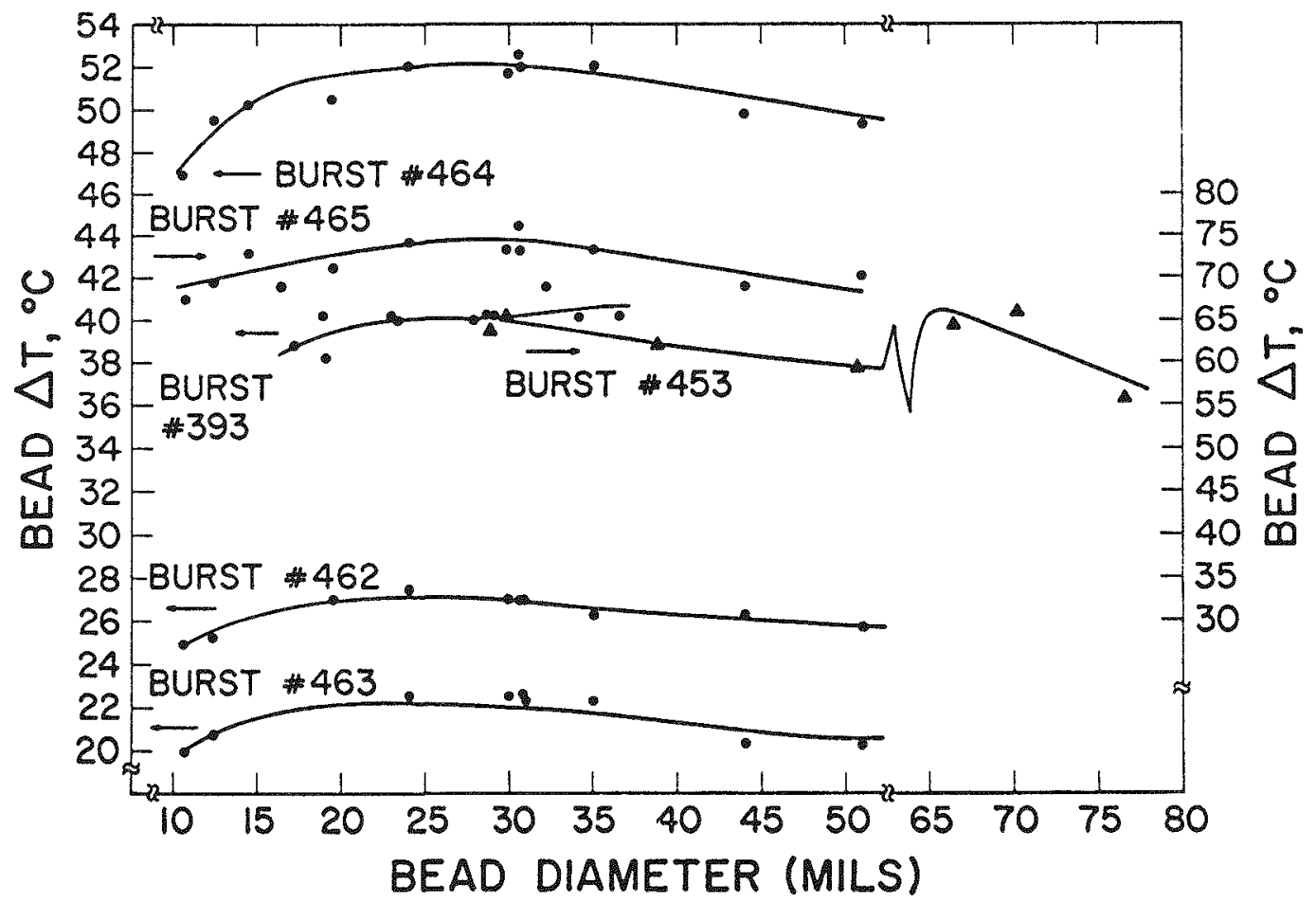

Fig. 5. ${ }^{235} \mathrm{U}$ bead $\Delta T$ vs bead diameter in the normal neutron leakage spectrum from the Super KUKLA Reactor (excluding neutron energies $<0.4 \mathrm{ev}$ ) (Case 2). 


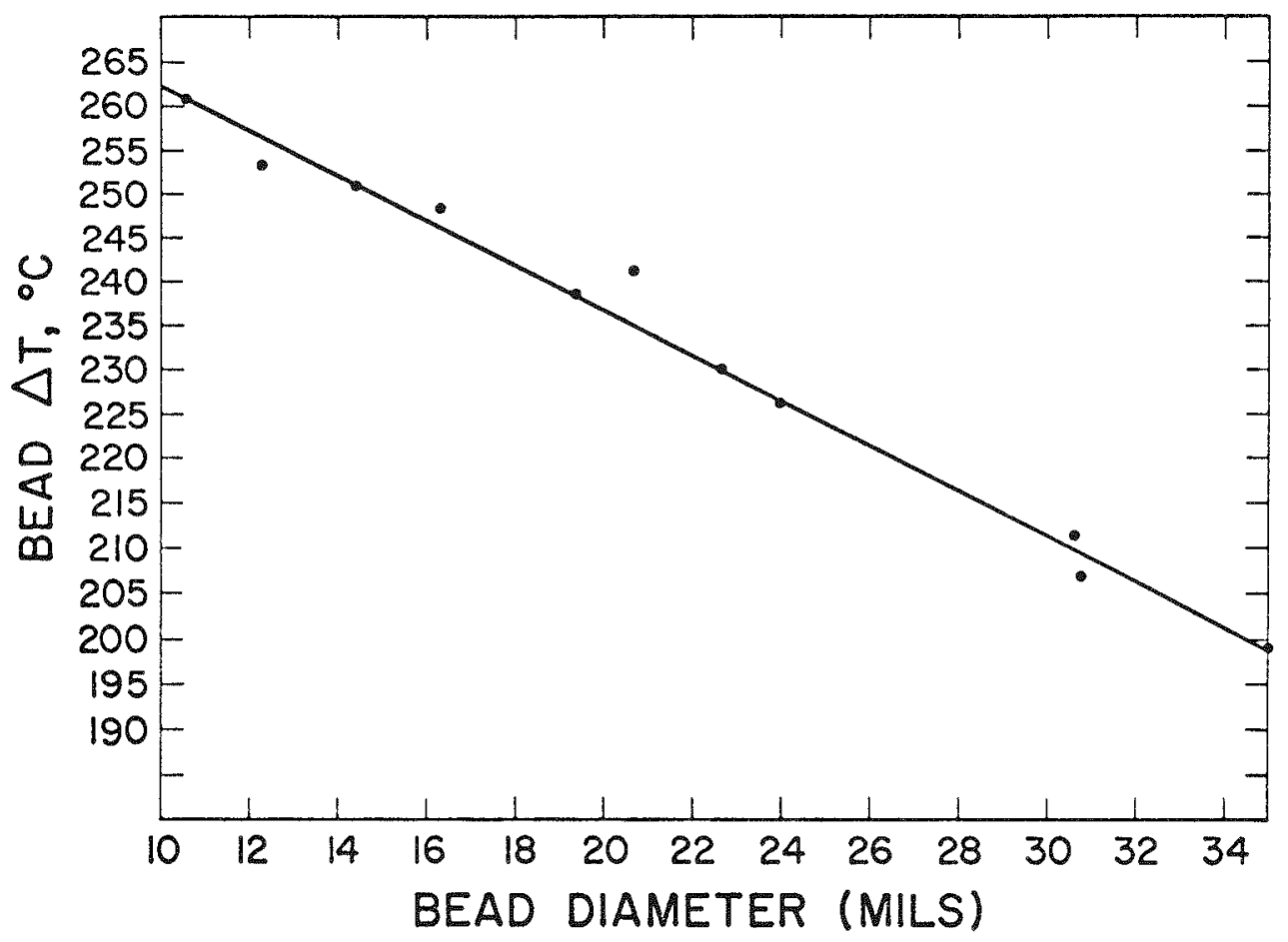

Fig. 6. ${ }^{23 b} \mathrm{U}$ bead $\Delta \mathrm{T}$ vs bead diameter in a thermal neutron environment (Case 3 ).

in Fig. $7(b)$ would show a higher voltage output than the fission couple in Fig. $T(a)$ if both fission couples were in the same thermal neutron flux. The experimental data in Fig. 6 shows the temperature dependence on the bead diameter as $2.5^{\circ} \mathrm{C} / 0.001 \mathrm{in}$. B. Conductive Heat Losses

Once the optimum bead size is determined, the conductive heat losses down the attached thermocouple lead wires can be investigated. The consideration is to keep the diameter of the thermocouple lead wires to a minimum while mantaining adequate mechanical strength of the junction at the bead and lead wires. This junction had previously been strengthened by the use of a plastic cement on the junction. However, experiments have shown that this material acts as a large heat sink (Fig. 8). strengthening the fission couple junction without a resultant heat sink effect remained a problem.

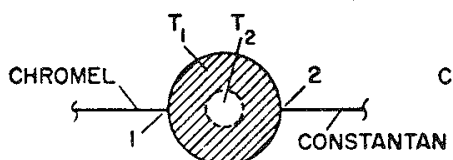

(a)

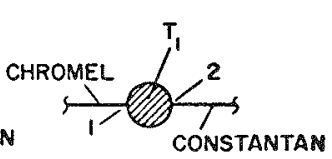

(b)

30-MIL-DIAM BEAD

10-MIL-DIAM BEAD

Fig. 7. jelf-shielding in a thermal neutron flux.

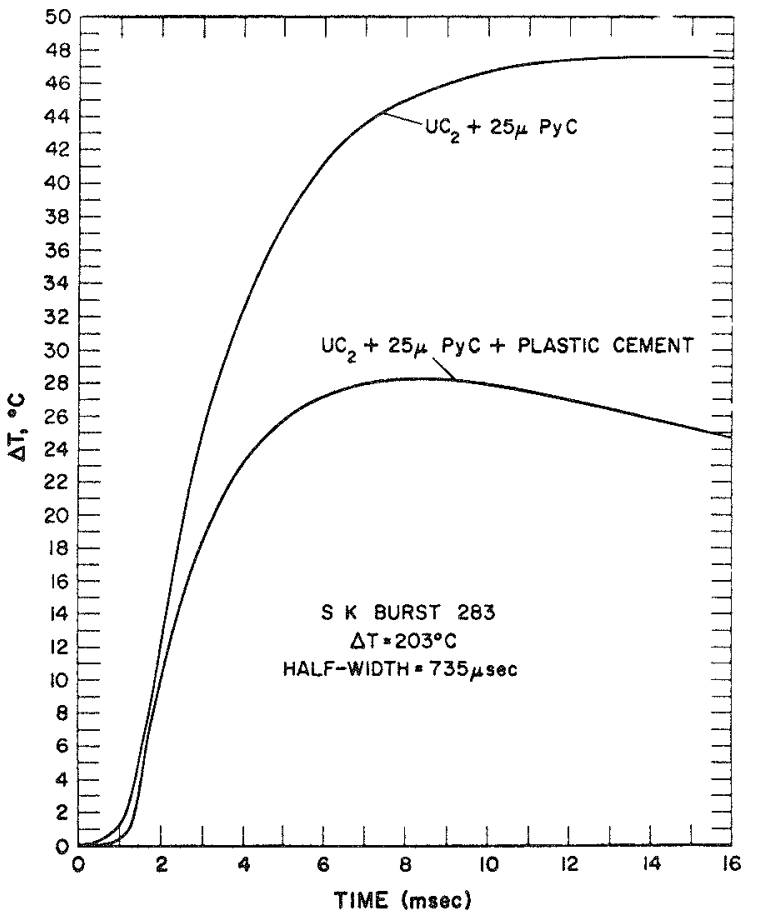

Fig. 3. Comparison of the effect of plastic cement. 
As the size of the bead is increased, the diamete. of the thermocouple lesd wire may also be increased without enlarging the relative heat sink. There is a practical limit to the maximum diameter for these lead wires, therefore an experiment was designed to determine this upper limit.

Chromel and Alumel thermocouple lead wires of $1 / 2,1,2,3$, and 5 mils diam were welded to their respective ${ }^{235_{U}}$ beads. All bead masses were approximately the same. The maximum diameter for the thermocouple lead wires was determined by comparing the relative temperature levels and the relative cooling rates of fission couples having various wire diameters. Figure 9 compares the response of five fission couples of essentially equal bead mass during a Super KUKIA reactor burst. There appeared to be no difference in the response and the temperature level reached for the 1/2-mil and I-mil-diam thermocouple lead wires. The 5-mil-diam lead wires definitely presented excessive heat transfer area to the pissile bead. The 2-mil and 3-mil-diam lead wires also exhibited excessive heat loss.

The heat conduction down the thermocouple lead wires is, in part, a function of the square of the wire diameter. Since there was no apparent difference in response and temperature level for the
$1 / 2-m 1 I$ and 1 -mil-diam lead wire, it appeared feasible to build fission couples using four $1 / 2$ mil-diam thermocouple lead wires (Fig. 10). An experiment showed that this structurally strengthened the fission couple junction as well as increased reliability in electrical continuity.

TABLE I

RELATION OF ${ }^{235} \mathrm{U}$ BEAD MASSES WITH LEAD WIRE DIAMETERS

\begin{tabular}{ll}
$\begin{array}{c}\text { Bead Mass } \\
\text { (mg) }\end{array}$ & $\begin{array}{c}\text { Thermocouple Lead } \\
\text { Wire Diameter } \\
\text { (mils) }\end{array}$ \\
\hline 3.83 & $1 / 2$ \\
3.84 & 1 \\
3.97 & 2 \\
4.08 & $1 / 2$ (four wires) \\
4.17 & 1 (four wires) \\
3.38 & $1 / 2$ \\
3.56 & 1 \\
5.24 & 2 \\
3.78 & $1 / 2$ (four wires) \\
5.39 & 1 (four wires)
\end{tabular}

Table I lists the ${ }^{235} \mathrm{U}$ bead masses which were welded to indicated diameters of Chromel-Alumel lead wire and were subjected to four reactor bursts. A plot of bead temperature vs time for one of the reactor bursts is shown for the various fission

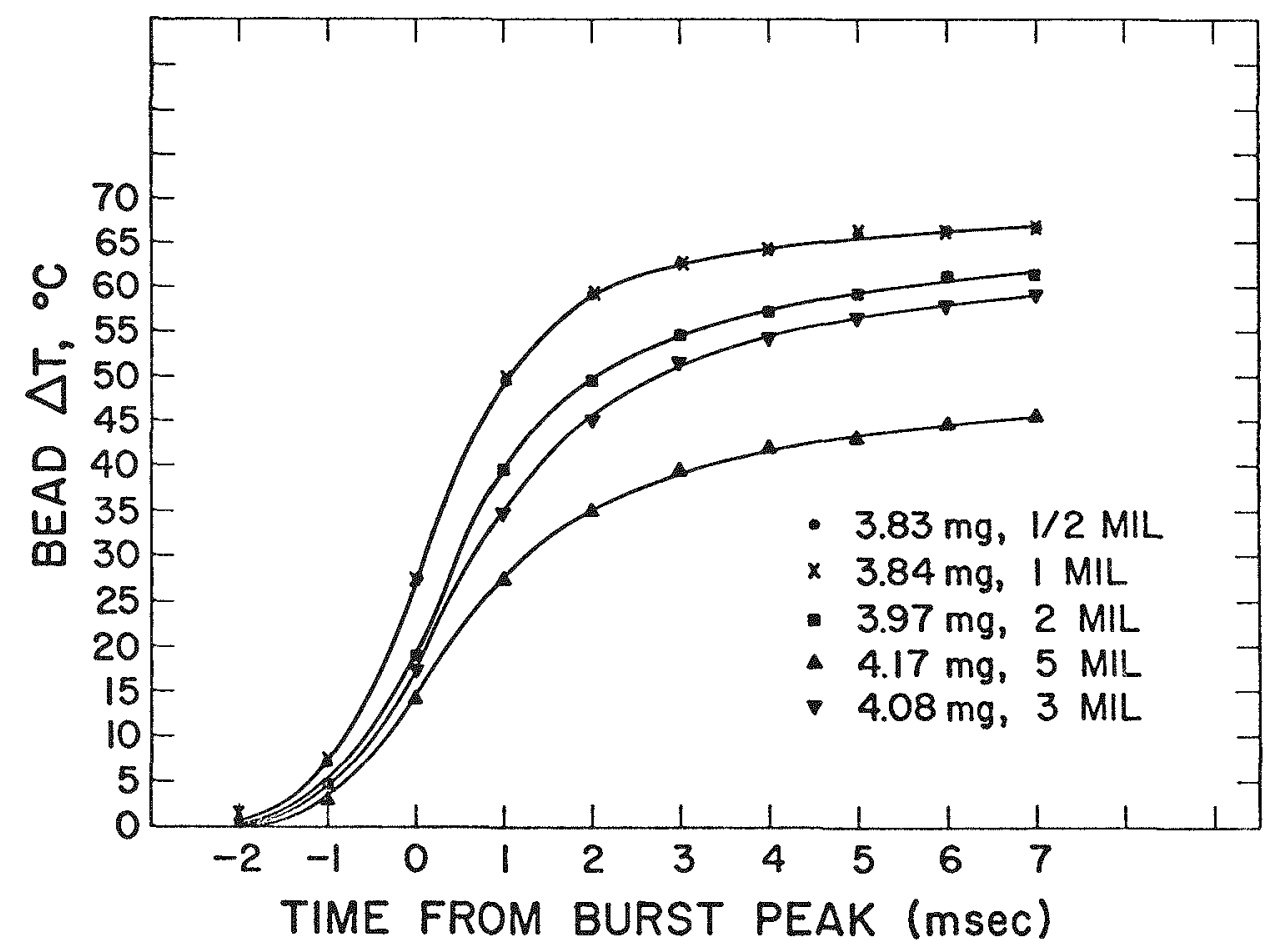

Fig. 9. ${ }^{235} \mathrm{U}$ beads of equal mass with varlous sized lead wires. 


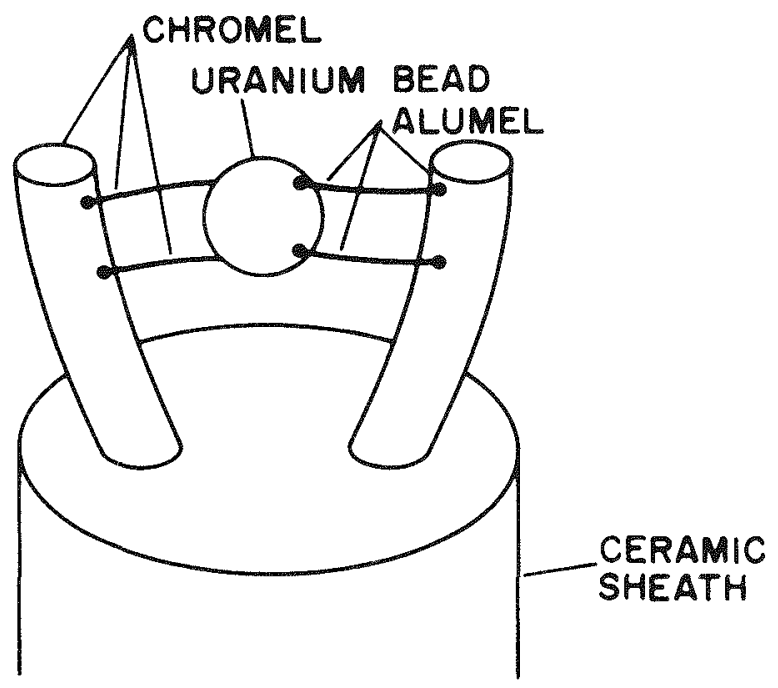

Fig. 10, Fission couple with four lead wires. couples in Figs. 11 and 12. As shown in Fig. 11, the two $1 / 2 \mathrm{mil}$ diam lead wires gave the best response in time and amplitude. The two 1 -mil and four 1/2-mil-diam lead wires were nearly identical in output. Their amplitude response, compared with the two 1/2-mil-diam lead wires, amounted to an error of only about $3 \%$.

Figure 12 shows that the two $1 / 2-m i l$-diam lead wires on a 3.381 -mg bead, and the four 1/2-mil-diam lead wires on a 3.775 mg bead showed identical outputs. The two 1-mil-diam lead wires, on 23.563 mg bead, and the four 1-mil-diam lead wires, on a $5.392-\mathrm{mg}$ bead, also showed identical outputs. The error between the outpur of the fission couple with

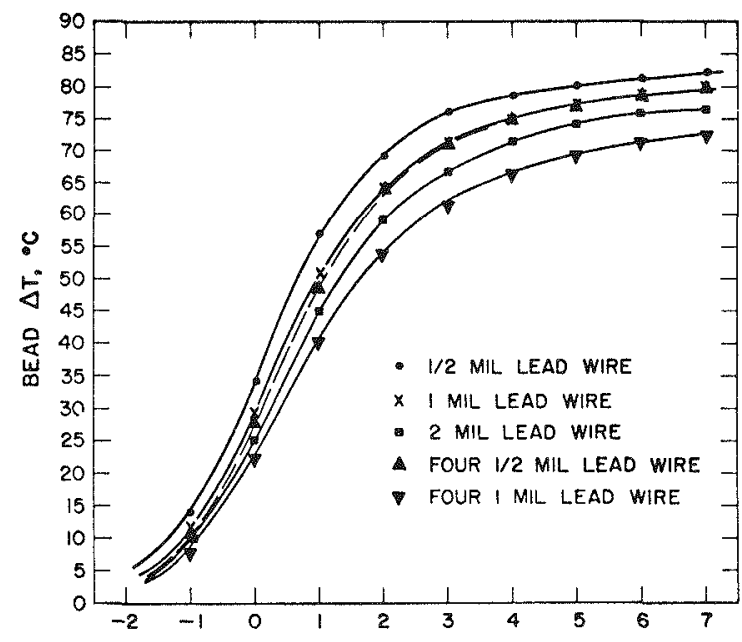

DIVISIONS FROM PEAK OF BURST (0.78125 msec/DIV)

Fig. 11. ${ }^{235} \mathrm{U}$ beads of $4 \mathrm{mg}$ with various sized lead wires.

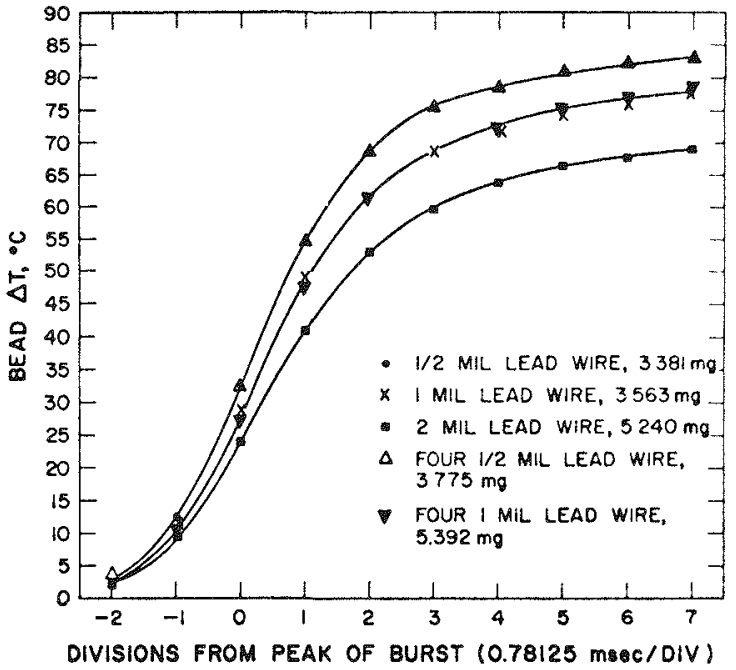

Fig. 12. ${ }^{235} \mathrm{U}$ beads with various sized lead wires.

1-mil-diam lead wires and that with $1 / 2 \mathrm{mil}$ diam

lead wires was only about $6 \%$.

The conclusion from these experiments is that, for bead diameters of $30 \mathrm{mils}$ or greater, two $1 \mathrm{mil}$ diam lead wires or four $1 / 2$ mil-diam lead wires can be used without appreciable error. Using four $1 / 2$ mil-diam lead wires would be the obvious choice since the fission couple junction would be physically stronger and thus more reliable. One wire (or one Chromel and one Alumel lead wire) could break and the electrical continuity would st 11 be maintained. c. Improvement of Packaging Techniques

It was a challenge to maise the itssion couplo unit into a rugged package without greatly increasinf its small size. Methods used to strengthen the package also had to be checked for heat sink effects. The small size of the fission couple is especially attractive because it facilitates the desired neutron flux measurement with minimum perturbation of the flux field.

Using four lead wires greatly improved the strength and reliability of the fission couple and evolved to another method of holding the fisilile bead (Fig. 13). The small lead wires show in Fir. 13 are $1 / 2$ mil-diam Chromel-Alumel welded to their respective larger lead wires. This technique has been tried and the response of such a fission couple is compared with fission couples of comparable mass with two 1/2-mil and two l-mil-diam lead wires (Fig. 14). Thus, the fission couple junction has been strengthened, with an increase in reliability, with no appreciable degradation in response time or 

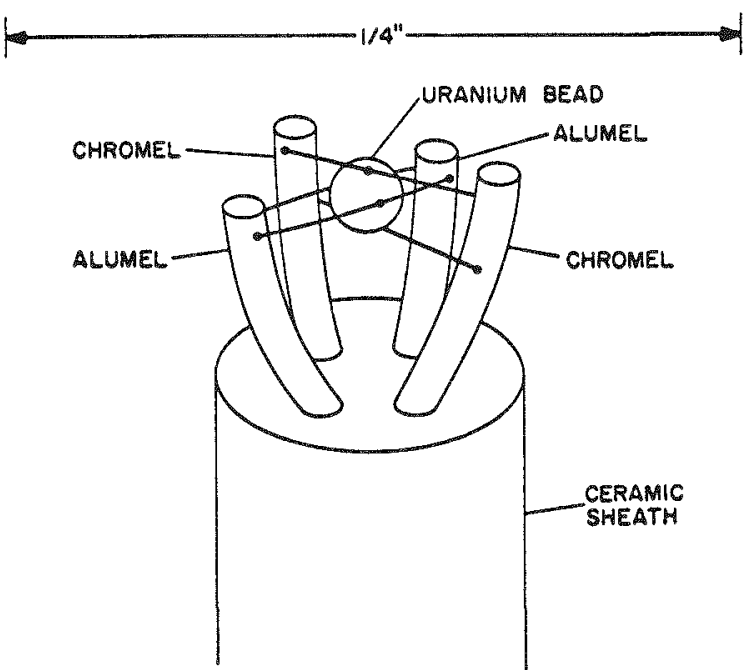

F18. 13. Four-lead wire fission couple design.

absolute output.

This four-lead configuration of the fission couple was the type tested in a nuclear weapons environment at the Nevada Test Site. The detector was placed $3 \mathrm{~m}$ from a nuclear weapon, and accurately followed the change in neutron population (alpha measurement) as the weapon exploded. This rapid change in neutron population had an e-folding tame of 20 nsec.
IV. OTHER AREAS OF INTEREST

A number of other experments have been attempted with fission couples such as investigating thermopile configurations of the fission couple, gamme bucking circuits, gamm heating in various elements and alloys, neutron energy spectral measurements (ratio of ${ }^{235} \mathrm{U}$ to ${ }^{238} \mathrm{U}$ fissiono), fission couple operation in a steady-state radiation environment, neutron flux mapping, and measuring the thermal conductivity of buffer coatings on buffercoated $\mathrm{UC}_{2}$ beads.

\section{A. Thermopile Configurations and Gama Bucksng Circuits}

Thermopile configurations are attractive when a higher signal level is desired whle monitorine a particular neutron flux level. The fission couple technique applied to a thermopile configuration is wired as shown in Fig. 15.

The advantage of using a themopile configuram tion of the fission couple is twofold. First, a greater sensitivity can be attanned since the output signal is multiplied by the number of parred junctions. Secondly, heating of the junction whether due to neutrons or gammas can be sorted out, thereby resulting in a neutron detector insensitive to ganms, that can operate in a mixed neutron and

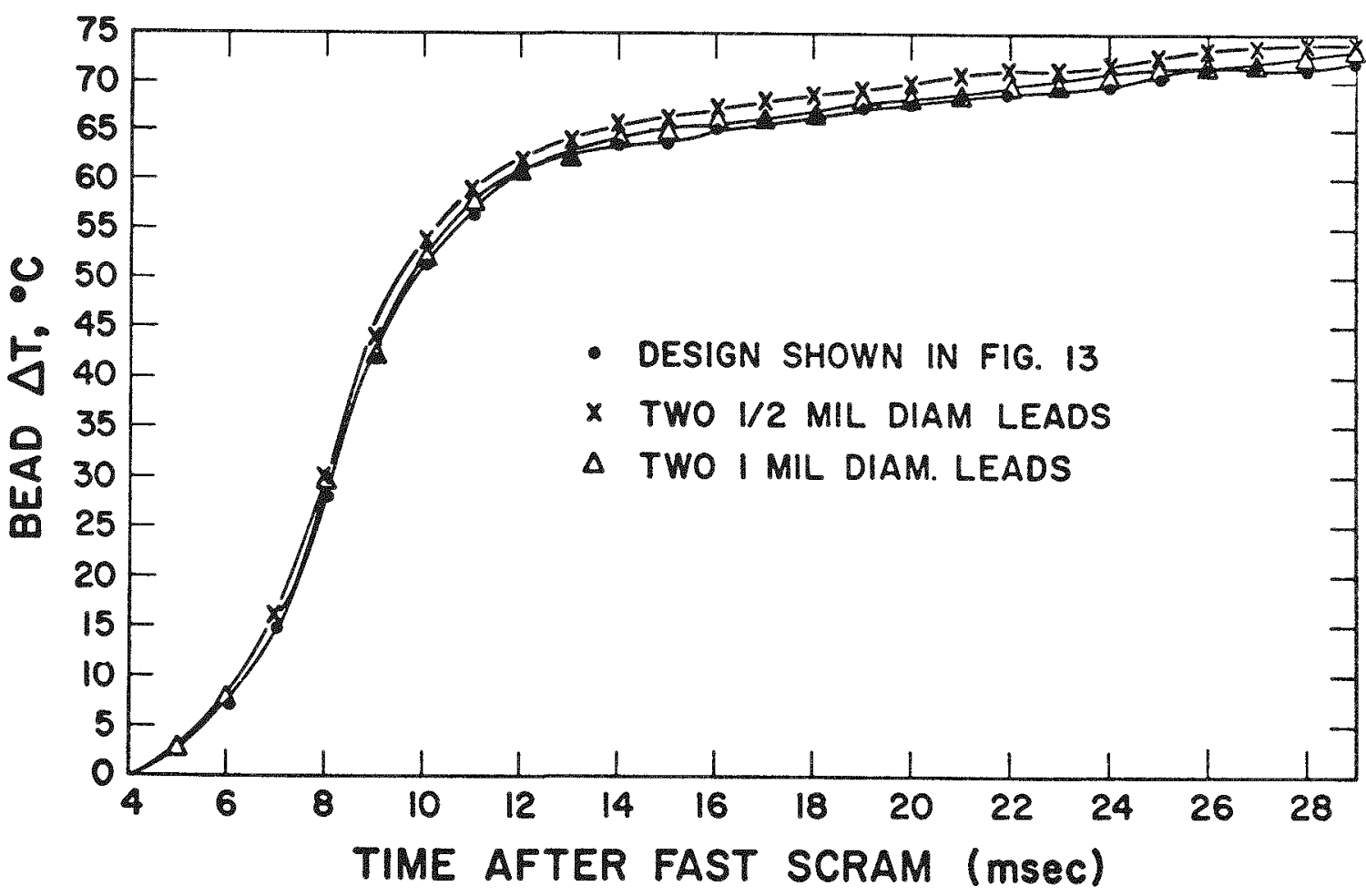

Fig. 14. Comparison of three different ${ }^{335} \mathrm{U}$ fission couple designs of equal ${ }^{35} \mathrm{U}$ masses. 
TABLE II

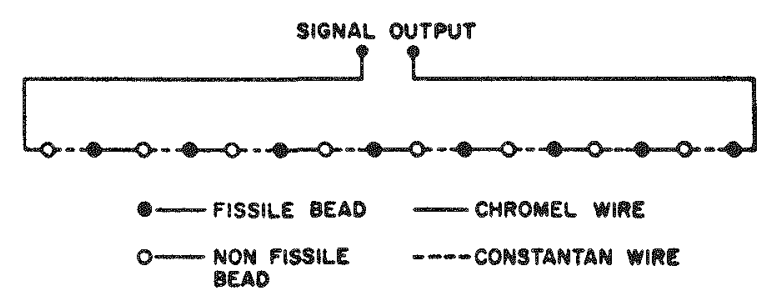

Fig. 15. Typical thermopile configuration of fission couples.

gamma enviroment. This occurs because the fissile bead (hot junction) will heat as a result of neutrons and gammas, whereas the nonfisslle bead (cold juncm tion) will heat only by gamme radiation. By wiring the beads alternately as hot (fissile bead) and cold (nonfissile bead) junctions, the gamm heating is subtracted from the total signal. The ideal circuit would require that the resulting temperature rise from ganma heating in the fissile bead would exactly match the temperature rise due to gamma heating in the nonfissile bead.

The mechanism of ganms heating in the materials is a combination of photoelectric absorption and of Compton effect whereby the gammas impart energy to electrons in the material. The resultant temperature rise may be calculated from the mass absorption coefficients and the specific heats of the materials in question.

A number of elements and alloys were considered to select the proper nonfissile material to match the uranium bead. Mass absorption coefficients correspondint to gama energies of $1 \mathrm{MeV}$ were used in the calculation since this is the average gamma energy from a normal ${ }^{235} \mathrm{U}$ fission spectrum. A result of the relative gamma heating of some elements (from the one-group energy calculations) are shown in Table II. The mass absorption coefficient is $\omega / \rho$ and the specific heat is $C_{p}$. The third column, labeled $A_{z} / A_{B_{i}}$, is the ratio of garma heating in the element $\mathrm{Z}$ to the gamma heating in bismuth. For example, gammo heating in uranium is $24 \%$ higher then the gamma heating in bismuth.

The elements shown in Table II were instmumented in the same manner as fission couples and subjected to reactor bursts to compare their relative gamma heating. The approximate values found from the gamma heating experiment are shown in the fourth colurn of Table II. The relative gamme heating of the selected elements were found to agree reasonably well with
REIATIVE GAMMA HEATTNG

\begin{tabular}{cccc} 
Element & $\mu / \rho C_{\mathrm{p}}=\mathrm{A}$ & $\begin{array}{c}\left(\mathrm{A}_{\mathrm{z}} / \mathrm{A}_{\mathrm{Bi}}\right) \\
\text { Theory }\end{array}$ & $\begin{array}{c}\left(\mathrm{A}_{\mathrm{z}} / \mathrm{A}_{\mathrm{Bi}}\right) \\
\text { Experimental }\end{array}$ \\
\hline Te & 0.54 & 0.39 & 0.36 \\
$\mathrm{BI}$ & 1.40 & 1.00 & 1.00 \\
Mo & 0.40 & 0.29 & 0.23 \\
W & 1.04 & 0.74 & 0.79 \\
Nb & 0.41 & 0.29 & 0.23 \\
U & 1.74 & 1.24 & $\ldots$
\end{tabular}

their calculated values based on a one-group energy. If the values in the second column of Table II are experimentally verified, a circuit as shown in F1g. 16 may be used to monitor only neutron heating.

Thermoplies, s shown in Fig. 15, have been used at the Super KUKIA reactor and the SNAPIRAN-2 destructive test to accurately monitor both the initial reactor period and the power profile of the reactor burst. The power profile of the reactos burst is obtained by differentiating the output signal of the themopile. In all cases, the power profile obtained from the themopile results in a narrower burst shape than that indicated from the conventional photocells.

When building a thermopile, the size of the unit should be minimized. It is desirable to keep all bead spacing as close to one another as possible so that the neutron flux measurements approach a measurement at a point in space. As the number of junctions increases, the overall size of the detector increases and the resultant signal then represents the average neutron flux value over the volume of the detector. One of the latest designs tested utilized thin ceramic disks with ten holes for the beads. These disks can be stacked to increase the number of junctions.

Another problem that arises when the number of junctions is increased is that the electrical resistance of the detector also increases. The length of the 1/2-mil-diam wires must be kept at a minimum to assure $20 \mathrm{~W}$ overall resistance. One of the latest thermopiles consisting of 20 junctions ( $20^{235} \mathrm{U}$ beads plus 20 Bi beads) had a resistance of only 3500

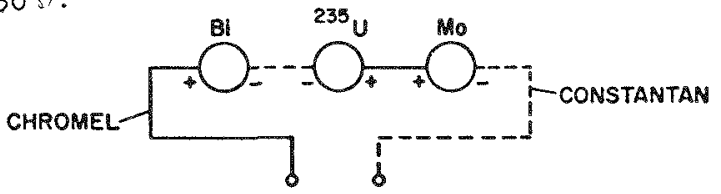

Fig. 16. Gema bucking circuit. 
B. Neutron Energy Spectrel Messurements and Neutron Flux Mepping

By making the fissionable junction of the fission couple from various materials such as ${ }^{236} \mathrm{U}$, ${ }^{238} \mathrm{U},{ }^{230} \mathrm{Pu},{ }^{237} \mathrm{~Np}$, etc., which possess fission cross sections of differing enerby dependence, it should be possible to determine the neutron energy spectrum produced within a particular reactor. A simpinfied experiment designed to demonstrate this ldea has been completed at the super KUKIA Reactor. Fission couples with beads of ${ }^{20} U$ and ${ }^{238} U$ were positioned inside and outside of the reactor to invest 2 gate the ratio of ${ }^{335} \mathrm{U} /{ }^{38} \mathrm{U}$ heating. The results are shown in Table III. The subseript I refers to the bead location inside the sample vold, and the subscript o refers to the bead location outside the reactor at a distance of $14 \mathrm{~cm}$ from the reactor fuel surface and $13 \mathrm{~cm}$ below the reactor core partang line.

\section{TABLE III}

RATIO OF ${ }^{235} \mathrm{U} /{ }^{38} \mathrm{U}$ HEATING IN THE SUPER KUKLA REACTOR

\begin{tabular}{lc} 
Ratio of Peak & $\begin{array}{c}\text { Approxim } \\
\text { Velta The }\end{array}$ \\
\hline$\left({ }^{230} \mathrm{U}\right)_{1} /\left({ }^{230} \mathrm{U}\right)_{0}$ & 3.2 \\
$\left({ }^{235} \mathrm{U}\right)_{1} /\left({ }^{238} \mathrm{U}\right)_{1}$ & 21 \\
$\left({ }^{235} \mathrm{U}\right)_{0} /\left({ }^{238} \mathrm{U}\right)_{0}$ & 30 \\
$\left({ }^{238} \mathrm{U}\right)_{1} /\left({ }^{28} \mathrm{U}\right)_{0}$ & 4.5
\end{tabular}

A plot of fissions per gram vs delta T* could be determined experimentally for various fissile materials by using fission couples. The fission couples could be subjected to a burst of neutrons and the resultent temperature rise recorded. Subsequent radiochemistry on the particular fission couple beads would then determine the number of fissions per gram that occurred in the bead. This information could hhen be used to generate a plot of fissions per gram vs delta $T$ for various fissile materials. once this relationship is established for the material of interest, voltage output of the fission couple would subsequently be sufficient to determine the number of fissions per gram thereby eliminating further radiochemistry. This would be a desirable feature considering the recovery problems involved

* Delta $T$ is the change in temperature from ambient. with conventional folis. The output of the fission couple, a dynamic measurement, would immediately determine the number of fissions per gram.

Once the curve of fissions per gram vs delta $T$ is established, a new method of obtaining $F$ numbers** would be possible. This would involve coating small insile junctions with materials of interest and subjecting them to bursts of neutrons from a prompt burst reactor. A comparison of the delta $T$ indicated on an uncoated fissile junction with that of a coated Junction, in the same location, would then provide the $F$ number for the coated material. C. Fission Couple Performance in a steady-State Radiation Environment

Experiments anvolving fission couples were performed in the steady-state radiation environment of the Omega West Reactor (OWR). More developmental work remains to be done in this area. In particular, the following problems are presently being investigated.

(1) Correlation of the temperature of the fission couple as a function of bead size, bead earichment, and the neutron flux.

(2) Determination of operating infetime of the fission couple as a function of total integrated neutron flux.

(3) Investigation of various packering techniques for the fission couple.

Four fission couples with junctions of $\mathrm{UC}_{\vec{B}}$, fully enriched in $^{235} \mathrm{U}$ and with a than tungsten costing, were irradiated in a neutron flux of approximately $3 \times 10^{23} \mathrm{n} / \mathrm{cm}^{2}$ sec at the OWR. The purpose of the test was to determine the fission couple output as a function of the bead diameter. The diameter of the $U C_{\text {ads }}$ bearled from $\approx 500 \mathrm{\mu m}$ to $\approx 900 \mathrm{~m}$. The fission couples were sealed in Pyrex glass envelopes which had been evacuated and then fllled with 1 atm of helium. The resultant data is hown in Fig. 17. The data contirmed that the equilibrium temperature reached by the beads was indeed a function of the bead diameter. The constant of proportionality was found to be $\approx 0.7^{\circ} \mathrm{C} / \mathrm{H}$

A correlation of reactor power level vs temper. ature output of a $\mathrm{UC}_{a}$ bead, fully enriched in ${ }^{33^{5}} \mathrm{U}$ and with a thin tungsten coating, is shown in Fig. 


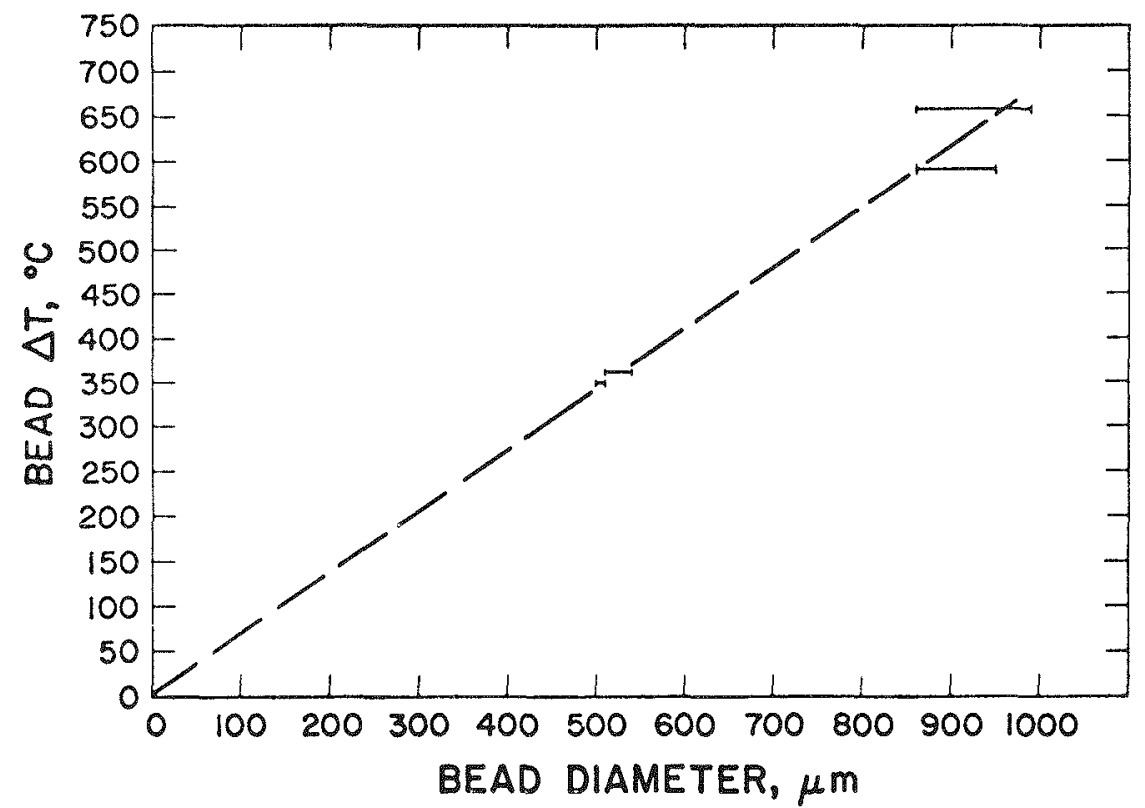

Fig. 17. UC bead temperature rise vs bead diameter.

18. This fission couple was also sealed in a Pyrex zlass envelope with a helium atmosphere as in the experiment described above. The owR power level was increased in steps from $10 \mathrm{~kW}$ to $4 \mathrm{MW}$ while the fission couple output was monitored. The change in temperature of the fission couple was linear with respect to reactor power with a proportionality constant of $60^{\circ} \mathrm{C} / \mathrm{MN}$.

Fission couples with junctions of $\mathrm{Nb} 20 \mathrm{wt} \%{ }^{235} \mathrm{U}$ are also presently being investigated in the OWR. The preliminary data indicates that lower enrichments of uranium would be desirable for neutron fluxes of $3 \times 10^{3} \mathrm{n} / \mathrm{cm}^{2} \mathrm{sec}$. The criterion is selection of both bead size and enrichment so that equilibrium temperatures of the fission couple junction are relative $1 \mathrm{y}$ low (approximately $150^{\circ} \mathrm{C}$ ) for the normal reactor power level. This will reduce oxidation of the bead junction and extend the lifetime of the detector.

\section{Thermal Conductivity Measurements}

The fission couple technique offers a unique method of obtaining thermal conductivity, ${ }^{6}$ of various materials. Pyrocarbon-coated nuclear fuel particles were overcoated with tungsten and the thermocouple wires were welded to the tungsten $180^{\circ}$ from each other. These fission couples were subjected to neutron bursts from a prompt burst reactor and the surface temperature responses were monitored. By knowing the particle dimensions and the burst shape, it was possible to calculate the effective thermal conductivities of the pyrocarbon coats. The good agreement between the thermal conductivity, measured by the fission couple method and the independent measurements on the dense isotropic pyrocarbon, indicates the general validity of the fission couple technique.

v. CONCLUSIONS

The initial goal for developing a fast response,

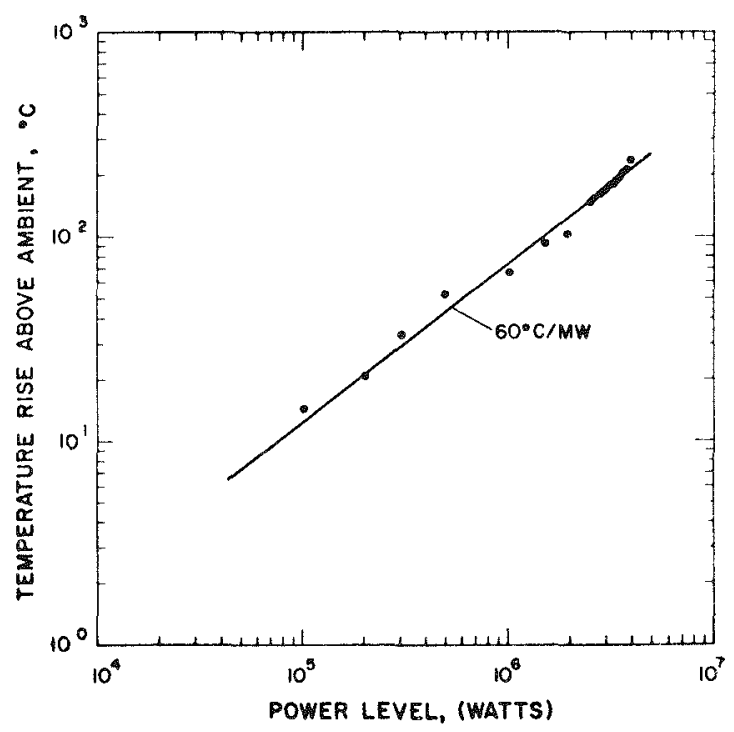

Fig. 18. Fully enriched $\mathrm{UC}_{\mathbb{R}}$ bead data of ${ }^{\circ} \mathrm{C}$ rise vs power level. 
minıature instrument capable of detecting neutrons In the presence of strong gamma radiation has been accomplished $w$ th the fission couple. The actual design of the fission couple, having the fissile bead as the junction of a thermocouple, allows the detector to be most accurate the more rapid the energy change occurs. This is unlque because most detectors have an inherent lag time while monitoring a rapidly changing environment.

In carrying out the experimental program, other possible applications for the fission couple detector were investigated. Among these, the most promlsing use for the detector appears to be in the area of neutron diagnostics and reactor controls. The minuature sıze and fast response of the fission couples combine to offer unique advantages over existing instrumentation in reactor diagnostics and control. ACKNOWLEDGMENTS

The development of the fission couple has required the assistance of many people within this Laboratory. In particular, we would like to thank L. D. P. King, P. G. Salgado, and T. F. Wimett for their many contributions toward the development of fission couples in our frequent discussions with them.

Particular thanks is given to R. G. Morrison, Phillips Petroleum Company, for his many suggestions and continued interest in the development of the fission couple and to $H$. Judge, Scientific Engineering Corporation in Las Vegas, Nevada, who was instrumental in developing the capability to manufacture the detectors.

We would also like to thank the personnel at the Super KUKIA Prompt Burst Reactor for thelr courteous attitude and helpful assistance durzng the many experimental setup procedures.

\section{RETERENCES}

1. R. G. Morrison and D. B. Stillman, "FissionCouples Applied toward Reactor Diagnostics and safety", LA-3470-MS, Los Alamos Scientific Laboratory (April 6, 1966).

2. F. A. Kloverstrom, "Operating Characteristics of the super KUKIA Prompt Burst Reactor", UCRLm 70138, Lawrence Radiation Laboratory (November $1,1966)$.

3. R. L. Coats and P. D. O'Brien, "SPR II Safety" Analysis Report", SC-RR-66-2706, sandia Corporation, Albuquerque (January 1967).
4. D. B. Stillman and S. W Mead, The FRAN Prompt Burst Reactor", UCRL 14413, Lawrence Radiation Laboratory (September 29, 1965)

5. I. D. Posey and J. V. Walker, 'The Fıssion Couple Detector: A Low-Resolution Neutron Spectrometer for Pulsed-Neutron Enviroments, Trans. Am. Nucl. soc. II (1), 342 (1968)

6. G. T. Brock, "Pyrocarbon Thermal Conductivity Measurement by the Fission Couple Method'. Masters Degree Thesis at New Mexico State University (November 1968). 


\section{Observation of Fuel Failure Behavior During. In-Pile Meltdown Tests Using High-Speed Photography and a Transparent Autoclave*}

John J. Barghusen, Lawrence J. Harrison, Richard O. Ivins and Lewis E. Robinson Argonne National Laboratory, Argonne, Illinois

P

\section{A. Introduction}

Over the past several years, the U. S. Atomic Energy Commission has sponsored an extensive program to investigate various aspects of the safety of water-cooled power reactor systems. As part of this program an experimental study was undertaken to provide information required for the analysis of excursion accidents in water-cooled thermal reactors. This study involved subjecting single fuel-rod specimens in a transparent autoclave to destructive nuclear transients, and observing, by photographic techniques, the behavior of the specimens during the transients. The principal objectives were to obtain sufficient data to define the failure threshold energy and mechanism of failure for Zircaloy-clad, $\mathrm{UO}_{2}$-core fuel rods. Since zirconium readily reacts with water at metal temperatures above $1000^{\circ} \mathrm{C}$, the contribution of the metal-water reaction to fuel failure behavior was assessed during this study.

The experimental program utilized the photographic capability of the TREAT (Transient Reactor Test) Facility. ${ }^{1}$ The reactor shown in Figure 1 is provided with a viewing channel 22 in. by $31 / 4$ in. extending from outside the reactor to the transparent autoclave located at the center of the reactor. To accommodate this channe1, special slotted fuel elements are employed. The channel terminates at a movable shielding block on which a series of mirrors is fastened. A camera, located outside the reactor shielding, views the specimen within the autoclave by means of the mirrors and channel--a distance of about 14 feet.

\section{B. Description of Transparent Autoclave}

The transparent autoclave assembly consists of three parts: a sample holder, a secondary capsule, and a slot liner, as shown in

* Work performed under the auspices of the U. S. Atomic Encrgy Commission 
Figures 2 and 3. The sample holder is an open-top stainless steel chamber, 17 in. tall by $1.5 \mathrm{in}$. Wide, with a high purity quartz (UV grade artificial fused silica) window affixed to the chamber by RTV silicone rubber. The chamber contains a graphite, test-specimen support and from 300 to $3000 \mathrm{ml}$ water. The size of the chamber can be varied depending upon the quantity of water required for the test. Usually, a separate stainless steel chamber is fabricated for each test, by bending a sheet of 304 stainless stee1 1/32 in. thick to form the three sides and then welding on a bottom plate.

The sample holder shown in Figure 2 is an early design which involved the use of high-purity quartz-glass tubes. Initial experiments with this design showed that the quartz darkened upon exposure to radiation. Apparently, impurities introduced into the quartz during the extrusion and drawing process resulted in radiation-sensitive tubes. Quartz-glass plates currently used for windows are not sensitive to radiation levels encountered in the TREAT facility.

The sample holder is inserted within the secondary capsule, 18.5 in. by 17.7 in. by $1.8 \mathrm{in.,} \mathrm{constructed} \mathrm{of} \mathrm{16-gauge} \mathrm{stainless} \mathrm{steel,} \mathrm{or} \mathrm{of}$ Zircaloy for tests with pre-irradiated fuel discussed later. The capsule window is sealed within a stainless steel frame by silicone rubber and the frame bolted to the capsule and sealed with a silicone rubber gasket.

The secondary capsule is placed within the stainless steel slot liner, $20.8 \mathrm{in}$. by $34 \mathrm{in}$. by $3 \mathrm{in.}$, fabricated from 14-gauge sheet metal. The rear portion of the slot liner which surrounds the secondary capsule is lined with reactor-grade graphite to act as a thermal barrier in the event fuel fragments melt through the capsule. The front window, constructed of 0.1 in. thick fused silica, is mounted at an angle of five degrees to prevent reflection of exterior light into the camera. The sides of the slot liner are reinforced with stainless steel plates to restrict pressure-induced bowing. The unit will accommodate internal pressures of up to about 30 psig; pressure relief is achieved by blow-out of the window frame gasket in the event of $a$ pressure excursion accident.

Two gas purge lines and an electrical connection are provided at the top of the slot liner. The electrical connection is potted in resin for a leak-tight seal. The entire autoclave assembly is provided with a helium gas purge system which is employed to leak-test the assembly at 7 psig and to reduce the oxygen content to 2 vol. \% prior to insertion of the assembly into the reactor. The quantity of hydrogen evolved from metal-water reaction during the transient is measured in post-transient gas samples.

Illumination of the autoclave and test specimen is provided by two 1000-Watt, quartz-iodine, "Sun-Gun" lamps. Each lamp is mounted in a stainless steel shield with a built-in reflector. A photograph of a specimen within the autoclave prior to insertion in the reactor is shown in Figure 4. 


\section{Fuel Element Design and Experimental Procedure}

In the course of this study over 25 photographic tests were performed with $5 \%$ and $10 \%$ enriched $\mathrm{UO}_{2}$ pellet fue 1 and with $5 \%$ and $10 \%$ enriched vibrationally-compacted (vibra-packed) $\mathrm{UO}_{2}$ powder fuel. Descriptions of the fuel types appear in Figures 5 . The $5 \%$ enriched pellet fuel specimens were nearly identical in composition and dimensions to the vibra-packed $\mathrm{UO}_{2}$ fuel rods, Type II. The pelletized $\mathrm{UO}_{2}$ fuel specimens containing $10 \% 235 \mathrm{U}$ were typical of PWR fuel, and were slightly shorter and of smaller diameter than the vibra-packed and $5 \%$ enriched pellet specimens.

Vibra-packed fuel specimens were prepared ${ }^{2}$ by crushing high-density oxide ( $298.6 \%$ theoretical density) into coarse particles, screening the particles into various size fractions, and blending appropriate size fractions into mixtures suitable for fabrication into Zircaloy clad fuel elements. The fabrication step was accomplished by vibrationalcompaction which principally involves special feeding methods coordinated with tamping by mechanical vibration. This fabrication method will consistently produce clad oxide fuels with smear densities of about $85 \%$ theoretical density.

The fuel element specimens are mounted in a graphite block placed on a stainless steel sheet which serves to maintain the specimen in the proper position within the sample holder. Cladding temperature profiles are obtained from two thermocouples (W-5Re/W-26Re) spot welded to the cladding. The response time of the thermocouples is about $15 \mathrm{msec}$. Figure 6 shows the thermocouples attached to a fuel rod after a transient experiment. Although in this photograph a bulge in the cladding appears coincidentally at the point of attachment of the thermocouple, no evidence has been noted relating fuel failure to the welding of thermocouples on the cladding.

Prior to placing the autoclave in the reactor, the entire assembly is leak checked and purged with helium to reduce the residual oxygen concentration to less than 2 vol. \%. Post-transient analyses of the gas for hydrogen are performed about 24 hours after the transient to allow for uniform diffusion of the hydrogen throughout the assembly. Samples of the gas are analyzed by mass spectrometry for hydrogen, helium, oxygen, nitrogen and carbon dioxide.

Calibration tests were performed with each type of fuel specimen in order to determine the factor relating reactor energy (MW-sec) to thermal or fission energy generated within the fuel specimen (cal/g $\mathrm{UO}_{2}$ ). A specimen is placed in the autoclave and subjected to a transient of known energy, usually $50 \mathrm{MW}-\mathrm{sec}$, which does not induce failure of the specimen, since the entire specimen is required for radiochemical analyses. Determination of fission energy input (fissions/ $\mathrm{g} \mathrm{UO}_{2}$ ) is made by direct measurement of the fission product $99 \mathrm{Mo}$. Assuming a prompt fission energy release of $172 \mathrm{Mev} / \mathrm{fission}$, the calibration factor is then readily calculated. Determination of the axial distribution of fission energy has been made by analyses of single pellets using counting techniques. 
Photographs of the specimens are obtained with a Fastax WF-14 camera using $400 \mathrm{ft}$ rolls of HS-Ektrachrome film at speeds of 1000 to 3000 frames/sec. Exposures are made with a 6 in. lens at an f-stop of 5.6. Superimposed on the photographs are two signals from an oscilloscope (see Figure 7): one signal provides timing lines each millisecond; the second signal generates a light pulse at a rate proportional to the reactor power (i.e., a neutron count). Initially, the neutron count on the film was used to correlate reactor time with film time. This method, obviously tedious to employ, was superseded by a method which involves noting on the oscillographic record of reactor power the instant the lamps are extinguished; this is readily observed on the film. The real time of events recorded on the film (such as cladding rupture) is determined by noting timing marks on the film in the interval.

When the autoclave is loaded in the reactor core, the reactor is manually brought to a steady-state power level of 50 Watts and the operation then transferred to an automatic control system. This system turns on the lights, starts the camera and recording oscillograph, and releases the control rod which initiates the transient. When a preset integrated reactor power value is reached, the reactor is scrammed. The oscillograph provides a continuous record of reactor power, integrated power, sample temperatures, timing lines, etc. The camera runs until the entire reel of film is exposed.

\section{Analysis of High-Speed Film and Experimental Data}

To illustrate the type of data obtained from the film six selected frames from a film record of a destructive-transient test are shown in Figure 8. In this test, a vibra-packed $\mathrm{UO}_{2}$ powder fuel specimen was subjected to a $391 \mathrm{MW}-\mathrm{sec}$ transient, equivalent to a total fission energy input of $257 \mathrm{cal} / \mathrm{g} \mathrm{UO} \mathrm{UO}_{2}$. The transient resulted in two separate failures, one at the bottom of the fuel rod at $220 \mathrm{cal} / \mathrm{g} \mathrm{U0} 2$, and one at the top of the rod at $255 \mathrm{cal} / \mathrm{g} \mathrm{UO} \mathrm{U}_{2}$. Classification of the two failures into separate events might not have been possible by postmortem examination of the residue shown in Figure 9 . By use of the photographic record, however, the sequence of the failures and the time at which they occurred were readily established. The first frame in Figure 8 shows the development of boiling at the surface of the cladding just after peak power. The subsequent frames show: the specimen one msec after the first failure (time $1.27 \mathrm{sec}$, see record in Figure 10); eighty msec later (1.35 sec); $2 \mathrm{msec}$ after the second failure at the top of the rod $(1.43 \mathrm{sec}) ; 10 \mathrm{msec}$ after second failure $(1.44 \mathrm{sec})$; and $60 \mathrm{msec}$ after the second failure ( $1.50 \mathrm{sec})$ illustrating the continued development of turbulence in the water by transfer of heat from the fuel although the transient at this time is completed.

The residue from this test is shown in Figure 9. Close examination of the rupture at the top of the specimen showed the presence of $\mathrm{UO}_{2}$ which had been fused. Both failures were probably the result of high internal pressures and intinate contact of the cladding with molten $\mathrm{UO}_{2}$ rather than failure by extensive metal-water reaction. Apparently, the 
pressure relief accomplished by the first failure was not effective in relieving high pressures in the upper portion of the fuel. The rupture in the middle of the cladding probably occurred at the time of the last frame shown in Figure 8, and was indicated by the development of intense heating at the central portion of the fuel specimen.

The significant data from the oscillograph record of this test are shown in Figure 10. The abscissa represents time, in seconds, after an arbitrary zero. The oscillograph output provides data on reactor power, two integrated-power circuits, and the two cladding thermocouples at $10-\mathrm{msec}$ intervals. Data from the two integrated-power circuits generally agree within 5 percent, and the energy of the transient is usually determined as the average of the two values.

Maximum cladding temperatures usually occurred 1.0 to $1.5 \mathrm{sec}$ after peak power. This behavior has also been observed in tests employing transients of short duration (reactor periods of 3 to $7 \mathrm{msec}$ ). 3 . In general, peak cladding temperatures were less than $1500^{\circ} \mathrm{C}$, although in a few tests temperatures in excess of the melting point of Zircaloy $\left(1852^{\circ} \mathrm{C}\right)$, up to $2100^{\circ} \mathrm{C}$, were recorded. In initial tests with the transparent autoclave attempts were made to obtain temperature data on the water coolant during the transient with the expectation of using these data to calculate energy balances. However, owing to the development of convective currents within the water, no valid data were obtained and such measurements were terminated.

Throughout the course of this investigation into fuel failure behavior during destructive transients, it was observed from analyses of the films that failures predominately occurred at either the top or bottom of the fuel rod, indicative, perhaps, of axial flux peaking at these positions. Several pellet fuel specimens were analyzed by counting techniques to establish the magnitude of flux peaking, and the results from analysis which appear in Figure 11 show a peak-to-average flux ratio of about 1.14 . The use of depleted $\mathrm{UO}_{2}$ pellets at the top and bottom of the specimens does not diminish the effect. The insertion of a 0.1 in. thick hafnium metal wafer at each end of the fuel column resulted in reducing the peak-to-average flux ratio to 1.07 . Although the existence of axial flux peaking should be considered in the interpretation of the fission energies associated with fuel failure threshold, all fission energies reported here are based on average values for the entire specimen, since there are no mechanisms available whereby the average values can be adjusted with certainty to account for flux peaking in every experimental test.

The TREAT facility provides $a_{4}$ technique for examining reactor fuel specimens by neutron radiography. ${ }^{4}$ Several post-transient specimens were examined by neutron radiography to assess the effects of a transient on the distribution and position of the fuel within the cladding. Neutron radiographs of pellet and vibra-packed $\mathrm{VO}_{2}$ fuel specimens are shown in Figure 12. The two holes in the cladding of the vibra-packed specimen are readily discernable. The pellet fucl specimen did not fail, but was warped as a result of a nuclcar transient equivalent to a fission energy input of $240 \mathrm{cal} / \mathrm{g} \mathrm{UO}_{2}$. The individual pellets are clearly defined in the radiograph. 
In the course of this work, sufficient information has been obtained to describe the nature of fuel failure during nuclear transients. By means of the photographic records, it was established that the failure threshold energy is between 230 and $290 \mathrm{cal} / \mathrm{g} \mathrm{UO}$, even though the total fission energy of the transient may exceed $290 \mathrm{cal} / \mathrm{g}$ $\mathrm{UO}_{2}$, and is nominally independent of fuel type and of reactor period between 40 and $120 \mathrm{msec}$. The magnitude of the failure threshold energies is largely related to the physical and thermal properties of the $\mathrm{UO}_{2}$ fuel, specifically the density of the oxide which decreases with an increase in temperature, and the enthalpy. Recent data 5 on the enthalpy of $\mathrm{UO}_{2}$ show that up to $267 \mathrm{cal} / \mathrm{g}, \mathrm{UO}_{2}$ is in the solid state; between 267 and $335 \mathrm{cal} / \mathrm{g}$, both solid and liquid phases exist; and above $335 \mathrm{cal} / \mathrm{g}$, the oxide exists as a liquid.

Photographs of residues from transient tests representative of various failure modes are shown in Figure 13. Depending upon the total energy, failures may appear as localized fractures or holes, severely broken cladding with some associated fuel, or nearly completely powdered material.

The mechanism of failure is by pressure rupture: high internal pressure coupled with high cladding temperatures. As energy is deposited within the oxide fuel, the fuel and any gases within the fuel element heat up, the volume occupied by the fuel increases, and thus very high internal pressures are quickly achieved. Since both the yield strength and ultimate strength of Zircaloy decrease markedly above $800^{\circ} \mathrm{C}$, internal pressures sufficient to induce rupture of the cladding may be produced without necessarily achieving high cladding temperatures or molten cladding.

The results of the TREAT photographic experiments are shown in Figure 14 as a plot of total transient energy versus reactor period. By this representation of the data, it can be seen that the nature of the residue can be classified into four categories: complete destruction, broken into large sections, holes or blisters, and intact. The fission energies which define these descriptive categories appear approximately independent of reactor period. At fission energies equivalent to 230 to $290 \mathrm{cal} / \mathrm{g} \mathrm{UO}_{2}$, fuel element failures are generally in the form of localized fractures, blisters, or holes in the cladding, with slight dispersal of fuel in the coolant. The fuel specimens remain essentially intact (the extent of metal-water reaction is less than $15 \%$ of the cladding in the fueled zone). At higher energies (total energy between 290 and $350 \mathrm{cal} / \mathrm{g} \mathrm{UO}_{2}$ ), the fuel element is fractured into several pieces, fuel is widely dispersed, and the specimen is further degraded owing to metal-water reaction. At energies exceeding $350 \mathrm{cal} / \mathrm{g} \mathrm{UO}$, only powder remains. The contribution of metal-water reaction to the mechanism of fuel failure becomes dominant only after fuel rods have been ruptured at energies above $290 \mathrm{cal} / \mathrm{g} \mathrm{UO} \mathrm{U}_{2}$. The extent of metal-water reaction (bascd on the cladding in the fuelcd zone) was found to increase lincarly with an incrcase in fission energy from 220 to about $500 \mathrm{cal} / \mathrm{g} \mathrm{UO}_{2}$. 
F. Transparent Autoclave for Photographic Tests with Irradiated Fuel

In preparation for an experimental program involving meltdown tests with pre-irradiated fuel specimens, the transparent autoclave was redesigned and modified to provide features which would facilitate remote assembly and remote handing of components, Since a complete description of this apparatus has been published, ${ }^{6}$ only a few salient features of the autoclave will be presented here. For ease of decontamination, the slot liner is lined with a zircaloy metal shell. Figure 15 shows the front of the slot liner with the test capsule partially inserted and the auxiliary flange removed to show its connections. The raised horizontal bands around the slot liner to stiffen the liner wall are offsets formed directly by rolling.

Figure 16 shows the disassembled test capsule. The window (made from UV grade artifical fused silica) slides down two tracks in front of the capsule. One lower corner of the window is removed to permit free circulation of the inert cover gas from the capsule to the remainder of the assembly. In front of the window in Figure 16 is the test sample load frame. The frame slides into the capsule and is latched in place by tabs. The rod extending from the front of the capsule is a handling "tee" for inserting the capsule into the slot liner.

The remote loading apparatus viewed through a cave window is shown in Figure 17. In the front, on the table, are welding clamps used for attaching thermocouples to the surface of the test specimen. The sample is already in the load frame, while the frame and zixcaloy capsule are fastened to the remote assembly jigs.

After assembly, the capsule is placed into the slot liner, the gas and instrumentation leads are plugged into the inside of the auxiliary flange (Figure 15), and the window flange is bolted into place. The slot linear flanges are leak checked with a helium mass spectrometer, and the entire unit is placed within a specially designed shipping coffin.

\section{$\underline{\text { References }}$}

1. G. H. Golden, C. E. Dickerman, and L. E. Robinson, "Facility for Photographing In-Pile Meltdown Experiments in IREAT," USAEC Report ANL-6457, January 1962 .

2. D. W. Brite and C. A. Burgess, "High Energy Rate Pneumatically Impacted $\mathrm{UO}_{2}-\mathrm{PuO}_{2}$ Fuels," Trans. Am. Nucl. Soc. 7(2): 408-409 (November 1964).

3. L. A. Stephan and C. S. Olsen, "Transient Irradiation of 0.466-inch OD Stainless Steel Clad Oxide Fuel Rods to $300 \mathrm{cal} / \mathrm{g} \mathrm{UO} 2$, "USAEC Report IDO-ITR-101, November 1968. 
4. James F. Boland, "Radiography--A Tool for Nuclear Materials Safeguards," Nucl. News 11(8): 48-50 (August 1968).

5. L. Leibowitz, L. W. Mishler, and M. G. Chasanov, "Enthalpy of Solid Uranium Dioxide from $2500^{\circ} \mathrm{K}$ to Its Melting Point," J. Wucl. Mater. 29: 356-358 (1969); and Reactor Development Program Progress Report December 1968, ANL-7527, p. 132 (Jan. 29, 1969).

6. L. E. Robinson and C. August, "Apparatus for High Speed Photographic Safety Experiments on Irradiated Fast Reactor Fuel Specimens," in "Reactor Physics Division Annual Report July 1, 1967 to June 30, 1968," USAEC Report AJL-7410, January 1969.

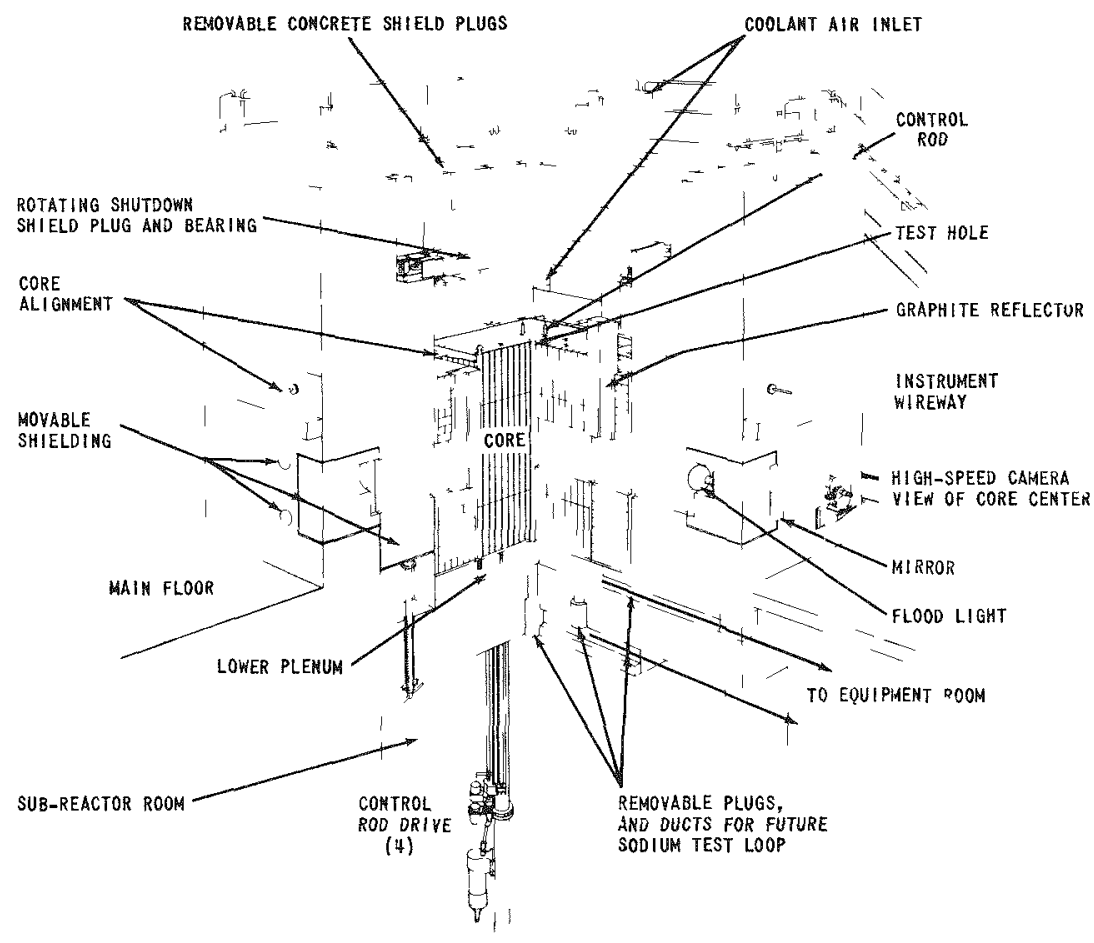

Figure 1. Cutaway Isometric of TREAT Reactor. 


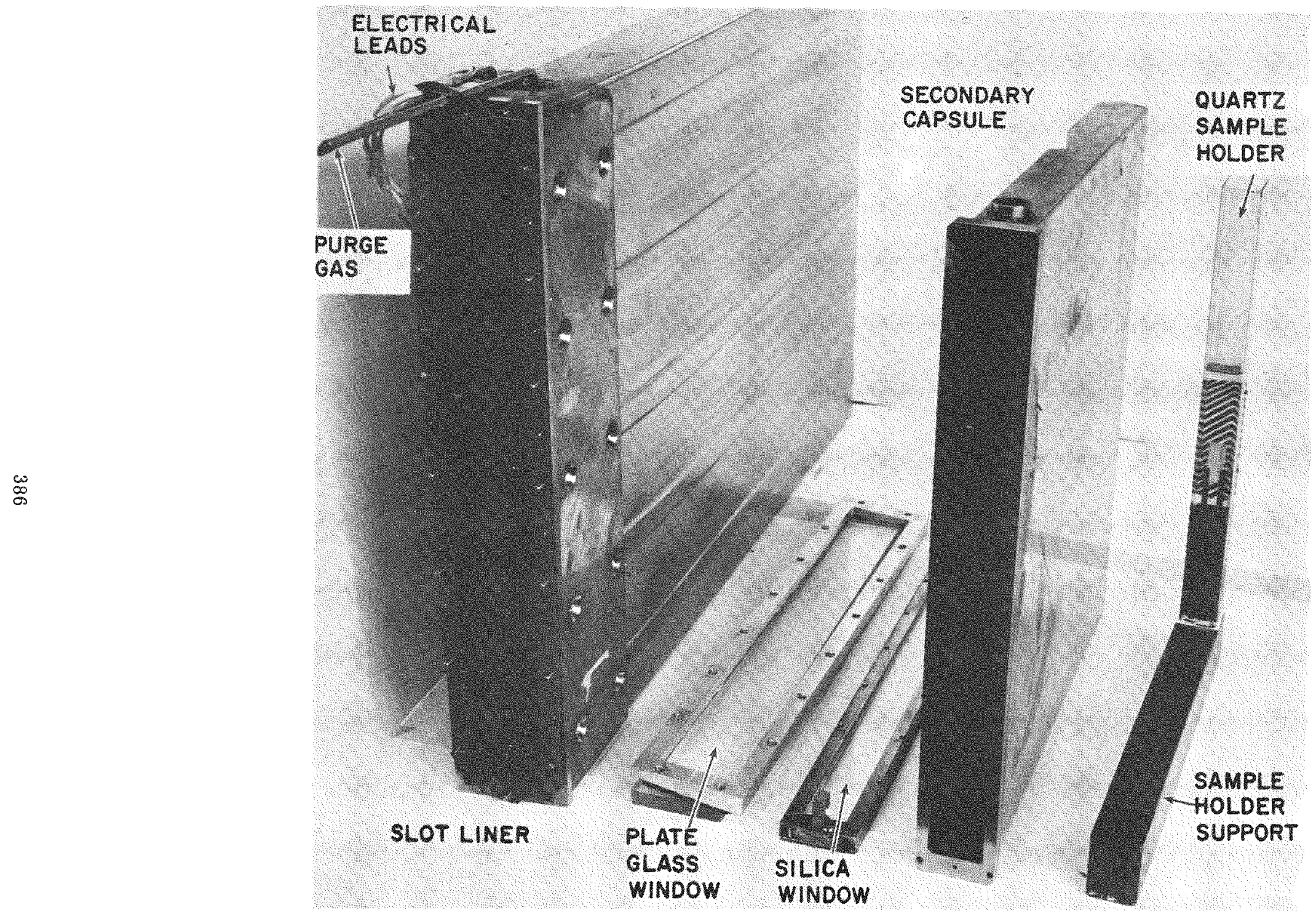

Figure 2. In-Pile Assembly for Photographic Studies. 


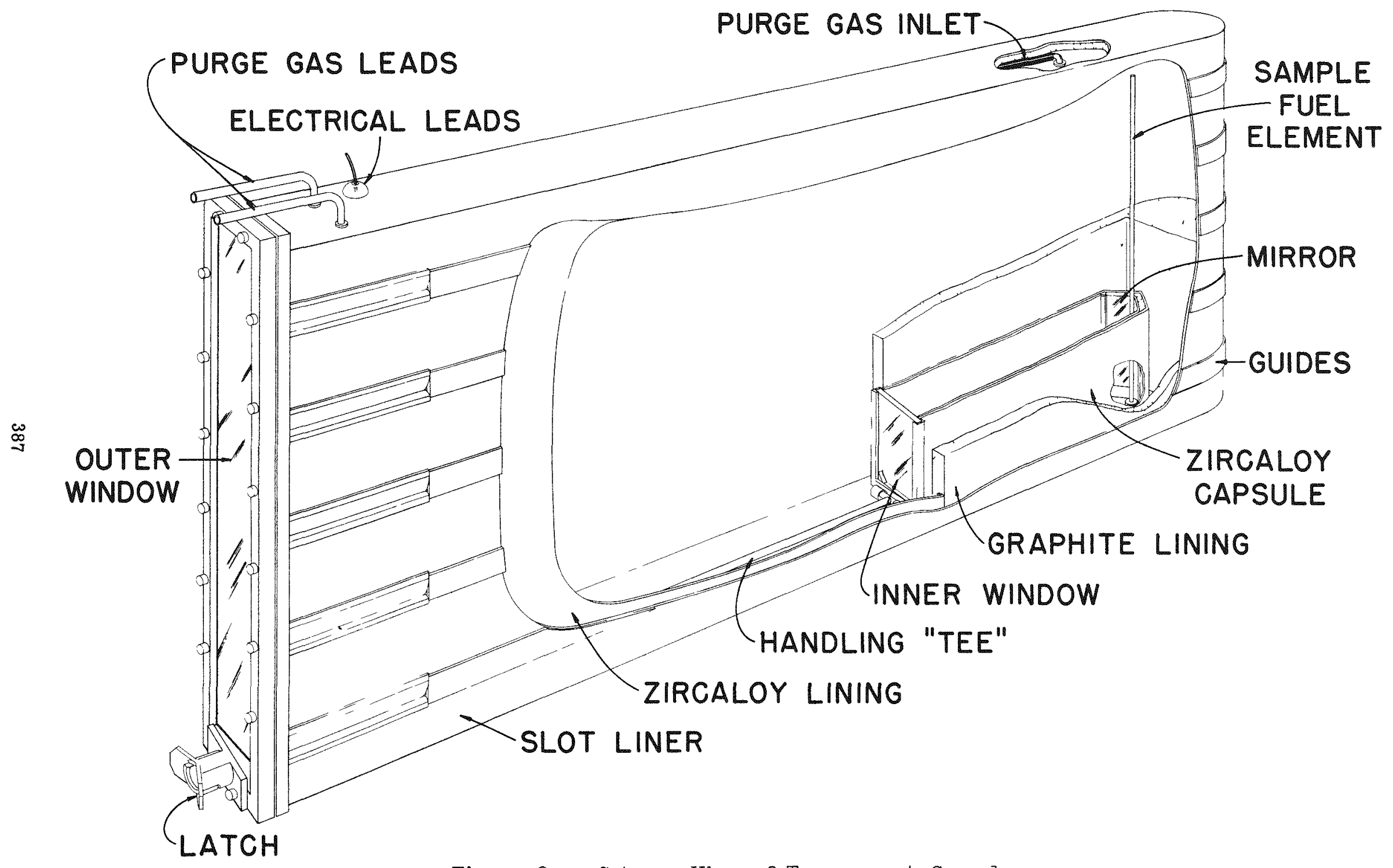

Figure 3. Cutaway View of Transparent Capsule. 


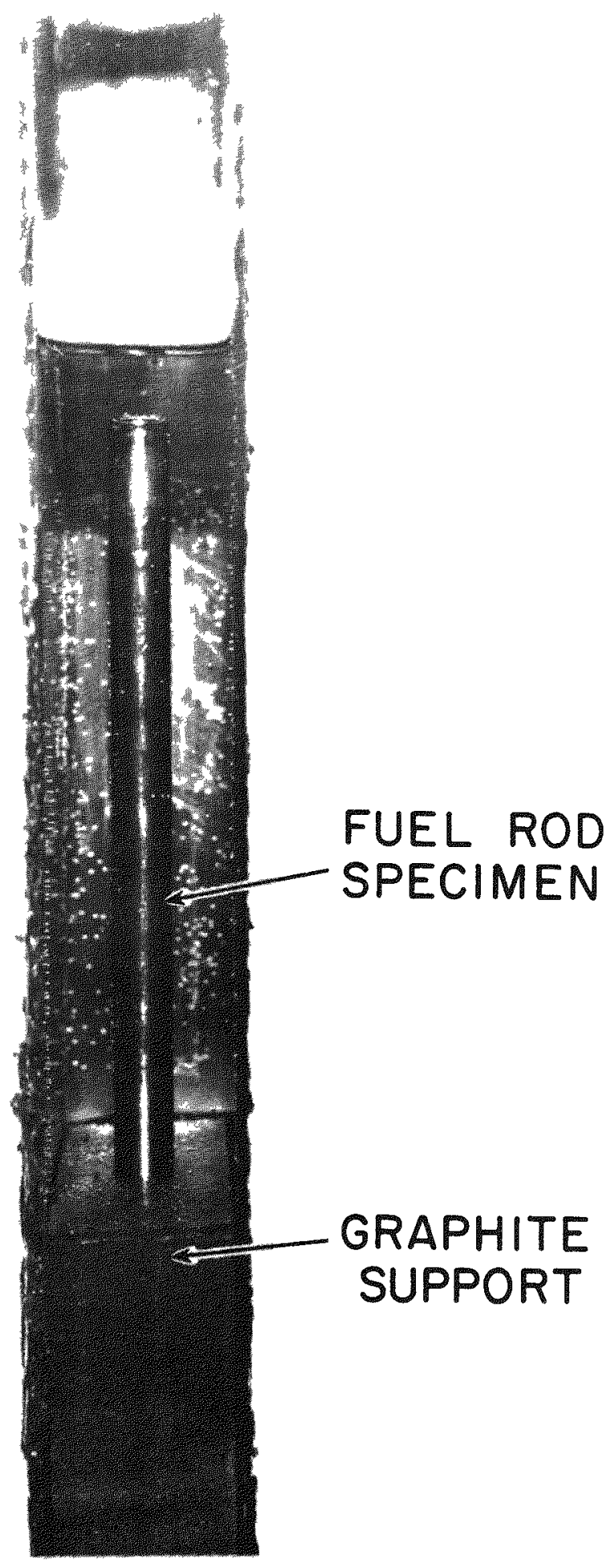

Figure 4. Fuel Rod in Photographic Capsule Before Experiment. 


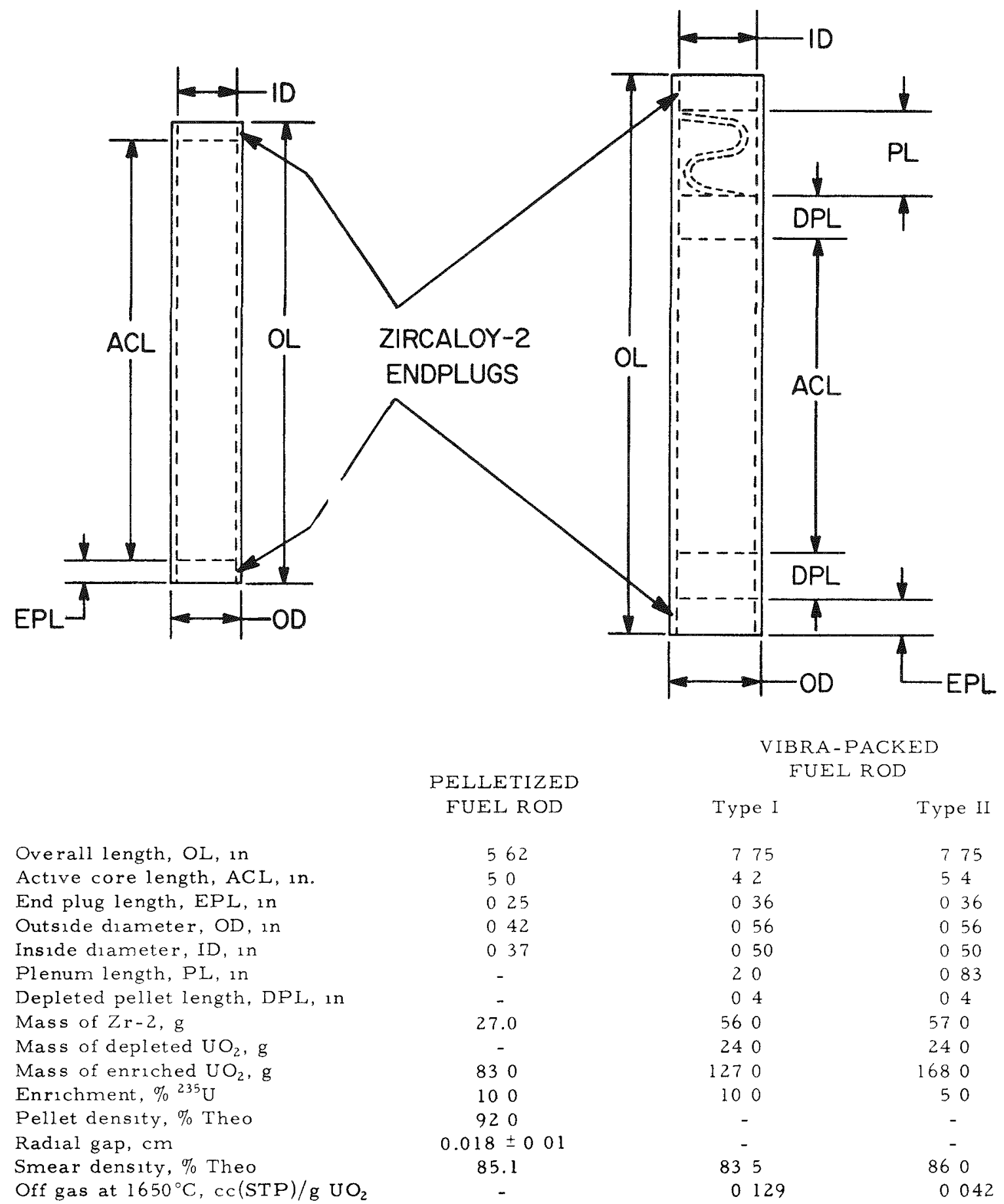

Figure 5. Fuel Rod Types Used in TREAT Excursion Simulations. 


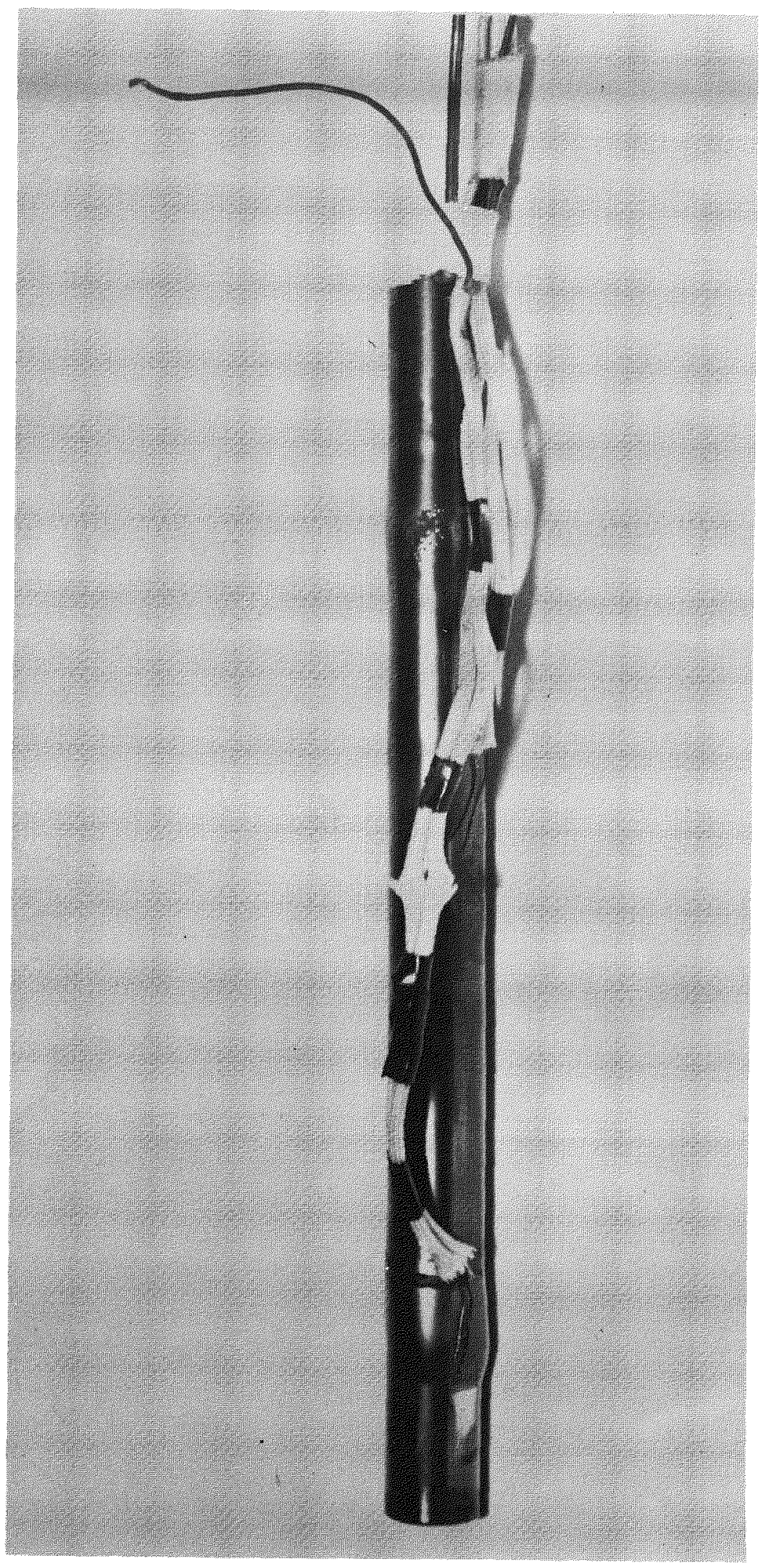

Figure 6. Fuel Rod After Transient Experiment Showing Thermocouple Attachment and Cladding Bulges. 


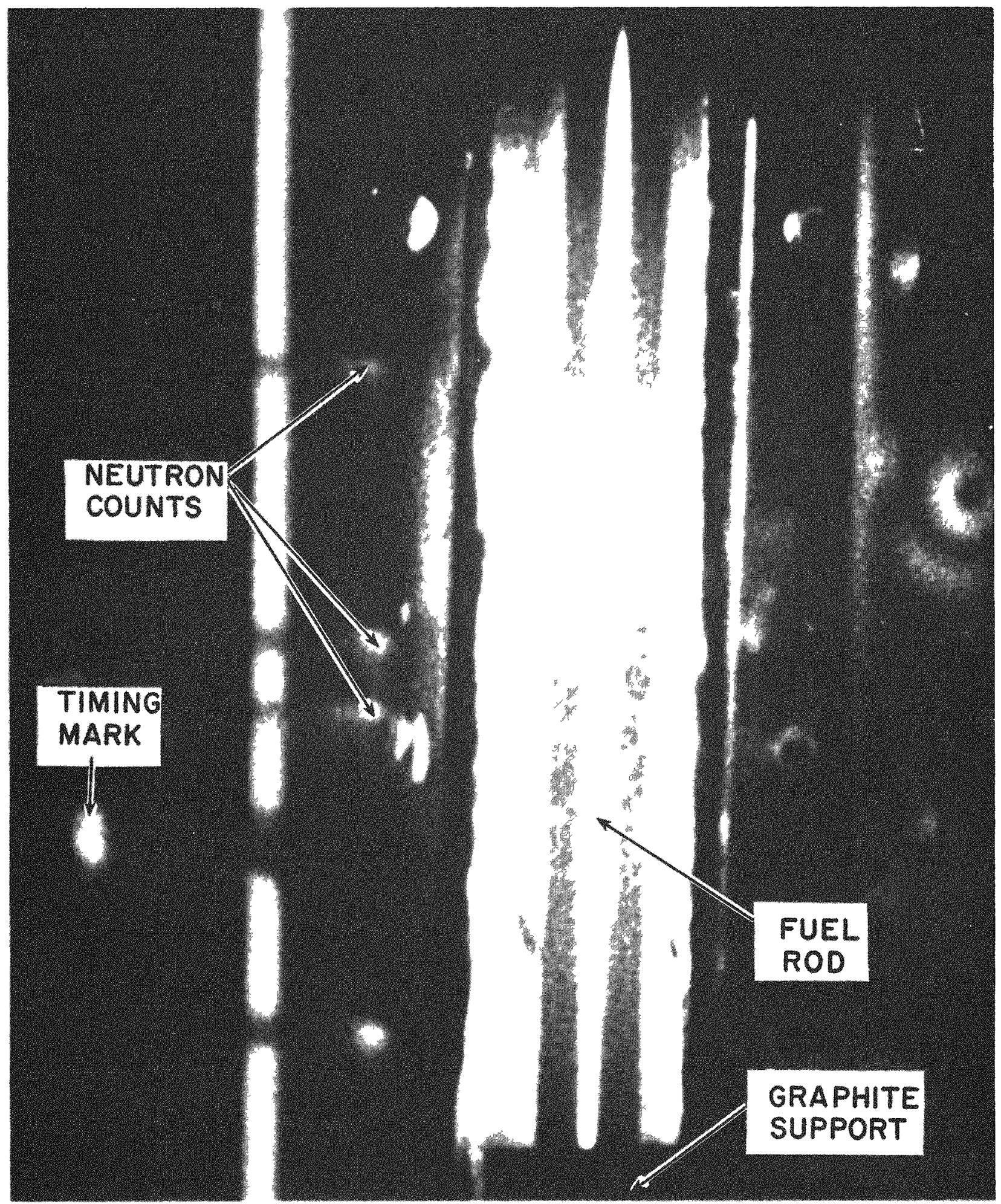

Figure 7. Single Frame From Film Showing Fuel Specimen Being Heated During Transient. 


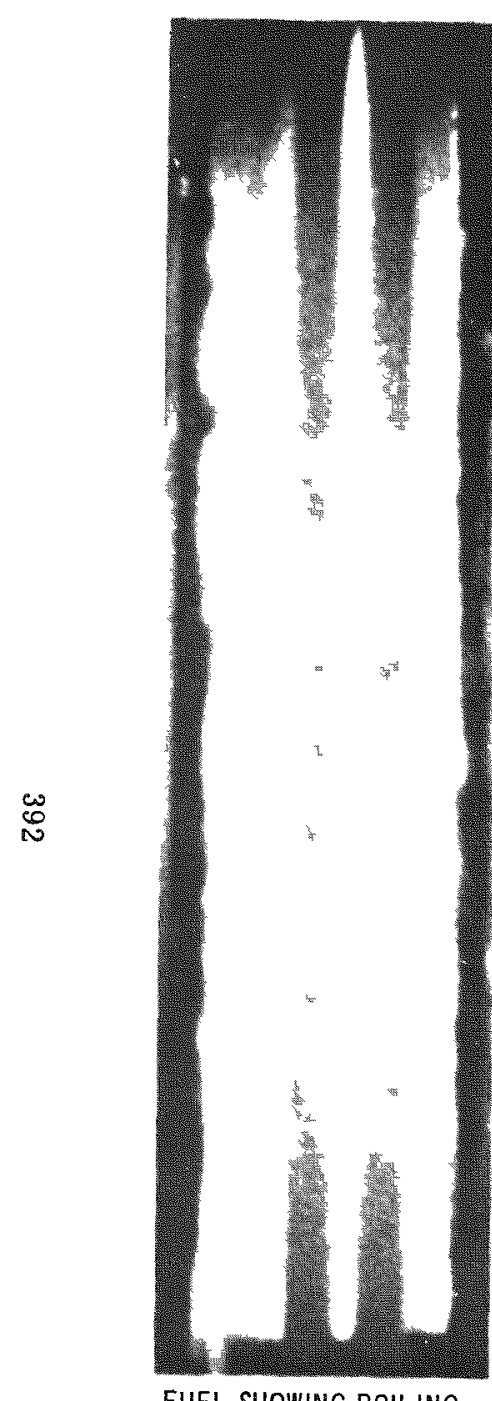

FUEL SHOWING BOILING CHERENKOV RADIATION

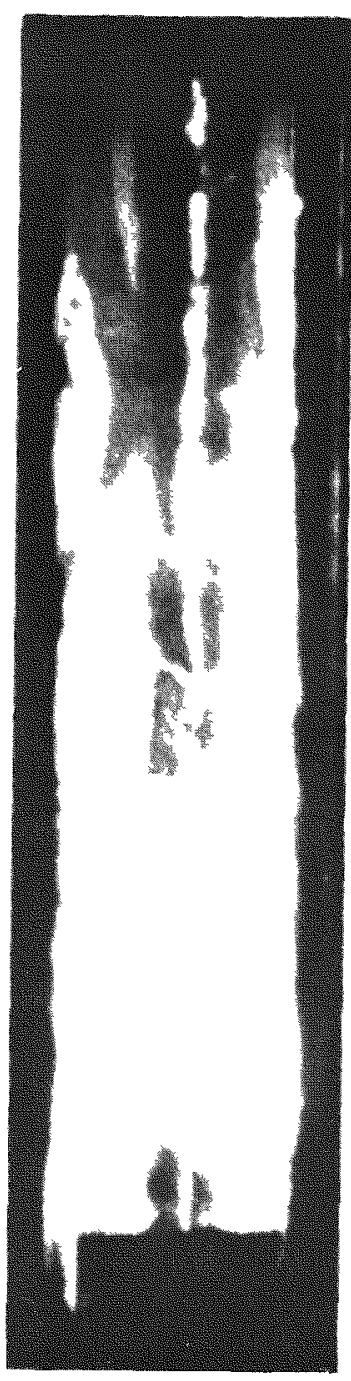

INSTANT OF FAILURE AT BOTTOM OF ROD $127 \mathrm{seC}$

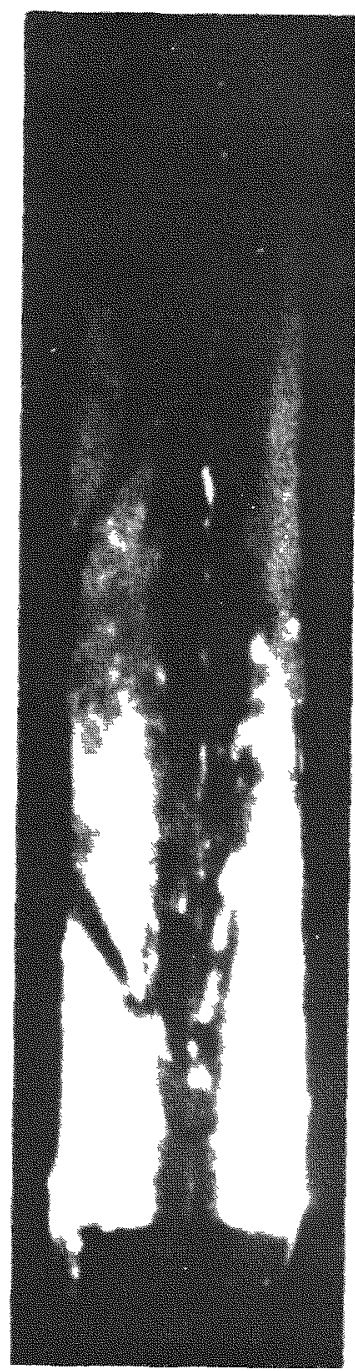

80 mSec AFTER CLAD RUPTURE $135 \mathrm{seC}$

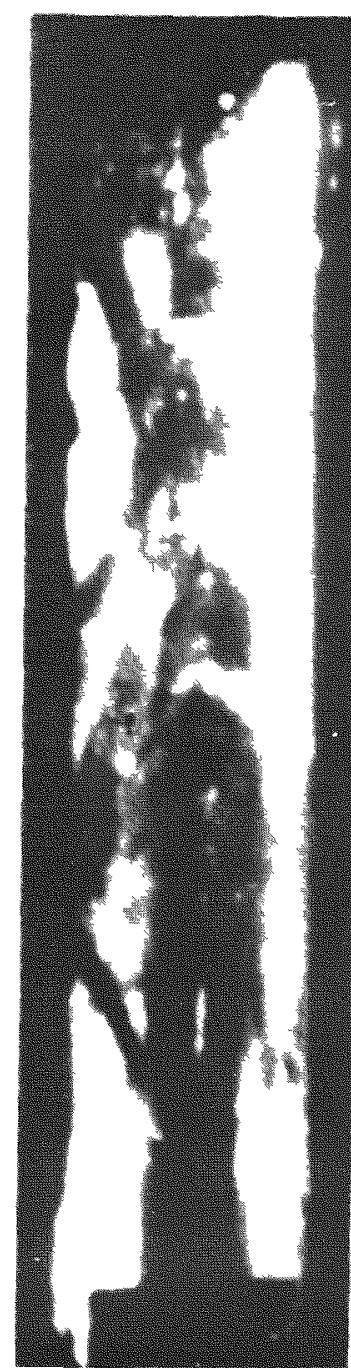

CLAD FAILURE AT TOP OF ROD $143 \mathrm{seC}$

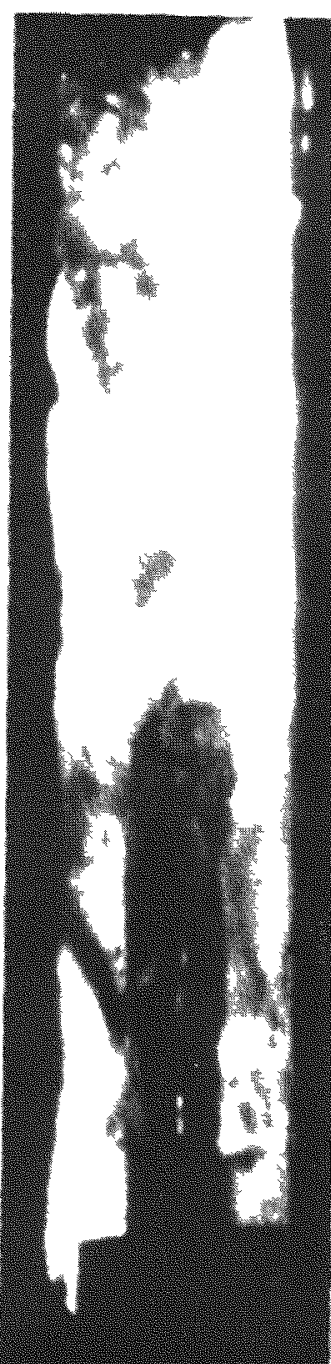

$10 \mathrm{msec}$ AFTER TOP RUTURE SHOWING HOT FUEL DISPERSION $144 \mathrm{sec}$

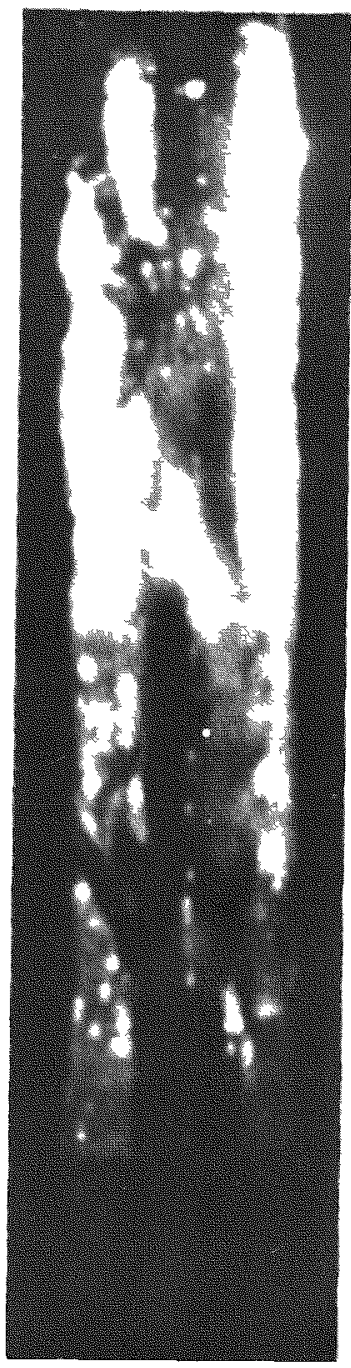

VIGOROUS HEATING AND

COOLANT BOILING CONTINUES $150 \mathrm{sec}$

Figure 8. Sequence of Motion Picture Frames Showing Two Separate Failures in Zircaloy-Clad $\mathrm{UO}_{2}$ Powder Fuel Experiment 244. 


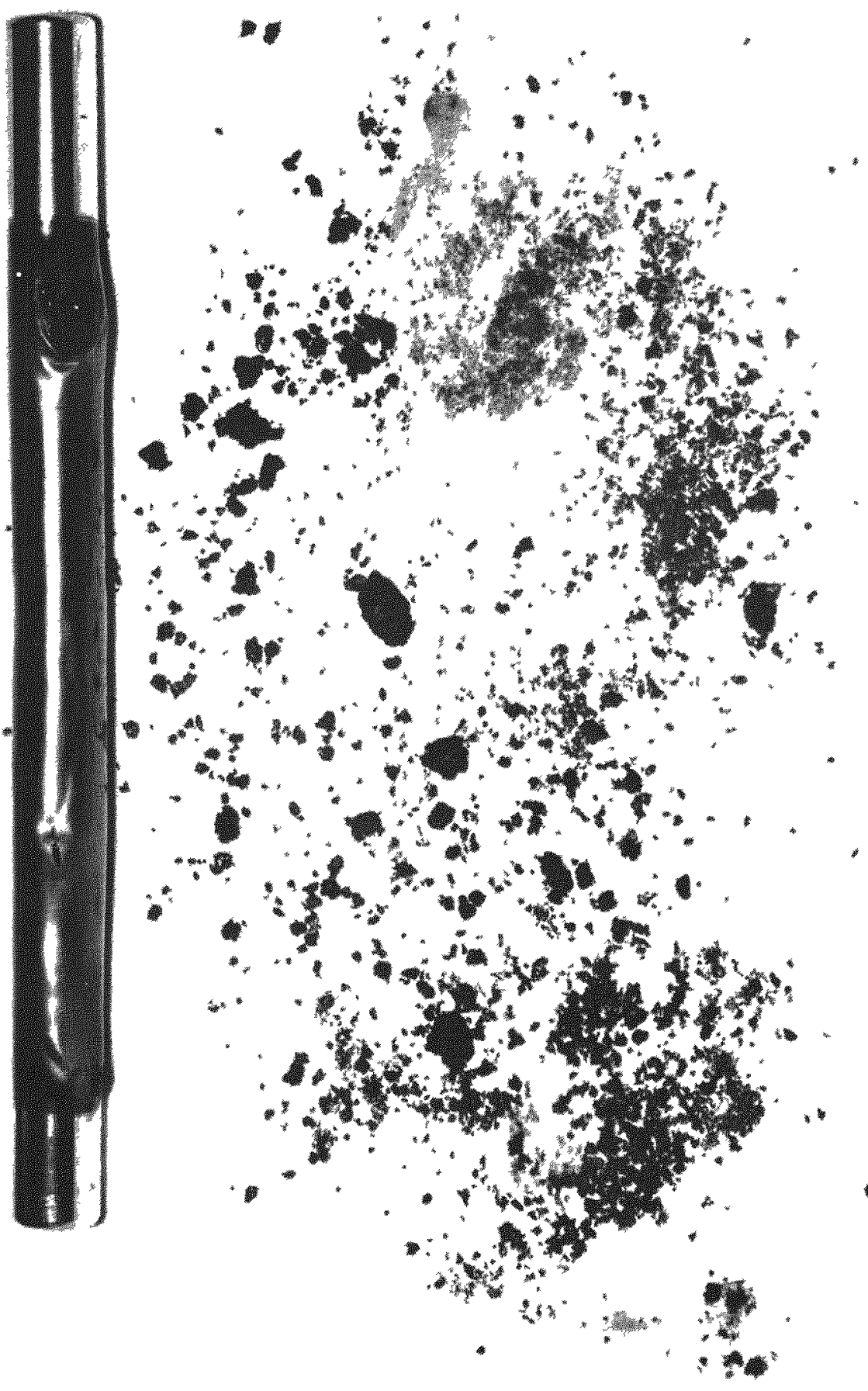

Figure 9. Residue From Experiment 244 Shown in Figure 8. 


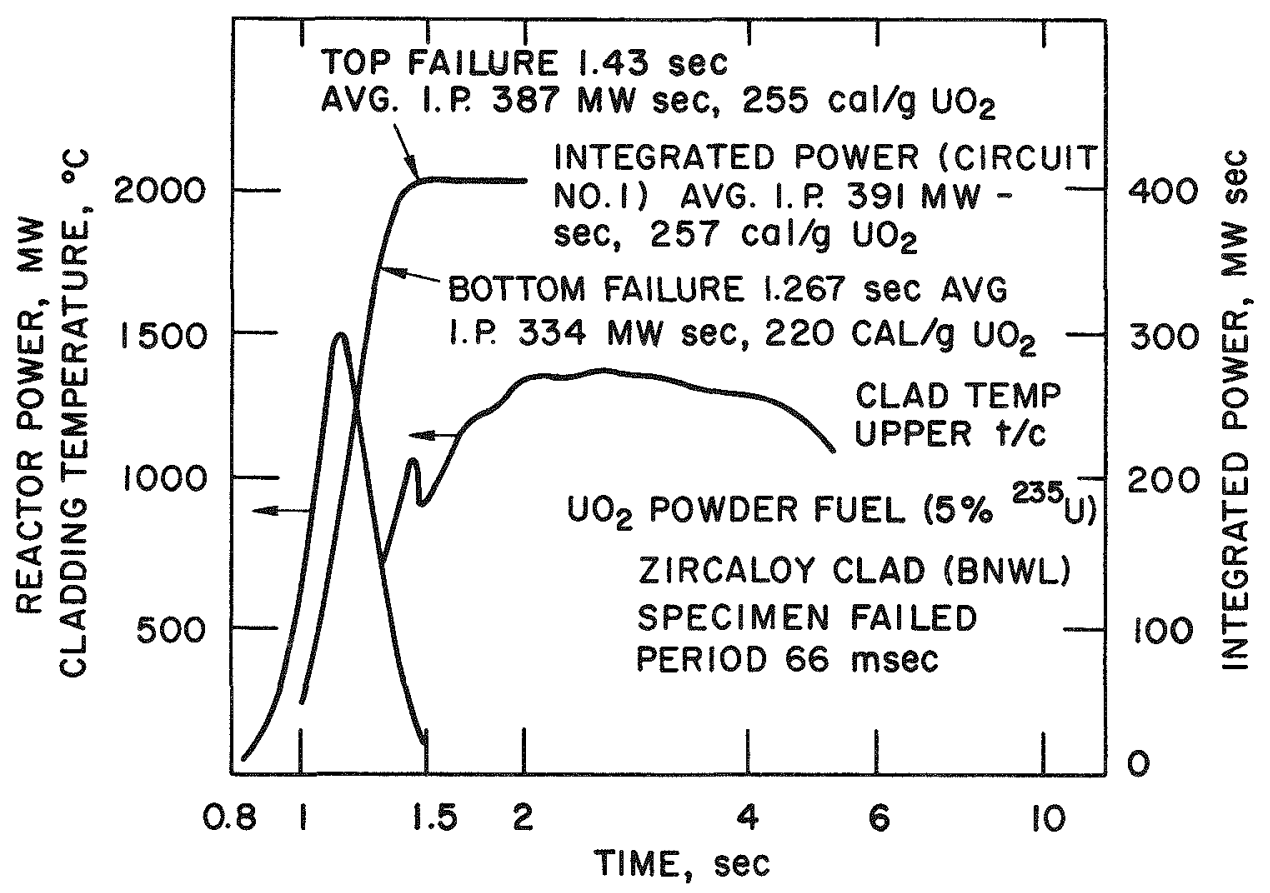

Figure 10. Reactor Power and Cladding Temperature Run 244.

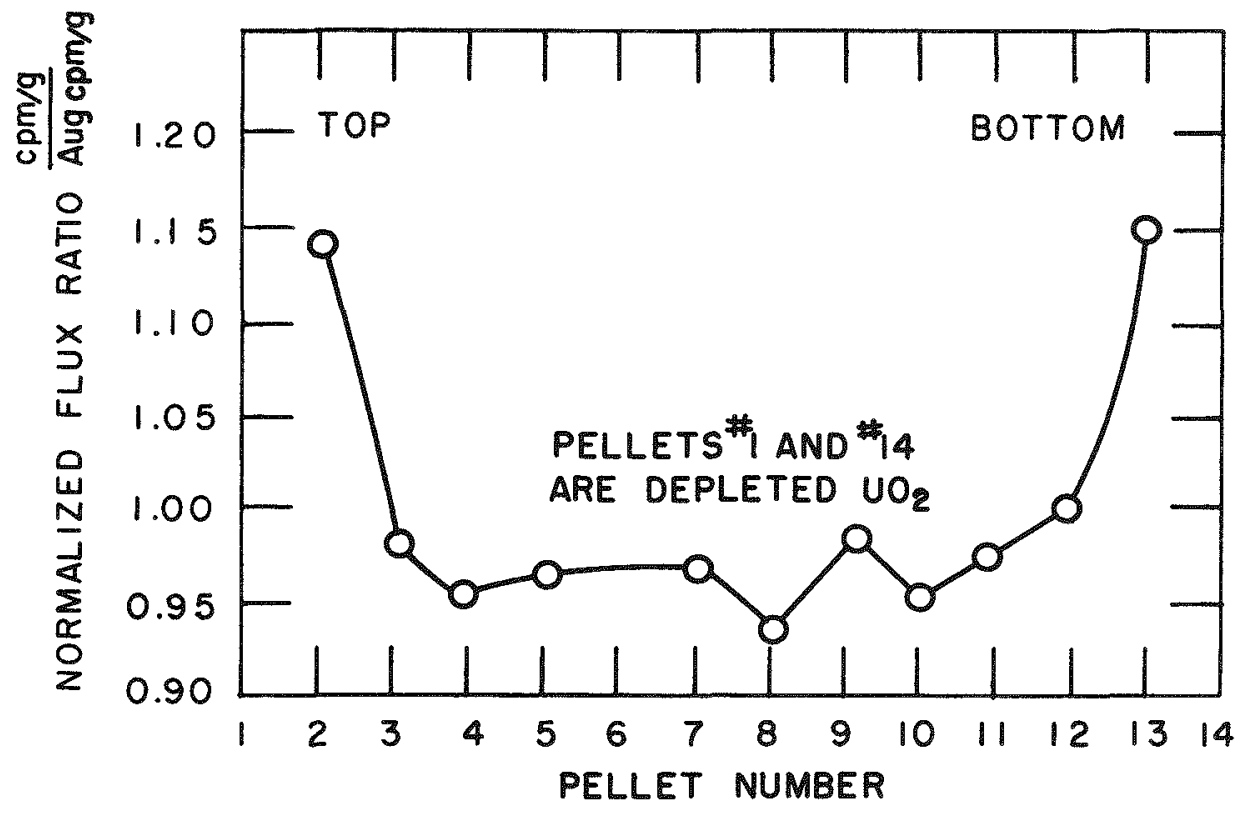

Figure 11. Axial Flux Profile for Pellet Fuel. 


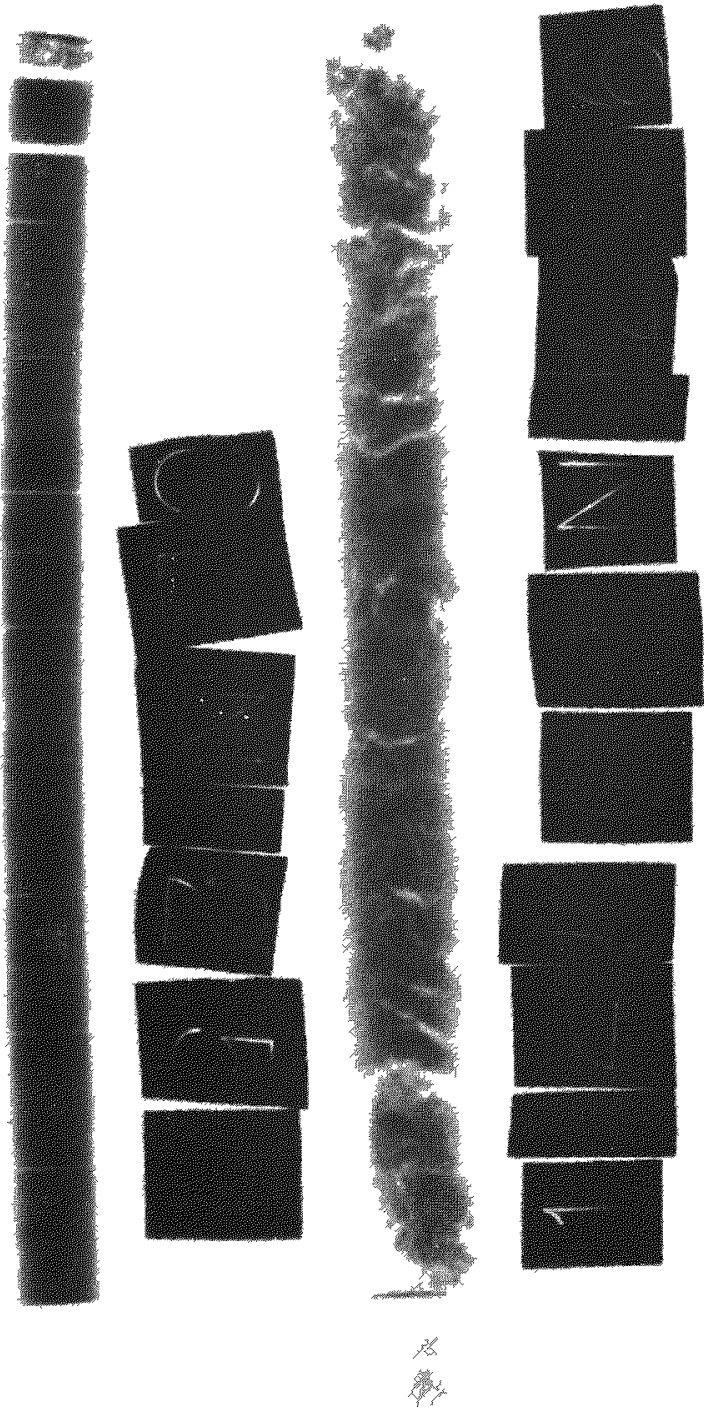

Figure 12. Veutron Radiograph of Pellet and Powder Fuel. 


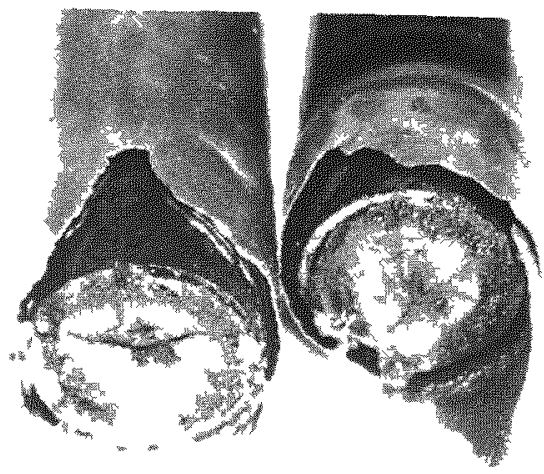

FAILURE OF PELLET FUEL BY CLADDING EMBRITTLEMENT

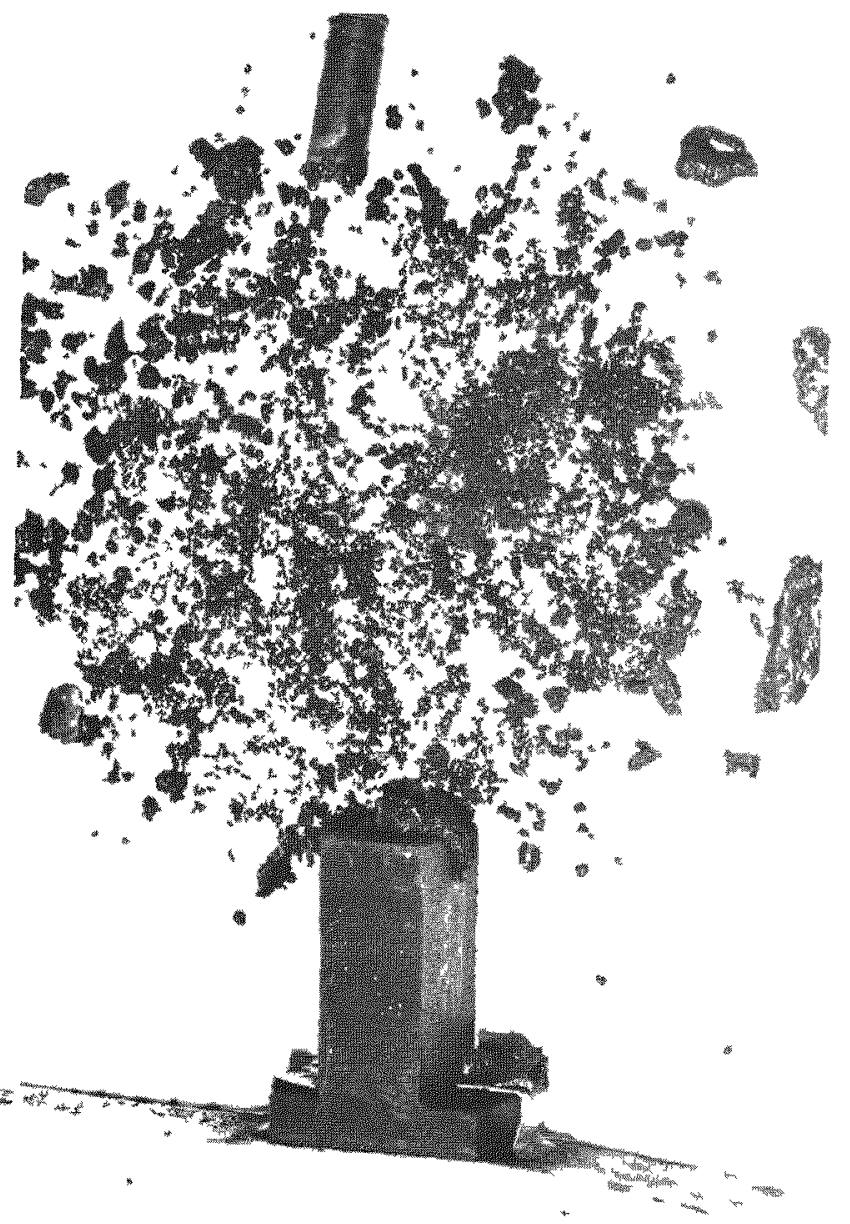

HIGH ENERGY FAILURE WITH COMPLETE DESTRUCTION OF FUEL
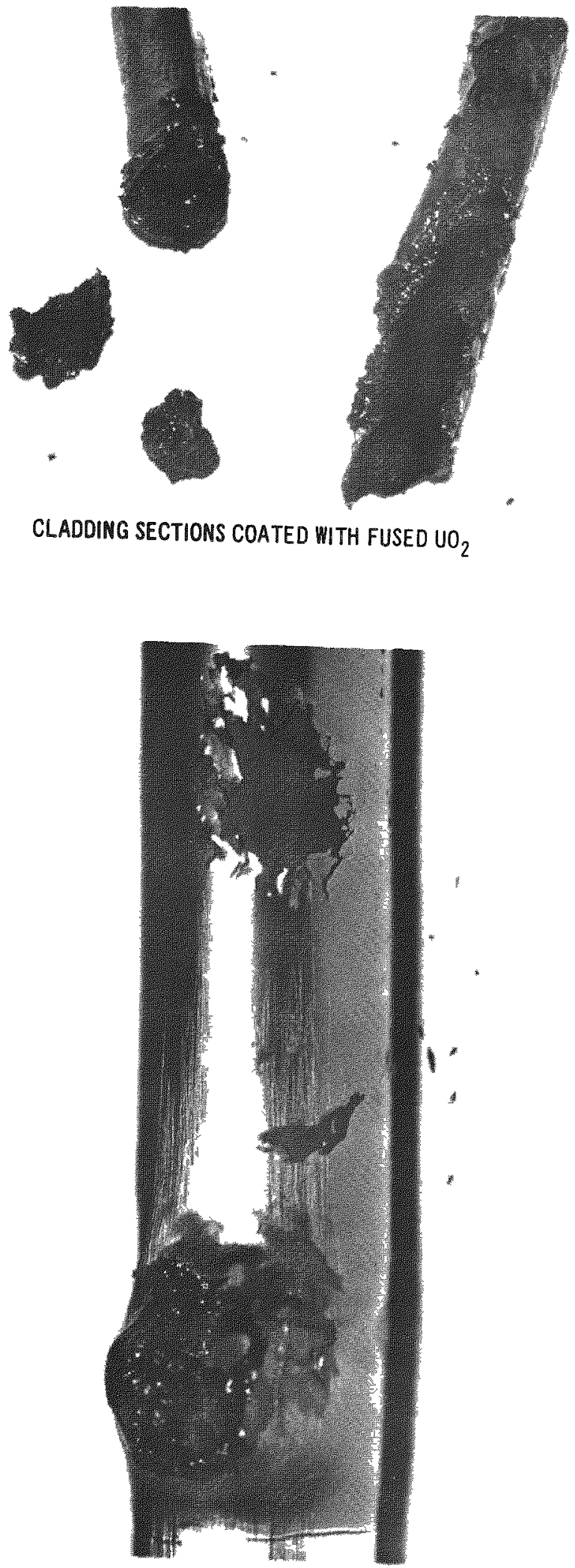

POWDER FUEL SPECIMEN SHOWING HOLE AND OXIDE SCALE

Figure 13. Types of Failure of Zircaloy-Clad $\mathrm{UO}_{2}$ Fuels. 


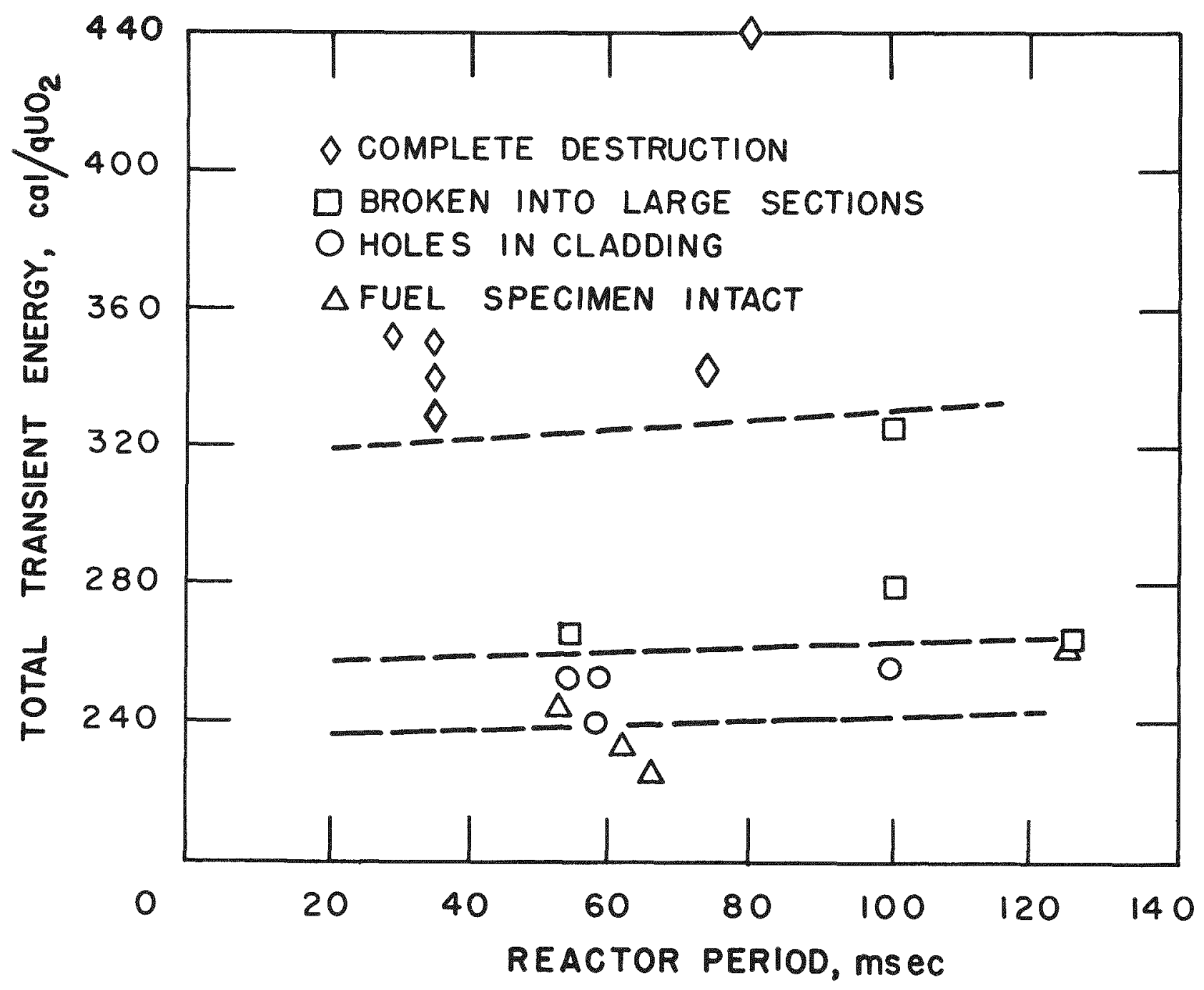

Figure 14. Summary of Fuel Behavior of Zircaloy Clad UO $\mathrm{U}_{2}$-Core Fuel. 


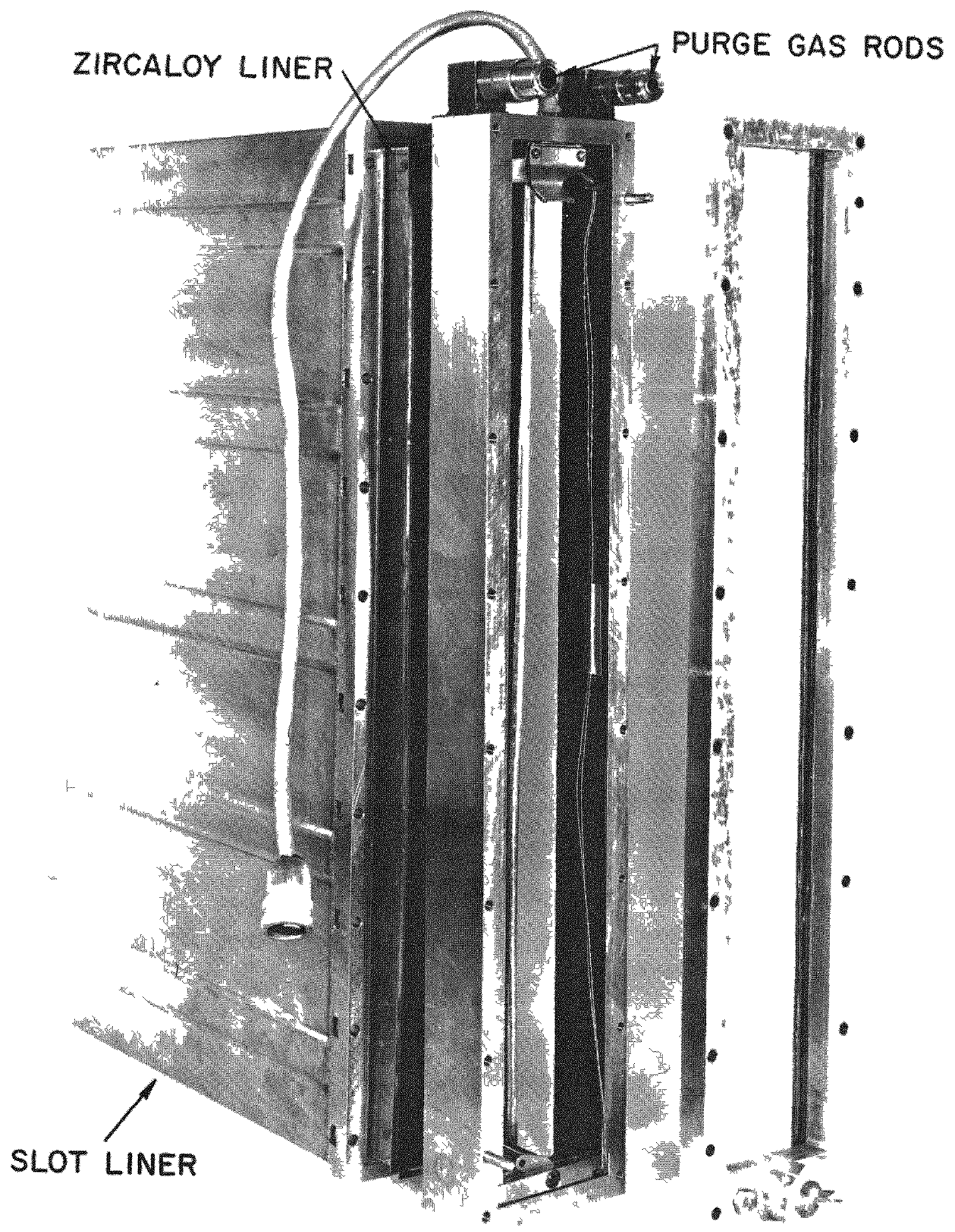

Figure 15. Exploded View of Slot-Liner. 


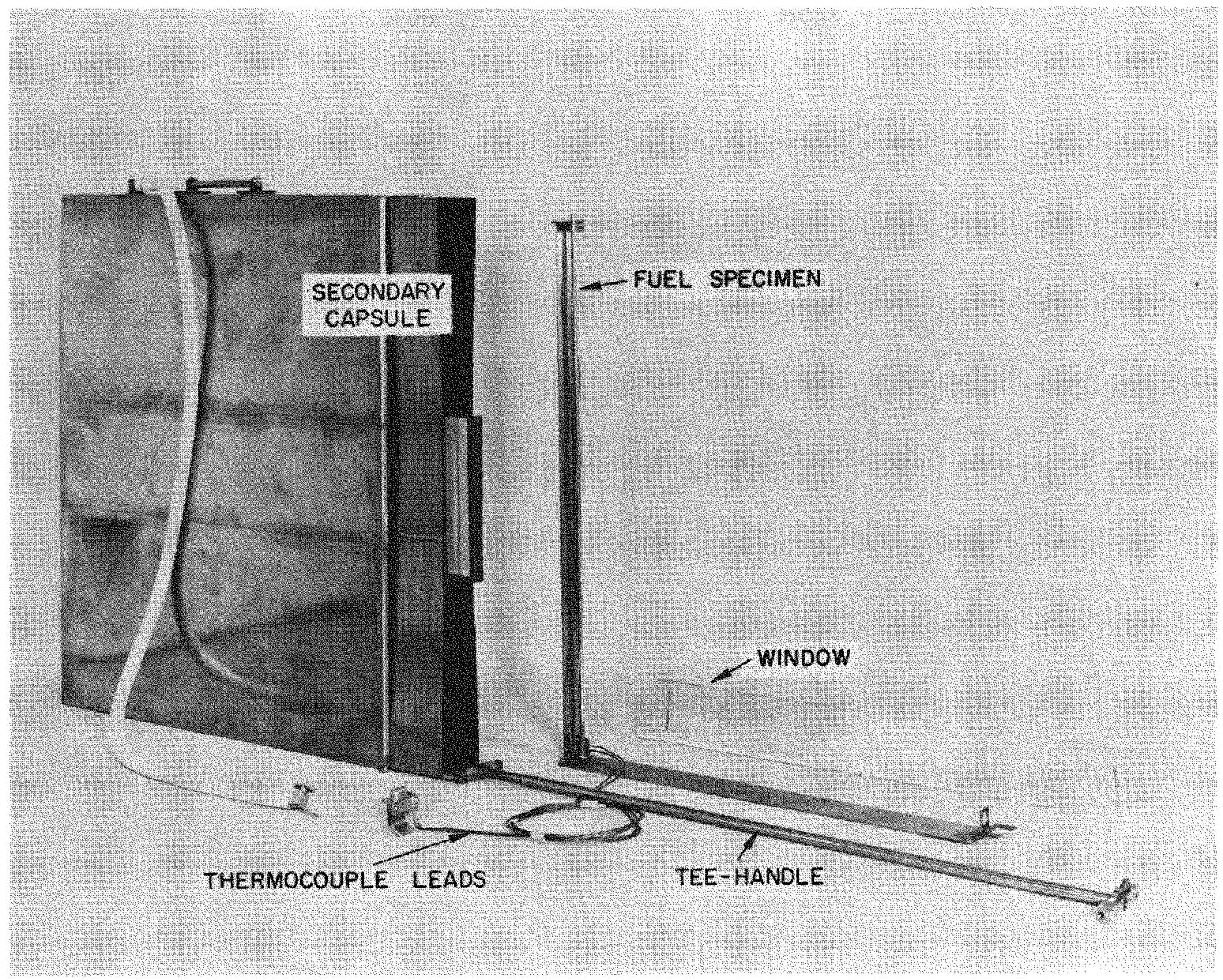

Figure 16. Disassembled Test Capsule. 


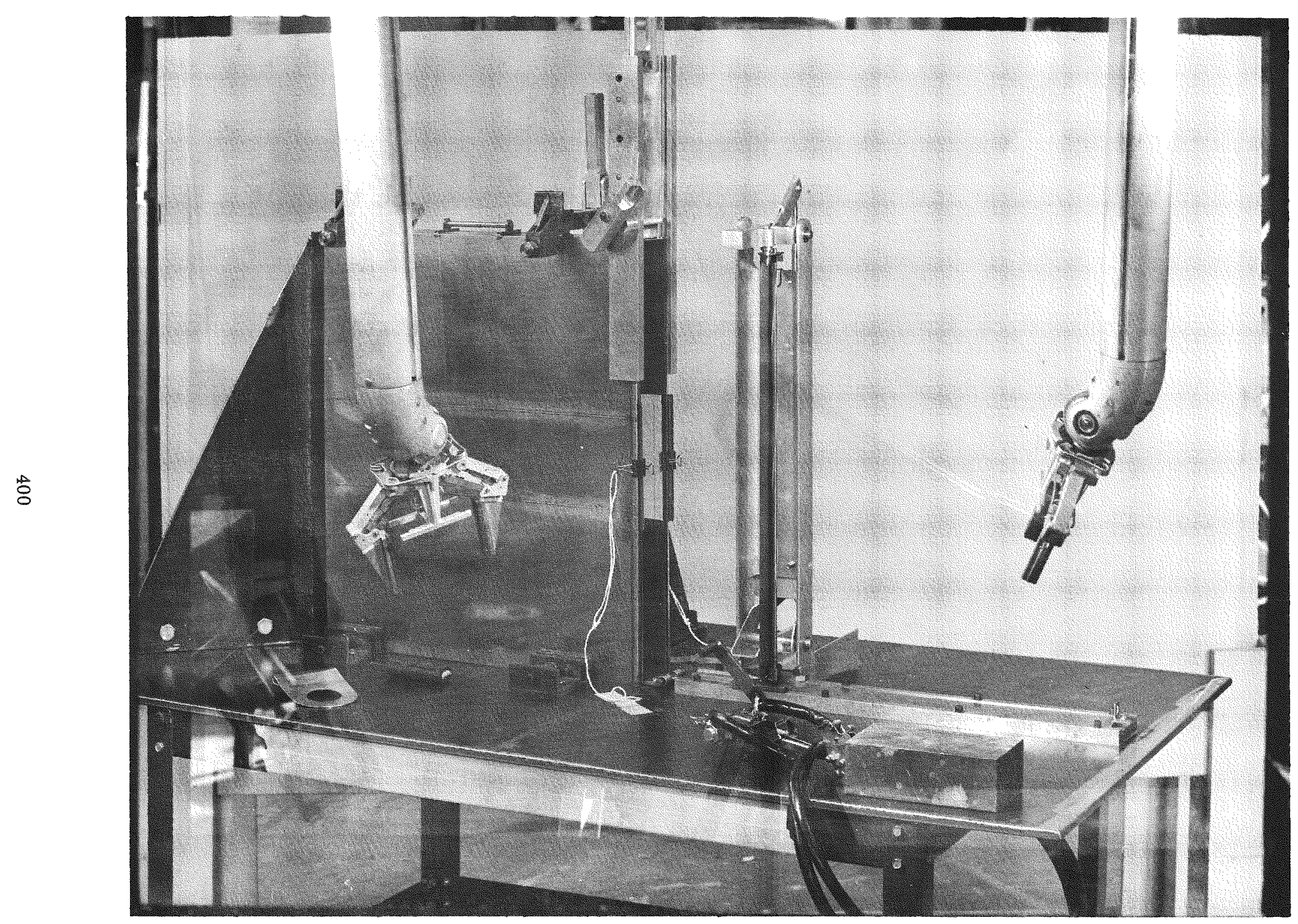

Figure 17. Remote Capsule Loading as Viewed Through Cave Window. 
Post-Irradiation Examination

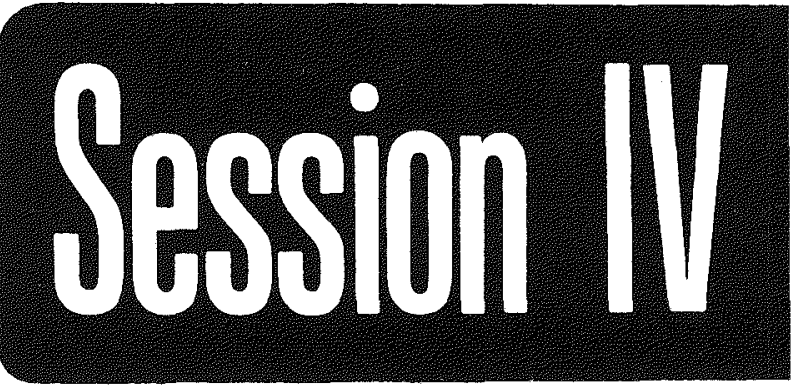




\title{
DUN.SA-108, 2
}

\section{IRRADIATED FUEL EXAMINATION AND MEASUREMENT}

\author{
K. B. Sowa \\ 26 Douglas United Nuclear, Inc.
}

\section{INTRODUCTION}

Underwater examinations and measurements of large quantities of irradiated fuel elements are performed at Hanford by the Fuel and Target Irradiation Technology Subsection of Douglas United Nuclear, Inc. In addition to special studies routine data are collected that include measurements of weight, length, inside and outside diameters, corrosion profiles and ultrasonic scanning. Over 7000 of these examinations and measurements are performed each month in support of programs to improve reactor operating parameters and fuel designs. A facility consisting of four underwater basins has been used to examine fuel elements from the older Hanford reactors since 1956; a separate facility was installed with the new $\mathbb{N}$ Reactor and is used for examinations and measurements of $\mathbb{N}$ fuels only. The large quantity of data from these facilities is occasionally supplemented by hot cell studies.

This report emphasizes two measuring techniques that approach a "zero wear" concept with respect to reliability in hostile environments. They are applicable to postirradiation measurements of test capsules.

DIMENSIONAL MEASUREMIENTS

At least five different methods are now used in the llanford basins for obtaining dimensional measurements.

1. Optical comparators

2. Hydraulic gaging

3. Ultrasonic transducers (N fuels)

4. Strain gage transducers

5. Variable reluctance transducers (N fuels) 
Of the five methods, the first two have demonstrated an operating reliability and inherent simplicity that are of special interest and are the subjects of this report. The three transducer gaging methods, ultrasonic, strain gage and variable reluctance are used in applications where optical or hydraulic gaging is not immediately feasible. These transducer systems generally require significant amounts of maintenance because of the problems caused by water immersion and radiation exposure. Also, the instrumentation is usually more complex than that required for hydraulic or optical gaging. This report suggests that optical and hydraulic gaging techniques should receive a "first consideration" in designing underwater measuring equipment.

\section{Optical Comparator}

The optical comparator is similar to those found in many machine shops except that it is adapted for underwater measuring. It consists of an above water, ground glass screen connected by an air tube to a lower projection lens and immersed projection lamp. The fuel specimen is placed in a manipulator between the projection lamp and the projection lens and its magnified profile is seen and measured on the ground glass screen. Two optical comparators are in use. One utilizes a double lens technique (See Fig. I) so that both sides of the object can be seen as an image overlap on the screen, thus enabling a wide range of diameters to be measured at $25 \mathrm{X}$ magnifications without limitations imposed by the screen diameter. The other comparator has a single projection lens and is used to view the end profiles and to obtain length measurements. (See Fig. II)

In the double lens comparator each projection lens is remotely adjusted in the horizontal axis until it is in focus with the center of a premeasured standard. The lenses are then adjusted vertically until the image of the top surface and the image of the bottom surface are seen on the ground glass screen. Because of the backlighting used, these images are seen as shadows, without surface color or details. Further vertical adjustment of the lenses causes the images, or shadows, to overlap. This produces a dark band of reinforced shadow on the screen. The device is calibrated by adjusting the shadow width to a predetermined value while the standard is being viewed. Placement of an unknown diameter fuel element in the manipulator will create a shadow width change that is proportional to the difference between the fuel diameter and the standard diameter. The shadow is measured on the screen with a sliding cursor that is mechanically linked to a digital counter. Each suel element is indexed so that diameter measurements are taken at five locations along the fuel axis. These measurements are referenced from a fixed plane so that warp values may also be calculated. The data are treated by a computer program which calculates fuel profile changes by comparison with preirradiation data. 
The single lens comparator is calibrated by placing a known length standard in the manipulator and adjusting a reference stop until the end profile of the standard is at a preselected location on the grid chart screen. The lengths of fuel elements are then determined by placing the fuel against the reference stop and measuring the change on the ground glass screen.

One principle advantage of these optical devices is the ability afforded the operator to observe and avoid surface anomalies that would otherwise affect a mechanical gage; the operator will take his measurements only from the base surface adjacent to the defect. If the anomaly is significant enough, it too can be measured in profile.

Light sources for the comparators consist of 1000 watt projection lamps with condensing lenses assembled in an underwater, removable lantern. The condensing lenses provide a parallel beam of back lighting that is necessary for sharp images. No attempt is made to insulate the lamps or condensing lenses from the water, the lantern housing must be electrically grounded, however. All underwater lenses and windows are made of nonradiation darkening glass.

Without mechanical contact with the part being measured, the optical comparator has a high degree of reliability. The double lens comparator has been in operation since 1956; eventual replacement of the fuel manipulator in 1967 was the only major maintenance required.

The optical comparator can be adapted for more generalized measurements of irradiated specimens through careful design of an $X-Y$ manipulator. The precision of this equipment is about equivalent to shop comparators of equal power and is somewhat dependent on the operator who measures the ground glass image. Standard deviations of approximately $.002^{\prime \prime}$ are normally encountered when measuring $1.5^{\prime \prime}$ diameter fuel elements.

\section{Hydraulic Gaging}

The principles of hydraulic gaging, similar to those of pneumatic gaging are applied at Hanford to obtain internal diameters of fuel elements. These measurements are obtained with acceptable precision (a standard deviation less than .001") and low capital investment (the original prototype was fabricated at a cost of approximately $\$ 200$, using standard pressure reducing valves and gages). This gaging method shows promise of application to other underwater measuring problems.

Hydraulic gaging is a means of measuring static or dynamic dimensions by the use of liquids in motion. Water is the liquid considered here because it is also the shielding medium for the fuel elements to be measured. The principle of water gaging is demonstrated when one notices the increase in back-pressure as a water jet nozzle approaches 


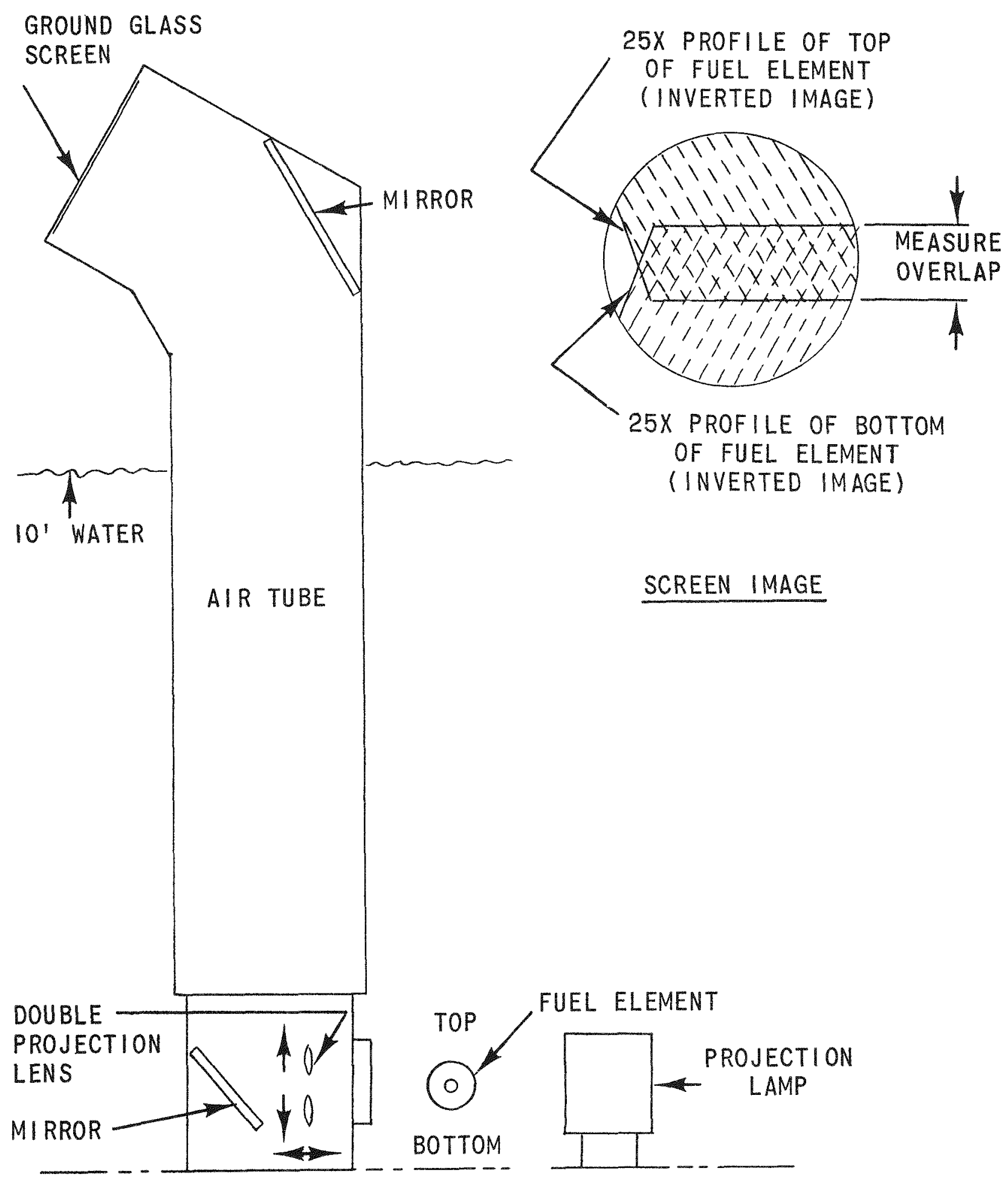

FIG. I 

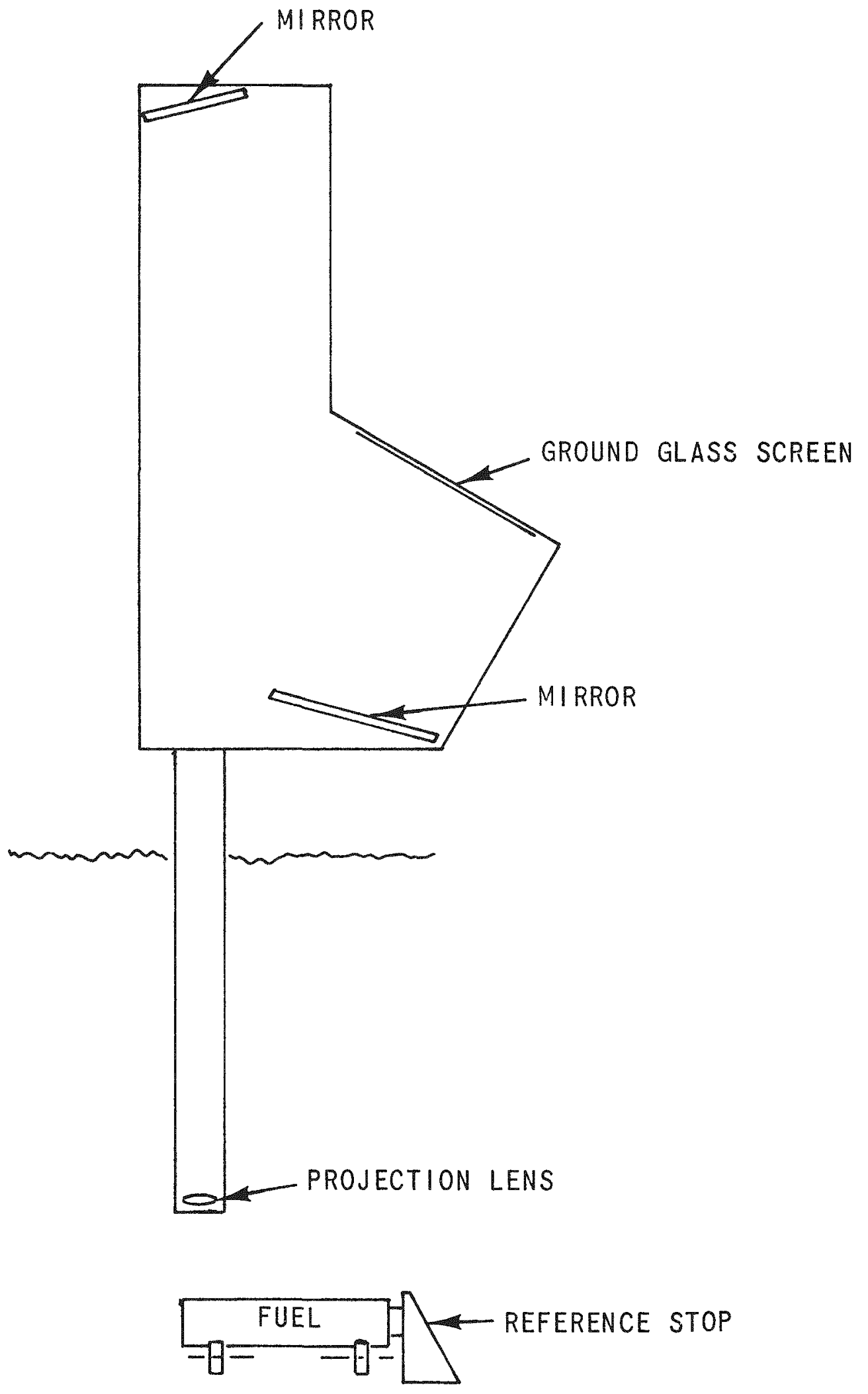

FIG. II 
a plane surface. Measuring this restrictive effect is readily accomplished with conventional hydraulic systems and gages. Because the signal pressure from the measuring nozzle can be sent through almost any hydraulic passageway to the readout system several feet away, hydraulic gages permit greater flexibility than mechanical gages. The ability to purge dirt or loose film is an obvious advantage in obtaining repeatable measurements with a water jet.

Utilizing the same principle as an orifice. a simple gaging nozzle has a water flow that varies proportionally with the outlet area. The nozzle in Figure III illustrates the variable area as an annular restriction. Since the flow of water through this restriction is proportional to its area it is also proportional to the quantity we wish measured, its clearance gap ' $X$ '. The size of the total variable area must remain small in comparison with the area of the nozzle bore in order for proportionality to remain well defined. In other words, if the variable area exceeds the nozzle area, the only significant pressure signal will be from within the nozzle. This relationship can be roughly illustrated by equating the nozzle area to the annular variable area; the nozzle shown in Figure III will be at the limit of its measuring range when the clearance gap is $1 / 4$ of the nozzle boxe diameter.

Figure IV shows the type of nozzle that is used to measure fuel element bores in the Fuel Examination Facility. In this application, the flow restriction is created between the cylindrical surface of the nozzle and the confines of the hole to be measured. The maximum difference between the bore diameter and the nozzle diameter that can be accurately sensed is approximately 0.020 " for bores that are $3 / 8$ of an inch in diameter. Centering of the nozzle is assured by the opposing outlets. Averaging the diameter of an elliptical hole is achieved by the water distribution groove that encircles the nozzle. Eliminating the water distribution groove, and using two opposing outlets, will permit measurements of elipticity.

Figure $V$ illustrates the hydraulic circuit that is presently used with the nozzles. In this system a constant pressure is maintained before an adjustable restriction. Bore restrictions encountered by the nozzle result in varying pressures downstream from the adjustable restriction. These pressure changes are seen on the dimension indicator, an ordinary Bourdon tube gage that can be calibrated to indicate total bore diameter.

If Delta $P$ denotes the difference between the free flow pressure and the measuring pressure, and Delta $D$ is the difference between the bore diameter and the probe diameter, then a log-log-plot of Delta D versus Delta $P$ is obtained as in Figure VI. It can be seen immediately that the slopes for all gages are equal. This slope bears out the basic orifice relationship. The displacement between the curves $(h)$ is due to the fact that the larger diameter probes encounter a 
smaller change in flow per mil of annulus, causing a smallex measuring range; hence, a shift in the curve toward the left is experienced. Gage No. 1 is smaller than gage No. 3, and so on.

Tests of precision and accuracy indicated a standard deviation capability of $0.0007^{\prime \prime}$ and a bias between operators of $0.0000^{\prime \prime}$. These results are well within the required precision of $0.002^{\prime \prime}$ required for valia data.

One disadvantage of a simple nozzle is apparent when widely varying dimensions need to be measured. Because of the narrow annulus measuring range of a single nozzle, several nozzles with overlapping measuring ranges are needed to measure the varying bores of the different fuel designs. This problem is solved, with a slight sacrifice in precision, by redesigning the nozzle to include a variable orifice. The orifice area is varied in proportion to the dimension being measured by the use of a tapered throttling needle. Using a flow measuring transducer instead of a pressure readout, and careful machining of the needle, will cause a linear output to be generated. The measuring stroke of the needle shown in Figure VII is approximately $120 \mathrm{mils}$, and allows the nozzle to measure bores from 380 to 500 mils. The precision of this device is dependent on the needle taper and the precision of the flow measuring technique; a long tapered needle will have less flow change per mil of measuring stroke than a short tapered needle and would require greater flow measuring precision. The flow transducer is a magnetic paddle wheel type that has an output of 55,000 pulses ( $5 \mathrm{mv}$ to $100 \mathrm{mv}$ ) per gallon of flow. The counting cycle time and supply pressure are adjusted so that one count is equivalent to a one mil change in needle stroke.

For the device show a count cycle time of approximately one second at a 30 psig supply pressure will provide a total count that is equivalent to the stroke in mils of the throttling needle. The count is accumulated on a $5 \mathrm{mv}$ sensitivity electronic counter and printed out. The precision of this device is limited primarily by the time cycle repeatability, which causes a standard deviation of $0.0017^{\prime \prime}$ for a.l1 measurements.

As might be expected with the addition of a throttling needle, some of the "zero wear" concept is sacrificed and the nozzle is more vulnerable to damage in an examination basin.

\section{CONCLUSION}

of the five techniques used at Hanford for underwater measuring, the underwater placement of electronic transducers will sometimes yield greater measuring precision but will also cause increased maintenance requirements. These maintenance requirements will seemingly increase in proportion to the "square" of the number of transducers placed underwater. On the other hand, optical and hydraulic gaging techniques have provided most of the large volume of post irradiation dimensional data at Fanford with a comparatively high degree of reliability. These two techniques can also be applied to underwater measurements of irradiated test capsules. 


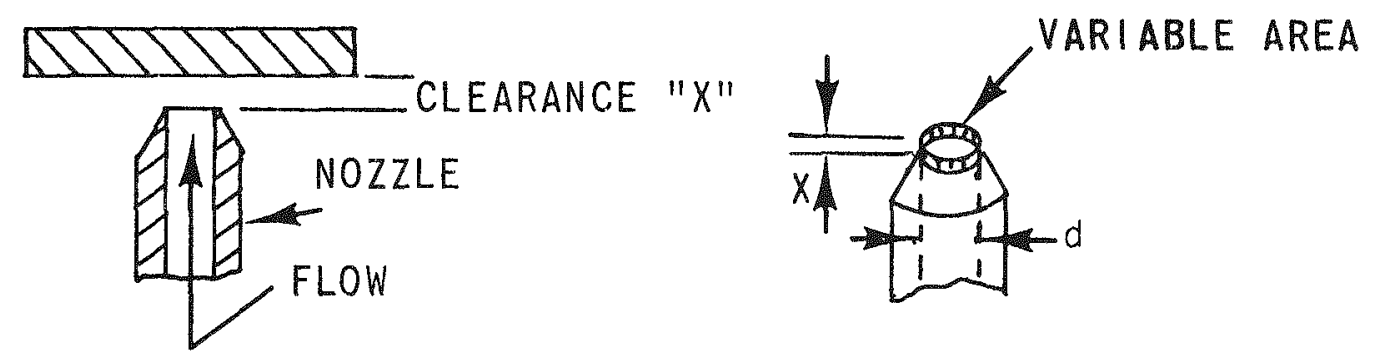

FIG. III

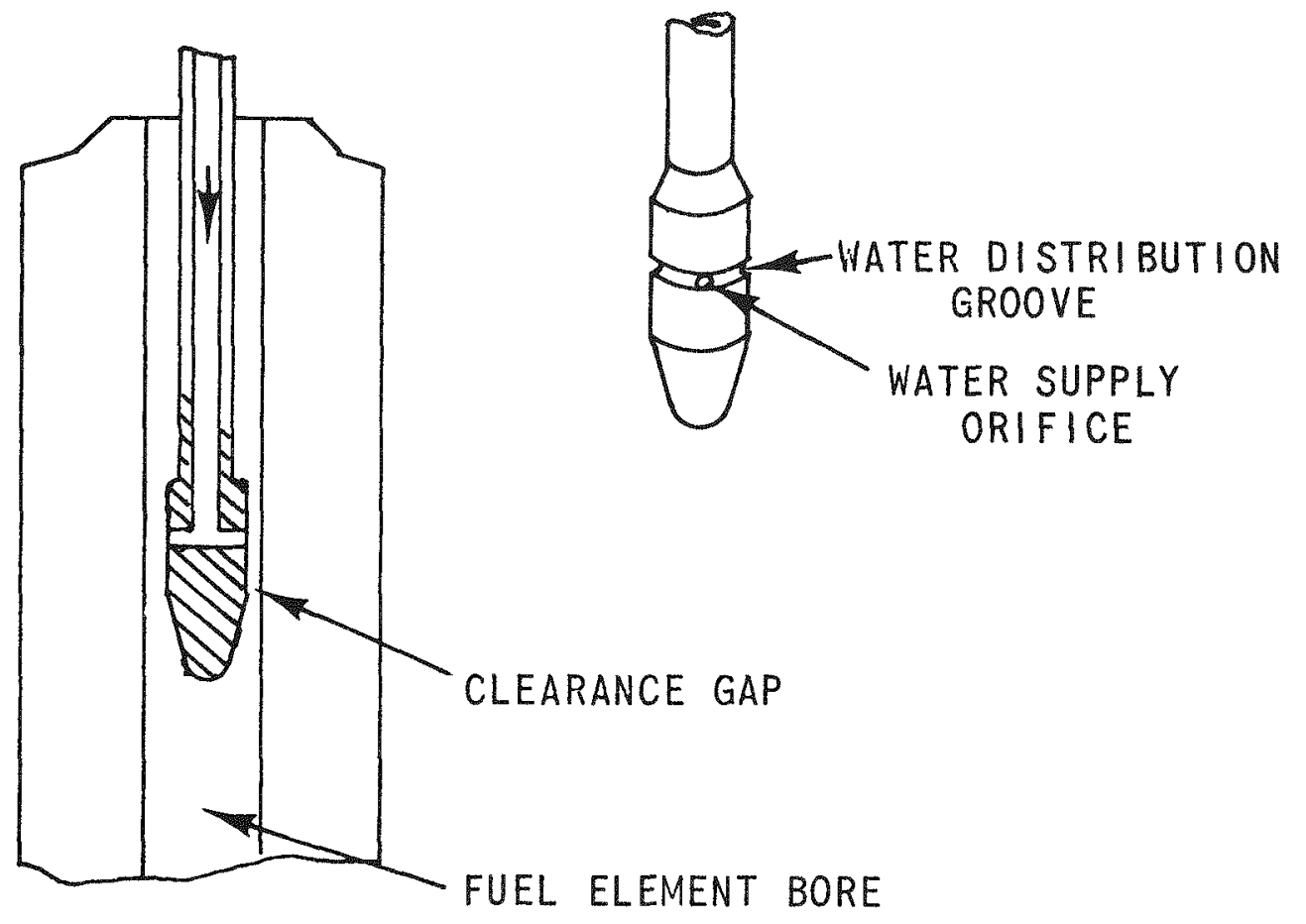

FIG. IV 


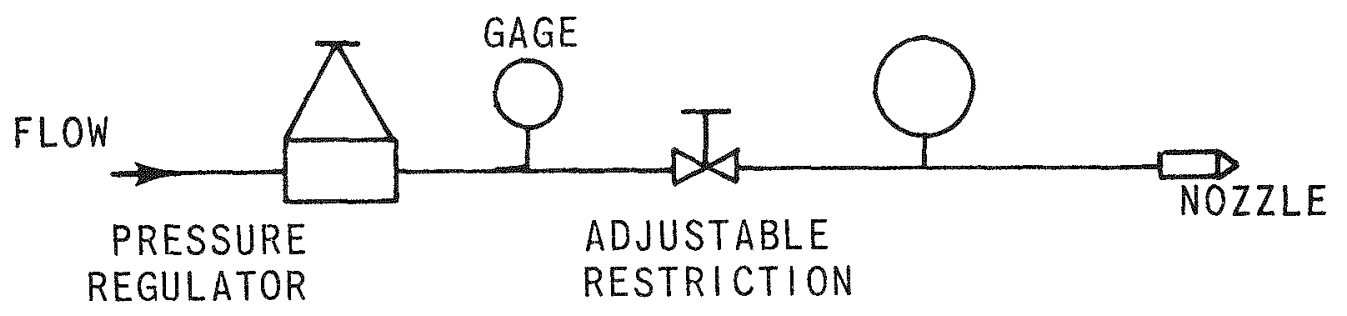

FIG. V

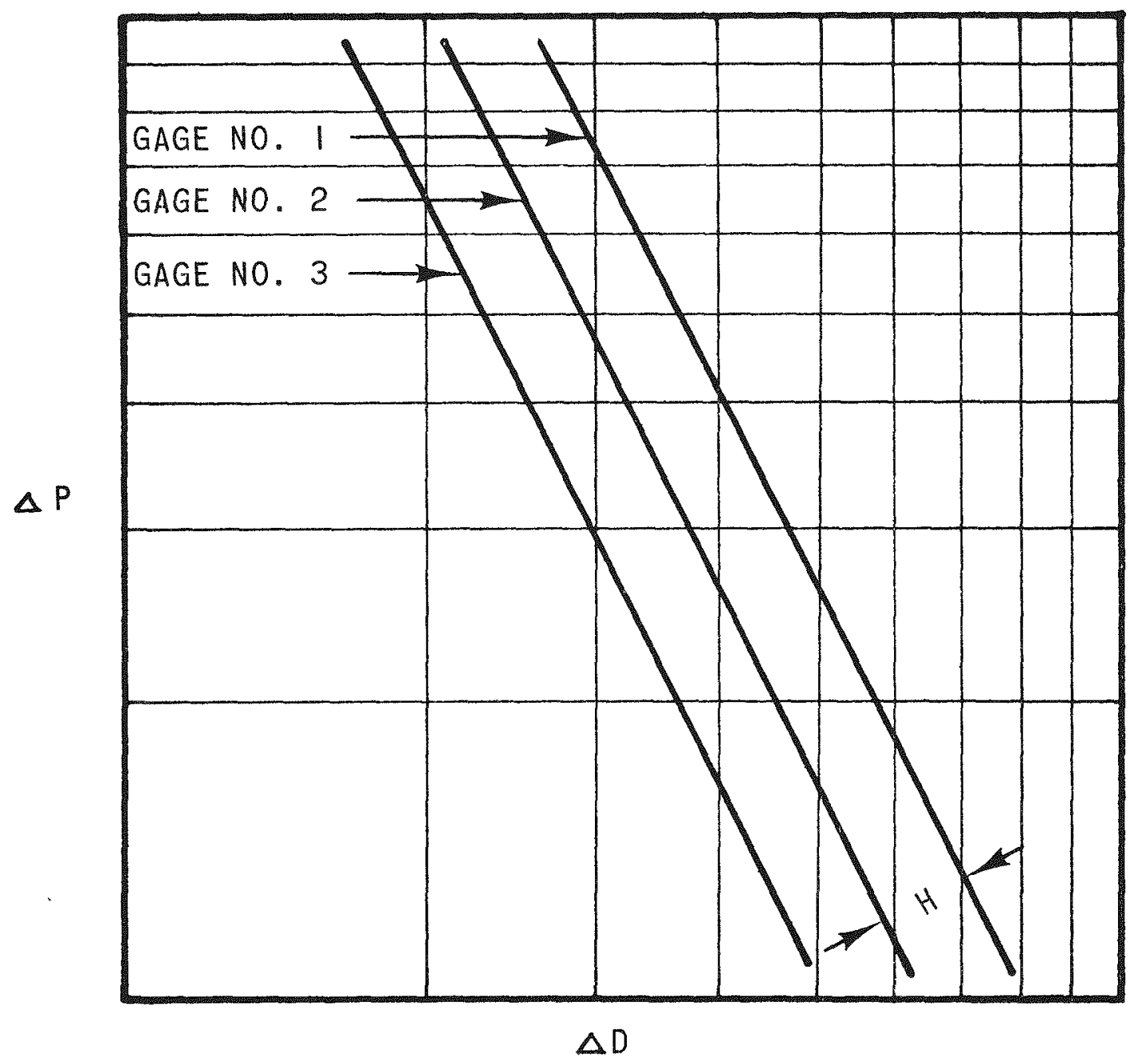

FIG. VI 

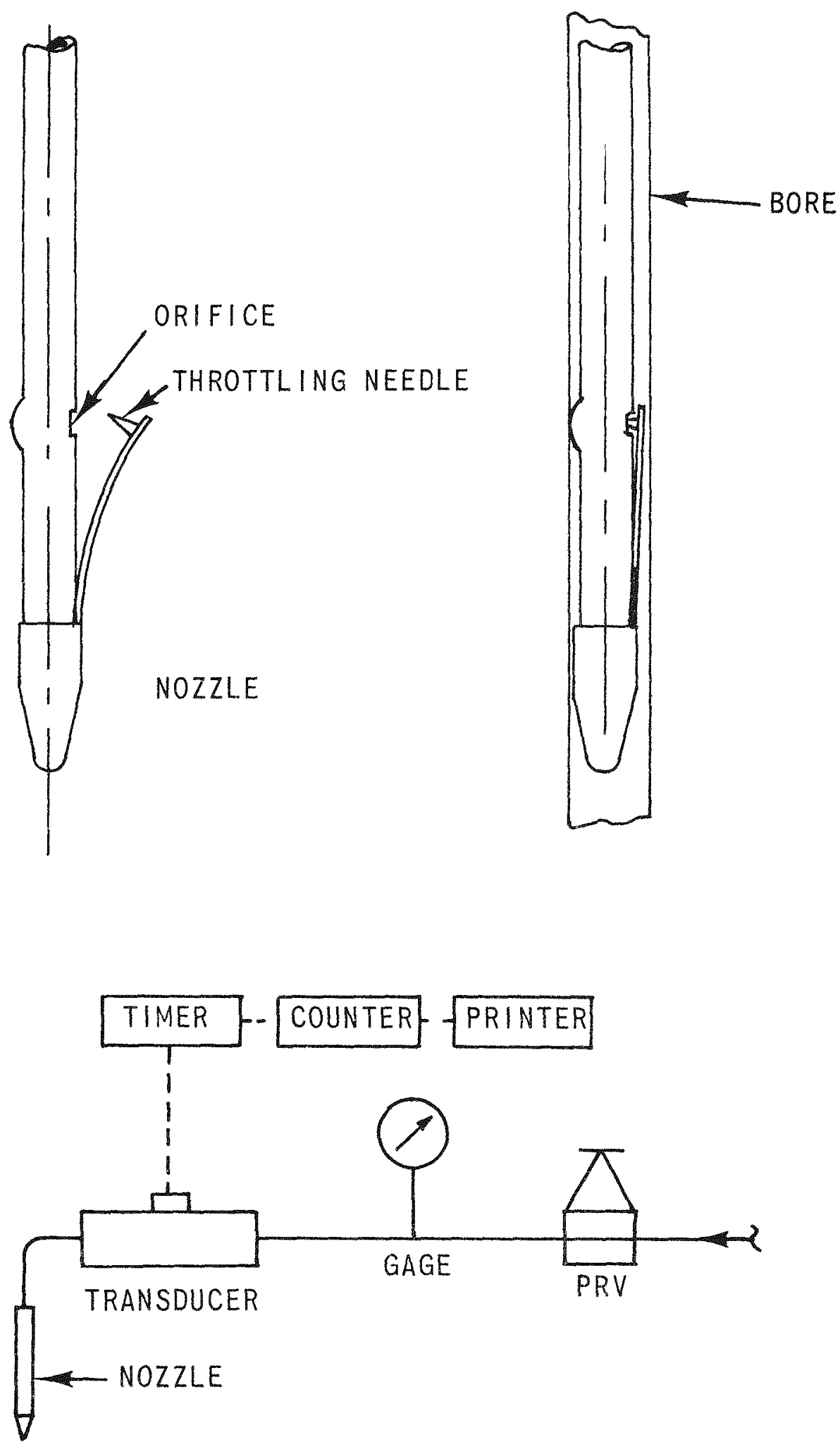

FIG. VII 


\title{
X PYCNOMETER, PROFILOMETER, AND LASER PUNCTURING \\ FOR SPACE REACTOR FUEL CAPSULE EXAMINATION
}

\author{
S. E. Dismuke
}

$6 / ?$ Oak Ridge National Laboratory, Oak Ridge, Tennessee, Operated by

Union Carbide Corporation for the United States Atomic Energy Commission

\section{INTRODUCTION}

Investigators at ORNL working on space reactor fuels have asked us for postirradiation data that could not be obtained with equipment on hand in the HighRadiation-Level Examination Laboratory. The first request was for the bulk density of swollen and cracked fuel pellets, the second was for continuous diameter and profile measurements on short ( $4 \frac{1}{2}$ in.) test capsules, and the third to puncture these capsules for gas collection without cracking the capsule wal1. Apparatus that was developed to obtain the required data is described in the following sections.

\section{PYCNOMETER}

This instrument was developed to measure the bulk volume as a step in obtaining the bulk density of porous fuel pellets in a master-slave manipulator equipped hot cell. The volume that is actually measured is estimated as that volume bound by the exposed surface of the sample and the area over cracks and pores less than $12 \mu$ wide.

Density measurements made using a low viscosity liquid are not always acceptable because the liquid penetrates the cracks and open pores resulting in a measured density approaching the material density rather than the bulk density. Mercury was chosen as the sample-enclosing liquid because it does not wet, amalgamate with, or react with the samples we wanted to measure. Mercury has a high surface tension; therefore, it does not penetrate the cracks and pores as much as most other liquids.

Figure 1 is a cross section of the pycnometer. A plunger, $P$, of known and uniform cross section is driven by a calibrated screw, $M$, into a mercury reservoir, $\mathrm{Hg}$, displacing mercury into an evacuated and sealed sample chamber and up a sight glass, S, to a mark placed above the level of the specimen chamber. Atmospheric pressure is applied to the mercury through the sight glass. The sample volume is determined by the difference in plunger travel between the filling of an empty sample chamber and one with a sample in it times the plunger cross-sectional area. In one model volumes up to $2.5 \mathrm{ml}$ are measured directly with an accuracy of better than $.001 \mathrm{ml}$ and a sensitivity of .0001 $\mathrm{ml}$. 
Effects caused by the high coefficient of thermal expansion of mercury have been minimized: (1) by leaving a large mass of metal around the mercury chamber; (2) by taking a zero measurement before each sample measurement.

The sight glass bore is selected so that the minimum measurable plunger movement causes about a millimeter movement of mercury in the sight glass. In the $2.5 \mathrm{ml}$ model, the minimum measurable plunger movement is $0.0001 \mathrm{~cm}$; the plunger area is one square centimeter, which required a sight glass bore of $0.0356 \mathrm{~cm}$.

By tilting the vacuum chamber seal assembly, the specimen chamber is exposed for loading specimens and cleaning.

Samples are cleaned to reduce the particulate material that could contaminate the mercury surface and surfaces of the sample chamber seal.

A coil spring is inserted into the specimen chamber to hold the specimen above the mercury during pump-down preventing intrapment of air. The spring is adjusted so that the specimen is held high enough to be easily grasped by tweasers for remova1.

In another model that we are evaluating, the sight glass is replaced by a pressure transducer; pressure is applied by driving the plunger in until a desired pressure is reached.

The volume that is measured depends upon how well the mercury outlines the specimen without being forced into the pores. Porosity measurements are based on the relation

$$
D=\frac{-4 \sigma \cos \theta}{P}
$$

where $\mathrm{D}$ is the largest pore diameter that mercury will not enter, $\mathrm{P}$ the pressure, $\theta$ the contact angle between mercury and the specimen material, and $\sigma$ the surface tension of mercury. Using commonly accepted values for $\sigma$ and $\theta$ this equation becomes $D=0.356 / P$ where $D$ is in inches and $P$ in torr. This equation is not used for pressures much below atmospheric. The pressure we have used is atmospheric, thereby defining a volume bound by the exposed specimen surface and the area over cracks and pores less than $0.00047 \mathrm{in}$. wide. The types of samples that we have measured so far have been adequately outlined with only atmospheric pressure above the mercury. Future use of this instrument will require extending the pressure range to bridge larger cracks with lower pressure and surround very small particles with higher pressures.

\section{PROFILOMETER}

The purpose of this device is to provide a means for measuring in-cell diameter and profile of small ( $3 / 8$ in.-OD x 4 l/2 in.-long) cylindrical specimens. The specimen is held rigidly at each end and rotated slowly. At the same time, pneumatic sensing units are moved along the axis of the specimen. The motions of the sample and the sensing units are synchronized so that the entire surface is covered; a continuous recording is made of the dimensions. 


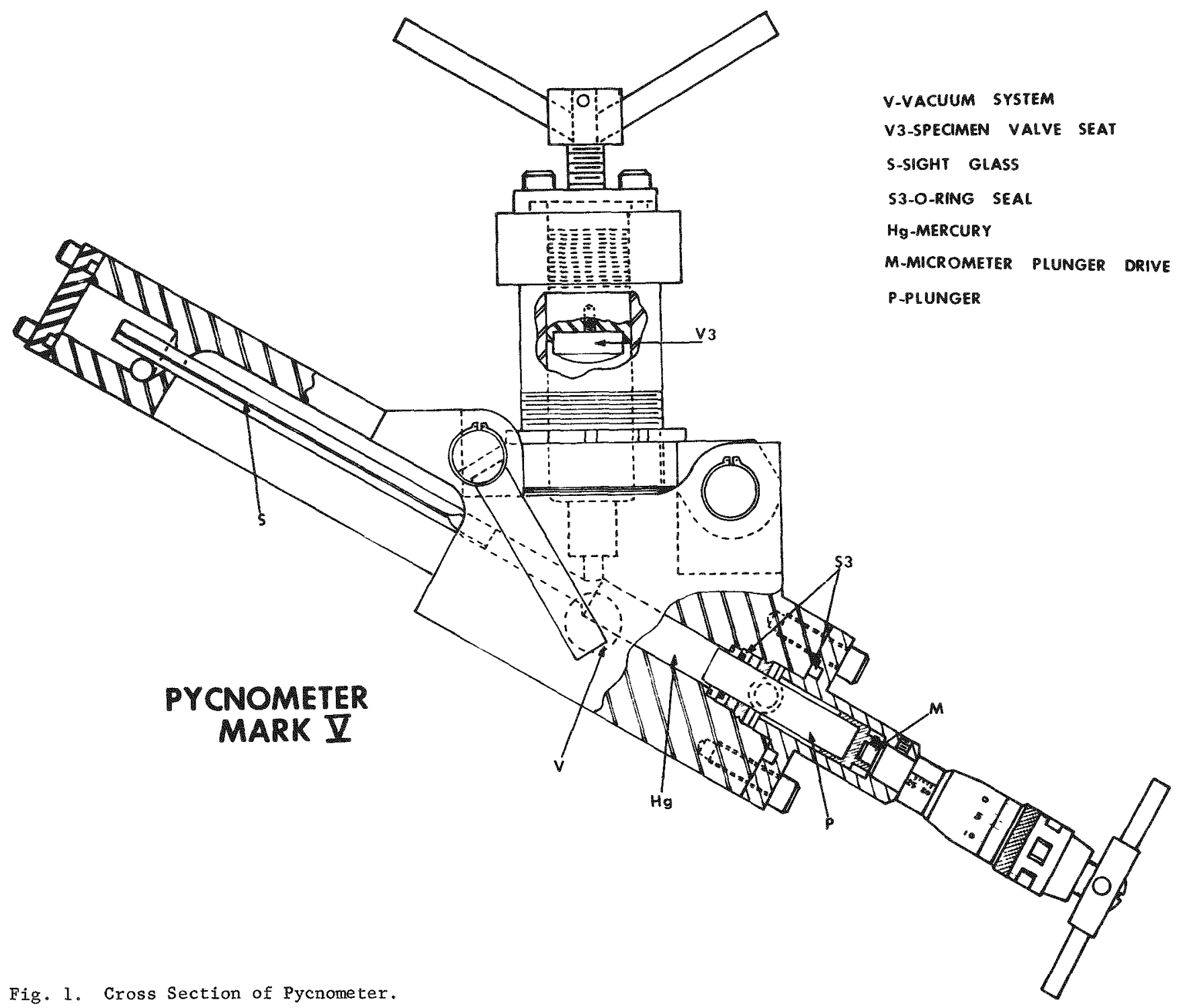


Figure 2 is a schematic drawing of the profilometer. The base of the positioning mechanism is of massive, one-piece construction machined from grey cast iron and stress relieved. This was done to obtain good dimensional stability. Both ends of the specimen are clamped to fixtures which are in turn held by magnetic chucks. The specimen is centered on the axis of rotation by adjusting the position of the fixtures on the magnetic chucks.

Motion of the carriage and specimen is provided by a lead screw with the drive motor at one end and a chuck holder at the other. The carriage (a bal1 slide assembly) is connected to the lead screw through a half nut that can be lifted up for disengagement and fast carriage travel. The chuck holder can be decoupled from the lead screw so that profiles parallel to the specimen axis may be recorded.

The sensors are mounted from the carriage at right angles to the motion of the carriage. One sensor is mounted rigidly to the carriage. It senses the position of the diameter measuring yoke, which corresponds to the profile of the capsule. The second sensor is mounted on the yoke. The yoke is supported by a bal1 slide assembly as shown in Fig. 2. Any side-to-side motion simply moves the entire yoke assembly and the diameter sensor sees no change. Any change in diameter does move the sensor stem and gives a signal change.

As shown in Fig. 3., the signal from a sensor goes to a booster relay, a variable ratio relay, and an adjustable bias relay. Then, finally it goes to the strip chart recorder. One cartirdge measures diameter and the other measures the profile.

The recorder chart drive is synchronized with the movement of the carriage and rotation of the specimen such that one revolution of the specimen gives $1 / 4$-in. chart level.

\section{LASER PUNCTURING}

An important part of the research for materials for reactor fuel and its cladding is the analysis of fission gases evolved from the fuel. The conventional method of puncturing a capsule has been either drilling or piercing.

With the investigation of brittle refractory alloys, it became necessary to find more suitable methods of puncturing. The piercing method is not practical because it can fracture or crush the capsule. Drilling was not considered desirable because of difficulties encountered with dynamic seals in the vacuum chamber and because of the difficulty of drilling through the hard capsule material. The laser beam proved to be ideally suited for piercing this type material.

The laser rod is in an elliptical cavity unit designed to operate with a single straight flash tube. The laser we used is a neodymium doped glass rod. The output energy is variable up to a maximum of 20 joules. Cooling is by forced air.

Figure 4 shows a schematic of the arrangement used to adapt the unit for hotcell use. The laser head is held by aiming screws attached to a bracket which is bolted to the cell wall. This provides a rigid mount and aiming adjustments. 


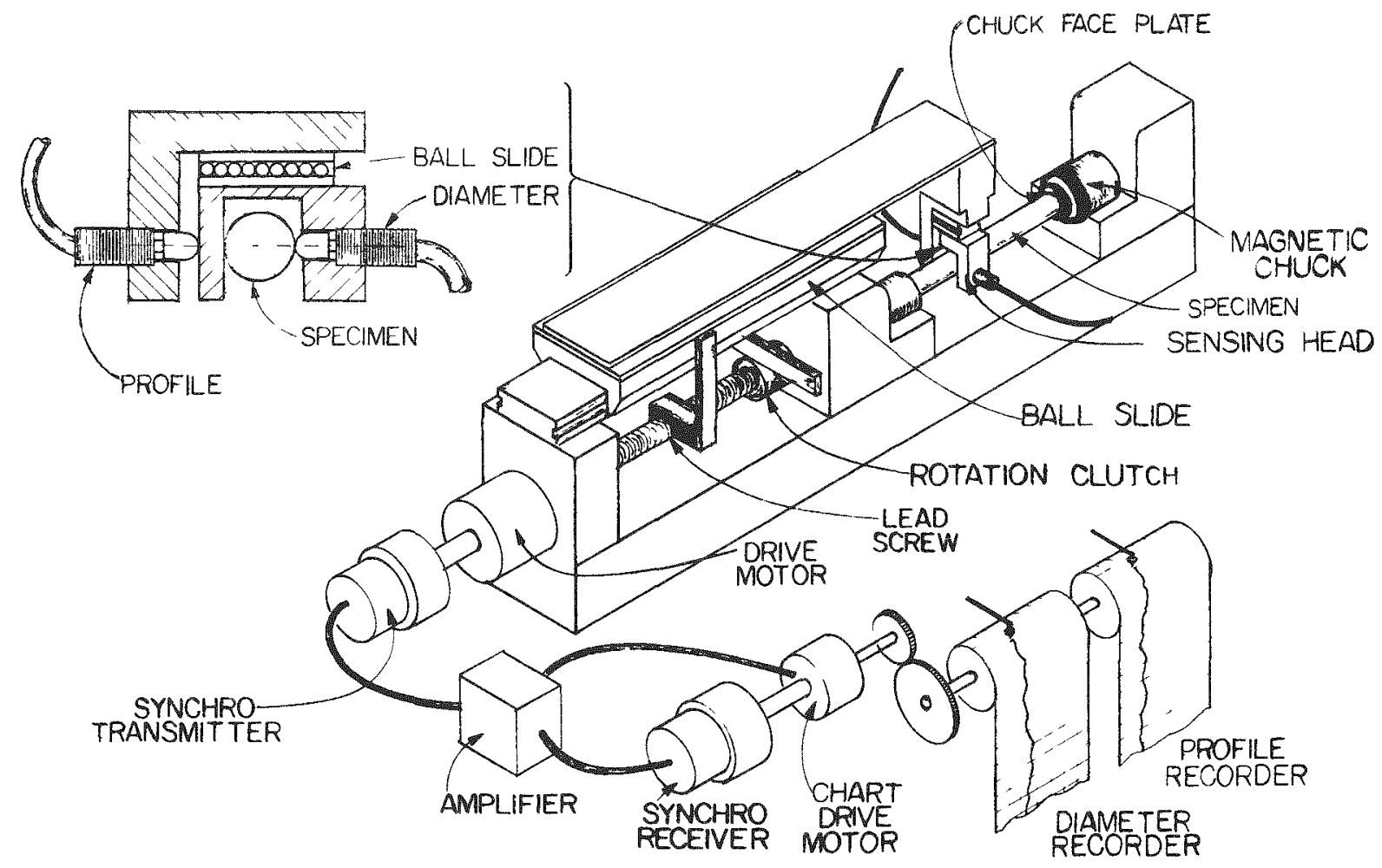

Fig. 2. Schematic Drawing of Profilometer.

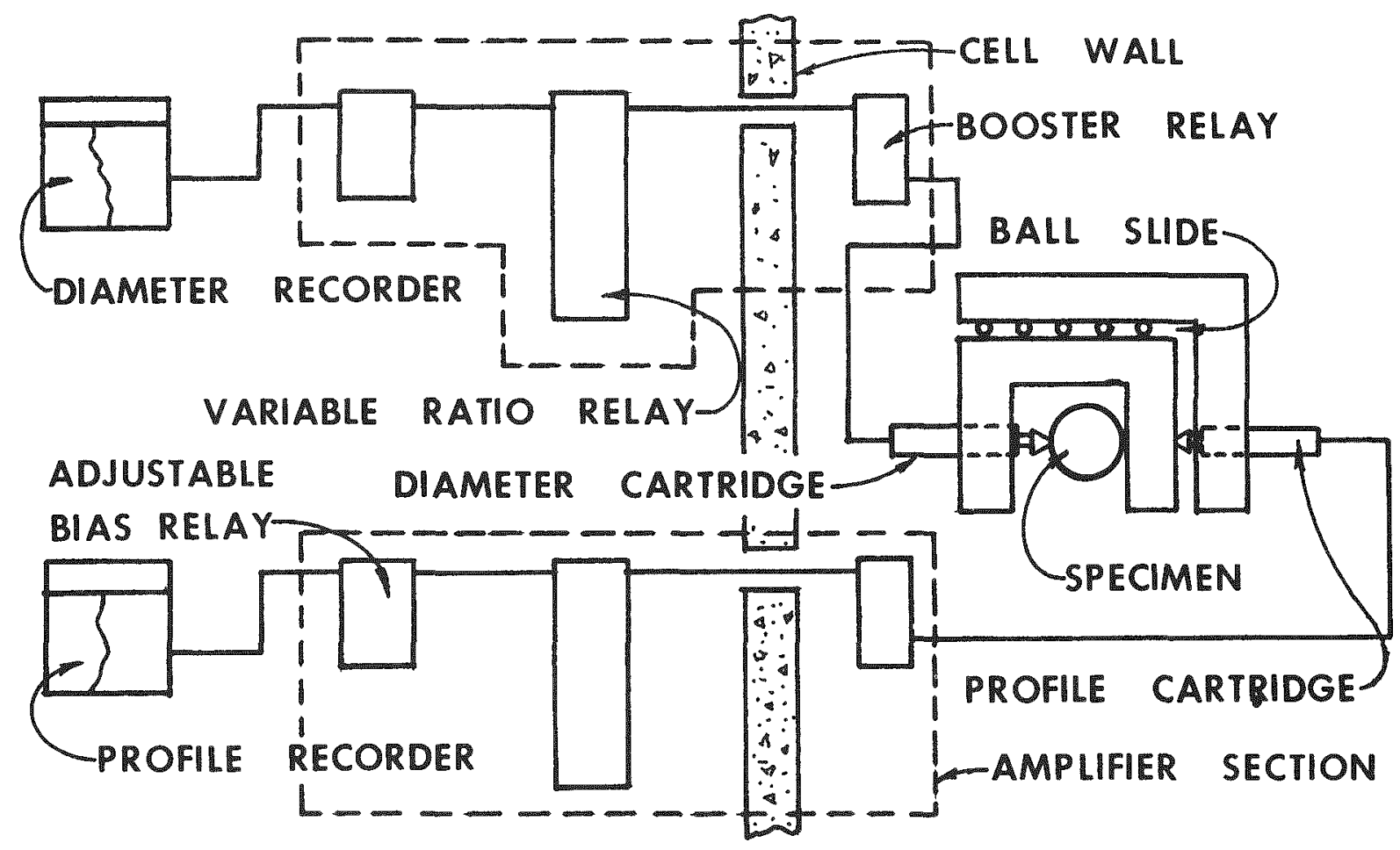

Fig. 3. Pneumatic Circuit Schematic for Profilometer. 


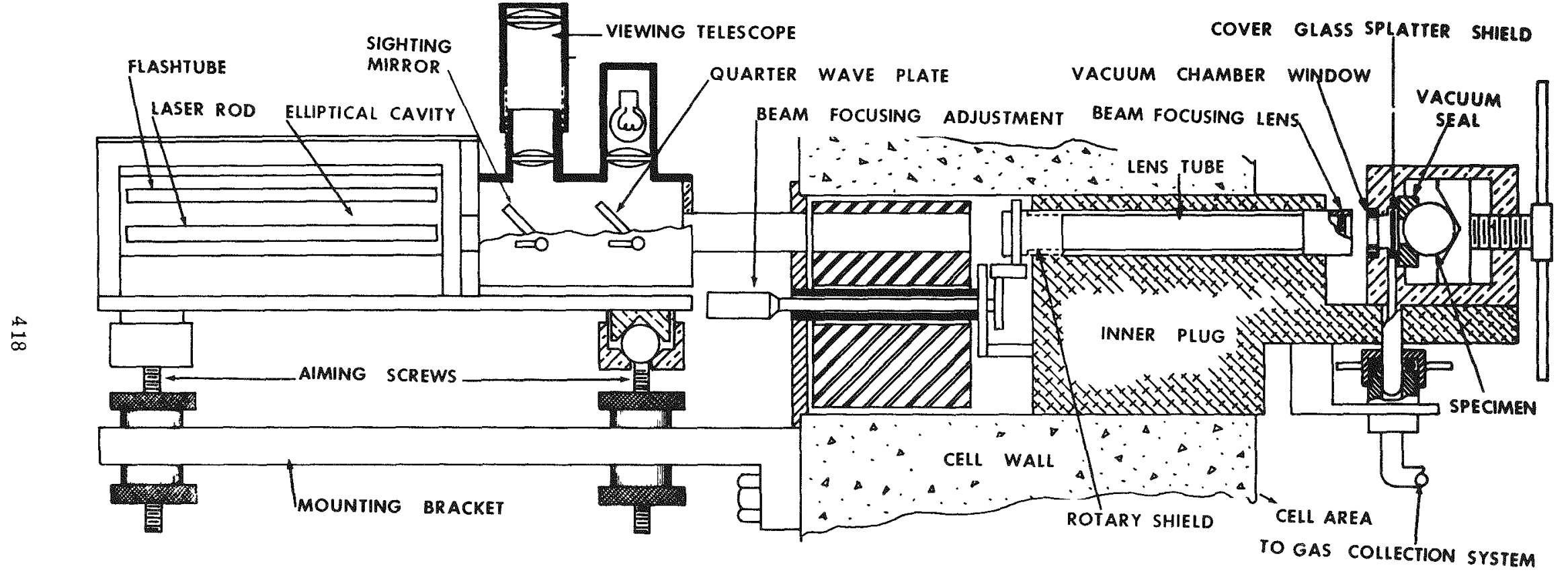

Fig. 4. Schematic of Arrangement for Laser Puncturing of Capsules. 
A telescope and lighting assembly was adapted to the laser head to facilitate aiming and focusing of the beam. A rotary steel plug (12 in. 1ong) is used to provide gamma shielding when the apparatus is not in use. The inner plug extends into the cell and forms a platform on which the specimen holder sits. The beam focusing lens is in the end of a tube that extends through the inner plug. The beam focusing adjustment consists of a micrometer screw linked through a gear train to the lens tube. The micrometer screw precisely locates the lens to focus the beam on the spot to be punctured.

The plug assembly is completely sealed for alpha containment. The specimen holder is designed to hold a specimen rigidly and to provide a vacuum chamber. Sealing the vacuum chamber to the side of the capsule makes the fixture readily adaptable to a wide range of capsule sizes. By contouring the sealing disc to fit the capsule, the holder will accommodate any diameter from $1 / 4$ in. to 2 in. The length of a capsule is unlimited as far as the holder is concerned. Sealing problems are minimized as there are no movable elements inside the vacuum chamber (i.e., drill or piercing needles). The splatter shield is necessary due to the splashback of molten and vaporized metal. After each puncture, the cover glass is coated with metal and must be moved away to be able to view the hole or fire the laser again. The vacuum connection is made when the specimen holder is positioned on the platform. A hollow stem, which doubles as a locating pin, slips into an o-ring sealed coupling. Fission gas is either expanded into, or pumped into, collection bottles for analysis. 


\section{K TECHNIQUES AND APPARATUS FOR INSPECTION, HANDIIING, AND ANNFALING HIGHLY RAD IOACTIVE HUEL MICROSPHERES * \\ 211,00 \\ M. T. Morgan and R. L. Towns \\ Oak Ridge National Laboratory \\ Oa.k Ridge, Tennessee}

\section{ABSTRACT}

Techniques and equipment have been developed for remote handing, inspecting, and annealing of coated nuclear fuel microspheres. The equipment is adapted for use in shielded glove boxes and includes a revolving microprobe stage with vibrator to turn particles over remotely, a suction pickup, wrist-action manipulators, a Geiger-Mueller tube probe, and inductiontype annealing furnace, and a separate gas collection and analysis system.

\section{INTRODUCTION}

One of the most promising nuclear fuels for high temperature gas-cooled reactors consists of pyrolytic carbon coated $\mathrm{UO}_{2}$ and $\mathrm{UC}_{2}$ particles in a graphite matrix. ${ }^{3}$ The coated fuel particles are microspheres, about 500 microns in diameter. The coatings serve to retain most of the fission products generated during irradiation. This function is dependent on various properties of the coatings, and techniques and apparatus have been developed for postirradiation study of the coated particles to aid in the development of better coatings. 2 These techniques can be applied to the remote handing and inspection of any small particles of similar size.

Postirradiation work on the coated particles includes transfer, separation, fission-product analysis, and heating to high temperatures to determine fission-product release through the coatings as a function of time and temperature. Microscopic inspection is necessary to count particles, to remove defective particles and trash from samples, or to separate coatings and cores of broken coated particles for individual analyses.

PREPARATION, HANDLING, AND INSPECTION OF IRRADIATED COATED PARTICIES

\section{Shielded Glove Boxes}

Postirradiation work on the coated fuel particles is performed in shielded glove boxes such as shown in Fig. 1. ${ }^{3}$ Two shielded glove boxes are used, one for preparation of samples and one for the annealing of samples. The glove boxes are shielded with four inches of lead and are

\footnotetext{
*Research sponsored by the U.S. Atomic Energy Commission under contract
} with Union Carbide Corporation. 


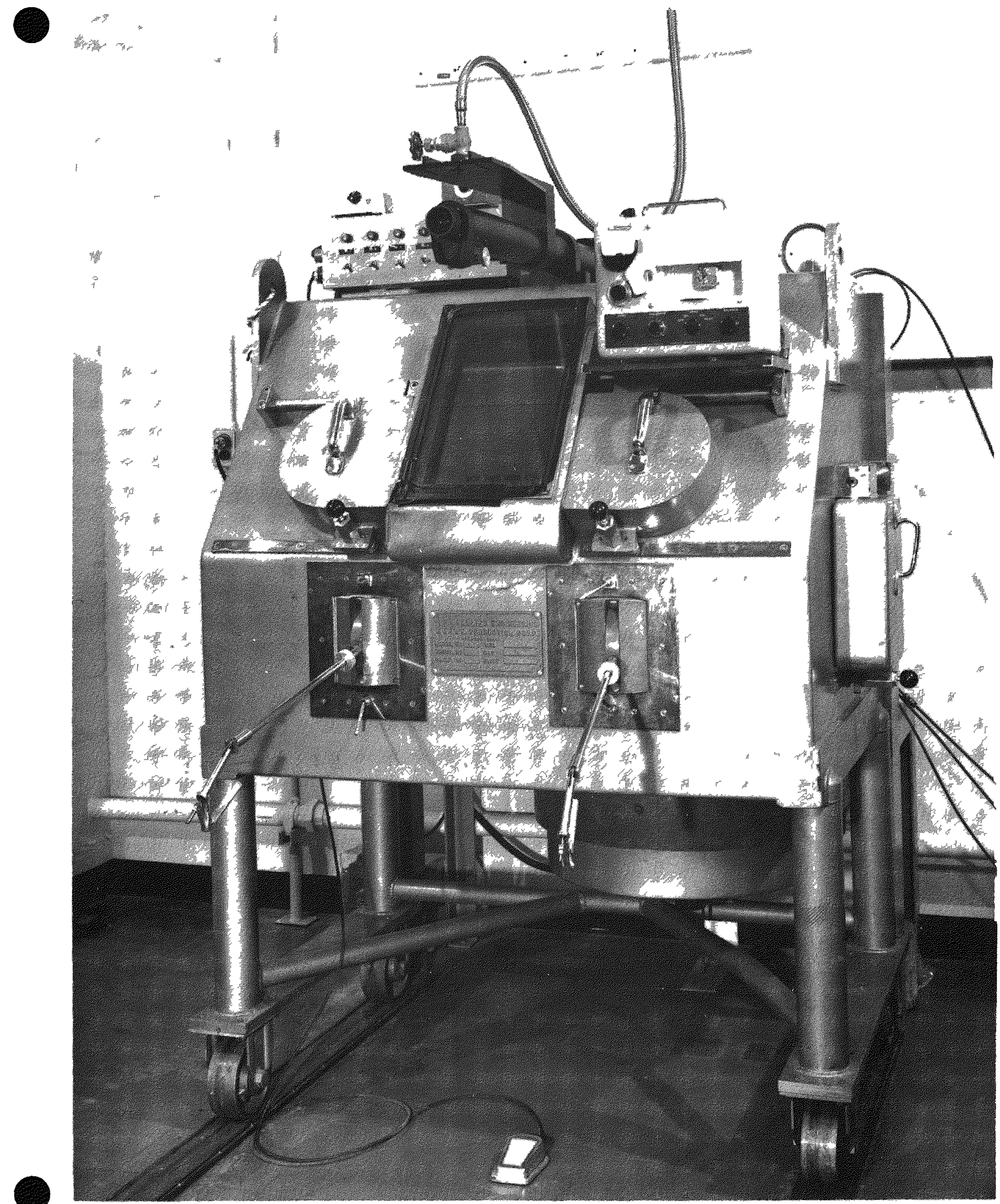

Fig. 1. Shielded Sample Preparation Box. 
Provided with special wrist-action manipulators. The effectiveness of the shielding was tested with a fuel sample containing 60 milligrams of ${ }^{235} \mathrm{U}$ irradiated to approximately $4 \%{ }^{235} \mathrm{U}$ burnup which had decayed for 38 days. The fuel sample had an activity of approximately 8 curies and was placed in the center of the floor of the box. Gamma activities at the outer surfaces of the box ranged from $10 \mathrm{mr} / \mathrm{hr}$ through the front to $4 \mathrm{mr} / \mathrm{hr}$ through the top of the box. Leakage through cracks was $20 \mathrm{mr} / \mathrm{hr}$ at the Castle joint of the manipulator and $44 \mathrm{mr} / \mathrm{hr}$ at the glove port shield. Higher activities were handled by using additional lead brick shields inside the glove box or by placing part of the samples in the storage facility. The glove ports are used only when radioactivity inside the box is below tolerance levels for the hands. The box interior ard the gloves are checked for beta-gamma activity before the gloves are used. Care is taken where tiny specks of concentrated radioactivity are present such as the irradiated coated fuel particles. The glove boxes are provided with a shielded well, originally intended for a centrifuge, but which was adapted for a sample storage facility. A cut-away sketch of the storage facility is shown in Fig. 2. The glove boxes are exhausted through a filter to a radioactive off-gas stack. The box interior is maintained at 1 -in. of water, negative pressure, and is exhausted to an off-gas stack through an absolute filter. The sample preparation glove box was placed on tracks as shown in Fig. I, so that it could be rolled forward to expos the interior box as shown in Fig. 3 for maintenance and for installation of equipment. The interior box is made of wood covered with a pheno.ic olastic. The shielded box as purchased is open at the back and is interued to be placed against some wall or structure for shielding. The rear wall shown in Fig. 3 was made of lead bricks supported in a steel framc. The shielded box as purchased weighed 9000 lbs.

The equipment for the sample preparation box shown from the back of the box can be seen in Fig. 4. In this view are shown the opening to the storage facility, the revolving periscope stage with built-in vibrator, the suction pickup, and the wrist-action manipulator. The objective end of the 30-power periscope above the revolving stage is not in the picture.

\section{Manipulators}

The manipulators are installed through Castle joints. The wrist action together with the special design of the fingers permits their use in opening screw-top bottles, and for handling tiny objects as well as large ones. These manipulators are not commercially available but were made locally.4 A cut-away sketch of the manipulators is shown in Fig. 5 .

\section{Transfer Cask}

Samples are transferred from the hot cells to the shielded glove box in a cylindrical shield shown in Fig. 6. This shield had 4-1/2 in. of lead shielding. To empty the carrier, the carrier is placed in line with the port of the shielded box and an extension rod is threaded into the transfer drawer and the drawer is pushed through the port of the shielded box. 


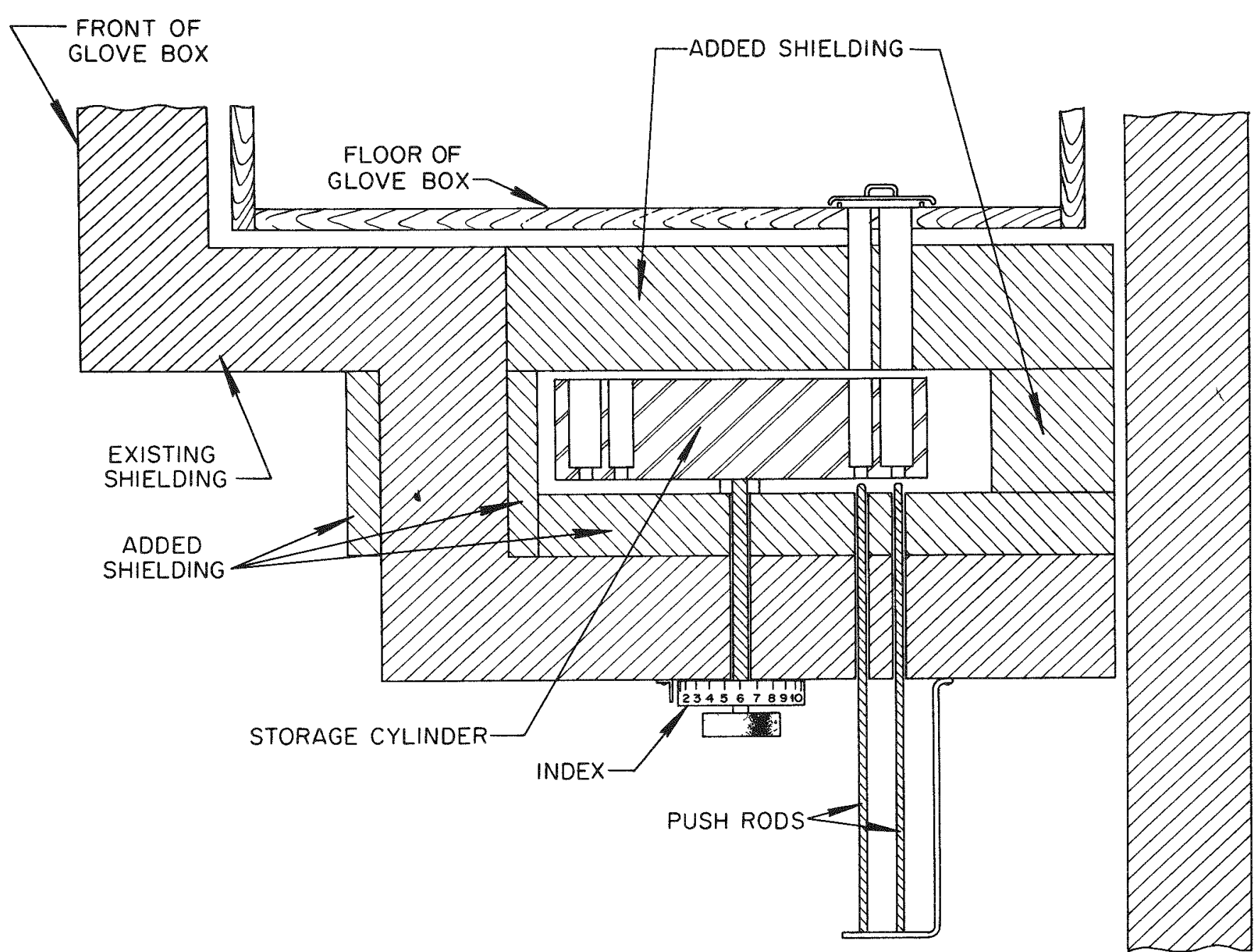

Fig. 2. Cross Section of Storage Facility Added to Centrifuge Well of Shielded Glove Box. 


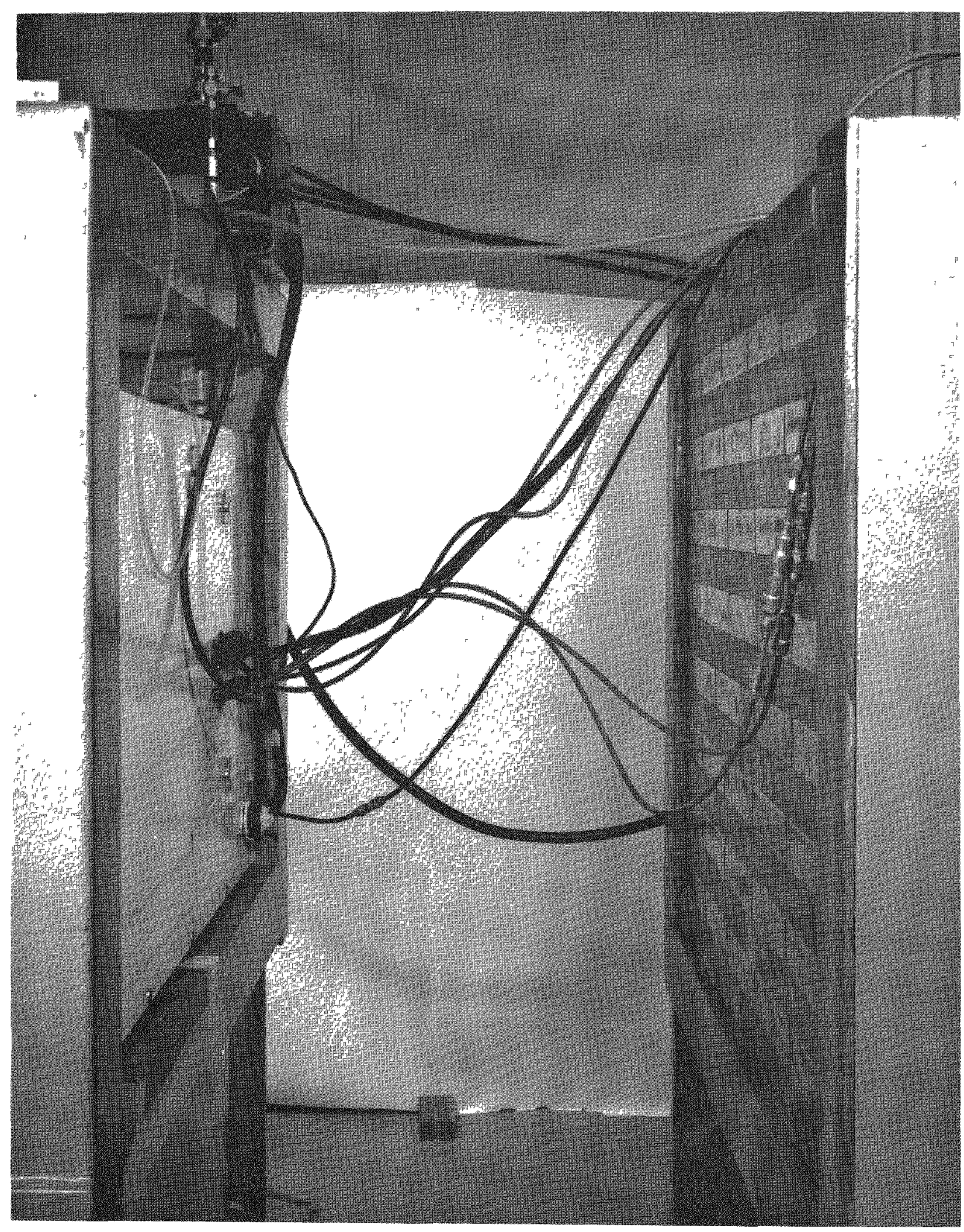

Fig. 3. Rear of Sample Preparation Box. 


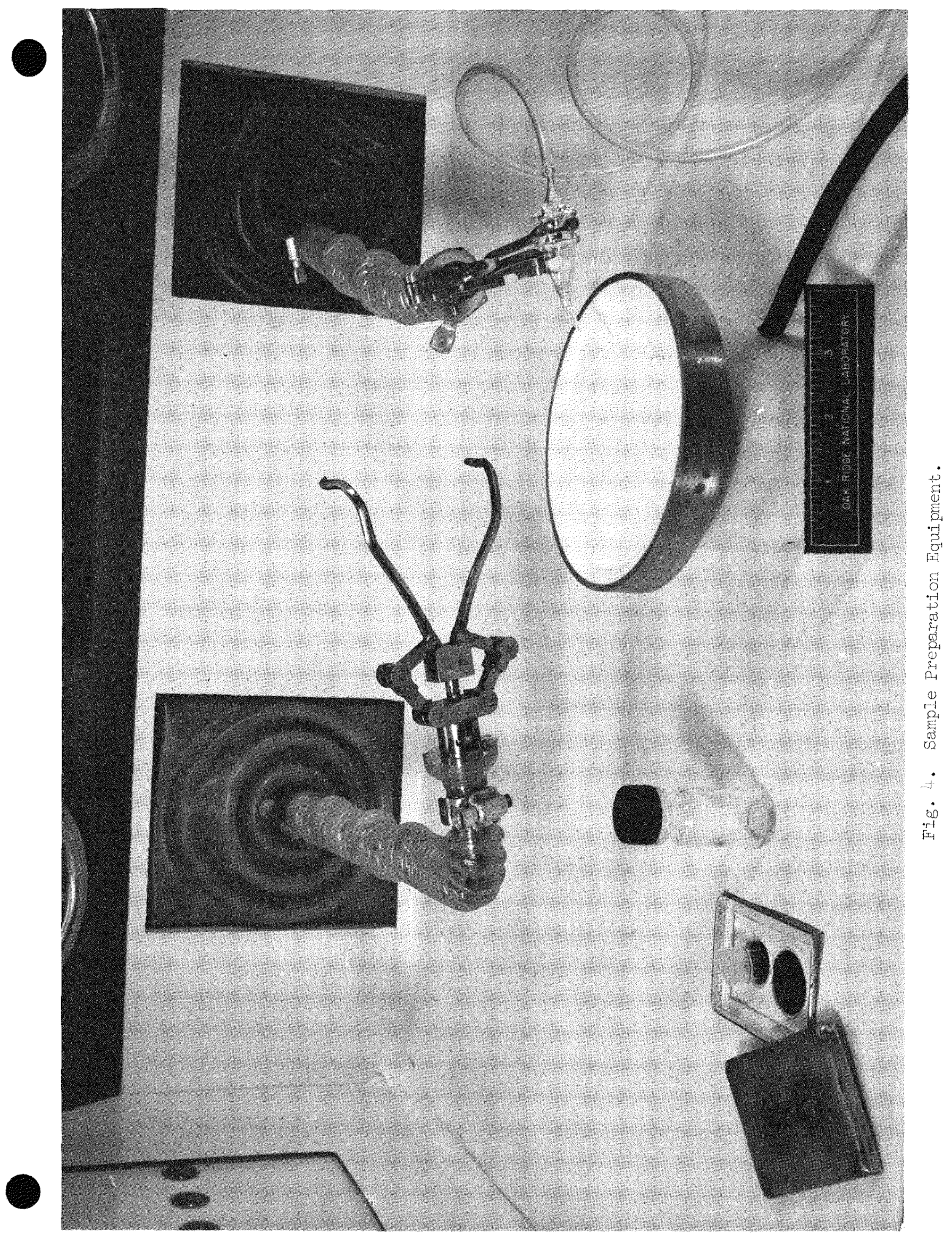


ORNL-DWG 69-387

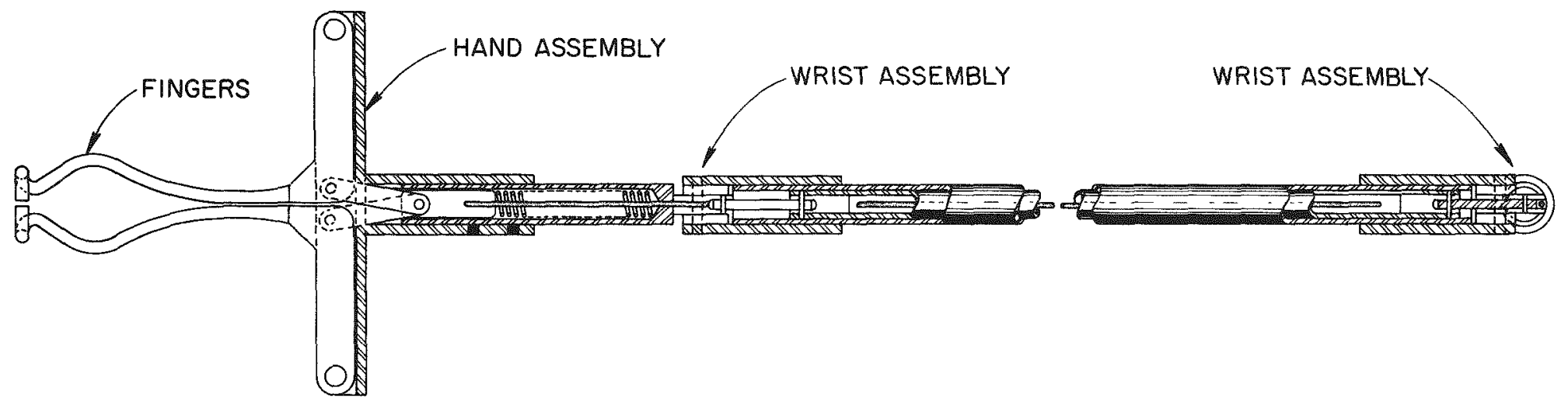

念
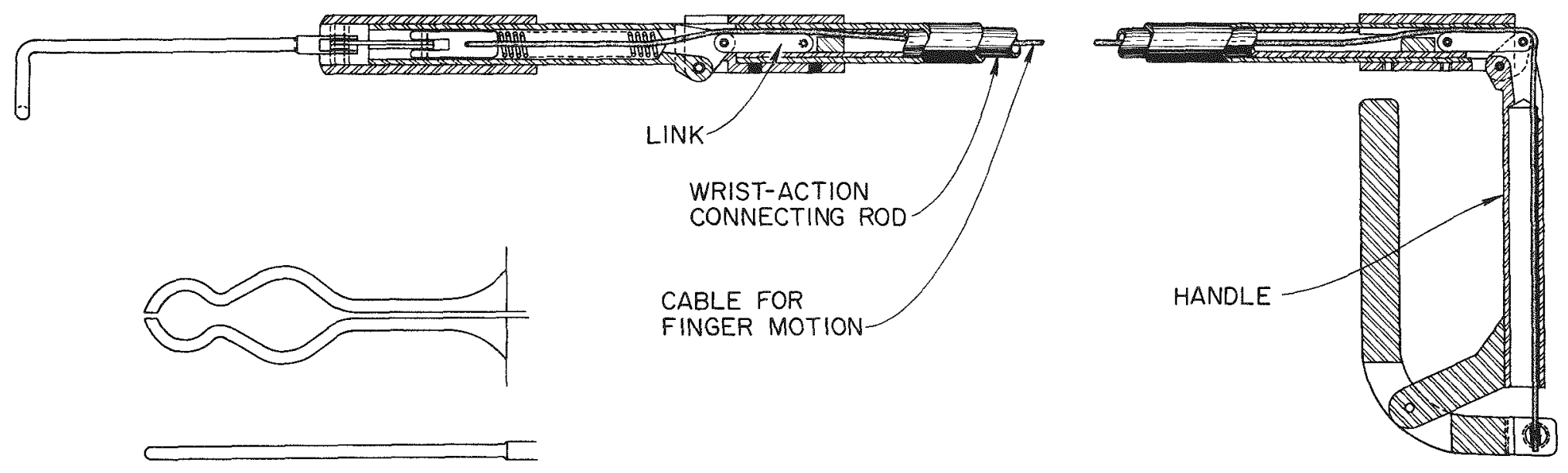

FINGERS FOR OPPOSITE ARM

Fig. 5. Cut-Away Sketch of Wrist-Action Manipulators. 

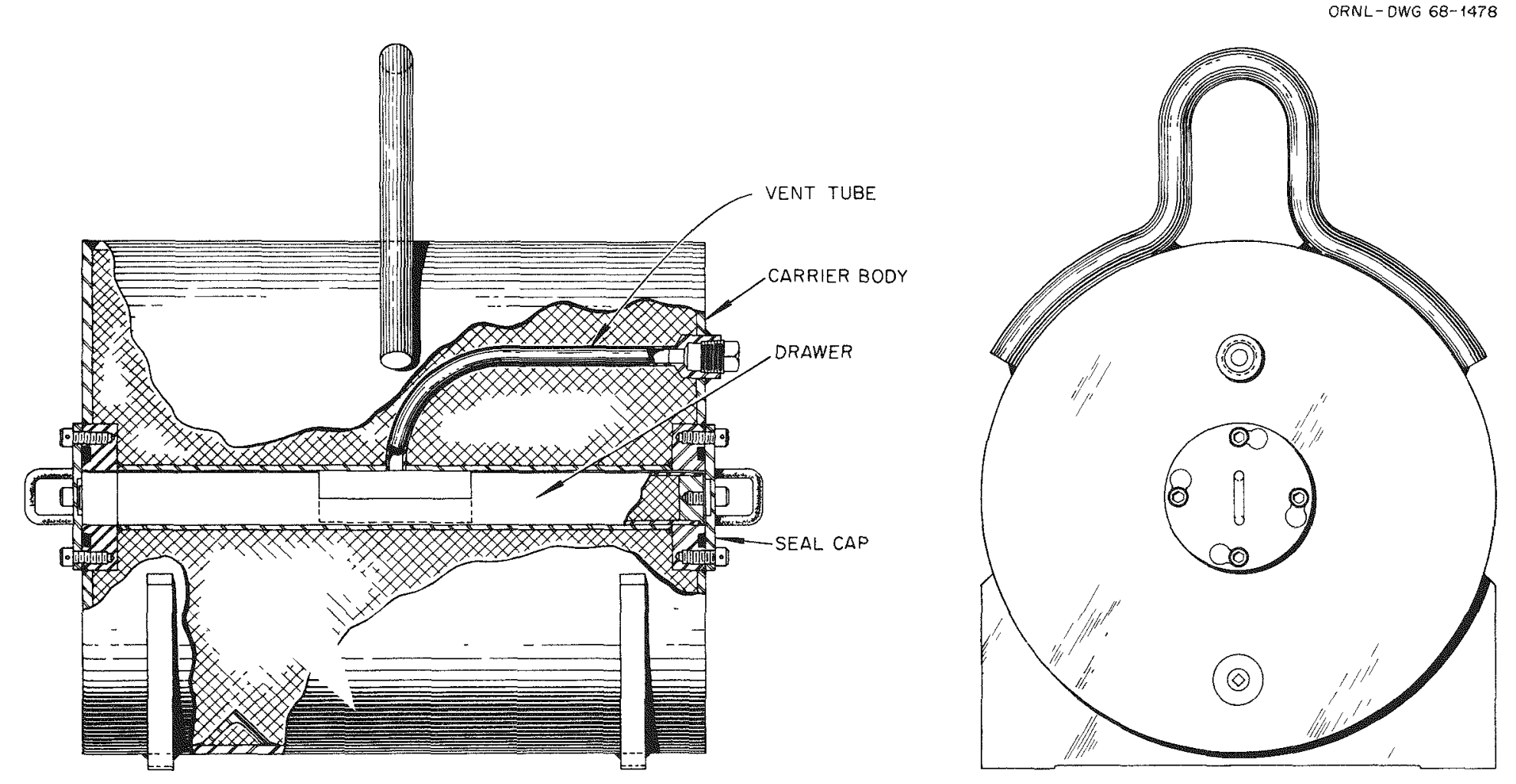

Fig. 6. Transfer Cask AssembIy. 


\section{Suction Pickup}

The coated particles are handled by means of a suction pickup shown in Fig. 7. An entire sample can be picked up and held in the pickup, then rolled out one particle at a time. As long as the coating is intact, no fission products are lost and the particles rarely become stuck in the pickup unless they have become coated with moisture or some other similar contamination. Carbon tetrachloride has been used to wash out particles and accumulated trash, but unless recovery of stuck particles is necessary, it is usually better to replace a contaminated pickup. If the coatings are fragmented and the particles exposed, the abrading action in the pickup removes some of the fission products and a small fraction of the coating fragments stick to the porous barrier in the pickup. Smaller tips to be slipped over the nozzle were made to pick up pieces of coatings and cores, one at a time at the end of the tip without abrasion. The pickup was proveded with flanges so that it could easily be held by the manipulators. The suction pickup is made from a glass filter tube with a 10-mm-diam fritted disk (porosity "B"), 5 and the end is drawn into a 1-mm-ID curved capillary tube.

\section{Periscope and Revolving Stage}

The coated-particle samples are inspected under a 30-power periscope. The outside end of the periscope installation can be seen in Fig. 1. The periscope focuses upon a revolving stage shown in Fig. 8, which has 36 hemispherical depressions around the periphery into which the coated particles may be separated. A vibrator located in the base of the stage will turn the particles over so that all surfaces may be inspected for cracks. Spherical particles turn over easily. Non-spherical particles may be bounced over by increasing the vibration amplitude. The stage is turned remotely by a servo motor. Two materials, teflon and alumina, have been tried for the surface of the stage. The teflon disk had microscopic fibers left in the surface after machining which sometimes interfered with the motion of the particles when they were vibrated to turn them over, but the teflon was still superior to the soft fired alumina disk which is hard to clean and which releases small specks of alumina to mix with the sample. Other materials may be better but the surface should be white and nonbrowning under irradiation.

\section{Lost-Particle Probe}

Particles lost on the floor of the shielded box are found with a Geiger-Mueller tube probe shown in Fig. 9. The lost-particle probe is made with a miniature Amperex $18508 \mathrm{GM}$ tube and is shielded with a 5/16-in. thickness of lead with a 1/8-in.-diam collimating hole. Small numbers of lost particles may be located and pinpointed by this instrument, but the probe becomes saturated near large numbers of particles.

\section{ANNEALING OF COATED FUEL PARTICLES}

The irradiated coated fuel particles are heated to reactor-operating temperatures and above to determine the fission-product release as a function of time and temperature. The range of temperatures used in our studies has been from $1000^{\circ} \mathrm{C}$ to $2000^{\circ} \mathrm{C}$. The length of annealing time was varied according to the amount of fission products released so that reasonable amounts could be collected for analysis, but where possible the same annealing periods were used so that direct comparisons could be made. The fraction 


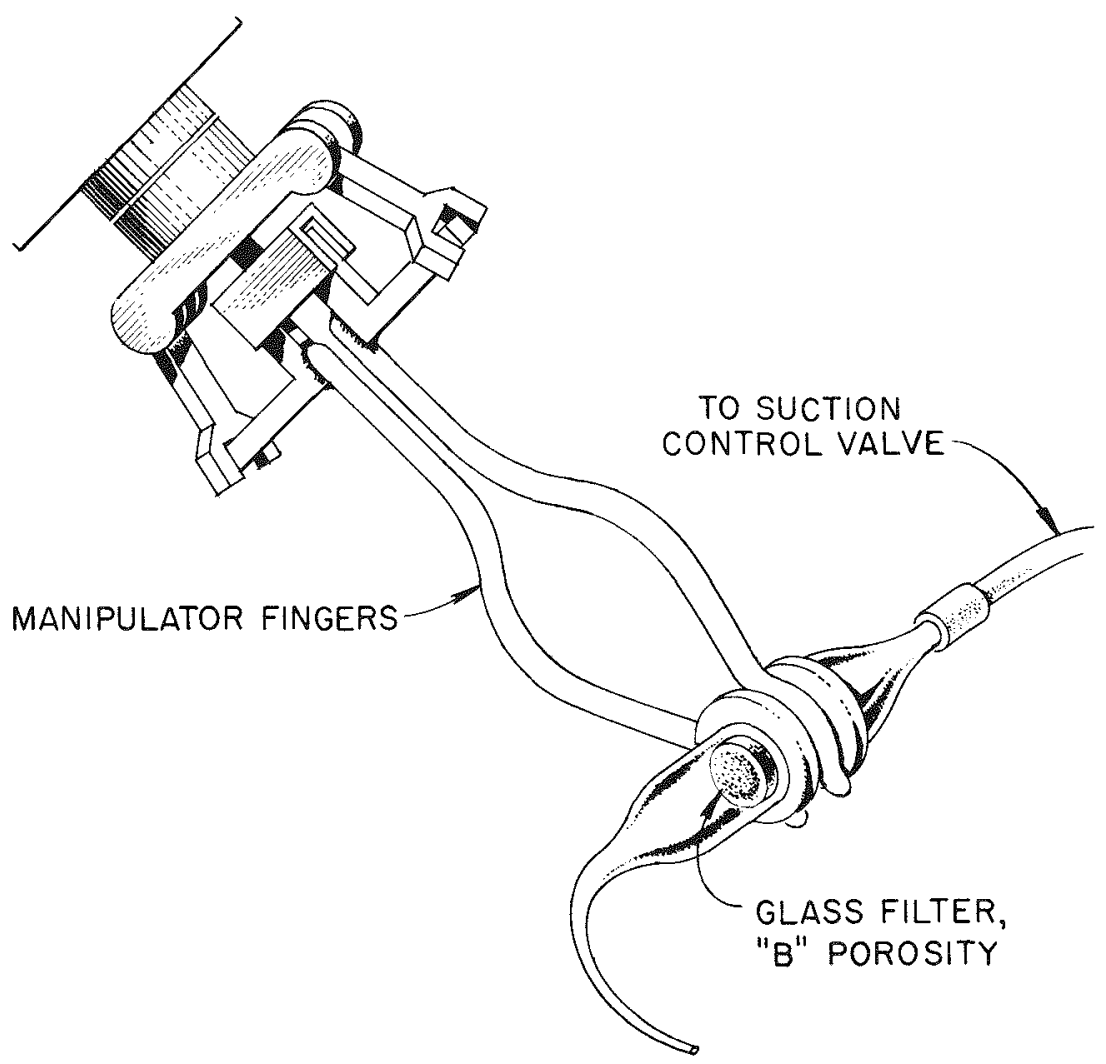

Fig. 7. Particle Pick-Up.

ALUMINA OR OTHER RADIATION

RESISTANT WHITE SURFACE

SUITABLE FOR MICRO-PHOTOGRAPHY BACKGROUND -

36 EACH HEMISPHERICAL

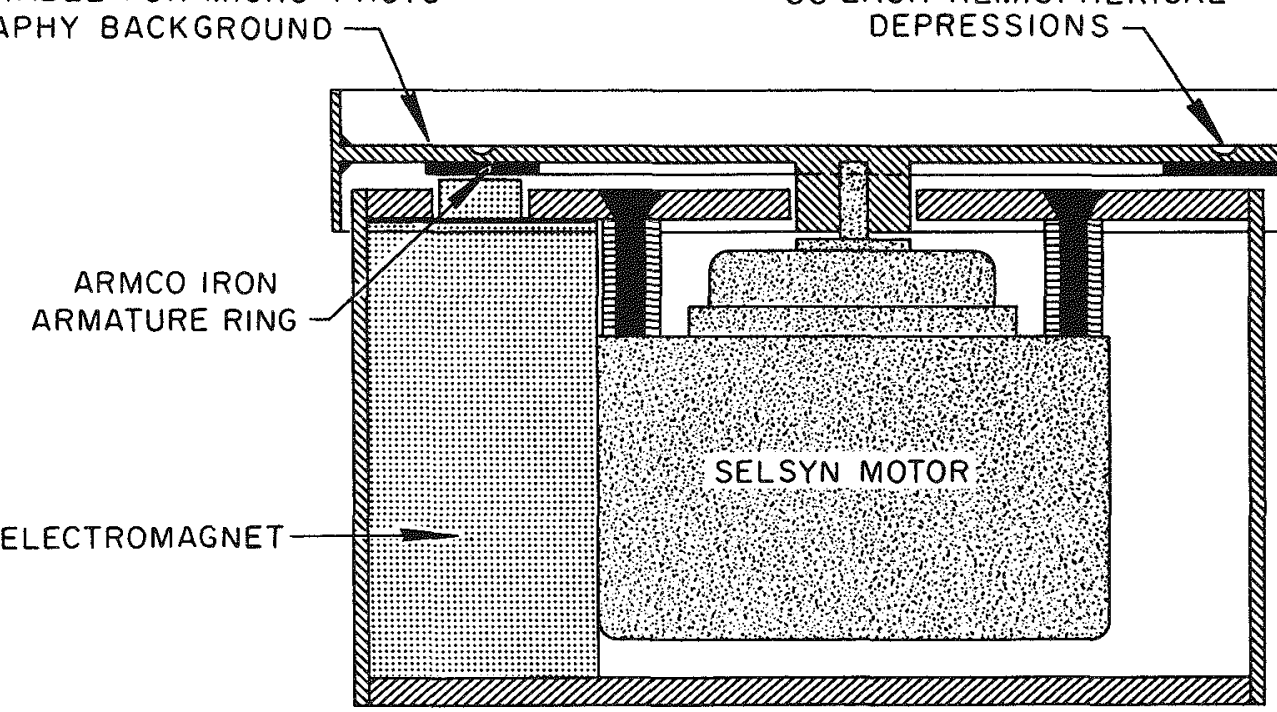

Fig. 8. Remote Revolving Stage with Vibrator for 3ox Periscope. 


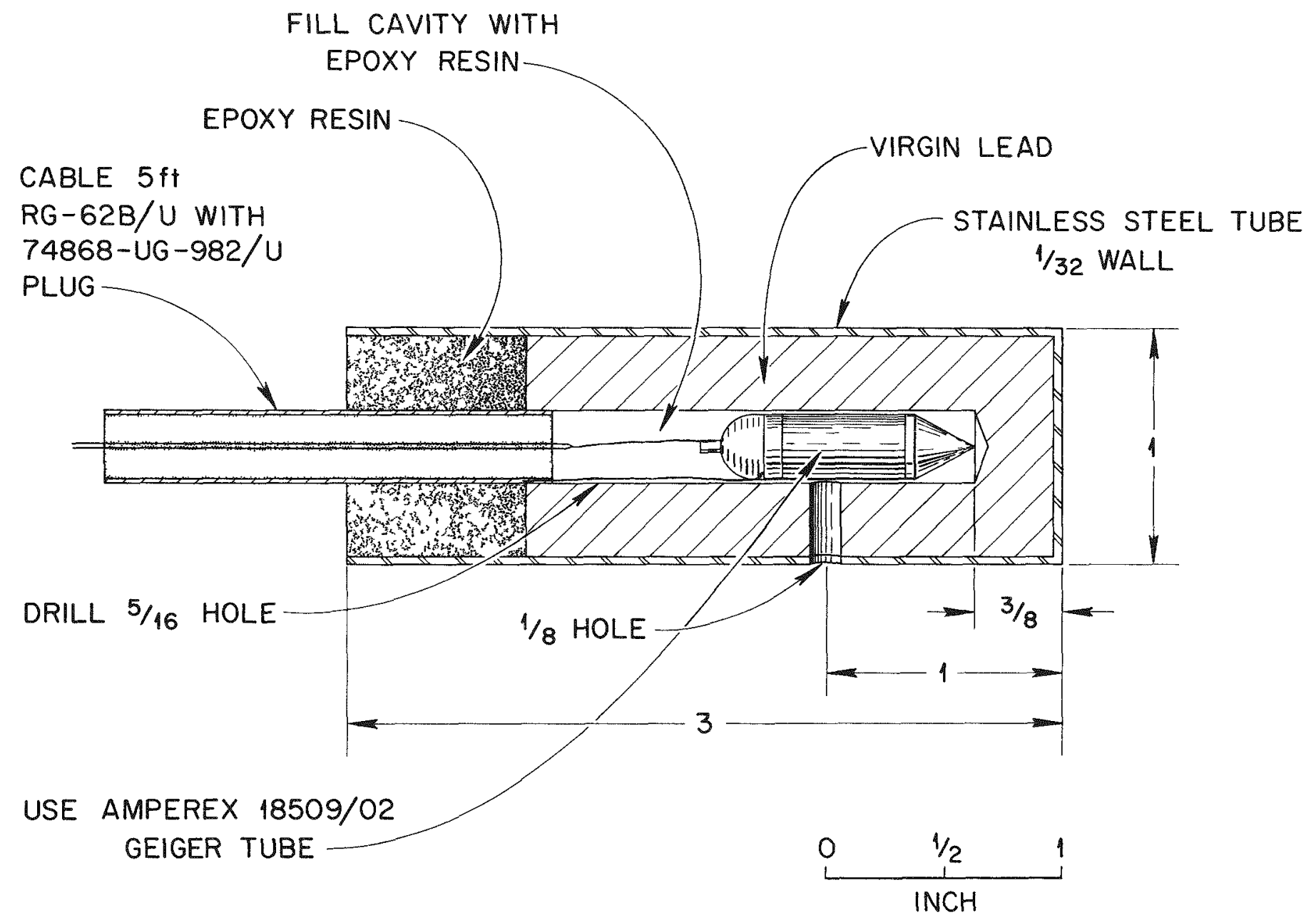

Fig. 9. Geiger Probe 
of fission products released is not a linear function of time. Ihe amount of fission product collected varied with time, temperature, fuel material, coating material, and fission-product isotope.

\section{Induction Furnace}

The RF generator for the induction furnace is a Lepel Model T-10-3S which has a power output of $10 \mathrm{kw}$ at a frequency of 250 to $600 \mathrm{kHz} .6$ This unit is controlled by a remote monitoring, indicating, and control servo, Lepel Model 7227 and a proportional current control with feedback, LepeI Mode1. 7228. The temperature is monitored by a Brown radiamatic pyrometer connected to a Brown electronic strip chart recorder. ${ }^{7}$ We wanted to use the pyrometer through the thick lead glass window of the shielded box, but the window absorbed too much light radiation to use the pyrometer as received. The lens system in the pyrometer was removed and replaced by a focusing system without a diaphragm so that more of the available light radiation could be used. This change made it possible to obtain a useable signal at temperatures as low as $1100^{\circ} \mathrm{C}$. Since the output signal of the pyrometer varied with distance and accuracy of focus, the radiamatic pyrometer was set for isothermal anneal by using an optical pyrometer which had been calibrated for the absorption of the lead window. The deviation from the set temperature could be read on the strip chart recorder, and the output of the strip chart recorder was used to control the Lepel units. This system controlled the temperature within $10^{\circ} \mathrm{C}$ of the set temperature over a temperature range of $1300^{\circ} \mathrm{C}$ to $2000^{\circ} \mathrm{C}$. The temperatures measured by the optical pyrometer were calculated to be within $\pm 30^{\circ} \mathrm{C}$ as judged to be the uncertainty of measurements and calibrations using a standard lamp and melting points of copper, platinum, and nickel.

\section{Gas Collection System}

The flow diagram for the system is shown in Fig. 10. The coated particles are heated in a flowing helium stream in a graphite crucible by induction heat. Purified helium sweep gas passes over the sample and carries the fission gases released through a filter to remove radioactive dust and into an enclosed vacuum system for counting and collection of the gas. The fission gas is continuously monitored as it flows through a coaxial Geiger-Mueller tube and is collected on a molecular sieve trap at liquid nitrogen temperatures. There are two traps which can be alternated; one being used for collection while the other is degassed by a Toepler pump into a total gas counting system. The gas can be re-pumped into bulbs for mass spectrometer or other analysis.

\section{Solid Fission Product Collection}

The quartz furnace tube is lined with a quartz sleeve as shown in Fig. 11. It was found that more than $99 \%$ of most of the solid fission products released from the fuel particles was retained in the crucible and on the quartz liners at sample temperatures up to $2000^{\circ} \mathrm{C}$. The crucibles and quartz liners are replaced periodically, and analyzed for fission products to get step-wise functions of fission-product release vs time. For collection of fission-product iodine, it was necessary to place a cold trap at the upper end of the furnace tube and to remove the entire furnace tube and trap for total iodine collection. The iodine trap consisted of a " $U$ " tube containing iodine-impregnated charcoal immersed in liquid nitrogen. 


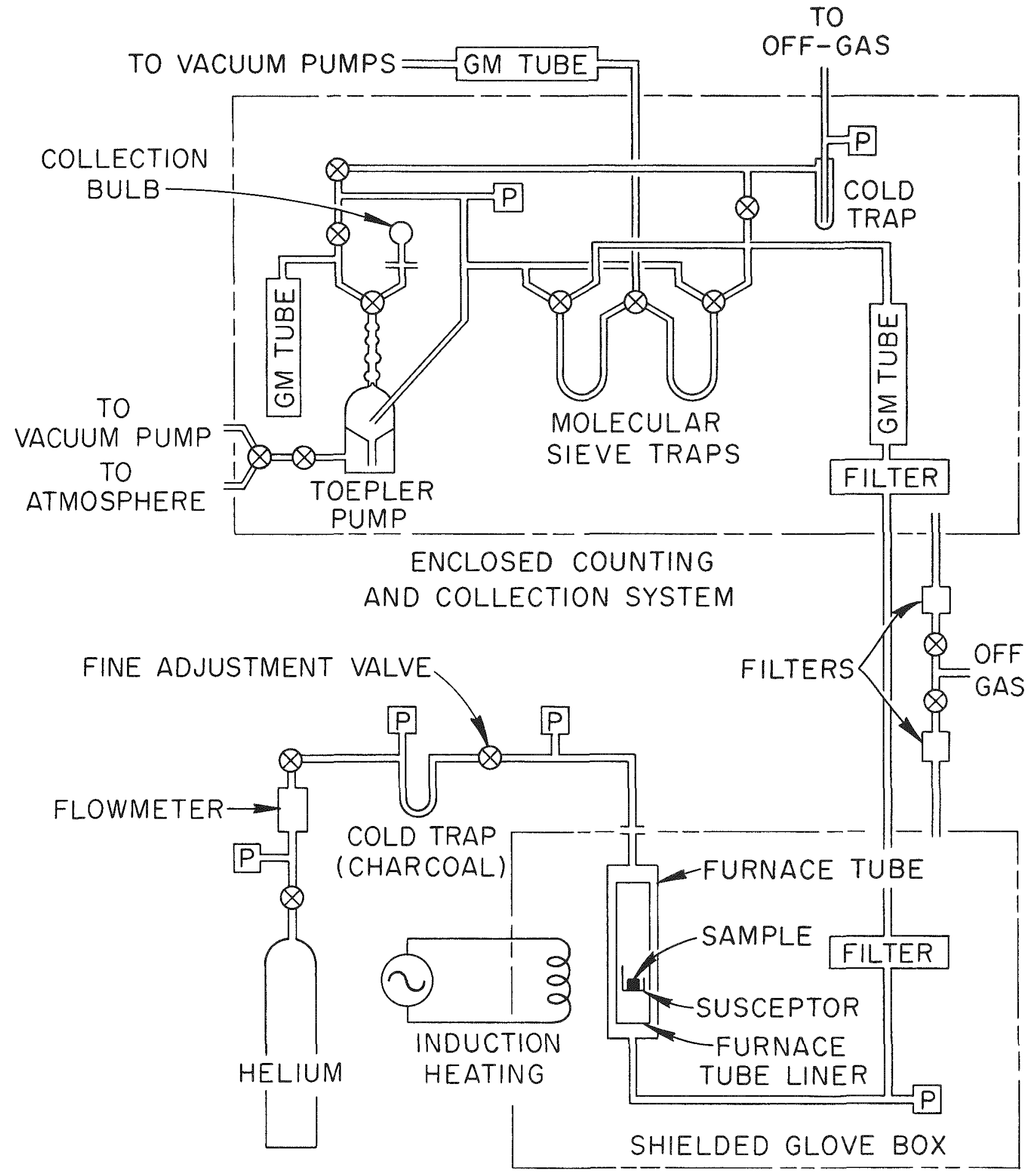

$P$ PRESSURE GAUGE

$\otimes$ VALVE

Fig. 10. Flow Diagram of Annealing System. 


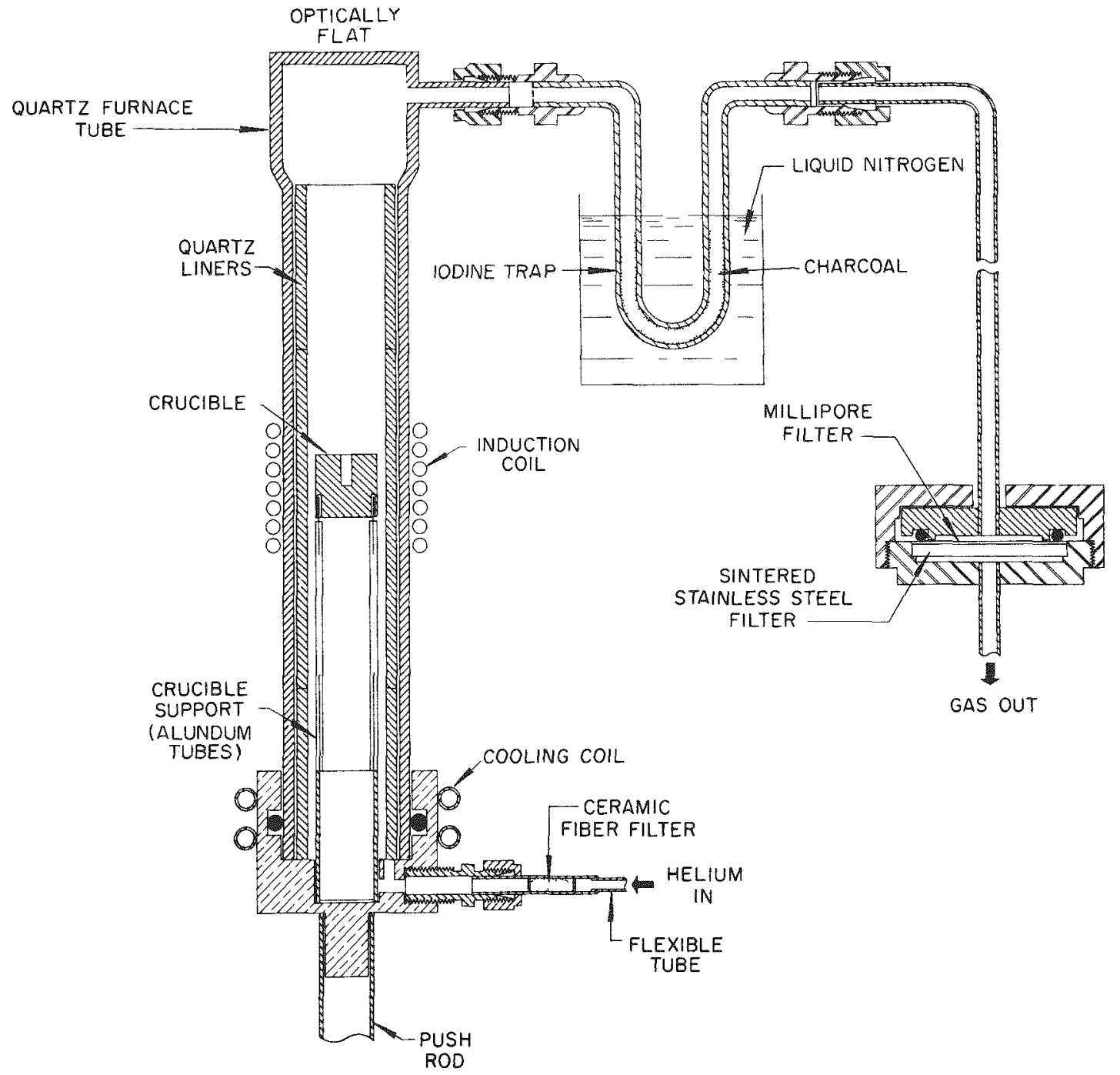

Fig. 11. Furnace Tube Assembly. 
Since the iodine trap also adsorbed krypton, it made the instant detection of coating failures by the release of ${ }^{85} \mathrm{Kr}$ impossible and, therefore, was not used except in experiments to determine iodine release from particles with no coatings or broken coatings. Iodine does not diffuse through intact particle coatings. The "U" tube was replaced by a straight connection for all other experiments. For future iodine collection it is planned to use silver zeolite in place of charcoal since it is reported to adsorb iodine at temperatures up to $500^{\circ} \mathrm{C} .{ }^{8}$ This would eliminate the use of liquid nitrogen.

\section{REFEREMCES AND FOOTNOTES}

1. W. V. Goeddel, "Coated-Particle Fuels in High-Temperature Reactors: A Summary of Current Applications," Nucl. Appl. 3, pp. 599-614, Oct. 1967.

2. M. T. Morgan, R. L. Towns, and J. G. Morgan, Fission-Product Release from High-Burnup Coated Fuel Particles by Postirradiation Annealing, pp 77-82, GCRP Semiann. Progr. Rept. Mar. 31, 1968, USAEC Réport ORNL-4266, Oak Ridge National Laboratory.

3. Allied Engineering and Production Corporation, Alameda, Califormia, Model S-4000.

4. M. T. Morgan, "Wrist-Action Manipulators for Shielded Glove Boxes," USAEC Report ORNL-TM-2512 (April, 1969) Oak Ridge National Laboratory.

5. Filter tube available from Scientific Glass Apparatus Co., Bloomington, N. J., Catalog No. ACE-8570.

6. LepeI High Frequency Laboratories, Inc., Woodside, N. Y.

7. Brown instruments are manufactured by Honeywell Industrial Products Group, Fort Washington, Pa.

8. W. J. Maeck, D. T. Pence, and J.H. Keller, "A Highly Efficient Inorganic Adsorber for Airborne Iodine Species (Silver Zeolite Development Studies)," USAEC Report IN-1224 (Oct. 1968). 
POST-IRRADIATION ANALYSIS OF HIGH TEMPERATURE, LOW BURNUP TUNGSTEN-UO 2 TEST PLATES

By L.L. Ball and D.W. Sheibley

Lewis Research Center

\begin{abstract}
Problems in determining fuel burnup of high temperature fueled plates were resolved by using tungsten-184 as a thermal flux monitor. Methods for determining fission product releases from fueled test plates were evaluated. The use of fission product $\mathrm{Cs}-137$ as a burnup monitor was also evaluated.
\end{abstract}

SUMMARY

The objective of these irradiations was to evaluate the uranium retention and iodine-and-xenon release qualities of test fuel plates. The plates contained uranium oxide dispersions which were fission-heated to anticipated rocket fuel element operating temperatures and nvt levels.

We found the following problems when we tried to use conventional methods for determining fission product releases and fuel burnup. Fission product recovery by leaching methods was unreliable. Burnup values obtained from the $\mathrm{U}^{236} / \mathrm{U}^{235}$ uranium ratio change were in many cases so small that results by mass spectrometry analysis were inaccurate. The high temperature of the irradiations precluded the use of standard thermal flux dosimeters.

Problems in measuring the fuel burnup were resolved by using tungsten-184 in the cladding of the test plates as a thermal neutron dosimeter. We calculated the neutron flux from the tungsten-185 activity, then used the neutron flux to calculate the fuel burnup. This 
measure of burnup then provided a sound basis for judging the effectiveness of fission product recovery and for determining the fission product release.

At the temperatures at which the irradiations occurred, most of the volatile fission products were released from the test plates. Therefore to recover all of the fission products, the interior of the stainless steel capsule containing the fueled test plate had to be either dissolved completely or leached.

Our first approach was to try to leach fission products from the capsule inner surface. Cesium-137 and iodine-131 were two fission products likely to be there. The fueled test plates were dissolved for complete accountability of the fission products.

Based on the number of fissions calculated from the tungsten-185 method and from the mass spectrometry analysis, on $1 y 70 \%$ of the cesium-137 produced was recovered by the leach method. The failure to recover all of the cesium-137 can be based on two reasons, both unique to this type of irradiation:

(1) The leaching and dissolution methods employed were not efficient due to the types of iodine and cesium compounds formed at the high temperatures, or

(2) the cesium-137 and iodine-131 as they deposited on the cooler interior portions of the capsule wall were covered by a layer of tungsten metal which had vaporized from the fuel test plates. Some test plates lost as much as $38 \%$ of their original weight including fuel.

The analysis of the fue 1 plates for iodine-131 indicated that approximately $98 \%$ of the iodine-131 produced had left the plates, based on the tungsten burnup numbers. The $\mathrm{NaOH}$ leach method indicated that only $20 \%$ of the iodine -131 produced had left the plates. This shows that total recovery of iodine by leaching only is unreliable. The method we recommend for iodine retention evaluation of a fueled test plate is to dissolve the test plate and determine the iodine present in the plate.

To determine the tungsten-185 activity samples of the tungsten picture frame surrounding the fueled plate were removed. The samples were dissolved and the tungsten-185 activity separated radiochemically and counted. 


\section{INTRODUCTION}

The irradiations discussed in this paper were part of a program to demonstrate the feasibility of refractory-metal-clad fuel elements for nuclear rocket application. The paper does not consider any aspects of the overall program or the programmatic results of the irradiations described here. Rather, we will talk about the methods we used and tried to use to measure fuel burnup and fission product release.

The total irradiation program consisted of approximately 40 capsule irradiations. All of these capsules were processed using the methods described in this paper for determining fission product release and fuel burnup. The data on fission product release from these irradiations were quite scattered. To determine the cause of the data scatter we initiated the work described in this report.

In addition to the tests scheduled for the program we irradiated four fueled plates to evaluate our methods for fission product recovery and for uranium burnup. This paper treats only the work on these four irradiations. We will discuss:

(1) The use of tungsten-184 as a thermal flux dosimeter in high temperature irradiations to determine the total fissions which occurred in fueled test plates.

(2) The use of fission-product cesium-137 recovered by radiochemical methods as a measure of total fissions which had occurred.

(3) The efficiency of various leaching methods used remotely to recover fission product iodine, cesium, and fission gases.

The development work related to establishing tungsten-184 as a thermal neutron dosimeter is described in reference 1 .

\section{EXPERIMENTAL DETAILS}

The fueled test plates were 2.54 by 2.54 by 0.127 centimeter thick. The fuel was enriched uranium oxide in a matrix of metaliic tungsten. 
The fuel content and homogenity of the test plates were checked and verified by chemical analyses. The experimenter supplied the fuel loading information. Al1 test plates were encased in a picture frame of tungsten and clad with 0.013 centimeter of tungsten. Each fueled test plate was surrounded by a thermal radiation shield and suspended inside an evacuated stainless steel capsule. Each of the four capsules was irradiated separately in the HT-1 test hole of the Plum Brook Reactor. The actual therma 1 neutron $f l u x e s$ which the test plates saw varied from $10^{13}$ to $10^{14} \mathrm{n} / \mathrm{cm}^{2}-\mathrm{sec}$.

Capsules $A$ and $B$ were longer irradiations ( $>12$ hours) so that fuel burnup could be determined from the change in the $\left(U^{236} / \mathrm{U}^{235}\right)$ ratio. The temperatures for capsules $A$ and $B$ ranged from $2600^{\circ} \mathrm{K}$ to $3000^{\circ} \mathrm{K}$, From this the thermal flux could be determined and compared with thermal flux values calculated from tungsten-185 activity. Capsules $C$ and $D$ were irradiated for four hours at $3300^{\circ} \mathrm{K}$.

Radiochemica1 Method - Capsules A and B, irradiated for burnup analysis, were stored for about two months to allow short-lived fission products to decay. Capsules $C$ and $D$ were processed in the hot cell within 24 hours after irradiation using the puncture rig described in ref. 2. Gas samples were collected and analyzed for xenon-135 to determine the iodine-135 release. Both capsules were leached with $0.1 \mathrm{~N} \mathrm{NaOH}$ solution to obtain the release of iodine-135 from the fuel.

After a decay period of approximately two months, we cut open a11 four capsules remotely in the hot cell and removed each component. We leached or dissolved the inside wall of each capsule with aqua regia in a glove box located in the cel1. The exhaust from the glove box was passed through liquid nitrogen cooled carbon traps, then into a radioactive gas collection system to collect iodine-131.

We partially dissolved the molybdenum-tungsten therma 1 radiation shields from each capsule with a mixture of hydrofluoric acid and nitric acid. This operation was also performed in the glove box connected to the radiation gas collection system.

Each leach solution was analyzed separately for cesium-137, iodine-131, and uranium. The carbon traps and radioactive gas collection 
tanks were sampled for iodine-131.

We weighed each fuel specimen after irradiation. Then we removed the corners of the picture frame cladding for tungsten-185 analysis.

We dissolved the tungsten corners in $\mathrm{HF}-\mathrm{HNO}_{3}$, then determined the tungsten-185 activity for each fuel plate.

Each specimen was then dissolved entirely in an acid mixture consisting of two parts $\mathrm{H}_{3} \mathrm{PO}_{4}$ and one part $\mathrm{HClO}_{4}$. The dissolution was performed in a reflux condenser connected to a $\mathrm{NaOH}$ scrubber trap and a carbon trap to retain any iodine. The resulting solutions were analyzed by radiochemica 1 methods for cesium-137 and iodine-131. Tota1 uranium was determined colorimetrically.

Mass spectrometric analyses for uranium burnup were performed on fuel specimens $A, B$, and $C$ using the change in the $U^{236} / U^{235}$ ratio.

\title{
DISCUSSION OF RESULTS
}

\author{
Burnup Ana1ysis
}

Cesium Recovery - One object of this study was to recover all of the cesium-137 produced during irradiation. If complete recovery is possible, then the number of fissions and hence, the burnup, can be calculated. The total cesium-137 found and the percentage of recovery for each irradiation are listed in table $I$. These recoveries are based on fissions calculated from the tungsten-185 activities. None of the recoveries for cesium-137 exceeded $76 \%$ 。

Most of the cesium-137 was found in the $0.1 \mathrm{~N} \mathrm{NaOH}$ solutions of the two capsules ( $C$ and $D$ ) which were caustic leached. In the other two capsules most of the cesium-137 was found in the leaches of the thermal radiation shields. As can be seen from table $I$, the fuel specimens contained less cesium-137 than the leaches. This means that during irradiation most of the cesium-137 escaped from the fuel. 
TABLE I. CESIUM-137 FOUND FROM LEACHING OF CAPSULES

\begin{tabular}{|c|c|c|c|c|}
\hline & $\begin{array}{c}\text { Capsule } \\
\text { A }\end{array}$ & $\begin{array}{c}\text { Capsule } \\
\text { B }\end{array}$ & $\begin{array}{c}\text { Capsule } \\
\text { C }\end{array}$ & $\begin{array}{c}\text { Capsule } \\
\text { D }\end{array}$ \\
\hline $\begin{array}{l}\mathrm{Cs}^{137} \text { total found, } \mathrm{d} / \mathrm{m} \\
\mathrm{Cs}^{137} \text { produced (calculated from } \\
\mathrm{W}-185 \text { fissions), } \mathrm{d} / \mathrm{m} \\
\mathrm{Cs}^{137} \text {, percent found of total } \\
\text { produced } \\
\mathrm{Cs}^{137} \text {, percent in fuel specimen } \\
\text { of total produced } \\
\mathrm{Cs}^{137} \text {, percent in baffle leach } \\
\text { of total produced } \\
\mathrm{Cs} 137 \text {, percent in capsule acid } \\
\text { leach of total produced } \\
\mathrm{Cs} 137 \text {, percent in } 0.1 \mathrm{~N} \mathrm{NaOH} \\
\text { leach of total produced }\end{array}$ & $\begin{array}{c}* \\
\text { not } \\
\text { leached }\end{array}$ & $\begin{array}{r}8.9 \times 10^{9} \\
12.2 \times 10^{9}\end{array}$ & $\begin{array}{l}4.5 \times 10^{9} \\
6.6 \times 10^{9}\end{array}$ & $5.7 \times 10^{9}$ \\
\hline
\end{tabular}

* Solution overturned in hot ce11 -- total unknown.

The fact that cesium-137 escapes from the fuel tends to rule out its use as a method for determining the total fissions occurring in a specimen irradiation at high temperature. The partial dissolution and acid leaching of the components was quite complicated with conditions difficult to control. Total dissolution of the capsule or the rmal radiation shields was out of the question because of the large amount of metal ions in solution. The metal ions interfere with the cesium recovery

There are two plausible explanations for incomplete recovery of both the cesium-137 activity and the iodine-131 activity (discussed in a later section). Both reasons are unique to these irradiations. 
Our leaching methods may have been ineffective due to the types of cesium and iodine compounds formed at the high temperatures of the irradiations. Or at these high temperatures, the cesium and iodine left the fuel and deposited on the cooler interior surfaces of the capsule. These fission products were then covered by a layer of tungsten metal which vaporized from the fueled test plate and deposited on the same cooler surfaces. We found evidence to support this latter mechanism. Some of the test plates lost as much as $38 \%$ of their original weight, including fuel. Analyses of the irradiated fuel plates showed fuel loss from the test plates was $33 \%$ for capsule A, $61 \%$ for capsule $B, 73 \%$ for capsule $C$, and $83 \%$ for capsule D. At the high irradiation temperatures, it is reasonable to assume the capsule contained a gaseous mixture composed of volatile fission products, including cesium and iodine, and vapors of tungsten and uranium dioxide. Hence either explanation is reasonable.

Flux Determined by Tungsten-185 Activity - We dissolved the tungsten corners of the specimens and determined the tungsten-185 activities. Using an effective cross-section of 1.94 barns (verified by cobalt-60 comparator in the Plum Brook Reactor and the Mock-Up Reactor) we calculated the integrated flux received by the tungsten metal. By using this data and the weight of uranium originally loaded, we calculated the total fissions for each fuel specimen. Table II 1ists the integrated flux from the tungsten-185, total fissions calculated, the fissions calculated from the cesium-137, and the fissions found by mass spectrometry.

We attribute the difference in fissions derived from $\mathrm{W}-185$ activation and from mass spectrometer burnup to using the average cross-section of $\mathrm{w}^{184}$ (1.94 barns) rather than the effective cross-section for $\mathrm{w}^{184}$ determined for each specimen by mass spectrometry.

The uranium in fuel specimen $D$ did not experience sufficient burnup to allow a calculation based on the changes in uranium-236 content. The $\mathrm{U}^{236}$ content of this specimen was high and could not be used for reliable burnup analysis. 
Data from the analysis of tungsten-185 in the cladding was used for al1 calculations of fissions in this study. These calculated fissions were confirmed by the burnup analysis by mass spectrometry.

TABLE II. - COMPARATIVE TOTAL FISSIONS

\begin{tabular}{|c|c|c|c|c|}
\hline & $\begin{array}{c}\text { Capsule } \\
\text { A }\end{array}$ & $\begin{array}{c}\text { Capsule } \\
\text { B }\end{array}$ & $\begin{array}{c}\text { Capsule } \\
\text { C }\end{array}$ & $\begin{array}{c}\text { Capsule } \\
\text { D }\end{array}$ \\
\hline $\begin{array}{l}\text { nvt calculated from } \\
\text { tungsten-185 activity } \\
\text { Fissions calculated } \\
\text { using tungsten-185 flux } \\
\text { Fissions using cesium-137 } \\
\text { found (line } 1 \text {, table } 1 \text { ) } \\
\text { Fissions determined * by } \\
\text { mass spectrometry } \\
\text { (burnup analysis) }\end{array}$ & $\begin{array}{l}4.5 \times 10^{18} \\
3.5 \times 10^{18}\end{array}$ & $\begin{array}{l}5.3 \times 10^{18} \\
4.2 \times 10^{18} \\
3.1 \times 10^{18}\end{array}$ & $\begin{array}{l}3.0 \times 10^{18} \\
2.3 \times 10^{18} \\
1.7 \times 10^{18}\end{array}$ & $\begin{array}{l}2.9 \times 10^{18} \\
2.0 \times 10^{18} \\
1.5 \times 10^{18}\end{array}$ \\
\hline
\end{tabular}

* Based on fuel loading.

** Solutions overturned in hot cell -- cannot be determined. xhr Burnup not determined.

Fission Product Iodine Release

Iodine Recovery - Iodine-131 concentrations and the percentages in each of the leach solutions are shown in table III. Also listed are the percent recoveries for iodine-131 based on the iodine-131 produced. No recovery exceeded 44\%. Most of the iodine-131 was probably lost during the leaching operations. The loss was due to deposition 
on the metal surfaces of the pipes leading from the glove box to the radioactive gas collection system, and on the inside surface of the gas tanks.

During the leaching of the capsules and baffles strong oxidizing acids had to be used to attack the metal to assure complete release of fission products. In this environment, iodine will become elemental and volatilize. This was evident when capsules and baffles were acid leached on capsules A and B (table II). Most of the iodine-131 (90\%) was found in the radioactive gas collection tanks. We found that acid leaching (with aqua regia) liberates large amounts of iodine.

Table III also shows that only a small percentage of iodine produced remained in the test specimens at these high temperatures.

TABLEIII. - IODINE-131 FOUND FROM LEACHING OF CAPSULES

\begin{tabular}{|c|c|c|c|c|}
\hline & $\begin{array}{c}\text { Capsule } \\
\text { A }\end{array}$ & $\begin{array}{c}\text { Capsule } \\
\text { B }\end{array}$ & $\begin{array}{c}\text { Capsule } \\
\text { C }\end{array}$ & $\begin{array}{c}\text { Capsule } \\
\text { D }\end{array}$ \\
\hline $\begin{array}{l}I^{131} \text { total found, } d / m \\
I^{131} \text { produced (calculated } \\
\text { using } W-185 \text { flux) } \\
I^{131} \text { percent found of produced } \\
I^{131} \text { percent in fuel specimen } \\
\text { of total produced } \\
I^{131} \text { percent in baffle leach } \\
\text { of tota } 1 \text { produced } \\
I^{131} \text { percent in capsule leach } \\
\text { of total produced } \\
I^{131} \text { percent in } 0.1 \mathrm{~N} \text { NaOH } \\
\text { leach of total produced } \\
I^{131} \text { percent in gas collection } \\
\text { tanks of } I^{131} \text { produced }\end{array}$ & $\begin{array}{c}* \\
\text { not } \\
\text { leached }\end{array}$ & $\begin{array}{l}1.8 \times 10^{12} \\
7.3 \times 10^{12}\end{array}$ & $\begin{array}{l}1.3 \times 10^{12} \\
4.0 \times 10^{12}\end{array}$ & $\begin{array}{c}1.5 \times 10^{12} \\
3.4 \times 10^{12} \\
44 \%\end{array}$ \\
\hline
\end{tabular}

* Solution overturned in hot cell. 
Evaluation of $0.1 \mathrm{~N} \mathrm{NaOH}$ Leaching \& Gas Evolution Methods - We were interested in evaluating our two methods for determining the iodine-135 release from fuel specimens during irradiation. of primary interest, the caustic leach method gave a $15 \%$ release for iodine-135 and a $20 \%$ release for iodine -131 on capsule C (table IV). The gas evolution method gave a release of $7 \%$ for capsule $C$. The results for capsule D were similar to capsule $C$.

By considering the ratio of iodine-131 remaining in the fuel specimens to the iodine-131 produced using the fissions calculated from the tungsten-185 activities, the releases are in the order of $99 \%$ for al1 specimens. There was approximately $1 \%$ iodine retention in the specimen.

TABLE IV. $-I^{131}$ AND $I^{135}$ RELEASES BASED ON FISSIONS
CALCULATED FROM $W^{185}$ FLUX VALUES

\begin{tabular}{|c|c|c|c|c|}
\hline & $\begin{array}{c}\text { Capsule } \\
\text { A }\end{array}$ & $\begin{array}{c}\text { Capsule } \\
\text { B }\end{array}$ & $\begin{array}{c}\text { Capsule } \\
\text { C }\end{array}$ & $\begin{array}{c}\text { Capsule } \\
\text { D }\end{array}$ \\
\hline $\begin{array}{l}\mathrm{I}^{131} \text { percent release with } 0.1 \mathrm{~N} \mathrm{NaOH} \\
\text { leach } \\
I^{131} \text { percent release considering } \\
I^{131} \text { remaining in fuel specimen } \\
I^{135} \text { percent release with } 0.1 \mathrm{~N} \mathrm{NaOH} \\
\text { leach } \\
I^{135} \text { percent release with gas } \\
\text { evolution method }\end{array}$ & $\begin{array}{c}\text { not } \\
\text { leached }\end{array}$ & $\begin{array}{c}\text { not } \\
\text { leached }\end{array}$ & $99 \%$ & $100 \%$ \\
\hline
\end{tabular}




\section{CONCLUSIONS}

We were able to account for approximately $70 \%$ of the cesium-137 produced based on the fissions calculated from the tungsten-185 and mass spectrometer burnup analysis. We could not recover all the cesium produced because the leaching methods we employed were not efficient in freeing the deposited fission product cesium-137.

Considering the cesium recovery and the problems in leaching, handling, and analysis of the leach solutions, it is our opinion that the method of determining the number of fissions from cesium-137 produced would be unreliable.

We found that the $0.1 \mathrm{~N} \mathrm{NaOH}$ leach used for iodine recovery in this particular application did not remove all of the iodine isotopes from the capsule interior as we had assumed. After analyzing the' fuel specimens for iodine-13I remaining, we found that approximately $99 \%$ of the iodine-131 had left the fuel specimens. Our $0.1 \mathrm{~N} \mathrm{NaOH}$ leach method indicated that only $20 \%$ of the iodine-131 produced had left the specimen. In future work to obtain more realistic iodine release data, the fuel specimen should be removed from the capsule and analyzed directly for iodine isotopes.

Tungsten-184 has been shown to be a reliable thermal flux dosimeter.

\section{REFEREIVCES}

1. Ball, Lauren I.; Richardson, Paul J.; and Sheibley, Dean W.: Use of Tungsten-184 as a Thermal Flux Monitor at High Temperatures. NASA TN D-5113, 1969.

2. Bonn, J. I.; and Kern, W.: Irradiated Gases Transferred Without Contamination or Dilution. NASA Tech. Brief B67-10044, Mar. 1967. 
$\checkmark$ DECAY HEAT GENERATION AND REMOVAL FROM FUEL TEST BUNDLES

H. F. Daugherty

R. M. Lieberman

M. Doumas

$12_{*}: *$ WESTINGHOUSE ELECTRIC CORPORATION

Bettis Atomic Power Laboratory

\section{ABSTRACT}

Simplified calculational techniques have been developed for estimating the decay heat power levels for irradiated fuel specimens, as a function of operating heat flux and effective full power days of operation. The calculated decay heat values were experimentally verified. In adoition, specimen surface temperatures have been measured in typical small test bundles exposed to stagnant air cooling.

\section{Introduction}

The radioactive decay of fission and activation products in irradiated fuel samples gives rise to heat generation within those samples. The heat generation, in turn, can cause elevated specimen temperatures in a restrictive heat transfer environment. To insure that high temperatures which may be detrimental to fuel samples are not attained, precautions such as extended coolant circulation after reactor shutdowns, wet test removal and transfer operations, and underwater examinations are often utilized. During hot cell examinations, however, precautions such as wet handling are not practical, and the experimenter must rely on heat transfer to ambient air to prevent overheating of fuel elements.

During test reactor shutdown periods, examinations of individual specimens or specimen bundles in hot cell facilities are a routine operation. The temperature of fuel samples during examination periods is of particular concern to the fuel element designer for the following reasons: 
1. Specimen cladding materials could exceed normal in-pile operating temperatures and may thus be degraded by exposure to air. Fuel materials may experience swelling not present in-pile.

2. Specimen surface condition and appearance may be altered significantly from that existing in-pile, thereby compromising visual examination information.

3. Remote handling equipment may be damaged by contacting specimens at high temperature.

4. The thermal expansion of fuel specimens must be considered in the evaluation of dimensional data.

As a result of these considerations the fuel element designer usually sets surface temperature limitations for fuel specimens during examination periods.

To evaluate the decay time or cooling period required to reduce specimen temperatures to an acceptable level prior to handling a specimen in a hot cell, simplified but reliable techniques were required for evaluation of the decay power and the resulting specimen surface temperatures. A technique for the calculation of fission product decay power was adapted from the data presented by Shure(1). Activation product decay power has been evaluated using appropriate activation rates and decay constants for those products contributing significantly to decay power. Decay power calculations using this technique were experimentally corroborated by calorimetric measurements using irradiated $\mathrm{UO}_{2}$ and $\mathrm{ThO}_{2}$ fuel samples.

Heat transfer measurements were conducted on mockup irradiation test bundles using electrically heated model fuel rods. These tests were conducted in a simulated hot cell environment. The results of these measurements are used to predict rod bundle specimen surface temperatures in a hot cell as a function of decay heat flux.

II. Decay Power Calculations

A. Fission Product Decay Power

Shure ${ }^{(1)}$ presented a review of data from several experimenters on the energy released from fission products in the form of beta and gamma radiation. As a result of this review for evaluation of total energy release, Shure recommended use of the results of Stehn and Clancy (2) for cooling times less than 103 seconds and data obtained from Perkins $(3)$ for cooling times in excess of $10^{3}$ seconds. Shure concluded that the data of several experimenters agreed within $20 \%$ for cooling times up to 400 days but recommended the results of Perkins for times in excess of $10^{3}$ seconds. Shure presented the data he considered most reliable in a manner suited for calculation. 
This calculational method was further simplified to develop design curves for the calculation of the released total, gamma, and beta energy as a function of in-pile fissioning rate, exposure time, and the cooling period after reactor shutdown. Only decay times in excess of 0.1 days were considered, as shorter times require further analysis of short lived products and are not relevant to the hot cell examination problem.

Figure 1 presents the total power production of fission products as a function of irradiation time and cooling time after reactor shutdown. The decay curves shown express the decay energy as a fraction of in-pile specimen fission power prior to reactor shutdown.

Presentation of the data in this manner permits the user to operate in the fission heat rate units of his choice, thus simplifying the decay power calculation. Figure 2 presents the fission product gamma decay power in the same format as the total decay power. The difference between the total and gamma decay powers from Figures 1 and 2 is the beta decay power for the fuel sample. The total, gamma, and beta fission product decay power is therefore given as

Equation (I) Total $P_{T}=F_{T} Q$

Equation (2) Gamma $P_{Y}=F_{Y} Q$

Equation (3) Beta $\quad P_{\beta}=\left(F_{T}-F_{\gamma}\right) Q$

where

$$
\begin{aligned}
& P_{T}=\text { Total Decay Power } \\
& P_{Y}=\text { Gamma Decay Power } \\
& P_{\beta}=\text { Beta Decay Power } \\
& F_{T}=\text { Total Decay Power Fraction } \\
& F_{Y}=\text { Gamma Decay Power Fraction } \\
& Q=\text { In-Pile Fuel Fissioning Rate }
\end{aligned}
$$


Since only a small fraction of the beta energy escapes from the specimen, all of the beta decay energy generated in the fuel specimen may be assumed to be absorbed within the fuel and cladding. A considerable portion of the gamma energy is not absorbed within the specimen with the fraction absorbed depending on fuel and cladding materibl, density, and geometry. The fraction of gamma energy absorbed within the specimen " $f$ " is estimated using shielding techniques. Gamma collision probability, which is plotted on Figure 3, is probability of absorption of gamma energy within a finite absorbing body if the gammas are borm within the body isotropically with a constant source density. The parameter "b" of Figure 3 is a distance characteristic of the body, having units of mean free path for energy absorption. The equations by which one estimates "b" are shown below. The constants used for the various materials of interest shown in Table I are computed from the energy absorption cross sections for $0.75 \mathrm{mev}$ gammas. This single energy will satisfactorily represent the gamma energy spectrum in this self-absorption process. For combinations of materials the constants should be volume averaged. The net gamma absorption may be evaluated for slabs or cylinders using Figure 3 and Equation 4 and 5.

$\begin{array}{lll}\text { Equation (4) } & \text { Slabs } & b=B p t \\ \text { Equation (5) } & \text { Cylinders } & b=B \rho R\end{array}$

Where

$$
\begin{aligned}
& b=\text { absorption parameter of Figure } 3 \\
& B=\text { material constant of Table } I \\
& \rho=\text { material density (gram/cc) } \\
& t=\text { slab thickness (centimeters) } \\
& R=\text { cylinder radius (centimeters) }
\end{aligned}
$$

The decay power generation for a fuel specimen therefore becomes Equation (6) $P_{\text {Decay }}=Q_{\beta}\left(F_{\beta}+f F_{\gamma}\right)+$ Activation Product Decay Power 
As previously noted the units of the in-pile heat rate determine the units of the calculated decay heating rate.

For cooling times in excess of 0.5 days fission product decay power is not sensitive to power history immediately preceding reactor shutdown. Fxperience indicates that good results may be obtained by using a two step decay power calculation with the first step including an average specimen power history to the reactor cycle prior to the cycle of interest, and the second step including the last cycle average power history.

B. Activation Product Decay Power

Among fuels of current interest, protactinium in thorium bearing fuels is the only activation product contributing significantly to decay power. $\mathrm{Pa}^{233}$ is formed by the capture of a neutron by Th232 and decays to U233 with a half-life of 27.0 days. However, the probability that $\mathrm{Pa}^{233}$ can capture a neutron is sizable and these two effects must be included in evaluating $\mathrm{Pa}^{233}$ decay power. These two competing effects cause the calculation of the $\mathrm{Pa} 233$ concentration at any given time to be sensitive to power history. A two step calculation of the $\mathrm{Pa}^{233}$ decay power is done, with the first step determining the equilibrium $\mathrm{Pa} 233$ using the average power prior to the most recent reactor cycle and the second step an explicit calculation for the most recent reactor cycle. For the period prior to the latest reactor cycle $\mathrm{Pa}^{233}$ decay power is calculated using the following equation:

Equation (7) $\left.P_{O_{1}}=\left\{\frac{.78\left[\left[596\left(1-F_{T}\right)\right]+\left[.1075 F_{T}\right]\right]}{\left[2930+.570 \mathrm{P}_{F}\left(1-F_{T}\right)+.0099 P_{F} F_{T}\right.}\right]\right\} W F_{R}$ where

$$
\begin{aligned}
& P_{O_{1}}=P a^{233} \text { decay power for Step I (watts } / \mathrm{cm}^{3} \text { ) } \\
& P_{F}=\text { Fission Power (watts/gm } \mathrm{U}^{235} \text { ) } \\
& F_{T}=\text { Fraction thermal fissions } \\
& F_{R}=\text { Fraction } \mathrm{Pa}^{233} \text { remaining } \\
& \left.W=\text { Thorium Ioading (grams } \mathrm{Th} / \mathrm{cm}^{3}\right)
\end{aligned}
$$


The above equation for $\mathrm{Pa}^{233}$ decay power for loop irradiations is related to the design curve technique developed by Lieberman (4). Specifically the loop power and the fraction fissions from thermal energy neutrons are used. In general both of these values are known for all conditions of test operation. The equation assumes equilibrium conditions, two energy groups of neutrons, $175 \mathrm{mev}$ per fission, a set of cross sections averaged over typical irradiation test neutron spectra and . $440 \mathrm{mev}$ of energy in the fuel element for each $\mathrm{Pa}^{233}$ decay.

For the explicit last cycle $\mathrm{Pa}^{233}$ decay power is calculated using the following equation:

Equation (8) $\quad \mathrm{P}_{\mathrm{o}_{2}}=\left(\mathrm{P}_{\mathrm{F}}\right)(\mathrm{W})\left(\mathrm{P}_{\mathrm{C}}\right)\left(\mathrm{F}_{\mathrm{R}}\right)$

where

$$
\begin{aligned}
P_{\mathrm{O}_{2}}= & \mathrm{Pa}^{233} \text { decay power for Step } 2 \text { (watts } / \mathrm{cm}^{3} \text { ) } \\
\mathrm{P}_{\mathrm{C}}= & \begin{array}{l}
\text { a design curve constant obtained from } \\
\text { Figure } 4
\end{array} \\
\mathrm{~W}= & \text { Thorium Loading (Grams } \left.\mathrm{Th} / \mathrm{cm}^{3}\right) \\
\mathrm{F}_{\mathrm{R}}= & \text { Fraction } \mathrm{Pa}^{233} \text { remaining }
\end{aligned}
$$

The above equation for $\mathrm{Pa}^{233}$ decay power includes the same assumptions as Equation (7). The decay power from $\mathrm{Pa}^{233}$ is the sum of the $\mathrm{Pa}^{233}$ decay powers from Equation (7) and (8).

Conservatism is included in this calculation in two ways: first the no-shutdown equilibrium calculation overpredicts the quantity of $\mathrm{Pa} 233$ and second it is assumed that no captures of the $\mathrm{Pa}^{233}$ present from previous cycles take place during the last cycle. To include the effect of shutdowns during the main portion of test operations and all $\mathrm{Pa}^{233}$ captures is beyond the ability of hand calculations, and is possible only with ex-post-facto computer calculations of the sort described in Reference 4. 


\section{Decay Power Mieasurements}

To corroborate experimentally the simplified decay power calculational technique, a series of decay power measurements were made for irradiated $\mathrm{UO}_{2}$ and $\mathrm{ThO}_{2}$ fuels. Measurements were made by calorimetric techniques using a cylindrical stainless steel calorimeter. The stainless steel calorimeters were fabricated to be 1.5 inches in diameter by six inches $10 \mathrm{ng}$ with a centrally drilled hole for rod type specimens or a machined slot for rectangular type elements. Stagnant natural convection conditions were achieved by utilizing a small plastic cover with several large holes, placed over the cylinder to eliminate air drafts which could alter temperature measurements. The cover thus provides similar heat transfer conditions for all measurements. The cylinders were calibrated by measuring the temperature difference between the cylinder and ambient air using electrical heaters with a known power output. The combined heat transfer coefficient applicable to the cylinder can thus be determined. These calibration runs showed that for the range of cylinder temperatures considered in our experiments an overall heat transfer coefficient of $\sim 2.3 \mathrm{Btu} / \mathrm{hr}-\mathrm{OF}_{\mathrm{F}}$ could be applied to the cylinder under natural convection conditions.

To measure decay power, specimens were placed in the cylinder and the steady state temperature of the cylinder above ambient was monitored. Using the heat transfer calibration curve for the cylinder the power developed was calculated. By correcting for the gamma absorbtions in the cylinder and the specimen, the total decay power and individual specimen decay power could then be calculated.

Table II presents a comparison of calculated and measured decay power for twelve fuel specimens of $\mathrm{UO}_{2}$ and $\mathrm{ThO}_{2}$ fuel types. The irradiation time for these specimens varied from 68 to 501 full. power days with cooling times from 0.8 to 18.7 days. These specimens operated at in-pile fission heat fluxes up to $750,000 \mathrm{Btu} / \mathrm{hr}-\mathrm{ft} \mathrm{t}^{2}$. Decay powers for the fuel specimens were calculated using Equations $(6),(7)$ and (8). 
The maximum positive and negative deviation of the measured versus calculated decay powers was $+18 \%$ and $-17 \%$ respectively. Overall the ratio of measured to calculated decay power is 1.01 indicating good general agreement between measurements and calculations. Since the fission heat flux uncertainty for the group of samples is of the order of +10 to $+15 \%$ it is felt that the measured data adequately corrroborate the decay power calculationel technique.

\section{Test Bundle Temperatures}

Within a currently used fuel development program, a series of small fuel rod bundles are irradiated in test reactor facilities. Typical irradiation test bundles consist of arrays of seven and ten .25 - . 30 inch diameter fuel rods arranged as shown in Figures 5 and 6. The fuel bundles are contained within an outer holder providing structural support. Some designs also include rod spacing grids for lateral fuel rod support within the bundle as shown in Figure 6.

In the hot cell environment heat transfer within the fuel bundle is accomplished by radiation and free air convection transfer to the external support holder. Transfer from the support holder is accomplished by free air convection, radiation, and conduction to the cell floor or examination support table. Heat transfer measurements were performed on two test bundle configurations under a simulated hot cell environment. Temperatures were monitored on electrically heated model fuel rods for various heating rates to generate design curves for rod temperatures within a fuel bundle under decay heating conditions. The configurations studied included the seven rod array of 0.250 inch diameter rods shown in Figure 5 , and a ten rod array of 0.250 inch diameter rods with and without a grid type rod spacer as shown in Figure 6.

A. Seven Rod Array

Tests were conducted on the seven rod array in the horizontal and vertical positions at rod surface heat fluxes to $350 \mathrm{Btu} / \mathrm{hr}-\mathrm{ft}^{2}$. The temperature rise of the central rod in the array exceeded the temperature rise of the surrounding rods by approximately $15 \%$ in both the horizontal and vertical orientations. For the same heat generation rates the observed individual rod temperatures were essentially the same in the horizontal and vertical orientations. Figure 7 shows the temperature rise above ambient of the central rod and the coolest outer rod. as a function of rod surface heat flux. 


\section{B. Ten Rod Array}

Tests were conducted on the ten rod array at rod surface heat fluxes to $550 \mathrm{Btu} / \mathrm{hr}-\mathrm{ft}^{2}$ for several configurations. The measured temperature rise above ambient was higher for the central two rods than the surrounding rods in all tests. For the same heating rates similar temperatures were observed on the central rods in both the horizontal and vertical orientations. For tests conducted in the vertical position the outer eight rods operated at relatively uniform temperatures with the central two rods exhibiting a temperature rise approximately $20 \%$ higher than the outer rods. For tests conducted horizontally the central rods operated at the highest temperatures, with the upper row next hottest, the lower row next, and the outer center row rods coolest. A summary of test configurations and results is presented below.

1. Ten Rods in Lateral Support Grid and External Support Holder - Horizontal and Vertical

Figure 8 shows the temperature rise above ambient of the hottest central rod in the horizontal and vertical as a function of rod surface heat flux.

2. Ten Rods in Lateral Support Grid without External Holder - Horizontal

Figure 9 presents the temperature rise above ambient as a function of rod surface heat flux for the hottest central rod in the support grid without external holder.

3. Ten Rods in External Support Holder without Lateral Support Grid - Horizontal and Vertical.

Figure 10 presents the temperature rise as a function of heat flux for the hottest central rod in the external holder without support grid.

The temperatures of the rods in the holder without support grid were approximately $3 \%$ higher than when the support grid was included. It is concluded that the support grid aids in the conduction of heat from the rods to the external support holder, thereby reducing the temperatures of the central rods. The above data is utilized in establishing when, after a given reactor shutdown, a bundle can be taken to a hot cell for detailed examination. 


\section{Summary}

The radioactive decay of fission and activation products may cause high temperatures in single fuel elements or fuel bundles in a hot cell environment. A method for calculating decay heat was developed and verified by decay heat measurements on typical irradiated fuel specimens. Equilibrium temperature curves for evaluating temperatures in small rod test bundles have been presented. The decay heat and bundle temperature curves are used to evaluate the cooling time required for such test bundles prior to hot cell examinations.

Acknowledgement

The authors wish to acknowledge Dr. W. A. Stewart and A. T. Pieczynski of the Westinghouse Research Laboratories who are responsible for the test bundle temperature data contained in this report.

\section{References}

1. K. Shure, "Fission Product Decay Energy" in "Bettis Technical Review, Reactor Technology", WAPD-BT-24, December 1961, pp. 1-17.

2. J. R. Stehn, E. F. Clancy, Fission-Product Radio Activity and Heat Generation in "Proceedings to the Second United Nations International Conference on the Peaceful Uses of Atomic Energy, Geneva 1958", Vol. 13, pp. 49-54, United Nations, Geneva, 1958.

3. J. F. Perkins, R. W. King, "Energy Release from the Decay of Fission Products", Nuclear Science and Engineering 3, 726-46 (1958).

4. Lieberman, R. M., "A System for Calculation of Fission Power in Irradiation Tests", Presented at: National Symposium on Developments in Irradiation Testing, Sandusky, Ohio, September 9-1I, 1969. 
Materials Factors for Gamma Absorption Probability

$\frac{\text { Liaterial }}{\mathrm{UO}_{2}}$
$\mathrm{ThO}_{2}$
$\mathrm{ZrO}_{2}$
$\mathrm{Zr}$
Al

Gamma Absorption
Naterial Factor (B)
.0743
.0743
.0286
.0283
.0280

TABLS II

Comparison of Decay Power Measurements and Calculations

\begin{tabular}{|c|c|c|c|c|c|c|c|c|}
\hline \multirow[b]{2}{*}{ Specimen } & \multirow[b]{2}{*}{ Geometry } & \multirow[b]{2}{*}{$\begin{array}{l}\text { Fuel } \\
\text { Miaterial }\end{array}$} & \multirow[b]{2}{*}{$\begin{array}{l}\text { Irradiation } \\
\text { Time (EFED)* }\end{array}$} & \multirow[b]{2}{*}{$\begin{array}{l}\text { Fission } \\
\text { Heat Flux } \\
\text { (Btu/hrmte }\end{array}$} & \multirow[b]{2}{*}{$\begin{array}{l}\text { Cooling } \\
\text { Time } \\
\text { (Days) } \\
\end{array}$} & \multicolumn{2}{|c|}{ Decay Power } & \multirow[b]{2}{*}{$\begin{array}{l}\text { Ratio } \\
\text { Measured } \\
\text { Calculated }\end{array}$} \\
\hline & & & & & & $\begin{array}{c}\text { Calculated } \\
(B t u / h r)\end{array}$ & $\begin{array}{l}\text { Measured } \\
(\mathrm{Btu} / \mathrm{hr})\end{array}$ & \\
\hline 1 & Rod & $\mathrm{ThO}_{2}$ & 232 & 420,000 & 1.6 & $6 I .6$ & 53.5 & 0.87 \\
\hline 2 & Rod. & $\mathrm{ThO}_{2}$ & 232 & 420,000 & 1.6 & 61.6 & 51.8 & 0.84 \\
\hline 3 & Rod & $\mathrm{ThO}_{2}$ & 232 & 420,000 & 1.6 & 61.6 & 51.5 & 0.83 \\
\hline 4 & Rod & $\mathrm{UO}_{2}$ & 260 & 295,000 & 4.7 & 13.5 & 14.6 & 1.08 \\
\hline 5 & Rod & $\mathrm{InO}_{2}$ & 257 & 420,000 & 18.7 & 34.8 & 29.3 & 0.86 \\
\hline 6 & Rod. & $\mathrm{UO}_{2}$ & 205 & 750,000 & 1.8 & 30.2 & 30.7 & 1.02 \\
\hline 7 & Siab & $\mathrm{UO}_{2}$ & 68 & 580,000 & 17.4 & 14.6 & 16.3 & 1.11 \\
\hline 8 & Slab & $\mathrm{UO}_{2}$ & 68 & 490,000 & 17.4 & 12.9 & 15.0 & 1.16 \\
\hline 9 & Slab & $\mathrm{UO}_{2}$ & 260 & 390,000 & 17.4 & 13.2 & 13.6 & 1.03 \\
\hline 10 & SIab & $\mathrm{UO}_{2}$ & 501 & 300,000 & 17.4 & 9.4 & 9.0 & 0.96 \\
\hline 11 & Slab & $\mathrm{UO}_{2}$ & 132 & 640,000 & 1.3 & 37.7 & 44.2 & 1.18 \\
\hline 12 & SIab & $\mathrm{UO}_{2}$ & 224 & 280,000 & 0.8 & 27.5 & 31.1 & 1.13 \\
\hline
\end{tabular}


TOTAL DECAY POWER FOLLOWING CONSTANT POWER OPERATION

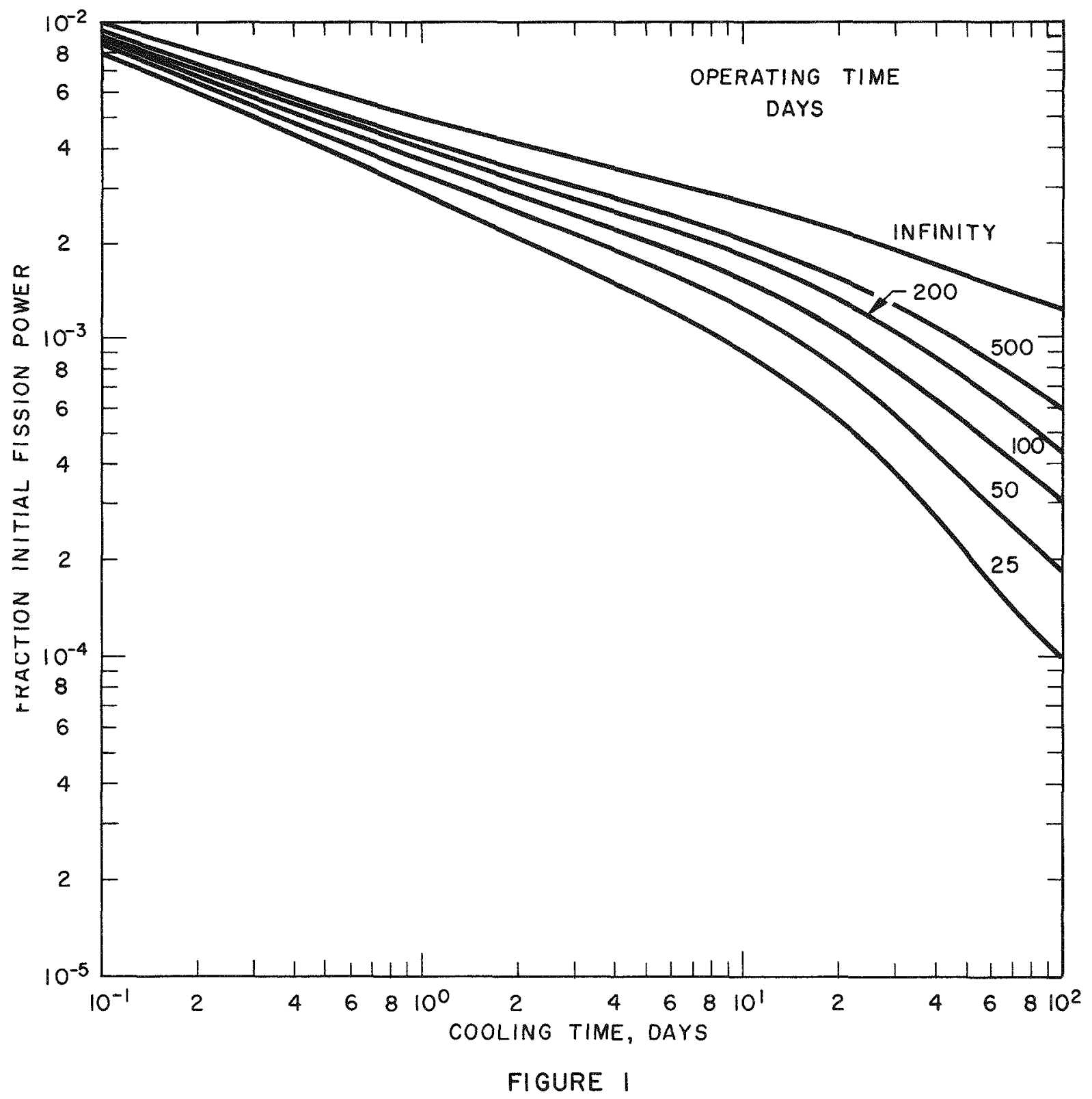


GAMMA DECAY POWER FOLLOWING CONSTANT POWER OPERATION

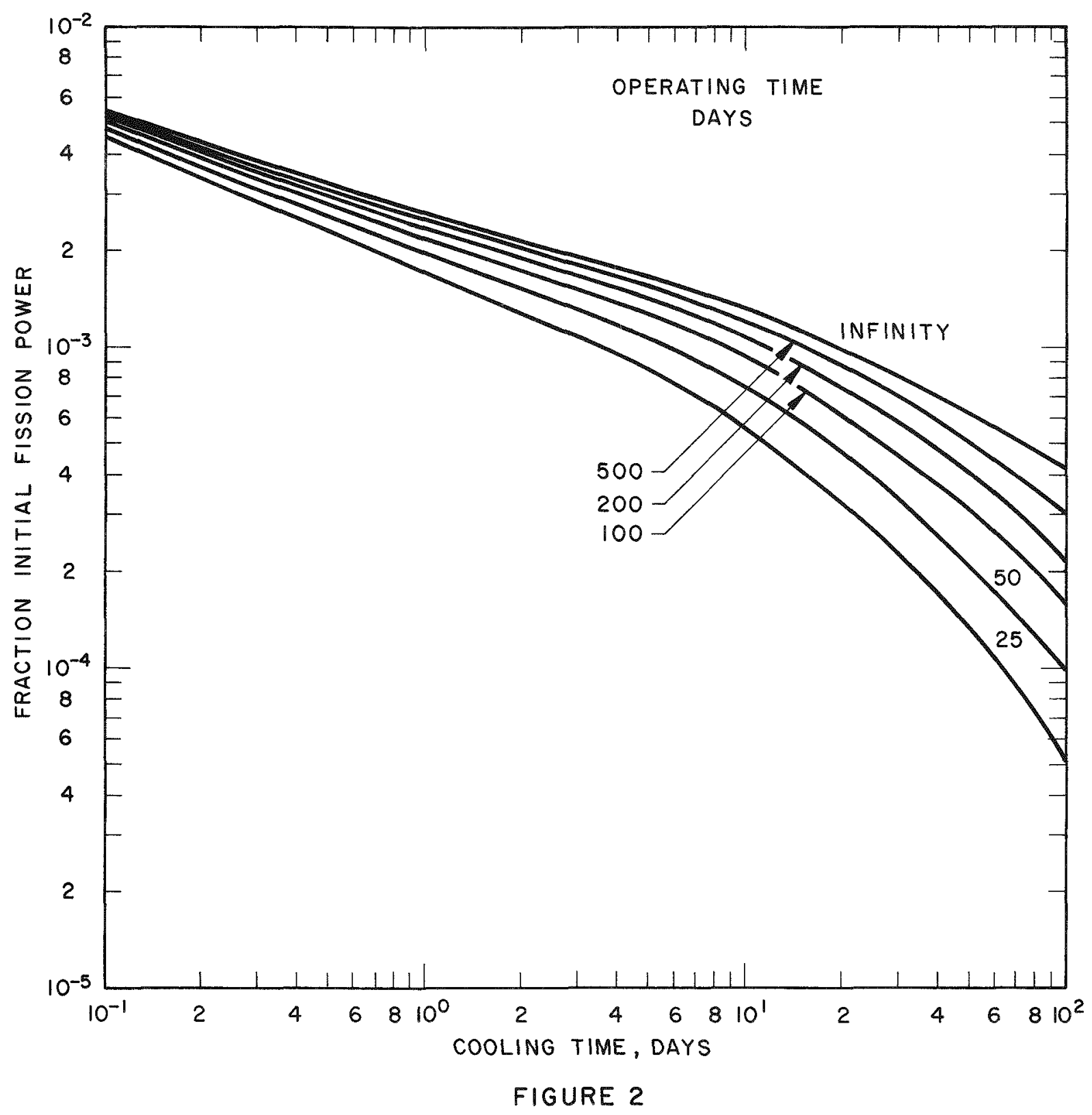




\section{GAMMA COLLISION PROBABILITY}

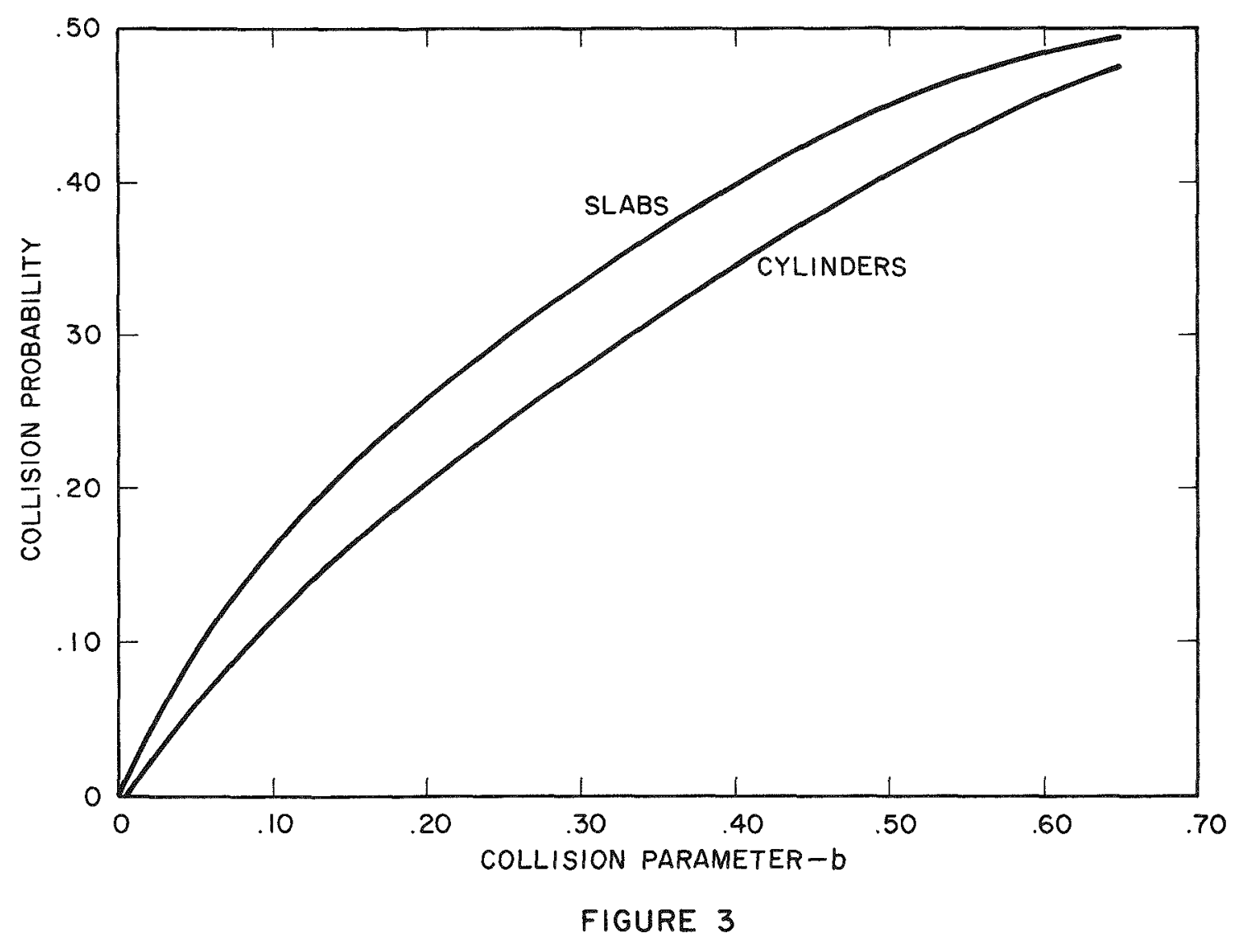




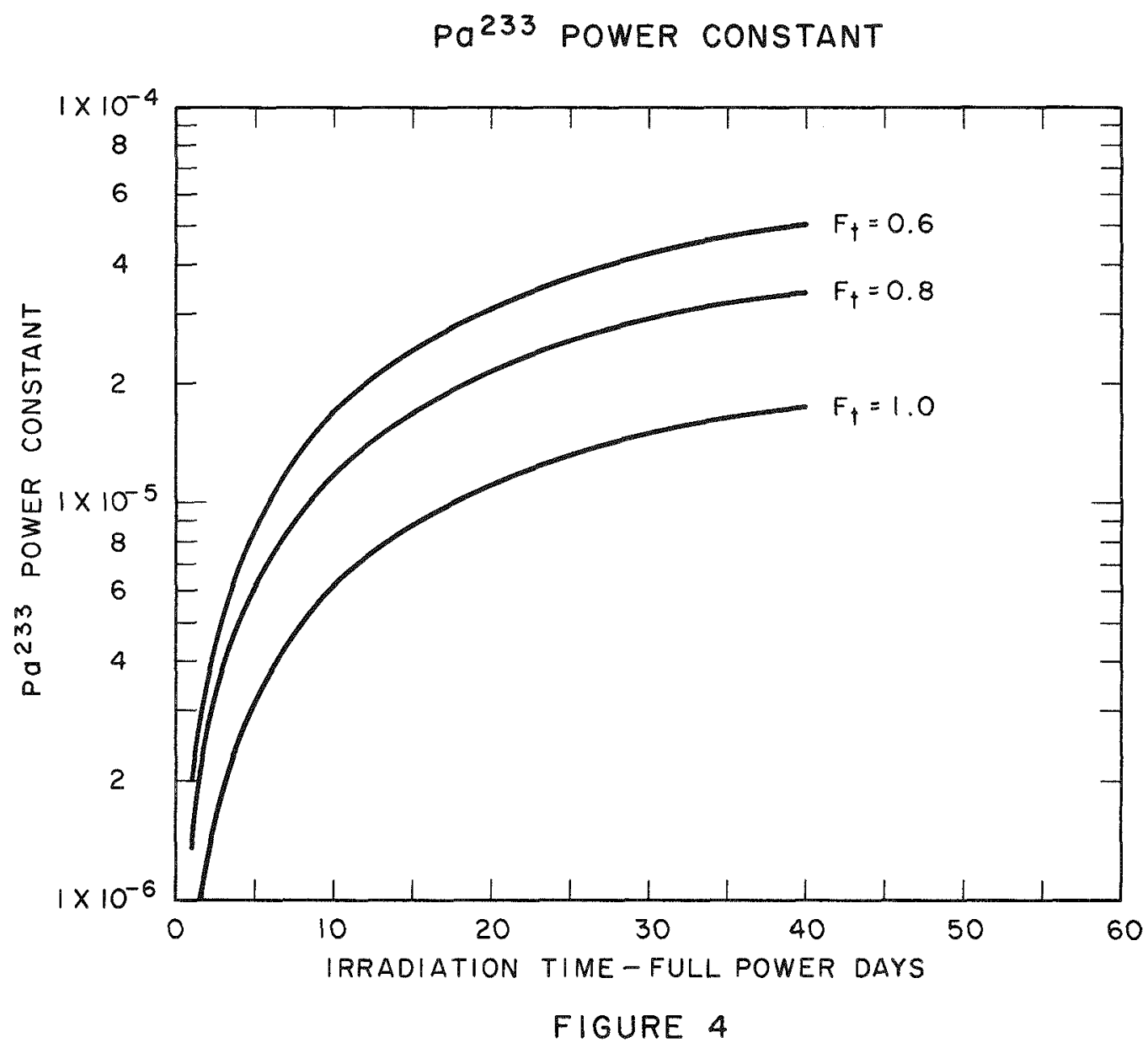




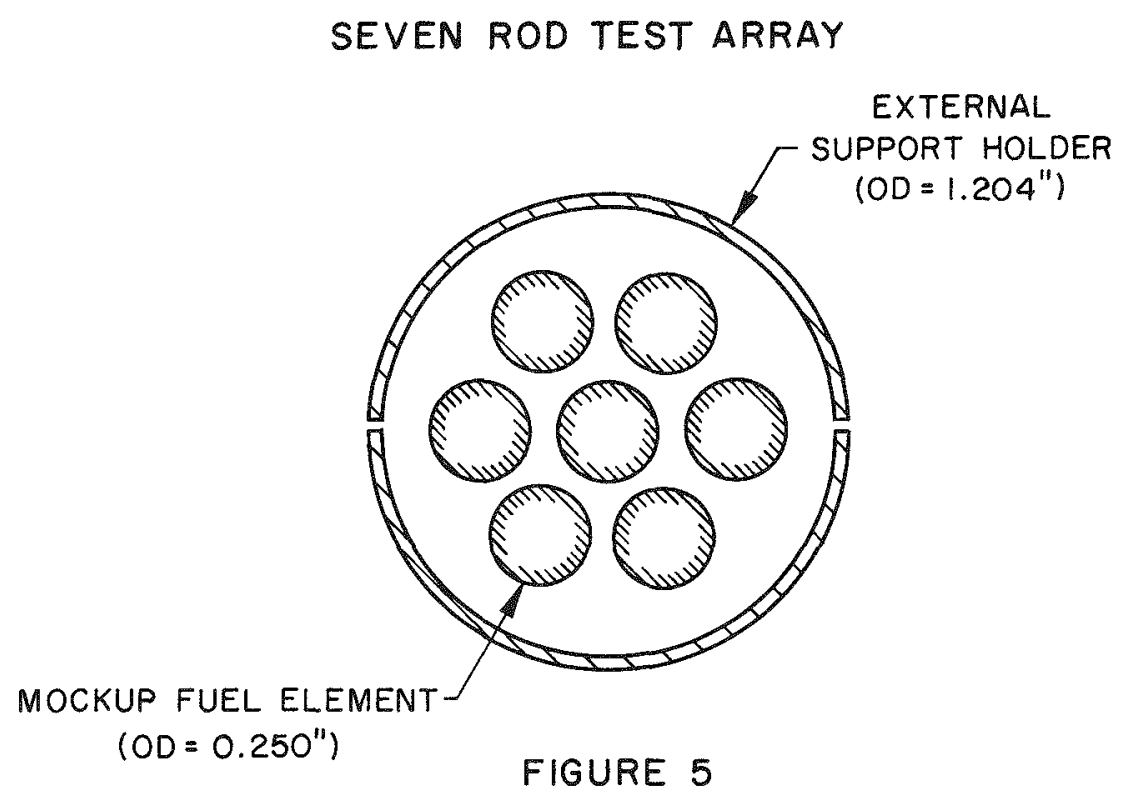

TEN ROD TEST ARRAY

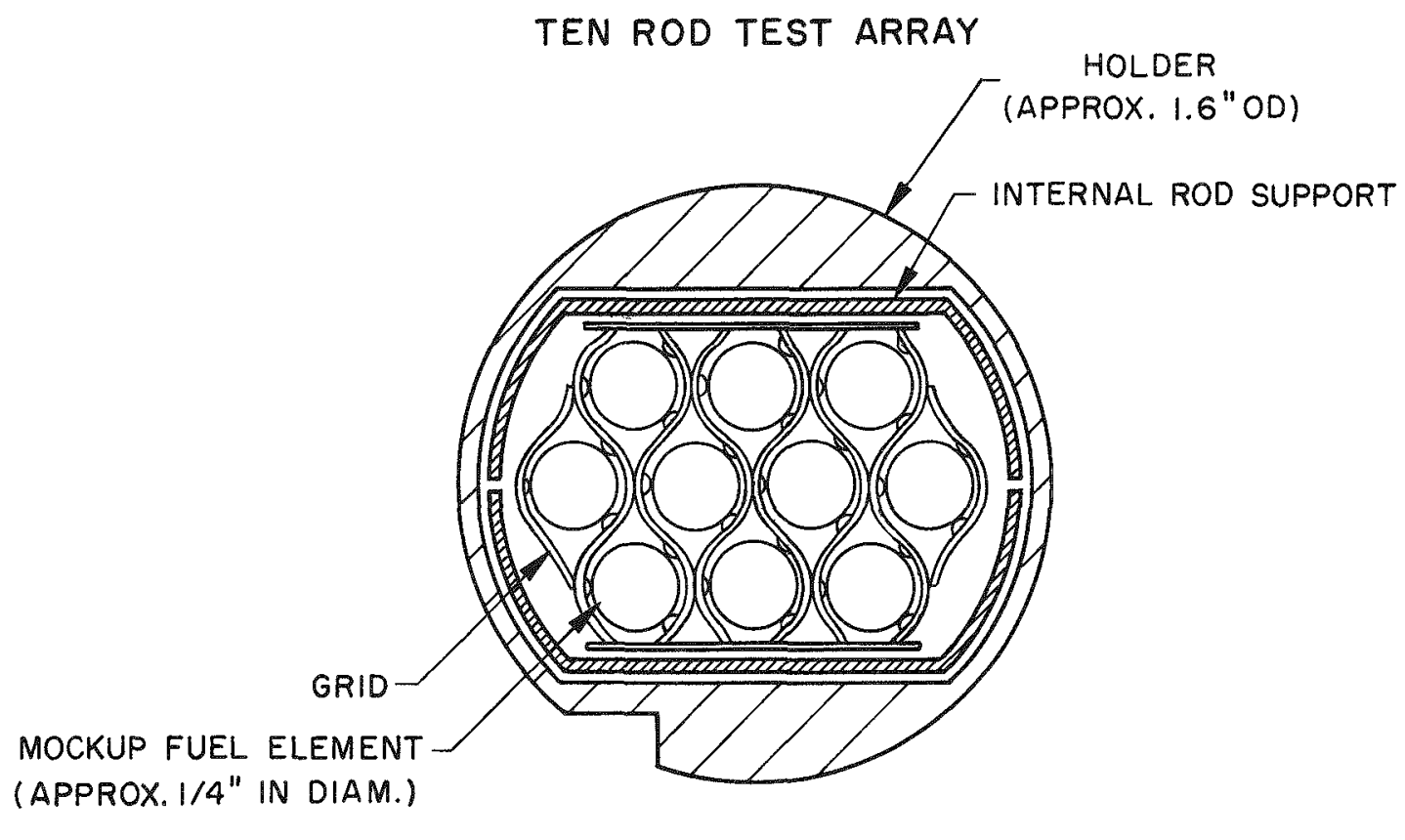

MOCKUP FUEL ELEMENT
(APPROX.1/4" IN DIAM.)

FIGURE 5

FIGURE 6 
HOTTEST ROD TEMPERATURE RISE -

SEVEN ROD ARRAY

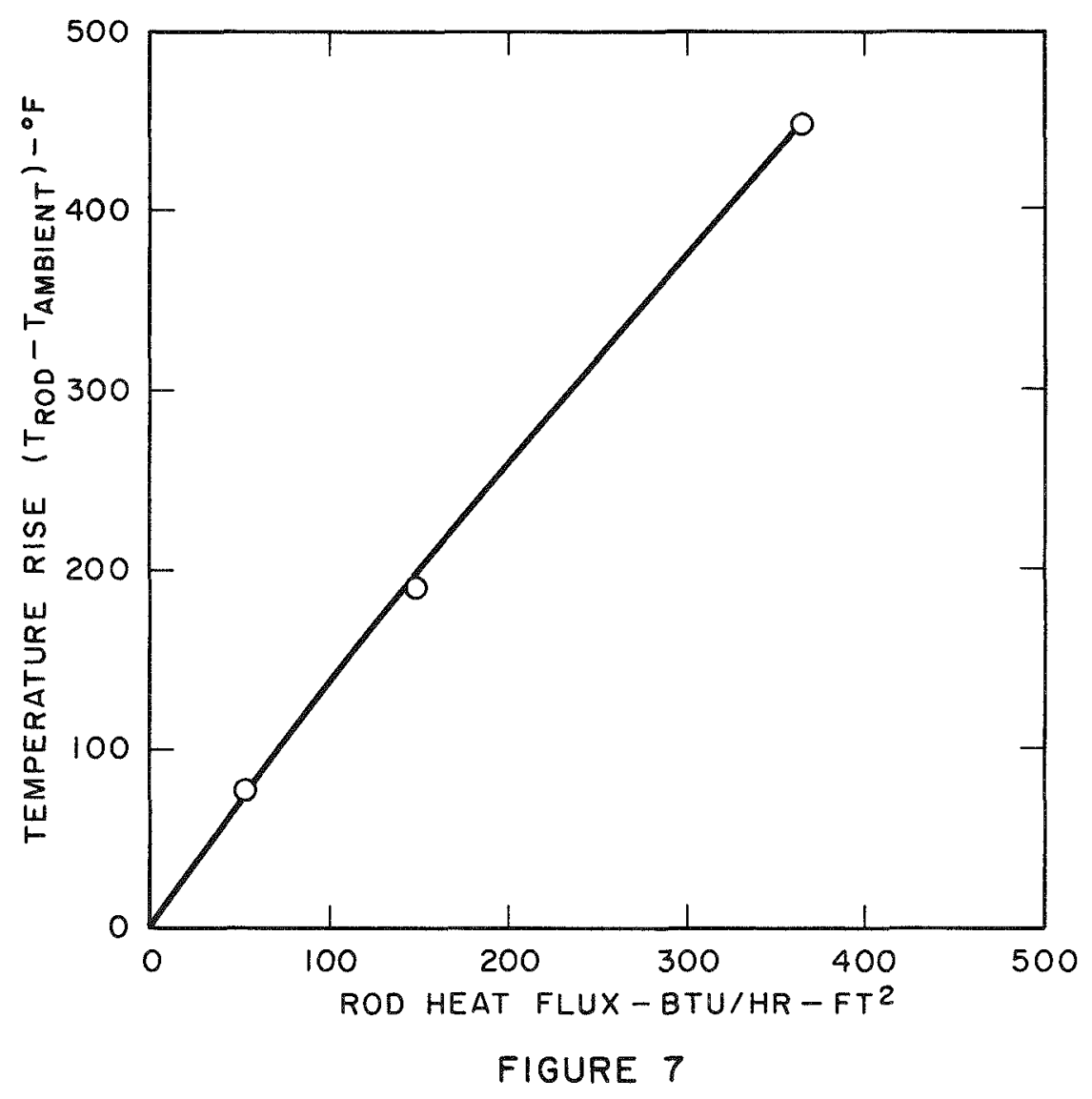


HOTTEST ROD TEMPERATURE RISE TEN ROD ARRAY

WITH GRID AND SUPPORT HOLDER

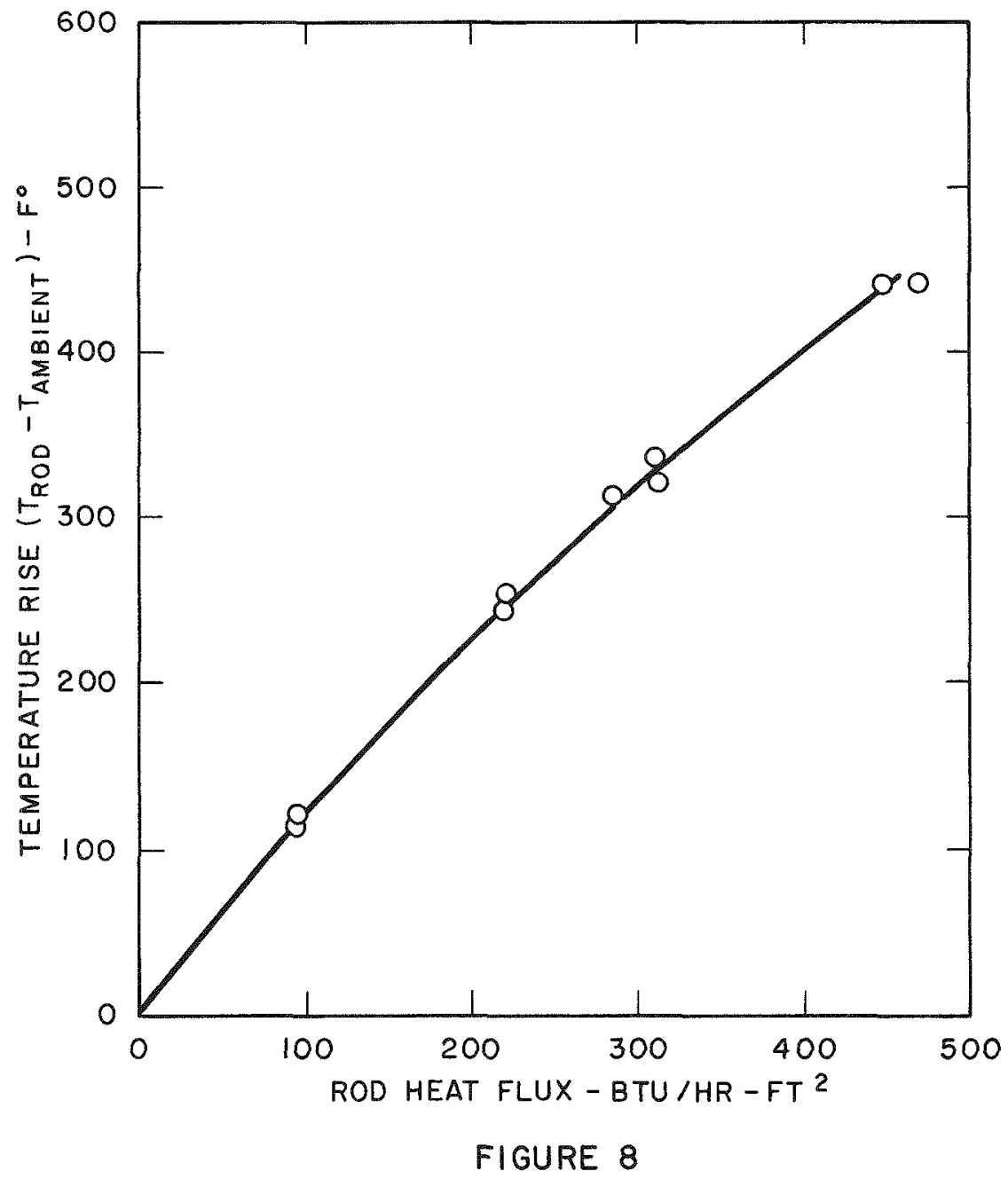


HOTTEST ROD TEMPERATURE RISE TEN ROD ARRAY

IN GRID AND WITHOUT SUPPORT HOLDER

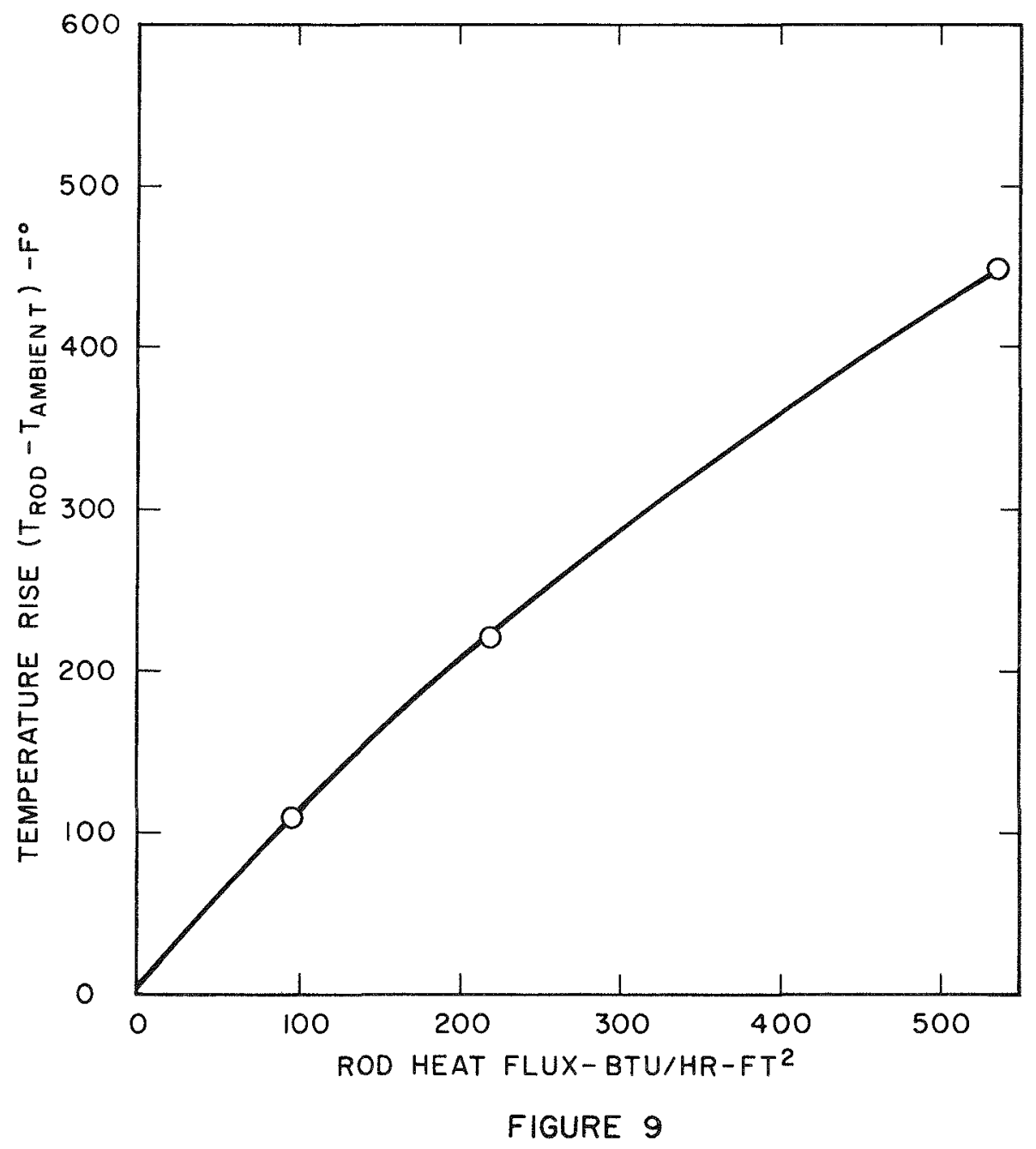




\section{HOTTEST ROD TEMPERATURE RISE- TEN ROD ARRAY IN SUPPORT HOLDER WITHOUT GRID}

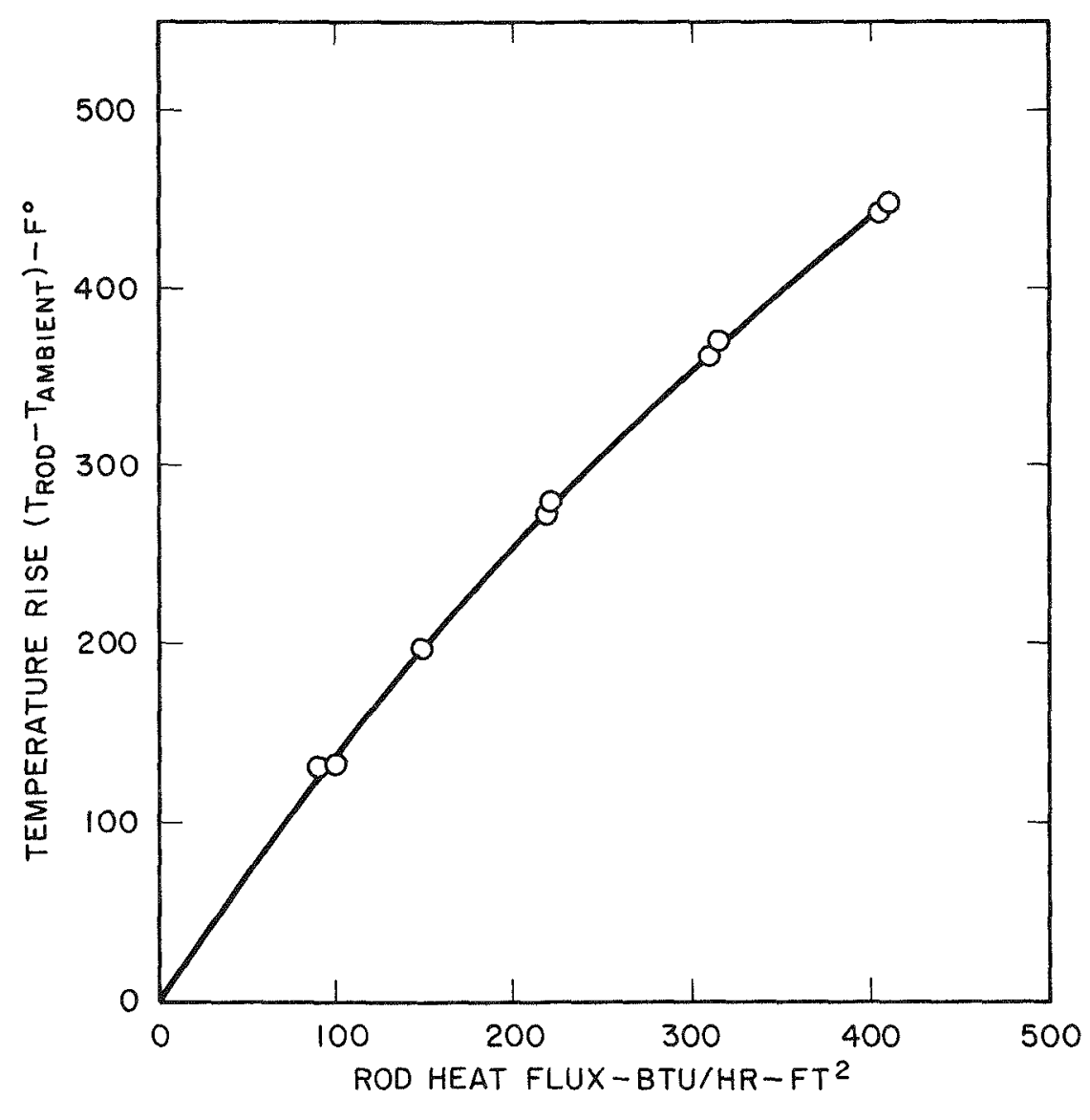

FIGURE IO 
1.

$$
\text { Post-Irradiation Microanalysis* }
$$

E. D. Albrecht, J. M. Johnson, and W. N. Hayes, Jr.

'Lawrence Radiation Laboratory, University of California

Livermore, California 94550

\begin{abstract}
A technique for examining 0.20 -in.-diam, by 0.75 -in.-long irradiated fuel specimens has been developed. Several pieces of specialized apparatus for de-cladding, subdividing and analyzing these specimens are described or referenced, and a systematic procedure is given for dimensional measurement and chemical and metallographic analysis of such specimens.
\end{abstract}

\title{
INTRODUCTION
}

A specialized post-irradiation examination facility was created at the Lawrence Radiation Laboratory, Livermore (LRL) for the purpose of sectioning, examining, and analyzing the pencil-size, $3 / 4$-in.-1ong fuel specimens that were irradiated in support of the Space Electric Power Program.

In this program there was considerable interest in the comparison of several different microstructures of the same reactor fuel. 1 For this reason, it was necessary to irradiate several fuel specimens under nearly identical environmental conditions to enable accurate comparison. This specification, as well as neutronic considerations, dictated that the irradiation specimens must be of a relatively small size. Furthermore, it was desired to have both chemical and metallographic analyses from specific areas of each specimen, which required the development of special saws and handling techniques.

This paper will describe the apparatus, techniques and procedures used to de-clad, subdivide, and analyze irradiated specimens in the LRL $\beta-\gamma$ Hot Cell Facility.

\footnotetext{
Work performed under the auspices of the U.S. Atomic Energy Commission.
} 


\section{PROCEDURE}

The specimens are removed from the irradiation capsule by normal hot cell operational procedures, using a remote pipe cutter, hack saw, and milling machine.

Slow-speed (300 r pm) diamond saws which have been described in detail by Albrecht, et al. ${ }^{2}$ were built for remote de-cladding and sectioning of irradiated specimens. Two saws were built, one for longitudinal cutting, the second for cutting cross-sectional diskshaped samples. These saws use 3-in.-diameter, 0.006-in.-thick diamond blades. This permits cuts to be made to predetermined depths for de-cladding without damaging the fuel. If there is a bond between the fuel and the cladding, the entire specimen can be sectioned without de-cladding. A typical specimen from a recent LRL irradiation is shown in Fig. 1, where the various steps of de-cladding and specimen sectioning are diagrammatically illustrated. In Fig. 1(a) the end cap is removed and the cladding has been longitudinally slit. At this point, the fuel specimen is available for macroscopic observation and dimensional measurement.

During all steps of de-cladding and analysis, a "windowless" macro-camera, described by Albrecht et al., 3 is available for photographically recording at magnifications from 2 to $30 \times$ any feature of interest. This camera is also used to photographically index all samples for later positive identification.

Dial micrometers accurate to \pm 0.0001 in. are used to determine the outside diameter, inside diameter (if specimen has a hole), and length. The outside diameter and length measurements are made with a micrometer equipped with a 1/8-in.-diameter ball contact on the plunger and a 1/8-in.-diameter tool steel rod as the anvil. Inside diameters are measured with standard machinist micrometers made expressly for this purpose.

However, more accurate volumetric data can be obtained by direct measurement using a mercury pycnometer. A mercury pycnometer described by Albrecht, et al." 4 was built with the capability of reproducing results accurate to 2 parts in 3000 and to accept specimens having a volume of 0.1 to $0.4 \mathrm{cc}$. Pycnometer-measured volumes eliminate the multiple error of lengths and extrapolation between diameters measured independently.

A semi-micro balance is available to determine weights of the de-clad specimens when it is desirable to know if the specimen composition has remained stable during the irradiation. Comparative pre- and post-irradiation densities can also be calculated from the weights and volumes measured.

When dimensional measurements have been completed, the specimen is returned to the low-speed diamond saws, where a 

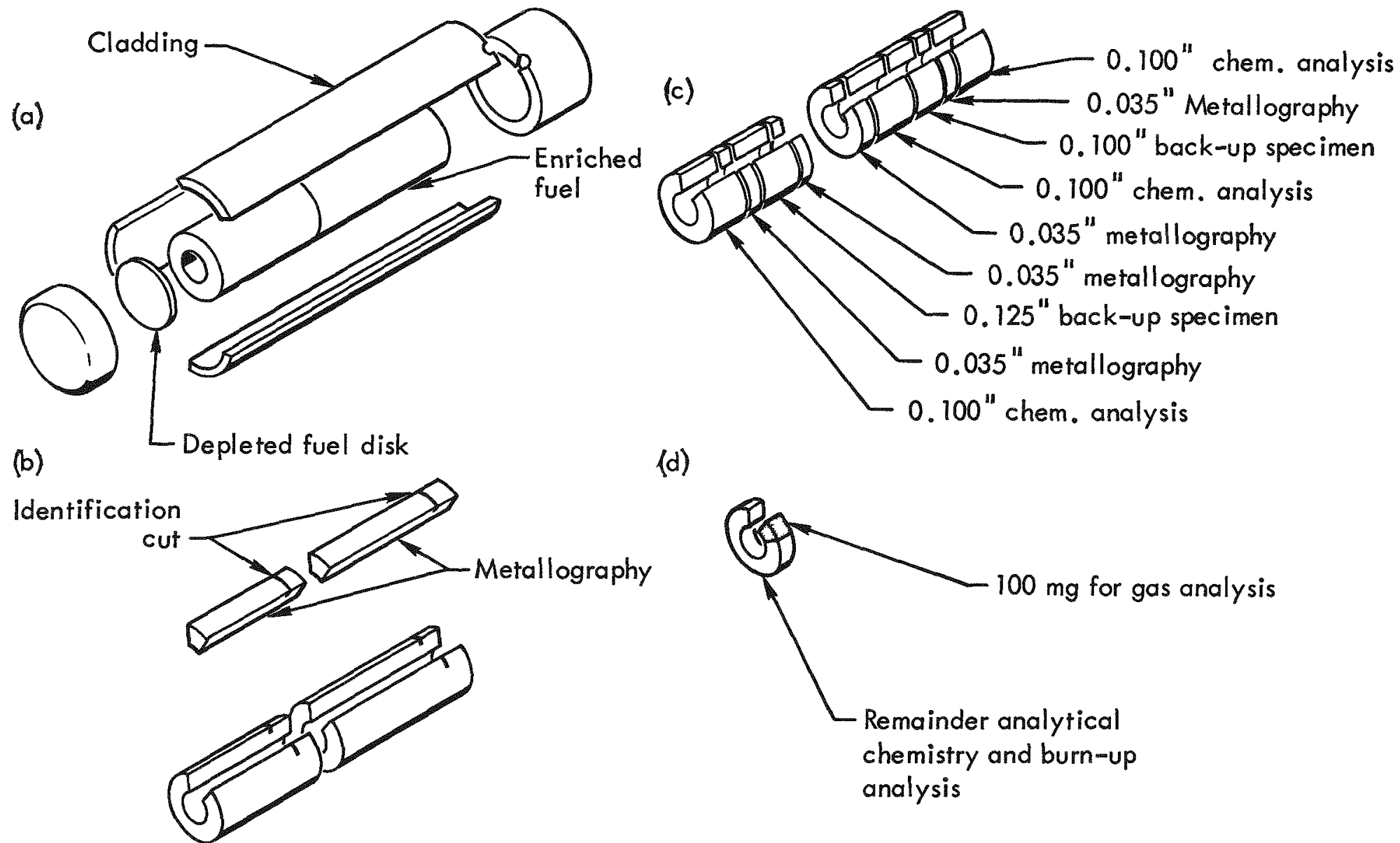

Fig. 1. Sequence of cladding removal and sectioning of fuel specimen for metallographic and chemical analvses. 
longitudinal metallographic specimen is removed, as shown in Fig. 1(b). This is a relatively simple task when a tubular specimen similar to that shown in the diagram is being examined. In the case of a solid cylinder, the specimen is either sectioned by a single lengthwise cut all the way through the specimen, with one-half being used for metallography, or this step is omitted, depending upon the interest in longitudinal irradiation effects in the specimen.

In Fig. 1(c) the sectioning sequence for chemistry, metallography, and back-up samples is shown. Samples as thin as 0.030 in. can be cut without fear of breakage. This sectioning procedure permits small samples to be removed for analytical chemistry from several locations within the specimen (as in Fig. 1(d)), making comparative analyses possible along the length or around the circumference of the specimen. Uranium analyses are determined by controlled-potential coulometric methods similar to ASTM Method D-217, and bomb-combustion techniques have been developed to handle milligram samples for gas analyses. 5 Remotely operated micro-pipettes permit accurate dissolution of samples for radiochemical analyses, which are performed outside the hot cells in a normal laboratory environment. Again, the technique permits com- 6 parative burn-up and radiochemical analyses by gamma spectroscopy from several locations in a tiny specimen.

Metallographic samples are generally removed adjacent to the chemistry samples. The metallographic samples are prepared by using remotized adaptations of mounting press, lapping and grinding machines, and vibratory polishers, with the resulting polished specimen being etched chemically or cathodically prior to microscopic examination. The remote metallographic facility has been described previously by Albrecht and Johnson. 7 The mounted metallographic samples can then be photographed with a remote metallograph or macro-camera and transferred to a remotized microhardness tester or removed from the hot cells via a shielded transfer cart to a shielded electron microprobe 8 for further examination of the microstructure.

All master-slave manipulators are equipped with rubber-tipped tweezers attached directly to the two-finger hands to facilitate the handling of the tiny samples during preparation. Each cell window is equipped with specially corrected short-focal-length binoculars, which are used to view the sample during preparation.

\section{SUMMARY}

A typical 4-g, 0.20-in.-diameter by 0.75-in.-long fuel specimen can be de-clad and sectioned into one longitudinal metallographic sample and ten cross-section samples, which are distributed: four to metallography, three to chemistry, and three to storage for duplicate analysis if necessary. The three chemistry specimens are again subdivided into three parts and distributed to analytical chemistry, 
gas analyses, and radiochemistry. A complete analysis of such a specimen, including preparation of cladding samples, will take approximately two man weeks using the system described herein.

\section{ACKNOWLEDGMENTS}

The authors wish to thank Edward H. Stearns and Bert J. Tuckey for their many contributions to the design and fabrication of the apparatus used in the examination of irradiated specimens.

\section{REFERENCES}

1. Albrecht, E. D., A. J. Rothman, J. D. Lee, J. M. Johnson, and W. N. Hayes, Jr., "Irradiation Studies in Uranium Nitride," Report UCRL-71703, Lawrence Radiation Laboratory, Livermore, August 1969.

2. Albrecht, E. D., E. D. Stearns, and F. J. Wittmayer, "LowSpeed Diamond Saw," Report UCRL-71611, Lawrence Radiation Laboratory, August 1969.

3. Albrecht, E. D., J. M. Johnson, and E. H. Stearns, "Windowless' Macro-Camera for Hot Cell Use," Report UCRL-71561, Lawrence Radiation Laboratory, Livermore, July 2, 1969.

4. Albrecht, E. D., W. N. Hayes, Jr., E. H. Stearns, and J. M. Johnson, "Use of Mercury Pycnometer in Volume Determinations," to be published.

5. Albrecht, UCRL-71703, op. cit.

6. Ibid.

7. Albrecht, E. D., and J. M. Johnson, "Remote Metallography Facility at Lawrence Radiation Laboratory," Report UCRL-71612, Lawrence Radiation Laboratory, Livermore, August 1969.

8. Scotti, V. G., J. M. Johnson, and R. T. Cunningham, "Electron Microprobe Analysis of Highly Radioactive Samples," Procedures of 14th Annual Conference on Application of x-Ray Analysis, 1965. 
Techniques and Applications - General 
A SYSTEM FOR CALCULATION OF FISSION POWER IN IRRADIATION TESTS

Richard M. Lieberman

WESTINGHOUSE ELECTRIC CORPORATION

Bettis Atomic Power Laboratory

\begin{abstract}
A system has been developed which will predict fission power for irradiation test fuel samples. This is possible because these samples are irradiated in a relatively stable, standard environment. Within this environment test fission power is controllable by the experimenter. The effect of system variables on fission power, determined by neutron diffusion and transport theory, was reduced to a set of design curves. Effective neutron fluxes are found from these design curves. Time dependent nuclear depletion equations are then solved for the isotopic content of both fertile and non-fertile test fuel materials. The results of the depletion calculation are converted to quantities of interest to the experiment designer such as fission power density, clad surface heat flux and burnup. This system has been applied and verified in the Engineering Test Reactor.
\end{abstract}




\section{Introduction - The Need for a System}

The Bettis Atomic Power Laboratory (BAPI) has a program for the irradiation testing of proposed fuel elements which features a large number of samples operating at conditions specified by the cognizant test designer. These samples begin life at fissionable material loadings which are functions of desired initial testing conditions and thus are not constant. During any test reactor cycle the samples being irradiated are at various stages of depletion. Samples may contain fertile material such as thorium, which after some irradiation can contribute significantly to the fission rate.

Individual samples are often interchangeable, allowing a continuous rearrangement of samples to take place. Irradiations take place in standard testing facilities which have some built-in capability to control fission power. The overall result is that the operating conditions of each sample in the BAPL irradiations program are maintained at closely controlled conditions. These conditions are specified by the needs and objectives of the experiment itself. As a result of the extreme flexibility which has been built into the irradiations program, there is a problem of the prediction of the operating conditions of each sample as new test arrangements begin successive cycles of test reactor operation. It is not desirable - in practice it is impossible - to do specific detailed nuclear and thermal calculations for every proposed or actual test configuration. A system has been developed which will allow cogmizant irradiation test designers to calculate operating conditions in a minimum of time. The irradiation test designer need only concerm himself with the conditions of the irradiations test once the effects of test variables have been reduced to clearly understandable functions. The development and utilization of the most important part of the design system - that of the calculation of fission power - will be described. 
II. Why a Calculation System is Possible

The fuel irradiations discussed here all take place in standard facilities which are portions of recirculating light water loops in the core of the Engineering Test Reactor (ETR). Figure I shows schematically the core of the ETR. Within the beryllium reflector is the active core which consists of 100 grid locations in a uniform square array of about three inch interval. A more detailed description of the ETR may be found in Reference 1. The figure shows the location of standard facilities. It also shows the location of other tests, reactor inserted control elements, and reactor fuel locations whether or not the fuel is associated with reactor control. In order to maintain a stable testing facility this core arrangement is changed very infrequently. Although the composition of the tests in the reactor is changed often, the location of these tests is maintained constant. Notice that with one exception there are no tests adjacent to standard loop facilities, an important contribution to stability.

The figure shows that the standard loop facilities are located at many radial core locations, from near the core center ( $\mathrm{J}-10)$ which is a maximum power location to the edge (N-14) which has the minimum power. Selection of the proper loop facility for an irradiation allows a variation in power level by a factor of about 3-I/2. Further power control can be obtained by proper axial location of a test within the selected radial loop location. The active core of ETR is three feet in height. For a test six inches high, changing axial location will allow a power variation of a factor of about three. There may be as many as five separa.te tests located in various axial positions in one radial core location.

A standard loop facility is that portion of a hot, high pressure, light water recirculating forced convection flow loop which is in the three feet active ETR core. Figure 2 shows a standard loop with a typical irradiation test. The power production in the test fuel elements is controlled to some extent by the power control shroud which is a .050 in. thick tube of $\mathrm{Zr}-\mathrm{H} f$ alloy. By variations of the hafnium content over the range of zero to 100 volume $\%$, the test fission power level can be changed by a factor of about two.

Thus the fission power level in a BAPL irradiation test is essentially within the control of the experiment designer, with few outside, uncontrollable effects to interfere. The attainment of this necessary condition allowed the calculation of irradiation test fission power to be reduced to a simple, rapid 
calculation system. The total capability of power variation is about a factor of 20 once a test arrangement has been fixed, which can give the test designer close control over test conditions.

III. The Generation of the System Functions

Fission power in an irradiation test in ETR is calculated by a complicated, computerized, but conventional nuclear computation technique. Essentially the normal methods of neutron diffusion and transport theory used for the nuclear calculations of light water moderated $U^{235}$ power reactors (Reference 2) are applied to the test reactor and associated experiments. Due to the complex and lengthy nature of this calculation it is not practical to compute every case which may be of interest in an extensive fuel irradiation program. For example to perform a survey of three possible arrangements of test fuel and power control shroud would require a total of about $1 / 2$ hour of time on a very fast CDC-6600 computer. More significant is the engineering time required to prepare the computer input and to analyze the output, which would be about three working days. Generally, a one week calendar time is required to analyze each set of possible test configurations. In order to handle a large number of samples with limited manpower, the simpler calculation system described here was developed.

Fission power in several typical irradiation tests was calculated using the lengthy nuclear design technique. The ETR core and control elements were represented after attainment of xenon equilibrium but prior to burnout of core burnable poison. This situation applies to most of the ETR core lifetime. The typical tests covered the full range of irradiation test variables fissionable material loading, power control shroud hafnium content, test radial and axial location in ETR for example. The results of these computer calculations were reduced to a set of design curves for rapid use by the experiment designer. Each design curve of the system will be described, both in terms of its use in the overall system and as to the nuclear phenomena it represents.

Figure 3 is the basic design curve of the system. It gives the relative power production of a gram of U235 as a function of the $\mathrm{U}^{235}$ content of a loop irradiation test. Curves applicable to three separate ETR irradiation facilities are shown. This figure and figures to be shown later are simplified versions of plots actually used by BAPL test designers. The power production figure shows that increasing the U235 loading of a loop test decreases the fission power per gram of U235. Stated another way the figure indicates 
that increasing absorption probability in a localized region of a reactor decreases the neutron flux in that region. If the curve were plotted in a different way - using watts/inch as the ordinate - it would be seen that for all locations increasing the $U 235$ content of a test increases the test power. The differences between the three curves indicate a typical expected effect of radial location in ETR on test fission power. The fission power values are applicable to nominal conditions of power control shroud, test water conditions, and axial location. The effect of these test variables will be shown in the following figures.

The upper portion of Figure 4 shows the effect of the hafnium content of the power control shroud on test fission power. As the material of the shroud tube is changed by adding absorbing hafnium the test power is reduced. The effect of test loading on the relative worth of the hafnium is illustrated by the differences between the three curves. The value of the shroud factor, SF, is set to unity at the nominal condition of no hafnium present.

The lower portion of Figure 4 shows the effect of test loop water conditions on test fission power. As the water content is increased test power increases due to improved local neutron moderation. Loop water temperature has a similar effect, with the decreasing density and the hardening thermal neutron spectrum which come from increasing temperature acting locally to lower the test power. The value of water factor, WE, is based upon nominal conditions of 15 volume \% water (based upon the area inside the power control shroud) and $500^{\circ} \mathrm{F}$ loop water temperature.

The effect on fission power of the location of the test axially in ETR is shown by the upper portion of Figure 5. This function was developed from measurements made in the ETR while the other system functions were calculated. It represents the time-averaged axial distribution during a typical ETR reactor cycle. The value of the axial factor, AF, is unity at the maximum point. Note that the nominal conditions of the functions SF, WF, and $\mathrm{AF}$ agree with the conditions specified for the fission power loading curves of Figure 3. Also used in the system is the function FTHERM, which is the fraction of $U^{235}$ fission power due to thermal energy neutrons. This is found on the lower portion of Figure 5. It can be seen that as absorption increases due to increased amounts of $\mathrm{U}^{235}$ or Hf, the neutron spectrum hardens and relatively less power results from fissioning due to thermal neutrons. 
IV. Calculation of Test Fission Power Conditions Using the System Functions

Fission power in an irradiation test is calculated from the functions evaluated from the figures previously described. The nominal fission power (from Figure 3) is multiplied by SF, WF and AF to obtain test fission power in watts per gram of $U 235$. In the restrictive case of only $U^{235}$ fuel and insignificant depletion, a power value of this sort would be sufficient to compute test power conditions. In order to calculate any depleting system the power value is converted to two group neutron fluxes, thermal and epithermal. The conversion for thermal flux is

$$
\varphi_{\text {Therm }}=(C)(P)(F \text { THERM }) /\left(\sigma_{\text {fiss, therm }}\right)^{235}
$$

where $P$ is the total power in watts/gram of $U^{235}$ and $C$ is a conversion factor. Note that the microscopic U235 fission cross section is used. The function FTHERM has been previously described. A similar equation exists for epithermal flux,

$$
\varphi_{\text {EPI }}=(C)(P)(I-F T H E R M) /\left(\sigma_{\text {fiss, }}^{235}\right)
$$

With two group fluxes and microscopic cross sections for all isotopes of interest the time dependent equations may be solved using a computer. Several computer programs have been written to do this; the one most often used at BAPL and most generally useful for this sytem is named CIVDER and is described in Reference 3.

The main result of the solution of a set of depletion equations is the timewise isotopic content of the fuel element. Using isotopic content, flux level, and microscopic cross section, the timewise power level and burnup can be calculated. In following changing test conditions or a varying test power level requirement, the system is used in a stepwise procedure. This is illustrated by Figure 6, a system schematic. Knowing the test conditions the functions are evaluated from the design curves.

Effective neutron fluxes are calculated as has been previously described. Knowing the power history of the EIR, the computer is used to calculate a new set of isotopic loadings for each test fuel element. Then changes are made if necessary to keep the test performing at desired conditions. 
Previously it was stated that the nuclear calculations from which the system was derived were applicable to the ETR core from the time of xenon equilibrium to the burnout of core burnable poison. Calculations supported by experience have shown that irradiation test power levels applicable to this period of ETR core life are adequate for depletion calculations. Further they represent power at any time in core life to about 10\%, with the greatest deviations coming as expected prior to xenon equilibrium and late in core life.

A typical application of the system is shown by Figure 7. This illustrates the operating conditions of a thorium fuel element with a small initial addition of $U^{235}$. Clad surface heat flux is shown as a function of fuel material burnup. The dotted line is the irradiation history which the irradiation test attempted to follow. In this case the only means of power control used was step changes in the hafnium content of the power control shroud. These changes were made at normal ETR refueling shutdowns. The figure shows how the step shroud changes closely follow the desired conditions. Also included on the figure is the contribution of the $U^{233}$ from the irradiation of the thorium. It is clear that in this case the fertility of the fuel material could not have been ignored.

The system described here is very useful in the interpretation of measured operating data. If power or heat flux are measured during the course of a test the depletion equations are solved for isotopic contents using heat flux rather than neutron flux as input. It is still necessary to know the test conditions of current loading and shroud hafnium content, since the function FTHERM must be accurately evaluated to attain a satisfactory solution.

\section{Verification of the System}

This calculation system was introduced to reduce the time necessary to calculate test fission power or similarly to allow the evaluation of more possible test configurations with existing time and manpower. Some reduction in the accuracy of the calculation results must be accepted in order to gain these benefits. Since the introduction of the system, test operational data have been used to verify that the system yields results of acceptable accuracy. 
Several different types of measurement are used to compare actual conditions to predicted conditions. The most sensitive of the instantaneous measurements is the center temperature of a fuel element under irradiation. Thermocouples are imbedded in the center of some fuel samples to give, the experimenter a direct measure and continuous record of fuel operating temperature. This measurement is compared to the temperature calculated from the heat flux predicted by the calculation system and calculated heat transfer coefficients. Another thermal measurement which is used is the total test heat output. Calorimetric calculations are made using measurements of loop water flow rates and water temperature rise. This measured heat input to the loop water is compared to the test heat output as predicted by the calculation system.

Time-integrated comparisons can also be used to verify the system calculation results. Post-irradiation destructive analysis of the isotopic content of the sample fuel is compared to the predicted value. This comparison is made for heavy elements such as $U^{235}, U^{236}$ or $U^{233}$ in the case of a thorium-bearing material. Fission product contents are also measured and used in the verification.

A comparison of measurement to prediction is shown by Figure 8. The lower portion of the figure shows a typical relationship between predicted and measured fuel element temperatures. The average difference is small and the $\sigma$ value is $13 \%$. The upper figure shows the comparison of predicted to mass spectrometric fuel burnup values from a different set of samples. Again the average difference is small; $\sigma$ is $15 \%$. Figure 9 shows the combination of both types of data. These data show a deviation from prediction of zero and a $\sigma$ value of $10 \%$. From these and other similar studies it has been concluded that the calculation system will predict most test operating conditions to within $10 \%$. Those tests which are outside this band are often characterized by samples which are not typical of BAPL irradiations. For such non-typical tests and for cases where great accuracy is necessary the time consuming computer calculations are performed.

VI. Extension to Similar Systems

The calculation system previously described is not peculiar to the ETR, but could be used for any test reactor which is reasonably independent of the tests in it. Quantities of 
interest to the experimenter, other than fission power, can be found from a similar system. At BAPL, gamma heating rates (which are often dependent upon local conditions) and fast flux levels ( $>1 \mathrm{MeV}$ ) are computed by very similar calculational techniques. For calculational simplicity these quantities are found from the same variables as the fission power. A complete irradiation test design package can be attained in this way.

VII. Summary

The system in use at BAPL for the calculation of ETR loop irradiation test fission power has been described. The system has been in use for several years, and has proven to be sufficiently accurate and rapid to perform the calculations necessary to support an extensive irradiation program.

\section{References}

1. J. Barnard, P. D. Bush, E. R. Kilsby, and W. M. Malott, "The Design and Construction of the ETR - A 175,000 kw Engineering Test Reactor" in Proceedings of the Second United Nations International Conference on the Peaceful Uses of Atomic Energy, Geneva, 1958. Vol. 10, Pg. 75-80, United Nations, Geneva, 1958.

2. A. Radkowsky Ed., "Naval Reactors Physics Handbook, Volume 1, Selected Basic Techniques", U. S. Atomic Energy Commission, 1964.

3. T. R. England, "CIVDER - A One Point Depletion and Fission Product System", WAPD-TM-334, August, 1962. 
ETR CORE

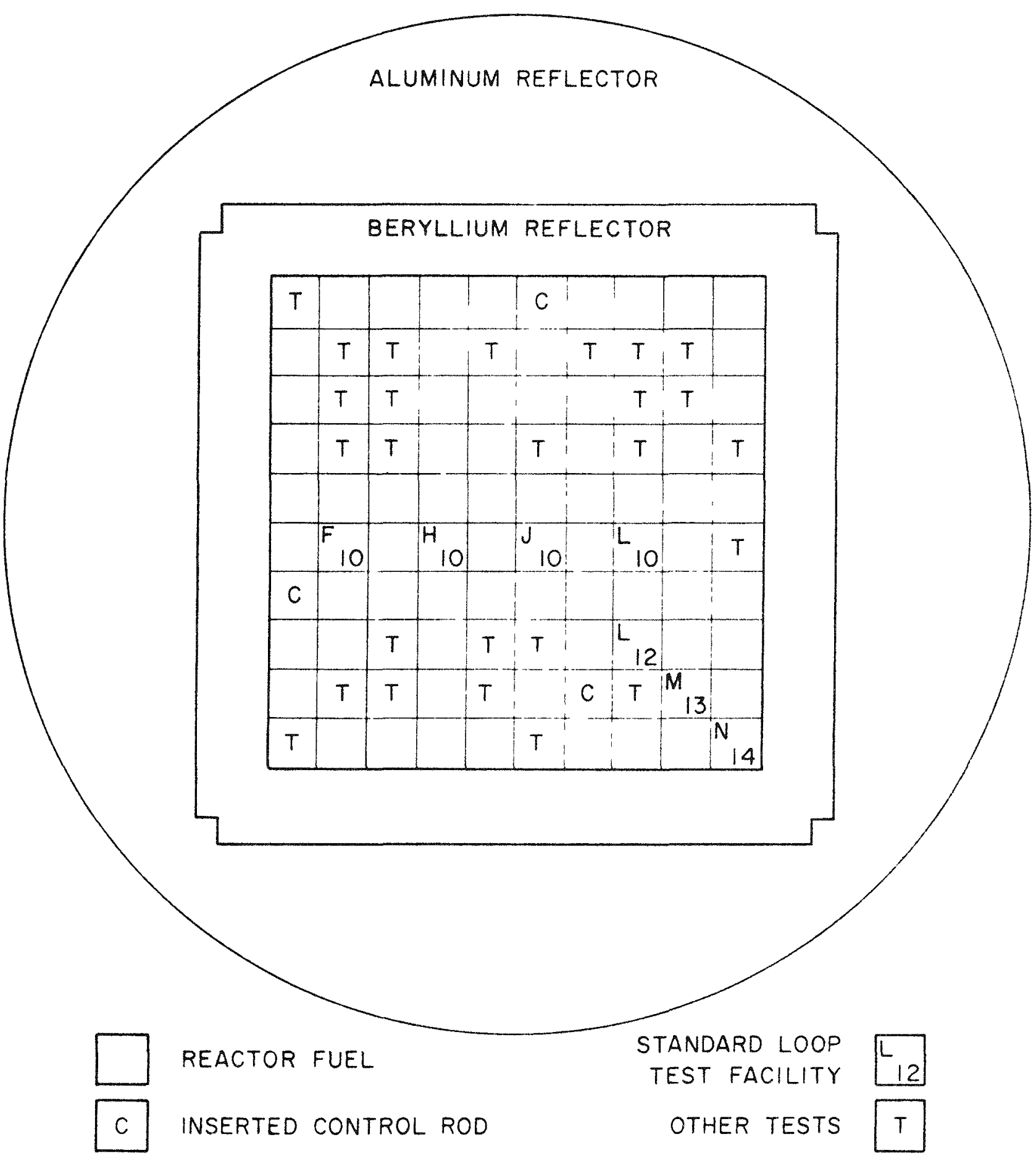

FIGURE 


\section{STANDARD LOOP FACILITY - WITH TEST}

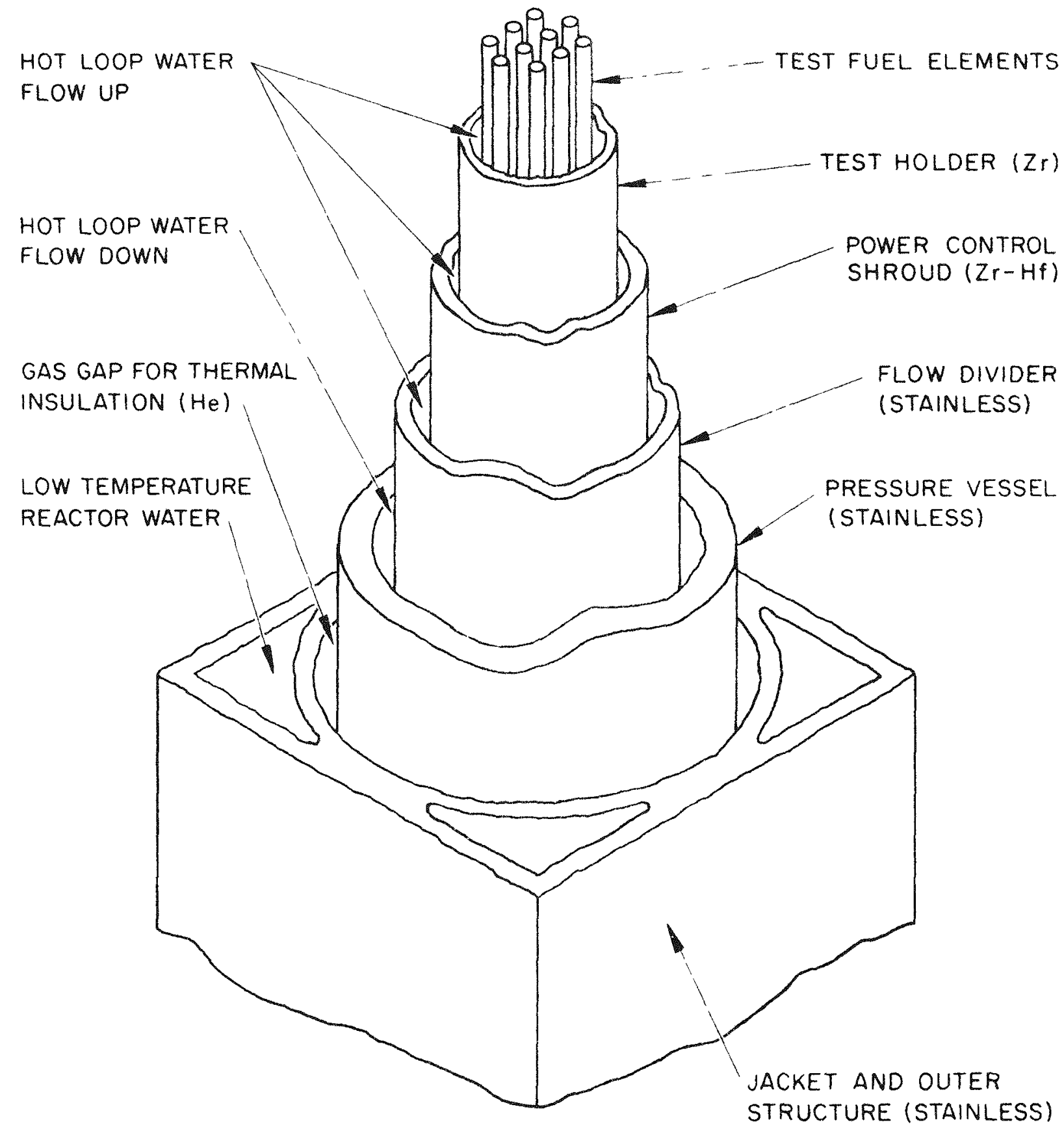

FIGURE 2 
FISSION POWER DESIGN CURVE

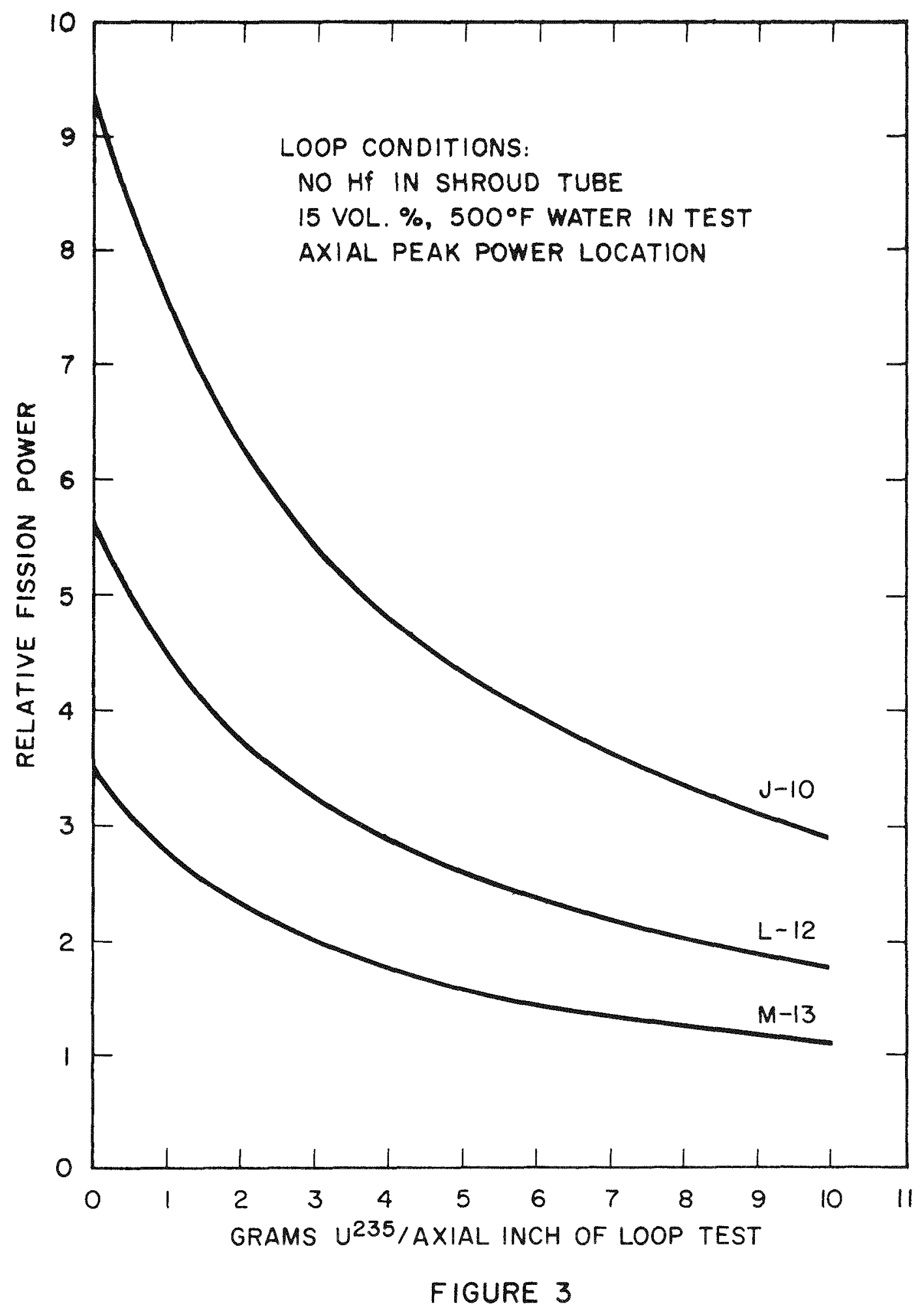


EFFECT OF HF IN POWER CONTROL. SHROUD ON FISSION POWER

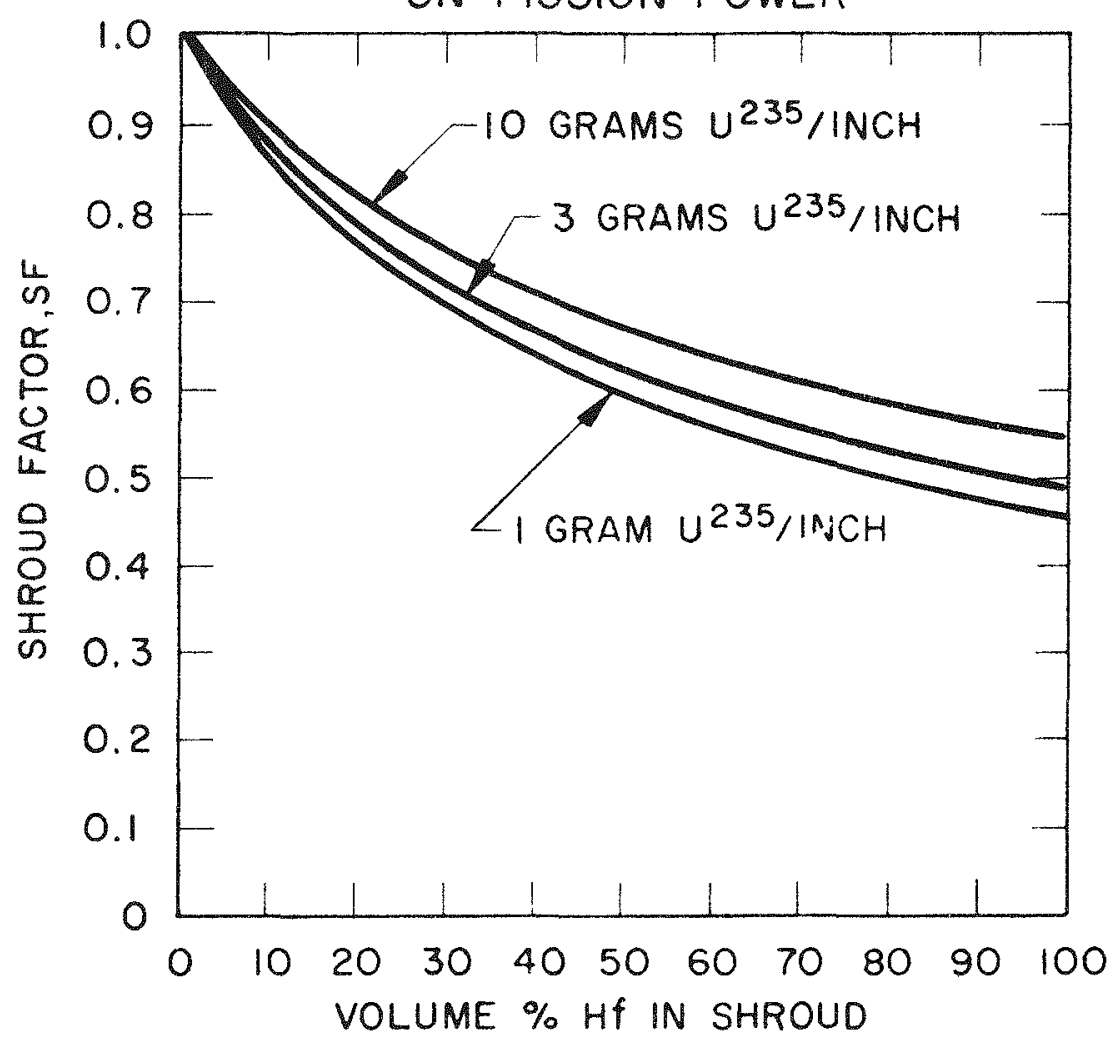

EFFECT OF TEST WATER CONDITIONS

ON FISSION POWER

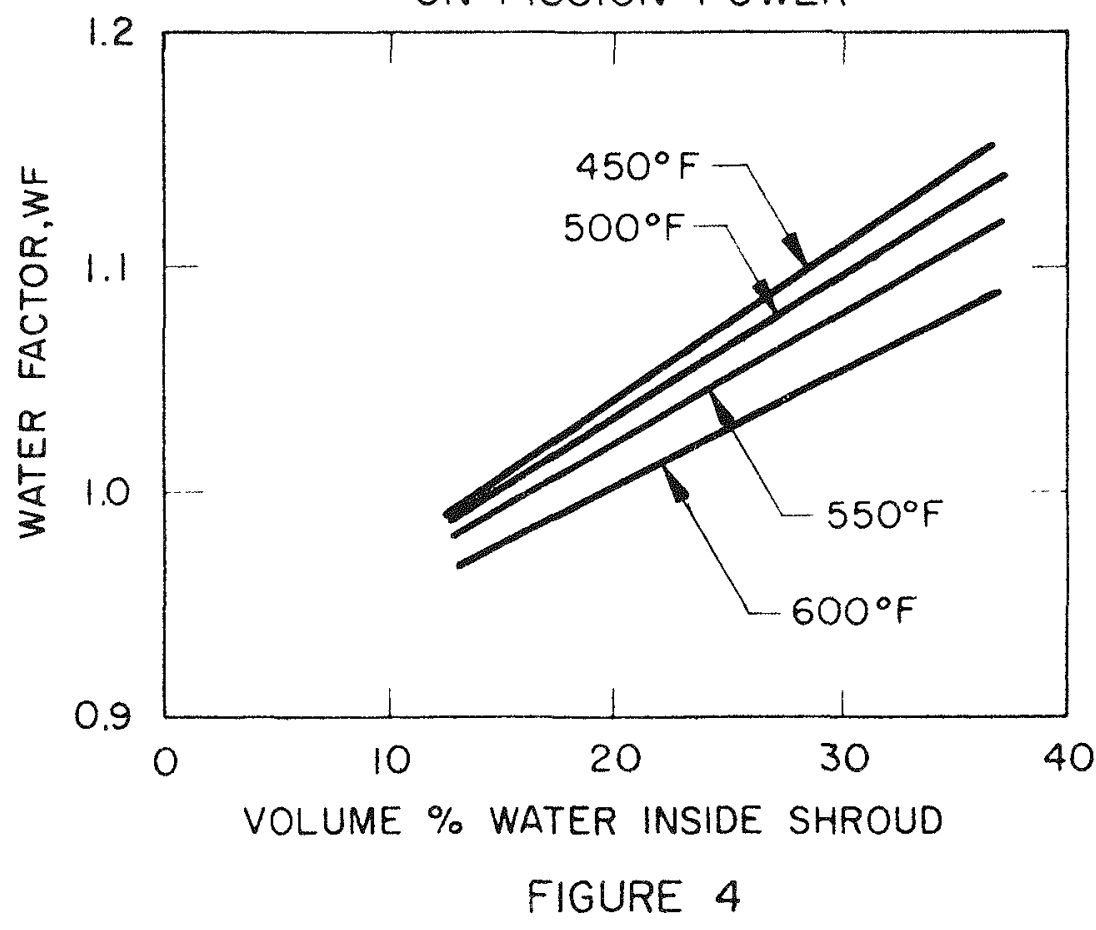



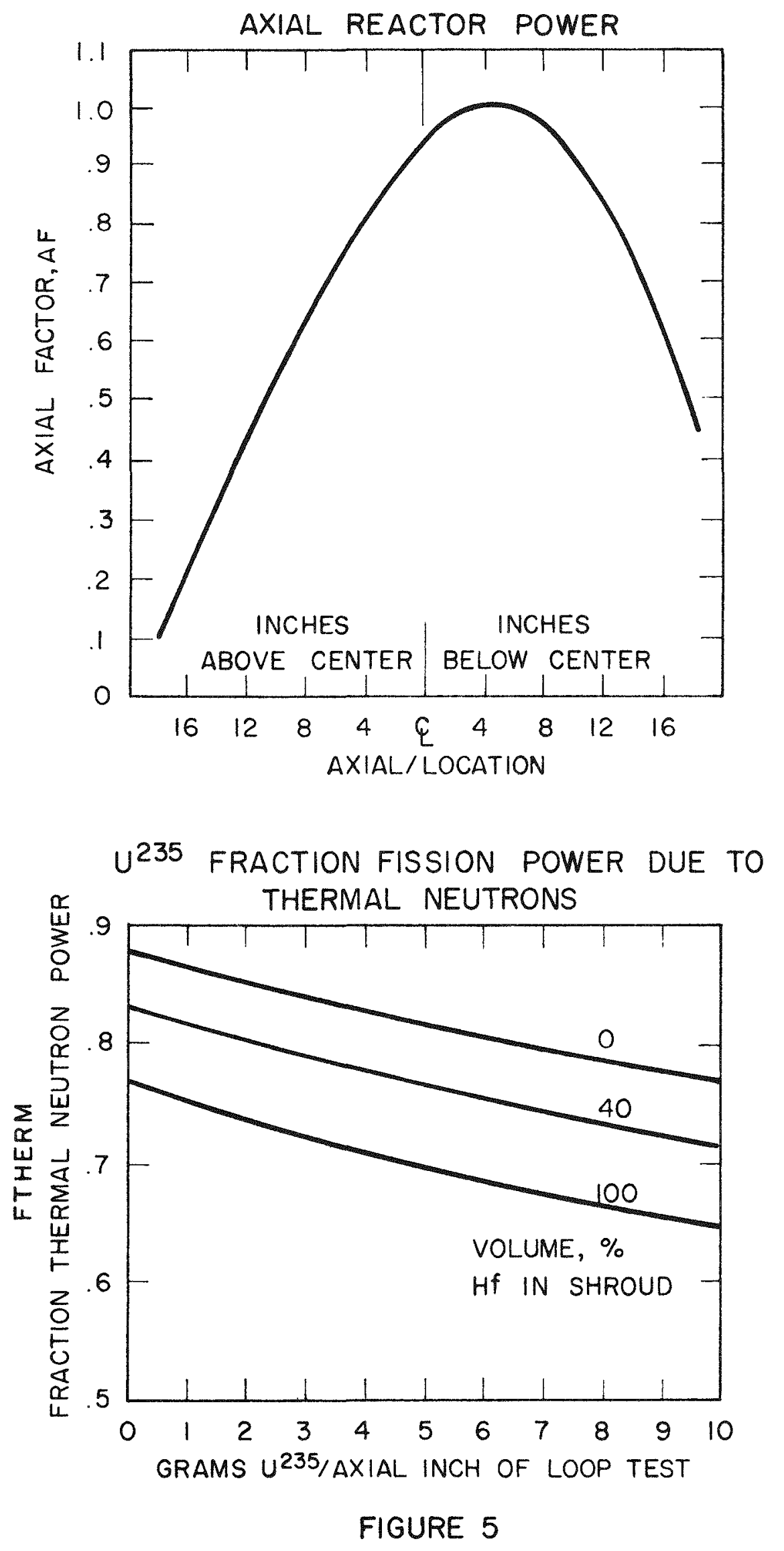


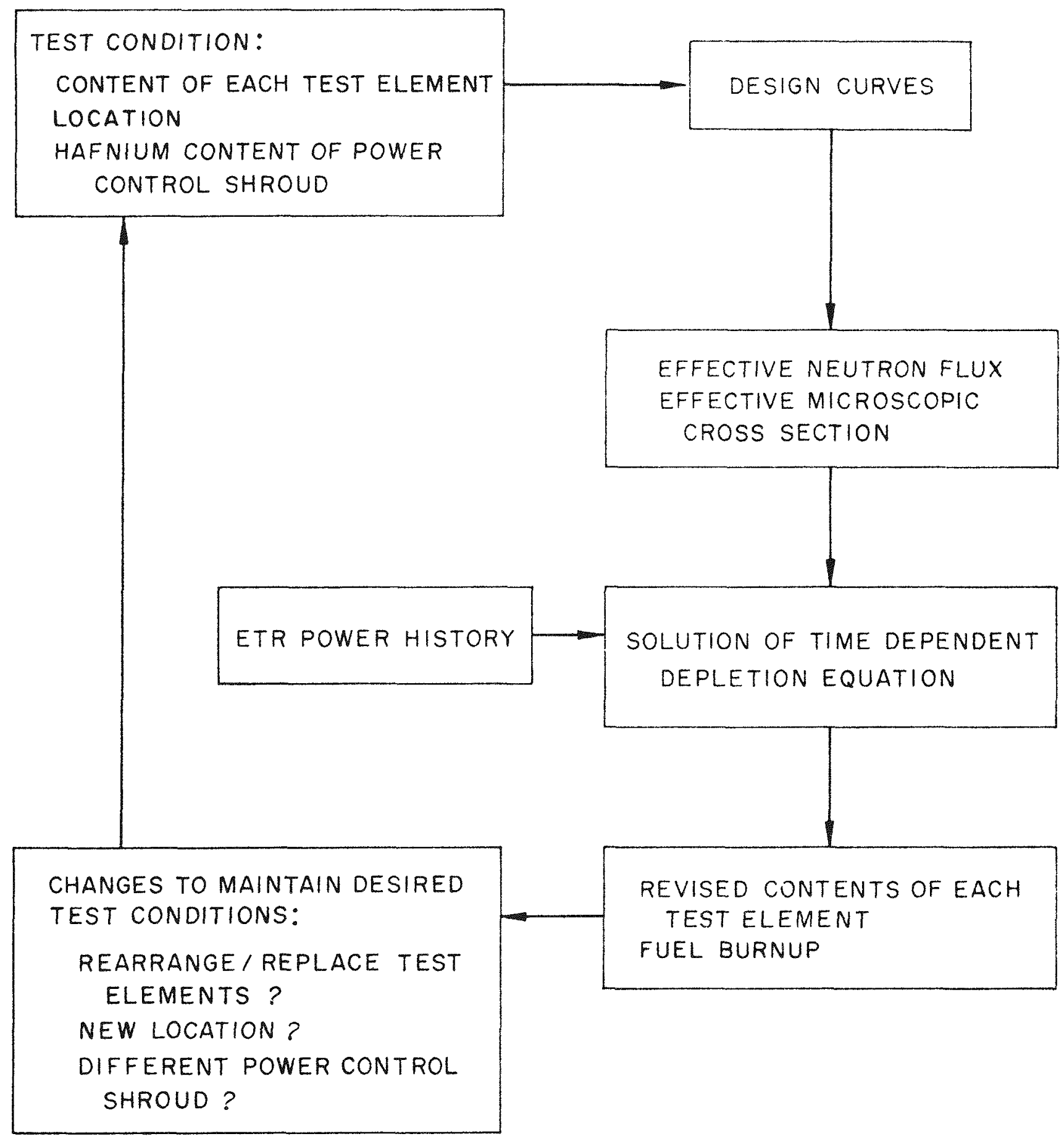

FIGURE 6 
TYPICAL IRRADIATION HISTORY

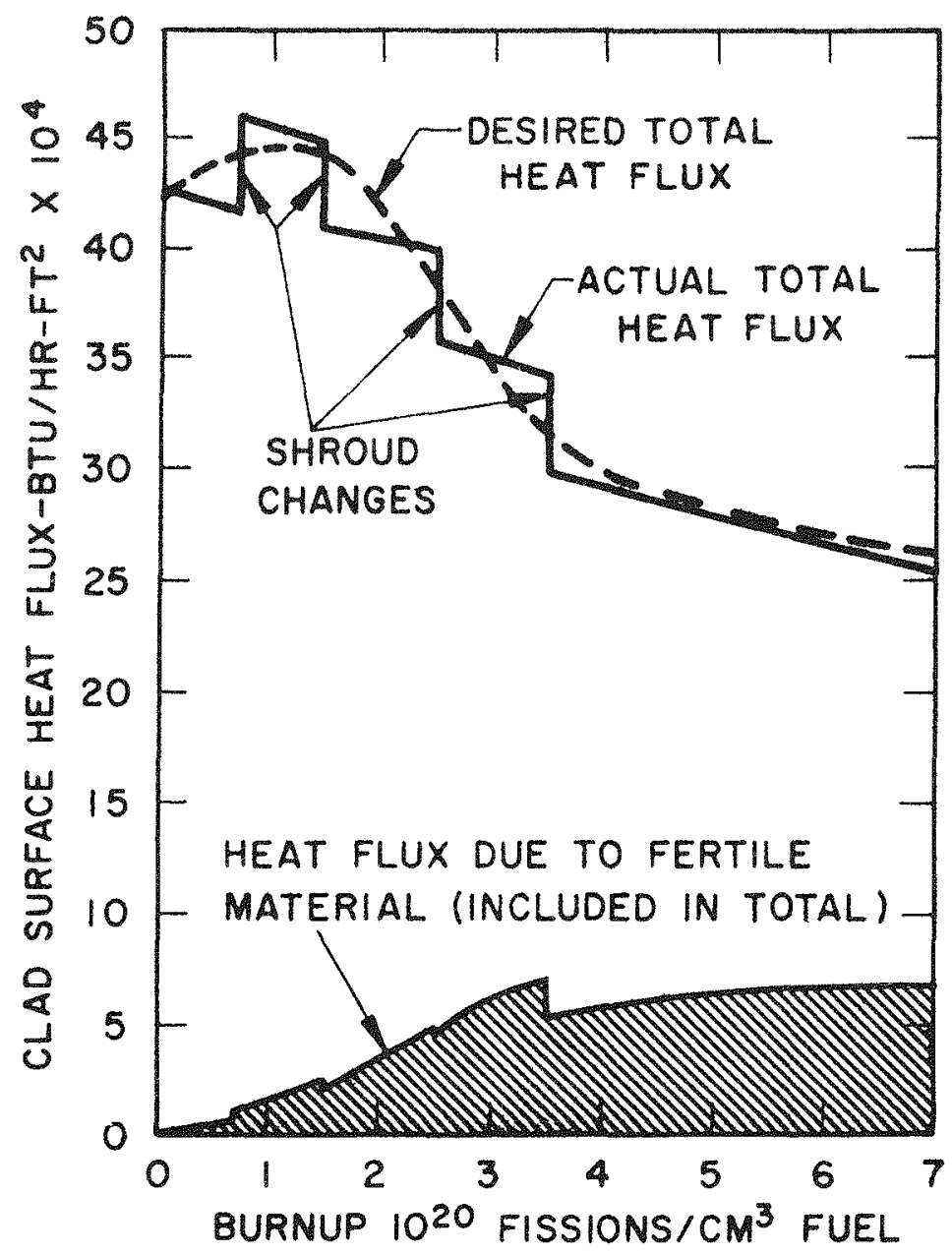

FIGURE 7 
COMPARISON OF PREDICTION TO MEASUREMENT
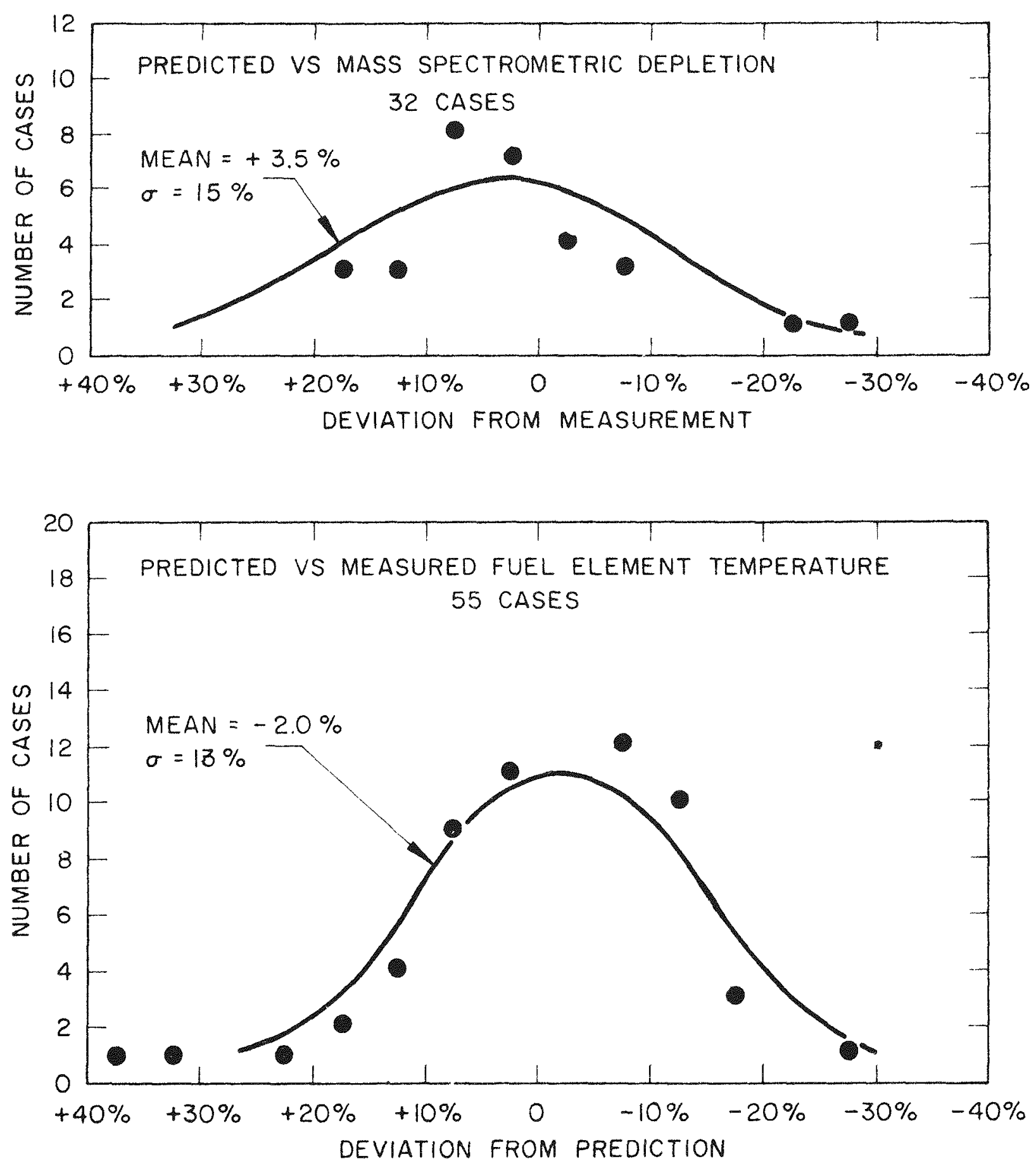

FIGURE 8 
COMPARISON OF PREDICTION TO MEASUREMENT

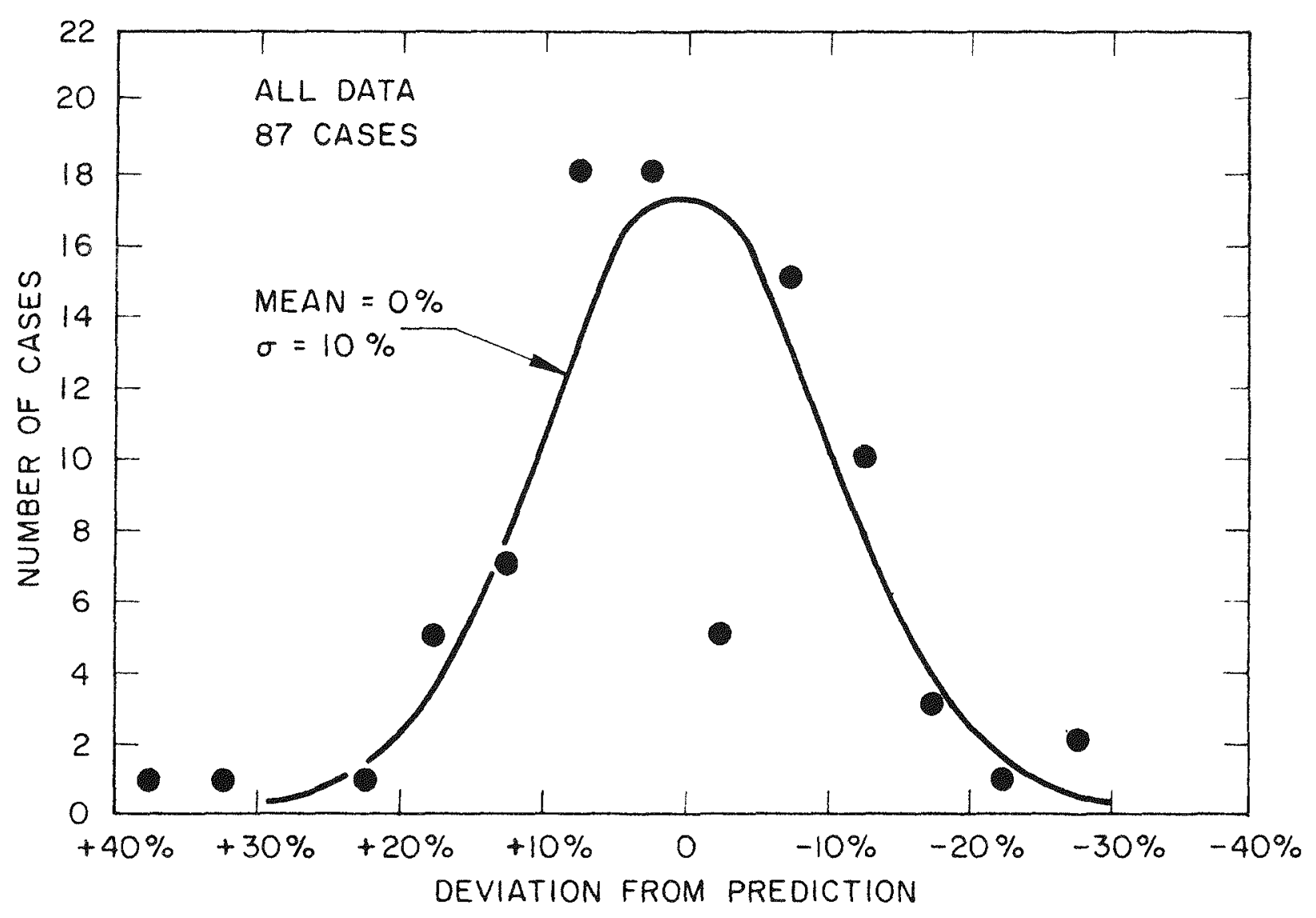

FIGURE 9 


\title{
$X$ Damage-Function Analysis of Neutron-Energy and Spectrum Effects Upon the Radiation Embrittlement of Steels
}

\author{
C. Z. SERPAN, JR. \\ Metallurgy Division \\ and \\ W. N. MCELROY \\ Battelle Northwest Laboratory
}

\begin{abstract}
Critical evaluations of irradiation effects data for steels exposed in different reactor environments depend upon fluence measurements that reflect the neutron population and the corresponding influence of damage mechanisms inherent to those neutron energy spectra. For the present research investigation, theoretical models describing neutron damage in reactors were adjusted with data on $\mathrm{mech}$ an ic al property changes of A302-B steel irradiated at $<450^{\circ} \mathrm{F}\left(232^{\circ} \mathrm{C}\right)$. As described previously, this procedure yielded a damage function that more properly accounts for the energy dependence of damage.

The present investigation was centered on a new comprehensive experiment which yielded wide variations in spectra and in corresponding measurements of neutron embrittlement. The experiment and the resultant data have validated the damage function. An important new part of the damage function analysis technique provides the percent contributions of neutrons of all energy levels to the embrittlement process. Values of the damage function, averaged for a typical reactor physics spectral calculation group structure, are presented with suitable descriptions of their applications to a wide variety of spectra.

One major conclusion reached in this study is that detailed as well as accurate neutron dosimetry measurements of fast and thermalfluxes, corrected to reactor operating temperatures, are necessary if good correlations among irradiation-effects data are to be obtained. This requirement applies to both experimental irradiations andirradiations at reactor component surveillance locations. The study has shown further that an independently derived damage function for irradiation of structural steels such as $\mathrm{A} 302-\mathrm{B}$ at $<450^{\circ} \mathrm{F}\left(232^{\circ} \mathrm{C}\right)$ is realistic and can be applied to new experimental data conforming to those conditions. The contributions of thermal and low-energy neutrons to the embrittlement process in low-alloy steel are shown to be of major importance to the interpretation of radiation-effects data.
\end{abstract}




\section{INTRODUCTION}

The deleterious changes in the mechanical properties of steels caused by the bombardment of neutrons within nuclear reactors become meaningful for immediate application or for projections of future behavior only if the damage-causing fluence of neutrons is well defined. This task is complicated because the neutrons in a flux spectrum create damage by many different mechanisms, all of which must be considered. Because the distribution of neutrons by energy groupings may change drastically for different reactors and reactor locations, it would appear to be impossible to determine a single fluence parameter for best correlation of radiation-effects data. Consequently, techniques have been developed to determine relative damaging effectiveness according to the best available theoretical estimates of the damage process or processes (1-6). Most of these techniques emphasize high-energy neutrons, and one study demonstrated improved correlation by use of damaging fluence $>0.5 \mathrm{MeV}$ (4). The more recent studies of Rossin (5) and Sheely (6) suggest, however, that thermal neutron damage may be significant, since even better correlation of neutron embrittlement in steel vs neutron fluence was attained with the increased importance placed on these neutrons in the respective damage models.

Because the correlation techniques developed so far have not provided for adjustment of the theoretical model by experimental data, a new approach was devised for this purpose (7). This new approach was demonstrated by using ductile-brittle transition temperature increase $(\triangle \mathrm{TT})$ data as functions of total neutron fluence, for many irradiations at $<450^{\circ} \mathrm{F}\left(232^{\circ} \mathrm{C}\right)$ of ASTM A212-B and A302-B steels in widely differing neutron environments. These data, coupled with their respective neutron spectra, were used to derive a damage function which presented a more realistic weighting of neutron population versus damaging potential for better correlation of experimental data. An additional important use of the damage function was to permit the calculation of total neutron fluence values required to produce a $200^{\circ} \mathrm{F}\left(111^{\circ} \mathrm{C}\right) \Delta \mathrm{TT}$ in $\mathrm{A} 302-\mathrm{B}$ steel in any spectral environment. An advantage of this semiempirical method is that damage caused by mechanisms other than displacement-related processes are included in the damage function.

This report presents the results of a new, comprehensive set of experimental data conforming to the irradiation conditions of the derived damage function (material, temperature, and fluence). The results of this experiment are discussed in terms of their establishing the validity of the damage function. Averaged values of the damage function are tabulated for a typical reactor physics calculation energy group structure, and their application to two different spectra is described. Finally, the damage-function fluences required to cause a $200^{\circ} \mathrm{F}\left(111^{\circ} \mathrm{C}\right) \Delta \mathrm{TT}$ in $\mathrm{A} 302-\mathrm{B}$ steel are presented for many different reactor locations. For each of the spectra involved, the upper and lower energy limits of significantly damaging neutrons are given, and some detail is also provided regarding the contribution of subgroups within the overall energy spectrum.

NOTE: This report was the result of a cooperative program between NRI and Battelle Memorial Institute, Pacific Northwest Laboratory, Richland, Washington. 


\section{EXPERIMENTAL DATA}

The transition temperature increase data used in this report were measured at the $30 \mathrm{ft}-\mathrm{lb}\left(5.2 \mathrm{kgm} / \mathrm{cm}^{2}\right)$ level, for a 6 -in.-thick $(152 \mathrm{~mm})$ plate of A302-B manganese-molybdenum pressure vessel steel. The chemical composition and heat treatment of this plate of steel are shown in Table 1. The irradiations were conducted in a graphite-moderated, water-cooled reactor, designated GMWC. Sealed capsules were contained within special shields that permitted $<240^{\circ} \mathrm{F}\left(116^{\circ} \mathrm{C}\right)$ water to pass over the capsules, but which altered the reactor spectrum incident upon the capsules to yield neutron fluence ratios of 1.8 , 5 , and 9 to 1 thermal to fast $(>0.5 \mathrm{MeV})$. Three distinct fluence levels were attained for each set of capsules, representing all three neutron energy ratios. The ITT values as functions of fast neutron fluence for the "tailored" GMWC irradiations are depicted in Fig. 1 and summarized in Table 2. (Included in this table for comparison are the A302-B irradiation data used to derive the damage function (7).)

Table 1

Chemical Composition and Heat Treatment of ASTM Reference A302-B Steel Plate 6 in. (152 mm) Thick

\begin{tabular}{|c|c|c|c|c|c|c|c|c|c|}
\hline $\mathrm{C}$ & $\mathrm{Mn}$ & $\mathrm{P}$ & $\mathrm{S}$ & $\mathrm{Si}$ & $\mathrm{Mo}$ & $\mathrm{Cu}$ & $\mathrm{V}$ & $\begin{array}{c}\mathrm{Al} \\
\text { Sol. }\end{array}$ & $\begin{array}{c}\mathrm{N} \\
\text { Total }\end{array}$ \\
\hline 0.23 & 1.35 & 0.015 & 0.022 & 0.022 & 0.52 & 0.22 & $<0.010$ & 0.02 & 0.008 \\
\hline
\end{tabular}

The Charpy-V curves of Fig. 1 clearly reveal three distinct levels of $\Delta T T$ corresponding well to the fluence levels. The specimens exhibited greater-than-average scatter, thus decreasing the overall accuracy of the final JTT values. The variations in $\triangle \mathrm{TT}$ at the separate levels are, nevertheless, in direct proportion to the fast neutron fluence $(>0.5 \mathrm{MeV})$ taken from Table 2 and do not appear to be caused by the differences in thermal-to-fast (>0.5 MeV) neutron fluence ratios. These results suggest then that thermal-to-fast $(>0.5 \mathrm{MeV})$ neutron populations in the range of $\sim 9.1$ for irradiations $<450^{\circ} \mathrm{F}\left(232^{\circ} \mathrm{C}\right)$ will have to be exceeded before embrittlement effects of engineering significance can be attributed to thermal neutrons.

The GMWC data have been plotted vs total neutron fluence in Fig. 2; the derivation of the total fluence values is described below. The fluences can be compared in this figure with the $\Delta \mathrm{TT}$ data used to derive the damage function. It is of particular interest to observe that the three data points for each of the three GMWC thermal-to-fast ratios align very well with the arbitrarily placed trend lines superimposed on the figure. The trend pattern is defined as a fourfold increase in total fluence for a $100^{\circ} \mathrm{F}\left(56^{\circ} \mathrm{C}\right) \Delta \mathrm{TT}$ in A302-B steel for irradiations $<450^{\circ} \mathrm{F}\left(232^{\circ} \mathrm{C}\right)$. This factor was suggested by Rossin's study (3) and was subsequently used as a reasonable basis for extrapolation of data to other fluence and embrittlement levels (7). Its use is well validated by the results of the GMWC data. Use of the straight-line trend behavior for data $\leq \triangle \mathrm{TT} 400^{\circ} \mathrm{F}\left(222^{\circ} \mathrm{C}\right)$ is further justified because full radiation embrittlement saturation for this steel irradiated at $<450^{\circ} \mathrm{F}\left(232^{\circ} \mathrm{C}\right)$ has not been observed.

The measured iron-wire fluence values $>1 \mathrm{MeV}$ in Table 2 are based on a fissionspectrum averaged cross section $\left(\bar{\sigma}^{\mathrm{fs}}\right)$ of 68 millibarns $(\mathrm{mb})$ for the ${ }^{54} \mathrm{Fe}(\mathrm{n}, \mathrm{p}){ }^{54} \mathrm{Mn}$ reaction; these values are included for reference only. Multigroup neutron spectra were calculated at Battelle-Northwest $(4,8)$ for each position using the diffusion theory code "2DB" (9). Spectra for other reactor locations used in this report were calculated using either the one-dimensional transport theory code "Program $S$ " (10) or the two-dimensional 

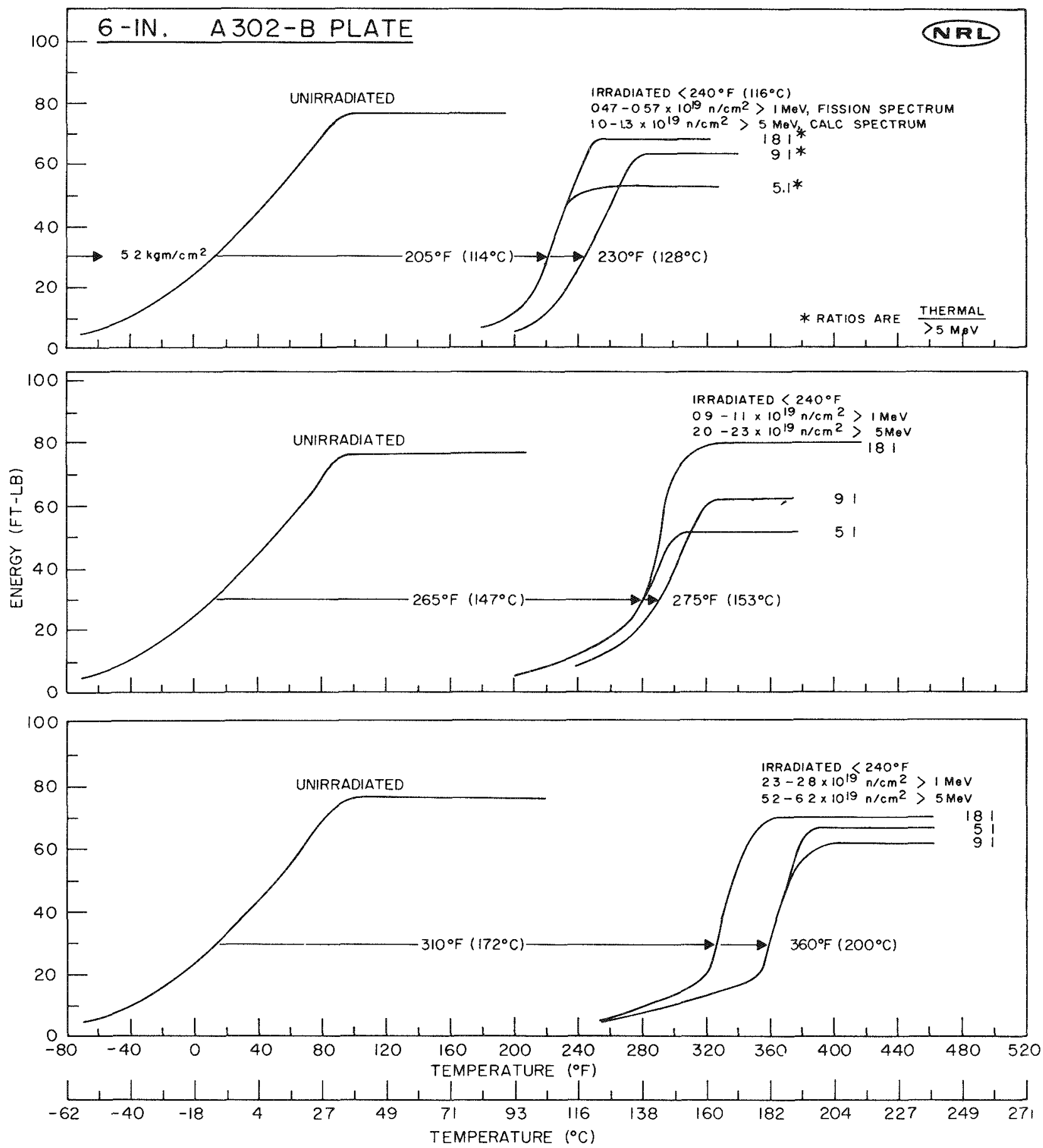

Fig. 1 - Charpy-V notch ductility characteristics of 6-in.-thick (152 mm) A302-B steel before and after irradiation in a graphite-moderated, water-cooled reactor. The neutron spectrum in the reactor location was a $\mathrm{t}$ ificially tailored to yield thermal-to-fast (>0.5 MeV) ratios of $1.8: 1,5: 1$, and $9: 1$. The transition temperature increases appear to be in direct relation to the fast $(>0.5 \mathrm{MeV})$ neutron fluence received. 
Table 2

Summary of Transition Temperature Increase and Neutron Fluence Data for $<450^{\circ} \mathrm{F}\left(232^{\circ} \mathrm{C}\right)$ Irradiations of 6-in.-Thick (152 mm) A.STM A302-B Steel

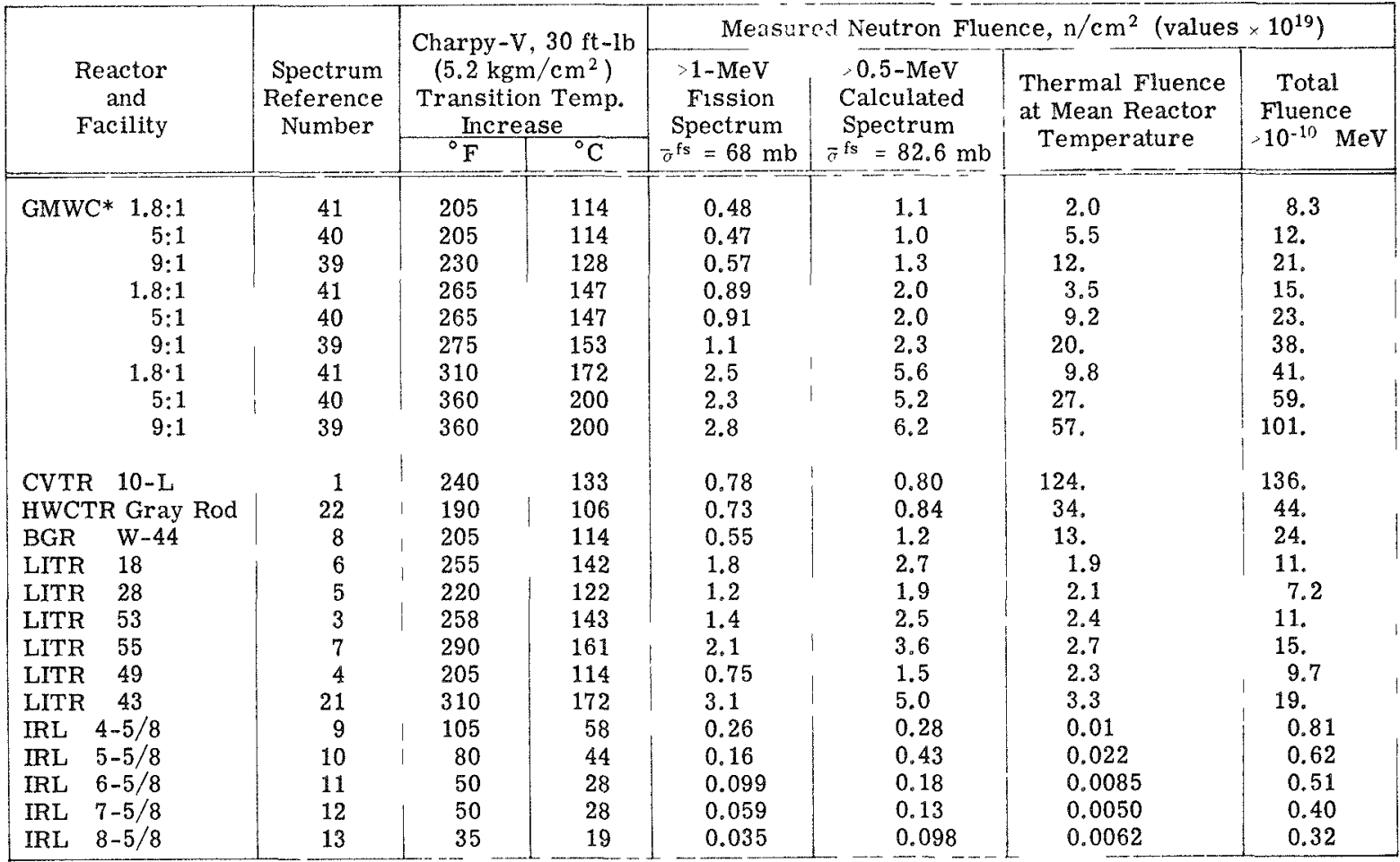

Neutron Spectrum Artificially Tallored Ratio is Thermal $>0.5 \mathrm{MeV}$

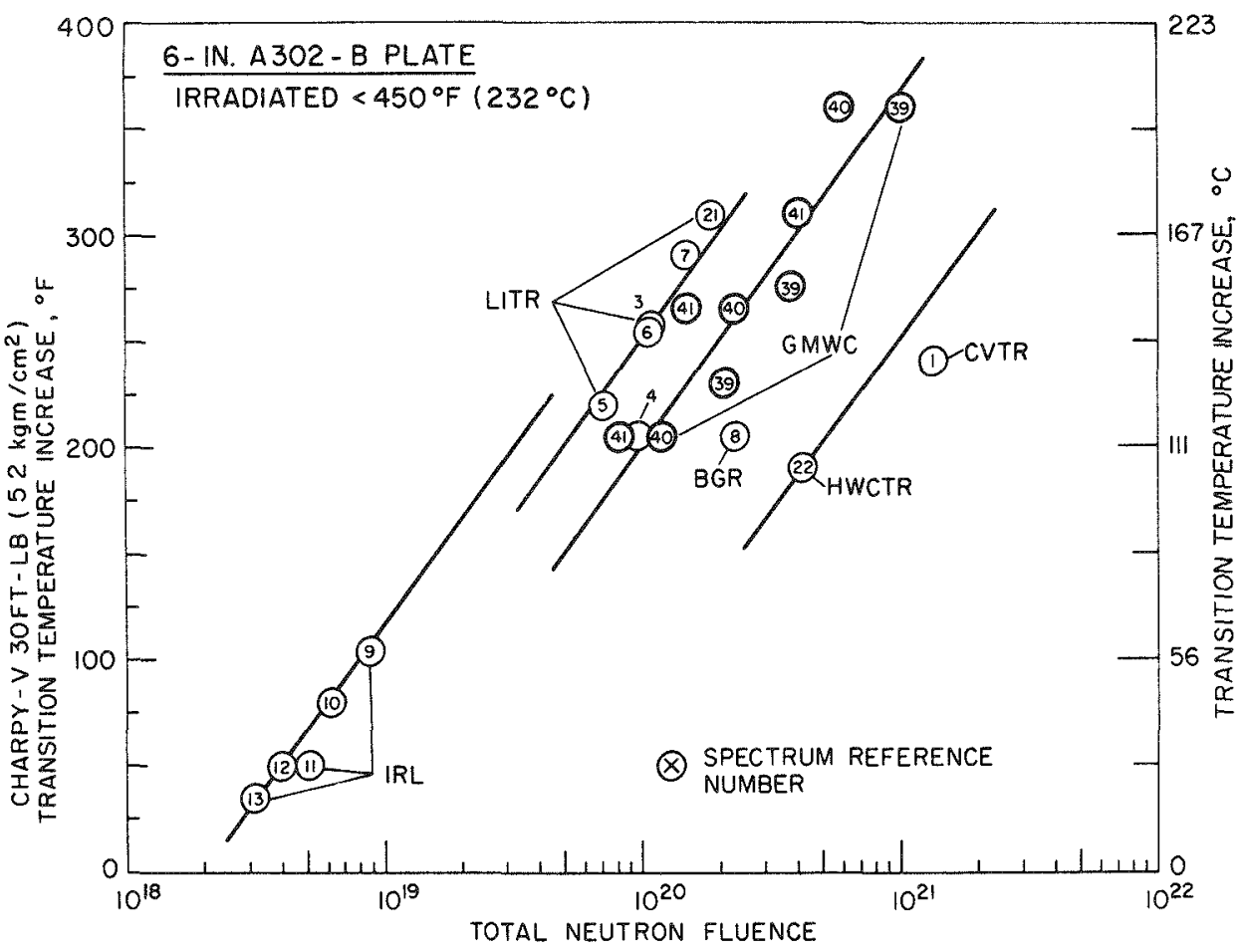

Fig. 2 - Charpy $-\mathrm{V} 30 \mathrm{ft}-1 \mathrm{~b}\left(5.2 \mathrm{kgm} / \mathrm{cm}^{2}\right)$ transition temperature increases vs total neutron fluence for irradiations $<450^{\circ} \mathrm{F}\left(232^{\circ} \mathrm{C}\right)$ of A302-B steel. The arbitrarily placed trend lines are used as guides to permit extrapolation of the embrittlement for each numbered spectrum to different levels such as $200^{\circ} \mathrm{F}\left(111^{\circ} \mathrm{C}\right)$. 
transport theory code "2DXY" (11). The calculated-measured* fluences $>0.5 \mathrm{MeV}$ of Table 2 were based upon ${ }^{54} \mathrm{Fe}(\mathrm{n}, \mathrm{p})$ measurements using an energy-dependent cross section with $\mathrm{a} \bar{\sigma}^{\mathrm{fs}}$ of $82.6 \mathrm{mb}(12)$. The use of different $\widetilde{\sigma}^{\mathrm{fs}}$ values has been discussed (13). For the present study, the thermal fluences were first calculated as $2200 \mathrm{~m} / \mathrm{sec}$ values from activities measured from the $1 / \mathrm{v}$ detector $59 \mathrm{Co}(\mathrm{n}, y)$; this was accomplished with wires of Al-0.1\% Co alloy which were exposed both bare and covered with 0.04 in. $(1 \mathrm{~mm})$ of cadmium. These values were then converted to actual thermal fluences by multiplying by $0.06592(T)^{1 / 2}$, where $T$ is an estimated mean reactor temperature in ${ }^{\circ} \mathrm{K}$ at the location of interest. The actual magnitudes of the thermal $(<\sim 0.4 \mathrm{eV})$ and fast $(>\sim 0.5 \mathrm{MeV}) \mathrm{com}-$ ponents of each calculated spectrum used in the damage-function analysis procedures were then determined by adjusting the calculated spectrum to agree with the activation detector measurements. The total fluences were then easily derived from the adjusted calculated spectra; the calculated-measured flux $>0.5 \mathrm{MeV}$ was divided by the percent flux $>0.5 \mathrm{MeV}$. These total fluences will be referred to as measured values in this report.

\section{DATA CORRELATION BY DAMAGE FUNCTION}

It can be seen from the ratio of thermal to fast $(>0.5 \mathrm{MeV})$ fluences, Table 2 , and from Fig. 2 that the embrittlement in A302-B steel has a neutron energy dependence. From such evidence, it is becoming clear that data obtained from diverse spectral environments can never be completely correlated in a simple plot of $\Delta T T$ versus neutron fluence for neutrons greater than any energy. However, for those systems in which $\geq 90$ percent of the damaging neutrons are predominantly those above a few keV, good correlation can be expected on a plot of $\Delta \mathrm{TT}$ versus neutron fluence for neutrons greater than a specific energy such as 0.5 or $0.1 \mathrm{MeV}$ (see Refs. 4 and 14). To achieve better correlations, it is necessary to plot the $\Delta \mathrm{TT}$ data vs another parameter which reflects those mechanisms other than displacements ${ }^{\dagger}$ in order to describe the spectral dependency of change (see Refs. 1 through 6). It should be recognized that within the displacement parameter concepts referenced, the measured property changes are not simply plotted vs fluences but vs calculated property changes in the form of displacements. An additional improvement in correlation can be made by use of a damage model that incorporates displacement effects as a starting point and includes provisions to integrate the effects of any other damage or "annealing" (correction) mechanisms. This improved correlation technique still does not permit the direct plotting of measured property changes vs fluences either, but it does allow the comparison of measured vs calculated total fluences for a given amount of neutron embrittlement.

The absolute damage function used to calculate the total fluence required to cause a $200^{\circ} \mathrm{F}\left(111^{\circ} \mathrm{C}\right) \Delta \mathrm{TT}$ in $\mathrm{A} 302-\mathrm{B}$ steel for irradiations at temperatures $<450^{\circ} \mathrm{F}\left(232^{\circ} \mathrm{C}\right)$ is presented graphically in Fig. 3 (7). A modified version of the SAND-II code (15) was used to obtain the damage function solution $G(E)$ for a set of integral equations of the form

$$
S_{j}=\iint G(E) \phi_{j}(E, t) d E d t
$$

where $S_{j}$ is a measured integral property change such as $\Delta \mathrm{TT}$ for a specified material, irradiated for a time $t$, in the jth neutron environment (at constant temperature $T$ ), and $\phi_{j}(E, t)$ is the corresponding $j$ th neutron differential spectrum.

\footnotetext{
*Multigroup spectra results were adjusted in magnitude by the activation detector measurements.

tDisplacements are generally thought to be associated with high-energy neutron effects.
} 


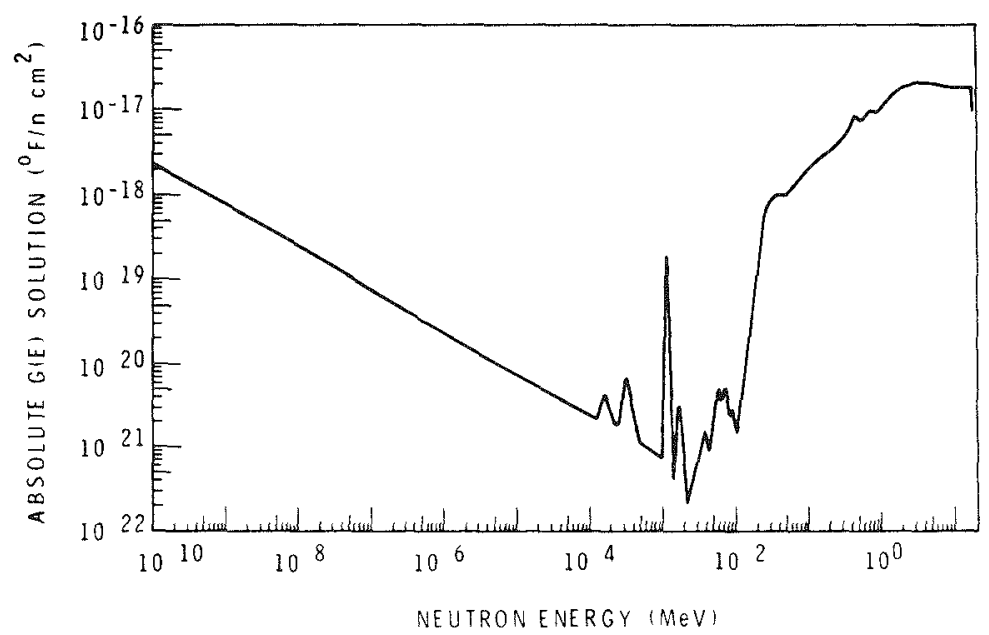

Fig. 3 - Absolute $G(E)$ damage-function solution for a $200^{\circ} \mathrm{F}\left(111^{\circ} \mathrm{C}\right)$ transition temperature increase in A $302-B$ steel for arradiations $<450^{\circ} \mathrm{F}\left(232^{\circ} \mathrm{C}\right)$

The derived damage function is used with multigroup reactor physics calculations to obtain the fluence (and appropriate energy limits of damaging neutrons) required to cause the property change at the irradiation location of interest. Equation (1) is rewritten:

where

$$
\mathrm{S}=\Phi \mathrm{t} \sum_{i=1}^{\mathrm{m}} \mathrm{G}_{1} \Phi_{1},
$$

$$
\sum_{i=1}^{m} \Phi_{1}=1 \text {, }
$$

$\Phi_{1}$ is the normalized integral flux within the ith group,

$\Phi^{t}$ is the fluence required to cause the $200^{\circ} \mathrm{F}\left(111^{\circ} \mathrm{C}\right) \Delta \mathrm{TT}$,

$S$ is the property change specified in the derivation of $G(E)$, the damage function, and

$\mathrm{G}_{1}$ is the corresponding group-averaged $\mathrm{G}(\mathrm{E})$ value.

To use the $G(E)$ function would then require knowledge of the neutron spectrum of the reactor location of interest. Group-averaged values of this (or any) spectrum would be multiplied by group-averaged values of the damage function and Eq. (3) would be solved for $\Phi \mathrm{t}$ according to

$$
\Phi t=S\left[\sum_{i=1}^{m} G_{1} \Phi_{2}\right]^{-1} ;
$$

the approximate energy limits of damaging neutrons could be inferred by a consideration of each group contribution, $\mathrm{G}_{1} \Phi_{1} / \mathrm{S} \Phi \mathrm{t}$, to the total property change. The fluence obtained would be that required to cause a change of $\mathrm{S}$, in this case a $200^{\circ} \mathrm{F}\left(111^{\circ} \mathrm{C}\right) \triangle \mathrm{TT}$ in $\mathrm{A} 302-\mathrm{B}$ steel, in the spectrum of interest. Fluences for $\Delta \mathrm{TT}$ values other than $200^{\circ} \mathrm{F}$ can also be calculated (Ref. 7). It was found that a single set of group-averaged values of the damage function (Fig. 3) could be used without introducing more than about a 5 -percent variation in the calculated values of fluences for many reactor spectra.

In the development of the damage function of Fig. 3 , it was recognized that the reactor environments were not sufficiently different to provide a reliable solution for 
the damage model in the intermediate energy region between $\sim 10^{-7}$ and $\sim 2 \times 10^{-2} \mathrm{MeV}$. In this region, therefore, the function is essentially a repetition of the assumed input model. For this reason, no attempt was made in this study either to generate or to use detailed structure in this region. Furthermore, for the thermal energy region, the magnitude of the derived damage function for A302-B steel may be too low.

The approximating procedure used in the GMWC experiment (and previously (7)) for defining the magnitudes of thermal, intermediate, and fast components of the neutron spectrum was studied. This approximating procedure has been compared with a more exact procedure based on the SAND-II, multiple-foil activation method (15). This comparison revealed that the approximating procedure may have yielded high thermal fluence results. This implies that the derived damage function may be too low in the thermal energy region. Future reevaluations of these data may reveal, therefore, that the damage from thermal neutrons may be greater than that suggested by the present results.

The foregoing discussion of the limitations of the damage-function analysis technique in no way detracts from the fact that it provides the very best means presently available for correlating radiation effects data from different reactor irradiations. Furthermore, it provides a strong analytical tool for improving the definition of the damaging function of a given spectrum of neutrons. Finally, it provides the only means for satisfactory evaluation of the damaging potential of a neutron spectrum in the absence of measured mechanical property data representative of that spectrum.

\section{ANALYSIS BY DAMAGE FUNCTION}

Application of Eq. (4) and group-averaged values, $\mathrm{G}_{\mathrm{i}}$, of the damage function will now be considered in Table 3 using the group integral flux values for two very different reactor irradiation locations. These group-averaged damage-function values are based upon the $<450^{\circ} \mathrm{F}$ irradiation behavior of a 6 -in. -thick plate of A302-B steel that has been. made available to many laboratories in different countries (16). Therefore, these $\mathrm{G}_{i}$ values could also be used for the analysis of data obtained by other laboratories for irradiations of this steel under the same temperature conditions. Total fluences, using temperature-corrected thermal values, would also have to be used.

In Table 3 , the left column under both spectral listings gives the group fluxes as normalized to one neutron, and the middle column is the product of the group flux and the group-averaged damage function. The summation of these products for both spectra are then divided into $200^{\circ} \mathrm{F}$ to yield the total fluence for a $200^{\circ} \mathrm{F} \Delta \mathrm{TT}$ in A302-B steel for irradiations $<450^{\circ} \mathrm{F}$. The right column under each listing shows the thermal $(<4.14 \times$ $\left.10^{-7} \mathrm{MeV}\right)$, intermediate $\left(4.14 \times 10^{-7} \mathrm{MeV}<\mathrm{E}<0.183 \mathrm{MeV}\right)$, and fast $(>0.183 \mathrm{MeV})$ energy region subtotals for this summation and the corresponding percent contribution to the total damage for these three larger groups. (Note that the total fluences in Table 3 are used to plot the abscissas of the respective data points appearing for these spectra in Fig. 4.)

The GMWC spectrum for the 9:1 thermal-to-fast $(>0.5 \mathrm{MeV})$ ratio was included in Table 3 because it represents the highest such ratio that does not appear to result in more embrittlement than could have been expected for the fast $(>0.5 \mathrm{MeV})$ fluence. It should be clear that the thermal population, even though it be almost an order of magnitude greater than the fast, nevertheless contributes only about 6 percent to the embrittlement. This amount of damage contribution could not be discerned except under the most favorable testing conditions. It is pointed out that the Charpy $-\mathrm{V}$ specimen evaluations of the GMWC irradiations revealed more data scatter than may be reasonably expected for this type of steel, and, more specifically, this plate of A302-B steel. On the other hand, three separate data points were developed for each of the fluence ratios in the experiment, and the same behavior pattern of no exceptional embrittlement was observed for every 
Table 3

Group-Averaged Values for Absolute $\mathrm{G}(\mathrm{E})$ for a $200^{\circ} \mathrm{F}\left(111^{\circ} \mathrm{C}\right) \triangle \mathrm{TT}$ in A302-B Steel Irradiated at $<450^{\circ} \mathrm{F}\left(232^{\circ} \mathrm{C}\right)$ and Application to Different Spectra

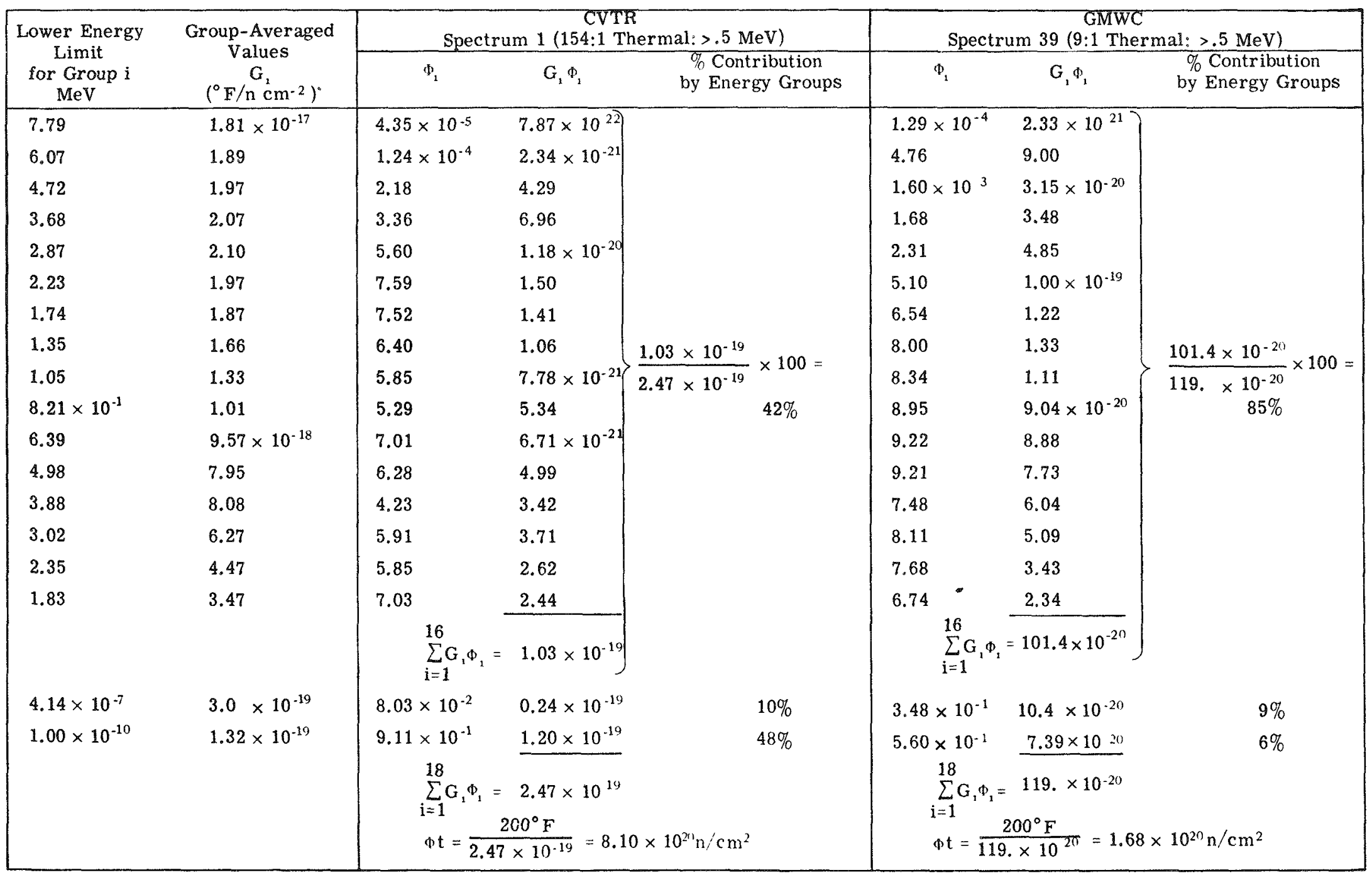




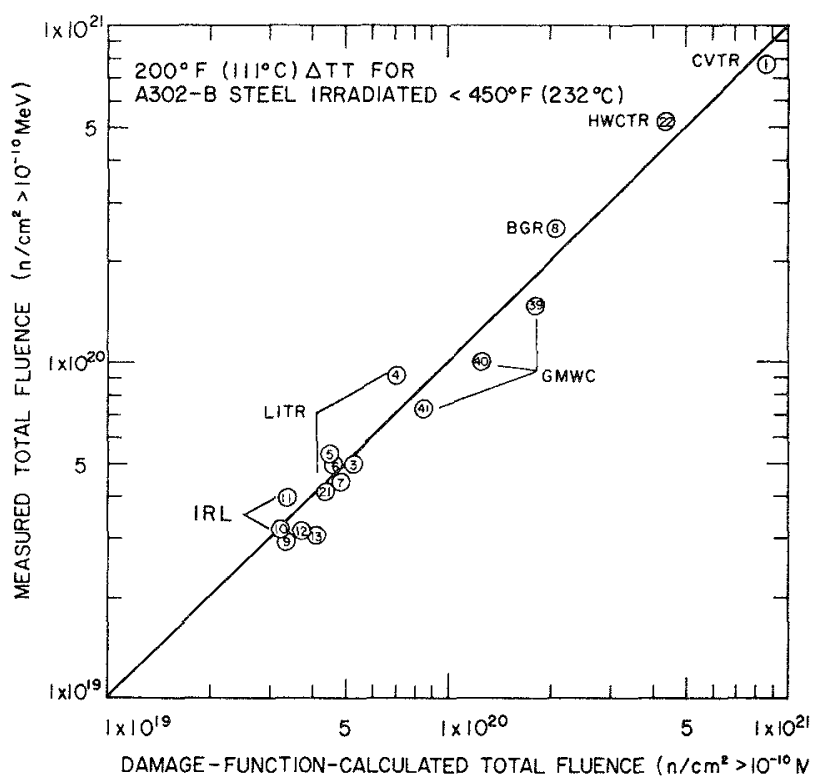

Fig. 4 - Verification of the $G(E)$ damage function by the graphite-moderated, watercooled reactor (GMWC) irradiation data. The damage-function-calculated fluence agrees very closely with the measured fluence for a $200^{\circ} \mathrm{F}\left(111^{\circ} \mathrm{C}\right)$ transition temperature increase in $\mathrm{A} 302-\mathrm{B}$ steel.

fluence and fluence ratio condition. When the techniques employed in this paper for evaluating and reporting all the fluence values are used, then a thermal-to-fast neutron ratio in the range of $9: 1$, as displayed by the GMWC data, does not appear to contribute increased embrittlement in A302-B steel for irradiations $<450^{\circ} \mathrm{F}$.

The spectrum from the heavy-water-moderated CVTR (Carolinas-Virginia Tube Reactor) was selected for Table 3 because it demonstrates exceptionally high thermal-tofast ( $>0.5 \mathrm{MeV}$ ) fluences. A ratio of $154: 1$ was measured at the irradiation location. The result of the irradiation was evidence of more embrittlement than would have been expected on the basis of the fast neutron fluence alone. Inspection of the damage percent contributions by energy ranges clearly reveals that thermal and intermediate energy range neutrons are responsible for over half of the embrittlement. The damage contribution percentages of Table 3 provide a means for understanding the increased embrittlement of the CVTR data, relative to that from a GMWC irradiation, which could not be gained simply from the embrittlement-vs fluence values alone.

The critical question of this investigation was to determine whether the damage function is applicable to the GMWC data. The means employed to effect this comparison was to determine from Fig. 2 the measured total fluences to cause a $200^{\circ} \mathrm{F} \Delta \mathrm{TT}$ in $\mathrm{A} 302-\mathrm{B}$ steel for all three GMWC spectra and plot them vs total fluence values determined by the damage-function-calculated process shown in Table 4. The measured and damage-functioncalculated total fluences are compared in Fig. 4. Perfect correlation is achieved when all points fall on the solid line; that is, the damage function can be used to calculate total fluences that agree exactly with measured fluence values for any reactor environment. The average value of ratios of measured to calculated fluences, Fig. 4, have a 15.2-percent standard deviation about a line representing perfect correlation. It can be seen that the GMWC data points (spectra 39, 40, and 41) compare favorably with that amount of deviation. This indicates that the damage function, considering the limitations previously 
Table 4

Prediction of Fluence and Energy Response Necessary to Produce a $200^{\circ} \mathrm{F}\left(111^{\circ} \mathrm{C}\right) \triangle \mathrm{TT}$ in $\mathrm{A} 302-\mathrm{B}$ Steel

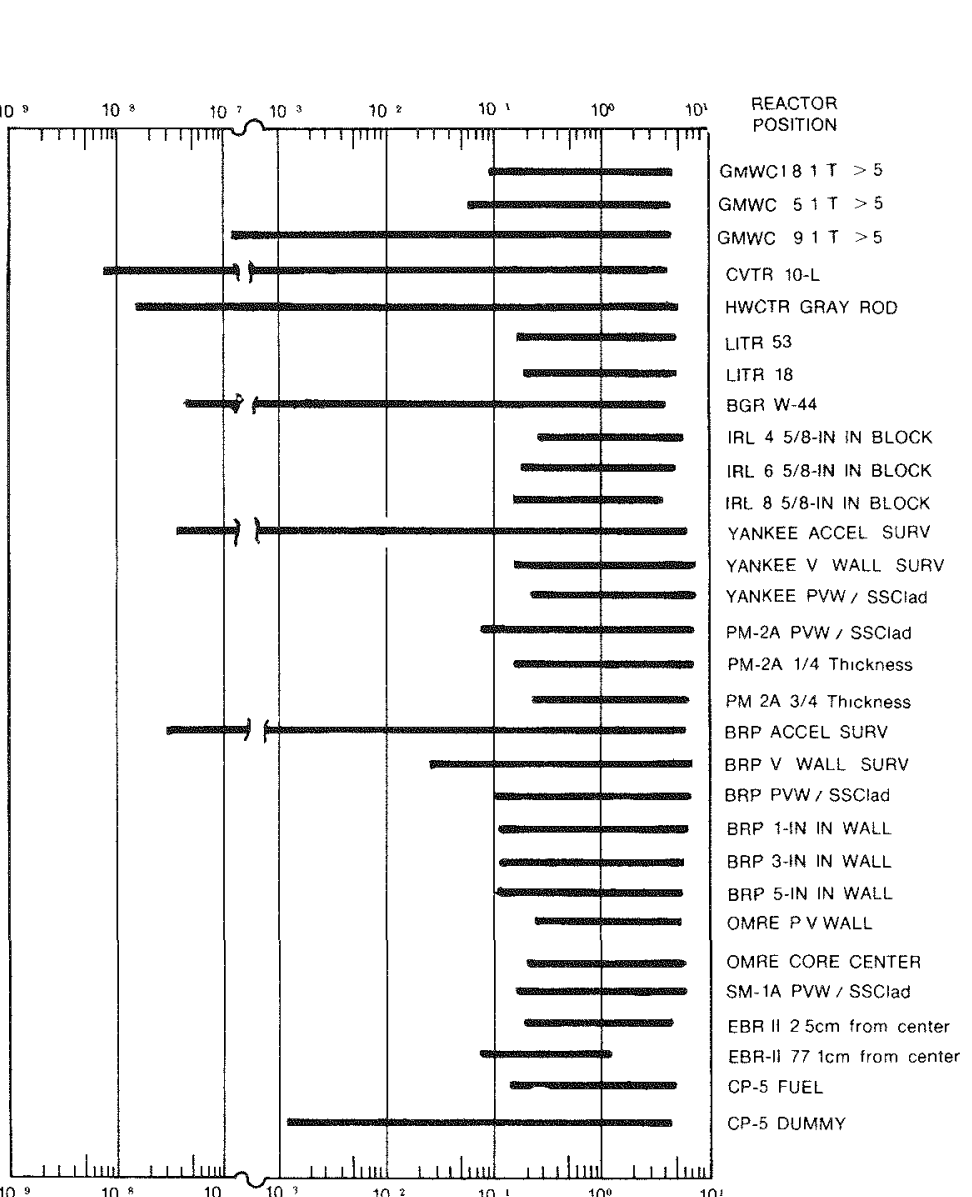

KEY TO REACTORS IN TABLE 4

BGR Brookhaven Graphite Reactor

BRP Big Rock Point Reactor

CP.5 Chicago Pile No. 5, heavy-water test reactor at Argonne National Laboratory. Fuel, within hollow center of fuel-bearing element; Dummy, within hollow center of non-fuel-bearing element.

CVTR Carolinas-Virginia Tube Reactor, posiłion 10-L EBR-II Experimental Breeder Reactor II

GMWC Graphite-Modulated, Water-Cooled Reat Heavy-Water Components Test Reactor,
irradiation location in "gray" control rod irradiation location in "gray" control
Industrial Reactor Laboratory, Inc. Industrial Reactor Laborato
$4-5 / 8$ in. from core face $6-5 / 8$ in. from core

LITR Low-Intensify Test Reactor, core lattice position 53, 18
DAMAGE CONTRIBUTION FROM
SPECIFIC ENERGY GROUPS

90\% DAMAGE
ENERGY LIMTS,

MeV

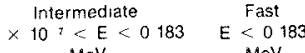

Exwe$$
E_{u, j}
$$

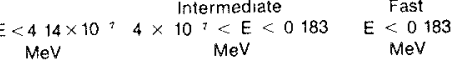

$58 \times 10^{2}$

$22 \times 10^{-}$

$72 \times 10^{9}$

$15 \times 10^{*}$

$17 \times 10$

$19 \times 10^{1}$

$45 \times 10^{1}$

$26 \times 10^{\prime}$
$18 \times 10^{1}$

$14 \times 101$

$34 \times 10^{\circ}$

$14 \times 10$

$21 \times 10^{1}$

$76 \times 10^{2}$

$14 \times 10$ 1

$22 \times 10^{1}$

$30 \times 10$ "

$26 \times 10=$

$10 \times 10^{1}$

$11 \times 10^{2}$

$11 \times 10^{1}$

$11 \times 10^{1}$

$26 \times 10^{1}$

$20 \times 10$ :

$16 \times 10^{1}$

$19 \times 10^{1}$

$69 \times 10$

$13 \times 10$

$$
\begin{gathered}
3 \\
6 \\
51 \\
21 \\
07 \\
05 \\
75 \\
03 \\
003 \\
004 \\
9 \\
25 \\
1 \\
4 \\
3 \\
1 \\
10 \\
5 \\
3 \\
5 \\
2 \\
15 \\
15 \\
04 \\
05 \\
- \\
- \\
03 \\
46
\end{gathered}
$$$$
\begin{gathered}
\mathrm{MeV} \\
1
\end{gathered}
$$$$
05
$$$$
75
$$$$
\begin{aligned}
& 03 \\
& 003
\end{aligned}
$$$$
004
$$$$
\begin{aligned}
& 9 \\
& 25
\end{aligned}
$$$$
3
$$$$
\begin{aligned}
& 2 \\
& 15
\end{aligned}
$$$$
\begin{aligned}
& 15 \\
& 04 \\
& 05
\end{aligned}
$$$$
\begin{array}{cr}
7 & 92 \\
8 & 89 \\
9 & 85 \\
5 & 44 \\
10 & 69 \\
53 & 94 \\
45 & 95 \\
85 & 84 \\
37 & 95 \\
6 & 94 \\
7 & 93 \\
3 & 88 \\
25 & 95 \\
4 & 95 \\
4 & 92 \\
4 & 93 \\
5 & 94 \\
5 & 85 \\
5 & 90 \\
5 & 92 \\
6 & 91 \\
7 & 91 \\
85 & 90 \\
35 & 95 \\
36 & 96 \\
55 & 94 \\
6 & 92 \\
26 & E>2\left\{\begin{array}{l}
94 \\
67
\end{array}\right. \\
14 & 93 \\
14 & 82
\end{array}
$$

$\begin{array}{cl}\text { OMRE } & \begin{array}{l}\text { Organic Moderated Reactor Experiment, } \\ \text { inner edge of pressure vessel wall }\end{array} \\ \text { PM-2A Army portable power reactor, Camp Century, } \\ \text { Greenland; } 1 / 4,3 / 4 \text { thicknesses are loca- } \\ \text { tions inside the steel pressure vessel wall. }\end{array}$

SM-IA Army stationary power reactor, Ft. Greely, Alaska 
indicated, is applicable to the GMWC spectral shapes for the characteristic embrittlement created within them and for the temperature-material conditions.

\section{DISCUSSION}

If the damage-function analysis technique is to be useful, it should permit the projection of the fluence required in a new spectrum to create a given amount of radiation damage and the percent contributions of neutrons in different energy regions. These damage functions should also permit the prediction of the amount of damage for a stated amount of fluence, provided these are within the validated limits (materials, temperature, and fluence) of the damage function. Using the damage function of Fig. 3, a large number of different neutron spectra were analyzed by computer to determine the total fluence required to produce a $200^{\circ} \mathrm{F} \Delta \mathrm{TT}$ in $\mathrm{A302}-\mathrm{B}$ steel if it could be irradiated at $\angle 450^{\circ} \mathrm{F}$ in the facility. The results of this analysis are presented in Table 4.

The energy limits corresponding to the neutrons responsible for causing 90 percent of the radiation embrittlement are tabulated and presented graphically in the table. The total fluence is presented as well as the percent contribution to damage by neutron energy regions. If correlations sufficiently accurate for engineering applications are to be achieved for reactor environments as diverse as shown here, it should be clear that accounting for neutrons of energies greater than $1 \mathrm{MeV}$ or even $0.5 \mathrm{MeV}$ will not be adequate. Further consideration of the graphical portion of Table 4, however, reveals that a threshold of about $0.1 \mathrm{MeV}$ could be used with a reasonable degree of effectiveness for correlations between many reactor environments. The danger inherent in universal employment of the ">1 MeV" concept for data correlation among diverse reactor envirm ments, however, is demonstrated by the energy response range of the "EBR-II $77.1 \mathrm{~cm}$ from center" data. This location is in the blanket of the Experimental Breeder Reacts II the upper limit for 90 -percent damaging neutrons is $1.2 \mathrm{MeV}$ based upon the damage functiv $1 \supset$ Fig. 3. To account for $\mathrm{n} / \mathrm{cm}^{2}>1 \mathrm{MeV}$ for this reactor location would be to ignore $\mathrm{m} n \rightarrow$ than 90 percent of the neutrons effective in causing radiation damage by mechanisms effective in causing $<450^{\circ} \mathrm{F}$ embrittlement in low-alloy steels.

Other data are included in Table 4 for interest, although the application of the $\left\langle x^{\prime},{ }^{\circ} \mathrm{F}\right.$ damage function is hypothetical. This is because the operating temperatures of these reactors are in excess of $450^{\circ} \mathrm{F}$ and thus permit simultaneous irradiation and annealing. The Yankee and Big Rock Point (BRP) relate to pressurized and boiling-water power reactors, respectively. The PM-2A and SM-1A are relatively compact, light-water reactors used by the U.S. Army for electrical power production at remote locations. The OMRE is the now-decommissioned Organic Moderated Reactor Experiment, and the CP-5 is a heavy-water-moderated and-cooled test reactor. In every case in which a large percentage of the damage is attributed to thermal neutrons, a relatively high ratio of thermal to fast neutrons exists.

As noted previously, increased radiation damage apparently can be caused by high populations of thermal neutrons relative to fast $(>0.5 \mathrm{MeV})$ when the ratio exceeds the range of $\sim 9: 1$, i.e., the GMWC data did not appear to show increased damage, but the 11.2:1 ratio of the BGR did appear to do so (14). On the other hand, the pressure-vessel wall locations of light-water power reactors operating at temperatures $>450^{\circ} \mathrm{F}$ appear to have ratios less than $\sim 9: 1$ but, nevertheless, seem to the associated with increased radiation embrittlement $(13,17)$. At present, no satisfactory explanation is known for this apparent anomaly. At elevated temperatures, however, there may be a significant reduction in displacement-related damage due to annealing effects. Accordingly, it may be postulated for elevated temperature tests that nondisplacement, low-energy neutron damage would provide a larger relative contribution to the total damage. This then suggests a possible explanation of the apparent power reactor embrittlement anomaly. 
The integral effect of all the damage mechanisms occurring in the embrittlement of low-alloy steel at irradiations $<450^{\circ} \mathrm{F}$ is exhibited by the differences in total fluences shown in Table 4. The exceptionally wide range of total fluences suggests that it may indeed be impossible to ever formulate a correlation technique based on an approach wherein the neutrons of energies greater than some threshold such as $1 \mathrm{MeV}$ are simply totaled by numbers with no accounting for their differing damage potential. This type of correlation would depict a single-trend, line behavior of increasing radiation damage for increasing total neutron fluence values regardless of the reactor environment. This type of trend behavior can only be used for carefully selected reactors wherein the spectral characteristics are quite similar. For reactors of significantly diverse spectral characteristics, such as in-core locations of light-water test reactors and vessel wall locations of light-water power reactors, it cannot be used.

\section{SUMMARY AND CONCLUSIONS}

This study has demonstrated the applicability of a damage function to the neutron embrittlement and related total fluence of three different neutron spectra. This damage function was developed to calculate the total fluence required in a given spectrum to cause a $200^{\circ} \mathrm{F}$ transition temperature increase at the Charpy- $\mathrm{V}, 30 \mathrm{ft}-\mathrm{lb}$ level in $\mathrm{A} 302-\mathrm{B}$ steel for irradiations at $<450^{\circ} \mathrm{F}$. The three GMWC spectra used to test the damage function were within the extremes of ratios of thermal to fast $(>0.5 \mathrm{MeV})$ neutrons used for the function development but are, nevertheless, significant because they cover the most reasonable range of neutrons in many test and power reactors. The good agreement between damage-function-calculated fluences and the measured fluences from the new experimental data establishes the damage-function technique as being valid for its intended purpose.

Group-averaged values for the damage function in terms of a typical reactor physics spectral calculation group structure are presented for use in analyzing $<450^{\circ} \mathrm{F}$ irradiation data for an ASTM A302-B steel plate, and the techniques are presented and described. Several cautions are noted, however, regarding the immediate use of the damage function. Most importantly, the total fluence must be used. For this study, foil measurements of iron, for fast neutron determinations, and bare plus cadmium-covered cobalt foils were used to define the thermal fluence. The thermal fluence was further adjusted for the mean reactor operating temperature. These foil measurements were then used to adjust the magnitude of the detailed reactor physics spectral calculations of the irradiation locations. On completion of the analysis discussed in this report, the neutron spectra for the GMWC experiments presented were reevaluated with the SAND-II code and the saturated activities of the iron and cobalt foils. The results strongly suggest that the thermal flux components may have been significantly overestimated. If this is the case, then the 9:1 ratio of thermal to fast $(>0.5 \mathrm{MeV})$ neutrons would not be a realistic upper limit for the absence of neutron embrittlement in excess of what could be expected from just the fast neutron fluence alone. The more accurate ratio would be a lower value.

The application of the damage function to neutron spectra from which there are no corroborating mechanical property measurements was also presented. Certain of these cases were hypothetical because it would have been difficult to effect irradiations at $<450^{\circ} \mathrm{F}$ in reactors whose operating temperatures were $100^{\circ} \mathrm{F}\left(56^{\circ} \mathrm{C}\right)$ above this level. These results dramatically revealed the need to account for all neutrons in many, if not most, reactor locations considered, if good correlations or predictions of data are to be made. This analysis also provided a fluence for a $200^{\circ} \mathrm{F} \Delta \mathrm{TT}$ in $\mathrm{A} 302-\mathrm{B}$ steel in these reactors, thereby providing a means to utilize the comparison of measured and calculated fluence values of Fig. 4. This comparison graphically displays the damaging potential of the spectrum in relation to other spectra. The potential of such comparisons is considerable if one considers that designers planning new reactors can simply test the spectrum calculated for their new reactor with the damage function and immediately 
know if the type of radiation damage they desire (for a test reactor) or do not desire (for a power reactor) will be present. If the damage function results suggest undesirable neutron embrittlement conditions, then changes can be made at the design stage, a new spectrum calculated for the modified reactor configuration, and the embrittlement conditions reevaluated with the damage function. The process can be repeated if necessary until the desired reactor conditions are achieved. Thus, it need not be necessary to construct a reactor before a realistic assessment of its potential for radiation damage can be made.

A final conclusion of this study is that detailed and accurate neutron dosimetry measurements are necessary if good correlations of irradiation effects data are to be obtained. It should be clear that good detail in spectral calculations and foil measurements is required for both low-and high-energy neutrons. The use of iron and cobalt foils without a spectral calculation will be inadequate. If a good spectral calculation is available, however, then iron and cobalt foils can be successfully used to define the magnitude of the spectral shape. If such a calculation is not available, then a suitable energy spectrum may be derived from the analysis of a number of different flux-detector foils with overlapping energy-response ranges. Experimenters and designers of pressurevessel surveillance programs should be sure to provide for the exposure of both highand low-energy neutron foils, such as iron and both bare and cadmium-covered cobalt foils, within irradiation experiments and at structural component surveillance locations. The availability of these data will be of significant value to the subsequent analysis of the data and will permit far more accurate assessments of the present component condition as well as permit better predictions of future conditions.

\section{ACKNOWLEDGMENTS}

This research was conducted at the Naval Research Laboraiory under the sponsorship of the Office of Naval Research and of the U.S. Atomic Energy Commission under contract AEC-AT(49-5)-2110; the research at the Battelle Memorial Institute was conducted under USAEC contract AT(45-1)-1830. The efforts of the staff members of the Reactor Materials Branch at NRL and of the Irradiation Damage Effects Unit at Battelle in support of this study are acknowledged with appreciation. Special thanks are accorded to C.H. Hogg and K.L. Rasmussen of the counting room staff of Idaho Nuclear Corporation, Materials Testing Reactor, for their efforts in counting and analyzing all of the neutronflux monitoring foils used for this study. The authors wish to note that studies of this kind cannot be accomplished in a short time, and thus, they are very appreciative of the long-term support that has been given by the sponsors for this purpose.

\section{REFERENCES}

1. Harries, D.R., Barton, P.J., and Wright, S.B., "Effects of Neutron Spectrum and Dose Rate on Radiation Hardening and Embrittlement in Steels," Symposium on Radiation Effects on Metals and Neutron Dosimetry, STP 341, Am Soc. Testing Materials (1963)

\section{Shure, $K_{\text {, " }}$ Radiation Damage Exposure and Embrittlement of Reactor Pressure Vessels," WAPD-TM-471, Bettis Atomic Power Laboratory, Nov. 1964}


3. Rossin, A.D., Nucl. Structural Eng. 1 (1965) 76

4. Dahl, R.E., and Yoshikawa, H.H., "Neutron-Exposure Correlation For Radiation-Damage Studies," Nucl. Sci. Eng. 21:312 (1965)

5. Rossin, A.D., "Reporting Neutron Exposure for Radiation Damage," in "Interaction of Radiation with Solids," Proc. Cairo Solid State Conference, Cairo, Egypt (Sept. 1966), New York:Plenum Press, 1967, p. 553

6. Sheely, W.F., Nucl. Sci. Eng. 29:165 (1967)

7. McElroy, W.N., Dahl, R.E., Jr., and Serpan, C.Z., Jr., "Damage Functions and Data Correlation," Nucl. Applications, (pending publication)

8. Dahl, R.E., Jr., Ulseth, J.A., and Busselman, G.J., Battelle-Northwest Laboratory, private communications to C.Z. Serpan, Jr., Naval Research Laboratory, Washington, D.C., 1968

9. Little, W.W., Jr., and Hardie, R.W., "2DB Users Manual," BNWL-831, BattelleNorthwest Laboratory, Aug. 1968

10. Duane, B.H., "Neutron and Photon Transport Plane-Cylinder-Sphere (GE-ANPD) Program S Variational Optimum Formulation," XDC-9-118, General Electric Company, Cincinnati, 1959

11. Bengston, J., and others, "2DXY Two-Dimensional, Cartesian Coordinate Sn Transport Calculation," USAEC Report AGNTM-392, June 1961

12. Helm, J.W., "High-Temperature Graphite Irradiations: 800 to 1200 Degrees C: Interim Report No. 1," BNWL-112, Battelle-Northwest Laboratory, Sept. 1965

13. Serpan, C.Z., Jr., and Hawthorne, J.R., Trans. Am Soc. Mech. Engrs. 89D4:897 (Dec. 1967)

14. Serpan, C.Z., Jr., and Steele, L.E., "Damaging Neutron Exposure Criteria for Evaluating the Embrittlement of Reactor Pressure Vessel Steels in Different Neutron Spectra," Symposium on the Effects of Radiation on Structural Metals, STP 426, Am Soc. Testing Materials, Pa., 1967, p. 594

15. McElroy, W.N., and others, "A Computer-Automated Iterative Method for Neutron Flux Spectra Determination by Foil Activation," AWFL-TR-67-41 Vols. I, II, III, and IV, Air Force Weapons Laboratory, Albuquerque, N.M., Sept. 1967

16. Landerman, E., "Surveillance Tests on Structural Materials in Nuclear Reactors," Symposium on Radiation Effects on Metals and Neutron Dosimetry, STP 341, Am Soc. Testing Materials, Philadelphia, Pa., p. 233, 1963

17. Serpan, C.Z., Jr., and Watson, H.E., "Notch Ductility, Tensile and Neutron Spectrum Analyses of the PM-2A Reactor Pressure Vessel," Symposium on the Effects of Radiation on Structural Metals, STP 457, Am Soc. Testing Materials, Philadelphia, $\mathrm{Pa}$. (pending publication) 1969 
USE OF NUCLEAR EXPLOSIONS FOR THE STUDY OF RADIATION DAMAGE RROCESSES*

\author{
Robert L. Carter \\ University of California \\ $4808=60$ Los Alamos Scientific Laboratory
Los Alamos, New Mexico**
}

and
University of Missouri 5
Columbia, Missouri

\title{
Introduction
}

The large flux of neutrons which accompanies chain fission reactions in nuclear reactors first led to extensive concern with the changes in the structure and properties of material subject to bombardment by energetic neutrons. Wigner's prediction ${ }^{1}$ of crystalline changes as a consequence of neutron irradiation led to intense engineering interest in possible modifications of fuel and moderator materials in thermal reactors. It was found that a large number of materials showed substantial changes in electrical, thermal, and chemical properties, along with profound distortions of crystalline microstructure following fast neutron irradiations of the intensities and for the times encountered in nuclear reactors. The obvious applications interest was coupled with the interest of solid-state physicists and physical metallurgists in the phenomenology of changes thus wrought in materials.

The changes brought about in the microstructure of a well-ordered crystal by the introduction of energetic neutrons are of interest because of the opportunity to test models of atomic interactions within the crystal. Stable configurations can be deduced through the use of $x$-ray and neutron diffraction techniques, and by small angle scattering experiments. Another powerful tool is lent by the study of thermal annealing phenomena, wherein the relaxation of the crystalline defects are observed to accompany an increase in temperature to a value substantially above the temperature at which the material was held during creation of the defects by neutrons. 2

\footnotetext{
* Work performed under the auspices of the U. S. Atomic Energy Commission. * Visiting Staff Member, June 1968 to September 1969.
} 
It should be pointed out that an almost equally interesting facet of such studies of microscopic changes which can be produced and healed in crystals of a material is the opportunity afforded to link the various physical properties of the bulk material to such controlled changes within the crystals. Changes occurring within such complex agglomerates as artificial graphite are not only of great practical interest; they permit a comparison of the roles played by the relatively orderly crystalline material and the disordered filler phase insofar as the various measurable properties are concerned.

An overly simplistic but useful model of neutron-induced radiation damage in a crystalline material may be envisaged as follows. The regular arxay of the perfect crystal is disrupted by collisions of fast neutrons and energetic displaced "recoil" atoms from the crystal, to yield a material with lattice vacancies ("Schottky" defects) and with displaced atoms wedged into high-energy locations among the remaining ordered atoms of the crystal ("Frenkel" defects). This model obviously is valid only when a relatively small fraction of the atoms have been displaced. If the displaced atoms are not in positions of chemical stability, they will continue to diffuse, provided that the lattice temperature is not too low to permit such diffusion. Such diffusing atoms constitute a sort of constrained gas, the partial pressure of which depends upon the intensity of the causitive neutron irradiation. Among the more likely targets for sorption of these gas atoms are crystal vacancies, crystal defects, crystal edges, or other gas atoms. Indication of the importance of the last sorption mechanism is believed to be provided by the phenomenon of "radiation annealing."

The bulk of the evidence for the radiation annealing phenomenon has come from studies of changes in the physical properties of graphite moderator in the long-lived production reactors at Hanford. ${ }^{3}$ A reproducible quantity of data had been collected describing changes in the properties of graphite occurring as a consequence of irradiation at moderate temperature followed by long-term thermal annealing at a given elevated temperature. It was clearly demonstrated that long-term annealing at the same elevated temperature, while simultaneously irradiating with neutrons, resulted in a markedly greater degree of recovery from the effects of irradiation damage, than did thermal annealing alone. Besides being of great practical usefulness in extending the useful lifetime of nuclear reactors utilizing graphite as a neutron moderator, this discovery put at the disposal of the solid-state physicist a powerful tool for studying the formation and destruction of interstitial complexes of displaced atoms within an irradiated material. The effects of such foreign structures upon thermal conductivity, specific heat, and electrical properties of crystalline materials are of great interest.

Neutron irradiation rate would be expected to play at least two roles in affecting the nature of defects remaining in a material following the termination of irradiation. The higher the irradiation rate, the higher the steady state density of diffusing displaced atoms, and the greater the probability of a second-order process leading to the formation of a stable two-atom complex. Often times, such a complex may serve as a "seed" for the subsequent condensation of other interstitial atoms into a larger complex. On the other hand, very high intensity irradiation with disruptive particles increases the probability that such complexes 
will fall victim to disruptive encounters. The effectiveness of such disruptive encountexs in destroying complexes might be expected to increase with the diffusion rate, and hence the temperature. However, experimental demonstration of the validity of such an assumption, as well as the measurement of the physical parameters governing the dynamics of the process awaits the availability of sources permitting a wide range of irradiation rates. The present papex explores the possible usefulness of nuclear explosive tests for this purpose.

Experiments Using Explosive Nuclear Sources

The intense bursts of neutrons obtained from underground nuclear detonations at the Nevada Test Site of the U. S. Atomic Energy Commission have been used to pexform cross section measurements upon a variety of nuclides, and the results have been reported in the open literature. 4 In Fig. I we see an underground enclosure for the explosive nuclear device centered at the "working point" 185 meters underground. The working point is connected by means of a large evacuated pipe with a collimator and expeximental area at the surface of the ground. The energy spectrum of neutrons drifting up the pipe is broadened by means of a polyethylene moderator placed $40 \mathrm{~cm}$ from the neutron source adjacent to the 180 -meterlong baffled pipe leading to the experimental area. The neutron source function for neutrons of less than $320 \mathrm{eV}$ leaving the moderator is shown in Fig. 2. Note that the half-width of the pulse is less than 5 usec.

The short duration of the neutron pulse at the source serves as a beam chopper, and the 180 meter drift tube serves to separate neutrons of varying energy in time at the experimental area. A simple calculation shows that neutrons of $1 \mathrm{MeV}$ energy require $13 \mathrm{usec}$ to traverse the tube, those of $10 \mathrm{keV}$ require $135 \mathrm{\mu sec}$, and those of $100 \mathrm{eV}$ requixe $1.35 \mathrm{msec}$. Energy dependence of fission cross section of material deposited on thin foils exposed to the beam of neutrons is readily obtained by observing time dependence of fission particles emitted by the foil deposit and collected on a solid state detector.

Character of a Nuclear Bomb as a Neutron Source

There is a dearth of material pertinent to the evaluation of a nuclear bomb as a source of radiation for experimentation in the open literature. As a starting point I quote from the introductory portion of the book "Neutrons from an Atomic Explosion," by P. A. Yampol'skiy. This is available in English translation from the office of Technical Services, U. S. Department of Commerce.

"The energy released by the explosion of one gram of TNT amounts to 1000 calories. [Therefore, the energy released by $1 \mathrm{kilo}$ tonne TNT equivalent is $-10^{12}$ calories.] ... An explosion equivalent to one $\mathrm{kilo}$ tonne of TNT requires the fission of $1.5 \times 10^{23}$ atoms, that is, about 60 grams of uranium or plutonium.... The fission process occurs within a very short period of time;... the time required for an explosion equivalent to $10 \mathrm{kilo}$ tonnes of TNT equals about $3.5 \times 10^{-7}$ second. In view of the exponential nature of the reaction development, the number of fission generations 
required for a $1,000 \mathrm{kilo}$ tonne explosion differs by only $10 \%$ from the number of generations required for a $10 \mathrm{kilo}$ tonne explosion. It may therefore be assumed that the duration of the explosions of different power is several tenths of a microsecond.

"Judging from press reports, the bombs exploded during tests vary a great deal in their power (from one kilo tonne to 10 mega tonnes). But regardless of the power of the explosions, their basic processes and the nature of their effect on the surrounding medium are the same. This applies not only to atomic explosions but also to hydrogen-bomb explosions.

"In a hydrogen-bomb explosion the thermonuclear reaction is used as an intermediate process whereby neutrons are initiated and bring about the [fast] fission of $238_{\mathrm{U}}$ atoms. The energy of uranium nuclear fission therefore accounts for a considerable portion of the power of the thermonuclear bomb.

"The number of neutrons released by an explosion of a purely thermonuclear [fusion] bomb may be several times greater than the number of neutrons released by an explosion of similar power based on fission reactions. If in a thermonucleax bomb use is made of the fusion reaction of tritium and deuterium, each reaction releases one neutron and an energy equal to $17.6 \mathrm{MeV}$. [Momentum considerations give the neutron $14.1 \mathrm{MeV}$ of this energy and the helium recoil $3.5 \mathrm{MeV}_{3}$ ] This energy is one tenth of the uranium or plutonium fission energy. Therefore, the number of neutrons per unit of released energy in such a bomb is considerably larger than in a bomb based on the fission reaction. Moreover, the neutron energy of a thermonuclear explosion is greater than the average neutron energy of an atomic fission explosion....

"The enormous energy of an atomic explosion is released within a bomb [assembly] weighing about one tonne in less than $10^{-6}$ second. It follows from [the preceding] that in the explosion of a $20 \mathrm{kilo}$ tonne bomb the average energy density equals $10^{7} \mathrm{cal} / \mathrm{g}$. Such a colossal energy density must result in the heating of matter within the bomb [assembly] to stellar temperatures in the neighborhood of tens of millions of degrees. Matter heated to such high temperature releases energy in the form of electromagnetic radiation of a very broad spectrum ranging from $x$-rays to longwave electromagnetic radiation."

Simple application of the stefan-Boltzmann radiation law to a blackbody at (say) $2 \times 10^{7} \mathrm{o}$ gives a radiation rate of $2.2 \times 10^{17} \mathrm{cal} / \mathrm{cm}^{2} \mathrm{sec}$. Material of moderate or large atomic number, at such a temperature, is ionized on the average to a degree of about +8 . The number of particles is therefore increased nine-fold, and the average specific heat may be 
taken to be nine times the low temperature value of about $0.3 \mathrm{cal} / \mathrm{g}^{\circ} \mathrm{C}$. This will give a heat capacity for a 1 ton assembly of about $2.7 \times 10^{6}$ cal/ ${ }^{\mathrm{K}}$. If the bomb assembly be assumed to be a 1 meter diameter sphere, its surface area is $31,000 \mathrm{~cm}^{2}$. The electromagnetic radiation rate at $2 \times 10^{7}{ }^{\circ} \mathrm{K}$ is $6.8 \times 10^{21} \mathrm{cal} / \mathrm{sec}$, under the assumption of a blackbody radiation law. These two numbers permit the calculation of an initial cooling rate of $2.5 \times 10^{15} \mathrm{~K} / \mathrm{sec}$. Of course, the emissivity of any plasma in the $x$-ray region is far less than one. Also, reflection and back radiation from the surrounding medium will further reduce the cooling rate. Nevertheless, it is evident that thermal quenching due to radiative energy losses is exceedingly rapid, and a steady state temperature will tend to be reached which depends upon the instantaneous rate of energy generation during the peak of the explosion.

Clearly the width of the intense pulse of blackbody electromagnetic radiation from the initial fireball will be determined by the length of the pulse of nuclear energy generated. This was above indicated to be of the order of a few tenths of a microsecond. The electromagnetic radiation (including prompt gammas) arrives at an experimental station distance $D$ meters from the working point in a time

$$
\mathrm{T}_{\text {e.m. }}=\left(3.3 \times 10^{-9}\right) \mathrm{D} \text { seconds }
$$

after the nuclear explosion. In the evacuated tube there is no dispersion, quanta of $x$-rays arriving at the same instant as quanta of infra-red radiation.

The radiant cooling rate of $6.8 \times 10^{21}$ calories per second previously estimated may be used to determine the electromagnetic radiation falling upon an experimental target a distance $D$ meters from the source. If isotropy and no reflection be assumed, we obtain a peak radiation intensity of

$$
I_{\text {e.m. }}=\frac{5.4 \times 10^{16}}{D^{2}} \mathrm{cal} / \mathrm{cm}^{2} \mathrm{sec} .
$$

If radiation emission is not a blackbody continuous spectrum, the intensity of electromagnetic radiation will be correspondingly lessened.

As was pointed out earlier, a range of neutron energies emitted leads to a separation of neutrons along the drift path following the explosion, so that neutrons of energy $E$ (electron volts) arxive at a position $D$ (meters) from the working point at a time

$$
T_{\text {neut }}=\frac{7.2 \times 10^{-5} \mathrm{D}}{\sqrt{\mathrm{E}}} \text { seconds }
$$

after the nuclear explosion.

If an excess neutron yield of 1.8 neutrons per fission be assumed, a $20 \mathrm{kt}$ device will yield $2.7 \times 10^{24}$ neutrons. If these are distributed isotropically (no scattering assumed) the integrated neutron flux in the 
pulse at a distance $D$ meters from the working point will be

$$
\phi_{\text {neut }}=\frac{4.5 \times 10^{19}}{\mathrm{D}^{2}}-\text { neutrons } / \mathrm{cm}^{2} .
$$

As was pointed out above, the neutron output of a purely thermonuclear source of the same energy yield may be several times greater than the above figure for a fission source.

Concommitant Mechanical Problems

An abrupt ground shock follows the detonation of the nuclear device beneath the ground, the time delay after the explosion and the acceleration experienced being a function of the properties of the earth intervering, and of the device yield.

For a line-of-sight tube extending from the working point to the experimental area, there will, of course, be a gaseous shock wave and particles of solid matter projected from the explosion at high velocities. The feasibility of detonation of underaround nuclear explosions without atmospheric contamination by radioactive debris necessitated the development of fast-acting closures. Such closures seal off the gases evolved from the nuclear bomb itself and from surrounding materials completely. shortly after the passage of the neutrons generated within the device.

The final environmental problem is the collapse of the pocket in the earth following the cooling of gases formed in the explosion. The size of the collapse crater, and the time required for collapse to occur depend upon the device yield, and upon the characteristics of the soil within which the explosion is set off. Typically, the surface of the ground above the working point will accelerate at $0.8 \mathrm{~g}$ within an hour after shot time for a distance of thirty feet, where it will rapidly decelerate at $50 \mathrm{~g}$ or more. The crater remaining after the collapse may be about 200 feet in diameter.

High Intensity Radiation Damage and Radiation Annealing Experiments

Passive experiments, with samples whose physical properties are to be measured before and after exposure, may be performed at distances from the working point ranging from about 50 meters to 200 meters or more. For the reference source we have developed expressions for, the resulting fast neutron exposures will range from $2 \times 10^{16}$ to $10^{15} \mathrm{~cm}^{-2}$. For a thermonuclear source of equal yield, fluxes of $14 \mathrm{meV}$ neutrons will be several-fold higher in intensity. For metal or ceramic targets, about 1\% of the neutron energy will be dissipated per millimeter traversed, For a $2 \times 10^{16} \mathrm{~cm}^{-2}$ burst of $1-\mathrm{MeV}$ neutrons, approximately $80 \mathrm{cal} / \mathrm{cm}^{3}$ will be dissipated, resulting in a temperature rise from neutron heating of about $80^{\circ} \mathrm{C}$ in a material having a specific heat of 0.2 and a density of $5 \mathrm{~g} / \mathrm{cm}^{3}$.

The heating due to the flash of electromagnetic radiation preceding the neutron burst must be considered. If the duration of the light flash 
is taken as $10^{-7}$ sec, use of the preceding expression for intensity gives a flash energy density of $2 \times 10^{6} \mathrm{cal} / \mathrm{cm}^{2}$ at a distance of 50 meters from the souxce. The seriousness of the heating effect depends upon the spectrum of the radiation. High energy quanta will penetrate the sample with little energy absorption. Low energy quanta will dissipate their energy in the first few microns of the exposed surface of the sample. Selective filtering may be employed to reduce the intensity of troublesome frequencies in the electromagnetic flash, while attenuating the neutron beam by a relatively small factor.

In order to observe property change transients immediately following irradiation, the sample may be instrumented for remote measurement of properties. Techniques developed at North American Aviation (now North American Rockwel1) by associates of the author in the early $1950^{\prime}$ 's for radiation damage studies using the Berkeley $60^{\prime \prime}$ cyclotron may be adapted. Electrical resistance and thermoelectric properties are easiest to measure, although Hall coefficient, magnetoresistance and thermal conductivity can also be measured remotely. The above-mentioned cyclotron work also successfully provided for helium gas cooling, permitting measurement of properties at controlled low temperatures. In the event that it becomes desirable to measure effects of irradiation intensities requiring exposure at distance less than 50 meters from the source, the difficulty of recovery for post-irradiation measurement necessitates instrumentation for in-place measurement of changes in the desired properties.

Should it be desirable to observe the differential effect of neutrons of various energies, a polyethylene moderator may be placed near the neutron source, and neutrons of energies ranging downward from those produced by the source will be obtained. At a distance of 100 meters from a thermonuclear source, $14 \mathrm{meV}$ neutrons arrive a little less than $2 \mu \mathrm{sec}$ after detonation, $1-\mathrm{MeV}$ neutrons require $7 \mu \mathrm{sec} ; 100 \mathrm{keV}, 23 \mu \mathrm{sec}$; and $10 \mathrm{keV}$, $70 \mathrm{\mu sec}$. It is feasible to mount samples to be exposed near the edge of a 1 meter diameter wheel, which is spun up to $1000 \mathrm{rpm}$ just prior to detonation time, The linear velocity of the samples being approximately $0.05 \mathrm{~mm} /$ usec, regions exposed to $1-\mathrm{MeV}$ neutrons will be separated by $025 \mathrm{~mm}$ from the onset of irradiation by $14 \mathrm{MeV}$ neutrons. Regions irradiated by 100 $\mathrm{keV}$ and $10 \mathrm{keV}$ neutrons are $1.1 \mathrm{~mm}$ and $3.5 \mathrm{~mm}$ displaced, respectively. Since it is not feasible to spin the wheel with a known phase relationship to the explosion time, it will be necessary to place samples of the material being studied around the entire perimeter of the wheel, then locate the exposed region by autoradiography of a thin foil placed adjacent to the material sample ring.

Direct Energy Conversion Studies

Many physicists feel that a more elegant procedure for the conversion of the energy of fission particles into useful forms of power should be possible. Efforts to produce large electrostatic potential differences in vacuo have been discouraging because of the large field emission electron currents which essentially short out the fission particle currents. 7 High fission particle irradiation rates occurring for very short times will permit the transient study of elemental processes in plasmas and in solids. A fission particle pulse can be initiated by a fast neutron burst 
impinging upon a fissionable material. Intense fission particle bursts of less than microsecond duration may be obtained by using a plate of a fissionable material having suitable cross section for fast fission. vacuum or semiconductox experimental arrays can be designed which permit a study of the dynamics of secondary electron production, and the measures which may be used to suppress or utilize these in energy conversion. Also, the rearrangement of cyclotron resonance state populations in materials within a magnetic field will shed light on the possible usefulness of the fission particle pumped cyclotron resonance maser concept.

The production of excited states by forbidden transitions induced by fission particle collisions within chemical systems could be explored at vexy high intensity fission particle irradiation intensity within a fluid containing a relatively small quantity of fissile material. Such fission recoil synthesis of chemical species has recently received attention $^{8}$ and is closely related to the new field of plasma Chemistry.

Accessibility of the N.T.S. Facility to Independent Researchers

To the writer's knowledge, no provisions have been made for independent investigators to undertake research unrelated to the A.E.C. programmatic projects at the Nevada Test Site. However, there are a number of contractors carrying out research, some of which has given results of sufficiently general interest to warrant open publication. opportunities for university faculty members and graduate students to participate in such programs, under sponsorship of one of the contractors, exist. The preceding paper has set forth the thesis that investigations of the effects of high intensity neutron ixradiation on the physical properties of solids, which can be conducted at the Nevada Test Site will yield useful, publishable data.

\section{References}

1. M. Burton, "Radiation Chemistry," J. Phys. and Colloid Chem. 51:618f (1947).

2. D. S. Billington and J. H. Crawford, Jr., Radiation Damage in Solids, Princeton University Press, (1961).

3. R. E. Nightingale, "Annealing Radiation Effects," Chapter 13, Nuclear Graphite, Academic Press: 376f, (1962).

4. P. A. Seeger, A. Hemmendinger, and B. C. Diven, "Fission Cross Sections of $241_{A m}$ and $242 m_{A m}, "$ Nuclear Physics A96: 605-616, (1967).

5. P. A. Yampol'skiy. Neutrons from an Atomic Explosion. English translation, document 62-15501, Office of Technical Services, U. S. Department of commerce.

6. H. P. Yockey, A. Andrew, F. L. Fillmore, L. E. Glasgow, C. d'A. Hunt and J.H. Pepper, "Cyclotron Techniques for Radiation Damage Studies," Rev. Sci. Instru. 25: 1011-1019 (1954). 
7. G. Safonov, "Direct Power Conversion - Part II, The Fission-Electric Reactor," ASTIA Document No. AD 144303, December 12, 1957.

8. Meyer Steinberg, "Fission Recoil Synthesis" BNL-9985. Paper presented at the 59th National Meeting of the AIChE Symposium on Chemical Synthesis Under Extreme or Unusual Conditions, May 15-18, 1966, Columbus, Ohio.

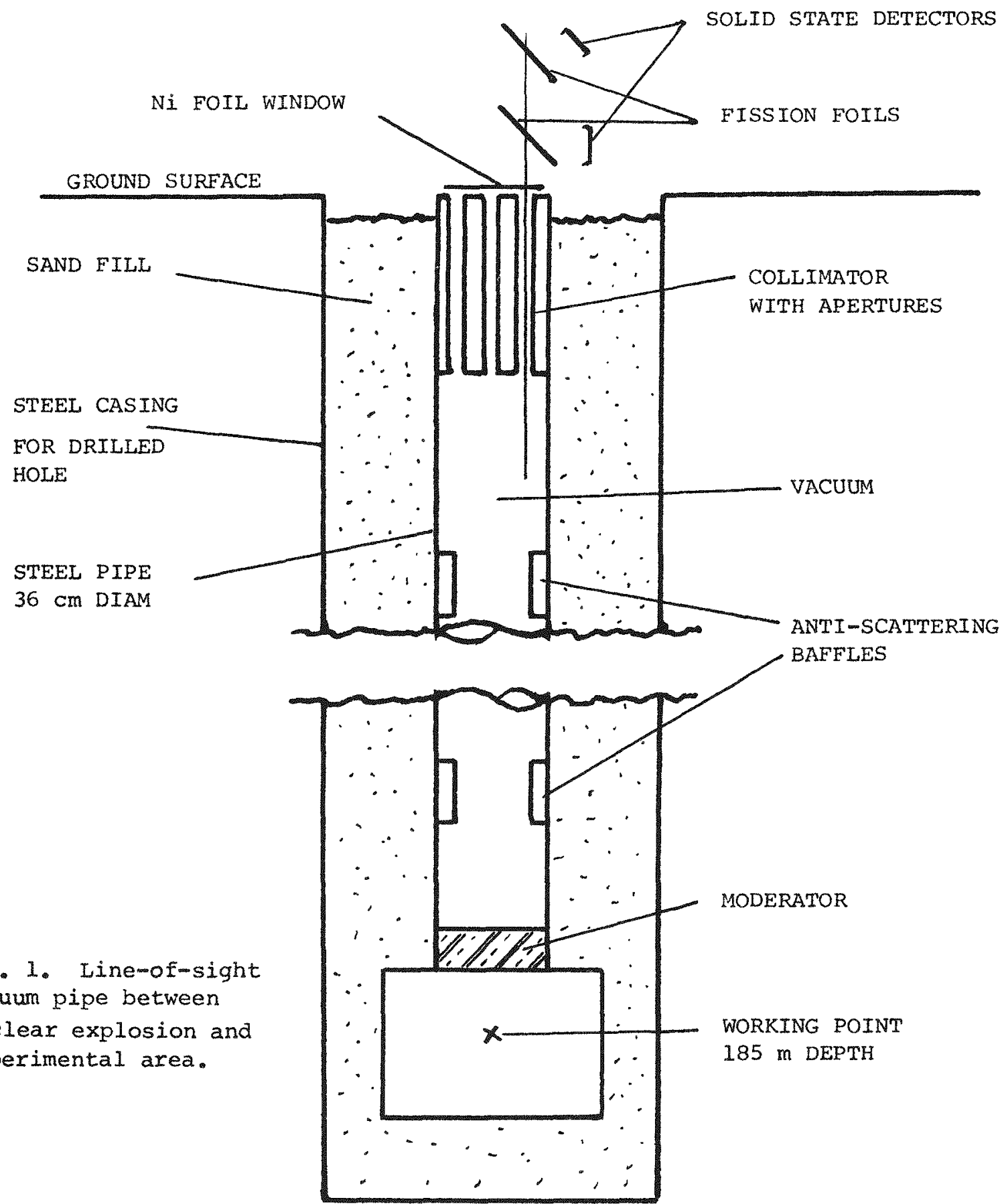




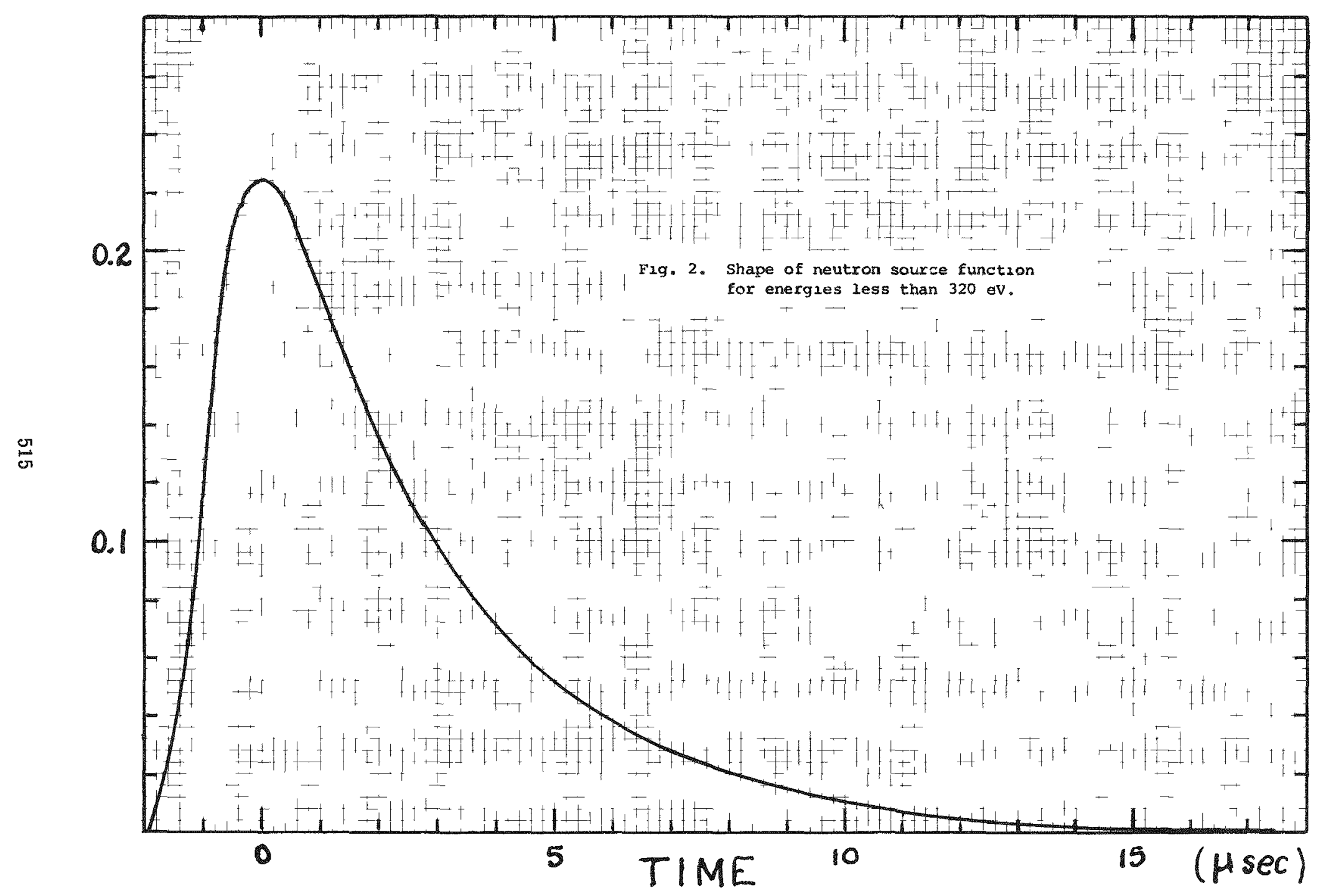




\author{
$y_{1}$ \\ A microprobe/Studu of THE RETENTION OF THE \\ FISSION GAS XENON IN IRRADIATED URANIUM FUELS \\ DISPERSED IN ALUMINUM CLAD NUCLEAR REACTOR FUEL PLATES* \\ By \\ W. F. Zelezny, G. W. Gibson, and M. J. Graber \\ Idaho Nuclear Corporation \\ P. O. Box 1845 \\ Idaho Falls, Idaho 83401
}

I. Introduction

The purpose of this paper is to describe (a) techniques which have been found useful in the examination of irradiated and highly radioactive materials with a microprobe which was unshielded at first and later had a limited amount of shielding added and (b) applications of these techniques in a study of the relative degree of retention of the fission gas, xenon, by irradiated dispersion fuel elements. These techniques include the preparation of specimens of limited size to decrease the total radioactivity, "counting-over-the-peak" to decrease the lower limit of detectability with an accompanying increase in the sensitivity of detection of trace elements in the presence of high background levels and the introduction of a limited amount of shielding in the microprobe. The discussion of the shielding includes a number of considerations which have a bearing on the examination of highly radioactive specimens by microprobe.

II. Techniques Useful in the Examination of Highly Radioactive Samples by Microprobe

1. Preparation of Small Specimens

Preparation of the specimens was accomplished entirely by remote control in the TRA Hot Cell. By the use of a micro-cutoff wheel, wafers 10 mils thick were made by cuts across the fuel sample, giving a a cross section of the core and its cladding as shown in the sketch in Figure 1. The wafers of fuel plates were held upright during the mounting process by brass specimen supports formed from pieces of brass sheet. The fuel plate wafer and its support were mounted in a standard 1-1/4 inch

* Work performed under Atomic Energy Commission Contract AT(10-1)-1230. 
diameter metallurgical mount. The first mounts were prepared using a mixture of 50 volume percent Fisher Quickmount (a liquid epoxy) and 50 volume percent lead powder. The lead powder was intended to provide shielding against the radioactivity of the specimen, but its use was discontinued when it was found to interfere with the polishing process of some materials.

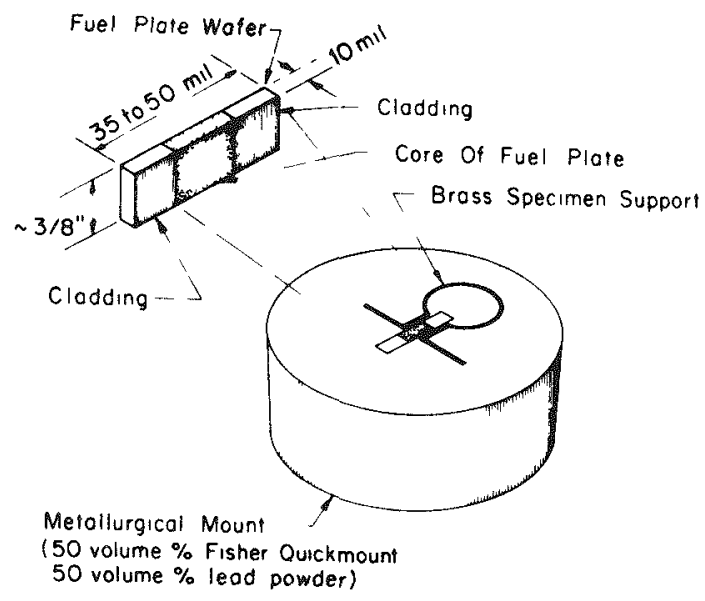

Fig. 1 Specimen mounts used in preparing irradiated specimens for microprobe examination.

2. Example of Results Obtained in the Examination of an Irradiated Dispersion Fuel

As an example, the results obtained by the examination of a UO2-A1 dispersion fuel material having a net fuel enrichment of 30 percent and which had been irradiated to the very high burnup of 83 percent will be described. The mounted specimen showed a radiation field of $>5 \mathrm{R} / \mathrm{hr}$ at "contact", and $100 \mathrm{mR} / \mathrm{hr}$ at a distance of one foot in air. The 30 percent enrichment was attained by mixing particles of fully enriched UO2 with the appropriate quantity of depleted $\mathrm{UO}_{2}$. The fact that the fuel particles are mixed, reveals in a most interesting manner, Figure 2, that the enriched UO2 particles have changed drastically in appearance in comparison with the depleted particles. This figure also shows an altered zone in the aluminum matrix surrounding the enriched, but not the depleted, UO2 particles. The altered zone polished to a characteristic appearance much lighter than that of the aluminum surrounding the depleted particles and at larger distances from the enriched particles. With Figure 2 is a key which points out these aforementioned characteristic regions shown in the photomicrograph. The portion of this photomicrograph which is enclosed within the small rectangle is reproduced on an enlarged scale in Figure 3. The entire photomicrograph is again reproduced in Figure 4 to show the distribution of the fission products.

The difference between the enriched and depleted particles is demonstrated further in Figure 3 which shows the $U M_{1} A_{1} A_{1} K_{\alpha}$, and Ce $\mathrm{L}_{1} 1 \mathrm{X}-\mathrm{ray}$ intensities obtained during a probe scan which crosses in succession (a) an enriched particle with its zone of altered aluminum, (b) a depleted particle, and (c) another enriched particle and accompanying altered zone. The depleted particle produced a considerably higher U MB 1 X-ray intensity which would be expected since it would have undergone no appreciable burnup. 


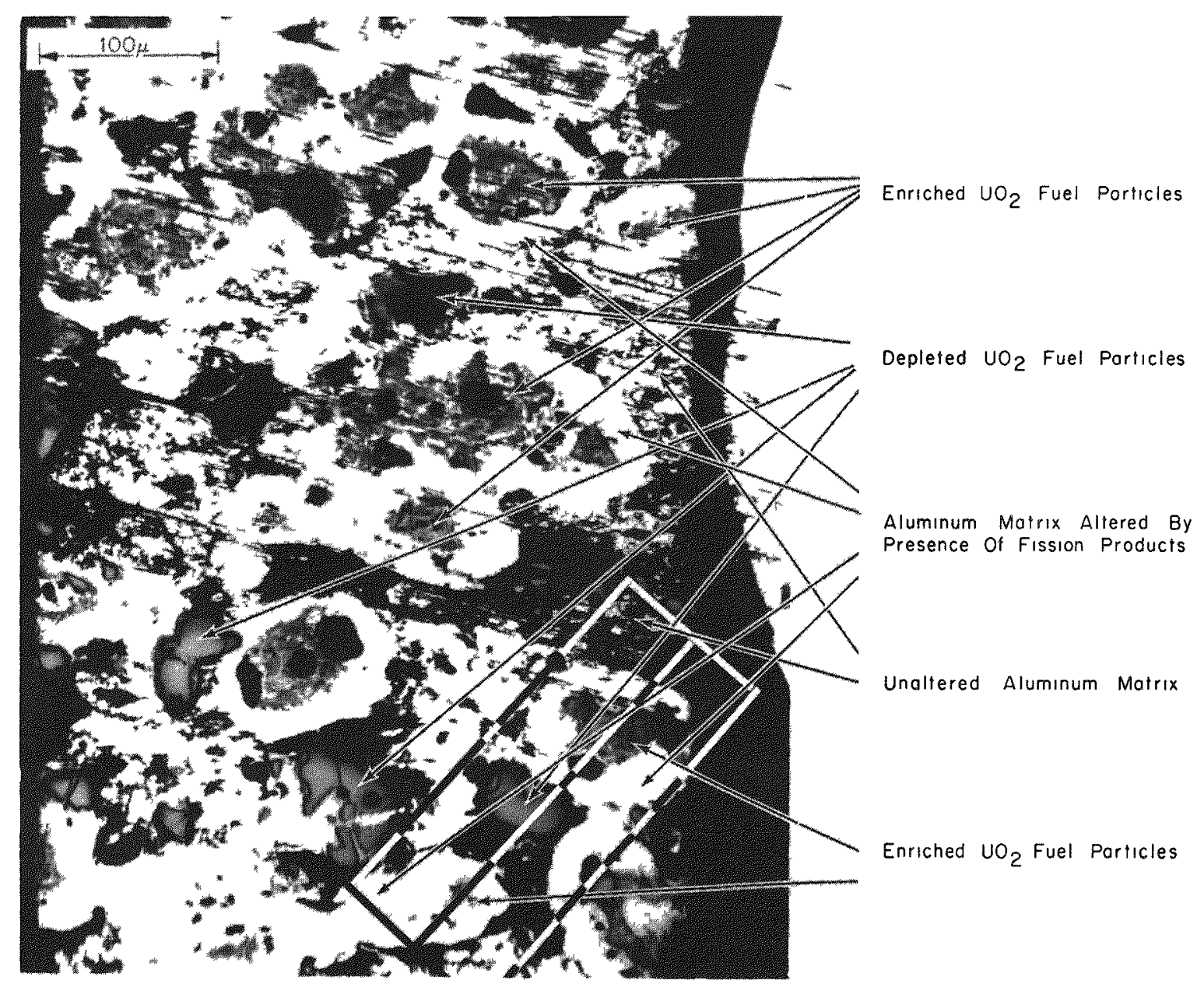

F1g. 2 Photomicrograph of specimen 28-3, together with key indicating characteristic regions shown in photomicrograph.
A. Photomicrograph; unetched. Area within small rectangle shows region chosen for probe scan shown in Figure 3.
B. Key to photomicrograph.

The Al $\mathrm{K}_{\alpha}$ trace (Figure 3) shows no aluminum at the centers of the particles (this was verified also by quantitative point counts) and pos sibly no aluminum in any place with the boundaries of the particles. The only region about which there could be any doubt regarding the presence of aluminum within the particles is in the immediate vicinity of the boundary in a region no wider than the limitation imposed on resolution by the finite diameter of the probe. From this, it is concluded that no indication of chemical reaction between the aluminum and the $\mathrm{UO}_{2}$ has been demonstrated by this investigation. If any such reaction has taken place, it is limited to a region around the periphery of the particle and nowider than the resolution of the probe. Under similar conditions in another $\mathrm{UO}_{2}-\mathrm{Al}$ fuel, appreciable reaction has been 

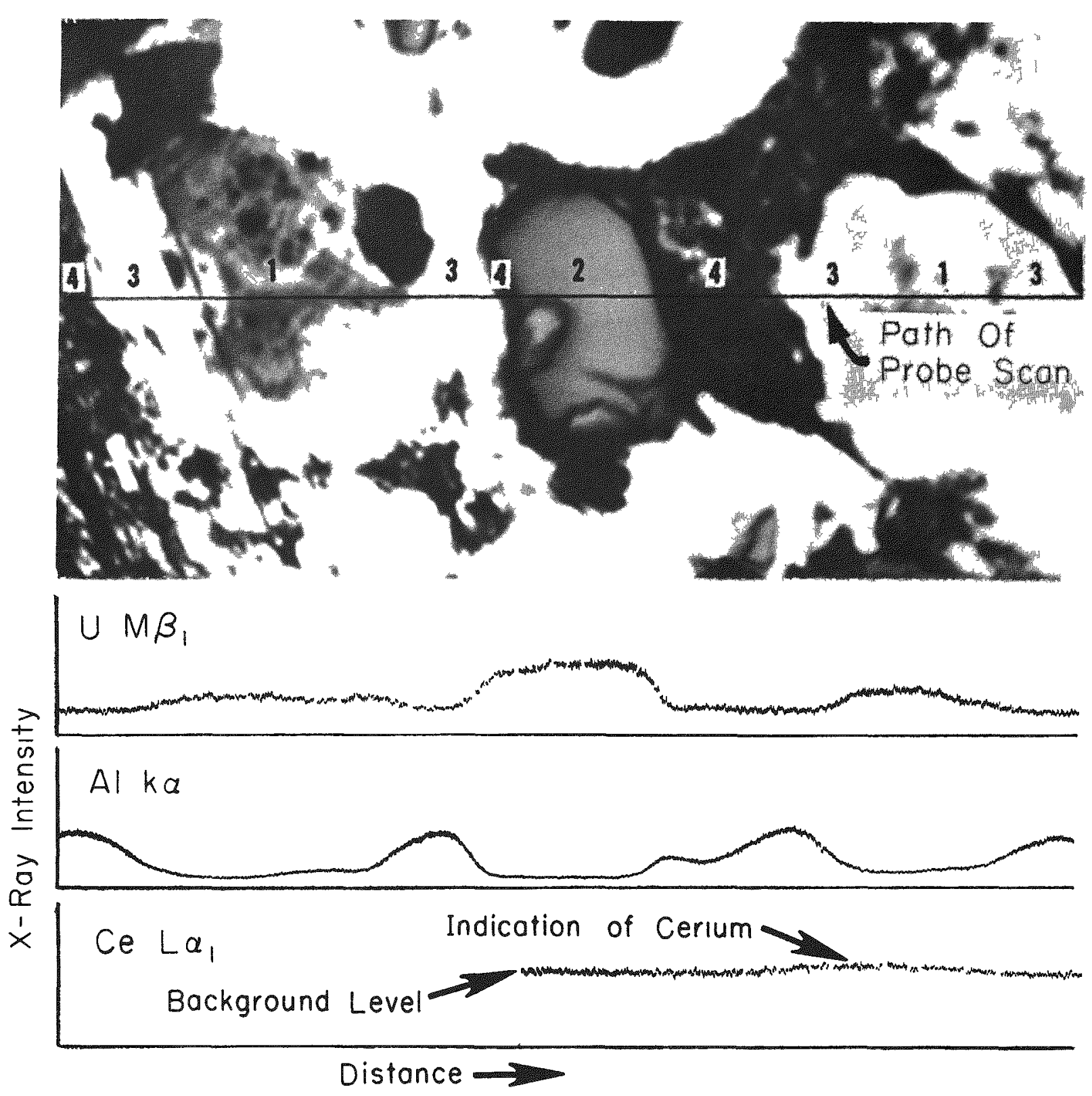

Fig. 3 Results of one-dimensional probe scan across region enclosed in small rectangle in Figure 2.

(1) Enriched $\mathrm{UO}_{2}$ Particles

(2) Depleted $\mathrm{UO}_{2}$ Particles

(3) "Altered" Alumınum Matrix

(4) Inaltered Aluminum Matrix 


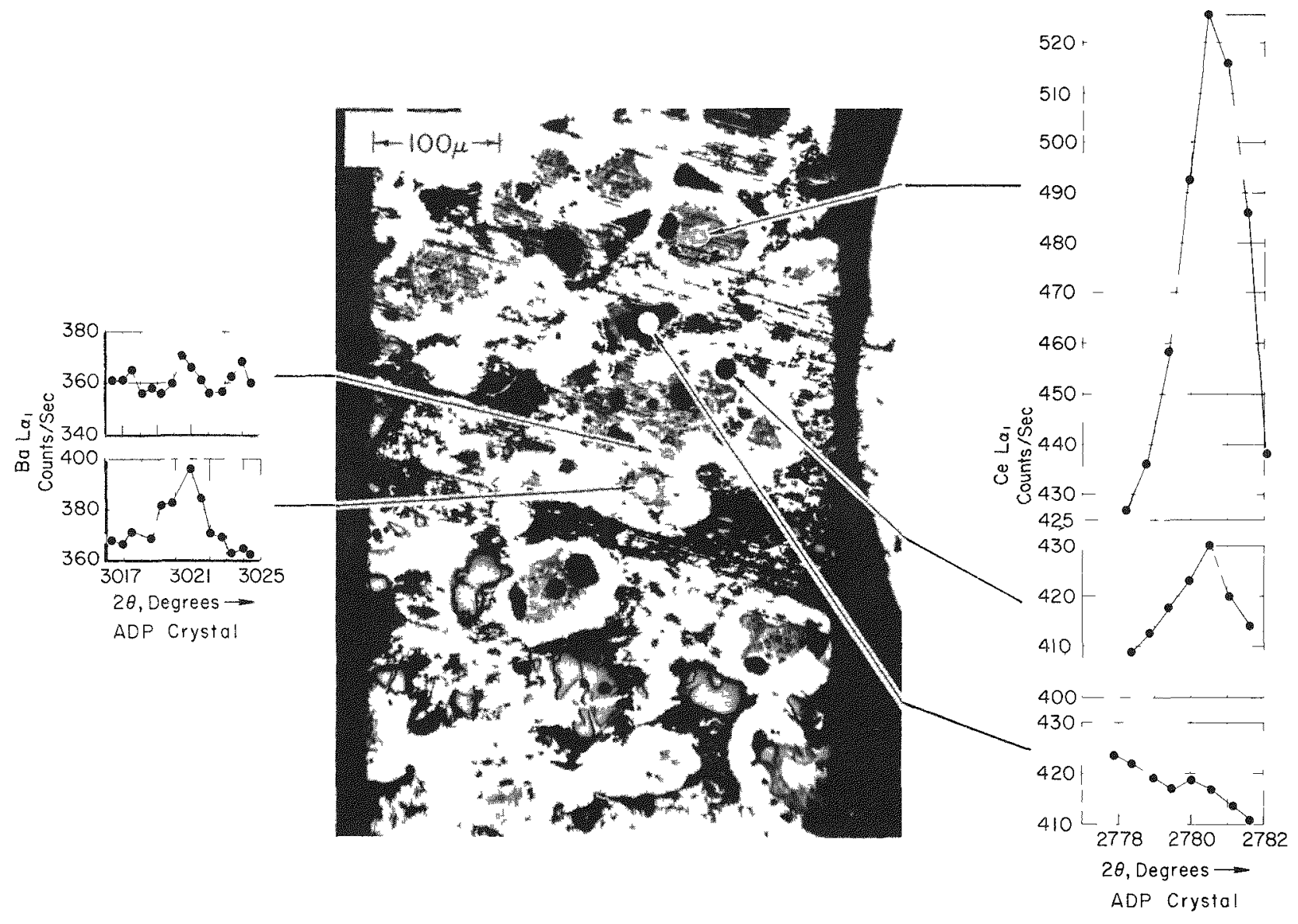

Fig. 4 Photomicrograph of $\mathrm{UO}_{2}-\mathrm{Al}$ fuel material and over-the-peak counts of $\mathrm{Ba} \mathrm{Ia}$ and Ce $\mathrm{La} \mathrm{X}-\mathrm{rays}$ at various points on the specimen.
A. Over-the-peak Ba I $\alpha$ count on depleted $\mathrm{UO}_{2}$ particle
B. Over-the-peak Ba $I_{\alpha}$ count on aluminum matrix adjacent to enriched $\mathrm{UO}_{2}$ particle
C. Over-the-peak $\mathrm{Ba} \mathrm{La}$ count on enriched $\mathrm{UO}_{2}$ particle
D. Photomicrograph; polished; unetched
E. Over-the-peak Ce $\mathrm{L}_{\propto}$ count on aluminum matrix adjacent to enriched $\mathrm{UO}_{2}$ particle
F. Over-the-peak Ce $\mathrm{L}_{\mathrm{x}}$ count on aluminum matrix adjacent to depleted $\mathrm{UO}_{2}$ particle
G. Over-the-peak Ce La count on depleted $\mathrm{UO}_{2}$ particle
H. Over-the-peak Ce $\mathrm{L} a$ count on enriched $\mathrm{UO}_{2}$ particle.

reported[1] between $\mathrm{UO}_{2}$ and $\mathrm{Al}$. Apparently a number of variables, including the stoichiometry of the $\mathrm{UO}_{2}$, influence the rate with which this reaction approaches completion. 
The chart recording for cerium (Figure 3) showed a very weak and uncertain indication for the fission product, cerium. This indication of the presence of cerium occurred only in the enriched particles and possibly in the altered zone of the aluminum matrix immediately adjacent to the enriched particles. This is exactly the distribution which would be expected since no fissioning would occur [a] in the depleted particle.

In order to establish more definitely the distribution of fission products in the fuel core, recourse was made to the technique of "countingover-the-peak". This consisted of a series of 100-second-long counts with the electron beam held at the same fixed point on the specimen. For each successive count, the spectrometer $2 \theta$ setting was advanced by an increment so that during the series of counts the spectrometer passed through the desired X-ray wavelength setting. Comparison of the chart recording weakly indicating cerium in Figure 3 with the "over-the-peak" counts in Figure $4 \mathrm{H}$ from a similar fuel particle will show vividly how the latter technique is able to reveal the presence of low percentages of elements in samples of very high background. An intensive series of over-the-peak counts for barium and for cerium were made in the various regions of the fuel plate core. Neither barium nor cerium was detected in the depleted fuel particles (Figure 4). Barium was found in the enriched fuel particles.

Strong cerium peaks were found in the enriched fuel particles while slightly less strong, but definite, peaks were obtained from the altered matrix near these particles. This is suggestive of concentration gradients of fission products from the fuel particles to the aluminum matrix. No cerium was found in or near the depleted particles.

3. Shielding and Instrumental Factors in the Microprobe Examination of Radioactive Materials

\section{A. Sample}

The radioactive sample just described was used in these experiments. Health Physics personnel monitored the fields around the specimen as $>5 \mathrm{R} / \mathrm{hr}$ at "contact", and $100 \mathrm{mR} / \mathrm{hr}$ at a distance of one foot in air.

\section{B. Apparatus}

The shielding illustrated schematically in Figure 5 was used in various combinations in a MAC Model 400 electron-probe microanalyzer. This consisted of a large lead shield within the specimen chamber and lead shielding in front of and behind the detector tube. The specimen-chamber shield interposed a maximum of $5.0 \mathrm{~cm}$ of lead between the specimen and the

[a] Strictly speaking, conversion of some of the U-238 in the depleted particle to $\mathrm{Pu}-239$ would occur; and part of the $\mathrm{Pu}$ would then undergo fission, thus producing its fission products. The extent of this conversion and fissioning may be considered negligible as far as the present investigation is concerned. 


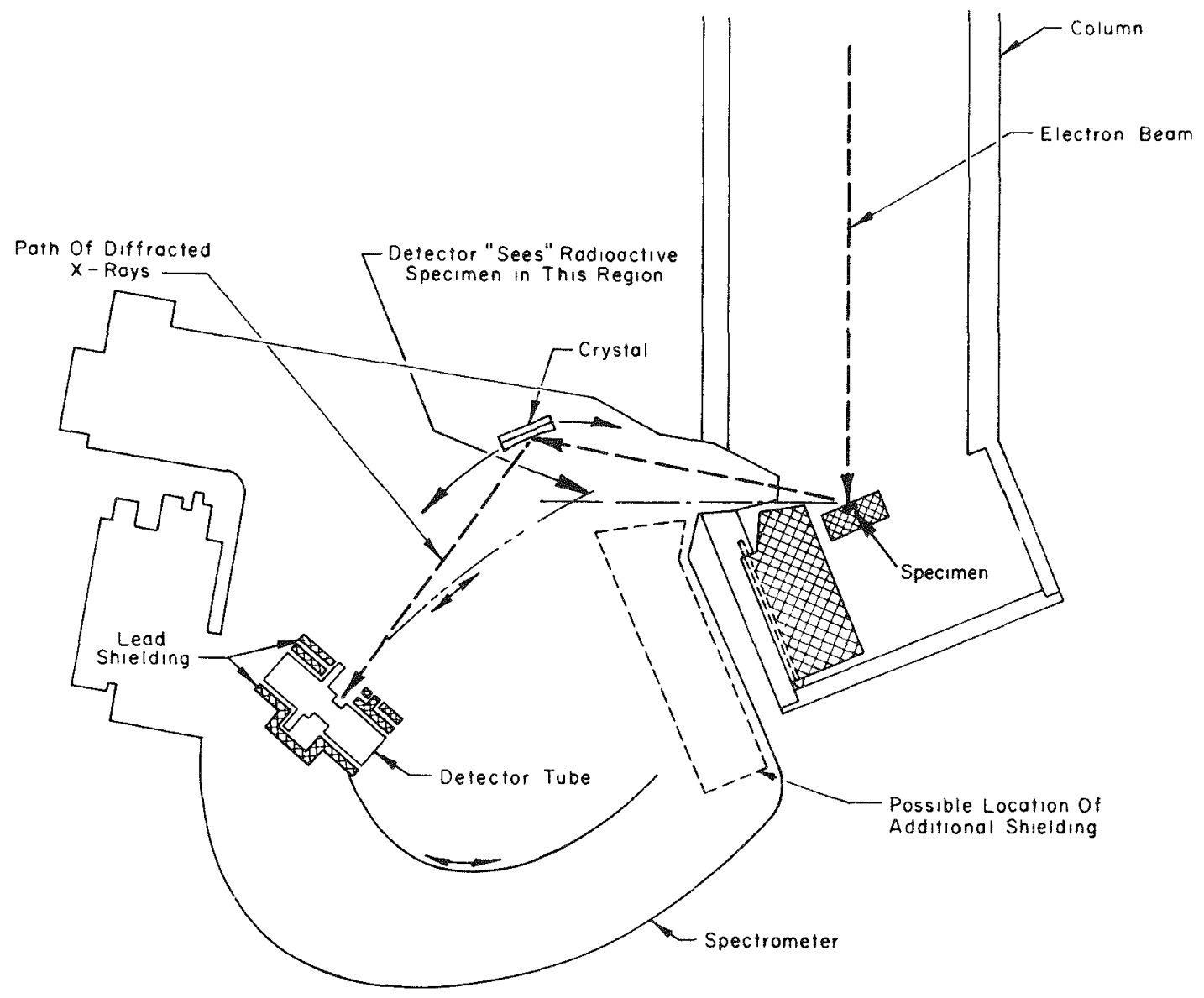

Fig. 5 Schematic diagram showing microprobe column and spectrometer, specimen-chamber shield piece, specimen mount containing lead and counter-tube shielding.

detector tube. The shield in front of the detector tube totaled $0.64 \mathrm{~cm}$ in thickness, while that behind the counter tube was one-half this thickness.

\section{Scattering from Spectrometer Crystal}

No shielding can be placed between the radioactive specimen and the spectrometer crystal without blocking the desired characteristic $\mathrm{X}$-rays. If the background radiation from the radioactive sample scatters appreciably from the crystal, this would limit severely the benefits to be gained from shielding that might be added elsewhere. In order to determine whether this type of scattering was appreciable, counts were made of the background from the radioactive specimen with the electron beam off and with the main specimen-chamber shield in place, both with the crystal in and with the crystal out. An ADP crystal was used with the spectrometer and pulseheight analyzer set for the $U$ MB, wavelength. The 100-second-long counts obtained were so similar in magnitude that it would have taken a greatly extended counting period to demonstrate any real difference. This was taken as reassuring evidence that the unavoidable non-diffraction scattering from the crystal was negligible. 
D. Attempts to Determine the Spectral Distribution of the Background - - Effect of Specimen-Chamber Shielding

An attempt was made to determine approximately the spectrum of the background from the radioactive specimen which appeared in the output of the detector tube [b]. This was accomplished by setting the amplifier gain at 960 and adjusting the high voltage to the detector tube (a flow proportional counter) to give a maximum output [c] for the $\mathrm{K}_{\alpha}$ line of titanium $a=2.75 \AA$ ). After this adjustment, the flow proportional counter high-voltage was held constant.

Since the voltage pulse from the flow proportional counter is inversely proportional to the $X-r$ ay wavelength which it is receiving, the following equation applies:

$$
\lambda=\frac{2 \cdot 75}{960} \times \text { Gain }
$$

where

$$
\begin{aligned}
\lambda & =\mathrm{X}-\text { ray wavelength in Angstroms } \\
\text { Gain } & =\text { gain setting of amplifier }
\end{aligned}
$$

Thus, using the equation, it is seen that a gain of 900 corresponds to a wavelength of $2.58 \AA$, a gain of 400 corresponds to a wavelength of $1.15 \AA$, etc.

With the spectrometer set for $\mathrm{Ti} \mathrm{K}_{x}(2 \theta=29.95 \mathrm{deg})$ and the electron beam off, counts were made of the background reading for various selected values of the gain setting between 1 and 960. This was done with no added shielding, and repeated with the specimen-chamber shield in place. The results indicated a relatively smooth and continuous background with no pronounced peaks and valleys. This background was level between amplifier gains of 400 to 900 and dropped uniformly as the gain was decreased below 400. In order to check on the possibility that peaks in the background may have occurred at gain settings intermediate to those actually used, the gain was manually varied in a slow and continuous fashion over the entire range while the ratemeter output was observed. This procedure did not reveal any peaks and provided additional verification of the results just described.

[b] The radiation detector and indicator system used consisted of the following: a flow proportional counter with its output going to a preamplifier, thence to an amplifier where the gain was variable, then to a pulse height analyzer, and finally either to a ratemeter and chart recorder, or to a scaler.

[c] Pulse-height analysis was used with the baseline set at 10 volts and a window width of 20 volts for all the measurements described in this report. 
These results are sufficient to give a measure of the shield effectiveness at this particular $2 \theta[d]$ setting of the spectrometer. The percentage reduction in the background produced by the shield under these conditions is around 50 percent and does not vary with the gain setting of the amplifier, i.e., with the wavelength chosen by the amplifier gain and pulse-height analyzer settings,

\section{E. Effectiveness of Various Combinations of Shielding as a Function of $2 \theta$ Setting of Spectrometer}

The fact that the radioactive background was constant over a wide range of gain settings gave confidence that the effectiveness of the shielding over the $2 \theta$ range of the spectrometer could be determined by keeping the amplifier gain constant and varying only the $2 \theta$ setting, thus saving considerable experimental effort.

Four series of counts of the radioactive specimen were made over a range of $2 \theta$ settings with the flow proportional counter high voltage and the pulse-height analyzer arbitrarily held constant for $\mathrm{Ti} \mathrm{K}_{x}$ and with:

(1) Only the front counter-tube shield $(0.64 \mathrm{~cm}$ of $\mathrm{Pb}$ sheet placed on the side of counter tube facing the radioactive specimen and the entrance window left uncovered) in place

(2) Only the large specimen-chamber shield in place

(3) Both the front counter-tube and specimen-chamber shields in place simultaneously

(4) No shields at all in place to give a basis for comparison of shielding effectiveness.

With the front counter-tube shield in place, the lower range of the spectrometer was limited, with the lowest reading being made at 34.7 deg $2 \theta$. Figure 6 shows that the counter-tube shield was most effective at the low end of the $2 \theta$ scale (where the specimen-chamber shield had the least effect) and that its effectiveness decreased with increasing $2 \theta$ angle. This behavior is just the opposite of that of the specimen-chamber shield; hence, the two shields complement each other in a desirable fashion. Figure 6 also shows the combined effect with both shields in place. The increasing effectiveness of the specimen-chamber shield at higher Bragg angles is explained by the fact that the counter tube is in the "shadow" of the shield for $2 \theta$ greater than about $48 \mathrm{deg}$, and "sees" the radioactive specimen at lower angles (Figure 5).

In order to further verify the validity of the above procedure of using detection system conditions for the arbitrarily chosen $\mathrm{Ti} \mathrm{K} \alpha$ wavelength regardless of the $2 \theta$ setting, a limited number of similar measurements of shielding effectiveness were made, this time with all conditions (2 $\theta$ setting and amplifier gain and pulse-height analyzer setting) corresponding exactly to the $\mathrm{Ka} X-\mathrm{ray}$ line of each of four elements. The choice of these four elements (Mn, Ti, Si, and $\mathrm{Al}$ ) resulted in spectrometer settings well spaced over

[d] $\theta$ is the Bragg angle of the diffracted $X-r$ ays . 
$2 \theta$ Angles

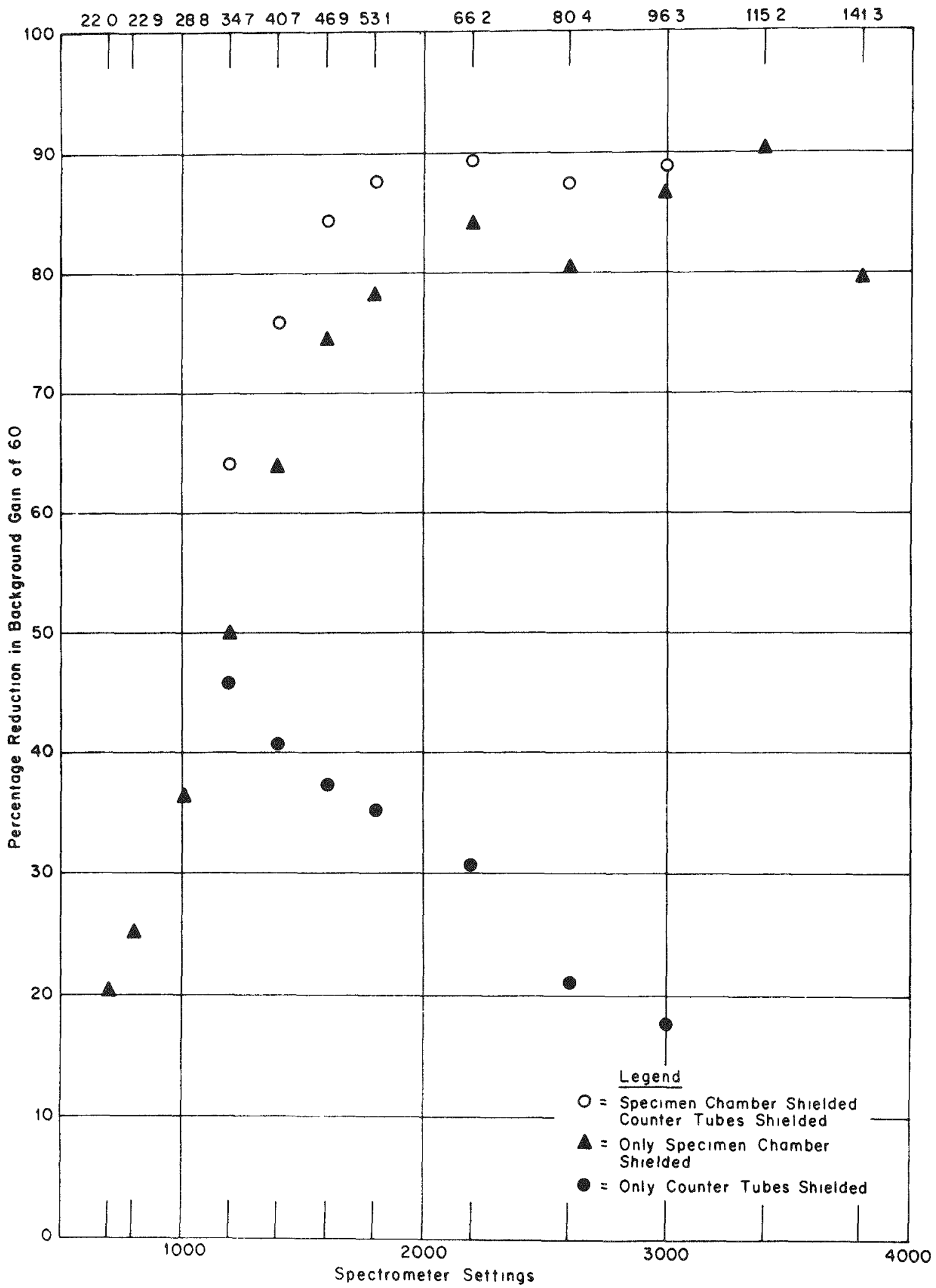

Fig. 6 Percent reduction in background at various spectrometer settings due to specimen-chamber shield alone, counter-tube shield alone, and to combined effects of both shields. Amplifier gain and pulse-height analyzer conditions held constant for $\mathrm{Ti} \mathrm{Ka}$. 
the $2 \theta$ range of the spectrometer. The results corresponded almost identically with Figure 6 and justified the procedure.

The effect of shielding behind the detector tube was determined by adopting an arbitrary set of operating conditions and measuring the counting rate due to the radioactive fuel specimen with and without a $0.32-\mathrm{cm}-$ thick sheet of lead in place behind the counter tube as illustrated schematically in Figure 5. The resulting counting rates were 180 counts per second with the sheet of lead absent and 191 counts per second with the lead sheet in place. This result is not surprising since in radiography[2] a lead sheet is placed behind the $X-r$ ay film to intensify the image. The result is similar here; the penetrating radiation which reaches the lead sheet gives rise to secondary radiation, some of which is scattered back into the counter tube. The conclusion is obvious that all shielding should be between the detector tube and the radioactive source, and no shielding should be placed behind the detector tube.

\section{F. Test for X-Ray Fluorescence of Elements in Sample}

Attention was next turned to answering the question of whether the background radiation caused elements present in the sample to fluoresce.

Aluminum was chosen as the element for examination in the sample, using the technique of "counting-over-the-peak", since it could be detected with the greatest sensitivity. The shape of the Al $K_{\alpha}$ count rate versus the spectrometer setting curve (see Figure 7) was established with the electron beam of the microprobe turned on. Then, with the electron beam off and the spectrometer "looking" at the aluminum matrix between the fuel particles in the core of the fuel plate, a similar count-over-the-peak was made. The results, plotted in Figure 7 , gave no detectable indication of fluorescent excitation of the aluminum in the sample. While no $\mathrm{X}$-ray fluorescence was observed in this example, it may be desirable to examine each highly radioactive sample for the possibility of fluorescent excitation before making extensive examinations by microprobe.

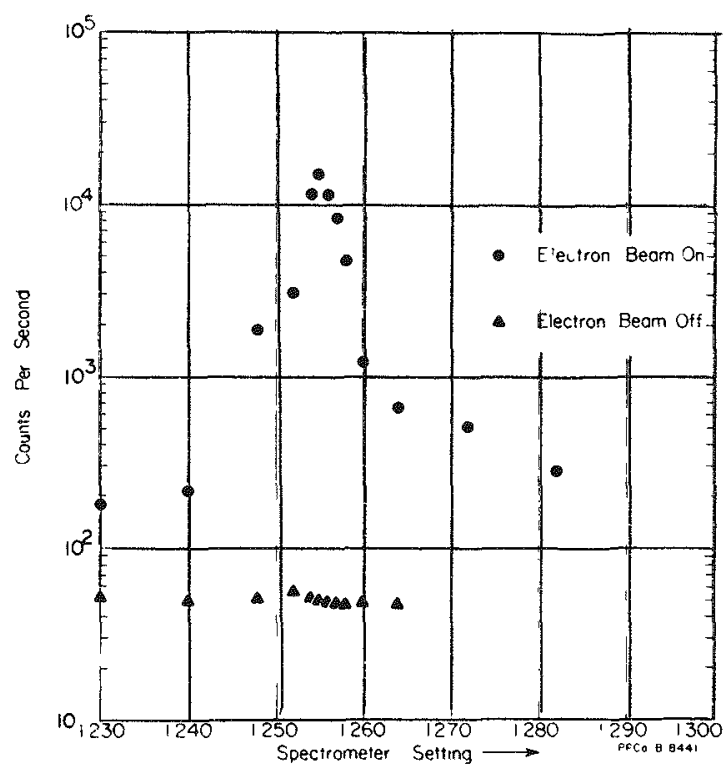

Fig. 7 Over-the-peak counting of Al Ka X-ray peak. 
III. Comparison by Microprobe of Retention of Fission Gas by Irradiated Oxide and Intermetallic Dispersion Fuels

Irradiation testing of nuclear fuel plates clad with aluminum has consistently shown that uranium aluminides have a superior ability to retain fission gases than do uranium oxides $[3,4,5]$. One explanation for this is thought to be that the stable uranium-aluminide compound, $\mathrm{UAl}_{4}$, has a structure which is deficient in uranium atoms and corresponds stoichiometrically to UA14.9[6]. This defect lattice structure could provide sites for the storage of elements produced during the fissioning of the U-235. One of these elements, of course, is xenon.

In order to experimentally compare the abilities of $\mathrm{UAl}_{4}$ and $\mathrm{U}_{3} \mathrm{O}_{8}$ nuclear fuel materials to retain fission gases (particularly xenon), sample fuel plates containing a mixture of particles of UAl4 and of $\mathrm{U}_{3} \mathrm{O}_{8}$, both dispersed in the same aluminum matrix, were prepared. The simultaneous presence of both fuels in the same fuel plate assured that each kind of fuel would experience the same thermal and radiation history, and give the best comparison of ability to retain fission gases.

These fuel plates were irradiated to a burnup of $5.5 \times 10^{20}$ fissions/ $\mathrm{cm}^{2}$, resulting in fissioning of 17.7 percent of the fissionable atoms present. A temperature of $125^{\circ} \mathrm{C}$ was maintained during the irradiation. A photomicrograph of a sample of the irradiated fuel prepared for microprobe examination is shown in Figure 8.

Fig. 8 Photomicrograph of dispersion fuel after irradiation at $125^{\circ} \mathrm{C}$ to a burnup of 5.5 $x 10^{20}$ fissions $/ \mathrm{cm}^{2}$.

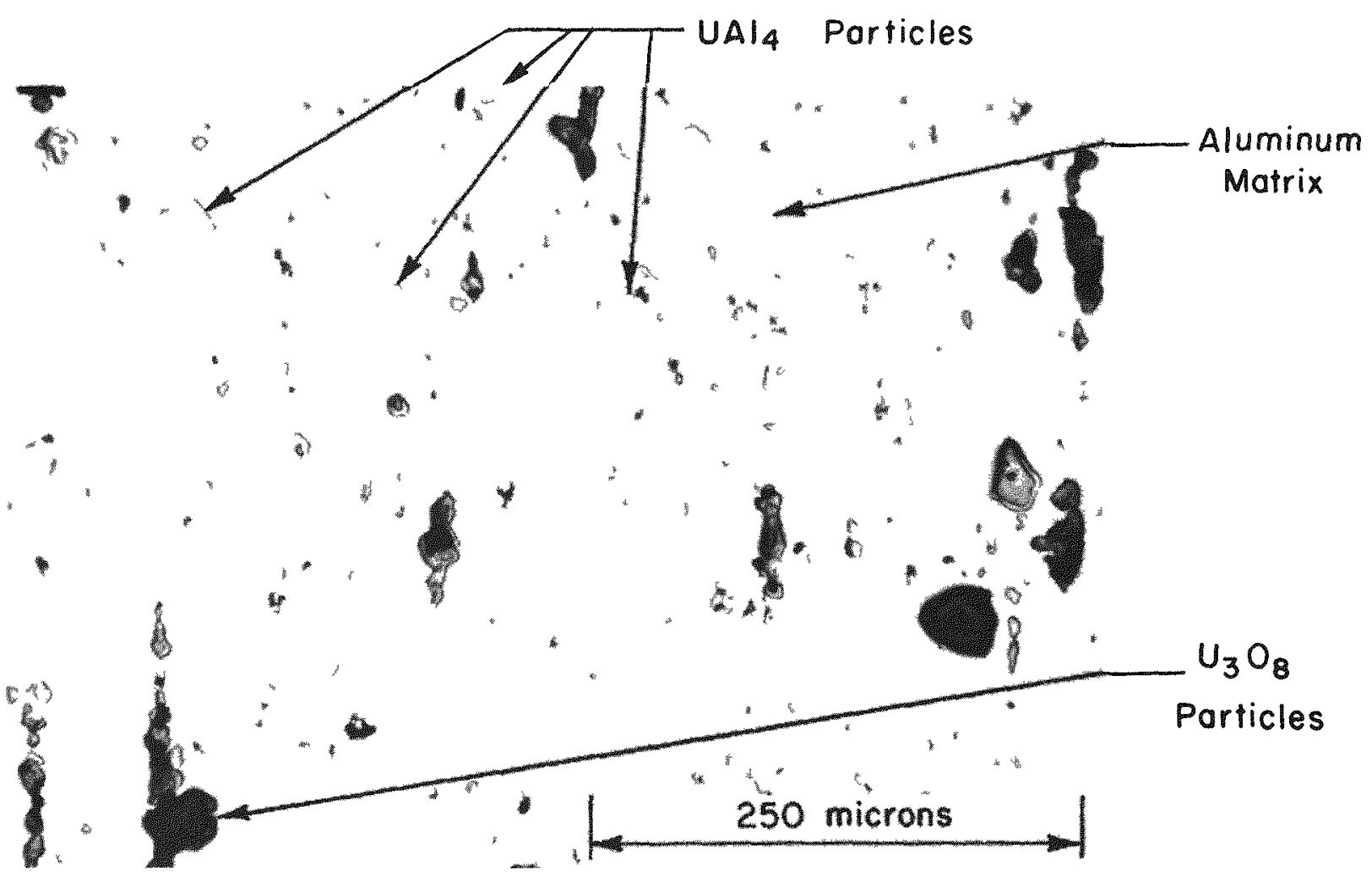


Because the irradiated fuel specimens were to be examined in an unshielded microprobe, radiation levels were minimized by using very small samples and by introducing two inches of leadshielding in the specimen chamber between the specimen and the spectrometers. Since the Xe Lo X-ray wavelength is not listed in the literature, the solid compound $\mathrm{Na}_{4} \mathrm{XeO}_{6}$ was excited in the microprobe to produce the characteristic xenon X-rays. Comparison with known wavelengths indicate that the $X e L_{1}$ wavelength is $3.010 \pm 0.005$ Angstroms.

Examination of the irradiated specimens revealed strongly detectable levels of xenon within the $\mathrm{UAl}_{4}$ fuel particles and rather weakly detectable levels of xenon in the $\mathrm{U}_{3} \mathrm{O}_{8}$ particles. Figure 9 shows an example of "overthe-peak" counts for Xe La X-rays under the same excitation conditions (20 Kilovolts and 0.115 microamps specimen current on aluminum) at fixed points on each of the two kinds of fuel particles. The markedly stronger indication of xenon in the $\mathrm{UAl}_{4}$ fuel particle is made all the more striking by the fact that the uranium density of $\mathrm{UAl}_{4}$ is $3.92 \mathrm{~g} \mathrm{U} / \mathrm{cm}^{3}$, compared to $7.04 \mathrm{~g} \mathrm{U} / \mathrm{cm}^{3}$ for $\mathrm{U}_{3} \mathrm{O}_{8}$. In addition, xenon was detectable to a distance of approximately 10 microns in the aluminum matrix surrounding the individual fuel particles. This is well within the estimated recoil range for fission fragments.

The comparison as in Figure 9 of $X-r a y$ peak intensities taken at single points on different fuel particles indicates roughly which kind of fuel particle contains the most xenon. It was felt desirable, however, to develop a method which would take a number of factors (including the relative production of xenon) into consideration and permit a more quantitative comparis on of the final retention of xenon by different kinds of fuel particles. One such factor is 'the self-shielding effect. Absorption of neutrons in the outer regions of fuel particles leaves a reduced number to reach the interior of the particle and results in a number of fissions in a given particle which is less than proportional to the size and the fuel density of the particle.

Other factors include the porosity of irradiated $\mathrm{U}_{3} \mathrm{O}_{8}$ which almost invariably contains pores and holes resulting from gas bubble formation. Also, both the $\mathrm{UAl}_{4}$ and $\mathrm{U}_{3} \mathrm{O}_{8}$ particles are much harder than the surrounding aluminum matrix and show a tendency to polish in relief, whereas a plane surface is desirable for accurate microprobe data. In addition, some effort should be made to correct for X-ray absorption effects which depend on the composition of the fuel particle.

The method adopted consisted of making simultaneous chart recordings of the $\mathrm{U}, \mathrm{Zr}$, and $\mathrm{Xe} \mathrm{X}-\mathrm{r}$ ay intensities during one-dimensionsal motor-driven scans across a number of fuel particles. The $U$ trace delineates the boundary of the fuel particle. The $\mathrm{Zr}$ (except for atoms reaching the recoil zone) is assumed to stay in place in the particle and serves as an internal standard to give a measure of the number of fissions which have occurred in each particle. This will allow an automatic compensation, in the procedure to be described subsequently, for the self-shielding effect. $\mathrm{Zr}$ was chosen as the internal standard since it is the most abundant fission product and is not expected to diffuse at the low temperature of this irradiation. 


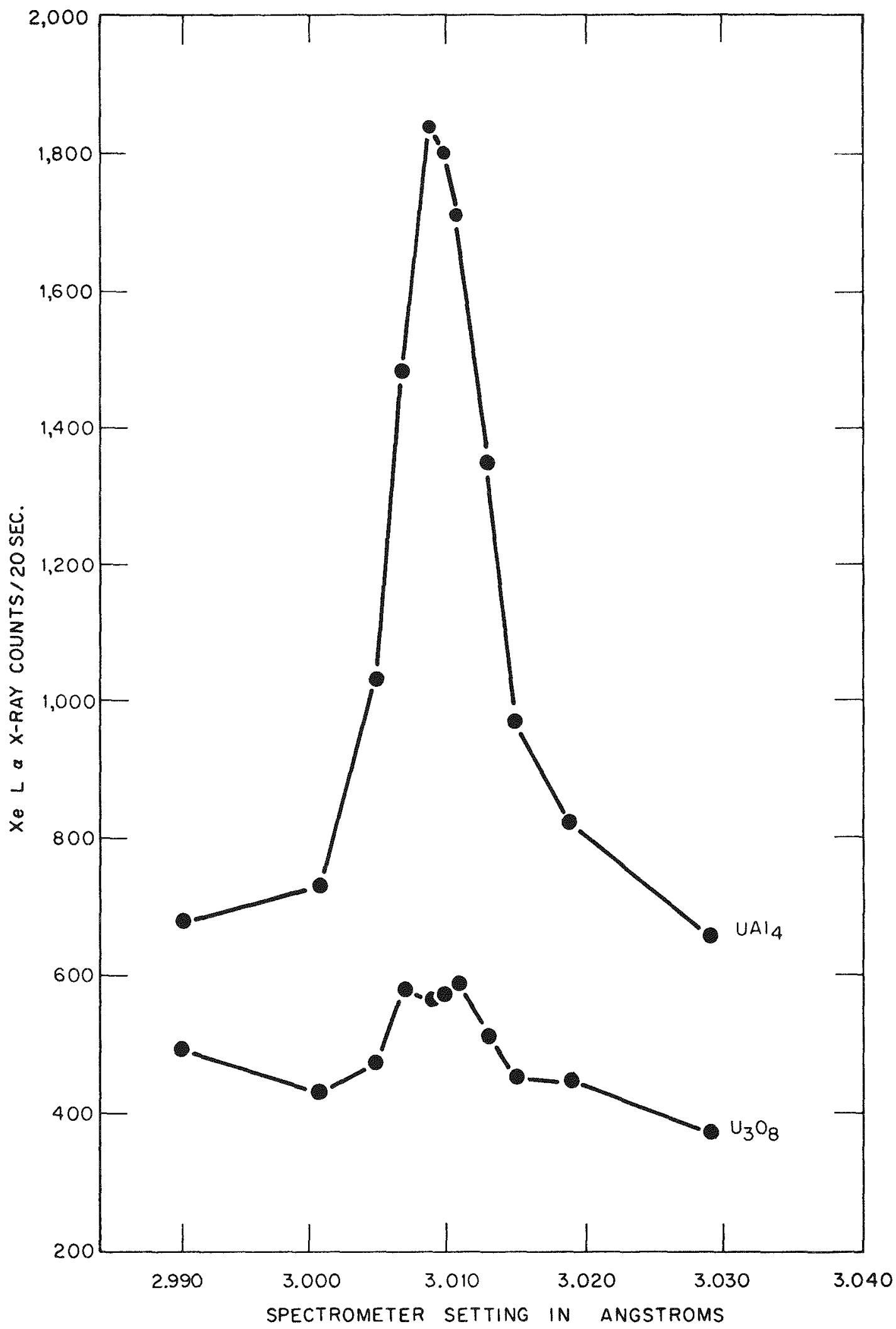

Fig. 9 Typical over-the-peak Xe $\mathrm{La} \mathrm{X}$-ray counts obtained from irradiated $\mathrm{U}_{3} \mathrm{O}_{8}$ and $\mathrm{UAl}_{4}$ fuel particles under the same excitation conditions $(20$ kilovolts and 0.115 microampers specimen current on aluminum). 
Another basic assumption is that since the $\mathrm{X}$-ray spectrometers "look at" a fuel sample from positions which are relatively close together, any curvature or irregularity (e.g., porosity) of the sample surface will affect in approximately the same way all the emergent X-rays simultaneously reaching the X-ray spectrometers and hence such effects will cancel out in making comparisons of $\mathrm{X}-\mathrm{r}$ ay intensities.

After suitable corrections for background, the areas under each set of $\mathrm{Zr}$ and $\mathrm{Xe} \mathrm{X}-\mathrm{ray}$ intensity curves from a given fuel particle are measured with a planimeter. Figure 10 illustrates the manner in which the areas under the $\mathrm{X}-\mathrm{ray}$ intensities curves are obtained.

The ratio

$$
\left(\frac{A_{X e}}{A_{Z r}}\right)
$$

where $A_{X e}=$ area under Xe X-ray intensity curve, etc.

is proportional (not equal) to the fraction of xenon retained in the particle.

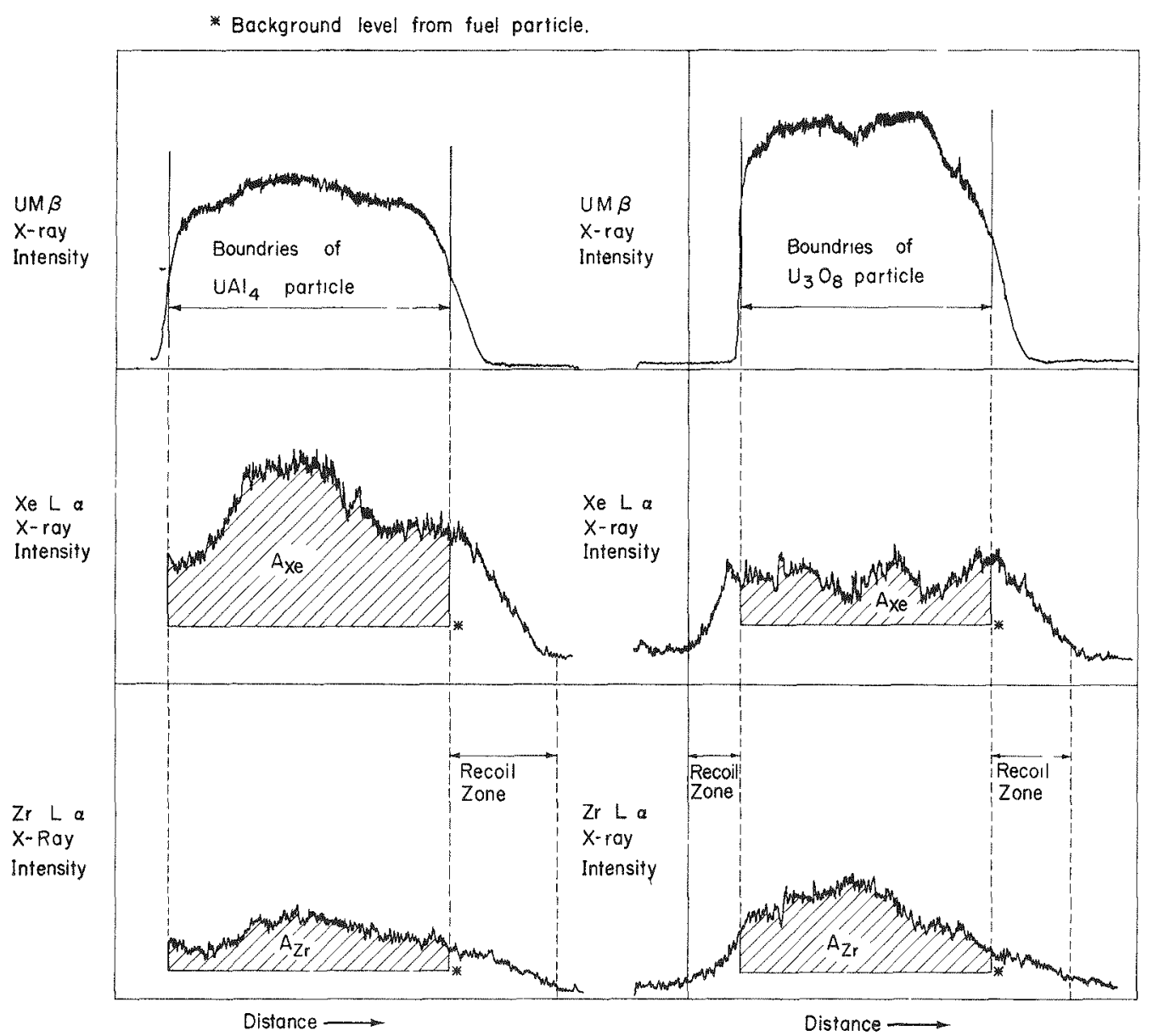

Fig. 10 Example of use of simultaneous chart recordings for $\mathrm{L}, \mathrm{Zr}$, and Xe X-ray intensities to obtain data for calculation of the proportion of xenon retained in the fuel particles. Top recordings show extent of fuel particles. Areas of cross-latched regions are measured by planimeter. 
The Xe La X-ray wavelength is absorbed slightly less by the UAl4 in comparison with the $\mathrm{U}_{3} \mathrm{O}_{8}$, while for the $\mathrm{Zr} \mathrm{La}_{\alpha} \mathrm{X}-\mathrm{ray}$ wavelength the reverse is true. By making use of Heinrich's absorption coefficients [7] and Birk's $F(X)$ value [8] it was found that, for the $20 \mathrm{kV}$ electron beam used in these experiments, unless correction was made for this difference in X-ray absorption, the number proportional to the fraction of xenon retained would be too high by a factor of 1.25 for $\mathrm{UAl}_{4}$ as compared to the $\mathrm{U}_{3} \mathrm{O}_{8}$.

Accordingly

$$
\frac{\left(\frac{A_{\text {Xe }}}{\mathrm{A}_{\mathrm{Zr}}}\right) \mathrm{UAl}_{\mathrm{X}}}{1.25\left(\frac{\mathrm{A}_{\mathrm{Xe}}}{\mathrm{A}_{\mathrm{Zr}}}\right) \mathrm{U}_{3} \mathrm{O}_{8}}
$$

where $\left({ }_{\mathrm{Xe}}^{\mathrm{A}_{\mathrm{X}}}\right) \mathrm{UAl}_{\mathrm{X}}$ is the previously described ratio of areas for the $U A 1_{X}$ $\overline{\mathrm{A}_{\mathrm{Zr}}} \mathrm{Xarticle}$, etc.

is a measure of the fraction of $\mathrm{Xe}$ retained by a $\mathrm{UAl}_{\mathrm{X}}$ particle in comparison with a given $\mathrm{U}_{3} \mathrm{O}_{8}$ fuel particle. Measurements made in this manner indicate that $\mathrm{UAl}_{4}$ retained xenon more effectively than $\mathrm{U}_{3} \mathrm{O}_{8}$ by a factor of 5.0 under irradiation at $125^{\circ} \mathrm{C}$.

\section{SUMMARY}

In summary, a number of techniques to enable the examination of irradiated and highly radioactive materials by microprobe have been described. A number of applications of these techniques for the location and identification of fission products in and around irradiated dispersion fuel particles are outlined, with special emphasis on a technique for the semi-quantitative comparison of the proportion of the fission gas xenon, retained by $\mathrm{UAl}_{4}$ and $\mathrm{U}_{3} \mathrm{O}_{8}$ dispersion fuel particles. It was found that $\mathrm{UAl}_{4}$ retained xenon five times as effectively as $\mathrm{U}_{3} \mathrm{O}_{8}$ under irradiation at $125^{\circ} \mathrm{C}$. It is believed that this technique could make a contribution in the study of fission gas (or other fission product) retention and migration in other fuel systems.

\section{ACKNOWLEDGMENTS}

The authors would like to thank Dr. J. M. Beeston and Mr. W. C. Francis for many helpful discussions in connection with this work. 


\section{REFERENCES}

(1) W. F. Zelezny and R. A. Moen, "Microprobe Investigation of Irradiated and Unirradiated Fuel Materials" in IDO-17104 Nuclear Technology Branches Quarterly Report, January 1 - March 31, 1965 (December, 1965) pp. 18-27.

(2) W. T. Sproull, X-Rays in Practice, McGraw-Hill Book Company, Inc., New York, 1946, pp. 181-182.

(3) M. J. Graber, G. W. Gibson, V. A. Walker, W. C. Francis, Results of ATR Sample Fuel Plate Irradiation Experiment, U.S.A.E.C. Report, IDO16958 (March, 1964).

(4) V. A. Walker, M. J. Graber, G. W. Gibson, ATR Fuel Materials Development Irradiation Results -- Part II, U.S.A.E.C. Report, IDO-17157 (June, 1966).

(5) G. W. Gibson, The Development of Uranium Aluminide Compounds for Use as Nuclear Reactor Fuels, U.S.A.E.C. Report, IN-1133 (December, 1967).

(6) B. S. Borie, "Crystal Structure of $\mathrm{UAl}_{4}$ ", Journal of Metals, Volume 3, September, 1951, p. 800 .

(7) Kurt F. J. Heinrich, "X-Ray Absorption Uncertainty" in The Electron Microprobe, John Wiley \& Sons, Inc., New York, 1966.

(8) L. S. Birks, "Electron Probe Microanalysis", Interscience Publishers, 1963. 
SOME APPLICATIONS OF NEUTRON RADIOGRAPHY IN IRRADIATION TESTING TECHNOLOGY *

by

\author{
J. W. Ray
}

BATTELLE MEMORIAL INSTITUTE

Columbus Laboratories

The ability of neutron radiography to provide high quality radiographs of highly radioactive specimens has led to its use in irradiation testing technology at several installations. It has been used routinely in conjunction with irradiation programs at the Battelle Research Reactor (BRR) for about four years. The principal applications of neutron radiography have been to determine fuel specimen dimensional changes periodically during irradiation and to examine experimental assemblies for determining integrity of key components.

\title{
Neutron Radiogxaphy Facilities
}

Neutron radiography equipment at the BRR includes two underwater facilities for radiography of radioactive specimens and a beamtube facility for radiography of non-radioactive specimens. One underwater facility consists of a parallel-wall collimator 1-1/2-in. square $x$ 144-in. 1ong with special hardware at the beam exit which permits specimen/detector foil combinations to be driven through the 1-1/2-in. square neutron beam. In this way the facility can be used to obtain radiographs of specimens up to $20 \mathrm{ft}$. in length.

The other underwater facility consists of a "conical" collimator with an entrance aperture 1-in. square, a length of 144-in., and a $14 \times 17$ in. beam exit area. A large be11-jar arrangement is mounted at the beam exit so that specimens $c$ an be mounted in a waterfree chamber during radiography. Thermal neutron shutters are located within the collimator to permit both fast and themal neutron radiography with this facility.

* Work performed under AEC Contract W-7405-eng-92. 
One of the more important applications of neutron radiography involves determination of the dimensional changes in nuclear fuel specimens during irradiation. This generally involves taking radiographs of an experiment periodically during irradiation. The radiographs are then examined to learn the condition of the fuel specimens, and dimensional measurements are made from the radiograph negative. Figure 1 shows a composite of neutron radiographs of an irradiation capsule taken at various times during irradiation. In this composite it is seen that the top specimen began to deteriorate fairly early in the irradiation as manifested by balloon-like swelling of the fuel region as well as a substantial increase in length. The fourth specimen from the top shows little radial swelling, but still exhibits an appreciable length increase. The bottom specimen exhibits a large length increase early in the irradiation, followed by a circumferential bulging near the top of the fuel and bowing of the specimen as it is constrained from further length increase by the mounting platforms above and below it.

Dimensional determinations can be made by visual measurements directly on the radiograph negative, or by scanning with a recording optical microdensitometer. Visual measurements are made on a light-table using a specially constructed micrometer to measure directly the diameter and length of the specimen image. Microdensitometer measurements are made by scanning the radiograph negative with a recording optical densitometer and calculating the specimen dimensions from the shape and dimensions of the densitometer trace. This is a particularly accurate method for obtaining the dimensions of specimens which have maintained their original cylindrical cross-section.

Comparison of dimensions obtained from post-irradiation neutron radiographs with subsequent micrometer measurements made in the hot cell indicate that quite good accuracy is obtained from the neutron radiographs. Figure 2 shows this comparison for a recent irradiation capsule. The post-irradiation swelling data from neutron radiographs and hot cell micrometer measurements agree within a few percent, lending confidence to the curves which are based on radiography data.

For experiments which are not irradiated at the BRR we routinely do pre-irradiation and post-irradiation neutron radiography of the capsules. This provides an early measurement of specimen dimensional changes and condition. Such information is useful in evaluating specimen performance as well as providing information useful in subsequent hot-cell disassembly of the experiment. Most of this radiography is done with thermal neutrons. However, in a program for irradiation of $(U, P u) N$ fuel rods in EBR-II, the use of an epithermal neutron beam has provided better information concerning fuel pellet condition. These specimens are fueled with fully enriched uranium and plutonium and consequently are essentially black to thermal neutrons. The use of epithermal neutrons provides greater specimen penetration and hence better definition of internal cracks or porosity in the fuel 


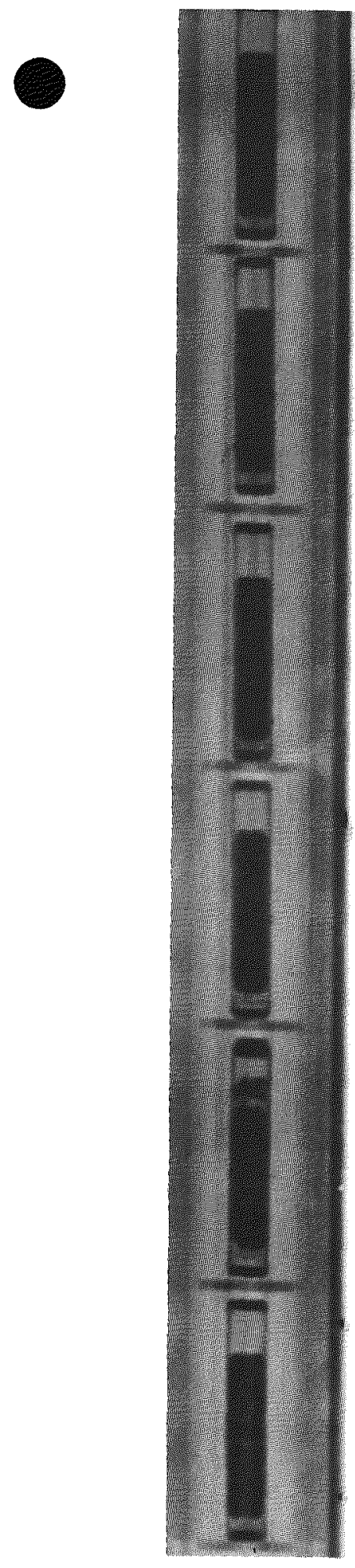

a

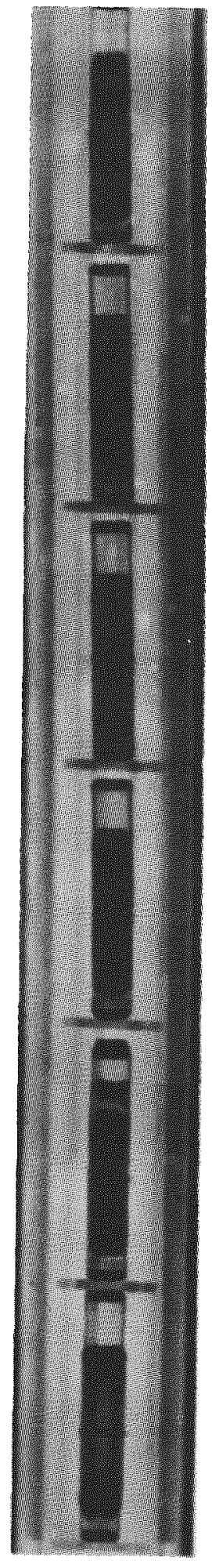

b

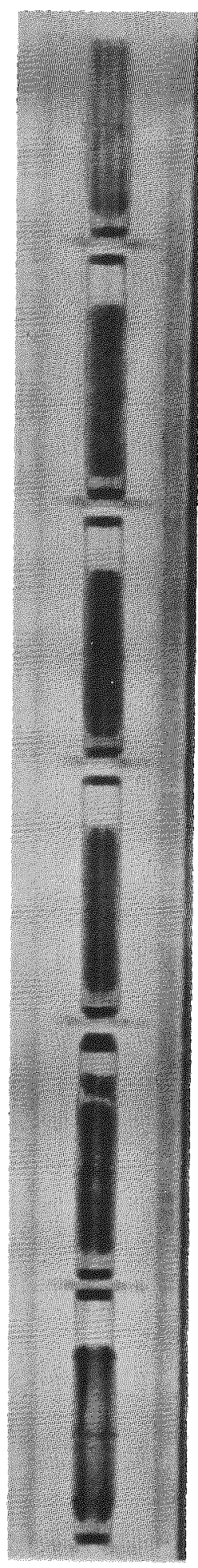

c

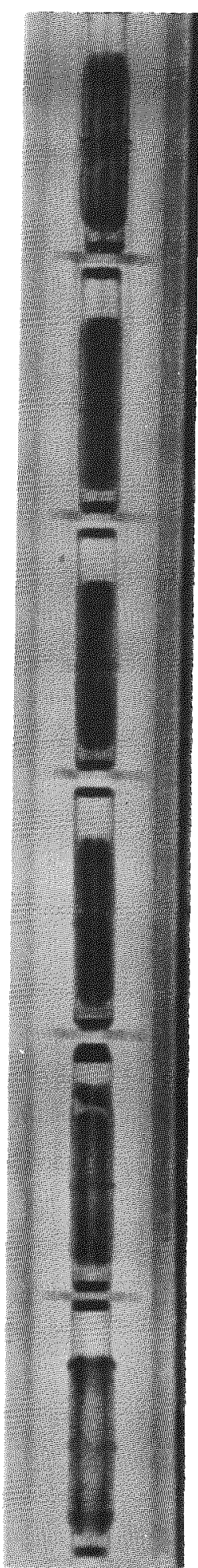

d

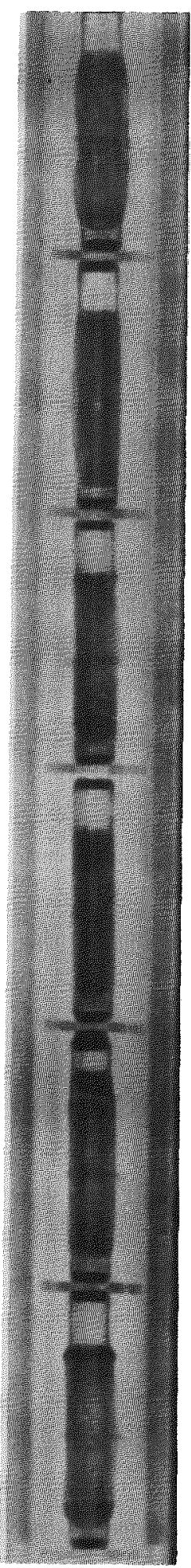

e

FIGURE 1. NEUTRON RADIOGRAPHS OF FUEL IRRADIATION CAPSULE AT SEVERAL TIMES DURING IRRADIATION 


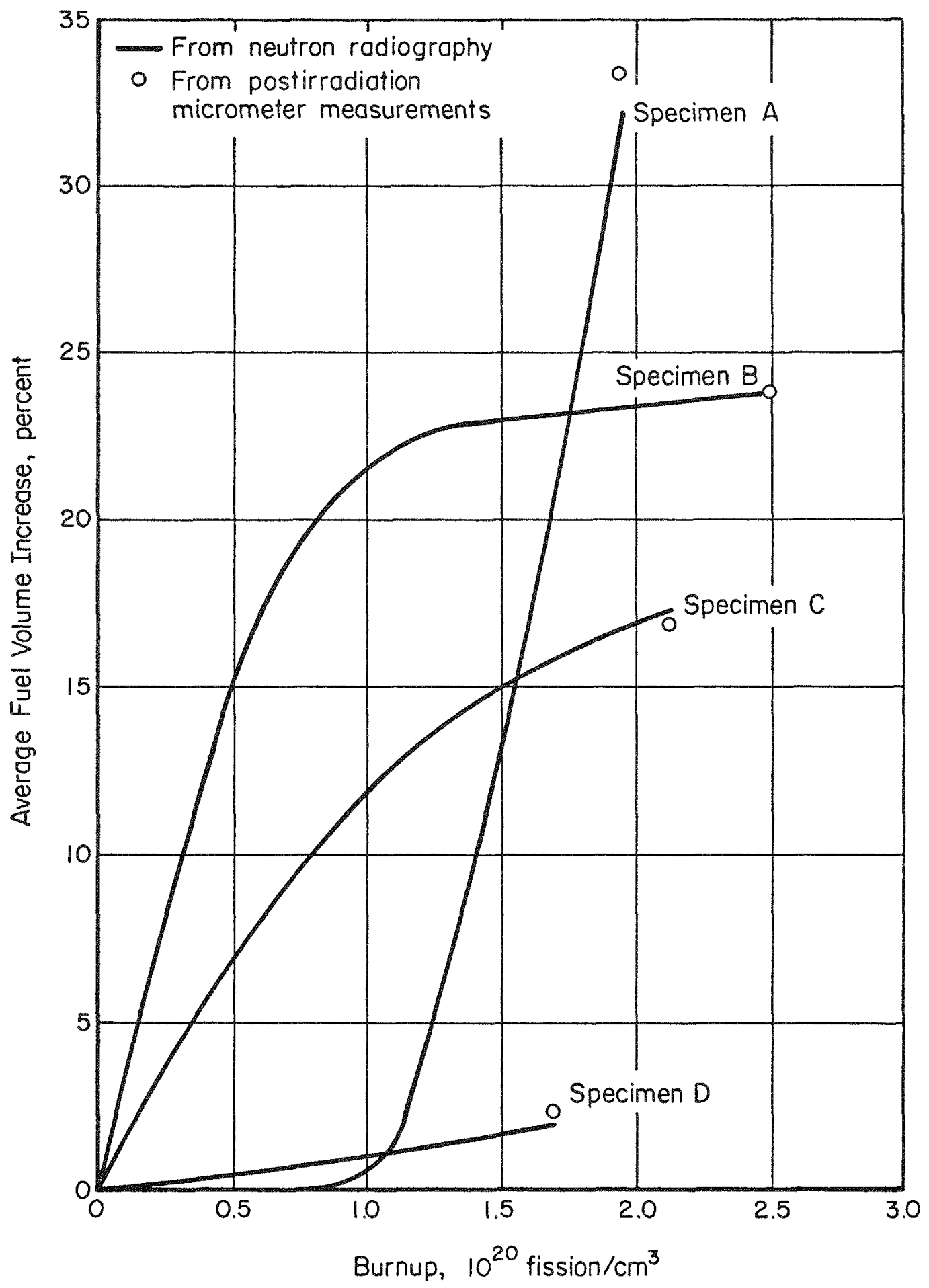

FIGURE 2. FUEL SPECIMEN SWELLING AS A FUNCTION OF BURNUP AS DETERMINED BY PERIODIC NEUTRON RADIOGRAPHY 


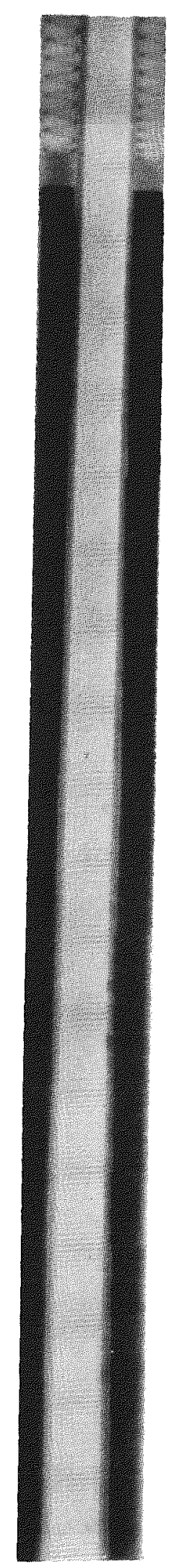

FIGURE 3. EPITHERMAL NEUTRON RADIOGRAPH OF $(U, P U) N$ FUEL RODS FOR IRRADIATION IN EBR-II 


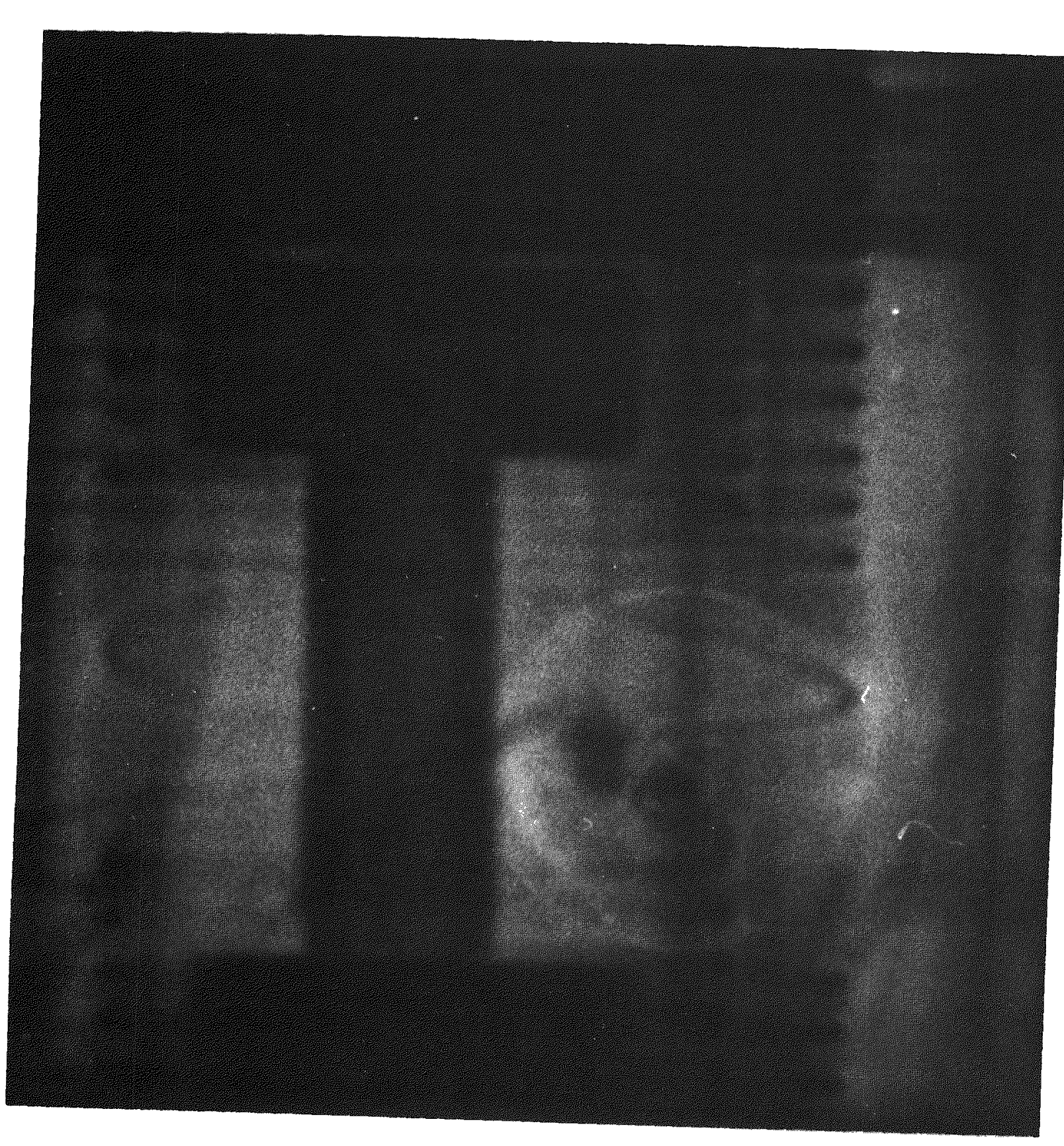
FIGURE 4. A NEUTRON RADIOGRAPH OF CREEP CAPSULE II TAKEN AT A SPECIMEN
STRESS OF 3500 PSI AND A TEMPERATURE OF $1300 \mathrm{C}$ 
pellets. Figure 3 shows an epithermal neutron radiograph of a fully enriched (U, Pu)N fuel pin prior to irradiation in EBR-II.

In another program neutron radiography is being used to obtain back-up data on dimensional changes of fuel specimens in a dynamic in-pile creep rig. It was desired to maintain the experiment at its operating temperature of approximately $1200 \mathrm{C}$ during neutron radiography to avoid potentially damaging thermal cycling of the specimen. Accordingly it was necessary to design a special collar for the experimental rig which would displace water from the vicinity of the rig while simultaneously providing a conduction path for heat rejection. Figure 4 shows a neutron radiograph of a $\mathrm{UO}_{2}$ creep specimen mounted in the irradiation creep rig and held at $1200^{\circ} \mathrm{C}$ by electrical heaters. The annular specimen is seen between the loading platens. The electrical heater wires are seen surrounding the specimen assembly. Measurements taken from radiographs like this before and after irradiation provide backup data on the total strain induced in the specimen during irradiation.

\section{Examining Experiment Integrity}

Neutron radiography is also useful in nondestructive examination of irradiation experiments prior to or during irradiation. At the BRR radiographs taken periodically during irradiation are examined for such things as specimen cladding failure, fuel redistribution, or capsule component relocation. This information is used in making decisions concerning the need or advisability of continuing the irradiation. In addition such radiographs may give early indication of incipient failure of a key capsule component.

Neutron radiography may also be useful as a diagnostic tool to help explain unusual or unexpected behavior of an experiment. For example, during an in-pile creep experiment a sudden change in indicated specimen creep rate may be due to an actual creep rate change; alternatively it may possibly be due to specimen failure or transducer difficulties. Subsequent neutron radiography of the specimen and transducer regions will show the condition of these parts and permit an evaluation of the cause of the change in indicated creep rate. This information then provides a basis for the decision to continue or terminate the experiment.

Another application of neutron radiography is as a nondestructive testing tool for examining an experimental rig prior to irradiation. It provides a valuable adjunct to $\mathrm{X}$-radiography for such things as examining braze joints, locating and evaluating of potted seals, determining of the location of certain fluids, and examining the alignment of key components in the experimental assembly. 


\section{NEUTRON RADIOGRAPHIC INSPECTION \\ OF IRRADIATED SNAP FUEL}
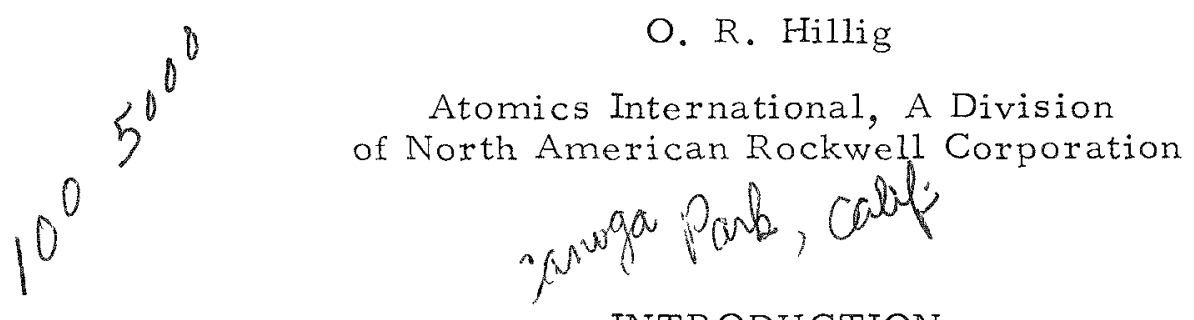

\section{INTRODUCTION}

Post-irradiation test samples of SNAP reactor fuel elements have been nondestructively examined using neutron radiography. The results of the neutron radiographic ( $N$-ray) examinations have been correlated with measurements made using standard hot cell destructive testing. Fuel swelling, cracking and decomposition were readily discernible in the first series of $N$-ray tests conducted at Atomics International (AI). Further examination of the Nrays revealed that information was available to determine hydrogen content in the uranium-zirconium hydride fuel and that the gap between the fuel and the cladding could be measured. Additional tests were performed using nonirradiated fuel samples of known hydrogen content and known gaps between fuel and cladding to establish procedures for neutron radiography of fuels and to provide standards for comparison with future tests. Hydrogen differences of $0.02 \mathrm{H} / \mathrm{Zr}$ atom ratio can be determined from the radiograph using known standards for comparison and gaps between cladding and fuel can be measured to 0.001 inch accurately.

Future AI fuel irradiation experiments will use neutron radiography as the principle tool for examinations. It is expected that enough information is available through neutron radiography that only a limited number of samples, selected by examining the radiographs, will be destructively examined. 
Three sets of SNAP fuel irradiation experiments either have been or will be neutron radiographed. The NAA 120-4 irradiation experiment has been examined. The NAA $121-1$ is being examined and two hundred and eleven SNAP 8 Developmental Reactor (S8DR) operating reactor fuel elements will be examined shortly after reactor shutdown. Both the 120-4 and 121-1 experiments are in support of the S8DR. The SNAP, S8DR type fuel element is approximately 17 inches in length by 0.53 inches in diameter. The $10 \mathrm{w} / 0$ highly enriched uranium alloyed with zirconium hydride fuel is contained within a 10 mil Hastelloy cladding and has a nominal 8 mil gap across the diameter. The $\mathrm{H}$ to $\mathrm{Zr}$ atom ratio is about 1.70. The 120-4 test samples are about 4 inches in length and the 121-1 and the S8DR fuel elements are 17 inches long. The 120 and 121 experiments were irradiated in the Hanford reactor.

\section{NEUTRON RADIOGRAPHY}

Neutron radiography, while being similar and complementary to $x$-radiography in many areas, has unique features which makes it particularly attractive to the examination of reactor fuel. Some of these features are: (1) the ability to distinguish between isotopes of an element such as U-235 from U-238, (2) the ability to radiograph irradiated fuel, and in the case of U-ZrH fuel, (3) the ability to distinguish and determine hydrogen content.

Figure 1 illustrates a comparison between $\mathrm{X}$-ray and $\mathrm{N}$-ray of the mass attenuation coefficients versus atomic number. One can see that elements of similar atomic mass which have essentially the same mass attenuation coefficient for X-ray or isotopes such as U-238 and U-235 quite often have good separation in thermal neutron mass attenuation coefficients. It also illustrates the neutron radiography advantage for some of the light elements that have high scattering coefficients (example hydrogen) and the rare earth elements that have high absorption coefficients.

For the work described in this paper the photographic method using standard $x$-ray film was used. The image definition available in neutron radiography is dependent on many factors. Some of these are: (1) neutron optics or resolution of the beam, (2) object to converter distance, (3) scattering within the object, 
(4) type of converter, (5) contact between converter and film, and (6) film emulsion type. We use a small aperture and separation between the aperture and object to provide good optics. Resolution $(R)$ is defined as $(L)$ the distance from aperture to object divided by (D) the aperture diameter. For objects such as the SNAP fuel elements a resolution of $\sim 300$ is required to resolve 0.001 inch since the object to converter distance is 0.300 inch. The U-ZrH fuel has considerable neutron scattering so some image definition is lost within the fuel. Thin ( 0.001 to 0.005 inch) metal converters were generally used. Dysprosium or Indium foils were used for irradiated fuel examination where the image was transferred from converter to film after neutron exposure. Gadolinium was used for the examination of unirradiated fuel where the film was sandwiched

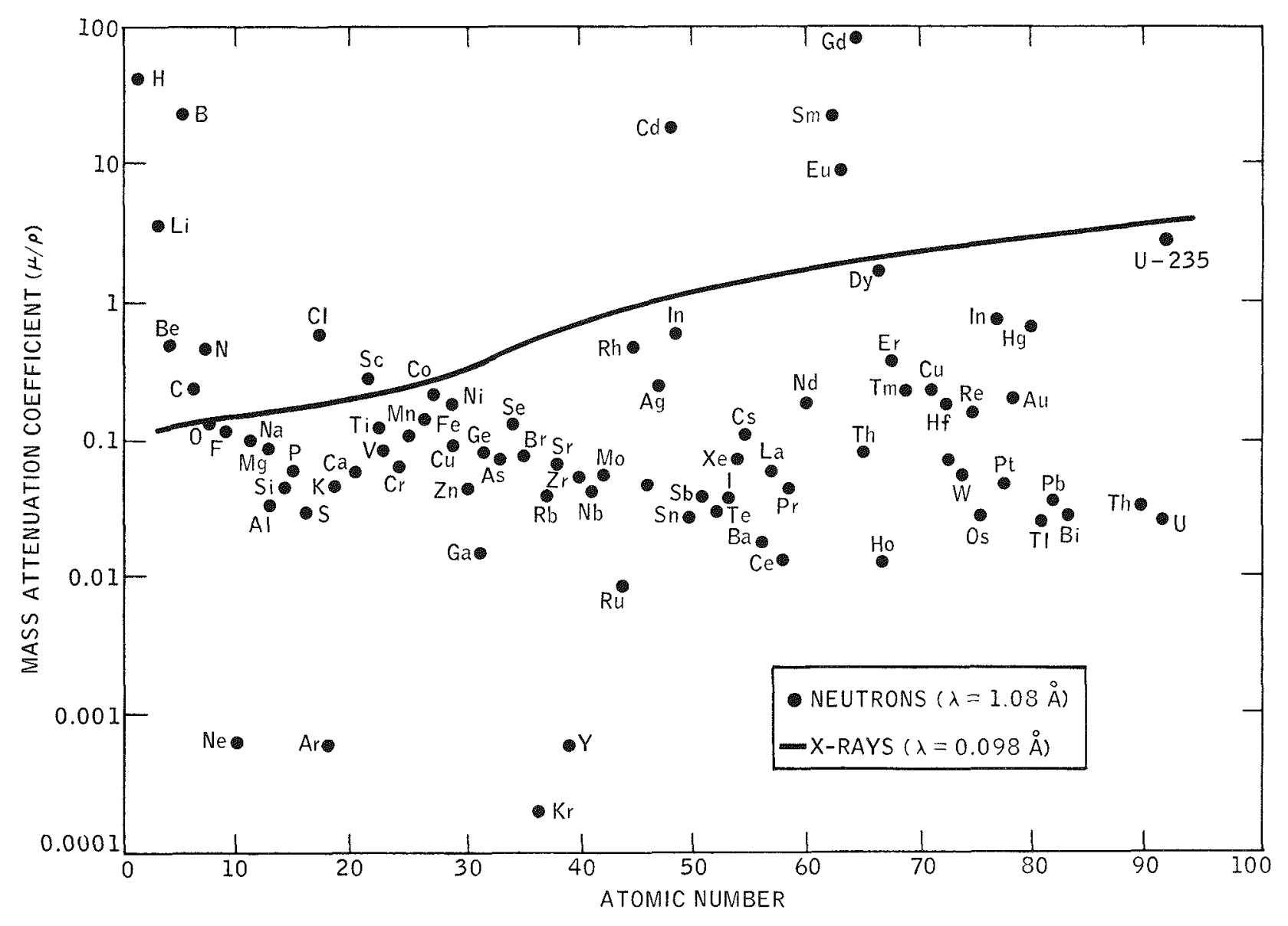

Figure 1. Mass Attenuation Coefficient vs Atomic Number 
with the converter to directly (during neutron exposure) transfer the image. Vacuum cassettes are used to provide intimate contact between the converter and film. Different speed films were used to answex different requirements: slow film for fuel to cladding gap detail and fast film to provide denser images in the difficult to penetrate areas for determining hydrogen content.

\section{NEUTRON RADIOGRAPHY FACILITY AND FUEL HANDLING}

The Shield Test and Irradiation Reactor (STIR), Figure 2, is used for neutron radiography. The 1 Megawatt pool type reactor provides the neutron source. Generally, thermal energy neutrons are used for radiography although some work has been done using neutrons of epithermal energy. In the former case a graphite thermal column is used to moderate the neutrons to the desired energy while for the epithermal neutron radiography a beam hole is provided in the graphite column to extract neutrons of higher energy directly from the core. The facility, due to the large test vault and the relatively intense neutron beam $\left(\sim 5 \times 10^{10} \mathrm{n} / \mathrm{cm}^{2}-\mathrm{sec}\right)$ at the aperture, allows for radiography of large objects with good resolution.

The STIR facility, not originally designed for neutron radiography, is not a part of the Atomics International Hot Laboratory. Therefore, neutron radiography of irradiated fuel requires transfer of fuel specimens from the hot cell to the reactor facility. To overcome this problem a reactor $(\mathrm{L}-88$ ) has been designed specifically for neutron radiography and for use in a hot cell facility. Presently special remote handling equipment and techniques are used to radiograph fuel. Figure 3 shows the fixture, fuel handling cask and converter cassette as used in this work. The elements are loaded at the hot cell into the four aluminum tube fixture and capped using O-ring seals to provide leak tightness. The fixture is raised out of the cask at the reactor facility and placed on a geared, motorized assembly for turning the elements for different views. Contact between the fixture and converter cassette is maintained to optimize image definition. After neutron exposure, the specimens are returned to the cask so the converter image detector can be changed or for return to the hot cell. The examination of the 211 S8DR elements will utjlize a 12 tube fixture and a means of remotely changing the image converters. 


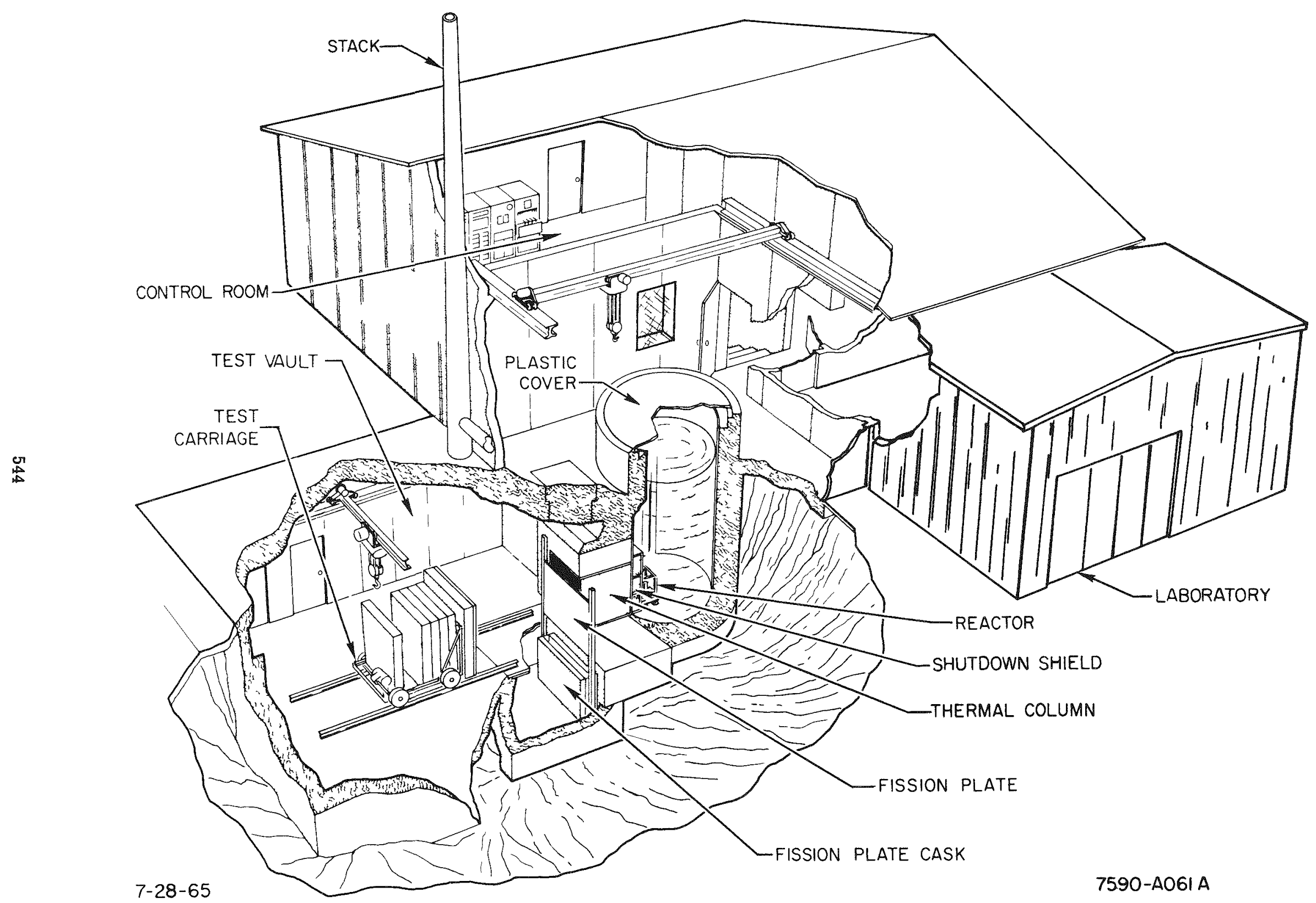

Figure 2. Shield Test and Irradiation Reactor 


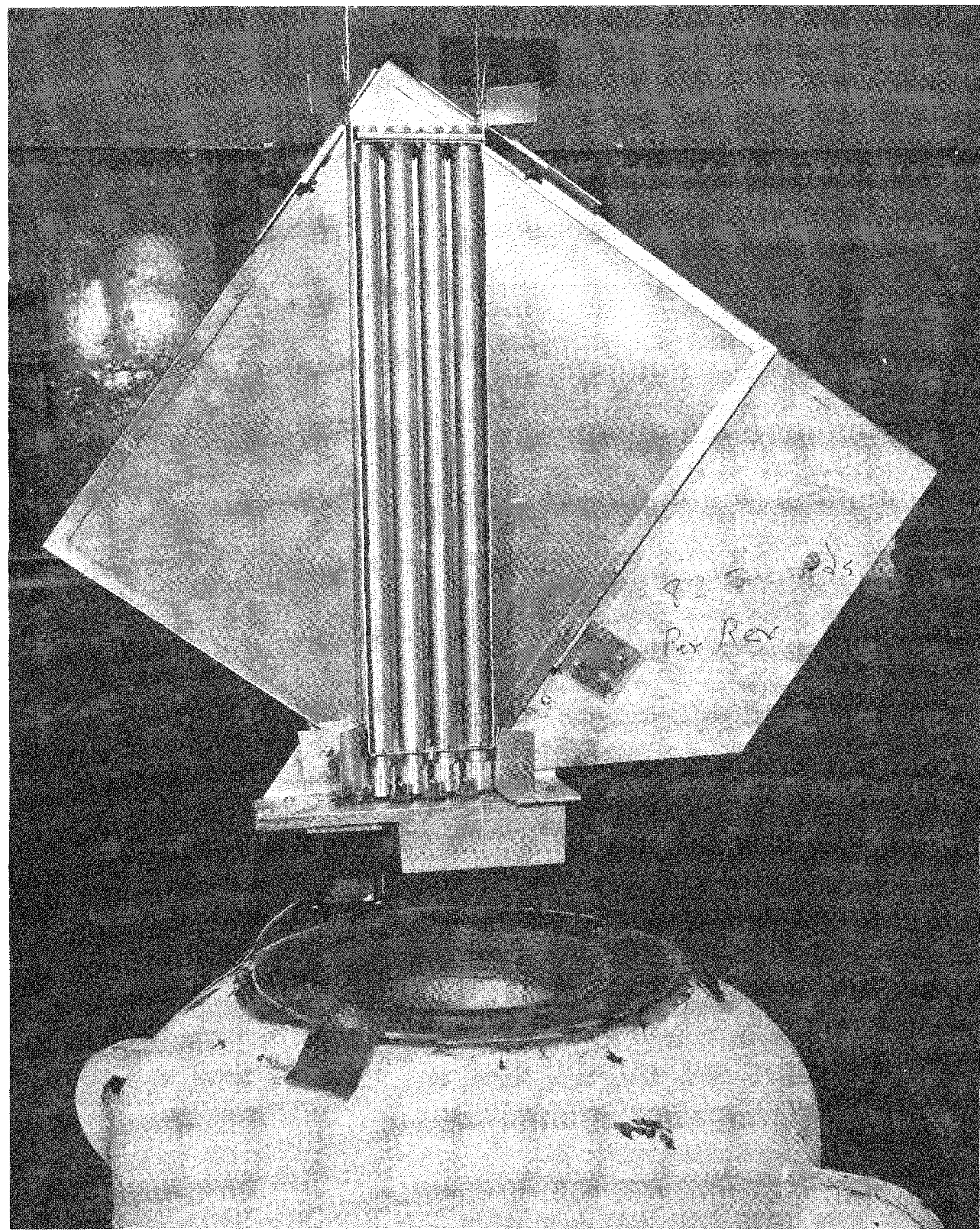

Figure 3. Fuel Handling Fixture for Neutron Radiography 
NEUTRON RADIOGRAPHY RESULTS *

The NAA 120 irradiation experiment was designed to provide a dissipation of SNAP fuel material irradiation stability as a function of burnup and operating temperatures. The objectives were to correlate the effects of irradiation temperatures, burnup, and selected compositional variables on fuel swelling behavior, and to evaluate the effects of phase transformation (due to hydrogen losses during irradiation) on the performance of the fuel material. Figure 4 shows radiographs of fuel materials before and after irradiation at Hanford. Although neutron radiography was initially intended as a replacement for pin hole auto radiography to assist in the destructive testing of the fuel samples, it proved to be of far greater value. The radiographic negatives, which are of considerably higher quality than Figure 4, revealed information on swelling, cracking and disintegration. Measurements of swelling made on the negatives agreed with later actual measurements of the fuel samples. The figure shows disintegration of the fuel at the sample ends and cracking in some samples. Optical densitometer measurements were made of the N-ray negatives. Regions of low hydrogen appear more dense in the negative, less dense on the positive (Figure 4). These density measurements correlated very well with measurements of the hydrogen content. The $\mathrm{H}$ to $\mathrm{Zx}$ atom ratio in fuel before irradiation is $\sim 1.70$. The densitometer measurement agreed to within $0.02 \mathrm{in}$. $\mathrm{H} / \mathrm{Zr}_{\mathrm{r}}$ with hydrogen measurements.

The results of the 120 series prompted further studies to improve the Nray technique for post-irradiation fuel examination. Indium converter foil ( 0.020 inch thick) had been used. Better image definition is available with thin metal converters such as gadolinium for the direct transfer method (cannot be used with irradiated fuel) and dysprosium for the indirect transfer method. Studies have been made with these and dysprosium (0.005 inch thick) will be used for future studies.

*Some of the results of these tests are classified. Only the use of neutron radiography and interpretation of the radiographs will be discussed. 

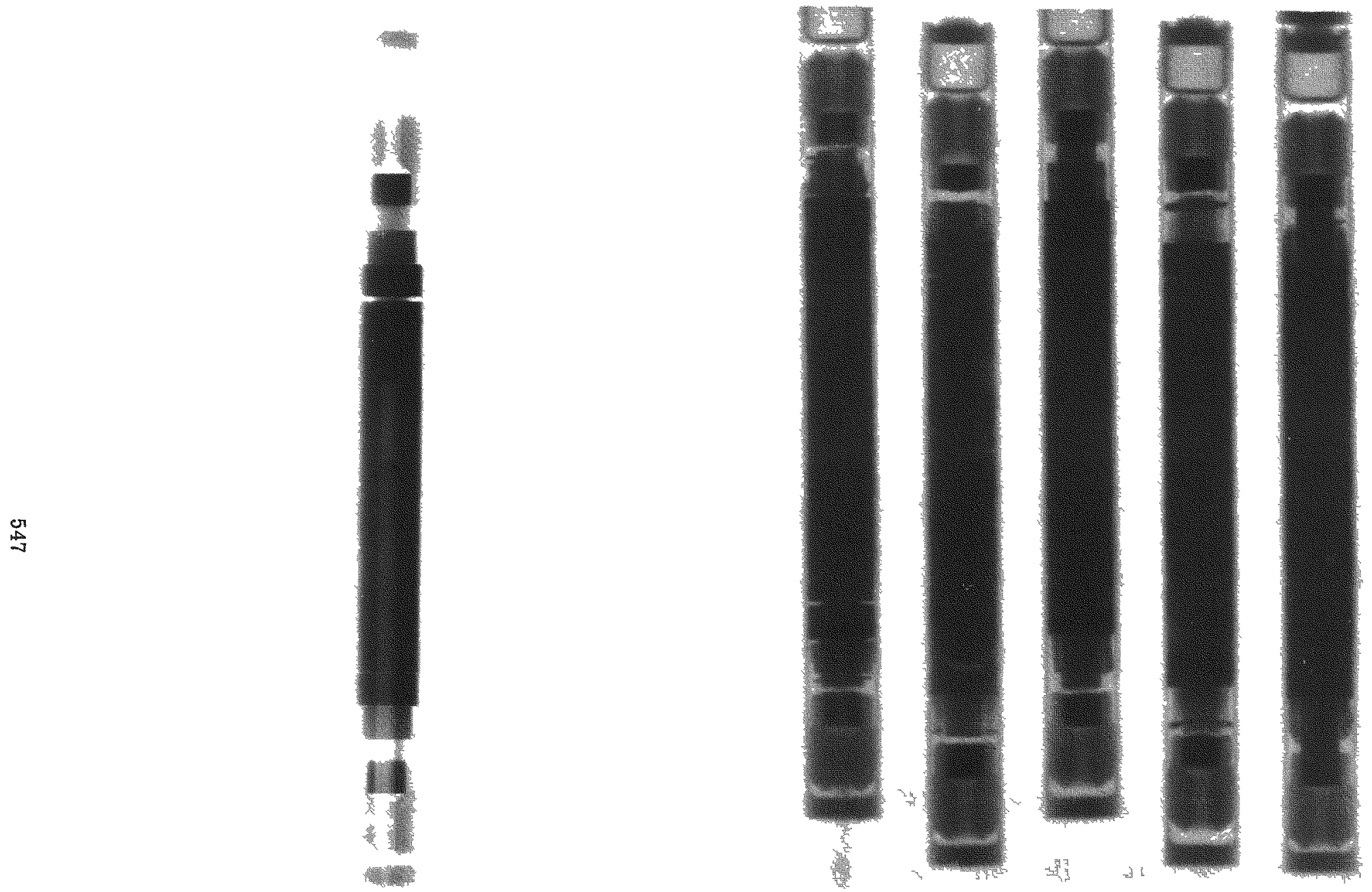

Figure 4. NAA-120-4 SNAP Fuel Irradiation Samples 
Two fuel elements were made with fuels of known $H / Z_{r}$ atom ratios. Each element contains fuel samples ranging in $\mathrm{H} / \mathrm{Z}_{\mathrm{r}}$ from 1.415 to 1.795. These standard elements will be radiographed with each batch of irradiated fuel studied and comparative densitometer measurements of the $N$-ray negatives will be used to determine hydrogen content. Figure 5 shows a densitometer trace of one standard and as can be seen, the results correlate with the $\mathrm{H} / \mathrm{Z}_{\mathrm{r}}$ atom ratios listed.

In addition to having known $\mathrm{H} / \mathrm{Z}_{\mathrm{r}}$ ratios, the standard fuel elements have known gaps between the fuel and cladding. Examination of the N-rays show that the gaps can be read to about \pm 0.001 inch accuracy. The gap measurements were made in two ways. They are: (1) an enlargement (4x) was made and a comparator magnifying glass was used to measure the gap, see Figure 6 , and (2) a densitometer with a 10 micron slit was used to traverse the gap at slow speed, see Figure 5. Both the traverse of Figure 5 and the enlargement of Figure 6 can be interpreted with good results, \pm 0.001 inch.

Future planned studies are: NAA 121 irradiation experiments in support of the S8DR test and examination of the 211 fuel elements from S8DR. Neutron radiography of the 121 series is now in progress. In each study full length, two view $\left(0^{\circ}\right.$ and $\left.90^{\circ}\right) \mathrm{N}$-rays will be made. It is expected that enough information (fuel swelling axially and radially, cladding condition, hydrogen content and gap) will be available so that only a small fraction of the elements will be destructively examined. 


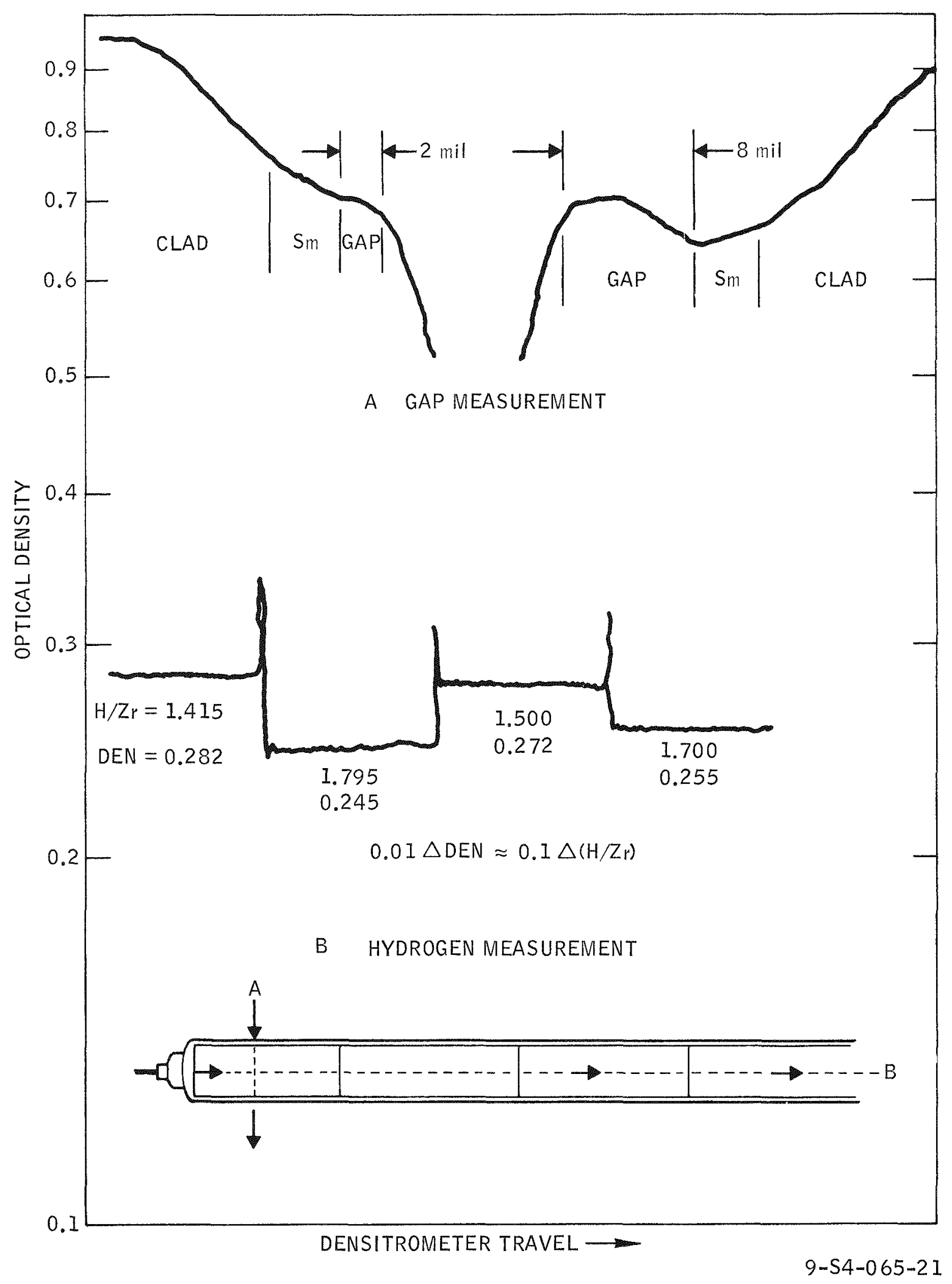

Figure 5. Results of Densitrometer Measurements 

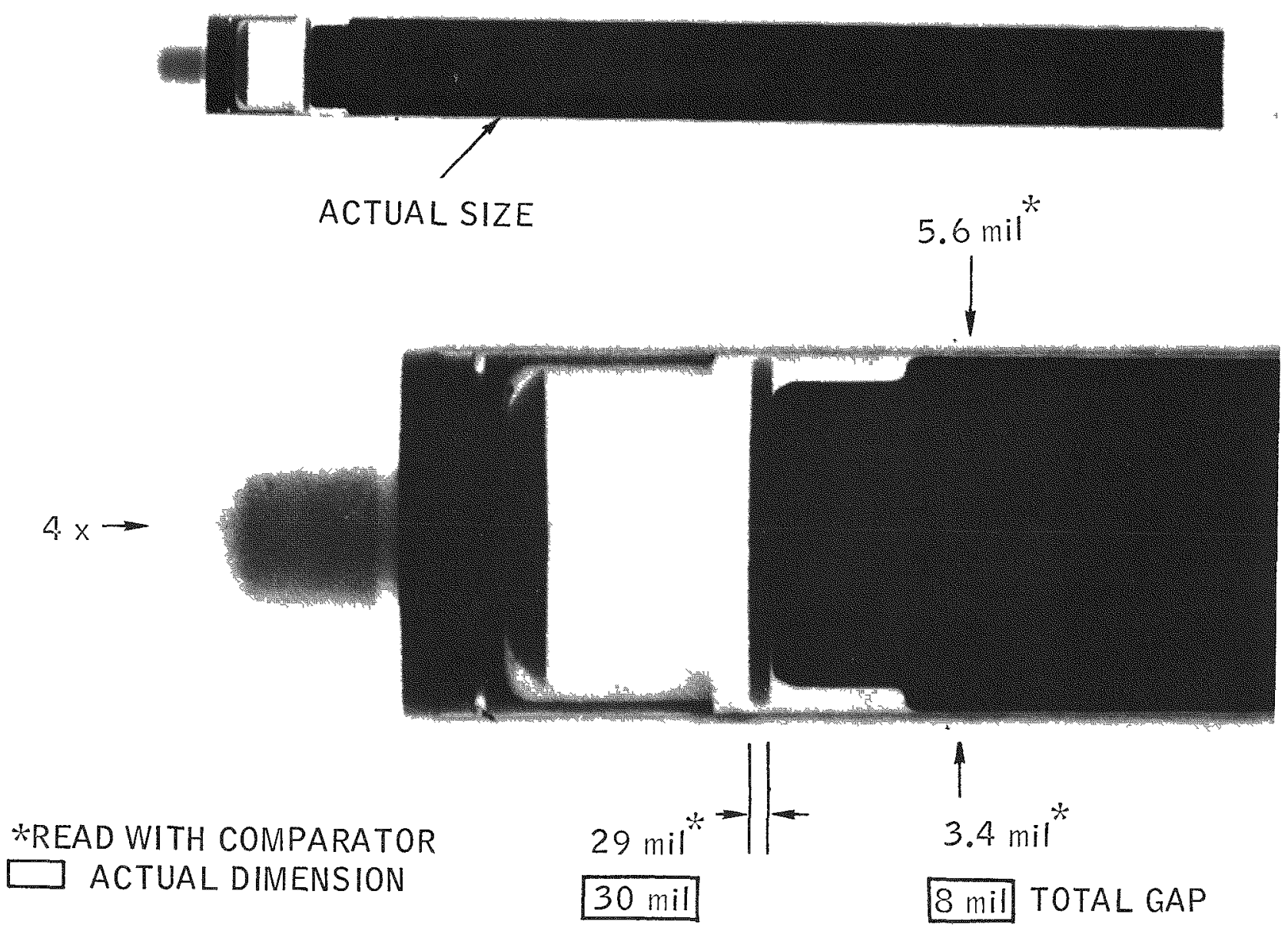

$9-54-065-22$

Figure 6. Fuel to Cladding Gap Measurement 


\title{
$\checkmark$ RELIABILITY OF HIGH-TEMPERATURE THERMOCOUPLES \\ IN IRRADTATION CAPSULES
}

N. L. Sandefur, F. D. Carpenter, R. J. Grenda, and J. S. Steibel Gulf General Atomic Incorporated $3722502 \quad$ San Diego, California

\begin{abstract}
A study of thermocouple behavior and reliability in irradiation capsules was conducted. The investigation covered 16 instrumented (lead-tube type) capsules, which contained a total of 200 Chromel-Alumel and tungsten-rhenium sheath-type thermocouples. The history of each thermocouple was recorded from the time of receipt to insertion in an experiment and during its operating life within the irradiation capsule. Testing conditions ranged from low neutron exposures to a total fluence of $4.5 \times 10^{21} \mathrm{n} / \mathrm{sq} \mathrm{cm}$ ( $\mathrm{E}>0.18 \mathrm{MeV}$ ) with operating temperatures to $1800 \mathrm{C}$. The rest data were tabulated into a computer code designed to evaluate thermocouple performance as a function of: (1) neutron exposure; (2) operating temperature; (3) time at temperature; (4) thermocouple type (Chromel-Alumel, tungsten-rhenium); (5) type of hot junction (capped or plugged, grounded, isolated); (6) manufacturer; (7) commercial construction versus construction to Gulf General Atomic specification; and (8) position of thermocouple within the capsule.

From these data, correlations were deduced concerning the in-pile behavior of the thermocouples and the probability of their successful operation. Postirradiation continuity and metallographic analyses were conducted on selected thermocouples to help establish location and/or causes of failure.

The computer analyses revealed that a $30 \%$ to $40 \%$ thermocouple failure rate can be expected under any set of conditions examined. The probability of failure was greater with increasing neutron exposure and longer times in the reactor. Thermocouples purchased to a GuIf General Atomic specification covering materials procurement and thermocouple manufacturing did not perform better than commercial production thermocouples for nuclear service.
\end{abstract}




\section{INTRODUCTION}

The coated particle irradiation program at Gulf General Atomic Incorporated requires temperature monitoring for the coated particle fuel and graphite irradiation tests. The temperatures fluctuate during a test and must be monitored continuously. The duration of an irradiation test extends several thousand hours. To determine these temperatures, sheath-type thermocouples are used because of their flexibility and compatibility with the particular experiment design.

The reliability of the thermocouples appeared completely unpredictable prior to this study. Thermocouples used in these irradiation capsules sometimes lasted throughout the irradiation. However, at other times, under exactly the same conditions, supposedly identical thermocouples failed at the beginning of the irradiation.

The problem of understanding thermocouple reliability is the objective of this study. This study evaluated thermocouple performance to determine which thermocouples perform best and also indicated which manufacturers construct the most reliable thermocouples. It was designed to show some of the factors that lead to better thermocouple performance or longer life. From the data, a reliability factor was determined which predicts how successful the use of a particular thermocouple may be.

\section{MATERIALS FOR STUDY}

Thermocouples of three basic types were used in this study: Chrome1Alume1, tungsten - 3\% rhenium/tungsten - 25\% rhenium, and tungsten - 5\% rhenium/tungsten - 26\% rhenium. The Chromel-Alumel (C/A) thermocouples contained a compacted magnesium oxide (MgO) insulation inside an Inconel sheath. The hot junctions were either of an isolated or a grounded type, several variations of which are listed in Table I. The length of the thermocouples was about $15 \mathrm{ft}$. The operating usage range assigned to these thermocouples was 200 to $1000 \mathrm{C}$.

The $W-3 \% \operatorname{Re} / W-25 \% \operatorname{Re}$ and the $W-5 \% \operatorname{Re} / W-26 \%$ Re thermocouples both contained compacted beryllium oxide (BeO) and magnesium oxide inside a tantalum sheath. The beryllium oxide extended from the hot junction end for $1 \mathrm{ft}$ followed by magnesium oxide for the remaining $3 \mathrm{ft}$. The hot junctions were made in the same manner and with the same variations as the Chromel-Alumel type (see Table I). The operating temperature of these thermocouples was 1000 to $1800 \mathrm{C}$.

Within the three basic types of thermocouples (C/A, W - 3\% Re/W $25 \% \operatorname{Re}$, and $W-5 \% \operatorname{Re} / W-26 \% \operatorname{Re}$ ), two major thermocouple processing variations were specified. The first of these variations specified that the thermocouple would be constructed for nuclear service by the manufacturer's usual method. No requirements from Gulf General Atomic other than the basic kind of wire, sheath, and insulation were requested. The second process specified that the basic materials (wire, sheath, and insulation) be of high purity (certified); also, the processing steps for constructing or assembling the thermocouple were specified as shown in the Appendix. 
TABLE I

MANUFACTURER AND CONSTRUCTION DATA ON THERMOCOUPLES USED IN GULF GENERAL ATOMIC PARTICLE IRRADIATION EXPERIMENTS

\begin{tabular}{c|l|l}
\hline & Chromel/Alume1 & $\begin{array}{l}W-3 \% \mathrm{Re} / \mathrm{W}-25 \% \mathrm{Re} \\
\mathrm{W}-5 \% \mathrm{Re} / \mathrm{W}-26 \% \mathrm{Re}\end{array}$ \\
\hline Size (in.) & 0.062 & $0.062,0.040$ \\
Insulation & Inconel 600 & Tantalum \\
Construction & $\begin{array}{l}\text { Swaged } \\
\text { Drawn } \\
\text { GGA specification } \\
\text { Grounded } \\
\text { Grounded plug } \\
\text { Ioslated }\end{array}$ & $\begin{array}{l}\text { Swaged } \\
\text { Grawn specification } \\
\text { Grounded } \\
\text { Grounded plug } \\
\text { Isolated }\end{array}$ \\
Manufacturers & $\begin{array}{l}\text { Aero Research } \\
\text { Continental Sensing } \\
\text { Thermo Electric Co. } \\
\text { Thermocouple } \\
\text { Products Co. }\end{array}$ & $\begin{array}{l}\text { Aero Research } \\
\text { Continental Sensing } \\
\text { Pyro Electric Co. } \\
\text { Thermo Electric Co. } \\
\text { Tuttle and Kift }\end{array}$ \\
\hline
\end{tabular}




\section{PRELIMINARY TESTING OF THERMOCOUPLES}

The thermocouples, after receipt from the manufacturers, were examined and tested before being incorporated into an irradiation capsule.

First, a check was made of the general appearance of the thermocouples, which would reveal obvious cracks or flaws in the sheath. The sealed hot-junction end was an important point in this visual inspection. The diameter of the sheath was checked for compliance with tolerance specification, which was usually $0.062 \mathrm{in.} \pm 0.001 \mathrm{in}$. The resistance of each wire and of the insulation was recorded. A spectrographic analysis was made on alternate batches of thermocouples to check for variation in impurities of the materials.

The first $2 \mathrm{ft}$ of each thermocouple was examined by radiography to show the amount of twist in the wires, the quality of the hot junction, and the condition of the wires. All thermocouples were leak checked with a helium mass spectrometer, and no thermocouple with a leak greater than $10^{-8} \mathrm{cc} / \mathrm{sec}$ was acceptable.

Next, each C/A thermocouple was thermally shocked from $820 \mathrm{C}$ to room temperature 25 times (in an induction furnace with a tantalum susceptor in flowing helium). The W/Re thermocouples were thermally shocked from $1200 \mathrm{C}$ to room temperature 15 times under the same conditions.

After the thermal shock testing, the thermocouples were calibrated to a maximum of $1000 \mathrm{C}$ for the $\mathrm{C} / \mathrm{A}$ and $1450 \mathrm{C}$ for the $\mathrm{W} / \mathrm{Re}$ with four additional points $2100 \mathrm{C}$ apart below these maximum temperatures.

\section{CAPSULE CONSTRUCTION}

Coated particle irradiation experiments are conducted with metallic capsules, which contain the nuclear materials for exposure to neutrons. These material capsules were the testing environment in which this thermocouple evaluation was conducted. The thermocouple is an intricate part of the capsule irradiation experiments.

Incorporating the thermocouples into the irradition capsule involves many construction processes, including mechanical- and thermal-type assembly processes, that the thermocouple must survive. The first phase of construction requires flexing and bending of the thermocouples. The most severe bending of the thermocouples occurs during threading of the thermocouples along and through close fitting holes. The smallest bend is about $1 / 2 \mathrm{in.}$ in diameter for a 0.062 -in.-diameter thermocouple. This is a $180^{\circ}$ bend.

The thermocouples are subjected to relatively high temperatures during brazing operations. When thermocouples are brazed to a primary containment can, the temperature of the $\mathrm{C} / \mathrm{A}$ thermocouple reaches $1210 \mathrm{C}$ for several minutes. 
The part of the thermocouple that is outside the reactor core, about $3 \mathrm{ft}$ from the hot junction and in the reactor tank, experiences at least two and sometimes four additional $1100 \mathrm{C}$ brazes during construction of the capsule. One of these areas is $4 \mathrm{in}$. above the reactor core and the other is $20 \mathrm{in}$. higher. These areas are shown in Fig. 1 as sections $G G$ and $H H$, respectively.

In the area between the two end caps (sections $\mathrm{GG}$ and $\mathrm{HH}$ ), a transition and braze weld is made to join the W/Re thermocouple to a compensating lead wire. An $1100 \mathrm{C}$ braze is performed at this point, and then the two ends are further welded.

From this area the compensating lead wire extends 10 ft through a steel tube to the area of the potted junction. Here the compensating lead wire is spot welded to a glass and/or asbestos insulated lead wire and the junction is encased in epoxy. The whip lead wire extends to the terminal head located on the side of the reactor tank.

\section{TESTING AND EXAMINATION}

\section{THERMOCOUPLE OPERATIONAL CONDTTIONS}

The thermocouples were operated in-pile in the Experimental Test Reactor (ETR) and the Materials Testing Reactor (MTR) at the National Reactor Testing Station (NRTS). About $80 \%$ of the thermocouples used in the experiments were tested in the ETR.

The fast-neutron exposure for the thermocouples ranged from nearly zero (failed during startup) to $4.5 \times 10^{21} \mathrm{n} / \mathrm{sq} \mathrm{cm}(E>0.18 \mathrm{MeV})$. The highest fast-neutron exposure accumulated in the MTR was $1.9 \times 10^{20} \mathrm{n} / \mathrm{sq} \mathrm{cm}$ $(\mathrm{E}>0.18 \mathrm{MeV})$.

The operating temperature for the thermocouples was 100 to $1800 \mathrm{C}$ in a helium and/or argon atmosphere. The most often used average temperature ranges were: $\mathrm{C} / \mathrm{A}, 400 \mathrm{C}$ on the containment cans and $900 \mathrm{C}$ in the fue1; W/Re, $1200 \mathrm{C}$ and $1400 \mathrm{C}$ in the fuel.

Thermal shocks were frequent because of normal reactor operating conditions. When the reactor "scrams," the temperature can drop from 1400 to $500 \mathrm{C}$ in a few seconds. Shocks such as these occur 5 to 20

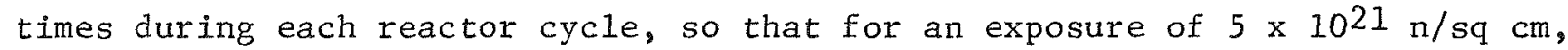
the reactor would operate 5 cycles with as many as 100 shocks recorded.

\section{THERMOCOUPLE EXAMINATION DURING OPERATION}

A record of the behavior of each thermocouple was kept for its entire operating life within the reactor. The records indicated thermocouple condition after its arrival at the reactor, after the capsule was inserted into the core, and during startup of the reactor. Each thermocouple was checked with a potentiometer before startup and at any time during the operation that the thermocouple behaved erratically. 


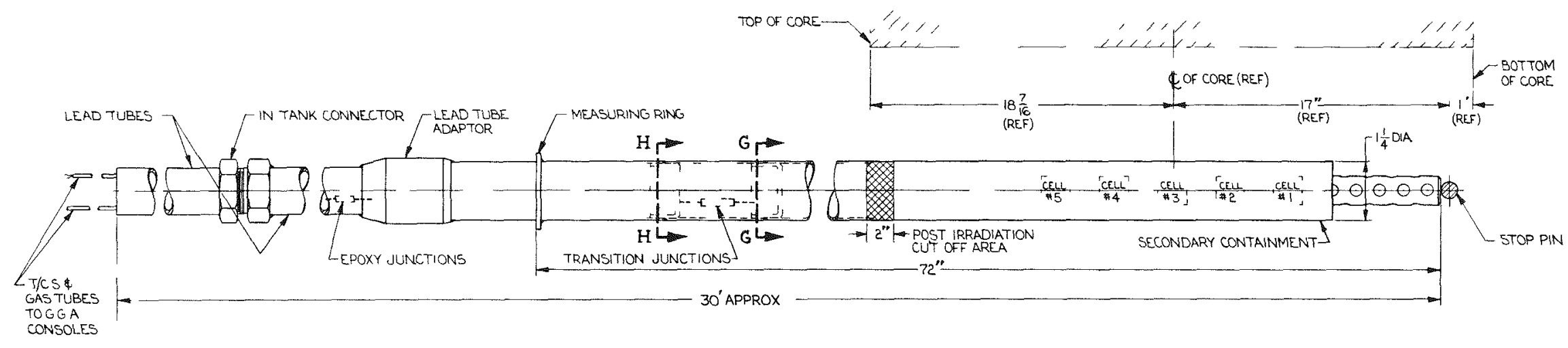

Fig. 1. Assembly drawing of fuel particle irradiation capsule

荝 
When a thermocouple failed, it was checked for an open circuit and the results were recorded. Each failed thermocouple was again checked for open circuits during the cycle shutdown period.

\section{POSTIRRADIATION EXAMINATION}

At the end of the irradiation period, the capsule was removed and cut in sections for shipment to the Gulf General Atomic hot cell. Two parts were shipped to the hot cell: the in-core fueled portion ( 35 in. Iong) and the 2-ft section just above the core. A third part, which included the epoxy transition junction, remained at the ETR. The epoxy transition junction section was only slightly radioactive, and a continuity check on this section could be done by hand at the ETR.

A continuity check was made at the "postirradiation" cut-off area of the 35-in. tube in the hot cell (see Fig. 1) by removing a portion of the secondary containment to expose the thermocouples in their sheathing. The sheathing was stripped off to expose the thermoelements. When the thermocouple could not be stripped, it was cut radially. A long, thin needle-like probe was used to check the continuity of the thermocouples. For a grounded thermocouple, continuity was checked 'through the wire and out the sheath. For an isolated junction, two probes were used. After the above check, the thermocouples were again cut just outside the primary end caps, and another continuity check was made of that portion of the thermocouple extending into the cell.

The 2-ft section above the core, which included the transition junction from the thermocouples to the compensating lead wire, was examined next. It also included the upper and lower braze areas (see Fig. 1, Sections $H H$ and $G G$ ).

This piece was clamped in place, and the outer can was cut back to expose the thermocouples. The sheath was stripped to expose the C/A and W/Re wires, which were probed for continuity in the same way as those at the postirradiation cut-off area. Sometimes one end was put in a mercury bath and the continuity was checked in this manner using mercury as contact at one end.

When the continuity checks had been made from one end cap through the other, the outer can was cut to expose the transition junctions on the $W / R e$ thermocouples. This area was removed by snipping the thermocouple on both sides of the junction. The thermocouple wires were then stripped and the junction was checked for continuity.

The next step was to open the primary cell and remove a 1/2-in. section of the thermocouple, including the hot junction, for a metallographic examination. The thermocouple pieces were encased horizontally in an epoxy mount and polished until the wires that connect to the hot junction showed. Polishing and grinding continued from the top of each thermocouple to the point where it was obvious that both wires were still connected to the hot junction or that one or the other was not. The condition of the insulation, sheath materials, and wires was also visible during the examination. 


\section{RESULTS AND DISCUSSION}

\section{AS-RECEIVED MATERIAL}

After the purchased thermocouples reached GuIf General Atomic, a quality control type examination was conducted. The most frequently found flaws were:

1. Outside diameter of the thermocouple was not as specified.

2. Length of the thermocouple was not as specified.

3. Hot junction was not helium leak-tight.

4. Cold end was not helium leak-tight.

5. Sheath was cracked.

6. Thermocouple was out of calibration.

7. Hot junctions broke off during the calibration run.

8. Thermocouple failed during thermal cycling test.

9. Thermocouple was damaged by poor packaging or shipping.

Only a small percentage of thermocouples were lost in calibration and thermal shock testing; the percentage of loss for most of the suppliers listed in Table I was less than 1\%. One group of $7 \mathrm{~W} / \mathrm{Re}$ thermocouples from a particular supplier performed especially well, so another group of 25 was ordered. However, 9 of 15 from this group failed during calibration. The problem appeared to be with the hot junction, which was loose or, in some cases, disconnected from the rest of the thermocouple. This illustrates a major problem with suppliers: inconsistency from lot to lot. Several of the flaws listed above can be corrected. However, an important question is if the manufacturer's quality control does not detect these obvious flaws, how many more are overlooked inside the sheathing where they cannot be easily checked.

\section{CAPSULE FABRICATION}

Of the 200 thermocouples installed in the 16 capsules, four failures occurred during capsule construction. The failures were attributed directly to construction techniques. All of the failed thermocouples were of the W/Re variety. Two of the failures occurred at the transition joint where the tungsten alloy legs are joined to the compensating lead wire. The other two discontinuities appeared after the thermocouples were bent at the primary containment. Any of the following could have been a contributing factor in the failure: high brazing temperature, impure atmosphere in the calibrating or shock-testing facility, or general handing. All thermocouples were repaired or replaced before the capsules were shipped. 
In this section all historical data on the thermocouples are compared with the length and severity of operation. There were numerous variables and the method used to find the best, most reliable thermocouple was a computer sorting of the variables. Data were collected as shown in Table II, and were recorded on computer cards for sorting. The following nine sortings were made on the initial run:

1. Manufacturer versus reliability factor (run A)

2. Reliability factor of Gulf General Atomic specification (GGA-spec) thermocouples versus commercial nuclear service thermocouples (run B)

3. Capsule position versus reliability factor (run C)

4. Increasing neutron exposure versus percent failed (run D)

5. $C / A$ versus $W-3 \% \operatorname{Re} / W-25 \% \operatorname{Re}$ versus $W-5 \% \operatorname{Re} / W-26 \% \operatorname{Re}($ run $E$ )

6. Type of hot junction versus reliability factor (run F)

7. Time of operation versus percent failed (run G)

8. Position (fuel or can) versus reliability factor (run $\mathrm{H}$ )

9. Operating temperature versus percent failed (run I)

The computer sorting runs were treated by calculating a $60 \%$ confidence level using the formula

$$
R=e^{-\lambda t},
$$

where $\mathrm{R}$ is the reliability confidence number, $\lambda$ is the number of failures, and $t$ is the total number of samples. This formula indicates the probability that the past performance of a group of thermocouples will be repeated in the future.

The results of the first computer run (run A, thermocouple manufacturer versus reliability factor) are shown in Fig. 2. This figure compares thermocouples made by five different manufacturers (designated A through $E$ ). The reliability factor varies from 0.90 for thermocouples produced by manufacturer $\mathrm{C}$ to 0.20 for thermocouples produced by manufacturer $\mathrm{E}$. These data point to large differences in successful operation for thermocouples of the same type but from different manufacturers. Reliability can be improved several fold by careful selection of the thermocouple manufacturer. 
TABIF II

THFROCOUPLI DAIA FROM IRRADIATION FXPI RIMI NIS OF PARTICLI

CAPSUIFS AND GRAPHITH CAPSULIS

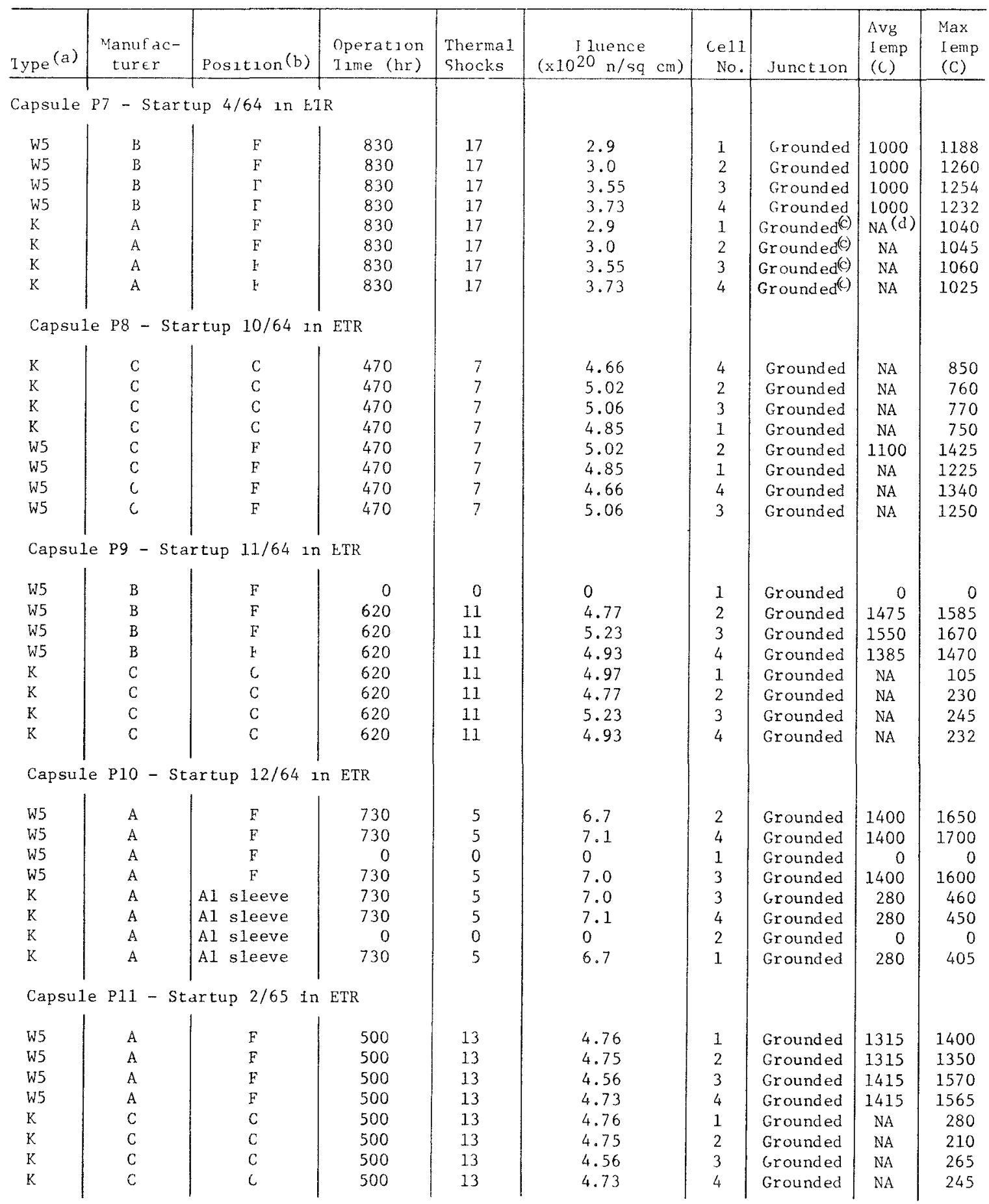


TABLE II (Continued)

\begin{tabular}{|c|c|c|c|c|c|c|c|c|c|}
\hline Type (a) & $\begin{array}{l}\text { Manufac- } \\
\text { turex }\end{array}$ & Position (b) & $\begin{array}{l}\text { Operation } \\
\text { Time (hr) }\end{array}$ & $\begin{array}{l}\text { Thermal } \\
\text { Shocks }\end{array}$ & $\begin{array}{c}\text { Fluence } \\
\left(\times 10^{20} \mathrm{n} / \mathrm{sq} \mathrm{cm}\right)\end{array}$ & $\begin{array}{c}\text { Cell } \\
\text { No. }\end{array}$ & Junction & $\begin{array}{l}\text { Avg } \\
\text { Temp } \\
\text { (C) }\end{array}$ & $\begin{array}{l}\text { Max } \\
\text { Temp } \\
\text { (C) }\end{array}$ \\
\hline \multicolumn{10}{|c|}{ Capsule P13G - Starcup $7 / 65$ in ETR } \\
\hline W5 & A & $\mathrm{F}$ & 672 & 1 & 0.6 & 2 & Grounded & NA & 850 \\
\hline W5 & B & $F$ & 672 & 1 & 0.6 & 3 & Grounded & NA & 850 \\
\hline W5 & B & F & 672 & 1 & 0.6 & 4 & Grounded & $\mathrm{NA}$ & 1350 \\
\hline W5 & $B$ & $F$ & 672 & 1 & 0.6 & 1 & Grounded & $\mathrm{NA}$ & 1300 \\
\hline $\mathrm{K}$ & B & C & 672 & 1 & 0.6 & 4 & Grounded & NA & 873 \\
\hline K & $B$ & C & 672 & 1 & 0.6 & 2 & Grounded & NA & 620 \\
\hline $\mathrm{K}$ & B & C & 672 & 1 & 0.6 & 3 & Grounded & NA & 775 \\
\hline $\mathrm{K}$ & $B$ & C & 672 & 1 & 0.6 & 1 & Grounded & NA & 633 \\
\hline $\mathrm{K}$ & B & C & 672 & 1 & 0.6 & $\mathrm{NA}$ & Grounded & NA & NA \\
\hline $\mathrm{K}$ & $B$ & $\mathrm{C}$ & 672 & 1 & 0.6 & NA & Grounded & $N A$ & NA \\
\hline $\mathrm{K}$ & A & Melt section & 672 & 1 & 0.6 & NA & Grounded & $\mathrm{NA}$ & NA \\
\hline$\overline{\mathrm{K}}$ & A & Melt section & 672 & 1 & 0.6 & NA & Grounded & $\mathrm{NA}$ & $\mathrm{NA}$ \\
\hline \multicolumn{10}{|c|}{ Capsule P12 - Startup 4/65 in ETR } \\
\hline W5 & $B$ & $\mathrm{~F}$ & 792 & 8 & 6.6 & 1. & Grounded & 1232 & 1350 \\
\hline W5 & B & $\mathrm{F}$ & 768 & 7 & 6.4 & 2 & Grounded & 1310 & 1345 \\
\hline W5 & D & F & 2080 & 15 & 9.0 & 3 & Grounded & 1277 & 1343 \\
\hline W5 & $\mathrm{D}$ & $F$ & 1102 & 15 & 9.3 & 4 & Grounded & 1288 & 1332 \\
\hline K & C & Al sleeve & 1978 & 22 & 16.5 & 1 & Grounded & 243 & 370 \\
\hline $\mathrm{K}$ & C & Al sleeve & 1978 & 22 & 16.5 & 2 & Grounded & 260 & 310 \\
\hline $\mathrm{K}$ & C & Al sleeve & 1978 & 22 & 16.5 & 3 & Grounded & 204 & 260 \\
\hline $\mathrm{K}$ & A & Al sleeve & 1978 & 22 & 16.5 & 4 & Grounded & 288 & 385 \\
\hline K & $\mathrm{C}$ & $\mathrm{C}$ & 1978 & 22 & 16.5 & 3 & Grounded & 488 & 593 \\
\hline $\mathrm{K}$ & $\mathrm{C}$ & C & 264 & 1 & 2.2 & 4 & Grounded & 343 & 343 \\
\hline \multicolumn{10}{|c|}{ Capsule P13E - Startup $6 / 65$ in ETR } \\
\hline W5 & B & F & 154 & 6 & 2.4 & 1 & Grounded & 800 & 975 \\
\hline W5 & B & F & 1718 & 46 & 27.0 & 2 & Grounded & 1250 & 1650 \\
\hline W5 & $B$ & $\mathrm{~F}$ & 1718 & 47 & 27.0 & 3 & Grounded & 1236 & 1525 \\
\hline W5 & $B$ & $\mathrm{~F}$ & 145 & 6 & 2.3 & 4 & Grounded & 600 & 910 \\
\hline W5 & B & $\mathrm{F}$ & 1718 & 47 & 27.0 & 5 & Grounded & 1366 & 1875 \\
\hline $\mathrm{K}$ & $\mathrm{E}$ & $\mathrm{C}$ & 60 & 2 & 1.1 & 1. & Grounded(c) & 320 & 438 \\
\hline $\mathrm{K}$ & $\mathrm{E}$ & $\mathrm{C}$ & 60 & 2 & 1.1 & 2 & Grounded & 350 & 485 \\
\hline $\mathrm{K}$ & $E$ & c & 260 & 8 & 4.0 & 3 & Grounded & 340 & 755 \\
\hline K & B & C & 260 & 8 & 4.0 & 4 & Grounded & 320 & NA \\
\hline $\mathrm{K}$ & B & $\mathrm{C}$ & 100 & 4 & 1.6 & 5 & Grounded & 380 & NA \\
\hline \multicolumn{10}{|c|}{ Capsule Pl3F - Starrup 4/66 in ETR } \\
\hline W5 & $F$ & F & 1888 & 30 & 27.0 & 2 & Isolated & 1278 & 1535 \\
\hline W5 & D & $\mathrm{F}$ & 1888 & 30 & 27.0 & 2 & Grounded & 1299 & 1555 \\
\hline W5 & B & F & 1888 & 30 & 27.0 & 2 & Grounded & 1308 & 1535 \\
\hline W5 & D & F & 1888 & 30 & 27.0 & 3 & Grounded & 1312 & 1615 \\
\hline W5 & F & $F$ & 1888 & 30 & 27.0 & 3 & Grounded & 1267 & 1615 \\
\hline W5 & D & F & 0 & 0 & 0 & 3 & Grounded & 0 & 0 \\
\hline พ5 & D & $\mathrm{F}$ & 1888 & 30 & 27.0 & 4 & Grounded & 1277 & 1325 \\
\hline W5 & F & F & 1888 & 30 & 27.0 & 4 & Grounded & 1302 & 1310 \\
\hline W5 & D & $F$ & 108 & 2 & 2.0 & 4 & Grounded & 1315 & NA \\
\hline W5 & $B$ & $\mathrm{~F}$ & 1888 & 30 & 27.0 & 5 & Grounded & 1191 & 1275 \\
\hline w5 & F & $F$ & 1888 & 30 & 27.0 & 5 & Grounded & 1199 & 1268 \\
\hline W5 & $\mathrm{D}$ & $\mathrm{F}$ & 1888 & 30 & 27.0 & 5 & Grounded & 1233 & 1315 \\
\hline $\mathrm{K}$ & B & $\mathrm{F}$ & 431 & 12 & 7.0 & 1 & Isolated & 939 & $\mathrm{NA}$ \\
\hline $\mathrm{K}$ & B & $F$ & 1016 & 20 & 15.0 & 1 & Swaged & 879 & $\mathrm{NA}$ \\
\hline K & B & $\mathrm{F}$ & 1888 & 30 & 27.0 & 1 & Grounded & 919 & NA \\
\hline $\mathrm{K}$ & B & $\mathrm{C}$ & 1888 & 30 & 27.0 & 2 & Grounded & 610 & $\mathrm{NA}$ \\
\hline $\mathrm{K}$ & B & $\mathrm{C}$ & 1570 & 26 & 22.0 & 3 & Grounded & 560 & $\mathrm{NA}$ \\
\hline $\mathrm{K}$ & B & $\mathrm{C}$ & 1888 & 30 & 27.0 & 4 & Grounded & 540 & NA \\
\hline $\mathrm{K}$ & NA & $c$ & 1386 & 22 & 20.0 & 4 & Grounded & 545 & $\mathrm{NA}$ \\
\hline
\end{tabular}


TABLE II (Continued)

\begin{tabular}{|c|c|c|c|c|c|c|c|c|c|}
\hline Type ${ }^{(a)}$ & $\begin{array}{l}\text { Manufac- } \\
\text { turer } \\
\end{array}$ & Position (b) & $\begin{array}{l}\text { Operation } \\
\text { Time (hr) }\end{array}$ & $\begin{array}{l}\text { Thermal } \\
\text { Shocks }\end{array}$ & $\begin{array}{r}\text { Fluence } \\
\left(\times 10^{20} \mathrm{n} / \mathrm{sq} \mathrm{cm}\right) \\
\end{array}$ & $\begin{array}{r}\text { Cell } \\
\text { No. } \\
\end{array}$ & Junction & $\begin{array}{l}\text { Avg } \\
\text { Temp } \\
\text { (C) }\end{array}$ & $\begin{array}{l}\text { Max } \\
\text { Temp } \\
(\mathrm{C})\end{array}$ \\
\hline \multicolumn{10}{|c|}{ Capsule } \\
\hline W3 & $\mathrm{B}$ & $F$ & 3164 & 71 & 45.0 & 5 & Swaged & 1245 & $\mathrm{NA}$ \\
\hline W3 & $\mathrm{B}$ & $F$ & 3164 & 71 & 45.0 & 5 & Swaged & 1260 & $\mathrm{NA}$ \\
\hline W3 & $\mathrm{B}$ & $F$ & 3164 & 71 & 45.0 & 5 & Swaged & 1230 & NA \\
\hline W3 & $B$ & $F$ & 3164 & 71 & 45.0 & 2 & Swaged & 1255 & NA \\
\hline W3 & B & $\mathrm{F}$ & 3164 & 71 & 45.0 & 2 & Swaged & 1270 & $\mathrm{NA}$ \\
\hline w3 & $B$ & $F$ & 28 & 1 & 0.7 & 3 & Swaged & 1310 & $\mathrm{NA}$ \\
\hline W3 & B & $F$ & 0 & 0 & 0 & 3 & Swaged & 0 & 0 \\
\hline W3 & $B$ & $\mathrm{~F}$ & 1880 & 39 & 27.0 & 3 & Swaged & 1250 & 1350 \\
\hline W3 & B & $\mathrm{F}$ & 0 & 0 & 0 & 2 & Swaged & 0 & 0 \\
\hline W3 & $B$ & $\mathrm{~F}$ & 36 & 1 & 8.0 & 4 & Swaged & 1295 & 1500 \\
\hline W3 & $B$ & $F$ & 2598 & 48 & 32.4 & 4 & Swaged & 1220 & 1400 \\
\hline W3 & $\mathrm{B}$ & $F$ & 23 & 1 & 0.7 & 4 & Swaged & 0 & 710 \\
\hline $\mathrm{K}$ & $B$ & $\mathrm{~F}$ & 1198 & 28 & 18.0 & 1 & Grounded & 950 & 1060 \\
\hline $\mathrm{K}$ & A & $\mathrm{F}$ & 675 & 16 & 10.0 & 1 & Grounded & 920 & 940 \\
\hline $\mathrm{K}$ & A & $\vec{F}$ & 2197 & 45 & 33.0 & 1 & Grounded & 998 & 1040 \\
\hline $\mathrm{K}$ & A & $\mathrm{C}$ & 3164 & 71 & 45.0 & 5 & Grounded & 250 & $\mathrm{NA}$ \\
\hline $\mathrm{K}$ & A & $\mathrm{C}$ & 3164 & 71 & 45.0 & 4 & Grounded & 525 & 700 \\
\hline $\mathrm{K}$ & $A$ & $\mathrm{C}$ & 3164 & 71 & 45.0 & 3 & Grounded & 493 & $\mathrm{NA}$ \\
\hline $\mathrm{K}$ & A & $C$ & 3164 & 71 & 45.0 & 2 & Grounded & 390 & NA \\
\hline $\mathrm{K}$ & A & $\mathrm{C}$ & 3164 & 71 & 45.0 & 1 & Grounded & 370 & 750 \\
\hline \multicolumn{10}{|c|}{ Capsule P13J - Startup $3 / 67$ in ETR } \\
\hline W3 & B & $F$ & 1530 & 32 & 22.6 & 2 & Grounded & 1316 & 1370 \\
\hline W3 & $B$ & $F$ & 2300 & 44 & 24.0 & 2 & Grounded & 1325 & 1363 \\
\hline W3 & $\mathrm{B}$ & $\mathrm{F}$ & 1837 & 41 & 28.4 & 2 & Grounded & 1307 & 1350 \\
\hline W3 & $B$ & $F$ & 2300 & 44 & 39.0 & 3 & Grounded & 1309 & 1360 \\
\hline W3 & B & $F$ & 2300 & 44 & 39.0 & 3 & Grounded & 1324 & 1365 \\
\hline W3 & B & $F$ & 2300 & 44 & 39.0 & 3 & Grounded & 1338 & 1390 \\
\hline W3 & B & $F$ & 2300 & 44 & 31.5 & 4 & Grounded & 1210 & 1320 \\
\hline W3 & $B$ & $E$ & 2300 & 44 & 31.5 & 4 & Grounded & 1218 & 1370 \\
\hline $\mathrm{K}$ & $B$ & $\mathrm{E}$ & 1611 & 35 & 17.8 & 1 & Grounded & 1038 & 1100 \\
\hline $\mathrm{K}$ & A & $F$ & 1617 & 35 & 17.9 & 1 & Grounded & 987 & 1070 \\
\hline K & $\mathrm{NA}$ & $\mathrm{F}$ & 944 & 26 & 10.5 & 1 & Grounded & 1031 & 1070 \\
\hline $\mathrm{K}$ & $E$ & $F$ & 1653 & 35 & 18.0 & 5 & Grounded & 635 & 670 \\
\hline $\mathrm{K}$ & A & $\mathrm{F}$ & 1293 & 31 & 14.1 & 5 & Grounded & 657 & 690 \\
\hline $\mathrm{K}$ & A & $F$ & 1850 & 42 & 20.1 & 5 & Grounded & 658 & 710 \\
\hline $\mathrm{K}$ & $\mathrm{NA}$ & $\mathrm{C}$ & 2200 & 42 & 24.3 & 1 & Grounded & $\mathrm{NA}$ & $\mathrm{NA}$ \\
\hline $\mathrm{K}$ & $\mathrm{NA}$ & $\mathrm{C}$ & 2000 & 42 & 29.6 & 2 & Grounded & NA & $\mathrm{NA}$ \\
\hline $\mathrm{K}$ & $\mathrm{NA}$ & $\mathrm{C}$ & 2300 & 44 & 39.0 & 3 & Grounded & $\mathrm{NA}$ & $\mathrm{NA}$ \\
\hline K & NA & $\mathrm{C}$ & 2300 & 44 & 31.5 & 4 & Grounded & NA & NA \\
\hline $\mathrm{K}$ & A & $\mathrm{C}$ & 2300 & 44 & 25.0 & 5 & Grounded & NA & $\mathrm{NA}$ \\
\hline W3 & $\mathrm{B}$ & $\mathrm{F}$ & 254 & 8 & 3.5 & 4 & Grounded & 1325 & 1350 \\
\hline \multicolumn{10}{|c|}{ Capsule P14B - Startup 4/66 in ETR } \\
\hline W3 & $\mathrm{B}$ & $\mathrm{F}$ & 16 & 0 & 0 & 2 & Grounded & 0 & $\mathrm{NA}$ \\
\hline W3 & $B$ & $\mathrm{~F}$ & 3138 & 45 & 41.0 & 2 & Grounded & 1325 & $\mathrm{NA}$ \\
\hline W3 & $\mathrm{B}$ & $\mathbf{F}$ & 3138 & 45 & 41.0 & 2 & Grounded & 1330 & $\mathrm{NA}$ \\
\hline W3 & $B$ & $\mathrm{~F}$ & 3138 & 45 & 41.0 & 3 & Grounded & 1285 & $\mathrm{NA}$ \\
\hline W3 & $\mathrm{B}$ & $\mathrm{F}$ & 2165 & 30 & 28.0 & 3 & Grounded & 1320 & $\mathrm{NA}$ \\
\hline W3 & $B$ & F & 3138 & 45 & 41.0 & 3 & Grounded & 1300 & $\mathrm{NA}$ \\
\hline W3 & B & $F$ & 2185 & 31 & 28.0 & 4 & Grounded & 1265 & NA \\
\hline W3 & B & F & 2185 & 31 & 28.0 & 4 & Grounded & 1210 & $\mathrm{NA}$ \\
\hline W3 & $B$ & $\mathrm{~F}$ & 3138 & 45 & 41.0 & 4 & Grounded & 1325 & $\mathrm{NA}$ \\
\hline W3 & $B$ & $F$ & 3138 & 45 & 41.0 & 5 & Grounded & 1310 & $\mathrm{NA}$ \\
\hline W3 & $B$ & $\mathrm{~F}$ & 2600 & 37 & 32.0 & 5 & Grounded & 1300 & $\mathrm{NA}$ \\
\hline W3 & B & $\mathrm{F}$ & 3138 & 45 & 41.0 & 5 & Grounded & 1300 & $\mathrm{NA}$ \\
\hline
\end{tabular}


TABLE II (Continued)

\begin{tabular}{|c|c|c|c|c|c|c|c|c|c|}
\hline Type (a) & $\begin{array}{l}\text { Manufac- } \\
\text { turer }\end{array}$ & Position $(b)$ & $\begin{array}{l}\text { Operation } \\
\text { Time (hr) }\end{array}$ & $\begin{array}{l}\text { Thermal } \\
\text { Shocks }\end{array}$ & $\begin{array}{l}\text { Fluence } \\
\left(\times 10^{20} \mathrm{n} / \mathrm{sq} \mathrm{cm}\right)\end{array}$ & $\begin{array}{c}\text { Cell } \\
\text { No. }\end{array}$ & Junction & $\begin{array}{l}\text { Avg } \\
\text { Temp } \\
\text { (C) }\end{array}$ & $\begin{array}{l}\text { Max } \\
\text { Temp } \\
\text { (C) }\end{array}$ \\
\hline \multicolumn{10}{|c|}{ Capsule P14B (Continued) } \\
\hline K & $\mathrm{B}$ & $\mathrm{F}$ & 1997 & 29 & 26.0 & 1 & Swaged & 935 & NA \\
\hline $\mathrm{K}$ & B & $\mathrm{F}$ & 400 & 6 & 3.0 & 1 & Isolated & 925 & NA \\
\hline $\mathrm{K}$ & B & $F$ & 1264 & 16 & 16.0 & 1 & Grounded & 895 & NA \\
\hline $\mathrm{K}$ & B & $\mathrm{c}$ & 1377 & 17 & 16.0 & 2 & Grounded & 505 & $\mathrm{NA}$ \\
\hline $\mathrm{K}$ & B & $\mathrm{C}$ & 2185 & 31 & 28.0 & 3 & Grounded & 555 & NA \\
\hline $\mathrm{K}$ & B & $\mathrm{C}$ & 240 & 3 & 3.0 & 4 & Grounded & 485 & NA \\
\hline $\mathrm{K}$ & B & $\mathrm{C}$ & 1057 & 14 & 14.0 & 5 & Grounded & 525 & $\mathrm{NA}$ \\
\hline \multicolumn{10}{|c|}{ Capsule P15 - Startup $7 / 66$ in ETR } \\
\hline W5 & B & F & 1801 & $4 i$ & 26.0 & 1 & Plugged (e) & 1295 & $\mathrm{NA}$ \\
\hline W5 & B & $\mathrm{F}$ & 134 & 8 & 3.0 & 1 & Plugged & 1225 & NA \\
\hline W5 & B & $\mathrm{F}$ & 2969 & 72 & 45.0 & 1 & Plugged & 1240 & NA \\
\hline W5 & B & F & 1900 & 45 & 31.0 & 2 & Plugged & 1275 & $\mathrm{NA}$ \\
\hline W5 & B & $\mathrm{F}$ & 1882 & 42 & 27.0 & 2 & Plugged & 1300 & $\mathrm{NA}$ \\
\hline W5 & B & $\mathrm{F}$ & 1801 & 41 & 26.0 & 2 & Plugged & 1275 & NA \\
\hline W5 & B & $\mathrm{F}$ & 2969 & 72 & 45.0 & 3 & plugged & 1260 & $\mathrm{NA}$ \\
\hline W5 & B & F & 2969 & 72 & 45.0 & 3 & Plugged & 1240 & NA \\
\hline W5 & B & F & 2969 & 72 & 45.0 & 3 & Plugged & 1210 & $\mathrm{NA}$ \\
\hline W5 & $\mathrm{F}$ & $F$ & 2969 & 72 & 45.0 & 4 & Plugged & 1230 & NA \\
\hline W5 & F & $\mathrm{F}$ & 2969 & 72 & 45.0 & 4 & plugged & 1220 & $\mathrm{NA}$ \\
\hline W5 & B & $\mathrm{F}$ & 1801 & 41 & 26.0 & 4 & Plugged & 1280 & NA \\
\hline W5 & B & $\mathrm{F}$ & 2969 & 72 & 45.0 & 5 & Plugged & 1160 & $\mathrm{NA}$ \\
\hline W5 & D & $\mathrm{F}$ & 2969 & 72 & 45.0 & 5 & Plugged & 1210 & $\mathrm{NA}$ \\
\hline W5 & F & $F$ & 2969 & 72 & 45.0 & 5 & Plugged & 1280 & $N A$ \\
\hline $\mathrm{K}$ & B & C & 2969 & 72 & 45.0 & 1 & Grounded & 420 & $\mathrm{NA}$ \\
\hline $\mathrm{K}$ & B & C & 2778 & 67 & 45.0 & 2 & Grounded & 540 & NA \\
\hline K & B & $\mathrm{C}$ & 2969 & 72 & 45.0 & 3 & Grounded & 480 & NA \\
\hline $\mathrm{K}$ & B & C & 2969 & 72 & 45.0 & 4 & Grounded & 550 & $\mathrm{NA}$ \\
\hline K & B & c & 2962 & 72 & 45.0 & 5 & Grounded & 560 & NA \\
\hline \multicolumn{10}{|c|}{ Capsule P16 - Startup $12 / 66$ in ETR } \\
\hline W3 & B & $\mathrm{F}$ & 4192 & 43 & 27.0 & 1 & Plugged & 1330 & NA \\
\hline W3 & B & $\mathrm{F}$ & 443 & 11 & 9.0 & 1 & plugged & 1287 & NA \\
\hline W3 & $\mathrm{B}$ & $\mathrm{F}$ & 1251 & 26 & 21.0 & 1 & Plugged & 1310 & NA \\
\hline W3 & $B$ & $\mathrm{~F}$ & 1492 & 43 & 27.0 & 2 & Plugged & 1310 & $\mathrm{NA}$ \\
\hline W3 & B & $\mathrm{F}$ & 1492 & 43 & 27.0 & 2 & Plugged & 1320 & $\mathrm{NA}$ \\
\hline W3 & B & $\mathrm{F}$ & 1492 & 43 & 27.0 & 2 & plugged & 1330 & $\mathrm{NA}$ \\
\hline W3 & $B$ & $\mathrm{~F}$ & 1492 & 43 & 27.0 & 3 & Plugged & 1330 & $\mathrm{NA}$ \\
\hline W3 & $\mathrm{B}$ & $\mathrm{F}$ & 15 & 3 & 1.0 & 3 & Plugged & 1300 & NA \\
\hline W3 & B & $\mathrm{F}$ & 1492 & 43 & 27.0 & 3 & Plugged & 1290 & NA \\
\hline W3 & B & $\mathrm{F}$ & 0 & 0 & 0 & 4 & Plugged & 0 & NA \\
\hline W3 & B & $\mathrm{F}$ & 1492 & 43 & 27.0 & 4 & Plugged & 1325 & NA \\
\hline W3 & B & $\mathrm{F}$ & 1492 & 43 & 27.0 & 4 & Plugged & 1290 & $\mathrm{NA}$ \\
\hline W3 & $B$ & $\mathrm{~F}$ & 1492 & 43 & 27.0 & 5 & Plugged & 1312 & $\mathrm{NA}$ \\
\hline W3 & $B$ & $\mathrm{~F}$ & 1170 & 23 & 18.0 & 5 & Plugged & 1070 & NA \\
\hline W3 & $B$ & $\mathrm{~F}$ & 1492 & 43 & 27.0 & 5 & Plugged & 1175 & NA \\
\hline $\mathrm{K}$ & B & $\mathrm{C}$ & 755 & 17 & 14.0 & 1 & Plugged & 530 & $\mathrm{NA}$ \\
\hline $\mathrm{K}$ & B & C & 1492 & 43 & 27.0 & 2 & Plugged & 616 & NA \\
\hline $\mathrm{K}$ & B & $\mathrm{C}$ & 1200 & 25 & 21.0 & 3 & Plugged & 488 & NA \\
\hline K & B & $\mathrm{C}$ & 1221 & 25 & 21.0 & 4 & Plugged & 482 & $\mathrm{NA}$ \\
\hline $\mathrm{K}$ & B & C & 1300 & 30 & 23.0 & 5 & Plugged & 463 & NA \\
\hline \multicolumn{10}{|c|}{ Capsule FM-1 - Startup $3 / 67$ in ETR } \\
\hline W3 & B & $\mathrm{F}$ & 1028 & 24 & 18.0 & 1 & Plugged & 1155 & NA \\
\hline W3 & $\mathrm{B}$ & F & 94 & 1 & $\begin{array}{r}1.0 \\
1.0\end{array}$ & 1 & Plugged & 1150 & NA \\
\hline W3 & $B$ & F & 1028 & 24 & 18.0 & 2 & Plugged & 1520 & $\mathrm{NA}$ \\
\hline W3 & $B$ & $\mathrm{~F}$ & 1028 & 24 & 18.0 & 2 & Plugged & 1540 & NA \\
\hline W3 & $B$ & F & 1028 & 24 & 18.0 & 3 & Plugged & 1415 & NA \\
\hline W3 & $B$ & $\mathrm{~F}$ & 1028 & 24 & 18.0 & 3 & Plugged & 1440 & NA \\
\hline
\end{tabular}


TABLE II (Continued)

\begin{tabular}{|c|c|c|c|c|c|c|c|c|c|}
\hline Type ${ }^{(a)}$ & $\begin{array}{l}\text { Manufac- } \\
\text { turer }\end{array}$ & Position (b) & $\begin{array}{l}\text { Operation } \\
\text { Time (hr) }\end{array}$ & $\begin{array}{l}\text { Therma } 1 \\
\text { Shocks }\end{array}$ & $\begin{array}{c}\text { Fluence } \\
\left(\times 10^{20} \mathrm{n} / \mathrm{sq} \mathrm{cm}\right)\end{array}$ & $\begin{array}{c}\text { Ce11 } \\
\text { No. }\end{array}$ & Junction & $\begin{array}{l}\text { Avg } \\
\text { Temp } \\
\text { (C) }\end{array}$ & $\begin{array}{l}\text { Max } \\
\text { Temp } \\
\text { (C) }\end{array}$ \\
\hline \multicolumn{10}{|c|}{ Capsule FM-1 (Continued) } \\
\hline $\mathrm{K}$ & A & $\mathrm{C}$ & 1028 & 24 & 18.0 & 1 & Plugged & 495 & NA \\
\hline $\mathrm{K}$ & $B$ & $\mathrm{C}$ & 1028 & 24 & 18.0 & 1 & Plugged & 560 & NA \\
\hline $\mathrm{K}$ & A & $\mathrm{C}$ & 1028 & 24 & 18.0 & 1 & Plugged & 620 & NA \\
\hline $\mathrm{K}$ & $A$ & $\mathrm{C}$ & 494 & 8 & 9.0 & 2 & Plugged & 515 & NA \\
\hline $\mathrm{K}$ & A & $\mathrm{C}$ & 1028 & 24 & 18.0 & 2 & Plugged & 750 & NA \\
\hline $\mathrm{K}$ & A & $\mathrm{C}$ & 0 & 0 & 0 & 0 & Plugged & 0 & NA \\
\hline $\mathrm{K}$ & $\mathrm{A}$ & $\mathrm{C}$ & 1028 & 24 & 18.0 & 3 & Plugged & 520 & NA \\
\hline $\mathrm{K}$ & $\mathrm{A}$ & $\mathrm{C}$ & 1028 & 24 & 18.0 & 3 & Plugged & 485 & NA \\
\hline $\mathrm{K}$ & A & $\mathrm{C}$ & 1028 & 24 & 18.0 & 3 & Plugged & 425 & NA \\
\hline $\mathrm{K}$ & $B$ & $F$ & 1028 & 24 & 18.0 & 4 & Plugged & 835 & $\mathrm{NA}$ \\
\hline $\mathrm{K}$ & B & $\mathrm{C}$ & 1028 & 24 & 18.0 & 4 & Plugged & 375 & NA \\
\hline $\mathrm{K}$ & B & $\mathrm{C}$ & 1028 & 24 & 18.0 & 4 & Plugged & 375 & NA \\
\hline $\mathrm{K}$ & A & $\mathrm{C}$ & 1028 & 24 & 18.0 & 4 & Plugged & 360 & NA \\
\hline $\mathrm{K}$ & A & $\mathrm{F}$ & 1028 & 24 & 18.0 & 4 & Plugged & 735 & NA \\
\hline \multicolumn{10}{|c|}{ Capsule F7 - Startup 4/66 in MTR } \\
\hline W5 & B & $F$ & 3659 & 58 & 1.9 & 1 & Grounded & 1421 & NA \\
\hline W5 & $\mathrm{D}$ & $\mathrm{F}$ & 3659 & 58 & 1.9 & 1 & Grounded & 1421 & NA \\
\hline $\mathrm{K}$ & $\mathrm{B}$ & C & 3659 & 58 & 1.9 & 1 & Swaged & 525 & NA \\
\hline $\mathrm{K}$ & B & $\mathrm{C}$ & 3659 & 58 & 1.9 & 1 & Grounded & 770 & NA \\
\hline $\mathrm{K}$ & B & $\mathrm{C}$ & 3659 & 58 & 1.9 & 1 & Isolated & 285 & NA \\
\hline $\mathrm{K}$ & $B$ & $\mathrm{C}$ & 3659 & 58 & 1.9 & 1 & Swaged & 720 & NA \\
\hline $\mathrm{K}$ & $\mathrm{B}$ & $\mathrm{C}$ & 3659 & 58 & 1.9 & 1 & Grounded & 175 & NA \\
\hline \multicolumn{10}{|c|}{ Capsule G } \\
\hline W5 & $B$ & $\mathrm{~F}$ & 0 & 0 & 0 & 2 & Plugged & 0 & NA \\
\hline W5 & B & $\mathrm{F}$ & Oper (f) & & & 2 & Plugged & & NA \\
\hline W5 & B & $\mathrm{F}$ & Oper & & & 3 & Grounded $(c)$ & & NA \\
\hline W5 & $B$ & $\mathrm{~F}$ & Oper & & & 3 & Isolated & & $\mathrm{NA}$ \\
\hline $\mathrm{K}$ & A & $\mathrm{C}$ & Oper & & & 5 & Grounded ${ }^{(c)}$ & & NA \\
\hline $\mathrm{K}$ & $\mathrm{A}$ & $\mathrm{C}$ & 508 & 10 & 8.0 & 5 & Grounded(c) & 300 & NA \\
\hline $\mathrm{K}$ & A & $C$ & Oper & & & 5 & Grounded & & NA \\
\hline $\mathrm{K}$ & A & $\mathrm{C}$ & Oper & & & 1 & Grounded $(c)$ & & NA \\
\hline $\mathrm{K}$ & A & $\mathrm{F}$ & 583 & 14 & 10.0 & 1 & Grounded $(c)$ & 330 & NA \\
\hline $\mathrm{K}$ & A & $\mathrm{F}$ & 273 & 8 & 4.0 & 4 & Grounded(c) & 275 & $\mathrm{NA}$ \\
\hline $\mathrm{K}$ & A & $\mathrm{F}$ & 265 & 8 & 4.0 & 4 & Grounded $d^{(c)}$ & 305 & NA \\
\hline K & A & $F$ & 505 & 10 & 8.0 & 5 & Grounded(c) & 315 & NA \\
\hline $\mathrm{K}$ & A & $F$ & Oper & & & 5 & Grounded $(\mathrm{c})$ & & NA \\
\hline $\mathrm{K}$ & A & $\mathrm{C}$ & 429 & 9 & 7.0 & 1 & Grounded $(\mathrm{c})$ & 310 & $\mathrm{NA}$ \\
\hline $\mathrm{K}$ & A & $\mathrm{C}$ & Oper & & & 1 & Grounded ${ }^{(c)}$ & & $\mathrm{NA}$ \\
\hline $\mathrm{K}$ & A & $\mathrm{C}$ & 508 & 10 & 8.0 & $\overline{1}$ & Grounded $(C)$ & 325 & NA \\
\hline $\mathrm{K}$ & B & $\mathrm{C}$ & 275 & 8 & 4.0 & 2 & Grounded $(\varphi)$ & 425 & $\mathrm{NA}$ \\
\hline $\mathrm{K}$ & A & $\mathrm{C}$ & Oper & & & 2 & Grounded(c) & & $\mathrm{NA}$ \\
\hline $\mathrm{K}$ & B & $\mathrm{C}$ & 215 & 8 & 4.0 & 2 & Grounded $(c)$ & 405 & $\mathrm{NA}$ \\
\hline $\mathrm{K}$ & B & $\mathrm{C}$ & 429 & 9 & 7.0 & 3 & Grounded $(\theta)$ & 385 & $\mathrm{NA}$ \\
\hline K & A & $\mathrm{C}$ & Oper & & & 3 & Ground ed (c) & & $\mathrm{NA}$ \\
\hline $\mathrm{K}$ & B & $c$ & 429 & 9 & 7.0 & 3 & Grounded $(c)$ & 445 & $\mathrm{NA}$ \\
\hline $\mathrm{K}$ & A & $\mathrm{C}$ & 429 & 9 & 7.0 & 4 & Grounded(c) & 300 & NA \\
\hline $\mathrm{K}$ & A & $\mathrm{C}$ & Oper & & & 4 & Grounded $(\mathrm{c})$ & & NA \\
\hline $\mathrm{K}$ & $\mathrm{A}$ & $\mathrm{C}$ & 266 & 8 & 4.0 & 4 & Grounded $(\mathrm{C})$ & 290 & $\mathrm{NA}$ \\
\hline
\end{tabular}

(a) ${ }^{2} 3=W-3 \% \operatorname{Re} / W-25 \% \operatorname{Re} ; W 5=W-5 \% \operatorname{Re} / W-26 \% \operatorname{Re} ; K=$ Chrome1-Alumel (a11 $\mathrm{K}$ thermocouples were in graphite).

(b) $\mathrm{F}=$ fuel; $\mathrm{C}=\mathrm{can}$.

(c) H, F. Bothman, Gulf General Atomic.

(d) Not avatlable.

(e) Plugged = grounded plug.

(f) Oper - still in operation at time data were recorded. 


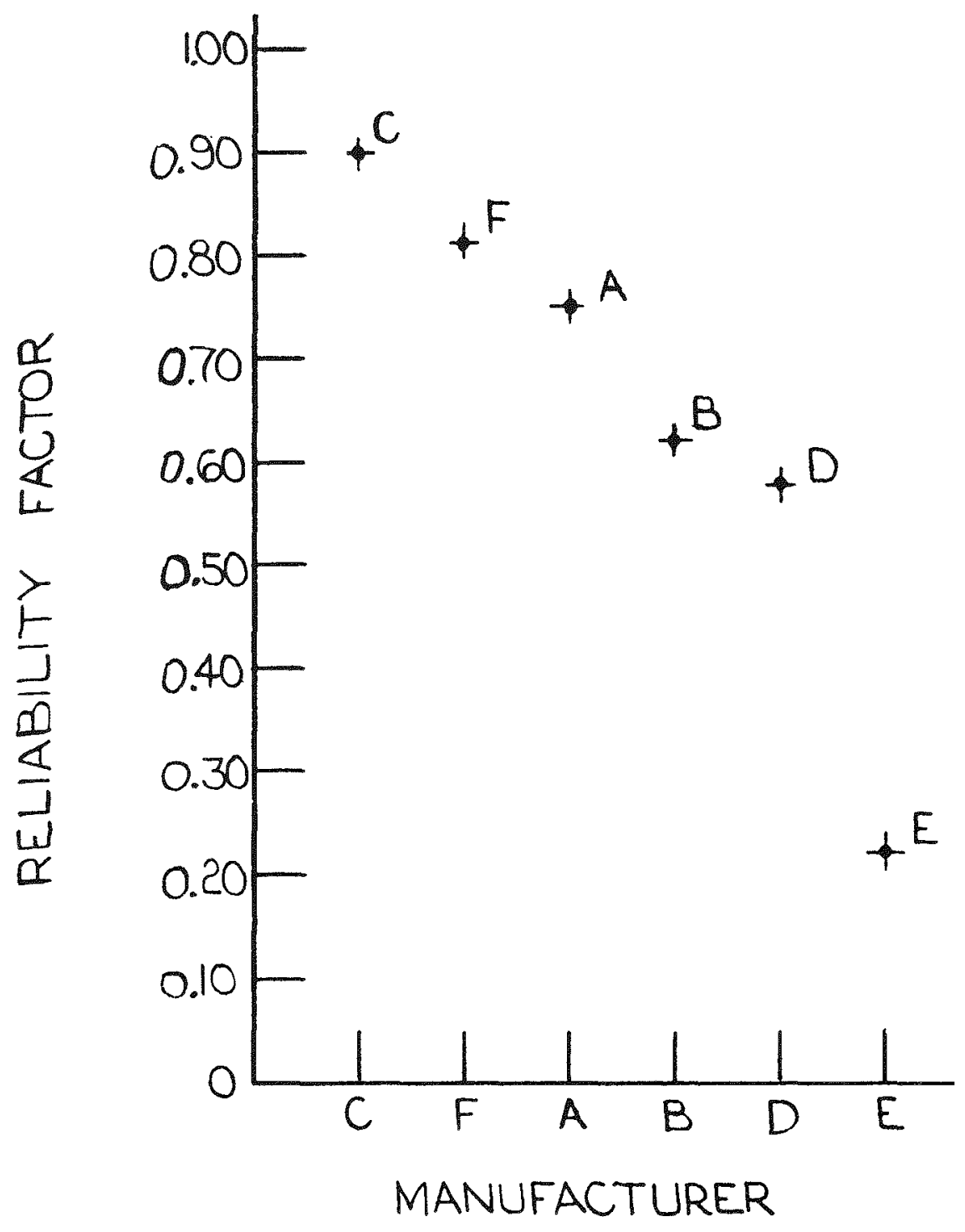

Fig. 2. Reliability factor versus manufacturer of $\mathrm{C} / \mathrm{A}, \mathrm{W}-3 \% \operatorname{Re} / \mathrm{W}-25 \% \operatorname{Re}$, and $\mathrm{W}-5 \% \operatorname{Re} / \mathrm{W}-26 \% \operatorname{Re}$ thermocouples, indicating performance during irradiation 
A comparison of GGA-spec thermocouples and commercial nuclear service thermocouples was completed in computer sorting run $B$. The GGA-spec is an attempt to control thermocouple quality by specifying high quality materials procurement and controlling thermocouple manufacturing processes. This kind of thermocouple was compared with a commercially available thermocouple that was specified for nuclear service.

The data from this sorting run (Table III) indicated that the commercial nuclear service thermocouples, which had a reliability factor of 0.69 , performed better during irradiation than the GGA-spec thermocouples, which had a reliability factor of 0.57 .

TABLE III

PERFORMANCE OF THERMOCOUPLES MADE TO THE GULF GENERAL ATOMIC SPECIFICATION AND COMMERICAL NUCLEAR SERVICE THERMOCOUPLES DURING IRRADIATION

\begin{tabular}{|c|c|c|c|c|c|}
\hline \multirow{2}{*}{$\begin{array}{c}\text { Thermocouple } \\
\text { Type }\end{array}$} & \multicolumn{2}{|c|}{ Good } & \multirow[b]{2}{*}{$\mathrm{Bad}$} & \multirow[b]{2}{*}{ Total } & \multirow[b]{2}{*}{ Reliability Factor, } \\
\hline & No. & $\%$ & & & \\
\hline $\begin{array}{l}\text { GGA-Spec } \\
\text { Commercial }\end{array}$ & $\begin{array}{l}40 \\
86\end{array}$ & $\begin{array}{l}50 \\
67\end{array}$ & $\begin{array}{l}40 \\
42\end{array}$ & $\begin{array}{r}80 \\
128\end{array}$ & $\begin{array}{l}0.57 \\
0.69\end{array}$ \\
\hline
\end{tabular}

The next sorting (run C) is plotted in Fig. 3, which shows the reliability factor versus the thermocouple position in the capsule. This graph illustrates the behavior of thermocouples that operate low in the reactor core (cell 1) and in higher parts (cells 2, 3, 4, and finally 5). The neutron exposure can vary by a factor of two if the thermocouple is moved from cell 5 to cell 2. A thermocouple in cell 1 would have $30 \mathrm{in}$. of thermocouple in the core, while a thermocouple in cell 5 would have only 6 in. in the core. Figure 3 shows that the position of the thermocouple in the capsule appears to have little effect on thermocouple performance.

Figure 4, sorting run D, shows a graph of accumulated fast-neutron exposure versus percent of failed thermocouples. Failures increased with increasing accumulated neutron exposure. This shows that neutron exposure is an important variable and indicates that a thermocouple tends to fail more of ten if given enough neutron exposure. This graph indicates that $40 \%$ of the thermocouples exposed to a fast-neutron exposure

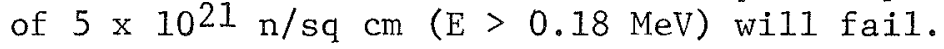

Computer sorting run $\mathrm{E}$ identified the performance of $\mathrm{C} / \mathrm{A}, \mathrm{W}-3 \% \mathrm{Re} /$ $\mathrm{W}-25 \% \mathrm{Re}$, and $\mathrm{W}-5 \% \operatorname{Re} / \mathrm{W}-26 \%$ Re thermocouples during irradiation tests. These data are listed in Table IV. The $W-5 \% \operatorname{Re} / \mathrm{W}-26 \% \operatorname{Re}$ thermocouples performed best with a reliability factor of 0.74 . The C/A and $\mathrm{W}-3 \% \mathrm{Re} / \mathrm{W}-25 \%$ Re thermocouples had reliability numbers of 0.64 and 0.62 , respectively, which indicates very little performance difference between the two. 


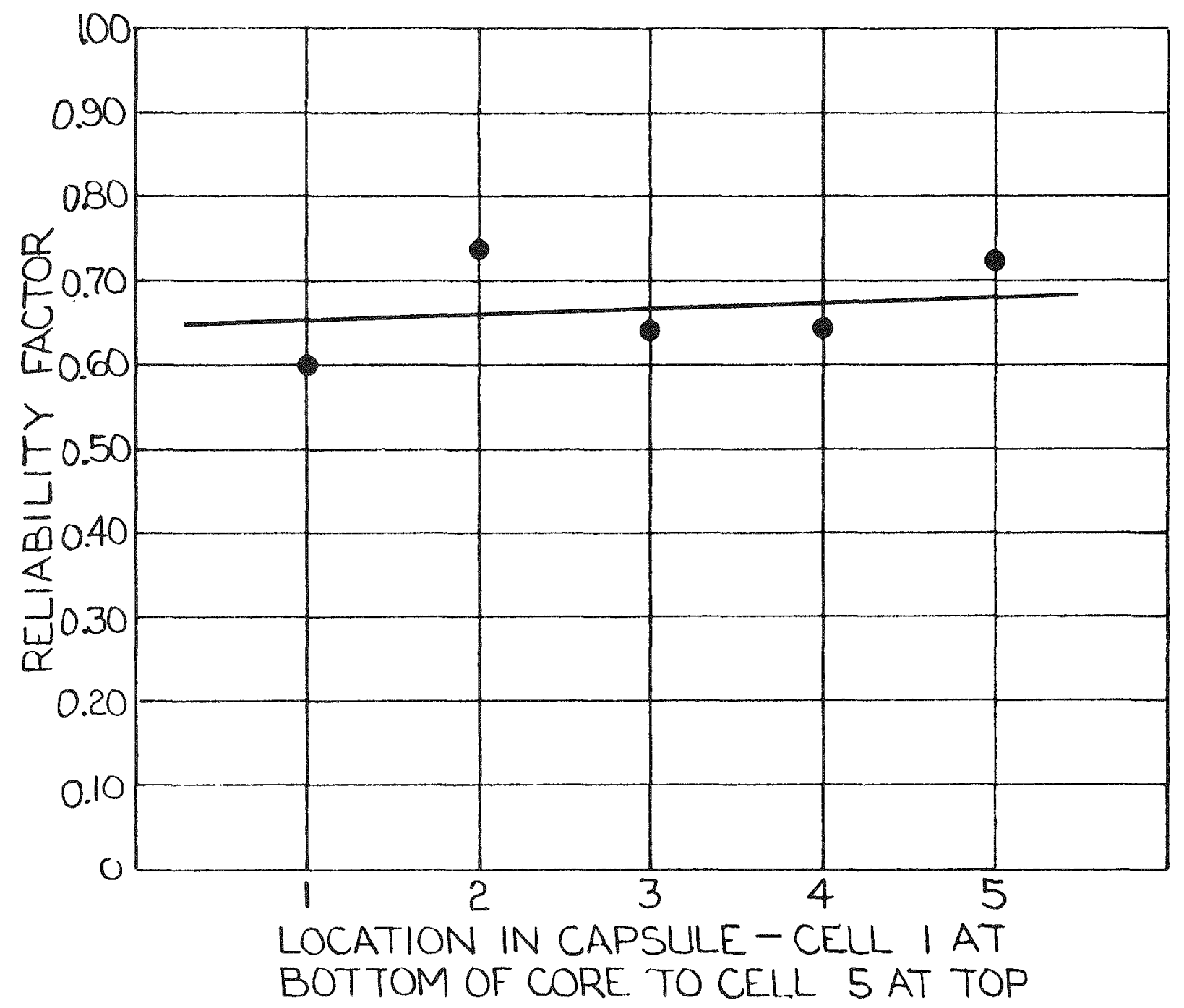

Fig. 3. Reliability factor versus location of thermocouples (C/A, W - 3\% $\operatorname{Re} / \mathrm{W}-25 \% \operatorname{Re}$, and $\mathrm{W}-5 \% \operatorname{Re} / \mathrm{W}-26 \% \operatorname{Re})$ within capsule 


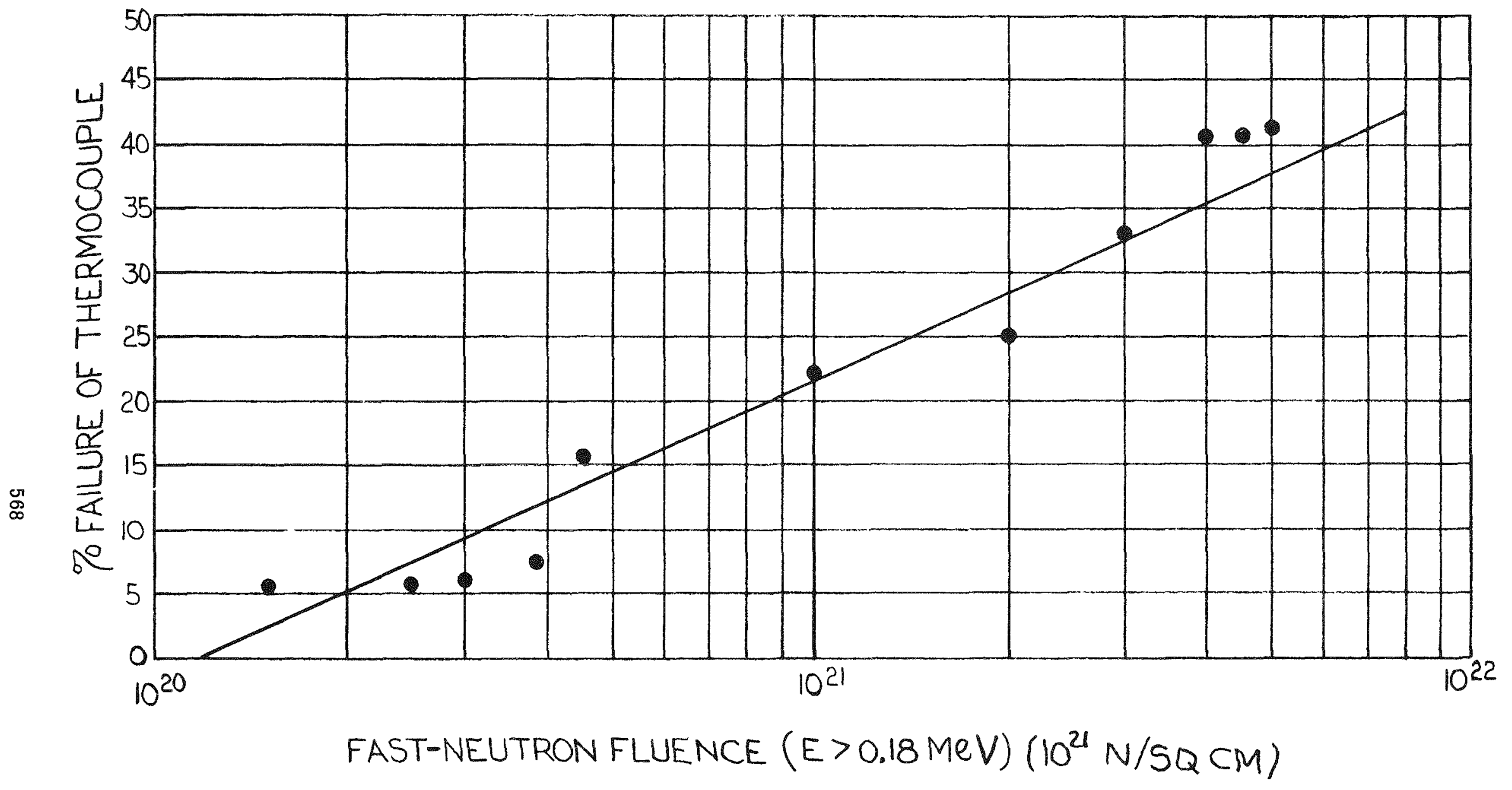

Fig. 4. Percent of failure (C/A, W - 3\% Re/W - 25\% Re, and $W-5 \% \operatorname{Re} / \mathrm{W}-26 \%$ Re thermocouples) versus accumulated fast-neutron fluence 
TABLE IV

BEHAVIOR DATA OF C/A, W - 3\% Re/W - 25\% Re, AND $W-5 \% \operatorname{Re} / W-26 \% \operatorname{Re}$ THERMOCOUPLES USED IN IRRADIATION CAPSULES

\begin{tabular}{c|c|c|c|c|c}
\hline \multirow{2}{*}{$\begin{array}{c}\text { Thermocouple } \\
\text { Type }\end{array}$} & \multicolumn{2}{|c|}{ Good } & & \\
\cline { 2 - 3 } & No. & $\%$ & Bad & Total & Reliability Factor, $R$ \\
\hline C/A & 58 & 58 & 42 & 100 & 0.64 \\
W - 3\% Re & 27 & 58 & 20 & 47 & 0.62 \\
W- $5 \% \operatorname{Re}$ & 46 & 70 & 20 & 66 & 0.74 \\
\hline
\end{tabular}

The next grouping (run F) compared the performance of different types of hot junctions. Four principal types of hot junctions were considered: grounded, grounded-swaged, grounded-plug, and isolated. Data from this run (Table V) indicate that thermocouples with a grounded-plug or a grounded hot junction perform best. The reliability numbers for the grounded-plug and grounded hot junctions were 0.70 and 0.68 , respectively, showing very little difference between these two. Thermocouples with isolated hot junctions showed the next best performance, followed by thermocouples with grounded-swaged hot junctions. However, only four samples of thermocouples with isolated hot junctions were used in obtaining these data.

TABLE V

PERFORMANCE OF THERMOCOUPLES WITH VARIOUS HOT JUNCTIONS DURING IRRADIATION

\begin{tabular}{l|r|r|r|r|r}
\hline \multirow{2}{*}{$\begin{array}{l}\text { Thermocouple } \\
\text { Type }\end{array}$} & \multicolumn{2}{|c|}{ Good } & \multirow{2}{*}{ Bad } & Total & Reliability Factor, R \\
\cline { 2 - 5 } & No. & $\%$ & & \\
Grounded & 98 & 64 & 56 & 154 & 0.68 \\
Grounded-swaged & 2 & 12 & 4 & 6 & 0.39 \\
Grounded-plug & 37 & 66 & 19 & 56 & 0.70 \\
Isolated & 2 & 50 & 2 & 4 & 0.45 \\
\hline
\end{tabular}

Data on hours of thermocouple operation versus percent failed were obtained in computer sorting run $G$. These data show the effect of long times in a neutron flux field on thermocouple performance. The graph of these data, Fig. 5, indicates that thermocouple failure increases as the time in a neutron flux environment increases.

The next sorting (run $H$ ) was done to show the effects of location of the thermocouple within a particular cell of a capsule. The data are listed in Table VI and show there is no preference for position in the capsule. 


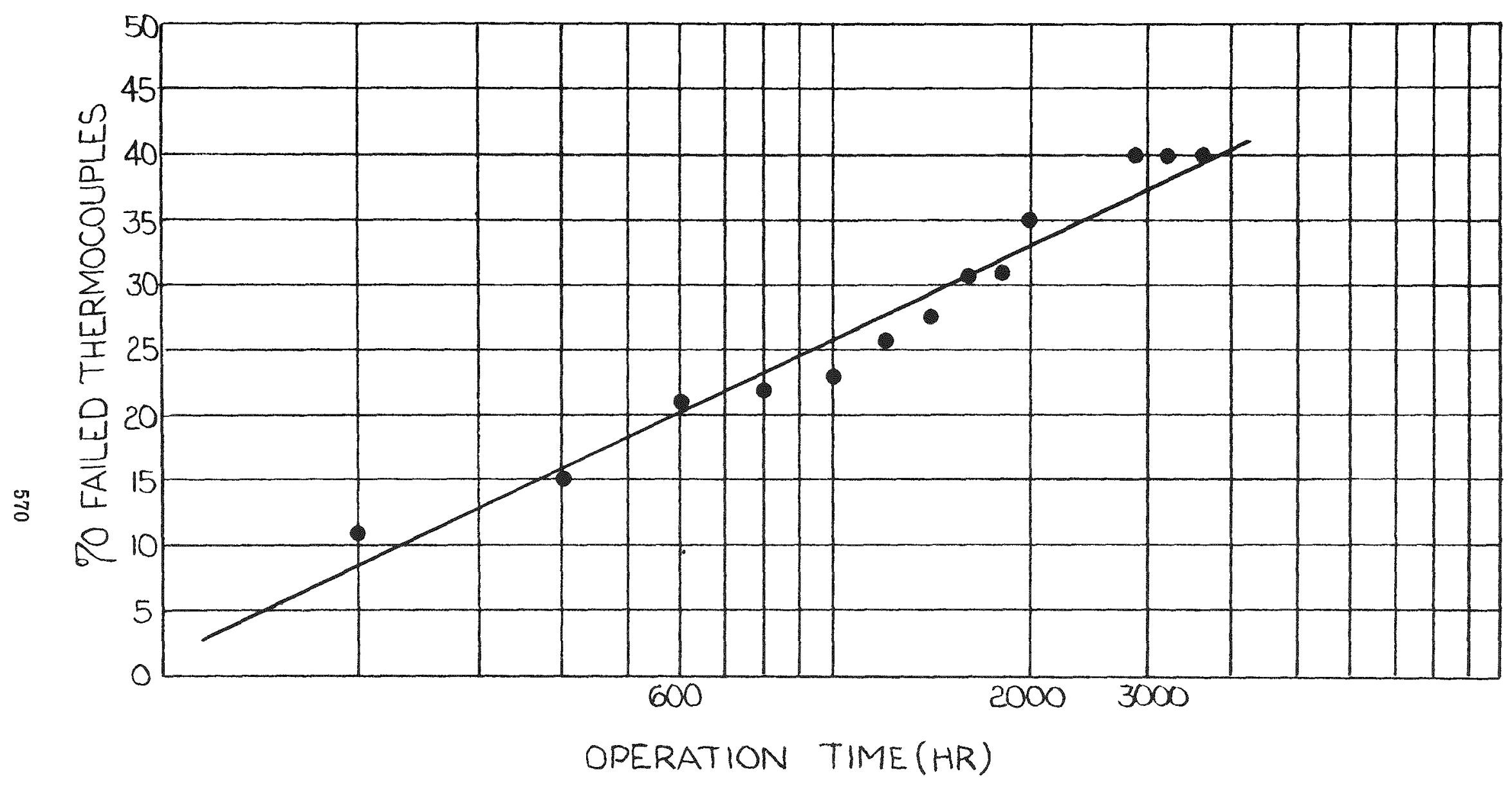

Fig. 5. Percent of failure (C/A, W - 3\% Re/W - 25\% Re, and $W-5 \% \operatorname{Re} / \mathrm{W}-26 \%$ Re thermocouples) versus hours of operation 
TABLE VI

PERFORMANCE OF THERMOCOUPLES POSITIONED IN FUELED SECTIONS AND OUTSIDE FUELED SECTIONS DURING IRRADIATION

\begin{tabular}{l|c|c|c|c|c}
\hline \multirow{2}{*}{ Location } & \multicolumn{2}{|c|}{ Good } & \multirow{2}{*}{ Bad } & Total & Reliability Factor, $R$ \\
\cline { 2 - 5 } Fuel & 76 & 60 & 52 & 128 & 0.64 \\
Can & 44 & 60 & 29 & 73 & 0.65 \\
\hline
\end{tabular}

Data from the final sorting (run I, operation temperature versus percent failed) is shown in Fig. 6. These data are scattered but the trend indicates that as the temperature of operation increases, the probability of a thermocouple operating successfully increases very slightly.

\section{COMPUTER SORTING CONCLUSIONS}

The computer sorting technique for analysis of thermocouple performance data has indicated several trends, as follows:

1. The manufacturer of the thermocouples is important. In this study the best thermocouple was obtained from manufacturer $\mathrm{C}$.

2. Commercial nuclear service thermocouples perform well, but each lot should be inspected and tested by the user before insertion into an experiment.

3. The position of a thermocouple in an irradiation capsule is not important. The study indicated that thermocouples in fueled and nonfueled areas perform equally well.

4. Thermocouple failure increases with increasing accumulated fast-neutron exposure. For an exposure of $5 \times 10^{21} \mathrm{n} / \mathrm{sq} \mathrm{cm}$, $40 \%$ failure should be expected.

5. In comparison with the other type thermocouples, the $\mathrm{W}-5 \% \mathrm{Re} / \mathrm{W}-$ $26 \% \mathrm{Re}$ thermocouples operate best in a neutron environment when measuring temperatures in the 1000 to $1800 \mathrm{C}$ range.

6. Thermocouples with a grounded-plug or grounded hot junction perform best in a neutron environment.

7. Thermocouple failure increases with increasing time in a neutron environment. For an operation time of $3500 \mathrm{hr}, 40 \%$ failure should be expected.

8. Thermocouple operation temperature does not appear important. 


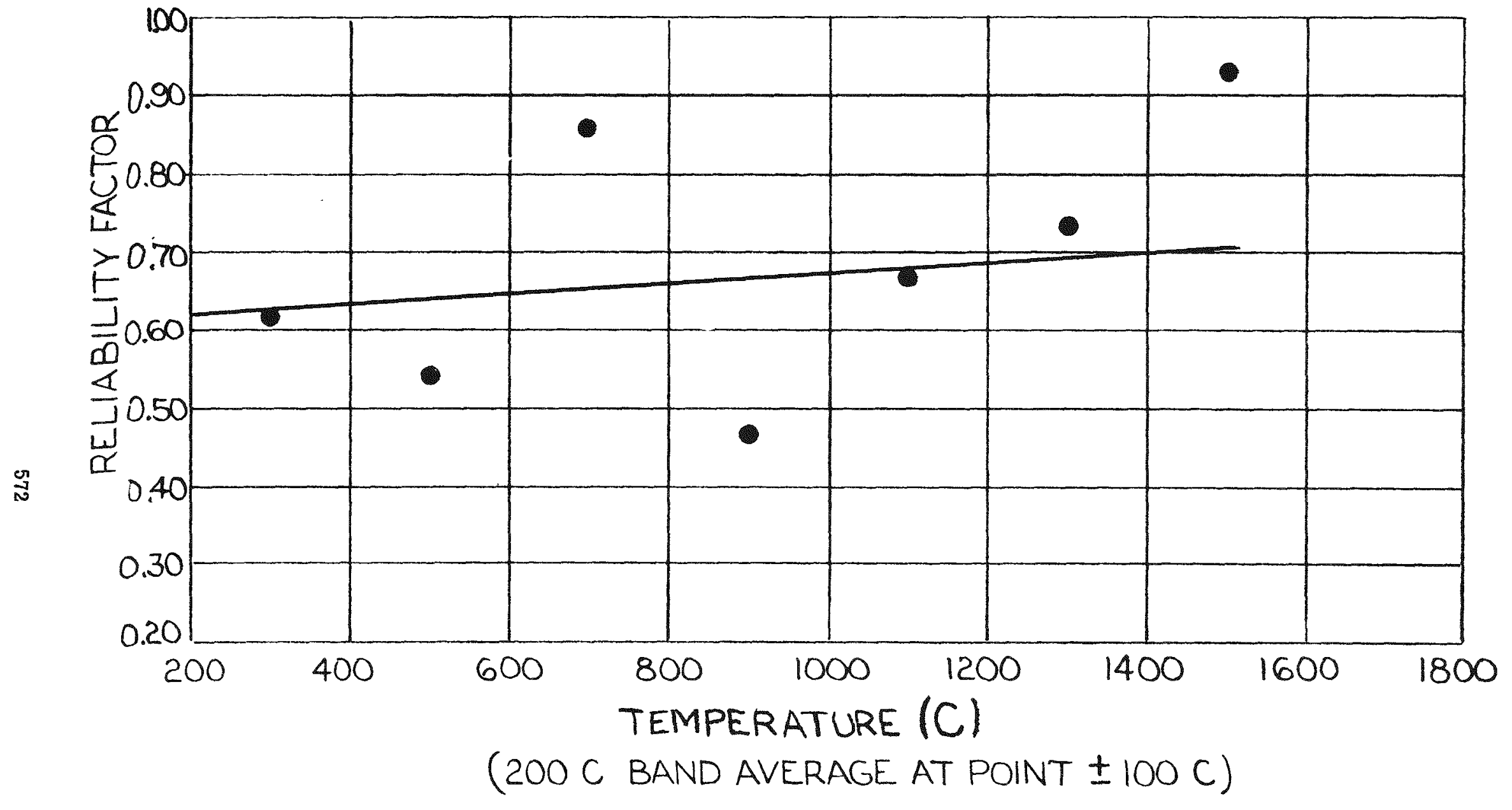

Fig. 6. Reliability factor versus temperature of operation for C/A, W - 3\% Re/W-25\% Re, and $W-5 \%$ Re/W - 26\% Re thermocouples 
A systematic program was initiated to investigate and identify the causes of thermocouple failure. Postirradiation continuity and metallographic analyses were conducted on selected thermocouples. Since the thermocouples were radioactively "hot," the Gulf General Atomic hot cell was used for the remote examination.

The capsule was divided into three areas (see Fig. 7). The first area included from the primary cells to the first upper end cap. The second area included both upper end caps. The third area extended from the second area to include the epoxy transition section and within $7 \mathrm{ft}$ of the junction box. These three areas include all parts that penetrate into the reactor tank and within the reactor core.

PRE-HOT-CELL WORK

Area 3, which includes the epoxy transition junction, was checked for continuity at the end of the irradiation period. Due to the low activity of this area, it could be handled without shielding. This area was checked on four capsules, and no failures were found in checks of 80 different thermocouples. Therefore, this area is considered trouble-free.

HOT CELL WORK

Area 2, which includes the transition junction, was examined on three capsules. This area, as well as area 1, was handled inside a hot cell with mechanical manipulators. Both continuity checks in succeeding lengths of the thermocouple and metallography technique were used to identify failures of broken wires.

Continuity Checks

To make the continuity checks, repeated attempts were made to strip the ends of the thermocouples and clip on lead wires; to cut the ends flush and probe with a needle-like contact point; or to put one end of the thermocouple in mercury, thereby leaving only one end to probe. None of these attempts was successful.

Metallography

Capsule P16 (fast-neutron exposure of $2.5 \times 10^{21} \mathrm{n} / \mathrm{sq} \mathrm{cm}$ ) was cut apart and seven hot junctions were removed. These were clipped with wire snips and then placed in a cup side by side for mounting and polishing. The mounted specimen was examined by meta1lographic techniques, and it was found that three of the seven thermocouples were broken at the hot junction (see Fig. 8). No correlation of the failures shown here to operational behavior, whether good or bad, could be made. 


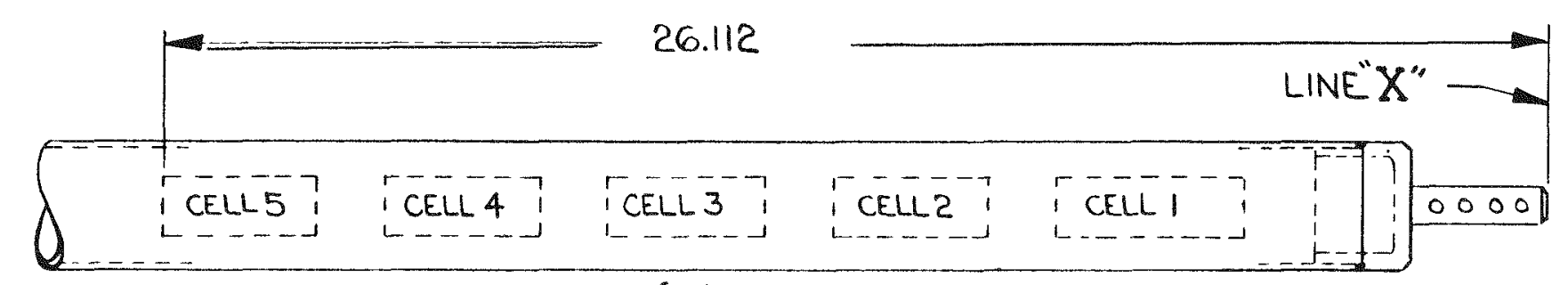

( 1 )

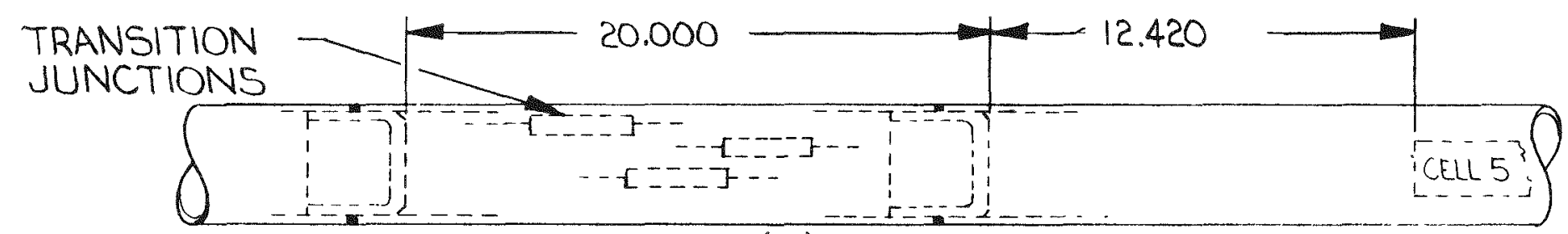

(2)

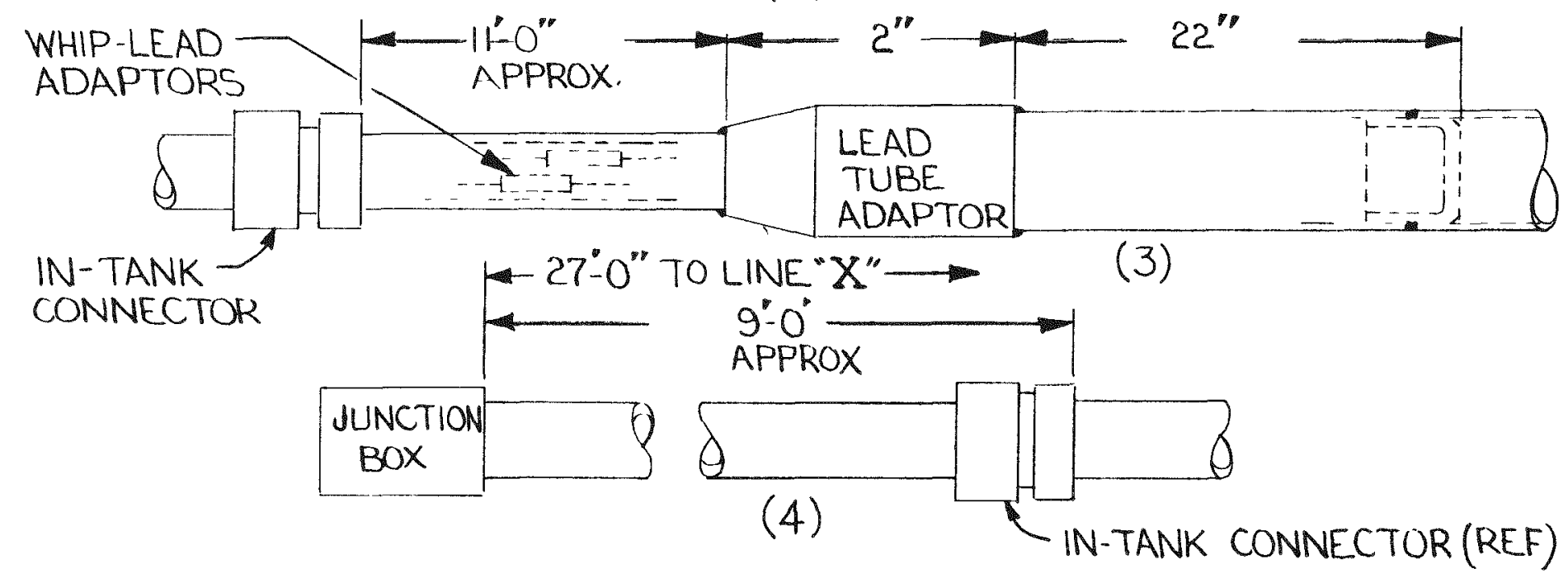

Fig. 7. Thermocouple examination areas for failure mode analyses of irradiation experiments (areas 1 through 4 equal the complete capsule) 

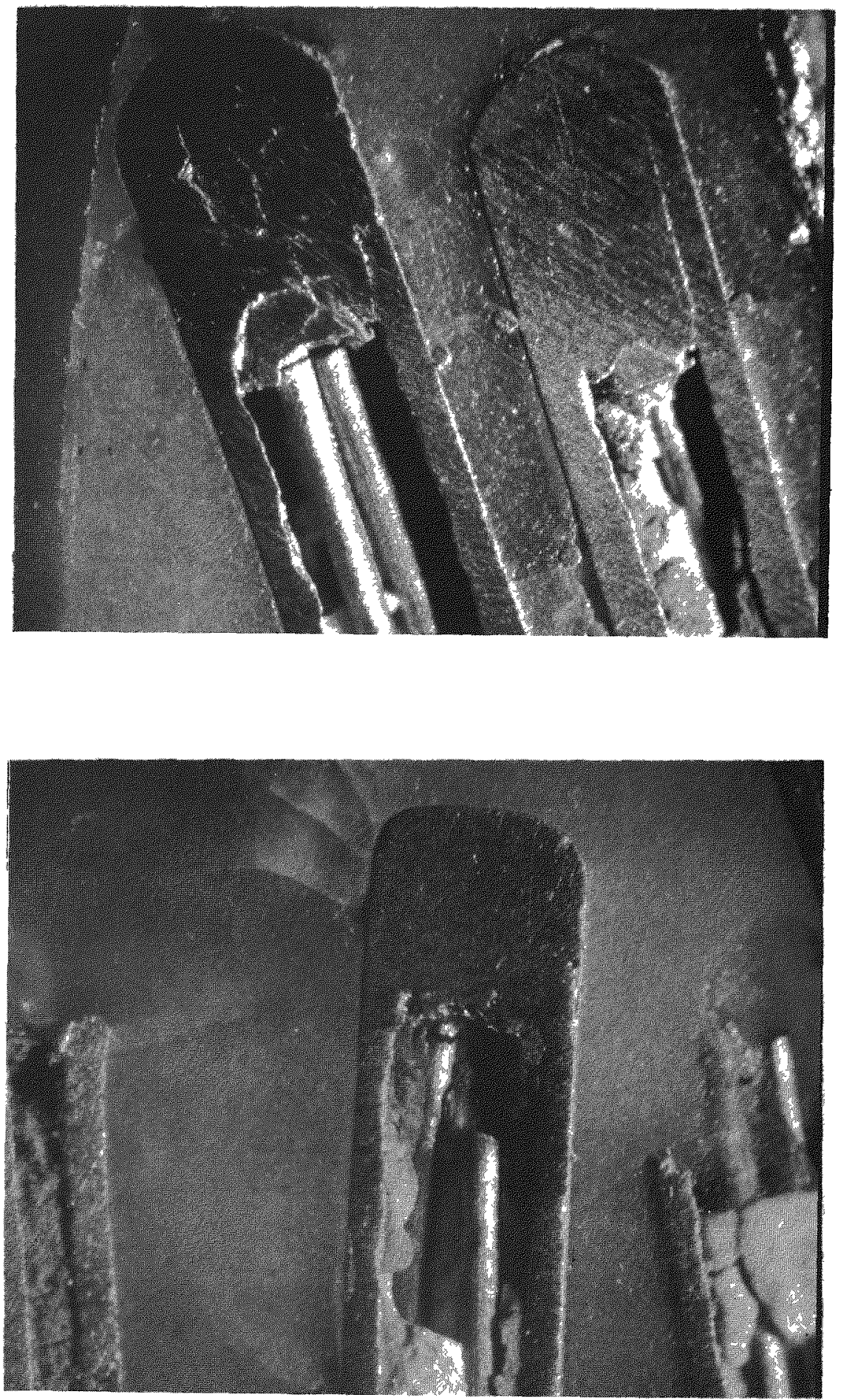

Fig. 8. Postirradiation photomicrographs of hot junctions showing failures (Capsule $\mathrm{P} 18)(18 \mathrm{X})$ 
To confirm these findings and to show whether hot junctions are the only problem area, another experiment was conducted with capsule P13J (fast-neutron exposure of $3.5 \times 1021 \mathrm{n} / \mathrm{sq} \mathrm{cm}$ ) and capsule $\mathrm{G}-12$ (fast-neutron exposure of $5.5 \times 1021 \mathrm{n} / \mathrm{sq} \mathrm{cm}$ ). Each thermocouple chosen for postirradiation metallography was known to have operated at the end of the irradiation cycle or to have failed pervious to this point. For further confirmation, a continuity check was made at the side of the reactor in the junction box. Al1 data were confirmed at this point.

In the Gulf General Atomic hot cell, 10 thermocouples including both $\mathrm{C} / \mathrm{A}$ and $\mathrm{W} / \mathrm{Re}$ types, some operative and some failed at the end of irradiation, were chosen from capsule P13J. In the same manner, seven were chosen from capsule G-12. Al1 17 thermocouples were identified and mounted as previously described for capsule P16.

The mounted specimens from capsules $P 13 \mathrm{~J}$ and $\mathrm{G}-12$ were examined for failed hot junctions. Photomicrographs of the P13J thermocouples showed one failed (see Fig. 9a) and nine good hot junctions. However, the failed one operated in the reactor to shutdown, while none of the nine good ones were operating before reactor shutdown. Photomicrographs of the G-12 thermocouple hot junctions (see Fig. 9b) indicated similar results. Of the seven thermocouples examined, none operated before reactor shutdown and only two failures could be found in the postirradiation metallography.

Postirradiation Conclusions

The conclusion of the hot cell metallography of hot junctions is that some failures may occur in this area, but it is not likely the primary cause of failure of thermocouples.

\section{ACKNOWLEDGMENTS}

Significant contributions to the development of the computer program and thermocouple data described in this paper were made by $R$. Abbey, H. Bothman, L. Burleigh, G. Chandler, B. Czech, G. Forbes, G. Graeber, and L. D. Scott. This work was supported by the U.S. Atomic Energy Commission under Contract AT(04-3)-167, Project Agreement No. 17. 

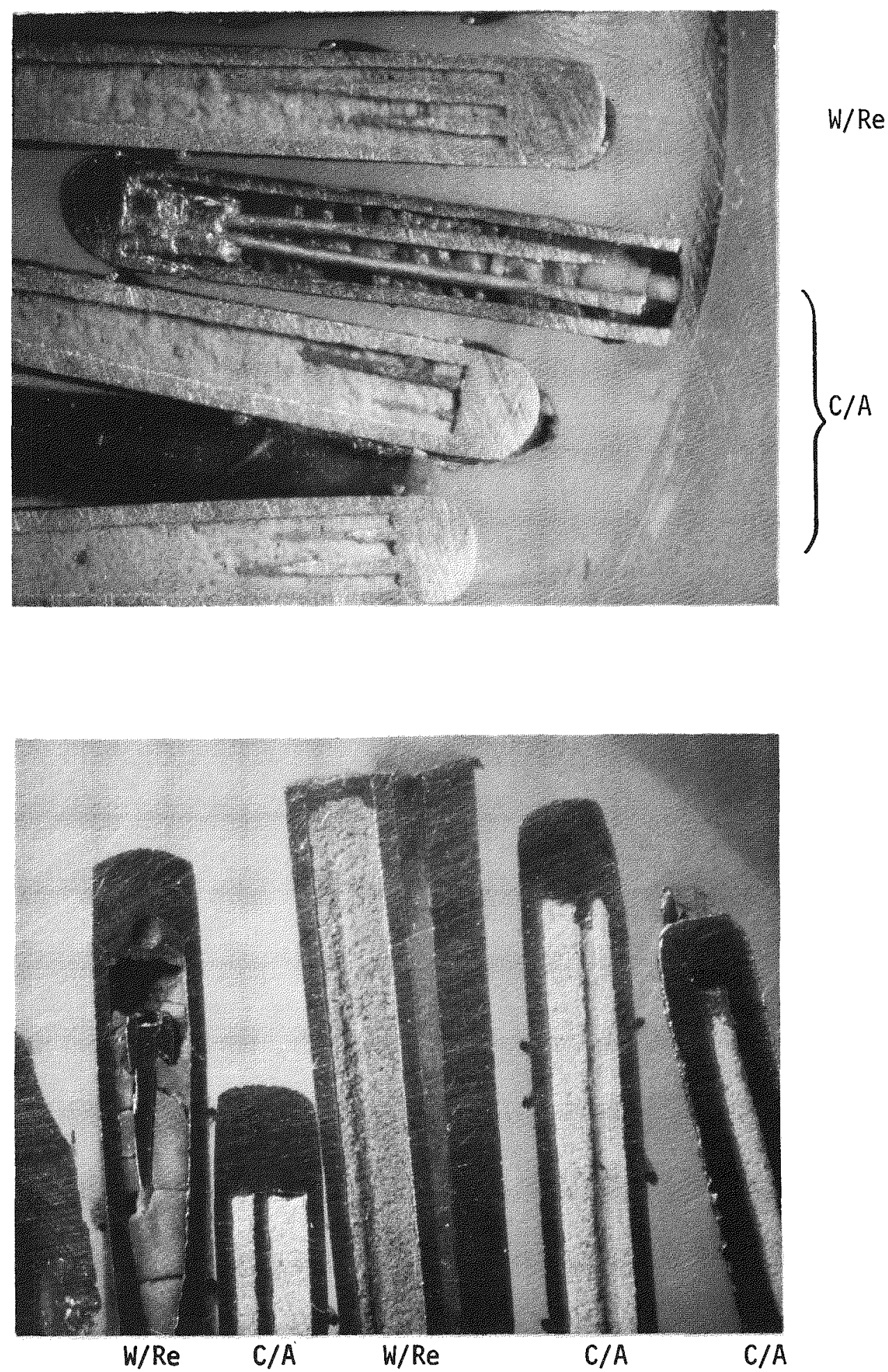

Fig. 9. Postirradiation photomicrographs of hot junctions showing good and failed junctions. Top, capsule P13J; bottom, capsule G-12 (9X) 
APPENDIX

GULF GENERAL ATOMIC SPECIFICATION FOR IRRADIATION CAPSULE THERMOCOUPLES

\begin{tabular}{|l|l|l|l|l|}
\hline \multicolumn{1}{|c|}{ THERMOCOUPLES FOR IRRADIATION CAPSULES } \\
\hline Reference Specification RI-4-1 & I s sue A & Date 3-5-69 & Page I of 5 \\
\hline
\end{tabular}

1. INFORMATION AND GENERAL REQUIREMENTS

1.1 SCOPE: This reference specification constitutes the standard requirements of Gulf General Atomic for thermocouples for ixradiation capsules.

1.2 CONFLICTS: Nothing in this reference specification sha11 relieve the Seller from compliance with the drawings and specifications specifically applicable to this procurement. Such drawings and specifications shall take precedence over this reference specification in the event of conflict.

1.3 SUMMARY OF WORK INCLUDED: The Seller shall provide all labor, material, equipment and services required for the fabrication, quality control, calibration, testing and shipment of a completely functional thermocouple assembly in accordance with this specification and other contract documents.

2. DIMENSIONAL REQUTREMENTS

2.1 OUTSIDE DIAMETER of thermocouple sheath sha11 be no more than 0.0630 inch and no less than 0.0610 inch when measured at any point along the length of the sheath.

2.2 WALL THICKNESS of the sheath shall be no more than 0.0120 inch and no less than 0.0080 inch when measured at any point along. the length of the sheath. No sharp discontinuities in thickness of the wall shall be present. Cracks in the sheath are not acceptable. Determination of cracks will be made visually, by fleurescent penetrant inspection or by helium leak testing.

2.3 LENGTH OF ASSEMBLIES sha11 be as specified on other contract documents $\pm 1 / 2$ inch.

2.4 WIRE DIAMETER shal1 be a minimum of 0.0080 inch and a maximum of 0.0110 inch when measured at any point along the length of the wire.

2.5 INSULATION LENGTH shall be as specified on other contract documents $\pm 1 / 2$ inch.

2.6 INSULATION DENSITY after fabrication shal1 be (a) approximately 80-90\% of theoretical density of ceramic used, or (b) as dense as possible without excessive work-hardening of the sheath or wires. Example: no "gripping" or "necking" down of wires by insulation material.

2.7 JUNCTIONS: The hot junction shall be a grounded plug junction unless exception is taken on other contract documents. The cold function shall be welded closed and shall be helium leak-tight.

3. PURITY REQUIREMENTS

3.1 THERMOCOUPLE WIRE shall be Type $\mathrm{K}$ conforming to Table III of ASTM E 230. Standard limits of error shall correspond to Table XV of ASTM E 230. Thermocouple wire shall meet the cleanliness requirements of ASTM E 235 .

\begin{tabular}{|l|l|l|}
\hline Classification Instruments & By N.Sandefur & Approved F.Carpenter \\
\hline
\end{tabular}




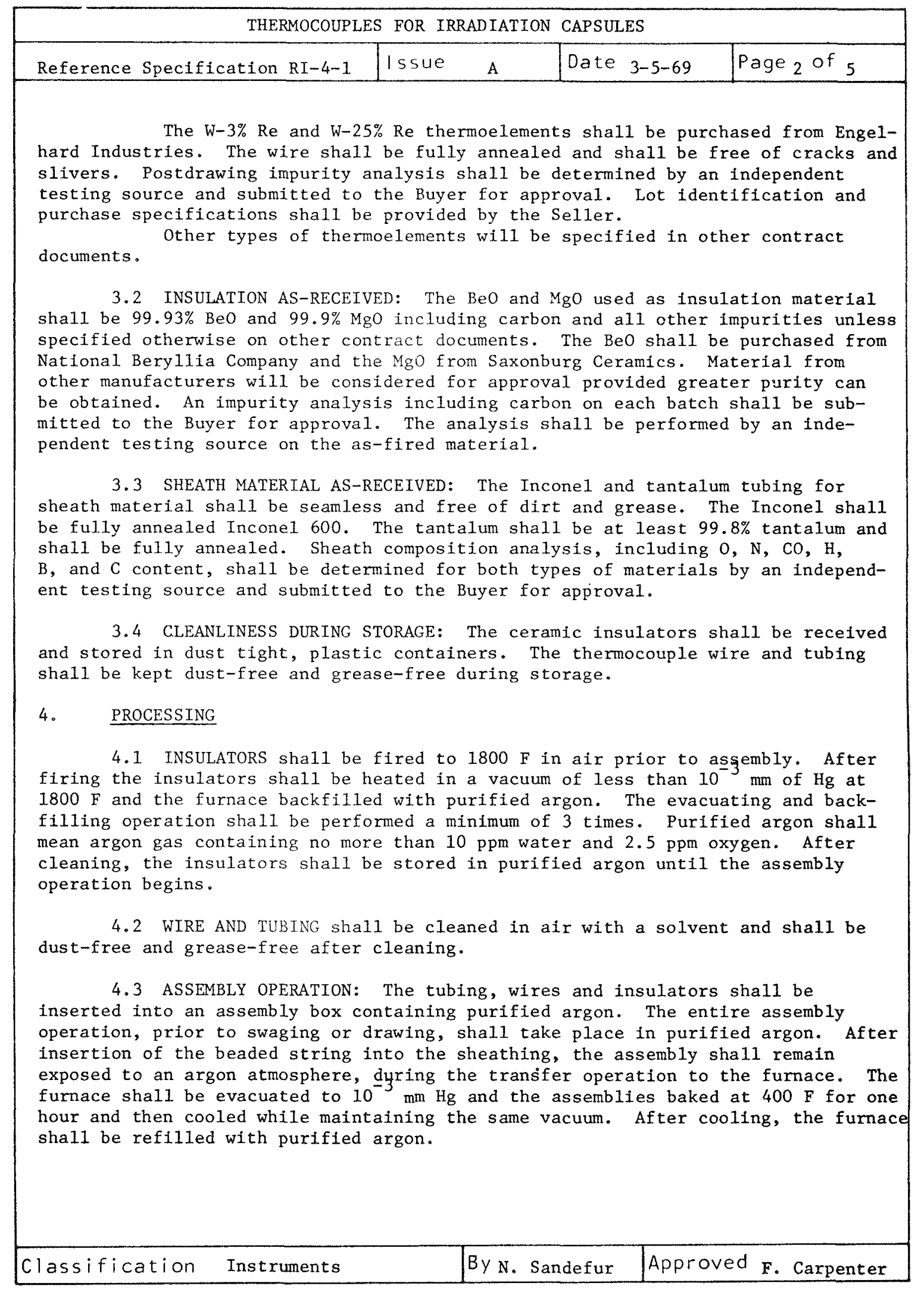




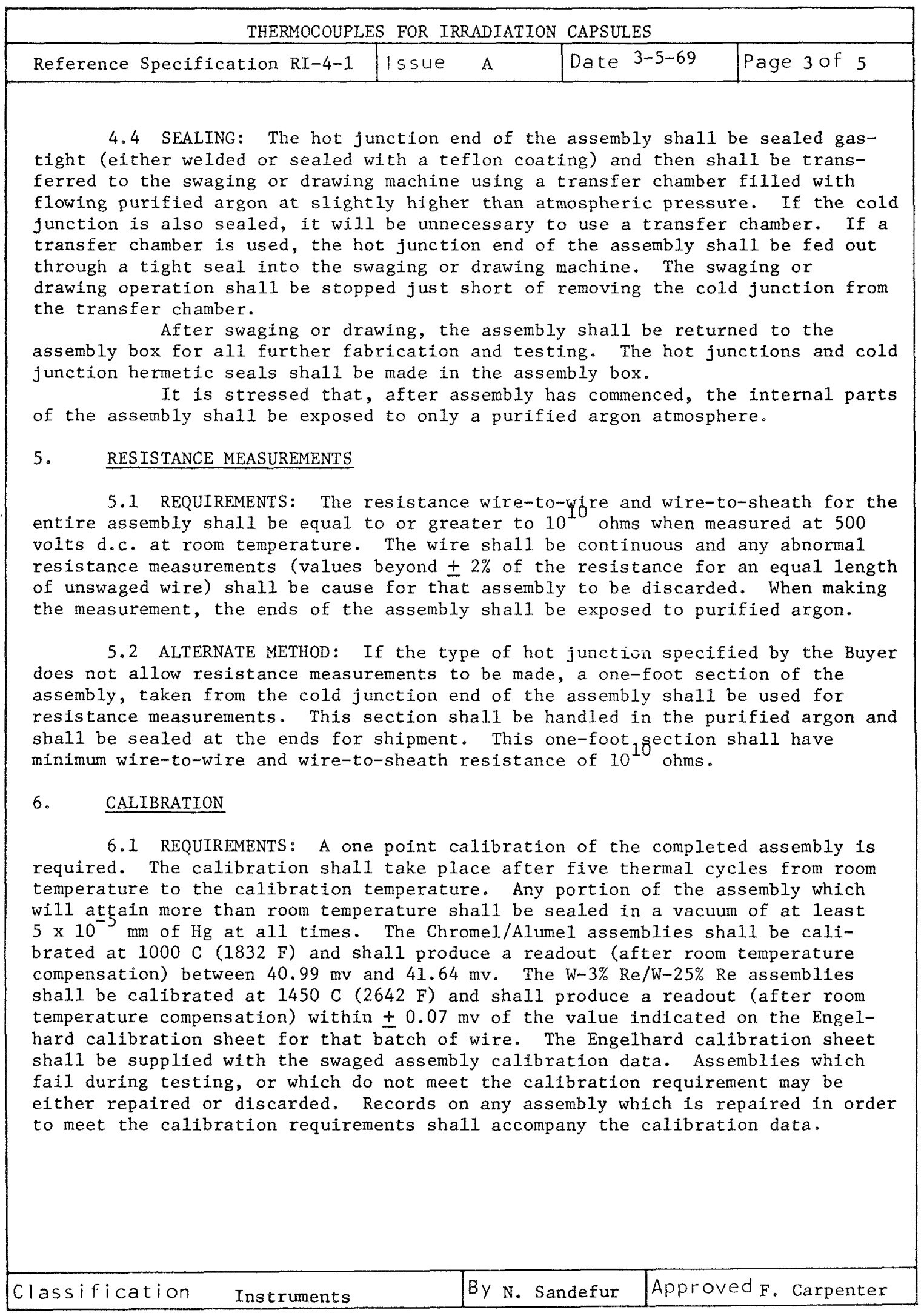




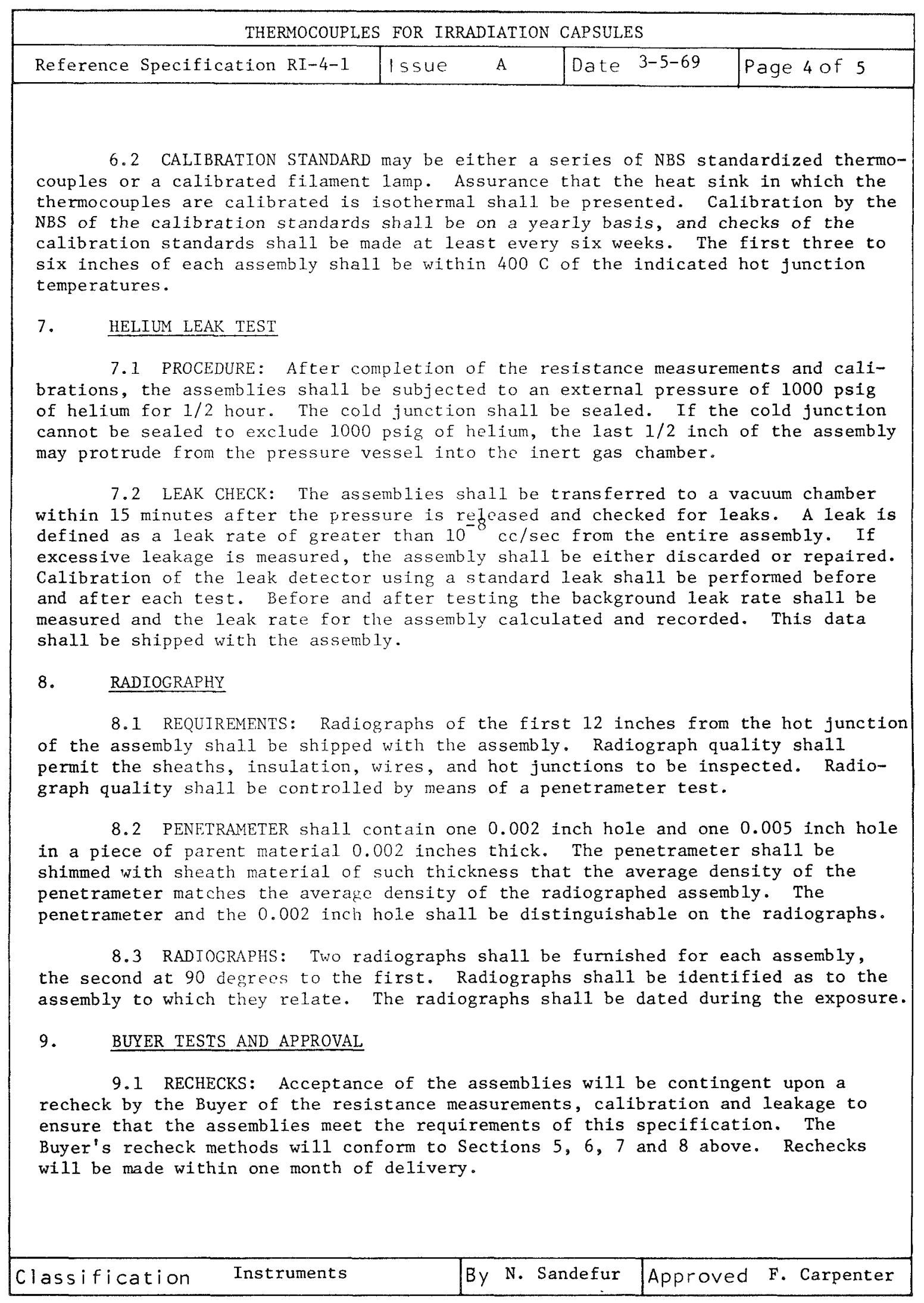




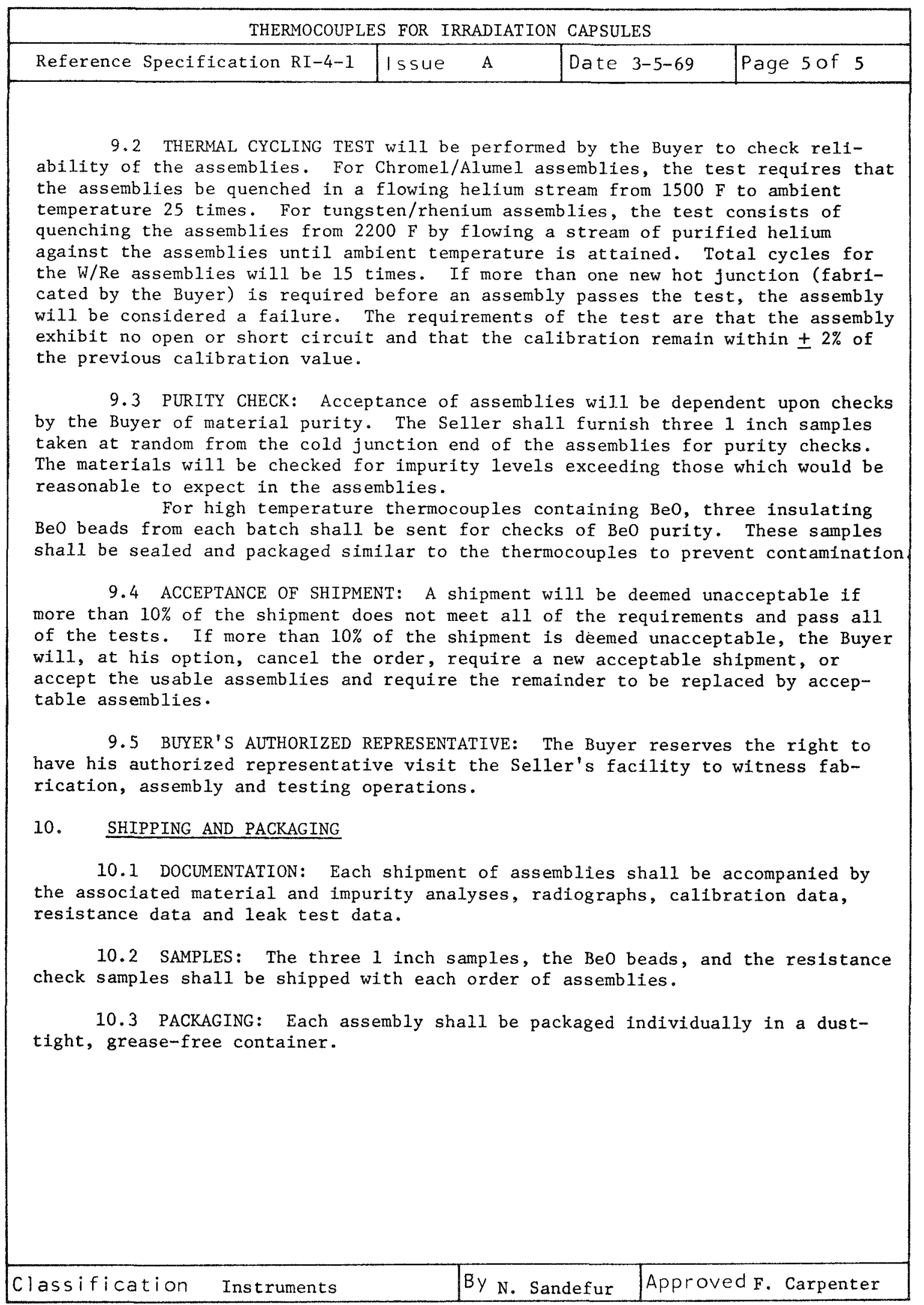




\section{CRICET, A SYSTEM FOR REMOTE MEASUREMENT \\ OF FUEL-ELEMENT TEMPERATURES *}

by

J. S. Buck

Atomics International Division of

North American Rockwell Corporation

The CRICET ${ }^{\dagger}$ system has been devised to remotely monitor temperature distributions within individual experimental fuel elements located in fast and thermal reactor cores. The system design is unique in that no in-vessel instrumentation leads or vessel penetrations are used to accomplish this function. Self-contained compact transmitter units are included within the structures of individual fuel elements. During reactor operation the transmitter sends a pulsed acoustic signal, with a pulse frequency proportional to the temperature measured, to acoustic pickups and noise analysis instrumentation outside the vessel, where the intelligence is interpreted and recorded.

In the situation where several driver elements of a power reactor core are outfitted with CRICET transmitters, the information measured will be valuable to reactor operation, reactor safety, and fuel-element engineering. The direct measurement of element hot-spot temperatures will reduce the limitations imposed by analytical safety factors and thereby allow operation at higher power. As a reactor safety aid the system will indicate off-normal temperature conditions which could cause a fission-product release incident, thereby allowing preventative measures to be taken. The information gained from continuous monitoring of temperature distributions with burnup can be used to optimize the element designs of future core loadings.

In many cases the CRICET system would be used to measure temperatures in advanced prototype test elements which have considerably different designs and operational conditions than the core driver elements. This information would be particularly valuable to fuel-element engineering since it can be used to closely define operational limitations for different designs.

* A proprietary development of North American Rockwell Corporation fContinuous Remote Instrumentation and Core Evaluation Technique 


\section{SYSTEM DESCRIPTION}

The CRICET system is comprised of the in-reactor sensor - transmitter unit and the ex-reactor receiver discriminator-recorder unit as shown in Figure 1. The transmitter unit is small and is subject to a hostile environment; the receiver unit is large and is subjected only to atmospheric conditions. Because of the hostile environment the trans mitter unit requires a large fraction of the effort in design and development of the system. Figure 2 shows the relationship of the CRICET components - sensors, power supply and amplifier, transducer, and receiver.

Thermocouples extend from the transmitter unit to the in-core positions in the fuel subassembly to be monitored. The electrical signals generated by the thermocouples are carried to the transmitter unit where they modulate the flow of power from the thermoelectric (TE) power supply to the acoustic transducer. This modulation causes the transducer to pulse at a frequency which is proportional to the value of the sensor signal. The resultant acoustical signal is received at the vessel exterior by sensitive pickups, and is amplified, analyzed, and translated into temperatures.

\section{Environmental Considerations}

Two environmental parameters are of concern, temperature and radiation. To minimize detrimental effects of temperature in reactors with large core temperature gradients, such as the LMFBR, the trans -mitter unit can be located in the lower end of the fuel subassembly where the coolant enters the reactor. It can be included at the upper end of the element if the gradients are small and it is more convenient from an element design standpoint. To minimize detrimental effects of radiation, the unit is located at a distance from the active core fuel. In the LMFBR the axial blanket and grid plates provide considerable shielding from the high core-neutron flux levels.

The neutron flux and gamma radiation levels beneath typical LMFBR and water reactor cores are shown in Figures 3 and 4, respectively.

The basic design has a wide degree of flexibility to (1) operate in gamma and neutron flux levels other than those shown, which might be present in different reactor designs, and (2) accommodate various radiation exposure limitations which might be imposed by the TE or electrical components. Factors which contribute to this flexibility are: (1) size of the gamma heater mass; by increasing its diameter or length beyond the reference design (1.8-in.-diam, 6-in. length) the gamma heater specific power requirement is decreased and consequently the transmitter can be located farther from the core in lower neutron flux levels; and (2) the transmitter thermoelectrics and electronics can be protected from radiation by shielding. With shielding, the transmitter could be located nearer to the core.

The location of the transmitter unit beneath the core at the coolant inlet specifies a temperature environment of 500 to $800^{\circ} \mathrm{F}$, and fast neutron fluxes $(>0.1 \mathrm{Mev})$ of $10^{10}-10^{11} \mathrm{nv}$. 


\section{Subsystem Functions}

Each of the subsystems is quite simple in design and flexible in operation. The operational characteristics of each subsystem can vary over a wide range while it is still performing its function satisfactorily. This is due to two system design specifications: (1) each subsystem will contain features that compensate for operational characteristic changes of input subsystems, and (2) the transmitter will be capable of being recalibrated periodically during operation.

Measurements are made using thermocouples and thermostat-type electrical switches which select the sequence of measurements taken. Information thermocouples and flux sensors extend from the transmitter unit to specified locations in the fuel element. A calibration thermocouple is contained within the transmitter unit.

The amplifier contains four types of components which use the sensor signal to modulate power from a silicon-germanium $T E$ power supply to pulse an acoustic magnetostrictive transducer at a frequency proportional to the sensor signal. The frequency will vary about a factor of two (e.g. from 0.25 to 0.5 rings/sec) as the sensor signal varies from minimum to maximum (see Figure 5). The amplifier circuit design is very basic and reliable. No tubes, transistors, or diodes are used. Because the circuit operates on low power ( 1.5 watt), the electrical components are physically quite small. The types of electrical components used have been tested at high temperatures and to high radiation exposures. These tests indicate that a design using operating temperatures of 500 to $800^{\circ} \mathrm{F}$ and total neutron exposures up to $5 \times 1018$ nvt is feasible for initial CRICET system demonstration tests. Examples of the electrical component state-of-the-art are given in Table I.

The reference power supply consists of a the rmally insulated tungsten gamma radiation heater and a 14-couple SiGe TE module. Gamma radiation from the reactor core is absorbed in the tungsten and generates thermal energy that causes a temperature gradient and heat flux across the TE couples, thereby producing electrical power. The amplifier circuit can operate effectively over a wide range of electrical power; hence, the $T E$ power supply can meet the circuit power requirements by operating at any level within a wide range of gamma heating. In actual practice, by conducting initial trials in a candidate reactor the gamma radiation levels can be determined quite accurately.

A key feature of the design is that the gamma radiation heat rates required for power supply operation are low $(\sim 0.01$ to $0.1 \mathrm{w} / \mathrm{gm}$ compared to normal in-core values (5 to $20 \mathrm{w} / \mathrm{gm})$. This characteristic allows the transmitter unit to be placed at a distance external to the active core fuel in a selected location.

The type of TE material used ( $\mathrm{SiGe}$ ) has been tested at high temperatures and to high radiation exposures. These tests indicate that a state-of-the-art design using operating temperatures up to $1800^{\circ} \mathrm{F}$ and total neutron exposures up to $5 \times 10^{18}$ nvt is feasible for initial system demonstration tests. 
TABLE I

DESIGN AND FABRICATION STATE-OF-THE-ART FOR ELECTRICAL COMPONENTS TO BE USED IN HIGH-TEMPERATURE/RADIA TION ENVIRONMENTS

\begin{tabular}{|c|c|c|c|c|c|c|c|}
\hline \multirow[t]{2}{*}{$\begin{array}{l}\text { CRICET } \\
\text { Component } \\
\text { Type }\end{array}$} & \multirow[t]{2}{*}{$\begin{array}{l}\text { Description of } \\
\text { Irradiation- } \\
\text { Tested Component }\end{array}$} & \multirow[t]{2}{*}{$\begin{array}{l}\text { Component } \\
\text { Manufacture } r\end{array}$} & \multirow[t]{2}{*}{$\begin{array}{l}\text { Test } \\
\text { Temp- } \\
\text { erature } \\
\left({ }^{\circ} \mathrm{F}\right)\end{array}$} & \multicolumn{2}{|c|}{$\begin{array}{c}\text { Type and Intensity } \\
\text { of Goal Neutron } \\
\text { Exposure } \\
(x 1018 \text { nvt }) \\
\end{array}$} & \multirow[t]{2}{*}{$\begin{array}{c}\text { Performance } \\
\text { During } \\
\text { Test }\end{array}$} & \multirow[t]{2}{*}{ Comments } \\
\hline & & & & Fast & Thermal & & \\
\hline \multirow[t]{3}{*}{$\begin{array}{l}\text { Magnetic } \\
\text { Amplifier }\end{array}$} & $\begin{array}{l}\text { Electromagnetic stepping } \\
\text { motors }\end{array}$ & AI & $\begin{array}{l}700 \text { and } \\
1000\end{array}$ & 5.0 & 3.0 & Excellent & $\begin{array}{l}\text { Wire wound (non-diode) magnetic } \\
\text { amplifiers should be operative to }\end{array}$ \\
\hline & $\begin{array}{l}\text { Rotary differential trans- } \\
\text { formers }\end{array}$ & AI & $\begin{array}{l}500 \text { and } \\
800\end{array}$ & 8.0 & 4.0 & Excellent & at temperatures of 900 to $1000^{\circ} \mathrm{F}$. \\
\hline & Wire-wound transformer & $\begin{array}{l}\text { General } \\
\text { Electric }\end{array}$ & 930 & 0.4 & - & Satisfactory & \\
\hline \multirow[t]{2}{*}{ Capacitor } & $\begin{array}{l}\text { Glass-Dielectric }(0.001 \\
\text { to } 0.01 \mu \mathrm{f})\end{array}$ & $\begin{array}{l}\text { Corning } \\
\text { Glass }\end{array}$ & 570 & 2.0 & 2.0 & $\begin{array}{l} \pm 3 \% \text { Capac- } \\
1 \text { tance } \\
\text { change }\end{array}$ & $\begin{array}{l}\text { Both types should be operative } \\
\text { in nontuned circuits in severe } \\
\text { environment }\end{array}$ \\
\hline & Mica-Dielectric $(0.5 \mu f)$ & $\begin{array}{l}\text { Bendix } \\
\text { Corp. }\end{array}$ & 750 & 0.1 & - & $\begin{array}{l}0 \text { to } 5 \% \\
\text { Capacitance } \\
\text { change }\end{array}$ & \\
\hline \multirow[t]{2}{*}{ Resistor } & Precision wire-wound & $\begin{array}{l}\text { U.S. Army } \\
\text { Engr. Lab }\end{array}$ & 100 & 2.0 & - & $\begin{array}{l}0 \text { to } 0.8 \% \text { Re- } \\
\text { sistivity } \\
\text { change }\end{array}$ & $\begin{array}{l}\text { Both types should be operative } \\
\text { to } 1019 \text { nvt at } 900^{\circ} \mathrm{F} \text {. }\end{array}$ \\
\hline & Metal-Oxide film & $\begin{array}{l}\text { Mallory \& } \\
\text { Co. }\end{array}$ & 930 & 0.02 & 2.0 & $\begin{array}{l}0.2 \text { to } 3.0 \% \\
\text { Resistivity } \\
\text { change } \\
\left(10^{3}-106\right. \\
\text { ohms) }\end{array}$ & \\
\hline $\begin{array}{l}\text { Chopper- } \\
\text { Relay }\end{array}$ & $\begin{array}{l}\text { Electromechanical } \\
\text { chopper }(400 \mathrm{~Hz})\end{array}$ & $\begin{array}{l}\text { Airpax } \\
\text { Electronics }\end{array}$ & 470 & 0.1 & 0.1 & Excellent & $\begin{array}{l}\text { Proper contact design must be } \\
\text { used to extend lifetime }\end{array}$ \\
\hline $\begin{array}{l}\text { Acoustic } \\
\text { Transducer }\end{array}$ & $\begin{array}{l}2 \mathrm{~V} \text { Permendur } \\
\text { (Magnetostrictive } \\
\text { material) }\end{array}$ & & 100 & 2.0 & - & $\begin{array}{l}1 \text { to } 3 \% \\
\text { Change of } \\
\text { electrical } \\
\text { character- } \\
\text { istucs }\end{array}$ & $\begin{array}{l}\text { Slight changes due to } 1 \text { rradiation } \\
\text { indicate probable operation to } \\
1019-10^{2} \text { nvt like Alnico Hard } \\
\text { magnetic materials. Operation at } \\
1000^{\circ} \mathrm{F} \text { verified by out-of-pile } \\
\text { tests. }\left(1710^{\circ} \mathrm{F} \text { Curie tempera- }\right. \\
\text { ture) }\end{array}$ \\
\hline
\end{tabular}


The transducer is an electroacoustic magnetostrictive device similar to those widely used in SONAR applications (transmitting, reception, torpedo guidance). These transducers are typically less than 2 in. ${ }^{3}$ and can operate at very low power levels. The capacitor discharge pulse technique used in the reference CRICET design has been used extensively in SONAR technology.

\section{Normal Operation}

The frequency of ringing of the acoustic transducer is proportional to the value of temperature or neutron flux measured as shown in Figure 5. The magnitude of the millivolt EMF (electromotive force) generated by the thermocouple is dependent upon the temperature differential between the hot-junction located at the point of measurement and the reference cold-junction located at the transmitter unit. The reference cold-junction in this system is at reactor-inlet-coolant temperature, compared to normal applications where the cold reference junction is at room temperature. The reference junction temperature is known accurately at all times since the reactor-coolant-inlet temperature is always measured at the inlet pipe.

By using bimetallic thermostat-type electrical switches as a multiple switching mechanism, several thermocouples within a subassembly can be sequentially measured by one transmitter unit. During a reactor startup a power level is reached at which the gamma radiation heating is sufficiently intense to produce the minimum electrical power required to operate the electrical circuit. At this point the acoustic transducer begins to transmit a signal as shown in Figure 6 . This signal is the "base signal" of the transmitter unit; none of the thermocouples is electrically coupled to the transmitter circuit yet. At approximately $90 \%$ of full power the reactor-coolant-inlet temperature has increased to a level sufficient to actuate a thermostat switch which couples the calibration thermocouple into the transmitter circuit. The transmitter then broadcasts a signal with a frequency of ringing higher than the base frequency by an amount proportional to the EMF of the calibration thermocouple. Since the calibration-thermocouple EMF is known accurately, the EMF-acoustic frequency relationship (Figure 5) can be checked and corrected if necessary. This in-situ calibration allows compensation to be made for any changes of overall operational characteristics whether they be due to the power supply subsystem, the amplifier subsystem, or the transducer subsystem. At approximately $95 \%$ of full power the coolant-inlet temperature has increased to a level sufficient to actuate thermostats which switch the calibration thermocouple out of the transmitter circuit and an information-measurement thermocouple into it. As the power increases, other thermocouples are switched sequentially into and out of the transmitter circuit. At full power and peak inlet temperature one thermocouple is measured continuously. In actual operation two or possibly more calibration thermocouples will be included in the circuit, to check the temperature signal-frequency relationship at different temperatures.

4. Transient Operation

The system is intended to function principally as a reactor steady-state temperature monitor; however, it will be able to 
provide information on reactor transient conditions within certain limitations. The main factors affecting ability to measure transient conditions are as follows:

\section{1) Local Power Density}

The gamma radiation heating, and hence the TE power supply output, is proportional to the local power density. The reference design transmitter unit does not operate at conditions equivalent to 0 to $60 \%$ of full reactor power.

\section{2) Amplifier Hysteresis}

The electrical circuits require a finite time to adjust to a new sensor signal level.

3) Transducer Rate of Ringing

It takes the time involved for several pulses of the transducer before a new signal can be recognized.

4) Reception Mode of Operation

The overall system response depends on the method of signal integration and data display used by the ex-reactor signal analysis instrumentation.

\section{Information Mapping}

Because thermostat switching is used and the reactor-inlet coolant maintains a constant temperature at full reactor power, one thermocouple or flux sensor is monitored continuously during normal full-power operation. To "interrogate" the transmitter unit and cause it to monitor the other information thermocouples, the coolant temperature must be reduced. In some reactor designs this can be done without reducing the power, by increasing the coolant flowrate or the reactor system heat rejection. The thermostats would be selected so that relatively small reductions of coolant temperature would cause them to sequentially switch through the thermocouples.

Transmitter units can be included in as many of the fuel subassemblies as desired. To identify individual transmitters the electroacoustic transducers are designed with slightly different physical sizes so that they pulse ("ring") with different inherent frequencies. Thus the frequency of pulsing is a measure of the temperature, while the frequency of an individual pulse (acoustic carrier frequency of the pulses) identifies the transmitter.

\section{Accuracy}

The principal factors which determine the overall accuracy of the CRICET System and their estimated uncertainties for short- and longterm operation are indicated below: 
Factor

1. Electrical Circuit Stability

2. Thermostat Repeatability

3. Thermocouple Indication

4. Inlet Coolant Temperature Stability

5. Signal Analysis and Display

$\begin{array}{cc}\begin{array}{c}\text { Short-Term } \\ \left( \pm^{\circ} \mathrm{F}\right)\end{array} & \frac{\begin{array}{c}\text { Long-Term } \\ \left( \pm^{\circ} \mathrm{F}\right)\end{array}}{3} \\ 2 & 5 \\ 2 & 2 \\ 2 & 2 \\ 2 & 3 \\ & 2\end{array}$

The designations "short-term" and "long-term" refer to the period of operational time from a specific time during the operation when the system is in-calibration and at its best accuracy. These specific times are either (a) at the initial startup of operation or (b) at points in the operation where the system has been recalibrated. Operation periods are considered short-term if one month or less, and long-term iflonger. Hence, if the system is recalibrated at one-month intervals, the accuracy corresponds to that listed for short-term operation.

Factors 1, 2, and 3 pertain to the operation of the transmitter unit, and their accuracy is estimated. The operation of the electrical circuit includes assumed effects of component degradation and drift, and power supply output variation; the thermostat accuracy includes assumed effects of drift of the actuation points with repeated operation; and that for the thermocouple includes as sumed effects of normal degradation due to thermal effects. Temperature indication inaccuracies due to radiation effects or improper thermocouple placement are not included in the estimate.

Factor 4 is based on measurement at the inlet pipes to the reactor, and Factor 5 pertains to the accuracy of the signal reception, analysis, and display instrumentation located outside the vessel. The inlet tem perature has a direct effect on the system accuracy since the transmitter unit is emitting a signal that is directly proportional to the difference between the known inlet temperature and the unknown information temperature. The signal received can be analyzed and translated into temperatures quite accurately by using signal integration techniques.

These estimated uncertainties are for a typical situation where the coolant-inlet temperature and transmitter-unit temperature are $600^{\circ} \mathrm{F}$, and the unknown temperature measured is approximately $700^{\circ} \mathrm{F}$. For temperature differences of less than $100^{\circ} \mathrm{F}$, the uncertainties would be less in absolute magnitude.

The uncertainties can be summarized by using the sum-of-thesquares statistical method widely used for evaluating reactor hot-channel uncertainty factors. This method assumes that the factors involved are statistically independent, which is true in our case.

By using this method it is calculated that the temperature indication displayed by the ex-reactor instrumentation would be accurate to within 5 and $7 \circ$ of the true temperature, for short-term and long-term operation respectively, with a $67 \%(1 \sigma)$ confidence level. Also, the instrumentation would be accurate to within 10 and $14^{\circ} \mathrm{F}$ for short-term and longterm operation, respectively, with a $95 \%(2 \sigma)$ confidence level. 
It is expected that the relative temperature differences measured by thermocouples of the same transmitter unit will be considerably more accurate than the absolute accuracies, thus allowing accurate relative temperature maps to be made.

\section{Transmitter Integration Into Fuel Subassembly}

The transmitter unit can be constructed in a wide variety of configurations. The only design criteria affecting the geometry and location of the transmitter unit are the requirements previously mentioned for neutron exposure levels and gamma heating rates.

The reference transmitter unit design, shown in Figure 7, has a 6-in. length of tungsten, which has a high thermal conductivity and high density, to absorb sufficient gamma radiation to produce approximately 100 watts of thermal power. A thermal resistance barrier comprised of four radiation baffles, with vacuum gaps separating them, is used to maintain the tungsten at a temperature of approximately $1600^{\circ} \mathrm{F}$. With this configuration approximately $50 \%$ of the thermal power flows through the TE module. The bulkhead between this module and the electrical circuit conducts the power directly to the reactor coolant. Because this bulkhead temperature is only slightly higher than the coolant temperature, it acts as a cold-plate for the TE module. The power is transferred from the tungsten to the hot-plate by radiation across a small vacuum gap.

\section{Summary}

Table II summarizes the important features of the CRICET system. TABLE II SUMMARY OF IMPORTANT FEATURES

\begin{tabular}{|c|c|}
\hline Feature & Significance \\
\hline 1. No instrumentation leads & $\begin{array}{l}\text { a. Practical for power reactor in- } \\
\text { core use }\end{array}$ \\
\hline $\begin{array}{l}\text { 2. Integral part of fuel } \\
\text { subassembly }\end{array}$ & $\begin{array}{l}\text { a. Can measure fuel subassembly } \\
\text { burnup } \\
\text { b. Can detect fuel subas sembly 'hot } \\
\text { spots" }\end{array}$ \\
\hline $\begin{array}{l}\text { 3. Utilizes core gamma radiation } \\
\text { for operating power }\end{array}$ & $\begin{array}{l}\text { a. Gamma heater mass can be easily } \\
\text { sized to give needed power } \\
\text { b. Contınuous power source }\end{array}$ \\
\hline 4. In-reactor calibration & $\begin{array}{l}\text { a. Enhances accuracy } \\
\text { b. Allows for modest amplifier tem - } \\
\text { perature sensitivity and drift }\end{array}$ \\
\hline 5. No intratransmitter interference & $\begin{array}{l}\text { a. Numerous transmitters will per- } \\
\text { mit "mapping" of core }\end{array}$ \\
\hline 6. Acoustic signal transmission & $\begin{array}{l}\text { a. Uses extensive existing SONAR } \\
\text { technology } \\
\text { b. Signal frequency with low back- } \\
\text { ground noise can be selected }\end{array}$ \\
\hline $\begin{array}{l}\text { 7. Demonstrated high-temperature, } \\
\text { high-exposure components }\end{array}$ & a. Feasible for reactor envionments. \\
\hline
\end{tabular}




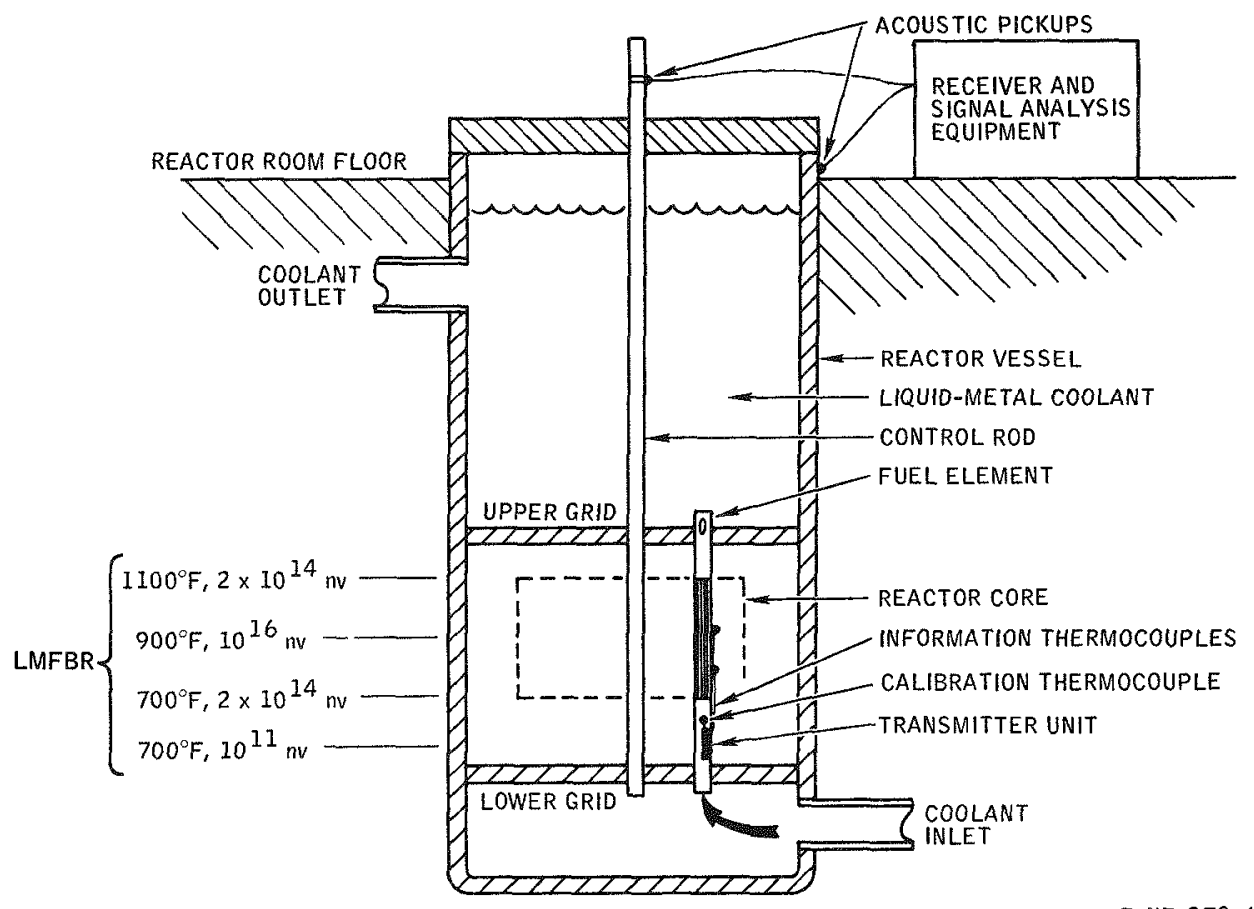

Figure 1. CRICET System Concept

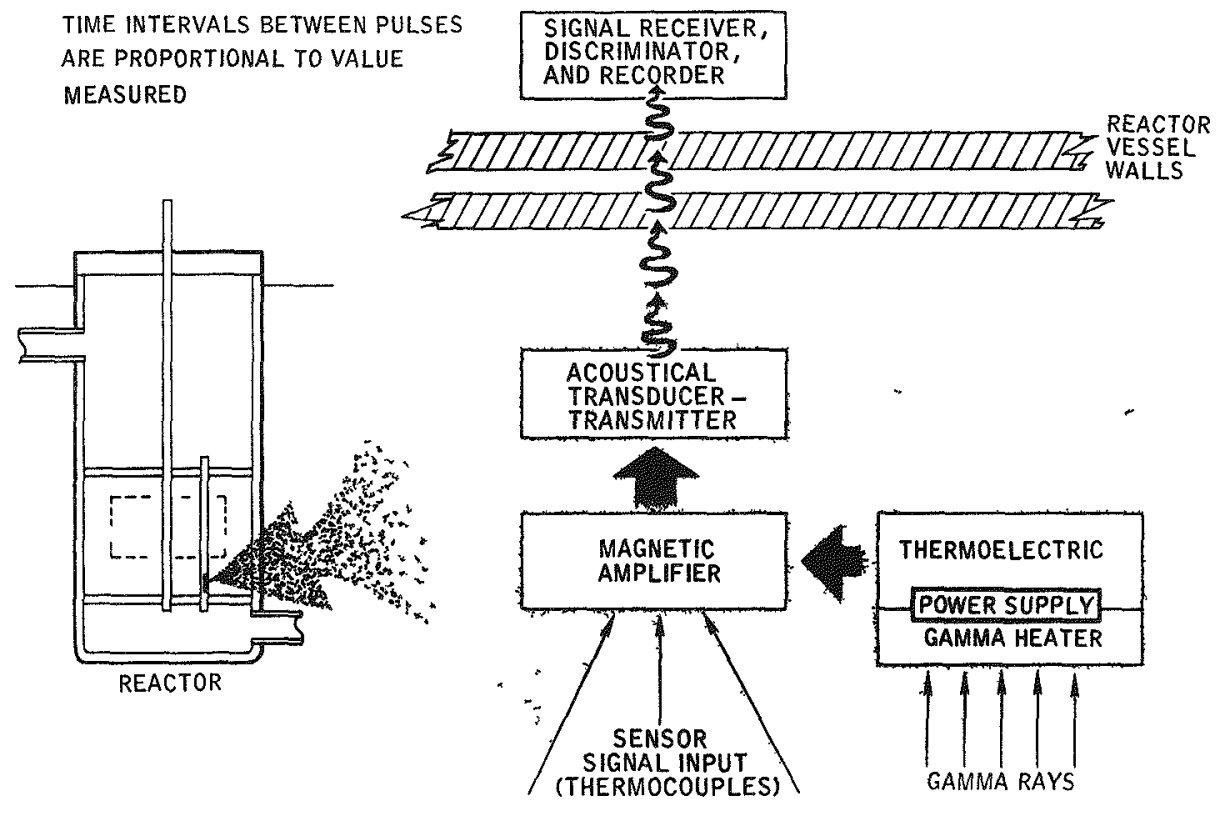

$7-N 7-272-9 B$

Figure 2. CRICET System Functional Diagram 


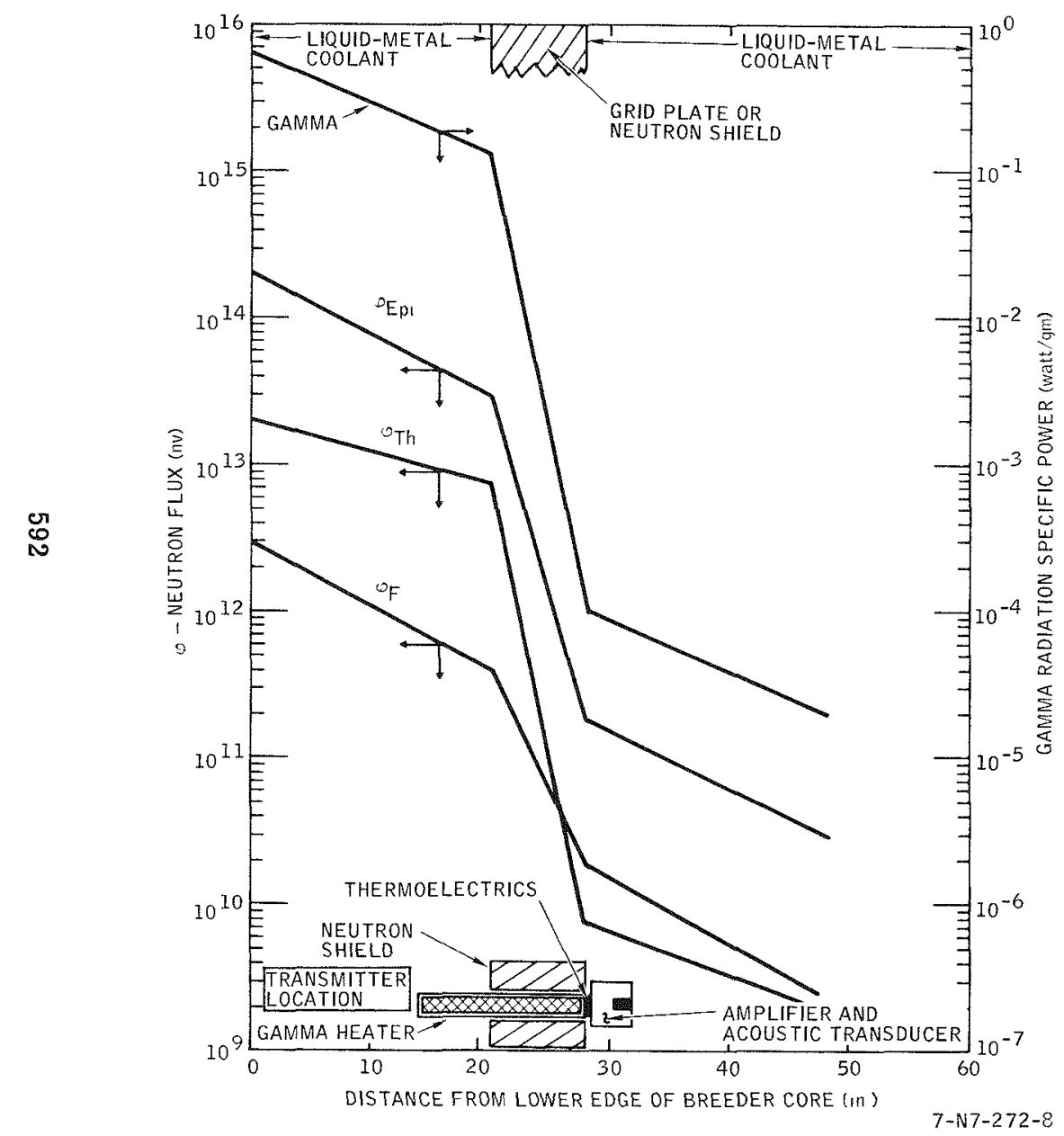

Figure 3. LMFBR Axial Radiation Profiles

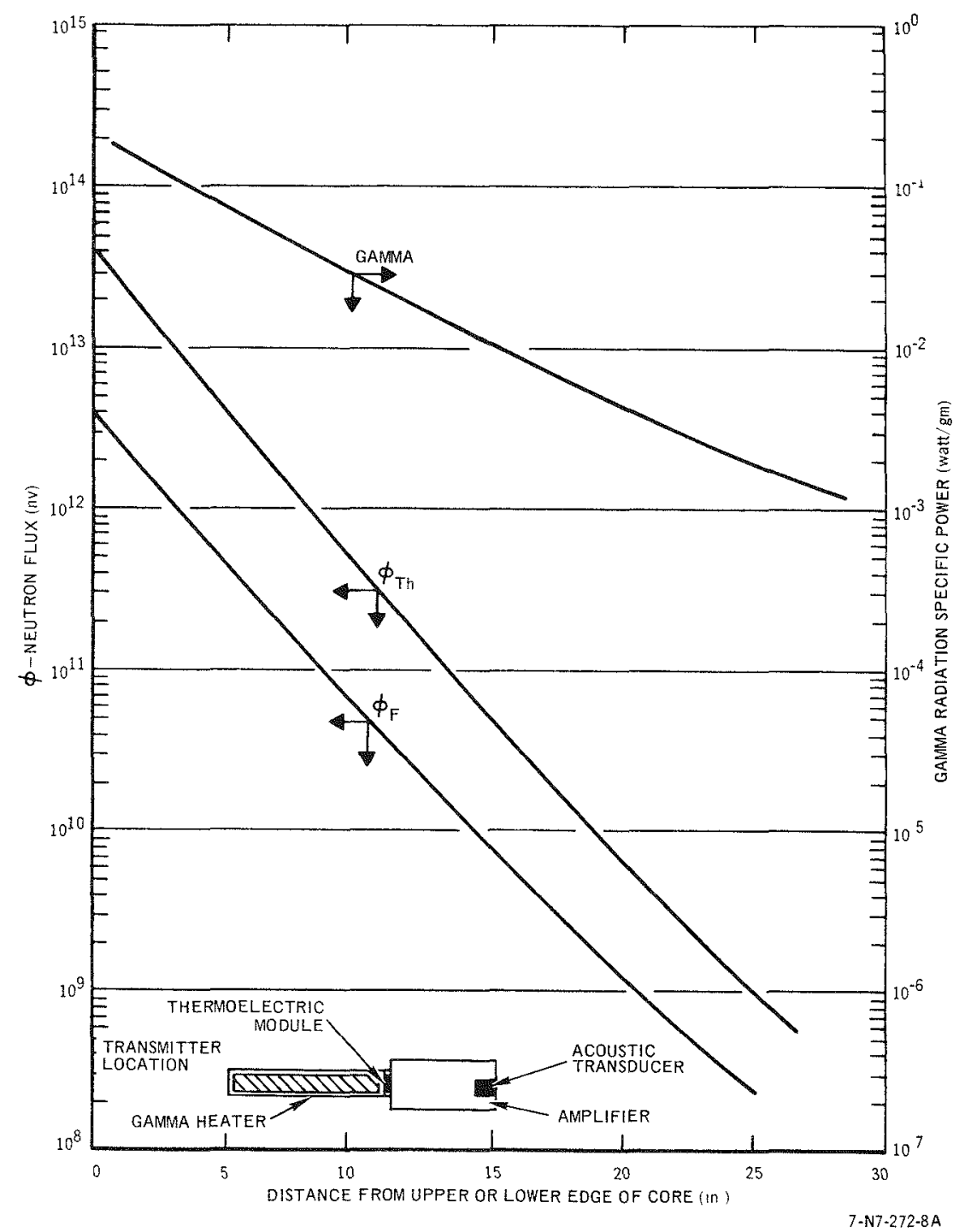

Figure 4. Water Reactor Axial Radiation Profiles 
TC HOT JUNCTION-COLD JUNCTION $\triangle T\left({ }^{\circ} \mathrm{F}\right)$

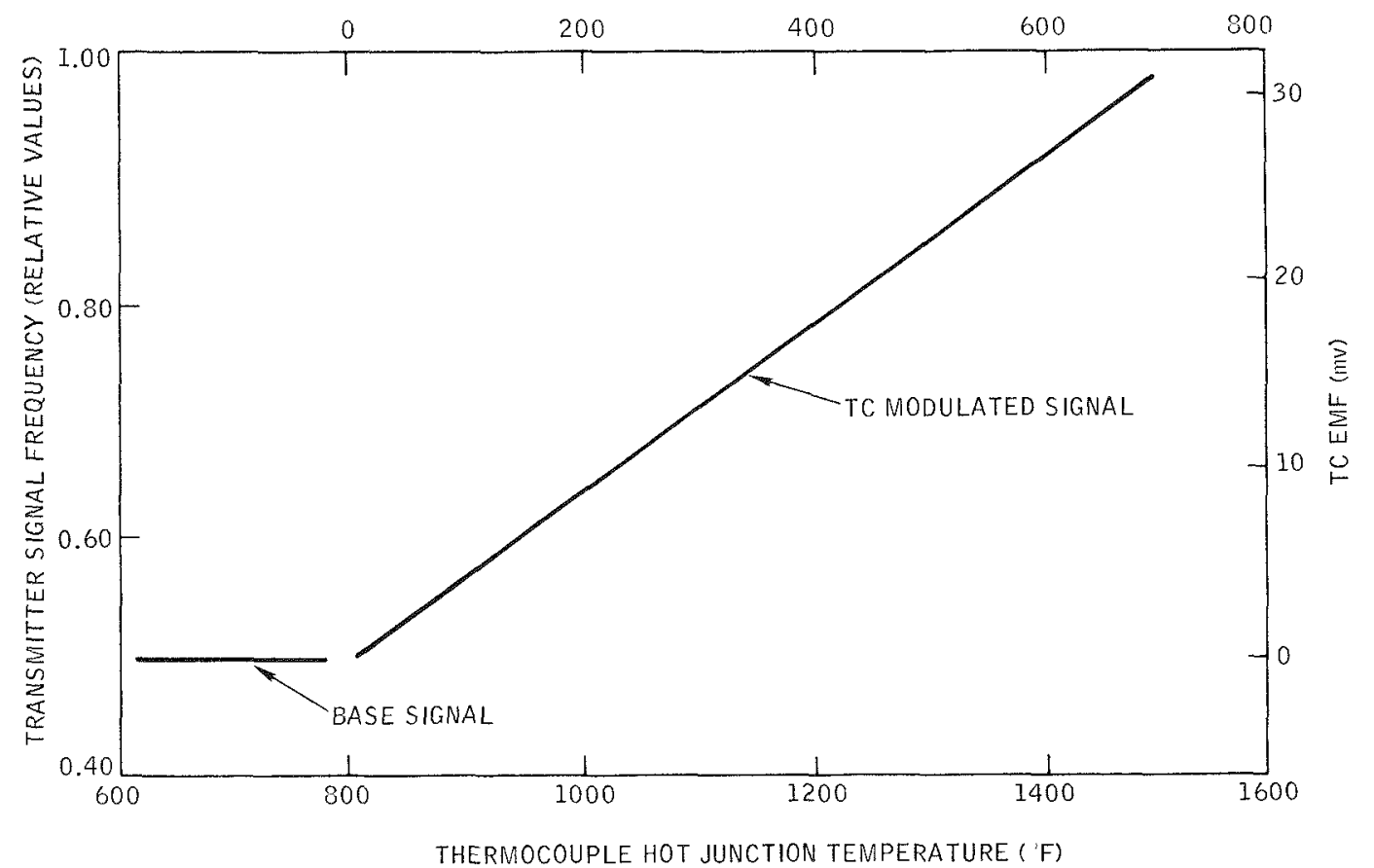

$7-N 7-272-10 A$

Figure 5. Thermocouple EMF-Transmitter Signal Relationship

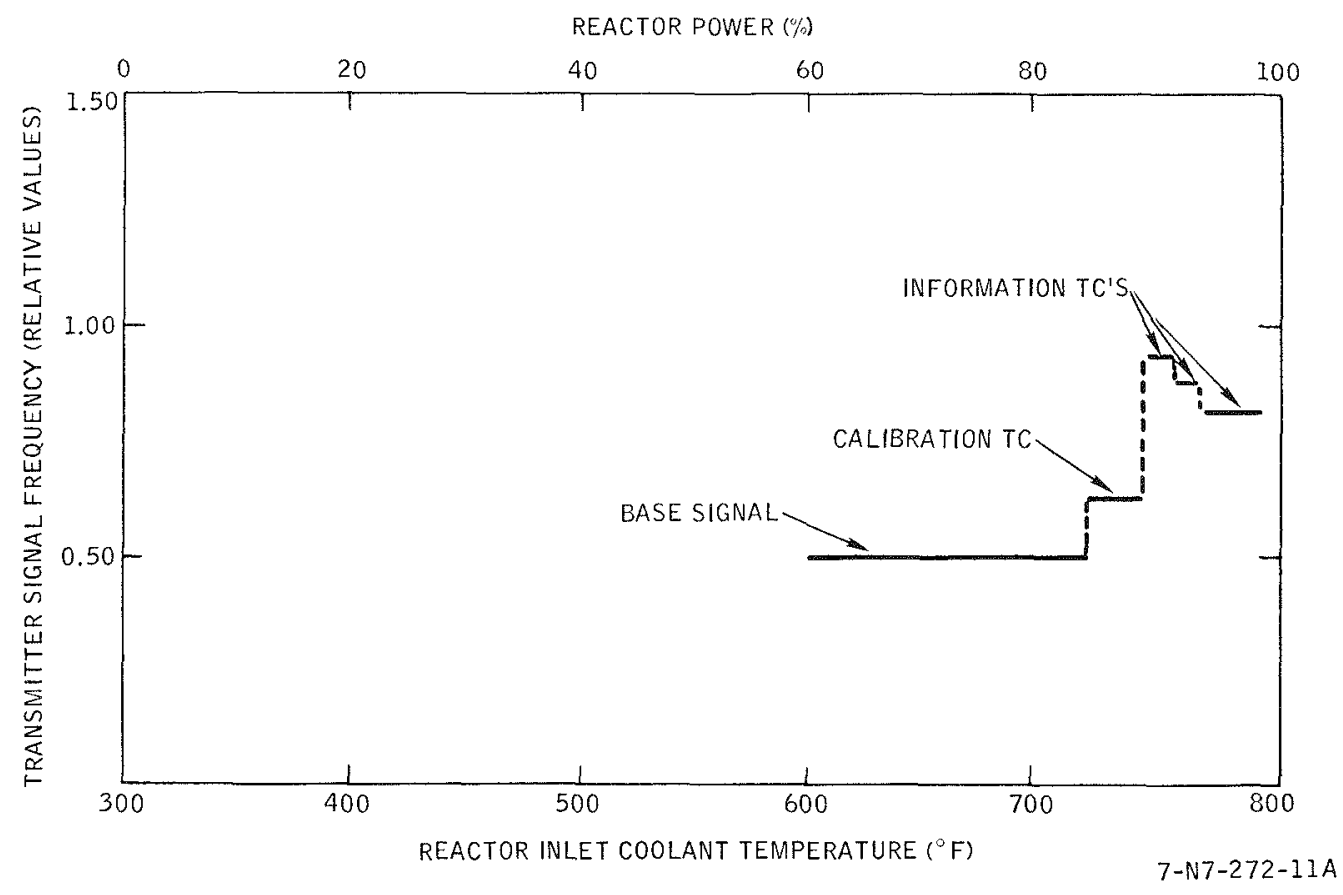

Figure 6. Thermocouple Selection Using Thermostats 


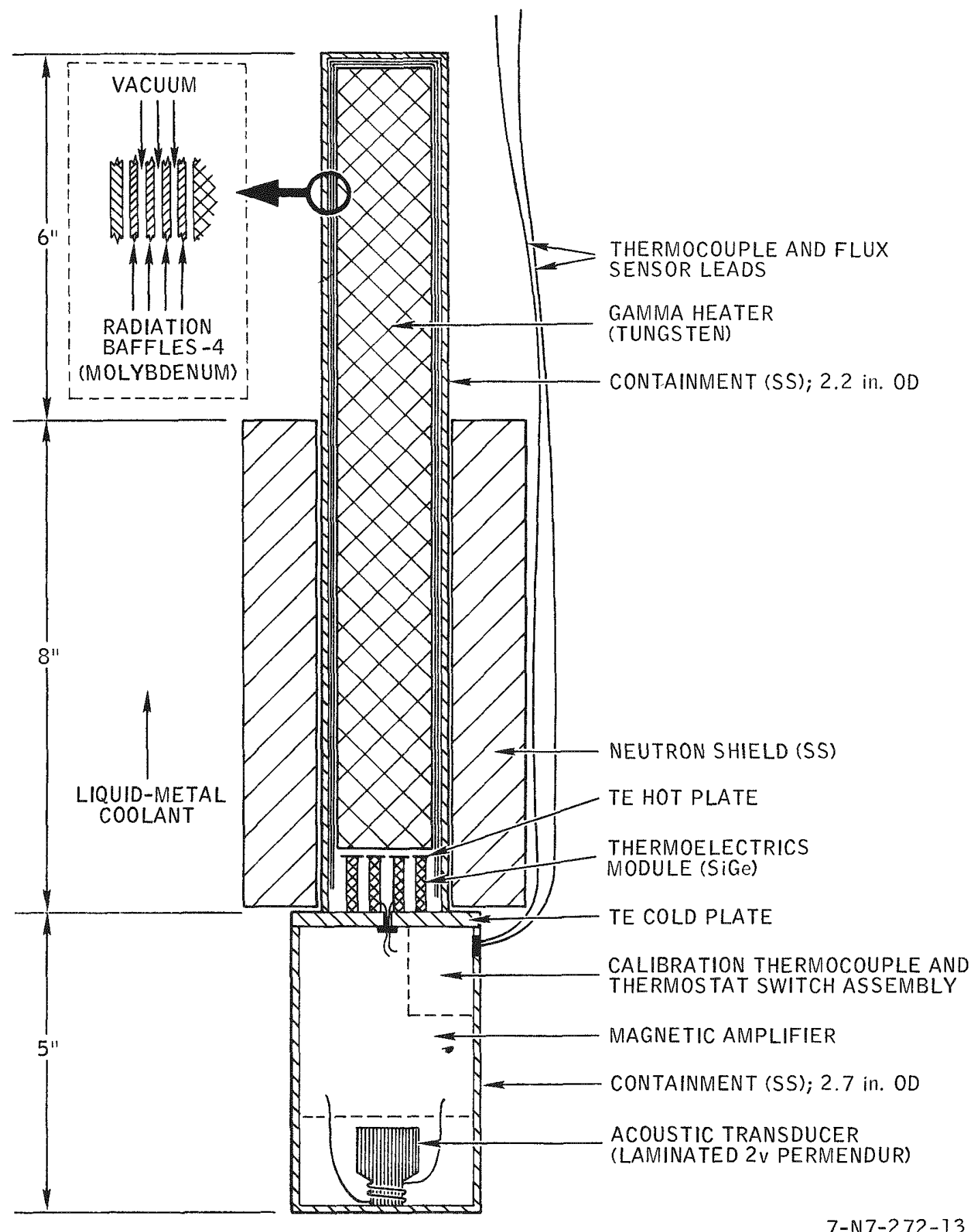

Figure 7. CRICET System Transmitter Unit 


\author{
Yrradiation Studies of Uranium Nitride* \\ E. D. Albrecht, A. J. Rothman, J. D. Lee, \\ J. M. Johnson, and W. N. Hayes, Jr. \\ Lawrence Radiation Laboratory, University of California \\ 8 \\ Livermore, California 94550
}

\begin{abstract}
A BSTRACT
A fission-heated, gas-bonded, binary gas controlled irradiation capsule containing eight uranium nitride specimens has been irradiated in the Battelle Research Reactor for eight cycles. The median specimen temperature ranged from 1307 to $1417^{\circ} \mathrm{C}$ during the irradiation and the average burn-up was $2.3 \times 10^{20}$ fissions/cc. Two specimens each of four different microstructures, including fine-grain (30 microns), 96\% dense; coarse-grain (105 microns), 97\% dense; single crystal, $100 \%$ dense; and fine-grain (30 microns), $78 \%$ dense fuel were irradiated for a total of 3558 hours. The $9.6 \%$ enriched specimens were nominally $0.750 \mathrm{in}$. in length and $0.200 \mathrm{in}$. in outside diameter, having a 0.100-in.-diameter axial hole. Each specimen was composed of from one to three segments, with the lower segment of the singlecrystal specimens remaining an undrilled, solid piece. The fuel specimens were clad in vented $W-25.5$ Re having a wall thickness of 0.020 in. To prevent decomposition of the uranium nitride, a partial nitrogen pressure was maintained during the irradiation by external heating of $\mathrm{U}_{2} \mathrm{~N}_{3}$ powder located at the bottom of the capsule. Neutron radiographs were made of the capsule before irradiation and at the conclusion of each cycle, in an attempt to acquire progressive swelling data during the irradiation. An extensive post-irradiation examination was made which included measurement of such physical dimensions as lengths, inside and outside diameters; density, metallography, chemical analyses for composition; and radiochemical analyses for determination of uranium burn-up. The only significant changes observed in the microstructure were the creation of fine porosity at the grain boundaries of all polycrystalline specimens and the preferential alignment of this porosity in those grain boundaries lying parallel to a specimen surface. The single-crystal specimens did not exhibit the porosity except in rare occasions along subgrain boundaries near the surface. Retained fission
\end{abstract}

\footnotetext{
*Work performed under the auspices of the U.S. Atomic Energy Cornmission.
} 
gas analyses by mass spectrometric techniques have shown that nearly $100 \%$ of the fission gas generated has been retained in each specimen, regardless of microstructure. The fine grain (30 microns), $96 \%$ dense UN swelled the greatest amount at $1417^{\circ} \mathrm{C}$ and to a lesser extent at $1347^{\circ} \mathrm{C}$, revealing an implied temperature dependence. The fine-grain (30 microns), 78\% dense and single-crystal, 100\% dense UN showed the least swelling under the conditions of this irradiation.

\section{Introduction}

Preliminary studies led to the selection of uranium nitride (UNN) as the prime fuel candidate for the Lawrence Radiation Laboratory (LRL) Space Flectric Power Program's reactor designs. From considerations of cycle thermodynamics, one develops the thesis that "hotter is better," so it was established that a high-temperature-fuel irradiation program was needed. And, since fuel swelling is a major problem in most reactor designs, it was given prime consideration.

Various theories of fuel swelling, or at least theories which relate to fuel swelling, have been propounded.1,2 Some studies and analyses on bubble migration in solids relating to fuel swelling have been made. 3-6 Even so, no satisfactory comprehensive theory of fuel swelling exists.

Some of the above studies suggest variables which may be important in influencing fuel swelling. If we assume that swelling is caused by the growth and migration of bubbles, 7 it is clear that diffusion of gas atoms and/or gas bubbles will affect swelling behavior. In turn, the diffusion rates should be affected by the thermodynamic activity of the fuel. In previous experiments no specific attempts have been made to fix the activity. In this experiment a fixed pressure of nitrogen over the UN was maintained by heating a $\mathrm{U}_{2} \mathrm{~N}_{3}$ source. Ultimately it was planned to change the uranium activity to other fixed values in future experiments.

Furthermore, there is excellent evidence that bubbles migrate in a temperature gradient. 8 Still other experiments show that bubbles tend to be trapped at defects, especially at grain boundaries. Thus, we were interested in fuel with no grain boundaries (single crystals) to see if the bubbles would then migrate out of the fuel without impedance by boundaries. Also we wished to determine the effect of few grain boundaries (coarse grain) and many grain boundaries (fine grain).

Once the fuel and temperature range of interest were determined, it was then necessary to establish certain desired conditions for the fuel samples to be irradiated. These conditions are specified below:

1. Several microstructures were to be compared, including single crystal, large-grain high-density, small-grain highdensity and approximately $75 \%$ dense uranium nitride.

2. Specimen surface temperature from 1350 to $1800^{\circ} \mathrm{C}$.

3. Radial temperature profile limited to less than $50^{\circ} \mathrm{C}$. 
4. Accurate temperature control and measurement.

5. Up to $6 \%$ total uranium burn-up.

6. Annular specimen design to be used to permit fission gas release and inner wall (center-line) temperature measurement. Solid specimens to follow permiting annular versus solid specimen comparison.

7. Nitrogen cover gas to be maintained at a fixed partial pressure and temperature to keep constant uranium thermodynamic activity and to prevent UN dissociation.

8. Optimum specimen design to be free standing or clad specimens which would allow approximately $30 \%$ unrestrained radial and axial growth.

9. Specimen inner to outer radius burn-up ratio to be greater than 0.8 to approximate fast reactor conditions.

10. Retention of fission gas from individual sample of secondary interest.

11. Eight or more individual samples per capsule.

Battelle Memorial Institute, Columbus, Ohio, (BMI-C) was selected to design, construct, and irradiate two capsules and to fabricate the fuel samples for each under contractural agreement with LRL. The selection of BMI was based primarily upon their extensive experience in the high temperature fuel irradiation field and the programmatic requirement for UN swelling data at the earliest possible date.

The first irradiation capsule was designated Capsule LRL-1 and was of the fission-heated type requiring no external heaters surrounding the fuel. The gas-bonded, binary gas-controlled capsule contained two specimens, each of four different microstructures. Capsule LRL-2 was of a similar design, but its fabrication lagged that of LRL-1 by sufficient time to permit design changes for the purpose of correcting inadequacies revealed during the irradiation of the first capsule. The irradiation of Capsule LRL-2 was delayed because the LRL Space Electric Power Program was cancelled by the Atomic Energy Commission (AEC). Had this program continued, our plan would have been to irradiate an LRL-designed gas-bonded and binary gas-controlled capsule in a higher power reactor such as the General Electric Test Reactor at Pleasanton, California (GETR). This was to have been followed by a liquid-bonded, binary-gas controlled capsule, and finally a capsule utilizing the annular heat-pipe concept of temperature control.

This paper will be limited to a description of the Capsule LRL-1 design, its irradiation, post-irradiation examination, and other related topics, as well as the reporting and discussion of irradiation results.

\section{Experimental Techniques}

The experimental techniques, calculations, and methods used to determine the capsule design, fabricate fuel and cladding, irradiate the 
capsule, calculate specimen temperatures, and perform the postirradiation examination are described in detail in the following paragraphs.

\section{A. Capsule Design}

In conjunction with the $A \mathrm{EC}^{\prime} \mathrm{s}$ high-temperature fuels program $\mathrm{BMI}-\mathrm{C}$ had designed and irradiated capsules containing up to six clad specimens in the 1600 to $2000^{\circ} \mathrm{C}$ range. 9 Because of this experience and LRL's immediate need for fuel swelling data, the BMI-C hightemperature capsule was selected as the design basis for a capsule to be altered to the specific needs of this program. A diagrammatic description of this capsule is shown in Fig. 1.

The clad fuel specimens were suspended end to end on a molybdenum and tungsten support structure along the centerline of the vertical capsule. This assembly was sealed in a molybdenum shell which formed the primary container. A helically wound nichrome heater having magnesium oxide insulation and sheathed in stainless steel was attached to the bottom end of the primary container to permit the external heating of depleted $\mathrm{U}_{2} \mathrm{~N}_{3}$ powder to $750^{\circ} \mathrm{C}$. The equilibrium $\mathrm{U}_{2} \mathrm{~N}_{3}=2 \mathrm{UN}+1 / 2 \mathrm{~N}_{2}$ at a fixed temperature maintained a fixed $\mathrm{N}_{2}$ pressure of $6 \mathrm{~mm}$ in the capsule. The remaining space in the primary container was filled with argon to minimize heat conduction. The predominate mechanism of heat transfer from the fuel was by thermal radiation. A 1-in.-diameter stainless-steel shell formed the outside or secondary containment. A smaller gas annulus between the primary and secondary containers was used to permit temperature selection and control during irradiation by varying the composition of the helium nitrogen gas mixture; i.e. changing the conductivity of the gas mixture.

Temperatures were monitored by W-5 Re versus W-26 Re thermocouples, each leg of which was composed of eight strands of $0.003-$ in.-diameter wire. One thermocouple was placed in a well receptacle in the top specimen cladding, permitting centerline temperature determination of that specimen alone. All specimens had surface temperature monitored by similar thermocouples tied to their cladding as shown in Fig. 2. The hot junction was simply a square knot. A similar thermocouple was inserted in the $\mathrm{U}_{2} \mathrm{~N}_{3}$ container to sense the temperature of that mass. Five chromel versus alumel thermocouples were attached to the outside of the primary container in the control gas gap.

The thermocouples from within the primary containment were insulated in BeO insulators and brought through a header assembly. Epoxy was used to make the final gas containing seal well above the elevated temperature zone.

Assuming that the fuel enrichment and density were to remain reasonably constant from one capsule to another, the capsule temperature could be varied by simple alteration of the primary and secondary gas gap dimensions. This would permit the same specimen configuration and arrangement for temperatures from 1200 to $2000^{\circ} \mathrm{C}$. 


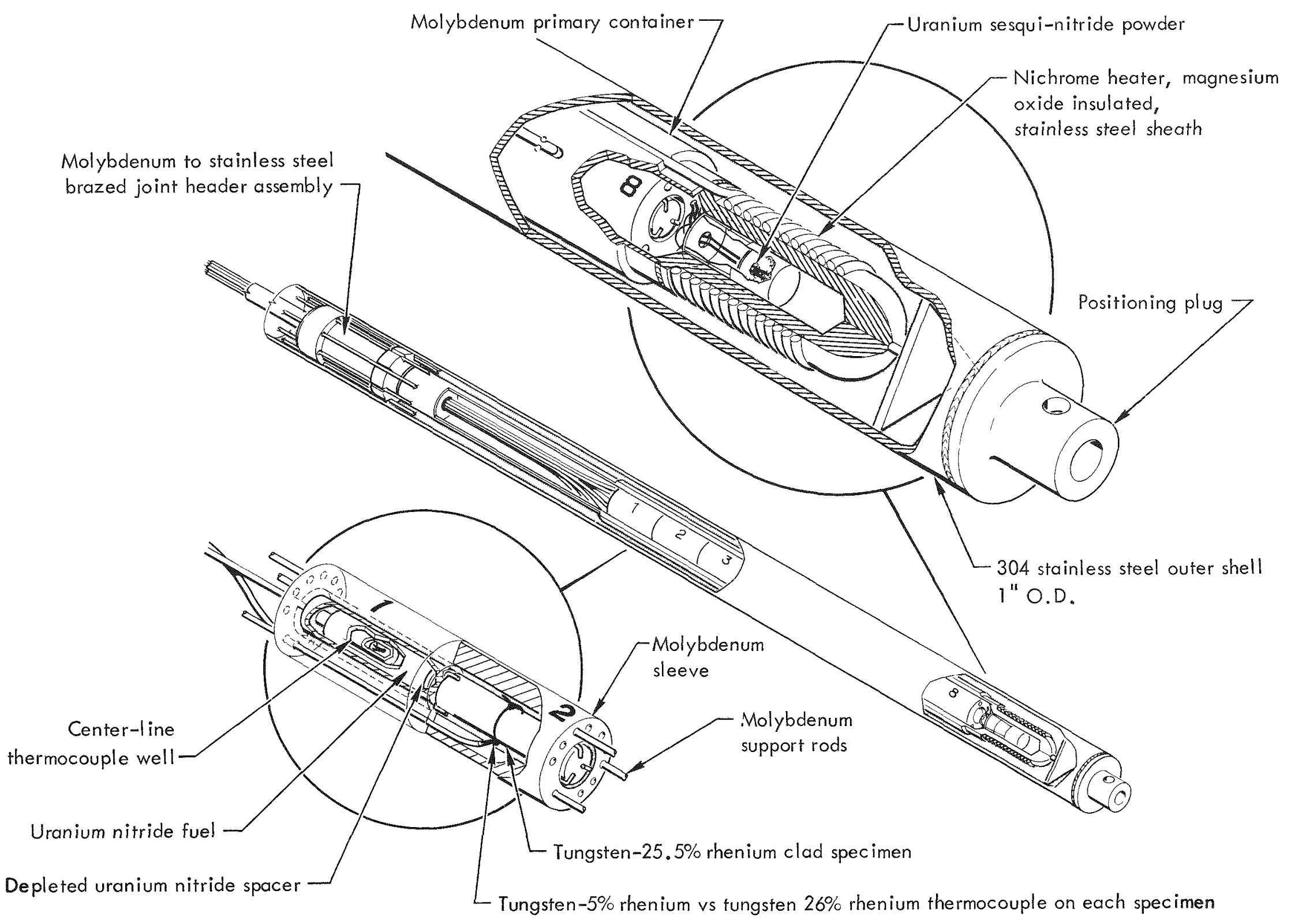

Fig. 1. Diagrammatic description of Irradiation Capsule LRL-1. 


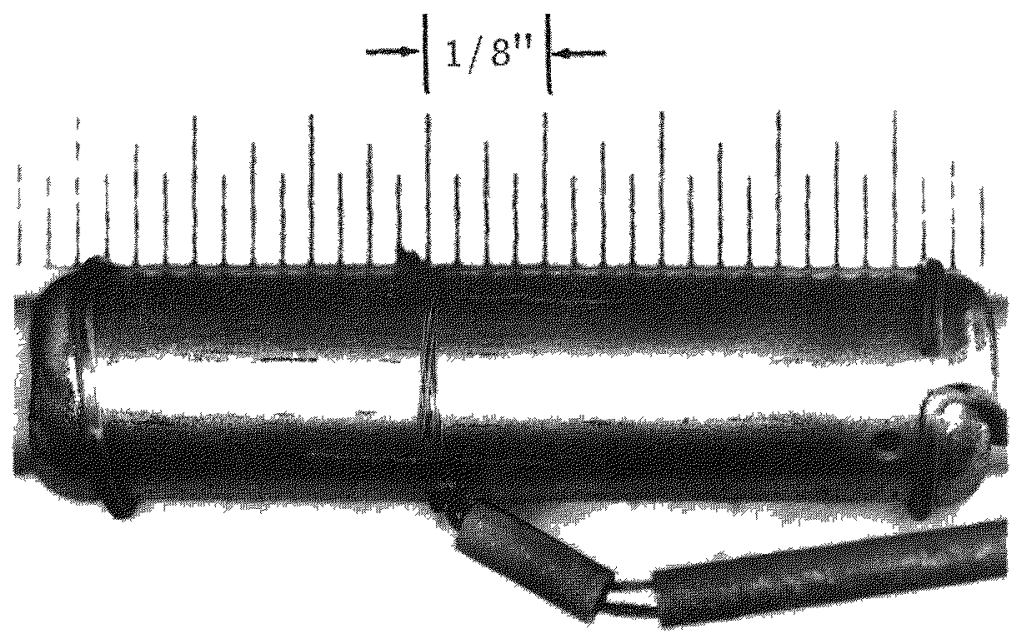

Fig. 2. Typical clad specimen from Capsule LRL-1 showing vent hole, positioning wres and W-5 Re vs. W-26 Re thermocouple attachment. 
Again, BMI-C was selected for fuel specimen fabrication for reasons of experience and immediate availability of fabrication facility. The uranium nitride fuel was to have certain variables in configuration, which are listed below in four categories:

1. Single-crystal, $100 \%$ dense

2. Large-grain $(150 \mu), 100 \%$ dense

3. Fine-grain $(25 \mu), 100 \%$ dense

4. Fine-grain $(25 \mu), 75 \%$ dense.

Duplicate specimens approximating each of these four types were irradiated in Capsule LRL-1.

Depleted and $93 \%$ enriched uranium metal were blended in an induction furnace for 20 minutes and the resulting $10 \%$ enriched metal. was cast in copper molds. Part of this product was fabricated into 0.25-in.-diameter rods for consumable arc melting under nitrogen, and the remainder was rolled and nibbled for making UN powder by the metal-gas reaction process.

Procedures for the preparation of UN single-crystal basically consisted of reacting a consumable electrode of uranium in about 15 atmospheres of nitrogen pressure, and, by arc-melting procedures, drip melting the UN into a water-cooled crucible. Melting conditions were adjusted to produce $\mathrm{UN}$ with a $\mathrm{U}_{2} \mathrm{~N}_{3}$ surface or grain boundary film to insure complete reaction. Heat treatment at $1650^{\circ} \mathrm{C}$ under a pressure of less than $1 \times 10^{-3}$ Torr for 8 hours was used to remove $\mathrm{U}_{2} \mathrm{~N}_{3}$ from the grain boundaries. At this point, large single grains were extracted from the casting and used as stock for the singlecrystal specimens.

The polycrystalline specimens were produced by powder metallurgical methods. Chunks of uranium approximately $1 / 4 \mathrm{in}$. each side were reacted with nitrogen at approximately one atmosphere pressure and $850^{\circ} \mathrm{C}$. $\mathrm{U}_{2} \mathrm{~N}_{3}$ was produced by this process and spalled from the surface yielding finely divided powder. A subsequent heat treatment at $1300^{\circ} \mathrm{C}$ under vacuum permitted conversion of UN. The UN powder was cold pressed into cylinders, which in turn, were placed in graphite-lined tantalum molds that were electron-beam welded closed under a pressure of $2 \times 10^{-5}$ Torr or less. This material was then hot isostatically pressed at $1650^{\circ} \mathrm{C}$ and 10,000 psi. The fine-grained, high-density materials were produced by this procedure. A lower pressing pressure was utilized to produce the $78 \%$ dense material.

Fine-grained, high-density UN was then shipped to LRL and annealed at $2350^{\circ} \mathrm{C}$ under $100-\mathrm{mm}$ nitrogen pressure for 115 hours to produce the large-grained UN, having an average grain diameter of 105 microns. 
All specimens were ground into right circular cylinders 0.200 in. in diameter and from $1 / 4$ to $3 / 4$ in. in length. A 0.095 -in.-diameter hole was machined by EDM drilling along the centerline and this was honed to a final diameter of 0.100 in. The single crystals were stacked in three pieces nominally $1 / 4 \mathrm{in}$. in length with the bottom segment remaining as a solid undrilled piece. All other specimens were composed of one or two pieces to make up their nominal $3 / 4$-in. length. The loading order for Capsule LRL-1 is given in Table 1 .

Table I

Specimen Loading Order for Capsule LRL-1

\begin{tabular}{ll}
\hline Specimen no. & \multicolumn{1}{c}{ Microstructure } \\
\hline $1-\mathrm{C}$ Top & Fine-grain $(30 \mu)$, high-density $(96 \%)^{*}$ \\
$2-\mathrm{A}$ & Single-crystal, high-density $(100 \%)^{*}$ \\
$3-\mathrm{B}$ & Single-crystal, high-density $(100 \%)^{*}$ \\
4-D & Fine-grain $(30 \mu)$, low-density $(78 \%)^{*}$ \\
$5-\mathrm{E}$ & Fine-grain $(30 \mu)$, low-density $(78 \%)^{*}$ \\
$6-\mathrm{G}$ & Fine-grain $(30 \mu)$, high-density $(96 \%)^{*}$ \\
$7-\mathrm{H}$ & Coarse-grain $(105 \mu)$, high-density $(97 \%)^{*}$ \\
$8-\mathrm{I}$ bottom & Coarse-grain $(105 \mu)$, high-density $(97 \%)^{*}$
\end{tabular}

"Density based upon percentage of theoretical density of $14.32 \mathrm{~g} / \mathrm{cm}^{2}$.

C. Cladding

W-25.5 Re material purchased from Chase Copper and Brass Company as 1/4-in.-diameter rod was used as the stock for the claddings. The rods were bored by EDM drilling, then honed to remove the layer of carbon contamination and damaged surface caused by the spark discharge. In the honing operation, $0.002 \mathrm{in.}$ of stock was removed from the tube wall and a mechanically polished surface was produced. Six claddings were used in the mechanically polished condition, while two were electro-polished on the inside surface to a mirror finish. The thermocouple well for the top specimen and the bottom. and top end plugs were machined from the same material. The bottom specimen surface was separated from the cladding by a 0.030-in.-thick depleted UN disc inserted for the purpose of better distinguishing the fuel during neutron radiograph measurements. Electron-beam welding was employed to weld the end plugs to the cladding tubes, thereby encapsulating the UN fuel. A single tapered vent hole, 0.010-in. diameter inside and $0.030-\mathrm{in}$. diameter outside, was located near the top of each encapsulated specimen.

\section{Irradiation}

Capsule LRL-1 was irradiated in the BMI-C Research Reactor for a total of 3558 hours. A 3 in. by 3 in. core position was used for 
the eight-cycle irradiation. The open-pool-type reactor operates at $2 \mathrm{MW}$, with a peak flux in excess of $1 \times 10^{13} \mathrm{nv}$ thermal. A continuous record of control gas composition and pressure, as well as temperature detection from each of the 15 thermocouples, was made during irradiation. A solid-state flux monitor with continuous current readout was installed on the outside of the capsule opposite the bottom specimen and moved to the top specimen near the end of each cycle to provide a relative axial flux profile of the capsule.

E. Neutronic and Thermal Analysis

Prior to irradiation of Capsule $L R L-1$, independent neutronic and thermal analyses were made at LRL and BMI-C to test the analytical methods employed, check capsule design, and reduce uncertainties in the predicted fission rates and temperatures of the fuel specimens.

Neutronic analysis of the capsule was performed using a $\mathrm{P}_{3}$ flux perturbation cell code which utilized specimen loading, capsule specifications, and an unperturbed flux profile provided by BMI-C.

Subsequently, a thermal analysis was performed using the fission rates calculated above, capsule specifications, and a two-dimensional $\left(\mathrm{R}\right.$ and $\mathrm{Z}$ ) nonlinear ( $\mathrm{T}^{4}$ radiation and $\mathrm{T}^{2}$ conduction) heat-transfer code called HRTZ.10 The analyses included determination of the relative temperature profile of a single specimen and absolute temperature profiles of the total capsule under various power levels and gas gap configurations. The resulting capsule profiles were then compared with measured capsule temperatures. The above analysis has been completely described by Lee. 11

During the first irradiation cycle it became obvious that the specimen clad thermocouples were not measuring actual specimen temperatures, since the hot junctions were large compared to the size of the annulus they were placed in, and because of the steep temperature gradient in this annulus. This made direct temperature measurement of specimens nos. 2-A through 8-I impossible. Fortunately, the top specimen, 1-C, also had a centerline thermocouple which appeared to record reasonable temperatures throughout the ir radiation. For this reason, it was necessary to calculate temperature histories of the remaining seven specimens during the eight irradiation cycles.

The temperature histories were calculated from available information including capsule specifications, one relative neutron flux profile measurement for each cycle except cycle no. 2, and the temperature history of the top specimen, $1-C_{3}$ as recorded by its centerline thermocouple. Assumptions made were that the properties and dimensions of the materials did not change during the irradiation; that the relative neutron flux profile was constant during a cycle and changed between cycles; and that variations in the top specimen's measured temperature during a cycle were caused by variations in reactor power and these variations were experienced by all specimens. 
Power profiles for each cycle were calculated by weighting the specimen powers in cycle no. 1 by the ratio of the specimen relative neutron flux in the cycle of interest to the same specimen's cycle no. 1 relative neutron flux. Detailed calculations of the power profile have been reported by Lee. ${ }^{12}$

The second step of the analysis was to run a heat-transfer problem for each different power profile to determine the effect of varying the relative neutron flux profile on the specimen train temperature profile. This heat-transfer analysis is also further described by Lee 13 and the results are given in Table II.

Table II

Calculated Specimen Temperature Profile $\left({ }^{\circ} \mathrm{C}\right)$

\begin{tabular}{cccccccc}
\hline & \multicolumn{7}{c}{ Cycle } \\
\cline { 2 - 8 } Specimen no. & 1 & 3 & 4 & 5 & 6 & 7 & 8 \\
\hline $1-\mathrm{C}$ & 1415 & 1390 & 1382 & 1370 & 1350 & 1371 & 1341 \\
$2-\mathrm{A}$ & 1485 & 1467 & 1470 & 1450 & 1430 & 1447 & 1417 \\
$3-\mathrm{B}$ & 1487 & 1483 & 1483 & 1472 & 1445 & 1466 & 1438 \\
$4-\mathrm{D}$ & 1436 & 1439 & 1439 & 1431 & 1404 & 1427 & 1407 \\
$5-\mathrm{E}$ & 1441 & 1459 & 1458 & 1455 & 1437 & 1454 & 1438 \\
$6-\mathrm{G}$ & 1469 & 1503 & 1500 & 1499 & 1490 & 1496 & 1492 \\
$7-\mathrm{H}$ & 1409 & 1460 & 1455 & 1454 & 1440 & 1443 & 1450 \\
$8-\mathrm{I}$ & 1417 & 1487 & 1476 & 1471 & 1465 & 1463 & 1469 \\
\hline
\end{tabular}

Step number three in the analysis relates the temperature histories of each specimen to that measured for specimen no. 1-C, weighted by the calculated temperature profiles for each irradiation cycle. This was done for each cycle using the following relationship:

$$
T_{n}=\frac{\left(\mathrm{TMI}_{1}-50\right)\left(\mathrm{TC}_{\mathrm{n}}-50\right)}{\left(\mathrm{TC}_{1}-50\right)}+50
$$

where

$$
\mathrm{n}=\text { specimen number }
$$

$T M_{1}=$ measured temperature of specimen no. $1-\mathrm{C}\left({ }^{\circ} \mathrm{C}\right)$

$\mathrm{TC}=$ Table II calculated temperatures $\left({ }^{\circ} \mathrm{C}\right)$

50 = temperature of heat $\operatorname{sink}\left({ }^{\circ} \mathrm{C}\right)$

The results of the temperature history calculation based upon the measured temperature history of specimen no. 1-C are given in Table III. A verage (mean) and median temperatures for each cycle and for the entire eight-cycle irradiation were calculated. 
Table III

Calculated Specimen Temperatures $\left({ }^{\circ} \mathrm{C}\right)$.

\begin{tabular}{|c|c|c|c|c|c|c|c|c|c|c|}
\hline \multirow{2}{*}{\multicolumn{2}{|c|}{$\begin{array}{c}\text { Specimen } \\
\text { no. }\end{array}$}} & \multicolumn{8}{|c|}{ Cycle no. } & \multirow[b]{2}{*}{ Overall } \\
\hline & & 1 & 2 & 3 & 4 & 5 & 6 & 7 & 8 & \\
\hline \multirow[t]{2}{*}{$1-\mathrm{C}$} & Average & 1381 & 1352 & 1302 & 1351 & 1327 & 1290 & 1268 & 1232 & 1317 \\
\hline & Media & 1417 & 1377 & 1297 & 1357 & 1307 & 1287 & 1287 & 1237 & 1307 \\
\hline \multirow[t]{2}{*}{$2-A$} & Average & 1449 & 1429 & 1372 & 1436 & 1401 & 1364 & 1337 & 1302 & 1388 \\
\hline & Median & 1487 & 1447 & 1367 & 1437 & 1377 & 1357 & 1357 & 1307 & 1377 \\
\hline \multirow[t]{2}{*}{$3-B$} & $A v$ & 1449 & 1431 & 1386 & 1449 & 1425 & 1379 & 1356 & 1321 & 1401 \\
\hline & Media & 1487 & 1447 & 1377 & 1457 & 1407 & 1377 & 1377 & 1327 & 1397 \\
\hline \multirow[t]{2}{*}{$4-D$} & Ave & 1401 & 1382 & 1343 & 1403 & 1387 & 1340 & 1318 & 1292 & 1359 \\
\hline & Median & 1437 & 1397 & 1337 & 1407 & 1367 & 1337 & 1337 & 1297 & 1357 \\
\hline \multirow[t]{2}{*}{$5-E$} & Average & 1401 & 1382 & 1360 & 1432 & 1408 & 1370 & 1345 & 1321 & 1376 \\
\hline & Median & 1437 & 1397 & 1357 & 1427 & 1387 & 1367 & 1367 & 1367 & 1377 \\
\hline \multirow[t]{2}{*}{$6-G$} & Aver & 1431 & 1411 & 1404 & 1464 & 1450 & 1421 & 1383 & 1369 & 1415 \\
\hline & Median & 1467 & 1427 & 1397 & 1467 & 1427 & 1417 & 1407 & 1377 & 1417 \\
\hline \multirow[t]{2}{*}{$7-\mathrm{H}$} & Average & 1371 & 1352 & 1363 & 1421 & 1408 & 1391 & 1335 & 1331 & 1369 \\
\hline & Median & 1407 & 1375 & 1357 & 1427 & 1387 & 1387 & 1357 & 1337 & 1367 \\
\hline \multirow[t]{2}{*}{$8-\mathrm{I}$} & Average & 1381 & 1362 & 1391 & 1441 & 1721 & 1399 & 1351 & 1349 & 100 \\
\hline & Med & 1417 & 1377 & 1387 & 1447 & 1397 & 1397 & 1367 & 1377 & 1387 \\
\hline
\end{tabular}

F. Neutron Radiography

A system of examining specimens by neutron radiography was utilized during this irradiation. Prior to irradiation and at the conclusion of each Battelle Research Reactor (BRR) cycle, Capsule LRL-1 was moved into a reactor pool position where the capsule was interposed between a collimated neutron beam and an indium foil. Variation in neutron attenuation of the capsule caused the indium to activate nonuniformly and an image of the capsule and its contents was formed on the foil. A photographic film was then brought into contact with the decaying foil to transfer the image. Since the film was not exposed to the highly radioactive specimen, it was not fogged by the radiation.

This technique was used in an attempt to observe the progression of swelling from one cycle to another and to thereby establish a swelling rate. Unfortunately, due to the presence of excessive amounts of Mo, $W$, and $R e$ in the capsule, the specimen image was not sharp and definitive dimensions were not readily obtained. 
At the conclusion of the final irradiation cycle (no. 8), Capsule LRL-1 was transferred to the BMI-C hot cells where the primary containment was pierced by drilling and the fission gases released during irradiation were pumped into glass collection vials of known volume, which later analyzed by mass spectrometry for Xe and Kr and their isotopic compositions.

The capsule was then cut to about $19 \mathrm{in}$. in length and shipped in a lead-lined cask to LRL for the remaining post-irradiation analyses, which were performed at the $\beta-\gamma$ hot cell facility described by Albrecht et al. 14

Capsule disassembly began with the removal of the secondary stainless-steel containment by cutting with a motorized pipe cutter just below the header. The tubular vessel was easily slipped off, revealing the primary molybdenum container which was removed by the same procedure. Figure 3 shows the disassembled capsule.

The specimen train was then sequentially disassembled from bottom to top, first the sleeve, followed by a clad specimen and its attached thermocouple. All parts were carefully numbered and placed in specially designed storage boxes for each specimen.

Each clad specimen was photographed macroscopically upon removal from the capsule. Diameter and length measurements were made of each clad specimen (following removal of the thermocouple), to determine dimensional changes. Specimen weights were also determined.

Cladding ends were then removed and the internal diameter of the specimens was measured. This was followed by removal of the remaining cladding. Diametral and length measurements of the fuel were then made.

The next step in post-irradiation examination was the determination of specimen density. Each piece of the one-, two-, or threesegment specimen was weighed on a semi-microbalance and its volume was determined by use of a mercury pycnometer described by Albrecht et al. 15 The density calculated from these weights and volume measurements was then compared to the pre-irradiation density to establish the gross volumetric swelling of the fuel.

Specimens were then sawed into several pieces for metallographic and chemical analyses. The procedure used for the microanalys is is described in detail by Albrecht et al. 16

Metallographic specimens were mounted in glass-filled diallyl phthalate using a remotized mounting press and then ground in a twostage grinding sequence using $12.5 \mu \mathrm{SiC}$ and $9 \mu \mathrm{Al}_{2} \mathrm{O}_{3}$ abrasives on 12 -in. Lapmaster lapping machines. After being ultrasonically cleaned, the specimens were polished on Syntron vibratory polishers 


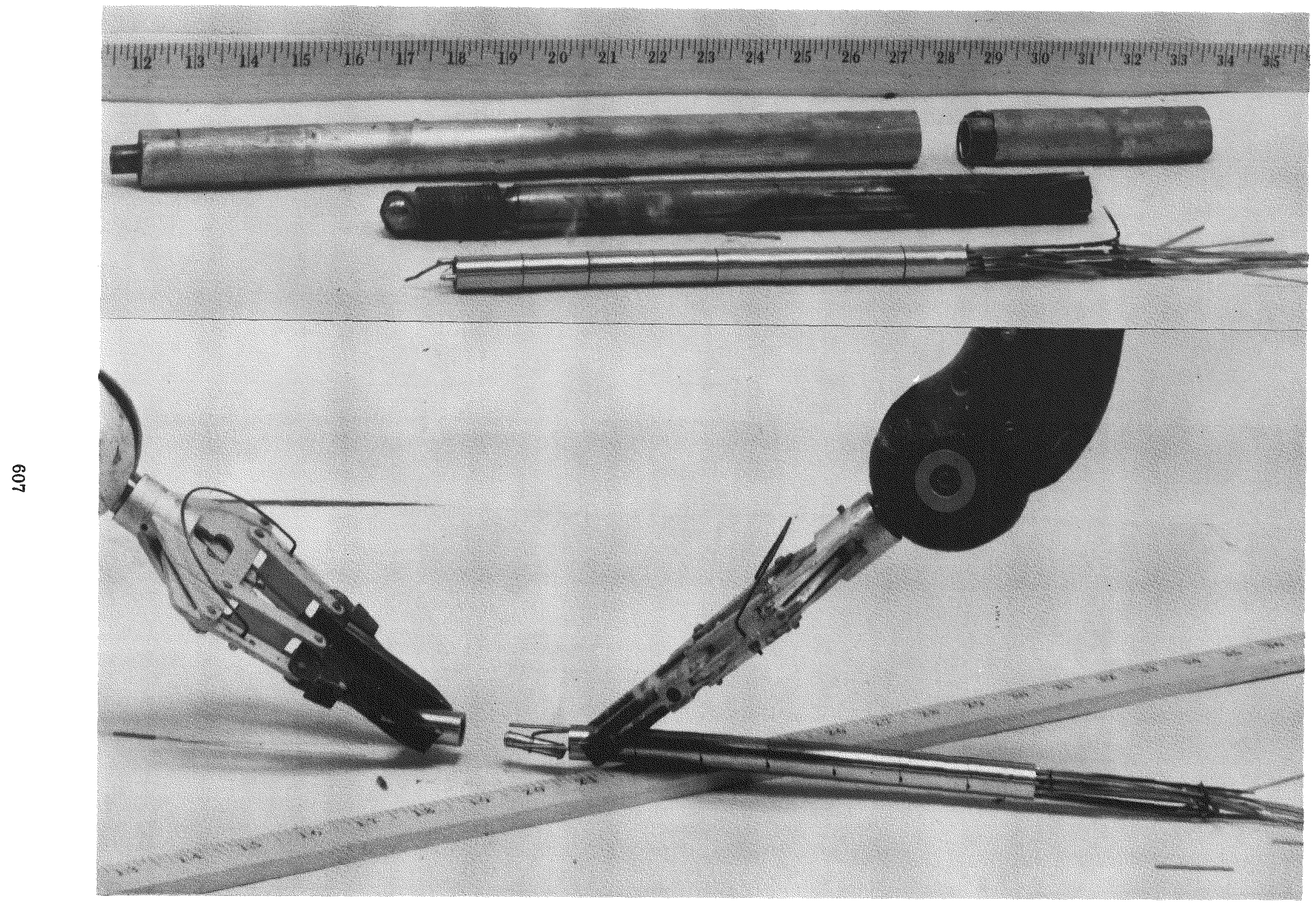

Fig. 3. Upper photo shows the primary and secondary containment removed from the buffered specimen train of Capsule LRL-1. Lower photo shows remote disassembly of specimen train. 
using $1-\mu$ diamond abrasive on silk or nylon cloth. The specimens were again ultrasonically cleaned and the entire surface was photographed in the as-polished condition at 50X magnification. Some areas were also photographed at $200 \times$ and $400 \times$. The specimens were then chemically stain-etched using 10-ml nitric, three drops hydrofluoric, $30-\mathrm{ml}$ lactic acid etchant and subsequently all areas were rephotographed at the same magnification used in the as-polished condition. The metallographic facility and procedures have been described in detail by Albrecht and Johnson. 17

At least three samples from, each specimen were analyzed for total uranium content by solution analytical chemistry. All analyses were run from two to five times for each sample. The procedure followed for this controlled-potential coulometric analysis is a slightly modified version of tentative ASTM Method D-217. which is based upon the method of Booman and Holbrook described elsewhere.18 The controlled-potential coulometer is described by Harrar and Behrin, 19 and the cell assembly by Harrar. 20

A bomb-combustion technique was used for the total nitrogen, xenon, and krypton determinations. This method, developed for the simultaneous determination of carbon, hydrogen and nitrogen, has been described by Frazer.21 The system used for UN was modified by the omission of a halogen trap and furnaces used for carbon and hydrogen analyses. Other modifications included a larger $(100 \mathrm{mg})$ sample size, higher firing temperature $\left(1000^{\circ} \mathrm{C}\right)$, and longer time (24 hours) to allow for complete sample combustion. The evolved gases were then pumped into bombs of predetermined volume which were attached to a mass spectrometer for total nitrogen and isotopic analyses of xenon and krypton.

Fuel burn-up was determined by radiochemical analysis where gamma spectroscopy was made on samples that were dissolved in nitric acid and extensively diluted. A carefully calibrated planar Ge(Li) detector 22 was used and the data analyzed by the GAMANAL computer code. $23,24,25$ Corrections to the activity were made for the effect of variations in flux during the irradiation, which was long in relation to the half-life of the investigated species. Zr 95 was used as the primary fission indicator with $\mathrm{Ce}^{144}$, which has the advantages of a longer halflife, being used as a secondary fission indicator. Uranium isotopic ratios were determined by standard mass-spectroscopic technique while the total uranium content was determined by controlled potential coulometric analysis described earlier.

Representative samples from the cladding on each specimen were mounted and metallographically prepared for inspection, in the aspolished, chemically etched or cathodically etched condition. The microstructures were examined and features of interest were photographed.

Two W-5 Re versus W-26 Re thermocouples, which were attached to the cladding surface during irradiation, were examined metallographically to determine if the bead knot sintered to the cladding and to 
see if any other microstructural changes, such as grain growth or appearance of a second phase, took place.

The centerline thermocouple was left intact and was calibrated at $1100^{\circ} \mathrm{C}$ in an argon-filled platinum-wound furnace. The activity level of the thermocouple was sufficiently low and swipe-free so that the calibration was performed in a hood outside the hot cells. A Pt versus $\mathrm{Pt} 10 \% \mathrm{Rh}$ standard thermocouple, an unirradiated $\mathrm{W}-5 \mathrm{Re}$ versus $W-26$ Re thermocouple of interest; and the irradiated thermocouple were bound together with beads intimate, but not in contact, and inserted into the argon-filled furnace tube. The free ends were attached to copper extension wire, and these cold junctions were immersed in ice water during the comparative emf readings.

III. Experimental Results

\section{A. Macroscopic Observations}

Disassembly of the capsule presented no particular difficulties, as both the secondary and primary containers were removed with ease. The primary molybdenum container was highly discolored, apparently caused by minor oxidation during irradiation. The five chromel versus alumel thermocouples were intact and in their proper positions at the conclusion of the irradiation. The specimen train, including the molybdenum buffers, was completely intact with all parts bright and apparently unreacted, and all $W-5$ Re versus $W-26$ Re specimen thermocouples were operational and correctly positioned. A single thermocouple running to the $\mathrm{U}_{2} \mathrm{~N}_{3}$ body at the bottom of the specimen train was also in proper position and operational. The auxiliary heater placed about the $\mathrm{U}_{2} \mathrm{~N}_{3}$ failed during the eighth and final cycle of irradiation; the cause of this failure was not investigated.

Each specimen cladding developed from one to three longitudinal cracks apparently caused by diametral fuel swelling, resulting in rupture stresses. These cracks, which varied in length and width, were directly related to the amount of fuel swelling. A typical cladding failure is shown in Fig. 4. The exterior cladding surface remained clean during irradiation and the vent holes were completely open. The W-5 Re versus W-26 Re thermocouples had sintered to the W-25.5 Re cladding in several spots on the higher temperature specimens.

After the cladding was removed it was seen that all eight specimens still retained their original configuration. No breakage, spalling, splintering, powdering, crumbling, or macroscopic cracks were observed in the fuel. All fuel specimens were readily removed from their cladding with no bonding or adherence, indicating no significant reaction. It was observed later, however, that a layer of UN approximately $0.001-$ in. thick was deposited on the inner cladding surface of both single crystal specimens nos. $2-A$ and $3-B$ and one high-density fine-grained (6-G) and one high-density coarse-grained specimen $(7-H)$ in the hottest locations in the capsule. The complementary specimens to No's, 6-G and 7-H which were in positions $1-\mathrm{C}$ and $8-\mathrm{I}$ 


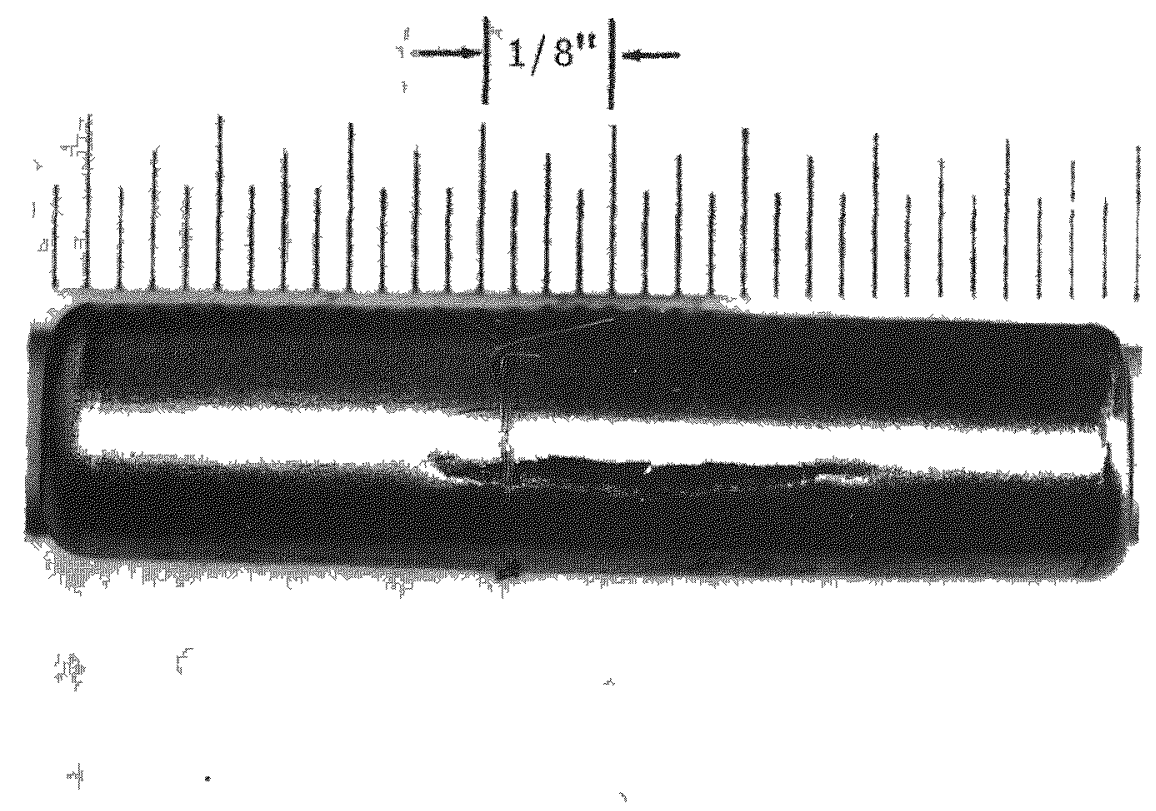

Fig. 4. Typical cladding failure of specimen 6-G, top of specimen to right, showing the low ductllity of the W-25.5 Re cladding used in this capsule. 
in the capsule and two fine-grain low-density specimens Nos. 4-D and 5-E, did not exhibit this UN deposit on the cladding. The 0.030-in.thick depleted UN disk used for delineation of the bottom edge of each specimen in neutron radiography was sintered to the fuel in specimen No. 6-G only, the specimen with the hottest thermal history. Specimen No. 6-G was composed of two segments which were also sintered together during irradiation. No other multiple-segment specimens showed any sintering or bonding between segments.

\section{B. Microscopic Changes}

Careful microscopic comparison of pre- and post-irradiation fuel microstructures were made for each of the four varieties of UN that were irradiated.

The fine-grain, low-density (78\% theoretical) microstructure was originally composed of polycrystalline high-density UN particles, having rather sharp angular surface features, sintered together in a hot pressed compact and retaining discontinuous angular voids at the particle boundaries. Figure 5 shows a typical pre-irradiated microstructure. The only significant change observed in the irradiated material was the collection of spherical porosity, approximately 0.5 to 1 micron in diameter, at the grain boundaries as shown in Fig. 6 . This porosity was heavily concentrated in grain boundaries parallel to the surfaces of the specimen; the concentration was greatest near the inside specimen surface. The grain diameter of this material was from 10 to 40 microns, while the particle size varied from 60 to 100 microns. The grain diameter and particle size were not altered during the irradiation. Minor amounts of a second phase identified as $\mathrm{U}_{2} \mathrm{~N}_{3}$ were observed and were attributed to fabrication rather than irradiation environment.

Fine-grained, high-density (96\% theoretical) UN having an average original grain diameter of 30 microns was the second of the four microstructures irradiated. A photomicrograph of a typical preirradiated microstructure is shown in Fig. 7. Again, rather angular voids were present at the grain boundaries which accounts for the density of the UN being nominally $4 \%$ less than theoretical. It should be noted in Fig. 7 that the voids are discontinuous and do not delineate the grains. Figure 8 shows the irradiated fine-grained, high-density microstructure. Two major changes in microstructure occurred within this material. The first was the collection of spherical porosity, approximately 0.5 to 1.0 micron in diameter, at the grain boundaries. The porosity was most predominate in grain boundaries parallel to the specimen surfaces and the concentration was again greatest at the inner surface. The second change was that longitudinal cracks appeared near the center of the top specimen No. 1-C which extended radially 0.003 to 0.007 in. into the UN from the outside surface. The complementary specimen, No. 6-G, which has a higher irradiation temperature history and exhibited greater swelling, did not show evidence of cracking which may be related to the presence of the centerline thermocouple well in specimen No. $1-\mathrm{C}$. 


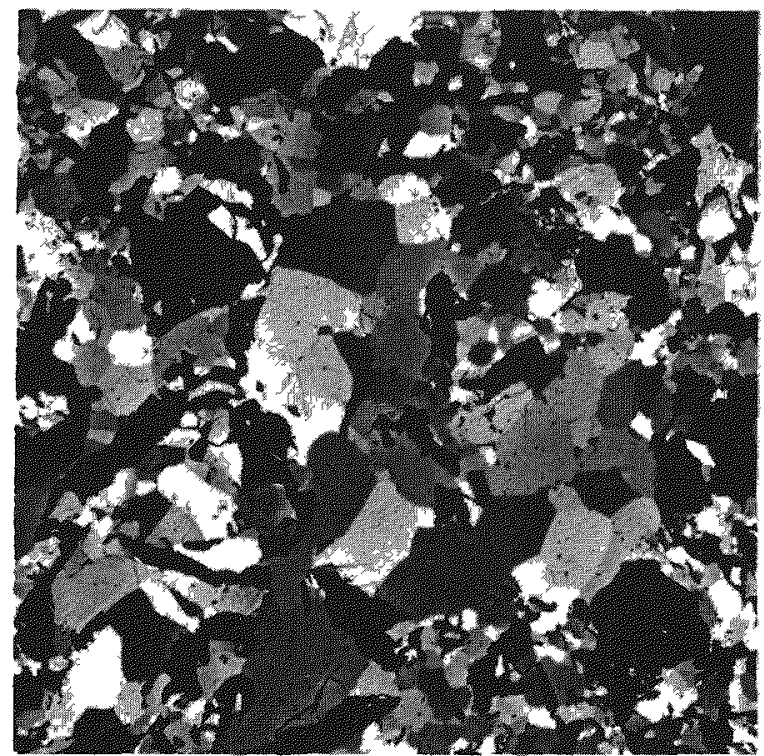

Fig. 5. Typical pre-irradiated $78 \%$ dense, fine-grained UN, $200 \times$ mag.

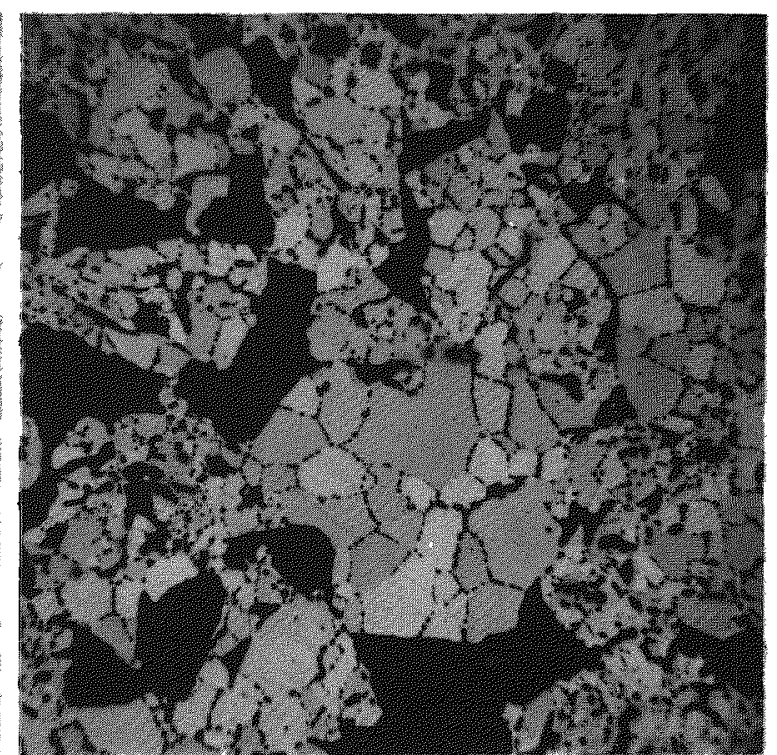

Fig. 6. Specimen 5-E, postirradiated, low density, fine-grained UN showing collection of spherical porosity at the grain boundaries, 200× mag.

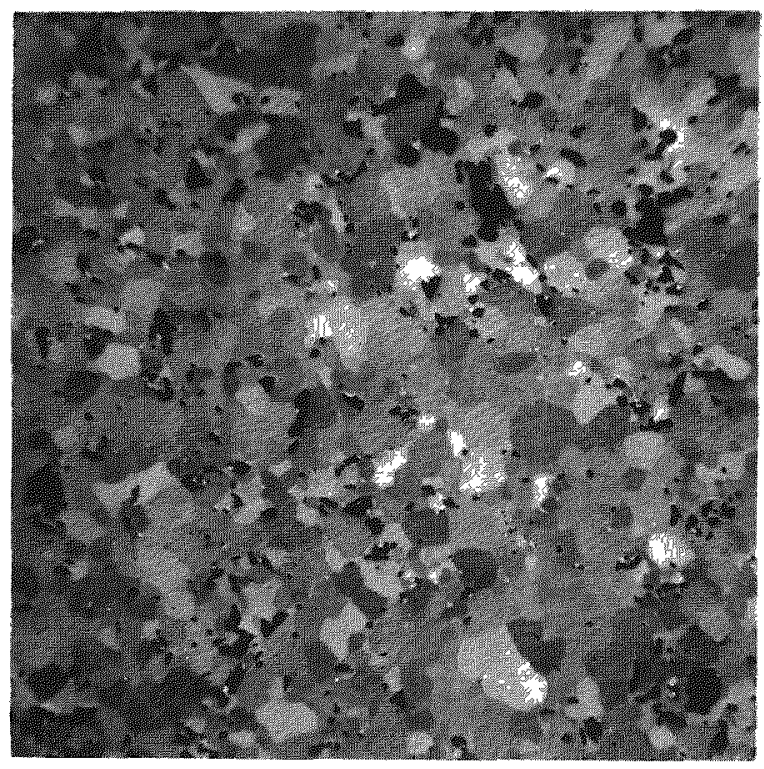

(a) Stain-etched, 200× mag.

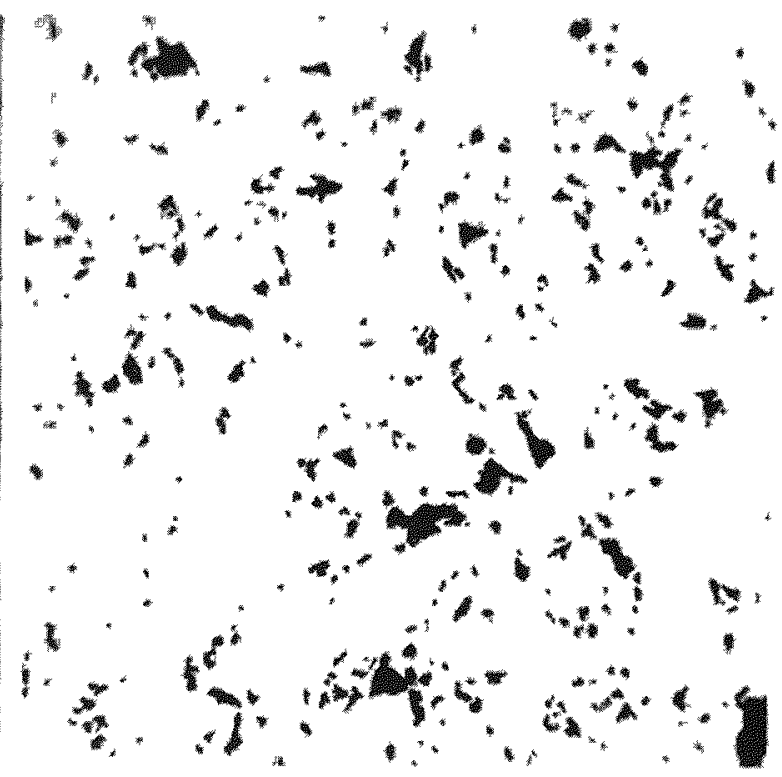

(b) As-polished, 200X mag.

Fig. 7. Typıcal pre-ırradiated $96 \%$ dense, fine-grained UN. 


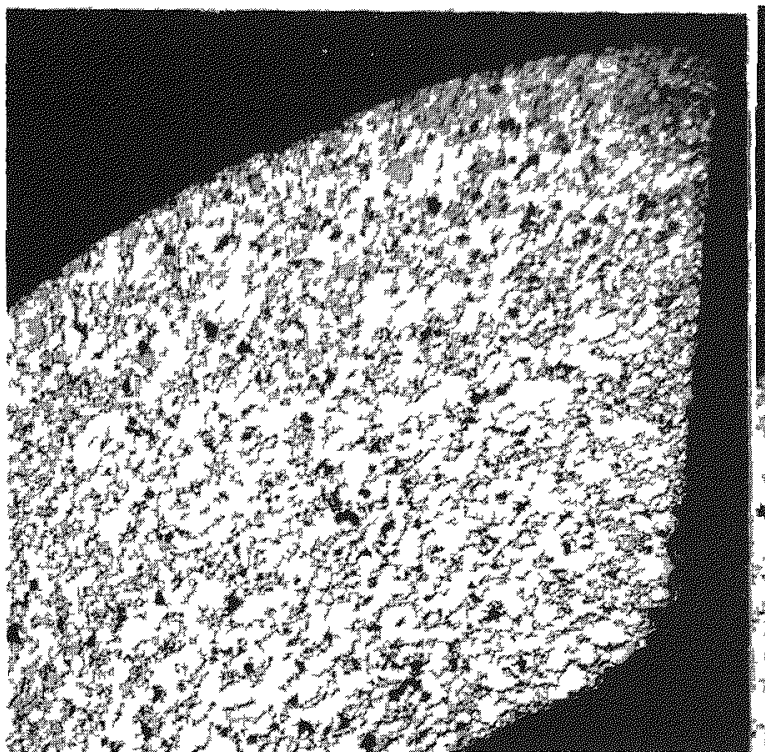

(a) Stain-etched, 50X mag.

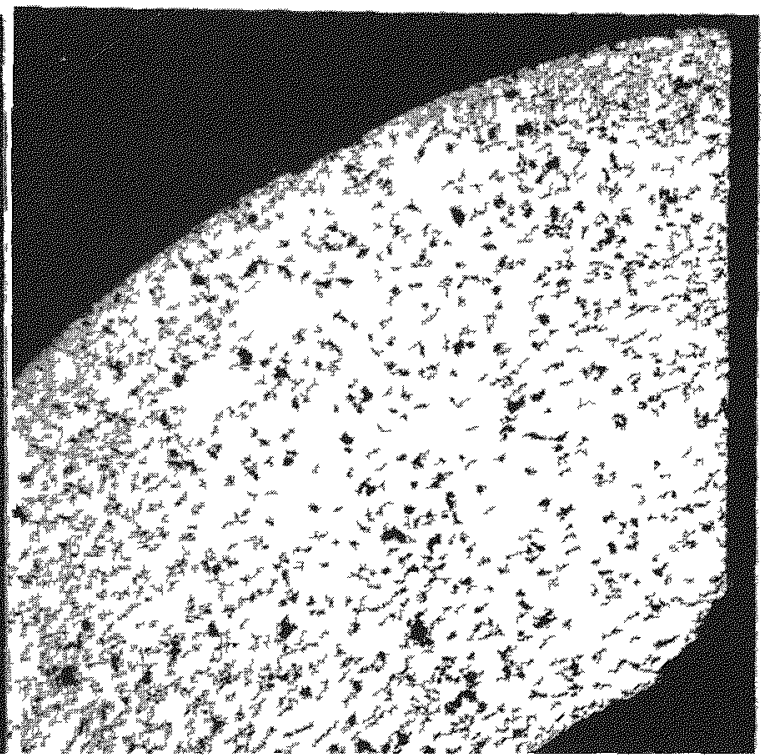

(b) As-polished, $50 \times$ mag.

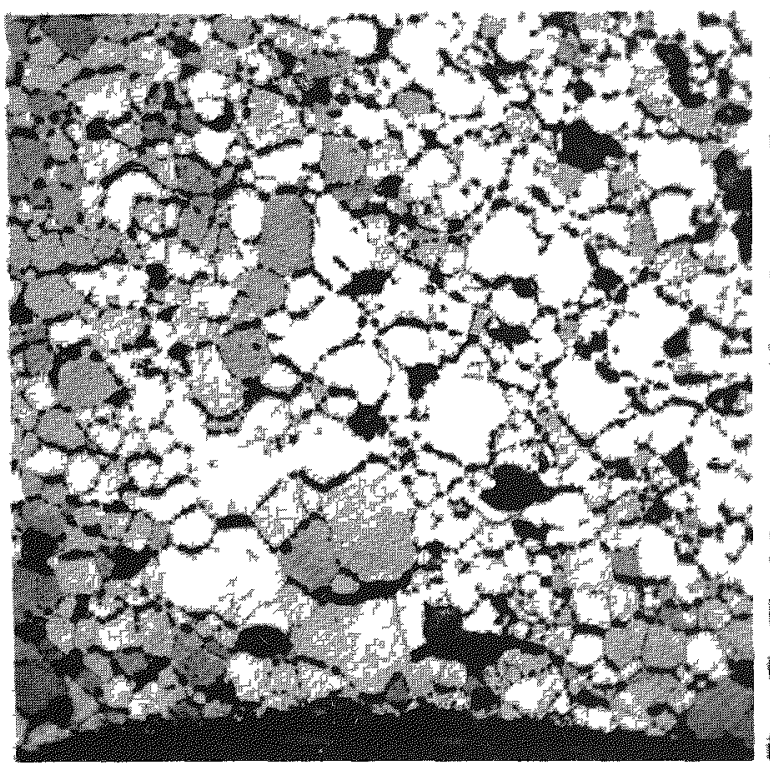

(c) Stain-etched, 200× mag.

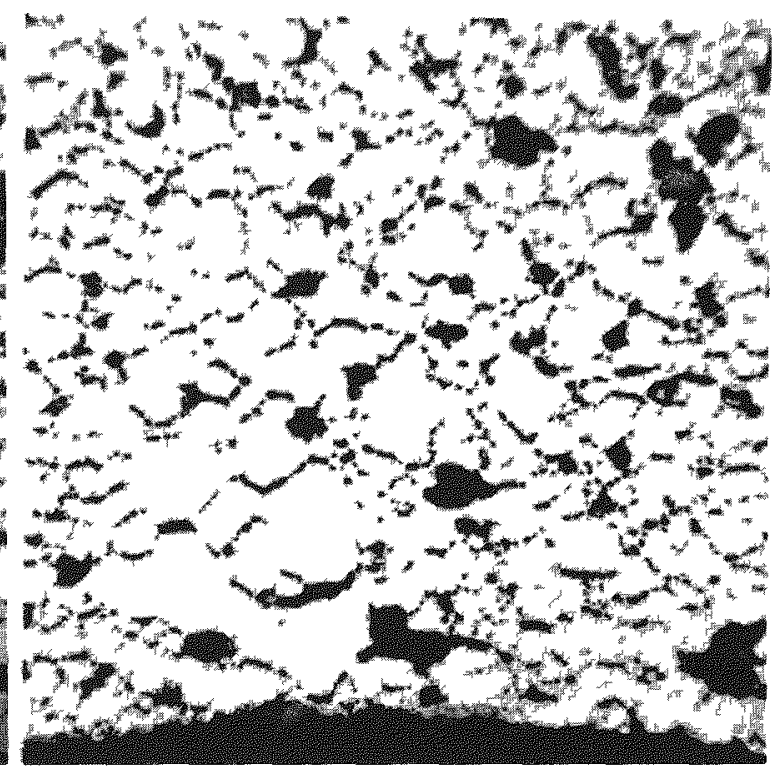

(d) As-pollshed, 200× mag.

Fig. 8. Specimen 6-G, high density, fine-grained UN after irradiation showing spherical porosity collected at the grain boundaries with greatest concentration in grain boundaries parallel to the surfaces of the specimen. 
The coarse-grained, high-density (97\% theoretica1) UN is shown in its unirradiated condition in Fig. 9. The original average grain diameter of 105 microns resulted from the annealing of fine-grained UN described earlier. This heat treatment caused the voids to become more equiaxed and to relocate at the triple points of the grain boundaries. A slight densification from 96 to $97 \%$ theoretical also resulted during the anneal. Figure 10 shows the post-irradiation microstructure. Nearly all grains are completely delineated by spherical porosity approximately 0.5 to 1.0 micron in diameter at the grain boundaries. This collection of porosity is a repeat of that previously described for the fine-grained high- and low-density UN. The porosity located in the grain boundaries parallel to the specimen surface is nearly continuous due to its abundance. By contrast, porosity in grain boundaries perpendicular to the surface are more sparsely distributed. The extensive porosity weakened the grain boundaries and thus caused the extraction of entire grains from the microstructure during metallographic specimen preparation.

Finally, the single crystal UN with a density of $100 \%$ theoretical is shown in the pre-irradiated condition in Fig. 11. The pre-irradiated microstructure reveals no voids or porosity, no grain or sub-grain boundaries, and no cracks or fissures. The post-irradiation microstructure shown in Fig. 12 reveals no significant change from that of the pre-irradiated UN. No porosity is seen in the bulk crystal at magnifications above $400 \times$. Some very fine porosity of a spherical nature, approximately 0.1 to 0.5 micron in diameter, has collected on sub-grain boundaries extending into the crystal from the external surface. No other changes in the microstructure were observed.

Figure 13 shows the microstructure of the W-25.5 Re cladding before irradiation. This material was single phase and cold worked with heat affected zones near the welds at the bottom and top of the cladding. Figures 14 through 16 show typical microstructures of the cladding after irradiation. The changes include creation of a stable microstructure of equiaxed grains through recrystallization and grain growth with an average grain diameter of 30 microns. A second phase (sigma) was precipitated from solution and occurred in the grain boundaries, especially at the triple points, indicating that the composition of the cladding was richer in Re than the amount that could be held in solution at the temperature of irradiation. There was no indication of any significant chemical reaction between the fuel and the cladding during this irradiation.

Electron microprobe examination revealed that no detectable uranium was present in the cladding and confirmed the presence of a second phase (sigma) having a higher rhenium concentration than the primary $\mathrm{W}-\mathrm{Re}$ solid solution.

C. Dimensional Changes

Neutron radiographs made before irradiation and at the conclusion of each cycle are shown in Fig. 17. Diameters of the clad speciments were measured from the neutron radiographs at the top, middle, and bottom of the cladding as well as lengths of the fuel and cladding. 


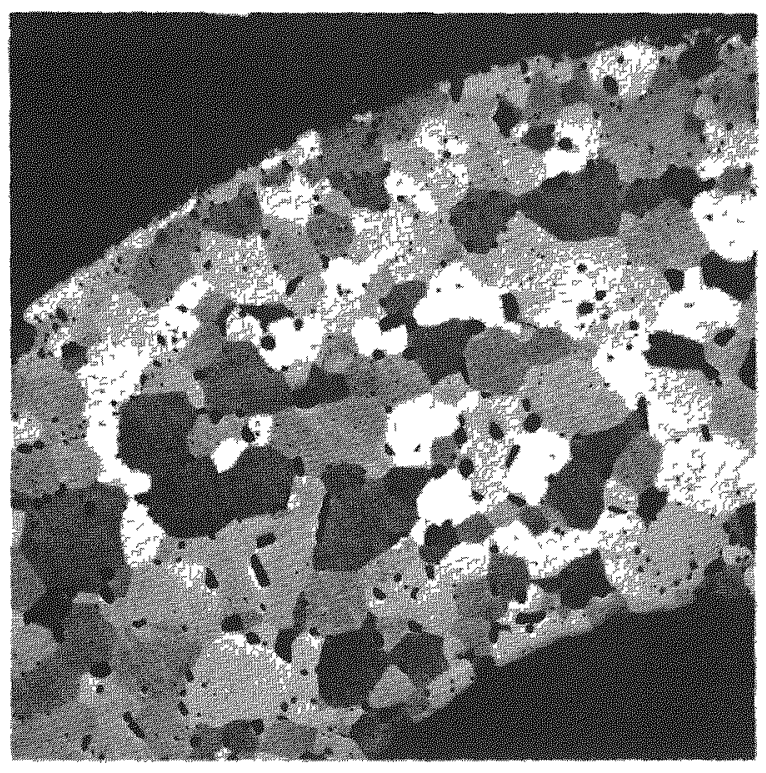

(a) Stain-etched, 50x mag.

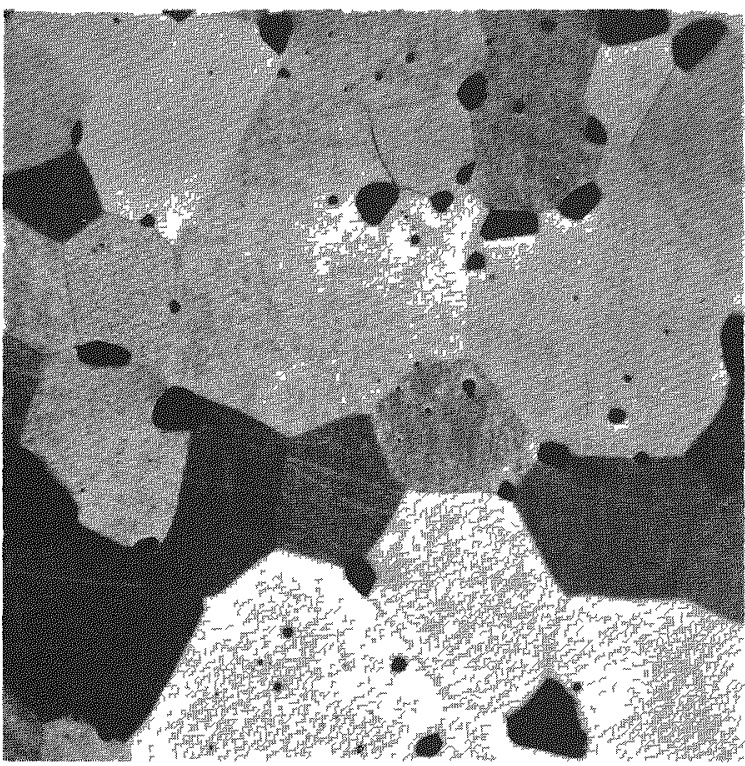

(c) Stain-etched, 200X mag.

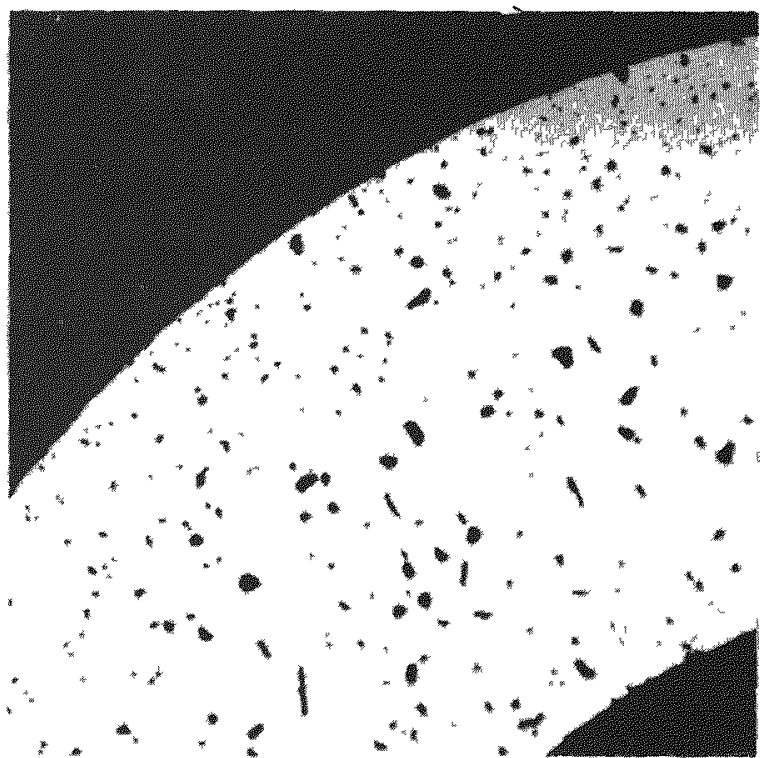

(b) As-polished, 50× mag.

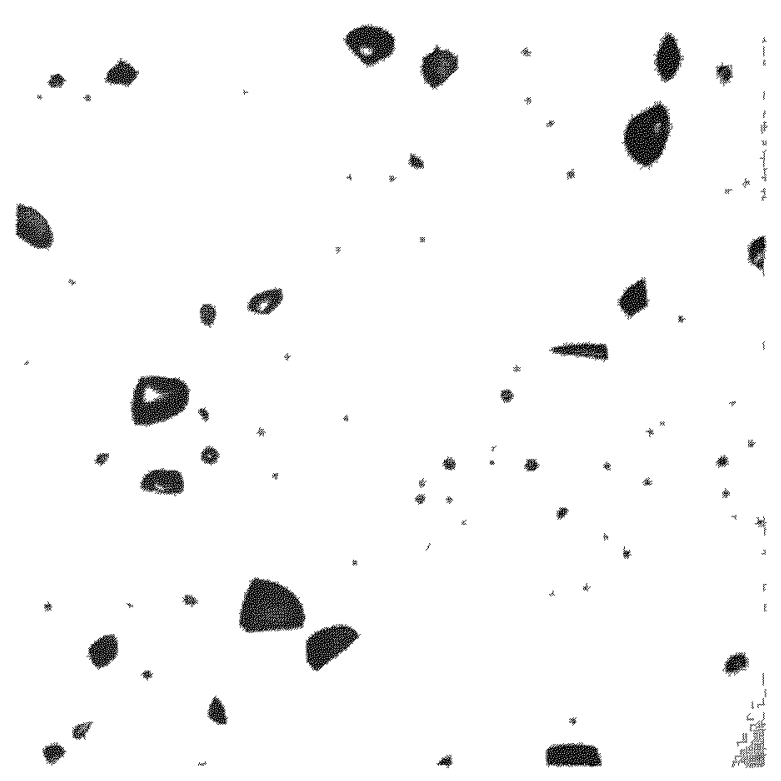

(d) As-polished, 200X mag.

Fig. 9. Typical pre-irradiated $97 \%$ dense, coarse-grained UN. 


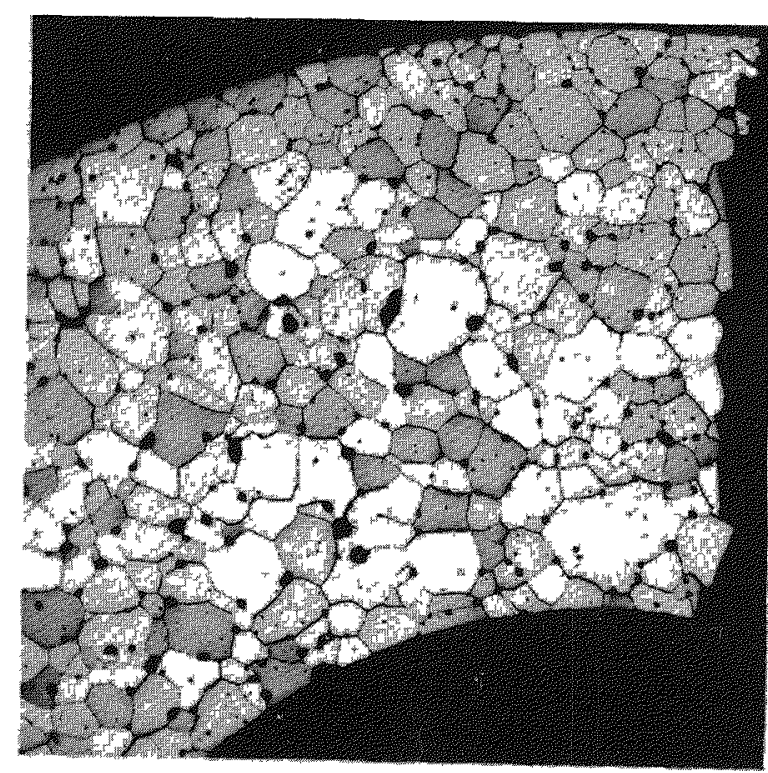

(a) Stain-etched, 50X mag.

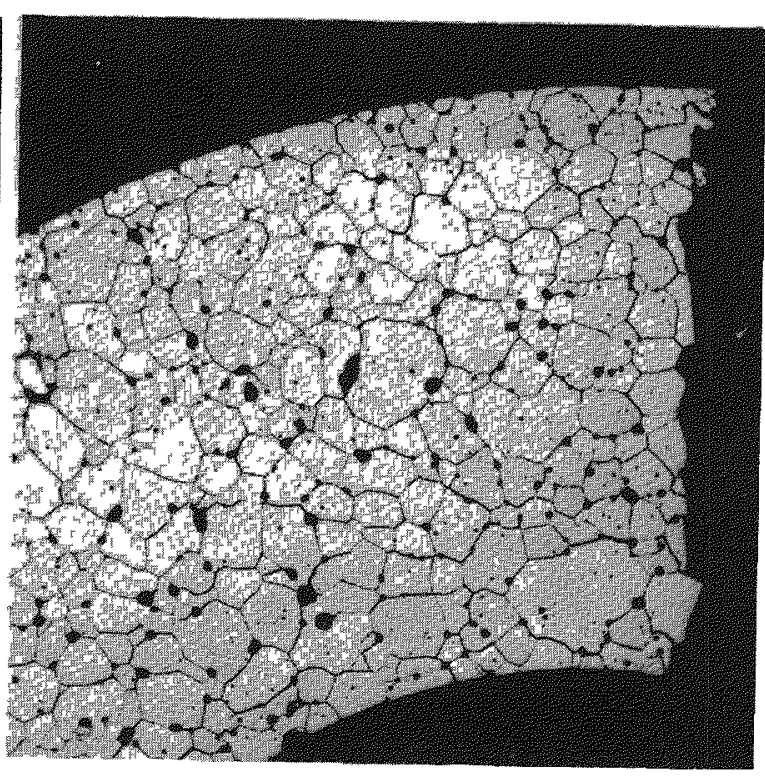

(b) As-polished, 50X mag.

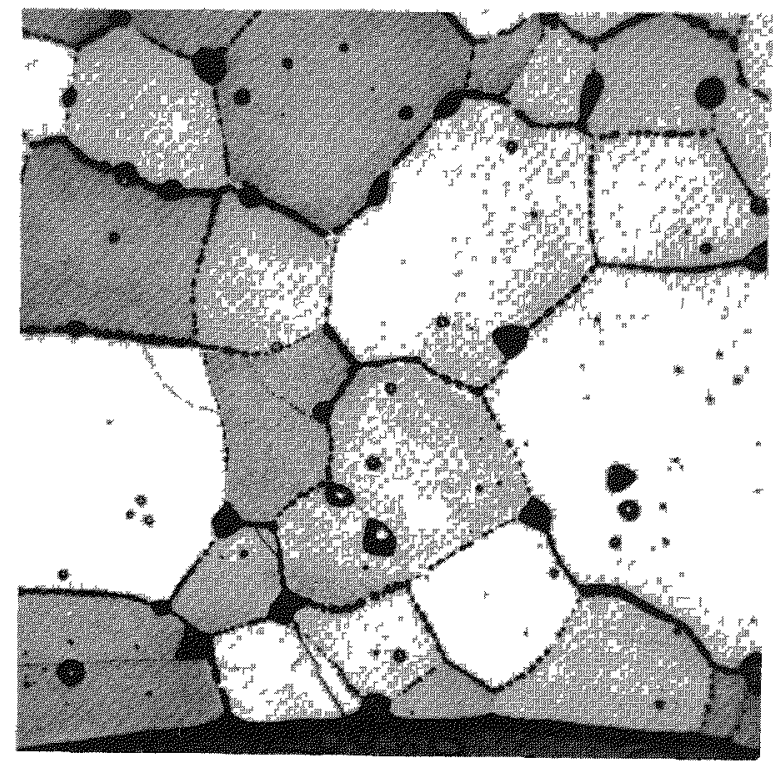

(c) Stain-etched, 200x mag.

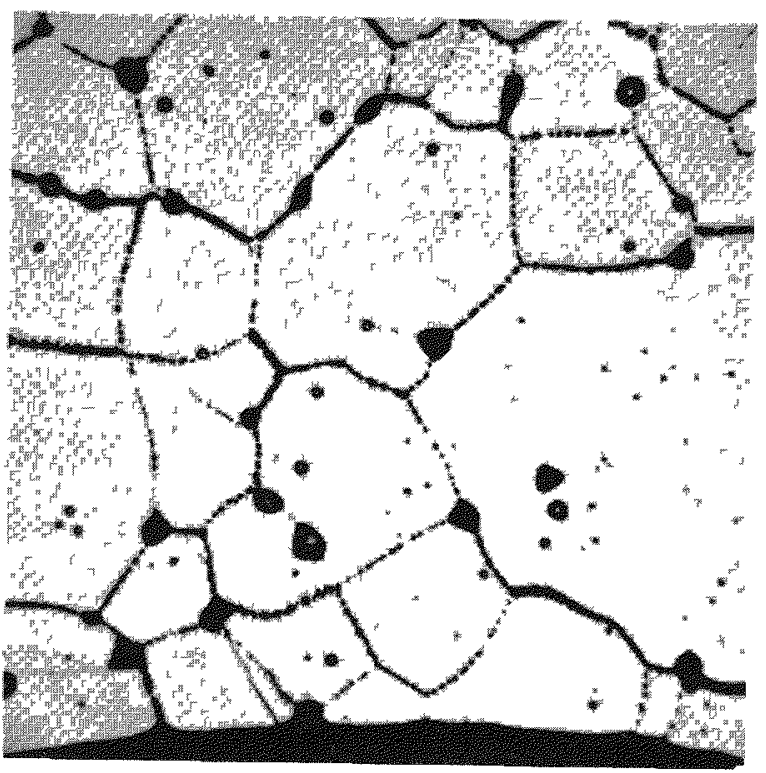

(d) As-polished, 200× mag.

Fig. 10. Specimen 7-H, high density, coarse-grained UN after irradiation showing a collection of spherical porosity in the grain boundaries with the highest concentration in those boundaries parallel to the surface of the specimen. 


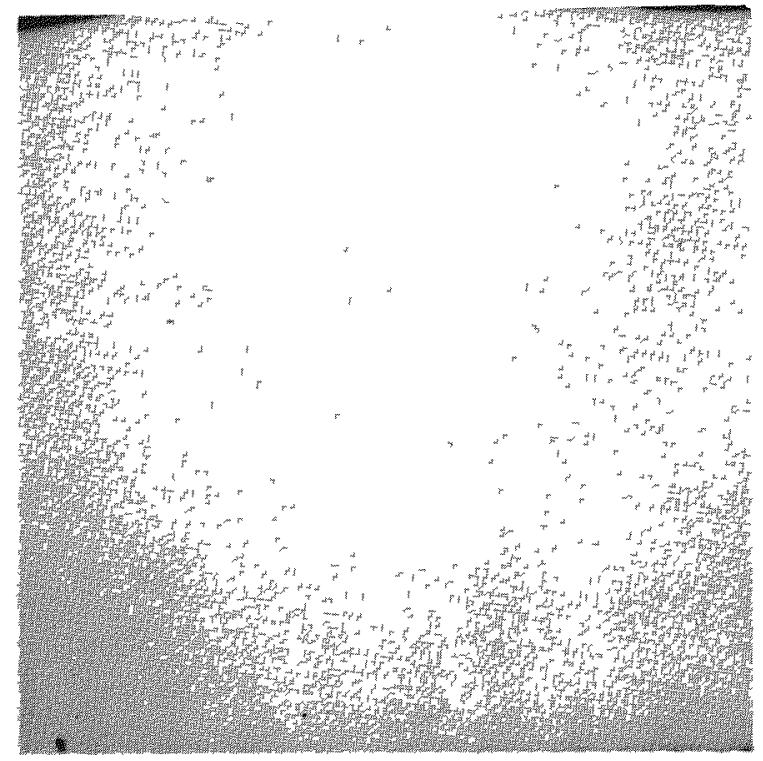

Fig. 11. Typical single crystal UN, pre-irradiation, strain etched, showing no porosity or other distinguishable features, $50 \times$ mag. 


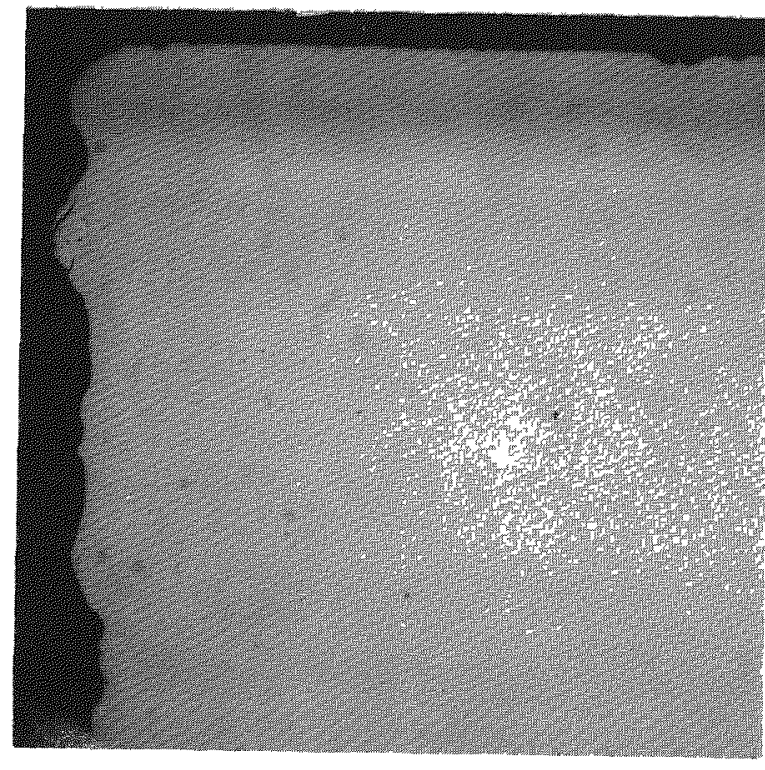

(a) As-polished, longitudinal section, $50 \times$ mag.

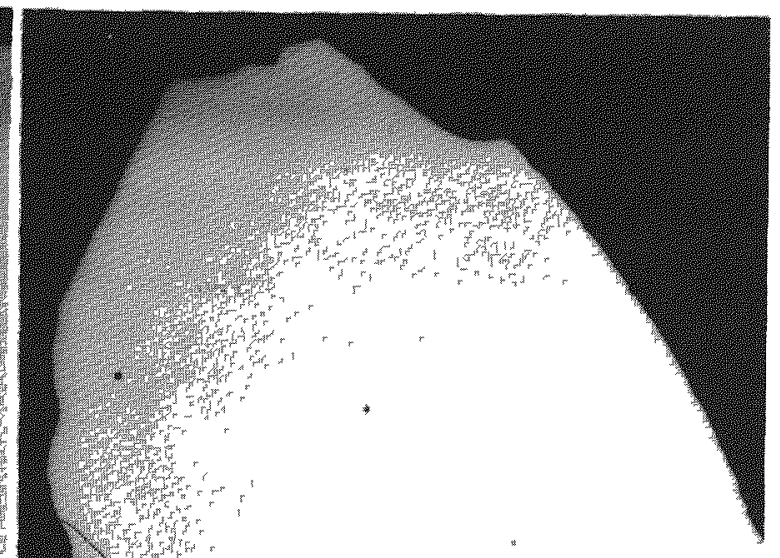

(b) As-polished, cross-section, $50 \times$ mag.

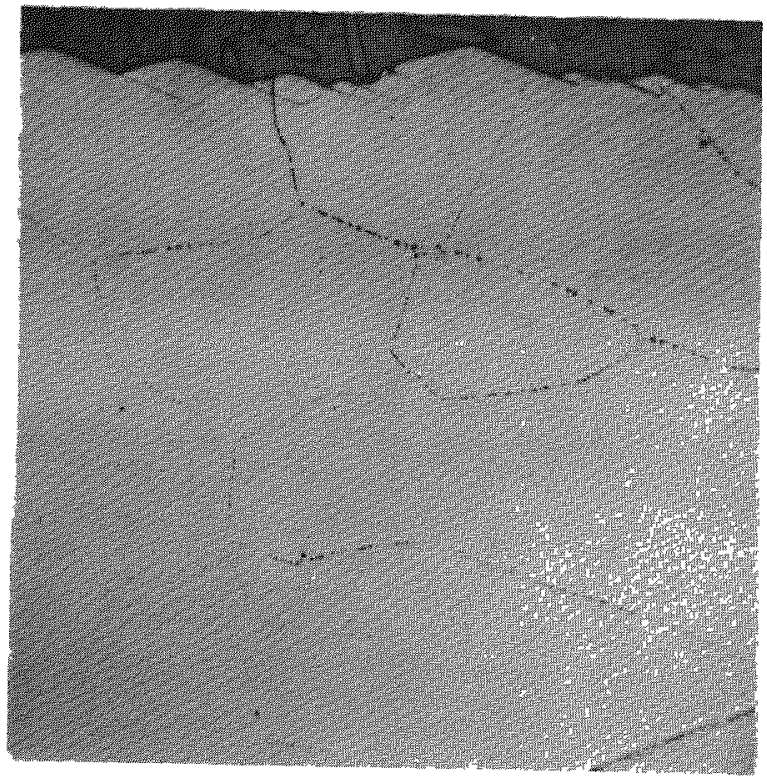

(c) Stain-etched, 200x mag.

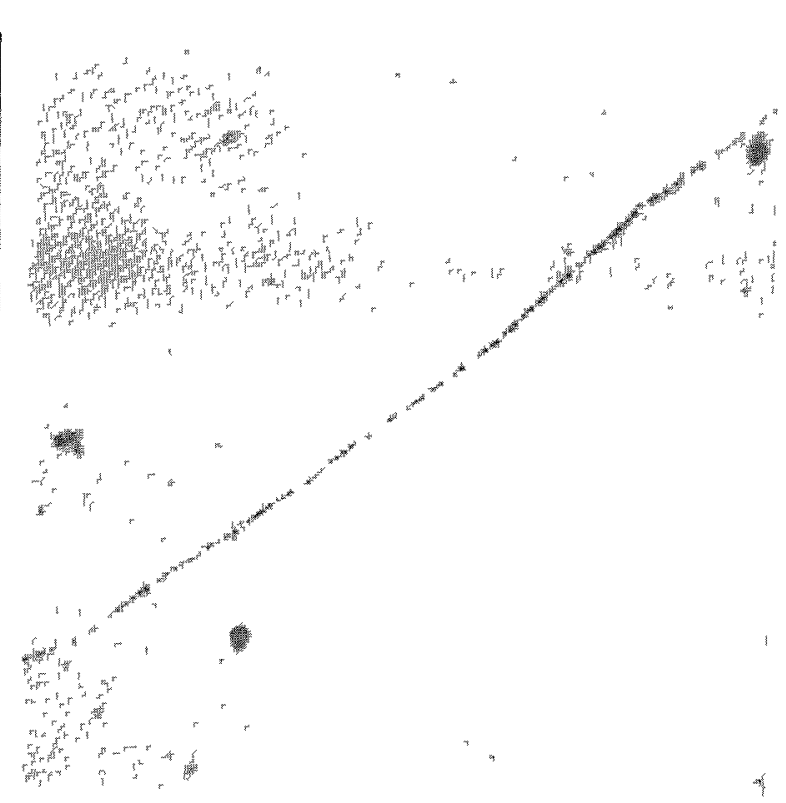

(d) As-polished, 400X mag.

Fig. 12. Specimens 2-A and 3-B, single crystal UN, post-irradiation microstructure showing no change in optically observed microstructure other than collection of small spherical porosity along sub-grain boundaries which rarely occurred. 


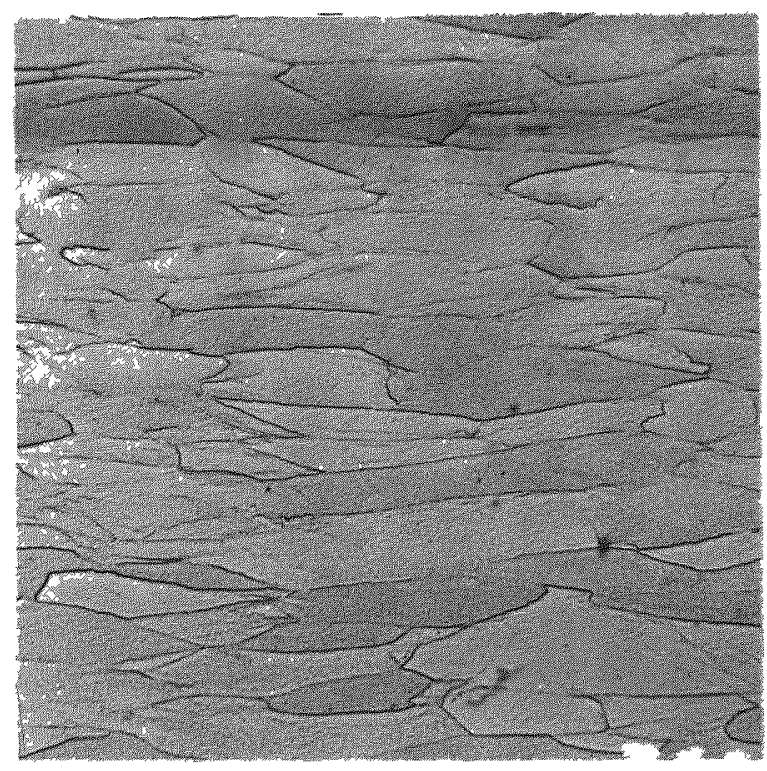

Fig. 13. W-25.5 Re cladding before irradiation showing worked structure, chemically etched, 200× mag.

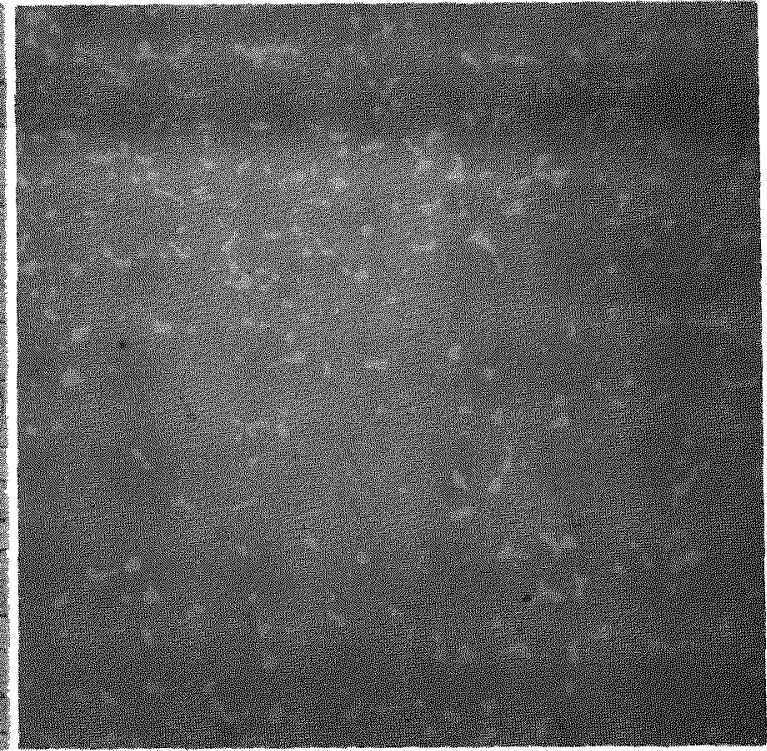

Fig. 14. W-25.5 Re cladding, postirradiation showing precipitation of sigma phase. As-polished, 200× mag.
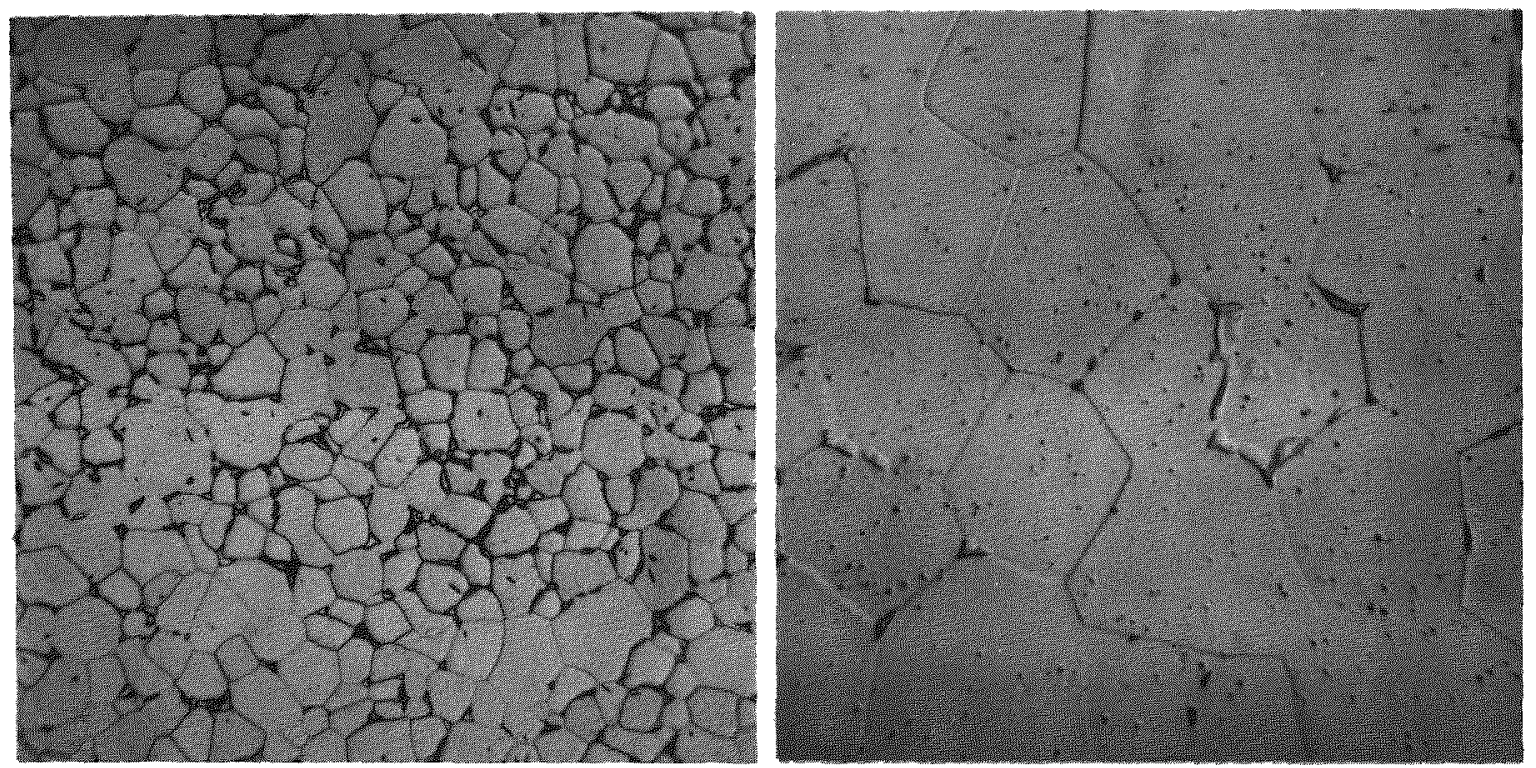

Fig. 15. W-25.5 Re cladding, post-Fig. 16. W-25.5 Re cladding, postirradiation showing sigma phase precipitation at triple-points in equi-axed grains of annealed microstructure. Chemically etched, 200X mag. irradiation showing precipitation of sigma phase at grain boundaries and within the grains. Cathodically etched, $400 \mathrm{X}$ mag. 


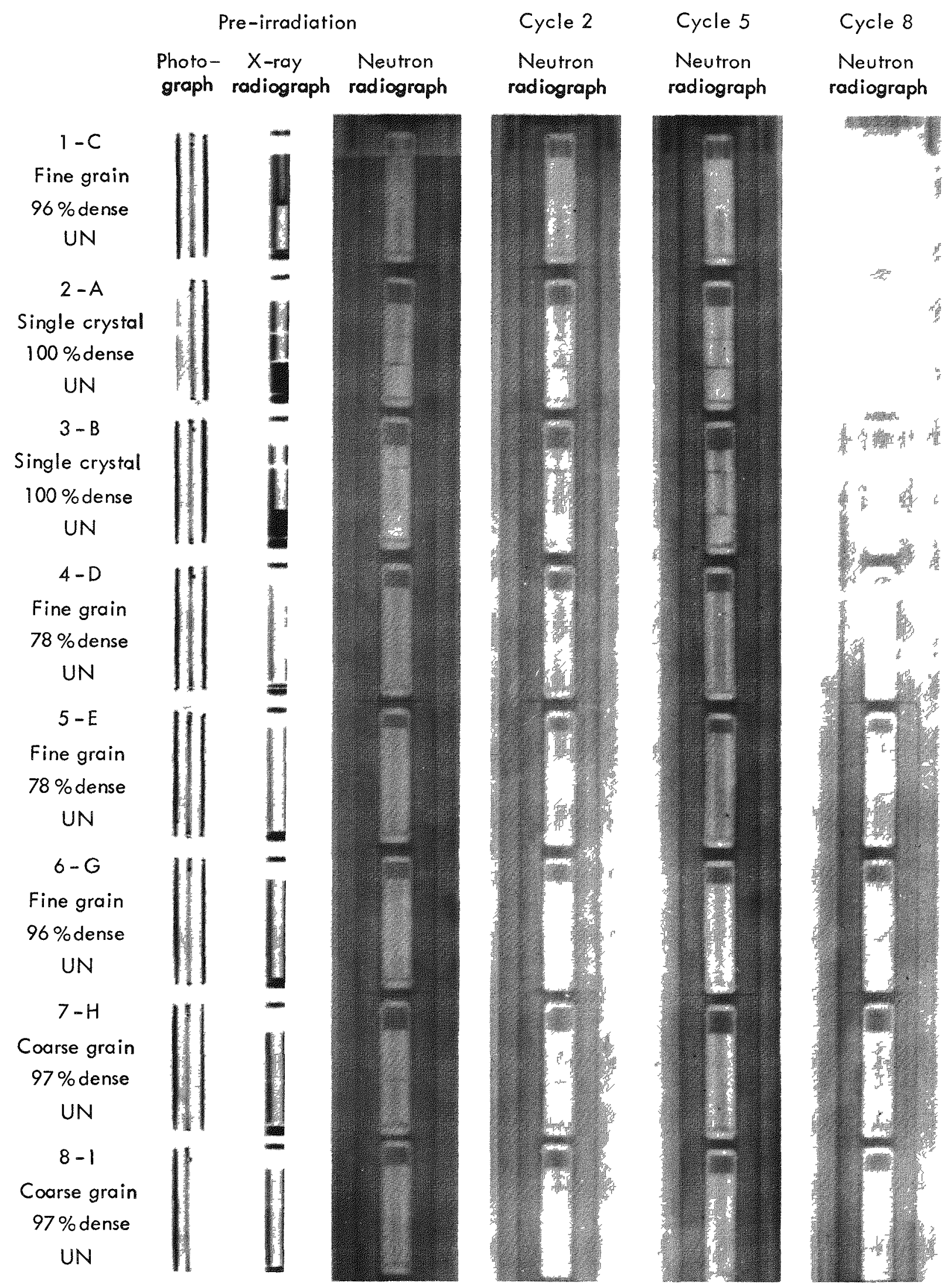

Fig. 17. Radiographs of Capsule LRL-1. 
Measurements were made at BMI-C by use of an $\mathrm{x}$-ray film reader with a micrometer-driven cross hair. The radiographs were re-measured at LRL by use of a two-coordinate comparator at $10 \times$ magnification. When scanning these measurements from beginning to final irradiation cycle it became evident that both the measurements made by BMI-C and LRL had sufficient random error associated with them to make the progressive swelling measurements uninterpretable. The presence of large amounts of $\mathrm{Mo}, \mathrm{W}$, and $\mathrm{Re}$ in the capsule contributed greatly to the poor resolution of the neutron radiographs. Another contribution to the error was the intensity variation within one film and from one film to another. For these reasons, and because the actual changes in dimensions during complete irradiation were small, it appears that the dimensional changes due to fuel swelling were no greater than the error limits of the method.

Pre-irradiation and post-irradiation weights of the clad and unclad specimens were made. Minor milligram discrepancies in the total clad specimen weights were observed and attributed to sintering of the thermocouple wires to the cladding and the subsequent loss or gain of particles of the cladding or when the thermocouple was removed from the specimen. The three longitudinal cladding cuts discussed earlier in the description of cladding removal frequently resulted in cuts or scores in the fuel. These cuts explain the small amount of weight loss measured in each fuel specimen.

Outside and inside diameters and lengths of each specimen segment were made repeatedly, as described earlier in this report. The fractional changes in lengths, diameters, and densities are given in Table IV.

\section{Table IV}

Post-Irradiation Dimensional Changes.

\begin{tabular}{|c|c|c|c|c|c|c|}
\hline $\begin{array}{l}\text { Specimen } \\
\text { no. }\end{array}$ & Material ${ }^{*}$ & $\begin{array}{l}\text { Median } \\
\text { temp. } \\
\text { (o C) }\end{array}$ & $\begin{array}{l}\text { Meas. vol. } \\
\text { increase } \\
(\%)\end{array}$ & $\begin{array}{c}\text { Theoret. } \rho \\
\text { decrease } \\
(\%)\end{array}$ & $\begin{array}{c}\text { Diameter } \\
\text { increase } \\
(\%)\end{array}$ & $\begin{array}{l}\text { Length } \\
\text { increase } \\
(\%)\end{array}$ \\
\hline $1-C$ & F.G. High $\rho$ & 1307 & 4.8 & 4.7 & 2.0 & 0.1 \\
\hline $2-A$ & Single Crystal & 1377 & 3.5 & 3.7 & 0.7 & 1.4 \\
\hline $3-B$ & Single Crystal & 1397 & 2.7 & 4.0 & 0.4 & 1.7 \\
\hline $4-1$ & $F \cdot G$. Low $\rho$ & 1357 & 3.6 & $3.0^{* * *}$ & 0.7 & 0.3 \\
\hline $5-\mathrm{E}$ & $\mathrm{F} \cdot \mathrm{G} \cdot \mathrm{L}, \mathrm{W} \rho$ & 1377 & 4.6 & $3.1^{*}$ & 0.9 & 0.4 \\
\hline $6-\mathrm{C}$ & F.G. Hligh $\rho$ & 1417 & 12.0 & 11.4 & 3.1 & 0.1 \\
\hline $7-\mathrm{II}$ & C.G. High $\rho$ & 1367 & 5.4 & 7.6 & 2.0 & 0.6 \\
\hline $8-I$ & C.G. IJigh $\rho$ & 1387 & 5.2 & 5.8 & 1.5 & 1.6 \\
\hline
\end{tabular}

F.G. = Fine grain size

C. $G .=$ Coarse grain size $\rho=$ Density

Based upon dry density pre-irradiation measurements at BMI-C. All others based upon wet pre-irradiation density at $\mathrm{B} . \mathrm{MI}-\mathrm{C}$. $\mathrm{Wet}=\mathrm{CCl}_{4}$. 


\section{Chemical Analyses}

All specimens were analyzed for $\mathrm{U}, \mathrm{N}_{2}$, Xe, and $\mathrm{Kr}$. Multiple uranium analyses were run for each specimen. No result below 92.0 or above $96.0 \%$ was accepted unless it could be duplicated. On a statistical basis, the uranium results were not significantly different from the theoretical value for pure UN of $94.44 \%$. The overall mean was $93.94 \%$ U. Uranium analys is was performed according to ASTM Method D-217 and all calculations were based upon an average atomic weight of 237.7 for uranium.

The nitrogen and retained fission gas analyses were troubled by the long distance separating the final Toepler pump from an intermediate mercury diffusion pump within the hot cell. Many of the first analyses were lost due to this pumping problem while others were incomplete and nitrogen content could not be determined. Since very small quantities of material were available for analyses, few specimens were large enough to permit duplicate runs. This accounts for unreported analyses in those cases where specimens were lost. When duplicate analyses were made they were in close agreement. The nineteen nitrogen determinations from mass spectrometer analyses that were made covered a range of 5.37 to $5.64 \%$ with a mean composition of $5.48 \%$, compared to $5.56 \%$ for the stoichiometric compound.

Post-irradiation fission gas release data were obtained by BMI-C as described earlier. Assuming equal gas release from each specimen and a total burn-up of $2.3 \times 10^{20}$ fissions/cc, it was calculated that $2.8 \%$ of the stable xenon is otopes and $2.2 \%$ of the stable krypton isotopes were released, respectively.

Retained fission gas analyses were made on from one to three samples from each specimen by the bomb-combustion techniques previously described. The average analysis of 15 specimens was $7.3-\mathrm{ppm}$ $\mathrm{Xe}$ and $1.0-\mathrm{ppm} \mathrm{Kr}$, to which an analytical error of $\pm 20 \%$ must be applied due to the small amount of the gas present. Therefore, an attempt in making a material balance of retained and released fission gas vs. calculated fission gas generated has failed due to an average yield in excess of $113 \%$ for $\mathrm{Xe}$ and $123 \%$ for $\mathrm{Kr}$ derived from retained fission gas analyses.

Fuel burn-up was obtained from 24 samples radiochemically analyzed by gamma spectroscopy, Each specimen was analyzed for $\mathrm{Zr}^{95}$, Ce141, Ce144, Cs137, Ru103, and $\mathrm{Ru} 106$. The combined results of $\mathrm{Zr} 95, \mathrm{Ce}^{141^{\circ}}$, and $\mathrm{Ce}^{144}$ were used in the final determination. Table $V$ gives the results of the burn-up analys is and the verification of the original estimate of total capsule burn-up of $2.3 \times 10^{20}$ fissions/cc.

Uranium isotopic ratios for all irradiated samples were measured but are not included in the report because these analyses were not obtained for the pre-irradiated fuel. 
Table V

Burn-Up Analyses. *

\begin{tabular}{ccccc}
\hline $\begin{array}{c}\text { Specimen } \\
\text { no. }\end{array}$ & $\begin{array}{c}\text { Kr } \\
\text { fissions/ } \\
\text { cc } \times 10^{20}\end{array}$ & $\begin{array}{c}\mathrm{Ce}^{141} \\
\text { fissions/ } \\
\mathrm{cc} \times 10^{20}\end{array}$ & $\begin{array}{c}\text { Ce } \\
\text { fissions/ } \\
\text { cc } \times 10^{20}\end{array}$ & $\begin{array}{c}3 \text { isotope } \\
\text { average } \\
\text { fissions/ } \\
\text { cc } \times 10^{20}\end{array}$ \\
\hline 1-C & 2.29 & 2.24 & 2.28 & 2.27 \\
$2-\mathrm{A}$ & 2.38 & 2.40 & 2.43 & 2.40 \\
$3-\mathrm{B}$ & 2.37 & 2.28 & 2.35 & 2.33 \\
$4-\mathrm{D}$ & 2.06 & 2.00 & 2.00 & 2.02 \\
5-E & 2.05 & 2.04 & 2.13 & 2.07 \\
$6-\mathrm{G}$ & 2.45 & 2.49 & 2.46 & 2.47 \\
$7-\mathrm{H}$ & 2.46 & 2.48 & 2.44 & 2.46 \\
$8-\mathrm{I}$ & 2.40 & 2.40 & 2.44 & 2.41 \\
\hline
\end{tabular}

*All analyses growth-corrected.

E. Thermocouple Calibration

A single thermocouple calibration point was measured at $1100^{\circ} \mathrm{C}$, as described earlier. Additional points were not measured, because the irradiated thermocouple failed when it was inserted into the furnace for the $1200^{\circ} \mathrm{C}$ calibration point. This failure was due to the loss of the inert furnace atmosphere and the rapid oxidation of the thermocouple during insertion. However, the one good calibration run indicated that the thermocouple was reading $15^{\circ} \mathrm{C}$ higher then the actual temperature.

V. Discussion

Paramount among the beginning goals of this investigation was the development of an understanding of the mechanism responsible for fuel swelling in a high-temperature irradiation environment. It was expected that whatever theory evolved could be substantiated through experiment. At the conclusion of only one relatively low burn-up irradiation, however, we are not in a position to generalize our results.

A. Swelling of UN

One of the major objectives of this irradiation was to compare variations in microstructure of the same basic fuel. Materials of high density (96 to 100\%) were compared to those of low density ( $78 \%$ ), and materials of high density were compared on a basis of grain sizes 30 microns, 105 microns, and single crystal. It can be seen from Table IV that the low-density, fine-grained UN showed less swelling than the high-density materials. This can reasonably be interpreted as accommodation of volume increase within the self-contained voids 
of the fuel. The superiority of the low-density (78\% theoretical) UN is quite clear when related to the fine-grain, high-density UN and the coarse-grain, high-density UN, but borderline when compared to the single crystal. Subsequently, it becomes obvious that the fewer the grain boundaries or the larger the grain size, the better the resistance to swelling exhibited in the high-density fuel. It is apparent in the photographs presented earlier in this report that porosity, presumably resulting from collection of fission gases, collects in the grain boundaries and is strikingly absent from the single-crystal microstructure.

Calculation reveals that a temperature drop of approximately $20^{\circ} \mathrm{C}$ was present between the centerline of the fuel and the cooler outside surface of each specimen. The temperature gradient within specimen creates a driving force along the gradient 26 causing the fission gas to migrate in the direction of higher temperature, i.e. the centerline of the specimen. In the case of the polycrystalline uranium nitride studied in this irradiation, the grain boundaries parallel to the cylindrical surface acted as barriers to the migration of fission gas and have become microscopically delineated by its collection and the concentrated formation of porosity in these grain boundaries.

Swelling was directly measured by comparing pre-irradiation dimensions to post-irradiation dimensions as described earlier. Of the many dimensional measurements made in the post-irradiation analysis, the lengths are subject to the greatest error because of indications that the original fuel segments, especially the single crystals, were not perfect right circular cylinders, i.e. both ends were not parallel. Therefore, post-irradiation lengths were measured on a basis of maximum length which, in the case of the shorter length threesegment crystals, could result in considerable error, as could the volume increases calculated from the lengths. The diametral increase is quite accurate and the volumetric increase was determined by immersion in mercury, where volumes can be measured accurate to two parts in 3000 .

The median temperature for each specimen was calculated as explained previously. From the information in Table III it can be seen that the duplicate pairs of fine-grain low-density, coarse-grain highdensity and single-crystal UN specimens were all irradiated within $\pm 20^{\circ} \mathrm{C}$ of the median irradiation temperature for the capsule. For this reason, the diameter increase and volumetric swelling were comparable for both specimens of a particular microstructure. The fourth pair of duplicate specimens was the fine-grain, high-density UN and they were in the positions of minimum and maximum median irradiation temperature, $110^{\circ} \mathrm{C}$ apart. They show diametral increase of 2 and $3 \%$, respectively, and volumetric increases of 4.5 and $11.0 \%$. From this information it is apparent that temperature has a strong relation to swelling, in the direction of increased swelling occurring at increased temperature.

As a result of a relatively constant reactor flux profile and nearly identical $\mathrm{U}^{235}$ enrichment of the fuel specimens, the burn-up for any one specimen was within $12 \%$ of the average measured burn-up for all 
eight specimens. This variation is insignificant and cannot be related to any differences in the irradiated microstructures.

All eight claddings failed by cracking, including those where the diametral increase was negligible. Therefore, it was concluded that the cladding offered no observable restraining effect upon the fuel. Additional confirmation of this statement is given in the negligible length increases shown in Table IV. Essentially, no indication of a "tooth-paste" extrusion of the fuel was seen in the microstructural analysis or in the length increase.

The dimensional changes and metallographic examination implied that fission gas retention might be considerably less in the single crystal than for the other microstructures due to the absence of poros ity and grain boundaries in the irradiated specimens. However, as can be seen in Table $V$, the fission gas retention in all samples was nearly identical, indicating that it was present on a sub-microscopic level within the structure of the single crystal, and that swelling was possibly more closely related to the size of the porosity.

The UN fuel was chemically stable during the irradiation, as electron microprobe analysis gave no indication of free uranium or $\mathrm{U}_{2} \mathrm{~N}_{3}$ in the fuel. No reaction between fuel and cladding was observed, suggesting that $W-25$ Re cladding or the $W$-Re family of materials is acceptable from the standpoint of chemical stability and fuel-cladding compatibility.

\section{B. Comparison of Results with Previous Data}

Uranium nitride irradiations have been made previously at Pratt and Whitney under the SNAP-50 program and have been described.27 Oak Ridge National Laboratory has continued much of the Pratt and Whitney work and are now generating their own program. The SNAP-50 capsules were irradiated under quite different conditions pertaining to cladding, nitrogen cover gas, temperature of irradiation, and total burn-up - which provides little information with which to compare the results of Capsule LRL-1. Oak Ridge has irradiated capsules at somewhat higher temperatures and higher burn-ups. These capsules are either in-pile or in post-irradiation analysis 28 at this writing, and direct comparison of data is impossible.

Battelle Memorial Institute, Columbus, has irradiated individual UN specimens in various capsules in their high-temperature fuels program and intends to expand these studies to include a study of the effect of grain size on swelling in UN.29 Again, the temperature of interest in this program is unfortunately not the same or near that of Capsule LRL-1 but much higher $\left(\sim 1700^{\circ} \mathrm{C}\right)$ which allows little direct comparison of results.

VI. Conclusions

The experimental results have shown that uranium nitride, enriched $9.6 \%$, irradiated at about $1400^{\circ} \mathrm{C}$ for 3558 hours to a burn-up of 
$2.3 \times 10^{20}$ fissions/cc, swelled at varying rates, depending upon the original microstructure of the fuel.

Uranium nitride having an original density of $78 \%$ theoretical swelled less than similar material having an original density of $96 \%$ the oretical.

Uranium nitride, having original densities of 96 to $100 \%$ theoretical, swelled at different rates, depending upon the original grain size of the fuel. Fine-grain 30- $\mu$ diameter, swelled more than coarsegrain, $105-\mu$ diameter, and single-crystal uranium nitride swelled least of the three grain sizes.

No significant differences in swelling of solid single crystals and single crystals with a 0.100 -in.-diameter axial hole were observed.

The results also show that the swelling rate of fine-grained, $96 \%$ dense uranium nitride is related to irradiation temperature: the swelling rate is greater at higher temperatures.

About $98 \%$ of the xenon and krypton generated in the fuel were retained in all microstructures. This indicates that fission gas retention was not dependent upon grain boundary effects under the conditions of this irradiation.

In this experiment there did not appear to be any relationship between amount of retained fission gas and fuel swelling.

Tungsten-25.5\% rhenium cladding was chemically compatible with uranium nitride under the conditions described above.

VII. Acknowledgments

An irradiation such as this is a formidable task and requires the assistance and cooperation of many individuals, both within and outside the structure of the responsible organization. Major recognition should be made of the capsule design and irradiation, for which N.E. Miller and Dr. J. W. Ray of BMI-C were responsible. Dr. D. E. Kizer and D. L. Lozier of BMI-C were responsible for specimen fabrication. Our sincere thanks goes to E. H. Stearns and B. J. Tuckey for their contribution to the design and fabrication of many pieces of specialized apparatus used in the post-irradiation analysis. The contributions of Mrs. J. C. Garrison in both metallographic consultation and photographic support are gratefully acknowledged. Our special thanks are extended to Dr. J. E. Harrar, Dr. J. E. Clarkson, Mrs. E. R. Smathers, Dr. R. Crawford, and J. W. Fischer for providing analytical chemistry techniques and support, and to J. B. Niday for the radiochemical analyses. Prof. D. Lazarus of the University of Illinois and C. F. Cline contributed to our program with their consultation and guidance. Finally, we would like to acknowledge the support and encouragement provided by Dr. J. S. Kane and Dr. J. W. Hadley throughout this research. 


\section{References}

1. Barnes, R.S. and R.S. Nelson, "Theories of Swelling and Gas Retention in Reactor Materials," AERE-R-4952, June 1965.

2. Barnes, R.S. and D.J. Mazey, "The Movement of Helium Bubbles in Uranium Dioxide," AERE-R-4570, March 1964.

3. Gruber, E.E., "On the Theory of Migration and Coalescence of Bubbles in Solids," ANL-7079, November 1965.

4. Shewmon, P.G., "The Movement of Small Inclusions in Solids by a Temperature Gradient," Transactions of the AIME, Vol. 230, August 1964, pp. 1134-1137.

5. Speight, M. V., "Bubble Diffusion and Coalescence During the Heat Treatment of Materials Containing Irradiation-Induced Gases," Journal of Nuclear Materials, Vol. 12, No. 2 (1964), pp. $216-220$.

6. Barnes, R.S. and D. J. Mazey, "The Migration and Coalescence of Inert Gas Bubbles in Metals," AERE-R-4223, January 1963.

7. Ibid.

8. Weaver, S. C., "Helium Gas Bubble Migration in Uranium Mononitride in a Temperature Gradient," ORNL-TM-2016, December 1967.

9. Zielenbach, W. J., "Irradiation Capsule Design for Operation to $2000^{\circ} \mathrm{C}$," presented at International Symposium on "Development in Irradiation Capsule Technology," Pleasanton, California, May 3-5, 1966.

10. Lee, J. D;, "HRTZ-A Two Dimensional Cylindrical Heat Transfer Code," UCID-15468 (1969).

11. Lee, J.D., "Neutronic and Thermal Analysis of the BMI-LRL UN Irradiation Capsule No. 1," UCID-15469 (1969).

12. Ibid.

13. Ibid.

14. Albrecht, E.D., J. M. Johnson, and E. H. Stearns, "Renovation and Equipping of LRL $\beta-\gamma$ Hot Cell Facility," UCRL-71558 (1969).

15. Albrecht, E.D., J M. Johnson, and W. N. Hayes, Jr., "PostIrradiation Microanalysis," UCRL-71704 (1969).

16. Albrecht, E.D., W. N. Hayes, Jr., E.H. Stearns, and J.M. Johnson, "Use of Mercury Pycnometer in Volume Determinations," to be published. 
17. Albrecht, E.D. and J.M. Johnson, "Metallographic Facility at LRL's $\beta-\gamma$ Hot Cells," UCRL-71612 (1969).

18. Booman, G. L. and W. B. Holbrook, Anal. Chem. 31, 10 (1959).

19. Harrar, J.E. and E. Behrin, Anal. Chem. 39, 1230 (1967).

20. Harrar, J.E., "Hg Pool Cell for Controlled-Potential Coulometry," UCRL-50335 (1967).

21. Frazer, J.W., "Simultaneous Determination of Carbon, Hydrogen and Nitrogen," UCRL-5134 (1958).

22. Gunnink, R., R.A. Meyer, J.B. Niday, and R. N. Anderson, Nuclear Instrumental Methods 65, p. 26-30 (1968).

23. Gunnink, R., H. B. Levy, and J.B. Niday, "Identification and Detection of $\gamma$-Emitters by Computer Analysis of (GeLi) Spectra," UCID-5140 (1967).

24. Gunnink, R. and J. B. Niday, "Quantitative Analyses of Unknown Mixtures by Computer Reduction of (Ge-Li) Spectra," UCRL-71226 (1968).

25. Gunnink, R., J.B. Niday, R. N. Anderson, and R.A. Meyer, "Gamma Ray Energies and Intensities," UCID-15439 (1969).

26. Barnes, op. cit.

27. De Cresente, M. A., M. S. Freed, and S. D. Caplow, "Uranium Nitride Fuel Development SNAP-50," (U) PWAC-488, October 1965 (Confidential).

28. Patriarca, P., "37th Monthly Report on UN Irradiation at the Oak Ridge National Laboratory for Period Ending March 19, 1969," (U) (Confidential).

29. Keller, D. L. and W. Chubb, "Developments in the Technology of Nuclear Fuels During August through October, 1968, "(U) BMI-1854, November 1, 1968. (Confidential). 


\section{Capsule Design, \\ Fabrication, and \\ Operating Experience}

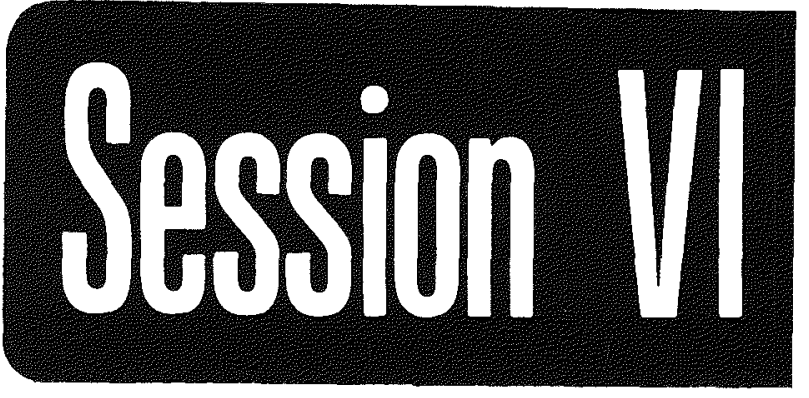


- 
EXPERIENCE WITH INSTRUMENTED CAPSULES FOR

HIGH TEMPERATURE IRRADIATION OE FUEL FORMS

By H.J. Reilly

$\because$, Lewis Research Center

INTRODUCTION

A contractor has designed and built for NASA a series of instrumented capsule experiments on the behavior of $\mathrm{UO}_{2}$ with tungsten clad. Two capsiles (which we will refer to as the first and second generation capsules) have been built and tested so far in the PBR. This paper will disciss the experience we had with the first capsule, how we used that experience to improve the design of the second capsule, and the programs and changes in practice that resulted from that experience.

\section{DISNCTION}

The purpose of the first capsule was to irradiate two annular cylindrical $\mathrm{UO}_{2}$ fuel forms clad with tungsten at high temperature. The capsule was to be operated in a vertically-adjustable facility tube (VAFT) to control the clad temperatures at $2073 \mathrm{~K}$. In this capsule there were two fuel specimens, one above the other. Figure 1 shows a sketch of one of the fuel specimen configurations. The fuel form is suspended inside the helium gas annulus by a tungsten stem. Because of the very high fuel and clad temperatures, no thermocouple was placed there. Instead, the clad temperature was to be inferred from the measured stem temperature with the aid of an out-of-pile calibration.

The operating power of the capsule was predicted using one-dimensional cylindrical geometry transport theory calculations. Combination of the calculated flux perturbation factor with measured unperturbed flux data for the intended irradiation position was believed adequate for the design.

When the capsule was first irradiated it was found that the indicated temperatures were greater than expected. In fact the VAFT facility with that capsule had to be moved to a different irradiation position to get the desired temperatures.

Investigation of the reasons for the error showed several things. First, it was found that the calculated flux perturbation factor was in error. 
In fact, it was found that calculated perturbation factors ranging from about 0.2 to 0.6 could be obtained using various plausible techniques. That is, a11 the techniques were sophisticated and consisted of various attempts to use transport codes to obtain a good answer. Even so, the uncertainty in such calculated powers was large, primarily because a short, small cylindrical fuel specimen placed next to a rectangular core simply did not look much like any of the geometries that were possible in the calculations.

The investigation also focused on the method used to determine clad temperature. Recall we said that the clad temperature was inferred from the temperature indicated by the stem thermocouple. This allowed use of a thermocouple that could withstand $1023 \mathrm{~K}$ for long periods of time to indicate clad temperatures around 2073K. The relationship between clad temperature and thermocouple temperature was determined by an out-of-pile calibration using an electrical heater. The clad temperature as read by an optical pyrometer was plotted as a function of stem thermocouple temperature. However, this curve then had to be corrected for the effects of internal heating in the stem which would be experienced in the reactor. (The irradiation position had gamma heat values of up to 10 watts/gram in water).

Figure 2 shows the distribution of temperature along a constant crosssection 2 "long stem. Curve 1 is what the distribution would be without radiation heat transfer or gamma heating. Curve 2 shows the effect of radiation and curve 3 that of gamma heat. Finally curve 4 shows the combined effects. It is obvious that, at an intermediate point such as at $1^{\prime \prime}$, the temperature can vary markedly for given end temperatures because of the effects of radiation and internal heating. Conversely, the end temperature ( 0 " on the curve) inferred for given temperature at $I^{\prime \prime}$ has uncertainty due to uncertainty in the amount of radiation and internal heating.

So we analyzed the uncertainty in the clad temperature when reading the stem temperature. We included uncertainties in neutron flux, gamma heat, conductivities and emissivities, dimensions and the optical pyrometer readings. We used the equation shown in figure 3. This is based on the equation for variance of a sum. It may not be exact due to the fact that not al1 the error distributions are normal. Nor are the uncertainty values for the variables known wel1. However, this was used, with the selection of uncertainty values corresponding to approximately a $95 \%$ confidence interval. The result was that the inferred clad temperature had an uncertainty of $\pm 230 \mathrm{~K}$ at $95 \%$ confidence level. This large value of uncertainty great ly compromised the usefulness of the results of the experiment and indicated that this method of clad temperature measurement was rea $11 y$ inadequate.

These studies induced us to require certain changes in the design of the second capsule which was to follow. First we moved the VAFT facility to 
a test position farther from the core where the gamma heat is smaller. This increased the fraction of the fuel heat due to fission power and also reduced the uncertainties due to gamma heat. We made measurements of the fission power in a nuclear mockup of the capsule in the Plum Brook Mock-Up Reactor. This insured that the capsule would reach power close to the expected position. We placed the two fuel specimens sideby-side instead of in-line, so they would be more likely to operate at the same power.

We also made changes in the fuel specimen. Figure 4 shows the new configuration. The best available high temperature thermocouple was placed deep in the stem next to the fuel. Heat transfer calculations using lumped parameter computer codes were used to determine the small (150K) difference between the temperatures of this thermocouple and the clad. One thermocouple was placed midway along the stem and one on the steel can wall opposite the helium gas annulus. The 1 ogic was that, even if the high temperature thermocouple did not last long, it would still allow in-pile calibration of the stem thermocouple at full operating conditions. The steel can thermocouple would give an. indication ( $\triangle T$ between can and water) proportional to capsule power. Thus, if the high temperature thermocouple had failed and the stem thermocouple temperature changed, the can wall thermocouple would tell us if the power had changed or if something had happened to the internal heat transfer path, such as fuel swelling to close the gas gap.

This capsule operated very successfully in the PBR. Both fuel specimens achieved the desired temperature in the selected test position. In one fuel specimen the high temperature thermocouple failed early but in the other fuel specimen the high temperature thermocouple lasted nearly to the end of the irradiation. When it was found that gradual insertion was required in order to continue to get constant temperature, the can wall thermocouple temperature showed clearly that power was being increased; thus fuel swelling was suspected immediately. And the uncertainty analysis of this capsule by the same methods described earlier showed that the clad temperature was within $\pm 40 \mathrm{~K}$ of the desired value. Even when the high temperature thermocouple eventually failed the uncertainty was less than $\pm 80 \mathrm{~K}$ 。

We considered that this experience proved the usefulness of doing an uncertainty analysis for every experiment during its design. Such analysis shows the amount of adjustment capability required for an experiment. It also gives a basis for deciding whether the indicated values from experiment instrumentation when first irradiated can be considered normal or not. So uncertainty analysis for each experiment has become standard practice at PBRF.

The analytical problems identified by this experience resulted in two other programs. The first of these was a program of 15 irradiations of cylindrical absorbers in the Mock-Up Reactor. This was done according 
to what is called a "central composite design" scheme for three variables. The result was a prediction equation for the rma 1 neutron flux perturbation in PBR reflector lattice test positions. The equation is shown in figure 5. We have found this to be useful in experiment design work at PBRF. The work is described in detail in reference 1. The other program consisted of one-dimensional transport theory calculations of gamma transport and heating for a point source-water shield w tungsten she11 geometry. This used the same central composite design scheme just referred to. The result was a prediction equation for the ratio between gamma heating in tungsten and that in water. To clarify, a piece of tungsten placed next to the reactor will experience different (usually more) heating per gram than the water due to its large photo electric cross-section. This equation (see figure 6) predicts the effect. This work is discussed in more detail in reference 2 .

While these polynomial equations are used at PBR, they also could have usefulness for other facilities; also, similar equations could be developed for other effects at other installations using similar techniques.

We started a program of calorimetric measurements of gamma heating in different materials. So far we have completed a set of irradiations in a gamma facility where the heating was due to used fuel elements that had been out of the PBR for 30 days. The specimens that were irradiated were 1.0 " $30.75^{\prime \prime}$ platelets, varying in material and in thickness. The results of this set of irradiations are shown in figure 7 . The results show strong effects of both atomic number and thickness. We are now doing measurements in the Mock-Up Reactor to determine whether the effects will be different with an actual operating reactor source spectrum.

\section{SUMMARY AND CONCLUSIONS}

Experience with a first generation $\mathrm{UO}_{2}$-fue1, tungsten-clad capsule was used to make substantial improvements in the second generation capsule. Also the experience resulted in two changes in PBRF practices in experiment design: Mock-Up Reactor measurements are required for prediction of flux perturbation factor for fueled experiments and uncertainty analyses are required for all experiments. An experimental program was initiated to give more accurate ways of predicting perturbation factors and a calculational program was initiated to give more information on gamma heating in tungsten. An experimental program to measure relative heating in specimens of varying atomic number and thickness is underway. 


\section{REFERENCES}

1. Lynch, John H., and Peters, Lawrence E.: Predictive Equations for Thermal Neutron Flux Perturbation Effects in Cylinders. NASA TN D-4949, December, 1968 .

2. Lynch, John H., Crum, Richard J., and Reilly, Harry J.: Gamma Heating in Thin Heavy-Element Absorbers. NASA TM X-1680, October, 1968.

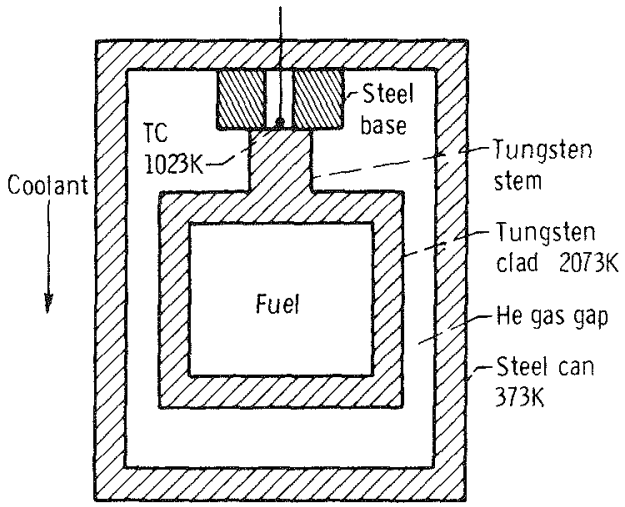

Figure I - Elevation view of first-generation capsule configuration (not to scale)

If $\left.\begin{array}{llll}Z & f\left(x_{1}\right. & x_{n} & x_{N}\end{array}\right)$

and $X_{1}$ are independent variables

then (approximately)

$\sigma_{2} \sqrt{\sum_{1} \frac{d Z}{d x_{1}} \sigma_{x_{1}}^{?}}$

where

$\sigma_{Z} \quad$ standard deviation of $z$

$Z$ nominal value of $Z$

$\frac{d z}{d X_{1}} \quad$ sensitivity of $z$ to error in $x_{1}$

$\sigma_{X_{1}} X_{1}$ as $Z$ above

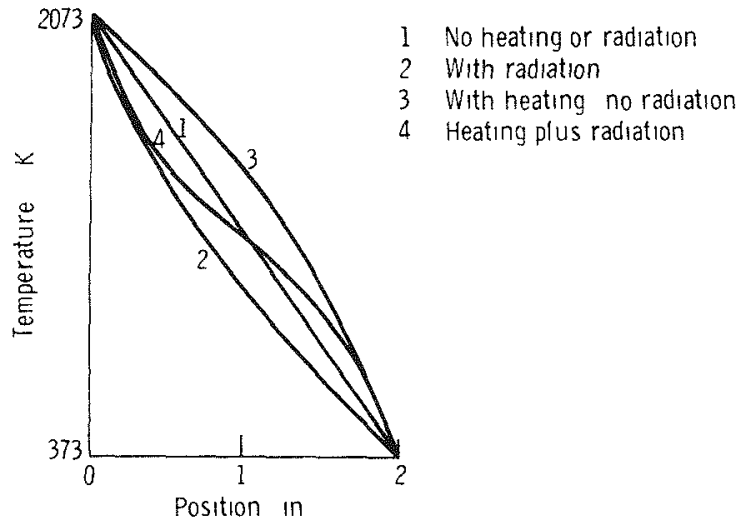

Figure? Calculated temperature distribution along a 2 inch stem with and without gamma heating and therma' radiation

Figure 3 


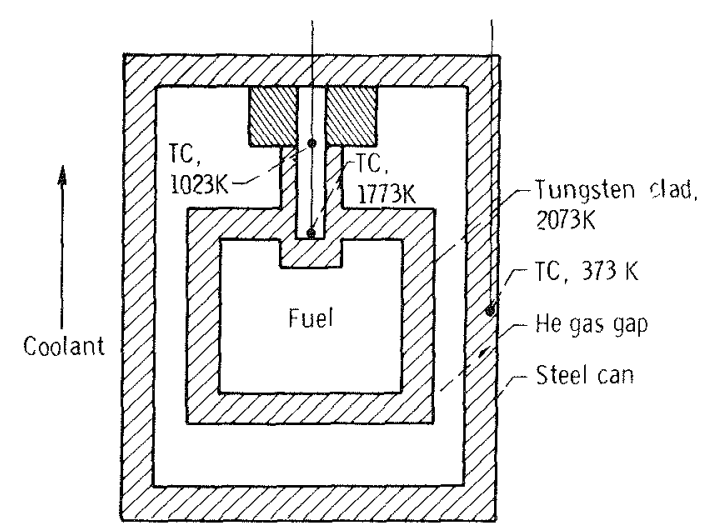

Figure 4. - Elevation view of second-generation capsule configuration.

$\ln Y=A_{0}+A_{1} X_{1}+A_{2} X_{2}+A_{3} X_{3}$

$$
\begin{aligned}
& +A_{11} x_{1}^{2}+A_{22} x_{2}^{2}+A_{33} x_{3}^{3} \\
& +A_{12} x_{1} x_{2}+A_{13} x_{1} x_{3}+A_{23} x_{2} x_{3}
\end{aligned}
$$

where

$Y$ ratio of gamma heat in tungsten to gamma heat in water

$x_{1}$ source energy

$x_{2}$ water shield thickness

$x_{3}$ tungsten thickness

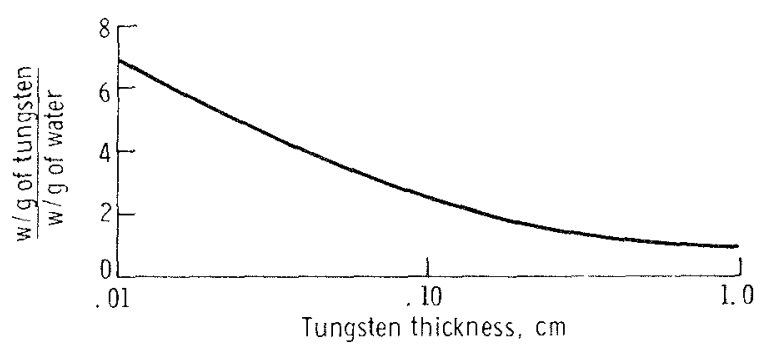

Figure 6. - Polynomial for gamma heating in tungsten.

$$
\begin{aligned}
\arcsin F^{1 / 2}=L_{0} & +L_{1} X_{1}+L_{2} X_{2}+L_{3} X_{3} \\
& +L_{11} X_{1}^{2}+L_{22} X_{2}^{2}+L_{33} x_{3}^{3} \\
& +L_{12} X_{1} X_{2}+L_{13} X_{1} X_{3}+L_{23} x_{2} X_{3}
\end{aligned}
$$

where

$F \quad$ thermal neutron flux perturbation factor

$x_{1}$ blackness coefficient

$x_{2}$ cylinder length

$x_{3}$ control rod shadowing variable

Figure 5. - Flux perturbation factor polynomial for cylindrical absorbers in PBR.

Thickness

\begin{tabular}{lccccc} 
& $1 / 32^{\prime \prime}$ & $1 / 16^{\prime \prime}$ & $1 / 8^{\prime \prime}$ & $1 / 4^{\prime \prime}$ & $1 / 2^{\prime \prime}$ \\
$\mathrm{Al}$ & $\ldots$ & $\ldots$ & 1.0 & 1.0 & 1.0 \\
$\mathrm{Zr}$ & $\cdots$ & 2.9 & 2.6 & 1.9 & $\ldots$ \\
$\mathrm{Sn}$ & $\cdots-$ & 4.5 & 3.4 & 2.5 & $\ldots$ \\
$\mathrm{Pb}$ & 7.25 & 5.6 & 4.15 & 2.5 & $\ldots$ \\
\hline \multicolumn{5}{l}{ Normalized to al uminum results. }
\end{tabular}

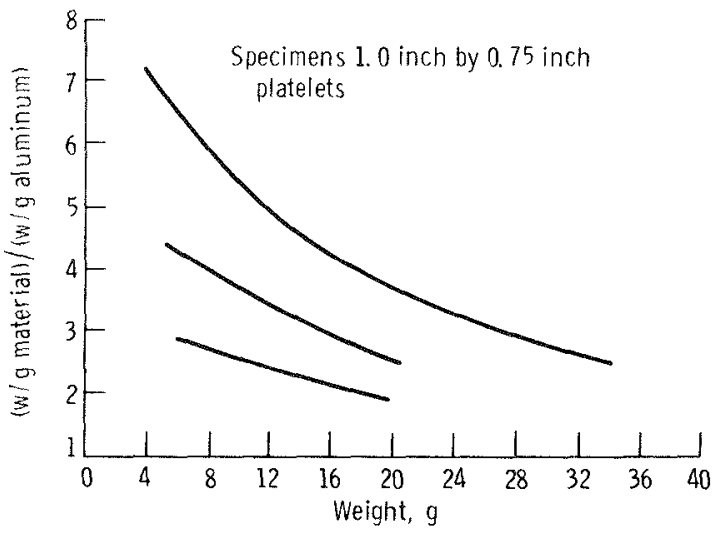

Figure 7. - Specific heating (W/g) relative to aluminum. 


\author{
X ASSEMBLIES FOR THERMAL AND FAST FLUX IRRADIATION OF (ŲPu)N FUELS* \\ by \\ E. O. Fromm and N. E. Miller \\ $1+460.6$ \\ BATTELLE MEMORTAL INSTITUTE \\ Columbus Laboratories
}

\begin{abstract}
The irradiation behavior of mixed uranium-plutonium fuels is being investigated as a part of the program on the development of mixed nitride fuels for high gain LMFBR's. Both thermal and fast neutron irradiations are being performed; irradiation conditions include fuel burnups to $150,000 \mathrm{MWD} /$ tonne, cladding temperatures to $700 \mathrm{C}$ and fission heat generation rates in excess of $40 \mathrm{kw} / \mathrm{ft}$. The thermal irradiation capsule experiments are being performed in the MTR and ETR to serve as a basis for planning the fast flux irradiations in EBR-II.
\end{abstract}

* Work performed under AEC contract W-7405-eng-92. 


\section{INTRODUCTION}

The Liquid Metal Fast Breeder (LMFBR) Reactor Program of the AEC is directed toward providing an economic power generating capability through development of a high gain breeder reactor. The first generation reactors in the LMFBR program will use the mixed oxide $\mathrm{UO}_{2}-\mathrm{PuO}_{2}$ as the reference fue 1 because of its more advanced state of development. However, $\mathrm{UO}_{2}-\mathrm{PuO}_{2}$ fuels have several inherent limitations which restrict their use in high gain breeders. These limitations are (1) relatively low density causing a low breeding ratio and (2) extremely poor thermal conductivity, which can lead to central fuel melting at relatively low fuel pin power ratings. The mixed nitrides and carbides of uranium and plutonium are considered as promising alternate fuels to the mixed oxide concept. The high fissile atom densities and high thermal conductivities of the nitrides and carbides can lead to substantial increases in breeding gain and fuel pin specific power levels compared to the mixed oxides. In addition, the mixed nitride fuel has the added advantage of chemical compatibility with potential IMFBR claddings, in the presence as well as absence of sodium bonding.

The irradiation behavior of the mixed uraniurn plutonium nitride fuels is being investigated at Battelle columbus Laboratories for the AEC Division of Reactor Development to obtain basic fuel performance data required for fuel element design. Since the objective is to demonstrate the feasibility of using mixed nitride fuels for use in a high gain LMFBR, irradiation conditions include fuel burnups to $150,000 \mathrm{MWD} /$ tonne, cladding temperatures to $700 \mathrm{C}$ and fission heat generation rates in excess of $40 \mathrm{kw} / \mathrm{ft}$. Initial irradiations have been performed in the predominately thermal neutron environment of the MTR and ETR in order to obtain early information on fuel behavior. In addition, experiments have been prepared for irradiation in the fast-

* Work performed under AEC Contract W-7405-eng-92. 
neutron environment of the EBR-II to obtain irradiation conditions which more closely match the environment of the fuel in a prototypical LMFBR. This paper will describe briefly the capsule designs used at Battelle Columbus for the irradiation of mixed nitride fuels in the MTR/ETR and in the EBR-II.

\section{THERMAL IRRADIATION CAPSULE DESIGN}

A difficult problem was presented in the design of the thermal test reactor irradiation capsules by the combination of high fission heat generation rate, and the relatively low fuel and cladding temperatures representative of LMFBR conditions, resulting in the low temperature drop to the reactor water to drive the heat through the capsule materials. The design which was settled upon is shown in Figures 1 and 2. The design is based upon a double-wall finned annulus concept utilizing electric heaters to melt the sodium in the various annuli of the capsule prior to reactor startup. The essential details of the capsule are as follows:

(1) The $20 \mathrm{w} / 0$ PuN-80 w/o UN fuel pins which are $0.225-$ inch in diameter by 0.75 -inch long are clad with 15 mils of Type 304 low carbon stainless steel and have either a helium bond, a 10-mil sodium bond, or a 20mil sodium bond between the fuel and the cladding.

(2) Two clad specimens with the same type of bond are immersed in sodium within a heavy wall nickel compartment, 0.720 inch $O D$ by 0.375 -inch ID by 4-3/4-inch long. Three such compartments are seal-welded together to form the inner capsule assembly.

(3) Holes for 6-thermocouples for monitoring the specimen temperatures are drilled by Electric Discharge Machining (EDM) in the wall of each nickel compartment; after the individual compartments are sealwelded together, the thermocouples are effectively sealed from the sodium which surrounds the inner assembly. Thermocouple protection tubes brazed into the top compartment protect the sheathed thermocouples from the sodium at the top of the assembly. One thermocouple is located opposite the axial midplane of each specimen.

(4) Heat transfer from the inner nickel assembly to the reactor water is through successively, a 25-mil sodium annulus, a Hastelloy- $X$ shell, another 25 mil sodium annulus, a Type A nickel finned she11 and an outer Type A nickel shel1. 


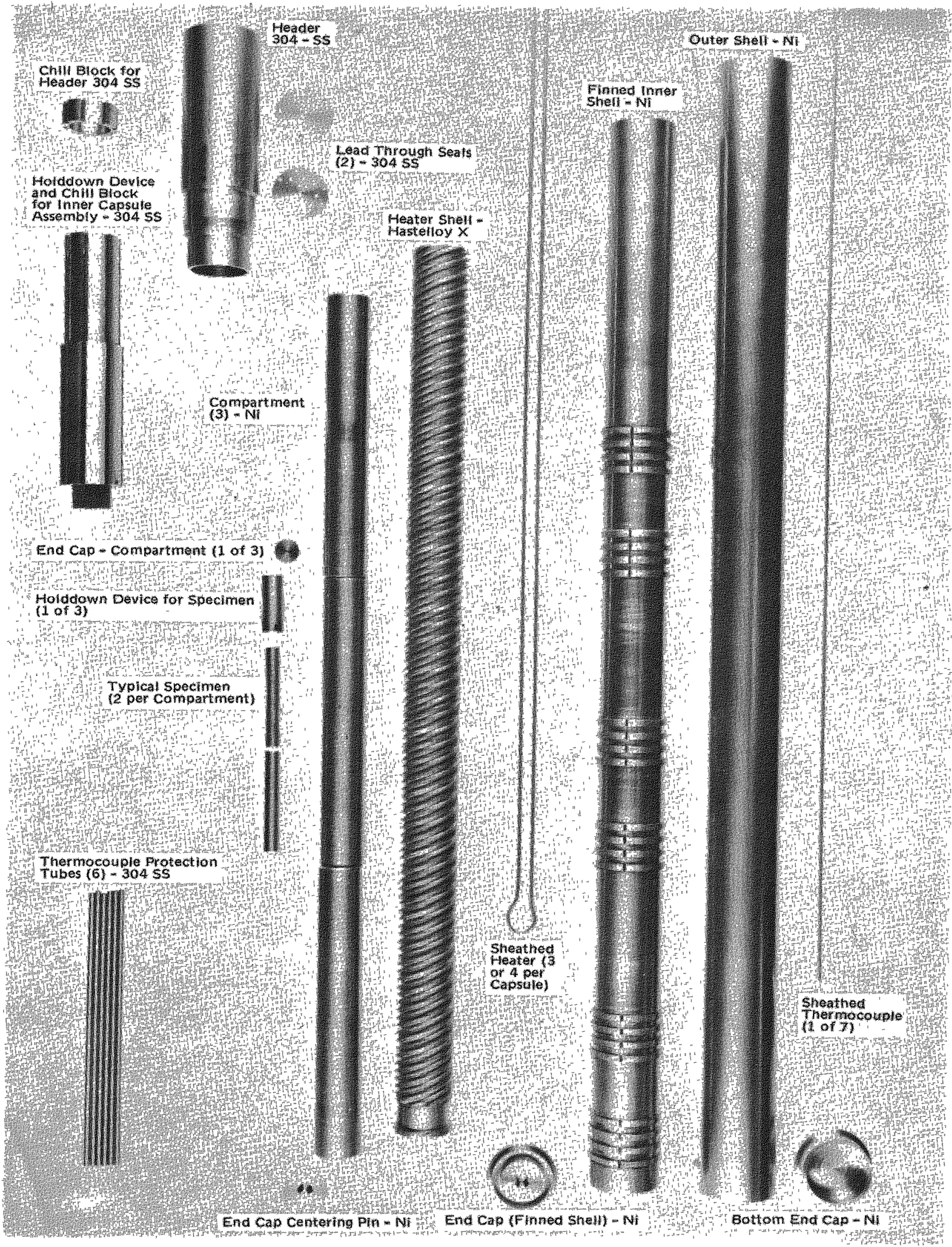

FIGURE 1. BMI-50 SERIES CAPSULE SUB-ASSEMBLY 

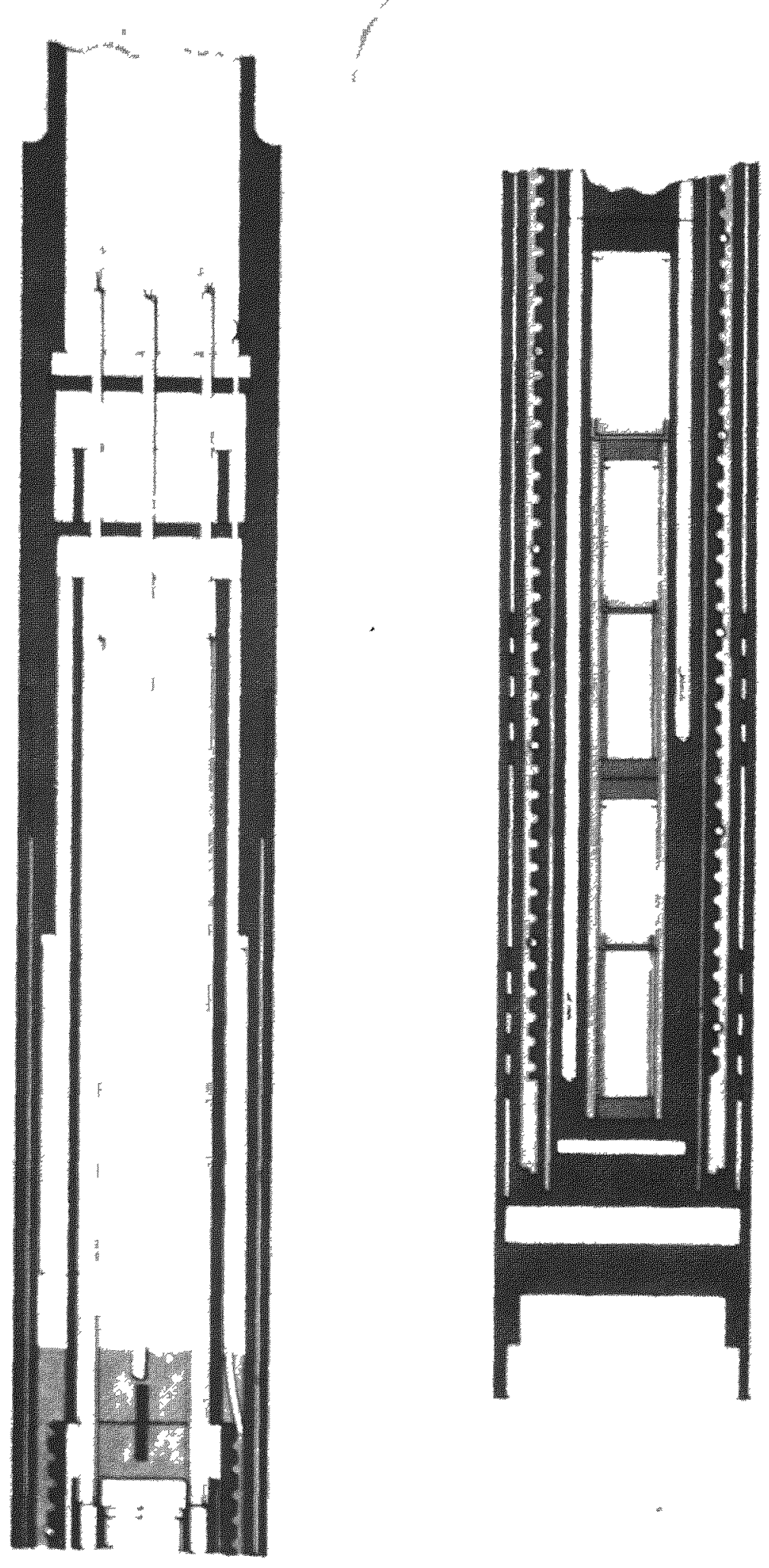

FIGURE 2. CROSSECTION OF BMI 50 IRRADIATION CAPSULE ASSEMBLY 
(5) The outside surface of the Haste11oy-X she11 is grooved to accommodate up to four 1/16-inch diameter incone 1 sheathed heaters which are used to melt the sodium in the capsule prior to reactor startup. The heaters, each capable of $2 \mathrm{kw}$, are brazed into the grooves using Nicrobraze 50 alloy.

(6) The outer surface of the fins in the finned she11 are precisely ground to a $0.4 \mathrm{mil}$ diametrical interference fit with the inside surface of the outer Type A nicke1 she11; the assembly of the two is accomplished by heating the outer shel1 to $370 \mathrm{C}$, cooling the finned shell assembly in liquid nitrogen, and rapidly inserting the finned she11 into the outer shel1. Since the fit must be extremely precise, special precautions are taken during shell fabrication procedures to assure that proper tolerances are maintained. Typical procedures for fabrication of the outer she11 are as follows:

(a) Anneal the 1.5-inch round bar of Type A nickel.

(b) Gun bore ID to within 2 mils of final dimension.

(c) Rough hone ID.

(d) Machine OD to within 20 mils of final size.

(e) Grind OD to final size

(f) Stress relieve.

(g) Final hone ID to a tolerance of $\pm 0.0001-$ inch.

(7) Fins are located only in the zones opposite the fueled region of the capsule. This directs most of the heat to flow radially outward, minimizing axial heat transfer, and leads to a more predictable heat transfer situation. This arrangement has been used very successfully in other capsule experiments at Batte1le.

(8) Special holddown devices are provided inside the specimen claddings, the individual compartments, and in the space above the inner assembly to prevent excessive axial movement of the components within the capsule.

(9) The heater leadouts above the sodium are inserted in axial grooves in a stainless steel chill block to dissipate the electric heat generated in the sheathed 
nickel leadout wire. This allows the heaters to be operated at higher specific power. Aluminum chill blocks are provided in the lead tube for the same reason.

(10) The sheathed heaters and thermocouples are double sealed into the header assembly using coast 52 brazing alloy, the brazing being done in a vacuum induction furnace.

(11) The finned shell and outer she11 are welded to the header assembly so that the sodium within the capsule is doubly contained from the reactor process water.

After assembly, the capsule is heated and centrifuged to obtain void free sodium bonds within the capsule. The bonding cycle consists of centrifuging for 10 minutes at $540 \mathrm{C}$ after which the electric heat is turned off and centrifuging continued till the sodium is solidified.

The heat transfer analysis for this capsule design was performed with a two-dimensional heat transfer code developed at Battelle-Columbus. The design curves obtained from this analysis are shown in Figure 3. For example, at a fission heat generation rate of $30 \mathrm{kw} / \mathrm{ft}$, the predicted temperature on the outer surface of the cladding is $615 \mathrm{C}$ for the helium bond specimen, $595 \mathrm{C}$ for the 10-mil sodium bond specimen, and $575 \mathrm{C}$ for the $20 \mathrm{mil}$ sodium bond specimen. The corresponding thermocouple temperature is $415 \mathrm{C}$ in each case.

\section{Operationa1 Experience}

Seven capsules of this design utilizing a specimen container of nickel have been assembled and sent to the NRTS for irradiation as indicated in Table 1. The first capsule, designated as BMI 50-4, began irradiation in the MTR during June, 1967, and was discharged in February, 1968, at burnups ranging from 38,000 to $62,000 \mathrm{MWD} /$ tonne. Capsule performance was very good with design temperature being maintained during most of the irradiation. The electric heaters, in addition to being used to melt the sodium prior to reactor startup were used at times to increase the specimen temperatures $30-55 \mathrm{C}$. This procedure has been utilized for all capsules irradiated thus far.

Capsule BMI-50-5 began irradiation in the MTR in July, 1967, and was discharged from the reactor in February, 1969, with a peak burnup of approximately 105,000 MWD/tonne as estimated from thermocouple data. This capsule operated at temperatures significantly less than those in Capsules BMI-50-4 and BMI-50-7 for comparable reactor positions for reasons which are not fully understood. 


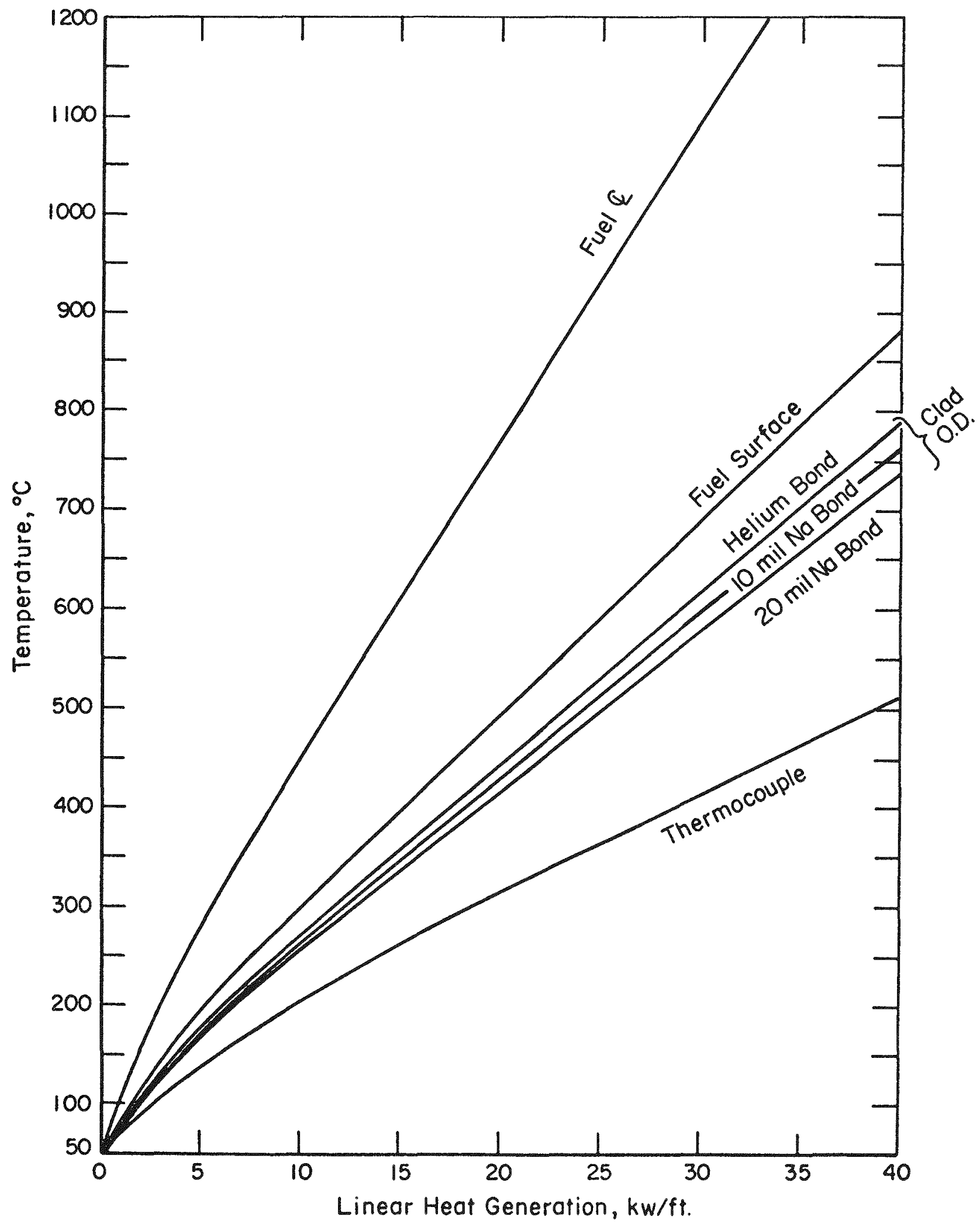

FIGURE 3. DESIGN CURVES FOR BMI 50 SERIES IRRADIATION CAPSULES (NICKEL COMPARTMENTS) 
TABLE 1. IRRADIAIION CONDITIONS FOR BMI 50 SERIES CAPSULES

\begin{tabular}{|c|c|c|c|c|c|c|c|}
\hline $\begin{array}{l}\text { Capsule } \\
\text { Number }\end{array}$ & Reactor & $\begin{array}{c}\text { Date } \\
\text { Inserted }\end{array}$ & $\begin{array}{c}\text { Date } \\
\text { Discharged }\end{array}$ & $\begin{array}{c}\text { Fue1 Surface } \\
\text { Temperature } \\
\text { Peak/Avg. } \\
\text { C }\end{array}$ & $\begin{array}{c}\text { Estimated Range } \\
\text { MWD/tonne } \times 10^{-3}(\mathrm{a})\end{array}$ & $\begin{array}{l}\text { f Burnup to } \\
\frac{69}{\text { Fiss/cc } \times 10^{-20}}\end{array}$ & $\begin{array}{c}\text { Average } \\
\text { Heating } \\
\text { Rate, kw/ft }\end{array}$ \\
\hline BMI $50-4$ & MTR & June, 1967 & Feb., 1968 & $720 / 610$ & $38-62$ & $13=20$ & 27 \\
\hline BMI $50-5$ & MTR & July, 1967 & Jan., 1969 & $630 / 500$ & $67-105$ & $24-34$ & 18 \\
\hline BMI $50-6$ & ETR & Nov., 1967 & $-\infty$ & $680 / 525$ & $45-59$ & $17-23$ & $21(30)^{(b)}$ \\
\hline EMI $50-7$ & MTR & Feb., 1968 & Jan., 1969 & $820 / 640$ & $84-94$ & $28-32$ & $24-30$ \\
\hline BMI $50-8$ & ETR & July, 1968 & $m$ & $700 / 580$ & $38-53$ & $13-16$ & $20-28(35)^{(b)}$ \\
\hline BMT $50-9$ & MTR & Jan., 1969 & Ju1y, 1969 & $760 / 675$ & 25 & 8 & 25 \\
\hline BMI $50-10$ & ETR & May, 1969 & $m$ & $770 / 695$ & 10 & 3 & $32(27)^{(b)}$ \\
\hline
\end{tabular}

(a) Based on 200 Mev/fission.

(b) Linear power ratings during July and August, 1969.

TABLE 2. BMI-1 SERIES DESTGN PARAMETERS

\begin{tabular}{|c|c|c|c|c|c|c|}
\hline \multirow{2}{*}{$\begin{array}{r}\text { Capsule } \\
\text { Number }\end{array}$} & \multirow{2}{*}{$\begin{array}{c}\text { Fuel } \\
\text { Diameter, } \\
\text { inch }\end{array}$} & \multicolumn{2}{|c|}{ Fue I Density, percent } & \multirow{2}{*}{$\begin{array}{l}\text { Fuel-clad } \\
\text { Radial } \\
\text { gap, inch } \\
\text { and bond }\end{array}$} & \multirow{2}{*}{$\begin{array}{l}\text { Maximum } \\
\text { Specific } \\
\text { Power, } \\
\text { Kw/ft }\end{array}$} & \multirow{2}{*}{$\begin{array}{c}\text { Fue 1 } \\
\text { Burnup, } \\
\text { a/o }\end{array}$} \\
\hline & & Theoretical & Smear & & & \\
\hline BMI 1-1 & 0.230 & 95 & 80.4 & $0.010 \mathrm{Na}$ & 24 & 5 \\
\hline BMI $1-2$ & 0.230 & 95 & 80.4 & $0.010 \mathrm{Na}$ & 24 & 9 \\
\hline BMI $1-3$ & 0.246 & 83 & 80.3 & $0.002 \mathrm{He}$ & 24 & 3 \\
\hline BMI $1-4$ & 0.238 & 95 & 86.1 & $0.006 \mathrm{Na}$ & 26 & 5 \\
\hline
\end{tabular}

TABLE 3. BMI-2 SERIES OF UN-PUN IRRADIATIONS IN EBR-II

\begin{tabular}{|c|c|c|c|c|c|c|}
\hline $\begin{array}{c}\text { Element } \\
\text { Identification } \\
\text { No. }\end{array}$ & $\begin{array}{c}\text { Cladding } \\
\text { Thickness, } \\
\text { in. }\end{array}$ & $\begin{array}{c}\text { Radial } \\
\text { Sodium Bond } \\
\text { Thickness, } \\
\text { in. }\end{array}$ & $\begin{array}{l}\text { Fue1 } \\
\text { Diameter, (2) } \\
\text { in. }\end{array}$ & $\begin{array}{l}\text { Fuel Smear } \\
\text { Density, }(3) \\
\text { percent }\end{array}$ & $\begin{array}{l}\text { Specific } \\
\text { Power, }(4) \\
\text { kw/ft }\end{array}$ & $\begin{array}{l}\text { Target } \\
\text { Burnup, (5) } \\
\text { a/o }\end{array}$ \\
\hline BMI 2-1 & 0.020 & 0.010 & 0.255 & 81.7 & $30.7-33.8$ & 6 \\
\hline BMI 2-2 & 0.020 & 0.010 & 0.255 & 81.7 & $30.7-33,8$ & 9 \\
\hline BMI 2-3 & 0.020 & 0.010 & 0.255 & 81.7 & $30.7-33.8$ & 12 \\
\hline BMI 2-4 & 0.015 & 0.015 & 0.255 & 76.1 & $30.7-33.8$ & 9 \\
\hline BML 2-5 & 0.015 & 0.015 & 0.255 & 76.1 & $30.7-33.8$ & 12 \\
\hline BMI 2-6 & 0.010 & 0.010 & 0.275 & 82.6 & $35.7-39.3$ & 6 \\
\hline BMI $2-7$ & 0.010 & 0.010 & 0.275 & 82.6 & $35.7-39.3$ & 12 \\
\hline BMI 2-8 & 0.010 & 0.010 & 0.275 & 82.6 & $35.7-39.3$ & 9 \\
\hline
\end{tabular}

(1) All cladding is Type 316 stainless stee1, $0.315 \mathrm{in.}$ OD.

(2) All capsules will contain a nominal 10-inch length of fuel axially centered within the EBR-II core.

(3) Based on fuel pellet density of 95 percent of theoretical, the theoretical density of (U $0.2^{\mathrm{Pu}} 0.8^{\mathrm{N}}$ is $14.3 \mathrm{~g} / \mathrm{cm}^{3}$.

(4) Range of specific powers in core Row 4 of EBR-II operating at $50 \mathrm{Mw}$. For the specified fuel this corresponds to $9.54-10.50 \times 10^{13}$ fissions $/ \mathrm{cm}^{3}-\mathrm{sec}$.

(5) For 95 percent theoretical density $\left(\mathrm{U}_{0} .8 \mathrm{Pu}_{0} .2\right) \mathrm{N}$, a heavy atom burnup of 1 percent equals $3.27 \times 10^{20}$ fissions per $\mathrm{cm}^{3}$. 
Capsule BMI-50-6, the first capsule in the series to be irradiated in the ETR, was inserted into a position ( $P-12-S W$ ) with a neutron flux less than required for design conditions to checkout the operation of the capsule. Subsequently the capsule has been moved to higher flux positions to obtain higher burnup rates and fue $I$ temperatures. The most recent move was into the beryllium reflector position 0-6 where design temperatures have been achieved. It is planned to irradiate this capsule to a very high burnup, approximately $150,000 \mathrm{MWD} /$ tonne.

Capsule BMI-50-7 began irradiation in the MTR in February, 1968, in the same position vacated by BMI-50-4 and was discharged at an estimated peak burnup of 92,000 MWD/tonne in February, 1969. Throughout the irradiation, the performance of the capsule was excellent with specimen heating rates near $30 \mathrm{kw} / \mathrm{ft}$ during most of the exposure. The capsule was returned to Battelle-Columbus along with BMI-50-5 for postirradiation examination.

Capsule BMI-50-8 was inserted into a high flux in the aluminum reflector region of the ETR in July, 1968. As the irradiation progressed some decline in fission rate was experienced and the capsule was moved into a higher flux position in the corner of the core (E-5-NE) during July, 1969. Fuel pin power ratings to $35 \mathrm{kw} / \mathrm{ft}$ are presently being experienced in this new position. The irradiation is planned to continue to a burnup of $100,000 \mathrm{MWD} /$ tonne.

Capsule BMI-50-9 was inserted into the same position in the MTR which was vacated by Capsule BMI-50-7. At startup the capsule performance was very good, but within a short time the indicated temperatures on the next-to-top specimen rose significantly above the other temperatures and it was necessary to raise the capsule to prevent an excessively high temperature on the specimen. Subsequently, a neutron flux shield was placed in front of the capsule to reduce the power in the top two specimens so the lower four specimens could operate near normal conditions. The irradiation of this capsule was terminated due to the forthcoming shutdown of the MTR. The accumulated burnup in the specimens is approximately 25,000 MWD/tonne.

Capsule BMI-50-10 began irradiation in the corner of the ETR reactor core $(\mathrm{E}-5-\mathrm{NW})$ in May, 1969. The performance of this capsule during the first cycle of irradiation was excellent with the specimens operating in the temperature range of 640 to $730 \mathrm{C}$ and linear heating rates to $32 \mathrm{kw} / \mathrm{ft}$. However, the temperatures of BMI-50-10 were decreased by approximately 15 percent during the second cycle as a result of the transfer of BMI-50-8 to the companion facility E-5-NE. The irradiation of BMI-50-10 will continue to an exposure of 100,000 MWD/tonne.

The thermal irradiation capsule was redesigned to permit the specimens for the next capsule, BMI-50-11, to operate at a heating 
rate of over $40 \mathrm{kw} / \mathrm{ft}$ without excessively high temperatures. The greatest temperature drop in the capsule occurs across the thick wall of the specimen container. It was found that increasing the thermal conductivity of this container by using $\mathrm{Nb}-1 \mathrm{w} / \mathrm{o} \mathrm{Zr}$ alloy rather than Type A-nickel would accommodate the desired design change. At $40 \mathrm{kw} / \mathrm{ft}$ the predicted temperature on the outer surface of the cladding is $710 \mathrm{C}$ for a helium-bond specimen, $682 \mathrm{C}$ for the 10-mil sodium-bond specimen, and $655 \mathrm{C}$ for the 20-mil sodium-bond specimens. Capsule BMI-50-11, presently under construction, will utilize this design change. It is scheduled for insertion in the ETR in October, 1969.

\section{EBR-II IRRADTATION EXPERIMENTS}

While thermal irradiations are a useful first step in the evaluation of any LMFBR fuel concept, the establishment of the feasibility of a particular fuel for LMFBR applications will require irradiation evaluation under LMFBR conditions, namely a fast neutron environment and prototypical temperatures and heat fluxes. The program of UN-PUN irradiations in EBR-II is designed to fulfill this need. Some of the factors associated with fast neutron spectrum irradiations which could influence the overall behavior of the fuel are:

(1) Heat generation and burnup in the fuel is uniform rather than being concentrated near the surface as in the thermal irradiation.

(2) Greater gas production from the $(n, \alpha)$ and $(n, p)$ reactions.

(3) A greater fast neutron exposure to the cladding.

In setting up the program for the EBR-II irradiations, the basic philosophy has been to limit the number and range of variables to be investigated to those which will make the maximum utilization of the nitrides performance potential. This led to the emphasis on the use of the highest practical densities in the fuel (to 95 percent of theoretical) and a sodium bond between the fuel and the clad.

Irradiation experience with other fuels, particular heliumbonded carbides, indicated that for extended burnups, stainless steel cladings would accommodate very little strain before failure. Thus, a criterion for the irradiations was to minimize cladding strain. This, of course, can be achieved by providing a substantial sodium bond thickness. However, such an approach, would decrease the smear density of the fuel pin. The conflicting requirements of ample fuel swelling space and a high smear density govern the range of sodium bond thicknesses to be investigated in the irradiations.

A sodium bond thickness of 6 mils was selected as the minimum practical size for the irradiation since the attainment and verification 
of bond thicknesses of this size and larger had been amply demonstrated by work at ANL. Calculations showed that sodium annuli of 6 and 10 mils would accommodate swelling of 10.4 and 18.2 percent of the fue 1 volume respectively. It was initially assumed that the swelling behavior of the nitrides is quantitatively similar to the carbides, thus indicating that sodium bond thicknesses of 6 and $10-\mathrm{mils}$ would accommodate 5 and 9 a/o burnup, respectively, before the fuel would contact the cladding. Subsequent data from the thermal irradiations of UN-PuN indicate that lower swelling rates can be expected. Design parameters for each of the capsules in the initial series of EBR-II irradiations are sumarized in Table 2. The smear density of the one helium bonded specimen BMI 1-3 was selected to be the same as for the reference sodium bonded specimen (10-mil sodium annulus). This required a fuel density of 83 percent of theoretical to obtain the desired smear density of 80.4 percent. Based on the assumption that 50 percent of the porosity in the fuel would be effective in accommodating swelling, the helium bonded specimen should undergo about 6 a/o burnup without cladding strain.

The four capsules in this series each contain a 14-inch long active fuel region within a 0.020 -inch cladding of Type 304 low-carbon stainless steel. The fuel consists of $20 \mathrm{w} / 0$ PuN-80 w/o fully enriched UN: fully enriched uranium is required to achieve the desired specific heat ratings in the EBR-II. The fuel is in the form of pellets prepared at the Battelle-Columbus Plutonium Laboratory by sintering techniques in which the as synthesized powders are ball-milled, cold pressed, sintered in a nitrogen atmosphere, machined to size and degassed in a vacuum. The clad fuel elements are immersed in sodium with in an outer capsule of Type 304 low-carbon stainless stee1 which is 0.375 -inch OD with a 0.020 -inch wall. Both the fuel cladding and outer capsule were obtained from ANL and are standard EBR-II components.

The capsule components underwent extensive characterization and nondestructive testing prior to final assembly. This included metallography, chemical analysis, radiography, ultrasonic testing and visual examination of the components.

\section{Assemb1y Procedure}

The general assembly procedure for the capsules is as follows: prior to encapsulation the bottom end-cap is heli-arc welded to the cladding and the weld is leak tested and radiographed. The fuel pin encapsulation is done in a dry box previously evacuated and backfilled with purified helium. Approximately 1.7 grams of purified sodium is loaded into the cladding, melted, and solidified. The pellets are inserted on top of the sodium, which is melted to allow the pellets to settle gently to the bottom. The sodium is solidified and the top end plug is heli-arc welded to the cladding. After decontamination, the clad assembly is leak checked, radiographed, and then centrifuged to bond the sodium. The bond is tested for soundness by eddy current techniques. For the helium bond assembly the loading is simplificd since all procedures involving sodium are eliminated. 
The encapsulation of the clad fuel assembly is done in a similar manner as for the fuel pellets. The bottom end-plug is heli-arc welded to the outer capsule she11 and the weld nondestructively tested for soundness and leak tightness. The encapsulation is again done in a vacuum dry box backfilled with purified helium. In the case of the sodium bonded clad assemblies it is necessary to melt the sodium preferentially from the top down before proceeding so as not to strain the cladding or disturb the sodium bond. This is not necessary for the helium bonded element. Approximately 9 grams of purified sodium is placed into the capsule shell, melted, and solidified. The clad assembly is lowered into the capsule and seated. The capsule is then cooled from the bottom up, the top end plug heli-arc welded to the capsule and the weld nondestructively tested for soundness. The capsule is centrifuged to eliminate voids in the sodium, and eddy current tested to verify soundness of the bond.

\section{Sodium Bonding and Eddy Current Testing}

The sodium bond in addition to accommodating fuel swelling, provides an excellent heat transfer path between the fuel and the cladding. Because of the high specific power levels at which these pins will operate, any substantial defects in the sodium bond will lead to local overheating and could precipitate cladding as we 11 as capsule failure. The achievement and verification of sound bonding is a prerequisite to the insertions of these experiments in EBR-II. Techniques were developed which led to satisfactory sodium bonds in every case with bond thicknesses as small as 6 mils. The centrifuge equipment used to remove voids from the sodium is shown in Figure 4. The centrifuge is equipped with heaters to maintain the elements at about $540 \mathrm{C}$ while rotating at $350 \mathrm{rpm}$. Provision is also made for temperature readout of each assembly during operation. Cooling of the element or cladding below $95 \mathrm{C}$ is always done from the bottom up to minimize shrinkage voids in the sodium bonds and to avoid stresses due to localized solidification of the sodium.

The equipment used to verify the soundness of the sodium bond utilizes the pulsed field reflected technique and is very similar to that used by ANL for the inspection of EBR-II fuel pins. The equipment is capable of detecting voids in the sodium bond annulus as smal1 as 1/16-in. diameter, the maximum size considered permissible for safe operation of the fuel elements at the high heat ratings desired. The sensitivity of the equipment is checked by the use of dummy capsules fabricated from materials closely approximating the electrical properties of the components in the actual irradiation capsules. Various void configurations in the sodium bond were simulated to provide a basis for the determination of the equipment sensitivity.

The four capsules in the BMI 1 series were assembled, tested, and sent to the EBR-II in February, 1969. Meanwhile, work was initiated 


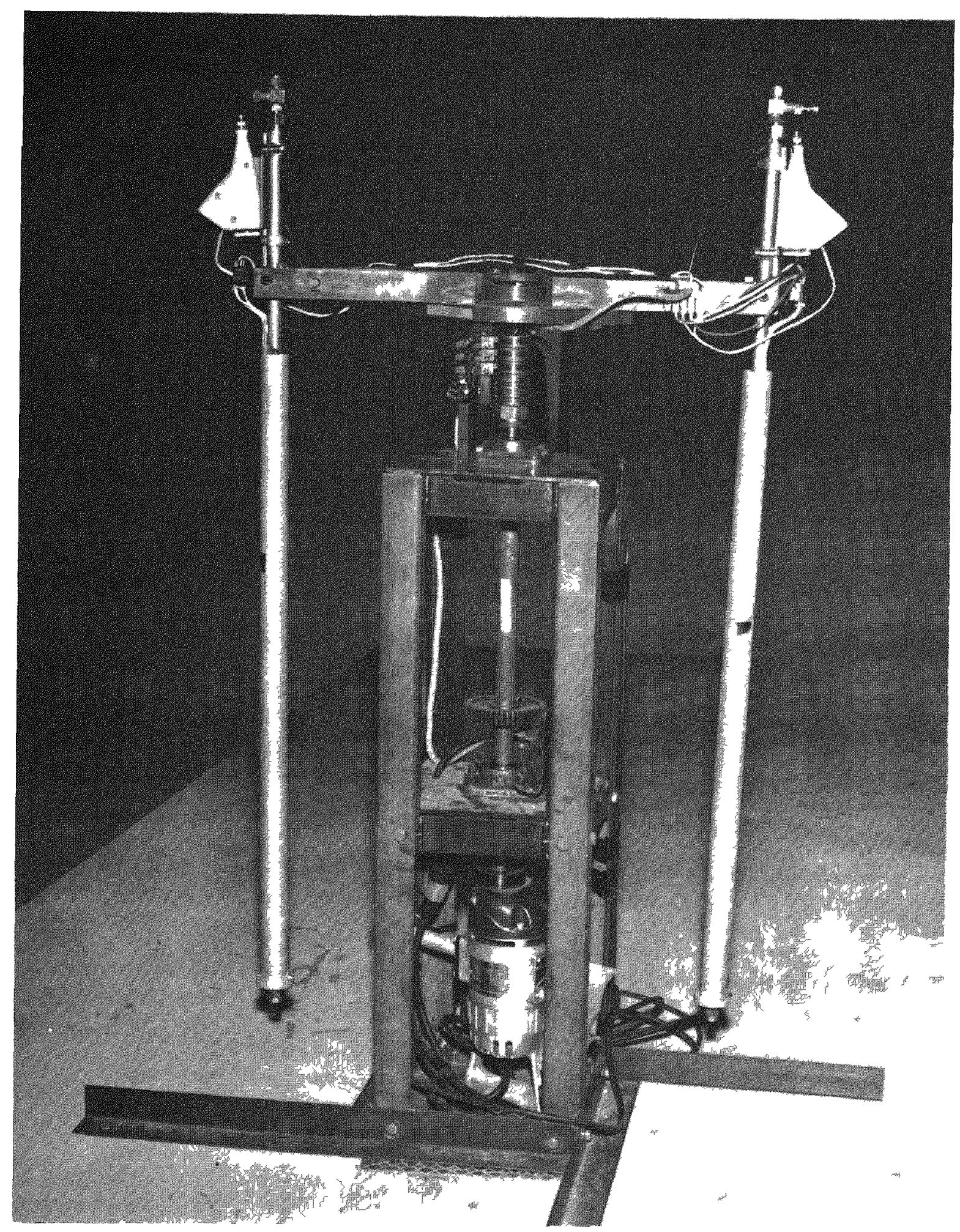

FIGURE 4. CENTRIFUGE EQUI PMENT FOR SODIUM BONDING EBR-II EXPERIMENTS 
on the second set of irradiation experiments consisting of eight (8) UN-PuN fue 1 pins and designated as the BMI 2 series. As before, the use of the highest practical fuel densities and of sodium bonding between the fuel and the cladding to provide for efficient heat transfer and accommodate fuel swelling were emphasized.

The experiments in this series are designed for specific power levels of $30-40 \mathrm{kw} / \mathrm{ft}$ and burnups up to $100,000 \mathrm{MWD} /$ tonne. In order to achieve the higher specific power levels without excessive cladding or capsule wall temperatures as well as to maintain the coolant temperature rise within limits prescribed by EBR-II, the active fuel length in these experiments was limited to 10 inches. Each capsule contains a nominal 10-inch active fuel length within a 0.315 in. OD Type 316 stainless steel cladding which has a 0.010 , 0.015 , or $0.020-i n$. wa11. The fuel consists of $20 \mathrm{w} / \mathrm{o}$ PuN-80 w/o fully enriched UN, prepared in the same way as that for the first series of EBR-II irradiations. All of the fuel pins have a sodium bond between the fuel and the cladding. The clad fuel elements are immersed in sodium within the standard EBR-II capsules of Type 304 low carbon stainless steel which is 0.375 -inch OD with a 0.020 -inch wall. The outer capsules were obtained from ANL and are standard EBR-II components; the inner stainless steel claddings were obtained commercially.

Table 3 summarizes the design details and desired irradiation conditions for each capsule. All of the experiments are designed to operate in any location within EBR-II core Row 4 with a maximum capsule surface temperature, including uncertainties, of less than $649 \mathrm{C}$ and a fuel cladding temperature of less than $760 \mathrm{C}$. The analyses on which this conclusion is based assumed $50 \mathrm{gpm}$ sodium flow rate per assembly, or 2.63 gpm per capsule.

The target burnups given in the table were selected to cover the range of principal interest at this time. In all cases, it is projected that there will be no cladding strain due to fuel swelling. As additional information on fuel swelling rates and other facets of UN-PuN behavior are developed from the thermal irradiation program, the target burnup values may be revised.

Assembly and testing procedures are essentially the same as for the initial series of irradiations. Extensive characterization and nondestructive testing of the materials utilized as well as strict control of the assembly and verification procedures will be utilized. It is anticipated the experiments will be delivered to EBR-II in late September, 1969. Eight additional capsules are planned for delivery to EBR-II during this fiscal year. Several helium bonded fuel pins will be included in these irradiations at fuel pin power ratings to $40 \mathrm{kw} / \mathrm{ft}$. 


\title{
A RETRACTABLE-REPLACEABLE THERMOCOUPLE \\ FOR IN-PILE EXPERIMENTS \\ by James $\mathrm{D}$. Heckelman \\ $S \operatorname{lin} 5$ \\ Lewis Research Center
}

\begin{abstract}
A design which allows the replacement of an in-core high temperature thermocouple located in an experiment capsule of a nuclear reactor has been developed, tested, and qualified for in-core use. The design is acceptable for fueled capsules, which require double containment. In addition, the thermocouple is remotely retractable so that it can be stored in a low temperature, low neutron flux zone, thus prolonging its 1ife.
\end{abstract}

SUMMARY

For practical thermionic nuclear power systems, fuel temperatures from 1500 to $2300^{\circ} \mathrm{K}$ are required. During the development and testing of fuel forms for this application, the required environment is frequently supplied by a nuclear reactor. During the irradiation, it is often necessary to measure the fuel temperature to within $\pm 100^{\circ} \mathrm{C}$ for up to 10,000 hours. Operating experience with tantalum sheathed, BeO insulated, $0.040^{\prime \prime}(0.102 \mathrm{~cm})$ to $0.062^{\prime \prime}(0.157 \mathrm{~cm})$ diameter tungsten rhenium alloy thermocouples used to measure fuel form temperatures at the Plum Brook Reactor and at other facilities has demonstrated that such thermocouples may not operate for the desired irradiation time. In addition, the drift in emf vs. temperature due to transmutation and radiation damage may not be known for a specific experiment because of geometry, shielding effects, or uncertainty in the nominal fluence values.

It is therefore desirable to have a thermocouple which can be replaced with a new one while the experiment capsule is in-core, underwater. An additional desirable feature is that the thermocouple be capable of being retracted (withdrawn) from the high temperature zone when not needed in order to prolong its 1ife. The Retractable-Replaceable Thermocouple (RRTC) was developed to fulfill this need. It satisfies the application since there is little need to continuously monitor specimen temperatures during long-term irradiations. 
The design of the RRTC utilizes a piston and cylinder placed about six inches $(15 \mathrm{~cm})$ above the test specimen in a cool region of the capsule. The thermocouple is attached to the piston which is operated with pressurized helium gas. A $2^{\prime \prime}(5.1 \mathrm{~cm})$ stroke is achieved with approximately a $40 \mathrm{lbs} / \mathrm{in}^{2}\left(27.6 \mathrm{~N} / \mathrm{cm}^{2}\right)$ pressure differential. An orifice and accumulator tank in the helium gas supply lines regulate the speed of insertion and withdrawal, which is typically $0.5 \mathrm{in.} / \mathrm{minute}$ ( 1.3 $\mathrm{cm} /$ minute). The entire assembly is attached to a flexible metallic hose and is guided into position in the capsule inside of a larger flexible metallic hose. The system is pressurized to prevent any possible entry of water.

Replacement of the thermocouple, with a new one is accomplished when the reactor is shut down by: (1) Purging the system with helium gas, (2) sampling the system for possible radioactive gases, (3) removing the smaller flexible metallic hose assembly, (4) disposing of the assembly underwater in the same manner as a fuel element, and (5) installing a new assembly. The system is then evacuated and back-filled with helium gas prior to operation. During the replacement of the RRTC, the piston and thermocouple tip are kept at least $80^{\prime \prime}$ (2 meters) below the surface of the water in the reactor tank. The water shields personnel from the possible 9600-curie source generated by activation of the materials in the RRTC assembly by a thermal neutron fluence of up to $1 \times 10^{22} \mathrm{n} / \mathrm{cm}^{2}$.

Extensive out-of-pile testing of a prototype RRTC indicated that the design was sound. The RRTC was then incorporated into an experiment and insta1led in-core. The purpose of this experiment is to determine incore reliability and radiation effects for a fluence of up to $10^{22}$ neutrons $/ \mathrm{cm}^{2}$ (therma 1) on W3Re-W25Re, $0.062^{\prime \prime}(0.157 \mathrm{~cm}$ ) diameter, tantalum sheated, BeO insulated thermocouples at a temperature of $2073^{\circ} \mathrm{K}$. The RRTC is very important in determining the true temperature of the thermocouples during irradiation and when the capsule is heated electrically during reactor shutdown.

\section{INTRODUCTION}

Accurate determination of temperatures in the 1500 to $2300^{\circ} \mathrm{K}$ range is important in the development of fuel forms for thermionic nuclear power systems and in many other applications. It is necessary to measure the fuel form temperatures to within $\pm 100^{\circ} \mathrm{K}$, and often desirable to measure it to within $\pm 50^{\circ} \mathrm{K}$ or less, for up to 10,000 hours of operation.

So far the only practical means of determining these temperatures in nuclear reactors is by the use of thermocouples. Environmental conditions usually dictate sheathed thermocouples of $0.040^{\prime \prime}(0.102 \mathrm{~cm})$ to $0.062^{\prime \prime}(0.157 \mathrm{~cm})$ diameter. During use, the thermocouples may experience drift in emf vs. temperature due to (1) chemical reactions caused by impurities in the thermocouple materials, (2) transmutation caused by thermal neutrons, and (3) radiation damage caused by fast neutrons and gamma radiation. For a specific experiment, large uncertainties may exist in these effects because of manufacture, geometry, shielding, or uncertainty in the nominal neutron fluence experienced by the the rmocouples. 
TABIE I. - SUMMARY OF IN-PILE OPERATING EXPERIRNCE WITH HIGH TEMPERATURE THERMOCOUPLES AT THE PLUM GROOK REACTOR

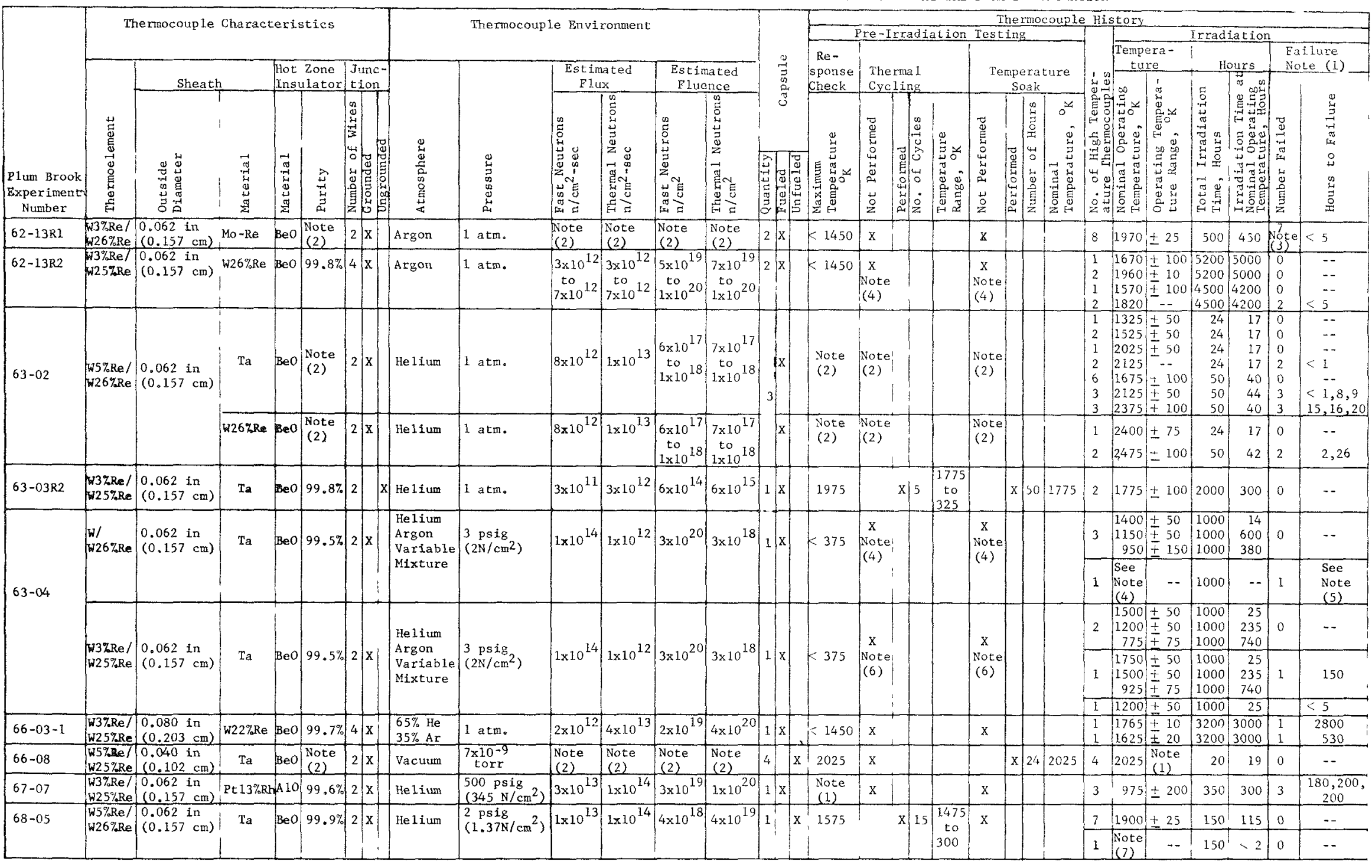

NOTES :

(1) Except for experiment 68-05, there are no direct means of determining the true temperature of the therwocouples for the experiments listed in this table. Therefore, "failure" means catastrophic failure or very significant drift as determined by other measured parameters.

(2) This information is not available at the time of publishing.

(3) The operating thermocouple was questionable at the end of the irradiation.
(4) Other thermocouples of the same lot were thoroughly tested out-of-pile. In general, a11 the rmocouples performed satisfactorily.

(5) Thermocouple failed prior to start of irradiation.

(6) Other thermocouples of the same lot were thoroughly tested out-of-pile. All thermocouples tested showed some sort of failure or significant change in emf vs. temperature.

(7) This is a retractable replaceable the rmocouple (RRTC) and is described in this report. 


\section{HISTORY OF HIGH TEMPERATURE THERMOCOUPLES}

\section{AT THE PLUM BROOK REACTOR FACILITY}

The in-pile long time reliability of high temperature thermocouples at the Plum Brook Reactor Facility (PBRF) and at other facilities is poor. A summary of our in-pile experience is provided in tabular form in table 1. Note that the operating temperatures are, in most cases, relatively low. Out-of-pile reliability of similar thermocouples is no better.

For long-term irradiations, during which time the true temperature must be known, redundant thermocouples are often used to obtain an improvement in the reliability. But this is not satisfactory in many cases since space is limited, additional instrumentation is costly, and the drift in emf vs. temperature due to transmutation and radiation damage is often too uncertain.

\section{DESIGN OBJECTIVES}

Considexing the above, a thermocouple which can be replaced with a new one while the experiment capsule is in-core, underwater, is highly desirable for long-term irradiations. An additional desirable feature is that the thermocouple be capable of being retracted, or withdrawn from the high temperature zone when not needed. Storing the thermocouple in this manner will prolong its life in two ways:

1. The chemical reactions within the thermocouple are occurring at much reduced rates, if at a11, since the thermocouple is at a much lower temperature, and

2. the neutron fluence experienced by the thermocouple may be significantly reduced in the stored position by distance from the core and/or by shielding.

In addition to the above, retracting the thermocouple also ensures that it can be replaced since the possibility of the thermocouple welding to the fuel form or to the thermocouple well is minimized. (Significant welding does not occur if the time at temperature is only in the order of a few hours.)

Such a design would permit the thermocouple to be inserted into the capsule whenever the true temperature of the test specimen is desired. It could also be used to check the accuracy of other thermocouples permanently installed in the capsule, and to recalibrate them at intervals during the irradiation. This mode of operation, which has been successfully used in at least one other type of experiment (ref. 1), satisfies long-term irradiations since there is little need to continuously monitor specimen temperatures at this level of accuracy.

The design proceeded with the intent of remotely retracting the thermocouple out of the high temperature zone when not needed in addition to making it possible to replace the thermocouple when desired. The specific design objectives were: 
1. To incorporate one replaceable and retractable thermocouple in the capsule at the test specimen center. This thermocouple must be sheathed, with a maximum diameter of $0.062^{\prime \prime}(0.157 \mathrm{~cm})$.

2. To develop a technique for replacement while the experiment remains in-core. Consideration must be given to the radioactivity from the activated thermocouple assembly after being irradiated for up to 5000 hours at a thermal neutron flux of up to $5 \times 10^{14} \mathrm{n} / \mathrm{cm}^{2}-\mathrm{sec}$.

3. To provide two containment barriers (in the case of fueled capsules) between the experiment fission products and the reactor containment vesse 1 atmosphere during irradiation of the experiment and yet provide means for replacing the thermocouple when the reactor is shut down. The fuel clad material cannot be considered a barrier since it is frequently under test. Consideration must be given to the possibility that the fuel cladding failed during irradiation and fission products (gases) are present, thus affecting the replacement of the thermocouple.

\section{DESIGN}

The design of the Retractable Replaceable Thermocouple (hereafter referred to as the RRTC) utilizes a piston and cylinder placed about six inches $(15 \mathrm{~cm})$ above the test specimen in a cool region of the capsule. This assembly is shown in figure 1. The thermocouple is attached to the piston which is operated with pressurized helium gas. Particles in the helium gas larger than 74 microns are removed by a 200 mesh screen. A $2 "(5.1 \mathrm{~cm})$ stroke is achieved with approximate $1 \mathrm{y}$ a $40 \mathrm{lbs} / \mathrm{in}^{2}(27.6$ $\mathrm{N} / \mathrm{cm}^{2}$ ) pressure differential across the piston. The range of diametrical clearance between the piston and the cylinder is $0.0010^{\prime \prime}\left(2.5 \times 10^{-3} \mathrm{~cm}\right)$ to $0.0015^{\prime \prime}\left(3.8 \times 10^{-3} \mathrm{~cm}\right)$. When the RRTC is inserted, the helium gas leakage rate past the piston is less than $0.4 \mathrm{ft} / \mathrm{min}$. $\left(1.13 \times 10^{4}\right.$ $\left.\mathrm{cm}^{3} / \mathrm{min}_{0}\right)$. The piston is returned to the stored position by a spring. The thermocouple is guided into the thermocouple wel1 (in the test specimen) by a stainless stee1 tube.

A simplified gas system schematic is shown in figure 2. An orifice and accumulator tank in the helium supply lines regulate the speed of insertion. An insertion speed of approximately $0.5 \mathrm{in./minute} \mathrm{(1.3}$ $\mathrm{cm} / \mathrm{minute}$ ) is necessary to allow the thermocouple to reach temperature slowly enough to avoid a thermal shock. Approximately the same length of time is required to withdraw the thermocouple for the same reason.

The piston, cylinder, and thermocouple assembly are attached to a $3 / 8^{\prime \prime}$ $(0.95 \mathrm{~cm})$ inside diameter flexible metallic hose, as is shown in figure 3 . The electrical leads from the thermocouple run to a hermetically sealed electrical connector on a flange located at the upper end of the $3 / 8^{\prime \prime}$ 
$(0.95 \mathrm{~cm})$ hose. The slack wire is taken up and coiled by a spring located near the flange. This spring is designed to maintain a slight force on the thermocouple lead wire to keep it tight, but not enough force to damage the wire, the insulation, or impair the operation of the piston. The travel of this spring assembly is approximately $12^{\prime \prime}$ (30.5 $\mathrm{cm}$ ), considerably more than the $2^{\prime \prime}(5.1 \mathrm{~cm})$ travel of the piston, to allow for changes in length of the flexible metallic hose when it is pressurized.

The 3/8" $(0.95 \mathrm{~cm})$ hose is guided into position in the capsule inside of a $1^{\prime \prime}(2.5 \mathrm{~cm})$ inside diameter flexible metallic hose, also shown in figure 3. This larger hose also provides a barrier to the primary water in the reactor tank. It is normally pressurized with helium gas to a pressure greater than the reactor tank pressure to prevent any possible entry of water into the RRTC system.

To enable the capsule to be removed from the reactor tank in case the RRTC lines contain radioactive gas, al1 hose connections are of the quick disconnect type with all ends self-sealing and helium leak tight.

\section{OPERATING PHILOSOPHY}

When used, the RRTC is inserted only for approximately five minutes -long enough to obtain a stable millivolt output -- and then withdrawn. The frequency of insertion would depend upon the neutron fluence and the anticipated drift in emf vs. temperature of fixed thermocouples in the capsule, the frequency or amount of capsule temperature changes indicated by other instrumentation, or upon other prevailing conditions if fixed thermocouples are not available.

After a time in-core, the accuracy of the RRTC may become questionable or it may have failed completely. At this time, the entire $3 / 8^{\prime \prime}(0.95 \mathrm{~cm})$ hose assembly is replaced with a new, recently calibrated thermocouple.

\section{RRTC REPLACEMENT TECHNIQUE}

The real importance of the design of the RRTC is the ability to replace the thermocouple with one which has been recently calibrated while a fueled or unfueled experiment is in the reactor core. Figure 4 shows a typical installation of a capsule which incorporates the RRTC in the Plum Brook Reactor. The replacement of the RRTC assumes the following:

1. The experiment has been in-pile and operating.

2. The irradiation time on the RRTC presently in the capsule is not greater than 5000 hours (for shielding requirements) at a $\phi_{\text {th }}$ of $<10^{14} \mathrm{n} / \mathrm{cm}^{2}-\mathrm{sec}$. 
3. The RRTC presently installed requires replacement.

4. The reactor is shut down and access to the reactor core is possible.

Prior to commencing the replacement of the RRTC, the system is purged with helium gas to remove all activated gases. The gas contained in the system is then sampled for radioactivity. To accomplish this, a radiation detector is placed in the helium gas exhaust line from the RRTC, and provision is made to take samples of the gas for laboratory analysis. If the capsule is not fueled, the purge should remove all activated gases, and there should be no problem in obtaining a clean system. If the capsule is fueled, a crack in the fuel cladding and the thermocouple well is credible (note that two failures are required). Thus, fission products could be present in the RRTC gas system. In such a case, the purging may not remove the radioactive gases, and replacement of the RRTC may not be possible.

After a satisfactory gas sample has been obtained, the replacement of the RRTC may begin. The top flange is unbolted and the $3 / 8^{\prime \prime}(0.95 \mathrm{~cm})$ hose is raised and fastened as shown in figure 5. The top flange assembly is then removed from the hose. A cable which runs inside of a $1^{\prime \prime}(2.5 \mathrm{~cm})$ interna 1 diameter by $8^{\prime}(2.4$ meters $)$ long flexible metallic hose (hereafter referred to as the "extension hose") is attached to an overhead crane. The other end of the cable is attached to a closed container filled with lead shot. This container is then attached to the $3 / 8^{\prime \prime}(0.95 \mathrm{~cm})$ hose, and the lead shot is expelled into the $3 / 8^{\prime \prime}(0.95$ $\mathrm{cm}$ ) hose, as shown in figure 6. The lead shot fills the $3 / 8^{\prime \prime}(0.95 \mathrm{~cm})$ hose to about $12^{\prime \prime}(30.5 \mathrm{~cm})$ above the piston and cylinder assembly, and serves as shielding to prevent streaming of the gamma radiation via the $3 / 8^{\prime \prime}(0.95 \mathrm{~cm})$ hose.

The extension hose has a flange which mates with the flange of the $1^{\prime \prime}$ $(2.5 \mathrm{~cm})$ hose leading to the capsule. A large ball valve with a 1-1/4" $(3.2 \mathrm{~cm})$ opening is located approximately $3^{\prime \prime}(7.6 \mathrm{~cm})$ above this flange. These two flanges are now bolted together, as shown in figure 7 . The $3 / 8^{\prime \prime}(0.95 \mathrm{~cm})$ hose is then lifted by the crane until the the rmocouple tip, which is at the end of the $3 / 8^{\prime \prime}(0.95 \mathrm{~cm})$ hose assembly, is $80^{\prime \prime}$ ( 2 meters) below the surface of the water in the reactor tank as illustrated in figure 7 .

The extension hose is then lowered until the large valve is below the tip of the thermocouple, as illustrated in figure 8 . The ball valve is then closed, and the extension hose is allowed to drop away from the RRTC assembly as illustrated in figure 9. The RRTC is disposed of in the same manner as a fuel element.

The extension hose is brought to the top of the reactor tank, the flanges are blown dry, and the extension hose is removed. Installing a new RRTC assembly, evacuation of the system with a vacuum pump, and backfilling the system with helium completes the replacement procedure. 
A 9600 curie source results from the irradiation if it is assumed that the RRTC assembly has been in the reactor for 5000 hours at $\phi$ th $=5 \times 10^{14}$ $\mathrm{n} / \mathrm{cm}^{2}$-sec. The gamma radiation energy is $1.23 \mathrm{Mev}$ maximum. The expected dose rate 80 inches ( 2 meters) from the assembly in air will be no greater than $1600 \mathrm{R} / \mathrm{hr}$. The 80 inches (2 meters) of primary cooling water will, however, serve as a shield for the 9600 curie thermocouple assembly. Twenty inches ( 0.5 meter) of water will act as a tenth value layer gamma shield. Therefore, the 80 inches (2 meters) of primary cooling water attenuates the source to $160 \mathrm{mr} / \mathrm{hr}$ at the surface of the water. It is expected that the thermocouple will be at the $80^{\prime \prime}$ location for no longer than 15 minutes. Therefore, the personnel replacing the thermocouple will receive not more than a $40 \mathrm{mr}$ dose.

In actual operation of an experiment, the RRTC would probably be replaced at least every 1000 hours of irradiation. In addition, the neutron dose rate to the material in the RRTC is a factor of two less than the maximum value assumed. Also, not all of the material is located at the point of maximum flux, as assumed above, since the thermocouple assembly extends out of the reactor core. Therefore, the dose rates above are conservative.

\section{AUXILIARY SYSTEMS REQUIRED}

Several auxiliary systems must be considered when using the RRTC. The basic requirements are 1 isted for reference; the specific pressures, etc. will, of course, depend on the particular installation.

1. A helium gas supply system to operate the piston is required. The helium gas must have less than $4 \mathrm{ppm}$ total impurities (primarily $\mathrm{O}_{2}$, $\mathrm{N}_{2}$, and $\mathrm{H}_{2} \mathrm{O}$ vapor) to prevent corrosion and subsequent embrittlement of the tantalum material in the hot zone.

The helium gas system can be of two types: (a) A self-contained system utilizing a pump to circulate the helium gas in a closed loop, or (b) a helium gas supply bottle and associated valves to provide the pressure required.

2. A radioactive gas detection system used during the replacement of the RRTC, as previously noted, is required.

3. A radioactive gas disposal system which will accept the exhaust from the RRTC is required. 
After the design of the RRTC was completed, two test assemblies were fabricated and are shown in figures 3 and 10 . Tests were devised to verify that the system was indeed operational. The test objectives were:

1. To test the replacement technique:

a) To actually perform the replacement of a thermocouple assembly under simulated reactor conditions.

b) To identify a11 problems with the RRTC assembly and/or the replacement procedure.

2. To test the mechanica1 operation at high temperatures:

a) To verify that the thermocouple inserted and retracted over the entire operating temperature range.

b) To find out if thermal cycling affected the mechanical operation of the thermocouple.

c) To verify that the thermocouple did not "weld" to the thermocouple well after one hour of operation at $2300^{\circ} \mathrm{K}$.

3. To evaluate the thermocouple performance at high temperatures:

a) To determine the accuracy of the thermocouple, using an optical pyrometer, without the presence of gamma heating. The effects of gamma heating on temperature may then be determined during an initial irradiation.

b) To determine the variance in the temperature as indicated by the retractable thermocouple after several insertions.

\section{RESULTS OF PERFORMANCE TESTS}

1. Replacement Technique:

The replacement technique was performed in a large pool of water used to test experiments prior to installation in the reactor tank. The test assembly shown in figure 3 was used. The procedure proved correct and replacement was easily accomplished.

2. Mechanical Operation at High Temperatures:

The mechanical operation of the piston and thermocouple assembly was tested using the apparatus shown in figure 10. The operation proved satisfactory up to temperatures of $2000^{\circ} \mathrm{K}$. There was no evidence of welding of the thermocouple to the well wall even after several hours of operation at temperature. 
Difficulty was experienced with the thermocouple failing to retract at temperatures in the $2000^{\circ} \mathrm{K}$ to $2300^{\circ} \mathrm{K}$ range after severa 1 minutes at temperature. This time seemed to be cumulative with each insertion within the above temperature range. Further investigation indicated that the thermocouple well was not in alignment with the axis of the piston, and the thermocouple was pressed fairly hard against the side of the well. Apparently at these higher temperatures, the thermocouple sheath, which was tantalum, did not have sufficient strength to resist this force. Consequently, the tip became bent and unable to be withdrawn.

To verify that this was the cause rather than internal stresses within the thermocouple, tantalum tubing was used to simulate a thermocouple and the tests were repeated. The same results were obtained, even when tantalum tubing, preheated to the same temperature for several hours to release any internal stress, was used. When the axis of the tantalum tube was altered so that it aligned with the axis of the wel1, the problem was eliminated. Therefore, mechanical operation of the RRTC at high temperatures proved satisfactory.

3. Electrica1 Operation at High Temperature:

The apparatus shown in figure 10 was also used to determine the accuracy and the variance in the RRTC in determining the temperature of the test specimen. As shown, an optical pyrometer was used to measure the temperature of the test specimen over a temperature range of $1500^{\circ} \mathrm{K}$ to $2100^{\circ} \mathrm{K}$. The graph shown in figure 11 compares this temperature with the temperature indicated by the RRTC. The points plotted represent the average of several observations. Except for one point (at approximately $1670^{\circ} \mathrm{K}$ ), the deviation is $5^{\circ} \mathrm{K}$ or less. The average deviation is negative, and is the result of heat loss via the thermocouple well and thermocouple.

The variance in the RRTC indication of temperature for several insertions and withdrawals was less than two degrees. In fact, the variance is hardly distinguishable.

The results of the above tests were quite satisfactory. The RRTC met the design objectives, and was qualified for use in-core.

\section{IN-CORE APPLICATION OF THE RRTC}

An experiment was designed at the PBRF to determine the in-core reliability and accuracy of high temperature measuring devices at temperatures up to $2300^{\circ} \mathrm{K}$. A sketch of the first capsule, which tests thermocouples, is shown in figure 12. The RRTC was incorporated into the capsule as a means of determining the true temperature of the thermocouples under test. 
The operating philosophy is to heat the capsule electrically when the reactor is shut down (or the capsule is withdrawn from the core), and to insert the RRTC to recalibrate the thermocouples under test. Electrical heating eliminates the errors caused by gamma heating, thus always performing the calibration under identical conditions. The capsule temperature may be varied so that a multi-point recalibration is obtained.

The RRTC may also be used during irradiation, but with greater uncertainty, since the gamma heating required to achieve a given temperature may vary because of changes in the heat transfer. This results in differences in the relative temperature of the thermocouples and cannot be distinguished from transmutation and radiation damage.

\section{CONCLUSION}

In conclusion, a design which allows replacement of a high temperature thermocouple located in a capsule in-core has been developed. The design is acceptable for fueled capsules which require double containment. Extensive out-of-pile tests qualified the design for in-core use. The design was incorporated into an experiment which determines the radiation effects on high temperature measuring devices at the PBRF. (This experiment has been irradiated, though a water leak terminated the irradiation prematurely. A second capsule will be fabricated and irradiated.) The RRTC performed very satisfactorily during the irradiation, and will be used in future experiments at the PBRF.

\section{REFERENCES}

1. Funston, E. S.; and Kuhlman, W. C.: High-Temperature Thermocouple and Electrical Materials Research. High-Temperature Materials Program. Rep. GEMP-63, General Electric Co., Dec. 3\%, 1966, pp. 129-135. 


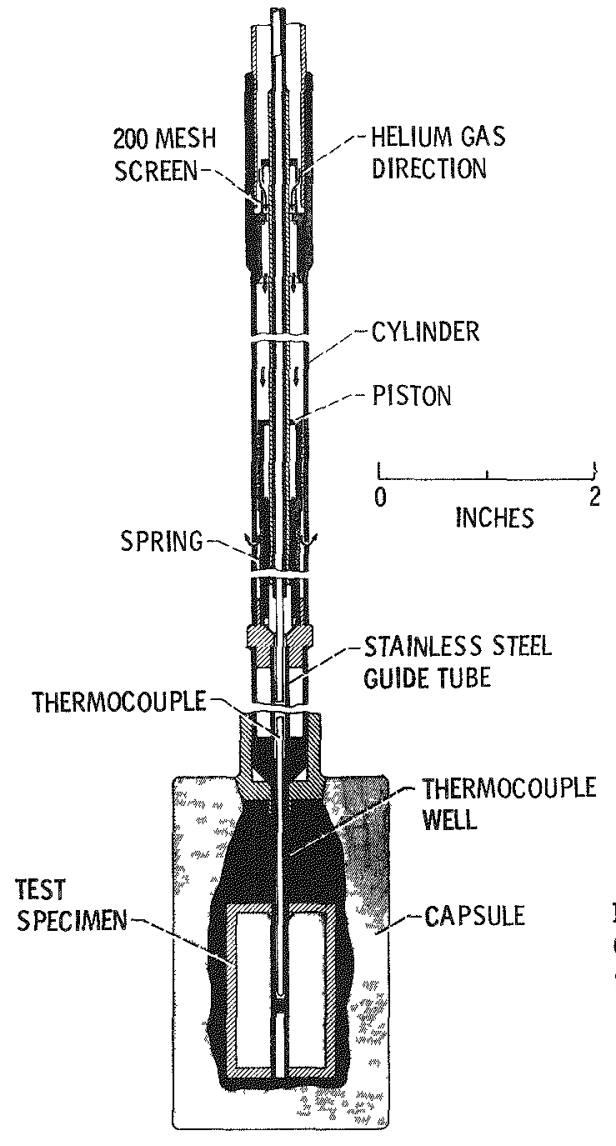

Figure 1. - RRTC piston and cylınder assembly.

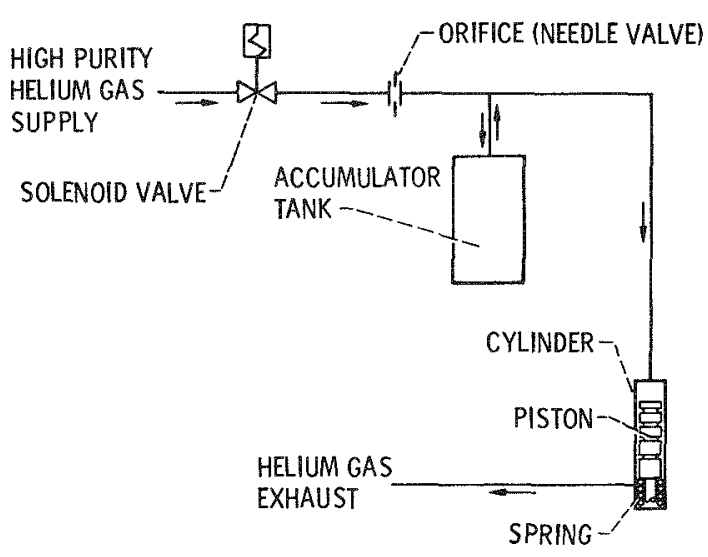

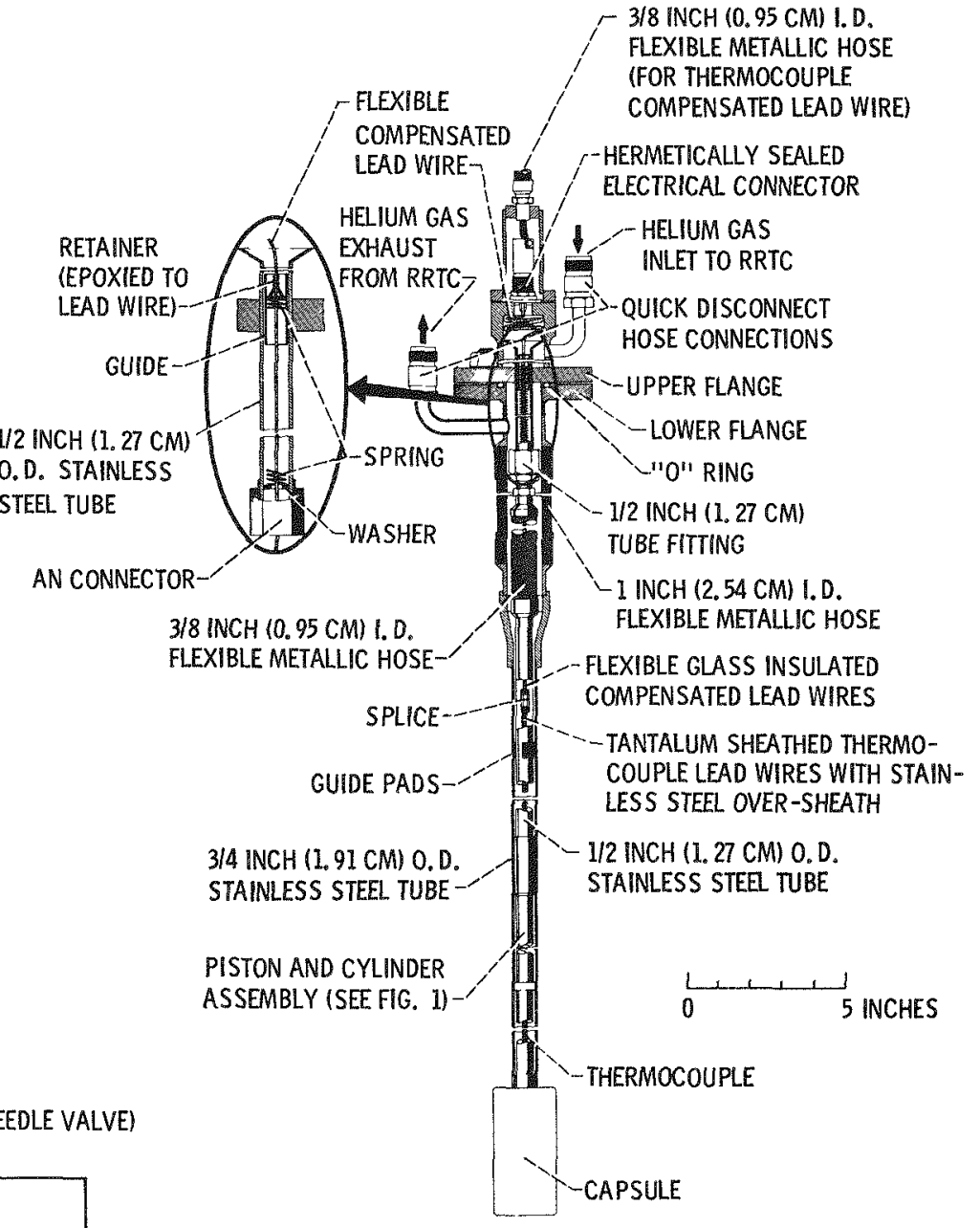

Figure 3. - RRTC Assembly.

Figure 2. - RRTC gas system schematıc 


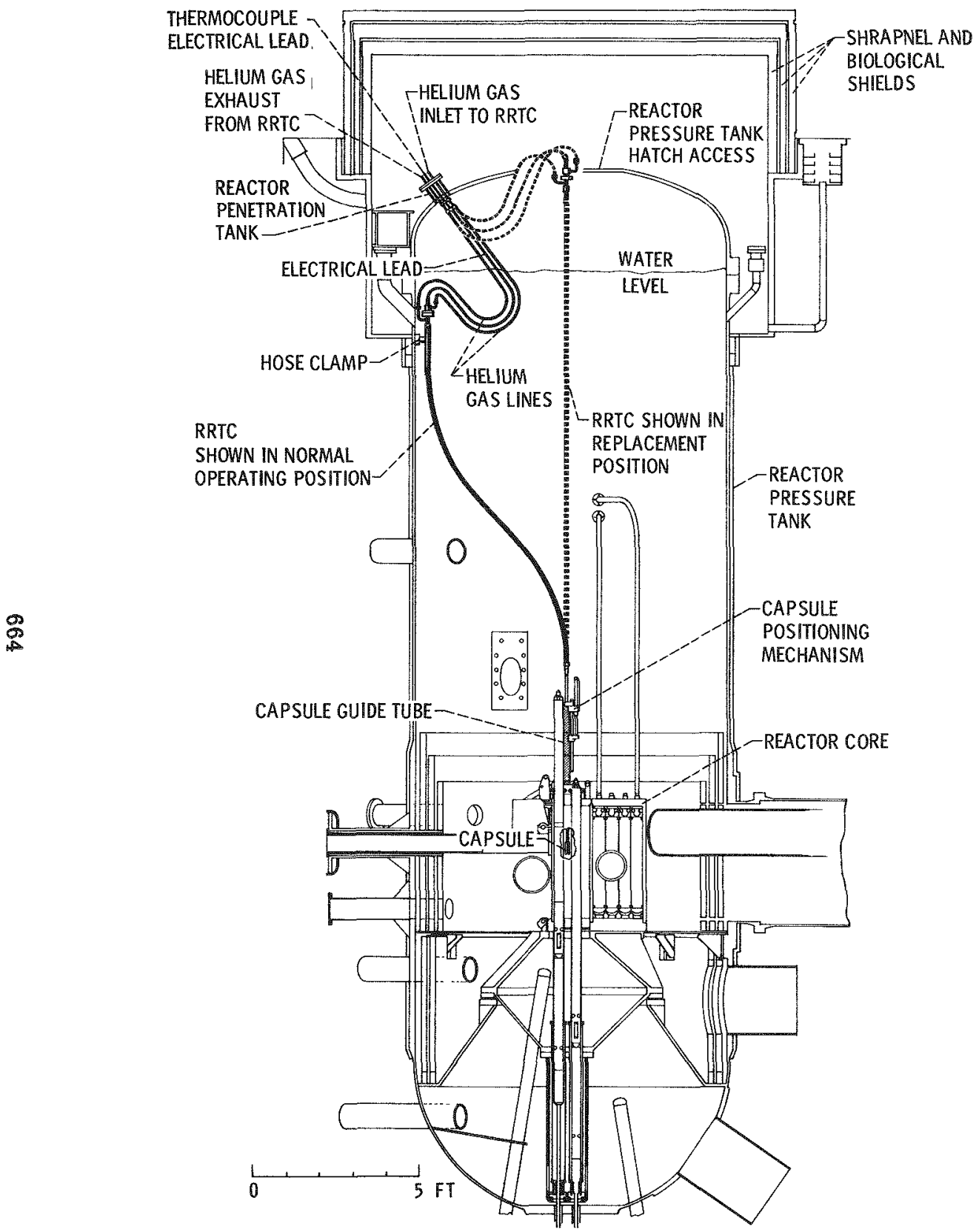

Figure 4. - Typical RRTC installation in the Plum Brook reactor.

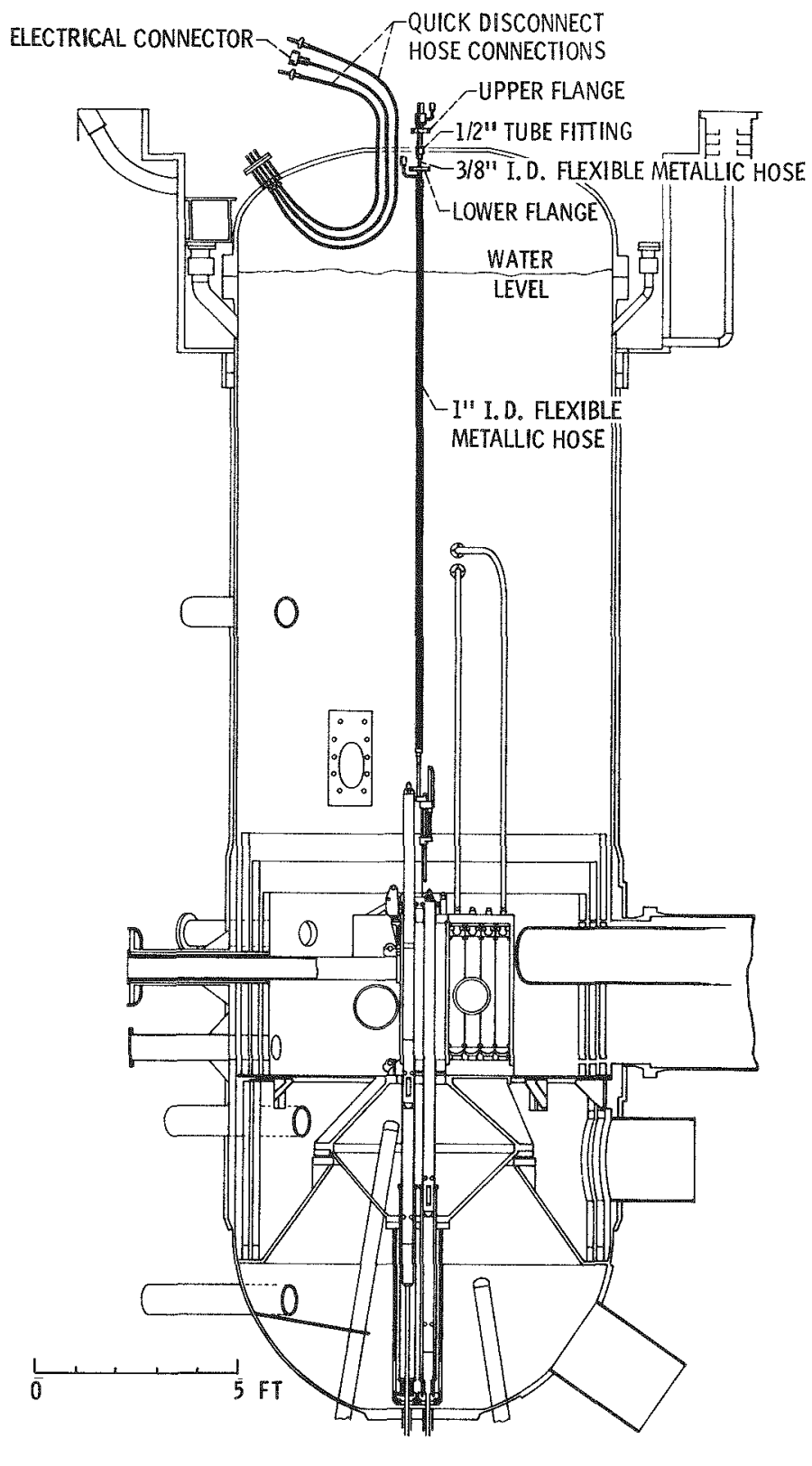

Figure 5. - RRTC replacement, step 1. 


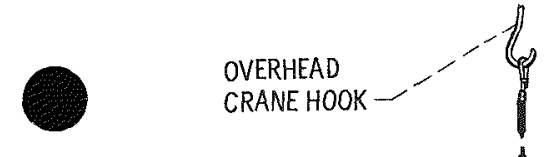

I' I. D. FLEXIBLE METALLIC

HOSE (EXTENSION HOSE)
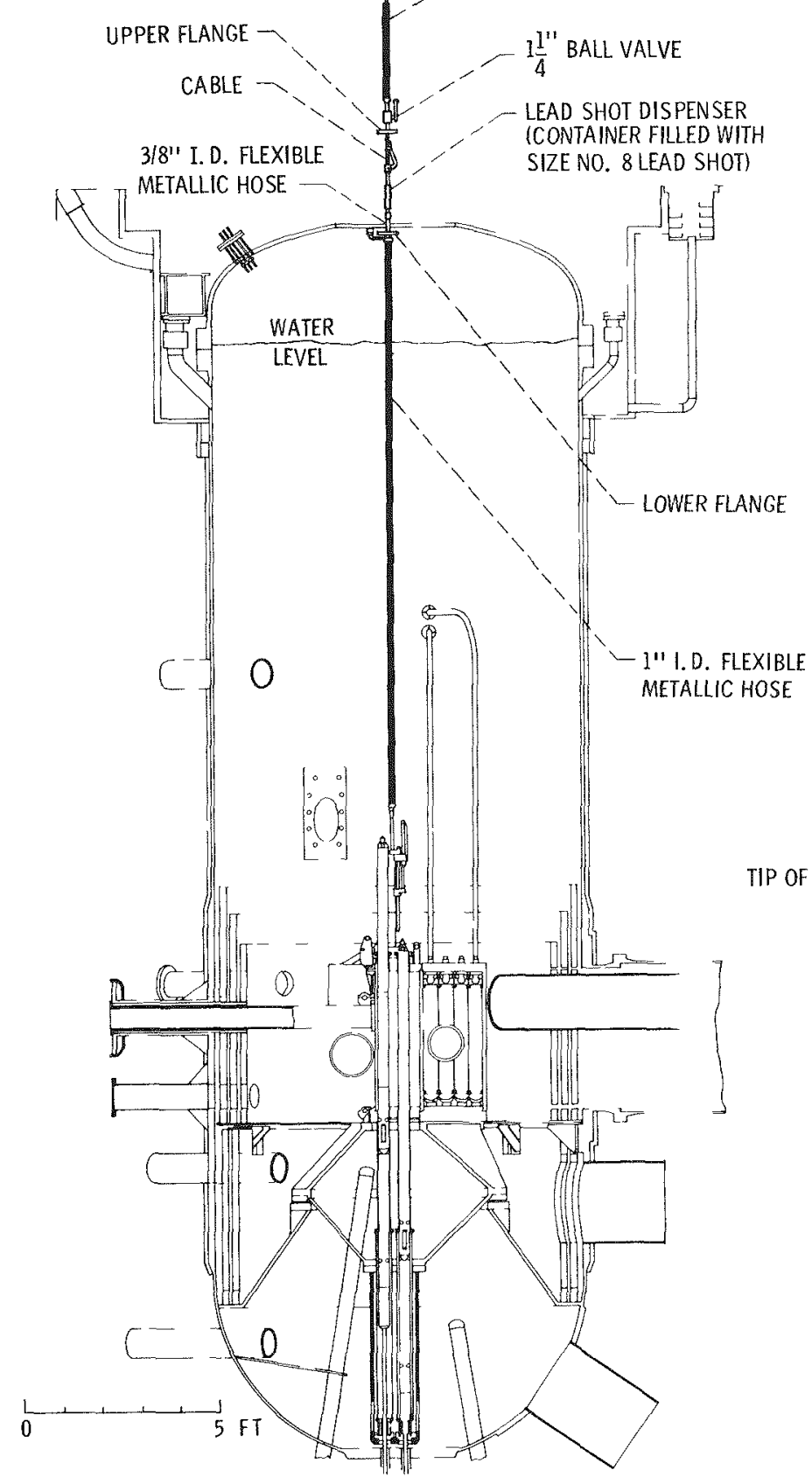

Figure 6. - RRTC replacement, siep 2.

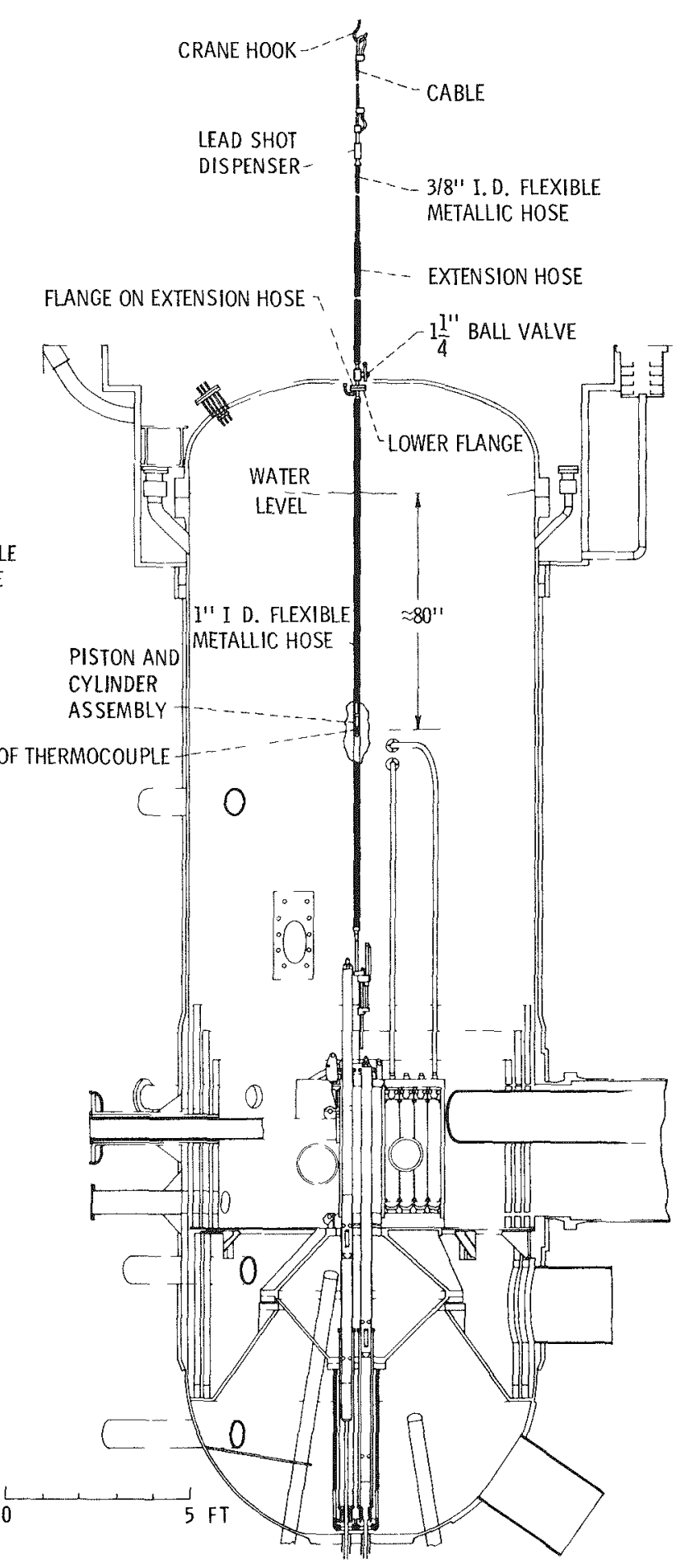

Figure 7. - RRTC replacement, step 3 


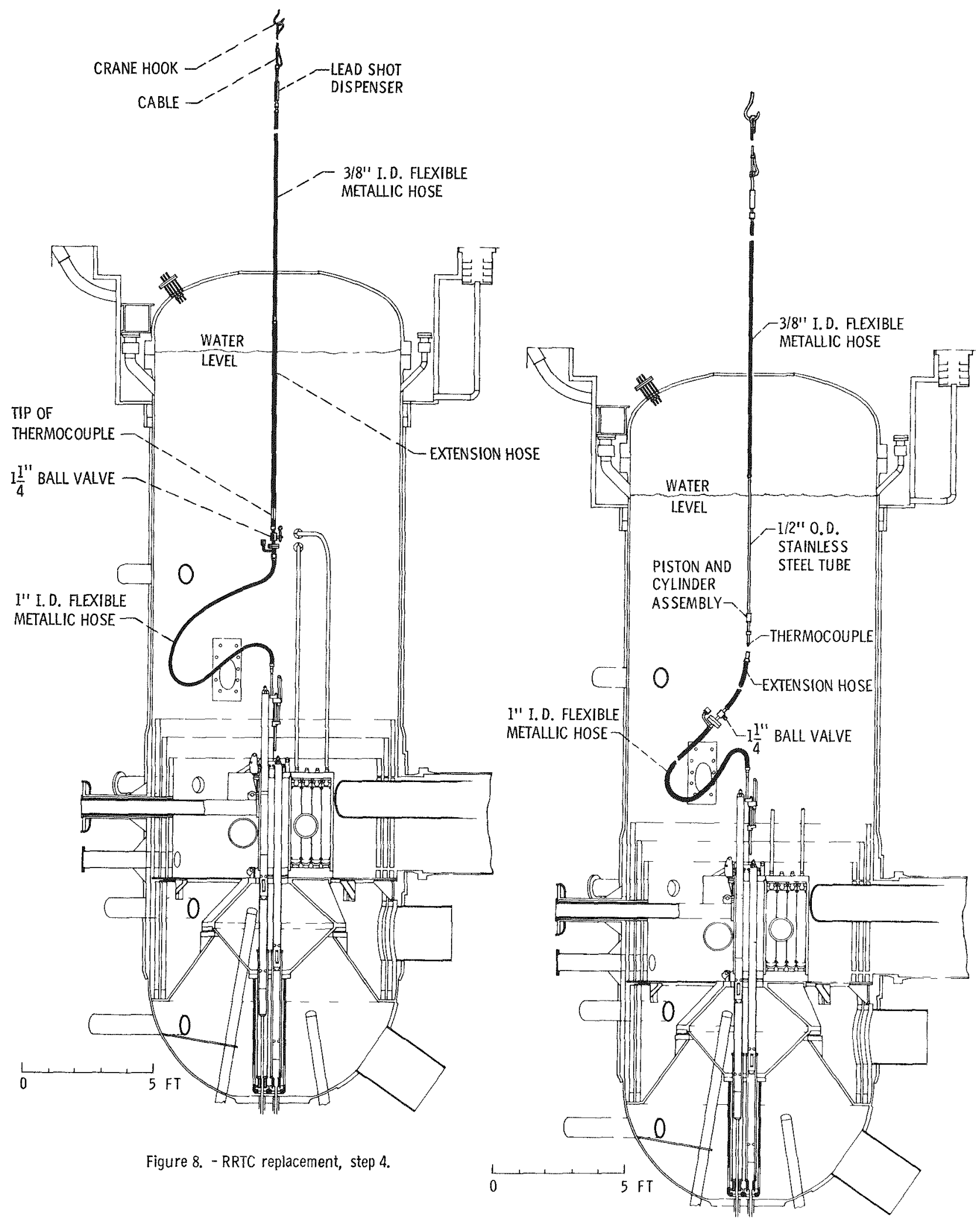

Figure 9. - RRTC replacement, step 5. 


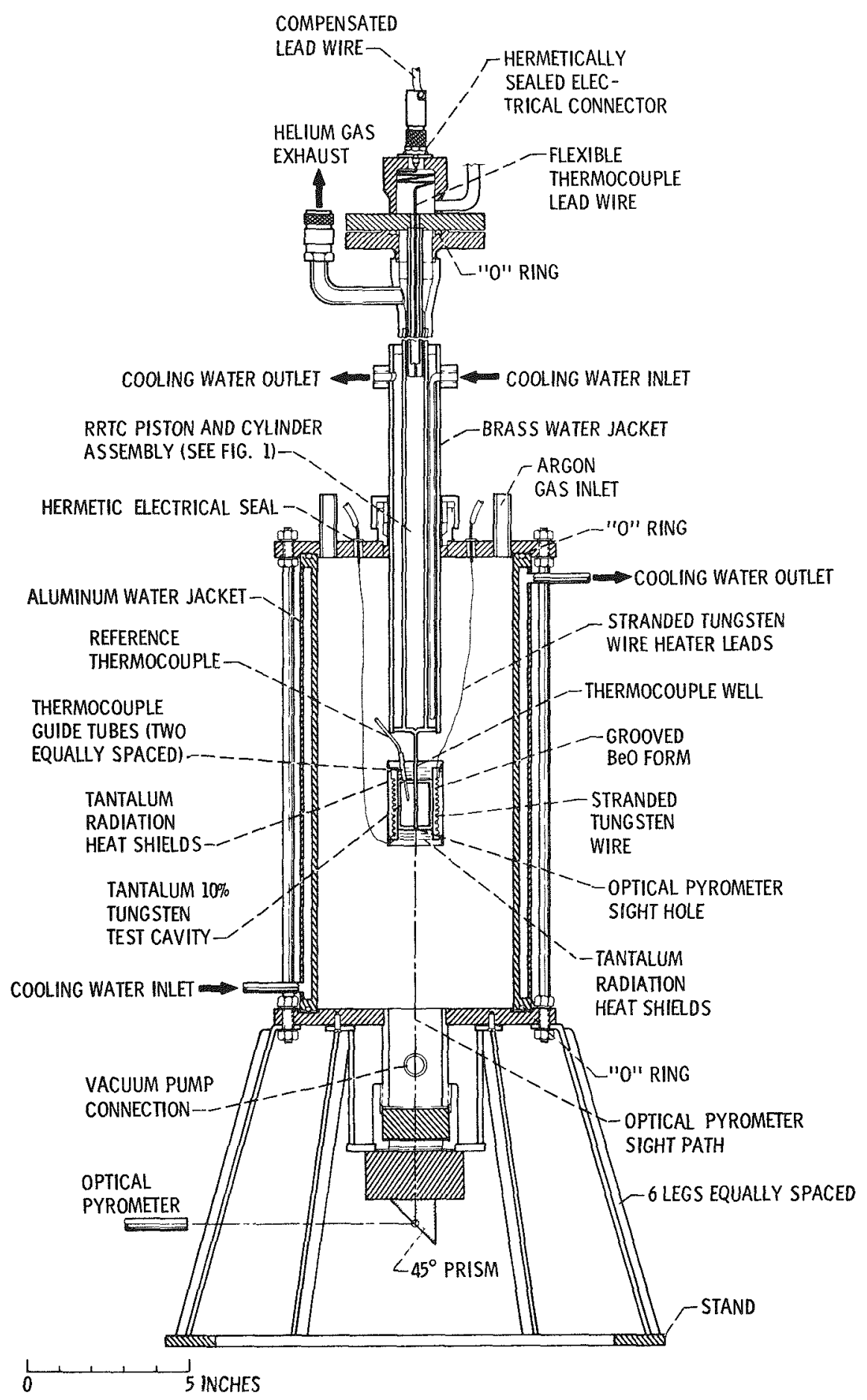

Figure 10. - Test furnace. 


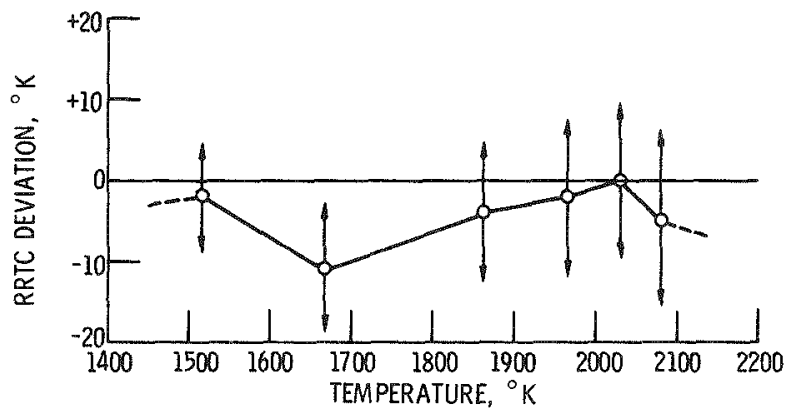

Figure 11. - Retractable replaceable thermocouple deviation vs temperature. (Uncertainty band for each observation is indicated by arrowsi.

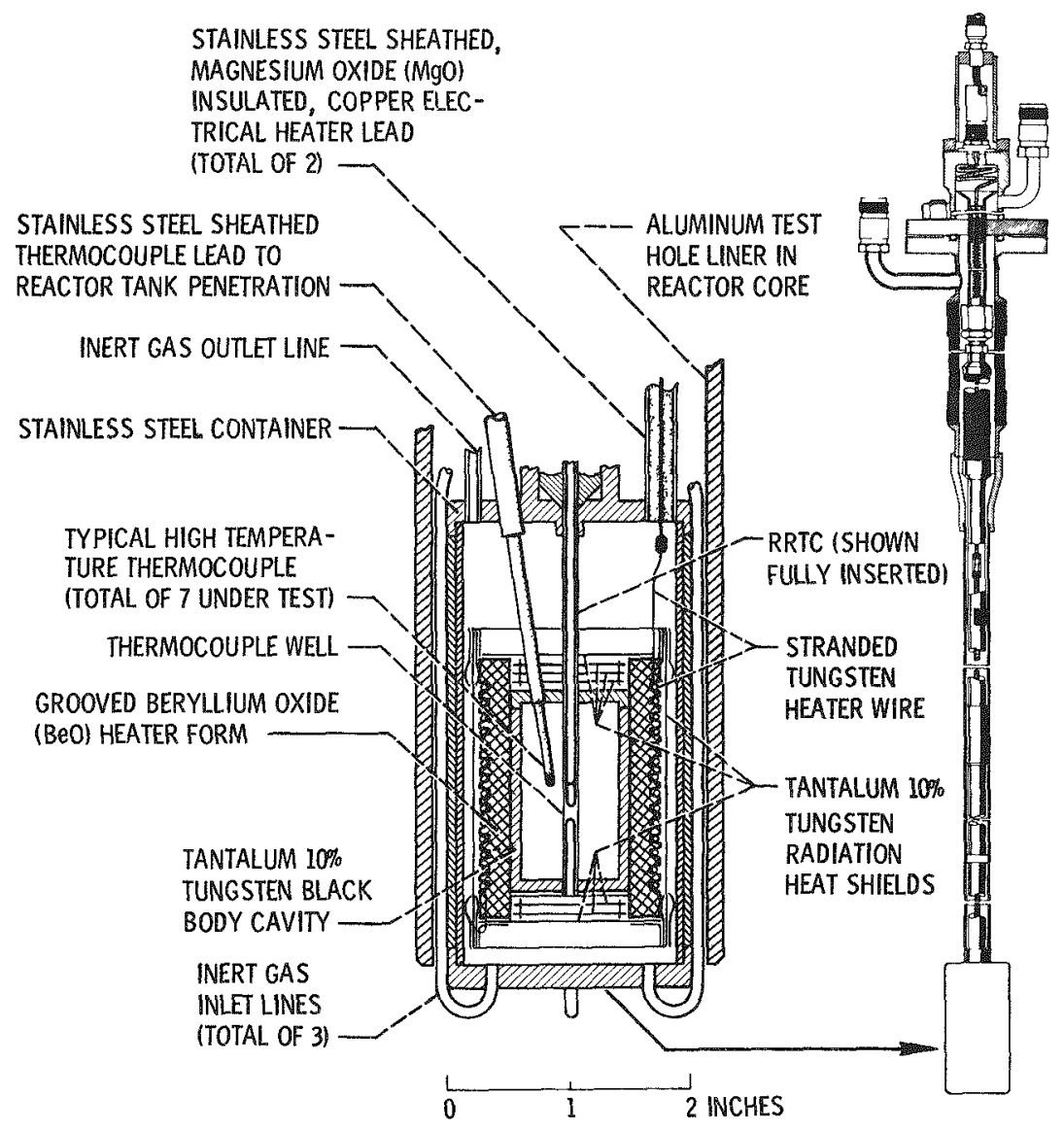

Figure 12. - Experiment capsule to irradiate high temperature thermocouples. (Experiment 68-05.) 
HFLOWING SODIUM CAPSULES IN THE GENERAL ELECTRIC TEST REACTOR

by G. W. Tunne 11 and D. L. Brown

330567 General Electric Company

Irradiation Processing Operation

Pleasanton, California

\subsection{INTRODUCTION}

This paper discusses the development of a facility to conduct fuel tests in a flowing liquid metal environment in a thermal test reactor. This facility allows fuel tests to be conducted at coolant velocities, powers, and temperature levels which represent operating conditions for both Liquid Metal Fast Breeder Reactors (LMFBR) and space nuclear power stations.

The experiment vehicle takes the form of a capsule in which concentric annuli provide a closed loop coolant flow path, hence the name "loopsule." The flexibility of the design allows particular experiments to meet specifications within wide ranges of power and temperature level, coolant flow rate, and neutron flux and spectrum. These loopsules, shown schematically in Figure 1, are suitable for a variety of tests, ranging from long exposures at a steady operating point to cyclic and transient experiments related to safety studies.

\subsection{CAPSULE DESCRIPTION}

Loopsule experiments exhibit a number of inherent advantages. The concentric annular flow circuit leads to a compact package which requires a minimum of reactor penetrations and is relatively easy to install and remove. This type of construction allows complete double encapsulation of both the liquid metal and the fuel, and lends itself to the use of an electromagnetic pump. With an electromagnetic pump, the windings may be kept entirely separate from the fluid-carrying pump duct, and the duct included inside the capsule's redundant containments. The necessary coupling between the stator and pumped fluid is supplied by the moving electromagnetic field. Electromagnetic pumps are available which give coolant velocities through the fuel test section which are representative of LMFBR's. A second characteristic of EM pumps, which is of considerable value, is their ability to operate continuously at any fraction of their rated output. This means that proper selection 
of a pump power supply allows complete control of the coolant flow rate without additional hardware. Loopsule performance is monitored with appropriate flow, temperature, pressure and nuclear instrumentation.

Capsule designs to date have focused on fuel specimens typical of those planned for LMFBR's and space applications. In these cases the pins have ranged from about $1 / 4$ to $1 / 2$ inch in diameter and from 1 to 3 feet in length. Loopsule experiments may be designed for single fuel pins or for multipin arrays. A cross section of a typical capsule is shown in Figure 2 .

Another attribute of the doubly-contained capsule type experiment is the ease with which a binary gas temperature control system may be implemented. The annulus between the two containment vessels is filled with a two-component flowing gas mixture which is coupled to a feedback control system to regulate capsule temperature level. Many factors combine to define the characteristics of a particular capsule control system, but experience shows that a control range of $\pm 300^{\circ} \mathrm{F}$ about a nominal temperature of $1000^{\circ} \mathrm{F}$ is easily attainable. Additionally, the complete feedback loop can be tuned to give a control band of $\pm 15^{\circ} \mathrm{F}$ about the same nominal temperature for steady experimental conditions. In some cases, such as transient or cyclic experiments, it may be desirable to partially disable the feedback control system and this can be accomplished without compromising the reactor safety standards.

The control system is set to answer unusual or unplanned circumstances with a fail-safe response and may employ a third gas to ensure the needed correction. For instance, a coolant high-temperature alarm can be set to trigger the control system for purge with a third high conductivity gas as well as initiating a reactor scram. Conversely, a reactor scram which is not related to the loopsule can trigger a purge with a third low-conductivity gas so that loopsule coolant remains molten.

\subsection{EXPERIMENT ENVIRONMENT}

\subsection{The General Electric Test Reactor (GETR)}

The GETR is a $50 \mathrm{MW}$ pressurized water reactor, the core of which is normally made up of 20 ETR type fuel assemblies. The pressure vessel is a 2 foot diameter aluminum tank which rests in a 9 foot diameter by 32 foot deep pool. The pool and core water systems circulate separately and provide cooling to the reactor and the various experiments.

Large liquid metal loopsules of the type considered here may be irradiated in either the GETR core or the pool as illus trated in Figure 1. This establishes two basic classes of experiments, because the experiment location determines the radiation environment, power level, heat rejection mode, pump size, and placement hardware. 


\subsection{Pool Experiments}

Experiments irradiated in the reactor pool can expect a flux of approximately $2.1 \times 10^{14} \mathrm{nv}$, of which about $58 \%$ is thermal and $42 \%$ is epithermal. Pool experiments are usually located in a movable facility which allows the capsule to follow the changing reactor flux profile and also allows the experiment power to be varied at will. Pool capsules are cooled by water at about $110^{\circ} \mathrm{F}$ and $25 \mathrm{psia}$ and may use either the forced or natural convection mode for heat rejection. The natural convection case is obviously the simplest, in which the capsule is submerged in a large, slowly circulating bath, but forced convection can be readily employed by the addition of a shroud around the capsule to which a constant water flow is supplied. The latter mode is more easily handled by the existing analytical tools, but the properly designed natural convection capsule is inherently safer because the loss of coolant accident need not be considered.

\subsection{In-Core Experiments}

Experiments placed in the reactor core can obtain an average flux of about $11.4 \times 10^{14} \mathrm{nv}$, of which $32 \%$ is therma $1,43 \%$ is epithermal, and $25 \%$ is fast ( $>.18 \mathrm{Mev}$ ). If the experiment contains a highly enriched fuel specimen, a significant flattening of the radial power profile can be achieved by screening out the thermal neutrons. The use of a flux filter can reduce the fraction of heat generated by thermal fission to 5 to $10 \%$ of the total fission heating. The axial peaking factor for both filtered and unfiltered experiments is about 1.7. Core capsules are cooled by the reactor primary water, which flows past the capsule at $120^{\circ} \mathrm{F}$ and 150 psia.

\subsection{CAPSULE THERMAL ANALYSIS}

The techniques used for the thermal analysis of liquid metal capsules have been developed within the Irradiation Processing Operation over a period of several years. The initial capsules used buoyancy-induced natural circulation of $\mathrm{NaK}$ as a heat transfer mechanism. The primary analytical method used for these capsules was a piece-wise continuous solution of the differential equations for the fluid channel temperature. The resulting algebraic equations for the fluid temperature were combined with the hydraulic description of capsule and programmed for digital computer solution as reported by Pfefferlen(1). This technique proved to be suitable for the natural circulation capsules. The addition of a pump to the concentric flow capsule system dramatically extended the range of test parameters available to the fuel experimenter, including the possibility for transient experiments. The out of pile experimental work of $\mathrm{R}, \mathrm{H}$. Leyse using this forced circulation loopsule concept demonstrated the potential of the system for reactor fuel testing.

The loopsule concept gives considerable flexibility for providing a range of experiment environments. The major parameters available to the experimenter and the experiment designer are: 
a. System heat generation rate

b. Sodium flow rate

c. Heat transfer area (loopsule diameter and length)

d. Gas annulus dimensions

e. Gas conductivity

f. Water side convective surface coefficients

g. Regenerative heat transfer, e.g., the rate of heat transfer to the down pass from the up pass as controlled by the diameter and length of the regenerative region.

System heat generation rates range from 20 to $70 \mathrm{KW}$ for single pin experiments, while for multipin experiments rates as great as $400 \mathrm{KW}$ are feasible. Sodium flow rates from 5 to 35 gallons per minute are available with existing pumps.

\subsection{Analytical Requirements}

The thermal analysis requirements for the loopsules fall into two general categories, design analysis and safety analysis. The two categories overlap somewhat but the distinction is useful. The design analysis includes the systems analysis required to size the system and select the operating parameters to obtain the desired performance for steady-state operation, for any planned transient operation, and shutdown and transfer conditions. This phase of the analysis provides the information for the operation of the system. The safety analysis predicts the performance of the system for abnormal and extreme operating conditions and provides the basis for evaluating the hazards associated with operating the experiment.

Two unique factors contribute to the complexity of predicting the performance of a loopsule. First, the short cycle time for the sodium (typically two seconds) and the regenerative heat transfer result in a strong thermal coupling of the system. Any change in the performance of the test section or the control system rapidly propagates throughout the experiment. This strong coupling requires the entire system to be modeled as a unit. Secondly, the thermal resistance of the gas annulus is a function of the system temperature and the gas composition. The thickness of the narrow gas annulus changes significantly with variations in the temperature of the sodium primary containment tube, and the gas composition is adjusted automatically to provide the system temperature control. While both of these factors complicate the prediction of the experiment performance, they result in a strong control system response and a negative feedback for temperature fluctuations. Any disturbance which tends to increase the temperature of the system is quickly followed by a decrease in the gas annulus thickness which improves the heat transfer from the loopsule, and provides a natural damping for a temperature transient. 


\subsection{Analytical Method}

The additional complexity of the forced convection loopsule, as compared to the natural circulation capsules, and the requirement of predicting transient temperature response dictated the need for a more general thermal analysis tool. The transient and steady-state heat transfer program THTD (Transient Heat Transfer - Version D) ${ }^{(2)}$ was chosen as a base for the analytical methods development project. The capabilities of the original program and familiarity with its predecessor THTB made it a logical starting point. The THTD program computes heat transfer by conduction, convection, diffuse grey body radiation, and energy transport by fluid flow. In addition, isothermal phase change is available to allow transient computations modeling fuel melting or solidification. The mathematical formulation used in the program is the conventional finite difference lumped parameter approach along with the first backward difference method for computing the transient thermal response. Additional features of the original, program include:

a. Arbitrary, three-dimensional geometrical input with admittances computed by the program from the geometry and thermal properties.

b. Temperature dependent material thermal properties.

c. Time and space dependent volumetric heat generation rates.

d. Time and space dependent surface heat flux input.

e. Interfacial thermal resistance between nodes.

f. Convective heat transfer coefficients for fluid nodes computed by several conventional techniques or input as functions of time.

g. Convective heat-transfer coefficients at system boundaries specified as functions of time.

The program has been extended to include the "at temperature" dimension of the binary gas gap and to compute the thermal properties of the binary gas mixture as a function of position and time. The gas annulus thickness is computed by the equation:

$$
\frac{\Delta x}{\Delta x_{0}}=\frac{R_{2}\left[1+\alpha_{2}\left(T_{2}-T_{\text {ref, }}\right)\right]-R_{1}\left[1+\alpha_{1}\left(T_{1}-T_{r e f, 1}\right)\right]}{R_{2}-R_{1}}
$$

where $\Delta x$ is the hot radial dimension of the gap

$\Delta x_{0}$ is the cold (reference) radial dimension of the gap

$R_{1}$ is the radius of the inner bounding surface

$R_{2}$ is the radius of the outer bounding surface 
$\propto$ is the mean coefficient of thermal expansion from $T_{\text {ref }}$ to the current centroid temperature, $T$, for the bounding node

The subscripts " 1 " and " 2 " refer to the inner and outer bounding surfaces or nodes, respectively. The coefficients for thermal expansion are entered as tabular functions of temperature.

The specific heat $C_{p m}$ and density $\rho_{m}$ for the binary mixture of gases are computed by the equations:

$$
C_{p m}=f_{1} C_{p 1}+\left(1-f_{1}\right) C_{p 2}
$$

and

$$
\mathrm{m}=\mathrm{f}_{1} \rho_{1}+\left(1-\mathrm{f}_{1}\right) \rho_{2}
$$

where subscript 1 and 2 refer to the first and second components respectively and $f$ is the mass fraction.

The mass fraction of the first component is computed from its mole fraction, $\mathrm{x}$, by

$$
\mathrm{f}_{1}=\frac{1}{1+\left[\frac{1-\mathrm{x}_{1}}{\mathrm{X}_{1}}\right] \frac{\mathrm{M}_{2}}{\mathrm{M}_{1}}}
$$

where $M$ is the molecular weight.

The thermal conductivity of the binary mixture, $k_{m}$, is computed from the following equation due to Wassiljewa, as given in Appendix 1 of Eckert and Drake:(3)

$$
\mathrm{k}_{\mathrm{m}}=\frac{\mathrm{k}_{1}}{1+\mathrm{A}_{1}\left[\frac{1-\mathrm{x}_{1}}{\mathrm{x}_{1}}\right]}+\frac{\mathrm{k}_{2}}{1+\mathrm{A}_{2}\left[\frac{\mathrm{x}_{1}}{1-\mathrm{x}_{1}}\right]}
$$

$A_{1}$ and $A_{2}$ are "Wassiljewa constants" defined by

$$
\begin{aligned}
& \mathrm{A}_{1}=\frac{1}{4}\left\{1+\left[\frac{M_{1}}{M_{2}}\left(\frac{\mathrm{M}_{2}}{\mathrm{M}_{1}}\right)^{3 / 4} \frac{1+\left(\mathrm{S}_{1} / \mathrm{T}\right)}{1+\left(\mathrm{S}_{2} / \mathrm{T}\right)}\right]^{1 / 2}\right\}^{2} \frac{1+\left(\mathrm{S}_{12} / \mathrm{T}\right)}{1+\left(\mathrm{S}_{1} / \mathrm{T}\right)} \\
& \mathrm{A}_{2}=\frac{1}{4}\left\{1+\left[\frac{M_{2}}{M_{1}}\left(\frac{\mathrm{M}_{1}}{\mathrm{M}_{2}}\right)^{3 / 4} \frac{1+\left(\mathrm{S}_{2} / \mathrm{T}\right)}{1+\left(\mathrm{S}_{1} / \mathrm{T}\right)}\right]^{1 / 2}\right\}^{2} \frac{1+\left(\mathrm{S}_{12} / \mathrm{T}\right)}{1+\left(\mathrm{S}_{2} / \mathrm{T}\right)}
\end{aligned}
$$


where $M$ is the dynamic viscosity

$S$ is the Sutherland constant

$\mathrm{S}_{12}=\sqrt{\mathrm{s}_{1} \mathrm{~S}_{2}}$ for ideal gases

$\mathrm{T}$ is the absolute temperature, ${ }^{\circ} \mathrm{K}$.

According to Eckert and Drake an approximate value of the Sutherland constant for the $i$-th pure component is given by

$$
\mathrm{s}_{i} \approx 1.5 \mathrm{~T}_{\mathrm{bi}}
$$

where $\mathrm{T}_{\mathrm{bi}}$ is the normal boiling point of the pure component.

The mole fraction of component 1 is specified as a tabular function of time as input to the program. In addition, a delay time may be specified as a function of position thus allowing the transient change in gas composition to be modeled.

The modified program has been used successfully to predict the performance of out of pile tests and the performance of the first in-pile test was predicted accurately. Figure 3 gives the temperature distribution computed for a typical in-core experiment.

\section{BIBLIOGRAPHY}

1. Knight, Pfefferlen, Leyse, "Design, Development and Operation of a NaK Filled Natural Convection Circulating Capsule," International Symposium on Developments in Irradiation Capsule Technology CONF-660511, May 1966.

2. S. C. Skirvin, "User's Manual for the THTD Computer Program (Transient Heat Transfer - Version D), P. O. No. 036-926052-T0602.

3. Eckert \& Drake, "Heat and Mass Transfer," McGraw-Hi11, 1959. 

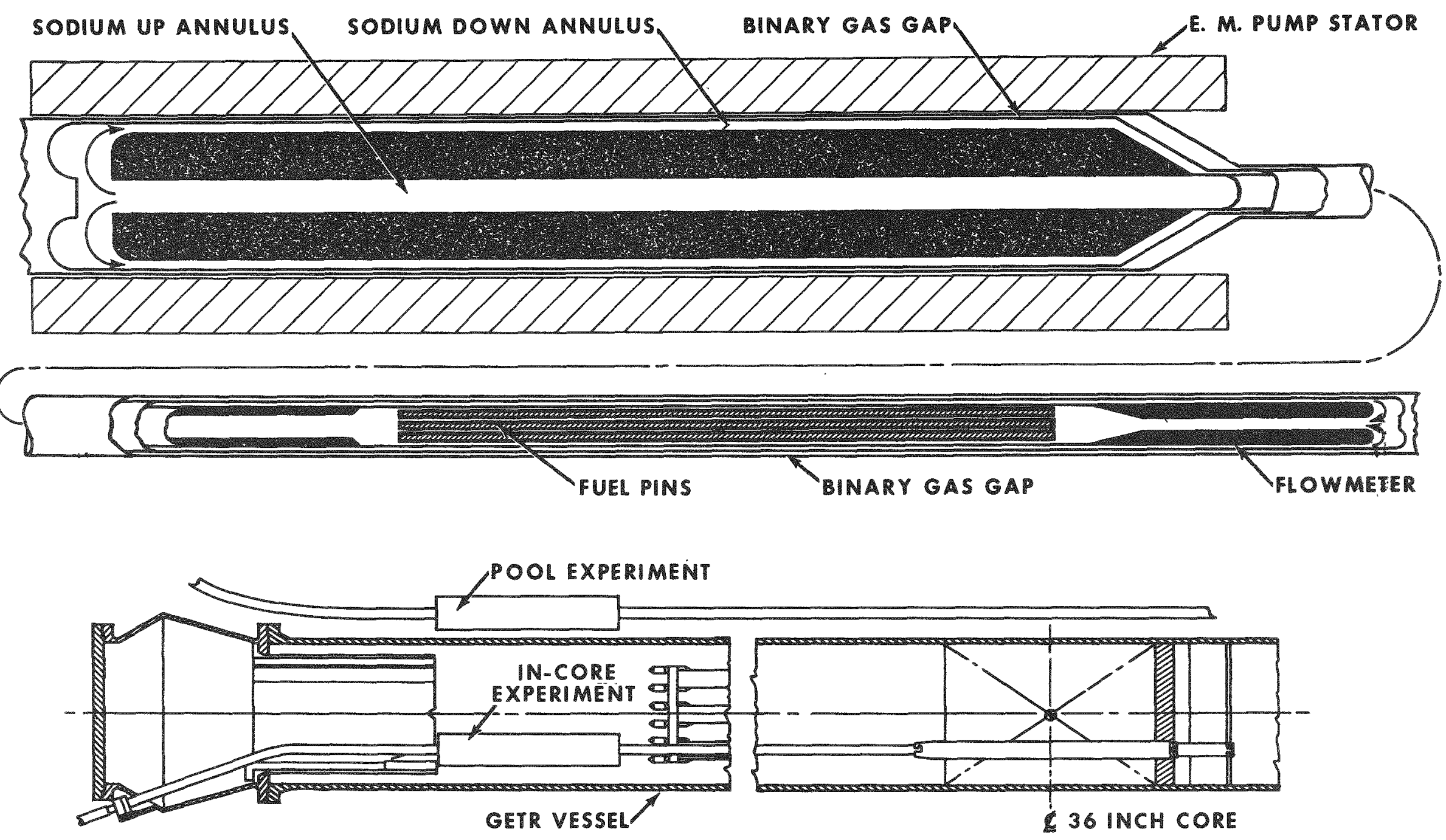

FIGURE 1. LIQUID METAL LOOPSULE AND THE GETR PRESSURE VESSEL 


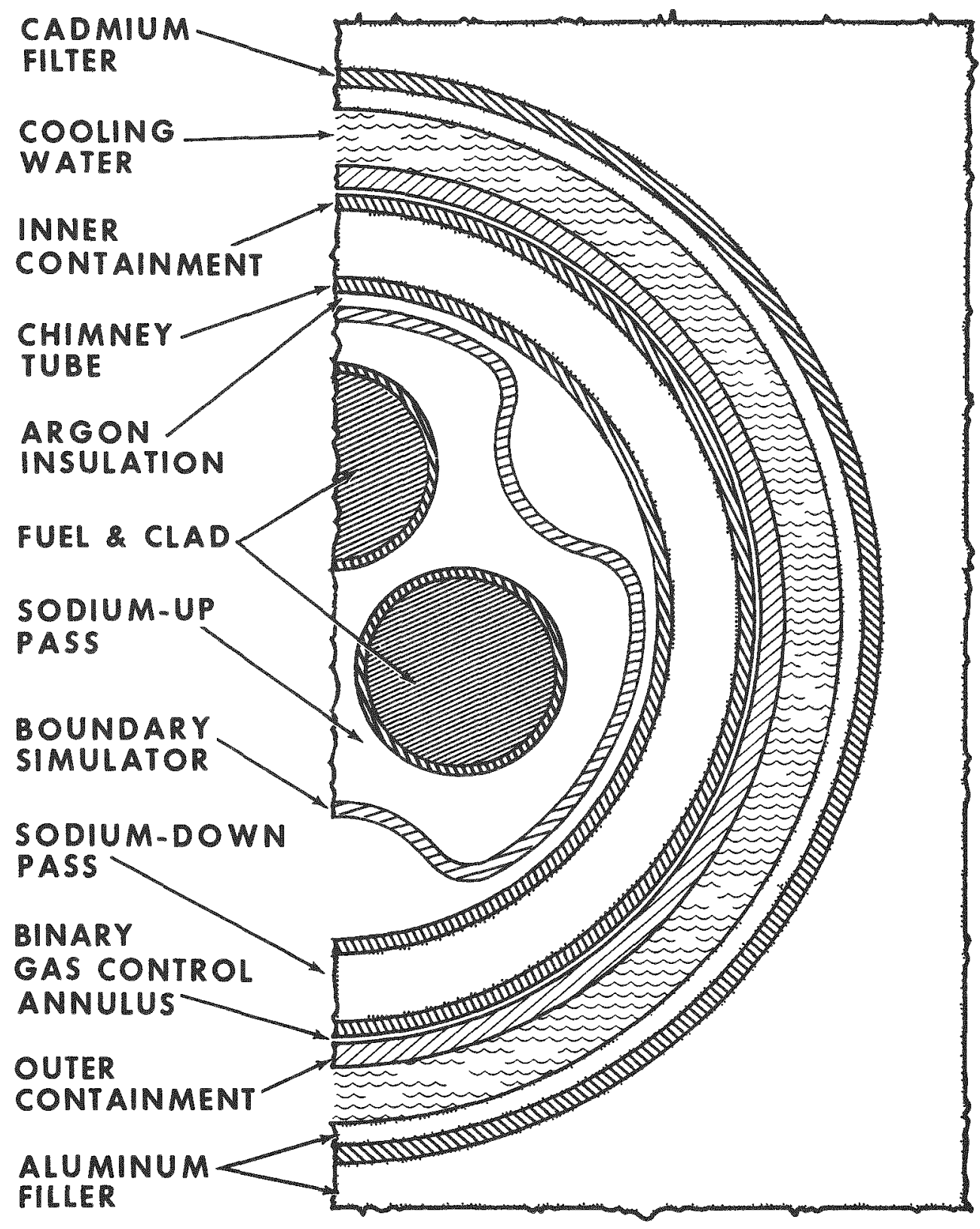

FIGURE 2. CROSS SECTION THROUGH A 3 PIN LOOPSULE 


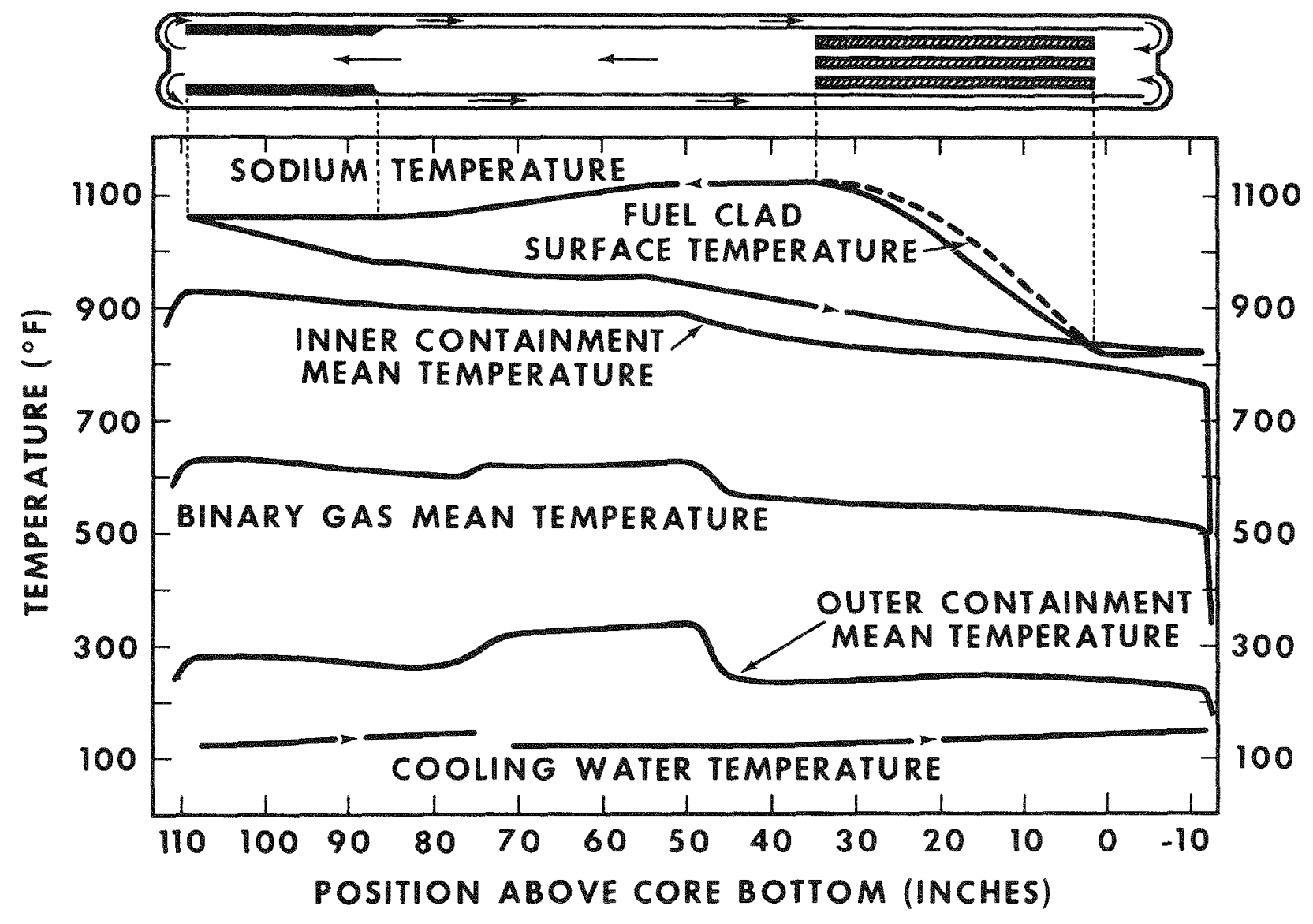

FIGURE 3. TYPICAL STEADY STATE TEMPERATURE DISTRIBUTION 


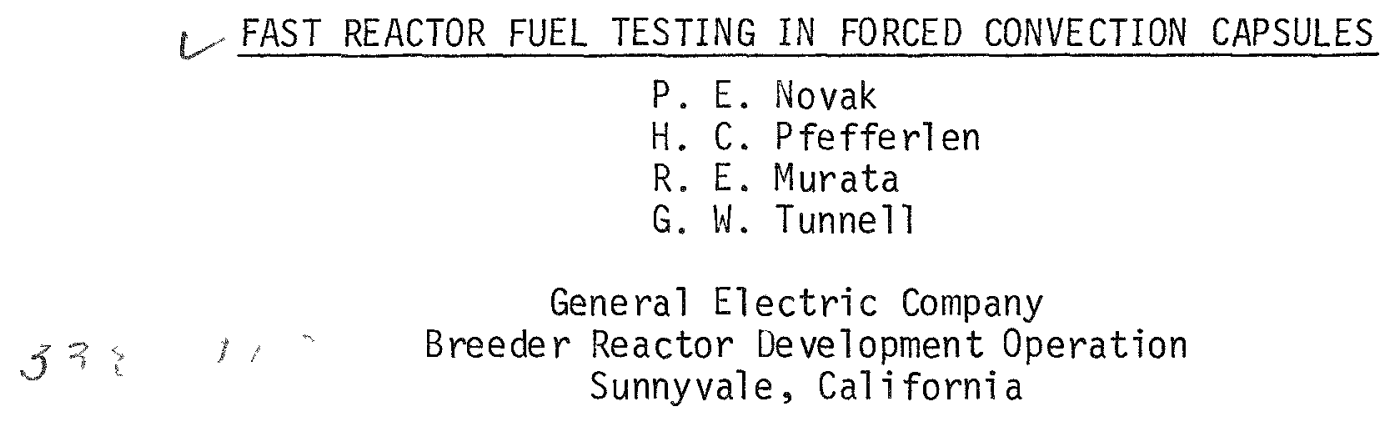

Accurate and timely information on the behavior of LMFBR fuel under casualty conditions is necessary to provide the answer to many pressing safety and design analys is questions for both demonstration plants and for test reactors. EBR-II currently has its own safety and operating limits that result in very 1 imited capabilities to test high risk pins, or continue operation of failed pins. A facility is needed which is capable of providing the required degree of containment along with a prototypic environment and the ability to control and measure the operating conditions. In-pile loops provide one means of achieving the above requirements. Unfortunately, in-pile sodium loops with external piping are quite expensive and must themselves be operated with such safety restrictions as to 1 imit their versatility for testing casualty conditions. Capsules, on the other hand, provide relatively low cost irradiation facilities which, by their expendable nature can accommodate casualty tests.

Capsules norma11y provide a static (non-flowing) sodium environment. Low velocity $\mathrm{NaK}$ flow has also been provided in natural circulation capsules (1). However, pumped flow is needed to produce higher velocities for realistic testing to and after failure. This permits good simulation of the effects of erosion, possible chemical reactions, and transport effects. A Forced Convection Capsule (FCC) has been developed

(1) "Design, Development, and Operation of a NaK Filled Natural Convection Circulating Capsule," F. W. Knight, H. C. Pfefferlen, H. C. Leyse, presented at the International Symposium on Capsule Irradiation Experiments, Pleasanton, California, May 3 - 5, 1966. (CONF 660511) 
and operated under sponsorship of the AEC Division of Reactor Technology (2). An accompanying paper discusses some features of the mechanical and thermal design of the FCC (3). It permits the use of extensive instrumentation and a range of temperatures and flow-rates of interest to reactor design.

This discussion deals with the operation of an initial test using a forced convection capsule (FCC). The objective of the test was to investigate the effect of stoichiometry on the swelling and/or erosion characteristics of defected mixed oxide fuel while operating at prototypic LMFBR conditions. In order to achieve this test objective, the following requirements had to be met:

1. Sodium velocity (14 fps)

2. Power and temperature typical of an operating LMFBR (18-20 Kw/ft, $1040^{\circ} \mathrm{F}$ outlet temperature)

3. Uniform operating conditions in each of two defect regions.

4. Sodium purity typical of that in an operating reactor.

5. Instrumentation to monitor flow rate and temperatures.

The details of how these requirements were met are discussed below.

The capsule test section contained three pins in a triangular array within a fluted liner. The cross-section was laid out to mockup a subassembly as shown in Figure 1. The three pins were each approximately one-foot long and spaced with spiders at three locations. Two of the pins were unfueled and had wire-wraps with a six-inch pitch. The purpose of the wire-wrap was to provide uniform spacing and to mix the coolant flow.

The active pin consisted of two axially-aligned five-inch-long sections of mixed oxide fuel of differing stoichiometries. The top section contained fuel with $1.990 / M$ and the bottom section had an $0 / M$ ratio of 1.95 . Except for this difference, both fuel pins were identical. The fuel pin fabrication parameters are shown in Table 1.

The defecting mechanism for both sections of the fuel pin cons is ted of three $1 / 32^{\prime \prime}$ diameter holes machined through the cladding and filled with a soluble braze. In addition, a groove $012 \mathrm{mils}$ deep was machined into the 15 mil thick cladding. The appearance of the defect region is shown in Figure 2. The braze material was braze alloy NWSA 680 whose major constituents are lead $(75 \%)$, zinc $(15 \%)$, and tin (10\%). The total impurity level introduced into the capsule sodium by the braze material was <20 ppm. The brazing alloy had a melting temperature of $514^{\circ} \mathrm{F}$ and was soluble in liquid sodium.

(2) Contract AT(04-3)-189, Project Agreement 10 between General Electric and USAEC.

(3) "Flowing Sodium Capsules in the G.E.T.R.," G. W. Tunnell and D. L. Brown, pres nted at the National Symposium on Developments in Irradiation Testing Technology, 9/9-11/69, Sandusky, Ohio. 
Relatively high coolant velocity through the test section $(\sim 14 \mathrm{ft} / \mathrm{sec})$ was provided by a helical induction pump. This pump type has a relatively high head at the flow rates of interest and thus permitted limiting the overall capsule sodium inventory to less than one $\mathrm{kg}$ to comply with safety requirements of the General Electric Test Reactor (GETR). Pump head - flow characteristics are shown in Figure 3.

Instrumentation consisted of thermocouples to monitor the inlet and exit bulk coolant temperature as well as a flowmeter to monitor the test section flow rate. The thermocouples were arranged to provide an accurate measure of the bulk coolant temperature. The electromagnetic flow meter was mounted at the inlet to the test section.

The bulk sodium temperature in the capsule was adjusted by varying the ratio of $\mathrm{N}_{2}$ to He in the gas filled annulus on the exterior of the capsule. This annutus also served as a second containment for this plutonium bearing experiment.

Controlled sodium chemistry was important to achieve the test objectives. Because of this, the sodium used to fill the FCC was first circulated and cold-trapped in an external "clean-up loop" until the desired oxygen level was obtained. The capsule was then filled with this high purity sodium and an integral cold trap provided in the capsule itself to maintain the desired purity level during operation (Figure 1). A gas "de-entrainer" was also provided to encourage gas bubble migration to the free surface since recirculating entrained gases released from the fuel after failure might disrupt the experiment.

The completed FCC was placed in a special facility in the GETR pool as shown in Figure 4 to permit power adjustment in the fuel test section during reactor operation. Adjustment was made by vertically and radially moving the capsule in the neutron flux gradient to maintain the desired power and power profile.

The operation of the experiment was designed to simulate conditions expected in a fast reactor. In addition, the experiment required the two fuel sections to operate at approximately the same power and temperature. The power level of 20-25 Kw/ft. was maintained at each defect by continuously positioning the fuel pin across the flux peak. The actual sodium temperatures were $951^{\circ} \mathrm{F}$ at the bottom defect and $993^{\circ} \mathrm{F}$ at the top defect.

In order to maintain the bulk sodium temperature constant, an automatic binary gas system was used. This system controlled the coolant outlet temperature to $1014 \pm 15^{\circ} \mathrm{F}$ by controlling the relative flow rates of helium and nitrogen through the binary gas annulus. Also, it provided the capability to raise or lower the bulk capsule temperature $2300^{\circ} \mathrm{F}$. Other pertinent capsule operating conditions are shown in Table 2.

The above systems were required to provide control and measurement of the fuel capsule environment. While none of these were particularly novel, the integration 
of the design and operation of these systems represented a major part of the total effort.

The experiment ran for one month in the GETR and included 25 full-power days and three power cycles. During the reactor shutdown periods, the sodium coolant was maintained molten by replacing the $\mathrm{N}_{2}$ and $\mathrm{He}$ in the binary gas annulus with xenon and operating the helical induction pump at full power. The pump heat input to the capsule under these conditions was sufficient to maintain the bulk sodium temperature at $450^{\circ} \mathrm{F}$.

After irradiation, the capsule was removed from the GETR pool and transferred to the radioactive materials laboratory for examination. In Figure 5 is shown the pre-irradiation and post-irradiation neutrographs. As can be seen, the high 0/M (1.99) fuel pin has expanded into the coolant stream. The lower $0 / M(1.95)$ fuel pin shows less apparent reaction. 

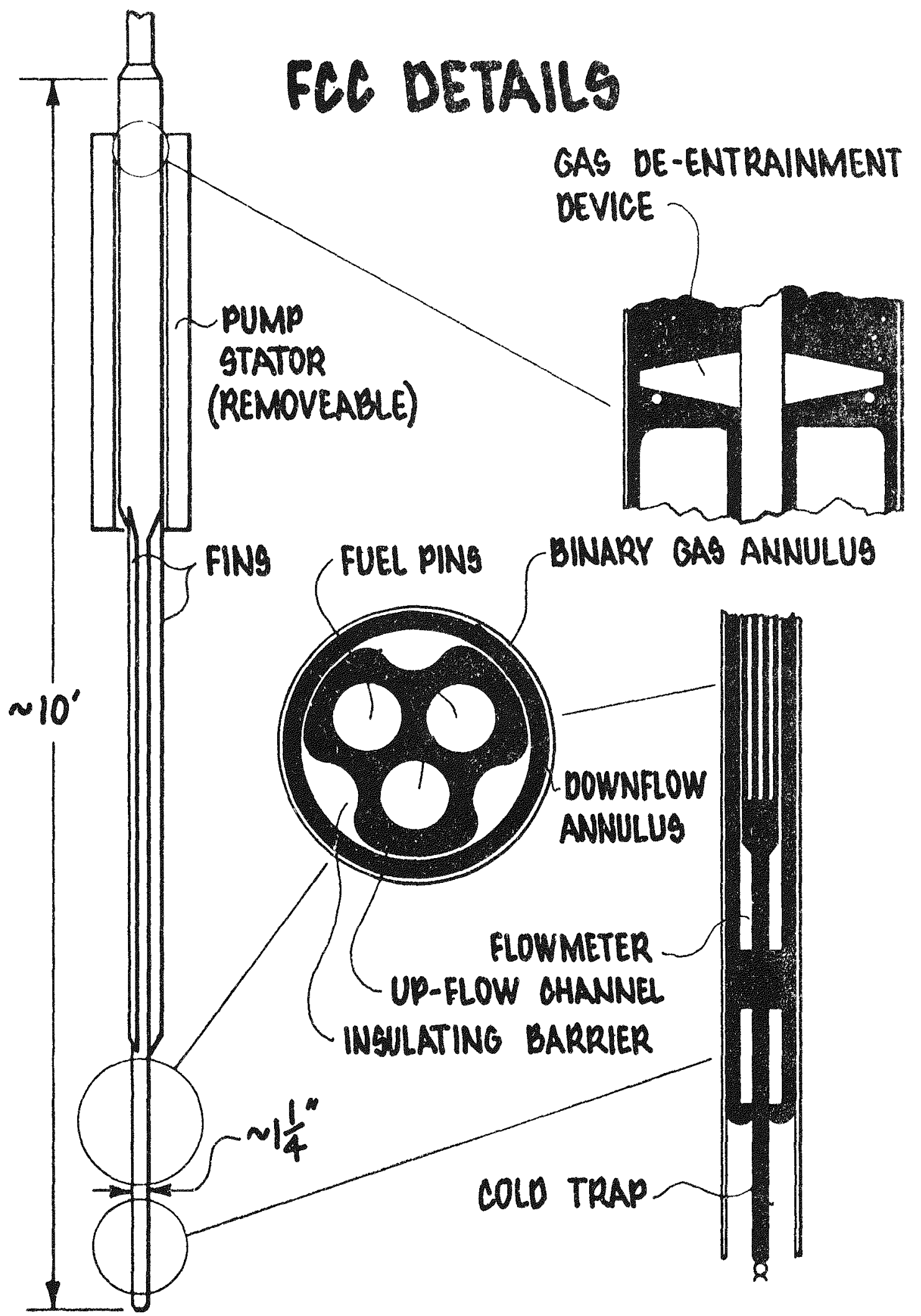

Figure 1 
TABLE 1

FORCED CONVECTION CAPSULE B9A

FABRICATION PARAMETERS

FUEL LENGTH

PELLET DIAMETER

PELLET DENSITY

SMEARED DENSITY

PU CONTENT

$U-235$

$0 / M$

DIA. GAP

CLAD O.D.

CLAD I.D.

FUEL RESTRAINT

BACK FILL ATM

DEFECT LOCATION

DESCRIPTION OF DEFECT $5^{\prime \prime} \pm 1 / 8^{\prime \prime}$

0.215

$94 \pm 2 \%$

$90 \%$

$25 \%$

$93 \%$

1.99

1.95

$$
\begin{aligned}
& 0.005^{\prime \prime} \pm 0.0025^{\prime \prime} \\
& 0.250 \\
& 0.220 \\
& \text { Axially Restrained } \\
& \text { Helium } \\
& C_{L} \text { of pin } \\
& \text { I" notch with } 31 / 32^{\prime \prime} \text { holes } \\
& \text { solder filled }
\end{aligned}
$$




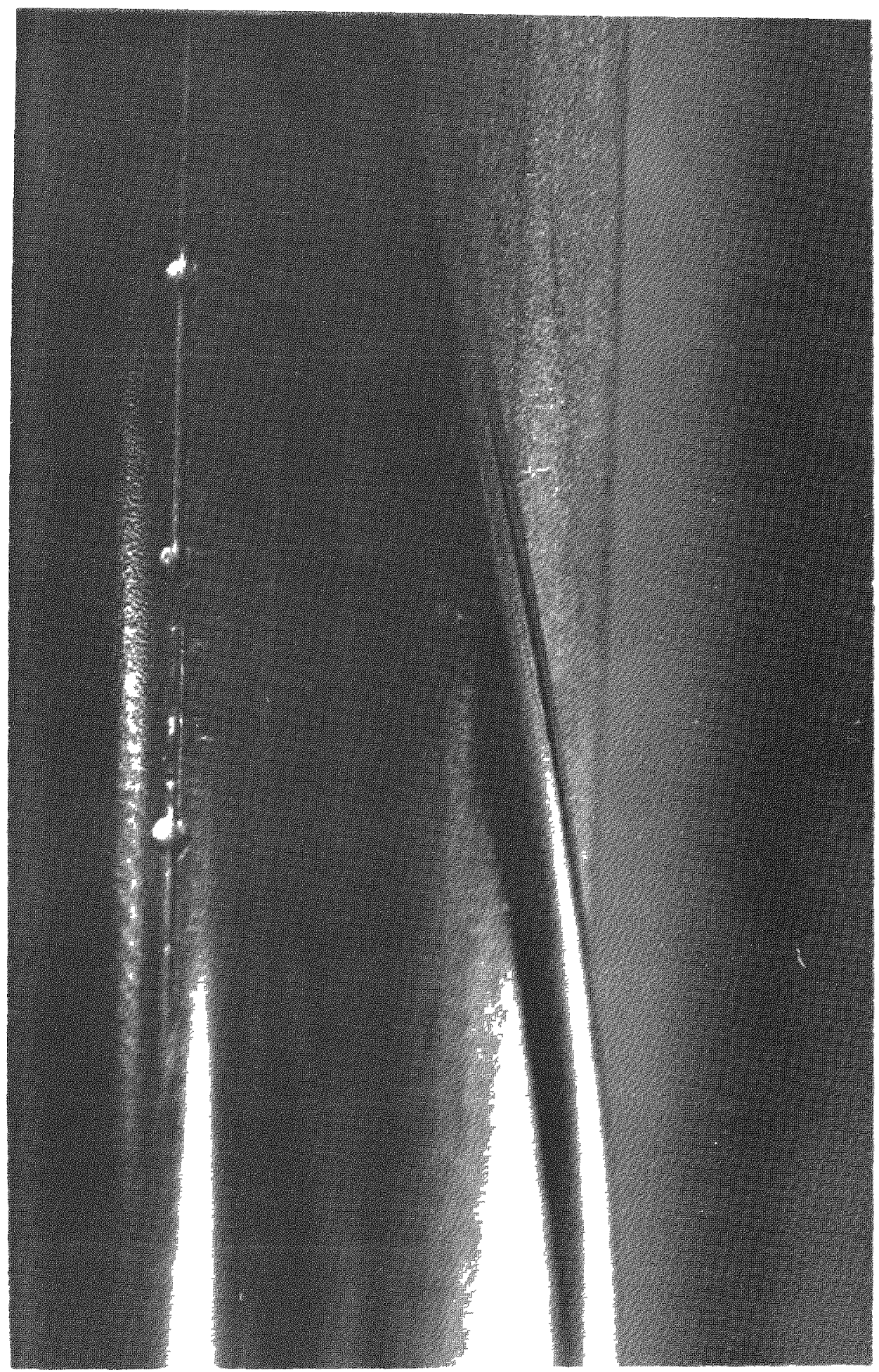

B9A-1 CLADDING DEFECT AREA B9A FABRICATION

Figure 2 


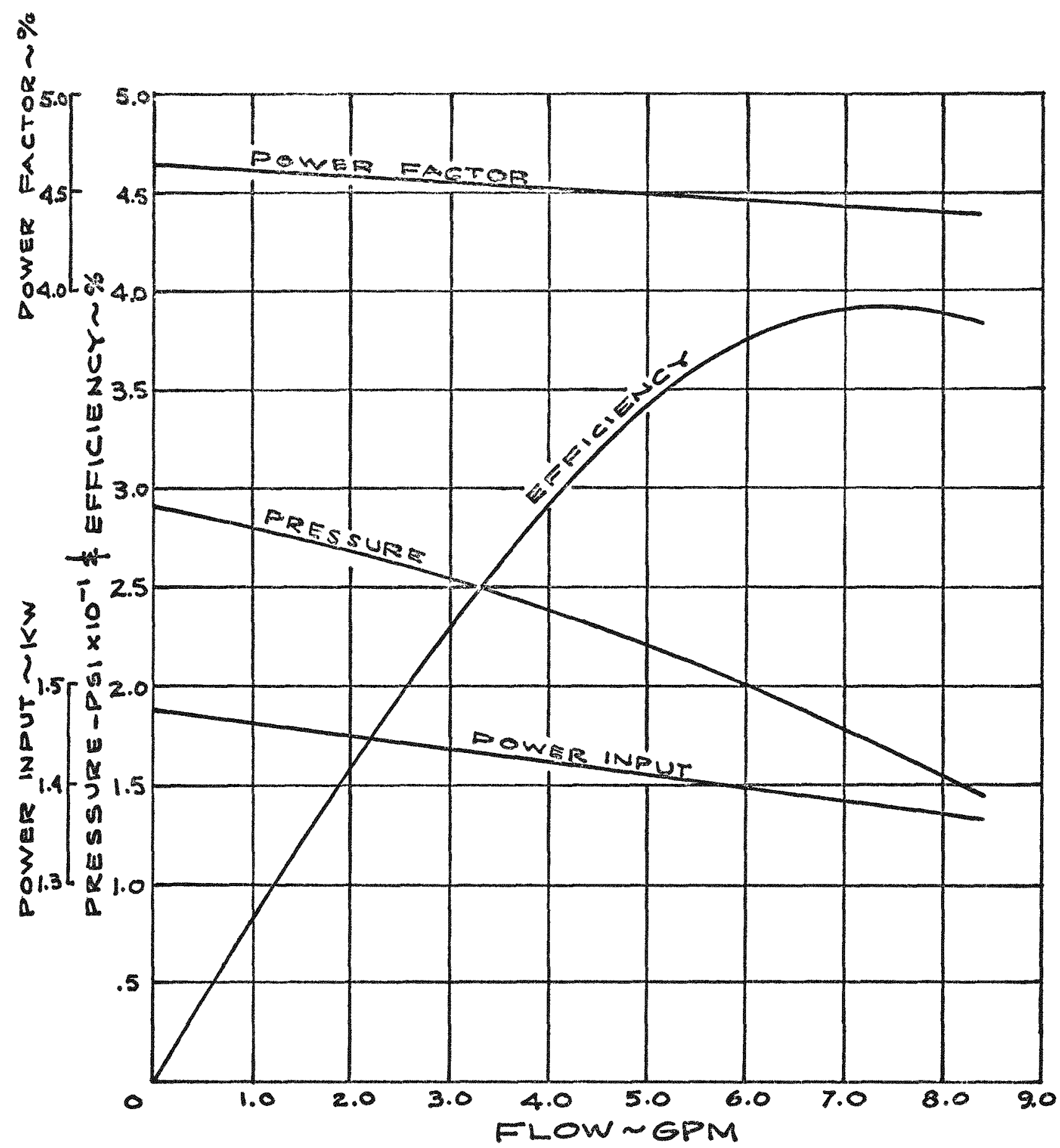

EM PUMP PERFORMANCE

FIGURE 3 


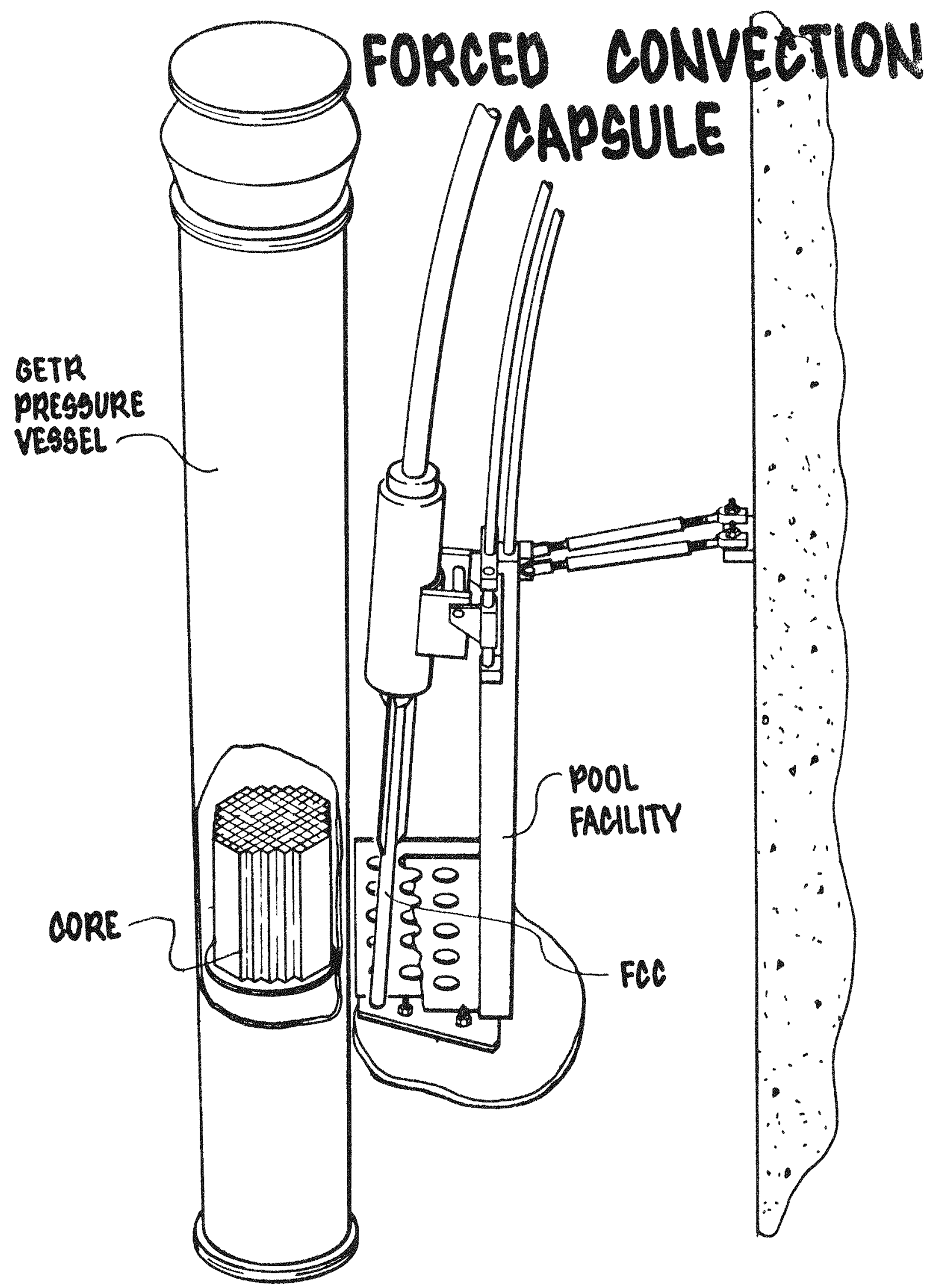

Figure 4 
TABLE 2

FORCED CONVECTION CAPSULE

SUMMARY OF OPERATION

POWER:

Fission

$22.5 \mathrm{Kw} / \mathrm{Ft}$.

Gamma*

$0.1 \mathrm{Kw} / \mathrm{Ft}$.

Total Fuel**

$22.6 \mathrm{KW} / \mathrm{Ft}$.

COOLANT: (Sodium)

Flowrate

$6.7 \mathrm{gpm}$

Inlet Temp.

$930^{\circ} \mathrm{F}$

outlet Temp.

$1014^{\circ} \mathrm{F}$

OPERATING DAYS:

25

NUMBER OF POWER CYCLES

3

TOTAL BURNUP**

$8200 \mathrm{MWD} / \mathrm{Te}$

* Gamma heating in ceramic fuel only.

** Based on analysis of capsule heat rejection to GETR Pool. 


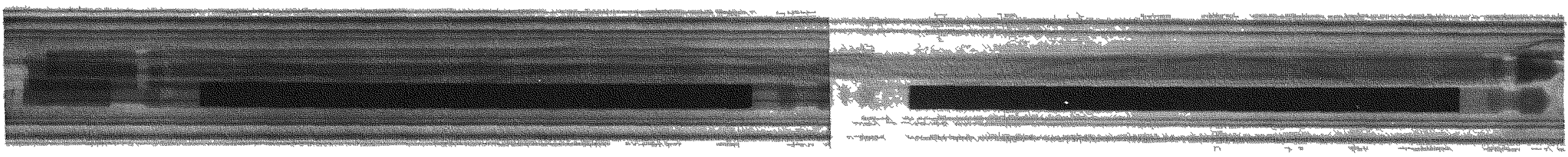

PREE-IRRADIATIOA NEUTROGRAPH

Defect area

Defect area.

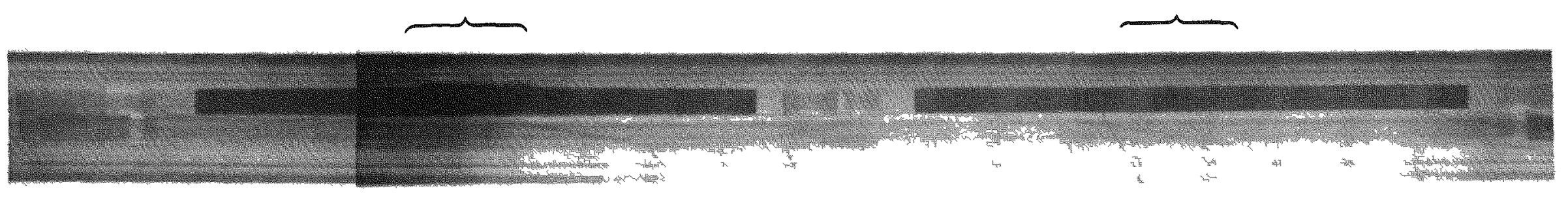

POST-ARRADIATION NEUTROGRAPI

\author{
FIGURE 5 PRE- AND POST-IRRADIATION
}




\section{DESIGN OF a LEAD-TYPE CAPSULE SYSTEM \\ TO INVESTIGATE THE RADIOLYSIS OF WATER \\ By Dean W. Sheibley \\ Lewis Research Center

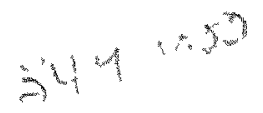 \\ ABSTRACT}

The capabilities and design requirements of the lead type capsule system are discussed. The system allows in-pile measurements of pressure, temperature, and neutron fluxes during reactor operation. The capsule system has been shown to withstand and contain a 15,000 psi reflected shock wave due to detonation of confined gases.

\section{SUMMARY}

A lead-type capsule system has been designed to investigate the radiolytic decomposition of water in aluminum capsules. The capsule system has the following proven capabilities. It permits:

1) Accurate delivery and return of small volumes of solution (5 $\mathrm{m} 1$ to $15 \mathrm{ml}$ ) to and from the in-pile capsule during reactor operation.

2) Introduction of pure gases or gas mixtures used as cover gases for experimental test runs.

3) Determination of fast and thermal neutron fluxes using cobalt and nickel dosimeter solutions.

4) Determination of the change in gas composition within the enclosed capsule system by gas chromatography.

5) Performing many experimental tests during a reactor cycle.

6) Continuous measurement of system pressure changes during in-pile tests.

7) Continuous monitoring of capsule wal1 temperature and sample solution temperature in the capsule.

8) Successful containment in the in-tank leads and capsule of an internal pressure shock wave of 15,000 psi resulting from detonation of confined gases.

The capsule system has provided valid test data on more than 100 test irradiations during more than 2500 hours of in-pile operation. 


\section{INTRODUCTION}

This report discusses a proven design of a lead type aluminum capsule which allows a quantitative in-pile study of radiolysis of water. The design of the hardware for this particulax experiment was predicated on two criteria: 1) A predetermined two-level factorial test program, and 2) the requirement of simplicity and flexibility of operation. Primary design emphasis was placed on minimizing system volume and on accurate delivery and recovery of test solutions.

Several types of lead tube-thermocouple capsule systems for monitoring pressure and temperature have been reported (ref. 1,2). These systems were used in empirical studies on the radiolysis of water.

The purpose of the experiment for which this capsule system was designed is to determine ways of inhibiting the pressure buildup due to the radiolysis of water in aluminum containers. The object of the investigation was to identify quantitatively the effects of gaseous, solid, and soluble inhibitors and to obtain information of the interaction effects of the variables selected for study. A two-level factorial experiment program design was used because of the large number of variables and the requirement to obtain interaction effects. The statistical considerations and unique problems associated with producing the factorial design for this experiment are discussed in reference 3.

The experiment uses an aluminum capsule containing three lead tubes and two thermocouples. The volume of the dry capsule is $27 \mathrm{ml}$. The entire in-pile, out-of-pile closed system volume is $90 \mathrm{ml}$.

This report discusses the design requirements and proven capabilities of the capsule system. The testing of the capsule system to contain a 15,000 psi shock wave without sustaining damage is also discussed.

\section{DESCRIPTION OF EXPERIMENT SYSTEM}

This lead type capsule simulates small experiment capsules used at the Plum Brook Reactor. Some of these small capsules contain water as a necessary environment during irradiation. The lead type capsule designed for this experiment physically resembles these small capsules. The lead-type capsule has a $2.57 \mathrm{~cm}$ ( 1.01 inches) internal diameter and a volume of $27 \mathrm{~m} 1$. It is fabricated of $6061-\mathrm{T} 6$ aluminum. The wal1 thickness is $0.79 \mathrm{~cm}\left(5 / 16^{\prime \prime}\right)$. Figure 1 shows the capsule mounted in a reactor test hole adaptor.

The capsule system contains two chromel alumel thermocouples. Both are stainless steel sheathed, mineral insulated and grounded. One thermocouple is imbedded in the capsule wall (see figure 1). The other senses the liquid temperature inside the capsule. Both temperatures are continuously recorded. The tip of the interior thermocouple is completely immersed during test runs. 
Three 304 stainless steel lead tubes are connected to the capsule. The lead tubes are approximately 15 meters $(48.7 \mathrm{ft})$ long and $0.1 \mathrm{~cm}(.040 \mathrm{in})$ inside diameter. One lead tube leaves the capsule at the top, one at the bottom, and one at the side above the solution level. This lead tube arrangement permits easy filling and emptying of the capsule. Also, the three lead tube arrangement assures that the water goes to the capsule and remains there during irradiation. The leads from the top and side are used to circulate the gases produced during irradiation for analysis by gas chromatography.

The three lead tubes and two thermocouples run from the capsule assembly in the reflector section of the reactor core to a flange in the dome of the reactor tank. This arrangement is shown in figure 2. At the flange, out of tank leads run approximately four meters (13 feet) to an experiment console.

The in-pile, out-of-pile test loop volume is approximately $90 \mathrm{ml}$. This is represented in figure 3 by the heavily inked portions of the schematic. Other features of the design such as valves, pump, and instrumentation are shown in figure 3 .

\section{Capsule. System Design Requirements}

The experiment test program to be performed with the capsule system required flexibility in handling and simplicity of operation of the system hardware, since many test runs are performed each reactor cycle, and capsule assemblies are sometimes changed between reactor cycles.

The capsule system design requires that the capsule assembly, that is, capsule plus adaptors, fit into any of the $6.35 \mathrm{~cm}(2-1 / 2 \mathrm{inch})$ I.D. test holes of the reflector section of the reactor. The stationary position of the capsule with respect to the core centerline is determined by the length of the adaptors.

The capsule is cooled by the primary water flow through the reactor core. The strength of the capsule necessary to withstand and contain a detonation pressure wave requires that an internal capsule temperature of $422^{\circ} \mathrm{K}\left(300^{\circ} \mathrm{F}\right)$ not be exceeded. The internal temperature of the capsule has not exceeded $350^{\circ} \mathrm{K}\left(170^{\circ} \mathrm{F}\right)$ in the nuclear environment described below:

NUCLEAR ENVIRONMENT OF RA-2 POSITION $+12 "$ ABOVE CORE CENTERLINE (MAXIMUM VALUES)

Gamma Heating

Fast Flux $(>0.1 \mathrm{Mev})$

Thermal Flux
3 to 4 watts/gram in water $4.3 \times 10^{13}$ neutrons $/ \mathrm{cm}^{2}-\mathrm{sec}$ $2.1 \times 10^{14}$ neutrons $/ \mathrm{cm}^{2}-\mathrm{sec}$

The data analysis requires that the system pressure during irradiation tests be continuously monitored. As a result, the closed loop capsule system $(90 \mathrm{ml})$ shown in figure 3 contains a pressure transducer to 
monitor pressure changes in the system during in-pile testing. The accuracy of the pressure transducer and hence pressure measurements, is $\pm 0.5 \%$ over the range 0 to $600 \mathrm{psig}$. The system a1so contains an attenuator circuit which permits pressure monitoring in the range of 0 to 60 psig with an accuracy of $\pm 5 \%$.

The factorial test program design specifies mixtures of hydrogen, oxygen, nitrogen, and helium gases which are flammable, and in some cases, detonable. As a result, the capsule engineexing design incorporates the capability of withstanding without damage a 15,000 psi reflected shock wave which could result from a gas mixture detonation. Prototype capsules with lead tubes attached were hydraulically tested + 15,000 psi. For this application the hydraulic testing was more severe than pressure shock testing due to the duration of high pressure.

To establish the operating pressure of gases in the capsule which would not exceed this 15,000 psi limit upon detonation, a series of detonation pressure tests were performed on a capsule and lead tube assembly. The tests were performed by Aeronautical \& Astronautical Engineering Department of Ohio State University. They performed these tests using mixtures of 2 volume parts hydrogen to one volume part oxygen (stoichiometric mixture) at initial pressures up to 600 psia. Similar tests were performed with the stoichiometric mixture diluted with helium. As a result of these tests an upper operating pressure limit of 600 psig was established.

A complete discussion of the detonation hazard and the detonation test results may be found in Appendices $C$ and $D$ of reference 4.

The capsule system closed test 1oop and the hold tank shown in figure 3 are designed incorporating safety systems which alarm at 600 psig and relieve at 700 psig.

The leak test requirement on the $90 \mathrm{ml}$ closed test loop is that no more than $1 / 2$ psi pressure loss occur during a minimum 4-hour test at 300 psig.

\section{System Capabilities}

The capsule system permits an accurate delivery and return of smal1 volumes of solution. The solution volumes used for the test program are 5, 10, and $15 \mathrm{ml}$ samples. Precision of solution delivery to the capsule is given below.

Volume Injected

$5 \mathrm{mI}$

$10 \mathrm{~m} 1$

$15 \mathrm{~m} 1$
Volume Delivered to Capsule (Std. Dev.)

$4.9 \mathrm{~m} 1 \pm 0.2 \mathrm{~m} 1$

$9.8 \mathrm{ml} \pm 0.2 \mathrm{ml}$

$15 \mathrm{ml} \pm 0.2 \mathrm{~m} 1$ 
The precision of removal of water from the capsule and recovery was determined for $15 \mathrm{ml}$ samples on $1 \mathrm{y}$. The result with the standard deviation based on six samples is: $14.6 \pm 0.6 \mathrm{ml}$. To achieve this capability the lead line tubing design minimizes the system volume and the use of tees and valves to and from the capsule.

The capsule system also permits the introduction of pure gases or gaseous mixtures which are used as cover gases for each experiment test run. Mixtures of helium, hydrogen, oxygen, and nitrogen at an initial pressure of 40 psia are used. The system is purged with the test gas mixture. The system design permits the water sample in the fill tank to be exposed to the gas mixture to achieve a gas phase-liquid phase equilibrium before the water is delivered to the capsule.

The thermal and fast neutron fluxes are determined using a liquid dosimeter technique. The dosimeters used are a cobalt solution and a nickel solution. These standard dosimeters were tested and found not to react with the interior of the capsule.

The system allows the change in the gas composition to be determined at the end of an irradiation test. This is done by incorporating a one milliliter gas sample loop of a gas chromatograph into the system.

The $90 \mathrm{ml}$ closed loop of the system is mixed using the bellows pump shown in figure 3. During the gas mixing process, the expansion tank is purged with helium gas and evacuated. The closed $90 \mathrm{ml}$ sample loop is sampled by expanding the tee volume between valves MV $-3, M V-4$ and $M V-5$ ( $T$ volume is $2.16 \pm 0.03 \mathrm{ml}$ ) into the expansion tank. Several tee volumes are expandē for each test run to obtain precise gas analysis data. The expansion tank is then pressurized with helium. After a delay to allow the gases to mix by diffusion, the entire expansion tank volume is purged through the gas chromatograph sample loop. The accuracy of this method of gas analyses is reasonably good. The analyses for hydrogen are typica11y $7 \%$ low; oxygen $\sim 11 \%$ low, and nitrogen $\sim 3 \%$ low.

\section{CONCLUDING REMARKS}

The design of the experiment system hardware was predicated on a factorial test program. The final assembled system was simple and flexible in operation. The primary design goals of minimum system volume and accurate delivery and recovery of test solutions have been achieved. The overall result of these efforts is an in-pile experiment which has operated satisfactorily for more than 2500 hours of in-pile testing and has provided valid test data for over 100 test irradiations. 


\section{REFERENCES}

1. Humphreys, J. R.; Abers, E. K.; and Solomon, Y.: Decomposition and Recombination in Irradiated Static Water Systems at High Temperatures. Rep. AllL-5004, Argonne National Lab., Mar. 1953.

2. Bebesel, P.; and Purica, I.: Separation of the Effect of Fast Neutrons and Gamma Radiation on the Radiolysis of Water. Acad. Rep. Populare Romine, Studii Cercetari Fiz. (Bucharest), vol. 12, 1961, pp. 557-562.

3. Holms, Arthur G.; and Sidik, Steven $M_{0}$ : Design of Experiments as "Doubly Telescoping" Sequences of Blocks with Application to a Nuclear Reactor Experiment. NASA TN D-5369, 1969.

4. Design Manual and Hazards Analysis for Experiment 67-04; Radiolysis of Water in Sealed Containers. Feb. 20, 1969. Unpublished manual. Available from Project Engineering Office of NASA Plum Brook Reactor, Sandusky, Ohio. 


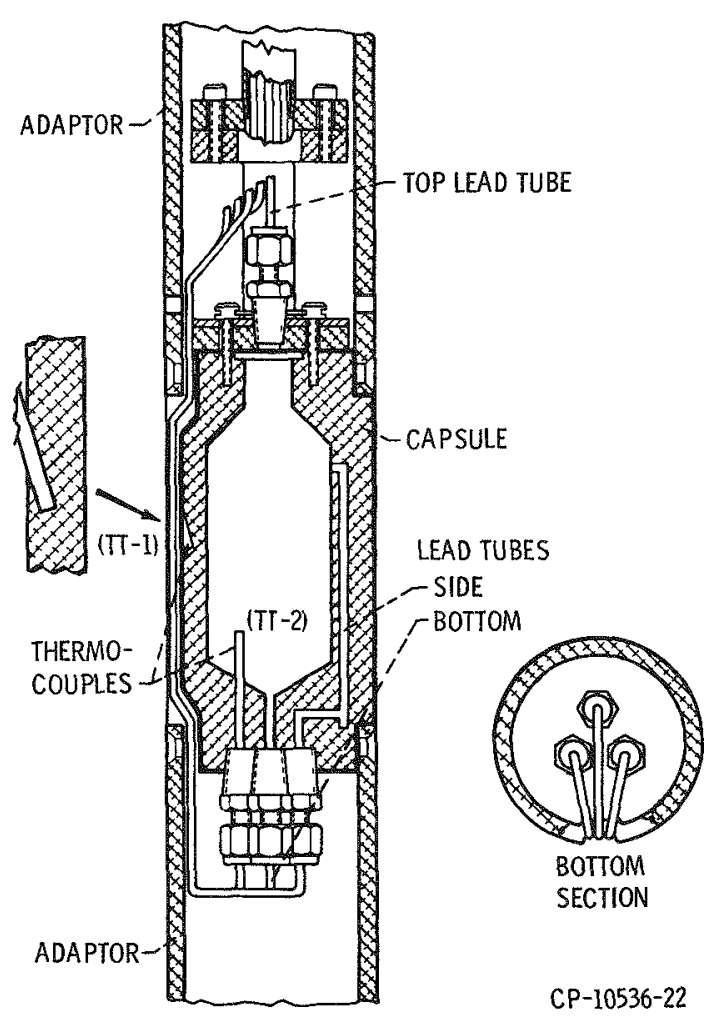

Figure 1. - Experiment 67-04 capsule and adaptors assembly.

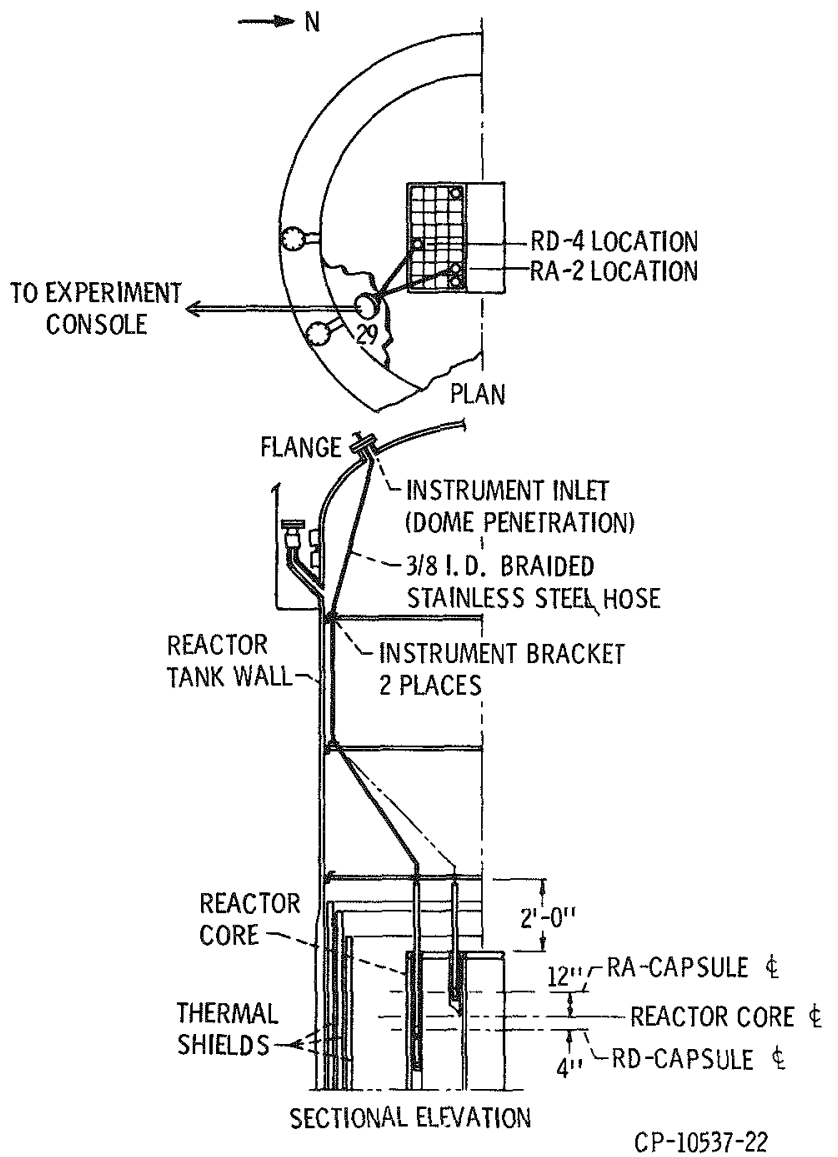

Figure 2. - Sectional elevation and location of 67-04 capsules in Plum Brook Reactor. 


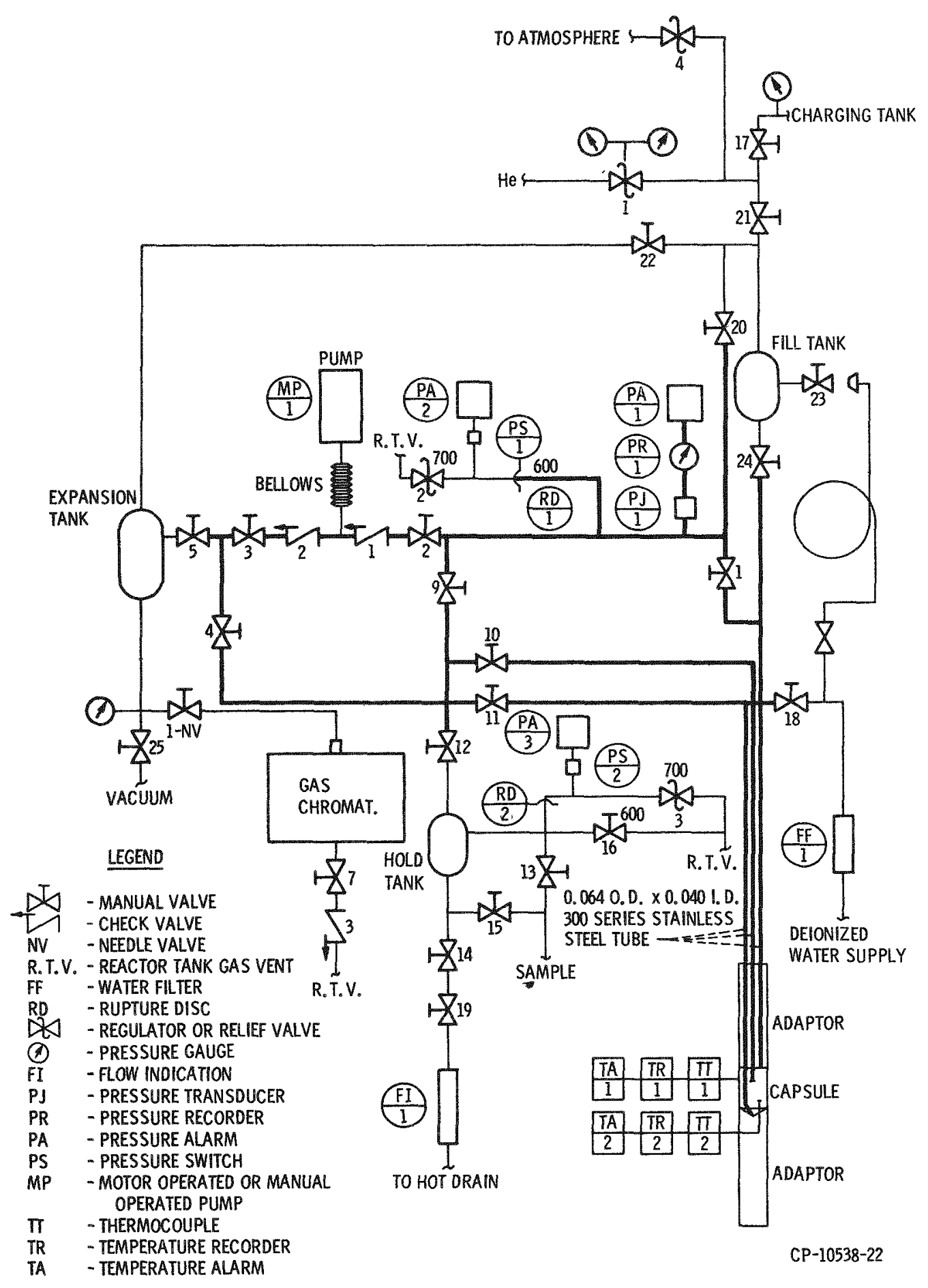

Figure 3. - System schematic, experiment 67-04. 


\section{f A "DOUBLY TELESCOPING" DESIGN OF A NUCLEAR REACTOR EXPERIMENT CONTAINING EIGHT VARIABLES}

By Dean W. Sheibley, Arthur G. Holms, Steve M. Sidik

$$
5490300 \text { Lewis Research Center }
$$

\section{ABSTRACT}

A blocked two-level factorial experiment was designed to measure the pressure buildup due to the radiolysis of water. Effects of controlled variables are influenced by changes in radiation environment with time and by equipment changes between groups of reactor cycles; the experiment is therefore doubly confounded. Experimenting is subject to unplanned curtailments with respect to the first influence, and might be expanded with respect to the second. So that the more important parameters can be estimated free of biases ordinarily resulting from the indefinite size of the experiment, the rationale is given for arranging the blocks of the experiment in a "doubly telescoping" (orthogona11y blocked) sequence.

\section{SUMMARY}

An experiment was conceived to investigate the pressure rise due to radiolysis of water in aluminum capsules. The design of the test program produced an experiment design technique termed "double telescoping".

Simple factorial experiment design techniques were not adequate. Seven variables were to be studied at two levels, one variable at eight levels.

The experiment design required flexibility in the number of tests to be performed during a cycle, and the number of reactor cycles. This 
flexibility was needed to accommodate unplanned reactor shutdowns, and/or additional tests at a later time.

For the variables chosen to define the experiment nuclear environment, certain unavoidable changes existed. The capsule was stationary in the reactor core. And as the reactor control rods were withdrawn during a cycle, changes in radiation component levels occurred. This produced one source of block effects.

A second source of block effects could exist because reactor or experiment equipment changes might be introduced between groups of reactor cycles.

Therefore, since the experiment contained two sources of block effects, the design is doubly confounded. The double telescoping technique allows the effects of the independent variables and their more important two- and three-factor interactions to be estimated free of block biases which would ordinarily result from an indefinite test program.

\section{INTRODUCTION}

The purpose of this report is to discuss the application of experimental design techniques to a particular nuclear reactor experiment. We hope that this discussion of design techniques for an irradiation experiment containing a large number of variables will prompt others to investigate and use this method of planning experiments wherever possible.

The particular problem needing experiment investigation was the pressure rise due to the radiolysis of water in an aluminum capsule in the NASA Plum Brook Reactor. The main effects of seven controlled variables and their more important two- and three-factor interactions were to be investigated.

The classical method of testing where one factor is tested at several levels while all others are held constant was inadequate for several reasons; primarily, data on interaction effects were desired. The factorial method of testing was used since it possesses the advantages of: 1) More efficiency than one-factor-at-a-time experiments; 2) all data are used in computing effects of the variables; and 3 ) 
information is obtained on possible interactions between independent variables. Webb (ref. 1) implies greater efficiency for factorial methods in the two criteria he proposes for orthogonal full factorial designs: a) Minimization of the volume of a confidence region of the estimated parameters, and b) minimization of the average variance of the predicted values. Other desirable statistical properties of these types of designs have also been discussed by Box and Hunter (ref. 2).

Parts of an experiment are sometimes performed in a sequence over differing time segments, over differing batches of raw material, or over differing pieces of equipment. The experimental units within the part are assumed to be relatively uniform within the part and such a part, or the set of treatments assigned to it, is called a block. The conditions that differ from one part to the next are assumed to affect the response by amounts that are not readily predicted or controlled. The responses to extraneous changes between the parts of the experiment are called block effects.

The novelty of the design of the present experiment lies in its treatment of two sources of block effects. The first source is created by the varying rod bank height. It has been assumed to exist at eight levels even though it is varied more or less continuously during a cycle. This variable is regarded as a block effect within the reactor cycles.

A second source of block effects can exist because reactor or experiment equipment changes might be introduced between groups of reactor cycles. Therefore, since the experiment contains two sources of block effects, the design is doubly confounded (ref. 3).

The experiment design allows flexibility in 1 ) the number of tests to be performed during a cycle, and 2) the number of cycles.

Because unplanned reactor scrams occur occasionally, cycles may be stopped short of the intended scheduled time, resulting in missing data. Or if the experimenter wishes to increase the scope of estimated interactions, the experiment program should be expanded to inçlude additional test runs at a later date, under new combinations of the controlled variables.

Thus, a strategy of experimenting is needed where curtailments of the experiment with respect to one source of block effects and expansion of the experiment with respect to the other possible source can be 
accepted without the estimates of the effects of independent variables being biased. A conventionally balanced experiment could introduce severe bias into results if the size of the experiment, as actually performed, did not conform to the original plan.

A telescoping design uses estimates of independent variable effects from the first block of data, retains these estimates, and combines them with estimates from additional blocks so that all estimates acquired are used cumulatively. An early discussion of this concept of single telescoping was given by Danie1 (ref. 4). Because the size of this experiment needs to be flexible with respect to the two sources of block effects, the experiment must be designed against such biases. A design which gives protection against double confounding is said to be "double telescoping". Designing for double telescoping with eight independent variables has been described by Holm and Sidik (ref. 5).

\section{SYMBOLS}

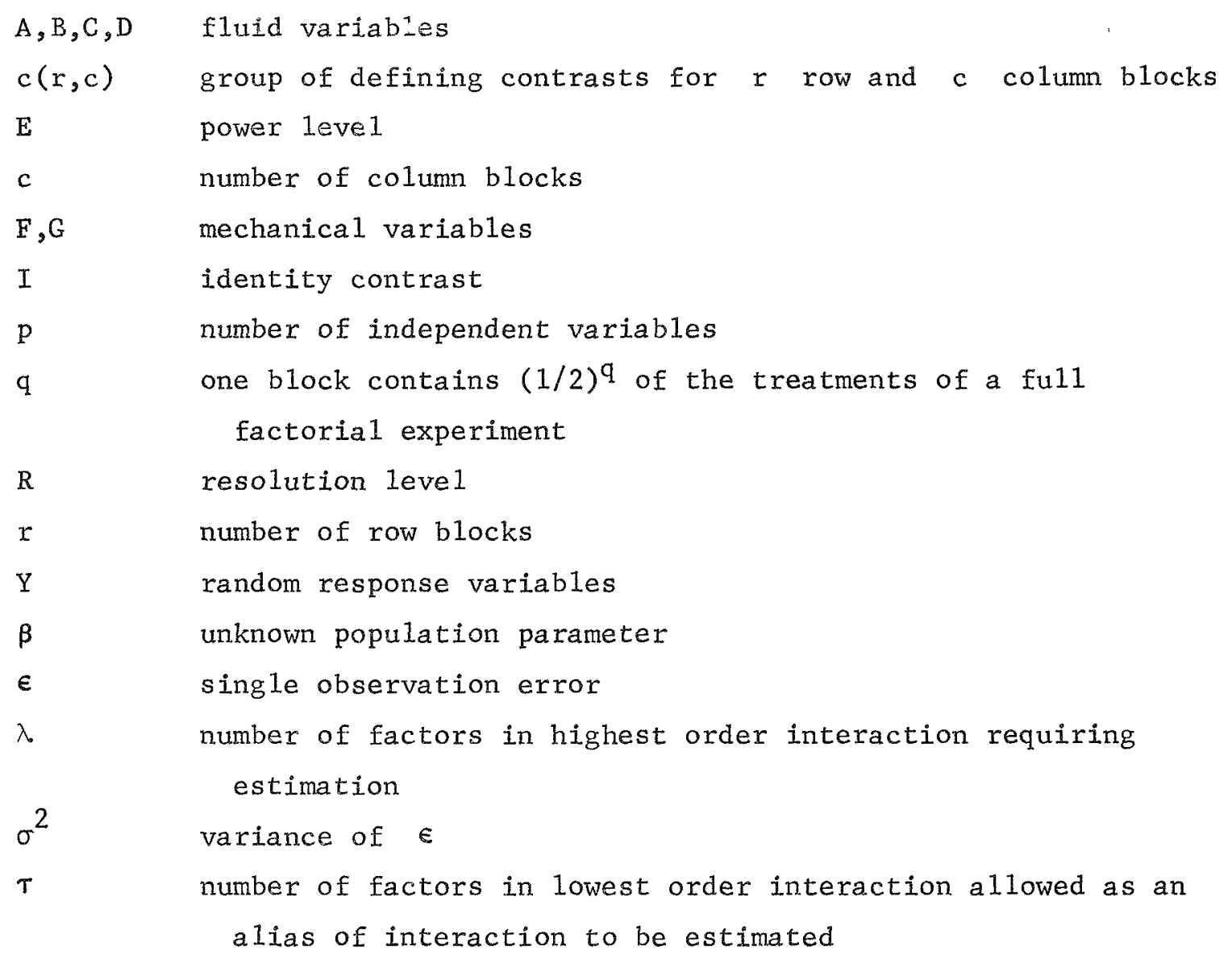


In brief, the purpose of the experiment is to estimate the coefficients in an equation that is assumed to represent the response as a function of the independent variables. The function together with the assumptions about the experimental error are called the model. The error of any particular observed response is called $\epsilon$, and it is assumed to have mean zero and constant variance $\sigma^{2}$.

The coefficients of the model equation are assumed to be initially unknown and as such are called parameters. The unknown parameters are represented by the symbol $\beta$. The performance of an experiment leads to numbers (estimates) that are assumed to approximate the true values of the parameters, and the error in the approximations is assumed to decrease with increasing numbers of observations. The estimates of the parameters are ordinarily obtained by the method of least squares. Estimation of $\sigma^{2}$ is outside the scope of the present discussion. An example of a model equation is

$$
\begin{aligned}
Y=\beta_{I} & +\beta_{A} x_{A}+\beta_{B} x_{B}+\cdots \cdot+\beta_{p} x_{p} \\
& +\beta_{A B} x_{A} x_{B}+\beta_{A C} x_{A} x_{C}+\cdots+\beta_{p-1, p^{x} p-1} x_{p} \\
& +\beta_{A B C} x_{A} x_{B} x_{C}+\cdots+\beta_{p-2, p-1, p}{ }_{p-2}{ }^{x}-1^{x} p \\
& +\ldots+\epsilon
\end{aligned}
$$

where the $\beta^{\prime}$ 's are the parameters to be estimated and the $x^{\prime}$ s are the independent variables. The parameter $\beta_{I}$ is called the constant term. The parameters with a single subscript are called main effects, and they give the magnitude of the first-degree effects on the response of $Y$ to the independent variables. The parameters with more than one subscript are called interactions, and they represent the fact that the response to one independent variable depends on the level of one or more other independent variables. 
The ful1 factorial experiment with conditions fixed at a11 combinations of just two levels of $P$ independent variables (factors) permits the estimation of parameters representing the constant term, the first-order (main) effects of the factors, and the results of factors interacting two at a time, three at a time, and in all combinations up to $\mathrm{p}$ at a time. If a fraction $(1 / 2)^{\mathrm{q}}$ of this experiment is performed, not all these parameters can be estimated. True response surfaces in physical investigations are typically smooth enough that the coefficients of the higher order terms of an approximating polynomial equation may be assumed negligible over a small enough range of the experimentation. Accordingly, only the lower order coefficients are estimated, and they are allowed to be biased by (aliased with) coefficients of higher order interactions because such coefficients are assumed to be negligible. A parameter of any order is called estimable if its estimate contains no aliased parameters, unless they are of higher order. Let the number of factors in the highest order interaction requiring estimation be $\lambda$, and let the number of factors in the lowest order interaction with which it is allowed to be aliased be $T$; then, the required resolution $R$ of the design is defined (ref. 4) to be

$$
R=\lambda+T
$$

As a minimum requirement on the first stopping point of an experiment, the first-order parameters are required to be estimable. They are allowed to be aliased with only the coefficients of two-factor or higher order interactions. This requires that $R=\lambda+T=1+2=3$. A somewhat improved design occurs if the first-order coefficients are estimated clear of two factor interactions. This requires that $R=\lambda+T=1+3=4$.

The requirement on the total experiment is that any possibly important two-factor interaction coefficients should be estimable (allowed to be aliased only with three-factor or higher order interaction 
coefficients). This requires that $\mathrm{R}=\lambda+\tau=2+3=5$.

The example of a mode1 equation shows on ly those coefficients that can be estimated by two-level factorial experiments. The estimation of coefficients of second degree terms (e.g., $x_{A}^{2}$ ) in each of the independent variables is efficiently done by adding "star" and "center" points to the design (ref. 2). Such points will actually be incorporated in the experiment, but a discussion of the details will be deferred to a report on the results of the experiment. The present report is concerned only with the problems of double confounding and fractional replication as they arise in the two-level factorial design.

\section{Notation for Treatments}

A combination of levels of the independent variables is called a treatment. The independent variables, which are written $\mathrm{x}_{A}, \mathrm{x}_{B}, \mathrm{x}_{C}$, etc., can be standardized (coded) so that the upper level can be represented by $\mathrm{x}_{\mathrm{A}}=+1, \mathrm{x}_{\mathrm{B}}=+1, \mathrm{x}_{\mathrm{C}}=+1$, etc. and the lower leve1 by $\mathrm{x}_{\mathrm{A}}=-1$, $\mathrm{x}_{\mathrm{B}}=-1, \mathrm{x}_{\mathrm{C}}=-1$, etc. Another notation for the levels of the independent variables is to use a 1 if the variable is at its lower level and to use the associated lower case letter if the variable is at its upper leve1. This letter notation is used in the design shown in table $I$. The equivalence of the notations is illustrated by the following example of a treatment:

$$
\left(x_{A}, x_{B}, x_{C}, x_{D}\right)=(1,1,-1,1)=a b l d=a b d
$$

The symbo1 (1) represents the treatment with a11 independent variables at the low leve1.

\section{Telescoping Blocks}

Experiments designed to estimate the parameters of the model equation without contamination from block effects are called orthogonally blocked 
designs. When a sequence of orthogonal blocks is designed so that observations from the first few blocks may be used to estimate the coefficients of a simple model and then be retained and combined with observations from new blocks so that al1 acquired observations are used cumulatively to estimate models of successively greater and greater generality, the blocks will be said to form a telescoping sequence.

\section{Crossed Blocking}

The subject of double confounding can be discussed from the point of view of two types of models for the block effects. One type is called nested blocking. An example of this type is given in reference 3 (p. 582). In the example, each of four units of a plant constitutes a block. Furthermore, if experimenting is done on each of two days, then the experimenting during one day is in a different block from the experimenting performed on another day. The blocking over days is thus nested within the blocking over units.

The particular problem that motivated the present investigation does not involve the nested type of double confounding but instead involves a type called crossed blocking. The general assumption of crossed blocking is defined to mean that there is an effect of column blocks, an effect of row blocks, and an effect of row column interaction.

\section{SOLUTION OF THE PARTICULAR PROBLEM}

The seven controlled independent variables selected for investigation were water volume, hydrogen gas, oxygen gas, nitrogen gas, reactor power, platinum metal, and cadmium sulfate solution.

These seven variables were to be tested at two levels. The eighth variable, reactor control rod bank height, was assigned eight levels.

The design of this experiment proceeded from the following logic: The controlled variables were separated into three classes; those 
variables which could be changed many times per cycle, called "fluid" variables; those variables which are changed only from cycle to cycle, called "mechanical" variables; and the reactor power leve1, which could be changed only once per cycle. Table I lists the controlled independent variables and their letter assignments.

There are two mechanical variables, $F$ and $G$. The levels of $F$ and $G$ cannot be changed during a reactor cycle. Therefore, main effects of these independent variables are estimated from the results of reactor cycles where these variables are at the levels (1), $f$, fg, and $g$ (see table I). This requires four reactor cycles. The reactor power leve1, $E$, may be changed only once during a cycle.

The levels of the fluid variables, A, B, C, D, are readily changed during the progress of a cycle. Economy in experimenting required that many fluid variable treatments be performed for each mechanical variable treatment. The time available during a reactor cycle allows about 16 fluid variable treatments to be performed. The reactor cycle was assumed to consist of eight column blocks so that a single block of a single cycle contains two treatments of fluid variables. Within four reactor cycles, a given block contains eight fluid variable treatments. (Example: Table I; block 1 contains eight treatments, abcd, (1), cef, abdef, adfg, bcfg, beg, and acdeg).

The remaining variable is rod bank height ( $\mathrm{RBH})$. The significant radiation environment components related to $\mathrm{RBH}$ are gamma heating, fast flux, and the gamma heating to fast flux ratio. Withdrawal of the control rods produces changes in the radiation component levels at the capsule, which is stationary in the reactor core。

In order to determine the effects of $\mathrm{RBH}$ and the interactions of RBH with the other independent variables, the reactor cycle is divided into eight $\mathrm{RBH}$ intervals or blocks (see table I). Actua11y, the RBH varies more or less continuously during the cycle. However, for convenience in the design and analysis of this experiment, the cycle was treated as though it was composed of eight blocks. The following prior physical knowledge justifies this. 
As indicated by dosimetry measurements, changes in radiation component levels within a $\mathrm{RBH}$ interval needed for two treatments are negligible, and changes at a given RBH from cycle to cycle, for a set of four cycles, are also negligible.

The treatments within a cycle were performed during the specified $\mathrm{RBH}$ intervals. These $\mathrm{RBH}$ intervals or blocks were not changed from cycle to cycle.

To insure orthogonality of the effects of RBH at eight leve1s with the effects of the other seven variables, the experiment was designed as if the $\mathrm{RBH}$ were a block effect; that is, an extraneous variation present in the experiment. If this block effect was not made orthogonal to the controlled variables, the effects of the controlled variables would then contain (be confounded with) the rod bank effects. The statistical technique for insuring the orthogonality is given in the Appendix, in which the final result is the set of treatments given in table $I$.

Table I shows the design used for the reactor experiment. Shown are the fluid variables and their letter designations, the mechanical variables and their letter designations. (Reactor power is listed with the mechanical variables). The table also shows the rod bank height boundaries for each of the eight blocks. Within the blocks are the fluid variables. The mechanical variable treatments and power level for each cycle are shown to the left of the sets of blocks. The 16 blocks shown represent the full factorial experiment, which is also called a full replicate.

The two-level full replicate experiment on seven independent variables contains $2^{7}$ treatments. If only one-half of these treatments are performed, the experiment is called a half replicate (written $2^{7-1}$ ).

The technique for insuring that the successive blocks of treatments of a telescoping sequence will form a sequence of fractional replicates with the desired statistical properties utilizes groups called defining contrasts, as discussed in the Appendix. These defining contrast groups serve to identify, and give the properties of, the successive stages of the telescoping sequence. The group for the stage consisting of $r$ row blocks and $c$ column blocks will be designated as $c(r, c)$. 
The conceivable arrangements of telescoping blocks for a $2^{7-1}$ experiment (two-level, seven controlled variables, a one-half replicate) with eight treatments per block are shown by figure 1 . Out of these conceivable arrangements, the options that are attractive for the reactor experimenting are shown by the solid line arrows.

To serve as a basis for checking subsequent tables, the defining contrasts appropriate to the reactor experiment are listed in table II. The information that can be obtained from the several stages of experimenting is displayed in tables III through $\mathrm{X}$.

The treatments needed for the several stages of telescoping have been listed in table $I$ in an order that is appropriate to the blocking, but is otherwise arbitrary. The same treatments are also 1 isted in tables III through $\mathrm{X}$ but in these tables the treatments are listed in an order such that the corresponding observations will be in the correct order for the application of Yates' method (ref. 3) of computing the parameter estimates. The output of Yates' method is a list of numbers that estimate combinations of the parameters of the model equation. Tables III through $X$ give the combinations of the model equation parameters in the order in which they are computed by Yates' method. The fact that one estimate is the estimate of a combination of parameters means that the experiment has not provided the information to evaluate either parameter separately. The parameters are said to be aliased.

The telescoping sequence of designs is intended to provide estimates of all the first order parameters at an early stage of the experimentation. The completion of al1 the intended stages of a sequence should provide estimates of all two-factor interaction parameters that were not confounded with block effects. Such parameter estimates might be aliased with three-factor and higher order interaction parameters. In the absence of prior knowledge to the contrary, the experimenter would assume that in any aliased set, only the lowest order parameters could be significant. On the other hand, the experimenter might have prior knowledge that tells him, for example, that in an aliased set consisting of a two-factor interaction and a three-factor interaction, it is the 
particular three-factor interaction, rather than the particular twofactor interaction, that is significant. The aliased three-factor coefficients are therefore tabulated for all the larger designs (designs containing four or more blocks -- see tables $\mathrm{V}$ through $\mathrm{X}$ ).

If an estimate does not estimate any combination of three-factor or lower order coefficients, the estimate is given a name by listing the lowest order set of interaction parameters that it does estimate.

The information obtainable at the several stages of the telescoping is summarized in table XI. It gives the number of two-and three-factor interactions that occur in a full factorial experiment on seven factors together with the numbers of these interactions that are estimable at various stages of completion of the blocks. As shown by the table, completion of eight blocks provides estimates of almost a11 of the twofactor interactions and about half of the three-factor interactions. Completion of 16 blocks provides estimates of almost al1 of the threefactor interactions.

\section{CONCLUDING REMARKS}

The sequence of designs has been formulated with the idea that only a small part of the total design is sufficient to estimate all of the main effect parameters and that the maximum contemplated sequence is sufficient to estimate almost all of the two-factor interactions. Such a design also provides estimates of some of the three-factor and higher order interaction parameters. This fact can be used to advantage if the experimenter has some prior knowledge of the interactions. He can then perform a strategic matching of the names of the physical variables to the letters that stand for the independent variables of the design. This should be done so that those interactions that are known to be negligible will be the interactions confounded with block effects. Those interactions that are likely to be important, according to prior knowledge, should be the interactions that become estimable at the earliest possible stage of the sequence of experiments. 


\section{APPENDIX \\ STATISTICAL CONSIDERATIONS}

\section{Defining Contrast Groups}

The technique for obtaining a design for an experiment with orthogonal block effects requires the use of a concept known as the defining contrast group. This concept, and the associated notation, was described by Davies (ref, 3). The concept and notation together with its extension to the general subject of doubly telescoping sequences of designs was given by Holms and Sidik (ref. 5). The application of these ideas to the reactor experiment with seven independent variables follows.

The first block contains $2^{3}$ treatments and is a $2^{-4}$ replicate of the full $2^{7}$ experiment, and as such requires four generators. The required independence is assured by annexing the letters $D, E, F$, and $G$, in succession, to combinations of $A, B$, and $C$. A block is made a principal block (Davies, ref. 3) by giving a generator a positive sign if it contains an even number of letters, and by giving it a negative sign if the number of letters is odd. The generators were chosen as:

$$
A D,-B C E,-A C F,-A B G
$$

The generators are multiplied together in all combinations to generate the complete group as listed for $c(1,1)$ in table II. The principal block defined by the $c(1,1)$ of table II occurs in the first row and column of table I.

The next step is to select the subgroups of $c(1,1)$ so as to provide the options indicated by the solid line arrows of figure 1. Each subgroup is to be selected so that every stage of the experimenting will have the highest possible resolution level. In particular, the desired resolution five design should occur at the earliest possible stage, and with seven independent variables the earliest stage is the half replicate. 
At any given stage of a doubly telescoping sequence, the experimenter should have the option of expanding the experiment either by doubling the existing number of columns or by doubling the existing number of rows. This option requires that the two subgroups of contrasts that define the two expanded experiments must both be subgroups of the group of contrasts defining the existing stage. The extension of this requirement to all of the conceivable options (figure 1) implies that the four defining contrasts for the half replicates, namely, $c(1,8), c(2,4), c(4,2)$, and $c(8,1)$ must be generators of $c(1,1)$.

Additional restrictions on the half replicate contrasts arise from the physical limitations of the experiment. The mechanical variables, $F$ and $G$, cannot be changed during a reactor cycle, and the power level, $E$, can only be changed at the midpoint (end of four column blocks) of a cycle; and therefore -EFG must be an element of $c(1,4)$. Words that meet all these restrictions on the half replicate defining contrasts are shown for $c(1,8), c(2,4), c(4,2)$, and $c(8,1)$ in table II.

The underlined words of table II show the manner in which the five independent defining contrasts for the half replicates have been used to generate the subsets that occur between the groups for the half replicates and the group consisting of $c(1,1)$.

Estimation of the Effects of Rod Bank Height

The effects of $\mathrm{RBH}$ and its interactions were obtained in the following manner.

The variable $\mathrm{RBH}$ was treated as though it had eight levels throughout a reactor cycle, that is, one level for each column block. Thus, RBH could conceivably be represented in a model equation by a polynomial of degree 7. In terms of the experimental design, however, only a polynomial of degree two was considered. We arranged the blocks of the seven-variable design in such a manner that a11 main effects and twofactor interactions are uncorrelated with the linear and quadratic effects of RBH. 
To see how to do this consider the following examples:

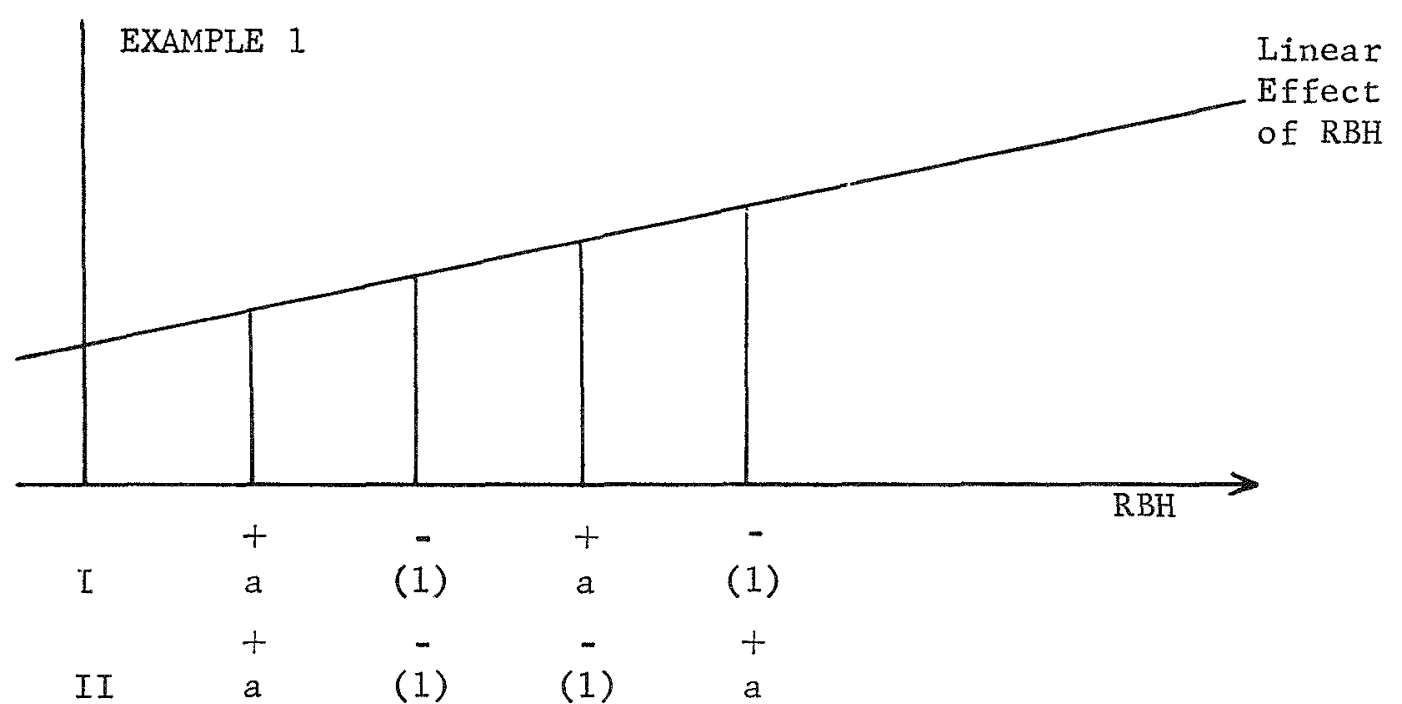

Example 1 shows that if the levels of variable A correspond to the pattern of $I$, then the $A$ effect (defined as the sum of responses at high level minus the sum of responses at low level divided by the number of responses) is perfectly negatively correlated with the linear effect of $\mathrm{RBH}$. The arrangement of II, however, gives a correlation between the two of zero.

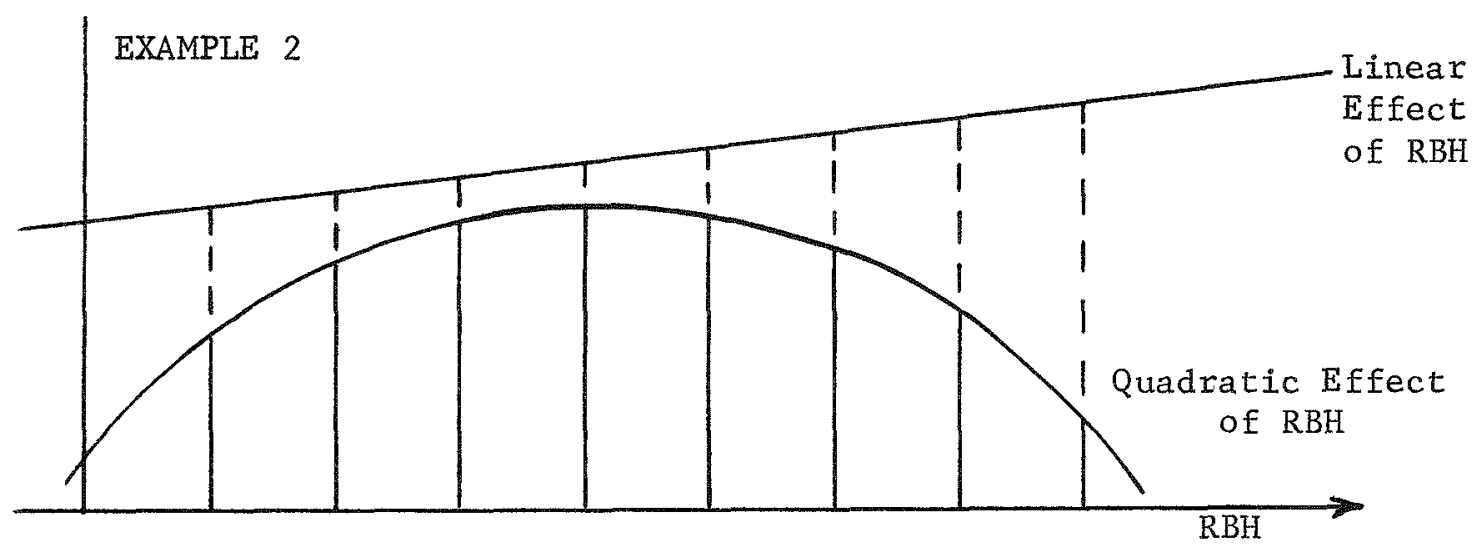

\begin{tabular}{|c|c|c|c|c|c|c|c|c|}
\hline \multirow[t]{2}{*}{$I$} & $a$ & $a$ & (1) & (1) & (1) & (1) & $a$ & $a$ \\
\hline & + & + & - & - & - & - & + & + \\
\hline \multirow[t]{2}{*}{ II } & $a$ & (1) & (1) & $a$ & (1) & $a$ & a & (1) \\
\hline & + & - & - & + & - & + & + & - \\
\hline \multirow[t]{2}{*}{ III } & $a$ & (1) & (1) & $\mathrm{a}$ & a & (1) & (1) & a \\
\hline & + & - & $=$ & + & + & - & - & + \\
\hline
\end{tabular}


In example 2, it can be seen that the arrangement of $I$ will give a positive correlation between the effect of $A$ and the quadratic effect of $\mathrm{RBH}$, while giving a zero correlation between the effect of $A$ and the linear effect of RBH. Arrangement II will give a zero correlation between both linear and quadratic effect. Arrangement III will give a low correlation with quadratic effect and zero with linear.

Thus to design the experiment so that linear and quadratic effects of RBH are as uncorrelated as possible with the main effects and twofactor interactions, it suffices to split into two parts each of the eight blocks of the seven-factor experiment and arrange the blocks in such a manner that as many of these effects have a high-low pattern such as arrangement II of example 1 or arrangement II or III of example 2 .

The choice of defining contrasts group (DCG) for the seven-factor experiment to get a block size of eight treatments is shown below.

\begin{tabular}{|c|}
\hline $\begin{array}{c}8 / \mathrm{Block} \\
\mathrm{DCG}\end{array}$ \\
\hline $\mathrm{AD}(2)$ \\
$\mathrm{BCE}$ \\
$\mathrm{ACF}$ \\
$\mathrm{ABG}$ \\
$\mathrm{CDF}$ \\
$\mathrm{BDG}$ \\
$\mathrm{EFG}$ \\
$\mathrm{ABEF}$ \\
$\mathrm{ACEG}$ \\
$\mathrm{BCFG}$ \\
$\mathrm{BDEF}$ \\
$\mathrm{CDEG}$ \\
$\mathrm{ABCDE}$ \\
$\mathrm{ADEFG}$ \\
$\mathrm{ABCDFG}$ \\
\hline
\end{tabular}

(2) indicates two-factor interactions 
From the defining contrasts it is evident that all main effects and two-factor interactions, except $A D$, are uncorrelated with the linear effects.

\begin{tabular}{|c|c|c|c|c|c|c|c|c|}
\hline & 1 & 2 & 3 & 4 & 5 & 6 & 7 & 8 \\
\hline $\mathrm{AD}$ & ++ & -- & -- & ++ & -- & ++ & ++ & -- \\
\hline
\end{tabular}

NOTE: The sign of a two-factor interaction is given by (e.g., AD)
a bc d efg
$(1)(\mathrm{xx})(1)(\mathrm{xxx})+$
$a(x x)(1)(x x x)-$
$(1)(x x)(d)(x x x)-$
$a(x x)(d)(x x x)+$

At the end of four blocks, $\mathrm{AD}$ is free of the linear effect of $\mathrm{RBH}$. At the end of eight blocks in either direction, $A D$ is free of linear and quadratic effects. This is summarized in the following table.

\begin{tabular}{|c|c|c|c|c|c|c|}
\hline & $\begin{array}{c}c(1,1) \\
1\end{array}$ & $\begin{array}{c}c(1,2) \\
2\end{array}$ & $\begin{array}{c}c(1,4) \\
4\end{array}$ & $\begin{array}{c}c(1,8) \\
8\end{array}$ & $\begin{array}{c}c(2,4) \\
8\end{array}$ & $\begin{array}{c}c(2,8) \\
16\end{array}$ \\
\hline $\mathrm{AD}$ & $*$ & $\mathrm{~L}$ & $\mathrm{Q}$ & & & \\
\hline$*$ & confounded with both linear and quadratic \\
$\mathrm{L}$ & confounded with linear \\
$\mathrm{Q}$ & confounded with quadratic
\end{tabular}




\section{REFERENCES}

1. Webb, Steve R.: Design, Testing and Estimation in Complex Experimentation. I: Fxpansible and Contractible Factorial Designs and the Application of Linear Programming to Combinatorial Problems. Rep. R-6078-1, Rocketdyne Div., North American Aviation (ARL-65-116, pt. 1, DDC No. AD-618515), June 1965.

2. Box, G. E. P.; and Hunter, J. S.: Multi-Factor Experimental Designs for Exploring Response Surfaces. Ann. Math. Statist., vol. 28, 1957, pp. $195-241$.

3. Davies, Owen I., ed.: The Design and Analysis of Industrial Experiments. Second ed., Hafner Publishing Co., 1956.

4. Daniel, Cuthbert: Fractional Replication in Industrial Research. Proceedings of the Third Berkeley Symposium on Mathematical Statistics and Probability. Vol. 5. Univ. California Press, 1956, pp. 87-98.

5. Holms, Arthur G.; and Sidik, Steven M.: Designing Experiments as

"Doubly Telescoping" Sequences of Blocks with Application to a Nuclear Reactor Experiment. NASA TN D-5369.

6. Box, G. E. P.; and Hunter, J. S.: The $2^{k-p}$ Fractional Factorial Designs. Part I. Technometrics, vol. 3, no. 3, Aug. 1961, pp. 31I-35I. 
TABLE I

ASSIGNMENT OF TREATMENTS TO BLOCKS

Rod Bank

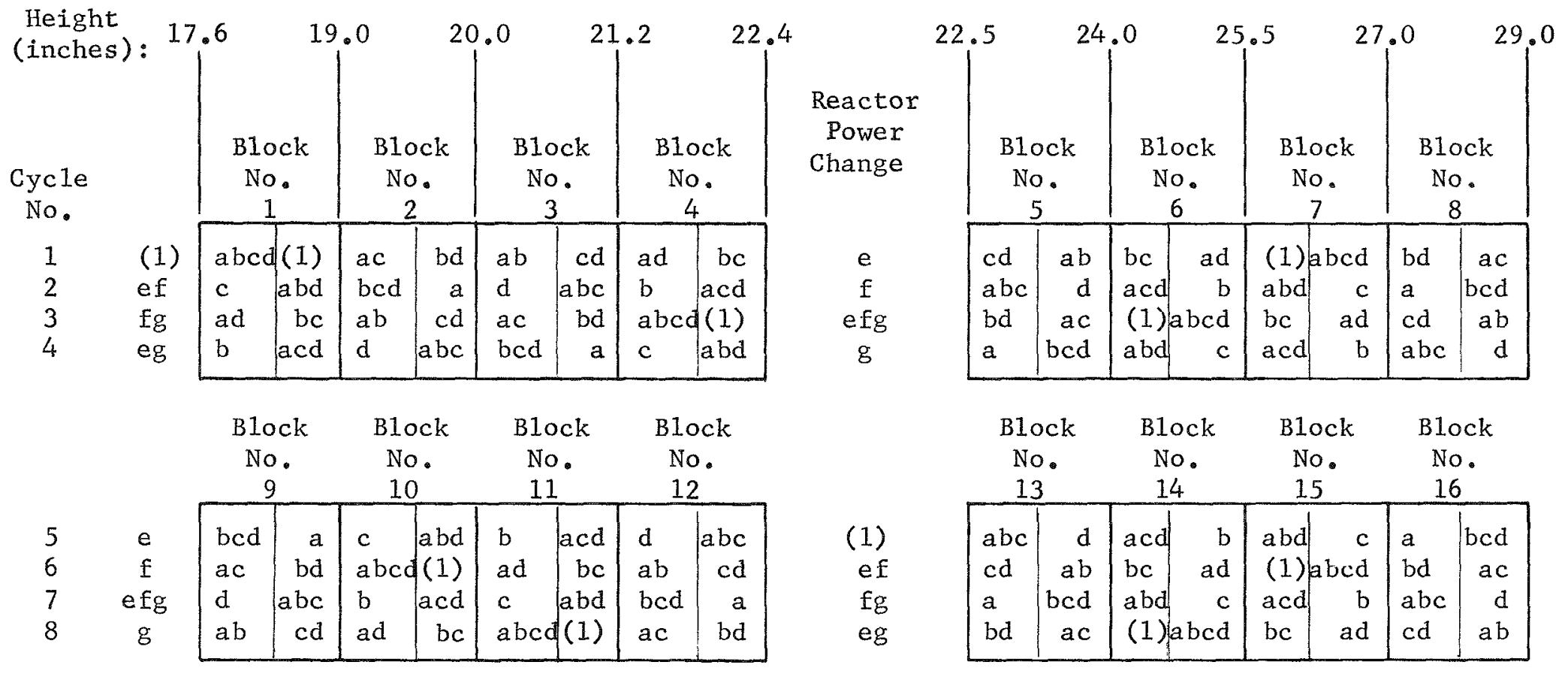

Fluid Variables

$$
\begin{aligned}
& \mathrm{A}=\mathrm{H}_{2} \mathrm{O} \\
& \mathrm{B}=\mathrm{H}_{2} \\
& \mathrm{C}=\mathrm{O}_{2} \\
& \mathrm{D}=\mathrm{N}_{2}
\end{aligned}
$$

Mechanica1 Variables

$$
\begin{aligned}
& \mathrm{E}=\text { reactor power } \\
& \mathrm{F}=\mathrm{Pt} \\
& \mathrm{G}=\mathrm{Cd}
\end{aligned}
$$


DEFINING CONTRAST GROUPS FOR TELESCOPING OPTIONS OF FIGURE 1

$\mathrm{C}(1,8)$

$I$

$\underline{A B C D F G}$
$C(2,4)$

$I$

$\underline{-A B C D E}$

$$
\begin{gathered}
C(1,4) \\
I \\
\frac{A B C D F G}{-A B C D E} \\
\frac{-E F G}{-E F G}
\end{gathered}
$$

$\mathrm{C}(4,2)$

I

$C(8,1)$

$\underline{A C E G}$

-ADEFG

$$
\begin{gathered}
C(1,2) \\
I \\
\frac{A B C D F G}{-A B C D E} \\
\hline-E F G \\
\frac{A C E G}{B D E F} \\
-B D G \\
-A C F
\end{gathered}
$$

$$
\begin{aligned}
& C(2,2) \\
& I \\
& \frac{-A B C D E}{\frac{A C E G}{-B D G}}
\end{aligned}
$$$$
C(4,1)
$$

ACEG

-ADEFG

$C(2,1)$

I

$-A B C D E$

ACEG

$-B D G$

-ADEFG

BCFG

$-\mathrm{CDF}$

$\mathrm{ABEF}$

$$
\begin{aligned}
& C(1,1) \\
& I \\
& A B C D F G \\
& \hline-A B C D E \\
& \hline-E F G \\
& \frac{A C E G}{B D E F} \\
& -B D G \\
& -A C F \\
& -A D E F G \\
& \hline-B C E \\
& B C F G \\
& A D \\
& -C D F \\
& -A B G \\
& A B E F \\
& C D E G
\end{aligned}
$$


TABLE III

\section{$(1,1)$ REPLICATE - DEFINING CONTRASTS GIVEN BY TABLE II}

\section{Block}

1

1

1

1

1

1

1

1
Treatment

(1)

adfg

beg

abdef

cef

acdeg

befg

abcd

\section{Estimate}

$\beta_{I}+\beta_{A D}$

$\beta_{A}+\beta_{D}$

$\beta_{B}-\beta_{C E}-\beta_{D G}-\beta_{A G}$

$\beta_{A B}-\beta_{G}+\beta_{B D}+\beta_{E F}$

$\beta_{C}-\beta_{B E}-\beta_{A F}$

$-\beta_{F}+\beta_{A C}+\beta_{C D}+\beta_{E G}$

$-\beta_{E}+\beta_{B C}+\beta_{E G}$

$-\beta_{A E}-\beta_{C G}-\beta_{D E}$ 


\section{TABLE IV \\ $(1,2)$ REPLICATE - DEFINING CONTRASTS GIVEN BY TABLE II}

\begin{tabular}{|c|c|c|}
\hline Block & Treatment & Estimate \\
\hline 1 & (1) & $\beta_{I}$ \\
\hline 2 & aef & $\beta_{A}-\beta_{C F}$ \\
\hline 1 & beg & $\beta_{B}-\beta_{D G}$ \\
\hline 2 & abfg & $\beta_{\mathrm{AB}}$ \\
\hline 1 & $\operatorname{cef}$ & $\beta_{C}-\beta_{A F}$ \\
\hline 2 & $a c$ & $-\beta_{F}+\beta_{A C}+\beta_{E G}$ \\
\hline 1 & $b c f g$ & $\beta_{\mathrm{BC}}$ \\
\hline 2 & abceg & $-\beta_{B F}-\beta_{D E}$ \\
\hline 2 & $\operatorname{deg}$ & $\beta_{D}-\beta_{B G}$ \\
\hline 1 & $\operatorname{adfg}$ & $\left(\beta_{A D}\right)^{c}$ \\
\hline 2 & bd & $-\beta_{\mathrm{G}}+\beta_{\mathrm{BD}}+\beta_{\mathrm{EF}}$ \\
\hline 1 & abdef & $-\beta_{C E}-\beta_{A G}$ \\
\hline 2 & $\mathrm{cdfg}$ & $\beta_{\mathrm{CD}}$ \\
\hline 1 & acdeg & $-\beta_{\mathrm{DF}}-\beta_{\mathrm{BE}}$ \\
\hline 2 & bcdef & $-\beta_{C G}-\beta_{A E}$ \\
\hline 1 & $a b c d$ & $-\beta_{\mathrm{E}}+\beta_{\mathrm{FG}}$ \\
\hline
\end{tabular}


TABLE V

$(1,4)$ REPLICATE - DEFINING CONTRASTS ARE I, -EFG, -ABCDE, ABCDFG

\begin{tabular}{|c|c|c|c|c|c|}
\hline Block & Treatment & Estimate & Block & Treatment & Estimate \\
\hline 1 & (1) & $\beta_{I}-\beta_{E F G}$ & 4 & (f) $g$ & $\beta_{G}-\beta_{E F}$ \\
\hline 2 & $a(e)(f)$ & $B_{A}$ & 3 & $a(e) g$ & $\beta_{A G}-\beta_{A E F}$ \\
\hline 4 & $b(e)(f)$ & $\beta_{B}$ & 1 & $b(e) g$ & $\beta_{B G}-\beta_{B E F}$ \\
\hline 3 & $a b$ & $\beta_{\mathrm{AB}}-\beta_{\mathrm{CDE}}$ & 2 & $a b(f) g$ & $\left(\beta_{A B G}+\beta_{C D F}\right)^{c}$ \\
\hline 1 & $c(e)(f)$ & $\beta_{C}$ & 4 & $c(e) g$ & $\beta_{C G}-\beta_{C E F}$ \\
\hline 2 & $\mathrm{ac}$ & $\beta_{A C}-\beta_{B D E}$ & 3 & $a c(f) g$ & $\beta_{\mathrm{ACG}}+\beta_{\mathrm{BDF}}$ \\
\hline 4 & $\mathrm{bc}$ & $\beta_{B C}-\beta_{A D E}$ & 1 & $b c(f) g$ & $\beta_{\mathrm{BCG}}+\beta_{\mathrm{ADF}}$ \\
\hline 3 & $a b c(e)(f)$ & $\beta_{\mathrm{ABC}}-\beta_{\mathrm{DE}}+\beta_{\mathrm{DFG}}$ & 2 & $a b c(e) g$ & $\beta_{D F}-\beta_{D E G}$ \\
\hline 3 & $d(e)(f)$ & $\beta_{D}$ & 2 & $d(\varepsilon) g$ & $\beta_{D G}-\beta_{D E F}$ \\
\hline 4 & ad & $\left(\beta_{A D}-\beta_{B C E}\right)^{c}$ & 1 & $\operatorname{ad}(f) g$ & $\beta_{A D G}+\beta_{B C F}$ \\
\hline 2 & bd & $\beta_{B D}-\beta_{A C E}$ & 3 & $b d(f) g$ & $\left(\beta_{B D G}+\beta_{A C F}\right)^{c}$ \\
\hline 1 & $\operatorname{abd}(e)(f)$ & $\beta_{\mathrm{ABD}}-\beta_{\mathrm{CE}}$ & 4 & $a b d(e) g$ & $\beta_{C F}-\beta_{C E G}$ \\
\hline 3 & $\mathrm{~cd}$ & $\beta_{C D}-\beta_{A B E}$ & 2 & $c d(f) g$ & $\beta_{\mathrm{CDG}}+\beta_{\mathrm{ABF}}$ \\
\hline 4 & $\operatorname{acd}(e)(f)$ & $\beta_{A C D}-\beta_{B E}+\beta_{B F G}$ & 1 & $\operatorname{acd}(e) g$ & $\beta_{B F}-\beta_{B E G}$ \\
\hline 2 & $\operatorname{bcd}(e)(f)$ & $\beta_{B C D}-\beta_{A E}+\beta_{A F G}$ & 3 & $\operatorname{bcd}(e) g$ & $\beta_{A F}-\beta_{A E G}$ \\
\hline 1 & abcd & $-\beta_{\mathrm{E}}+\beta_{\mathrm{FG}}$ & 4 & $\operatorname{abcd}(f) g$ & $\beta_{F}-\beta_{E G}$ \\
\hline
\end{tabular}

${ }^{c}$ Confounded with column block differences. 
TABLE VIa

$(1,8)$ REPLICATE - DEFINING CONTRASTS ARE I, ABCDFG

\begin{tabular}{|c|c|c|c|c|c|}
\hline Block & Treatment & Estimate & Block & Treatment & Estimate \\
\hline 1 & (1) & $\beta_{I}$ & 7 & e & $\beta_{E}$ \\
\hline 5 & ag & $\beta_{\mathrm{A}}$ & 3 & aeg & $\beta_{\mathrm{AE}}$ \\
\hline 7 & bg & $\beta_{B}$ & 1 & beg & $\beta_{\mathrm{BE}}$ \\
\hline 3 & $a b$ & $\beta_{\mathrm{AB}}$ & 5 & abe & $\beta_{A B E}$ \\
\hline 6 & $\mathrm{cg}$ & $\beta_{C}$ & 4 & ceg & $\beta_{C E}$ \\
\hline 2 & $a c$ & $\beta_{A C}$ & 8 & ace & ${ }^{\beta} A C E$ \\
\hline 4 & bc & $\beta_{B C}$ & 6 & bce & $\left(\beta_{\mathrm{BCE}}\right)^{\mathrm{c}}$ \\
\hline 8 & abcg & $\beta_{\mathrm{ABC}}+\beta_{\mathrm{DFG}}$ & 2 & abceg & $\beta_{\mathrm{ABCE}}+\beta_{\mathrm{DEFG}}$ \\
\hline 8 & $\mathrm{dg}$ & $\beta_{D}$ & 2 & deg & $\beta_{\mathrm{DE}}$ \\
\hline 4 & ad & $\left(\beta_{A D}\right)^{c}$ & 6 & ade & $\beta_{\mathrm{ADE}}$ \\
\hline 2 & $\mathrm{bd}$ & $\beta_{\mathrm{BD}}$ & 8 & bde & $\beta_{\mathrm{BDE}}$ \\
\hline 6 & abdg & $\beta_{\mathrm{ABD}}+\beta_{\mathrm{CFG}}$ & 4 & abdeg & $\beta_{A B D E}+\beta_{C E F G}$ \\
\hline 3 & $\mathrm{~cd}$ & $\beta_{C D}$ & 5 & cde & $\beta_{\mathrm{CDE}}$ \\
\hline 7 & acdg & $\beta_{\mathrm{ACD}}+\beta_{\mathrm{BFG}}$ & 1 & acdeg & $\beta_{\mathrm{ACDE}}+\beta_{\mathrm{BEFG}}$ \\
\hline 5 & bcdg & $\beta_{\mathrm{BCD}}+\beta_{\mathrm{AFG}}$ & 3 & bcdeg & $\beta_{\mathrm{BCDE}}+\beta_{\mathrm{AEFG}}$ \\
\hline 1 & $a b c d$ & $\beta_{\mathrm{FG}}$ & 7 & abcde & $\left(\beta_{E F G}\right)^{c}$ \\
\hline
\end{tabular}

${ }^{c}$ Confounded with column block differences. 
TABLE VIb

$(1,8)$ REPLICATE - DEFINING CONTRASTS ARE I, ABCDFG

\begin{tabular}{|c|c|c|c|c|c|}
\hline Block & Treatment & Estimate & Block & Treatment & Estimate \\
\hline 4 & $\mathrm{fg}$ & $\beta_{F}$ & 6 & efg & $\beta_{\mathrm{EF}}$ \\
\hline 8 & af & $\beta_{A F}$ & 2 & aef & $\beta_{\mathrm{AEF}}$ \\
\hline 6 & $\mathrm{bf}$ & $\beta_{\mathrm{BF}}$ & 4 & bef & $\beta_{\mathrm{BEF}}$ \\
\hline 2 & $a b f g$ & $\beta_{\mathrm{ABF}}+\beta_{\mathrm{CDG}}$ & 8 & abefg & $\left(\beta_{\mathrm{ABEF}}+\beta_{\mathrm{CDEG}}\right)^{\mathrm{c}}$ \\
\hline 7 & cf & $\beta_{\mathrm{CF}}$ & 1 & cef & $\beta_{\mathrm{CEF}}$ \\
\hline 3 & $\operatorname{acfg}$ & $\left(\beta_{\mathrm{ACF}}+\beta_{\mathrm{BDG}}\right)^{\mathrm{c}}$ & 5 & acefg & $\beta_{\mathrm{ACEF}}+\beta_{\mathrm{BDEG}}$ \\
\hline 1 & bcfg & $\beta_{B C F}+\beta_{A D G}$ & 7 & bcefg & $\beta_{\mathrm{BCEF}}+\beta_{\mathrm{ADEG}}$ \\
\hline 5 & $a b c f$ & $\beta_{\mathrm{DG}}$ & 3 & $a b c e f$ & $\beta_{\mathrm{DEG}}$ \\
\hline 5 & $d f$ & ${ }^{\beta}{ }_{D F}$ & 3 & $\operatorname{def}$ & $\beta_{\mathrm{DEF}}$ \\
\hline 1 & $\operatorname{adfg}$ & $\beta_{\mathrm{ADF}}+\beta_{\mathrm{BCG}}$ & 7 & adefg & $\beta_{\mathrm{ADEF}}+\beta_{\mathrm{BCEG}}$ \\
\hline 3 & bdfg & $\beta_{\mathrm{BDF}}+\beta_{\mathrm{ACG}}$ & 5 & bdefg & $\left(\beta_{\mathrm{BDEF}}+\beta_{\mathrm{ACEG}}\right)^{c}$ \\
\hline 7 & $\operatorname{abd} f$ & ${ }^{\beta}{ }_{C G}$ & 1 & abdef & $\beta_{C E G}$ \\
\hline 2 & $\operatorname{cdfg}$ & $\left(\beta_{\mathrm{CDF}}+\beta_{\mathrm{ABG}}\right)^{\mathrm{c}}$ & 8 & cdefg & $\beta_{\mathrm{CDEF}}+\beta_{\mathrm{ABEG}}$ \\
\hline 6 & $\operatorname{acd} f$ & $\beta_{B G}$ & 4 & acdef & $\beta_{B E G}$ \\
\hline 8 & bcdf & $\beta_{A G}$ & 2 & bcdef & ${ }^{\beta}{ }_{A E G}$ \\
\hline 4 & abcdfg & $\beta_{G}$ & 6 & abcdefg & $\beta_{E G}$ \\
\hline
\end{tabular}

${ }^{\mathrm{C}}$ Confounded with column block differences. 
$(2,1)$ REPLICATE - DEFINING CONTRASTS GIVEN BY TABLE II

\section{Block}

1

9

1

9

1

9

1

9

9

1

9

1

9

1

9

1
Treatment

(1)

a(e)

$b(e)(g)$

$\mathrm{ab}(\mathrm{g})$

$c(e)(f)$

$\operatorname{acf}$

bcfg

abcefg

defg

adfg

bd $f$

abdef

cdg

acdeg

bcde

abcd

\section{Estimate}

$\beta_{I}$

$\mathrm{B}_{\mathrm{A}}$

$\beta_{B}-\beta_{D G}$

$\beta_{A B}+\beta_{E E}$

$\beta_{C}-\beta_{D F}$

$\beta_{\mathrm{AC}}+\beta_{\mathrm{EG}}$

$\beta_{B C}+\beta_{F G}$

$-\beta_{\mathrm{DE}}$

$\beta_{D}-\beta_{C F}-\beta_{B G}$

$\left(\beta_{A D}\right)^{r}$

$\beta_{B D}-\beta_{G}$

$-\beta_{A G}-\beta_{C E}$

$\beta_{C D}-\beta_{F}$

${ }^{-\beta_{A F}}-\beta_{B E}$

$-\beta_{A E}-\beta_{B F}-\beta_{C G}$

$-\beta_{E}$

${ }^{\mathrm{r}}$ Confounded with row block differences. 
$(2,2)$ REPLICATE - DEFINING CONTRASTS ARE I, -BDG, ACEG, -ABCDE

\section{Block Treatment}

1

9

1

9

10

2

10

2

$2 d(e)(g) \quad \beta_{D}-\beta_{B G}$

10

2

$$
c(e) \quad \beta_{C}+\beta_{A E G}
$$$$
\text { ac } \beta_{A C}+\beta_{E G}-\beta_{B D E}
$$$$
b c(g) \quad \beta_{B C}-\beta_{C D G}-\beta_{A D E}
$$

$$
a b c(e)(g) \beta_{A B C}+\beta_{B E G}-\beta_{D E}
$$

$$
\operatorname{ad}(\mathrm{g}) \quad\left(\beta_{\mathrm{AD}}-\beta_{\mathrm{ABG}}-\beta_{\mathrm{BCE}}\right)^{\mathrm{rC}}
$$$$
\text { bd } \quad \beta_{B D}-\beta_{G}-\beta_{A C E}
$$$$
\text { abd (e) } \quad \beta_{A B D}-\beta_{A G}-\beta_{C E}
$$

$9 \quad \mathrm{~cd}(\mathrm{~g}) \quad \beta_{\mathrm{CD}}-\beta_{\mathrm{BCG}}-\beta_{\mathrm{ABE}}$

$1 \operatorname{acd}(e)(g) \beta_{A C D}+\beta_{D E G}-\beta_{B E}$

$9 \operatorname{bcd}(e) \quad \beta_{B C D}-\beta_{C G}-\beta_{A E}$

1 abcd

$$
-\beta_{E}-\beta_{A C G}
$$

\section{Block Treatment Estimate}

10

$\begin{array}{rcc}2 & a(e) f & \beta_{A F} \\ 10 & b(e) f(g) & \beta_{B F}-\beta_{D F G} \\ 2 & a b f(g) & \beta_{A B F}\end{array}$

1

$$
c(e) f \quad \beta_{C F}
$$$$
\operatorname{acf} \quad\left(\beta_{\mathrm{ACF}}+\beta_{\mathrm{EFG}}\right)^{\mathrm{r}}
$$

$1 \quad \operatorname{bcf}(g) \quad \beta_{B C F}$

$9 \quad a b c(e) f(g) \quad-\beta_{D E F}$

$\begin{array}{ccc}1 & \operatorname{adf}(g) & \beta_{\mathrm{ADF}} \\ 9 & \operatorname{bdf} & \beta_{\mathrm{BDF}}-\beta_{\mathrm{FG}} \\ 1 & \operatorname{abd}(e) f & -\beta_{\mathrm{AFG}}-\beta_{\mathrm{CEF}}\end{array}$

$2 \operatorname{cdf}(g) \quad\left(\beta_{\mathrm{CDF}}\right)^{\mathrm{c}}$

$10 \operatorname{acd}(e) f(g) \quad-\beta_{B E F}$

$2 \operatorname{bcd}(e) f \quad-\beta_{C F G}-\beta_{A E F}$

10

${ }^{c}$ Confounded with column block differences.

${ }^{\mathrm{r}}$ Confounded with row block differences.

${ }^{r c}$ Confounded with row-column block interactions. 
$(2,4)$ REPLICATE - DEFINING CONTRASTS ARE I, -ABCDE

\begin{tabular}{|c|c|c|c|c|c|}
\hline B1ock & Treatment & Estimate & B1ock & Treatment & Estimate \\
\hline 1 & (1) & $\beta_{I}$ & 10 & $\mathrm{f}$ & $\beta_{F}$ \\
\hline 9 & $a(e)$ & $\beta_{\mathrm{A}}$ & 2 & aef & $\beta_{\mathrm{AF}}$ \\
\hline 11 & $b(e)$ & $\beta_{B}$ & 4 & bef & $\beta_{B F^{\prime}}$ \\
\hline 3 & $a b$ & $\beta_{\mathrm{AB}}-\beta_{\mathrm{CDE}}$ & 12 & $a b f$ & $\beta_{\mathrm{ABE}}$ \\
\hline 10 & $c(e)$ & $\beta_{C}$ & 1 & cef & $\beta_{\mathrm{CF}}$ \\
\hline 2 & $a c$ & $\beta_{A C}-\beta_{B D E}$ & 9 & $\operatorname{acf}$ & $\left(\beta_{\mathrm{ACF}}\right)^{r \mathrm{C}}$ \\
\hline 4 & $\mathrm{bc}$ & $\beta_{\mathrm{BC}}-\beta_{\mathrm{ADE}}$ & 11 & bcE & $\beta_{\mathrm{BCE}}$ \\
\hline 12 & $a b c(e)$ & $\beta_{\mathrm{ABC}}-\beta_{\mathrm{DE}}$ & 3 & $a b c(e) f$ & ${ }^{-\beta} \mathrm{DEF}$ \\
\hline 12 & $d(e)$ & $\beta_{D}$ & 3 & $d(e) f$ & $\beta_{\mathrm{DF}}$ \\
\hline 4 & ad & $\left(\beta_{A D}-\beta_{B C E}\right)^{r c}$ & 11 & $\operatorname{adf}$ & $\beta_{\mathrm{ADF}}$ \\
\hline 2 & $\mathrm{bd}$ & $\beta_{B D}-\beta_{A C E}$ & 9 & $b d f$ & $\beta_{\mathrm{BDF}}$ \\
\hline 10 & $\operatorname{abd}(e)$ & $\beta_{\mathrm{ABD}}-\beta_{\mathrm{CE}}$ & 1 & $a b d(e) f$ & ${ }^{-\beta} \mathrm{CEF}$ \\
\hline 3 & $\mathrm{~cd}$ & $\beta_{C D}-\beta_{A B E}$ & 12 & $\operatorname{cdf}$ & $\left(\beta_{C D F}\right)^{c}$ \\
\hline 11 & $\operatorname{acd}(e)$ & $\beta_{A C D}-\beta_{B E}$ & 4 & $\operatorname{acd}(e) f$ & $-\beta_{\mathrm{BEF}}$ \\
\hline 9 & $\operatorname{bcd}(\epsilon)$ & $\beta_{\mathrm{BCD}}-\beta_{\mathrm{AE}}$ & 2 & $\operatorname{bcd}(e) f$ & $-\beta_{\mathrm{AEF}}$ \\
\hline 1 & $a b c d$ & $-\beta_{\mathrm{E}}$ & 10 & abcd $f$ & $-\beta_{\mathrm{EE}}$ \\
\hline
\end{tabular}


TABLE IXb

$(2,4)$ REPLICATE - DEFINING CONTRASTS ARE I, -ABCDE

\begin{tabular}{|c|c|c|c|c|c|}
\hline Block & Treatment & Estimate & B1ock & Treatment & Estimate \\
\hline 11 & $g$ & $\beta_{G}$ & 4 & $\mathrm{fg}$ & $\beta_{\mathrm{FG}}$ \\
\hline 3 & $a(e) g$ & $\beta_{A G}$ & 12 & $a(e) f g$ & $\beta_{\mathrm{AFG}}$ \\
\hline 1 & $b(e) g$ & $\beta_{B G}$ & 10 & $b(e) f g$ & $\beta_{\mathrm{BEG}}$ \\
\hline 9 & $a b g$ & $\left(\beta_{A B G}\right)^{r c}$ & 2 & $a b f g$ & $\beta_{\mathrm{ABFG}}$ \\
\hline 4 & $c(e) g$ & $\beta_{C G}$ & 11 & $c(e) f g$ & $\beta_{C F G}$ \\
\hline 12 & $\operatorname{acg}$ & $\beta_{A C G}$ & 3 & acfg & $\beta_{A C F G}$ \\
\hline 10 & bcg & $\beta_{\mathrm{BCG}}$ & 1 & bcfg & $\left(\beta_{B C F G}\right)^{c}$ \\
\hline 2 & $a b c(e) g$ & ${ }^{-\beta_{D E G}}$ & 9 & $a b c(e) f g$ & $-\beta_{\mathrm{DEFG}}$ \\
\hline 2 & $d(e) g$ & $\beta_{\mathrm{DG}}$ & 9 & $d(e) f g$ & $\beta_{\mathrm{DFG}}$ \\
\hline 10 & $\operatorname{adg}$ & $\beta_{A D G}$ & 1 & $\operatorname{adfg}$ & $\beta_{\mathrm{ADFG}}$ \\
\hline 12 & bdg & $\left(\beta_{B D G}\right)^{c}$ & 3 & $\mathrm{bdfg}$ & $\beta_{\mathrm{BDFG}}$ \\
\hline 4 & $a b d(e) g$ & ${ }^{-\beta} \mathrm{CEG}$ & 11 & $\operatorname{abd}(e) f g$ & $-\beta_{\mathrm{CEEG}}$ \\
\hline 9 & $\mathrm{cdg}$ & $\beta_{C D G}$ & 2 & $c d f g$ & $\beta_{\mathrm{CDFG}}$ \\
\hline 1 & $\operatorname{acd}(e) g$ & $-\beta_{B E G}$ & 10 & $\operatorname{acd}(e) f g$ & $-\beta_{B E F G}$ \\
\hline 3 & $\operatorname{bcd}(e) g$ & ${ }^{-\beta} \beta_{A E G}$ & 12 & $\operatorname{bcd}(e) f g$ & ${ }^{-\beta}$ AEFG \\
\hline 11 & abcdg & $-\beta_{E G}$ & 4 & $\operatorname{abcd} f g$ & $\left(-\beta_{E F G}\right)^{r}$ \\
\hline
\end{tabular}

${ }^{c}$ Confounded with column block differences.

${ }^{r}$ Confounded with row block differences.

${ }^{r c}$ Confounded with row-column block interactions. 
TABLE Xa

$(2,8)$ REPLICATE - FULL FACTORIAL DESIGN

\begin{tabular}{|c|c|c|c|c|c|}
\hline Block & Ireatment & Estimate & Block & Treatment & Estimate \\
\hline 1 & (1) & $\beta_{I}$ & 7 & e & $\beta_{\mathrm{E}}$ \\
\hline 16 & a & $\beta_{A}$ & 9 & ae & $\beta_{\mathrm{AE}}$ \\
\hline 14 & $\mathrm{~b}$ & $\beta_{B}$ & 11 & be & $\beta_{\mathrm{BE}}$ \\
\hline 3 & $a b$ & $\beta_{A B}$ & 5 & $a b e$ & $\beta_{\mathrm{ABE}}$ \\
\hline 15 & c & $\beta_{C}$ & 10 & ce & ${ }^{\beta} \mathrm{CE}$ \\
\hline 2 & $a c$ & $\beta_{\mathrm{AC}}$ & 8 & ace & $\beta_{\mathrm{ACE}}$ \\
\hline 4 & $\mathrm{bc}$ & $\beta_{\mathrm{BC}}$ & 6 & bce & $\left(\beta_{\mathrm{BCE}}\right)^{\mathrm{rC}}$ \\
\hline 13 & $a b c$ & $\beta_{A B C}$ & 12 & $a b c e$ & $\beta_{\mathrm{ABCE}}$ \\
\hline 13 & d & $\beta_{\mathrm{D}}$ & 12 & de & $\beta_{\mathrm{DE}}$ \\
\hline 4 & $\mathrm{ad}$ & $\left(\beta_{\mathrm{AD}}\right)^{\mathrm{rc}}$ & 6 & ade & $\beta_{\mathrm{ADE}}$ \\
\hline 2 & $b d$ & $\beta_{\mathrm{BD}}$ & 8 & bde & $\beta_{\mathrm{BDE}}$ \\
\hline 15 & $a b d$ & $\beta_{\mathrm{ABD}}$ & 10 & abde & $\beta_{\mathrm{ABDE}}$ \\
\hline 3 & cd & $\beta_{C D}$ & 5 & cde & $\beta_{\mathrm{CDE}}$ \\
\hline 14 & acd & $\beta_{A C D}$ & 11 & acde & $\beta_{\mathrm{ACDE}}$ \\
\hline 16 & $\mathrm{bcd}$ & $\beta_{B C D}$ & 9 & bcde & $\beta_{\mathrm{BCDE}}$ \\
\hline 1 & abcd & $\beta_{\mathrm{ABCD}}$ & 7 & abcde & $\left(\beta_{A B C D E}\right)^{c}$ \\
\hline
\end{tabular}

${ }^{\mathrm{c}}$ Confounded with column block differences.

${ }^{r}$ Confounded with row block differences.

${ }^{r c}$ Confounded with row-column block interactions. 
TABLE Xb

\section{$(2,8)$ REPLICATE - FULL FACTORIAL DESIGN}

\begin{tabular}{|c|c|c|c|c|c|}
\hline Block & Treatment & Estimate & Block & Treatment & Estimate \\
\hline 10 & $\mathrm{f}$ & $\beta_{F}$ & 15 & ef & $\beta_{E F}$ \\
\hline 8 & af & $\beta_{\mathrm{AF}}$ & 2 & aef & $\beta_{\mathrm{AEF}}$ \\
\hline 6 & $\mathrm{bF}$ & $\beta_{\mathrm{BF}}$ & 4 & bef & $\beta_{\mathrm{BEF}}$ \\
\hline 12 & $a b f$ & $\beta_{A B F}$ & 13 & abef & $\left(\beta_{\mathrm{ABEF}}\right)^{c}$ \\
\hline 7 & $\mathrm{cf}$ & $\beta_{C F}$ & 1 & cef & $\beta_{\mathrm{CEF}}$ \\
\hline 9 & $\operatorname{acf}$ & $\left(\beta_{A C F}\right)^{r C}$ & 16 & acef & $\beta_{A C E F}$ \\
\hline 11 & $\mathrm{bcf}$ & $\beta_{\mathrm{BCF}}$ & 14 & bcef & $\beta_{\mathrm{BCEF}}$ \\
\hline 5 & $a b c f$ & $\beta_{A B C F}$ & 3 & abcef & $\beta_{\mathrm{ABCEF}}$ \\
\hline 5 & $\mathrm{df}$ & $\beta_{\mathrm{DF}}$ & 3 & def & $\beta_{\mathrm{DEF}}$ \\
\hline 11 & $\operatorname{adf}$ & $\beta_{\mathrm{ADF}}$ & 14 & adef & $\beta_{\mathrm{ADEF}}$ \\
\hline 9 & $\operatorname{bdf}$ & $\beta_{\mathrm{BDF}}$ & 16 & bdef & $\left(\beta_{\mathrm{BDEF}}\right)^{r \mathrm{c}}$ \\
\hline 7 & abdf & $\beta_{\mathrm{ABDF}}$ & 1 & abdef & $\beta_{\mathrm{ABDEF}}$ \\
\hline 12 & $\operatorname{cdf}$ & $\left(\beta_{\mathrm{CDF}}\right)^{\mathrm{c}}$ & 13 & cdef & $\beta_{\mathrm{CDEF}}$ \\
\hline 6 & $\operatorname{acdf}$ & $\beta_{\mathrm{ACDF}}$ & 4 & acdef & $\beta_{\mathrm{ACDEF}}$ \\
\hline 8 & bcdf & $\beta_{\mathrm{BCDF}}$ & 2 & bcdef & $\beta_{\mathrm{BCDEF}}$ \\
\hline 10 & abcdf & $\beta_{A B C D F}$ & 15 & abcdef & $\beta_{A B C D E F}$ \\
\hline
\end{tabular}

${ }^{c}$ Confounded with column block differences.

${ }^{r}$ Confounded with row block differences.

${ }^{r c}$ Confounded with row-column block interactions. 
TABLE $X_{C}$

$(2,8)$ REPLICATE - FULI FACTORIAL DESIGN

\begin{tabular}{|c|c|c|c|c|c|}
\hline Block & Treatment & Estimate & Block & Treatment & Estimate \\
\hline 11 & $g$ & $\beta_{G}$ & 14 & eg & $\beta_{E G}$ \\
\hline 5 & ag & $\beta_{A G}$ & 3 & aeg & $\beta_{A E G}$ \\
\hline$?$ & bg & $\beta_{B G}$ & 1 & beg & $\beta_{B E G}$ \\
\hline 9 & abg & $\left(\beta_{A B G}\right)^{r C}$ & 16 & abeg & $\beta_{A B E G}$ \\
\hline 6 & $\mathrm{cg}$ & $\beta_{\mathrm{CG}}$ & 4 & ceg & $\beta_{\mathrm{CEG}}$ \\
\hline 12 & $\operatorname{acg}$ & $\beta_{A C G}$ & 13 & aceg & $\left(\beta_{\mathrm{ACEG}}\right)^{\mathrm{c}}$ \\
\hline 10 & bcg & $\beta_{B C G}$ & 15 & bceg & $\beta_{\mathrm{BCEG}}$ \\
\hline 8 & $\mathrm{abcg}$ & ${ }^{\beta} \mathrm{ABCG}$ & 2 & abceg & $\beta_{\mathrm{ABCEG}}$ \\
\hline 8 & $\mathrm{dg}$ & $\beta_{D G}$ & 2 & deg & $\beta_{\mathrm{DEG}}$ \\
\hline 10 & $\operatorname{adg}$ & $\beta_{A D G}$ & 15 & adeg & $\beta_{A D E G}$ \\
\hline 12 & bdg & $\left(\beta_{B D G}\right)^{c}$ & 13 & bdeg & $\beta_{\mathrm{BDEG}}$ \\
\hline 6 & abdg & $\beta_{A B D G}$ & 4 & abdeg & $\beta_{\mathrm{ABDEG}}$ \\
\hline 9 & $\mathrm{cdg}$ & $\beta_{\mathrm{CDG}}$ & 16 & cdeg & $\left(\beta_{\mathrm{CDEG}}\right)^{\mathrm{rc}}$ \\
\hline 7 & $\operatorname{acdg}$ & $\beta_{\mathrm{ACDG}}$ & 1 & acdeg & ${ }^{\beta} \mathrm{ACDEG}$ \\
\hline 5 & bcdg & $\beta_{\mathrm{BCDG}}$ & 3 & bcdeg & $\beta_{\mathrm{BCDEG}}$ \\
\hline 11 & abcdg & $\beta_{A B C D G}$ & 14 & abcdeg & $\beta_{A B C D E G}$ \\
\hline
\end{tabular}

${ }^{c}$ Confounded with column block differences.

${ }^{\mathrm{r}}$ Confounded with row block differences.

${ }^{\mathrm{rC}} \mathrm{Confounded}$ with row $\mathrm{column}$ block interactions. 
TABLE Xd

$(2,8)$ REPLICATE - FULI FACTORIAL DESIGN

\begin{tabular}{|c|c|c|c|c|c|}
\hline Block & Treatment & Estimate & Block & Treatment & Estimate \\
\hline 4 & $f g$ & $\beta_{F G}$ & 6 & efg & $\left(\beta_{\mathrm{EFG}}\right)^{\mathrm{rc}}$ \\
\hline 13 & $a f g$ & $\beta_{\mathrm{AFG}}$ & 12 & aefg & $\beta_{A E Z G}$ \\
\hline 15 & $\mathrm{~b} f \mathrm{~g}$ & $\beta_{\mathrm{BEG}}$ & 10 & befg & $\beta_{B E F G}$ \\
\hline 2 & $a b f g$ & $\beta_{\mathrm{ABFG}}$ & 8 & abefg & $\beta_{A B E D G}$ \\
\hline 14 & $c f g$ & $\beta_{\mathrm{CFG}}$ & 11 & cefg & $\beta_{\text {CEFG }}$ \\
\hline 3 & acEg & $\beta_{A C F G}$ & 5 & acefg & $\beta_{\triangle C E F G}$ \\
\hline 1 & bcfg & $\left(\beta_{\mathrm{BCFG}}\right)^{\mathrm{c}}$ & 7 & bcefg & $\beta_{\text {BCEFG }}$ \\
\hline 16 & $a b c f g$ & $\beta_{A B C F G}$ & 9 & abcefg & $\beta_{\mathrm{ABCEFG}}$ \\
\hline 16 & dfg & $\beta_{D F G}$ & 9 & defg & $\beta_{\mathrm{DEFG}}$ \\
\hline 1 & $\operatorname{adfg}$ & $\beta_{A D F G}$ & 7 & adefg & $\left(\beta_{\mathrm{ADEFG}}\right)^{\mathrm{c}}$ \\
\hline 3 & $\mathrm{bd} f g$ & $\beta_{\mathrm{BDFG}}$ & 5 & bdefg & $\beta_{\text {BDEEG }}$ \\
\hline 14 & abdfg & $\beta_{\mathrm{ABDFG}}$ & 11 & abdefg & $\beta_{\mathrm{ABDEFG}}$ \\
\hline 2 & $\operatorname{cdfg}$ & $\beta_{\mathrm{CDEG}}$ & 8 & cdefg & ${ }^{\beta} \mathrm{CDEFG}$ \\
\hline 15 & $\operatorname{acd} f g$ & $\beta_{\mathrm{ACDFG}}$ & 10 & acde $f g$ & $\beta_{\mathrm{ACDEFG}}$ \\
\hline 13 & bcdfg & $\beta_{\mathrm{BCDEG}}$ & 12 & bcdefg & $\beta_{\text {BCDEFG }}$ \\
\hline 4 & abcdfg & $\left(\beta_{A B C D F G}\right)^{r}$ & 6 & abcdefg & $\beta_{A B C D E F G}$ \\
\hline
\end{tabular}

${ }^{\mathrm{c}}$ Confounded with column block differences.

${ }^{r}$ Confounded with row block differences.

${ }^{r c}$ Confounded with row-column block interactions. 
TABLE XI

\section{NUMBER OF INTERACTION PARAMETERS ESTIMABLE ${ }^{a}$ \\ AT SUCCESSIVE STAGES OF THE TELESCOPING SEQUENCE}

Interaction

Coefficient

Number of

Interactions

in the

Complete

Mode 1

Two-factor

Three-factor

\section{1}

35
Number of Interaction Parameters Estimable After Completing the Following Number of Blocks

First Row Two Rows

$\begin{array}{cccccc}\frac{4}{b_{18}} & b_{21} & & 12 & 21 & 21 \\ 0 & c_{15} & & b_{6} c, d_{25} & c_{35}\end{array}$

assumes row-column interactions to be negligible

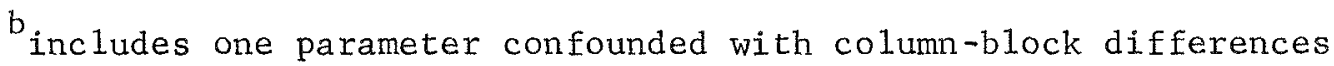

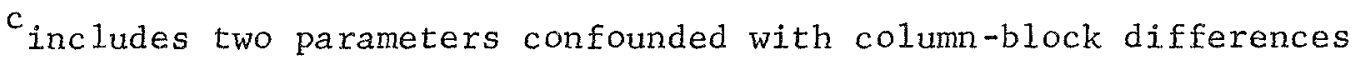

dincludes one parameter confounded with row-block differences 


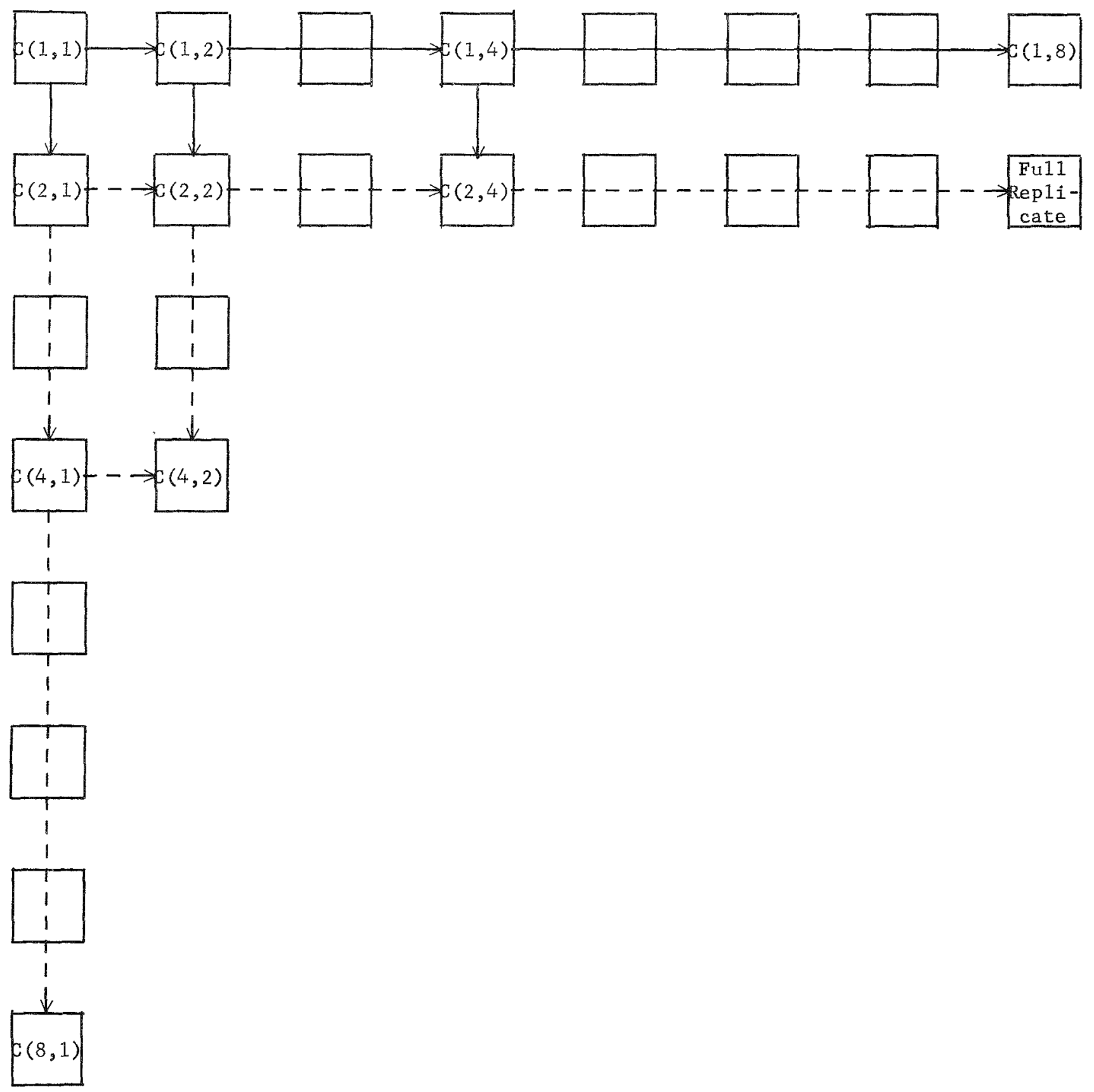

FIGURE 1

DEFINING CONTRAST AND BLOCK TELESCOPING OPTIONS FOR A $2^{7}$ EXPERIMENT (solid arrows show options contemplated for the reactor experiment; dashed arrows show other possible options)

(the $c(r, c)$ are the defining contrast groups

for the stages of telescoping consisting of $r$ row blocks and $c$ column blocks) 
FAST REACTOR SODIUM-BONDED FUEL ROD EVALUATION IN A THERMAL REACTOR

Michael H. Montgomery, Carl J. Grando, and Alfred A. Strasser

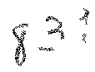

United Nuclear Corporation

The objective of this irradiation program is to demunstrate the performance of sodium-bonded, uranium-plutonium carbide fuel rods at high performance. In particular, the compatibility between fuel, sodium. and clad, the ability of a sodium bond to accommodate fuel swelling, and the integrity of the sodium bond over long periods of time at high heat fluxes are to be demonstrated. An instrumented sodium-bonded, uranium-plutonium carbide fuel capsule has been designed țo operate at a minimum linear power of $820 \mathrm{w} / \mathrm{cm}(25 \mathrm{kw} / \mathrm{ft})$ to an exposure of $35 \times 10^{20} \mathrm{fiss} / \mathrm{cm}^{3}(100,000 \mathrm{Mwd} / \mathrm{T})$. The design of the experıment is such that the power level of the capsule can be expanded to higher linear powers.

The performance of sodium-bonded fast reactor fuels can be simulated in thermal reactors more effectively than the performance of helium-bonded fast reactor fuels. The effectiveness of the two types of reactors is compared below.

Test Parameter

Fuel-Na-clad compatibility

Integrity of $\mathrm{Na}$ bond during fuel lifetime

High heat transfer
Thermal vs Fast Test Reactor

Thermal reactor is effective simulator of fast reactor.

Thermal reactor is effective simulator of fast reactor. The thermal reactor low-temperature shutdown freezes the sodium, creating a more severe test than the fast reactor high-temperature shutdowns which keep the sodium molten.

Thermal reactors currently can generate high heat fluxes anticipated in LMFBR's better than the currently operating fast reactors. 
Test Parameter

Fuel swelling

Fuel-clad interaction
Thermal vs Fast Test Reactor

The flux depression in fuels in thermal reactors gives a different temperature distribution and lower average fuel temperature than in fuels tested in fast reactors. The average temperature of (UPu)C ir radiated at $820 \mathrm{w}$, $\mathrm{cm}$ in thermal reactors is $200 \mathrm{C}$ lower than in a fast reactor. The much lower temperature of sodium compared to helium-bonded fuels makes the difference less significant. because the rate of swelling as a function of temperature is less.

The major advantage of sodium-bonded fuels is that they can be designed not to strain the clad. In that event, the different effects of thermal and fast flux on clad properties would not affect fuel rod performance.

Since space is limited in fast reactors and instrumentation is unavailable, a thermal test reactor was chosen for testing. The GETR V-RAFT facility was chosen to help maintain constant test conditions during the irradiation.

The capsule concept, as used successfully in the past by United Nuclear Corporation up to $114,000 \mathrm{Mwd} / \mathrm{T}(9200 \mathrm{hr})$ and $875 \mathrm{w} / \mathrm{cm}$. was selected as the basis for the design. The capsule is illustrated in Figs. 1 and 2. Double encapsulation is required for the irradiation of plutonium-bearing fuels in the GETR; therefore, the capsule design incorporates an inner and outer stainless steel containment shell around the fuel rod. Each containment shell is individually sealed at top and bottom by welded end plugs.

The relatively high linear power of the fuel $(820 \mathrm{w} / \mathrm{cm}$ and up) requires a low overall thermal resistance through the various capsule regions in order to satisfy the desired clad surface temperature of $600 \mathrm{C}$. Eutectic $\mathrm{NaK}$ would be desirable as the thermal bond material throughout the capsule since. unlike sodium, it is liquid at room temperature, and ther efore. would not undergo a 


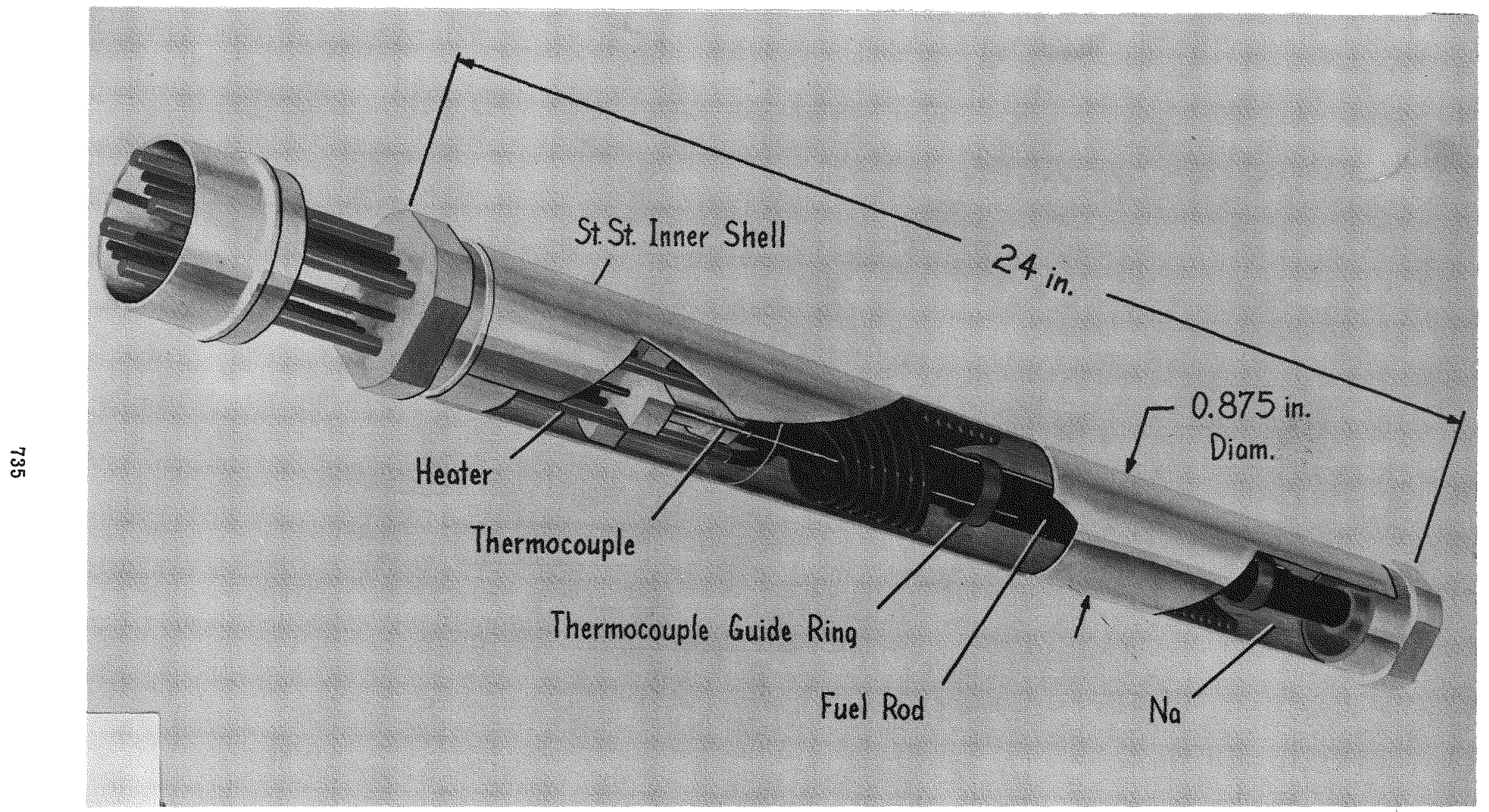

Fig. 1 - Sodium-Bonded Carbide Fuel Irradiation Experiment - Inner Shell Assembly 


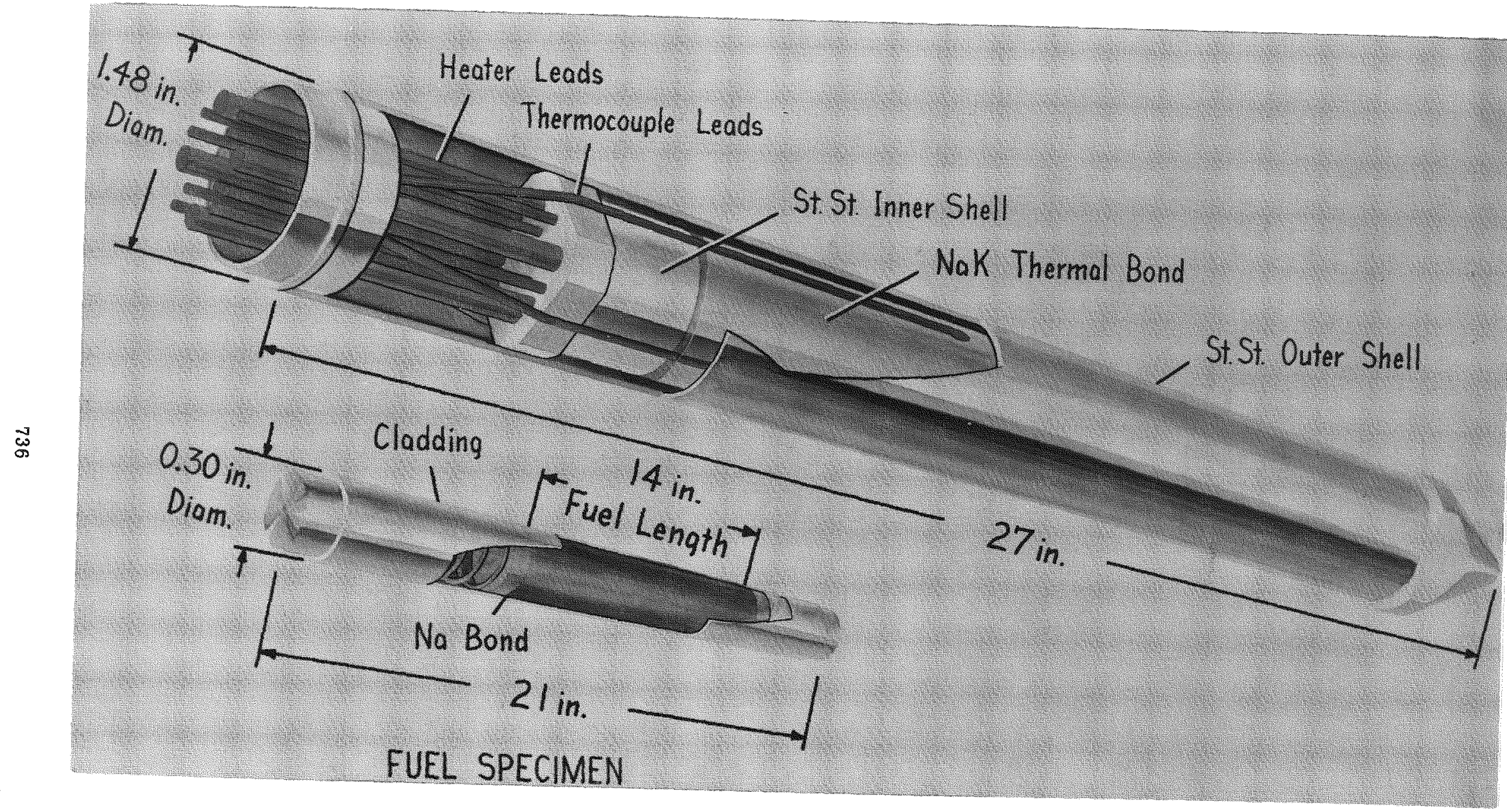
Fig. 2 - Sodium-Bonded Carbide Fuel Irradiation Experiment - Capsule
Assembly 
change of phase on startup. The relatively low thermal conductivity of NaK, how ever, would lead to a clad surface in excess of the desired $600 \mathrm{C}$ if NaK were used throughout the capsule. Sodium, therefore, with its high thermal conductivity, was selected as the thermal bond material between the specimen clad and inner shell.

Since sodium is solid at room temperature, it will undergo a large volume change at startup. The uncontrolled volumetric expansion of sodium on startup or shutdown in the fuel-clad bond and the annulus between the specimen clad and irner containment could result in damage to the components.

Various modes of damage could occur. If the fuel-clad bond melted first locally and the volume expansion were restricted by frozen sodium. the fuel clad could bulge or rupture. If a shrinkage void were formed in the fuel-clad bond or around the outside of the clad on shutdown, a rapid startup could cause local fuel or clad melting.

A heaterless design would require a more complex, careful movement of the GETR V-RAFT device to facilitate controlled melting of the sodium by fission heat. Controlled melting can be accomplished by initially positioning the V-RAFT bearing the capsule below the axial flux peak in the core, raising the facility vertically to center the fuel midplane to proceed from top to bottom, and thereby eliminate the possibility of sodium melting in confined locations. Such a design would not be satisfactory in a fixed reactor position. Also, controlled melting of the sodium can be accomplished by an electrical heater in the sodium annulus. The advantages and disadvantages of both methods are as follows:

Advantages

No Heater Simplest design to fabricate.
Disadvantages

Depends strongly on the operator for startup. Proven design not developed. 
Advantages

With Heater
Concept has been used successfully at UNC. Can melt sodium prior to startup. Simple startup procedure.
Disadvantages

Continuation of irradi ation uncertain if heat er fails.

As stated above, the startup procedures with a heater are simple, essentially requiring only the use of the heater to melt the sodium prior to startup. The added thermal resistance of a heater requires a relatively thick sodium annulus to maintain the clad surface temperature at $600 \mathrm{C}$. Failure of the heater in this case could jeopardize the experiment because of shrinkage voids in the sodi um annulus.

The thermal bond between the inner and outer containment shells presented a choice between helium, sodium, and eutectic NaK. The use of NaK offers two advantages:

1. More economical capsule fabrication; the tolerances in the thick $\mathrm{NaK}$ annulus $(0.05$ in.) can be large and easy to fabricate. A helium gas annulus must be in the order of 0.001 in. with very tight tolerances for good temperature control. The gas annulus capsule is difficult to assemble and is limited in power to about $700 \mathrm{w} / \mathrm{cm}$ if clad surface temperatures are to be kept down to $600 \mathrm{C}$.

2. More accurate determination of capsule temperatures by calorimetric means, since the thermal resistance of a thick NaK annulus remains essentially constant, and the thermal resistance of a thin gas gap can vary considerably due to thermal expansion effects.

A sodium-filled annulus between the inner and outer shells is not desirable because there is insufficient space for an electric heater to melt the sodium prior to startup. A NaK thermal bond between the inner and outer containment shells, 
however, did present the possibility of failure due to the inclusion of voids or bubbles during handling of the experiment. Experiments were performed which mocked up the inner and outer containment shells with the NaK annulus to determine if significant size bubbles or voids would remain in this region. The experiments demonstrated that bubbles could be easily removed by external vibration.

The capsule contains 11 Chromel-Alumel type thermocouples. The position of each thermocouple is illustrated in Fig. 3. Six thermocouples are attached along the length of the fuel clad and four are in the outer $\mathrm{NaK}$ annulus. The pur pose of the thermocouples is to measure the clad surface temperature as well as verify design calculations. To date, three of the four capsules have achieved a goal burnup of $50,000 \mathrm{Mwd} / \mathrm{T}$ while the fourth experiment is at $\sim 40,000 \mathrm{Mwd} / \mathrm{T}-$ all at the design power of $820 \mathrm{w} / \mathrm{cm}(25 \mathrm{kw} / \mathrm{ft})$. None of the 44 thermocouples has failed during the course of the experiment which represents $\sim 23,000$ total hours of operation. Each thermocouple operated for an approximate $6000 \mathrm{hr}$. Fig. 4 shows the operating data for Capsule UNC 127, which is typical of all four capsules. Table 1 shows that there is very good agreement between the design and measured temperatures throughout the capsule. The predicted $\Delta t$ between the clad material and the eutectic $\mathrm{NaK}$ bond was $720^{\circ} \mathrm{F}$ at the fuel midplane and compares excellently to the measured value of $745 \mathrm{~F}$ at the same point. 
TABLE 1 - TYPICAL OPERATING DATA FOR CAPSULE UNC 128

\begin{tabular}{|c|c|c|}
\hline $\begin{array}{c}\text { Thermo } \\
\text { couple } \\
\text { No. }\end{array}$ & $\begin{array}{c}\text { Predicted } \\
\text { Temp. } \\
\text { }{ }^{\circ} \mathrm{F} \\
\end{array}$ & $\begin{array}{c}\text { Measured } \\
\text { Temp. } \\
{ }^{\circ} \mathbf{F} \\
\end{array}$ \\
\hline 1 & 960 & 900 \\
\hline 2 & 1000 & 975 \\
\hline $3^{*}$ & 1060 & 1060 \\
\hline 4 & 960 & 880 \\
\hline 5 & 1000 & 1020 \\
\hline $6 *$ & 1060 & 1020 \\
\hline 7 & 370 & 320 \\
\hline 8 & 370 & 325 \\
\hline 9 & 370 & 325 \\
\hline 10 & 370 & 320 \\
\hline
\end{tabular}

${ }^{*}$ Control thermocouple located on the clad at the fuel midplane.

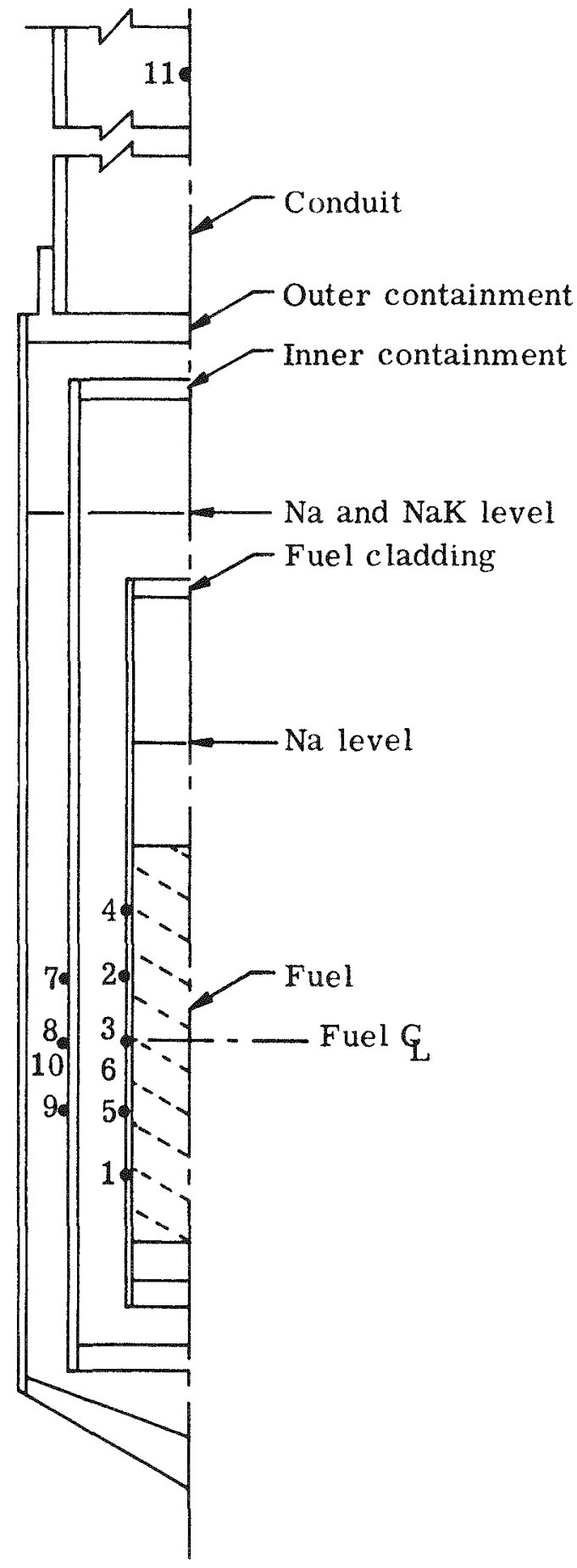

Fig. 3 - Capsule Thermocouple Locations 


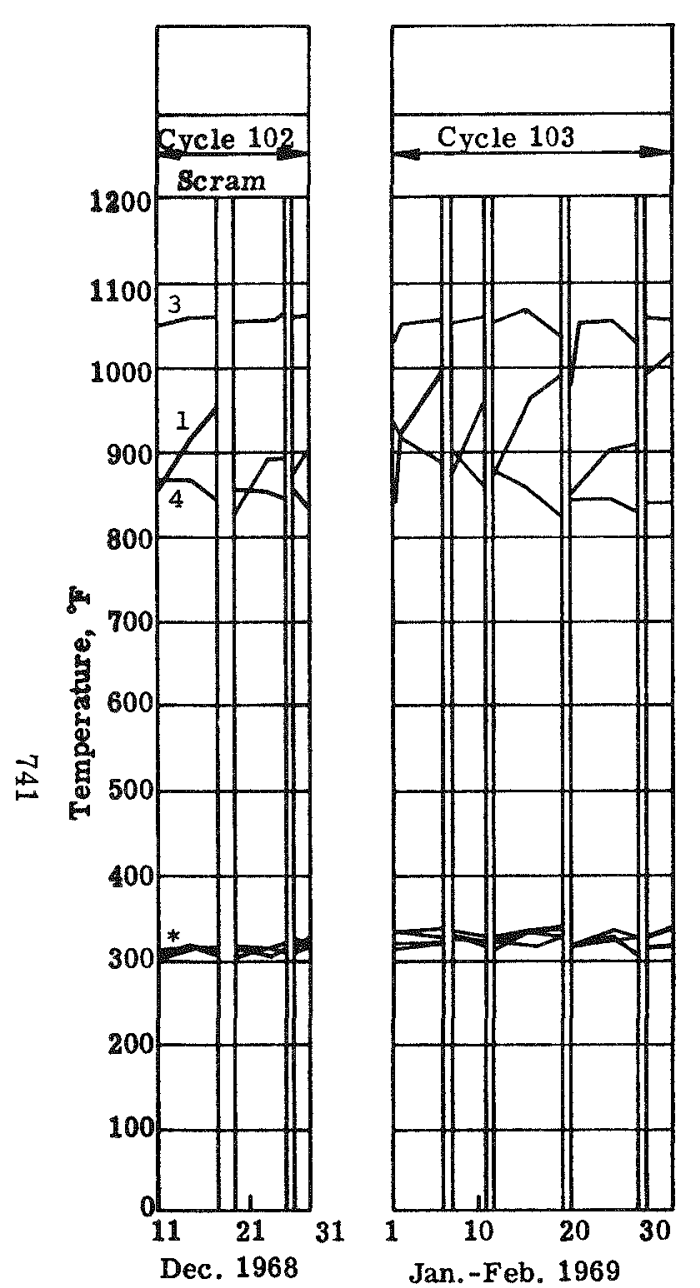

*Thermocouples 7 through 10.
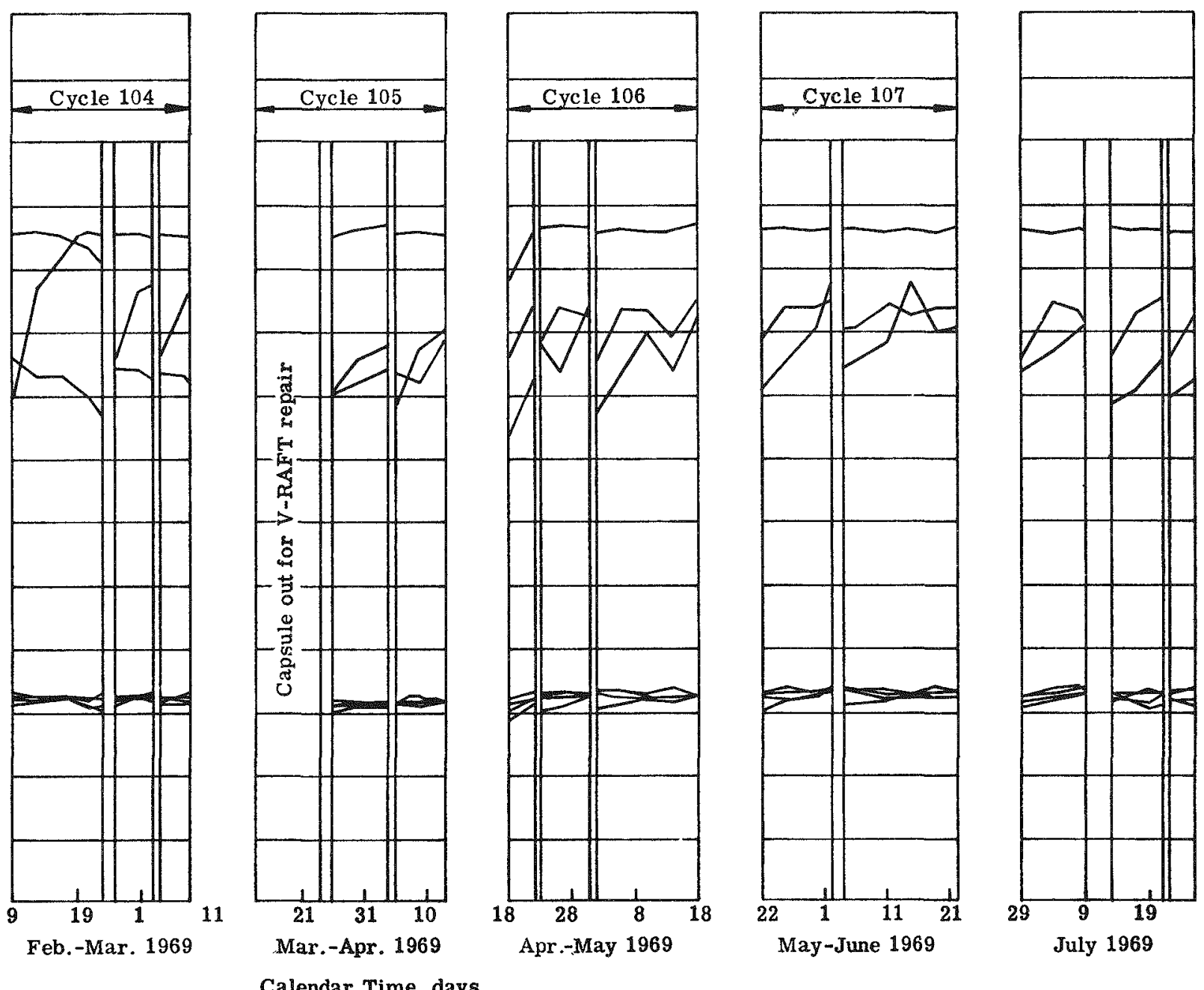

Fig. 4 - Thermocouple Readings vs

Time for Capsule UNC 127 


\section{A CAPSULE DESIGN FOR DYNAMIC IN-PILE FRICTION EXPERIMENTS}

by T. P. Jacobson, R. E. Kerkhoff, and H. H. Christenson

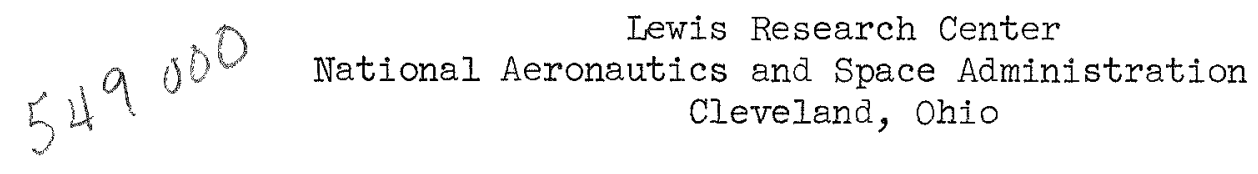

\section{ABSTRACT}

A capsule design is described for a lubricant irradiation experiment at the NASA Plum Brook Reactor Facility. The capsule contains a friction and wear test apparatus for conducting dynamic experiments in reactor radiation up to $5 \times 10^{12} \mathrm{n} / \mathrm{m}^{2}-\sec (E>1 \mathrm{Mev})$. Techniques that increase the reliability of these experiments and improve the hot lab capability of making repairs are illustrated. These techniques include special methods of measuring and controlling test specimen temperature and a modular design using linear variable differential transformers to measure friction coefficients during radiation exposure. Initial inpile operations are also discussed. 


\section{SUMMARY}

The effects of fast neutron flux and fluence on solid lubricants are being investigated in an experimental program at the NASA Plum Brook Reactor Facility. The objective of this experiment is to measure the friction force between a rider pin loaded against a rotating disk during exposure to a fast neutron flux ( $\mathrm{E}>1 \mathrm{MeV}$ ) and to measure the wear after. The effect of fluxes from $3 \times 10^{11}$ to $5 \times 10^{12} \mathrm{n} / \mathrm{cm}^{2}-\mathrm{sec}$ and fluences from $3 \times 10^{16}$ to $5 \times 10^{18} \mathrm{n} / \mathrm{cm}^{2}$ on friction and wear will be studied.

In order to make these dynamic, in-pile friction experiments, the pinon-disk specimen configuration, standard at the Iewis Research Center, had to be adopted to a capsule designed to fit a horizontal beam hole. Techniques were developed that increase the reliability of the experiment capsule and improve the capability of making hot lab repairs. These techniques include special methods of specimen temperature measurement and control and a modular design using linear variable differential transformers to measure friction coefficients during radiation exposure. Initial, in-pile operations have generated satisfactory lubrication data in spite of some unexpected difficulties which are discussed.

\section{INTRODUCTION}

A research program is being conducted to evaluate solid lubricants during exposure to nuclear reactor radiation. These data are required for nuclear propulsion and power systems for projected applications in aircraft and space vehicles. The experiment objective is to study the effect on friction and wear of fast neutron fluxes from $3 \times 10^{11}$ to $5 \times 10^{12} \mathrm{n} / \mathrm{cm}^{2}-\mathrm{sec}$ after exposure to fluences from $3 \times 10^{16}$ to $5 \times 10^{18} \mathrm{n} / \mathrm{cm}^{2}$ $(\mathrm{E}>1 \mathrm{MeV})$.

To provide these dynamic, in-pile friction and wear experiments a pinon-disk specimen configuration, standard at the Lewis Research Center, had to be adopted to a capsule to fit into the underwater horizontal 
beam hole designated HB-1. Although simple in concept, this adaptation is not easily accomplished. Complications arise due to the special requirements and uncertain factors normaliy associated with reactor experiments.

Previous studies reported in the literature of radiation effects on solid lubricants have largely been confined to post-irradiation tests. Permanent radiation effects on the lubricant can be determined by post-irradiation testing, but the transient radiation effects cannot. In order to achieve a capability for studying sliding interface phenomenon during exposure to reactor radiation, the present effort, Plum Brook Experiment 62-04, has continued.

The objective of this paper is to describe the capsule designed and used for in-pile friction and wear experiments. The development of the experiment capsule, referred to as the test section, has produced several techniques which may be useful in other in-pile applications. These techniques include test temperature control by varying the cooling of the nuclear radiation-heated test specimens and a modular design concept that permits hot lab in-cell repairs. Before discussing the test section in detail, a general description is presented of the friction and wear experiment and of the supporting systems that also had to be developed.

\section{FRICTION AND WEAR EXPERIMENT}

The friction and wear test apparatus consists of a hemisphericallytipped (3/16-inch radius) rider pin which is loaded against the flat of a rotating disk specimen. The friction and wear experiment involves recording the friction force between the rider and disk during the experiment and measuring the rider wear scar and disk wear track after the experiment. When using this test to evaluate solid film lubricants, the experiment is terminated when the friction increases to a pre-set value. The length of this experiment, or the number of cycles to failure, determines the wear life of the film. The operating conditions initially desired in this experiment include rider loads to 1000 grams (intial Hertz surface stress of 140,000 psi), disk speeds to 5000 rpm and test temperatures to $1500^{\circ} \mathrm{F}$ in a dry air environment.

The test specimens are shown inside their polystyrene storage case in Figure 1. To provide more efficient data generation, two rider pins are consecutively loaded against the same disk. One rider is loaded. against the disk at a $l$-inch radius; the other rider displaced $180^{\circ}$ is loaded at $3 / 4$-inch radius. For a larger range of obtainable test temperatures, two disk thicknesses are used; a 0.1 inch thick disk for low temperatures and a 0.3 inch thick disk for higher temperatures. In the same radiation field, the 0.3 inch disk generates about three times the heat of the 0.1 inch disk. Two chromel-alumel thermocouples are imbedded $1 / 4$ inch deep into the rim of the disk 0.050 inch from the surface. These termocouples measure disk temperature during irradiation until they break upon disk rotation. 
The experiment facility includes (1) an insertion system, (2) a cooling system, and (3) an experiment control system as well as four test sections. The location of these systems at Plum Brook is shown in Figure 2 .

The insertion system consists of a drive table, located underwater on the floor of Quadrant $D$; the $\mathrm{HB}-1$ beam hole seal assembly; and the control console, located on the grating above Quadrant D. The insertion system permits the test section to be inserted or withdrawn independently of reactor operation. The proximity of the test section to the reactor core can be adjusted by increments of 0.01 -inch. This allows a fine control over the radiation exposure.

The cooling system is a recirculating air loop driven by two 50 scfm diaphragm-type compressors located in the test corridor behind Quadrant D. Compressed air is stored in a 6 cubic foot receiver tank at pressures to 500 psig. A 10 cubic foot receiver tank, kept at partial vacuum, is used to collect the exhaust air during purging of the test sections and to permit emergency coolant flow in case of compressor failure.

The cooling system in the test section consists of two independent flow paths: disk cooling air and secondary cooling air. The test temperature of the disk is maintained over a range of gamma heating rates by varying the disk air flow from 6 to $60 \mathrm{scfm}$. This flow rate can be controlied either manually, based on the test specimen temperature, or automatically, based on a nearby reference thermocouple signal. The secondary cooling air, set at 15 to $30 \mathrm{scfm}$, provides constant cooling of the remaining test section parts. A constant 60 psia pressure is maintained inside the test section by a backpressure valve controlled by a pressure transducer located inside the test section. To approximate the chemical kinetics of specimen oxidation in air at standard pressure, a synthetic air mixture of $95 \%$ nitrogen and $5 \%$ oxygen is used as the coolant. Besides providing an oxygen partial pressure of 0.2 atmospheres, this mixture has a reduced argon content of less than 10 parts per million compared to 1 percent in normal air. The resulting reduction of radioactive argon eliminates shielding requirements for the air loop equipment.

The experiment control console is remotely located in the Experiment Control Room Annex. The lubricant test conditions are controlled and monitored at this console. These conditions include the test section pressure, the disk and secondary air flows, the disk motor speed, and the rider pin load. The friction force and the rider load are recorded on a strip chart. An existing data logging system, EDLAS, provides a computer print-out of all other experiment data. A remote read-out for the EDIAS data points is located in the control console.

TEST SECTION DESCRIPTION

Radiation effects on the friction and wear apparatus are minimized by positioning the test specimens close to the reactor core while keeping the support hardware as far away as possible. The drive motor and 
loading system are located away from the core but the friction measuring system could not easily be remote from the test specimens. Thus, a radiation resistant linear variable differential transformer (IVDT) was chosen as the friction measurement transducer. To ensure reliability two redundant IVDT units are used.

Because of repeated exposures to high radiation levels, the friction measuring system could fail to function while the rest of the apparatus is still operating normally. In grder to extend the usefulness of the test section, this system was designed as a replaceable module. This modular approach to capsule design resulted in the test section, shown in Figure 3, consisting of an outer tube, an inner assembly, and a friction module.

\section{OUTER TUBE}

The outer tube is 9 feet long with an in-pile section diameter of 6 inches and a 1/4 inch wall. This shape was chosen so that other facilities such as the HB-2 hot cave could be used. The tube is 6061-T6 aluminum with a hard anodize surface. Reactor primary cooling water flows along the in-pile surface. The outer tube temperature is measured by eight thermocouples equally spaced around the inside front rim of the tube. The resulting temperature distribution indicated provides an approximate measurement of the magnitude and gradient of the gamma heating rate. A front end cap, sealed by a polyurethane "O" ring, provides access to the test specimens and the friction module. (Because of the operating experience discussed below, polyurethane "O" rings are used only for low fluence experiments. At higher fluences, metal seals will be used.) Two different end caps can be bolted onto the tube. One is designed to fit around the 0.1 inch thick disk specimen; the other, around the 0.3 inch thick disk. The appropriate fit provides the proper air gap around the disk to maintain the same cooling characteristics for both disks. The front end cap contains four sets of nickel and cobalt flux wires. When analyzed after an insertion, these wires indicate the fast and thermel fluences, from which the flux and flux gradients can be determined. The back flange, sealed by a neoprene "O" ring provides access to the inner assembIy. The inside of this flange contains terminal strips for connecting the lead wires from the friction module. The outside of this flange contains a lifting ring and a " $V$ " block, which connects to the insertion drive universal joint.

The two upper flanges at the back, sealed by neoprene "O" rings, contain the test section penetrations. Cooling air is provided through three quick-disconnect couplings: two inlets and a common return. The pneumatic loading system requires four quick-disconnect couplings: one for each rider, one for the reference pressure, and one to unlock the system. Three 37 pin electrical connectors are required: two for the chromel-alumel thermocouples and one for the copper electrical leads. The electrical connectors are each connected to cables installed in air pressurized flexible conduit to prevent shorting due to quadrant water. 
The inner apparatus is cooled by two separate air flows. The disk cooling air is ducted to the front of the test section and around the drive shaft. The air passes over the test specimens and returns inside the shaft and through the perforated coupling; from there it is ducted past the motor. The secondary air is also ducted to the front end of the test section. This air then passes over the friction measuring IVDT's and returns past the front bearing, loading system, shields, rear bearing, and mixes with the disk air behind the motor. The return air exits the test section through a 0.3 micron particulate filter. The filter restricts radioactive wear debris from being exhausted out of the test section.

The disk drive motor is a compact 1 HP induction motor, 3.75-inches in diameter and $10-$ inches long. It is radiation resistant up to $10^{9}$ rads, the limitation of the polyimide insulation. Speed is varied from 900 to $5000 \mathrm{rpm}$ by frequency control. The hollow drive shaft is fabricated. from 600T nickel alloy. The shaft is supported by two bearings. The front bearing is a carbon bushing which is held by a shrink fit in a. titanium housing. The rear bearing is a singie row, deep groove ball bearing with 4400 stainless steel balls and a stamped retainer.

The rear bearing and the two motor bearings are lubricated with a dyethickened polyphenyl ether grease. This grease can withstand temperatures to $500^{\circ} \mathrm{F}$ and radiation exposure to $10^{9} \mathrm{rad}$. In order to extend the life of this grease, two inches of lead shielding is located in front of the rear bearing. Three and a half inches of stainless steel is located in front of the lead to reduce the internal heat generation in the lead. A 0.02 -inch thick sheet of chemically pure cadmium, sandwiched between aluminum, is located ahead of the steel to reduce the activation of the shield assembly.

Both bearing supports fit with close clearance $( \pm 0.002-$ inch $)$ into the outer tube, which has steps machined inside to locate the supports. This close fit provides a rigid support for the drive shaft bearings and a good heat conduction path to the water-cooled outer tube. An induction proximeter probe, located 0.030 -inch away from the shaft at the midpoint between the bearings, measures shaft vibration in case of unstable operation.

The loading system consists of three pneumatic piston-cylinder assemblies. The upper and lower assemblies load the lower and upper riders at $3 / 4$ and 1 -inch, respectively. A pressure difference of 47 psia across the piston produces a 1000 gram tension in the cable connected. to the pivoted rider holder. The third assembly retracts a springloaded locking pin which prevents undesired rider contact before the test load is applied.

The load is measured by an LVDT-proving ring assembly located between the load piston and the cable and shown in Figure 4. A thermocouple is welded to the ring to provide for temperature calibration. A change was made from the original test section design because of difficulties with binding of the piston and with calibrating the IVDT. 
This change enables the loading system to be calibrated before installation in the test section and to be replaced if necessary. Special electrical terminal blocks, shown in Figure 5, were made for the IVDT and thermocouple leads. The blocks were made from lava (hydrous aluminum silicate) and fastened to the inner tube wall.

\section{FRICTION MODULT}

The friction module is bolted onto the front rim of the test section outer tube. The friction module parts, shown in Figure 6 , include a baffle tube, yoke ring, IVDT plate, rider holder, and a baffle plate, all anodized aluminum, plus four inconel leaf springs and two flexure pivots. The arms of the yoke ring hold the rider holder in a trunion arrangement of the flexure pivots. The rider holder is shown in Figure 7 with the upper rider pin installed. Pulling back on the lower cable slot will load the upper rider against the disk at a 1 inch radius. The wires attached to the rider holder are for the rider holder temperature thermocouple and for the upper and lower rider temperature thermocouple connections.

The rider pin thermocouple arrangment is shown in Figure 8. A chromelalumel thermocouple is imbedded 0.050 inch from the tip of the roider. The thermocouple wires are threaded through a lava insert and are welded to the chromel and alumel rings at the base of the insert. The rider holder connector consists of three lava wafers which locate the alloy wires on the back of the holder. The thermocouple connection is made simply by inserting the rider into the rider holder. The tip of the rider is grooved to make this insertion (and removal) easier in the hot lab.

The thermocouple wires from the rider holder are connected to alloy pins located on the front side of the IVDT plate shown in Figure 9. Connector pins for the two disk thermocouples are also on this plate. The other wires on the IVDT plate are for the two LVDT plate thermocouples, the two friction measuring IVDT thermocouples, and the baffle plate thermocouple. All wires are five mil diameter alloy wire with a half mil baked on ceramic insulation. Two-hole ceramic bead is used for plate penetration and clamping. The baffle plate thermocouple is swaged in a stainless steel sheath bent to produce a slight spring force to locate the thermocouple firmly into a counter-sunk hole in the baffle plate. Signals from the baffle plate, rider holder, and. IVDT plate go to the four safety channel recorders located in the Experiment Control Room. These channels control alarm, automatic experiment abort, and reactor scram in case of over-temperature. The baffle plate thermocouple signal is also used to automatically control the disk cooling air flow in order to maintain a fixed disk temperature after disk rotation.

The thermocouple wires penetrate the LVDT plate and are connected to a lava terminal block on the back side of the plate. The terminal block, shown in Figure 10, is attached to the cable harness which is fastened to the IVDT plate. The LVDT wires from the friction module cable are connected to the IVDT units through two other terminal blocks made from lava. 
The IVDT terminal block is shown in Figure 11, both before and after design changes to the friction measuring system were made. These changes were required to eliminate binding of the core inside the LVDT and to simplify core adjustment. The changes to the LVDT clamp and terminal block has resulted in an improved method of replacing the IVDT unit. As shown in figure 12, the only disassembly required is the removal of the IVDT clamp screw, the terminal block screw, and half of the terminal block. This operation can be made in the hot lab with the friction module completely assembled.

The assembly of the friction module is facilitated by using a universal mount as shown in Figure 13. The yoke ring, with the four leaf springs attached, is about to be connected to the IVDT plate by fastening the leaf springs inside the plate mounts. The arms of the yoke ring then extend through holes in the plate and the IVDT core is connected to the yoke ring arm.

The assembled friction module is shown in Figure 14. The presence of the hex driver illustrates the clearance with the thermocouple wires. The friction force, indicated in Figure 14, is transmitted to the yoke ring which is restrained by the four leaf springs. The resulting movement of the yoke arms is measured by the change in position of the LVDT cores connected to the yoke arms. The baffle plate is required to prevent the high velocity disk cooling air from striking the lubricated surface of the disk and to thermally shield the rider holder from the disk during high temperature tests. In order to install the friction module in the test section, the leads are grouped in a cable which is snaked through the test section and connected to the terminal strips on the back flange. Another cable then goes from these terminal strips to the appropriate electrical connectors on the inside of the upper flange.

\section{CALCUIATIONS}

To have a good degree of confidence that the test section would operate in $\mathrm{HB}-1$ without exceeding its capabilities and would have an adequate lifetime for data acquisition, the test section was designed to operate in the "worst case" conditions and to have a minimum operating lifetime of six months.

The worst case conditions were determined by the uncertainties of both the reactor parameters and test section design factors.

The maximum reactor parameters that were used are the radiation values inside the $\mathrm{HB}-1$ beam hole next to the reactor core. These values are:

$$
\begin{array}{ll}
\text { Fast neutron flux: } & 7.5 \times 1012 \mathrm{n} / \mathrm{cm}^{2} / \mathrm{sec} \\
\text { Thermal neutron flux: } & 2.4 \times 1014 \mathrm{n} / \mathrm{cm}^{2} / \mathrm{sec} \\
\text { Gamma heating: } & 6 \text { watts } / \mathrm{gram}
\end{array}
$$

Of all the test section design factors, the contact resistance for heat transfer, and the actual air flow rates in various channels, were identified as the two factors having the greatest uncertainty. These two factors were assigned uncertainty values of $25 \%$ and $10 \%$. 
Using the uncertainties of the input parameters, a computer program determined temperature distributions in the test section during various off-normal transient conditions and established the operating envelope of the apparatus.

The items that would determine the operating lifetime of the Test Section were identified as:

\section{The front carbon bearing.}

2. The grease in the rear ball bearing.

3. The LVDT units.

4. The bearing grease and insulation of the disk drive motor.

Because of the sparse radiation damage information available for these items for this particular application, the operating lifetime that will be actually achieved is not known at this time. However, a useful lifetime is anticipated based on the chosen combination of design and material. Furthermore, contributions to the state-of-the art radiation damage information for these items is expected by performing thermal, epithermal, and fast flux mapping, and by using "lifetime" dosimeter wires locations in each test section.

\section{IN-PIIE OPERATTON}

Prior to the initial in-pile operation, an expected temperature band was calculated based on the uncertainties of the reactor input parameters, for the disk and other selected parts of the test section, in order to varify proper operation of the temperature readout systems. During the first irradiation of the test section, temperatures fell below this calculated uncertainty band. Apparently, the test section uncertainty values, especially the contact resistances and the cooling air flow distribution, were too conservative. Comparisons of component temperatures obtained during operation to those calculated by the computer were difficult to make reliably. The appropriate air flows could not be obtained during irradiation due to control problems with the cooling air system. Based on the thermocouple readings from the front end of the water cooled outer tube, the gamma heating rate could be estimated. For the test section fully inserted and the reactor operating at $60 \mathrm{MW}$, the gamma heating rate was 2 watts/gram.

After about 20 hours in-pile, the experiment equipment suddenly shut down and the test section automatically aborted from the beam hole. The abort was caused by failure of the end cap polyurethane "O" ring, which allowed water to leak into the test section. Iater inspection revealed that the "O" ring, which had received a dose of about $10 \times 10^{9}$ rads, had taken a set, become brittle, and had cracked. Subsequent tests in the Plum Brook Reactor rabbit tube facility have shown that for this application polyurethane is reliable up to about $7 \times 10^{8}$ rads but doubtful above $4 \times 10^{9}$ rads. The test section was successfully dried out by alternately flowing hot dry nitrogen gas 
through it and by pulling a vacuum in it. Resistance values of the motor and instrument leads eventually returned to their original values after which power was applied to verify proper operation.

While recalibrating the IVDT units, the upper loader failed to operate. Rather than disassembling the test section to repair this loader, the irradiation program was continued even though only one wear track could be made. The intent was to use this test section for further design check-out so that corrections could be incorporated in the other test sections before irradiation.

The radiation exposure of the second irradiation was limited because of the radiation damage threshold of the polyurethane " $O$ " ring. The test section was inserted into the beam hole position 6 inches back from full insertion. After an hour and twenty minutes of irradiation with the reactor operating at $40 \mathrm{MW}$, a friction experiment was successfully performed. An Mos 2 solid lubricant coating, bonded to a sand-blasted surface of a $440-C$ stainless steel disk, was evaluated under the following conditions: a disk temperature of $140^{\circ} \mathrm{F}$, a rider load of 500 grams, and a speed of $1000 \mathrm{rpm}$. The friction coefficient was 0.10 , a typical value for nonirradiated coatings. No coating failure occurred during the 45 minutes of dynamic operation. The fast neutron fluence received was $8.3 \times 10^{15} \mathrm{n} / \mathrm{cm}^{2}$ ( $\mathrm{E}>1 \mathrm{Mev}$ ) at a flux of $1.7 \times 10^{12} \mathrm{n} / \mathrm{cm}^{2}-\mathrm{sec}$. The gamma heating rate was 0.5 watts/ gram and the dose received was $3.6 \times 10^{8} \mathrm{rad}$.

\section{CONCLUDING REMARKS}

In the design, fabrication, and operation of the dynamic, in-pile friction experiment capsule, several useful techniques have been developed.

1. Test temperatures are controlled by varying the cooling air flow to the nuclear radiation-heated test specimens.

2. Temperature measurements are facilitated by specially designed thermocouples and thermocouple connectors.

3. Linear variable differential transformers (IVDT) operate successfully as transducers in reactor radiation.

4. The usefulness of the capsule and the ability to make capsule repairs in the hot lab are improved by a modular design of the capsule. 


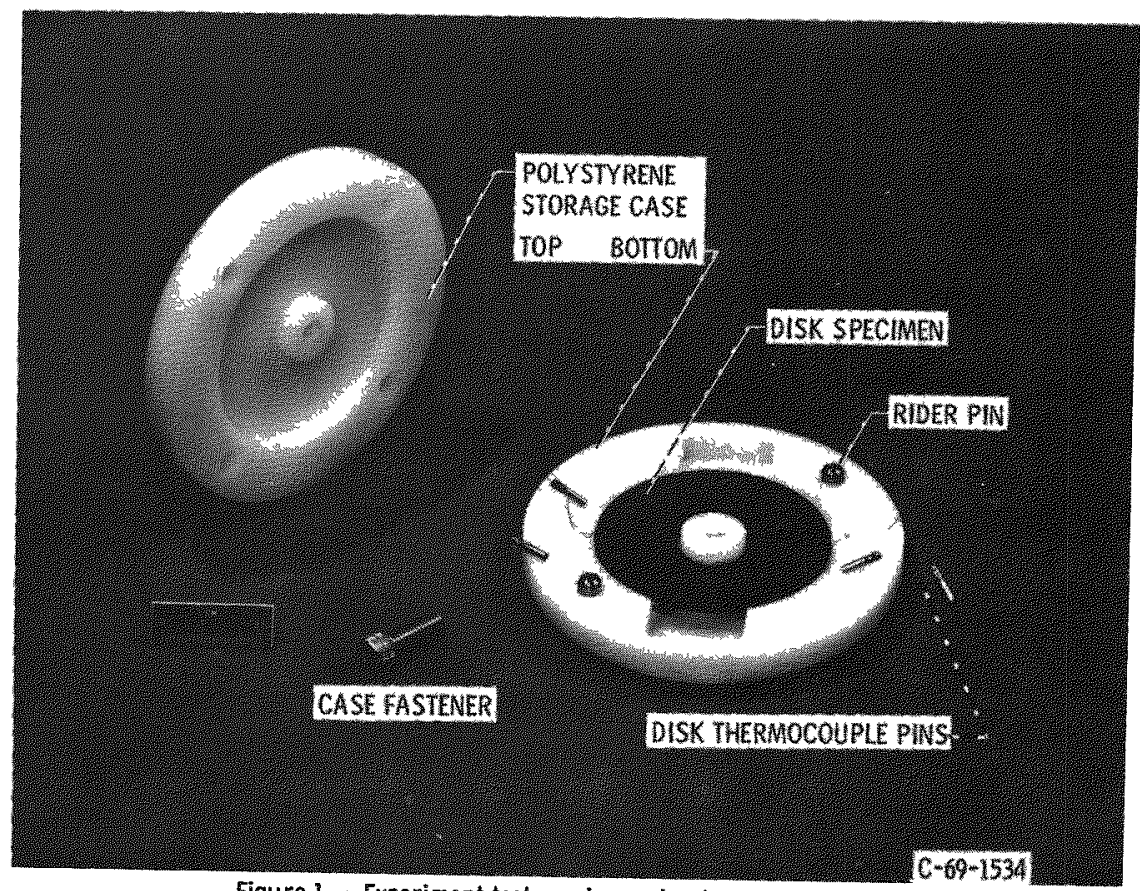

Figure 1. - Experiment test specimens in storage case.

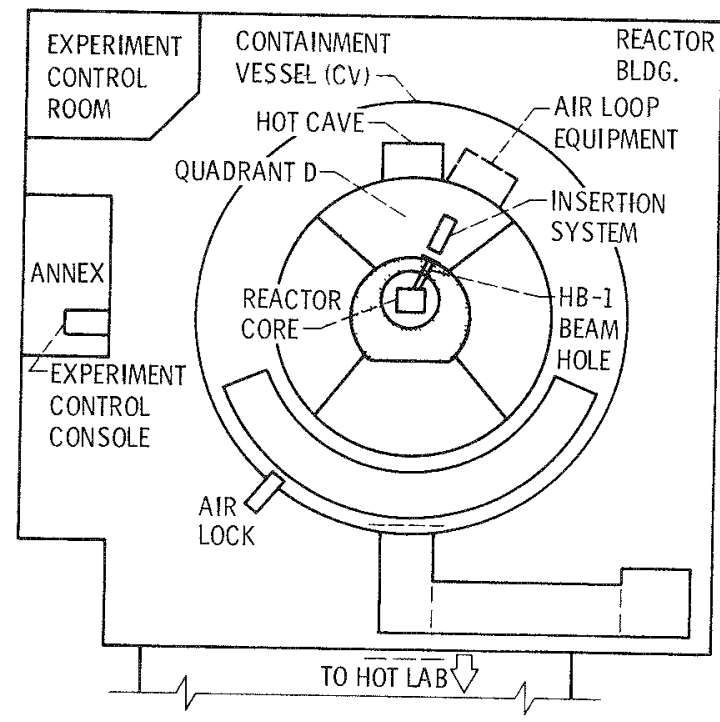

Figure 2. - Equipment location in Plum Brook Reactor Facility. 


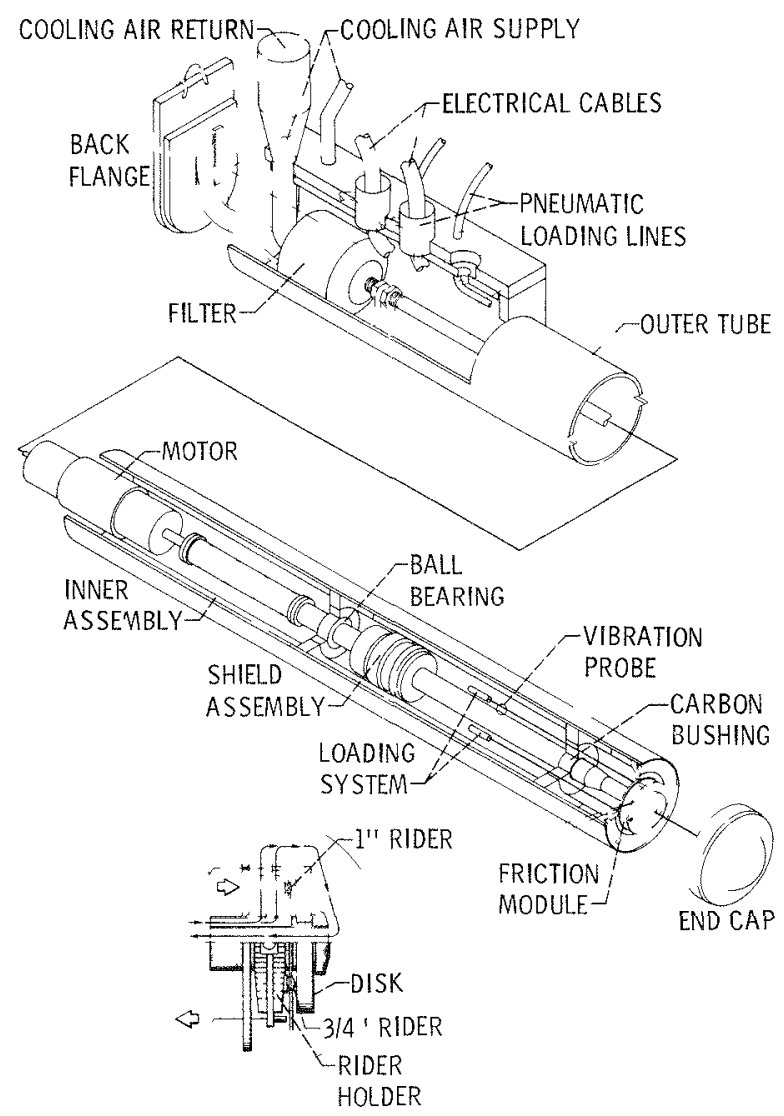

Figure 3 - Perspective drawing of the test section for dynamıc, in-pile friction and wear experiments

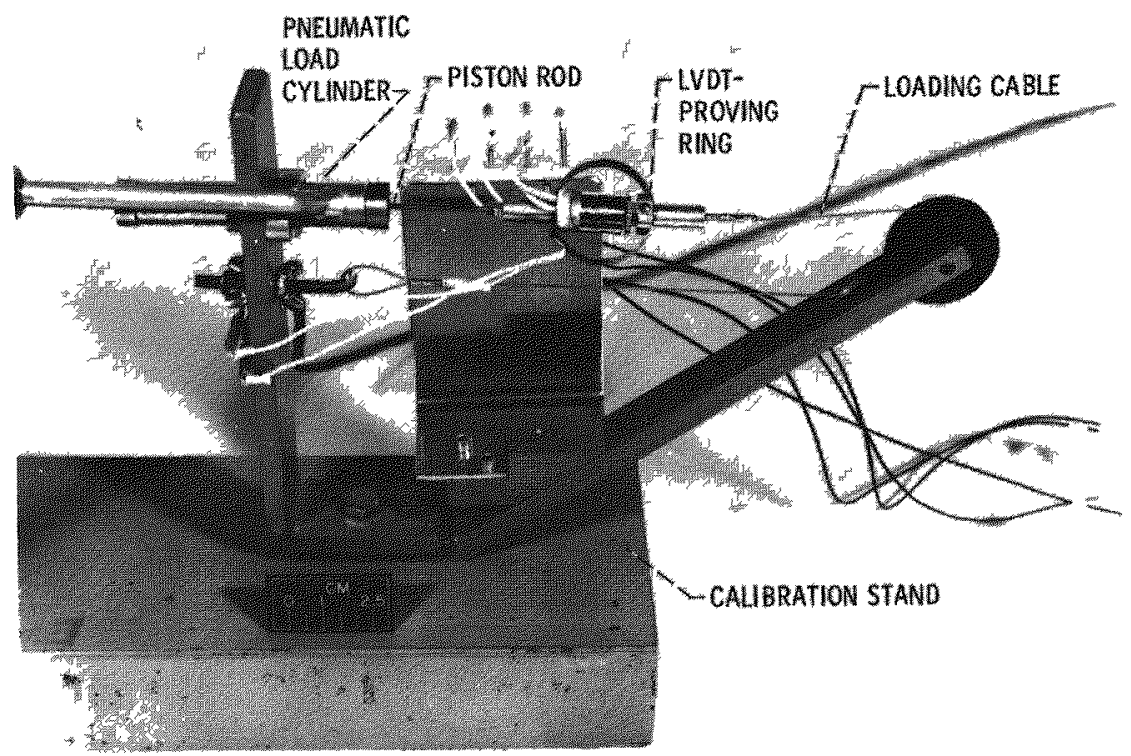

Figure 4 - Experıment loading system on calıbration stand 


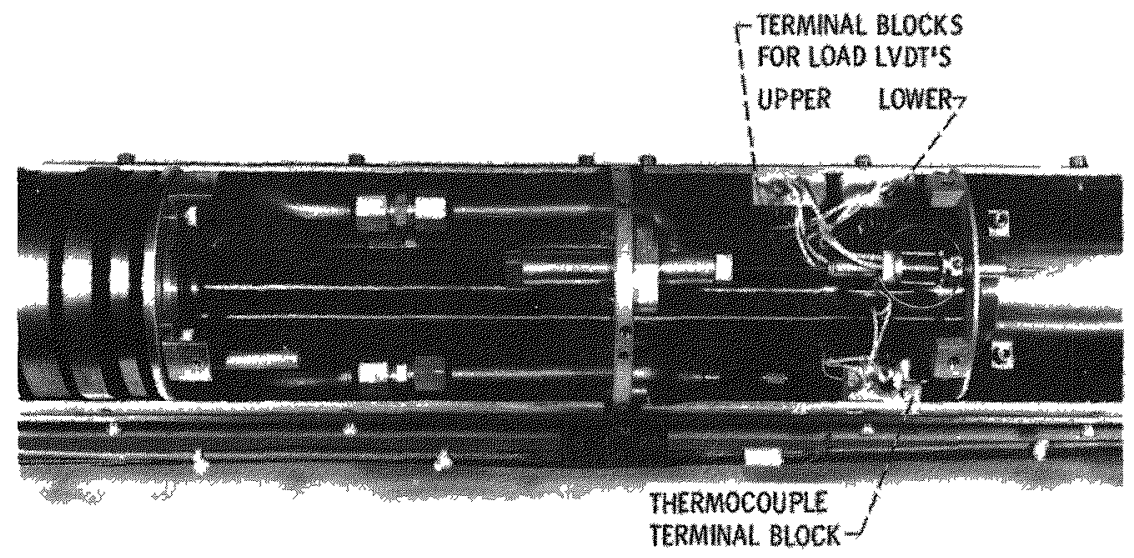

C. $69-1834$

Figure 5 - Loadıng system installed in experiment test section

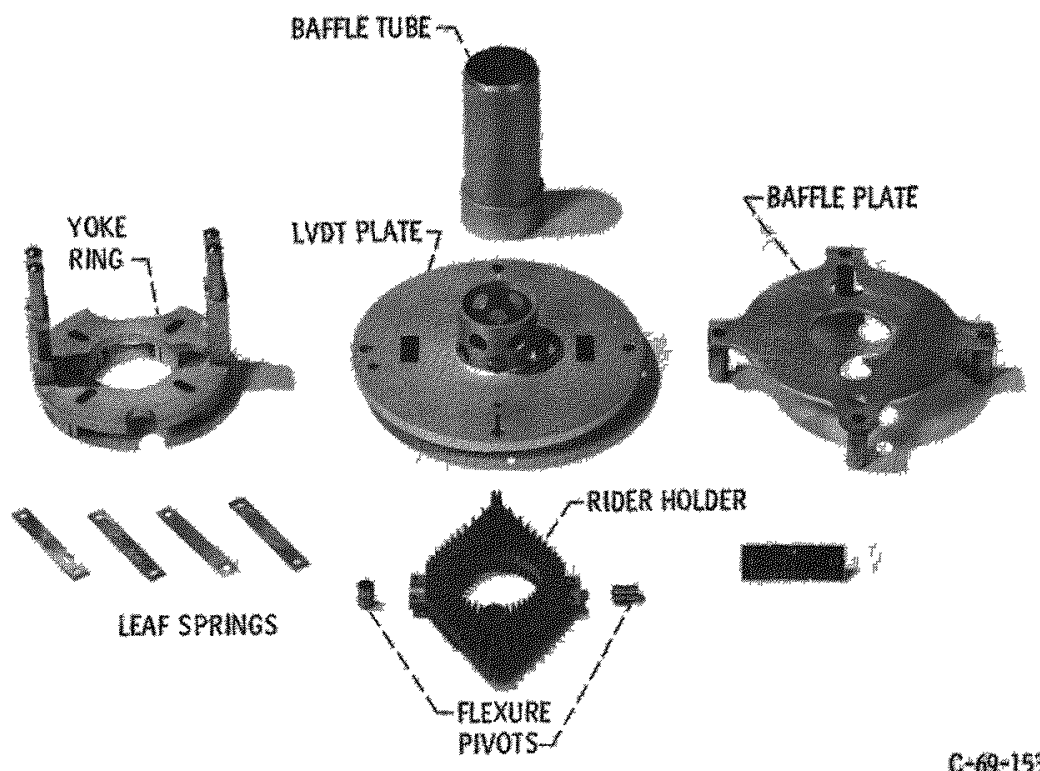

Figure 6 - Parts of friction module for experiment test section

$6+69+1532$ 


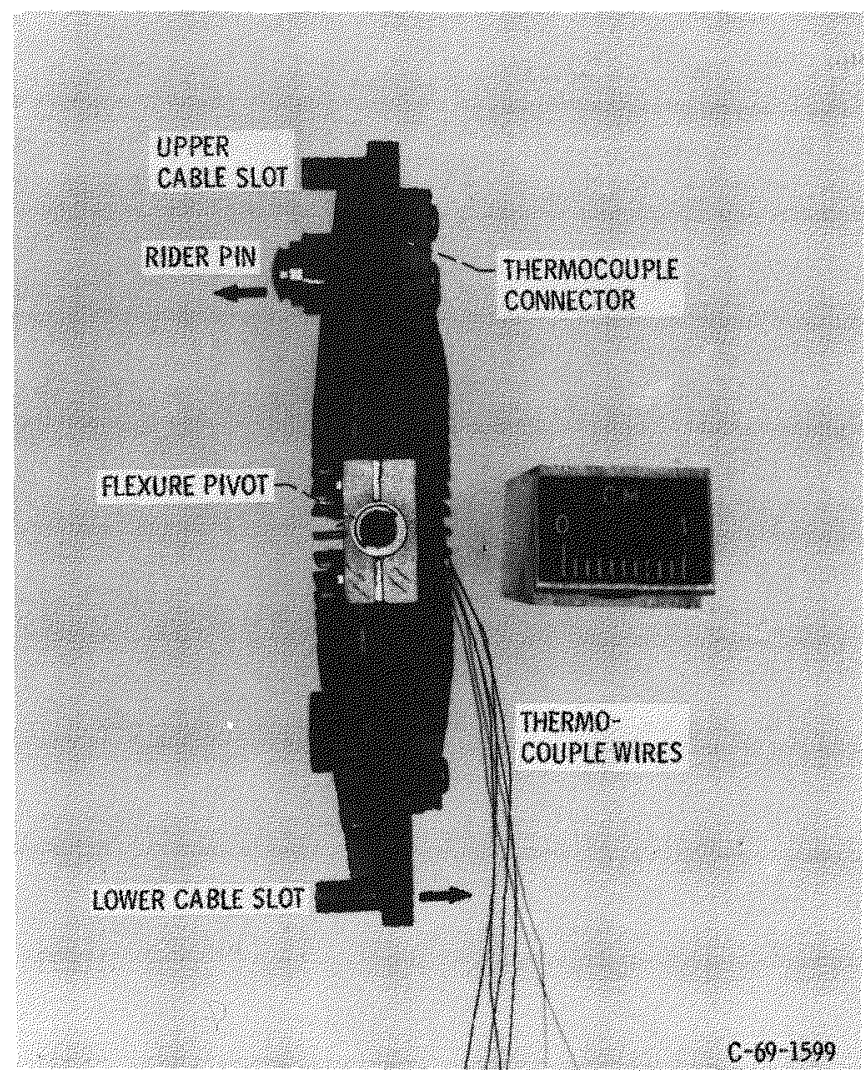

rigure 1. - Rider pin installed in rider holder of experiment test section.

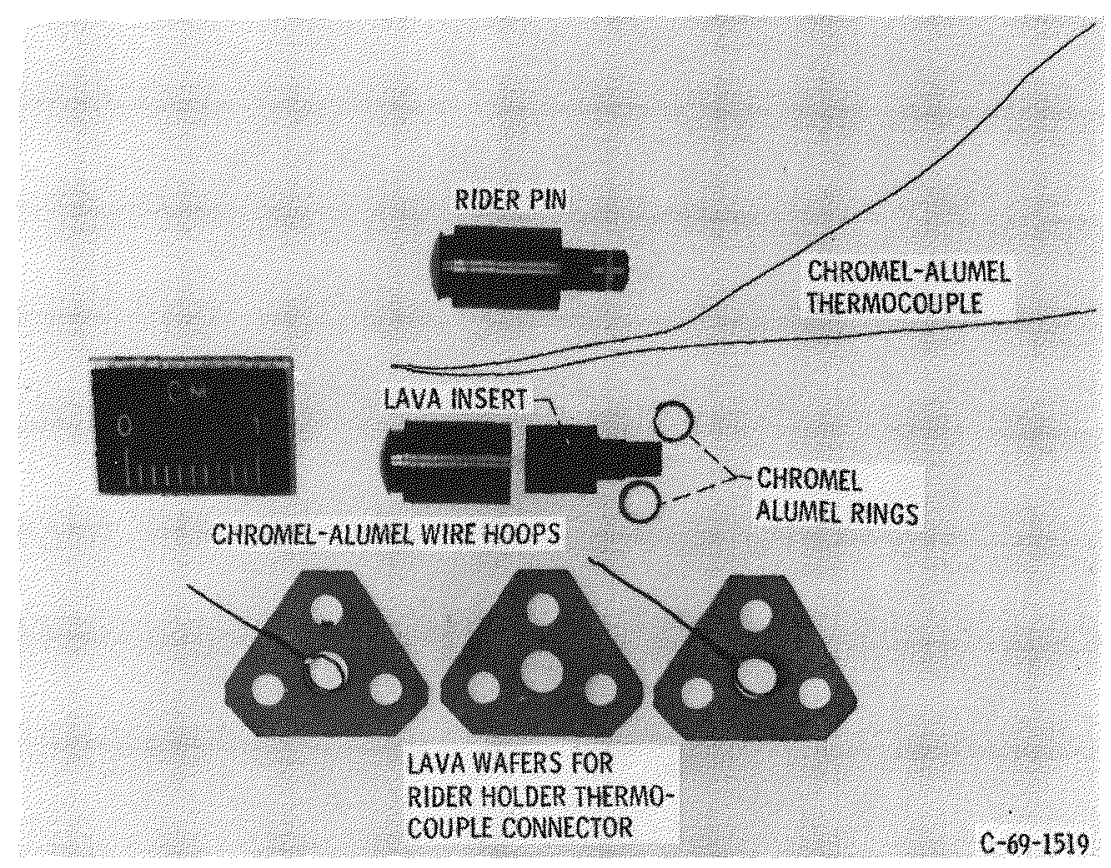

Figure 8. - Rider pin thermocouple arrangement in experiment test section. 


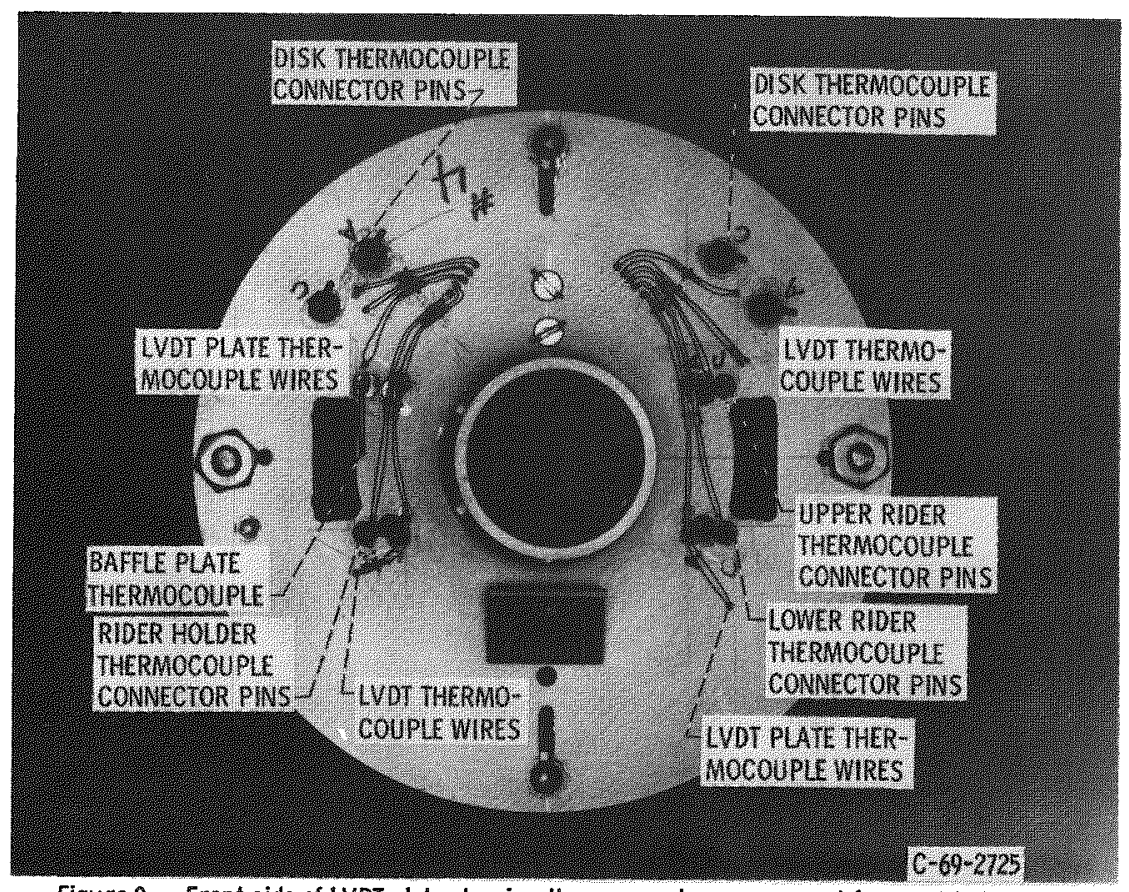

Figure 9. - Front side of LVDT plate showing thermocouple arrangement for the friction module of the experiment test section.

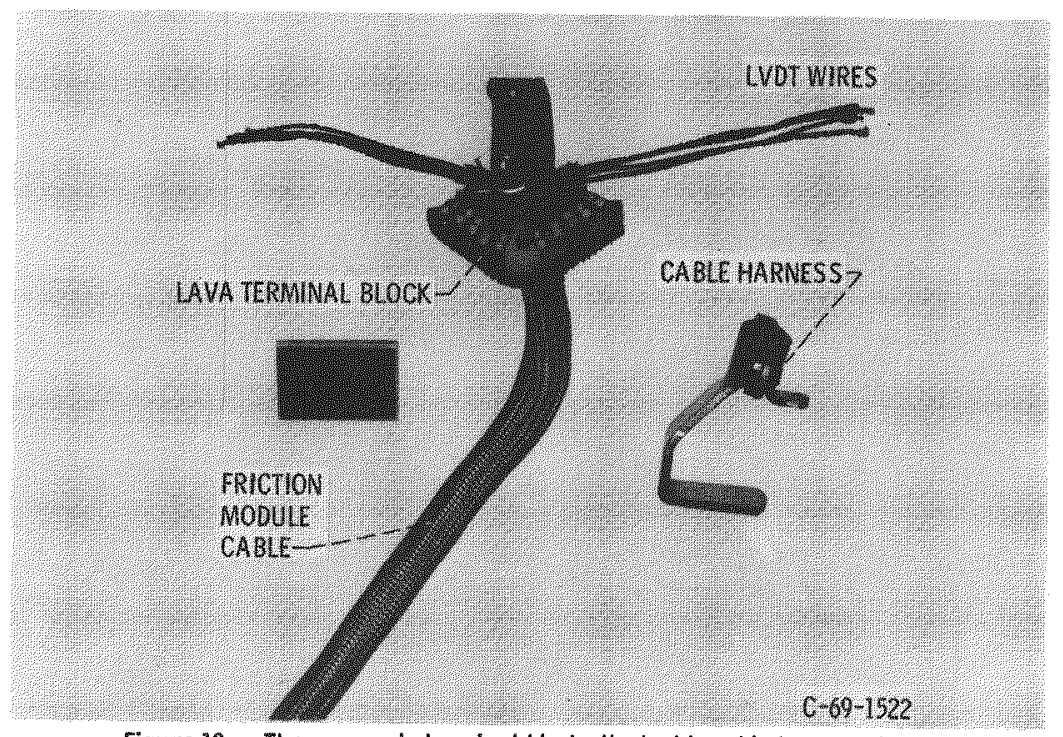

Figure 10. - Thermocouple terminal block attached to cable harness for the friction module of experiment test section. 


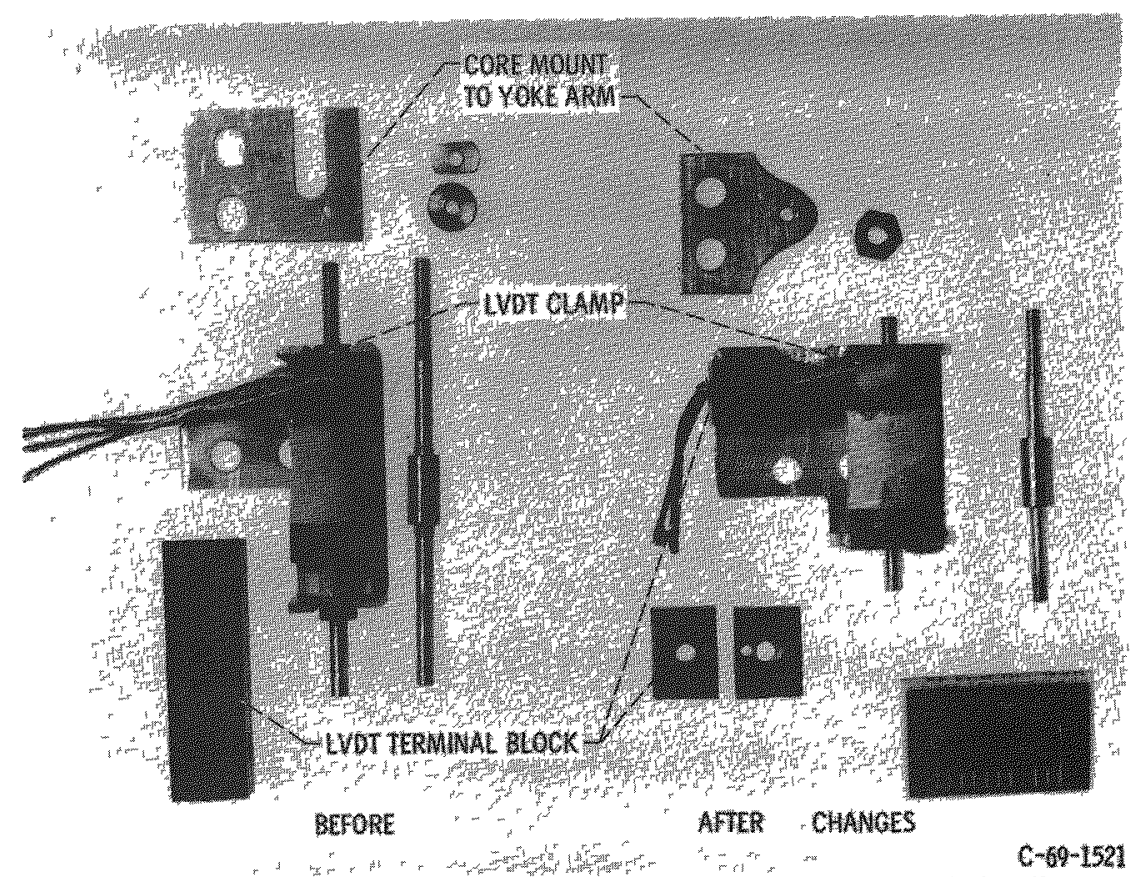

Figure 11. - Friction IVDT assembly changes for friction module of experiment lest section.

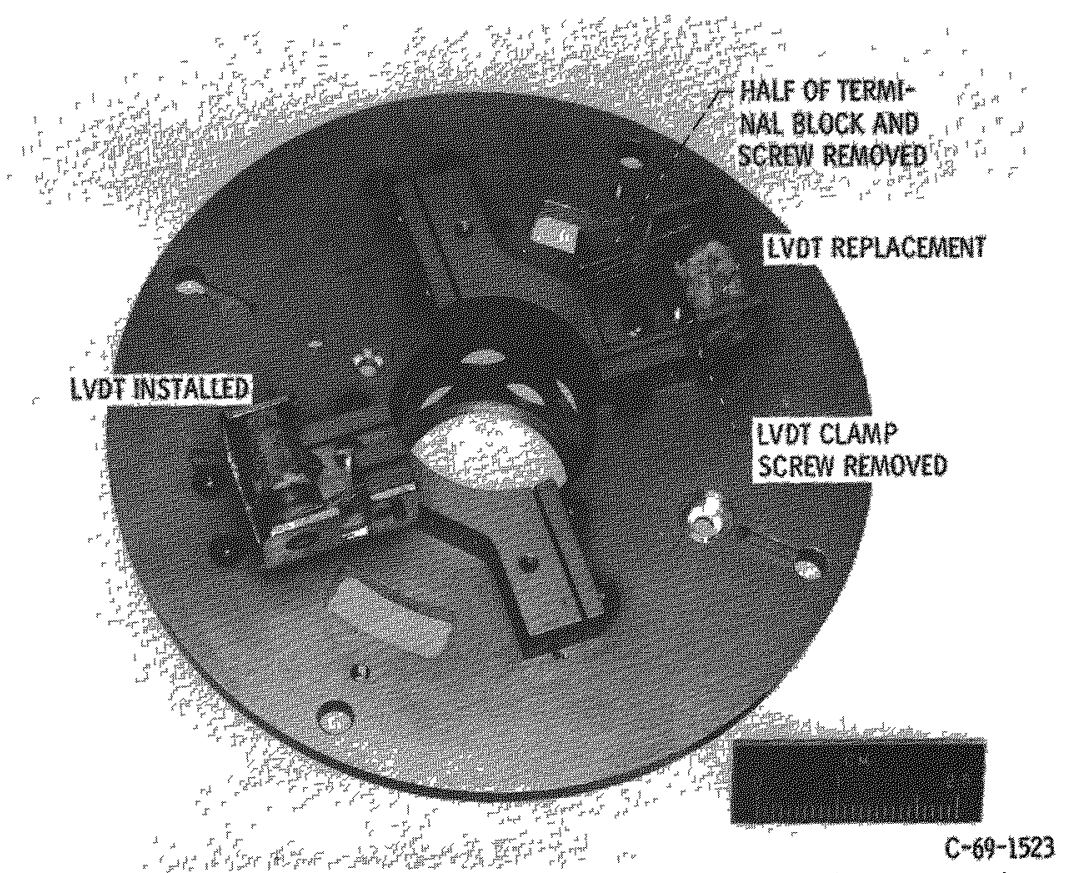

Figure 12. - Friction LVOT replacoment of LVOT plate of friction module for the experiment test section. 


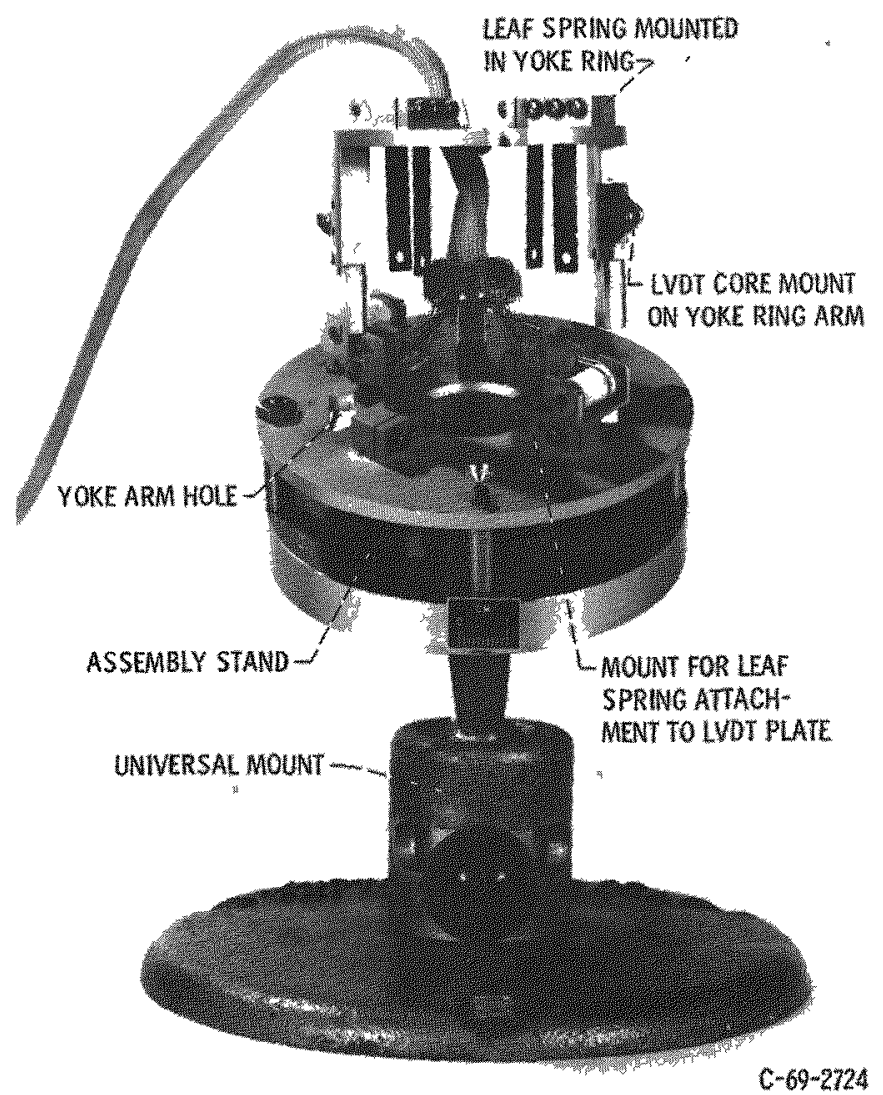

Figure 13. - Partial assembly of friction module for experiment test section.

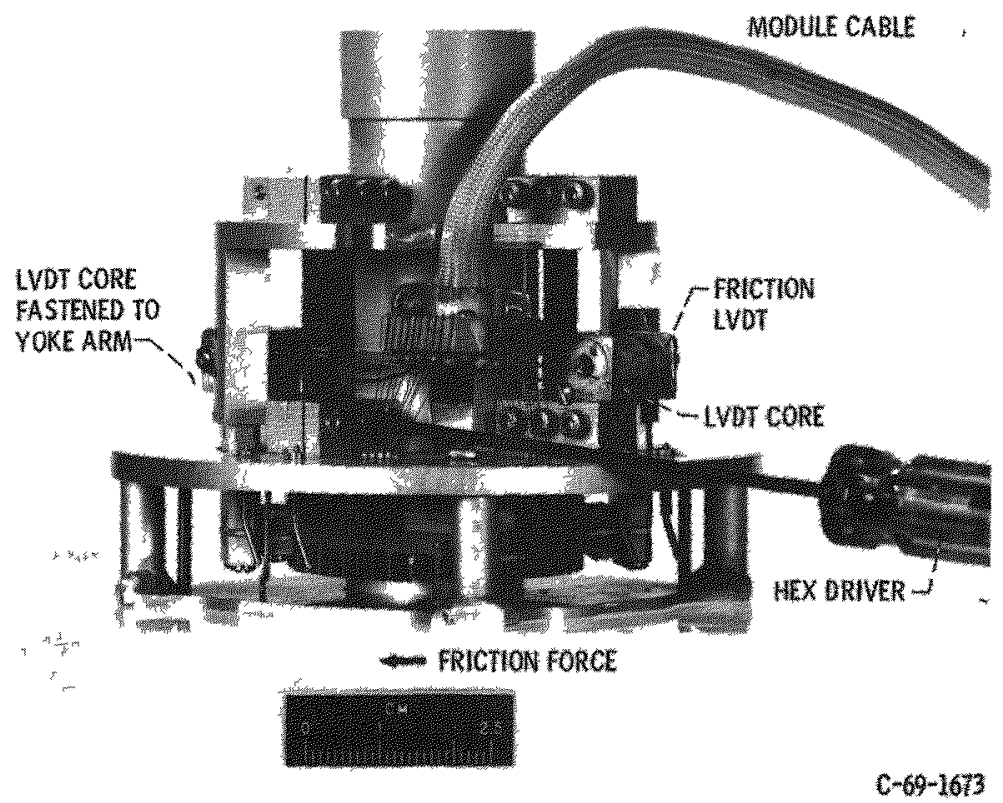

Figure 14. - Assembled friction module for experiment test section. 
Remote Encapsulation and TREAT Capsule Handling Techniques

G. E. Culley and M. E. McMahan

Battelle Memorial Institute

Pacific Northwest Laboratory

Richland, Washington

\section{ABSTRACT}

Equipment and procedures have been developed at PNL for the remote encapsulation of pre-irradiated fuel pins to be transient irradiated in the TREAT facility in support of the FFTF driver fuel development program. This equipment is located in the Shielded Materials Facility (SMF) located in the 324 Building at PNL.

The fuel pins to be encapsulated are steady-state irradiated in either the GETR or EBR-II. The GETR type fuel pins are irradiated in an instrumented NaK-bonded capsule which is remotely encapsulated by inserting the GETR capsule into a pressure vessel known as a TREAT capsule. The EBR-II type fuel pins are removed from the sodium-bonded capsules used during steady-state irradiation and re-encapsulated into an instrumented capsule very similar to the GETR capsule which is, in turn, inserted into the TREAT capsule.

The remote equipment utilized during re-encapsulation include a fuel pin profilometer, a gamma scanner, a programmed welding station, a leak detection system, and a NaK filling system. The equipment used in encapsulating into the TREAT capsule include a positioning table, a torque wrench, a leak detection system, and an instrumentation and heater checkout system. Shipping cask handling facilities which enable the use of the T-2 cask horizontally include a motorized dolly and turntable. 
Transient irradiations of prototypic fuel pins are being conducted in support of the Fast Flux Test Facility (FFTF) driver fuel development program. These tests are designed to evaluate the fuel pin response and performance limits under simulated accident conditions as required for the plant safety analysis. A series of steady-state irradiations in both fast and thermal test reactors is being conducted to obtain test fuel pins with fuel and cladding properties characteristic of actual fuel pins at various burnup levels. Following the steady-state irradiations and nondestructive tests, the fuel pins are encapsulated into a testing vehicle (TREAT capsule) that meets the safety and handiing requirements of TREAT, the transient testing facility. Due to the highly radioactive condition of the fuel pins following the steady-state irradiation, this encapsulation process must be performed remotely in a shielded facility. At Battelle-Northwest, the east cell of the Shielded Materials Facility (SMF) has been adapted and equipped for this purpose.

The test fuel pins are composed of mixed oxide (25\% $\left.\mathrm{PuO}_{2}-75 \% \mathrm{UO}_{2}\right)$ pressed and sintered pellets clad in 0.250 inch 0.D. type 304 stainless steel tubing. In the case of the thermal flux steady-state irradiations in GETR (General Electric Test Reactor), the fuel pin is contained in a NaK-bonded instrumented capsule that is 1-1/8 inches in diameter, approximately five feet long and known as the GETR capsule. Figure 1 is an artist's conception of this capsule. The fast flux irradiated fuel pins are contained in a sodium-bonded non-instrumented capsule, $3 / 8$ inch in diameter, of which 19 are used to make up an experimental subassembly for EBR-II.

When transient testing in TREAT, the central fuel element is removed and the TREAT capsule is inserted. Figure 2 illustrates this capsule which is basically three inches in diameter and nine feet long. This capsule is interchangeable with a standard driver element in that the head block contains the gripping notch above the four inch square section, and there is a guide pin on the bottom of the capsule. The metal "0" ring sealed, bolted flange in the square section allows the TREAT capsule to be assembled and disassembled nondestructively. The capsule contains heaters and thermocouples for maintaining and monitoring an isothermal temperature up to $800^{\circ} \mathrm{F}$ prior to the transient test. 
As shown in Figure 2, the fuel pin is contained in an inner capsule inside the TREAT capsule. In the case of the thermal flux pre-irradiated fuel pins, this is simply the GETR capsule itself. The fast flux irradiated fuel pins, on the other hand, must be recovered from their capsules and re-encapsulated into an inner capsule prior to insertion into the TREAT capsule.

Figure 3 is a flow diagram showing the major steps in assembling a TREAT capsule from either the GETR or EBR-II type capsules. The GETR capsule, after minor hardware removal, neutron radiography and gamma scanning is ready for encapsulation into the TREAT capsule. The fast flux irradiated pin must be re-encapsulated to get to the inner capsule stage. This remote re-encapsulation requires specialized-equipment and techniques for handling the components and assuring a sound capsule. Starting with the fuel pin recovered from the EBR-II capsule, it is first profilometered to determine any changes in cladding diameter during the steady-state irradiation and to characterize the pre-transient diameter profile. An in-cell view of the profilometer is shown in Figure 4. The measuring head consists of dual opposing linear motion transducers. The fuel pin is positioned in a rotating chuck which translates the fuel pin with respect to the stationary transducer head. Continuous lengthwise diameter measurements are made at several orientations around the pin. The profilometer is calibrated with a standard before and after each pin is measured. The accuracy of the system is within +0.0002 inch. The profilometer controls and recorder are shown in Figure 5. Following the profilometry, the fuel pin is gamma scanned to evaluate fission product distribution within the fue 1 pin and to determine the steady-state axial power profile. The in-cell portion of the gamma scanner, which consists of the translating mechanism and collimator, is shown in Figure 6. A Tithium-drifted germanium-diode detector is located outside the cel1, and the collimator aperture can be varied. The electronics system consists of translator controls and nuclear instrumentation and is shown in Figure 7. The system uses a 2,048 channel analyzer which can be operated in the spectral scan mode or to digitize the axial scan data. A CRT display, an X-Y plotter and a digital printer are used to record the data. The gamma scanning operation involves obtaining continuous axial scans and spectral scans at selected positions. Axial scans of the gross gamma activity, the $\mathrm{Zr}-\mathrm{Nb}$ and the $\mathrm{Cs}-137$ photopeaks are taken.

At the completion of these nondestructive tests, the fuel pin is prepared for re-encapsulation by cleaning and drying. The inner capsule, which has been fabricated complete with instrumentation, is ready for insertion of the fuel pin and filling the assembly with NaK. The fuel pin is attached to the fuel pin pedestal by a "T" bar arrangement that utilizes the EBR-II capsule end cap that is still attached to the fuel pin. The fuel pin - pedestal - bulkhead combination is remotely welded to the inner capsule assembly with the remote welding fixture shown in Figure 8 . The in-cell portion of the welding fixture consists of a rotating chuck and a TIG welding torch. Outside the cell is a programmed power supply. To select the proper welding parameters and qualify the welding procedure, a test series of welds were made and examined metallographically. The programmable feature assures that the selected welding parameters will be 
duplicated on the actual capsule welds. A helium leak check is made on the weld by attaching a mass spectrometer type leak detector through the cell wall to the NaK fill tube located on the bottom of the inner capsule. The maximum allowable leak rate, while covering the weld area with helium, is $1 \times 10^{-8} \mathrm{cc}$ (STP) $\mathrm{sec}^{-1}$.

Assuming that the weld passes the leak test, the next step is to fill the capsule with NaK to the proper level. The NaK fill system is shown in Figure 9. The capsule is positioned vertically as shown with the level detector in place and the fill tube attached to the system. The principal of operation is as follows:
a. Evacuate inner capsule.
b. Fill and evacuate inner capsule with helium three times.
c. Fill capsule with NaK for flush operation.
d. Obtain first NaK sample.
e. Fill capsule to desired level with Nak.
f. Obtain second NaK sample.
g. Backfill capsule with helium to one atmosphere (absolute) pressure.
h. Perform pinch and cutoff operation on fill tube.

The NaK level within the capsule is determined with an eddy current device. The sensing coil can be moved up and down the capsule to verify the desired level. The pinch and cutoff operation does not form a leak tight seal on the fill tube and a minimum volume end cap is immediately welded into place. The same welding fixture with slightly different welding parameters is used to make this weld.

The minimum volume end cap contains a vent hole so that increase of pressure during welding does not cause any welding problems. This hole is used to fill the end cap with helium before it is welded closed. These last two welds are then helium leak checked by slipping a vacuum-tight enclosure over the bottom of the capsule and attaching this to the leak detector. The helium located inside the end cap is used as the source. The maximum allowable leak rate on these welds is also $1 \times 10^{-8} \mathrm{cc}$ (STP) $\mathrm{sec}^{-1}$.

The re-encapsulation of the EBR-II type fuel pin is now completed, and for encapsulation purposes this inner capsule is identical to the GETR capsule. The encapsulation process begins with an out-of-cell check out of the TREAT capsule to insure that the heater elements are in working order and that a leak-tight seal at the bolted flange can be obtained. The capsule to introduced into the cell and positioned on the work table with two pipe vises. The head block is positioned to one side and the inner capsule inserted into the heater can in the TREAT capsule. The instrumentation connector on the inner capsule is aligned with the mating connector in the head block by rotating the inner capsule. The head block is aligned with the capsule body, and the bolts are torqued to the specified values using both air and conventional torque wrenches. The seal at the bolted flange is prepared for leak checking by evacuating and filling the TREAT capsule with helium to a pressure of two atmospheres (absolute). 
The valve located at the bottom of the TREAT capsule is used to introduce and hold the gas in the capsule. A clam-shell type enclosure is then placed around the bolted flange and the gas present in this enclosure is sampled for the presence of helium using a "sniffer" probe attached to the leak detector. If any helium is detected, the seal is rejected and must be redone.

The leak tight capsule is then ready for the electrical checkout. The instrumentation cable is attached to the connector in the head block, and the capsule is placed in a vertical position. The heater power supply and thermocouple monitoring system is located out of cell. The three heaters are operated both singly and in series to a temperature of $800^{\circ} \mathrm{F}$. The thermocouples are monitored and any anomalies noted. This completes the encapsulation process.

Shipments of the TREAT capsules between BNW and TREAT utiTize the $T-2$ radioactive shipping cask. This cask was specifically designed to handle the TREAT capsule and is compatible with the facilities available at TREAT. The cask is normally loaded vertically, but due to the layout of the cells in the SMF, horizontal loading must be performed. The cask, located in the air lock, is positioned against an opening in the east cell door, and the TREAT capsule is positioned into the cask using the in-cell overhead crane and the power manipulator. The cask is handled in and out of the air lock cell with a large self-propelled dolly, mounted on a track (Figure 10) and the ninety degree turn necessary to mate with east cell is accomplished with a motorized turntable (Figure 11). When the cask is out of the air lock cell, it is placed in the fire and crash shield located in the building cask handling area utilizing a 30 -ton overhead crane. The completed shipping container containing the TREAT capsule is then shipped by motor freight to the TREAT facility located at the National Reactor Testing Station, Idaho Falls, Idaho. 


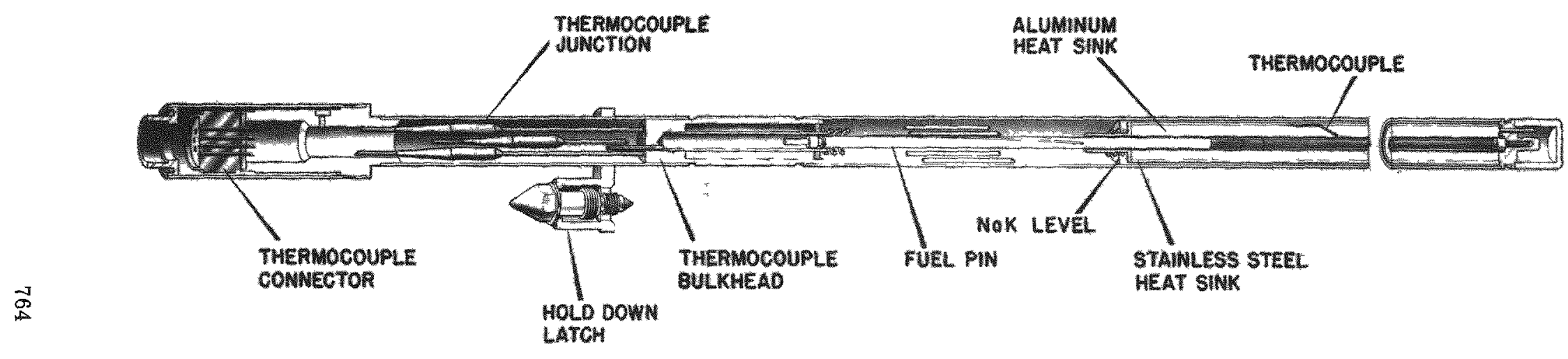

FIGURE 1

GETR-TREAT

Inner Capsule Assembly 


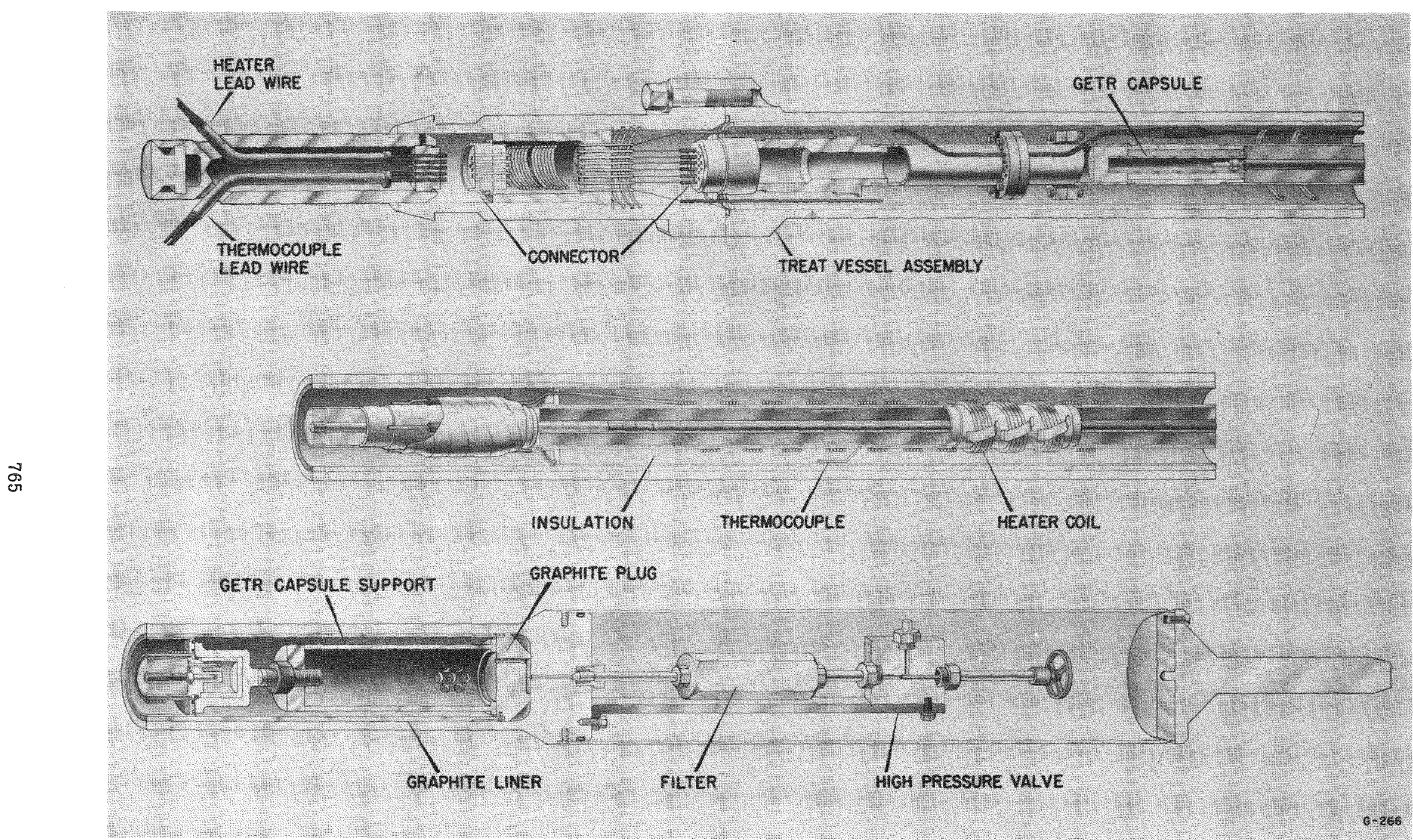

FIGURE 2

TREAT Capsule Assembly 


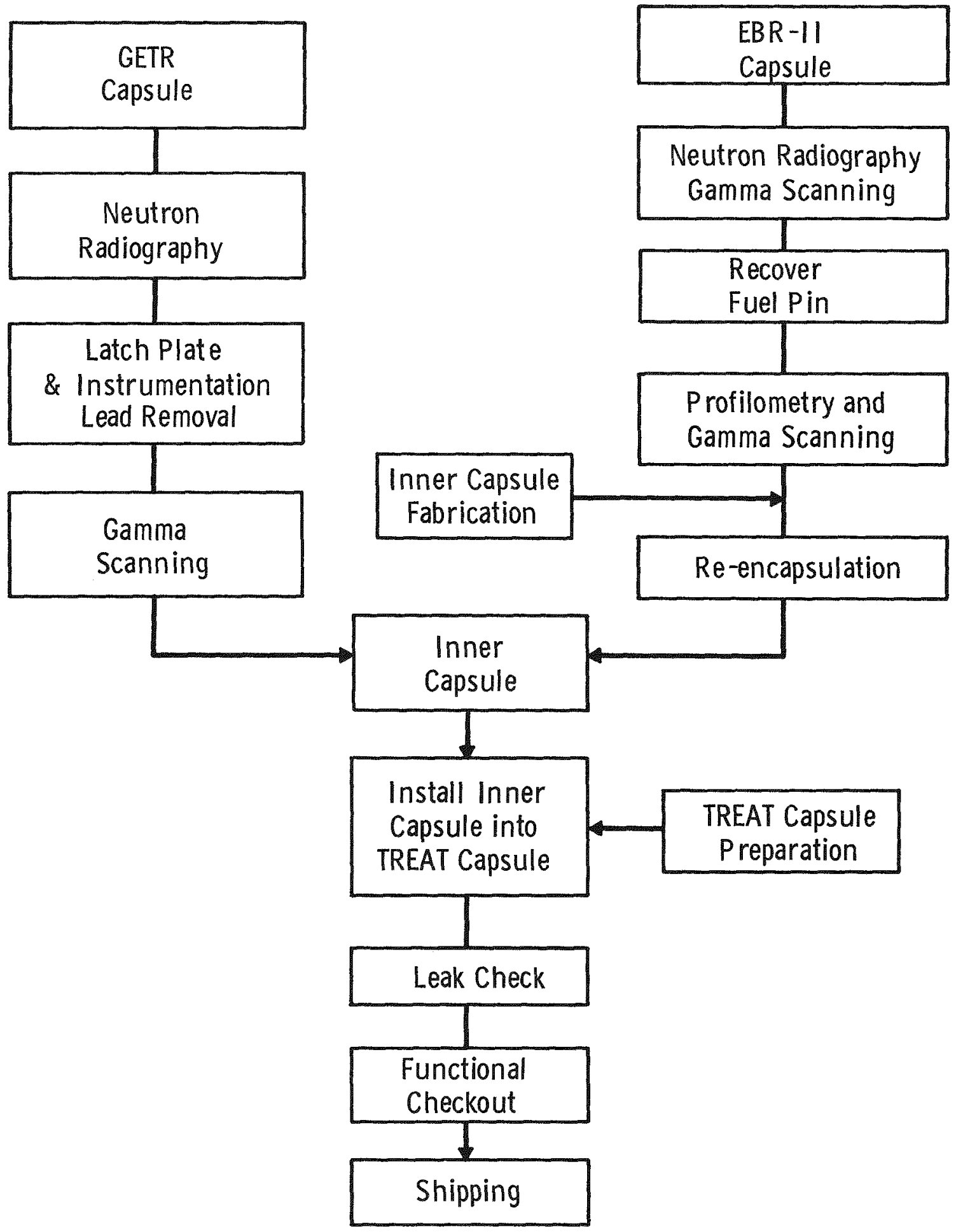

FIGURE 3

Flow Diagram for TREAT Encapsulations 


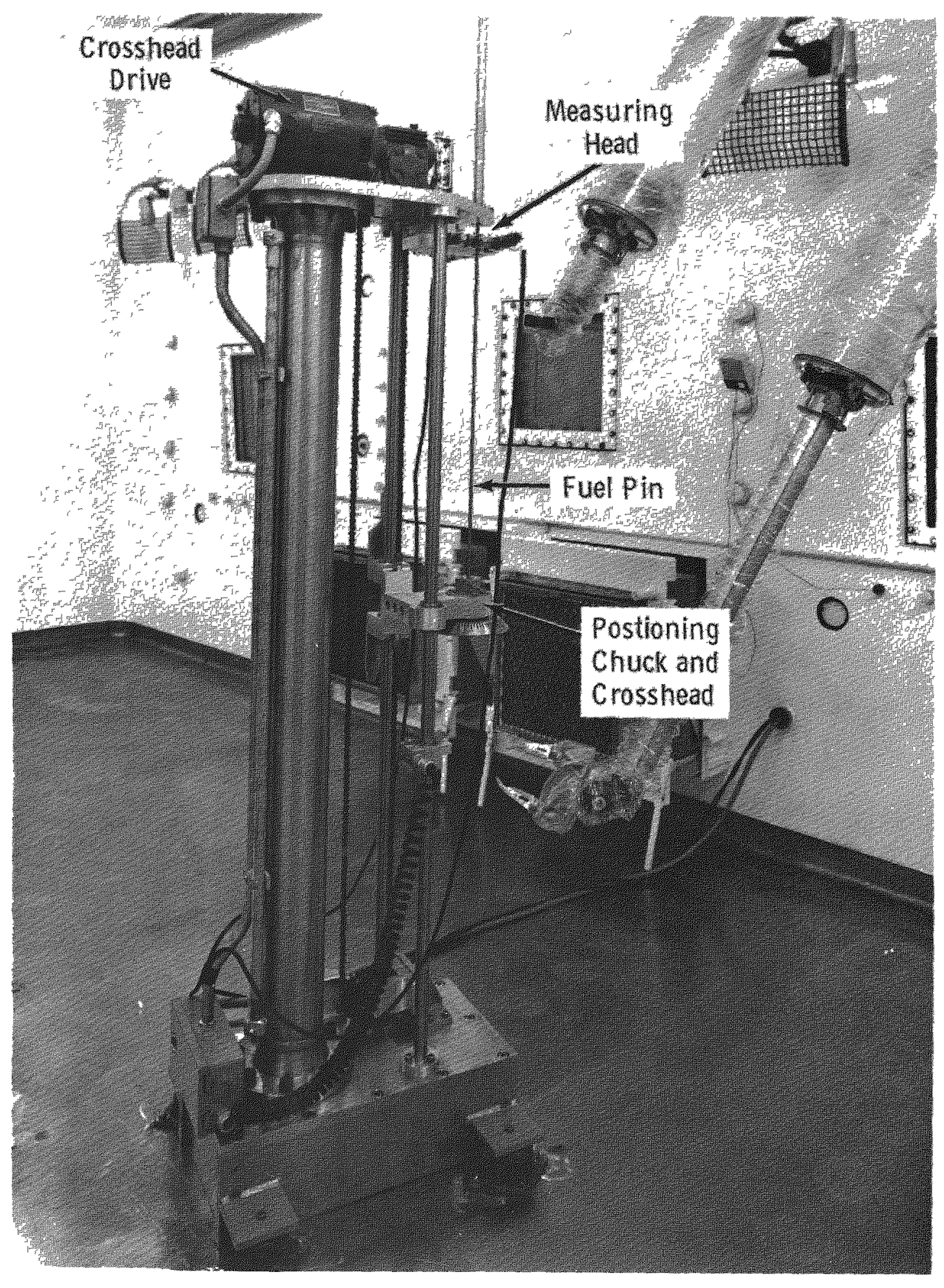

FIGURE 4

In-Cell View of Profilometer 


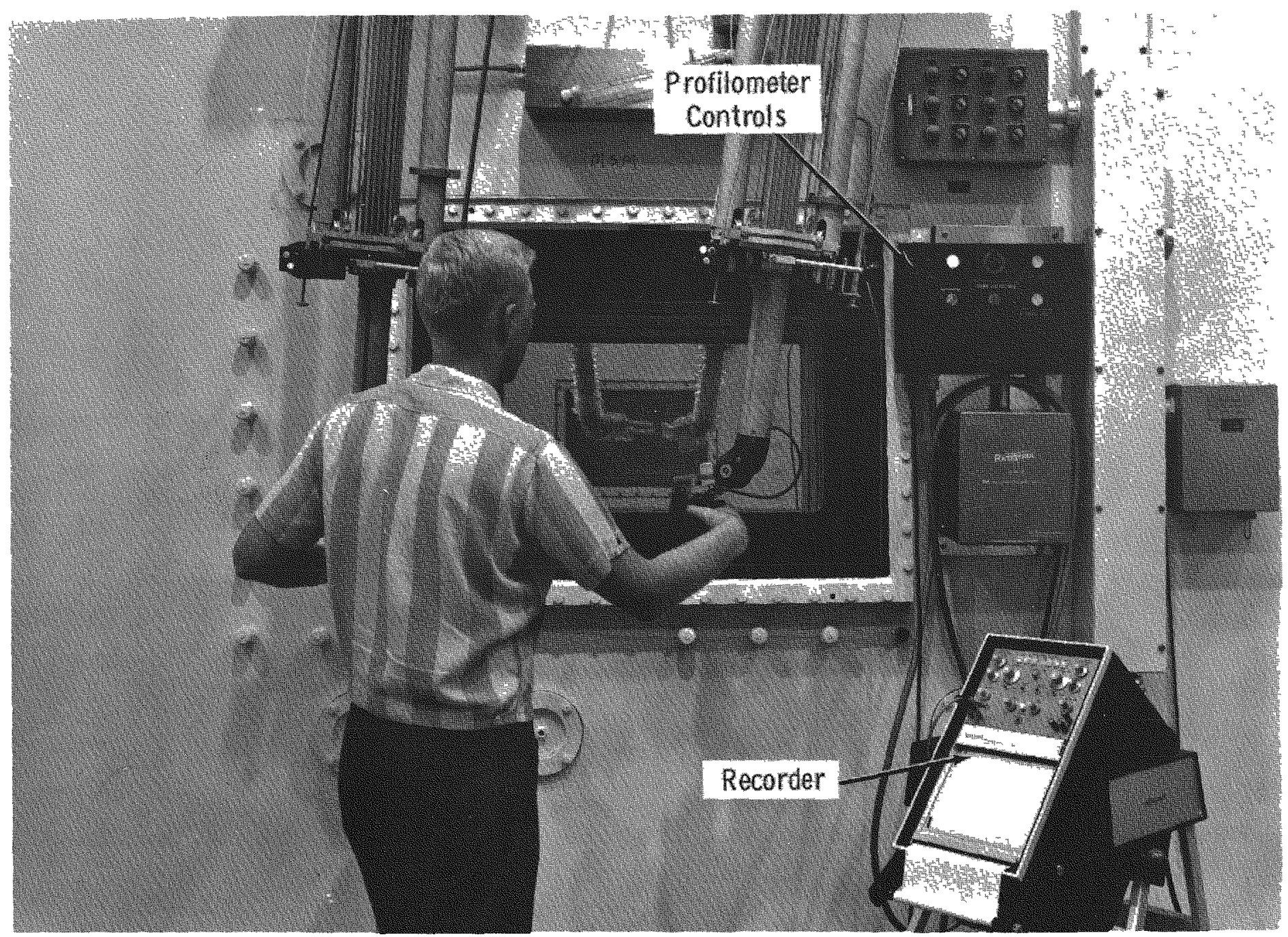

FIGURE 5

Profilometer Recorder and Controls 


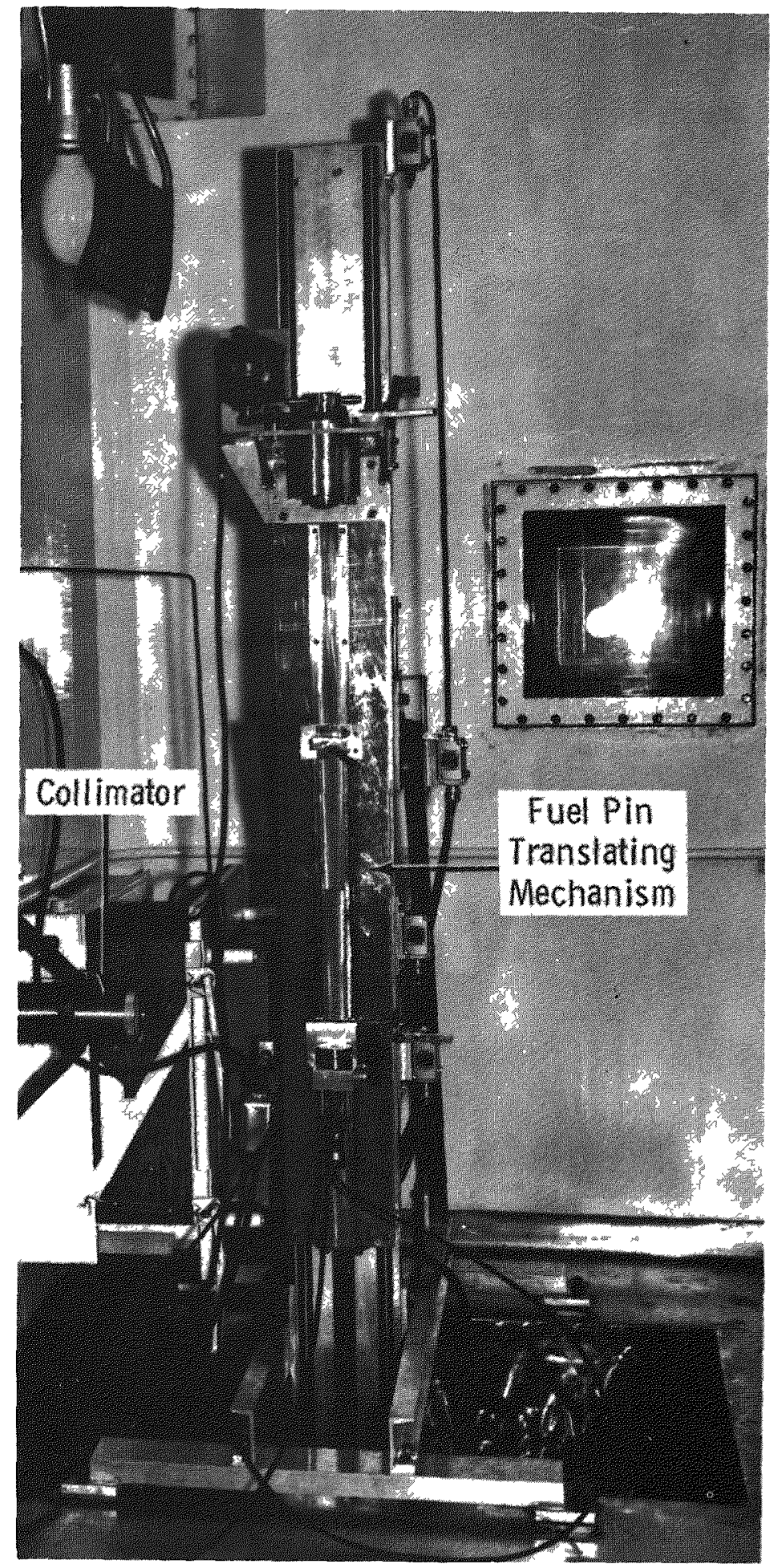

FIGURE 6

In-Cell Portion of Gamma Scanner 


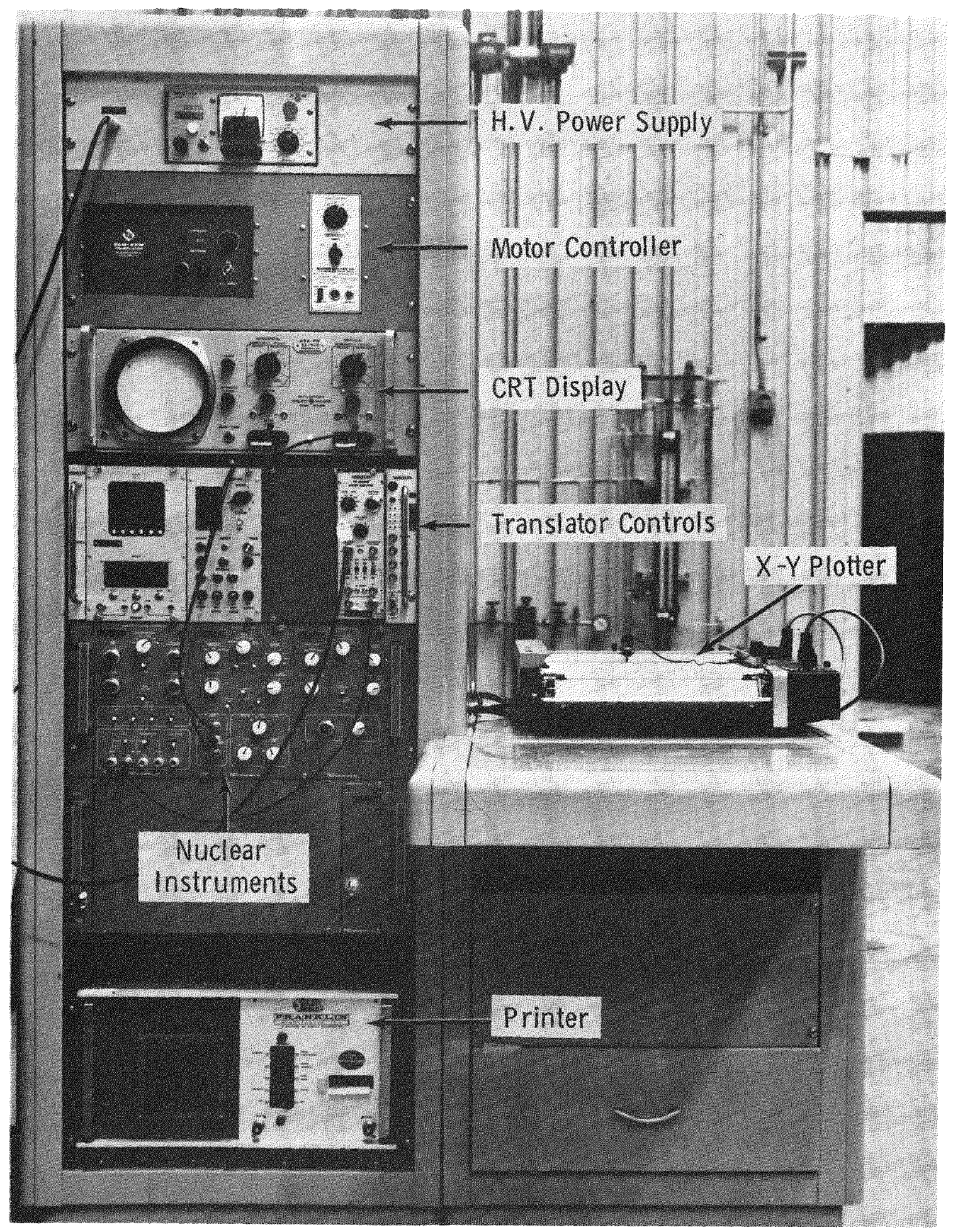

FIGURE 7

Gamma Scanning Electronics 


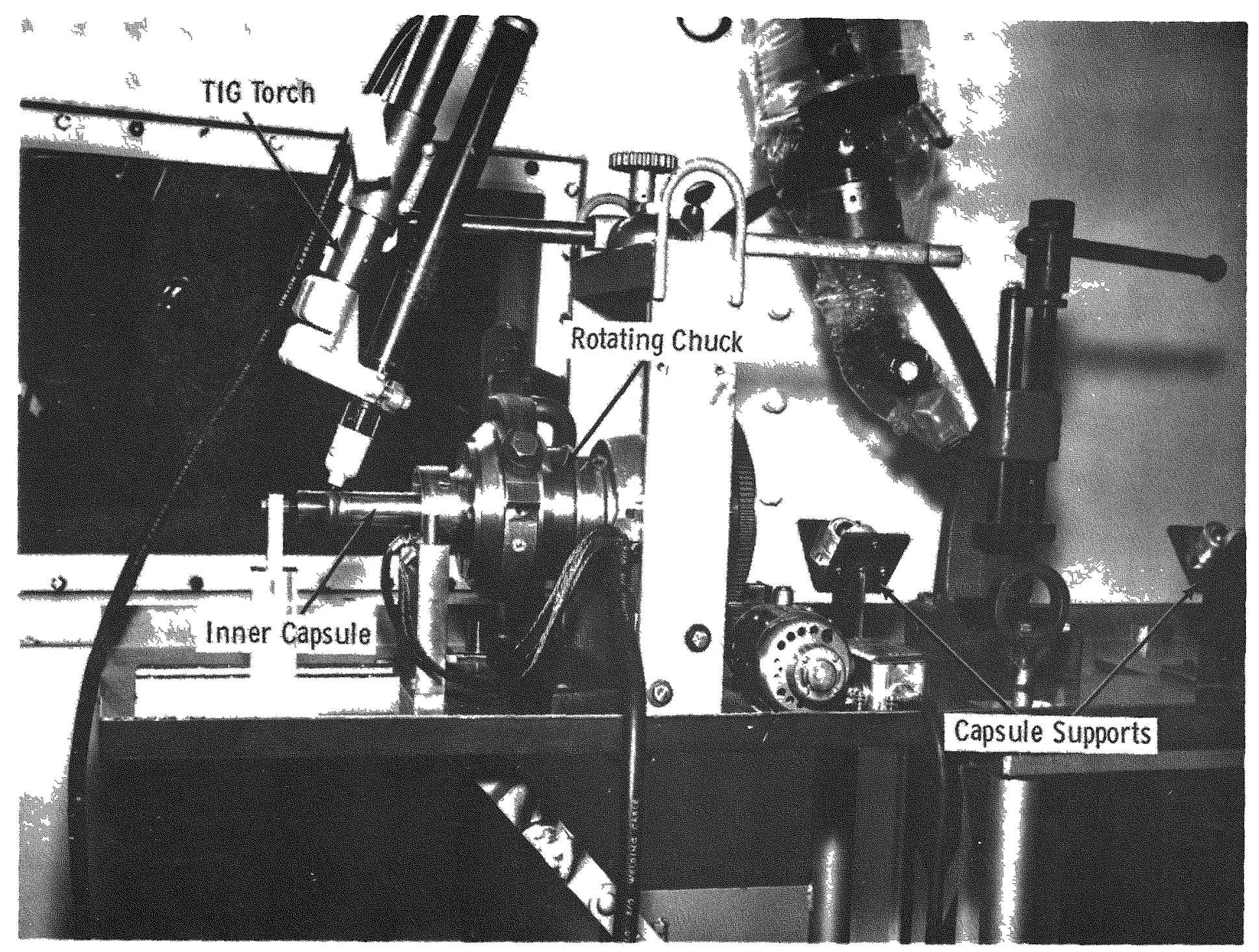

FIGURE 8

Remote Welding Fixture 


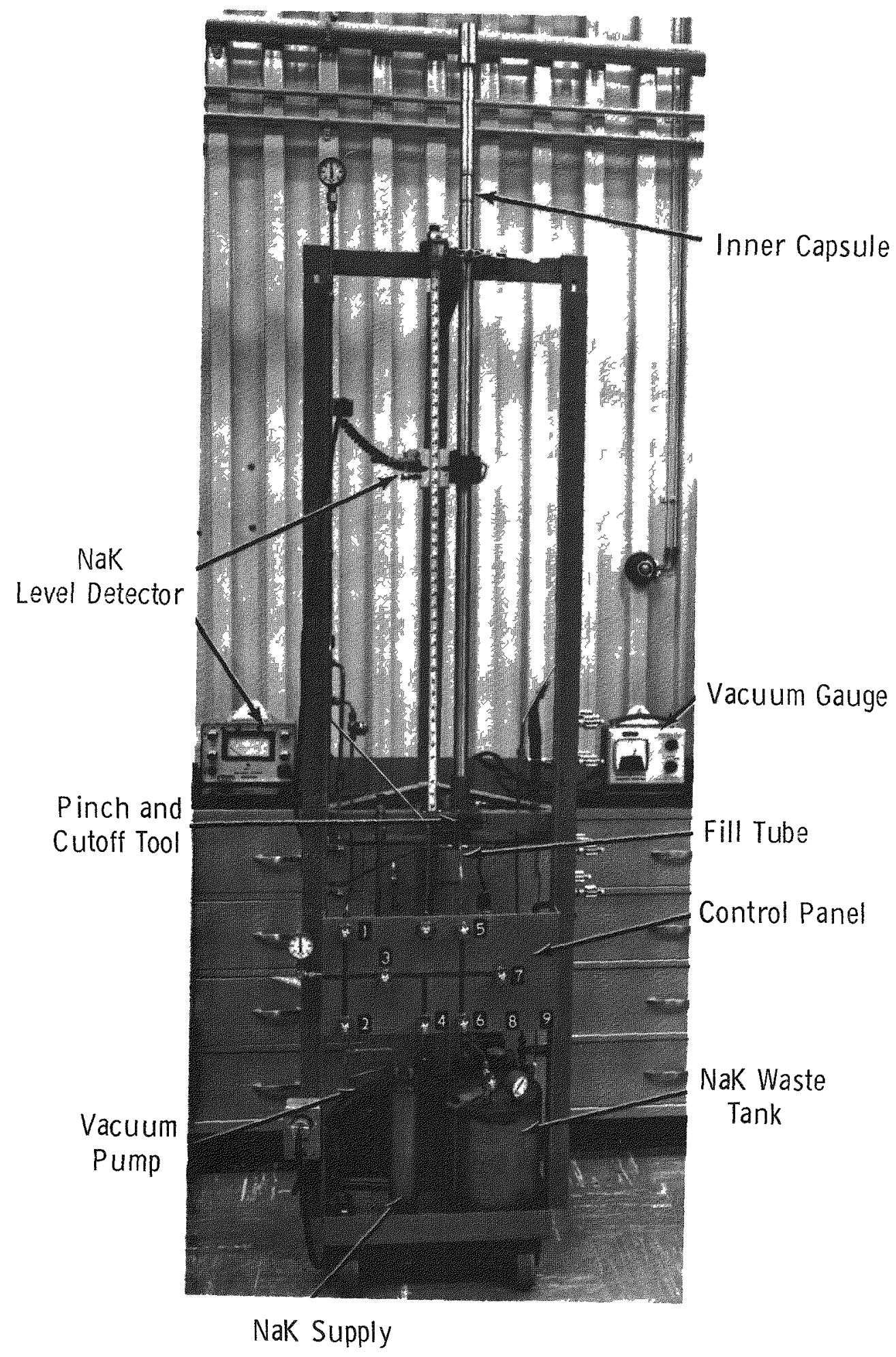

FIGURE 9

NaK Fill System 


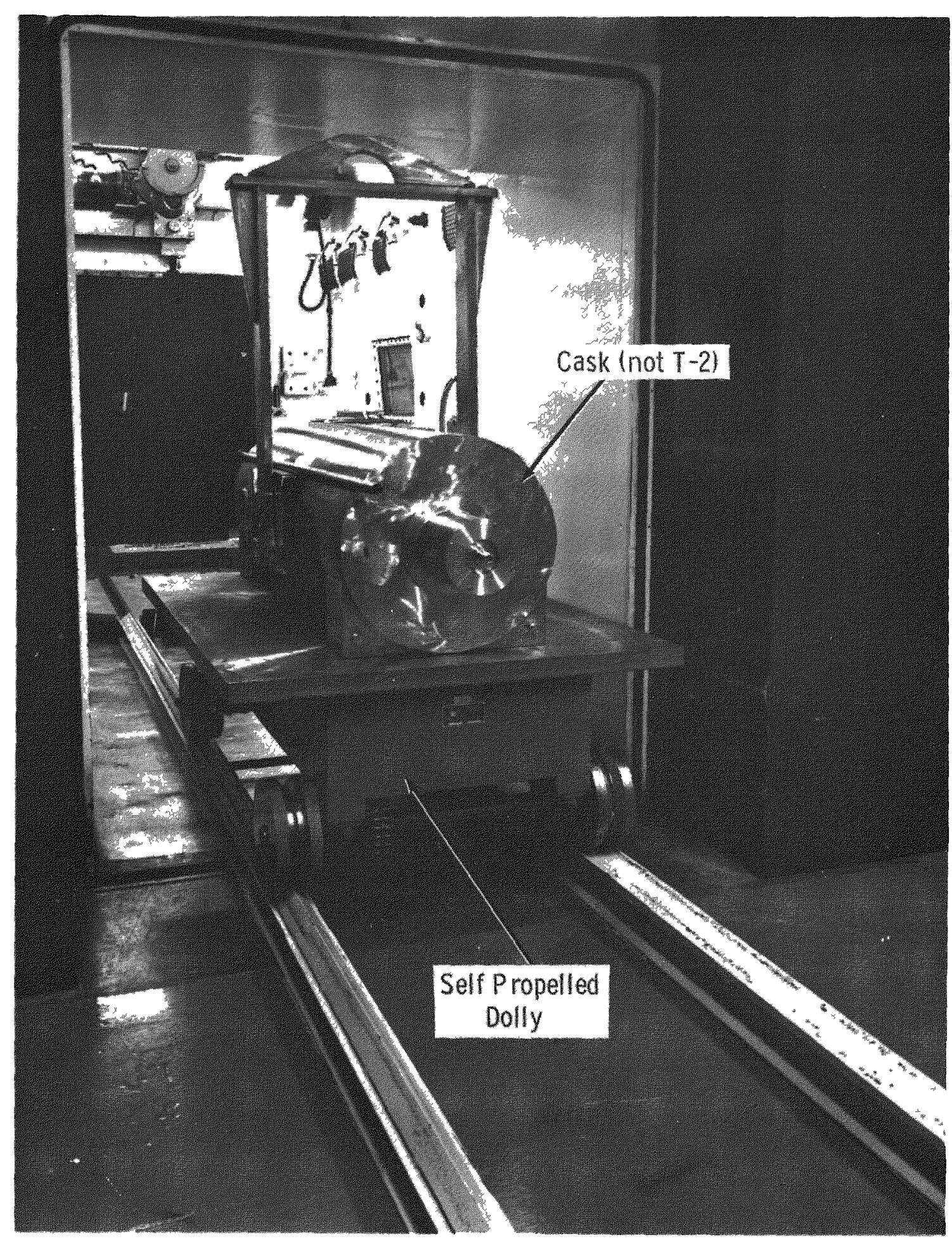

FIGURE 10

Cask Transfer into Air Lock 


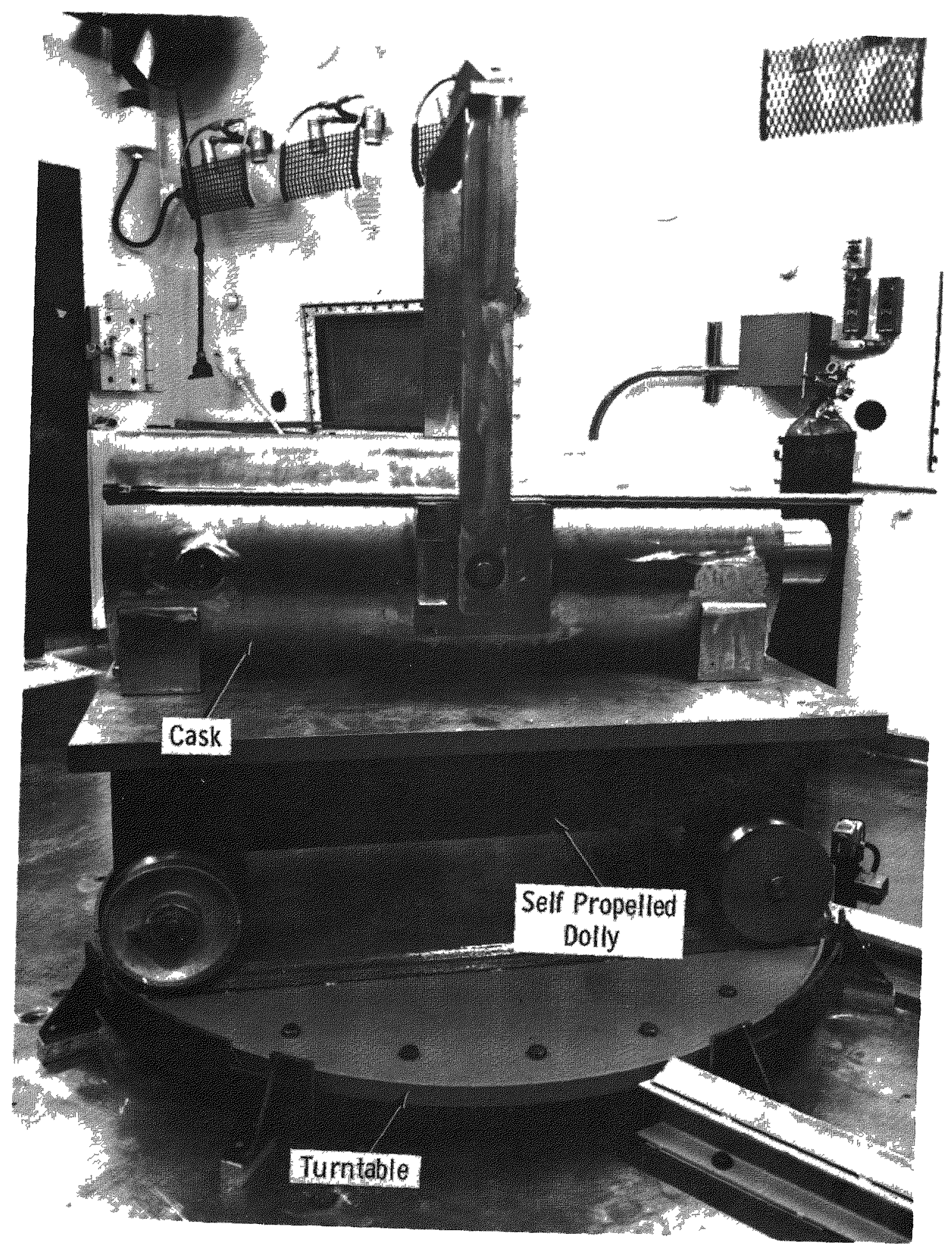

FIGURE 11

Cask Positioning Turntable 
THE DESIGN AND IN-PILE OPERATION OF INSTRUMENTED HYDRAULIC CAPSULES

\author{
by Robert G. Rohal \\ National Aeronautics and Space Administration \\ Lewis Research Center \\ Cleveland, Ohio
}

\begin{abstract}
$\underline{A B S T R A C T}$
An in-pile hydraulic capsule facility was designed, built, and tested which permits temperature readings within the capsule to be obtained without the use of leads coming directly from the capsule. This is accomplished by using pins which penetrate the capsule walls thru an insulating ceramic seal. The portion of the pin inside the capsule is connected to one leg of a thermocouple. The exterior portion has a spring attached which contacts a commutator permanently installed in the in-pile facility. The other leg of the thermocouple is grounded to the capsule body which is in turn grounded to the facility. The thermocouple signal is transmitted from the capsule thru the pin to the commutator by the spring, and from the commutator to recorders by permanent leads. Results obtained thus far indicate such an in-pile testing concept is both feasible and practical.
\end{abstract}


This report describes an irradiation capsule and commutator assembly design for converting a hydraulically-operated, uninstrumented, capsule facility into an instrumented capsule facility. The design will permit continuous capsule data readout, without leads attached directly to the capsule. This retains the simplicity of capsule assembly and insertion inherent in uninstrumented capsules, while adding the data readout capability of more complex and costly lead capsules.

In the design described, the temperature signal is brough through the capsule wall by a ceramic-insulated pin having a spring at the outer end. The spring engages a commutator ring as the capsule reaches the fullyinserted position. The commutator ring is connected by a permanent lead to a recorder.

This report shows a six-capsule assembly based on this design which was used in a recent facility modification. The problems encountered in the design and operation are discussed, and some in-pile test results presented.

This design offers a relatively simple and inexpensive means of modifying a conventional, hydraulically-operated, uninstrumented capsule facility into an instrumented facility.

\section{INTRODUCTION}

Hydraulically-inserted capsule facilities are used at many research reactor installations because of their simplicity. The capsules used in such facilities are relatively easy and inexpensive to assemble. Insertion and removal of the capsules is relatively simple, and can be done in many cases while the reactor is operating. However, this type of facility has one major disadvantage: no data can be obtained while the capsule is being irradiated. Important test conditions such as specimen temperature might be determined at best after the test.

Because of the inability to obtain data during irradiation, the hydraulically-operated capsule facility is often not adequate for performing in-pile irradiation tests. The need for continuous data readout usually requires a lead-type capsule facility with long continuous leads coming directly from the capsule. Such an arrangment results in a more complicated and expensive capsule than the capsules used in the hydraulic facilities. The lead type capsules also require more complicated procedures for handling and usually restricts insertion or removal to reactor shut down periods.

In this report an in-pile hydraulic capsule facility is discussed which combines the best features of both the hydraulically-operated facilities and the lead-type capsule facilities. A hydraulic capsule facility has been designed which provides continuous data readout but does not require leads directly attached to the capsule. Instead, the signals from the sensors are 
connected upon capsule insertion and therefore the capsules can be handled as is done in a simple hydraulic capsule facility.

This so-called non-lead instrumented capsule design is being reported for two reasons. First, the data obtained thus far clearly indicates that such a facility is feasible and practical. Second, there may exist other uninstrumented capsule facilities which could be modified to accommodate the type of facility described herein. This would greatly improve the data obtainable in these facilities and minimize the need for new and costly lead capsule facilities.

The principle of operation of such a facility will first be discussed. Then the important parts of the facility will be described with respect to their purpose and salient features. Problems inherent to this particular design will also be discussed since many of these problems could occur in facilities employing a similar design. Finally, some in-pile test data will be presented to show that the facility will retrieve data as can be done with the conventional lead-capsule facility.

\section{DESCR IPTION}

The Instrumented Non-Lead Hydraulic Capsule Facility

Facility - A typical hydraulically-operated capsule facility is shown schematically in figure 1. The capsule is inserted into or retracted from its full-in or stop position in the facility inner tube by the flow of Primary Cooling Water (PCW). The capsule is also held in the stop position and cooled by the PCW flow. The PCW returns from the facility to the PCW system thru an annulus formed by the inner and outer snout tubes of the facility. In this and similar facilities, the capsule is not instrumented to provide continuous readout during the in-pile test.

A modification of this hydraulically-operated capsule facility is shown schematically in figure 2. In the facility under consideration, six capsules are tested concurrently but this is not pertinent to the principle of operation. In this facility when the capsule (or capsule assembly) is propelled hydraulically down the facility tube it comes to rest against a special type of stop which also serves as a commutator. The commutator and capsule are designed so that when the capsule comes to rest at the commutator, electrical connections are made between the capsule lead pins and commutator rings. The capsule lead pins are electrically-inswlated from the capsule body and attach to sensors (e.g. thermocouples) in the capsules. The commutator rings are electrically-insulated from each other and have fixed leads running to recorders. The commutator rings and fixed leads are part of the facility and not part of the capsule, so they can be used for numerous capsule tests.

The contact between each capsule lead pin and commutator ring is accomplished by a specially-designed spring. The spring deforms as it rides up an approach ramp on the commutator. The force associated with this deflection provides sufficient force for good electrical contact ( $<1$ ohm resistance) 
against a commutator ring. The components shown on figure 2 will be discussed in greater detail in the following sections.

Capsule - As mentioned earlier six capsules are tested concurrently in the facility under investigation. One of the capsules used in the facility is shown in figure 3 which illustrates the features of an instrumented hydraulically operated capsule.

The capsule bodies are made of aluminum and the sensors are thermocouples. At one end of the capsule is a double, ceramic seal assembly. The assembly provides a leak tight, radiation-resistant, insulated penetration for an electrical signal. Two seals are used because it is felt that this component is the weakest part of the capsule from the standpoint of containment. In the arrangement used in these tests, the negative leg of the thermocouple is grounded to the capsule body while the positive leg is connected to the capsule lead pin which penetrates the capsule wall via the double ceramic seal. Both the positive and negative legs of the thermocouple are attached to their respective compensating alloy leads at the commutator so that the dissimilar metal junctions are self-compensating with junctions made in a region of low temperature.

Six capsules of the type shown in figure 3 are assembled in a hexagonal array in a stainless steel holder as shown in figure 4 to form a capsule assembly. A rod protrudes from the center of the capsule holder which serves to stop the capsule assembly at the full-in or stop position. This rod will be referred to as the stop-rod.

Because each capsule is provided with a spring, six separate signals can be transmitted from the capsule assembly. All negative legs of the thermocouples are grounded to the capsule body. Therefore, the ground leg will be the same for all six capsules. The capsules are not insulated from the holder. The ground contact for the assembly is provided for by the stoprod coming in contact with the commutator.

Commutator - The commutator is permanently attached to the inner in-pile snout tube. The commutator used in the facility under consideration is shown in figure 5. The commutator body and contact rings are made of stainless steel. The contact rings are insulated from the commutator body and each other by high purity $(99.9 \%)$ aluminum oxide $\left(\mathrm{Al}_{2} \mathrm{O}_{3}\right)$ insulators. The commutator also incorporates a specially designed rotation-ramp which serves to rotate the capsule assembly to a predetermined angular orientation as the stop-rod engages with the commutator. Correct angular orientation of the capsule assembly is necessary in this design because the neutron flux across the in-pile tube is not uniform. Therefore each capsule of the assembly must be designed for a given neutron flux level. A small pin in the capsule holder stop-rod (figure 4 ) contacts the rotation ramp to rotate the capsule assembly to the correct angular position. A thermocouple extension lead spot welded to each commutator ring servesto transport the signal to recording instrumentation. 
In developing a workable facility of the type described, a number of problems arose. Much time and effort was expended in overcoming these problems. The problems inherent to this facility will be discussed in this section in the hope that it will save time and effort on any similar facility design.

Capsule-commutator springs - One of the most critical design problems of this type of facility is the design of the spring that provides contact between the capsule lead pin and the commutator ring. The spring must exert enough force when deflected to provide a low resistance contact $(<1$ ohm resistance in air). However, the spring must not exert so much force that it prevents the capsule assembly from being fully inserted against the commutator stop. The fully-inserted position is shown in figure 6 . In this figure, the lucite tube simulates the inner facility tube. The size (width and thickness) and design of the spring was such that its elastic limit would not be exceeded when the spring underwent the necessary deflection.

Because of the commutator design, another problem was encountered with the capsule-commutator springs. The capsule assembly in moving to the final longitudinal and angular position, causes both a longitudinal and circumferential force on the spring. The circumferential force associated with angular orientation requires that the capsule-commutator spring be sufficiently wide to prevent the spring from "cocking". Such cocking causes excessive friction between the springs and commutator, and can prevent the capsule assembly from completely seating against the commutator stop. A typical spring design is shown in figure 7 .

Contact resistance - A problem which arose after about six months of operation was that contact between the capsule-commutator springs and the commutator rings began to fail. The problem became more serious as time went on. After about nine months contact could not be obtained on some rings. A specially-constructed stainless steel wire brush was used to clean the commutator. After this cleaning, tests revealed that contact between the capsule-commutator springs and the commutator rings was again obtained. Thus it appears that periodic cleaning with a wire brush is necessary in order to provide for satisfactory operation of the facility.

Because of present program requirements, complete and satisfactory analysis of this problem has not been obtained. The problem may be caused either by corrosion of the stainless steel commutator rings or a deposit from the PCW sys tem.

Capsule body welds - Another problem involved welding of the aluminum capsule bodies. It was found that satisfactory welds (welds with leak rates $<10^{-9}$ cubic feet/hour of helium) were very hard to obtain with 6000 series aluminum alloys. Satisfactory welds could be obtained, however, if 5083 
aluminum alloy was welded to 1100 aluminum alloy or 1100 aluminum alloy was welded to 1100 aluminum alloy. Welds 1 and 2 on figure 3 employ the first type weld whereas weld 3 employs the second type. In welding the capsule bodies, it was also found that using a "trepan" arrangement at the weld eliminated cracks in this area.

Another weld problem was associated with welds near the ceramic seals. It was found that ordinary electron beam welding of welds 2 and 3 (see figure 3) caused the ceramic seals to crack. These cracks led to unacceptable helium leak rates. The cracking was finally traced to the heating and expanding of the aluminum when it was welded. This caused the aluminum to expand away from the seal, stressing the seal and causing it to crack. Conventional heat sinks were tried in order to absorb the welding heat loads. These did not work because of insufficient heat transfer surface area and poor contact. A special heat sink was designed to improve the heat transfer between the heat sink and the aluminum body. This heat sink with a capsule inserted in it is shown in figure 8. The heat sink is designed with a small space (approximately $10 \mathrm{mils}$ ) between the capsule wall and the heat sink. This space is filled with water to improve surface contact between the capsule body and the heat sink. This type of heat sink was satisfactory. All welds were made with an electron beam welder. All welds on the capsules were made in a short period of time (welding time at any seam is about 5 seconds).

\section{IN-PILE TEST RESULTS}

To illustrate operation of the facility, a representative in-pile test of a multiple capsule assembly will be presented. Each capsule, as described earlier, contained a single fuel pin. Each pin had a $W 26 \% \operatorname{Re} / W 5 \% \operatorname{Re}$ thermocouple brazed to one end. The fuel pin clad material was TZM. The fuel in the fuel pin was fully enriched $\mathrm{UO}_{2}$ powder. The heat generated by the fuel pin was transmitted across a helium gap between the pin and capsule wall, and then to the Primary Coolant Water flowing past the capsule.

The results of a typical test are shown in figure 9 . It can be seen from figure 9 , that a given thermocouple reading is quite stable meaning that good uniform contact was obtained through the test period which lasted 200 hours.

\section{CONCLUDING REMARKS}

A non-instrumented capsule facility has been converted into an instrumented capsule facility. The design, revision, and operation can be achieved rather quickly, with relatively little expense, and with no major compromise in capsule simplicity and cost. The facility has operated satisfactorily for periods as long as 200 hours. 


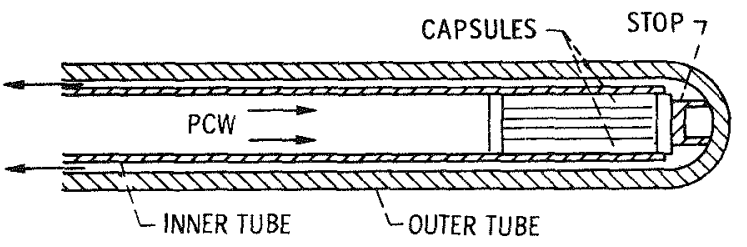

$\operatorname{CS}-51556$

Figure 1 - A typical hydraulically-operated capsule facilıty.

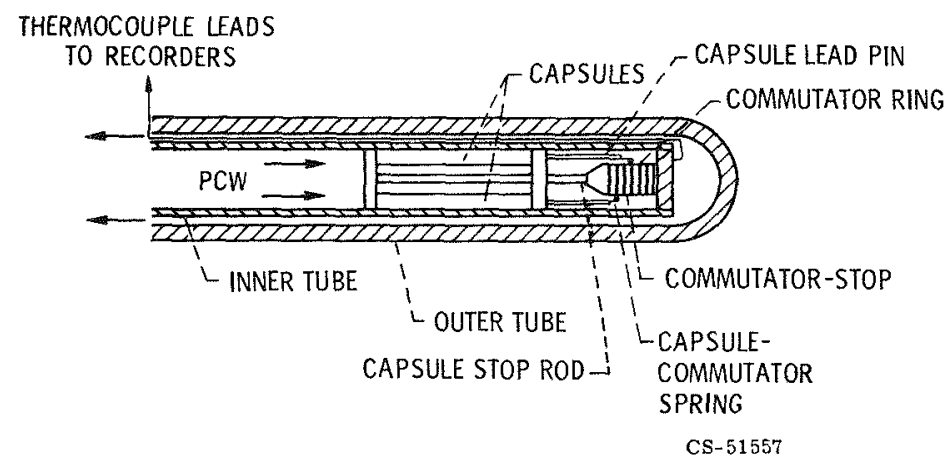

Figure 2 - A typical instrumented hydraulıc capsule facilıty.

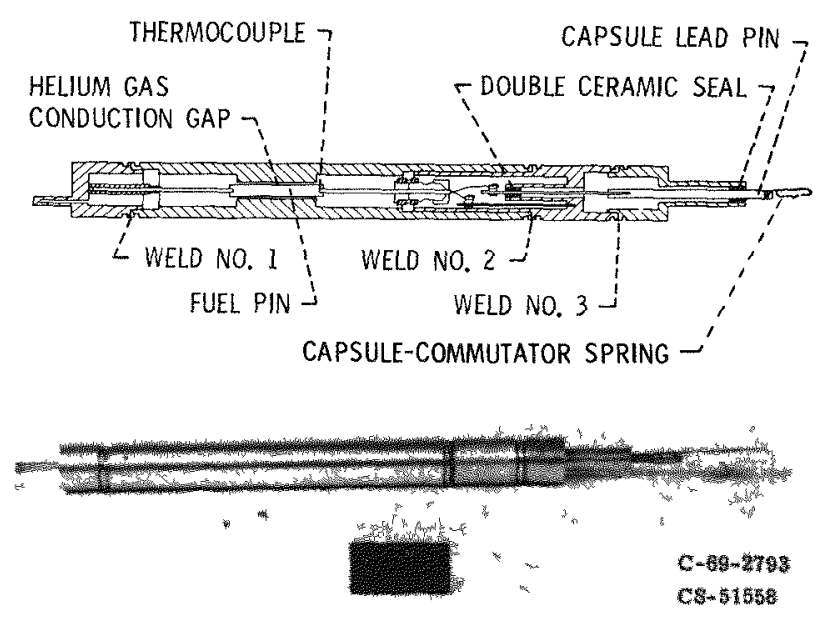

Figure 3. - Sketch and photograph of a typical capsule 


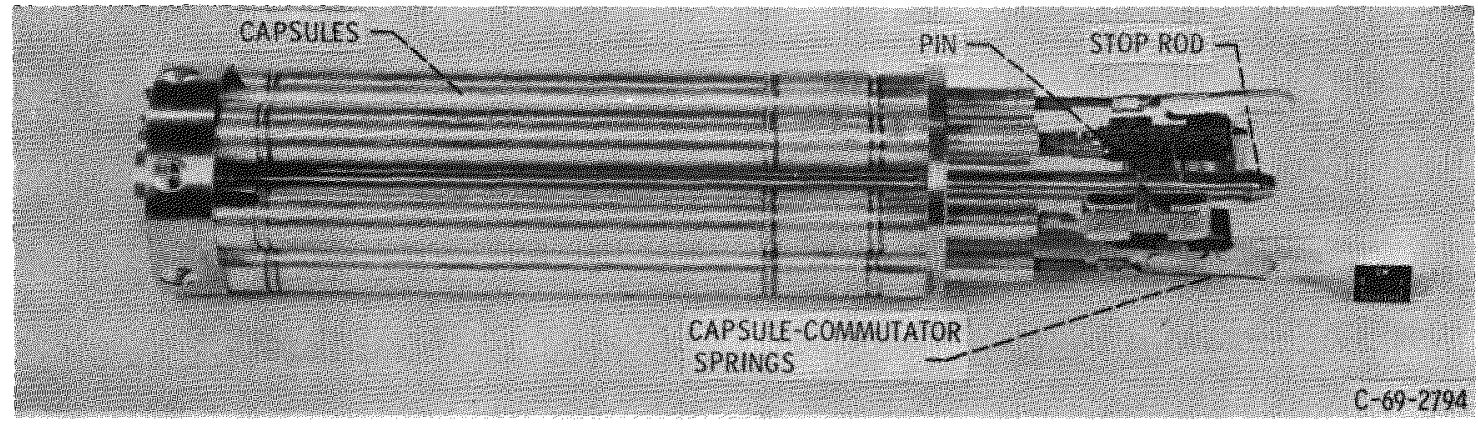

Figure 4. - Photograph of the capsule assembly.

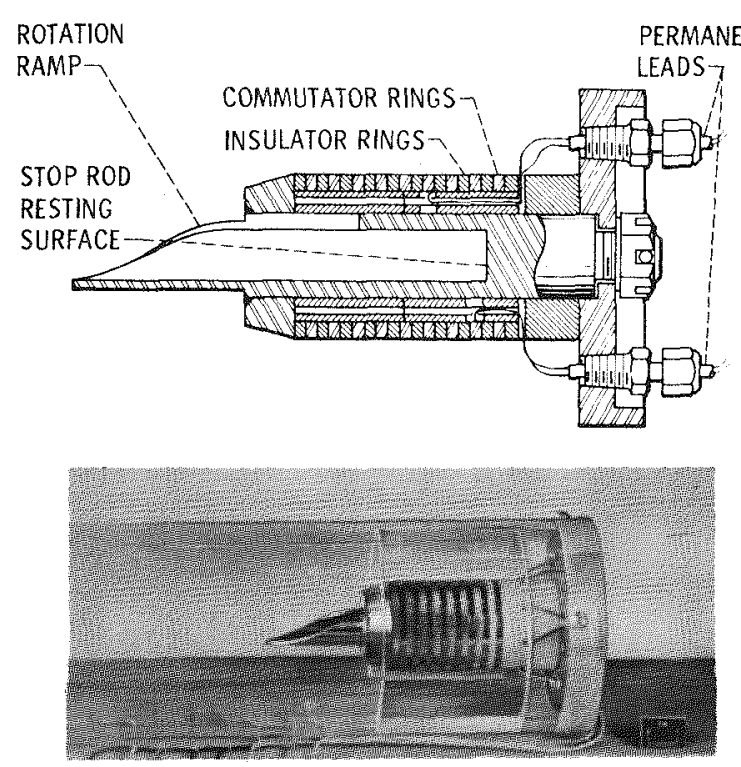

CS-51561

Figure 5. - Sketch and photograph of the commutator.

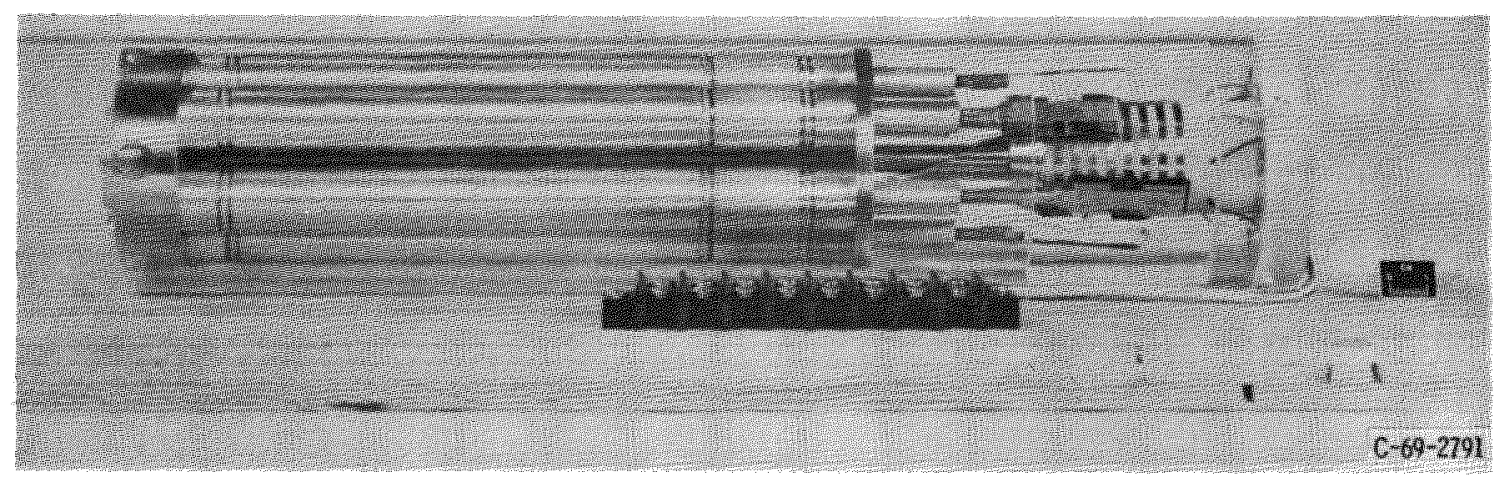

Figure 6. - Photograph of the capsule assembly inserted on the commutator. 


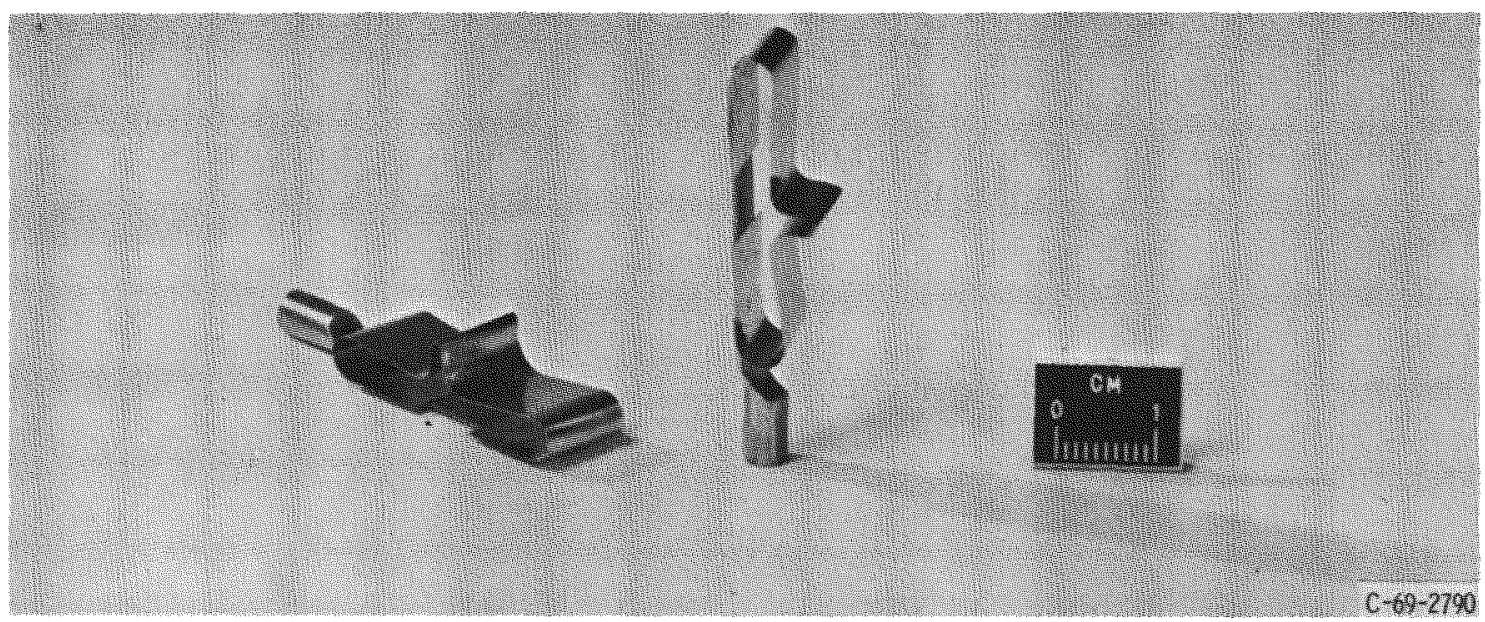

Figure 7. - Photograph of the capsule-commutator springs.
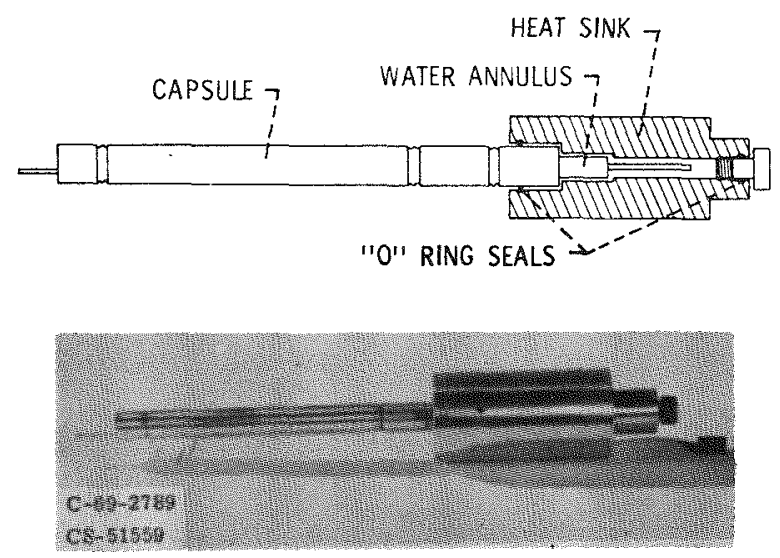

Figure 8. - Sketch and photograph of the capsule heat sink.

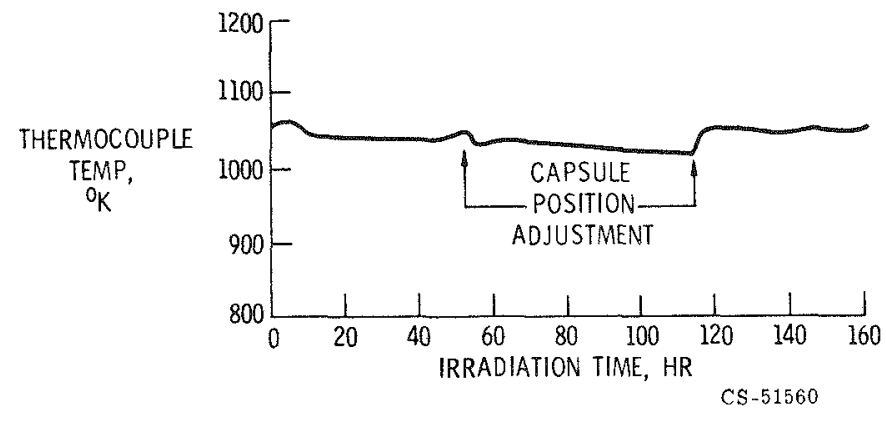

Figure 9. - Typical inpile test results for the non-lead instrumented hydraulic capsule facility. 


\section{LIST OF ATTENDEES}

Agee, Jesse R.

National Aeronautics \& Space Administration

Plum Brook Station

Sandusky, Ohio 44870

Allen, Donald

General Electric Company

Anderson, Ronald E.

U. S. Atomic Energy Commission

Washington, D. C. 20545

Bahorich, Donald J.

National Aeronautics \& Space Administration

Plum Brook Station

Sandusky, Ohio 44870

Bal1, Lauren L.

National Aeronautics \& Space Administration

Plum Brook Station

Sandusky, Ohio 44870

Barghusen, John J.

Argonne National Laboratory

Argonne, I11inois 60439

Barkley, Jr. H. B.

National Aeronautics \& Space Administration Plum Brook Station

Sandusky, Ohio 44870

Barnett, Edward

Massachusetts Institute of Technology

Cambridge, Massachusetts 39021

Barnett, Roger $M$.

Jet Propulsion Laboratory

Pasadena, California 09911
Basham, Sam J.

Battelle Memorial Institute

Columbus Laboratory

Columbus, Ohio 43201

Baston, Forest

Idaho Nuclear Corporation

Idaho Falls, Idaho 83401

Baughman, George

National Aeronautics \& Space Administration

Kennedy Space Center, Florida

Beck, Nelson

Argonne National Laboratory

Argonne, Illinois 60439

Beeston, J. M.

Idaho Nuclear Corporation

Idaho Falls, Idaho 83401

Biancheria, Amilcare

Westinghouse Electric Corporation

Advanced Reactors Division

Waltz Mills Site

Madison, Pennsylvania 15663

Boitel, Earl C.

National Aeronautics \& Space Administration Plum Brook Station

Sandusky, Ohio 44870

Bowles, Kenneth $\mathrm{J}$.

National Aeronautics \& Space Administration Lewis Research Center

CTeveland, Ohio

Bradley, John G.

Battelle-Nor thwest

Richland, Washington 99352 
Bray, John D.

Westinghouse Electric Corporation

Advanced Reactors Division

Waltz Mills Site

Madison, Pennsylvania 15663

Brickley, R. H.

National Aeronautics \& Space Administration

Plum Brook Station

Sandusky, Ohio 44870

Brown, Robert L.

National Aeronautics \& Space Administration

Lewis Research Center

Cleveland, Ohio

Buck, John

Atomics International

Sherman Daks, California 97043

Burns, George

National Bureau of Standards

Washington, D. C. 20545

Butler, Robert E.

Genera1 Electric Company

Vallecitos Nuclear Center

Pleasanton, California 94566

Carter, Robert L.

University of Missouri

Columbia, Missouri 65201

Case, David R.

Air Force Nuclear Engineering Center

Wright-Patterson Air Force Base, Ohio

Caye, Thomas

Westinghouse Electric Corporation
Chandler, B. A.

General Electric Company

Cincinnati, Ohio

Chaplin, Richard C.

National Aeronautics \& Space Administration Lewis Research Center

Cleveland, Ohio

Cipollone, Peter

National Aeronautics \& Space Administration Lewis Research Center

Cleveland, Ohio

Coats, Richard L.

Sandia Lab

Albuquerque, New Mexico 87114

Conant, C.W.

National Aeronautics \& Space Administration Plum Brook Station

Sandusky, Ohio 44870

Conlin, John

Oak Ridge National Laboratory

Oak Ridge, Tennessee 37830

Coombe, John

Westinghouse Electric Corporation

Pittsburgh, Pennsylvania

Corne 11, Dona 1d $H$.

General Electric Company

Schenectady, New York

45433

Crabtree, Robert

General Dynamics

Fort Worth, Texas 01761 
Cranda11, Don

National Aeronautics \& Space Administration

Plum Brook Station

Sandusky, Ohio 44870

Crawford, A. Clegg

Atomics Internationa 1

Sherman Oaks, California 91043

Crooks, John L.

National Aeronautics \& Space Administration

Plum Brook Station

Sandusky, Ohio 44870

Daugherty, Hugh F.

Westinghouse-Bettis

Ontario, Canada

Davis, A. B.

National Aeronautics \& Space Administration

Plum Brook Station

Sandusky, Ohio 44870

Davidson, Melvin

Aerojet General Corporation

Cleveland, Ohio

DeFayette, Robert

National Aeronautics \& Space Administration

Plum Brook Station

Sandusky, Ohio 44870

DeMastry, John A.

Battelle Memorial Institute

Columbus Laboratories

Columbus, Ohio 43201

Deobald, Theodore $L$.

Douglas United Nuclear

Richland, Washington 99352
Didelot, Robert $C$.

National Aeronautics \& Space Administration

Plum Brook Station

Sandusky, Ohio 44870

Dismuke, S. E.

Oak Ridge National Laboratory

Oak Ridge, Tennessee 37830

Divita, Edward L.

Jet Propulsion Laboratory

Pasadena, California 09911

Donoughe, Patrick L.

National Aeronautics \& Space Administration

Lewis Research Center

Cleveland, Ohio

Duncan, Robert $N$.

General Electric Company

Atomic Power Equipment Dept.

San Jose, California

Durney, John L.

Idaho Nuclear Corporation

Idaho Falls, Idaho 83401

Dyslin, Dale

Oak Ridge National Laboratory

Oak Ridge, Tennessee 37830

Eggen, Dona 1d J.

Northwestern University

Evanston, I11inois 30111

Ehde, Chester L.

General Electric Company

Evendale, Ohio

Eldred, Dona ld

General Electric Company

Pleasanton, California 94566 
Fasano, Anthony

Air Force Nuclear Engineering Center

Wright-Patterson Air Force Base, Ohio

45433

Fecych, WiTliam

National Aeronautics \& Space Administration

Plum Brook Station

Sandusky, Ohio 44870

Finnegan, P. M.

National Aeronautics \& Space Administration Lewis Research Center

Cleveland, Ohio

Fitts, Robert $B$.

Oak Ridge National Laboratory

Oak Ridge, Tennessee 37830

Floyd, John J.

Brookhaven National Laboratory

Upton L.I., New York

Folkrod, John R.

Argonne National Laboratory

Argonne, Illinois

Fromm, Earle 0.

Battelle Memorial Institute

Columbus Laboratories

Columbus, Ohio 43201

Galbo, Robert J.

National Aeronautics \& Space Administration

Plum Brook Station

Sandusky, Ohio 44870

Glawe, George E.

National Aeronautics \& Space Administration

Lewis Research Center

Cleveland, Ohio
GTuyas, R. E.

National Aeronautics \& Space Administration

Lewis Research Center

Cleveland, Ohio

Goda, Kermit

Carpenter Technology Corporation

Reading, Pennsylvania

Goldsmith, Stanley

Battelle-Northwest

Richland, Washington 99352

Grando, Carl

United Nuclear Corporation

Elms ford, New York 10523

Grant, Samue]

Babcock \& Wilcox

Lynchburg, Virginia

Green, Farno L.

General Motors Corporation

Warren, Michigan

Gwinn, David A.

Massachusetts Institute of Technology

Cambridge, Massachusetts 39021

Haley, Fred A.

National Aeronautics \& Space Administration

Lewis Research Center

Cleveland, Ohio

Hanny, Joseph A.

Idaho Nuclear Corporation

Idaho Falls, Idaho 83401

Hanson, John $E$.

Battelle-Northwest

Richland, Washington 99352 
Harbinson, E. Neil

Babcock \& Wilcox Company

Lynchburg, Virginia

Hasse, R. A.

National Aeronautics \& Space Administration

Plum Brook Station

Sandusky, Ohio 44870

Heckelman, J. D.

National Aeronautics \& Space Administration

Plum Brook Station

Sandusky, Ohio 44870

Helms, Ira L., Jr.

U.S. Atomic Energy Commission

Space Nuclear Propulsion Office

Washington, D. C. 20545

Hillig, Oscar R.

Atomics International

Sherman Oaks, California 91043

Homyak, Len

National Aeronautics \& Space Administration

Plum Brook Station

Sandusky, Ohio 44870

Howard, William

Jet Propulsion Laboratory

Pasadena, California 09911

Hurst, Wiibur

National Bureau of Standards

Washington, D. C. 20545

Hutter, Ernest

Argonne National Laboratory

Argonne, Illinois
Ikeuye, Da Te T.

General Electric Company

Jacobson, Thomas $P$.

National Aeronautics \& Space Administration Lewis Research Center

Cleveland, Ohic

Jankins, Thomas

National Aeronautics \& Space Administration

Plum Brook Station

Sandusky, Ohio 44870

Keating, John $\mathrm{J}$.

Atomic Energy Commission

Idaho Falls, Idaho 83401

Kersher, Carl

Monsanto Research Corp.

Mound Laboratory

Miamisburg, Ohio 45432

Kirchgessner, Thomas A.

National Aeronautics \& Space Administration

Lewis Research Center

Cleveland, Ohio

Kittel, J. H.

Argonne National Laboratory

Argonne, Illinois

Kolodjeski, Victor L.

National Aeronautics \& Space Administration Plum Brook Station

Sandusky, Ohio 44870

Lanzo, Chester $D$.

National Aeronautics \& Space Administration Lewis Research Center

Cleveland, Ohio 
Legler, Frank $C$.

U.S. Atomic Energy Commission

Washington, D. C. 20545

Lieberman, Richard $M$.

Westinghouse Electric Corp.

Bettis Atomic Power Laboratory

West Mifflin, Pennsylvania 15122

Lietzke, A. F.

National Aeronautics \& Space Administration Lewis Research Center

Cleveland, Ohio

Maag, William L.

National Aeronautics \& Space Administration

Lewis Research Center

Cleveland, Ohio

Malone, Jerry $H$.

National Aeronautics \& Space Administration

Lewis Research Center

Cleveland, Ohio

Mather, Roger $F$.

National Aeronautics \& Space Administration Lewis Research Center

Cleveland, Ohio

Mattson, William F.

National Aeronautics \& Space Administration Lewis Research Center

Cleveland, Ohio

McCauley, Edward W.

Lawrence Radiation Laboratory

Livermore, California 94550

McCracken, Robert

Lawrence Radiation Laboratory

Livermore, California
McDonald, Glen

National Aeronautics \& Space Administration Lewis Research Center

Cleveland, Ohio

McKenzie, Jerry L.

Air Force Nuclear Engineering Center

Wright-Patterson Air Force Base, Ohio 45433

Miller, Charles

Jet Propulsion Laboratory

Pasadena, California

Miller, Ernest $W$.

Miller, Neil E.

Battelle Memorial Institute

Columbus Laboratory

Columbus, Ohio

Miller, Richard W.

Idaho Nuclear Corporation

Idaho Falls, Idaho 83401

Minderman, E. John

National Aeronautics \& Space Administration Plum Brook Station

Sandusky, Ohio 44870

Mittendorf, R. J.

National Aeronautics \& Space Administration

Plum Brook Station

Sandusky, Ohio 44870

Mixon, William

Oak Ridge National Laboratory

Oak Ridge, Tennessee 37830 
Mondt, Jack

Jet Propulsion Laboratory

Pasadena, California

Montgomery, Michael

United Nuclear Corporation

Elmsford, New York

Moorhead, Paul E.

National Aeronautics \& Space Administration Lewis Research Center

Cleveland, Ohio

Morgan, Marvin $T$.

Oak Ridge National Laboratory

Oak Ridge, Tennessee 37830

Morley, James A.

National Aeronautics \& Space Administration Plum Brook Station

Sandusky, Ohio 44870

Moteff, Dr. J.

Genera1 Electric Company

Nuclear Systems Programs

Cincinnati, Ohio

Murata, Ronald

GeneraT Electric Company

Sunnyvale, California

Neimark, Lawrence

Argonne National Laboratory

Argonne, Illinois

Nettles, J. Cary

National Aeronautics \& Space Administration

Plum Brook Station

Sandusky, Ohio 44870
Nu11, Clyde R.

National Aeronautics \& Space Administration Plum Brook Station

Sandusky, Ohio 44870

01sen, Arnold R.

Oak Ridge Nationa 1 Laboratory

Oak Ridge, Tennessee 37830

Panasik, Paul

National Aeronautics \& Space Administration Lewis Research Center

Cleveland, Ohio

Peacock, D. W.

Douglas United Nuclear

Richland, Washington 99352

Pinkel, Benjamin

RAND Corporation

Santa Monica, California

Pitner, A. L.

Battelle-Northwest

Richland, Washington 99352

Power, John L.

National Aeronautics \& Space Administration Lewis Research Center

Cleveland, Ohio

Pugh, R.

General Electric Company

Cincinnati, Ohio

Ray, Joseph

Battelle Memorial Institute

Columbus Laboratory

Columbus, Ohio 43201 
Reuscher, Jon

Sandia Laboratories

Albuquerque, New Mexico

Rice, Edward A.

Air Force Nuclear Engineering Center

Wright-Patterson Air Force Base, Ohio

45433

Richardson, P. J.

National Aeronautics \& Space Administration

Plum Brook Station

Cleveland, Ohio

Riel, Dr. Gordon

Naval Ordnance Laboratory

United States Navy

White Oaks, Maryland 21502

Roake, William E.

Battelle-Northwest

Richland, Washington 99352

Robichaud, Gilbert G.

United Nuclear Corporation

Elmsford, New York

Robinson, Richard $A$.

Battelle Memorial Institute

Columbus Laboratories

Columbus, Ohio 43201

Robinson, Richard A.

National Aeronautics \& Space Administration

Plum Brook Station

Sandusky, Ohio 44870

Rohal, R. G.

National Aeronautics \& Space Administration Lewis Research Center

Cleveland, Ohio
Rothman, Alan

Argonne National Laboratory

Argonne, Illinois

Rubenstein, Lester

U.S. Atomic Energy Commission

Washington, D. C. 20545

Salgado, Peter

Los ATamos Scientific Laboratory

Los Alamos, New Mexico

SaTmon, Benjamin

Carpenter Technology Group

San Diego, California

Sams, Eldon W.

National Aeronautics \& Space Administration Lewis Research Center

Cleveland, Ohio

Sandefur, Nelson L.

Gulf General Atomic

San Diego, California

Saunders, Neal

National Aeronautics \& Space Administration Lewis Research Center

Cleveland, Ohio

Schuh, R. M.

National Aeronautics \& Space Administration

Plum Brook Station

Sandusky, Ohio 44870

Schulman, Dr. Fred

National Aeronautics \& Space Administration Headquarters

Washington, D. C. 20545 
Schwartz, Jerome N. D.

1321 Club Drive

Hewlett Harbor, New York

Schwarzer, Donald E.

Gulf General Atomic

San Diego, California

Scotti, Vincent

Battelle Memorial Institute

Columbus Laboratories

Columbus, Ohio 43201

Seidel, Robert C.

National Aeronautics \& Space Administration

Plum Brook Station

Sandusky, Ohio 44870

Seim, Orville s.

Argonne National Laboratory

Argonne, Illinois

Senkfor, $M$.

National Aeronautics \& Space Administration

Plum Brook Station

Sandusky, Ohio 44870

Senn, Ron

Oak Ridge National Laboratory

Oak Ridge, Tennessee 37830

Serpan, Charles Z., Jr.

Naval Research Laboratory

Washington, D. C. 20545

Sheibley, Dean W.

National Aeronautics \& Space Administration

Plum Brook Station

Sandusky, Ohio 44870
Simmons, C. R.

Westinghouse Electric Corporation

Atronuclear Core Operations

Cheswick, Pennsylvania 15024

STaby, Jack G.

National Aeronautics \& Space Administration Lewis Research Center

Cleveland, Ohio

Sliney, Harold E.

National Aeronautics \& Space Administration Lewis Research Center

Cleveland, Ohio

Smaardyk, Abe

Argonne National Laboratory

Argonne, 117 inois

Smith, John R.

National Aeronautics \& Space Administration Lewis Research Center

Cleveland, Ohio

Smith, Roger L.

National Aeronautics \& Space Administration Lewis Research Center

Cleveland, Ohio

Snyder, Gary

National Aeronautics \& Space Administration

Plum Brook Station

Sandusky, Ohio 44870

Sowa, Kenneth

Douglas United Nuclear

Richland, Washington 99352

Stein, Ralph

National Aeronautics \& Space Administration

Plum Brook Station

Sandusky, Ohio 44870 
Steinke, Lester A.

National Aeronautics \& Space Administration Plum Brook Station

Sandusky, Ohio 44870

Stewart, John P.

U.S. Atomic Energy Commission

Richland Operations Office

Richland, Washington 99352

Sti11man, Danny

Los Alamos Scientific Laboratory

Los Alamos, New Mexico

Stoker, John 0.

Jet Propulsion Laboratory

Pasadena, California

Strasser, Alfred

United Nuclear Corporation

Elmsford, New York

Struss, Roland G.

Ames Laboratory

U.S. Atomic Energy Commission

Ames, Iowa

Sudsina, Michael $W$.

National Aeronautics \& Space Administration

Plum Brook Station

Sandusky, Ohio 44870

Suhanic, James $E$.

National Aeronautics \& Space Administration

Plum Brook Station

Sandusky, Ohio 44870

Sweeney, James

General Electric Company

Pleasanton, California
Tambling, T.N.

National Aeronautics \& Space Administration Plum Brook Station

Sandusky, Ohio 44870

Thoms, Kenneth

Oak Ridge National Laboratory

Oak Ridge, Tennessee 37830

Toenshoff, Donald A.

Engelhard Ind.

East Newark, New Jersey

Tunne11, George W.

General Electric Company

Pleasanton, Cal ifornia

Wachter, William J.

$F$. Wachter Company

Pittsburgh, Pennsylvania

Waide, Chrarles H.

Brookhaven National Laboratory

Upton, New York

Walker, David E.

Argonne National Laboratory

Argonne, I1] ino is

Wallace, Jack L.

U.S. Atomic Energy Commission

Idaho Operations Office

Idaho Falls, Idaho 83401

Washburn, Troy N.

Oak Ridge National Laboratory

Dak Ridge, Tennessee 37830

Weaver, Cliff $V$.

Los Alamos Scientific Laboratory

Los Alamos, New Mexico 
Weinstein, Michael $B$.

National Aeronautics \& Space Administration Lewis Research Center

Cleveland, Ohio

Wilkinson, Larry

General Electric Company

Pleasanton, California

Williams, James $M$.

Oak Ridge National Laboratory

oak Ridge, Tennessee 37830

Winters, Glenn

National Aeronautics \& Space Administration

Plum Brook Station

Sandusky, Ohio 44870

Woodward, William

National Aeronautics \& Space

Administration - Headquarters

Washington, D. C. 20545

Yarger, Larry L.

Battelle Memorial Institute

Columbus Laboratories

Columbus, Ohio 43201

Zelzny, William F.

Idaho Nuclear Corporation

Idaho Falls, Idaho 83401

Zielenbach, William J.

Battelle Memorial Institute

Columbus Laboratories

Columbus, Ohio 43201

Zimmerman, Paul

Douglas United Nuclear

Richland, Washington 99352
Zulliger, Albert $F$.

Oak Ridge National Laboratory

Oak Ridge, Tennessee 37830

Zysk, Edward D.

Engelhard Minerals and Chemicals Corp.

Newark, New Jersey 